\title{
Floods of March-May 1965 in the Upper Mississippi River Basin
}

GEOLOGICAL SURVEY WATER-SUPPLY PAPER 1850-A

Prepared in cooperation with the States of Minnesota, $W$ isconsin, Iowa, Illinois, and Missouri and with agencies of the Federal Government

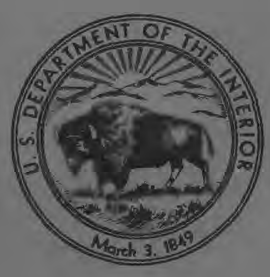




\section{Floods of March-May 1965 in the Upper Mississippi River Basin}

By D. B. ANDERSON and I. L. BURMEISTER

FLOODS OF 1965 IN THE UNITED STATES

GEOLOGICAL SURVEY WATER-SUPPLY PAPER 1850-A

Prepared in cooperation with the States of Minnesota, $W$ isconsin, Iowa, Illinois, and Missouri and with agencies of the Federal Government

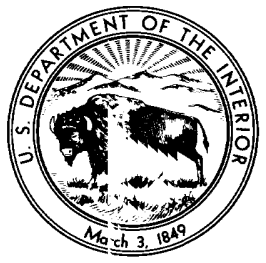


UNITED STATES DEPARTMENT OF THE INTERIC $Q$

WALTER J. HICKEL, Secretary

\section{GEOLOGIGAL SURVEY}

William T. Pecora, Director 


\section{CONTENTS}

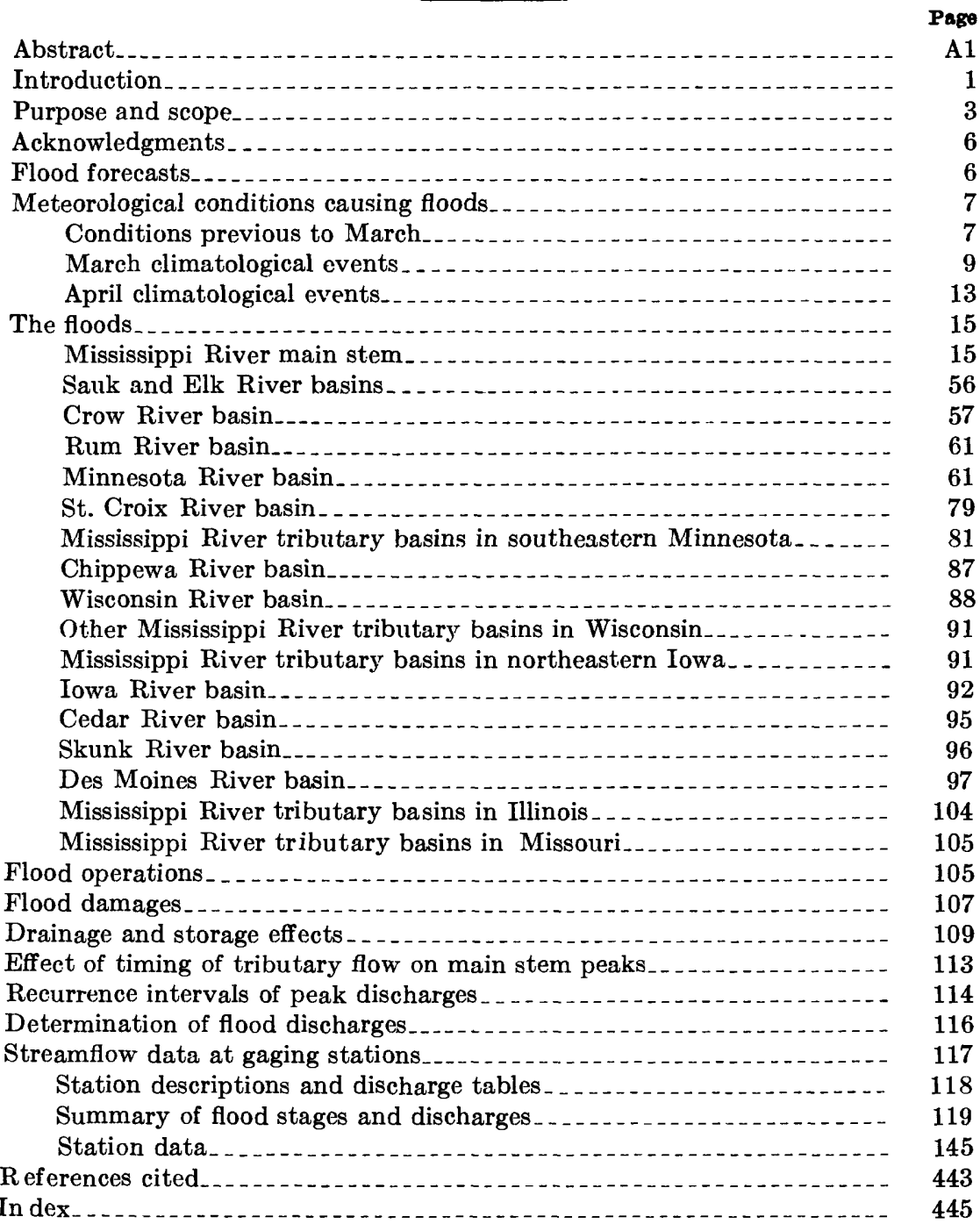




\section{ILLUSTRATIONS}

Figure 1. Map showing area covered by this report

2. Map showing location of flood determination sites........

3. Chart showing daily range in temperature at four U.S. Weather Bureau stations in Minnesota and Iowa for the period December 1, 1964, to April 15, 1965

4. Map showing snow cover on the ground as of March 181965.

5. Map showing water equivalent of snow on the ground at the end of March 1965

6. Isohyetal map showing precipitation during the period April 3-7, 1965

7. Isohyetal map showing precipitation during April 1965....

8. Photographs of flood damage caused by the Mississippi River at Elk River, Minn.

9. Photograph of flood-damaged home in Lilydale, Mirn...-

10. Map of flooded area in St. Paul, Minn . .

11. Photograph of the St. Paul, Minn., municipal airport covered by flood waters

12. Map of flooded area in Wabasha, Minn

13. Map of flooded area in Winona, Minn ...................

14. Photograph of flood damage to railroad trackage near Winona, Minn

15. Photograph of a flooded area in LaCrosse, Wis

16. Map of flooded area in LaCrosse, Wis.

17. Photograph of flooded interstate bridge approach $\varepsilon$ nd an area in Praire du Chien, Wis.

18. Photograph of flooded industrial area in Dubuque, Jowa

19. Map of flooded area in Dubuque, Iowa.......... 35

20. Map of flooded area in Clinton, Iowa...... 36

21. Photograph of flooded area in Fulton, Ill ........ 38

22. Map of flooded area in Davenport and Bettendorf, Iowa, and Rock Island, Moline and East Moline, Ill ........

23. Photograph of flooded area in Davenport, Iowa ..........

24. Map oi flooded area in Muscatine, Iowa..............

25. Photograpn of flooded area in Hannibal, Mo

26. Flood-erest profiles of Mississippi River....................

27. Comparative discharge hydrographs at selected gaging stations on the Mississippi River, April-May 1965.....-

28. Discharge hydrographs at selected gaging stations on Mississippi River tributaries upstream from St. Paul, Minn., April 1965.

29. Flood-crest profiles of the North and South Forks of Crow

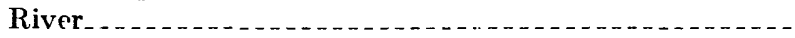

30. Map of flooded area in Mankato, Minn . . .

31. Photograph of flooded area in Mankato, Minn ........

32. Map of flooded area in Henderson, Minn . ..............

33. Photograph of flooded area in Henderson, Minn

34. Map of flooded area in Carver, Minn . .

35. Photograph of flooded area in Carver, Minn ...........

36. Map of flooded area in Chaska, Minn 
Figuee 37. Photograph of flooded area in Chaska, Minn

38. Flood-crest profiles of Minnesota River.

39. Discharge hydrograph at selected gaging stations in tho

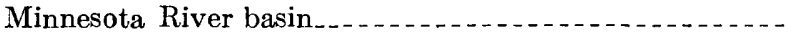

40. Discharge hydrographs at selected gaging stations on the Mississippi River tributaries in Wisconsin and south-

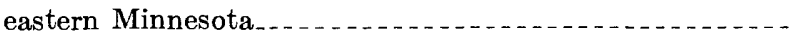

41. Map of flooded area in Hastings, Minn

42. Photograph of flooded area in Hastings, Minn

43. Flood-crest profiles of South Fork Zumbro River and Zumbro

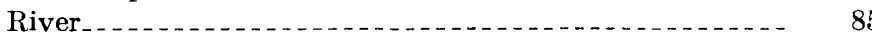

44. Photograph of flooded area in Schofield, Wis._. 90

45. Discharge hydrographs at selected gaging stations in Iowa

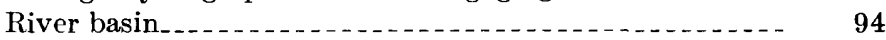

46. Photograph of flooded area in Jackson, Minn_._. 98

47. Flood-crest profile of West Fork Des Moines River._._. 100

48. Discharge hydrographs at selected gaging stations in Der

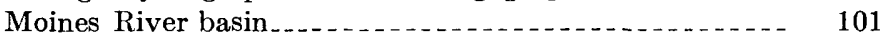

49. Effect of timing of Minnesota River peak on maximum discharge of Mississippi River at St. Paul, Minn

50. Flood frequency curves for selected Mississippi River gaging

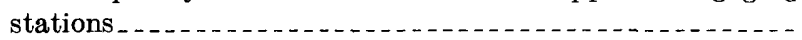

\section{TABLES}

TABLES 1-5. Flood-crest elevations:

1. Mississippi River

2. North and South Forks Crow River._........ 60

3. Minnesota River....................... 71

4. South Fork Zumbro River and Zumbro River ...... 84

5. West Fork Des Moines River, April 6-13, 1965_... 101

6. Flood damages by States........................ 107

7. Communities where flood damages exceeded \$1 million ..... 109

8. Effectiveness of reservoirs in upper Mississippi River basir during flood of $1965 \ldots$

9. Summary of flood stages and discharges........... 120 


\title{
FLOODS OF 1965 IN THE UNITED STATES
}

\section{FLOODS OF MARCH-MAY 1965 IN THE UPPEP MISSISSIPPI RIVER BASIN}

\author{
By D. B. ANDerson and I. L. Burmeister \\ ABSTRACT
}

The floods of March-May 1965 in the upper Mississippi River basin ocerrred as two different events, one during the latter part of February and early March and the other starting early in April and extending into May. Factors ccntributing to the floods were rapid melting of the winter accumulation of snow, heavy rains on the snow pack, and deeply frozen ground throughout much of the basin, which made the soil almost impervious and thereby greatly incres sed the amount of runoff.

Peak stages and discharges during the floods exceeded previous known maxima at many points. The maximum discharge at St. Paul, Minn., for example, was 137 percent of the previous known maximum, and the stage was 4 feet higher than the previous maximum during a period of known floods extending back to 1851 . Flood magnitudes exceeding those corresponding to a 50-year recurrence interval occurred at 37 sites where discharge records have been collected.

Flood damage was estimated to be about $\$ 160$ million in a five-State area comprising Minnesota, Wisconsin, Iowa, Illinois, and Missouri. Fifteen lives were lost and over 700 persons were injured or became ill as a result of the floods. More than 11,000 homes were flooded, and many thousand acres of rich agricultural land was inundated.

This report summarizes peak stages and discharges for this flood at 333 sites in the upper Mississippi River basin and presents the previous maxima at these sites where such data are available. The recurrence interval (the average period of time in which the 1965 flood is apt to be equaled or exceede once) is tabulated for many sites. The report discusses flood damages and tr.bulates damage figures by States and by type of damage incurred. It also d'scusses briefly the effect of drainage and storage on flood peaks and describes the operations of the U.S. Geological Survey and other Federal agencies during the flood emergency.

\section{INTRODUCTION}

The floods of March-May 1965 were the most devastating in the history of the upper Mississippi River basin. Five States-Minnesota, Wisconsin, Iowa, Illinois, and Missouri-were affected (fig. 1). The 


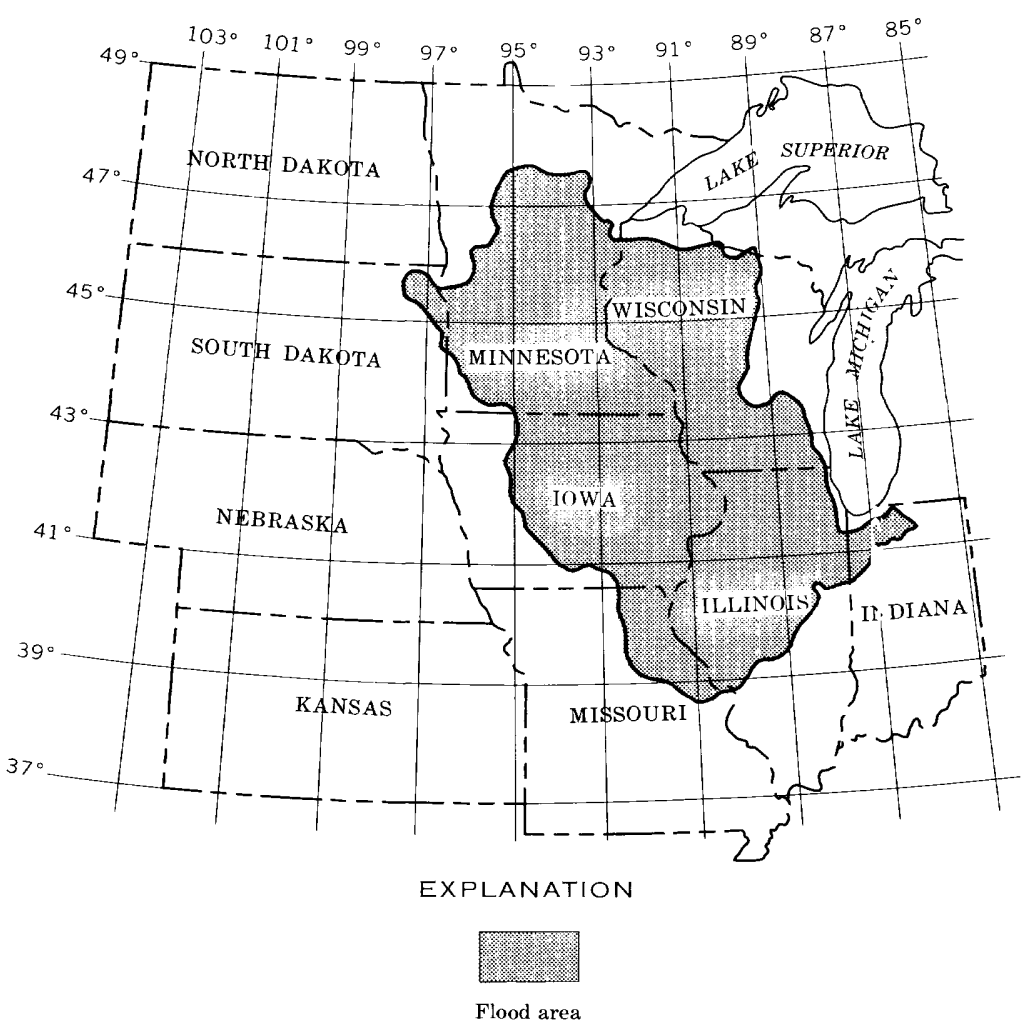

Figure 1.-Area covered by this report.

Mississippi River crested at stages higher than any ever recorded throughout a 680-mile reach between Fort Ripley, Minn., and lower lock and dam 22 near Saverton, Mo. From the mouth of the Minnesota River at St. Paul, Minn., to Muscatine, Iowa, the crest was 3-4 feet higher than any previously recorded. Recordbreaking floods occurred on the Minnesota River and its tributaries from Mankato to the mouth and on many other Mississippi River tributaries in Minnesota and Wisconsin.

The great volume of runoff was a significant feature of this flood. The Mississippi River remained above flood stage for almost a month. From Royalton, Minn., to the mouth of the Missouri River and in the Minnesota River, from Mankato to the mouth, the flood runoff was more than 25 percent greater than that of any previously recorded flood.

Throughout most of the upper Mississippi River basin, flooding occurred during two separate periods almost a month apart. During the first period warm temperatures during the latter part of February 
and early March melted the winters accumulation of snow in southeastern Minnesota, northeastern Iowa, southwestern Wisconsin. The runoff from snowmelt and rainfall, which in some areas exceaded 2 inches during the first 3 days in March, caused severe flooding in the Zumbro and Root River basins in Minnesota and in the Cedar River basin in Minnesota and Iowa. During the second period, which started early in April and extended into May, floods occurred in the entire upper Mississippi River basin. These floods were caused by the rapid melting of the winter's accumulation of snow in northern Mir nesota and Wisconsin and the March accumulation of snow in northern Iowa, southern Minnesota and Wisconsin. The water equivalent of snow in the upper part of the basin was as much as 11 inches. Warm temperatures moved northward into Minnesota during the last days of March and nighttime temperatures remained above freezing. As much as 3 inches of rain fell during the period April 3-7; it accelerated the snowmelt and increased the runoff. Frost penetrated the ground deeply, as a result of the severe winter, and consequently much of the rain and snowmelt ran off. Those streams which flooded early in March flooded again, but this time more extensively. Many of the streams which were covered by snow and ice in March were raging torrents in full flood in April. Fifteen lives were lost and more than 700 person were injured as a result of the floods or associated events. Property damage was estimated at $\$ 160$ million. Damage would have more than doubled had it not been for the flood warning and crest predictions issued by governmental agencies and the enthusiastic response of thousands of volunteers who built emergency flood-protection works.

\section{PURPOSE AND SCOPE}

The purpose of this report is to provide a historical and statistical record of the extreme 1965 floods in the upper Mississippi River basin. Data presented may be used for planning and designing structures affected by floodwaters and for studying hydrologic events. Flood data are provided for 333 sites (fig. 2). Discharges at 192 sites are tabulated on a daily basis or for increments of time within each day during the flood period so that the discharge hydrograph can be accurately reconstructed. The antecedent and immediate conditions, flood descriptions, and damages are discussed by basins. If available, pictures of inundated areas in cities and towns are shown. Discharge hydrographs at selected gaging stations are presented. The recurrence interval of the peak discharge or the ratio of the peak discharge to the theoretical flood having a 50-year recurrence interval ${ }^{1}$ is shown for many of the gaging

\footnotetext{
1 For simplicity of expression in this report, a flood with a given recurrence inte-val, say 50 years, is expressed as a 50 -year flood.
} 


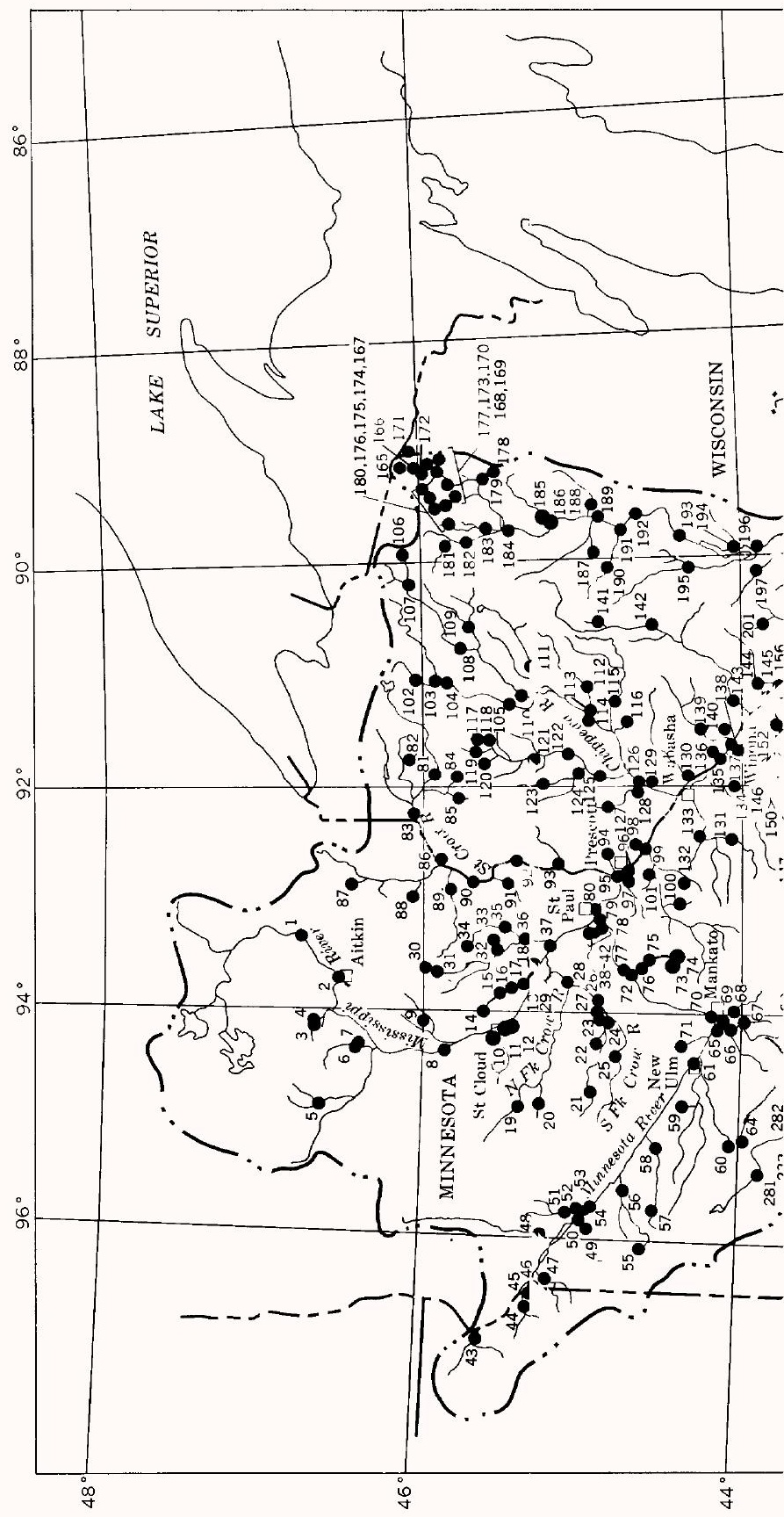


stations. Flood profiles for both the 1952 and 1965 floods in the Mississippi River are shown from Royalton, Minn., to St. Louis, Mo., and flood profiles of some Mississippi River tributaries are shown for the 1965 flood.

\section{ACKNOWLEDGMENTS}

Discharge records and other flood data appearing in this report were collected as part of cooperative programs between tho U.S. Geological Survey and the States of Minnesota, Wisconsin, Iowa, Illinois, and Missouri, the St. Paul, Rock Island, and St. Louis Districts of the U.S. Army Corps of Engineers; and the U.S. Bureau of Sport Fisheries and Wildlife. Other Federal and State agencies, municipalities and corporations gave assistance, financial or otherwise, in the collection of flood data. Credit is given for this assistance ir the appropriate station description.

Isohyetal maps were prepared from data furnished ky the U.S. Weather Bureau, and damage figures were furnished by the U.S. Army Corps of Engineers. Aerial photographs taken near the flood crests in the Minnesota and Mississippi River basins were obtained from Mark Hurd Aerial Surveys Inc. These photographs vere helpful in defining inundated areas in some urban communities.

The basic data for this report were computed and compiled under the direction of the following district engineers or acting district engineers of the Surface Water Branch, Water Resources Division: D. B. Anderson, Minnesota; K. B. Young, Wisconsin; H. H. Snhwob succeeded by S. W. Wiitala, Iowa; W. D. Mitchell, Illinois; and A. Homyk, Missouri.

During the flood emergency, engineers and technicians of the U.S. Geological Survey were detailed to the Minnesota district from Nebraska, South Dakota, Ohio, California, and Michigan. Their assistance is gratefully acknowledged.

\section{FLOOD FORECASTS}

On March 19, the ESSA-Weather Bureau Office at Minneapolis-St. Paul, Minn., forecast spring floods for the Mississippi River and its tributaries in Minnesota and in parts of Wisconsin and Iowa. At this time, although it was recognized that there was a flood potential, the condition was not considered serious. For example, the peak stage of of the Mississippi River at St. Paul, was predicted to be 16.5 feet, 2.5 feet above flood stage and 9.5 feet lower than the actual peak which occurred on April 16. On March 30, the Weather Bureau stated that "if snowmelt takes 3 days or more, Minnesota streams will not crest as high as 1952 even though the water content of snow on the ground is comparable." On April 7, owing to warm temperatures and exces- 
sive rainfall, the Minnesota River at Mankato, Minn., was predicted to crest at 26.5 feet on April 9, and the Mississippi River at St. Paul, Minn., was predicted to crest at 21.0 feet on April 14 or 15. Facause on-the-spot streamflow measurements indicated runoff greater than anticipated, on April 8, these predictions were revised to 3C.0 feet at Mankato to occur April 10 and 27.0 feet at St. Paul to occur April 16. Actual peaks were 29.09 feet at Mankato and 26.01 feet at $\mathrm{S}^{+}$. Paul on the dates predicted. On April 9, the Weather Bureau predicted that crests on the Mississippi River below St. Paul would exceed tra, 1952 flood peaks at Winona, Minn., and La Crosse, Wis. A later prediction was issued on the morning of April 16 that the Mississippi $R$ ver at St. Paul would crest at 26.9 feet on April 20. Actually, the crest at St. Paul occurred later in the day on April 16 and the additional C.9 foot stage did not materialize.

Throughout the period in which the preceding predictions were being made, the Weather Bureau stressed that rapid thawing and rainfall during the snowmelt would intensify the flood threat. Temperatures were above freezing throughout most of the flood area during the first part of April when the Minnesota River and its tributaries reached their crests. During the first 14 days of April, precipitation ranged roughly from 2 to 4 inches over the Minnesota River basin and over much of the basin of the Mississippi River and its trib itaries downstream from Minneapolis. Normal precipitation during April for this area is about 2 inches.

\section{METEOROLOGICAI CONDITIONS CAUSING FLOODS}

\section{CONDITIONS PREVIOUS TO MARCH}

Precipitation in the upper Mississippi River basin during the fall of 1964 was considerably higher than normal. For example, in Soptember 1964, the U.S. Weather Bureau station at the Minneapolis-S+. Paul airport recorded precipitation on 16 of the 30 days. The departure above normal at this station was 2.78 inches for the month. T'a station at Mason City, Iowa, recorded 7.96 inches of precipitation in September, which was 4.76 inches above normal. Generally, above normal precipitation was recorded throughout the basin during September. Precipitation was below normal during October and N'ovember, but because of the heavy rains in September, the ground was well saturated when winter set in.

Temperatures through the first half of November were unseasonably mild; then they plunged and severe freezing ensued. Temperatures ranged widely during the month. Britt, Iowa, for example, observed a high of $78^{\circ}$ and a low of $-10^{\circ}$. Record low temperatures for November were observed at many Weather Bureau stations. Temperatures re- 

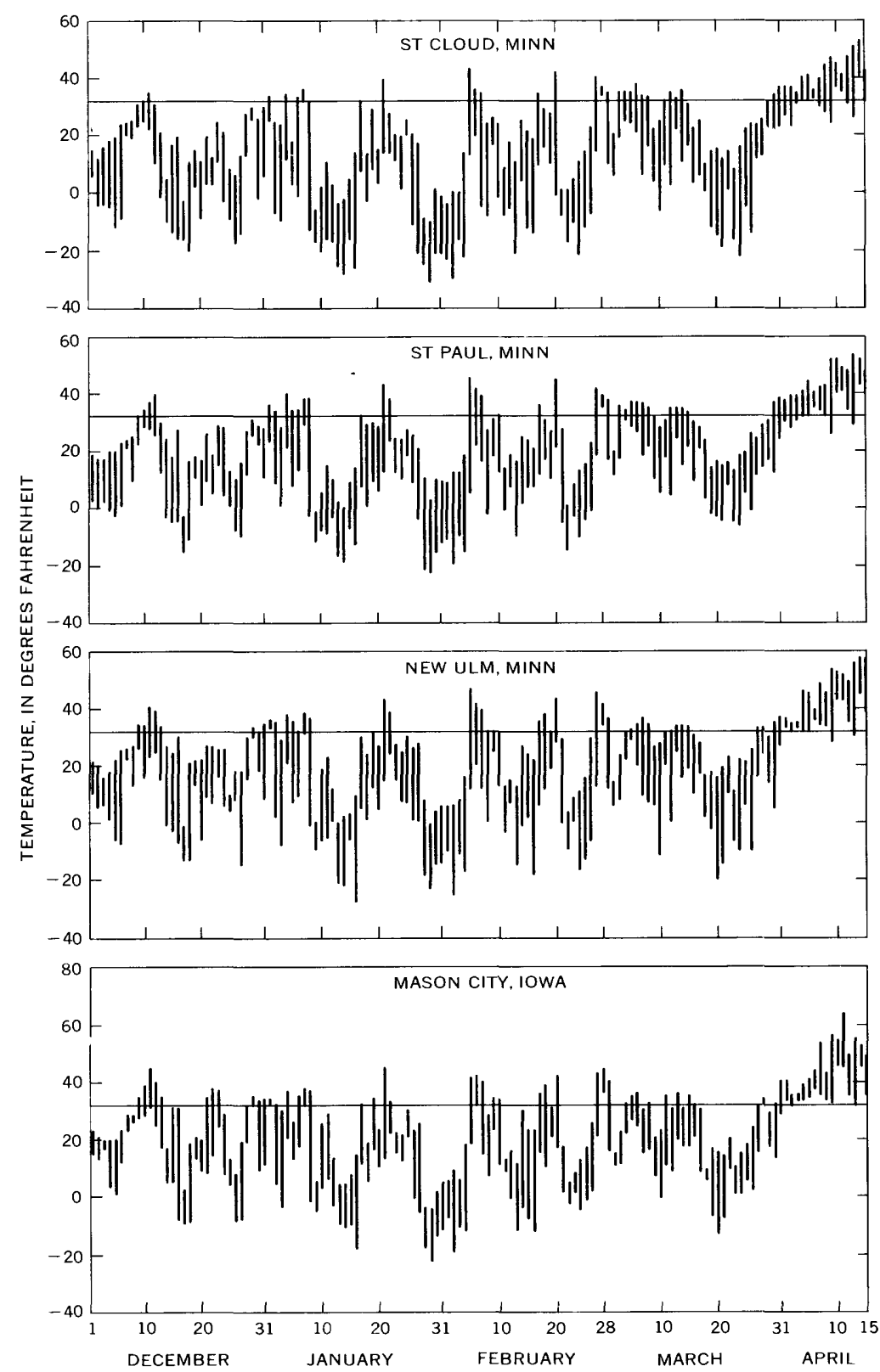

Figure 3.-Daily range in temperature at four U.S. Weather Bureau stations in Minnesota and Iowa for the period December 1, 1946, to April 15, 1965. 
mained exceptionally low for much of the remainder of the winter (fig. 3). Frost penetration was unusually deep throughout most of the upper Mississippi River basin.

During December 1964 and January 1965, there was a normal amount of snowfall. An exceptional amount of sleet fell and glaze formed in Iowa during these 2 months. A January 24-hour rainfall record was broken when 5.11 inches of rain fell at Keokuk, Iowa, on New Year's day. The monthly precipitation at this station we $\$ 8.58$ inches; it was the wettest January in 95 years. February precipitation in the southern half of Minnesota and Wisconsin exceeded the rormal by an inch or more. The rain that fell early in February in this area compacted the winter snowpack and materially increased its water equivalent. Most of the rain was absorbed in the snowpack or was retained on the ground as a heavy glaze. The February precipitation in Illinois was generally below normal, but snow drifted heavily over most of the State on the 23d and 24th. A considerable amount of sleet fell and glaze formed in Iowa during February. Rain fell in the Southern counties and snow fell heavily in the northern and western counties, much of it over the glaze. A storm on the 11th, the worst in years, deposited 10 inches of snow over glaze in the north-central part of the State. Frost penetration was deeper than normal and extended to 5 feet in this area. In Iowa, the winter of 1964-65 was the fourth coldest since 1935-36. Temperatures rose however, at the end of February and started melting the snow as far north as the Minneapolis-St. Paul area in Minnesota, but did not begin to thaw the ground in the upper reaches of the Minnesota River basin.

\section{MARCH CLIMATOLOGICAL EVENTS}

The relatively warm temperatures at the end of February and during the first part of March created heavy snowmelt runoff in the Mississippi River basin in southeastern Minnesota, northeastern Iowa, and southwestern Wisconsin. Rainfall exceeded 2 inches at several Weather Bureau stations during the first 3 days of March. This combination of snowmelt and rainfall caused some of the highest floods of record in certain of the Mississippi River tributaries in southern Minnesota. The Cedar River at Austin, Minn., and the Root River near Houston, Minn., had the highest stages of record during this flood.

Heavy snowfall continued throughout March. A blizzard on I Tarch 17-18 spread over northern Iowa, Wisconsin, Minnesota, and enstern South Dakota depositing up to 18 inches of snow. The snow on the ground after this storm totaled up to 45 inches in the upper Rum, Snake, and Kettle River basins in Minnesota (fig. 4). The snow cover diminished slowly after this date in southern Minnesota and Wis?onsin 


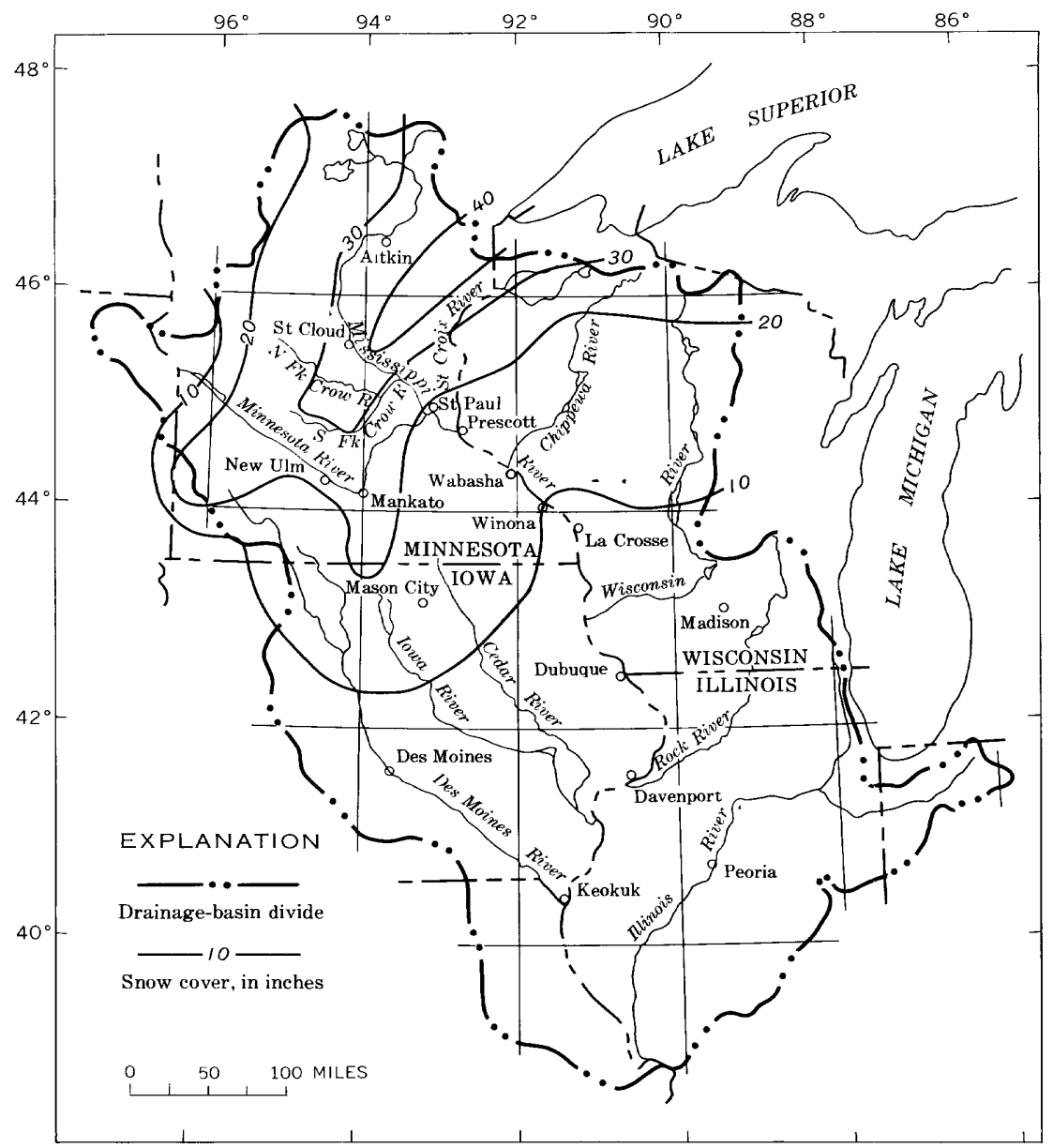

Figure 4.-Snow cover on the ground as of March 18, 1965.

and northern Iowa, but additional storms late in March maintained or increased the accumulation in northern Minnesota and Wisconsin. The water equivalent of the snow on the ground and the rate of thawing are important factors in forecasting runoff from snowmelt. The later that warm temperatures arrive in the spring, the greater the probability of rapid thawing and heavy runoff. On the basis of snow surveys conducted by the Weather Bureau and Corps of Engineers during the last 2 weeks in March, the water equivalent ranged from 2 inches in central Iowa to 11 inches in the vicinity of St. Cloud, Minn. (fig. 5). 


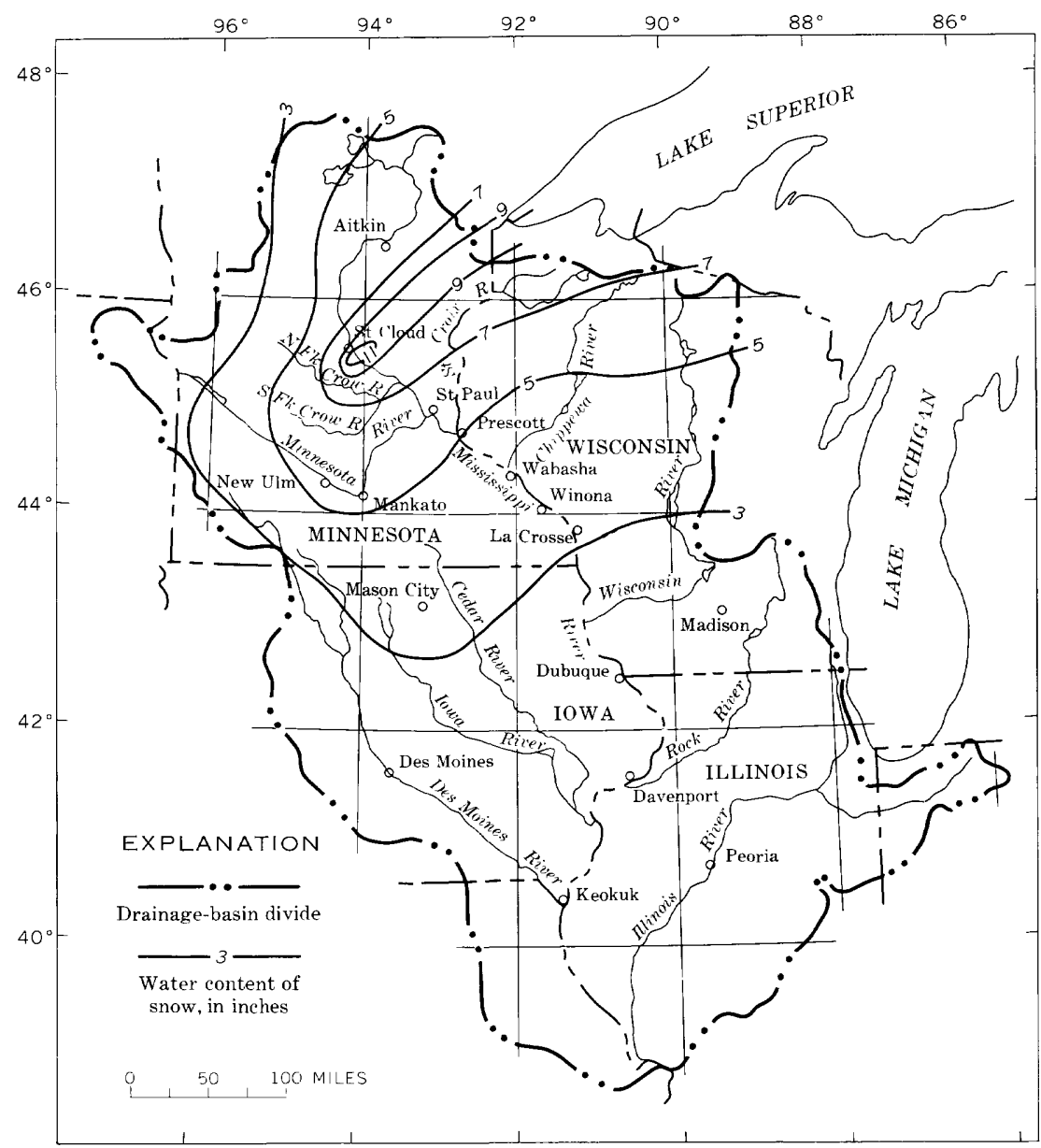

Figure 5.-Water equivalent of snow on the ground at the end of Marcl 1965.

Records at the Weather Bureau station in St. Cloud showed 35 inches of snow on the ground on March 29. A record 51.7 inches of snow fell in this area during the month. Early in March, the Army National Guard was called out to remove snow from the streets of St. Cloud to expedite the flow of vehicular traffic. Where the snow had not been hauled away, traffic moved through deep snow chasms higher than an automobile. As a safety measure, automobile drivers tied brightly 
colored streamers to their auto aerials to warn other drivers of their approach at intersections.

In many places in northern Wisconsin, the depth of snow on the ground on March 31 was the maximum recorded and was 2-4 inches above normal in water equivalent.

A study of Weather Bureau climatological data collected at Minneapolis, Minn., reveals some of the conditions prevalent in the upper Mississippi River basin during March. Precipitation at this station for the month was 4.75 inches or 1.38 inches more than tho previous March high in 1949. Snowfall amounted to 37.1 inches, which was only exceeded by 40 inches in March 1952. Snowfall for the season at the end of March was 71.7 inches compared to the seasonal record of 88.9 inches in 1950-51. Precipitation, most of it as rain, amounted to 1.62 inches on March 1. This was a new 24-hour record for March. Average temperature was $19.4^{\circ}$, which was $8^{\circ}$ below normal, the sec nnd coldest average temperature on record, and the coldest since March 1899. Record-low daily minimum temperatures were equaled or broken on 5 days during March.

The extremely cold temperatures during most of March caused deep frost penetration. In the extreme upper part of the basin where heavy snow cover existed, the average penetration was 1 foot. Farther south, where little or no snow cover existed, frost penetrated to 5 feet in some areas. There was heavy precipitation during the first part of March, followed by below freezing temperatures which reached the subzero range during the period March 19-26. In some $\varepsilon$ reas, there was a layer of ice on the ground from the storm on March 1 and previous storms. The frozen ground covered with glaze was a very important factor in intensifying the spring flood of 1965 .

In Iowa, two snowstorms with heavy snowfalls occurred on March 1-2 and on March 17-18. In the southern and central countios of Iowa, considerable sleet fell and ice formed as a result of the first storm, and in the northern counties, from 6 to 10 inches of snow fell. The second storm was concentrated in the northern part of Iowa and resulted in additional snow cover ranging from 4 to 12 inches. Snowfall in central and northern Iowa during the month of March was the fourth highest of record, and the average temperature at Des Moines for March was the second coldest of record. Below-normal temperatures continued until April 1. That part of Illinois which lies in the Missiscippi River drainage basin had weather conditions similar to those in Iowa. As a result of the rainfall, snowfall and freezing temperatures, many of the small streams were locked with snow and ice in southeastern Minne- 
sota and northeastern Iowa. This obstruction of the smaller c'rainage channels had an effect on the timing of runoff and may have increased the flood intensity on some streams.

\section{APRIL CLIMATOLOGICAL EVENTS}

By the second week of April, a warming trend had advanced northward in the upper Mississippi River basin into Minnesota and Wisconsin. Nighttime temperatures remained above freezing and daily maximums exceed $50^{\circ}$ throughout most of the basin. Above-normal precipitation accompanied the warmer weather. Snow fell in the upper part of the basin in Wisconsin on April 1. Up to 31/2 inches of rain fell over most of the basin during the period April 3-7 (fig. 6). Runoff

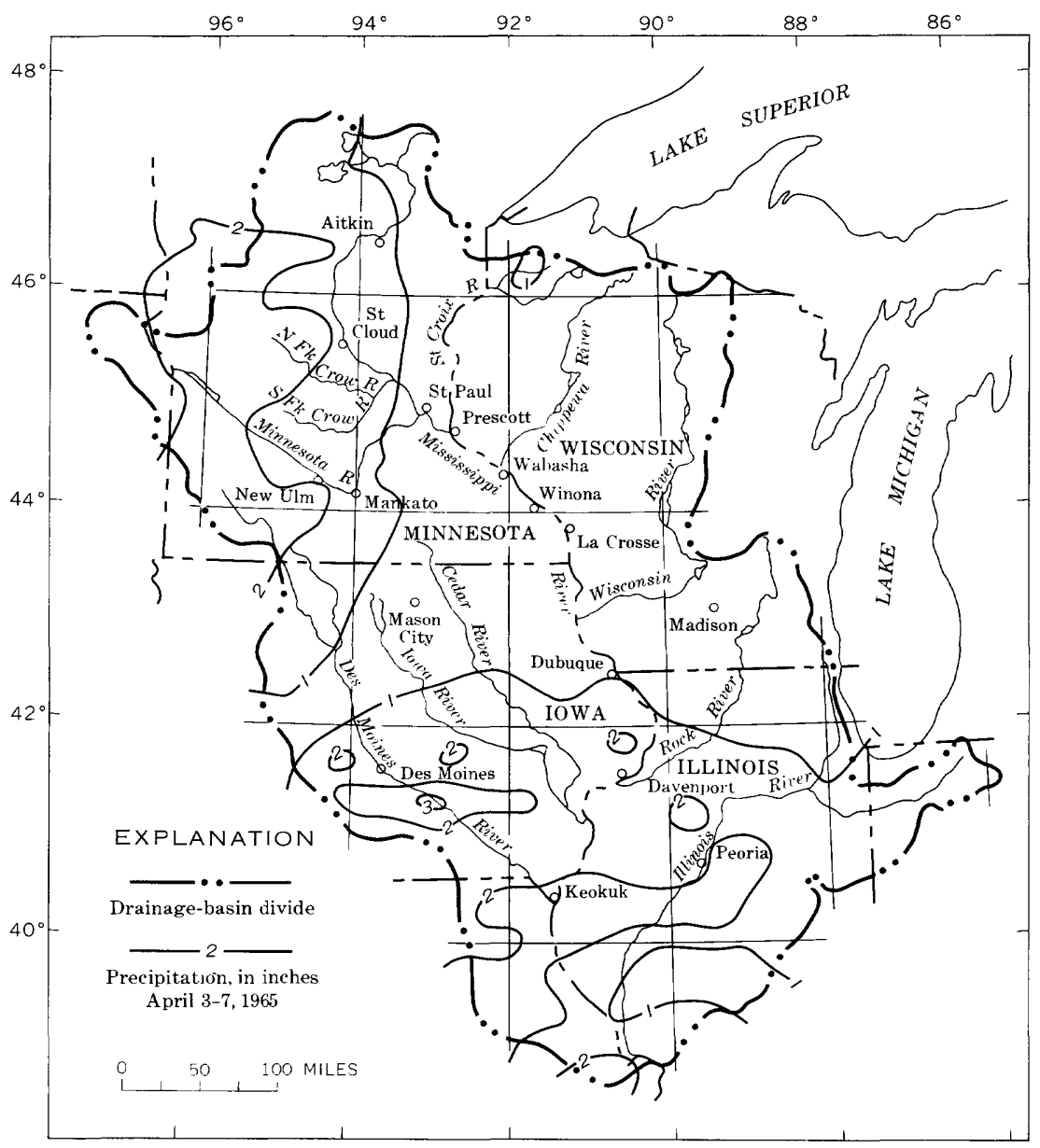

Figure 6.-Precipitation during the period April 3-7, 1965. 
from these widespread rains was intensified by snowmelt runoff. Snow melted steadily and was gone in Iowa and Illinois by April 6 and in the area south of St. Paul, Minn., by A pril 11. The snow melted slower in the extreme north. A 17 -inch snow cover remained at Eagle River, Wis., on April 14, and a 10 -inch cover remainec at Grand Rapids, Minn., on April 11. All of the snow had melted by April 29.

The combination of rainfall and snowmelt during the first few days in April set the stage for the severe floods which were to follow. Large amounts of ponded water were visible in the Minnesota River basin downstream from New Ulm and also in the basins of the small tributaries of the Mississippi River downstream from St. Paul. Personnel of the Corps of Engineers estimated from aerial observation that 40 50 percent of the Crow River basin in Minnesota was covered by water. This ponded condition, which was accentuated by the flat topography of the basin, resulted from rainfall and snowmelt which corld not run off freely through normal channels because they were obstructed by snow and ice. The ponding affected the timing of runoff from many of the smaller basins. There were extremely high rates of runoff because of the abnormally high precipitation, rapid snowmolt, and the impervious condition of the soil which was still deeply frozen and covered with ice throughout much of the basin.

Precipitation for April at North Mankato and New Ulm, Minn., was nearly 5 inches, which was $21 / 2$ inches above normal. In Iowa, the departure above normal was 1 inch in the north-central part of the State and 5 inches in the southeastern part. Exceptionally sovere thunderstorms, accompanied by hail and tornadoes, dumped up to 6 inches of rain over an area between Marshalltown and Muscatina, Iowa, on the $23 \mathrm{~d}$. This storm caused additional flooding in the area and hampered the Mississippi River flood fight on the already water-soaked levees. In Missouri and Illinois, showers and thunderstorms, during the period April 3-7, produced some rises on streams tributary to the Mississippi River. In general, these streams did not exceed bank-full stage. The widespread rains that fell in Illinois on April 15, 24, and 25 caused the Illinois River to rise above flood stage. Total precipitation during the month in the upper Mississippi River basin is shown in figure 7 . 


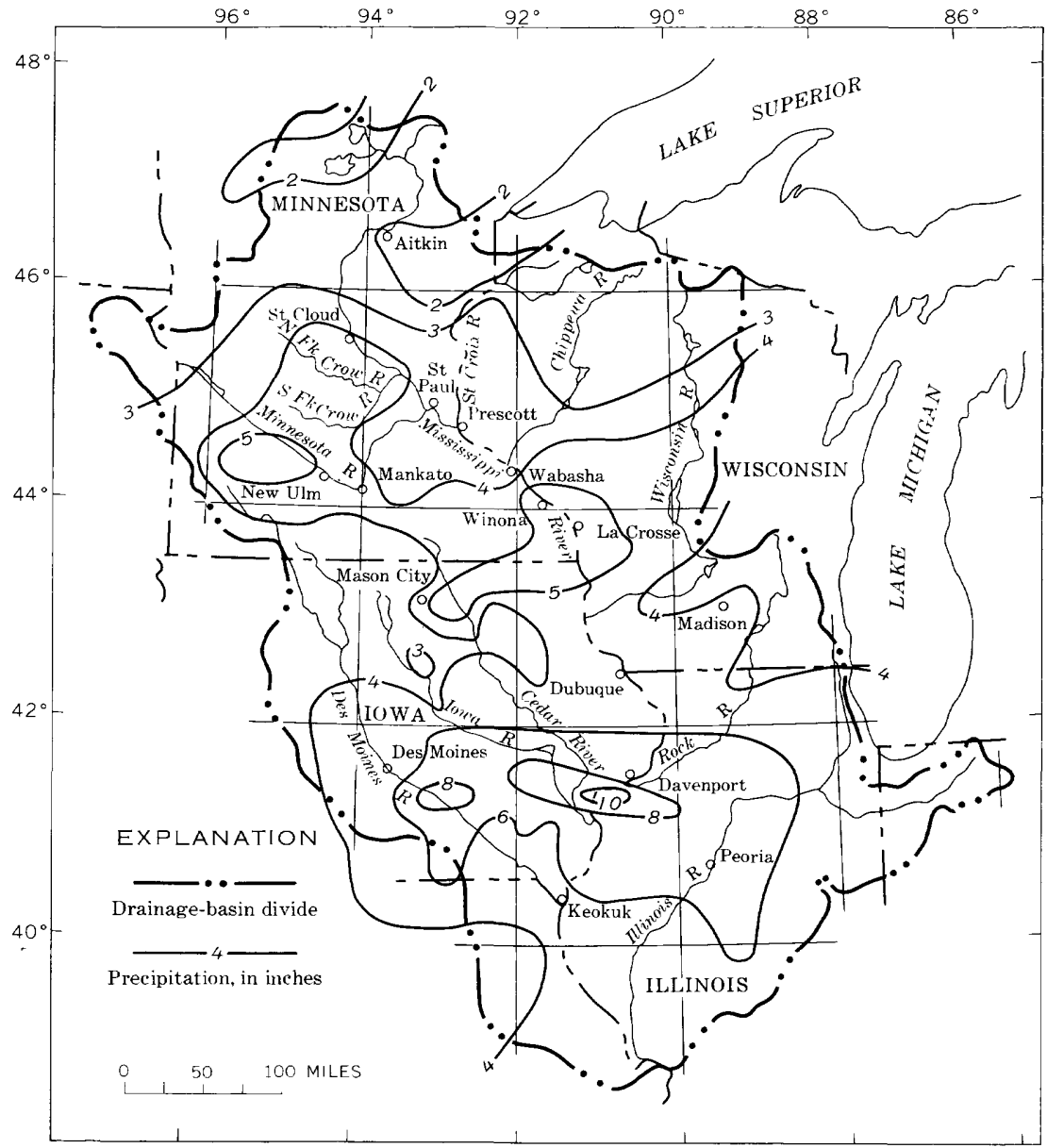

FTgure 7.-Precipitation during April 1965.

\section{THE FLOODS}

\section{MISSISSIPPI RIVER MAIN STEM}

There was no severe flooding in the upper 350 miles of the Mississippi River basin. The farthest upstream discharge record in this re, port was collected at the gaging station near Libby, Minn., about 260 miles downstream from the source of the Mississippi River. In 25 years of record at this site, three previous floods exceeded the 1965 peak. 
There are six headwater reservoirs on the main stem of the I Iississippi River or its tributaries. These reservoirs, all in Minnesota, are Winnibigoshish Lake near Deer River, Leech Lake at Federal Dam, Pokegama Lake near Grand Rapids, Sandy Lake at Libby, Pine River Reservoir at Cross Lake, and Gull Lake near Brainerd. Data from Pine River Reservoir and Gull Lake are included in this report, and they show only a moderate amount of storage from the spring runoff. A diversion channel at Aitkin, 310 miles downstream from the source, handled the spring runoff well and reduced flood damages considerably in Aitkin and the surrounding area. The recurrence intel val of the peak discharge at Aitkin was 13 years.

In the 100-mile reach between Aitkin and Royalton, flood runoff became severe. At Fort Ripley, Minn., about 25 miles upstream from Royalton, the maximum stage was higher than any preriously recorded. This situation prevailed for about 680 miles downstream to lower lock and dam 22 near Saverton, Mo.

Flood damage resulted from Mississippi River overflow in St. Cloud, Minn. Ice started breaking up above the mouth of the Sauk River and moved downstream to two bridges in St. Cloud where it jammed and caused a sudden rise of $3-4$ feet in river stage, which flooded 17th Street North to Third Street North. Fifth Avenue North was inundated with 5 or 6 feet of water between Third Street and the water treatment plant. There was $\$ 50,000$ worth of damage to the bridge at Sartell, just upstream from St. Cloud.

At the town of Elk River, Minn., the Mississippi River makes a sharp horseshoe bend. Water cut across the open end of the horseshoe washing out several hundred yards of highway, flooding 26 homes and severely damaging 14 of them (fig. 8). The stage at Elk River was 2.7 feet higher than the highest stage of the 1952 flood, which was the previous maximum during the period of record. The discharge was 1.23 times that of a 50-year flood. Downstream, at the gaging station near Anoka, the 1965 flood peak was 2.0 feet higher than the 1952 peak, the previous maximum in 34 years of record.

In Minneapolis, severe damage was inflicted on the Great Northern Railway stone arch bridge just downstream from St. Anthony Falls. The scouring of the flood waters caused one of the piers to settle; as a result the bridge needed extensive repairs. Lilydale, (fig. a) a small, unincorporated community across the river from St. Paul and just downstream from the mouth of the Minnesota River, was flooded extensively. Floodwaters covered the town to a depth of 6-8 feet and swept some homes from their foundations. Many of the 33 homes in this community were damaged beyond repair.

At St. Paul, downstream from the mouth of the Minnesota River, 
the peak stage of 26.01 feet was the highest since at least $1851 .^{2}$ It exceeded by 4 feet the 1952 peak stage which was the previous maximum. The 1965 peak discharge was 171,000 cfs (cubic feet per second); in 1952 it was $125,000 \mathrm{cfs}$. The recording gaging station on Shepard Road was inundated and rendered inoperative, but stages were read at frequent intervals on a temporary staff gage. Flooding in St. Paul was intensified by the high discharge of the Minnesota River; the peak discharge of the Minnesota River was greater than the peak discharge of the Mississippi River above the mouth of the Minnesota River. It was fortunate, indeed, that the Minnesota and Mississippi Rivers did not crest concurrently at St. Paul; the Mississippi River peak above St. Paul occurred 6 days after the Minnesota River peak.

Damages, including the cost of flood fighting, exceeded $\$ 4$ million in St. Paul. If flood protective works on both banks of the Mississippi River had not been constructed, additional damages would have been about $\$ 8$ million, according to Corps of Engineers' estimates. About 800 persons were evacuated from low-lying areas in St. Paul. Local overflow, undermining, and seepage on the left river bank along Shepard Road caused damage to industrial properties. Five of the floodaffected industries on Shepard Road, Northern States Power Co., Industrial Steel Container Co., West Publishing Co., The Farmers' Union Grain Terminal Association, and Pure Oil Co., shared in the cost of reinforcing the dikes. Shepard Road and several other streets were inundated and closed to traffic (fig. 10). The Union Depot was closed to rail traffic for more than 2 weeks and the Minneapolis-St. Paul sewage treatment plant could not operate for 4 weeks. Water lapped at the foot of the Post Office Building and prevented the handling of mail. The Golden Garter, a night club atop the 95-year old Minnesota Boat Club on Navy Island, wanted to stay open but was closed by police order. The morning after it was closed, part of the upstream wall of the boat club gave way to the rushing waters which flowed across the island. The Navy Island Bridge, a small, 150-foot long, timber bridge, broke loose from its moorings and was carried downstream. In order to prevent the St. Paul Yacht Club on Harriet Island from becoming a hazard to two downstream bridges and many moored boats, it was dynamited. Not according to plan, the building caught fire, and burned to the waterline thus averting the threat that it posed.

2 Previous reports state that the period of known stages at St. Paul is "since at least 1870." A recent study by the Corps of Engineers of 1870 newspaper accounts and 1952 flood records reveals, however, that the 1870 peak stage and discharge were probably exceeded by the 1952 flood and were certainly exceeded by the 1965 flood. The 1870 newspaper accounts state that "a flood prevalls which was only surpassed by that which occurred in the spring of 1851"; so it seems safe to say that the 1965 flood was the greatest since at least 1851. Newspaper accounts of 1851 are also available, and they state that the 1851 peak was the greatest since 1832 . 


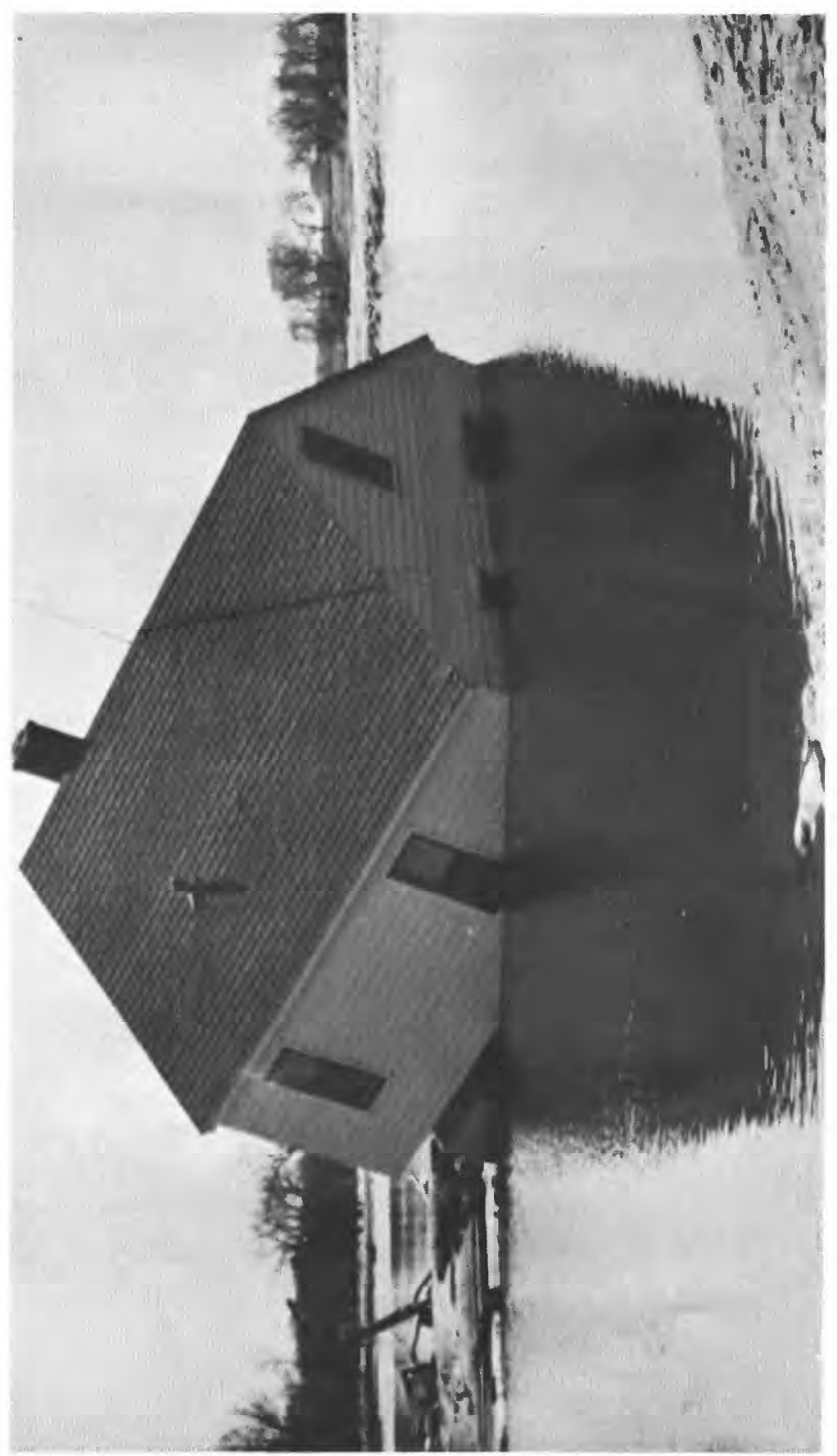



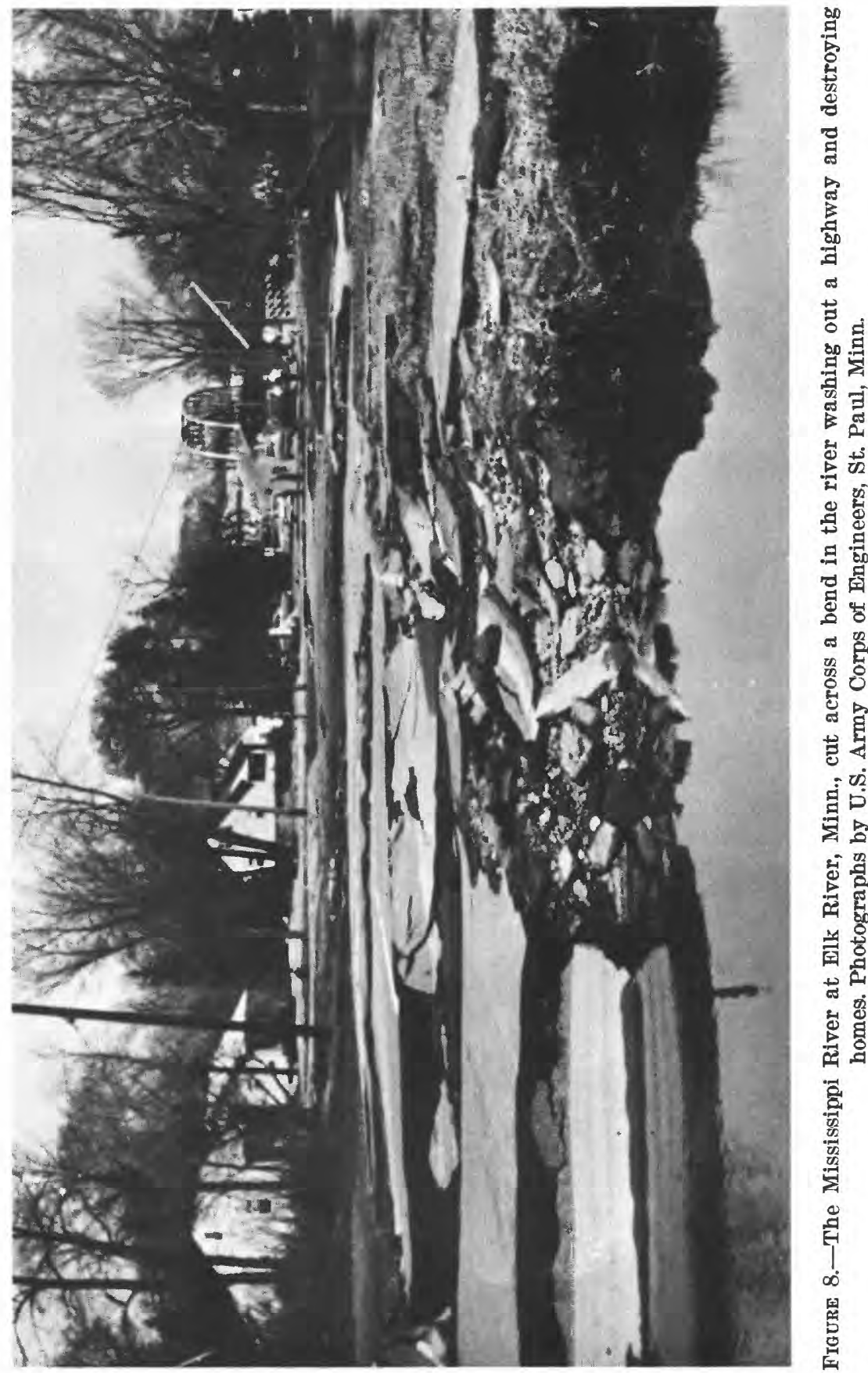


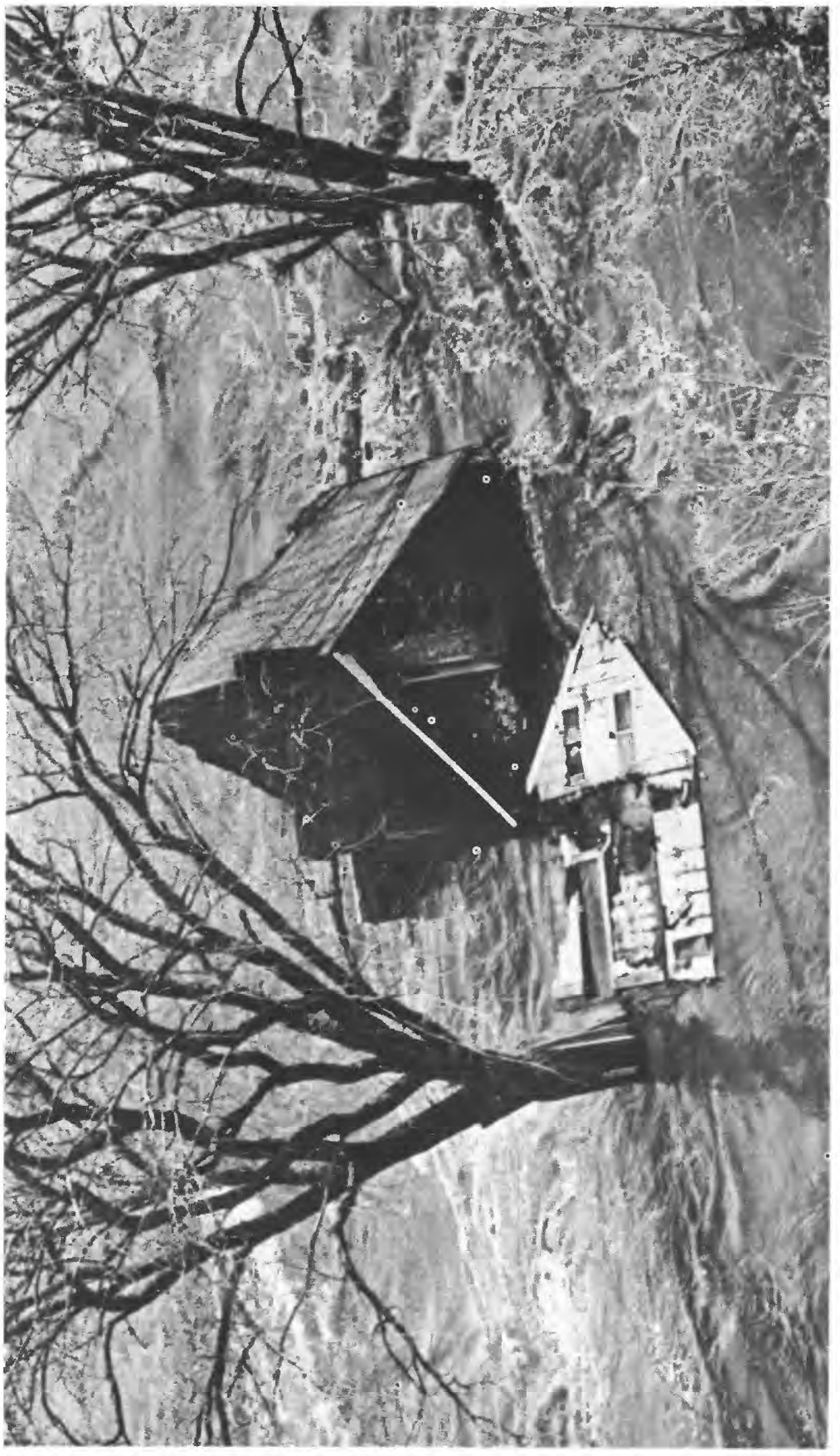

을 
On the right bank across the river from downtown St. Paul, the protected industrial area escaped damage; but the St. Paul municipal airport, which was outside the protective works, was flooded to a depth of 6-8 feet and was out of operation for 4 weeks (fig. 11).

In South St. Paul, a dike, which was built for the 1951 flood, was raised about 3 feet as an emergency measure to keep the 1965 floodwaters out of the stockyards and meatpacking plants. This dike, which was $31 / 2$ miles long, protected many South St. Paul facilities; nevertheless, considerable damage was done and many operations were disrupted. Railroad service was suspended for about 3 weeks. A number of storm sewers and sanitary sewers were damaged. The sewage treatment plant was inoperative for about a month, although a sandbag barrier minimized damage to the plant. Urban damage and the cost of emergency flood protection work in South St. Paul amounted to about $\$ 4$ million.

Prescott, Wis., at the confluence of the St. Croix River and the Mississippi River was flooded by both rivers, and both rivers were in extreme flood. The maximum discharge of the St. Croix River was about $44,000 \mathrm{cfs}$, and the timing of its peak coincided very closely with that of the Mississippi River upstream from Prescott. At its peak the St. Croix River contributed 19 percent of the flow in the Mississippi River immediately downstream from Prescott. The discharge of Mississippi River at Prescott was 1.57 times that of a 50-year flood.

The Mississippi River at Red Wing crested at 20.72 feet on April 18, almost 4 feet above the crest of the 1952 flood. Most of the city was safe on high ground, but some levees were sandbagged and some residents were evacuated. One of three municipal water pumps and the sewage treatment plant were put out of operation. Floodwaters swirled around several grain elevators located on low ground near the river. U.S. Highway 63 was inundated on April 16, and a temporary ferry service was put into operation between Red Wing and the Wisconsin side of the river.

Between Red Wing and Wabasha the Mississippi River flows through Lake Pepin. At Lake City, on the shore of Lake Pepin, the crest was about 3 feet higher than the previous maximum in 1952 . Local residents constructed about 2 miles of sandbag dikes, but in spite of this a number of homes and businesses suffered flood damage. Fifty summer homes on Central Point, north of town, were sheared off by ice floes or damaged by high water. Several boat marinas also were damaged.

On April 19 the Mississippi River crested at 20.05 feet at Wabasha, Minn. The crest was 8 feet over flood stage and 3.3 feet higher than the previous maximum which occurred in 1952 . Wabasha became an island as the rampaging waters inundated a third of the city and 


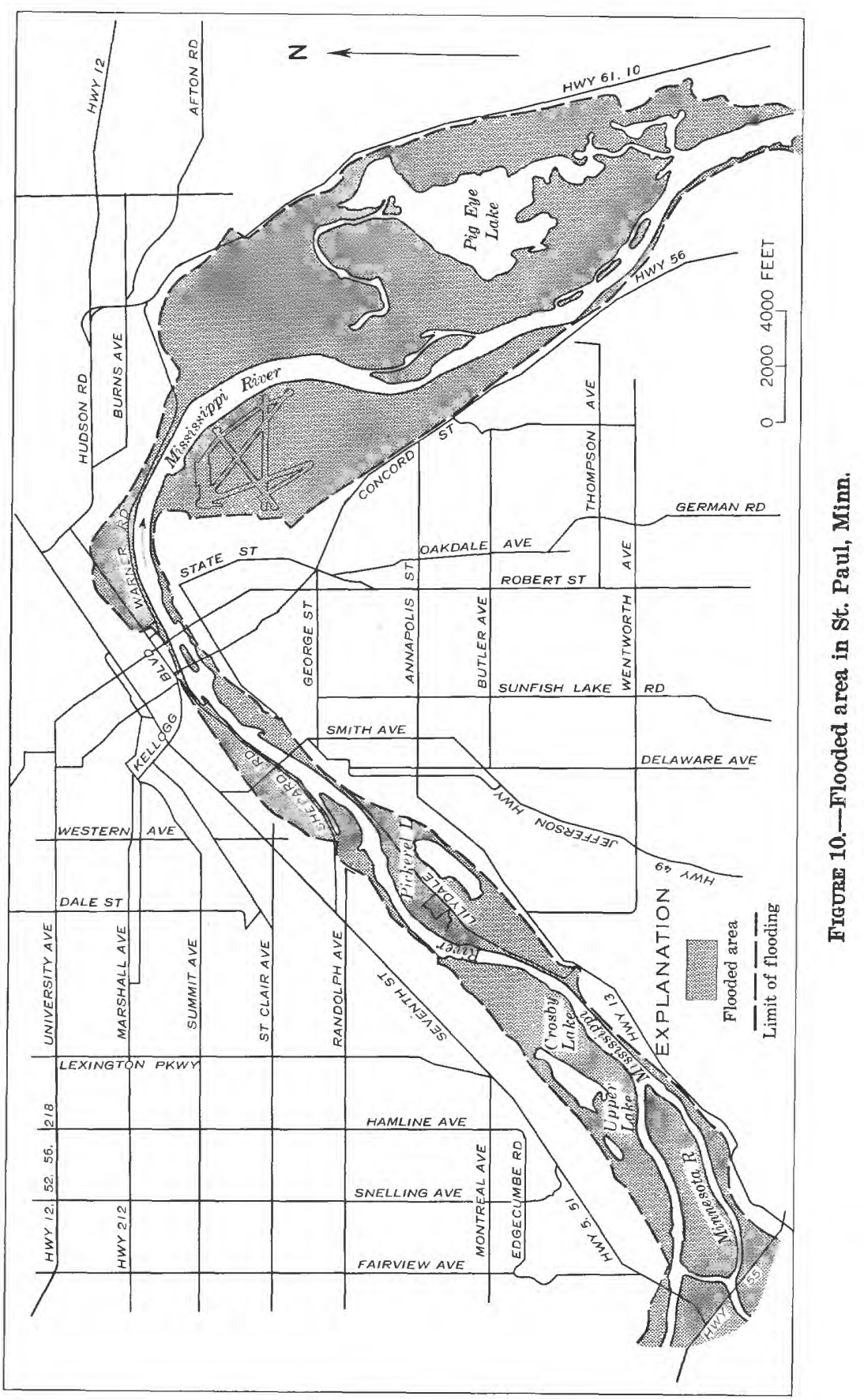



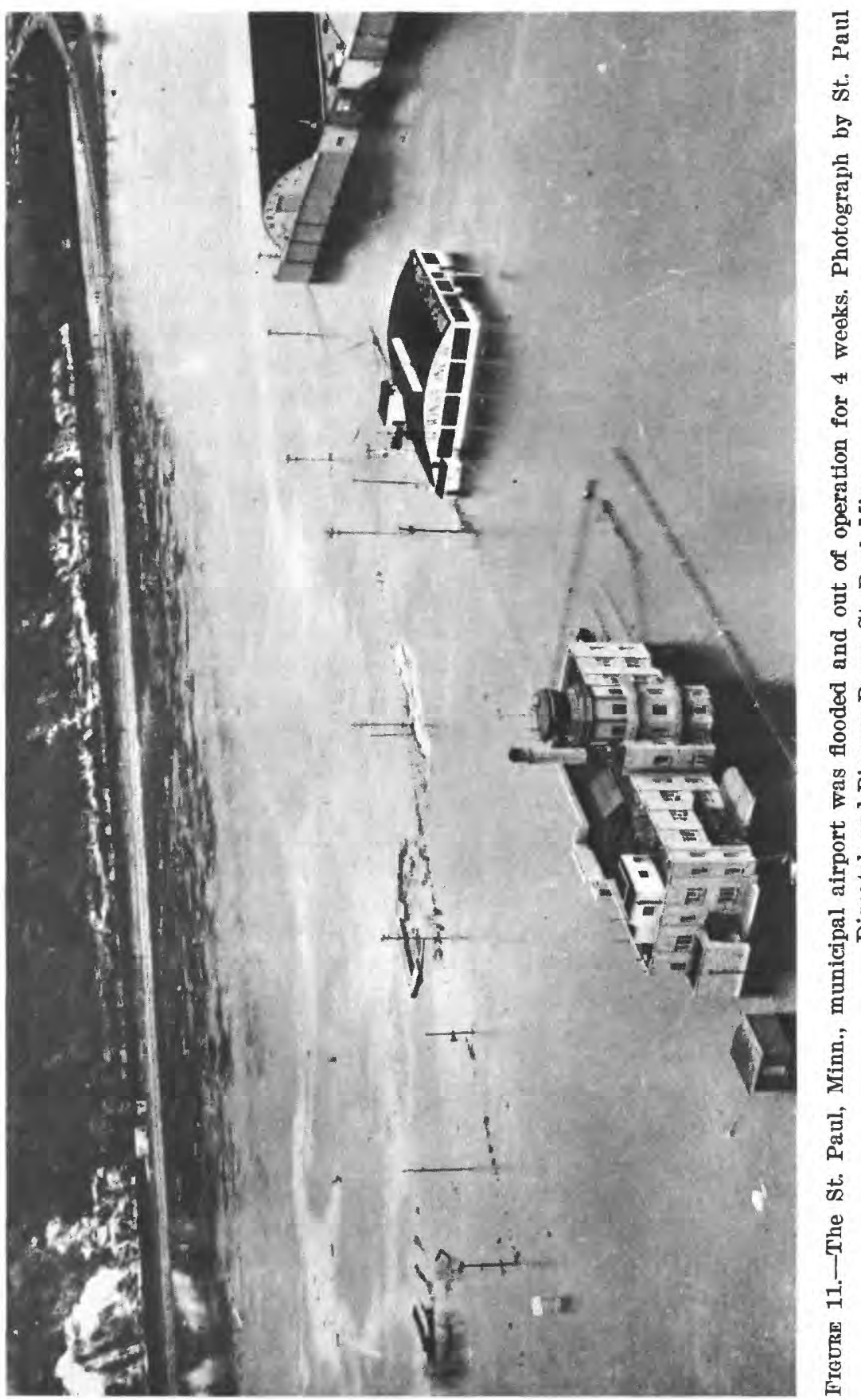
encircled a large part of it. U.S. Highway 61 , the main route through Wabasha, was closed for over a week, and Minnesota State Highway 60 , leading to the interstate bridge and Nelson, Wis., was also closed. Water was almost 2 feet deep over U.S. Highway 61 in town and a greater depth occurred south of town. Highway 60, at the railroad underpass, was flooded to a depth of almost 7 feet (fig. 12). Heavy ice floes from Lake Pepin, just upstream from Wabasha, and from the Chippewa River in Wisconsin threatened the town. An ice floe, almost as wide as the river and a quarter of a mile long, smashed into a wooded area above town splintering and grinding the trees which absorbed its impact. After the 1952 flood, the Nelson-Wabasha dike was raised 4 feet. About 3,000 feet of the 21/2-mile-long, 4-foot raise was washed away in the 1965 flood. Many business places were closed during the flood, and about 50 families were evacuated. Total damages to the city were about $\$ 350,000$.

Four feet of water covered the lock walls at lock and dam 4 near Alma, Wis. The pool elevation was 676.45 feet, 3.15 feet higher than the 1952 flood which was the maximum previously recorded at the dam since it was completed in 1935 .

In Buffalo and Trempeleau Counties, between the Chippewa and Black Rivers in Wisconsin, flood damages were estimated at $\$ 1.5$ million. Cochrane, Fountain City, and Trempeleau all suffered damages in excess of $\$ 200,000$. Water covered State Highway 35 at the south end of Alma, and forced the residents of Front Street to evacuate their homes. The switches on the power exchanges which direct power to Midwest States were endangered at the Alma substation. About 150 of Cochrane's 458 people were evacuated as backwater surged into town through Indian Creek. Dikes hurriedly erected at Buffalo also protected Cochrane about a mile distant and farther from the river but 18 feet lower. In Fountain City, 11 families and 10 businesses were evacuated. Almost 1,400 flnod fighters, employed by the U.S. Boatyard in Fountain City, and 150 National Guardsmen transported sandbags by barge from the boatyard and emplaced 190,000 sandbags at lock and dam 5A. The Fountain City boatyard dispensed more than a million sandbags and sent pumps and men to other cities and villages to help fight the flood.

The river crested at Winona, Minn., on April 19 at 20.77 feet, almost 3 feet higher than the flood of April 20, 1952. The maximum discharge of $268,000 \mathrm{cfs}$ was 1.34 times that of a flood of 50-year recurrence interval. Flood stage at Winona is 13.0 feet; the river remained above this stage for 24 days. Volunteer workers in Winona waged a tremendous battle against the flood. There was much at stake. Had Winona's dike system failed, only two small islands would have remained above water and about two-thirds of the city would have been flooded. The 


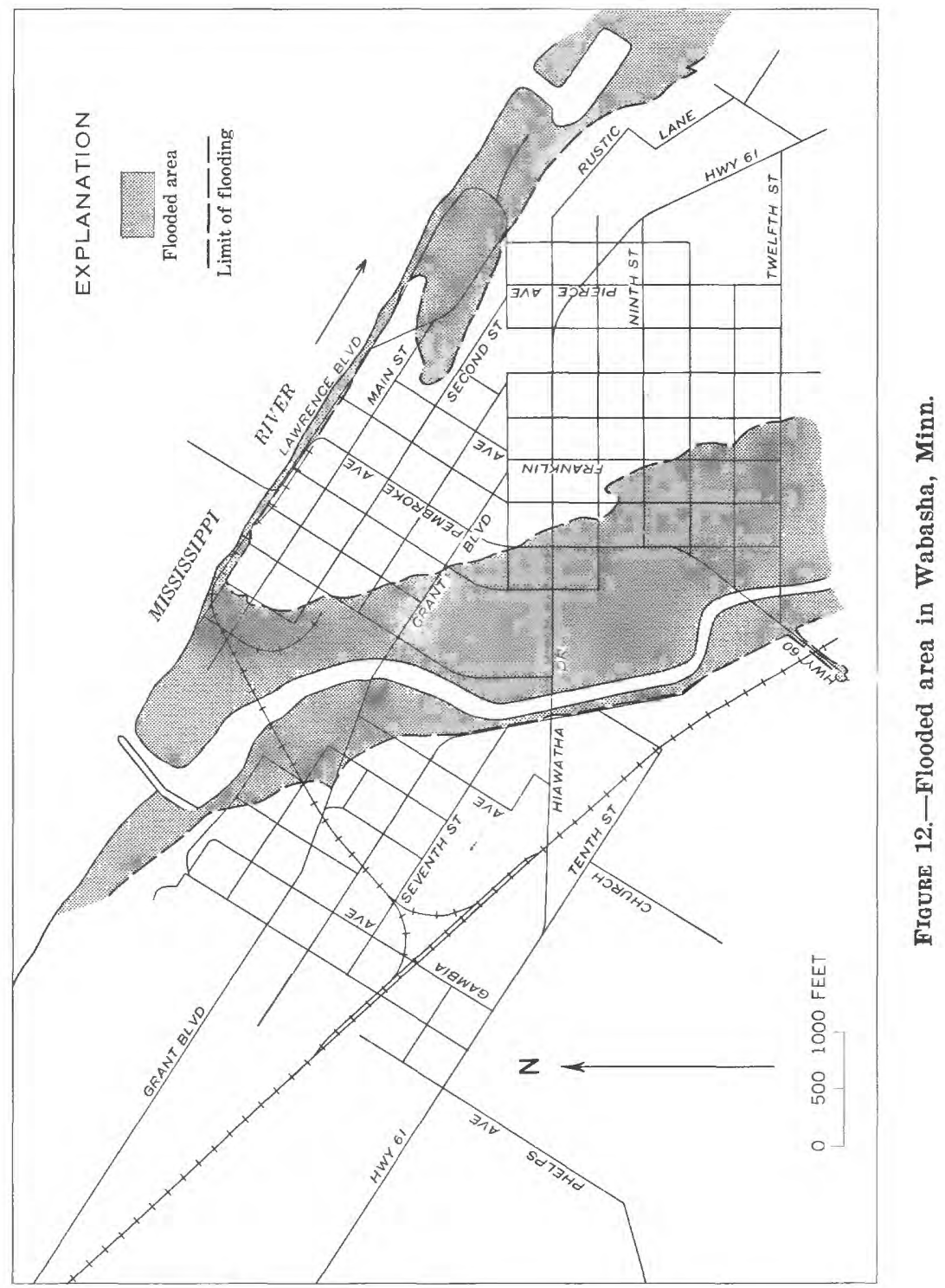


flood-fighting effort was costly, about $\$ 2.6$ million, but it probably prevented about $\$ 20$ million in damages. Total damages to the city including the flood-fighting effort were $\$ 3.7$ million.

The massive effort at Winona involved ringing the city with 9 miles of dikes at the 22 -foot gage elevation. Some existing dikes were raised and new ones were built. Over 250,000 cubic yards of earth was required to build the dikes, and during peak periods of operation, over 400 trucks, bulldozers, earthmovers, power shovels, and draglines were used at one time. Dozens of pumps were required to remove water seeping through and under the floodwalls. On April 18 and 19 high winds and lapping waves threatened to destroy some critical sections of the dikes, but because sandbagging and earthfilling operations were accelerated the dikes held. Though there was no physical loss to the 510 -city-block residential and business area, there was a substantial loss to business because of the disruption of transportation facilities and because of the diversion of many residents into flood-fighting work.

There was great danger also when storm sewers seemed about to erupt from the high water pressures. To counteract this threat, a pioneering effort was made by city officials who hired a professional diver to seal off critical storm sewers with inflatable rubber bags. This technique proved successful and was later used by several cities farther downstream. During the period when the eruption of storm sewers threatened, over a thousand persons were evacuated from the danger areas.

The area inundated in Winona at the flood crest is shown in figure 13.

The flood prevented rail and air travel to Winona, and by April 15 only buses remained in operation. Max Conrad Airport was under water. Railroad trackage suffered heavy damage. A break in the roadbed of the Burlington track on the Wisconsin side upstream from Winona released water to a flood plain area of 5,000 acres on the Delta Fish and Fur Farm lands and may have reduced the peak at Winona somewhat. The water which poured into the flood plain undermined the dike which carried the tracks of the Green Bay and Western Railroad (fig. 14) and rejoined the main channel just across the river from Winona. The Burlington Railroad lost additional trackage farther downstream near Trempealeau. The Chicago and North Western Railway and the Milwaukee Railroad also suffered severe flood damage. Rail transportation into Winona resumed on April 27 during the flood's recession, but it was several days before all the railroads were back in operation.

The two main highways through Winona, U.S. Highways 61 and 14, remained open although Highway 61 was flooded to the north in the Wabasha area, Across the river from Winona, State Highway 
35 in Wisconsin was flooded both north and south of the interstate bridge.

At La Crosse, Wis., the Mississippi River crested at 17.96 feet on April 22, 2.6 feet higher than the 1952 flood and 1.5 feet higher than the flood of 1880 . The maximum discharge of $278,000 \mathrm{cfs}$ is 1.34 times as great as a flood of 50-year recurrence interval.

Extensive flood protection measures were undertaken in La Crosse. Construction of 5 miles of emergency dike required 350,000 sandbags and 59,000 tons of material. In La Crosse and its environs, over 600 homes and about 80 businesses were flooded. Over 1,200 persons were evacuated from their homes. Total damages at La Crosse, including the cost of flood fighting, were about $\$ 7.6$ million; no other municipality except Dubuque, Iowa, suffered greater damage. A photograph of part of the flooded business area in La Crosse and a map showing the inundated area of the city are shown in figures 15 and 16.

At Lansing, Iowa, floodwaters cut off the south residential area from the rest of the town and closed the bridge across the Mississippi River. A mobile power unit, readied when floodwaters threatened the local power substation, supplied electrical power to the community when flooding occurred.

A peak stage of 25.38 feet, 7.38 feet above flood stage, occurred at McGregor, Iowa, on April 24. It exceeded all stages since at least 1828. An emergency levee on " $A$ " Street and sandbags along some properties on Main Street confined the flooding to the immediate riverfront area. Marquette, Iowa, just 2 miles north, was isolated when all highways leading into the city were inundated. The city was separated into three parts by the floodwaters and water stood $31 / 2$ feet deep at the Main Street intersection. Supplies and emergency aid were brought in by National Guard "ducks" and other boats. At Prairie du Chien, Wis., just across the river, about 250 houses and 25 business establishments were flooded. One-third of the town was under water and over 1,000 persons were homeless. The approaches to the Mississippi River bridge between Marquette and Prairie du Chien were inundated and closed to traffic on April 19 (fig. 17). The topography at Prairie du Chien does not lend itself to flood protection works because an old channel runs behind the city ; consequently dikes would have to encircle the city to protect it. Flood losses in Prairie du Chien were great-almost $\$ 21 / 2$ million, of which over $\$ 1$ million was to residential property.

The stage at Guttenberg, Iowa, reached 23.65 feet on April 24, about $81 / 2$ feet above flood stage. About 70 percent of the town was protected from flooding by about 1 mile of emergency levees. At Cassville, Wis., 9 miles downstream from Guttenberg, the flood crest 


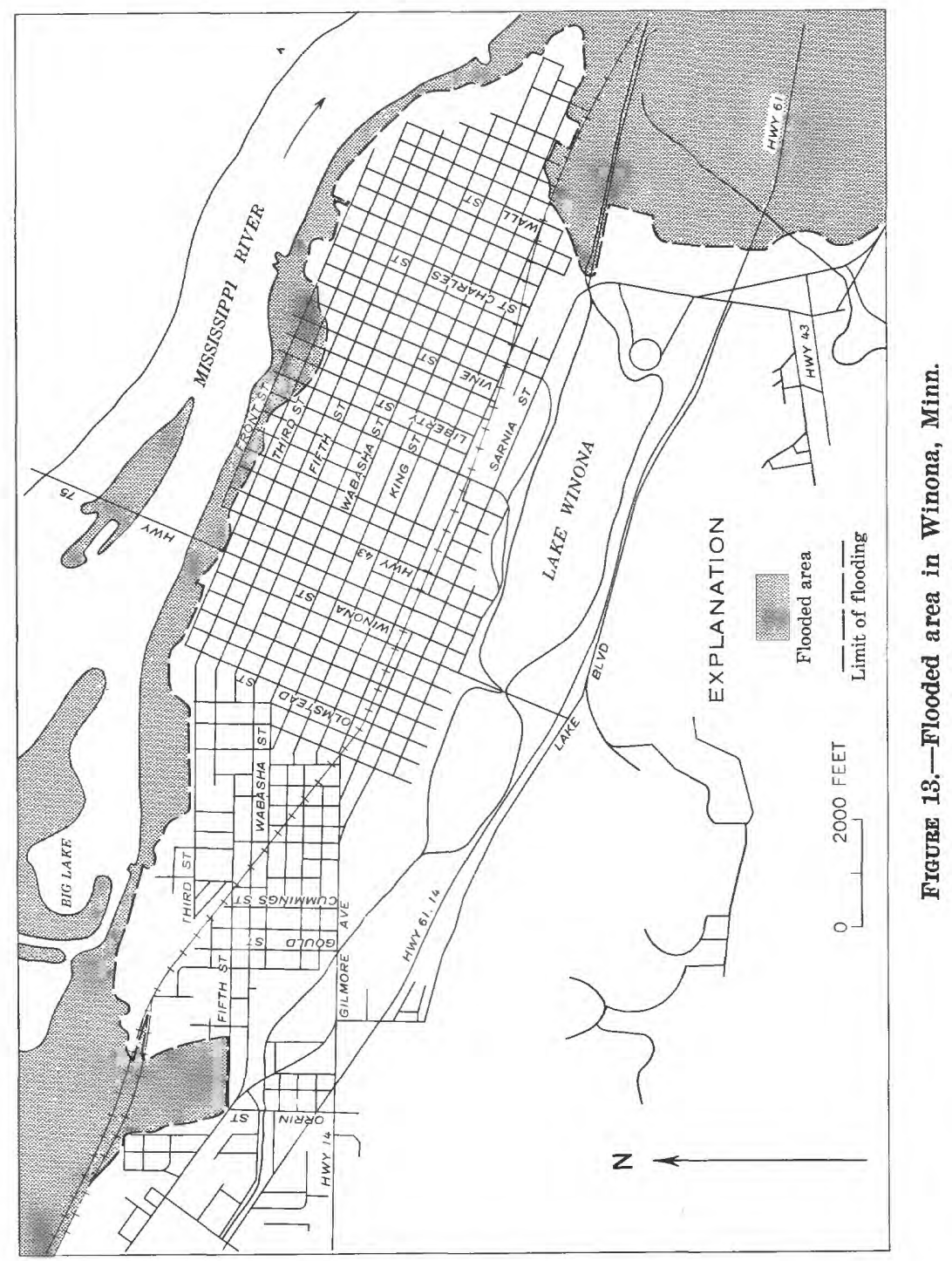




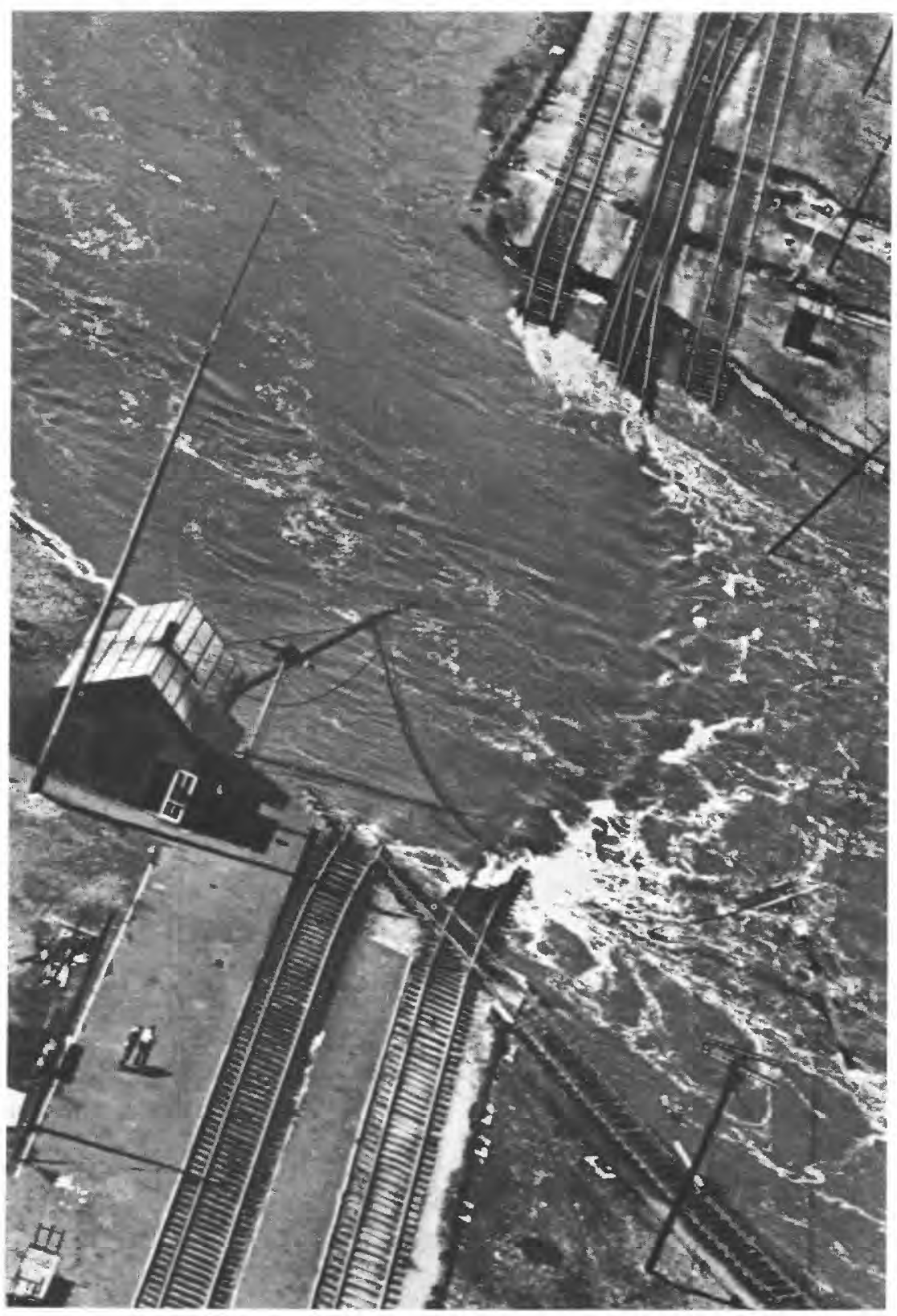

FIGURE 14.-Railroad trackage near Winona, Minn., suffered heavy damage. Photograph by Minneapolis Star and Tribune, Minneapolis, Minn. 


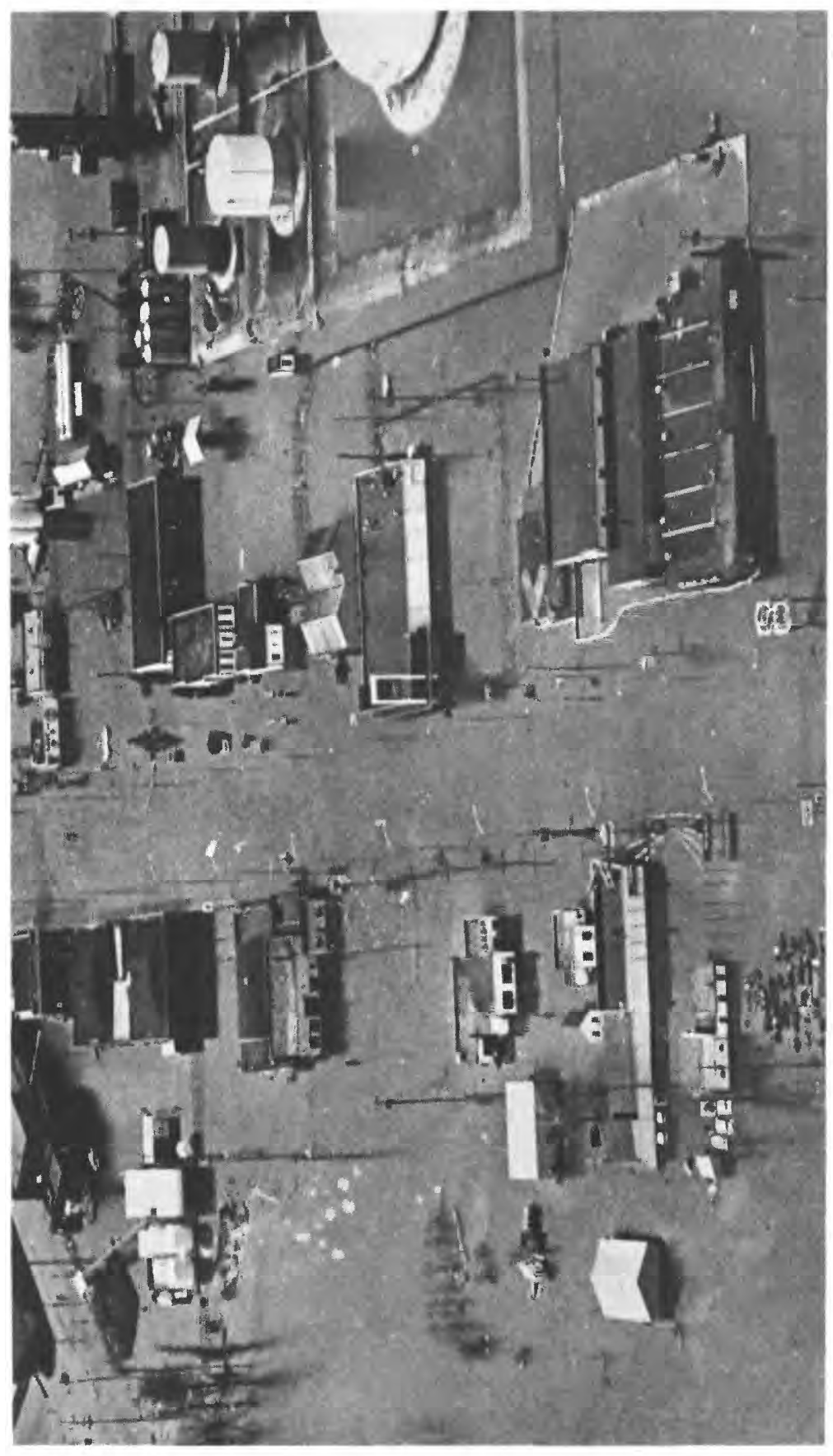

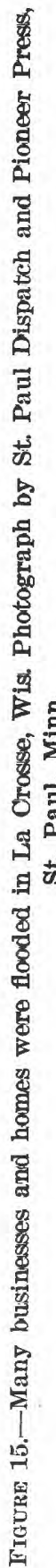




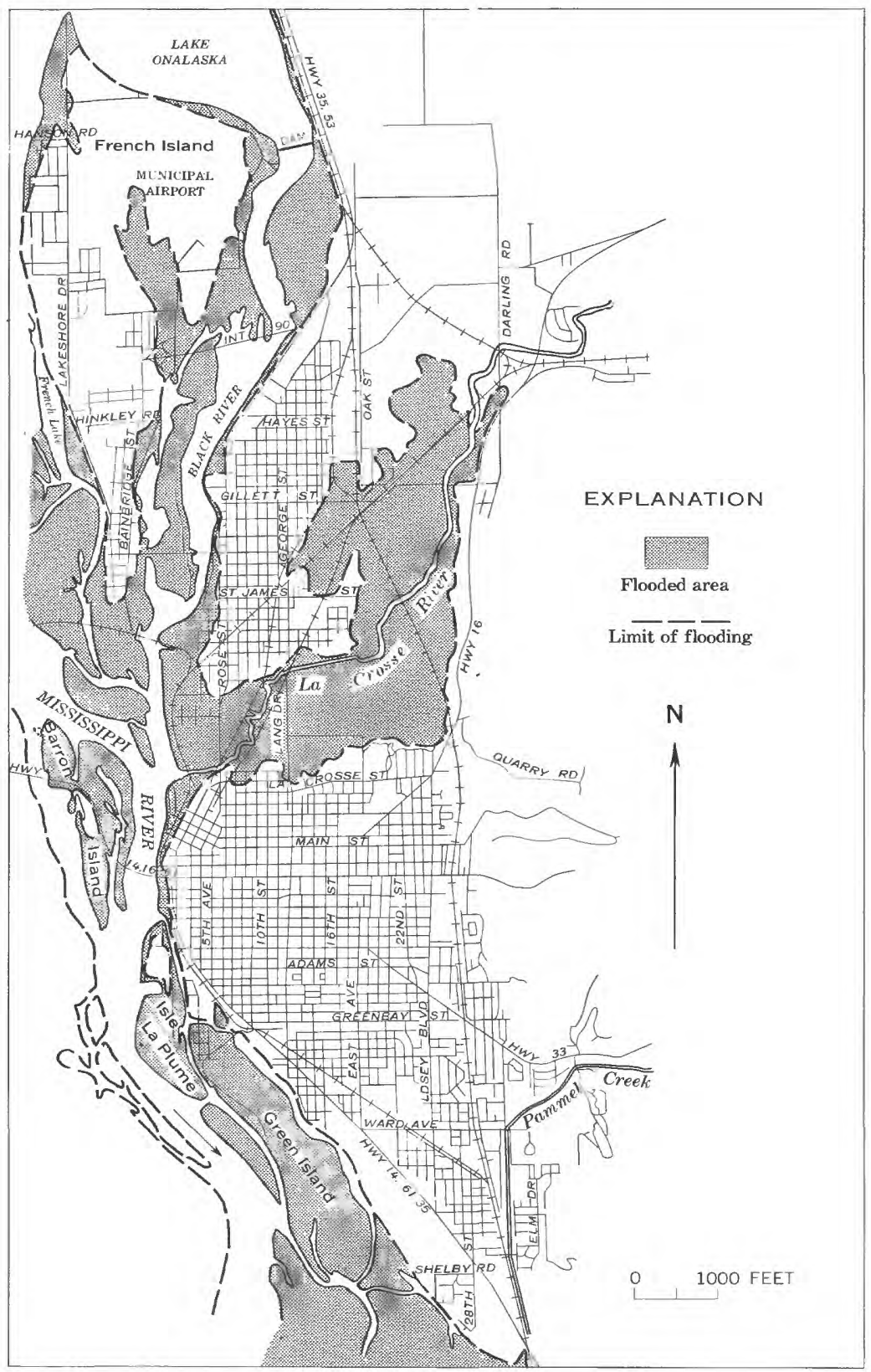

Figure 16.-Flooded area in La Crosse, Wis. 
exceeded the previous maximum stage by about 4 feet. About 220 persons were evacuated from this small town of 1,290 population. Flood damage was estimated to be about $\$ 300,000$.

The stage at Dubuque, Iowa, was above the 17-foot flood stage for 28 days. The peak stage of 26.71 feet on April 26 was 4 feet higher than the previous maximum of record which occurred in 1952. The southern tip of the business district and several industrial areas were flooded (fig. 18). Most of the residential areas were protected from the floodwaters by dikes along the riverfront and on Eighth Street, but about 350 persons were evacuated from their homes. About 3,500 volunteers placed 400,000 sandbags on $31 / 2$ miles of emergency dikes. This effort saved 450 houses and many industries from inundation and kept the Julien Dubuque Bridge open to traffic. For several days, at the height of the flood, this bridge was the only one open across the Mississippi River in the 200-mile reach between La Crosse, Wis., and Davenport, Iowa. The Illinois Central Railroad jacked up about $11 / 2$ miles of tracks to keep them above the rising waters, but the company had to discontinue service on the west side of the Mississippi River on April 23. The Iowa-Wisconsin bridge was closed on April 19 and the Milwaukee Railroad Company suspended service in the Dubuque area on April 21. The Dubuque sewage treatment plant was out of operation for several days beginning on April 24. Nineteen business and industrial concerns were closed, putting many people temporarily out of work. Flood protection works at John Deere and Dubuque Packing Plants successfully withheld the floodwaters and the plants remained open. A second line of dikes was constructed on 12th Street, Fengler Street, and Kerper Boulevard in case the first line was breached, but all dikes held. The water from seepage under the dikes and from local drainage was collected in a storage lagoon behind the dikes and was pumped into the river. Over $\$ 3$ million was spent on flood protection which prevented about $\$ 71 / 2$ million of damages. The Corps of Engineers estimated the flood damages in Dubuque to be almost $\$ 7.7$ million, the greatest loss sustained by any metropolitan area. The flooded area in Dubuque is shown in figure 19.

The Mississippi River crest, 3.6 feet higher than that of the 1952 flood, reached Clinton, Iowa, on April 28. The peak stage was the highest one recorded since 1828. Emergency dikes and strengthened levees saved the city's main business district and most of the residential and industrial areas from inundation (figure 20). Failure of the dikes would have caused 150 blocks to be flooded and an estimated additional $\$ 9.5$ million in damages. In spite of the many flood protection structures built, over $\$ 5$ million in damages occurred. The Lyon's business district in Clinton and over 50 industrial and commercial concerns were flooded (fig. 20). The Lyon's-Fulton Bridge and Gateway Bridge 


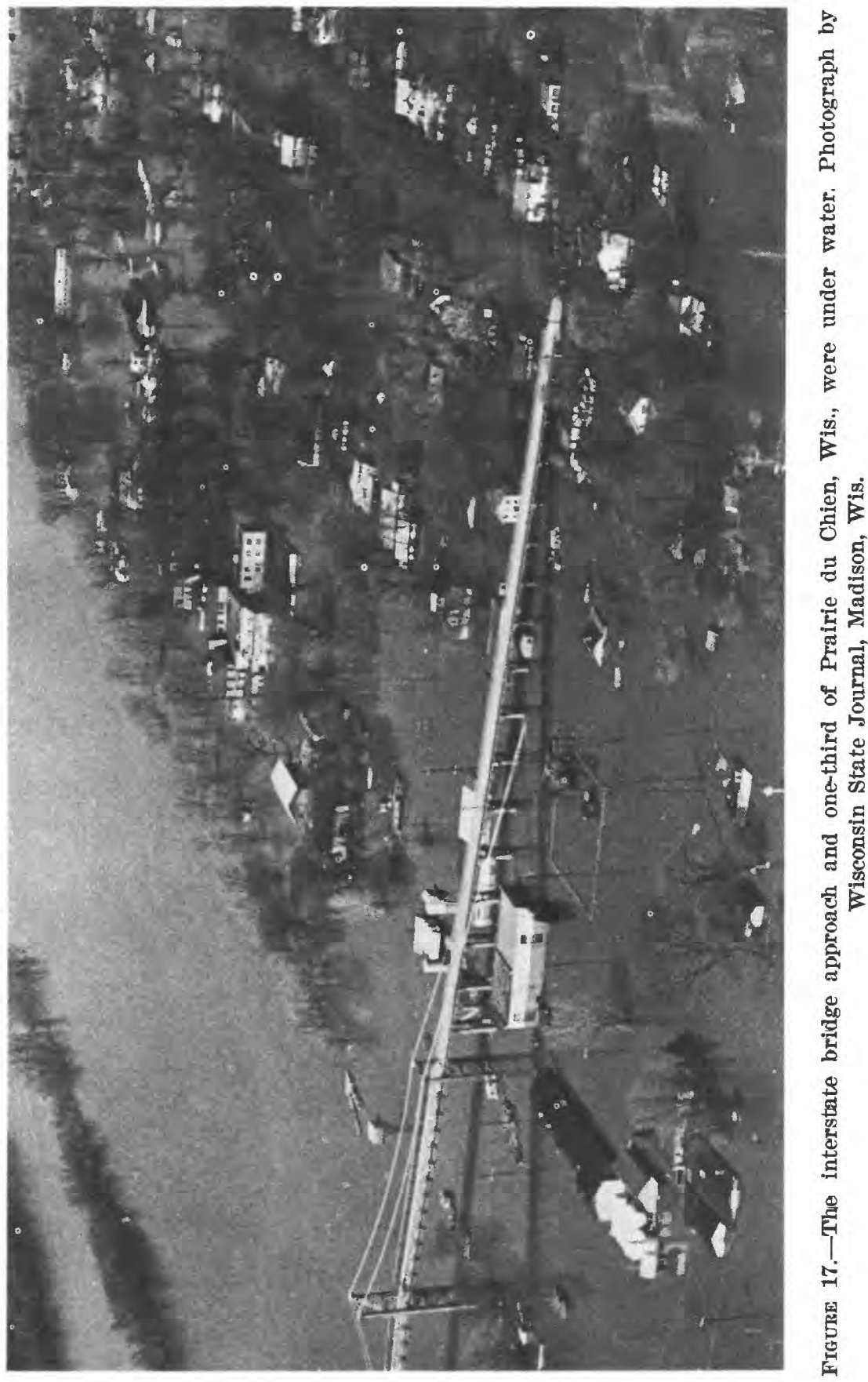




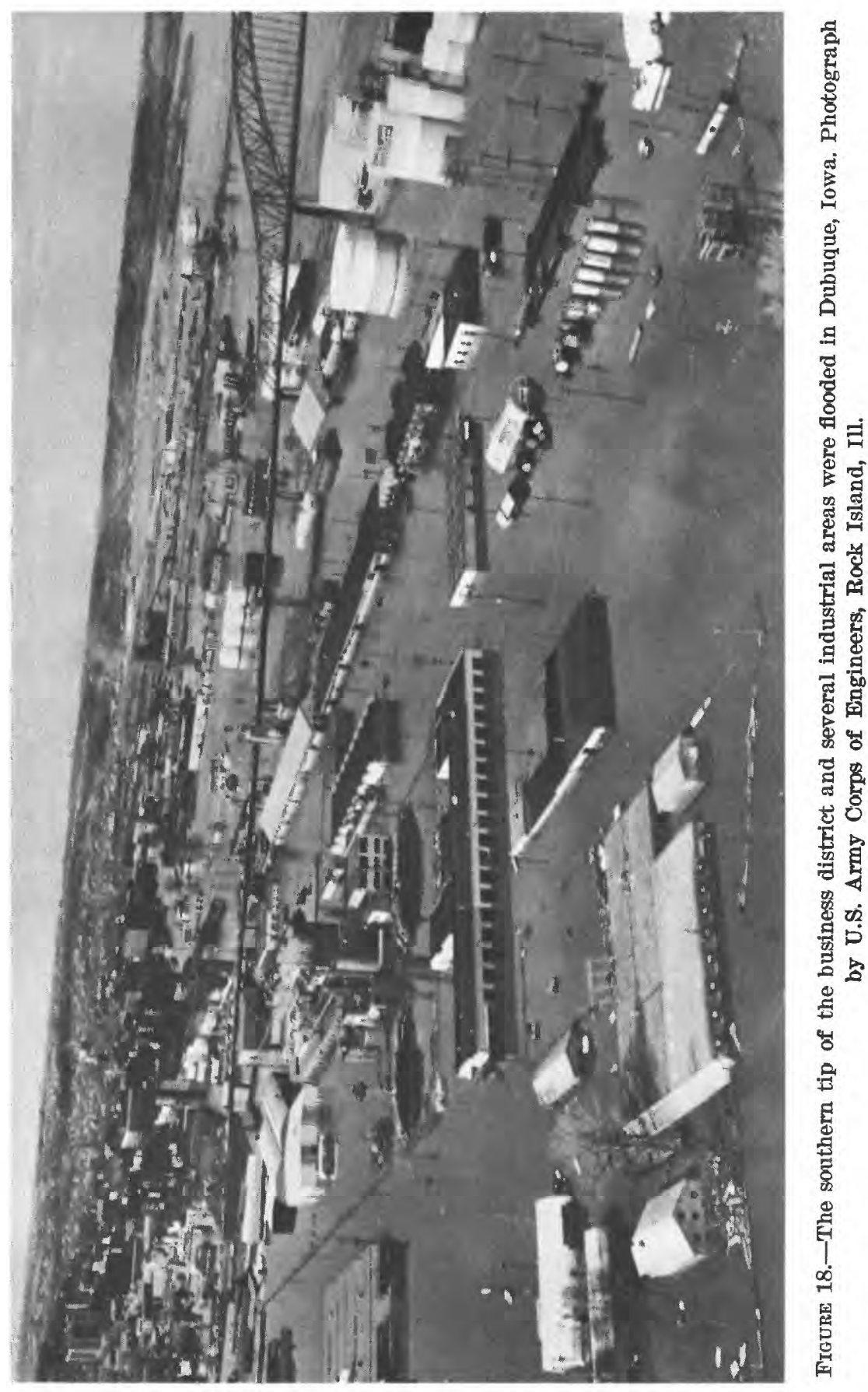




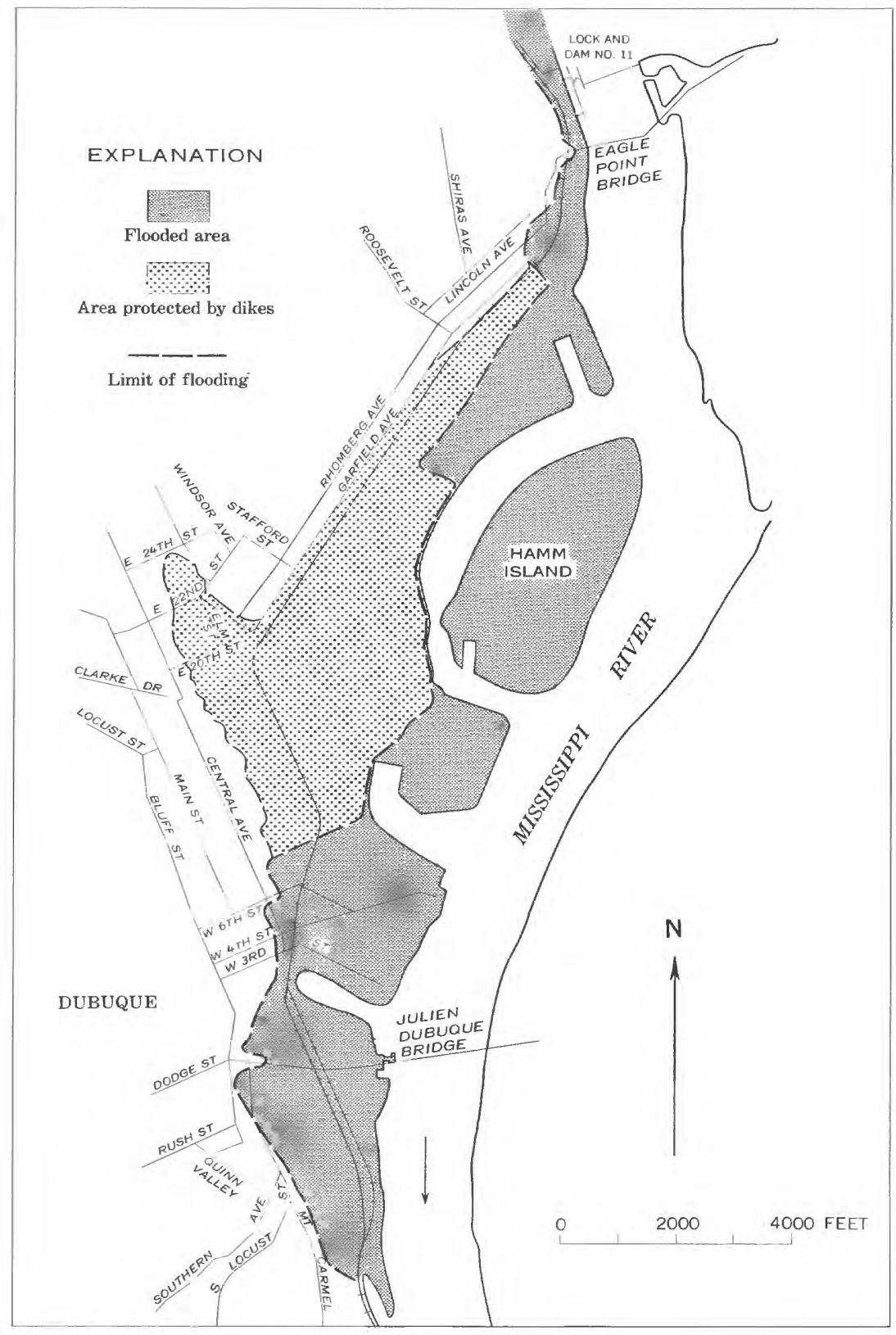

Figure 19.-Flooded area in Dubuque, Iowa. 


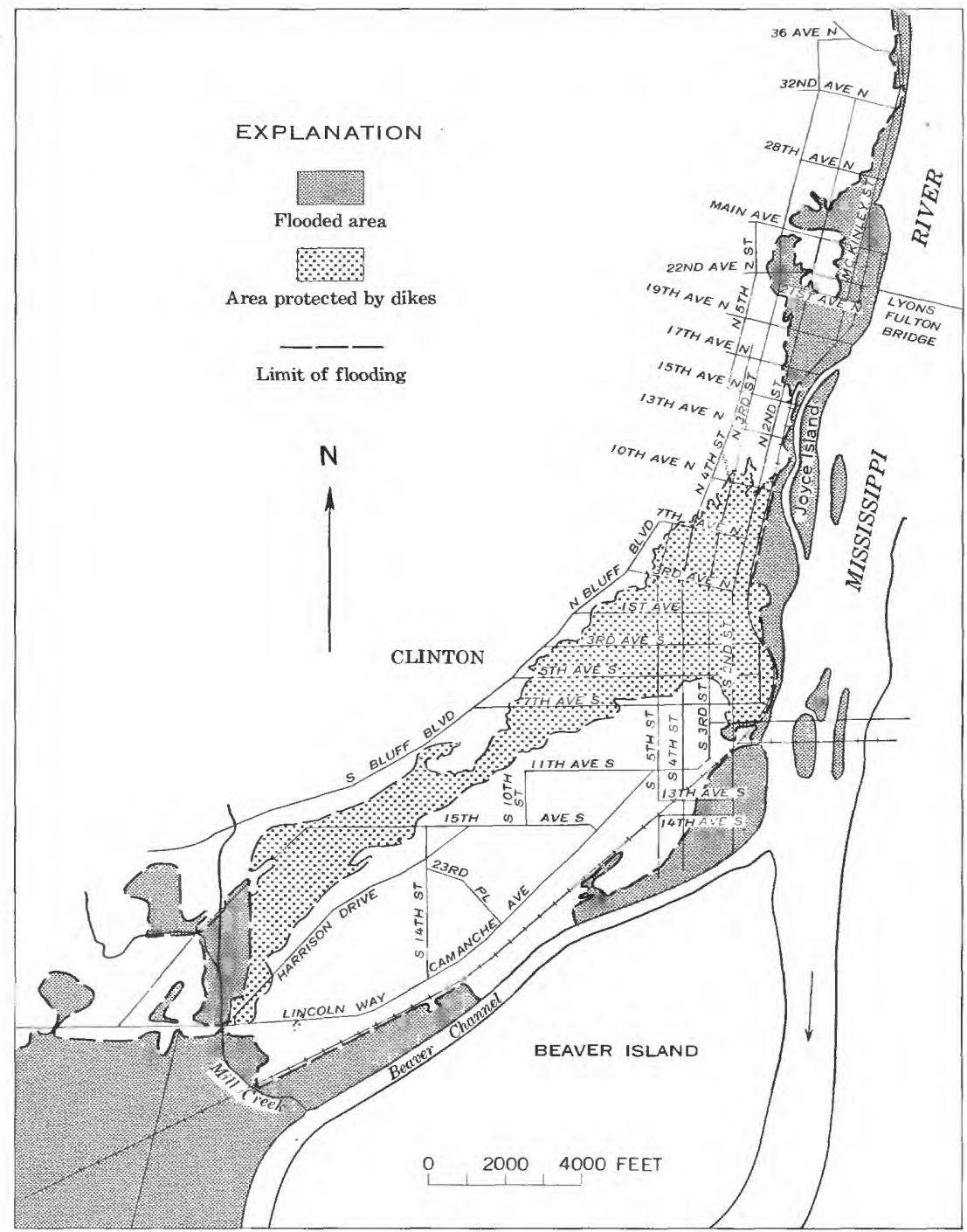

Figure 20.-Flooded area in Clinton, Iowa.

were closed April 23 for about 2 weeks and the Chicago and North Western Railway Company had to suspend operations through this area because of the floodwaters. Fourteen youths were injured when a cinder-block wall, being reinforced to serve as a floodwall, collapsed from the weight of earthfill placed against it.

An interesting story can be told about the efforts to save the Clinton First Avenue pumping station. Much of the city behind the levee would have been flooded from seepage water if the pumps had failed. 
The sandibagged station, resembling a fortified island in the rampaging waters became known as Fort Johannsen, named after Lt. Johannsen of the Iowa National Guard. He, along with a band of fellow National Guardsmen, manned the station day after day and kept the pumps operating and the dikes intact.

Another interesting story was that of "Silent Henry" Steele. Silent Henry, about 72 years old, had lived for 30 years on his small island near Clinton. Throughout the 1965 flood, he floated above his shack on a raft tied to the top of a tree; he not only refused evacuation but also any assistance for food and supplies.

In Whiteside County, Ill., the Johnson Creek and Cat Tail Drainage District levees were breached, flooding more than 9,000 acres. Fulton, Ill. was completely surrounded and engulfed by these floodwaters (fig. 21). Damages were about $\$ 1.7$ million, and more than 2,000 persons were forced to leave their homes. Downstream, at Albany, Ill., the 12-mile section of Meredosia Dike threatened to fail. If it had, 19,000 acres of farmland and neighboring communities would have been flooded. Hundreds of volunteers worked against tremendous odds and successfully reinforced the dike and Meredosia pumping station.

The metropolitan area of Davenport and Bettendorf, Iowa, and Rock Island, Moline and East Moline, Ill., was inundated on both banks along more than 10 miles of the Mississippi River (figs. 22 and 23). The 1965 flood in this entire area caused about $\$ 12$ million in damages and drove 12,000 persons from their homes. According to Corps of Engineers estimates, $\$ 101$ million in damages were prevented by the tremendous flood emergency measures. The peak stage of the tailwater at dam 15 at Davenport on April 28 was 22.48 feet, 7.48 feet above flood stage.

At Bettendorf, Iowa, emergency dikes built across River Drive and State Street confined street flooding to less than three blocks. The failure of a 25-foot section of dike in Rock Island caused floodwaters to inundate many blocks of houses and industrial buildings. Throughout the metropolitan area, many industrial plants had to shut down. A few industries succeeded in keeping the floodwaters out of their buildings, but in many plants water was waist deep or higher. More than 200 houses were flooded in the Garden Addition in Davenport as well as some businesses in the downtown area. The Government Bridge was closed April 26 for several days when floodwaters inundated the approach.

At Muscatine, Iowa, the flood fight was hampered by 6 inches of rain which fell a few days before the flood crested. Several industries and business houses, protected from the river by dikes and levees, were flooded by the ponding of interior drainage when the power failed for 5 hours at the Mad Creek pumping station. More than 3,500 feet of 


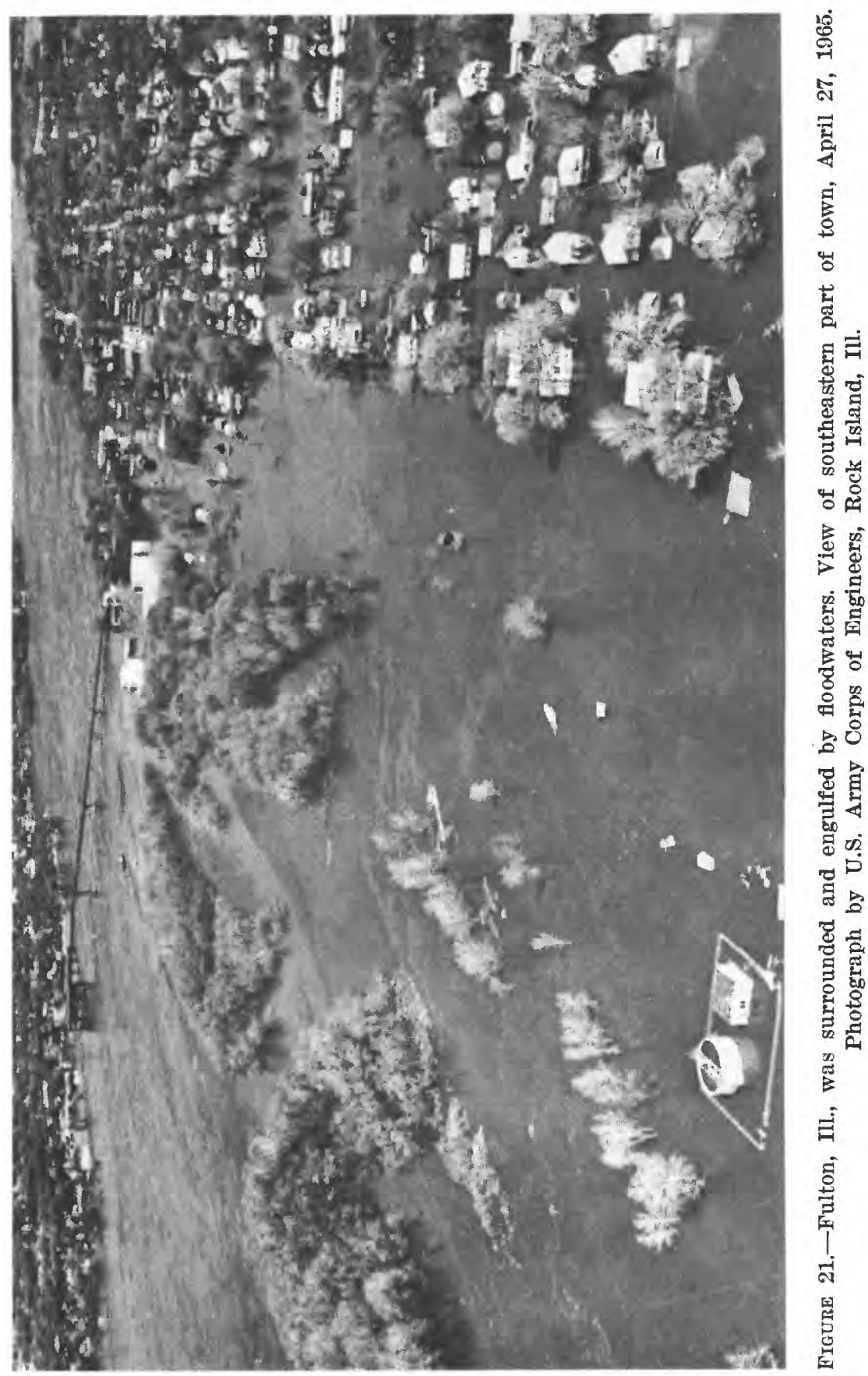


earthen dikes were built in 10 days in the Port Louisa and Michael Creek areas south of the city. These dikes prevented the flooding of 47,000 acres of farmland and a huge industrial and residential area valued at $\$ 50$ million. Damages and flood-fight costs in the Muscatine area were about $\$ 628,000$. The closure structures, from Second Street to Seventh Street in the Mad Creek levee system, were very successful in sealing off floodwaters. Other than a section of floodwall that gave way on April 26, all the structures and rain-soaked levees and dikes held and confined most flood damages to the riverfront areas and about 500 houses (fig. 24). The Muscatine High Bridge was closed on April 23 for over 2 weeks. The peak stage of 24.8 feet, which was 8.8 feet above flood stage and 3.8 feet above the 1952 flood, occurred on April 29.

Most businesses at Buffalo, Iowa, were forced to close because of the floodwaters. Damages in the Buffalo area were approximately $\$ 167,000$. At Keithsburg, Ill., over half the business district was engulfed by floodwaters in spite of the more than 40,000 sandbags used to contain the flood.

The peak stage of 21.0 feet, 6 feet above flood stage, occurred at Burlington, Iowa, on April 30 and May 1. The previous maximum stage of record was 18.9 feet in 1851. The river remained above flood stage for more than 50 days, and many of the riverfront industries and streets were flooded. A 1,000-foot break in the Tama Levee north of the city flooded 3,200 acres of farmland. Just north of this area, the Yellow Springs Levee was raised and strengthened so that it kept 20,000 acres from flooding. Across the river from Burlington, floodwaters poured through several levee breaks and spread over 28,400 acres in Henderson County, Ill., creating a floodway 8 miles wide in places. Gulfport, Ill. was entirely flooded up to rooftop levels. U.S. Highway 34 underpass east of the city was washed out, thus cutting the Burlington Railroad main line.

Several levees of Federal organized levee and drainage districts failed between Dubuque, Iowa, and Quincy, Ill. The Green Island Levee in Jackson County, Iowa, failed on April 15; 7,200 acres were flooded. The Henderson County, III., levees failed April 21, 26 and 27; a total of 15,200 acres were flooded. The Des Moines County, Iowa, levee failed April 26 flooding 3,800 acres. When the Indian Grave and South Quincy Levees failed, 17,800 and 5,500 acres, respectively, were flooded in Adams County, Ill.

Damages, including flood-fight costs, at Fort Madison and Keokuk, Iowa, amounted to about $\$ 690,000$. Most of the flooding was in the heavy industrial areas of both communities. At Fort Madison, the railroad yards were flooded, but the main line track was raised about 3 feet to enable rail service to continue. Riverview Park was under 


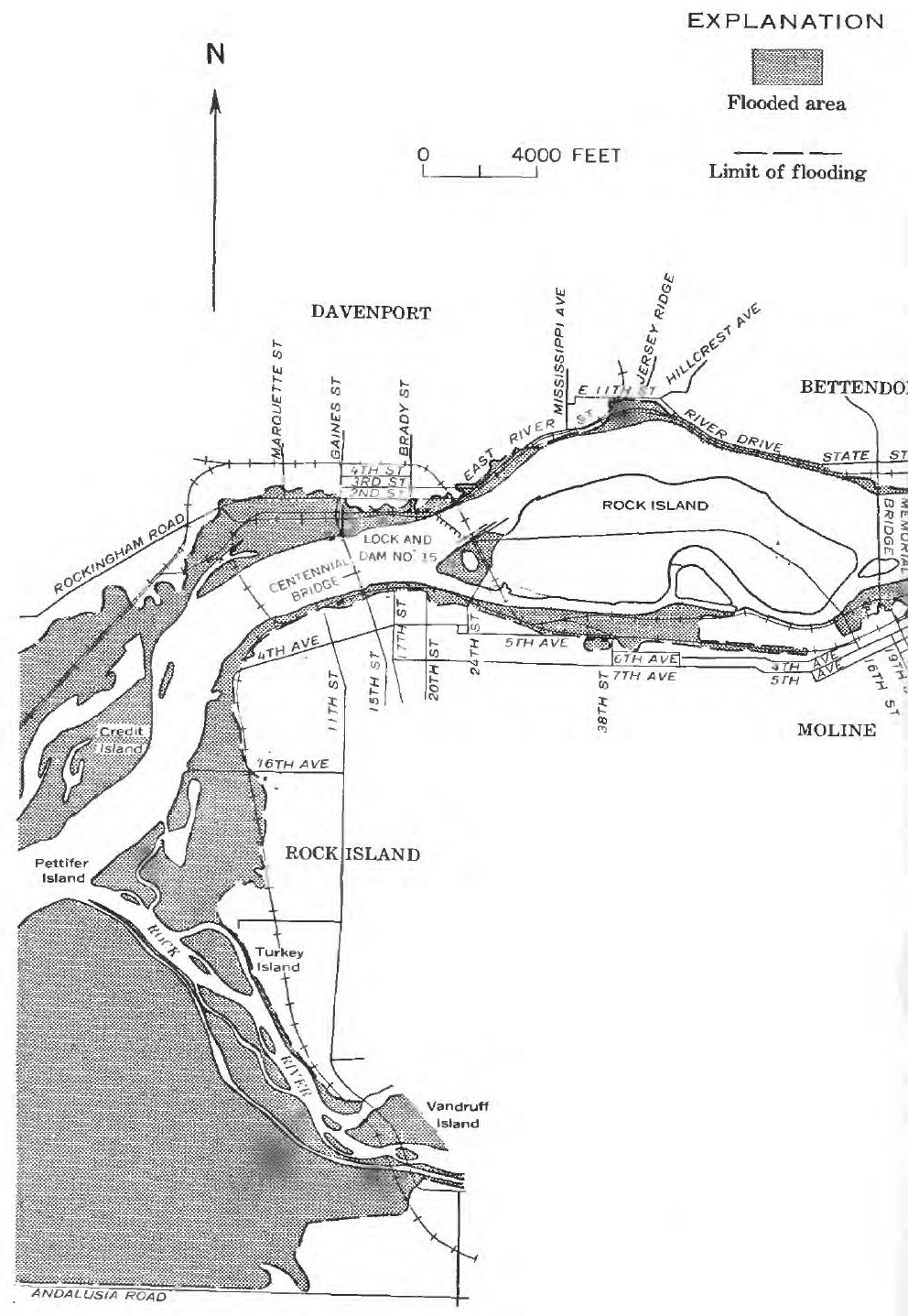




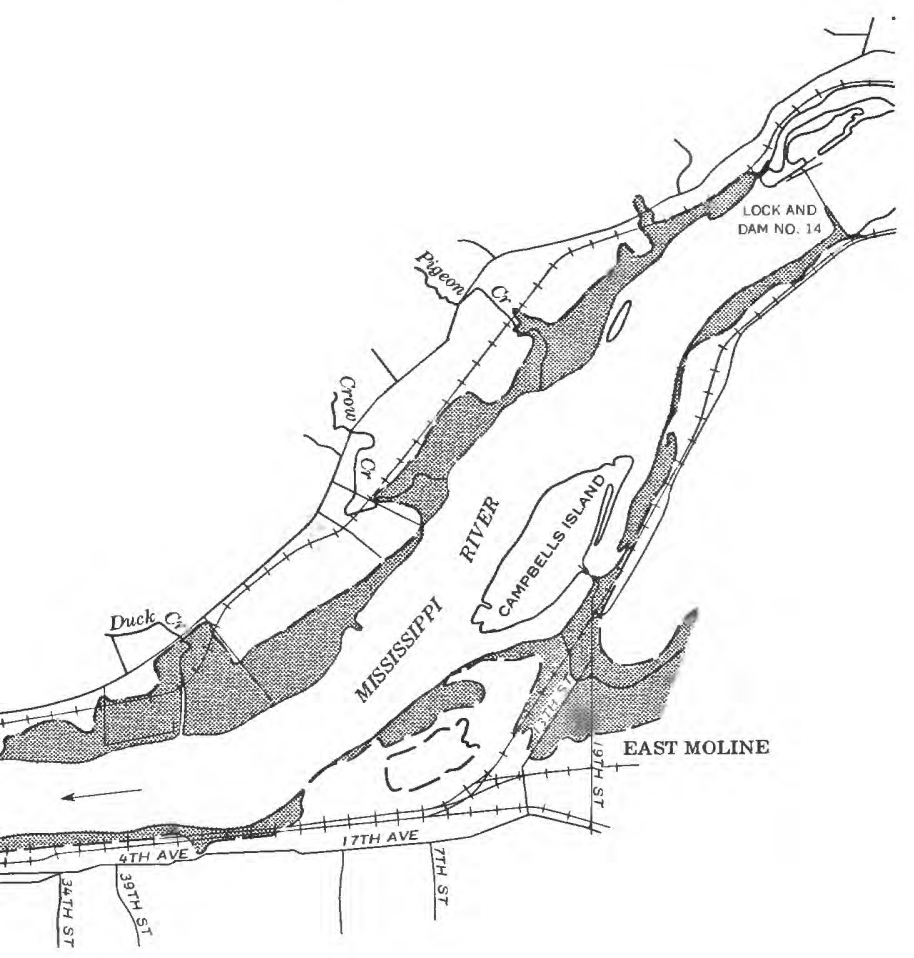

Figure 22.-Flooded area in Davenport and Bettendorf, Iowa, and Rock Island, Moline and East Moline, Ill. 


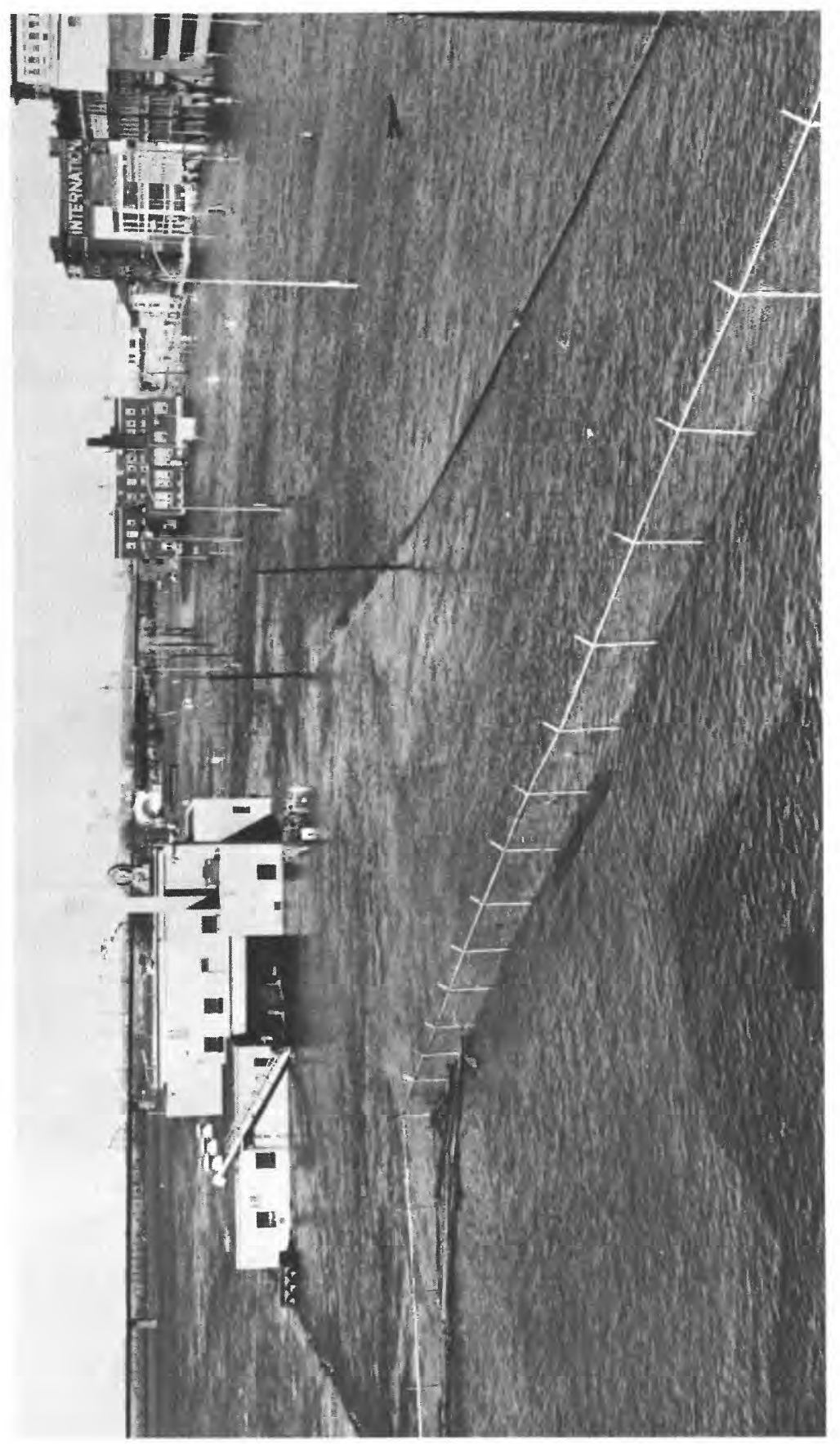

है

를

$>$

बें

密

है

要

密

을 핻

范南

ॠ岁

in

능

ने 믐

क

舟车

蛋

등

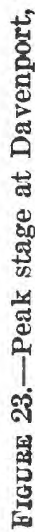




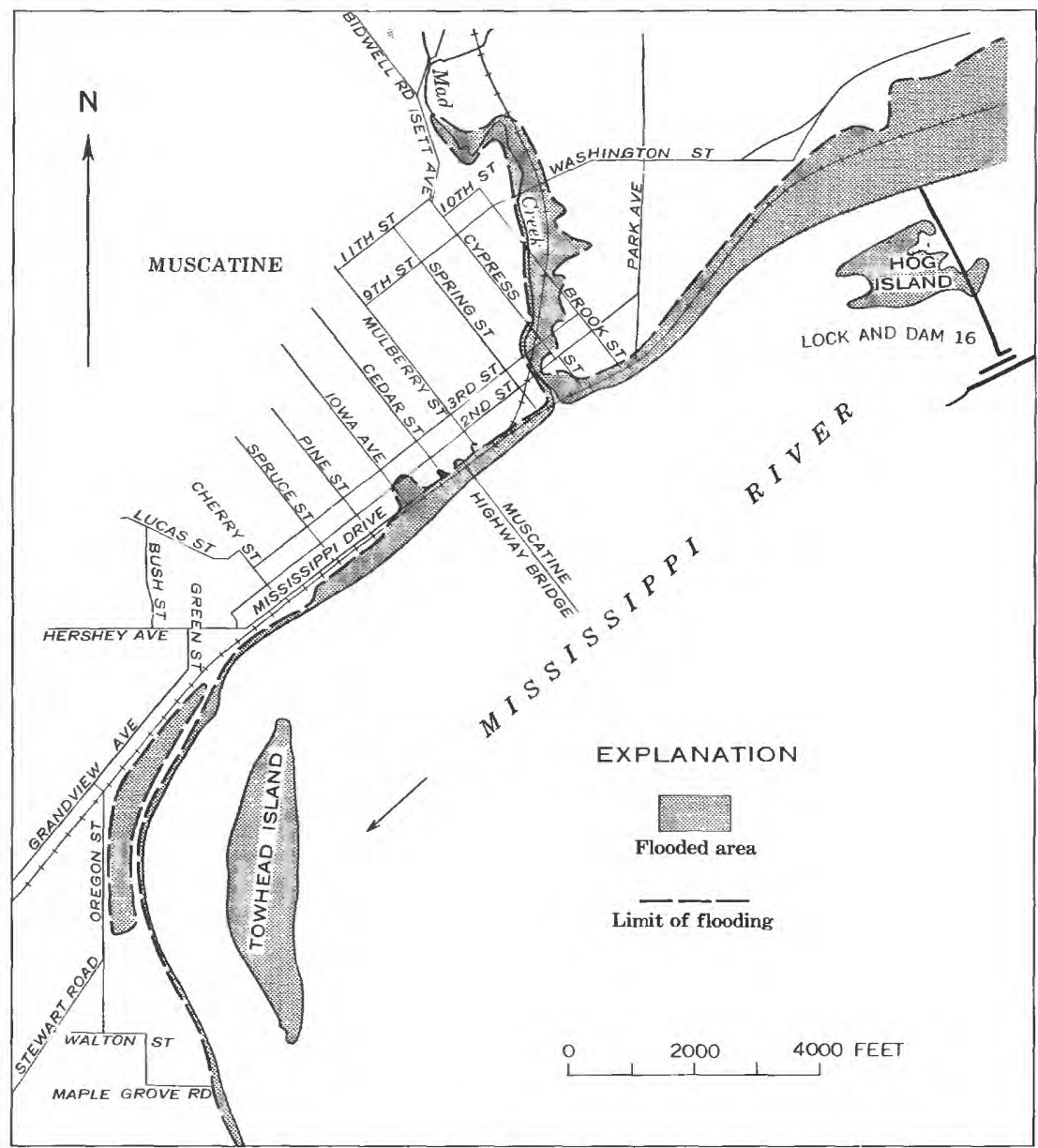

Figure 24.-Flooded area in Muscatine, Iowa.

water. During the extremely high discharge period, the pool elevation of the Union Electric dam at Keokuk was more than half a foot lower than the elevation a month earlier at much lower discharges. Many of the gates of the dam were open during the peak discharge permitting the natural flow of the river to pass. The maximum stage of 22.14 feet, which was 6.14 feet above flood stage, occurred on May 1 at Keokuk. This stage, which is the tailwater elevation at the Union Electric dam, exceeds the previous maximum historic flood stage of 21.0 feet which occurred in June 1851. The 1965 maximum discharge is the highest since May 1878. Keokuk's waterfron't industrial area, Commercial Alley, was flooded during both the April and May high water periods. 


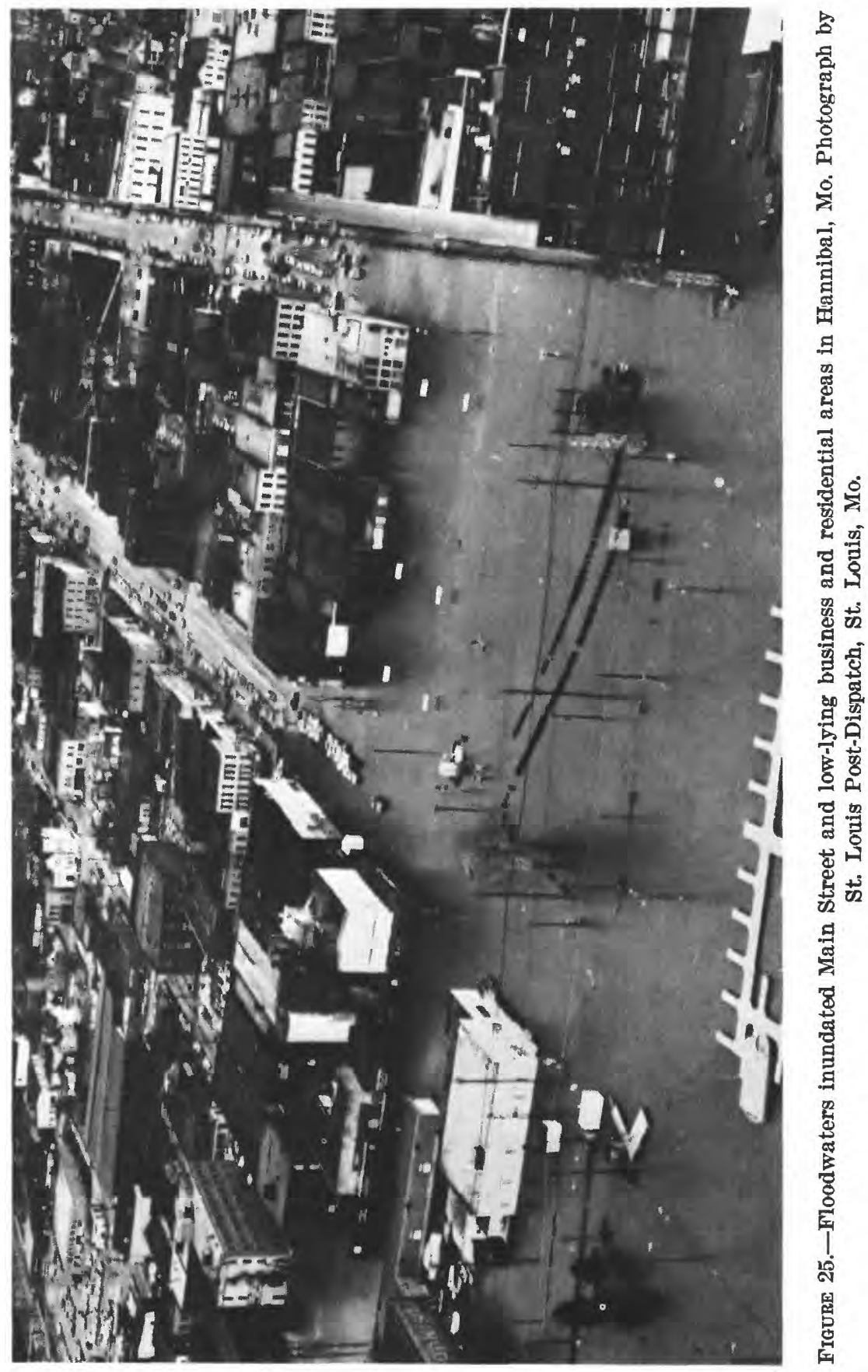


All waterfront industries except one closed for 2-3 weeks. Over 1,000 persons were temporarily dismissed from work. Downstream from the Union Electric dam at Keokuk, Iowa, there were two major crests on the Mississippi River during the period March-May 1965. The first crest occurred in April as a result of high inflow from the Des Moines River and other tributaries. The second crest occurred early in May. The May crest exceeded the April crest except in the reach between La Grange and Palmyra, Mo., where levee breaks lowered the peak stage in May, and in the reach downstream from Grafton, Ill., where less backwater from the Missouri River, occurred in May.

Downstream from Mundy's Landing, Mo., the 1965 crest was lower than the previously known maximum at each point of determination. Two peaks about equal in discharge occurred at Alton, Ill., on April 19 and May 13. Higher peaks discharged at Alton in 1943 and 1944, and a peak discharge in 1947 was equal to that of 1965 , but in each of the 3 earlier years, overflow from the Missouri River, caused by levee breaks, entered the Mississippi River upstream from Alton. After deducting this overflow, the peak discharges in 1943, 1944, and 1947 were lower than the peak discharge in 1965 when there was nc overflow. The peak discharge of $380,000 \mathrm{cfs}$ at Alton has a recurrence interval of 11 years.

The 1965 maximum discharge at St. Louis, below the mouth of the Missouri River, was 525,000 cfs on April 16, about equal to a 2-year flood. This peak was relatively small because the Missouri River, which is now extensively regulated, did not contribute greatly to the flood condition. The Missouri River inflow was greater in April than in May, and as a result the maximum discharge at St. Louis occurred during the April peak. The highest discharge at St. Louis in May was 431,000 cfs on May 1.

Most of the flood damage below Keokuk, Iowa, resulted from inundation of farmland, river cottages, highways, and business houses. The residents of Alexandria, Mo., were evacuated during both the April and May crests. The system of previously constructed levees, floodwalls, pumping plants, and closure structures successfully prctected Canton, Mo., and prevented more than $\$ 2$ million damages. Quincy, Ill., incurred damages of about $\$ 367,000$ including cast of flood fighting. The floodwaters in April and May inundated Main Street and lowlying business and residential areas in Hannibal, Mo. (fig. 25). Sandbagged storefronts and improvised walkways permitted most stores to remain open for business. Hannibal incurred damages and floodfighting costs of about $\$ 875,000$. The Mark Twain Memorial Bridge at Hannibal and U.S. Highway 36 in Kinderbrook, Ill., were closed. The main river levee failed near Saverton, Mo., and the floodwaters spread 

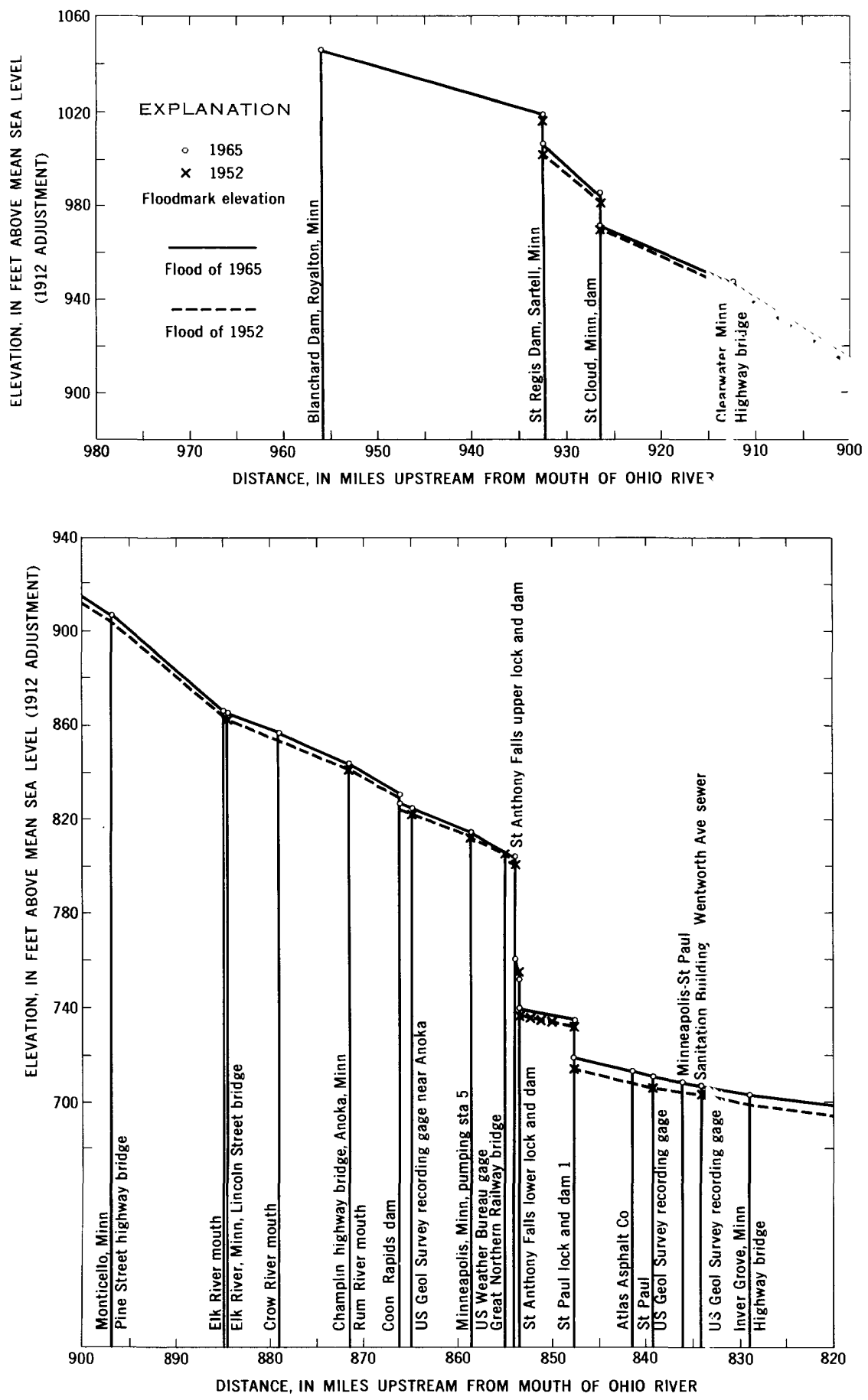

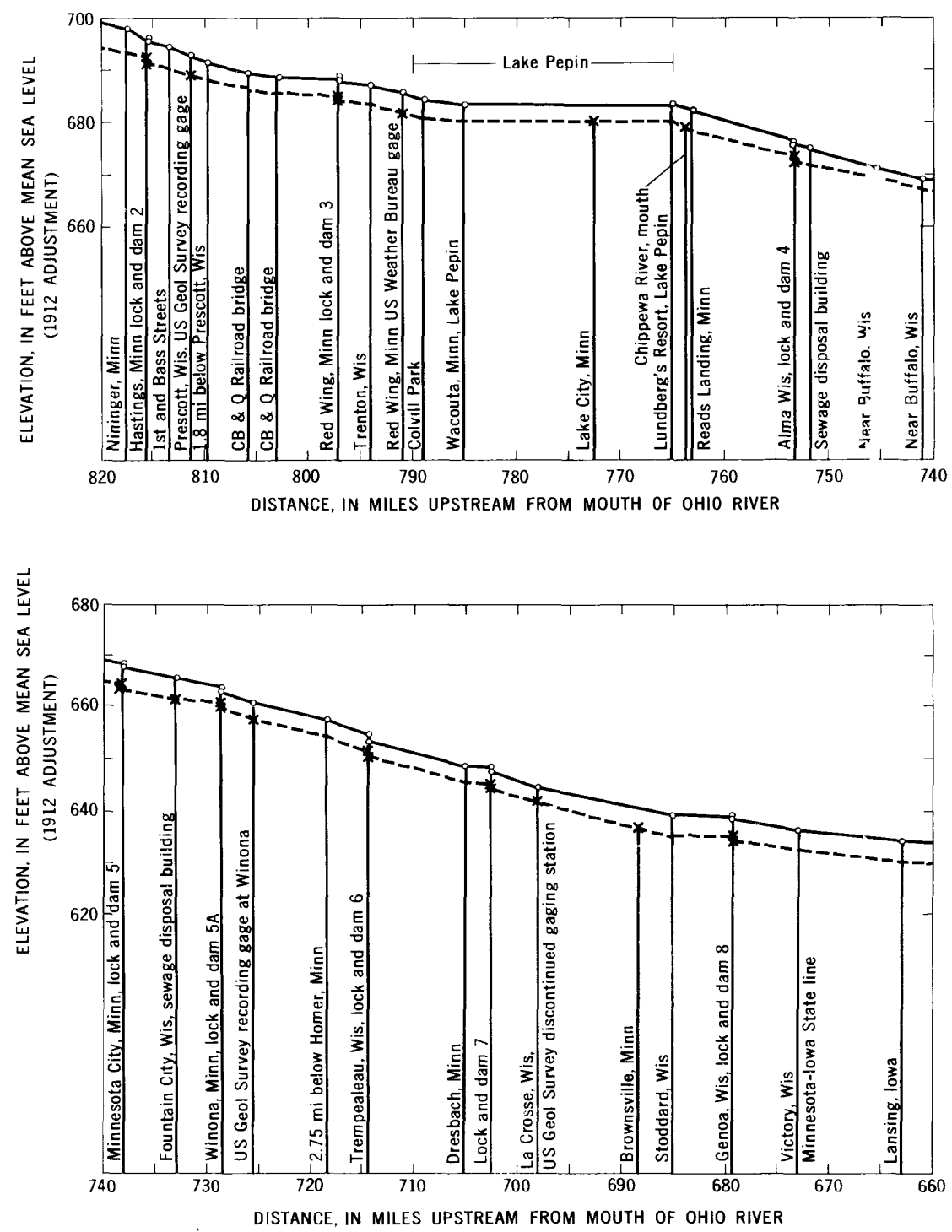

Frgure 26.-Flood-crest profiles of Mississippi River. Water-surface elevations were obtained at U.S. Geological Survey gages or were selected ffrom high-water data furnished by the U.S. Army Corps of Engineers.

(Figure 26 continues on next page.) 

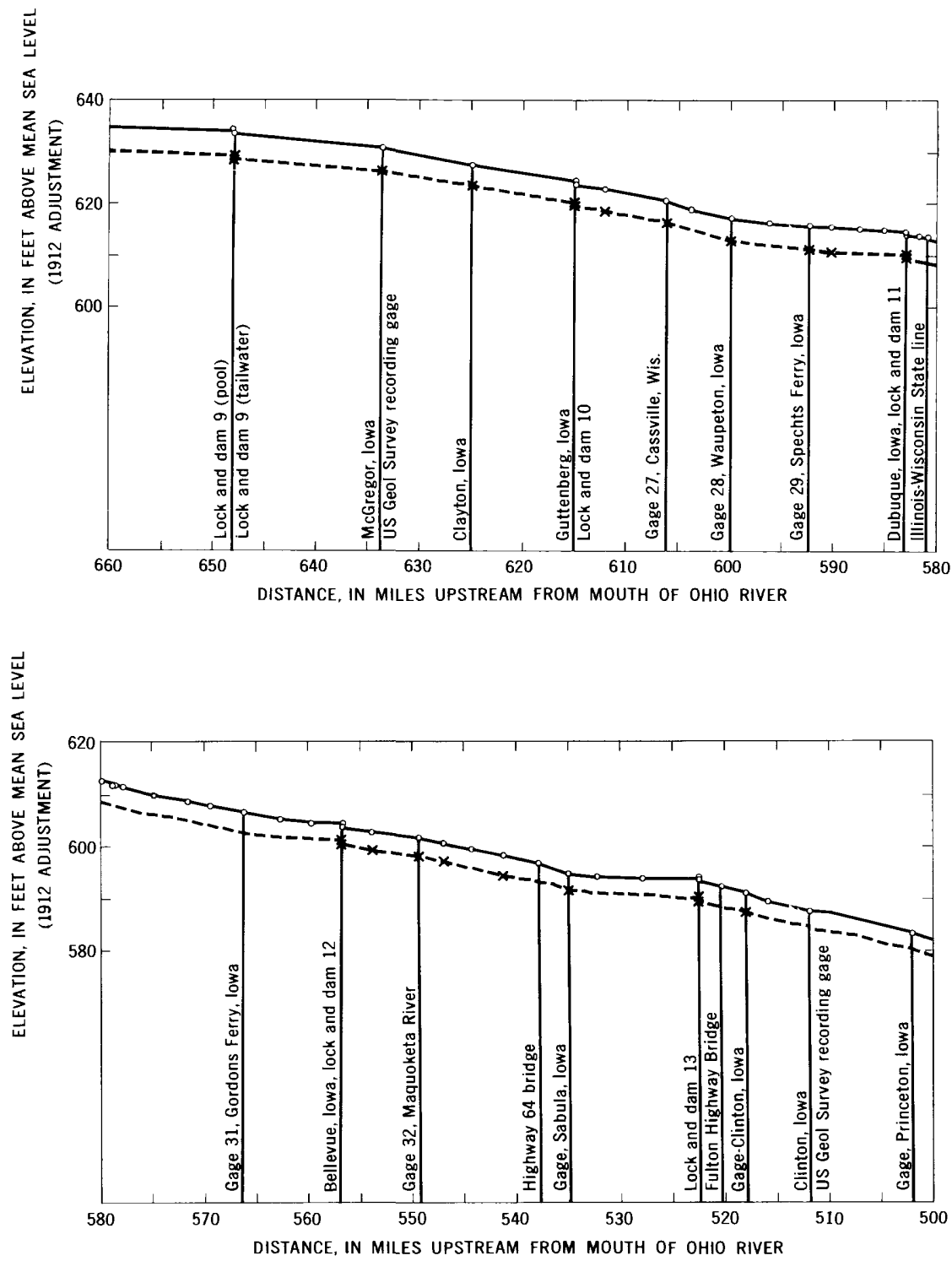

Figure 26.-Continued. (Flood-crest profiles of Mississipp: River. Water-surface elevations were obtained at U.S. Geological Survey gages or were selected from high-water data furnished by the U.S. Army Corps of Engineers.) 

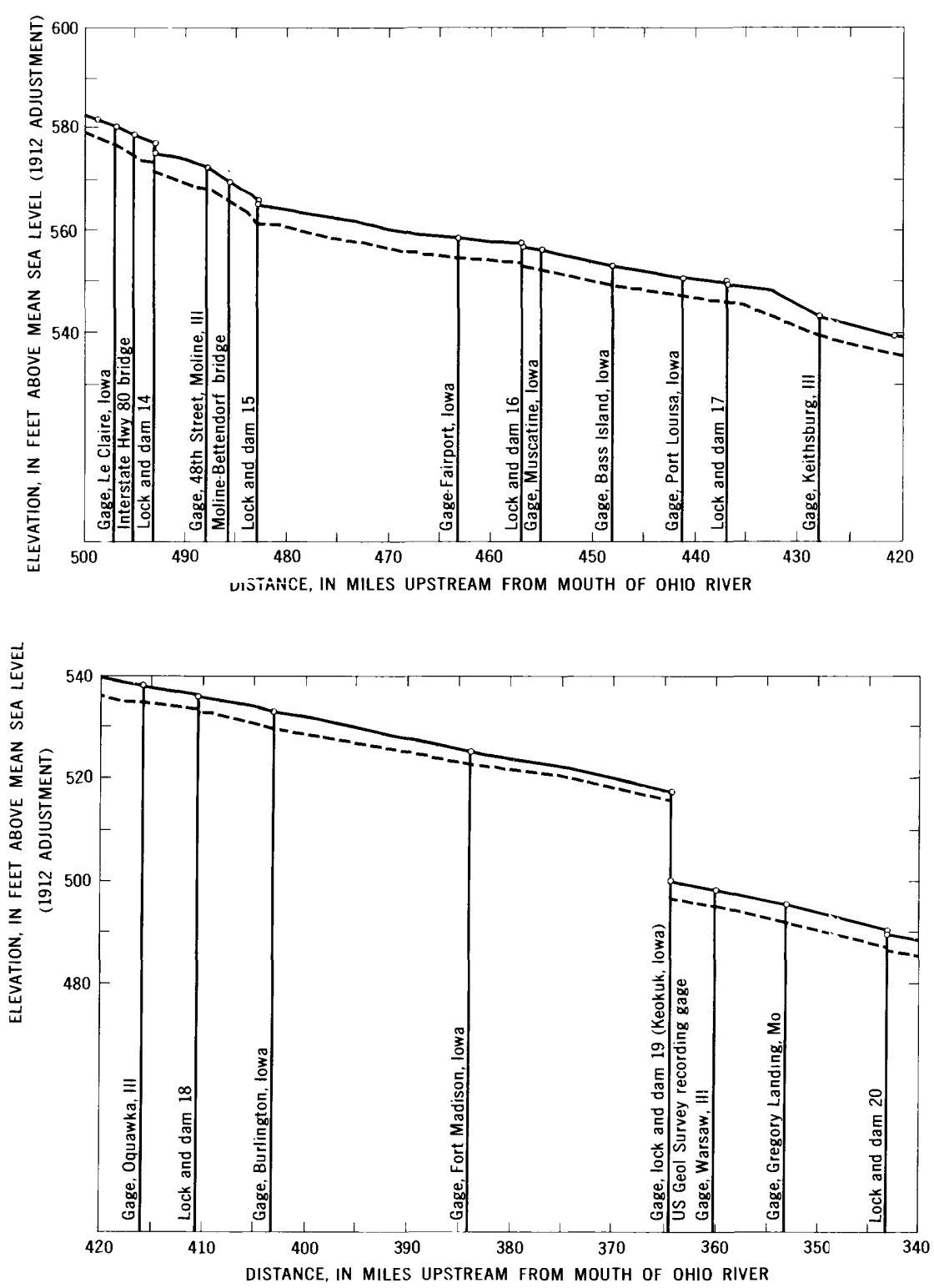

Figure 26.-Continued.

(Figure 26 continues on next pag?.) 

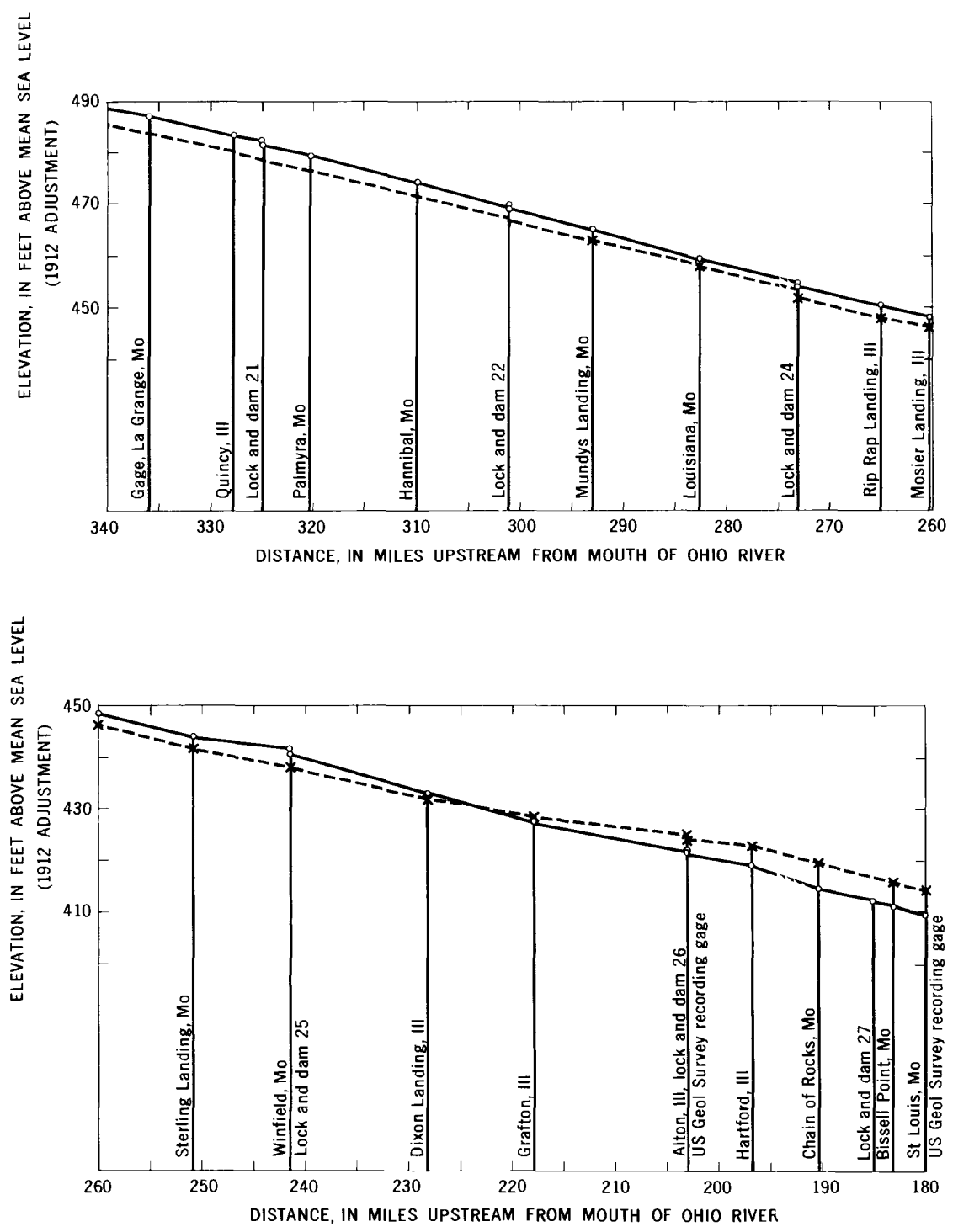

Frgure 26.-Continued. (Flood-crest profiles of Mississippi River. Water-surface elevations were obtained at U.S. Geological Survey gages or were selected from high-water data furnished by the U.S. Army Corps of Engineers.) 
over 1,000 acres of farmland engulfing the small community of Hull, Ill., to depths of $4-6$ feet. The 535 residents had been evacuated earlier.

Flood crest profiles, which are graphs of maximum flood elevations plotted against river miles, are shown for the Mississippi River in figure 26. The 1965 maximum elevations are compared with those which occurred in the 1952 flood. Table 1 describes and tabulates the 1965 flood elevations used in plotting the profiles. Comparative discharge hydrographs for the 1965 flood are shown for Mississippi River gaging stations in figure 27.

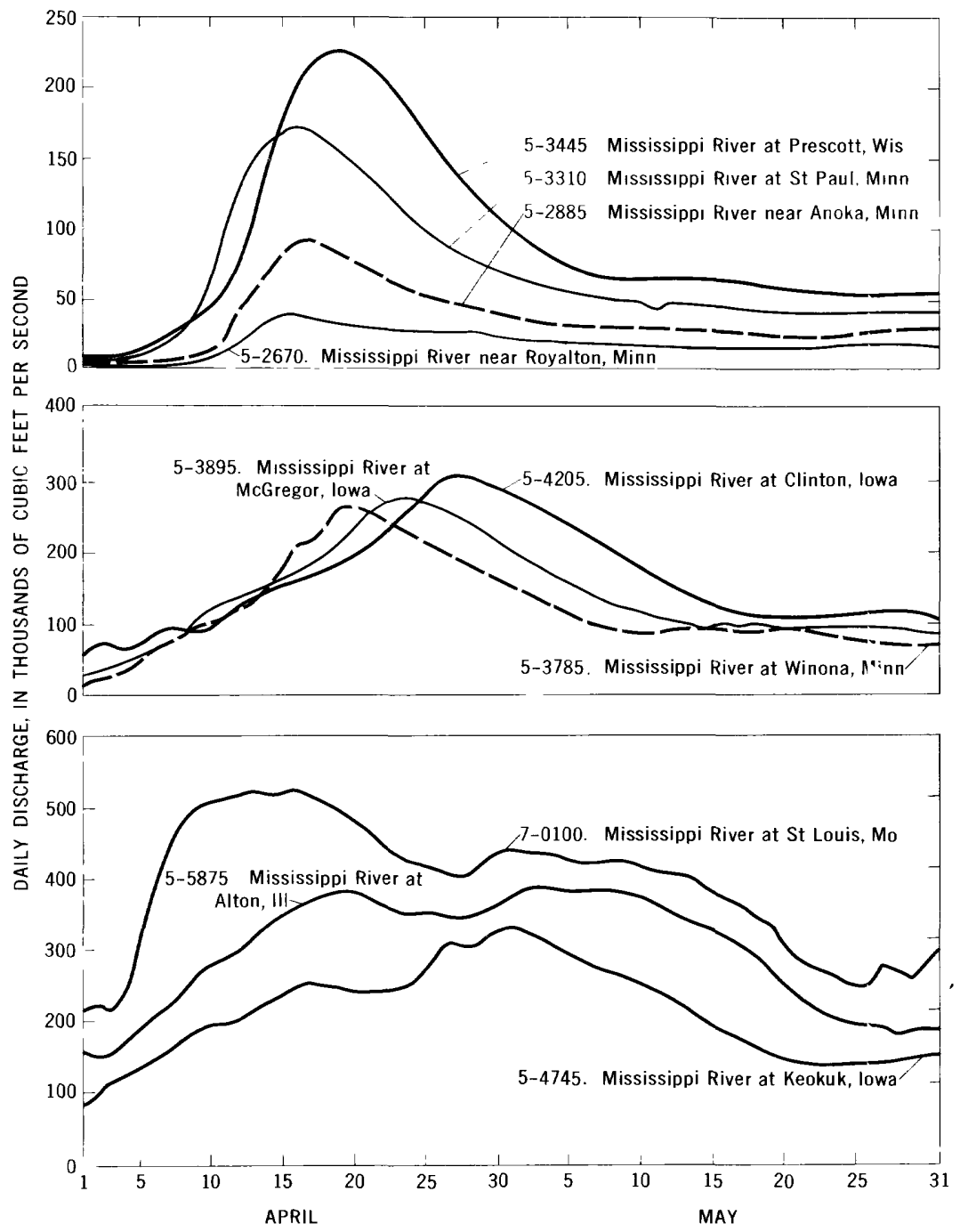

Figure 27.-Comparative discharge hydrographs at selected gaging stations on the Mississippi River, April-May 1965. 


\section{TABLE 1.-Flood-crest elevations, Mississippi River}

[Based on data furnished by U.S. Army Corps of Engineers except at U.S. Geological Survey gaging stations]

\begin{tabular}{|c|c|c|c|c|c|}
\hline \multirow[b]{2}{*}{ Location } & \multirow[b]{2}{*}{$\begin{array}{c}\text { Miles } \\
\text { above } \\
\text { mouth } \\
\text { of } \\
\text { Ohio } \\
\text { River }\end{array}$} & \multicolumn{2}{|c|}{1952} & \multicolumn{2}{|c|}{1965} \\
\hline & & Date & $\begin{array}{l}\text { Eleva- } \\
\text { tion in } \\
\text { feet } \\
(1912 \\
\text { adjust- } \\
\text { ment) }\end{array}$ & Date & $\begin{array}{c}\text { Eleva- } \\
\text { tion in } \\
\text { foet } \\
\text { (1912 } \\
\text { adjust- } \\
\text { ment) }\end{array}$ \\
\hline
\end{tabular}

Blanchard Dam, tailwater

Sartell, Minn.:

St. Regis Paper Co. dam, pool..

St. Regis Paper Co. dam, tailwater

St. Clond, Minn.:

Northern States Power Co. St. Cloud dam,

Northern States Power Co. St. Cloud dam,

tallwater

Clearwater, Minn., at Minnesota State Highway

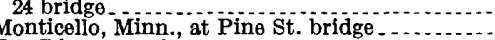

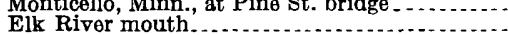

Elk Rivor, Minn., at Lincoln st. bridge.

Crow River mouth . . . Anoka, Minn.:

Upstream side of U.S. Highway 52 bridge... Downstream side of U.S. Highway 52 bridge

Northern States Power Co. Coon Rapids dam, pool ................................

U.S. Geological Survey recording gage near Anoka, Minn .................. Minneapolis, Minn.:

Pumping station 5 and U.S. Weather Bu-

reau gage -..... bridge, west ond, down-
Plymouth Ave.

stream
Plymouth Ave. bridge, east end, downstream.

Left end Great Northern Railway bridge ..

Right end Great Northern Railway bridge

St. Anthony Falls upper lock and dam, pool.

St. Anthony Falls upper lock and dam, tailwater.

St. Anthony Falls lower lock and dam, pool St. Anthony Falls lower lock and dam, tailwater.

Minneapolis barge terminal

Franklin Ave. bridge, east end, upstream..

Franklin Ave. bridge, west end, downstream.

Lake St.-Marshall A ve. bridge

Lock and dam 1, pool.

Lock and dam 1 , tailwater

Ford Bridge, west end, upstream . .......

St. Paul, Minn.:

Ford Bridge, east end, upstream.............

Atlas Asphalt Co. garage, $375 \mathrm{ft}$ upstream from railway bridge.

U.S. Geological Survey recording gage

Minneapolis-St. Paul Sanitary District building.

Chicago Great Western Ry. bridge, southwest corner.

Wentworth A ve sewer outlet

South St. Paul, U.S. Geological Survey recording gage.

Inver Grove, Minn., at highway bridge.........

Nininger, Minn

Hastings, Minn. at:

Lock and dam 2, pool.

Lock and dam 2, tailwater

Northeast corner East and Bass St..........

Prescott, Wis., U.S. Geological Survey rocording gage.

Profile point, 1.8 miles below Prescott, Wis

Riverward side and upstream end of CB\&QRR bridge.
956. 0

Apr. 15, 16_ _ 1, 045.4

Apr. 12 . . . 1, 015.87 Apr. 14 .... 1,019.10 932.5 Apr. 13.... 1,001.95 Apr. 14.... 1,005.95

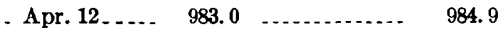

926. 5 Apr. 12.... 971.0 Apr. 14_... 971.2

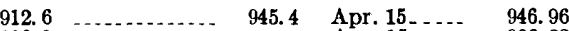

896. 9 .

896.8
884

884.6 Apr. $12 \ldots . .862 .42$ Apr. 16.... 865.12

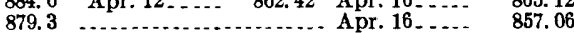

871.46 Apr. 11-18_ 842.1 Apr. 17 . 844

843. 8

$866.3 \quad \ldots 31.4$

$866.3 \quad 326.6$

864.8 Apr. 14_... 822.53 Apr. $17 \ldots \ldots$. $\quad 824.55$

858.6 Apr. $14 \ldots \ldots 813.80 \ldots \ldots \ldots \ldots$

855.0 Apr. $15 \ldots . .805 .98 \ldots \ldots$

854.8 Apr. $15 \ldots 802.14 \ldots \ldots \ldots \ldots$

854.8 Apr. $15 \ldots 802.54 \ldots 8$

853.85 Apr. $14 \ldots . . .802 .6$ Apr. $17 \ldots \ldots$

853.85 Apr. 14 ............... Apr. $17 \ldots \ldots$

853. 5 Apr. $12 \ldots 753.20$ Apr. $17 \ldots \ldots \quad 751.42$

853. 5 Apr. $12 \ldots$ 737.10 Apr. $17 \ldots \ldots$ 739. 02

852.6 Apr. $14 \ldots . .736 .70 \ldots \ldots \ldots \ldots$

851. 46 Apr. $15-734.86$

851.48 Apr. 15

849. 55 Apr. $15 \ldots 733.20 \ldots \ldots$

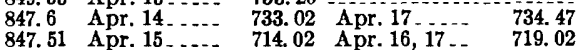

847.40 Apr. $15 . . .-732.71$....

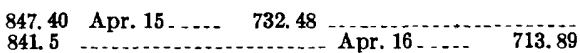

839.3 Apr. 16 . ... 706.18 Apr. 16 . . $\quad 710.17$

836. 35 Apr. $16 . . . .508 .5$

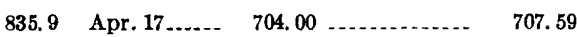

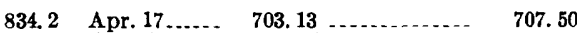

833.7 Apr. $16 \ldots \ldots$..... $703.15 \ldots$

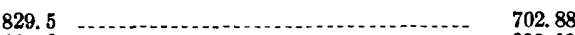

817.6 .

815. 42 Apr. 16, 17 . 692.40 Apr. 17.... 697.07

814.99 Apr. 16.... 691.59 Apr. 17..... 696.13

813.45 A

811. 4 Apr. 16-18... 689.03 Apr. 18...

$809.7 \quad \ldots \ldots \ldots 1.21$

$805.79 \ldots 96$ 
TABLE 1.-Flood-crest elevations, Mississippi River-Continued

\begin{tabular}{|c|c|c|c|c|c|}
\hline \multirow[b]{2}{*}{ Location } & \multirow[b]{2}{*}{$\begin{array}{c}\text { Miles } \\
\text { above } \\
\text { mouth } \\
\text { of } \\
\text { ohio } \\
\text { River }\end{array}$} & \multicolumn{2}{|c|}{1952} & \multicolumn{2}{|c|}{1965} \\
\hline & & Date & $\begin{array}{c}\text { Eleva- } \\
\text { tion in } \\
\text { feet } \\
\text { (1912 } \\
\text { adjust- } \\
\text { ment) }\end{array}$ & Date & $\begin{array}{l}\text { Eleva- } \\
\text { tion in } \\
\text { feet } \\
(1912 \\
\text { adjust- } \\
\text { ment) }\end{array}$ \\
\hline
\end{tabular}

Downstream landward end of CB\&QRR bridge.

Lock and dam 3, pool, near Red Wing, Minn....

Lock and dam 3, tailwater, near Red Wing,

Minn.

Trenton, Wis., at Evert's Resort.

Red Wing, Mínn.

U.S. Weather Bureau gage

Colvill Park, upstream riverward corner of tennis court.

Wacouta, Minn., on right bank of Lake Pepin.. Lake City, Minn., on right bank Lake Pepin, on bridge $\mathrm{L} 136$ at Lund berg's resort.

Chippewa River mouth . . . . . . .

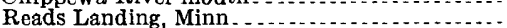
Alma, Wis.:

Lock and dam 4, pool.

Lock and dam 4, tailwater

年

Sewage disposal building

Profile point nar Buffalo, Wis

Profile point near Buffalo, Wis

Lock and dam 5, pool

Lock and dam 5 , tailwater

Fountain City, Wis., at sewage disposal building Winona, Minn.:

Lock and dam $5 \mathrm{~A}$, pool.

Lock and dam $5 \mathrm{~A}$, tailwater

U. S. Geological Survey recording

Railroad bridge on right bank, $2.75 \mathrm{mi} \mathrm{be}$ low Homer, Minn.

Lock and dam 6, pool, near Trempealeau, Wis Lock and dam 6, tailwater near Trempealeau, Wis

Lock and dam 7, pool, near Dresbach, Minn ...

Lock and dam 7, tailwater, near Dresbach, Minn La Crosse, Wis

Discontinued U.S. Geological Survey gaging station.

Brownsville, Minn

Stoddard, Wis

Genoa, Wis.:

Lock and dam 8 , pool

Lock and dam 8, tailwater

(n)

Minnesota-Iowa State line

Upper

Uper lowa River mouth . ...........................

Lansing, Iowa.

Lynxville, Wis:

Lock and dam 9, pool.

Lek and dam $\theta$, tallwater.................... Lock and dam

U.S. Geological Survey recording gage......

Clayton, Iowa

Guttenberg, Iowa:

Lock and dam 10, pool

Lock and dam 10, tailwater

Profile point .

Turkey River mouth

Gage at Cassville, Wis

Profile point

Gage at Waupeton, Iows

Profile point

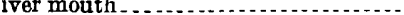

Gage at Spechts Ferry, Iowa

Profile point ....

Platte River mouth

Profile point

Little Maquoketa River mouth

Profile point.
803. 00

688.86

797.06

688.22

796. 69 Apr. 17...... 684.14 Apr. 18.....

687.93

794, 10

687.10

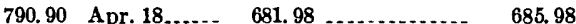

788. 92 Apr. $18 \ldots \ldots . . .684$

$785.0 \quad \ldots \ldots \ldots \ldots . .41$

764.9

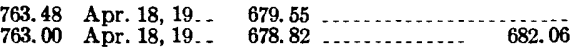

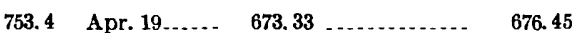

$753.2 \quad$ Apr. 19..... $672.33 \ldots \ldots \ldots \ldots \ldots$

751.8 Apr.

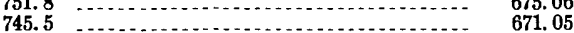

$741.3 \quad 369.46$

738.27 Apr. 19, 20 - 664.56 Apr. $19 . . .668 .73$

737.90 A pr. $20 \quad 663.85$ Apr. 19

732. 7 665. 74

728.65 Apr. 19, 20.- 660.40 Apr. 19..... 663.74

728. 28 Apr. 20.... 659. 88 Apr. 20.... 663.35

725.7 Apr. 20 ... 658.03 Apr. 19 $\quad 660.89$

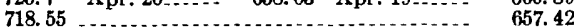

714. 44 Apr. 20_... 651.07 Apr. 20 ... 654.65

714. 07 Apr. 20..... 649.97 Apr. 20 .... 653.02

702.61 Apr. 20 644.85 Apr.21 648.18

702.24 Apr. 20 644.30 Apr. $21 \cdots$

697.78 Apr. 20.... 641.64 Apr. 22 .... 644.28

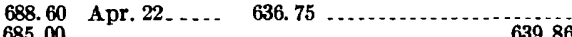

679. 39 Apr. 22 .... 635.30 Apr. 22 _... 639.05

679.02 Apr. 22.... 634.52 Apr. 22, 23.. 630.40

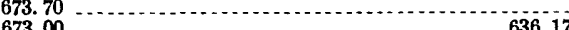

663.00 ............. 636.17

663. 0 Apr. $23 . \ldots 630.40$ Apr. $24 \ldots \ldots$

648.1 ................... Apr. 24 633.8

647. 7 Apr. $24 \ldots \ldots$ 633. 2

633.4 Apr. $24 \ldots \ldots$ 626.19 Apr. $24 \ldots \ldots 630.7$

624. 8 Apr. $24 \ldots . .623 .15$ Apr. $24 \ldots . .6627 .2$

615. 3 Apr. $24 \ldots \ldots 620.20$ Apr. $24 \ldots \ldots$.... 624.2

615.1 Apr. 24.... 619.68 Apr. 24_... 623.6

612.0 Apr. 24.... 618.93 ........

608.1 Apr. $24 \ldots .616 .47$ Apr. $25 . . .620 .4$

603. 7 Apr. 618.9

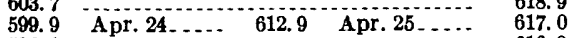

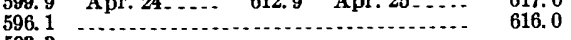

593.3 Apr. 24.611 .19 Apr. $25 . \ldots 615.5$

$590.2 \quad$ Apr. $24 . \ldots-610.80 \ldots \ldots$

$588.3 \quad \ldots$

586.5

585.2

614.8 
TABLE 1.-Flood-crest elevations, Mississippi River-Continued

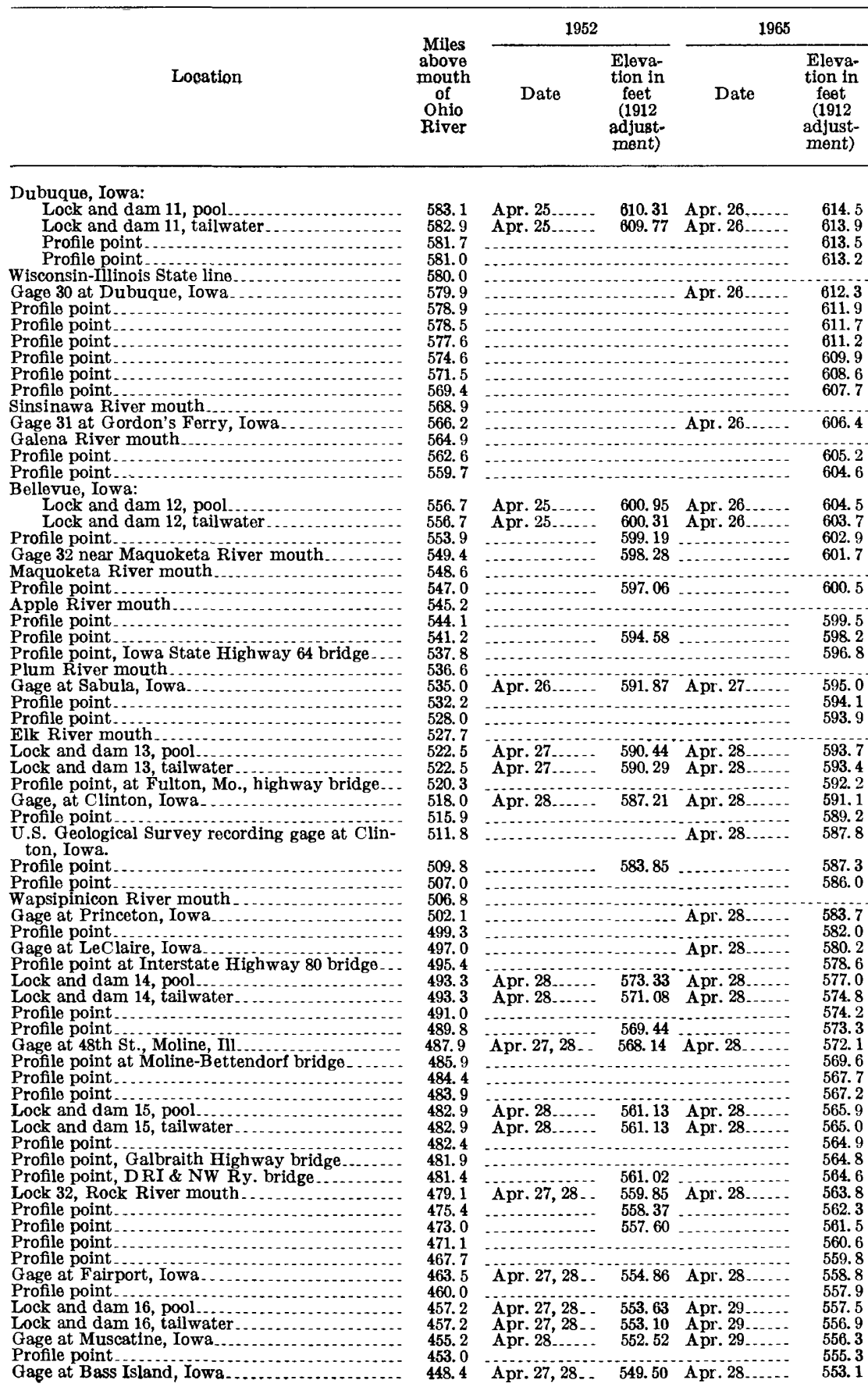




\section{TABLE 1.-Flood-crest elevations, Mississippi River-Continued}

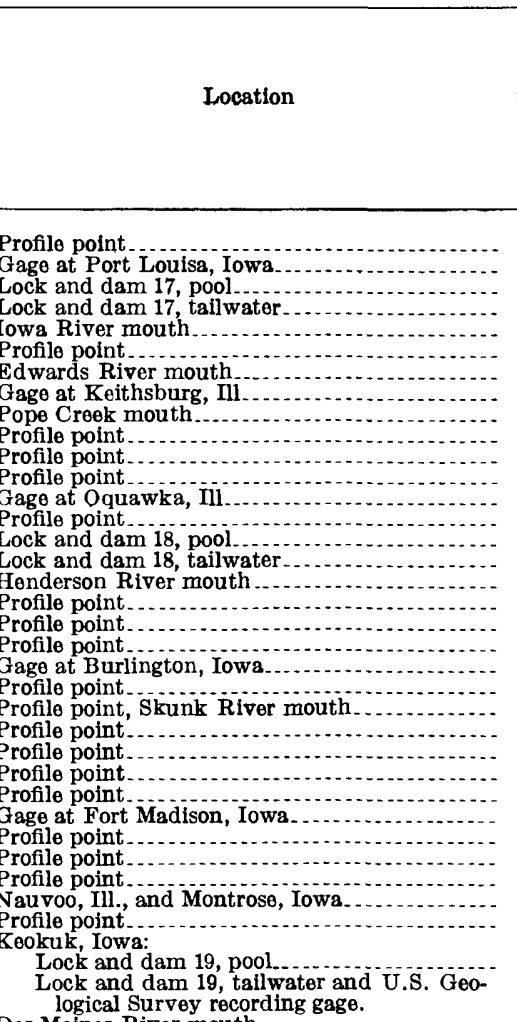

logical Survey recording gage.

Des Moines River mouth.

Gage at Warsaw, Ill

Fox River mouth.

Gage at Grogory

Lock and dam 20 ,anding

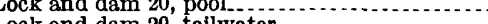

Water...................

Gage at LaGrange, Mo.

Qunicy, Ill

Lock and dam 21, pool....

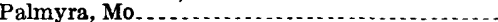

Saverton, Mo.:

Lock and dam 22 , pool...........................

Lock and dam 22 , tailwater

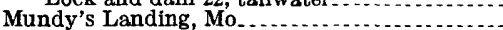

Louisiana, Mo

Clarksville, Mo.:

Lock and dam 24, pool..

Lock and dam 24, tailwater

Rip Rap Landing, II

\begin{tabular}{|c|c|c|c|c|}
\hline \multirow[b]{2}{*}{$\begin{array}{c}\text { Miles } \\
\text { above } \\
\text { mouth } \\
\text { of } \\
\text { Ohio } \\
\text { River }\end{array}$} & \multicolumn{2}{|c|}{1952} & \multicolumn{2}{|c|}{1965} \\
\hline & Date & $\begin{array}{l}\text { Eleva- } \\
\text { tion in } \\
\text { feet } \\
\text { (1912 } \\
\text { adjust- } \\
\text { ment) }\end{array}$ & Date & $\begin{array}{l}\text { Eleva- } \\
\text { tion in } \\
\text { feet } \\
\text { (1912 } \\
\text { adjus t- } \\
\text { ment) }\end{array}$ \\
\hline
\end{tabular}

443. 7

441. 3

437.2

437.0

433. 5

432.9

431.3

428.0

427.9

425. 9

420. 6

418. 2

415. 9

413.0

410. 5

410.5

409. 9

408. 4

406. 5

404.1
403. 1

400.5

396. 0

392.6

389.5

388.1

387.0

383.9

382.7

381.5

379. 0

364. 3

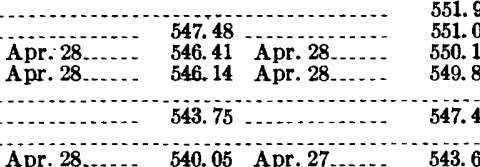

Apr. 28... 540.05 Apr. $27 \ldots$

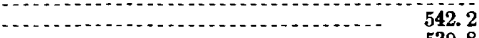

$\begin{array}{lll} & 0.0 & 539.8\end{array}$

Apr. $28,29.534 .64$ Apr. $30.5 \%$

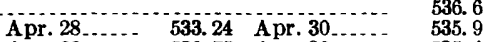

$\begin{array}{llll}\text { Apr. 28.... } & 532.75 & \text { Apr. } 30 \ldots . . & 535.4\end{array}$

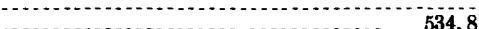

$\begin{array}{lll} & & \end{array}$

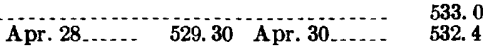

$\begin{array}{lll}\text { Apr. } 28 . \ldots . & 532.4 \\ & \end{array}$

530.1

(528.5

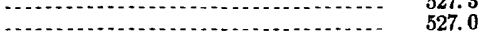

$\begin{array}{lll}\text { Apr. } 29 & 522.67 \text { May } 1 \ldots \ldots & 526.6\end{array}$

$\begin{array}{lll} & 524.7\end{array}$

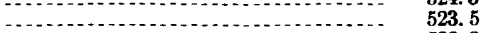

522.2

$\begin{array}{rrr} & & \end{array}$

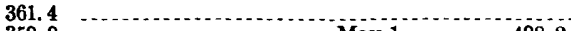

359.9 May $1 \ldots \ldots .2$

353.6 (352.9

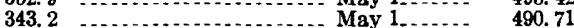

$343.2 \quad$ May 1... 489.92

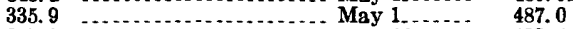

327.9 . .

324.9

324.9 $9 . \ldots \ldots \ldots$ Apr. $28 \ldots \ldots \ldots$

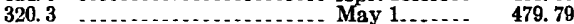

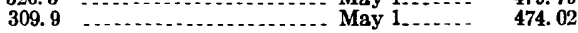

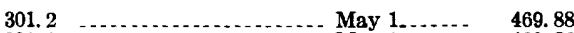

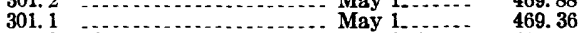

293. 0 Apr. 26... 463.0 May 1,4... 465.2

282.9 Apr. 26.... $457.5 \quad$ May 1, 4.... 459.6

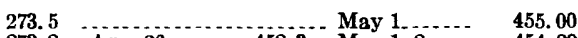

273.2 Apr. 26_... 452.3 May 1,2 2... 454.29

265. 0 Apr. 27..... 448.4 May 2..... 450.7

260.3 Apr. 27..... 446.4 Apr. 18, 4

(n)

Sterling Landing, Mo

Apr. 27.....- 446. 4 Apr. 18,
$\begin{aligned} & \text { May 2, } \\ & \text { May 4-6. }\end{aligned}$

250.8

Apr. 27..... $441.8 \quad$ May $8 . .$.

444. 0

241.5 - 5 May 2_... $\quad 440.85$

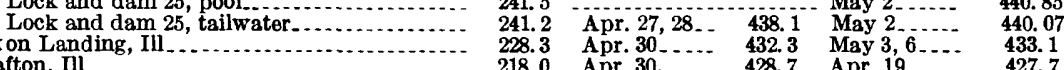

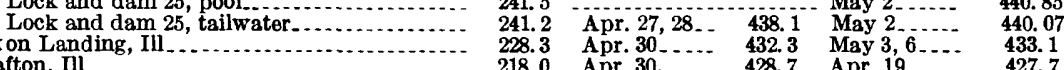

Dix on Landing, Ill

Aay 1.

428. 7 Apr. 19

427.7 
TABLE 1.-Flood-crest elevations, Mississippi River-Continued

\begin{tabular}{|c|c|c|c|c|c|}
\hline \multirow[b]{2}{*}{ Location } & \multirow[b]{2}{*}{$\begin{array}{c}\text { Miles } \\
\text { above } \\
\text { mouth } \\
\text { of } \\
\text { Ohio } \\
\text { River }\end{array}$} & \multicolumn{2}{|l|}{1952} & \multicolumn{2}{|l|}{1965} \\
\hline & & Date & $\begin{array}{c}\text { Eleva- } \\
\text { tion in } \\
\text { feet } \\
\text { (1912 } \\
\text { adjust- } \\
\text { ment) }\end{array}$ & Date & $\begin{array}{c}\text { Eleva- } \\
\text { tion in } \\
\text { feet } \\
\text { (1912 } \\
\text { adjust- } \\
\text { ment) }\end{array}$ \\
\hline \multicolumn{6}{|l|}{ Alton, Ill.: } \\
\hline Lock and dam 26, pool & 203.0 & & & Apr. 16 & 421. 90 \\
\hline $\begin{array}{l}\text { Lock and dam } 26 \text {, tailwater and U.S. Geolog- } \\
\text { ical Survey recording gage. }\end{array}$ & 202.7 & Apr. $30 \ldots$ & 424.75 & Apr. 16... & 421.03 \\
\hline 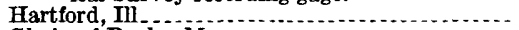 & 196.8 & Apr. 30. & 423. 58 & Apr. 16. . . & 419. 11 \\
\hline $\begin{array}{l}\text { Chain of Rocks, Mo } \\
\text { Lock and dam } 27 \text {, pool }\end{array}$ & $\begin{array}{l}190.4 \\
185.3\end{array}$ & Apr. $30 \ldots$. & 420.0 & Apr. 16.... & 414. 6 \\
\hline 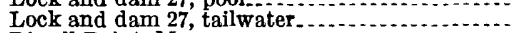 & 185.1 & & & Apr. $16 \ldots$ & 412.23 \\
\hline Bissell Point, Mo ..................... & 183.3 & Apr. 30, & 416.1 & Apr. 16..... & 411.1 \\
\hline $\begin{array}{l}\text { St. Louis, Mo., U.S. Geological Survey recording } \\
\text { gage. }\end{array}$ & 180.0 & May ... & 414.05 & Apr. 16.... & 408.05 \\
\hline
\end{tabular}

\section{SAUK AND ELK RIVER BASINS}

The Sauk River was the farthest upstream tributary in which major flooding occurred. Maximum water equivalent of snow in the Sauk River basin at the end of March was 10-11 inches (fig. 5), more than anywhere else in the upper Mississippi River drainage basin.

The maximum discharge of the Sauk River at St. Clond was 9,100 cfs, and the maximum daily discharge was $7,940 \mathrm{cfs}$ (fig. $2 \mathcal{2}$ ), by far the greatest during the 35 years of record. The stage exceeded the previous maximum, which occurred in 1951, by 2.8 feet. The maximum discharge was 1.82 times that of a 50-year flood. In spite of the heavy runoff in the Sauk River basin, there was not a great deal of flood damage. The Sauk River banks in the city of St. Cloud are relatively high, and water did not overflow them. Five counties in the Sauk River basin reported a total of $\$ 165,000$ damage to public property such as roads. streets, and bridges. Further damage was sustained at Melrose where the flood destroyed a recreational dam. There was also damage to homes, agricultural land, fences, and other private property throughout the basin.

Flooding in the Elk River basin resulted principally from the melting of a heavy snow cover. The Elk River and its trikitaries flow through flat, marshy land in a sand plain area throughout most of their reaches. There is little cultural development along the river; so flood damage was small. The maximum discharge at the gaging station near Big Lake was 7,360 cfs, 1.47 times the 50-year flood, and the highest in 40 years of record. 


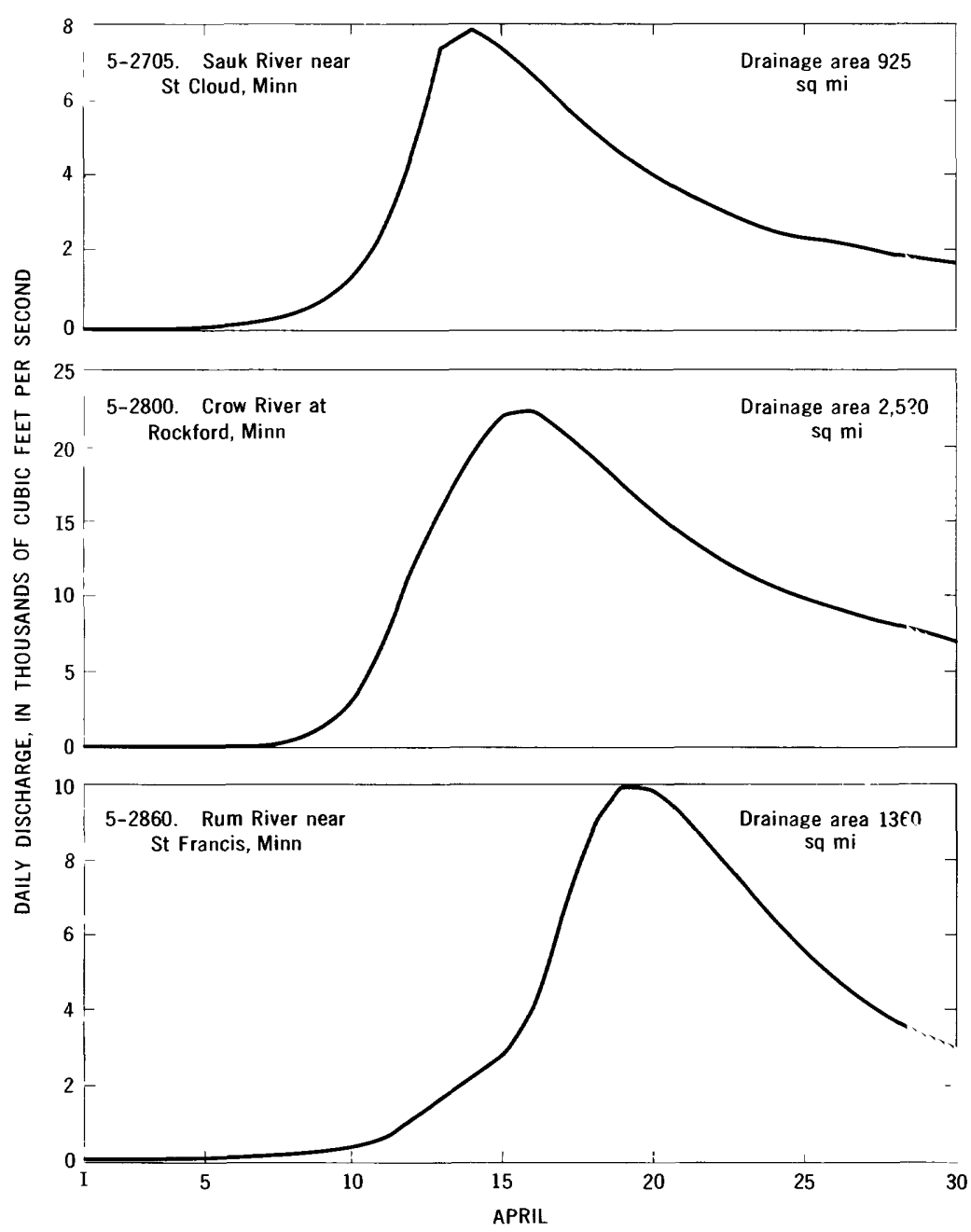

Figure 28.-Discharge hydrographs at selected gaging stations on Missirsippi River tributaries upstream from St. Paul, Minn., April 1965.

\section{CROW RIVER BASIN}

Flooding in the Crow River basin was very heavy as the result of the melting of a very dense snow cover. On April 1, the water equivelent of the snow in the basin ranged from 6 to 8 inches. Two to three inches of precipitation, most of which was rain, fell in the basin during the period April 1-12 and intensified the flood condition.

Flooding was heavy at the South Fork from Cosmos, Minn., to the confluence with the North Fork and throughout the entire reach of the main stem. At the discontinued gaging station on the North Fork 
near Regal, Minn., the stage and discharge were about the same as those in the 1951 and 1952 floods. The discharge was 1.39 times a 50 year flood. Flood-crest profiles of the North and South Forks of the Crow River are shown in figure 29, and flood-crest elevations are tabulated in table 2.

At Hutchinson, Minn., the South Fork crested on April 11 about 4 feet above flood stage, exceeding by 1.3 feet the previous record set by the 1952 flood. To prevent extensive flooding of the town, flood workers constructed emergency sandbag dikes which they connected to each abutment of a small recreation dam located upstream from State Highway 15 bridge. These sandbag dikes extended upstren m about 800 feet along each riverbank and prevented flow around the dam. By preventing this flow, the dikes kept much of the business district from being flooded. The Great Northern Railway crossing, about 1 mile downstream from the dam, was breached to prevent backwater from raising the stages of the river in Hutchinson. In spite of these measures, three highway and two railway bridges were severely damaged. Also, about 50 homes and 22 businesses between the dam and the railway

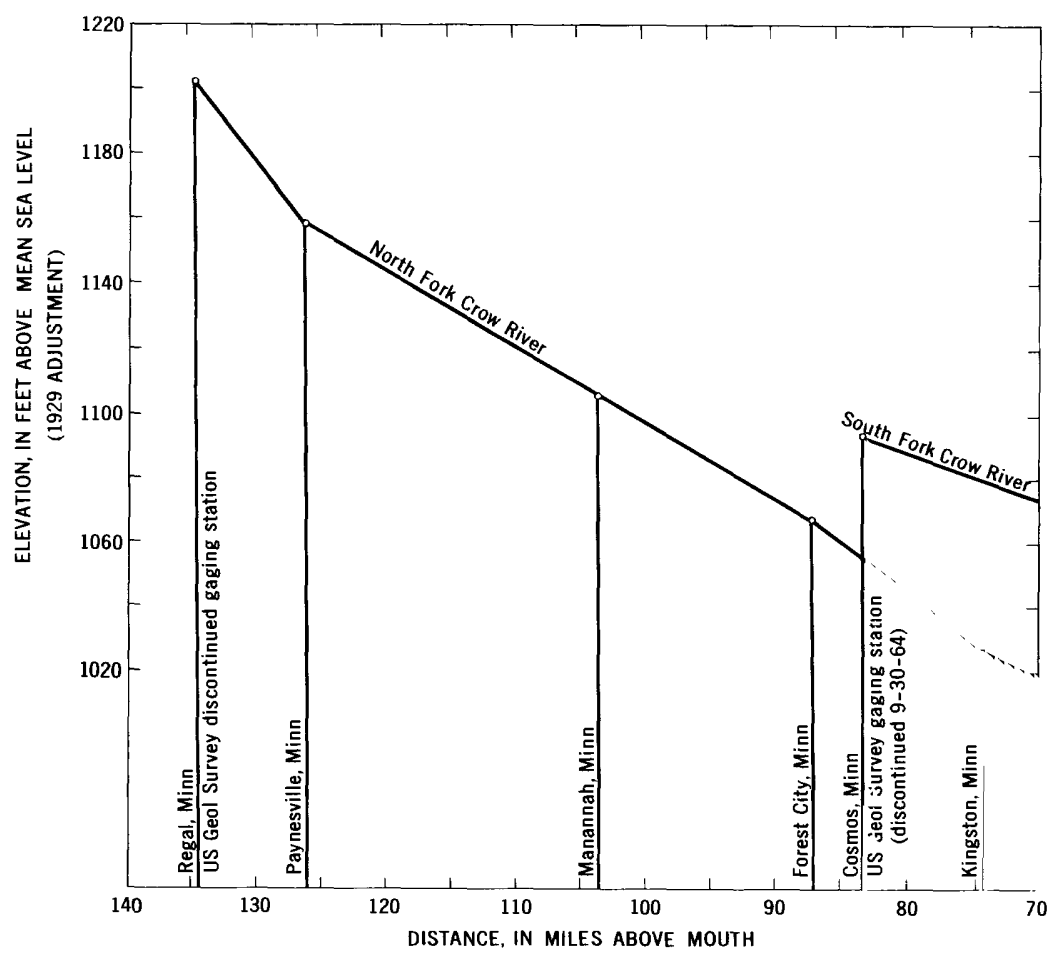

Frgure 29.-See explanation on next page. 
crossing sustained flood damages; the residents of these homes were evacuated. The continuous force of the floodwaters finally destroyed the recreational dam on April 19, as the water was receding. It was fortunate, however, that this dam remained in place during the earlier higher stages, because at the time of the dam's destruction, flood levels were too low to cause much damage. The dam provided the means for computing the maximum discharge by indirect methods.

The crest of the South Fork occurred at Watertown, Minn., on April 13, it was about 4.5 feet above flood stage and 3.5 feet above the previous record set in 1952 . Watertown was effectively protected by an emergency sandbag-and-earth dike, and only three homes outside the protected area were flooded.

The South Fork Crow River at Delano, Minn., crested on April 13, reaching a level 8.5 feet above flood stage; it was 3.0 feet higher than the 1925 flood, the previous maximum. Emergency sandbagging measures protected many residences and businesses of Delano. The first-

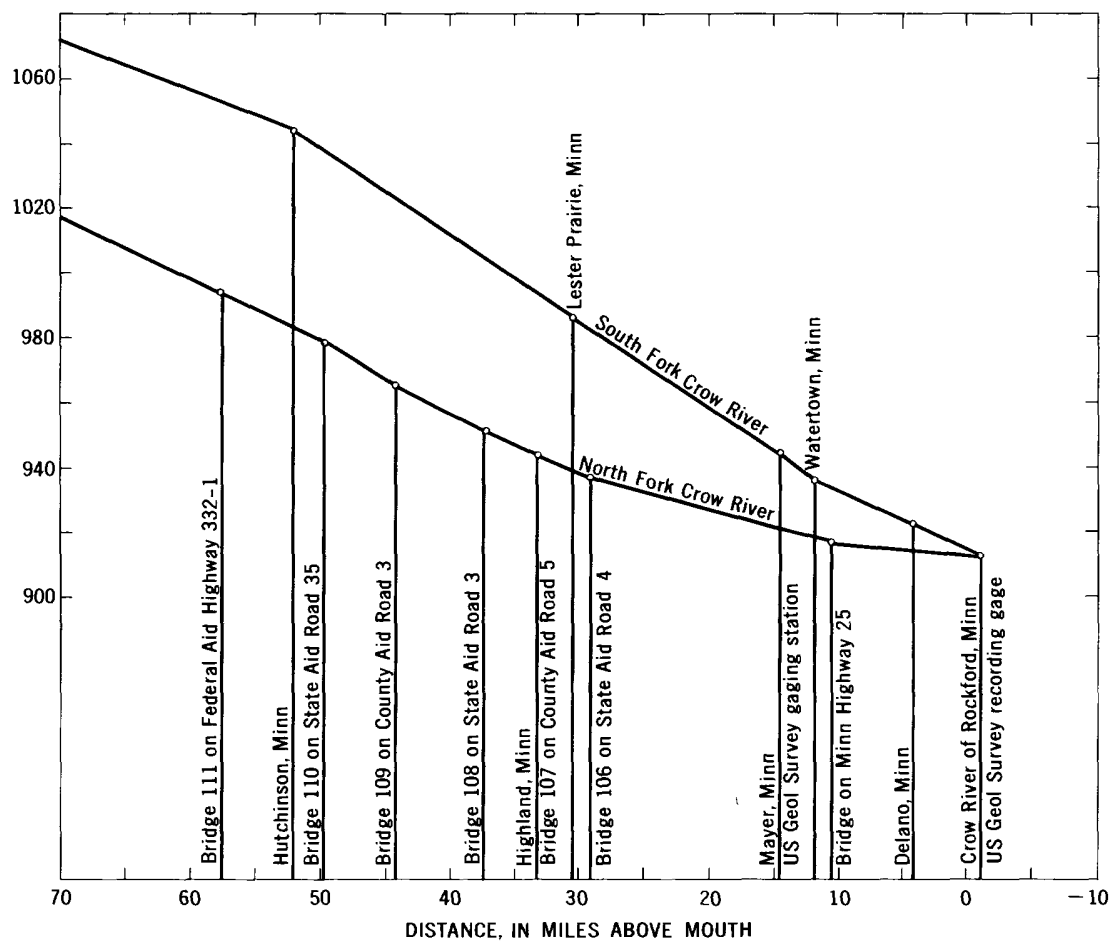

Figure 29.-Flood-crest proflles of the North and South Forks of Crow River. Water-surface elevations were obtained at U.S. Geological Survey gages or were selected from high-water data furnished by the U.S. Army Corps of Engineers. 
TABLE 2.-Flood-crest elevations, North Fork and South Fork Crow River [Based on data furnished by U.S. Army Corps of Engineers except at U.S. Geological Survey gaging stations]

Miles
above
confluence $\quad$ Date $\begin{gathered}\text { Elevation } \\ \text { in feet } \\ \text { (datum of 1929) }\end{gathered}$

\section{North Fork Crow River}

Regal, Minn., at U.S. Geological Survey discontinued gaging station

Paynesville, Minn., at bridge on Minnesota Highways 4 and 55

Manannah, Minn., at bridge on County Road $30_{-}$

Forest City, Minn., at bridge on Minnesota Highway $24 \ldots$

Kingston, Minn., at bridge on Minnesota Highway 15

Bridge 111 on Federal Aid Highway 332-1.....

Bridge 110 on State Aid Road 35....

Bridge 109 on County Aid Road 3.........

Bridge 108 on State Aid Road 3.

Highland, Minn., at bridge 107 on County Aid Road 5 .

Bridge 106 on State Aid Road 4

Bridge 103 on Minnesota Highway $25 . . . . .$.

Rockford, Minn., at U.S. Geological Survey re-

recording gage

\begin{tabular}{|c|c|c|}
\hline 4.5 & Apr. 9 & \\
\hline $\begin{array}{l}126 \\
103.5\end{array}$ & $\ldots$ & $\begin{array}{l}1,157.7 \\
1,105.7\end{array}$ \\
\hline 86.8 & & $1,066$. \\
\hline $\begin{array}{l}74 \\
57 . \\
49 . \\
44 . \\
37 .\end{array}$ & & $\begin{array}{r}1,025 . \\
994.5 \\
978.9 \\
965.8 \\
951 .\end{array}$ \\
\hline $\begin{array}{l}33.2 \\
29.2 \\
10.4\end{array}$ & & $\begin{array}{l}944 . \\
936 . \\
917 .\end{array}$ \\
\hline
\end{tabular}

-1.0 Apr. 16

912.93

\section{South Fork Crow River}

Cosmos, Minn., at U.S. Geological Survey discontinued gaging station . . .

Hutchinson, Minn

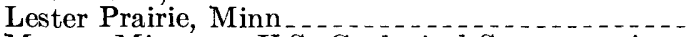

Mayer, Minn., at U.S. Geological Survey gaging station

Watertown, Minn

Delano, Minn.

Rockford, Minn., at U.S. Geological Survey recording gage

$\begin{array}{rcc}83.0 & \text { Apr. } 11 & \text { 1, 092.14 } \\ 51.9 & \text { Apr. } 11 & \text { 1, 044. 10 } \\ 30.4 & --.-- & 986.2 \\ & & \\ \text { 14. } 4 & \text { Apr. } 13 & 944.96 \\ 11.9 & \text { Apr. } 13 & 936.04 \\ 4.3 & \text { Apr. } 13 & 923.3 \\ & & \\ -1.0 & \text { Apr. } 16 & 912.93\end{array}$

floors of about 90 homes and 20 businesses were floodec as were the basements in a number of other homes and businesses. Abo't 90 families were evacuated.

The Crow River at Rockford, Minn., crested at 19.27 feet on April 16, 9.3 feet above flood stage and about 3 feet above the previous maximum which occurred on April 13, 1952. Maximum discharge was $22,400 \mathrm{cfs}$, 1.78 times a 50-year flood. Daily discharges during April are shown in figure 28. Almost 6 inches of runoff occurred from the basin upstream from Rockford during the months of April and May. The runoff in April from the basin was 4.00 inches, exceeding the April precipitation of about 3.5 inches. This fact points out the substantial ccntribution of snowmelt runoff to the flood situation. In Rockford, there was no 
community-wide project to protect the town against flood damage, but local residents endeavored to protect individual homes and businesses. About 45 families were evacuated. First floors were flooded in 35 homes and 15 businesses, and basements flooded in an additional 10 homes and five businesses.

At Hanover, Minn., ice floes destroyed a new timber bridge on County Highway 19. Other bridges, roads, and urban property in the lower part of the basin were damaged. Extensive inundation of agricultural lands damaged crops, and the floodwaters destroved fences.

The total damage in the basin was estimated at $\$ 2.9$ million by the Corps of Engineers; a major share represents costs for flood-fighting activities in the river communities.

\section{RUM RIVER BASIN}

Flooding in the Rum River basin was caused principally by the melting of heavy snow cover augmented by more than an inch of rain which fell during the first part of April. At the end of March, water equivalent of snow in the basin ranged from 6 to 9 inches (fig. 5). The heaviest snow cover was in the upstream or north end of the basin. Daily discharges at the gaging station near St. Francis are shown in figure 28. The maximum discharge of $10,100 \mathrm{cfs}$ was 1.11 times a 50 year flood and was the maximum of record during a continuous period since 1929. Mille Lacs Lake, a natural reservoir in the upper part of the basin, partly regulated the floodflow.

The floods inundated agricultural lands, roads, and bridges in the reach between Princeton and Isanti. Residences in the community of Isanti were also damaged by floodwaters. Total damage in the basin was estimated at $\$ 200,000$ by the Corps of Engineers.

\section{MINNESOTA RIVER BASIN}

The upper part of the Minnesota River basin is formed by the Little Minnesota River, which originates on the eastern slope of the Dakota foothills (Coteau des Prairies) in South Dakota about 30 miles west of Lake Traverse. The Little Minnesota River flows into the upper end of Big Stone Lake on the South Dakota-Minnesota border. Big Stone Lake, which is only about a mile and a half in width, ertends southeastward for 26 miles. Although the extreme upper end of the Minnesota River basin is in South Dakota, modern maps indicate that the source of the Minnesota River is at the lower end of Big Stone Lake. The river flows southeastward through Minnesota for 225 miles to Mankato where it turns abruptly to the northeast and flows another 106 miles to its mouth near Fort Snelling, Minn. on the southern outskirts of the Twin Cities. 
During the glacial epoch, the River Warren, which fowed in the present Minnesota River ralley, drained glacial Lake Agassiz, a rast body of water in the Northwestern part of the State. As a consequence of the great volume of this early drainage, the Minnesot: River flows through wide flood plains, which intensify flood problems in the basin. At the downstream gaging station near Carver, Minn., measurements of flood discharge were made by boat across a valley 2 miles wide. In other locations the valley is as much as 3 miles wide, but throughout much of its reach it is from 1 to $11 / 2$ miles in width. Tl $\circ$ Minnesota River itself has little slope, but its tributaries are comparetively steep; their steepness compounds the flood problem.

The flood in the upper part of the Minnesota River Easin was not severe. In fact, the Little Minnesota River had its annual peak in June, after the flood period. The recurrence intervals of maximum discharges in tributaries upstream from the Lac qui Parle River varied from 4 to 11 years. Peak discharge of the Lac qui Parle River, which joins the Minnesota River 41 miles downstream from Big Stone Lake, was $8,370 \mathrm{cfs}$, a 40 -year flood, and the second highest peak discharge in 38 years of record.

The next major tributaries downstream from the Lac qui Parle River are the Chippewa River, draining an area on the north, and the Yellow Medicine and Redwood Rivers, which drain areas on the south. The recurrence interval of peak discharges in these tributaries in their lower reaches varied from 8 to 20 years. The Minnesota River is gaged at Montevideo, Minn. just downstream from the mouth of the Chippewa River. Flood discharge at this location was $12,900 \mathrm{cfs}$, a 13-year flood. Records have been collected at this site for 56 years. The 1965 flood was exceeded only by the thunderstorm flood of June 1919 and the snowmelt flood of April 1952. The dischargo of the 1952 flood, however, was almost twice that of the 1965 flood.

The discharge of the Minnesota River and its tributaries from New Ulm Minn., to the mouth 145 miles downstream, exceeded all previously known peak discharges. The runoff from the area south of New Ulm and Mankato, Minn., was particularly intense. The April runoff from the Blue Earth and Le Sueur River basins was 6.08 inches and 6.66 inches, respectively, which was greater than that from any other large gaged area in Minnesota with the exception of the Cannon River basin.

Severe urban and agricultural damages occurred in the Minnesota River basin downstream from New Ulm. Because of tho wide river valley, a great deal of rich agricultural land was flooded. Flandrau Dam on the Cottonwood River and Rapidan Dam on the Blue Earth River were damaged. The greatest damage by far, however, was to 
communities along the Minnesota River at Mankato and downstream.

Le Hillier, on the outskirts of Mankato, suffered heavy damages when a temporary levee was overtopped and all the community's business establishments and 125 residences were flooded by the Blue Earth River. Floodwaters in Le Hillier were as much as 12 feet deep. Another levee protecting Mankato failed, and a large business area and about 225 residences were flooded by the Minnesota River (figs. 30 and 31). A strenuous effort was carried on in North Manke,to to protect that community from the floodwaters. This effort was largely successful, but its cost over $\$ 250,000$ and about 5,000 persons were eracuated because of the flood danger. Total damages, including the flood-fighting effort in Mankato and its environs, Le Hillier and ${ }^{\top}$ orth Mankato, were about $\$ 7$ million and more than 8,000 persons were evacuated.

The large flow contributed by the Blue Earth and Cottonwood Rivers to the Minnesota River did much to increase the flooding at Mankato. The mouth of the Cottonwood River is only 33 miles upstream from the Blue Earth River, which joins the Minnesota River s.t the southwest edge of Mankato. The major tributaries of the Blue Earth River are the Watonwan and Le Sueur Rivers and major tributaries of the Le Sueur River are the Cobb and Maple Rivers. All these rivers drained areas where the runoff was exceptionally high.

Records from gaging stations on the Minnesota River at Mankato and on tributaries in the vicinity of Mankato gave evidence of the great volume of runoff. The Cottonwood River near New Ulm crested on April 8 at 26,000 cfs, almost twice the previous maximum during the 38 years records have been collected. Discharge of the Blue Farth River was over $11 / 2$ times the previous maximum in 23 years of record, and discharge of the Le Sueur River was greater than the maximum in 22 years of record. The maximum discharge of the Minnesota Piver at Mankato, 94,100 cfs, was greater than the previously known maximum, 90,000 cfs, which occurred in 1881. The maximum stage in 1881, however, was about 0.8 foot higher. The recurrence intervals of the flood discharges of the Mimnesota River at Mankato and its tributaries in the vicinity varied from 1.20 to 3.30 times the 50-year flood.

In communities downstream from Mankato, flood damage was also heavy. Residential areas and city streets were damaged in St. Peter, Minn., not from Minnesota River floodwaters, but from a small drainage basin which released its runoff through the town. Damages were about $\$ 500,000$. In the small town of Henderson (population 765), about 400 persons were forced to exacuate their homes. Water was 6-8 feet deep in some areas and half the town was under water (fics. 32 and 33). Total damages exceeded $\$ 600,000$. 


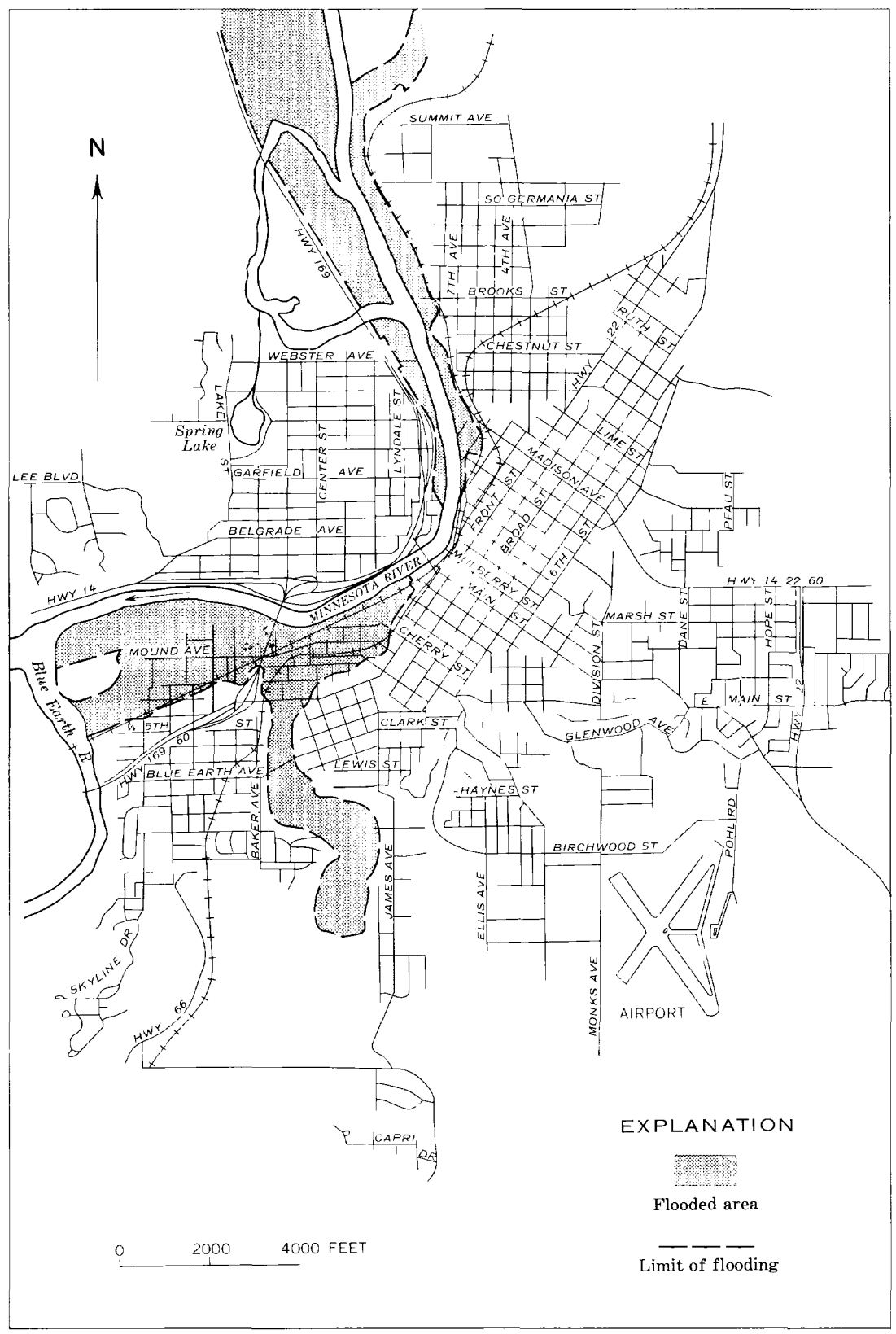

Figure 30.-Flooded area in Mankato, Minn. 


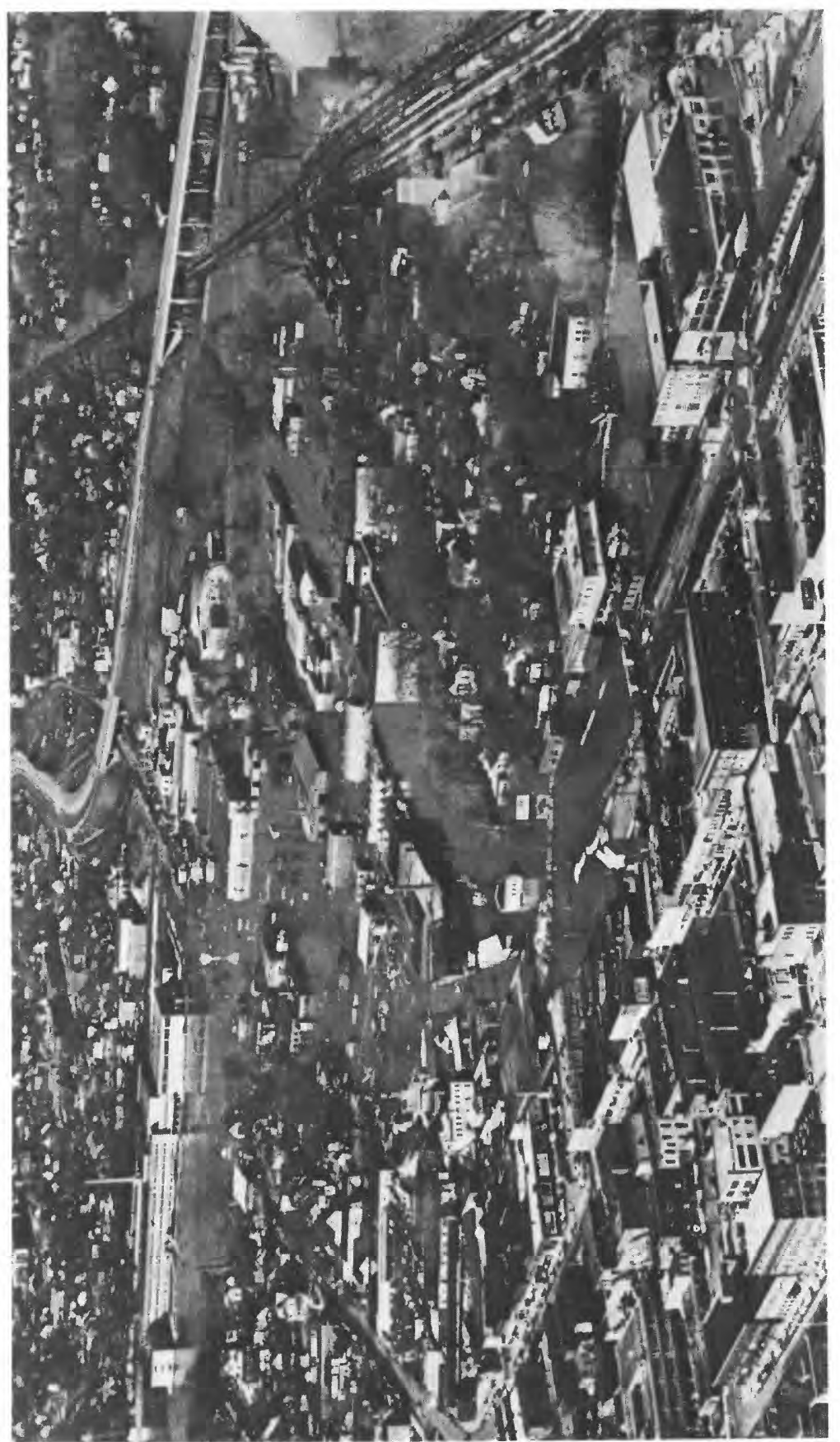

 $\because$ 总 도 동 o 영 突 혼 $\rightarrow$ 5 율 这 을 还 递 \& ลิง 일 䆠

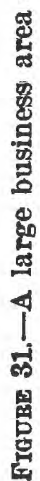




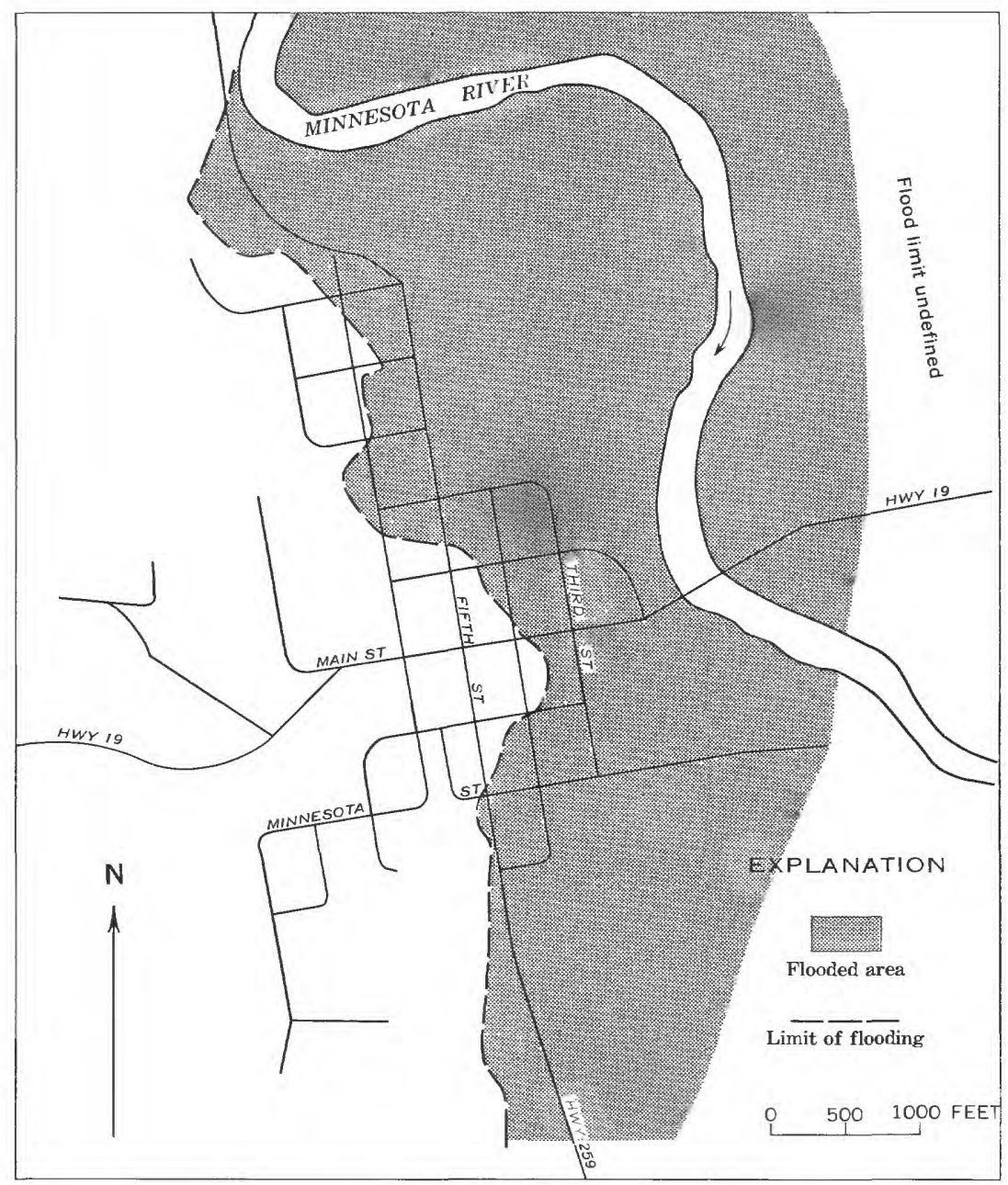

Figure 32.-Flooded area in Henderson, Minn.

At Carver, Minn., the Minnesota River crested 16 feet over flood stage and damaged a large part of the town's business district and residential area (figs. 34 and 35). About 60 families were forced to leave their homes. The gaging station on the Minnesota River, 3 miles upstream from Carver, was inundated, and the concrete structure was severely damaged when struck by trees and debris which were carried downstream by the current. The maximum stage at this site, determined from floodmark, was 34.37 feet, more than 6 feet higher than the maximum previous stage of record which occurred in 1952. The maximum discharge, $117,000 \mathrm{cfs}$, was 1.83 times that of a 50 -year flood. 


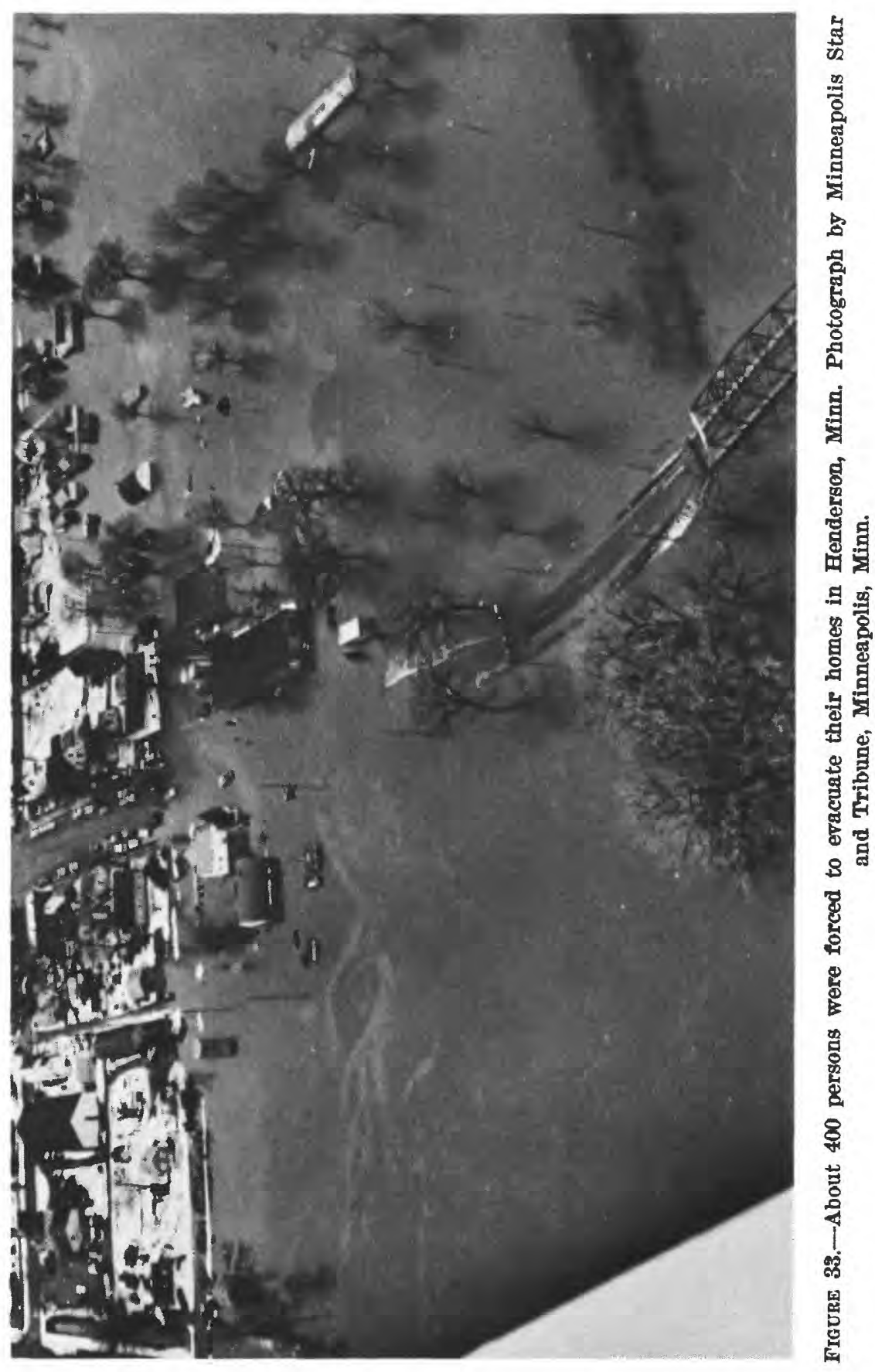




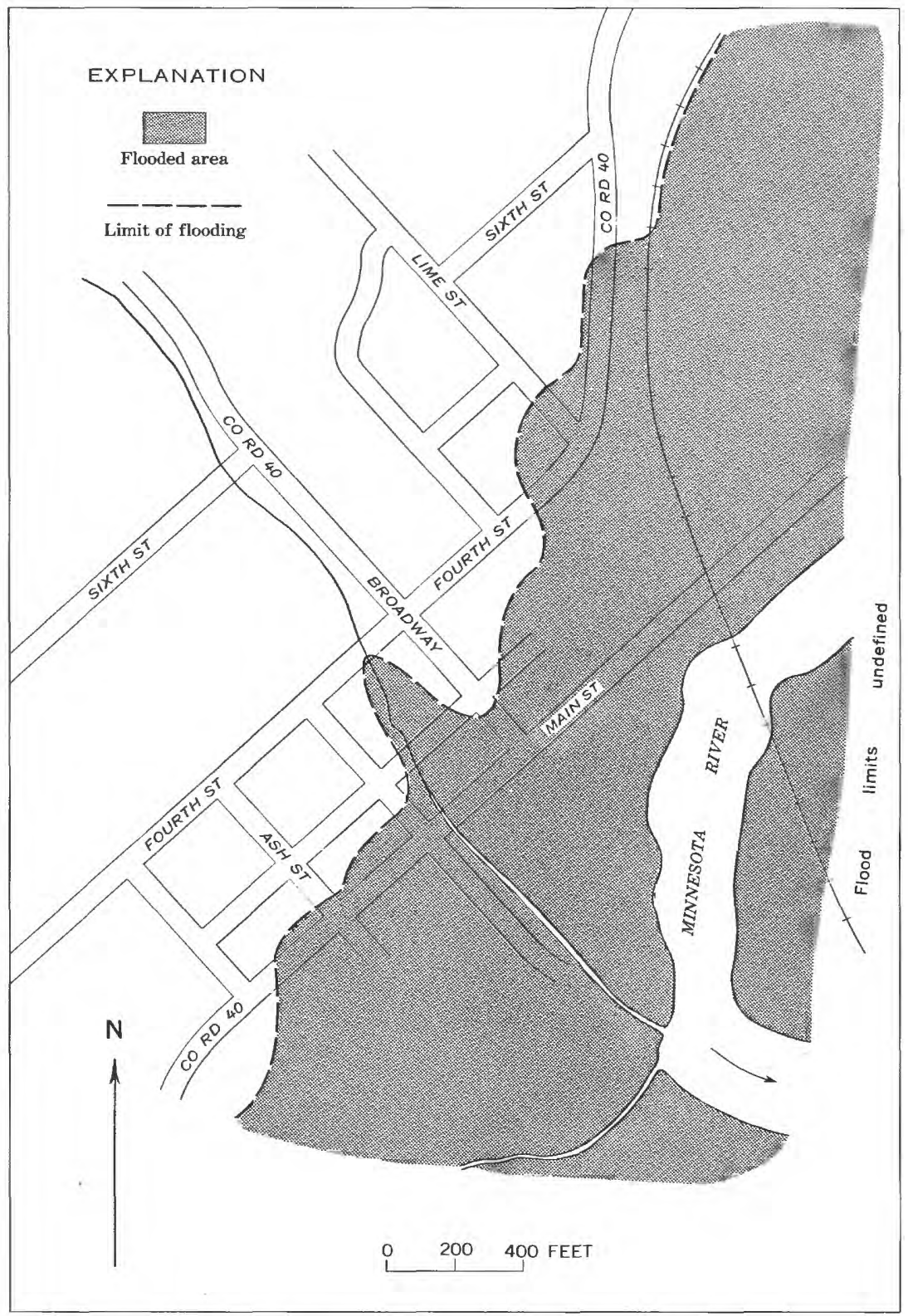

Etgure 34.-Flooded area in Carver, Minn. 


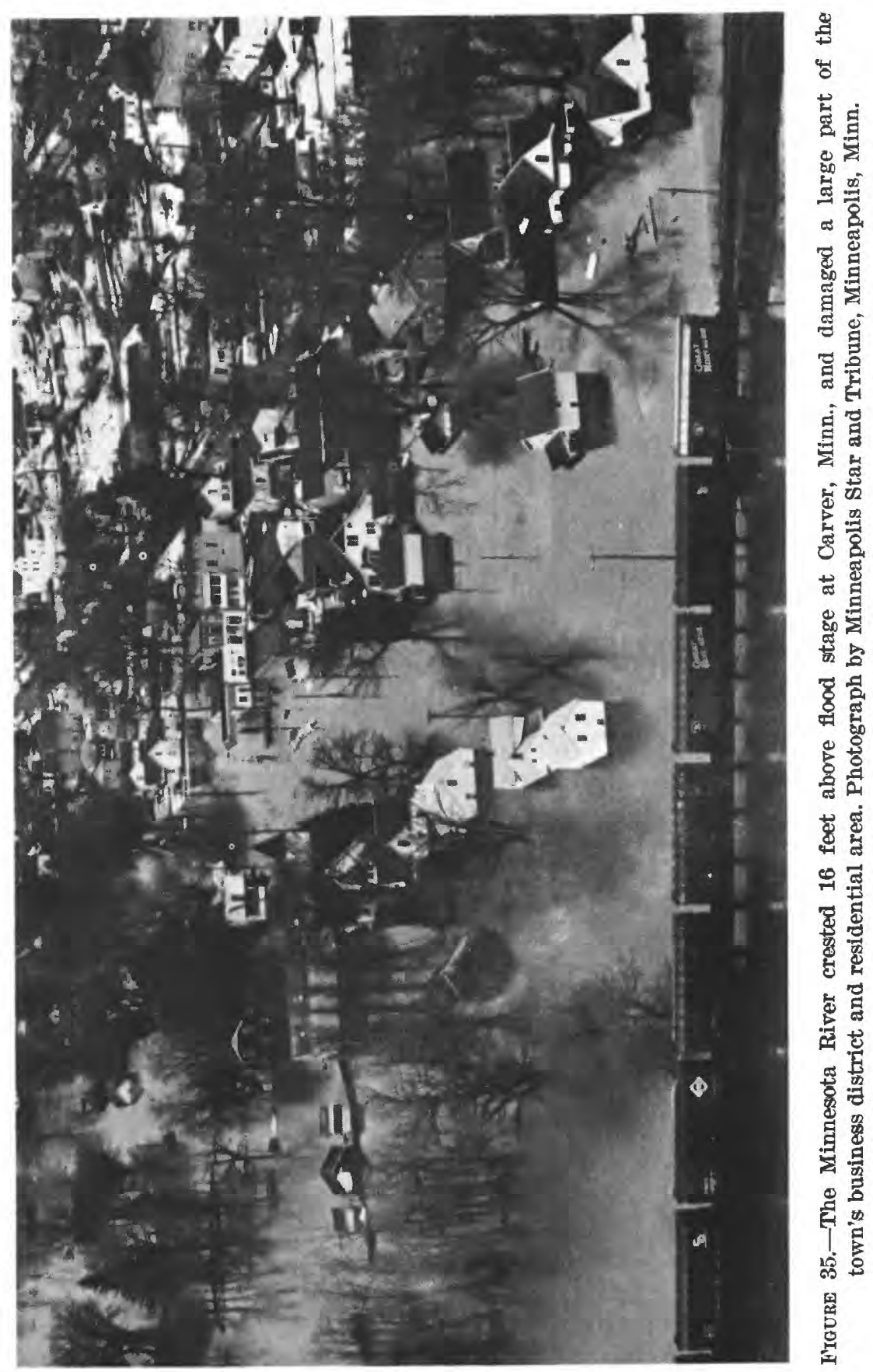


About 500 persons in Chaska, Minn., were forced to leave their homes when the floodwaters overtopped a 1,600-foot-long dike constructed by local residents (figs. 36 and 37). The new Carver County Courthouse and sewage disposal plant were protected, however, by emergency sandbag dikes.

Several oil and grain terminals at Savage, Minn, suffered severe damage from the recordbreaking floods, which exceeded the 1952 stages by almost 5 feet. The residential part of the community and a 14-million-bushel grain elevator complex were protected by emergency sandbag dikes.

There was a great deal of damage to highways and railways in the Minnesota River valley. For several days during the flood, only one highway, U.S. Highway 169 at Le Sueur, was open across the Minnesota River in the area from the mouth to Mankota, 106 miles upstream. Of the three bridges crossing the Minnesota River in the Twin City area, only Mendota Bridge at the mouth was open. It was choked by commuter traffic for hours each day. Water was about 12 feet deep on Cedar Avenue near Cedar Avenue Bridge, and Interstate Highway $35 \mathrm{~W}$ was inundated by about 2 feet of water south of the bridge. Railroads suffered losses to track, roadbed, and bridges as vell as operational losses from interruption of service.

Total damages in the Minnesota River basin, including the cost of flood fighting, were estimated by the Corps of Engineers to be over $\$ 38$ million.

A profile of 1965 flood elevations for the Minnesota River is shown in figure 38, and tabulated elevations are given in table 3. Discharge hydrographs for Le Sueur, Blue Earth, and Minnesota Rivers are shown in figure 39 . 
TABLE 3.-Flood-crest elevations, Minnesota River

[Based on data furnished by U.S. Army Corps of Engineers except as noted]

\begin{tabular}{|c|c|c|c|}
\hline Location & $\begin{array}{l}\text { Miles } \\
\text { above } \\
\text { mouth }\end{array}$ & Date & $\begin{array}{c}\text { E evation } \\
\text { in feet } \\
\text { (do.tum of } \\
\text { 1929) }\end{array}$ \\
\hline
\end{tabular}

Profile point on left bank $100 \mathrm{ft}$ northeast of bridge

263. 6

920.83

at Wegdahl, Minn.

Granite Falls, Minn.:

Gage on left bank at crest of city waterworks dam.

Right bank $30 \mathrm{ft}$ upstream from U.S. Highway 212.

Gage at right upstream corner of Northern States Power Co. Minnesota Valley Steam Plant.

Highwater mark on left bank in pool at NSP Minnesota Falls Hydro Plant.

Highwater mark at Pete's Point Resort downstream from NSP Minnesota Falls Hydro Plant.

In NW $1 / 4$ sec. 34 , T. $115 \mathrm{~N}$., R. $38 \mathrm{~W}$., near bridge on road from Sacred Heart, Minn., to Minnesota State Highway 67 at Eugene Eber farmhouse.

At bridge on Redwood County Highway 7, 6 miles north of Belview, Minn.

At bridge on Redwood County Highway 6, 4 miles north of Delhi, Minn.

At downstream side of bridge on Redwood County Highway $17,21 / 4$ miles northwest of Delhi, Minn.

At bridge on Redwood County Highway 21, 0.8 mile northwest of North Redwood, Minn.

At bridge on U.S. Highway 19 near Morton, Minn -

At bridge on Redwood County Highway 11, 1 mile south of Franklin, Minn.

At right downstream side of bridge on Minnesota State Highway 4.

New Ulm, Minn.:

Downstream left abutment of bridge on U.S. Highway 14.

Right bank, $250 \mathrm{ft}$ west of Courtland bridge in southeast New Ulm.

Right bank on line between sec. 11 and 12, T. 109 N., R. 30 W., near New Ulm, Minn., 1 mile north of Minnesota State Highway

253. 0 905. 04

252. 2

250. 3 Apr. 15 888. 6

249.5 887. 45

249.0 880. 00

238. 3 871. 86

228. 5

223. 7

219. 6

849. 87

212. 2

840. 7

202. 9

831. 20

192. 4

823. 43

175. 5

814. 14

146. 8 Apr. 9

806. 26

143. 8 Apr. pr. 9

or 10 .

805. 05 801. 48 68. 
TABLE 3.-Flood-crest elevations, Minnesota River-Continued

[Based on data furnished by U.S. Army Corps of Engineers except as noted]

Location $\quad \begin{gathered}\text { Miles } \\ \text { above } \\ \text { mouth }\end{gathered} \quad$ Date $\begin{gathered}\text { Elevation } \\ \text { in foet } \\ \text { (datum of } \\ 1929)\end{gathered}$

Courtland, Minn., $50 \mathrm{ft}$ east of center line of road which leads to Minnesota State Highway 68.

Cambria, Minn., on right bank at farthest downstream house on street closest to river.

Judson, Minn., on west side of land leading to bridge near school.

Mankato, Minn.:

Right bank Blue Earth River at southwest cor. of Sibley Park.

U.S. Geological Survey recording gage at left downstream side of Main Street bridge.

Right bank, $1000 \mathrm{ft}$ downstream from main building of sewage plant.

On left bank in NW1/4 sec. 24, T. 109 N., R. 27 W., near Mankato.

Kasota, Minn., near downstream limits of town . - -

St. Peter, Minn.:

Gage on powerhouse

Terminal power pole for new St. Peter sewage plant.

On left bank 3 miles downstream from St. Peter, at NW cor. sec. 4, T. 110 N., R. 26 W.

On right bank, 1.2 miles upstream from Le Sueur, Minn., along railroad from Le Sueur Post Office Bldg., at same location as USC and GS BM D-3.

At left downstream corner of bridge on U.S. Highway 169 north of Le Sueur, Minn.

Henderson, Minn.:

Right bank, $115 \mathrm{ft}$ upstream and $40 \mathrm{ft}$ riverward from C\&NW Ry overpass on Minnesota State Highway 19.

Downstream side of above bridge

134. 3

797. 14

130. 6

793. 98

120. 3

787. 6

108. 2 Apr. 10_- 781.66

106. 4 Apr. 10_.- ${ }^{1777.01}$

105. 3 Apr. 10_.. 774. 08

99. 8 Apr. 10_- 768.1

$92.5 \quad \ldots \ldots \ldots 2.01$

90.1 _ $\ldots \ldots$. 758.27

89. 2 _...

85. 2 _...... 754.0

76. 9

$72.2 \ldots \ldots \ldots 743.67$

$67.1 \ldots \ldots$

$67.1 \ldots \ldots \ldots 739.63$

See footnote at end of table. 
TABLE 3.-Flood-crest elevations, Minnesota River-Continued

[Based on data furnished by U.S. Army Corps of Engineers except as noted]

\begin{tabular}{|c|c|c|c|}
\hline Location & $\begin{array}{l}\text { Miles } \\
\text { above } \\
\text { mouth }\end{array}$ & Date & $\begin{array}{c}\text { E'evation } \\
\text { in feet } \\
\text { (datum of } \\
1929)\end{array}$ \\
\hline
\end{tabular}

On right bank at C\&NW Ry bridge 269 , in $\mathrm{NW} 1 / 4$

62.8

739. 18

sec. 31 , T. 113 N., R. $25 \mathrm{~W}$.

On right bank at C\&NW Ry bridge 264, near east line sec. 24, T. 113 N., R. $26 \mathrm{~W}$

Blakeley, Minn., on right bank, $63 \mathrm{ft}$ west of railroad and $60 \mathrm{ft}$ south of road to bridge.

Belle Plaine, Minn.:

Upstream side of bridge on Minnesota State Highway 25.

Downstream side of above bridge

On right bank on Richard Klehr farm near Belle Plaine, Minn., in SE1/4 sec. 21 , T. 114 N., R. $24 \mathrm{~W}$.

On right upstream bank at Corps of Engineers gage site near Jordan, Minn.

U.S. Geological Survey recording gage near Carver, Minn.

Carver, Minn., at County Road 40 opposite railroad water tank.

Chaska, Minn., at Corps of Engineers gage . . . . . .

Shakopee, Minn., on right bank, $100 \mathrm{ft}$ upstream from bridge on U.S. Highway 169.

Savage, Minn., on left bank, downstream from combination highway and railroad bridge.

60. 7 _

$56.4 \ldots 734.95$

48. 9 Apr. 11.. 730.84

48. 9 _.

44.3 _. 328.63

39.4 - 425.06

36. 0 Apr. $12 \ldots{ }^{1} 724.37$

31. 9 Apr. 12_- 723. 46

29. 6 Apr. 13_. 722.25

$25.2 \ldots 721.27$

14. 3 _ _ _ 719.15

Bloomington, Minn., at bridge on Interstate 11.0 Apr. 15_._ 1718. 20 Highway $35 \mathrm{~W}$.

Cedar Ave. at Nicols railroad siding, Dakota County.

Mendota, Minn.:

Downstream landward corner of $\mathrm{C} \& \mathrm{NW}$ Ry bridge.

Mouth of Minnesota River

7. 4 _

1.0

0.0 Apr. 16

1 From U.S. Geological Survey. 


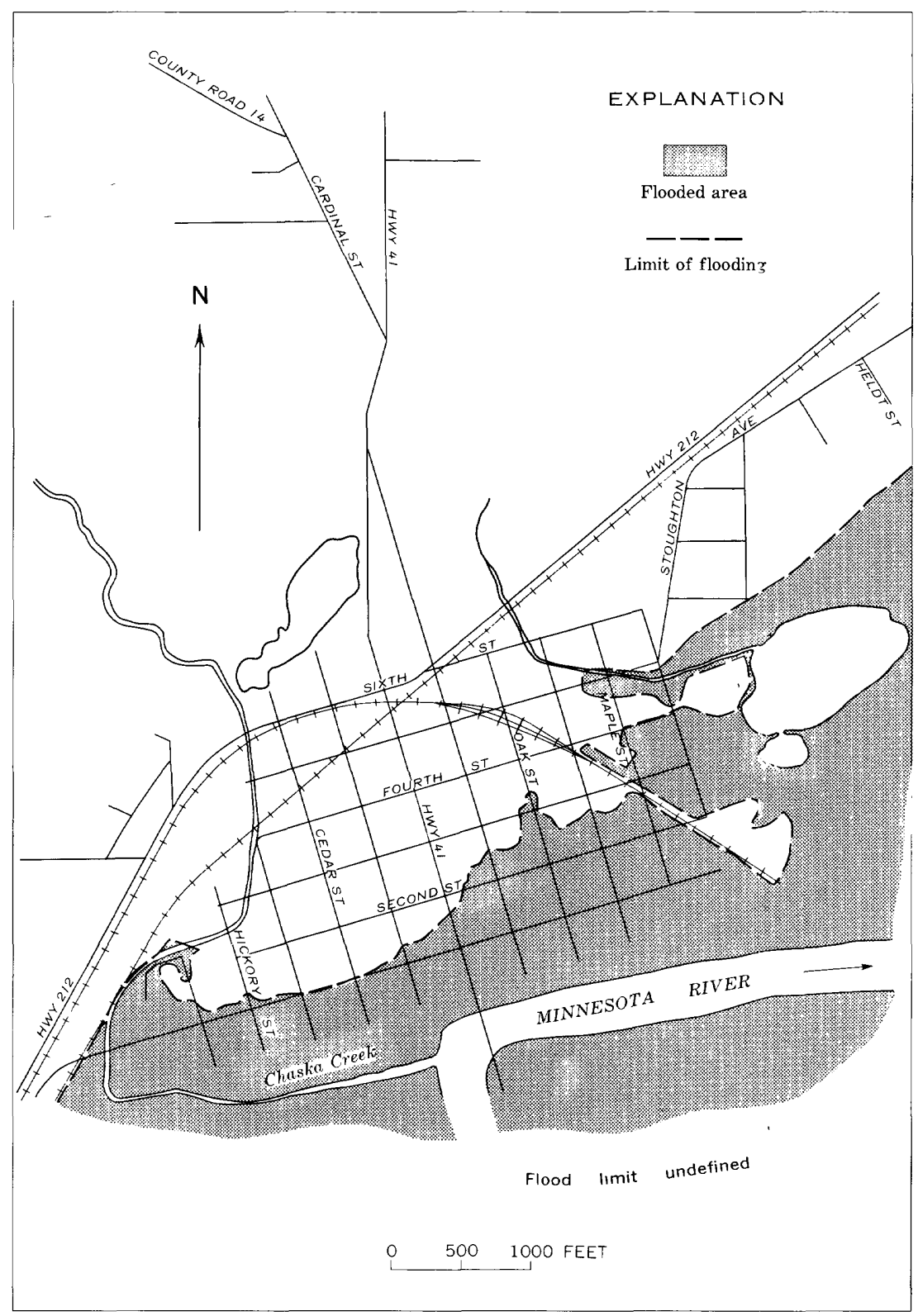

Frgure 36.-Flooded area in Chaska, Minn. 


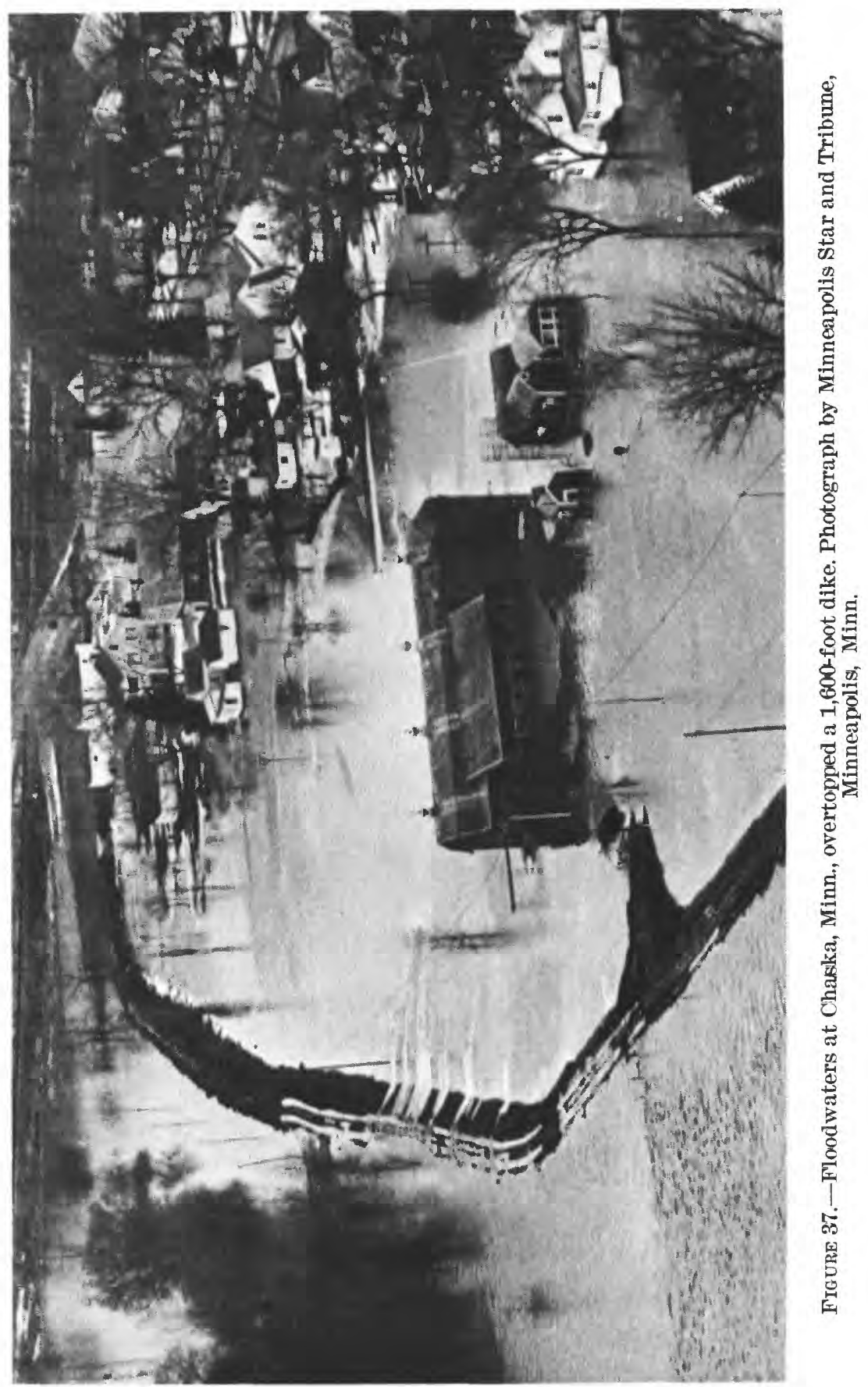



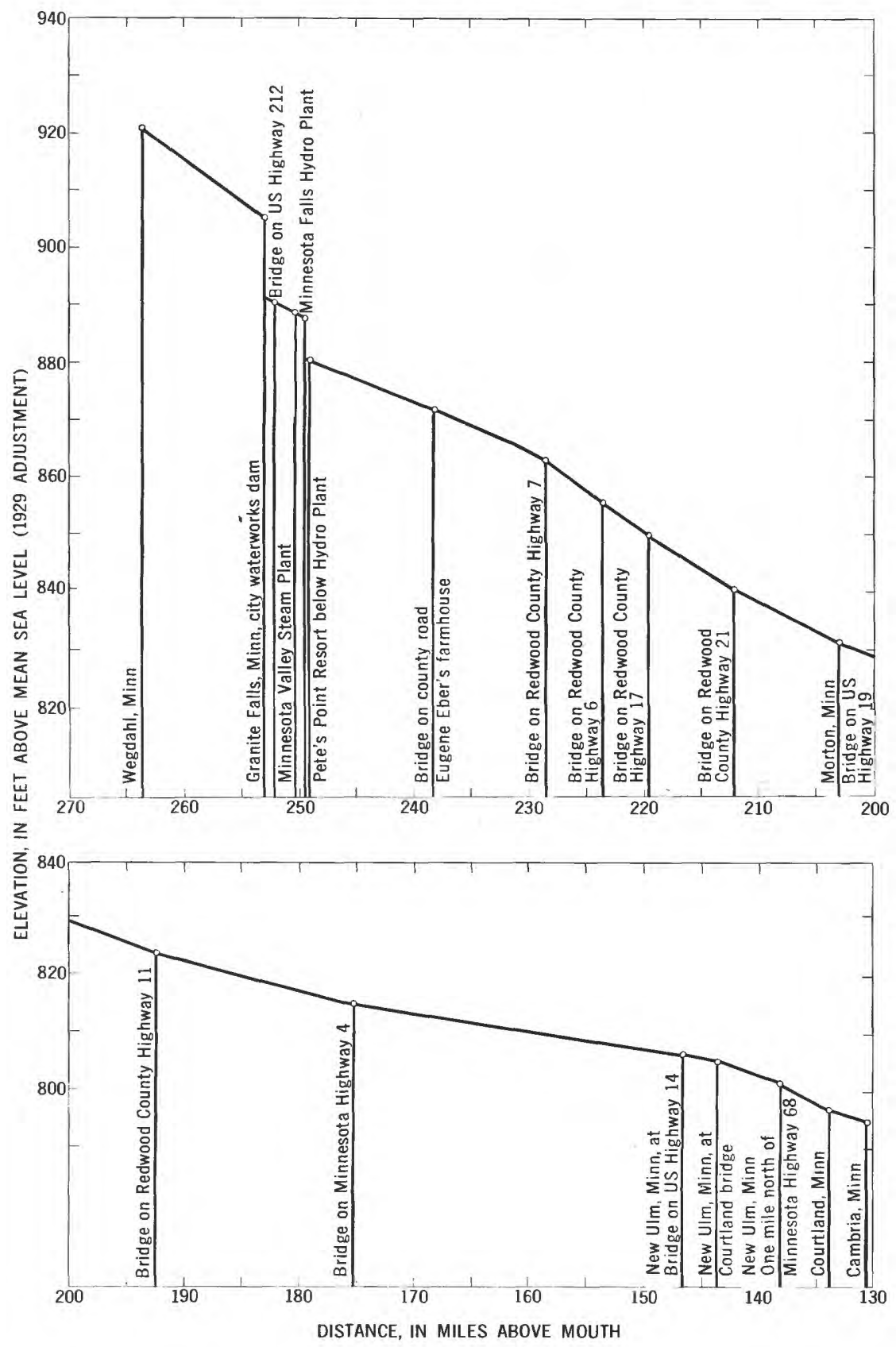

FTgure 38.--See explanation on next page. 


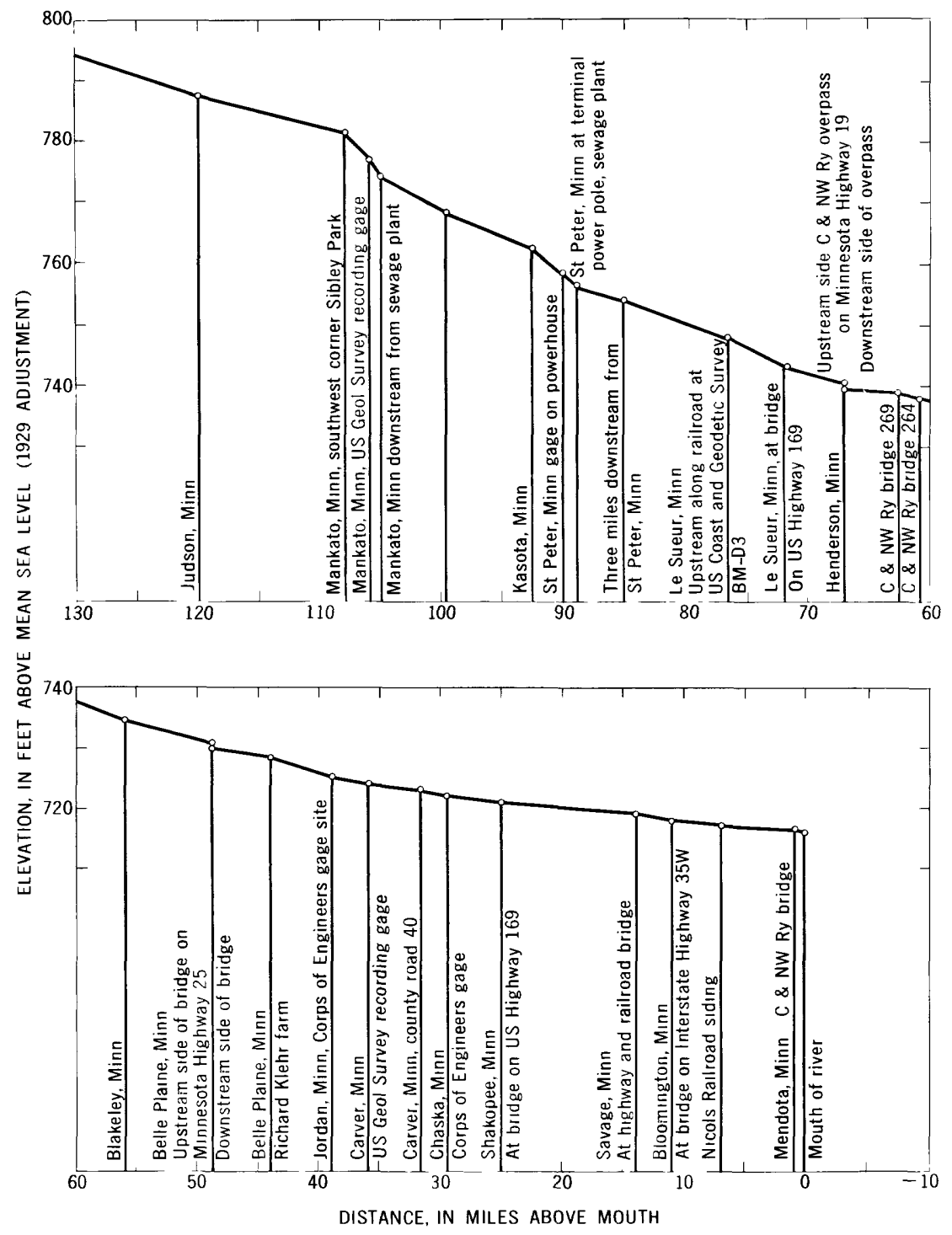

Figure 38.-Flood-crest profiles of Minnesota River water-surface elerations were obtained at U.S. Geological Survey gages or were selected from high-water data furnished by the U.S. Army Corps of Engineers. 


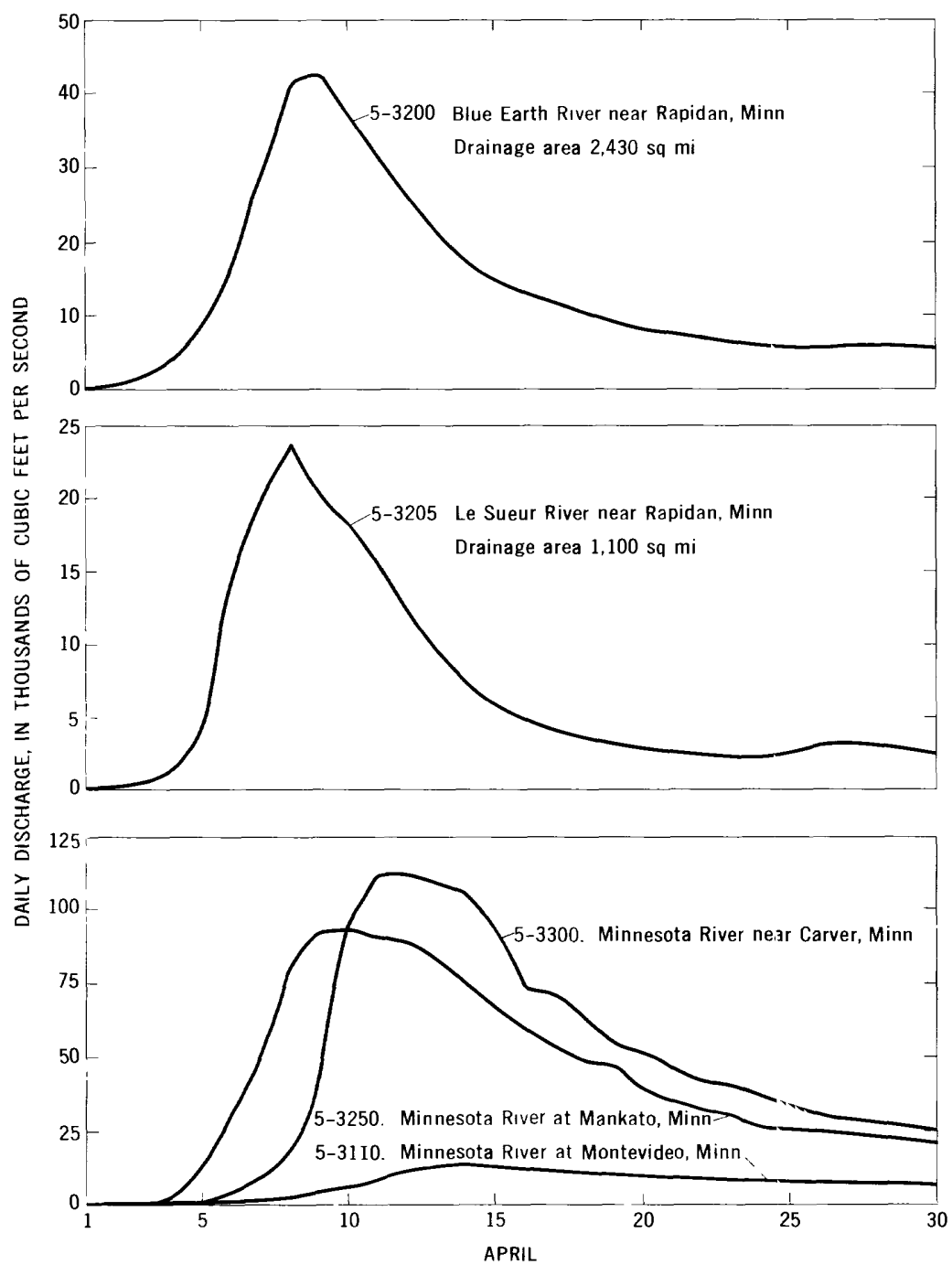

Frgure 39.-Discharge hydrographs at selected gaging stations in the Minnesota River basin. 


\section{ST. CROIX RIVER BASIN}

Most of the winter precipitation was still on the ground in the St. Croix River basin at the end of March (figs. 4 and 5) in the form of 20-30 inches of wet snow and ice (water equivalent, 5-7 inches; 2-4 inches above normal). Snow on April 1 and rain on April 6 and 7 contributed approximately another inch of precipitation. An average of almost half an inch of rain fell April 10, followed by a lighter rain April 15. Snow on the ground gradually melted from early April to the middle of the month. Runoff over the deeply frozen ground was rapid.

Small streams began rising by April 6, and the St. Croix River main stem, by April 10. Because the cool nights caused a diurnal pattern of snowmelt, the runoff from the small basins extended over a long period of time; thus, the weather prevented extremely high instantaneous peaks, but the discharge on the main stem increased rapidly for a week to 10 days after April 10. Peak discharges between Danbury and St. Croix Falls, Wis., occurred at approximately the same time, but they increased in magnitude in a downstream direction. Recurrence intervals were 5 years, 16 years and 25 years, respectively, for the peaks near Danbury on April 19, near Grantsburg on April 18, and at St. Croix Falls on April 18. The 1965 maximum discharges of $6,460 \mathrm{cfs}$ near Danbury and $45,700 \mathrm{cfs}$ at St. Croix Falls were the highest at these stations since the 1950 maxima of $10,200 \mathrm{cfs}$ and $54,900 \mathrm{cfs}$, respectively. 'The river remained high at St. Croix Falls for a long period. Daily discharge exceeded 40,000 cfs for 1 week (April 16-22) and exceeded 25,000 cfs for 2 weeks (April 14-27) as shown in figure 40. From Stillwater, Minn., to the mouth, stages were about $41 / 2$ feet higher than any previously racorded. Because the high stage of the Mississippi River prevented the movement of ice near the mouth, large ice rafts were created there.

In the small basins, many culverts and small bridges were danaged, and roadways and shoulders suffered wash damage. Heavy ice damaged farm fences and outbuildings. In the large basins, many parks, recreation areas, sewage disposal plants, and summer homes were inundated or damaged. Records for the gaging station on the Apple River in the southern part of the basin showed that the peak daily discharge was the highest in 65 years. Two small dams on the Apple River were washed out, as were several bridges. Water damaged generators at one small power plant. On the lower St. Croix River, a hugh ice pack threatened the Minnesota-Wisconsin interstate bridge. The greatest damage in the St. Croix River basin was along St. Croix Lake at Hudson and Prescott, Wis., and at Stillwater, Bayport, Lakeland, St. Croix Beach, St. Mary's Point, and Afton, Minn. Damage 

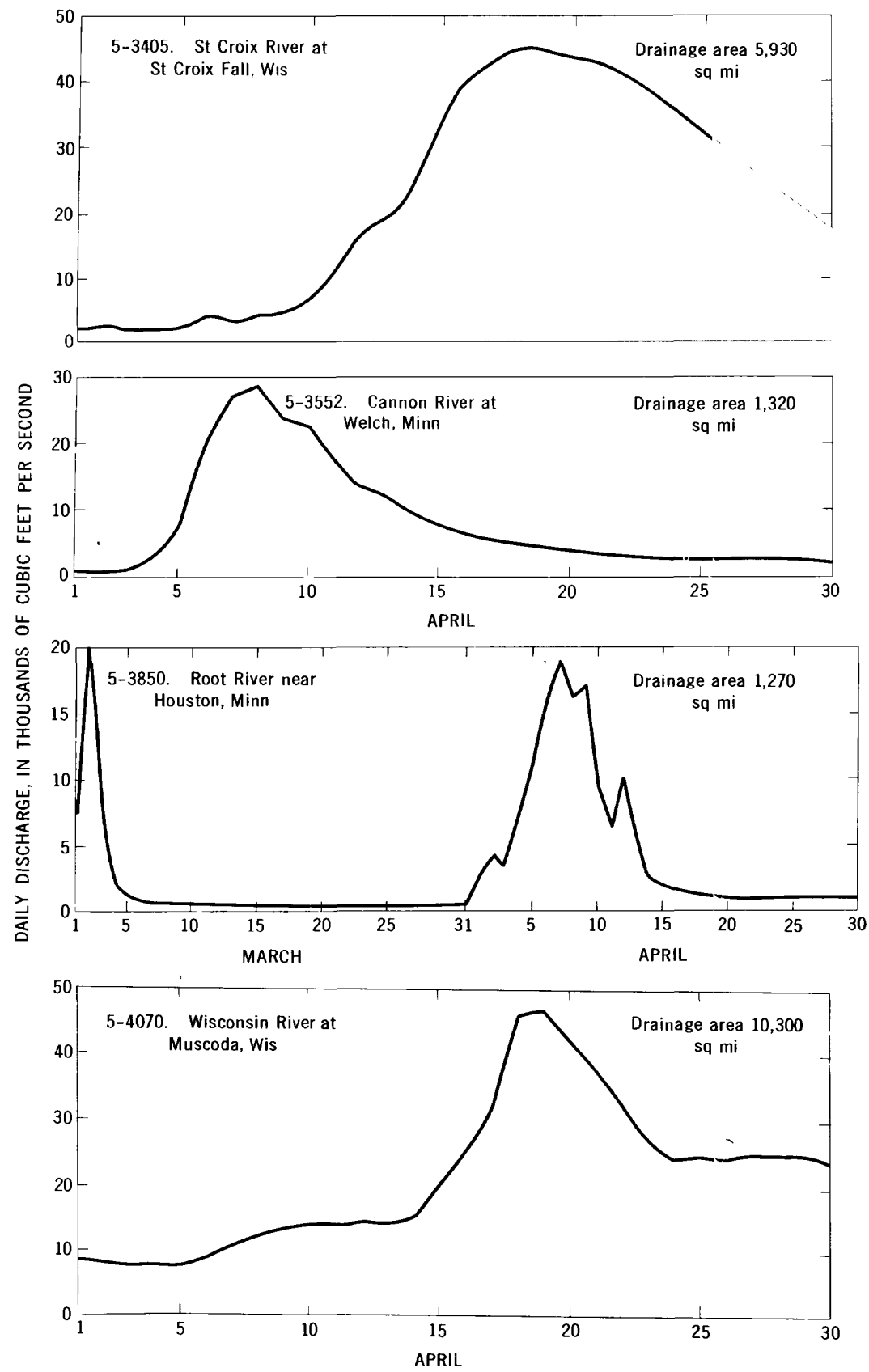

FIgURe 40.-Discharge hydrographs at selected gaging stations on the Mississippi River tributaries in Wisconsin and southeastern Minnesota. 
also occurred to homes, cottages, resorts, and boat marinas along the St. Croix River upstream from Stillwater.

The total damage in the St. Croix basin was over $\$ 5$ million, about half of which occurred in Stillwater. Temporary levees at Stillwater, Bayport, and Hudson prevented additional damages which might have amounted to several million dollars.

The flood damaged about 200 homes and caused the closing cf two major highways across the St. Croix River. Rail travel was affected, but the Chicago and North Western Railway bridge at Hudson, Wis., remained open and provided a crossing for trains of four railroad companies operating between the Twin Cities and Chicago. About $\$ 500,000$ damage occurred to railroads, highways, and railroad and highway bridges.

\section{MISSISSIPPI RIVER TRIBUTARY BASINS IN SOUTHEASTERN MINNESOTA}

The Vermillion River is an ungaged stream which flows into the Mississippi River at Hastings, Minn. Floodwaters from this river caught many residents by surprise during the night of April 5-6 when the normally placid stream overflowed its banks and inurdated much of the Westwood Addition, a new housing area which had been built since the 1952 flood (figs. 41 and 42 ). Overflow from the Vermillion River backed up behind County Highway 47 and increased the flood threat to residents in the southwestern part of Hastings. To alleviate this situation, county highway crews breached a 37-foot section of highway so that the impounded water could return to the channel. About 280 families were evacuated, many of whom were previously unaware that their homes were subject to flood inundation. About 42 city blocks were flooded, and water was as much as 5 feet deep in some of the streets. Total damages from the Vermillion River flood were more than $\$ 725,000$. The Mississippi River crest arrived at Hastings almost 2 weeks later on April 18, but damages from the main stem, about $\$ 50,000$, were minor when compared with those resulting from the flooding Vermillion River.

Runoff from the Cannon River basin was exceptionally heavy. During April, it amounted to 6.96 inches in the basin upstream from Welch, Minn. This was the maximum computed from data collected at any of the more than 100 gaging stations in Minnesota, although it probably was exceeded in some small basins where continuous records of discharge were not maintained. The maximum discharge of 36,100 cfs at the Welch gaging station on April 8 was more than twice that of the 1952 flood and 1.54 times the discharge of a 50-year flood. 


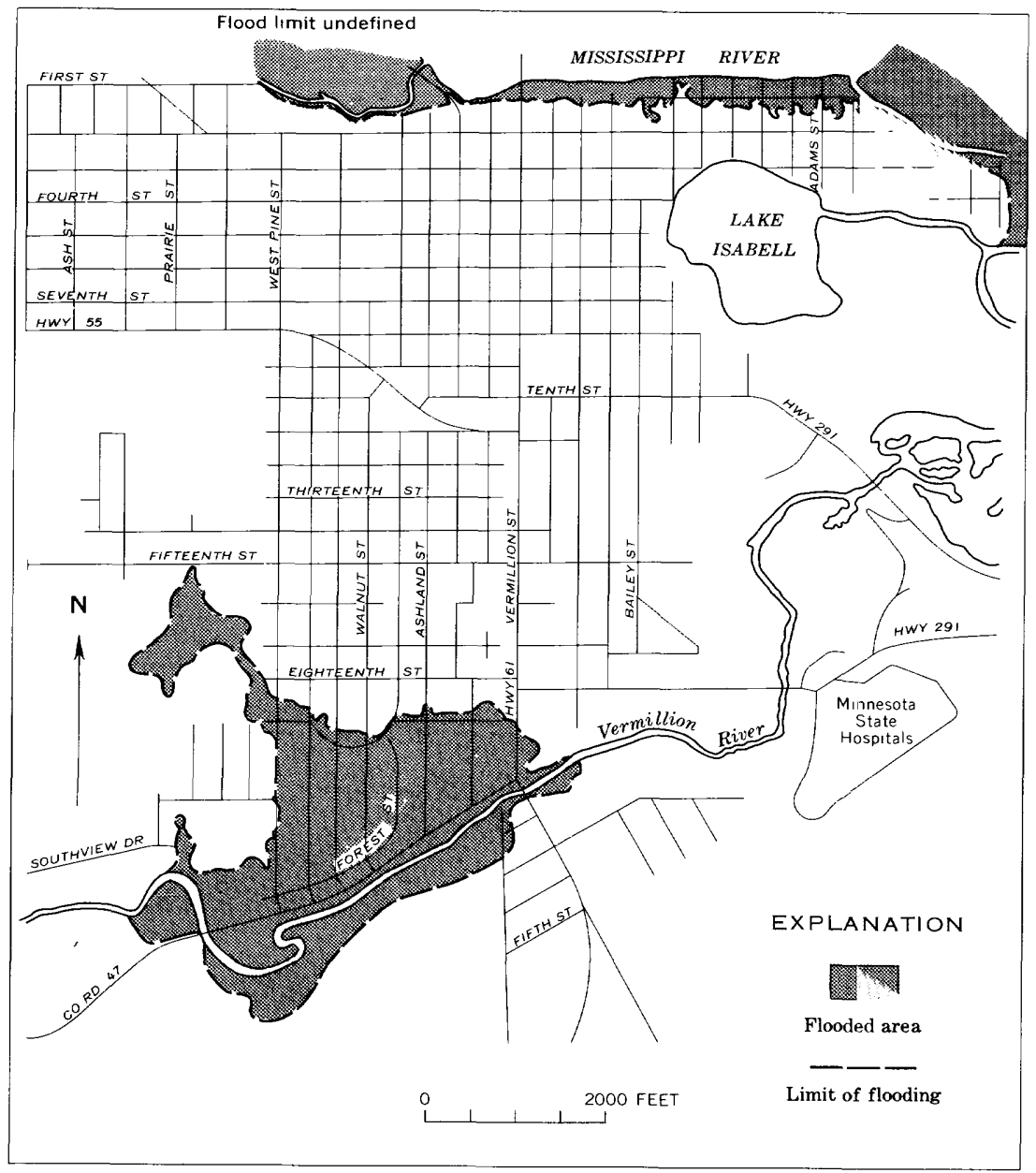

Frgure 41.-Flooded area in Hastings, Minn. 


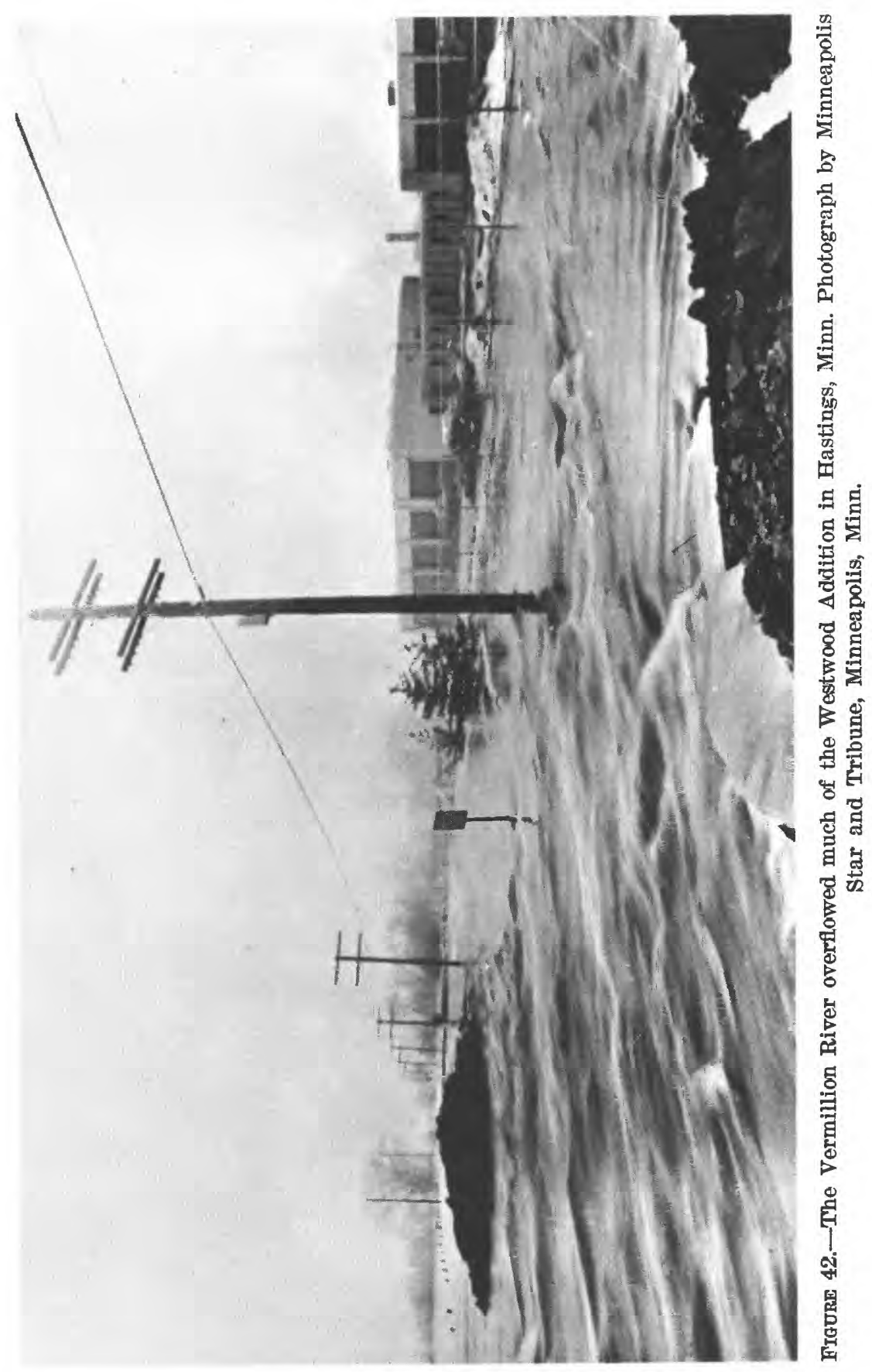


The flood hydrograph of daily discharges is shown in figure 40. Urban damage in the basin was about $\$ 182,000$, and agricultural damage was $\$ 97,000$. Greatest urban damage occurred in the towns of Faribault, Northfield, and Cannon Falls, Minn.

Most streams in Minnesota crested in April, but a few streams in the southeastern part of State crested in early March (table 4). The Zumbro River, which flows into the Mississippi River downstream from Lake Pepin and lock and dam 4, is the northernmost of these streams. On the South Fork Zumbro River near Rochester, Minn., the 1965 maximum stage of 19.12 feet, 7 feet over flood stage, was 0.66 foot higher than the March 1962 peak and more than $11 / 2$ feet higher than any other peak which has occurred since 1908. At the downstream gaging station at Zumbro Falls, Minn. the maximum discharge on March 2 was $29,600 \mathrm{cfs}$, a 29 -year flood.

Another peak occurred on April 7, but the April flooding was not as severe because the winter snow cover had already melted and run off during the March flood. Urban damages in the Zumbro River basin, most of which occurred in Rochester, were over $\$ 458,000$, and agricultural damages were about $\$ 78,000$. Flood-crest elevations in the Zumbro River basin are shown in profile (fig. 43) and described and tabulated (table 4).

TABLE 4.-Flood-crest elevations, South Fork Zumbro River and Zumbro River [Based on data furnished by U.S. Army Corps of Engin $\theta \oplus r s$ except at U.S. Geological Survey gaging stations]

\begin{tabular}{lccc}
\hline Location & $\begin{array}{c}\text { Miles } \\
\text { above } \\
\text { mouth }\end{array}$ & Date & $\begin{array}{c}\text { Elevation in } \\
\text { foet (datum } \\
\text { of 1929) }\end{array}$ \\
\hline
\end{tabular}

Rochester, Minn.:

U.S. Weather Bureau gage at bridge on U.S. Highways 52 and 14

U.S. Geological Survey recording gage.....

Rochester power dam, pool

80. 5 Mar. 1 1, 002. 20

75. 1 Mar. 1 968. 188

Rochester power dam, tailwater.

Zumbro Falls, Minn., at U.S. Geological Survey recording gage . continued gaging station

Kellogg, Minn., at bridge on U.S. Highway $6 \overline{1}_{\ldots}$

Zumbro River mouth.

59. 0 Mar. 2 925.75

59. 0 Mar. 2 873. 2

48. 5 Mar. $2 \quad 839.66$

24. 5 Mar. $2 \quad 745.27$

4. 5 Mar. $2 \quad 687.90$

0 Mar. 3 664.52 


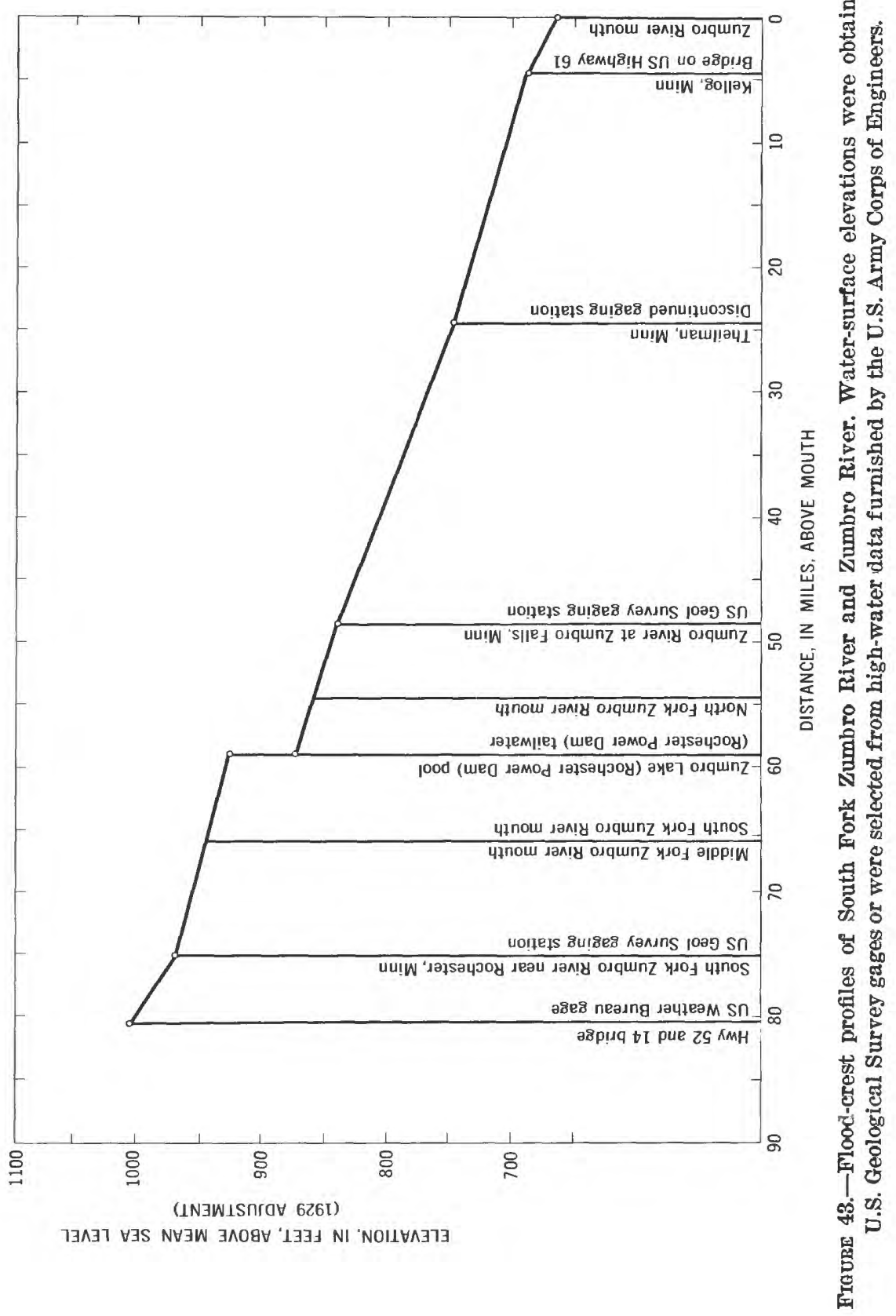


The only gaging station in the Whitewater River basin is on a relatively small drainage area on the South Fork Whitewater River near Altura, Minn. Flooding and damages in this basin were not severe. The maximum stage at the Altura gaging station, with occurred on March 1, was largely the result of rapid snowmelt runoff and ice backwater. The maximum discharge of $2,360 \mathrm{cfs}$ which occurred during a later peak on April 7 was only a 2 -year flood.

The Root River, which joins the Mississippi River about 3 miles downstream from La Crosse, Wis., drains an area in extreme southeastern Minnesota and also a few square miles on northeastern Iowa. The topography of the Root River basin is characteristic of southeastern Minnesota. It is rolling and undulating and contains a number of steep bluffs. The greater part of the basin is under cultivation, but the plateaus at the top of the bluff and many of the hillsides are forested. The topographic relief varies more than 650 feet within the basin, and as a consequence, runoff is more rapid than in many areas in Minnesota.

Runoff from snowmelt started in the Root River basin at the end of February, as the result of warm temperatures, and was intensified by a heavy rain which fell on March 1 . This condition created very sharp ice-a ffected peaks in the basin on March 1 and 2 (fig. 40). Stages exceeded any previously known from Lanesboro, Minn., to the mouth. At the gaging station near Houston, Minn., the maximum stage of 18.32 feet was more than 3 feet higher than the previous maximum stage in 44 years of record, but the maximum discharge of the 1965 flood was exceeded twice during the period of record. Recurrence intervals of the maximum discharge varied from 19 years at Lanesboro to 30 years near Houston. Paradoxically, the recurrence intervals of tributary peaks on Rush Creek and South Fork Root River were only 5 years and 3 years, respectively.

The economy of the Root River basin, which depends largely upon agriculture, was not seriously affected because most of the damage was urban. Greatest damage occurred in Houston where most of the business establishments and about 255 homes were flooded. Streets and sewerlines also were damaged. In Rushford, 150 homes and 40 businesses were flooded, and in Peterson, 35 homes were flooded. Total damages in the basin exceeded $\$ 2$ million to urban property and about $\$ 320,000$ to agricultural property. 


\section{CHIPPEWA RIVER BASIN}

The major flooding in the Chippewa River basin occurred in the lower reaches of the main stem and along the Red Cedar River, the second largest tributary, which flows into the Chippewa River 23 miles above its mouth. The most significant flooding in the upper part of the basin occurred in medium-sized subbasins. Reservoirs stored much of the northern runoff and greatly reduced the flood threat on the upper Chippewa River and its major tributary, the Flambean River.

On April 14, the Chippewa River at Durand, Wis., near the mouth, peaked at $66,200 \mathrm{cfs}$, which is a flood of 5-year recurrence interval. The river crested and receded slowly, it remained above $50,000 \mathrm{cfs}$ for about 8 days. The Red Cedar River, which flows into the Wisconsin River 9.5 miles above Durand, peaked at Menomonie, Wis., on April 12 at $28,700 \mathrm{cfs}$, which is 1.24 times a 50 -year flood. The major tributary of the Red Cedar River, the Hay River, peaked near its mouth on April 11 at 10,900 cfs, a 22 -year flood.

High runoff occurred later in the headwaters of the Chippewa and Flambeau Rivers. The big reservoir, Lake Chippewa, began storing water on April 5 and rose steadily until it became full in mid-May. The Flambeau Flowage filled between April 10 and May 10.

Lake Wissota on the Chippewa River, just upstream from Chippewa Falls, Wis., stored over 3,200 million cubic feet of water during the period April 10-14, or an equivalent average discharge of 7,400 cfs. The head on the powerplant service pond exceeded its normal operating elevation throughout the period of maximum discharge April 14-18. At the gaging station at Chippewa Falls, the maximum discharge, which occurred on April 16, was 44,700 cfs, a 3-year flood. Daily discharge exceeded 35,000 cfs during the period April 14-20.

Huge ice cakes damaged many business places in Durand. The floodwaters filled many basements, snapped telephone and electric power poles, and lifted propane tanks from their foundations. Most communities in the low areas along the Red Cedar River experienced minor damage from the floodwaters and floating ice cakes. The Red Cedar River passes through Tainter Lake between Colfax and Menomonie, Wis., and the Hay River joins the Red Cedar River in Tainter Lake. Here flood and ice damage to cottages and homes was extensive. Many students from Stout State University in Menomonie joined homeowners on Tainter Lake in building sandbag barricades and breaking up threatening ice rafts. 


\section{WISCONSIN RIVER BASIN}

Flood severity during the period March-May 1965 varied considerably throughout the Wisconsin River basin. Early in March, little runoff occurred in the northern part of the basin, but heavy runoff from snowmelt and rainfall occurred in the southern part. Major runoff began in the upper basin about April 7, but backwater from ice jams was still in effect on some streams as late as April 14. Numerous reservoirs in the north and the Big Eau Pleine Reservoir and power reservoirs in the central part of the basin reduced peak discharges on the main stem of the Wisconsin River.

On the main stem at Merrill, Wis., the mean flow for March was less than that for February. The reservoir system registered a slight gain, which was almost entirely in the Big Eau Pleine Reservoir during the first week of March. Streamflow at Rothschild and Wisconsin Rapids was up slightly the first 2 weeks of March, but daily discharges did not exceed $4,500 \mathrm{cfs}$. The maximum daily discharge in March of the Lemonweir River was about 1,600 cfs compared with almost 3,000 cfs in April. The Lemonweir River enters the Wisconsin River below Castle Rock power dam and upstream from Wisconsin Dells. At Wisconsin Dells, daily discharges ranged from 8,000 to 10,000 cfs during the period March 4-26 as a result of increased runoff from the midbasin tributaries and lowering of Petenwell and Castle Rock power reservoirs in anticipation of high streamflow in April. In the southern part of the basin, the March floods on the tributaries exceeded the April floods. The Baraboo River near Baraboo, Wis., peaked on March 6 at 4,500 cfs, a 7-year flood. The highest crests on the Kickapoo River, the farthest downstream major tributary of the Wisconsin River, also occurred in March, but frequency intervals were only 3-4 years.

Peak discharges in the mid-basin and upper-basin tributaries and the Wisconsin River downstream from the mouth of the Eau Claire River occurred in April. The maximum discharge of 6,980 efs on April 12 on the Eau Claire River at Kelly, 5 miles southeast of Wausau, was the second highest in the periods of record, 1914-26, 1940-65. Recurrence intervals of the April maximums on the tributaries on the upper part of the basin ranged from 6 to more than 50 years, and those in the lower part of the basin ranged from 3 to 14 years. The highest runoff in the basin occurred on the Eau Claire and Rib Rivers, which enter the Wisconsin River just upstream from Rothschild. 
At Rothschild, the Wisconsin River crested on April 12 at a discharge of 49,200 cfs, a 12-year flood. The maximum discharge known at this station was $75,000 \mathrm{cfs}$ in September 1941. At Wisconsin Rapids, the next gaging station downstream, the river crested at $64,000 \mathrm{cfs}$, a 10-year flood, on April 13. Farther downstream at Wisconsin Dells, the peak discharge was 50,200 cfs, a 4-year flood, which occurred on April 15. Pentenwell and Castle Rock Reservoirs, between Wisconsin Rapids and Wisconsin Dells, stored almost 7,600 mef (million cubic feet) in the 5 days April 11-15, which is the equivalent of an average discharge of $17,600 \mathrm{cfs}$ during the 5-day period. The equivalent average discharge for the maximum 2-day rate of storage is over $25,000 \mathrm{cfs}$. The crest continued to attenuate as it moved downstream. The peak discharge at Muscoda, 44 miles upstream from the mouth, was 48,500 cfs, a 3-year flood, occurring on April 18. A hydrograph of the daily discharges at the Muscoda gaging station is shown in figure 40.

Discharges in the reach of the Wisconsin River upstream from the mouth of the Eau Claire River peaked in May after the major flood crest in the lower reach. At Merrill, the peak discharge, which occurred on May 18, was $12,300 \mathrm{cfs}$, a 2-year flood. An earlier peak of 11,300 cfs occurred on April 17. Storage in the 20 upstream reservoirs impounded about 6,700 mcf during the period April 8-25 and another 2,300 mef during the last week of April. The Big Eau Pleine Reservoir stored an additional 3,000 mef during the period April 4-18.

Flood damage in the Wisconsin River basin was generally on the main stem and on the tributaries joining the main stem between Wausau and Wisconsin Rapids. Ice was thick as a result of the long, cold winter, and the breakup was quite rapid. Some destruction was caused by large ice jams. Flood conditions in Schofield, Wis. (fig. 44), at the mouth of the Eau Claire River were intensified by ice backwater. The flood disrupted the operations of many paper mills. It threatened power dams and destroyed a 100-foot section of timber dam at the Mosinee Paper Mills Company. Business places and residences in the Wausau, Schofield, and Antigo areas suffered flood damage. Many highway pavements were undermined and shoulders washed out, but early warning provided time for precautionary measures which kept property damage low in the Wisconsin River basin. 


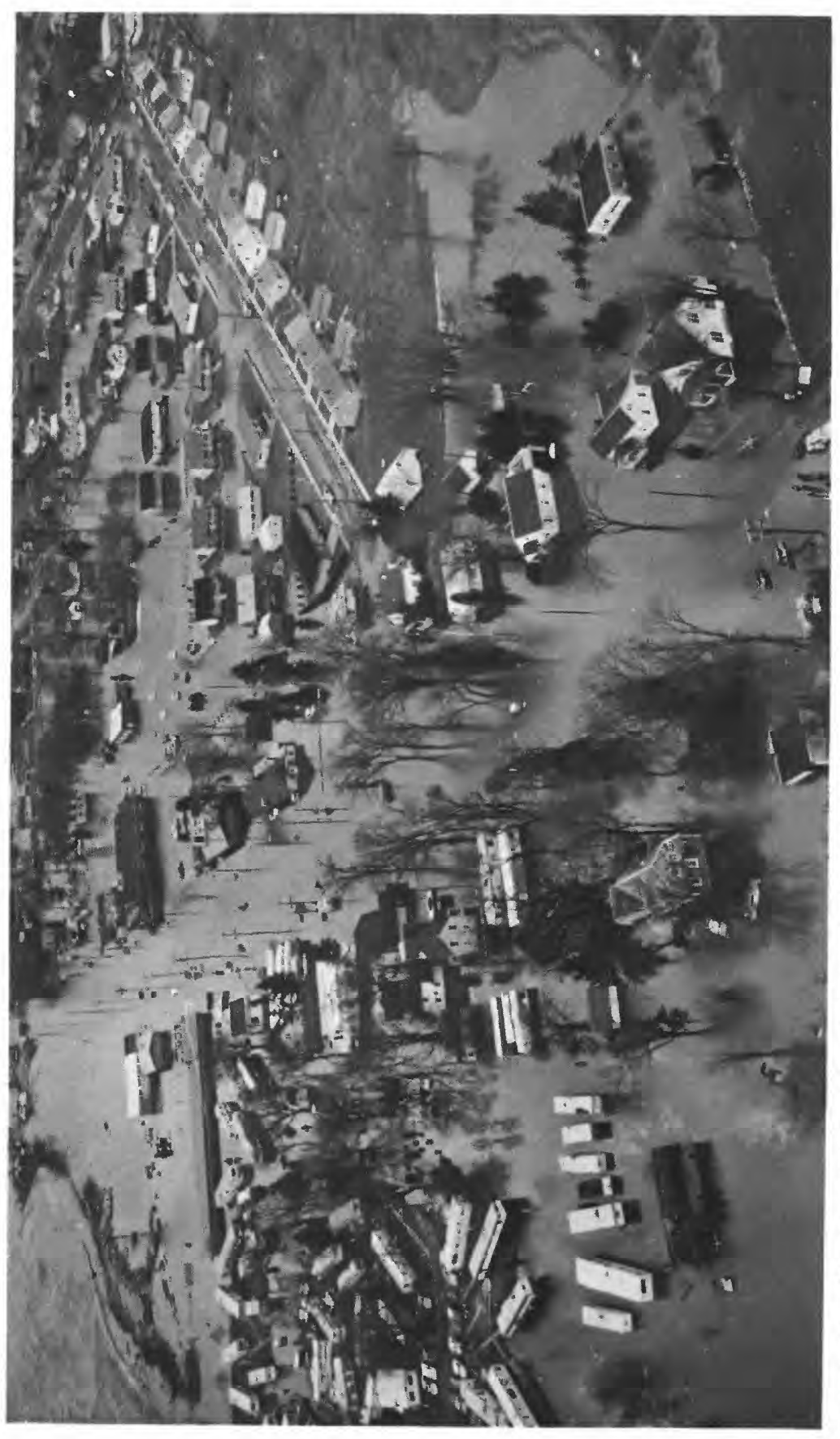




\section{OTHER MISSISSIPPI RIVER TRIBUTARY BASINS IN WISCONSIN}

The magnitude of floods in the smaller basins in Wisconsin, tributary to the Mississippi River, varied considerably. The recurrence intervals of peak discharges on the Trempealeau River were about 30 years, on the Black River about 4 years, and in the southern basins such as the Platte and Galena Rivers less than 2 years.

Severity of peak flow varied with drainage area. On several small basins (drainage areas less thn 30 square miles) between the St. Croix and Trempealeau Rivers, the recurrence intervals of the maximum discharges were from 2 to 4 years, but in the larger basins they were considerably higher. This phenomenon is characteristic of snowmelt runoff. In the small basins, runoff increased during the day, peaked in late afternoon, then decreased sharply as night temperatures dropped below freezing. Daily minimums were reached in midmorning and runoff increased again as temperafures rose. In the larger basins, however, these diurnal fluctuations were less extreme as the flow from individual tributaries was integrated into the larger systems.

The Trempealeau River at Dodge, Wis., about 9 miles upstream from the mouth, peaked on April 7 at 12,100 cfs, a 7-year flood. This flood was considerably less severe than the maximum of record, which was 17,400 cfs on April 4, 1956.

Near the mouth of the Black River the maximum discharge, which occurred on April 13, was 33,000 efs, a 3-year flood. At Neillsville, Wis., where the drainage area is about one-third that at the mouth, a maximum discharge of $18,300 \mathrm{cfs}$ on April 12 was also a 3-year flood. Flood damage in the Black River basin was confined mostly to country roads, but several highways were inundated for a short time.

The La Crosse River peak on April 12 was slightly lower than an earlier peak of 2,610 cfs on March 3, which was a 2-year flood. South of the La Crosse River basin in Wisconsin, major snowmelt runoff occurred in February and March, and April runoff was consequently reduced. Rivers in small basins in southwestern Wisconsin such as the Grant, Platte, and Galena Rivers rose several times in January, February, and March. Maximum discharges generally occurred March 1 or March 31, and recurrence intervals were less than 2 years. The relatively low peaks were due to the frequent melting periods and resultant runoff in January and February.

\section{MISSISSIPPI RIVER TRIBUTARY BASINS IN NORTHEASTERN IOWA}

Temperatures in the forties in the latter days of February melted the 1-5 inches of snow on the ground in northeastern Iowa. This snowmelt, which was accompanied by some rainfall, produced sharp rises on 
the tributary streams from the upper Iowa River basin to the Wapsipinicon River basin. Maximum discharges, however, had recurrence intervals of only $2-4$ years.

Streams also crested at about the same magnitude at the end of March as a result of the melting of snow that had accumulated to depths of 6-12 inches during the March 16-18 storms. The 1/2-2 inches of rain which fell during the period April 3-7 in the Maquoketa and Wapsipinicon basins produced only minor crests.

All the tributaries had peaked well before the Mississippi River crested in this reach. The Upper Iowa River, Paint Creek, and Little Maquoketa River had no significant rises after April 1. The latest crests on the Turkey and Maquoketa Rivers occurred about April 6. The Wapsipinicon River discharged its latest crest into the Mississippi River on April 14, 2 weeks before the peak flow on the main stream.

\section{IOWA RIVER BASIN}

Minor crests occurred the first week in March as a result of snowmelt in the upper part of the basin. The most significant runoff was in April. Ten to eighteen inches of snow had accumulated in the upper part of the basin in March. The melting of this snow during the period March 27 to April 5 and rainfall of 1-11/2 inches on April 3-7 caused extreme floods. The East Branch Iowa River at Klemme, Iowa, crested at 10.67 feet on April 6 during a period of ice backwater. The maximum discharge of 4,090 cfs, 1.33 times the 50-year flood, occurred 2 days later. Downstream at Rowan, the maximum discharge of the Iowa River was 1.05 times the 50-year flood. An ice jam $21 / 2$ miles long formed upstream from Iowa Falls. When it broke loose, the surging water deposited huge ice cakes in the city and inundated some areas to depths of 5 feet. The communities of Tama and Chelsea were cut off or inundated by the rampaging waters. Coralville Reservoir was used very effectively to control the floodflow on the Iowa River. During the period April 2-17, storage in the reservoir eliminated flood damages between the reservoir and the mouth of the Cedar River and reduced the Mississippi River stage at Burlington by about 0.4 foot.

The second crest in April occurred during the third week. Heavy rains of 11/2-51/2 inches on April 24-25 produced sharp rises on all tributaries of the Iowa River. The Iowa River did not crest nearly as high as earlier in the month, but this flow was more significant because the timing coincided with the Mississippi River crest. The Coralville Reservoir storage on this occasion reduced the Mississippi River crest at Burlington by 0.3 foot. Inflow from tributaries downstream from the reservoir, principally the Cedar River, produced a peak discharge of $38,000 \mathrm{cfs}$ on the Iowa River at Wapello during the second crest on 
April 26, just 3 days prior to the arrival of the crest of the Mississippi River at the mouth of the Iowa River. At the time of the Mississippi River crest, discharge from the Iowa River was approximately 5 percent of the total discharge of the Mississippi River below the mouth of the Iowa River.

Discharge hydrographs at selected gaging stations in the Iowa River basin are shown in figure 45 . 

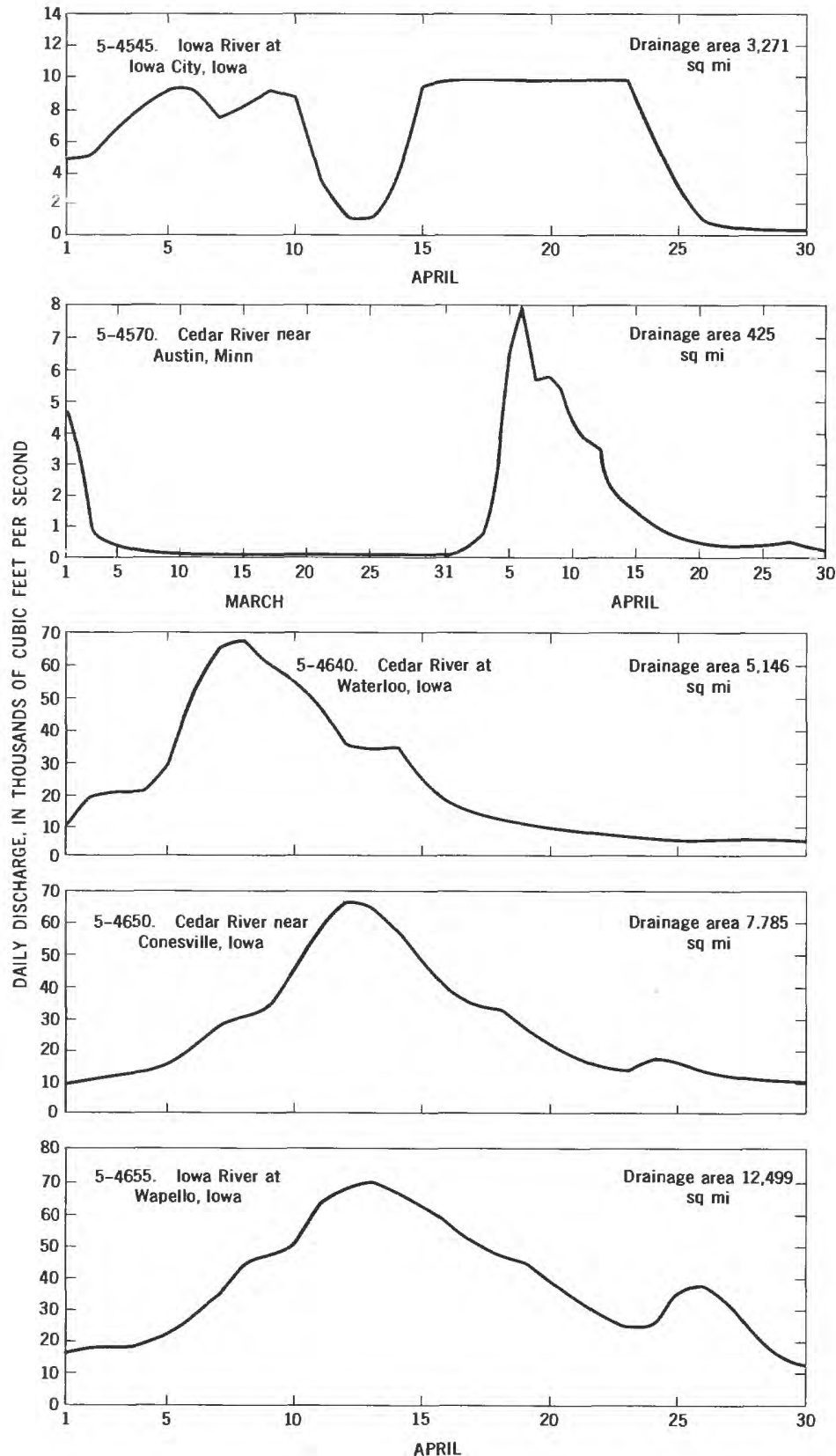

FIGURE 45.-Discharge hydrographs at selected gaging stations in Iowa River basin. 


\section{CEDAR RIVER BASIN}

Rain which fell during the first part of February created a very dense snow pack in southeastern Minnesota; at the end of February, warm weather occurred and on the 1st of March about 11/2 inches of rain fell. These factors caused rapid runoff, which commenced in the upper part of the Cedar River basin on February 28. The rapidity of runoff is indicated by the fact that the daily mean discharge increased from $100 \mathrm{cfs}$ on February 28 to 5,820 $\mathrm{cfs}$ on March 1, at the gaging station near Austin, Minn. The peak instantaneous discharge of 9,400 cfs on March 1 was almost equal to the maximum for the 26-year period of record. The peak discharge at the Austin gaging station was affected by backwater, and the stage was about a foot higher than any previously recorded. The recurrence interval of the March peak at Austin was 5 years.

Adding to the flood flow on the Cedar River were crests from several large tributaries in northern Iowa. The Little Cedar River at Ionia, Iowa, peaked the same day as the Cedar River at Charles City, Iowa. The Winnebago River, Shell Rock River, Beaver Creek, and Blackhawk Creek all crested within a 2-day period. High flows during the early March peak from Cedar River tributaries just upstream from Waterloo, Iowa, contributed to a crest on March 4 at Waterloo of 14.72 feet, 0.28 foot below flood stage.

Later in March, snowfall accumulated to a depth of 6-15 inches over the basin upstream from Waterloo. The melting of this snow in late March and early April plus additional precipitation during the first few days of April created a second peak at Austin of 8,400 cfs on April 6. At this time, however, no ice backwater was present at Austin and the maximum stage was 2.67 feet less than that recorded on March 1 . Farther downstream in northern Iowa, ice jams formed during the early April flood, and caused much destruction at Floyd, Charles City, Nashua, and Waverly, Iowa. Backwater flooded homes and businesses and huge ice cakes caused much damage as they moved with crushing force through the inundated areas. Additional damage resulted from the grinding, crushing force of the ice as it raced downstream after the jam broke. The rapid rise of the river at Charles City, caused by the heavy runoff and an ice jam, necessitated the evacuation by boat of ovnr 40 families. Many highways throughout the area were closed and damaged. An ice jam at Greene, Iowa, on the Shell Rock River backed water into the business district.

The Cedar River and its several major tributaries upstream from Waterloo, Iowa, had significant floodflows early in April. The peak discharge on the Cedar River at Janesville was 29,200 cfs, a 14-year 
flood. The recurrence interval of the peak discharges on the West Fork Cedar River at Finchford, Iowa, and Beaver Creek at New Hartford, Iowa, were 30 and 32 years, respectively. Shell Rock River at Shell Rock, Iowa, had a 7-year flood and Blackhawk Creek at Hudson, Iowa, had a 16-year flood. Fortunately, the crests from all the tributaries did not occur simultaneously with the crest on the Cedar River, but the combined flow on the Cedar River at Waterloo, Iowa, was $69,500 \mathrm{cfs}$ almost 80 percent of the potential peak discharges from the upstream stations. The recurrence interval of the Waterloo peak discharge was 34 years. The peak stage reached 21.67 feet, 6.67 feet above flood stage and only 0.19 foot below the record flood of 1961. More than 400 families were forced from their homes in Waterloo, Cedar Falls, and Evansdale. Many homes were saved from the flood by the gallant efforts of the local residents and a detachment of the Iowa National Guard, who carried out the flood fight as planned by the respective city officials.

The crest at Cedar Rapids, Iowa, was 18.51 feet on April 10, 1.49 feet below the record stage of March 1929 and 1.15 feet below that of March 1961. The Cedar River joins the Iowa River downstream from Cedar Rapids. The Iowa River floodflow above the junction was controlled by Coralville Reservoir, but the Cedar River crest continued down the Iowa River below the junction and reached the mouth of the Iowa River 2 weeks before the crest on the Mississippi River arrived. High flow continued in the Iowa River for the remainder of the month.

\section{SKUNK RIVER BASIN}

A blizzard in the upper Skunk River basin and a 1-inch rain in the lower basin occurred during the period March 16-18. The runoff from the snowmelt and rain produced peaks with recurrence intervals up to $21 / 2$ years. Crests in the lower part of the basin were more significant than those upstream. The stage at the downstream station at Augusta, Iowa, reached 16.99 feet, 2 feet above flood stage, on March 18.

During the storm of April 3-7,11/2-3 inches of rain fell in the basin. The recurrence intervals of flood peaks resulting from this storm ranged from 3 to 5 years. The crests at the upstream stations were more significant than those in March, but the Augusta crest in April was 0.54 foot lower. This crest reached the mouth 2 weeks before the Mississippi River crest.

A minor flood crested the last week in April as a result of heavy rains in the southeastern part of Iowa. This crest was perhaps more significant in relation to the Mississippi River flood because the peak 
outflow at the mouth occurred almost simultaneously with that of the Mississippi River crest. The discharge from the Skunk River, however, was only about 3 percent of the peak flow of the Mississippi River at the mouth of the Skunk River.

\section{DES MOINES RIVER BASIN}

Minor flooding of the Des Moines River and its tributaries between the North Raccoon River and Cedar Creek was caused by the 1-11/2 inches of rain that fell during the middle of March. The recurrence interval of the tributary floods ranged from 3 to 18 years.

The outstanding flood occurred early in April. At the end of March, snow on the ground in the West Fork Des Moines River basin in Minnesota varied from 7 to almost 30 inches. Warm temperatures beginning March 27 began to melt this snow. During the period April 3-7, rainfall, varying from 11/2 to 2 inches in the upper part of the basin, increased the amount of runoff and intensified the flood condition. A rainfall of up to 3 inches in the lower part of the basin produced floods on the tributaries and a rise on the Des Moines River ahead of the main crest moving downstream. Rapid runoff over the frozen ground caused streams to rise, breaking up the heavy ice cover. Numerous ice jams caused flooding and damage.

A maximum stage of 18.62 feet, determined from floodmark occurred on West Fork Des Moines River at Jackson, Minn., on April 6. Heavy ice floes destroyed the gaging station at this time. The maximum discharge of $9,530 \mathrm{cfs}, 1.23$ times the 50 -year flood, was greater than any previous discharge during the 30 years of record, and the maximum stage was 1.2 feet higher than any previously recorded (fig. 46). The flood on the West Fork Des Moines River at Estherville, Iowa, was 1.17 times the 50-year flood; on the East Fork Des Moines River near Burt, Iowa, the recurrence interval was 50 years; and on the East Fork at Dakota City, Iowa, it was 1.03 times the 50-year flood.

At Fort Dodge, Iowa, a large ice jam on the Des Moines River broke and damaged the hydroelectric power dam necessitating hurried construction of a rock fill to prevent failure of the dam. The maximum discharge of $35,600 \mathrm{cfs}$ was greater than any previous discharge during 34 years of record, but the stage was 1.83 feet lower than the flood of June 1947. The recurrence interval of the 1965 flood is 17 years.

The peak stage of Boone River near Webster City, Iowa, reached 15.91 feet, 2.64 feet lower than the flood of June 1954 and 3.2 feet lower than the flood of 1918 . The peak discharge had a recurrence interval of 21 years.

The Raccoon River flows into the Des Moines River at the City of Des Moines, Iowa. Fortunately, the flood crest on the Raccoon River preceded the crest on the Des Moines River by about 5 days. The peak 


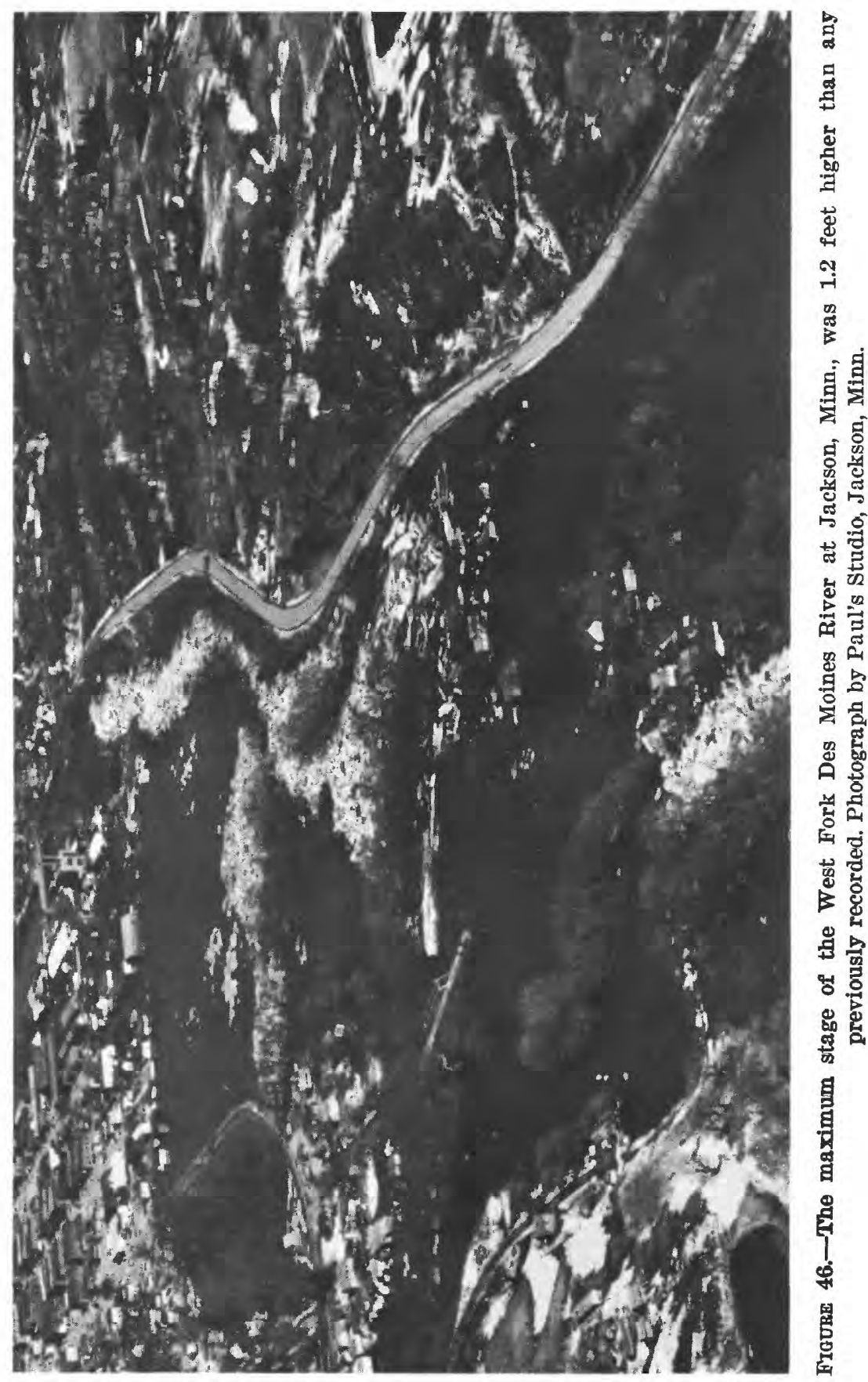


discharge of the Des Moines River below the Raccoon River had a recurrence interval of 19 years and has been exceeded in 1947, 1954, and 1960. Considerable areas adjacent to the Raccoon and Des Moines Rivers were flooded in Des Moines. Many streets, including Riverside Drive, University Avenue, and Vandalia Road, were closed to traffic. The 63d Street Bridge over the Raccoon River was closed because of road damage on each side of the bridge. On April 17, a 100-foot section of the Sixth Avenue Bridge collapsed into the channel of the Des Moines River. The failure was due to the poor condition of the aging concrete bridge as well as to the swollen floodwaters. " "ore than 10,000 telephone circuits and vehicular traffic were cut off when the bridge collapsed. Total damages to city property and cost of the flood fight were about $\$ 671,000$.

As the crest moved downstream from Des Moines, the peak discharge continued to increase as a result of the high runoff from the lower tributaries. At Keosauqua, Iowa, on April 12 the peak discl 'rge was $79,800 \mathrm{cfs}$ at a stage of 19.36 feet, 4.35 feet above flood stage. The recurrence interval of the flood at this location was 9 years. The Des Moines River crest reached the mouth 2 weeks before the Mississippi River crest, but the discharge from the Des Moines River remained high for the remainder of the month. Another minor crest occurrad at Keosauqua on April 26 as a result of heavy rains in the lower part of the basin. The peak discharge of this flood was 33,000 cfs, about 10 percent of the peak flow of the Mississippi River below the morth of the Des Moines River.

A profile of flood stages in the Des Moines River basin and discharge hydrographs at selected gaging stations are shown in figures 47 and 48 . Descriptions and elevations of profile points are given in table 5. 
FLOODS OF 1965 IN THE UNITED STATES
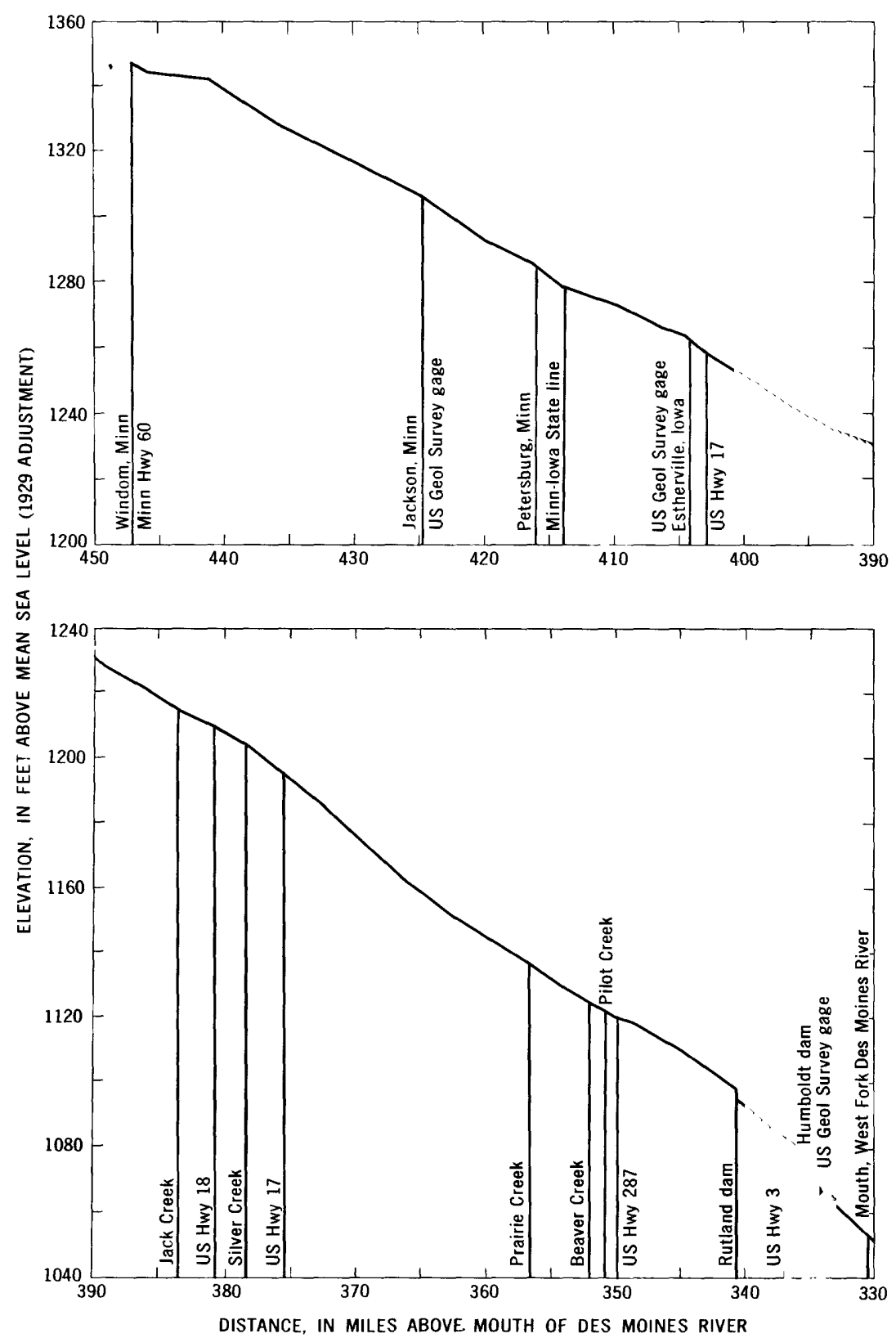

F'IGURE 47.-Flood-crest profile of West Fork Des Moines F 'ver. 


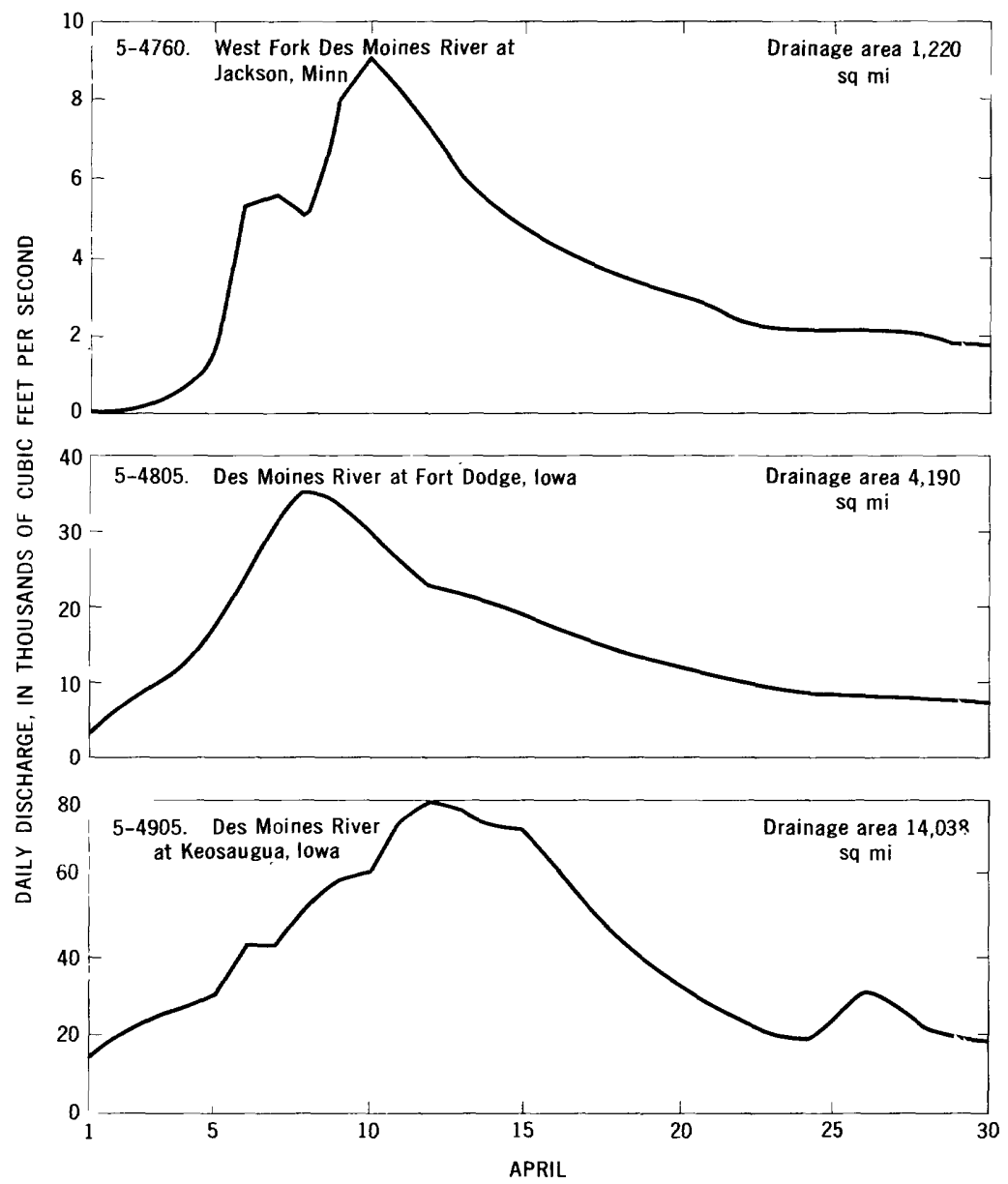

Fraune 48.-Discharge hydrographs at selected gaging stations in Des Moines River basin.

TABLE 5.-Flood-crest elevations, West Fork Des Moines River, April 6-18, 1965

\begin{tabular}{|c|c|c|}
\hline Location & $\begin{array}{c}\text { Miles } \\
\text { above } \\
\text { mouth of } \\
\text { Des } \\
\text { Moines } \\
\text { River }\end{array}$ & $\begin{array}{l}\text { Elevation in } \\
\text { feet (datum } \\
\text { of 1929) }\end{array}$ \\
\hline
\end{tabular}

\section{Windom, Minn.:}

Upstream side of bridge on State Highway 60

447. $0 \quad 1,348.1$

Downstream side of bridge on State Highway $60 \ldots-447.0 \quad 1,347.1$

Downstream side of county bridge in $\mathrm{NE} 1 / 4$, sec. 1, T. 104

N., R. 36 W., Jackson County

Upstream side of county bridge in $\mathrm{S} \mathrm{W} 1 / 4$, sec. 19 , T. $104 \mathrm{~N}$.,

445. $661,344.0$

R. 35 W., Jackson County

441. $181,342.8$ 
TABLE 5.-Flood-crest elevations, West Fork Des Moines River, April 6-13, 1965Continued

Location \begin{tabular}{c} 
Mres \\
above \\
mouth of \\
Des \\
Moijes \\
River (datum \\
of 1929) \\
\hline
\end{tabular}

Upstream side of county bridge near center of line between sec. 16 and 17, T. 103 N., R. 35 W., Jackson County -

County bridge in SE1/4, sec. 28 , T. 103 N., R. 35 W., Jack-

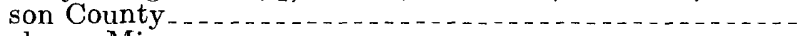
Jackson, Minn.:

Upstream side of bridge on U.S. Highway 16

Downstream side of bridge on U.S. Highway 16

Upstream from

U.S. Geological Survey recording gage downstream from powerplant dam

Upstream side of county bridge at south edge of Jackson

Downstream side of county bridge on line between sec. 7 and 8, T. 101 N., R. 34 W., Jackson County .........

Downstream side of county bridge at Petersburg, Minn...

Minnesota-Iowa State line

Upstream side of county bridge in NW1/4, sec. $28, \mathrm{~T} .100$ N., R. 34 W., Emmet County _._.

Downstream side . . .

Upstream side of county bridge in $\mathrm{SE}_{1} / 4$, sec. $34, \mathrm{~T} .100$ N., R. 34 W., Emmet County _... 407.40

Downstream side. . . . . . . . .

Estherville, Iowa:

Upstream side of Fifth Street Bridge

Downstream side

435. 16

1, 327. 2

431. 69

1, 321. 2

425. 59

429. 59

424. 79

424. 59

424. 59

$1,308.5$

1, 307. 9

1, 306. 9

1, 306. 7

424. 14

1, 306. 37

419. 55

415. 98

1, 304. 1

$1,292.3$

$1,285,6$

$1,278.5$

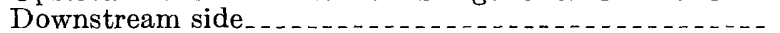

Upstream side of bridge on State Highway 9

Downstream side. . .

U.S. Geological Survey recording gage

Upstream side of bridge on State Highway 17

Downstream side

Upstream side of CRI\&PRR bridge

$1,274.0$

$1,273.2$

Downstream side

Upstream side of county bridge in NW $1 / 4$, sec. $31, \mathrm{~T} .99 \mathrm{~N}$.,

R. 33 W., Emmet County

Downstream side _...

$1,268.5$

$1,266.9$

405. 05

405. 05

404. 60

404. 60

404. 35

404. 35

404. 20

402.90

402. 90

402. 75

402. 75

1, 265. 0

1, 264 . 7

1, 263. 9

$1,263.9$

$1,263.8$

$1,263.5$

$1,263.16$

1, 259. 3

1, 259. 1

1, 258. 6

1, 257.9

Upstream side of county bridge in SE $1 / 4$, sec. 7, T. $98 \mathrm{~N}$., R. 33 W., Emmet County -

396. 65

$1,250.8$

$1,250.5$

Downstream side

Upstream side of county bridge in NE $1 / 4$, sec. $29, \mathrm{~T} .98 \mathrm{~N}$.,

R. 33 W., Emmet County

Downstream side. 396. 65

$1,243.6$

$1,242.8$

393. 10

$1,235.6$

Emmet-Palo Alto County line

$1,235.0$

1, 231. 7

Upstream side of county bridge " $J$ " in NE1/4, sec. 9 , T. 97 N., R. 33 W., Palo Alto County _._ 389.70

Downstream side $\ldots$

Upstream side of CRI\&PRR bridge

$1,230.9$

$1,230.0$

$1,228.6$

$1,227.5$

Upwnstream side of county bridge in $\mathrm{SE} / 4$, sec. $27, \mathrm{~T} .97 \mathrm{~N}$.,

R. 33 W., Palo Alto County

Downstream side

Below mouth of Jack Creek

Upstream side of bridge on U.S. Highway 18_..... 380

$1,219.0$

1, 218.8

$1,215.1$

1, 210.1 
TABle 5.-Flood-crest elevations, West Fork Des Moines River, April 6-19, $1965-$ Continued

\begin{tabular}{|c|c|c|}
\hline Location & $\begin{array}{c}\text { Miles } \\
\text { above } \\
\text { mouth of } \\
\text { Des } \\
\text { Moines } \\
\text { River }\end{array}$ & $\begin{array}{l}\text { Elevation in } \\
\text { feet (datum } \\
\text { of 1929) }\end{array}$ \\
\hline
\end{tabular}

Downstream side

380. 60

$1,209.6$

Upstream side of CMStP\&PRR bridge

1, 208. 0

ownstream side of county bridge in $\mathrm{NW}^{1 / 4}$, sec. $26, \mathrm{~T}, 96$

N., R. 33 W., Palo Alto County.

379. 55

1, 206. 8

Above mouth of Silver Creek

378. $40 \quad 1,203.6$

Below mouth of Silver Creek._._._.

$1,203.8$

Upstream side of county bridge in SE1/4, sec. $35, \mathrm{~T} .96 \mathrm{~N}$., R. 33 W., Palo Alto County _..... 377.65

Downstream side

377. 65

1, 202. 0

Upstream side of bridge on State Highway 17

Downstream side

375. 55

Upstream side of county bridge in NE1/4, sec. $21,7.95 \mathrm{~N}$., R. 32 W. Palo Alto County

Downstream side

375.55

$1,201.4$

$1,195.5$

$1,194.8$

Upstream side of county bridge in $\mathrm{N} \mathrm{W} 1 / 4$, sec. $25, \mathrm{~T} .95 \mathrm{~N}$. R. 32 W., Palo Alto County

372. 10

1, 184. 3

372. 10

$1,183.3$

Downstream Side

Upstream side of county bridge "C" in $\mathrm{SW} 1 / 4$, sec. $29, \mathrm{~T} . \overline{95}$ N., R. 31 W., Palo Alto County

Downstream side

Below mouth of Cylinder Creek

369. 15

1, 173. 2 369. 15

1, 172.9

Upstream side of county bridge in NE1/4, sec. $8, T .94 \mathrm{~N} ., \mathrm{R}$. 31 W., Palo Alto County -

367. 10

367. 10

1, 166. 3

$1,164.9$

365. 20

$1,159.9$

Downstream side

Upstream side of county bridge in SE $1 / 4$, sec. $17, \mathrm{~T} .94 \mathrm{~N} ., \mathrm{R}$. 31 W., Palo Alto County.

364. 95

$1,159.3$

364. 95

$1,158.4$

Downstream side

Upstream side of county bridge in $\mathrm{NE} / 4$, sec. $29, \mathrm{~T} .94 \mathrm{~N}$. R. 31 W., Palo Alto County

Downstream side

363. 40

1, 154. 6 363. $40 \quad 1,154.3$

Palo Alto-Pocahontas County line

361. 95

1, 151. 2

361. $95 \quad 1,149.7$

358. $601,142.2$

Upstream side of county bridge in $\mathrm{NW} / 4$, sec. $1, T .93 \mathrm{~N} ., \mathrm{R}$. 31 W., Pocohantas County.

357. 80

357.80

Downstream side.

Pocahontas-Humboldt County line

Downstream side

Humboldt-Pocahontas County line.

Upstream side of county bridge in $\mathrm{NW} / 4$, sec. $25, \overline{\mathrm{T}} .93 \mathrm{~N}$., R. 31 W., Pocahontas County. 355.70

Downstream side

Below mouth of Beaver Creek $\ldots \ldots$. 352.00

1, 140. 5

1, 139.3

$1,137.0$

$1,137.0$

$1,136.7$

$1,134.2$

$1,128.1$

$1,127.3$

Upstream side of county bridge "T" in $\mathrm{NE} / 4$, sec. $1, \mathrm{~T} .92$ N., R. 31 W., Pocahontas County $\ldots$

Downstream side

Below mouth of Pilot Creek

Pocahontas-Humboldt County line

Upstream side of C\&NWRy bridge

Downstream side._._._.

Upstream side of bridge on State Highway 287

Downstream side. 349.90

Upstream side of county bridge in $\mathrm{NW} / 4$, sec. $17, \mathrm{~T} .92 \mathrm{~N}$., R. $30 \mathrm{~W}$. Humboldt County. 348.45

Downstream side _._.

Upstream side of county bridge in $\mathrm{NW} 1 / 4$, sec. $23, \mathrm{~T} .92 \mathrm{~N}$., R. 30 W., Humboldt County -

1, 124.8

$1,122.7$

$1,122.3$

$1,122.2$

$1,122.0$

1, 121.4

$1,120.7$

$1,120.2$

$1,119.5$

$1,118.0$

$1,117.6$

1, 108.0 
Table 5.-Flood-crest elevations, West Fork Des Moines River, April 6-13, 1965Continued

\begin{tabular}{|c|c|c|}
\hline Location & $\begin{array}{c}\text { Milss } \\
\text { abore } \\
\text { mouth of } \\
\text { Des } \\
\text { Moines } \\
\text { Rirer }\end{array}$ & $\begin{array}{l}\text { Elevation in } \\
\text { feet (datum } \\
\text { of 1929) }\end{array}$ \\
\hline Downstream side_. & 344.25 & $1,107.8$ \\
\hline Upstream of Rutland Dam. & 340.70 & $1,098.6$ \\
\hline Downstream of Rutland Dam & 340.70 & $1,095.1$ \\
\hline $\begin{array}{l}\text { Upstream side of county bridge in } \mathrm{SE} 1 / 4 \text {, sec. } 29, \mathrm{~T} .92 \mathrm{~N} \text {, } \\
\text { R. } 29 \text { W., Humboldt County }\end{array}$ & & 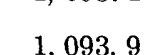 \\
\hline Downstream side & 340. 30 & $1,093$. \\
\hline $\begin{array}{l}\text { Upstream side of country bridge in SE1/4, sec. } 34, \text { T. } 92 \text { N., } \\
\text { R. } 29 \text { W., Humboldt County }\end{array}$ & 337.50 & 1.084 \\
\hline $\begin{array}{l}\text { Downstream side. } \\
\text { Humboldt. Iowa: }\end{array}$ & 337.50 & 1,084 . \\
\hline $\begin{array}{l}\text { Humboldt, Iowa: } \\
\text { Upstream side of bridge on State Highway } 3\end{array}$ & 20885 & 10834 \\
\hline Downs & $\begin{array}{l}336.85 \\
336.85\end{array}$ & $1,083.0$ \\
\hline Upstream of Humboldt Dam & 335.15 & $1,078.2$ \\
\hline Downstream of Humboldt Dan & 335.15 & $1,073.1$ \\
\hline Upstream side of bridge on State Highway 169 & 334.85 & $1,070.5$ \\
\hline Downs & 334.85 & $1,070.5$ \\
\hline Upstream side of Sumner Avenue Brid & 334.40 & $1,069.1$ \\
\hline Downstream side & 334.40 & $1,068.0$ \\
\hline $\begin{array}{l}\text { U.S. Geological Survey wire-weight gage on upstream } \\
\text { side of First Avenue Bridge. }\end{array}$ & 334 & \\
\hline Downstream side & $\begin{array}{l}334.30 \\
330\end{array}$ & 1,067 . \\
\hline $\begin{array}{l}\text { Upstream side of county bridge in SW } 1 / 4 \text {, sec. } 9 \text {, } \\
\text { R. } 28 \text { W. Humboldt County }\end{array}$ & & \\
\hline Downstream side & 330.85 & $1,053.9$ \\
\hline Mouth of West Fork Des Moine & 330.40 & \\
\hline
\end{tabular}

MISSISSIPPI RIVER TRIBUTARY BASINS IN ILIINOIS

Minor floods occurred on streams tributary to the Mississippi River in Illinois. Flooding along these streams was confined principally to the lower reaches and was due almost entirely to backwater from the Mississippi River.

At the gaging station on Mill Creek at Milan, Ill., the peak stage of 11.53 feet exceeded the previous maximum by 2.17 feet. Conditions at this station were unique in comparison with other tributary streams in Illinois because it was the only station at which both the peak stage and discharge exceeded previous maxima.

Backwater on the Rock River caused flooding at Rock Island. About 300 homes were flooded and about 1,000 persons were evacusted.

Extensive flooding occurred along the Mississippi River particularly in the reach from the Illinois-Wisconsin State line downstream to Quincy. Many families were evacuated when some levees failed after withstanding floodwaters for nearly 3 weeks. An area of about 60,000 acres, most of which was rich farmland, was inundated. 


\section{MISSISSIPPI RIVER TRIBUTARY BASINS IN MISSOURI}

During the period March-May 1965, only minor crests occurred on the streams in Missouri that are tributary to the Mississippi Piver upstream from the mouth of the Missouri River. Peak discharges having recurrence intervals of 1 or 2 years occurred in March or April prior to the time of the peak discharge on the Mississippi River. The runoff from the tributary basins was less than 1 percent of that $\mathrm{c} f$ the Mississippi River at Alton, Ill., during the period March-May 1965.

\section{FLOOD OPERATIONS}

The President of the United States declared a total of 183 counties in the five-State area eligible for natural-disaster assistance under Public Law 875. Eighty-seven of these counties were in Iowa, 65 in Minnesota, 14 in Wisconsin, 10 in Illinois, and seven in Missouri.

The Office of Emergency Planning set up disaster field offices in Mankato and St. Paul, Minn., La Crosse, Wis., and Iowa City, Iowa, to provide information for local communities on the procedures for applying for Federal disaster assistance under Public Law 875 .

All the military services were involved in the flood-fighting effort. The Army National Guard expended 7,186 man-days on flood-fighting activities in Minnesota and 2,410 man-days in Wisconsin. Records show that 2,830 guardsmen were called to duty during the flcod in Iowa; 612 were called in Illinois, and 279 in Missouri. In addition, the National Guard furnished mobile equipment, airplanes, and helicopters. The U.S. Navy and U.S. Air Force provided helicopters for reconnaissance and rescue missions and the Coast Guard provided emergency communications equipment and patrol boats for rescue and security missions. Navy and private planes obtained aerial photographs of the Mississippi River and the Minnesota River at or near their crests.

Early in March the U.S. Army Corps of Engineers made snow surveys in the Minnesota and upper Mississippi River basins. During the flood, the Corps of Engineers North-Central Division office in Cl icago and the district offices in St. Paul, Minn., and Rock Island, Ill., established emergency operations centers in 60 major disaster areas. These centers assisted the local communities and provided vital information concerning flood-fighting techniques. The Corps also made surveys of damage during and after the flood.

The U.S. Bureau of Public Roads, in cooperation with the State Highway Departments, provided cost estimates for emergencr road repairs and restoration of damaged highway structures. The Bureau of Public Roads also provided assistance to States and counties in implementing the provisions of Public Law 875. 
The Department of Health, Education, and Welfare, in cooperation with State Departments of Health, inspected public water supply and sewage disposal systems and evaluated applications for disaster relief from the stricken communities.

The Federal Housing Conmission surveyed damage to public buildings in the flood-stricken area and informed flood-affected communities on its programs for granting low-interest loans.

The National Red Cross provided staff workers in the flooded areas to assist local chapters. Total expenditures by the National Red Cross were about $\$ 1,800,000$. More than 150,000 flood victims and workers received Red Cross care and assistance.

Recognition should be given to the inhabitants of the stricken communities for the great effort they put forth to diminish damages to public property, businesses, and homes. Volunteer flood wcrkers turned out by the thousands and great quantities of equipment and supplies were donated to the flood-fighting effort. High school and college students, in particular, endeared themselves to many communities by their selfless and tireless efforts to control and minimize the damage caused by floods of unprecedented magnitude.

In the five-State flood-affected area, about 50 engineers and technicians of the Water Resources Division, U.S. Geological Survey, collected the stage and discharge data which are tabulated in this report. Many of the discharge measurements upon which the computation of data depends were made under extremely adverse conditions involving inclement weather, impassable roads, ice floes, submrrged gages, high stream velocities, deep soundings, and wide overflow channels. Discharge measurements in channels and overflows up to a mile and a half wide had to be made by boat. The U.S. Geological Survey Flood Coordinator in St. Paul forwarded discharge data as soon as they were available to the Weather Bureau and the Corps of Engineers. These data were very useful in forecasting flood stages.

In addition to data obtained at gaging stations, flood information was obtained at many other sites. At the request of the Corps of Engineers district office in St. Paul, the U.S. Geological Survey made discharge measurements at lock and dams $3,4,5$, and 7 at the peak and during the recession. Measurements made at gaging stations at Prescott, Wis., and Winona, Minn., provided discharges for dams 2 and $5 \mathrm{~A}$, respectively. Discharges at dams 3 and 7 were measured from highway bridges in Red Wing, Minn., and La Crosse, Wis., respectively. Discharges at dams 4 and 5 were obtained by working over the upstream side of the dam in a basket suspended from a crane located on the dam. The discharge at Lansing, Iowa, was measured from the highway bridge and from a boat and the inflow of the Wisconsin River measured from the highway bridge near its mouth was added to it. 
In spite of the hazardous conditions under which flood data were collected, no U.S. Geological Survey employees were injured excepting one engineer assigned to the St. Paul office, whose arm was hurt when a power sounding crane collapsed.

\section{FLOOD DAMAGES}

Hundreds of communities and several million persons were affected directly or indirectly by the 1965 spring floods in the five-State area of Minnesota, Wisconsin, Iowa, Illinois, and Missouri. For floods of this magnitude, it is difficult to assess flood damages, and estimates ranged widely from $\$ 100$ million to $\$ 190$ million. A summation of total damages prepared from figures furnished by the Corps of Engineers appears in table 6 . In addition to the direct and tangible damages, the economy of the affected region suffered in many ways and the flood caused much human anguish. The losses to the economy and the $\mathrm{r}$ uman suffering can never be evaluated in terms of dollars and cents.

In the upper Mississippi River Basin, 15 lives were lost boause of the floods or flood-associated events, 13 lives were lost in Minnesota, and one life each was lost in Wisconsin and Illinois. More than 700 persons were injured or became ill as a result of the floods. Of the injured, about 60 were hospitalized. More than 11,000 homes were flooded and of these, more than 2,000 suffered major structural demage. Damages to agriculture due to loss of crops, land erosion, deposition of debris, wrecked fences, and destruction of soil conservation structures were great.

The 1965 flood damages in the upper Mississippi River basin totaled almost $\$ 160$ million, far exceeding those caused by any previous flood. In comparison, damages resulting from the 1952 flood were only about

\section{TABLE 6.-Flood damage by States in thousands of dollars}

[Compiled from figures provided by St. Paul, Rock Island, and St. Louis districts, U.S. Army Corps of Engineers]

\begin{tabular}{|c|c|c|c|c|c|c|c|c|c|}
\hline \multirow[b]{2}{*}{ State } & \multicolumn{5}{|c|}{ Urban communities } & \multirow{2}{*}{$\begin{array}{l}\text { Agri- } \\
\text { cul- } \\
\text { ture } 1\end{array}$} & \multirow{2}{*}{$\begin{array}{l}\text { Trans- } \\
\text { porta- } \\
\text { tion }\end{array}$} & \multirow{2}{*}{$\begin{array}{l}\text { Locks, } \\
\text { dams, } \\
\text { levees, } \\
\text { and } \\
\text { navi- } \\
\text { gation }\end{array}$} & \multirow[b]{2}{*}{ Total } \\
\hline & $\begin{array}{l}\text { Com- } \\
\text { mer- } \\
\text { cial }\end{array}$ & $\begin{array}{c}\text { Resi- } \\
\text { den- } \\
\text { tial }\end{array}$ & Public & $\begin{array}{l}\text { Flood } \\
\text { fight } \\
\text { and } \\
\text { cleanup }\end{array}$ & $\begin{array}{l}\text { Sub- } \\
\text { total }\end{array}$ & & & & \\
\hline $\begin{array}{l}\text { Minnesota } \\
\text { Wisconsin.-. } \\
\text { Iowa } \\
\text { Illinois... } \\
\text { Missouri }\end{array}$ & $\begin{array}{r}\$ 14,143 \\
6,844 \\
8,417 \\
7,186 \\
767\end{array}$ & $\begin{array}{r}\$ 9,536 \\
3,578 \\
2,097 \\
2,387 \\
37\end{array}$ & $\begin{array}{r}\$ 6,209 \\
1,457 \\
1,162 \\
1,087 \\
17\end{array}$ & $\begin{array}{r}\$ 9,577 \\
1,320 \\
7,665 \\
8,180 \\
559\end{array}$ & $\begin{array}{r}\$ 39,465 \\
13,199 \\
19,341 \\
18,840 \\
1,380\end{array}$ & $\begin{array}{r}\$ 26,033 \\
557 \\
23,521 \\
211,408 \\
1,431\end{array}$ & $\begin{array}{r}\$ 17,197 \\
2,740 \\
723 \\
603 \\
250\end{array}$ & $\$ 2,728$ & $\begin{array}{r}\$ 82,695 \\
16,496 \\
23,585 \\
30,851 \\
3,061 \\
2,728\end{array}$ \\
\hline Total ............ & $\$ 37,357$ & $\$ 17,635$ & $\$ 9,932$ & $\$ 27,301$ & $\$ 92,225$ & $\$ 42,950$ & $\$ 21,513$ & $\$ 2,728$ & $\$ 159,416$ \\
\hline
\end{tabular}

1 Includes cost of fiood fight in agricultural areas.

2 Organized levee districts suffered about $\$ 2.4$ million damage in Iowa and $\$ 10$ million damage in Illinois. 
$\$ 20$ million. It is interesting to conjecture as to the reasons for the eight-fold increase in flood damages. One of the reasons for this great increase in damage is that the magnitude of the 1965 flood was greater than any other previously known. Also property values have increased over the years, and the cost of materials and labor for flood-fighting has also increased. Perhaps the greatest consideration is the increased occupancy of flood plains. The tremendous property damage that results from major floods points out the need for flood-plain zoning.

A special congressional subcommittee headed by Congressman John Blatnik of Minnesota was appointed by the chairman of the House Committee on Public Works to visit the flood-devastater areas, the committee inspected the damages and met with Federal, State, and local officials. The information obtained was provided to the Committee on Public Works so that this committee could consider legislative matters pertaining to flood control and water resources development (89th Cong., 1st sess., House Committee Print No. 13). 'The subcommittee made early estimates of flood damages including the cost of flood fighting.

Extensive flood damages occurred in many communities. Twentythree urban and industrial areas suffered damages in excess of $\$ 1$ million each (table 7). More detailed information concerning flood losses in the individual basins is presented in the discussion partaining to the basin.

The losses arising from the 1965 floods would have been much greater had not many communities, industries, and individuals undertaken emergency flood-protection measures which curtailed flood damages by many millions of dollars. The flood-fighting effort in itself was costly. In the urban areas flood-fighting costs amounted to over $\$ 27$ million, about equal to 30 percent of total urban damages. According to Corps of Engineers estimates, additional losses of almost $\$ 300$ million would have occurred if emergency flood-fighting moasures had not been undertaken. 
TABLE 7.-Communities where flood damages exceeded $\$ 1$ million

[Based on data furnished by St. Paul and Rock Island districts, U.S. Army Corps of Enginfers]

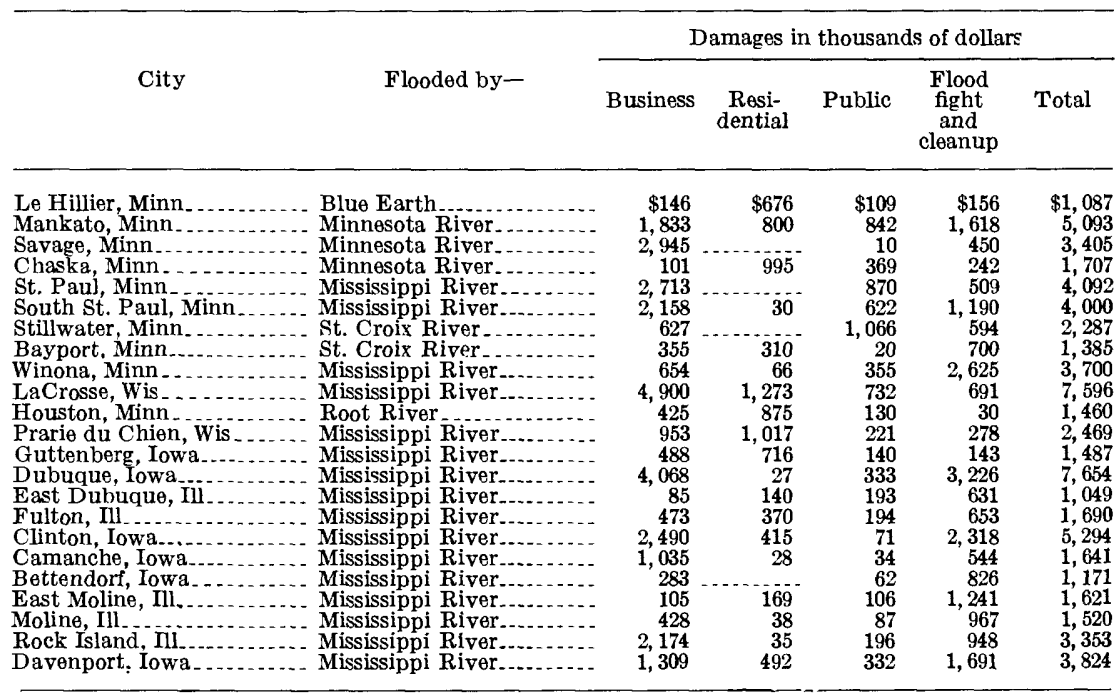

\section{DRAINAGE AND STORAGE EFFECTS}

Whenever a major flood occurs, the causes of the flood and the measures that can be taken to diminish or eliminate future flood losses become topics of interest. The effect of agriculture, drainage ditches, and drain tile often are discussed. Although it might $k_{3}$ safe to say that the existence of drainage ditches and drain tiles has some effect on local peak discharges of short duration, there is little basis for assuming that such drainage works had much effect on the perk discharges of large streams in this flood. In the areas of heaviest runoff during the 1965 flood, water was temporarily impounded in thousands of small depressions and did not run off freely because snow and ice obstructed the smaller drainage channels and drain tiles. This condition created the same effect as that which would result from numerous farm ponds, and no doubt played a small part in actually decroasing the magnitude of flood peaks on some of the major streams. Many peak discharges on streams draining small basins of 10 square miles or less occurred about the same time as the crests on major streams.

Another controversial issue is the effectiveness of farm ponds and small reservoirs in eliminating or decreasing flood damage. Certainly such reservoirs can play a part in changing the runoff pattern in small and moderate floods, and any effect they have would tend to dininish the magnitude of flood peaks. There is a tendency, however, to overemphasize the effectiveness of such reservoirs, especially in major 
floods. For example, consider a hypothetical effort to store the floodflows of the Minnesota River and its tributaries between Montevideo and Mankato, Minn. The area drained in this part of Minnesota, 8,720 square miles, is almost entirely agricultural land of good quality and is worth about $\$ 300$ to $\$ 400$ per acre. Flood stage at Mankato is 19.0 feet and the corresponding discharge is about $30,000 \mathrm{cfs}$. Flood stage on the Minnesota River at Mankato was exceeded for 17 days in April. During this time $1,108,000$ acre-feet in excess of the discharge which occurs at flood stage passed the Mankato gage. During the s?me period, 265,000 acre-feet ran off at Montevideo. Therefore, the area between Montevideo and Mankato contributed 843,000 acre-feet in excess of the discharge at Mankato flood stage. If this runoff excess were stored in the basin between Montevideo and Mankato, 97-acre-feet would have to be stored for each square mile of drainage area.

Most conservation reservoirs or farm ponds cover an acre or two in area and probably would not exceed a usable capacity of 6 acre-feet on the average. Their cost of construction is from about $\$ 2,000$ to $\$ 3,000$ each. It follows, then, that to contain the discharge in excess of flood stage which occurred at Mankato, about 140,000 farm fonds would have to be constructed at a cost of about $\$ 350$ million. In addition, about 200,000 acres of land worth about $\$ 70$ million would be removed from agricultural use. Perhaps the total storage volume in the preceding computation could be reduced somewhat because storing water in headwaters basins would have the effect of flattening out the crest of the unstored water which found its way into the Minnesota River. Nevertheless, providing the farm-pond storage capacity which would have maintained the flow below flood stages at Mankato would have been a formidable undertaking and would have been economically infeasible. It is a fallacy to contend that farm ponds can prevent floods of great magnitude. Knowledgeable proponents of farm-pond construction make no such claim. They do claim, with justification, that farm ponds are an important conservation measure. Proper upstream land management is very effective in minimizing the damages which occur in the many floods of low recurrence intervals.

Large storage reservoirs played an important part in minimizing flood damages. In the Wisconsin River basin, Pentenwell and Castle 
Rock Reservoirs stored about 174,000 acre-feet during the 5 days of maximum flow and materially reduced the discharge of the Wisconsin River below the reservoirs. The peak discharge at Wisconsin Dells was $50,200 \mathrm{cfs}$, a 4 -year flood; it would have been over 70,000 cfs, a 15 year flood, without the storage in Pentenwell and Castle Rock Reservoirs. It is apparent that these reservoirs performed an important floodcontrol function although they were designed primarily for power production.

A further example of the effect of large reservoirs on the magnitude of flooding is illustrated by the Coralville Reservoir on the Iowa River 9 miles upstream from Iowa City. This reservoir is designed to store floodflows temporarily during high-water periods. Its capacity for flood storage is 458,000 acre-feet, equivalent to $23 / 4$ inches of runof". The reservoir is operated to minimize flood damages along the Iowa River and the Mississippi River below the mouth of the Iowa River.

During the period April 1-15, the reservoir stored about 300,00 acrefeet. Maximum discharge above the reservoir at Marengo, Iowa, was $19,900 \mathrm{cfs}$; below the reservoir at Iowa City it was $9,4 \mathrm{CO}$ cfs. Reservoir release was maintained at about 10,000 cfs to draw down the reservoir to provide more storage for possible future floods. On April 23, storage was resumed, and during the period April 23 to May 5, an additional 123,000 acre-feet was stored. During this period, the peak discharge of the Iowa River upstream from the reservoir was 7,770 cfs, not nearly so high as early in April; so it was possible to reduce the release from the reservoir to about $150 \mathrm{cfs}$. The operation of this reservoir during these two periods reduced the flood crest by 0.4 foot on the Mississippi River downstream from the mouth of the Iowa River.

Two reservoirs (Red Rock and Saylorville) are under construction on the Des Moines River. The effect on the Mississippi River stage by the Coralville Reservoir operation during the 1965 flood period, and the predicted future effect that Coralville, Red Rock, and Saylorville Reservoirs would have on the same flood conditions are shown in table 8 . 


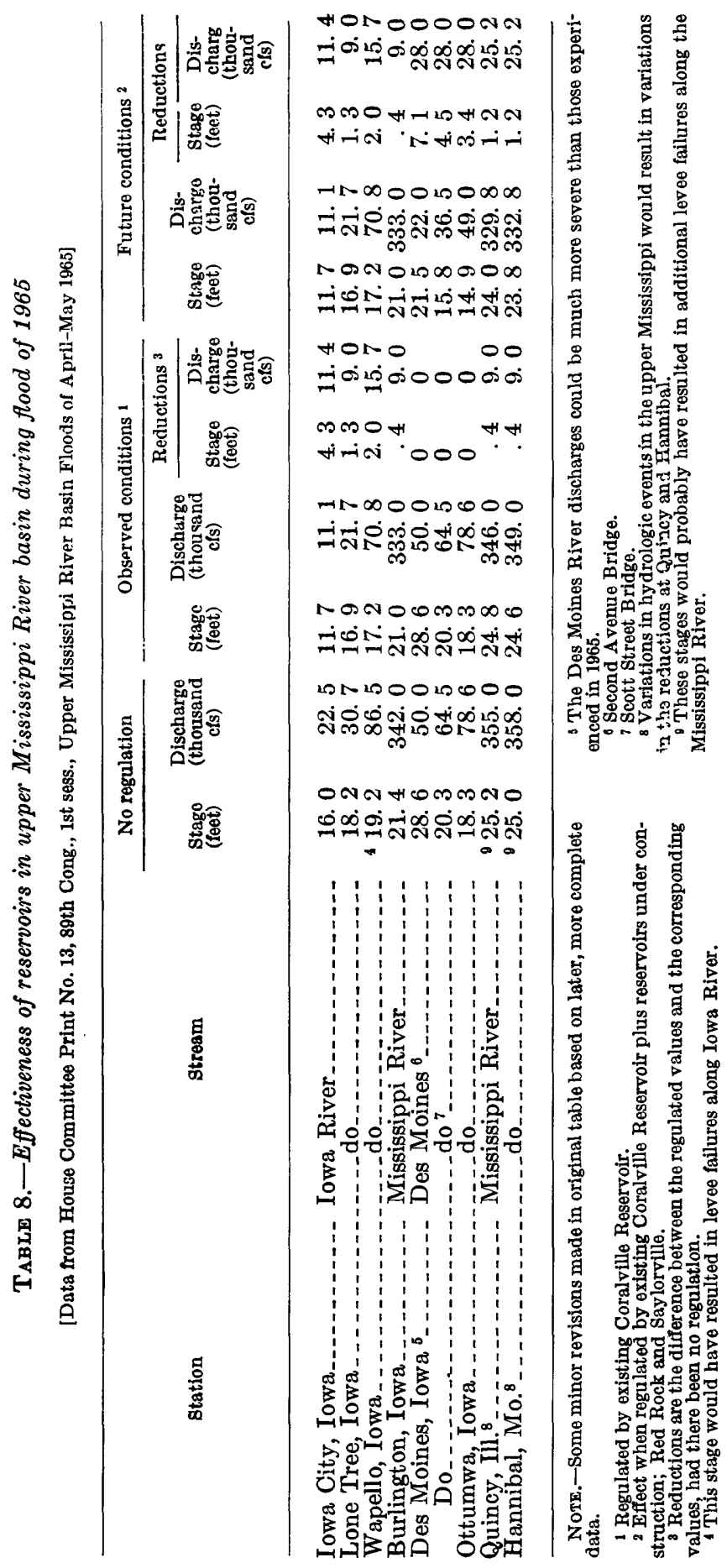




\section{EFFECT OF TIMING OF TRIBUTARY FLOW ON MAIN STEM PEAKS}

Under slightly different meteorological conditions some of the flood crests could have been considerably higher, a disconcerting thought.

Since March 1951 there have been 5 years in which the annual maximum discharges resulted from snow-melt floods in both the Mississippi River and the Minnesota River above their confluence at the southwest edge of St. Paul. During these five periods of runoff, which include the three highest floods of record at most gaging stations in the upper Mississippi River basin, the crest on the Minnesota River arrived at the confluence before the crest on the Mississippi River on three occasions and after the crest on two occasions. In April 1965, the Mississippi River above the Minnesota River crested 6 days after the Minnesota River at its mouth. Meteorological conditions could be such that crests would occur simultaneously in the Minnesota and Mississippi Rivers above St. Paul. If this had occurred in 1965, peak discharge of the Mississippi River at St. Paul would have been more than 200,000 cfs and the stage would have been 2 or 3 feet higher (fig. $49)$. Other examples could be cited where slightly different meteorological conditions would have caused much greater flood stagas and discharges. 


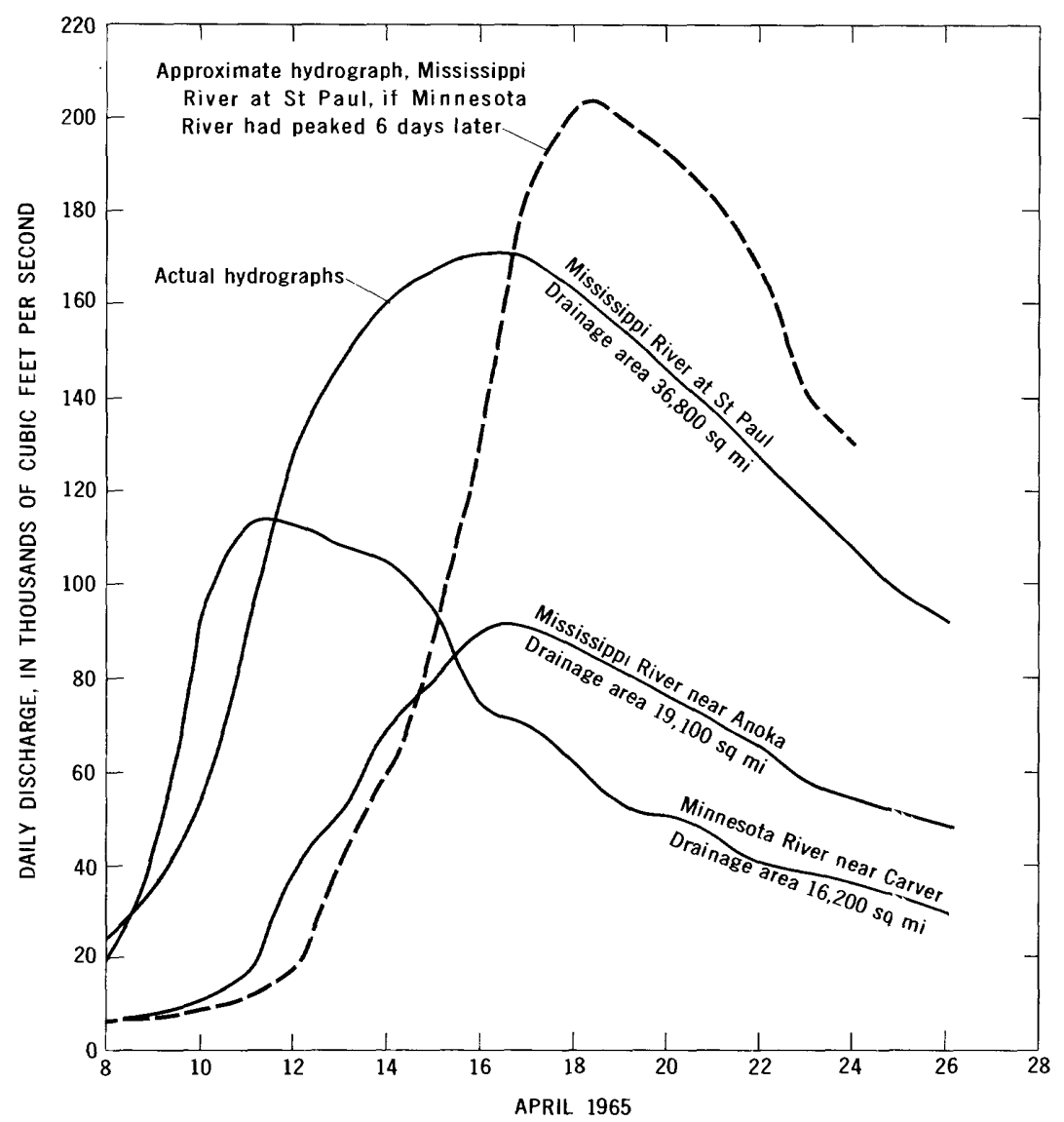

FIGURE 49.-Effect of timing of Minnesota River peak on maxinum discharge of Mississippi River at St. Paul, Minn.

\section{RECURRENCE INTERVALS OF PEAK DISCFARGES}

The frequency of flooding is a major factor in the economical design of structures and in the use of land on or near a stream. Tl sre must be some balance between the cost of flood protection and the potential flood damages or liabilities. Because floods are caused primarily by nature's unpredictable events, this balance can only be ertimated on the basis of average recurrence intervals. The "recurrence interval" of a flood of a given magnitude is defined as the average interval of time within which that flood will be equaled or exceeded once. The methods used by the U.S. Geological Survey to determine flood magnitude and frequency have been described by Dalrymple (1960) and Benson (1962a, 1962b, 1964). These methods have been used in the analyses 
of flood data on streams in the upper Mississippi River basin. Magnitude and frequency relations have been analyzed and published on a regional basis (Patterson and Gamble, 1968) ; some have been published by the U.S. Geological Survey on a State basis, Wis onsin (Ericson, 1961) and Missouri (Searcy, 1955); and some have been published by States, Illinois (Mitchell, 1954), Minnesota (Prior, 1949; Prior and Hess, 1961), and Iowa (Schwob, 1953, 1966). Combining records for all gaging stations in an area with homogenic hydmogic characteristics provides a better basis for determining these relations than individual analyses of relatively short-term records. Flood discharges exceeding the 50-year recurrence interval are expressed as a ratio to the 50-year flood. The records on the Mississippi River main stem have ben analyzed on a station basis (fig. 50) because $\varepsilon$ large river does not have the same runoff characteristics as a smaller tributary.

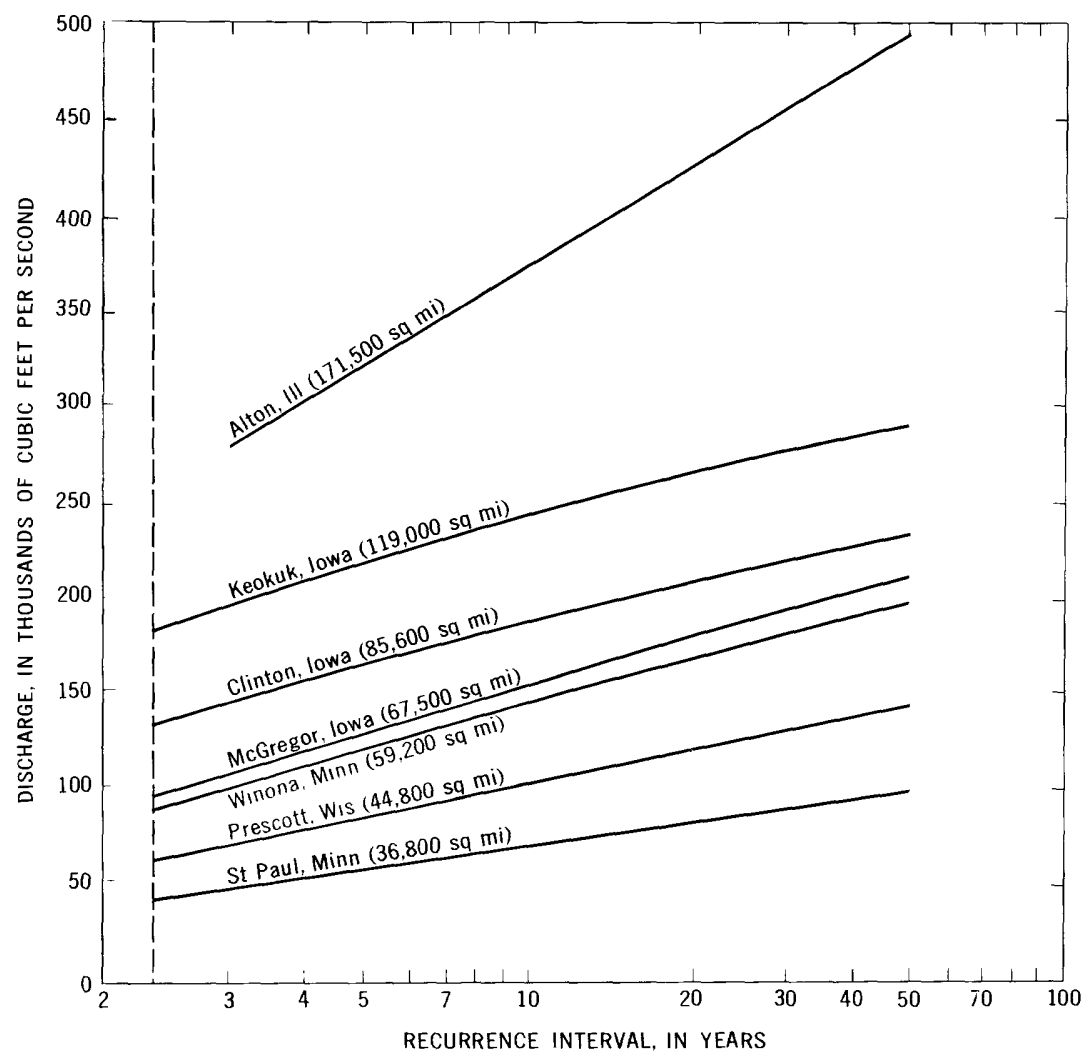

FIGURE 50.-Flood frequency curves for selected Mississippi River gaging stations. 
The recurrence intervals for the floods during the period March to May 1965 on streams throughout the upper Mississippi River basin varied considerably. The Mississippi River main stem and its tributaries, originating mostly in Minnesota and northwesterr Wisconsin, had the greatest recurrence intervals. On the main stem, these values increased from 4 years downstream from the Sandy River near Libby, Minn., to 1.68 times the 50-year flood at St. Paul, Minn., then diminished slowly to 11 years at Alton, Ill., and to only 2 years at St. Louis, Mo., below the mouth of the Missouri River. This pattern of floodfrequency values indicates or substantiates the flood-producing areas. The upstream tributaries, almost all having floods of 1-2 times the 50 -year recurrence interval, added their flows to the main-stem flood. Fortunately, peaks from these tributaries did not occur simultaneously with the main-stem peak, but those entering the main stem upstream from the Root River, which flows into the Mississippi River in southeastern Minnesota, were close enough to contribute an abnormal share to the Mississippi River flow. On tributaries downstream from the Root River, the recurrence intervals of flood peaks were either less than those for upstream tributaries or the timing of the peaks was such that they did not contribute significantly to the main-stem flood. The upper parts of the Cedar and Des Moines River basins experienced floods having recurrence intervals of 5 years to 1.23 times the 50 -year flood, but they attenuated considerably before they enterid the Mississippi River ahead of the main-stem peak. The other tributaries generally had minor floods to 1-10 year recurrence intervals.

\section{DETERMINATION OF FLOOD DISCHARGES}

The flood data in this report were obtained principally at streamgaging stations which are operated to provide stage and discharge data at the gaging site. A stage-discharge relation is developed by measuring discharge at the site at various stages throughout the range of recorded stages. A rating curve is then drawn by plotting discharge against stage, and the curve is translated into a table so tl at the discharge can be determined easily from the recorded or observed stages collected at the gaging site.

Discharges are usually measured by current meter, but during floods they may be determined indirectly by running surveys to high water marks and applying slope-area methods or by computing flow over dams or through contracted openings. These indirect mothods are considerably more expensive and time-consuming than current-meter measurements, but they are often necessary because of the difficulties of obtaining current-meter measurements at all sites during flood 
periods. Such difficulties are created by impassable roads to gagirg stations, running ice floes and debris which make it impossible to make soundings and velocity observations, destruction of bridges and cableways from which discharge measurements are made, and rapid cresting of streams (many crest about the same time) so that there is insufficient time and manpower to obtain current-meter measurements near the crest. Most discharge measurements at gaging-station sites during the 1965 spring flood were made by current meter. Some indirect measurements were obtained, particularly at miscellaneous sites and at crest-stage partial-record stations, where only flood-peak information is obtained.

If a rating curve is fairly well defined throughout most of its range by discharge measurements, either direct or indirect, short extensions may be permissable. Such extensions are usually based on logarithmic plotting or velocity-area studies. At most gaging sites on alluvial streams, the stage-discharge relationship frequently changes as a result of varying control features or shifting control in the channel below the gage; consequently, it is necessary to make discharge measurements at gaging sites at fairly regular intervals even though the stagedischarge relationship is apparently well defined throughout the range in stage.

\section{STREAMFLOW DATA AT GAGING STATIONS}

One of the purposes of this report is to present detailed hydrologic information for streams in the upper Mississippi River basin during the period March-May 1965. Streams on the fringes of the flooded area are included so that the flood can be well defined on an areal basis. Detailed discharge data are presented so that flood hydrograrhs can be accurately reproduced and the data used in flood-control and watershed development studies. Some streams, particularly those in Minnesota and parts of Wisconsin, had only normal, low-flow discharges during March and for these no March discharge data are provided in this report.

The operation of a stream-gaging station requires a stage record, discharge measurements at various stages, and other information pertinent to the computation of the discharge record such as in formation on channel changes or ice formation. The stage record may be obtained from an automatic water-stage recorder which provides a continuous graphic record, from a digital recorder which punches stage on a paper tape usually at 15 -minute intervals, or by reading a nonrecording gage. At many gaging sites which are included in this 
report, the automatic gages were inundated and it was necessary to manually record the flood stages at these sites from nonautomatic temporary gages.

Included in this report are data collected at crest-stage stations which are generally on streams having small drainage areas. The primary function of these stations is to provide data pertaining to peak stages and discharges, and consequently a continuous record of discharge throughout the flood period is not available at these site.

\section{STATION DESCRIPTIONS AND DISCHARGE TABTES}

The description of the gaging station gives information concerning location, datum and type of gage, area of the drainage basin, details of gage-height and discharge records, and miscellaneous remarks. The paragraph on discharge records briefly explains the mathods used to define the stage-discharge relation over the range in stage that occurred during the flood period, use of auxiliary gages or other methods used to obtain discharge, and conditions that may have affected the stage-discharge relation.

The maximum stage and discharge are given for most stations for the flood period March-May 1965, for the previous period of gaging station record, and for any known maximum floods that occurred outside the period of record. Remarks on cooperation, regulation, and other pertinent information are included where applicable.

The table of daily mean discharge gives data for the period MarchMay 1965, a sufficient length of time in most cases, to show discharges during antecedent and recession periods. The table also shows the monthly mean discharge, in cubic feet per second, and the runoff, in inches, from the drainage basin.

The table of stages and discharges at indicated times defines the stage and discharge during significant flood flows during the report period so that accurate hydrographs can be drawn.

The stages of most stations were obtained from records cf digital or continuous water-stage recorders. For a station with an incomplete record, the gage height for an indicated time may be selected from a graph which has been constructed from supplemental gage readings, high-water marks, and other pertinent information. Des ription of the methods used in defining the stage are given in the section of the description concerning gage-height record.

The stations are numbered and arranged in downstream order from headwater to mouth, with stations on tributaries inserted in corresponding order following the order in which the tributaries enter the main stream. The reference number in parentheses preceding the name of the gaging station is the same as that used in figure 2 and will aid in identifying the site. 
SUMMARY OF FLOOD STAGES AND DISCHARGES

Maximum stages and discharges at stream-gaging stations, creststage stations, and miscellaneous sites are summarized in table 9. Most of the column headings are self explanatory. The numbers in the first column correspond to those designating the site in figure 2. Except for a few miscellaneous stations, all stations in the report have permanent station numbers (column 2) as used in the annual streamflow reports of the Geological Survey. Drainage-area determinations are not available for a number of streams.

The first column under maximum previously known floods shows the period prior to March 1965 for which information is available concerning the magnitude of previous floods. The length of this period may exceed that in which continuous records of discharge we ${ }^{r e}$ obtained. More than one period is shown for some stations differentiating between the period of known maximum stages or discharges and the period of known maximum stages and discharges. At stations where the maximum discharge of the period did not occur simultar eously with the maximum stage, the maxima are given on separate lines and a dash inserted in the respective gage-height or discharge column.

The last column shows the average interval of time in which the peak discharge of the 1965 flood can be expected to be equaled or exceeded once. Whenever the interval exceeds 50 years, the ratio of the peak discharge to the discharge of the 50-year flood for that strition is shown. 


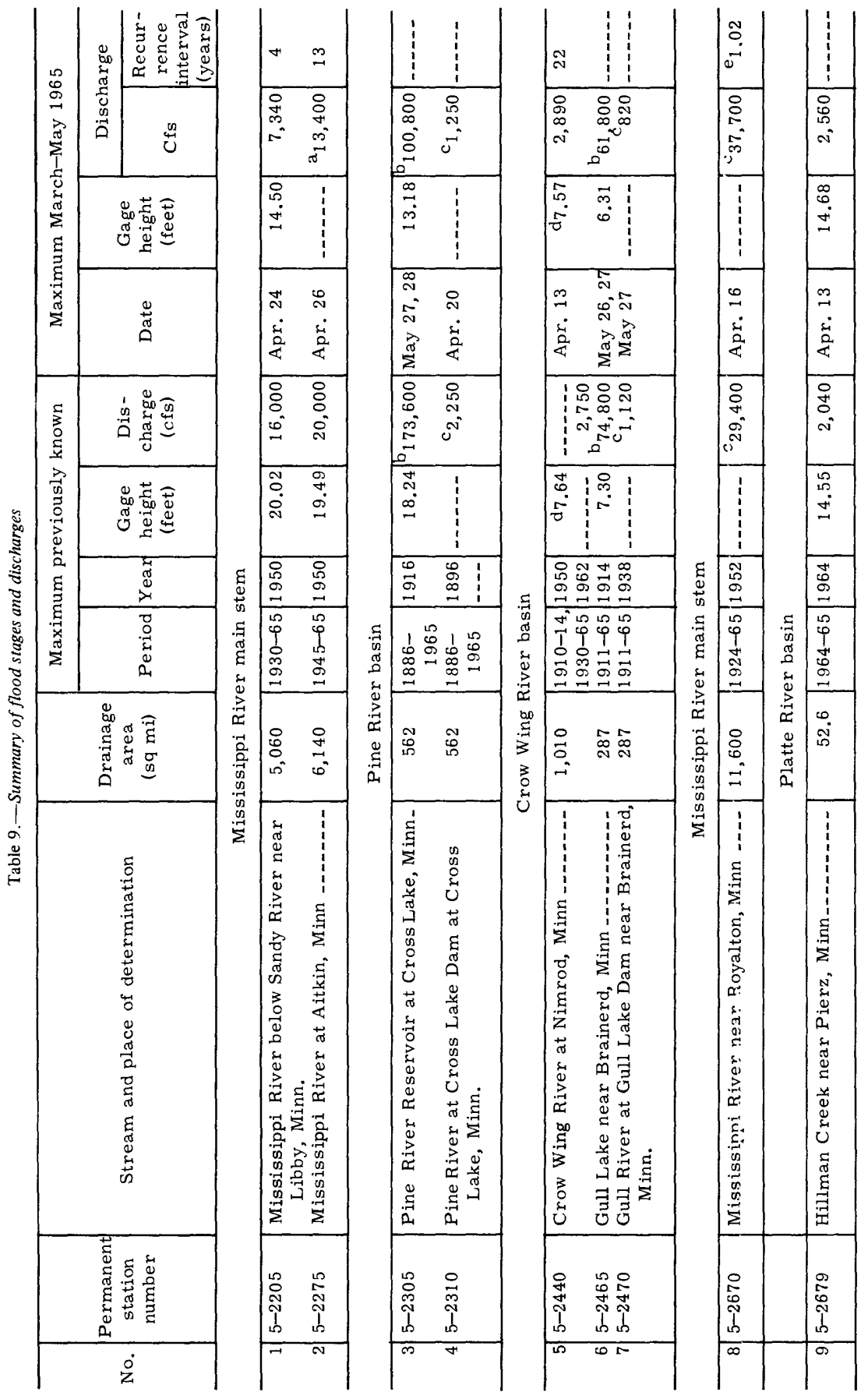


MARCH-MAY, UPPER MISSISSIPPI RIVER BASIN

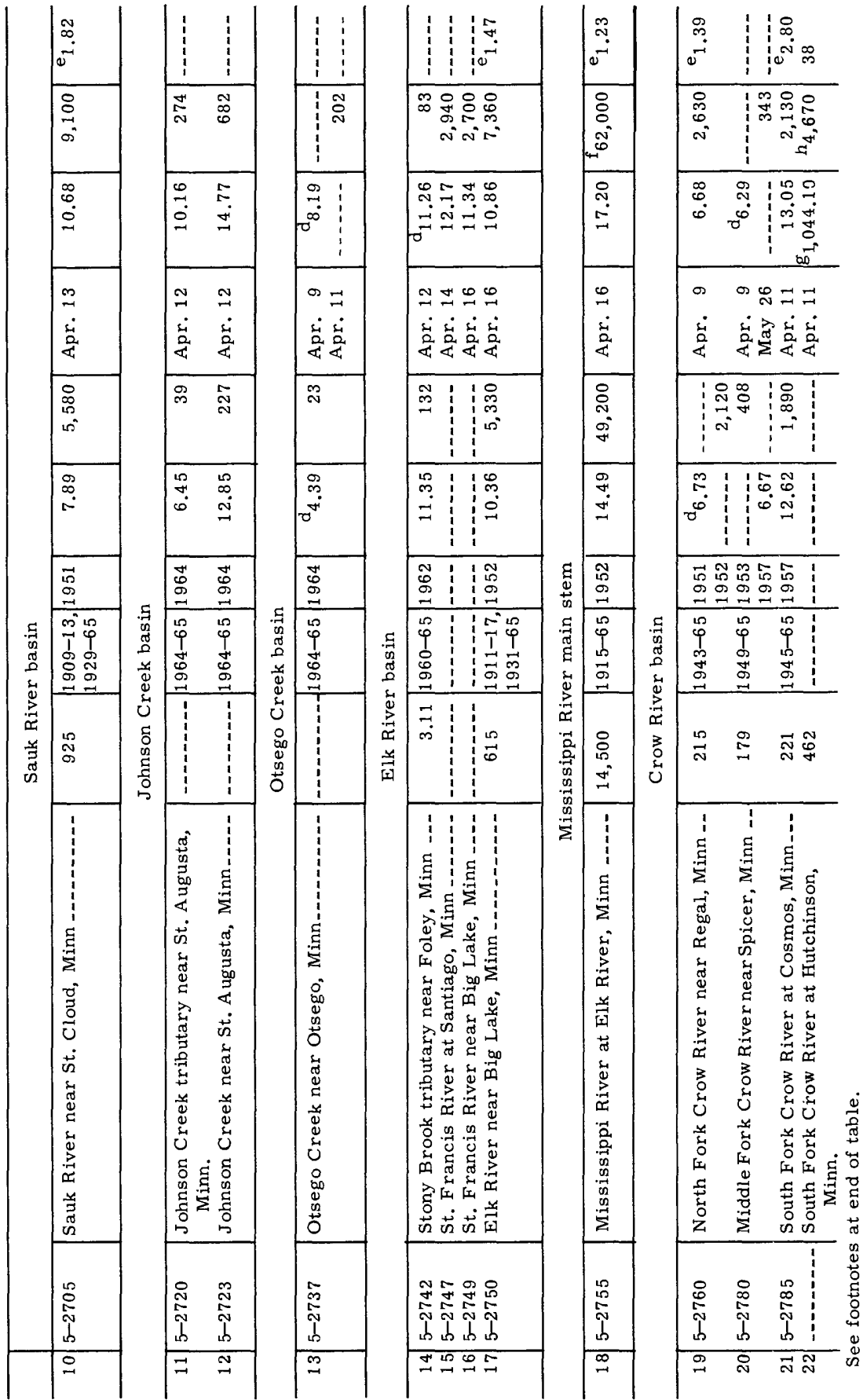




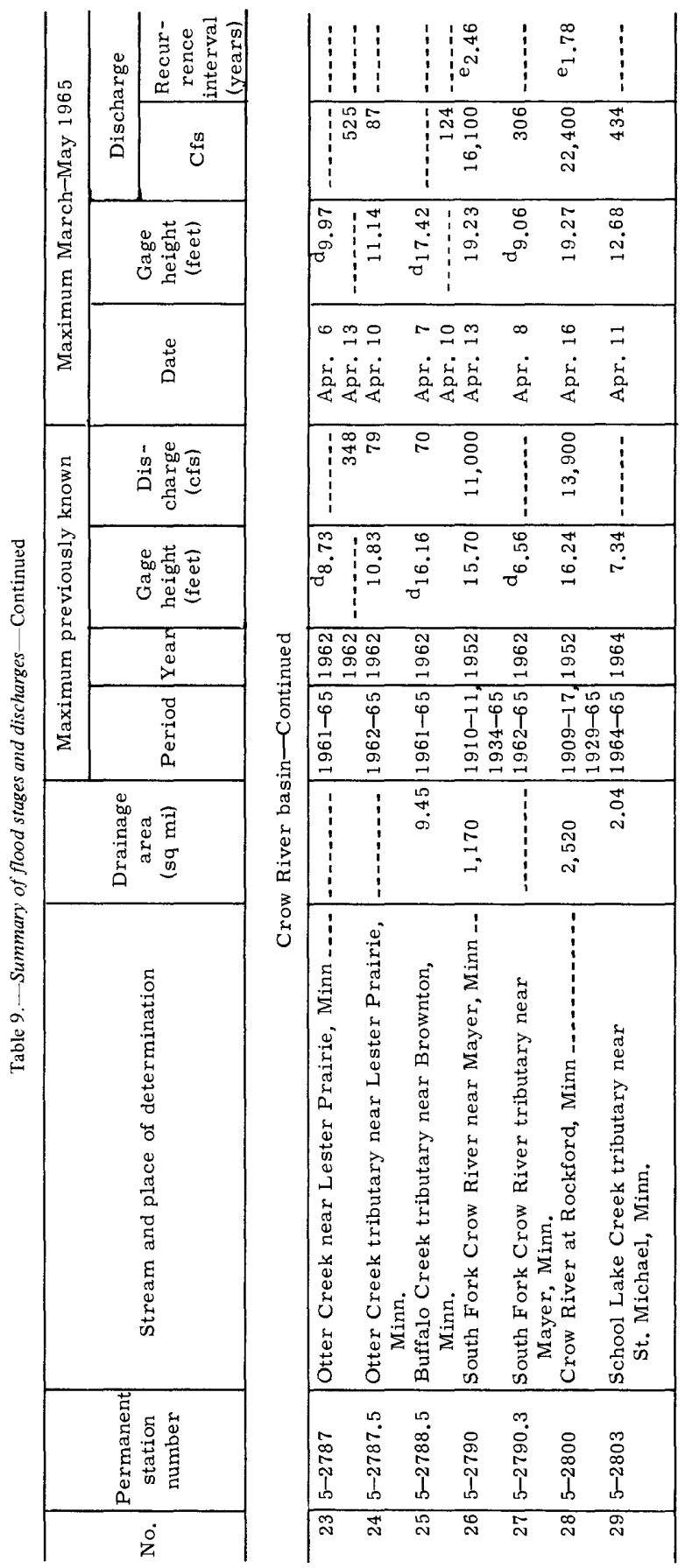

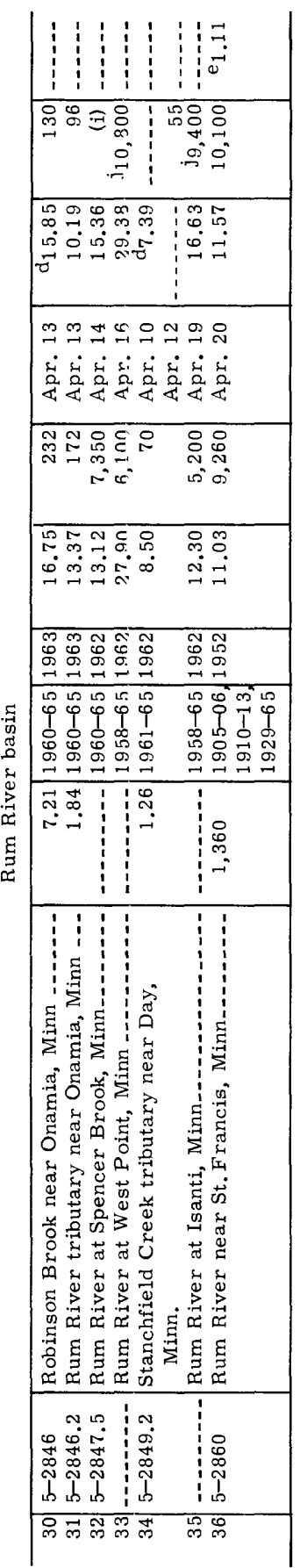


MARCH—MAY, UPPER MISSISSIPPI RIVER BASIN
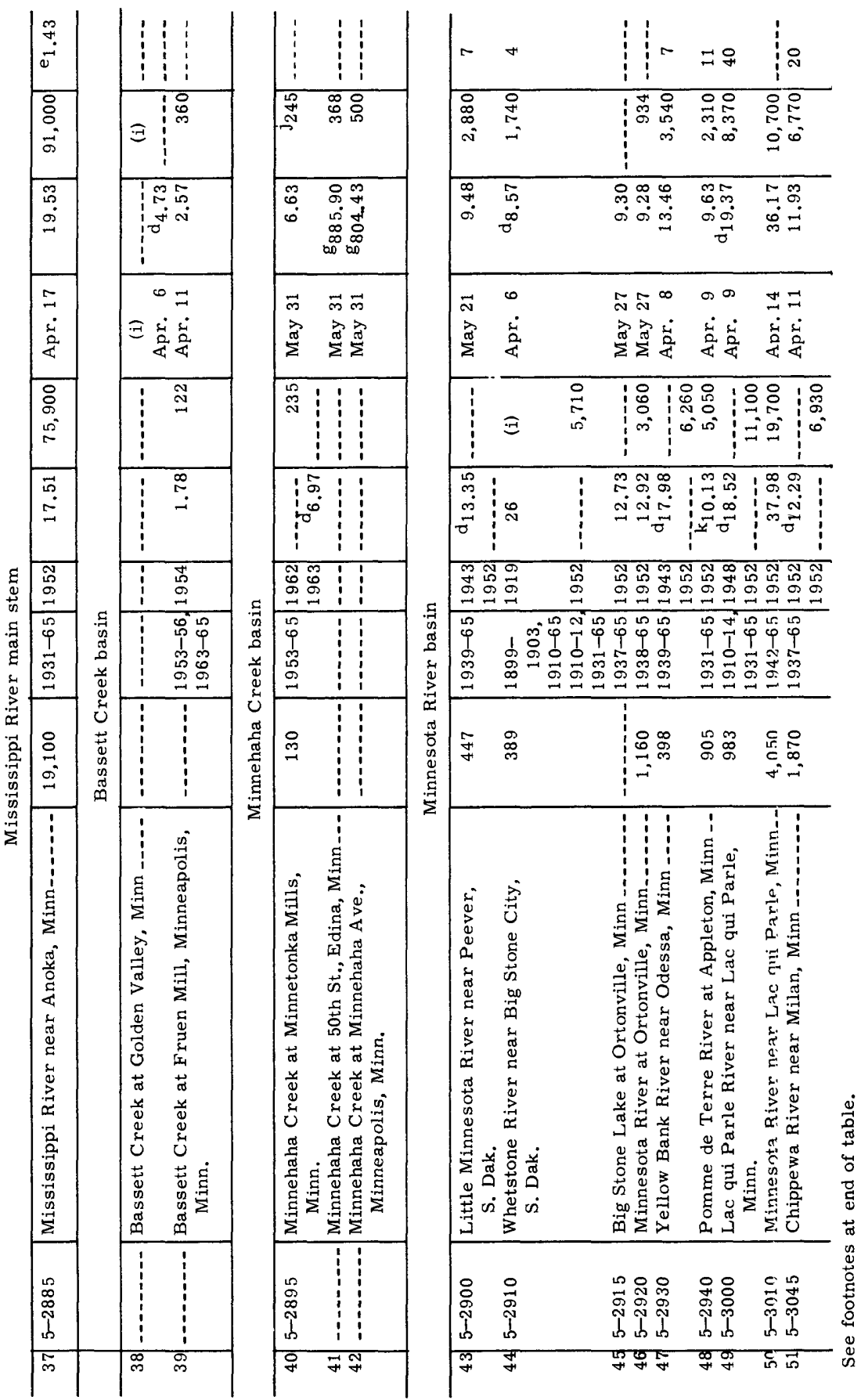


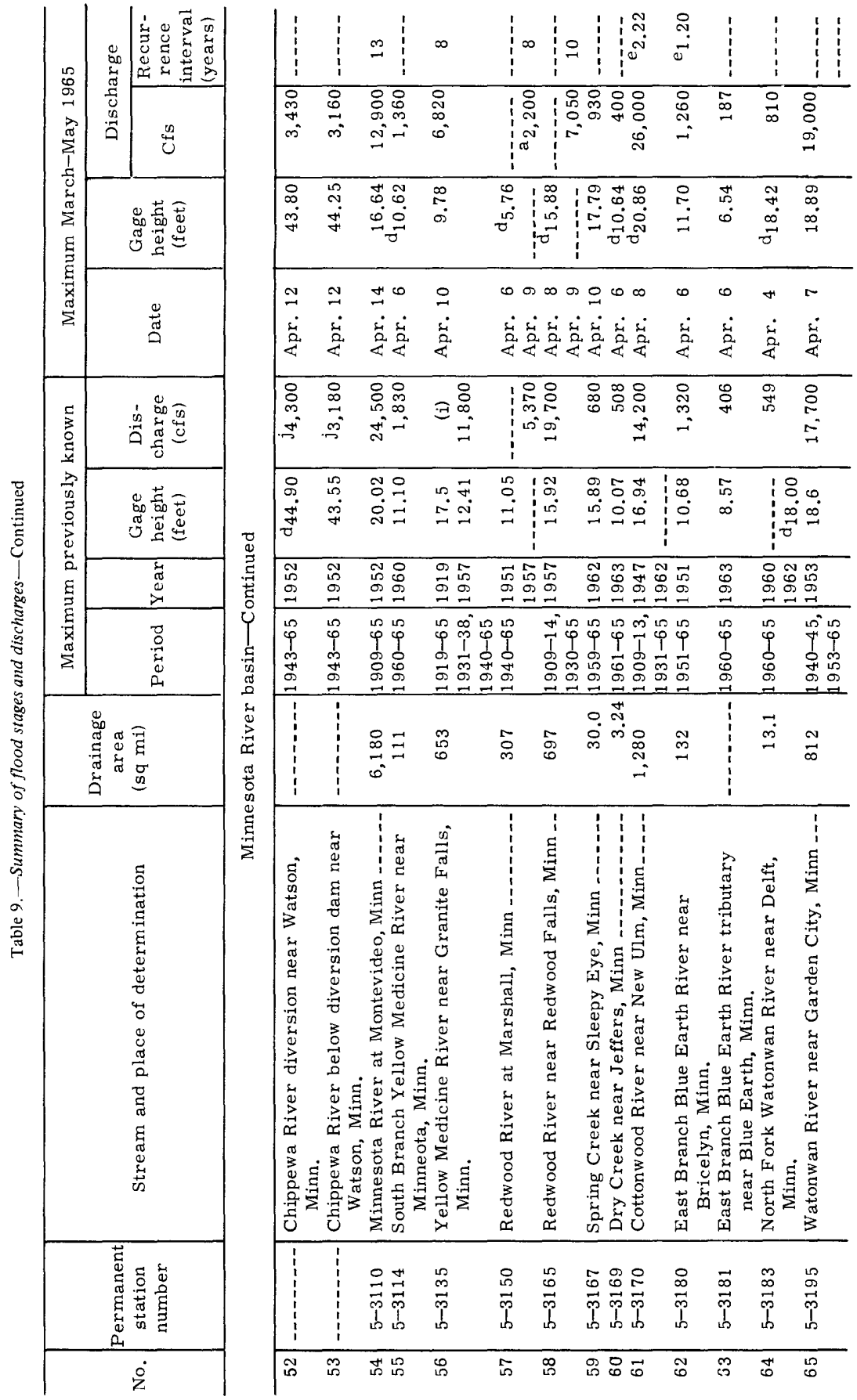


2

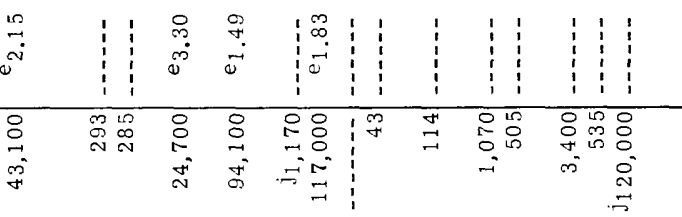

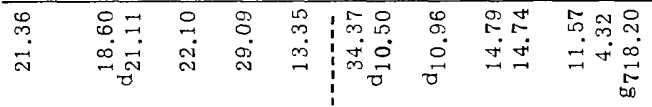

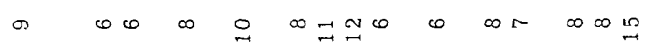

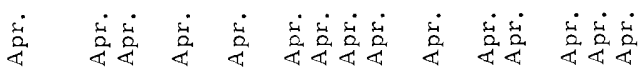

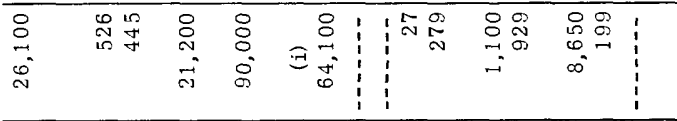

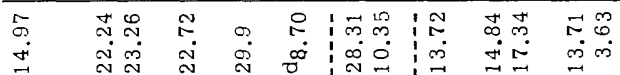

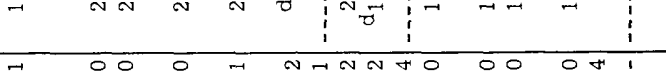

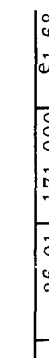

$\mid$\begin{tabular}{l}
$\infty$ \\
0 \\
0 \\
0 \\
\hline 0 \\
0 \\
0 \\
0 \\
0 \\
\hline-1 \\
0 \\
0 \\
0 \\
\hline
\end{tabular}

象

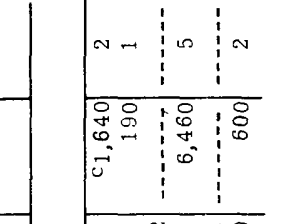

8
8
0
2
2

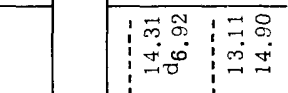

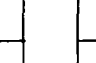

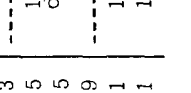

का

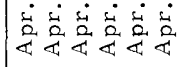

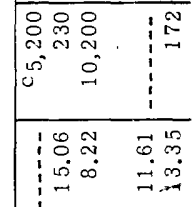

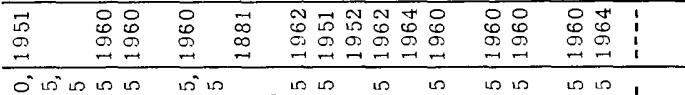

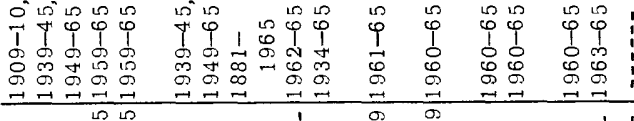

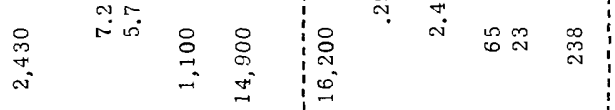

站

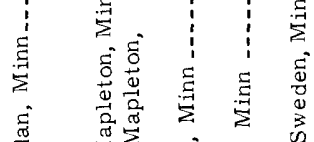

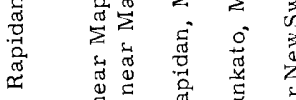

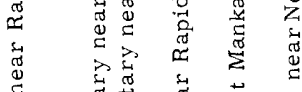

范

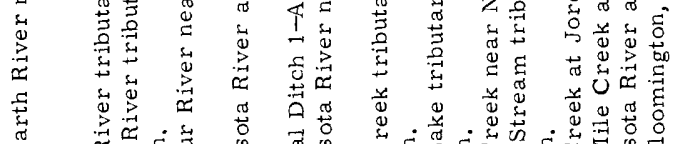

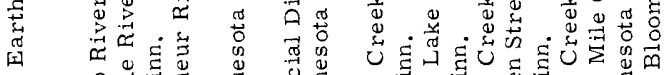

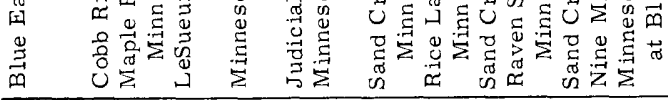

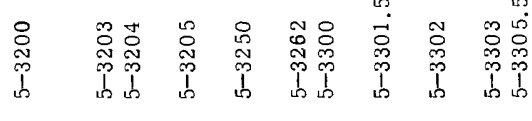

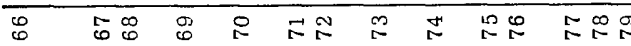

in

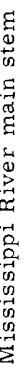

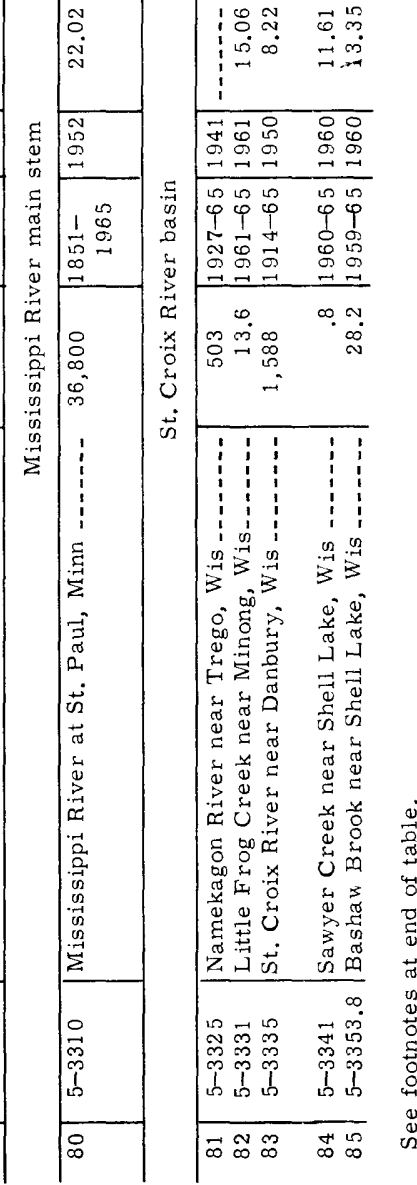




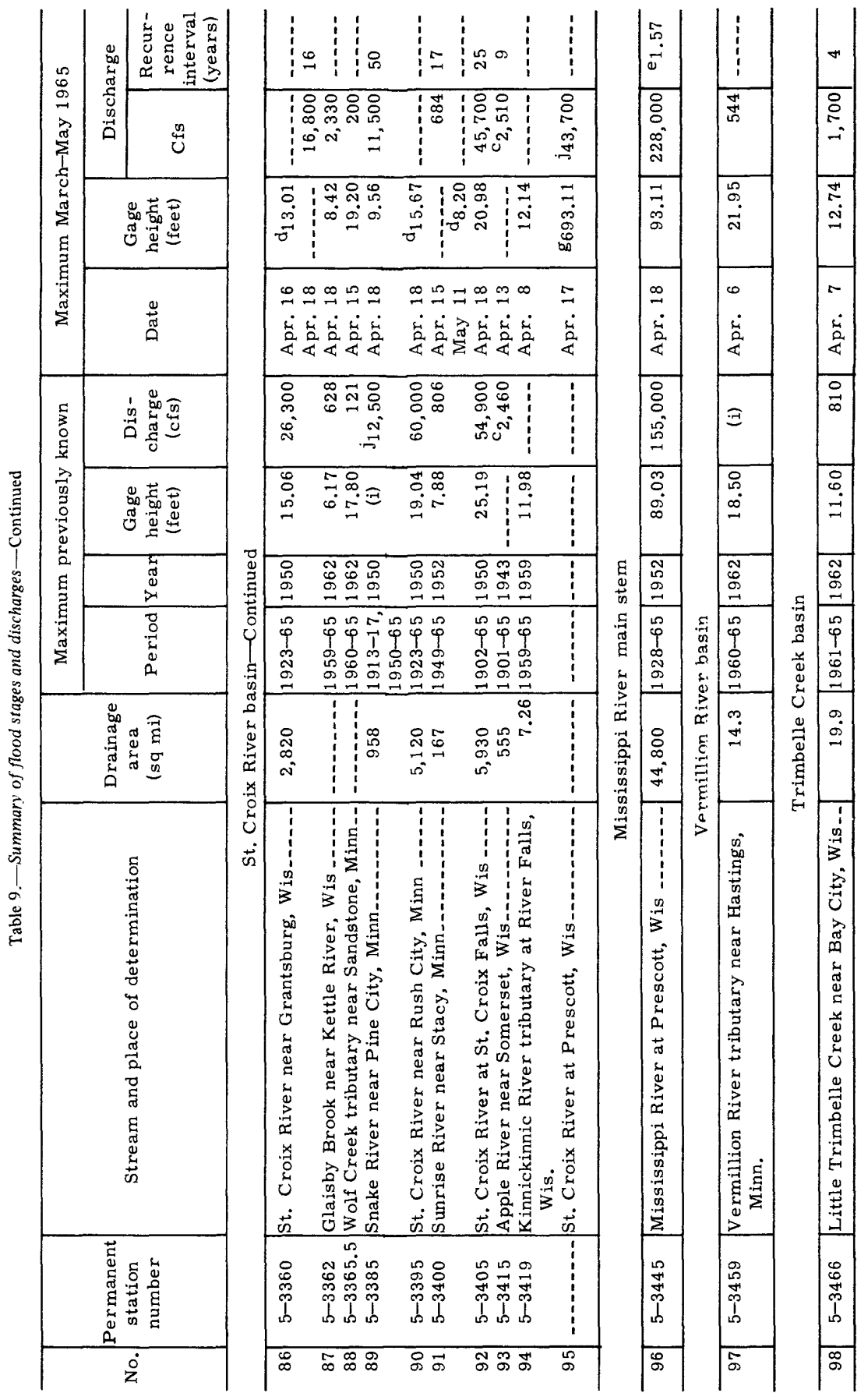




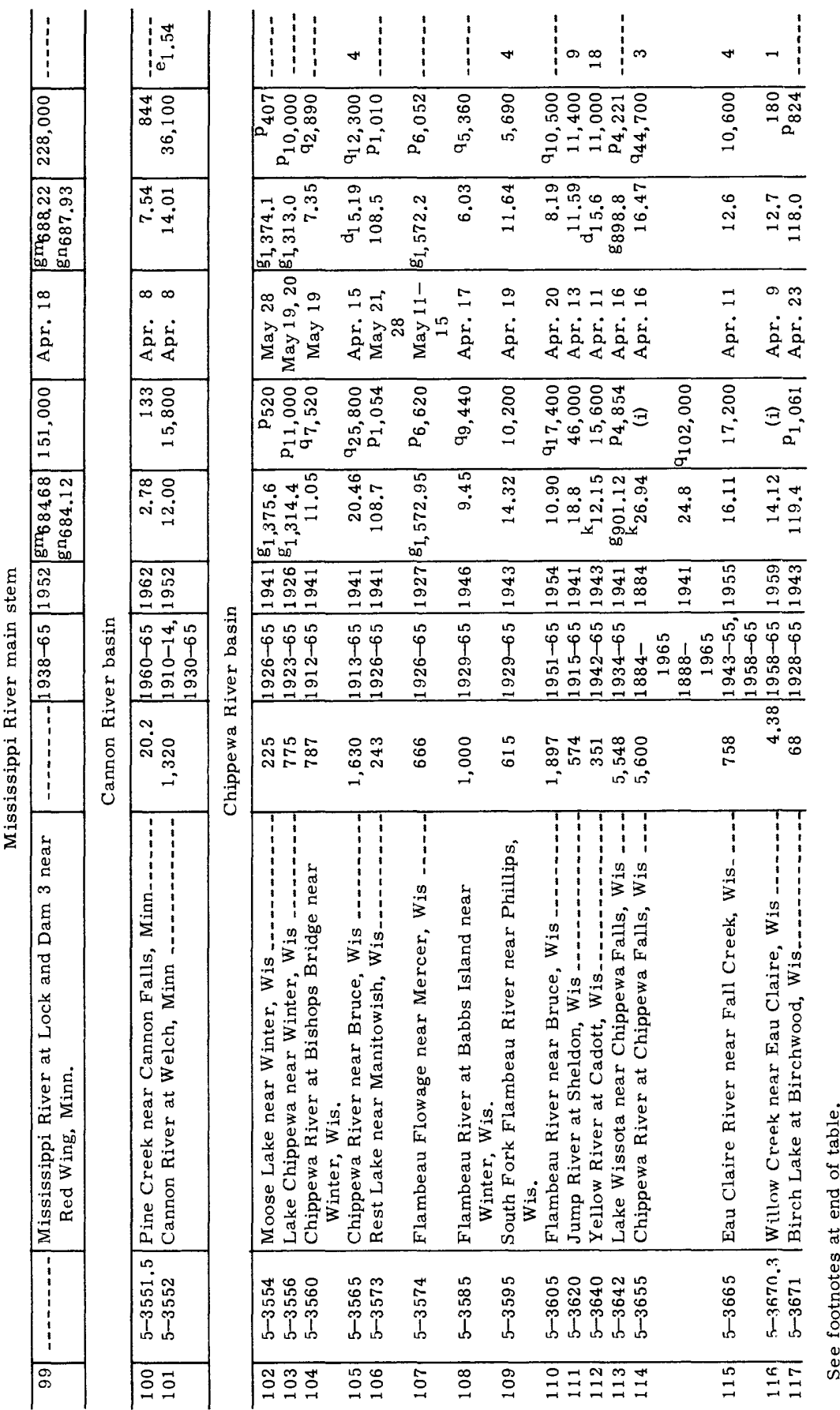




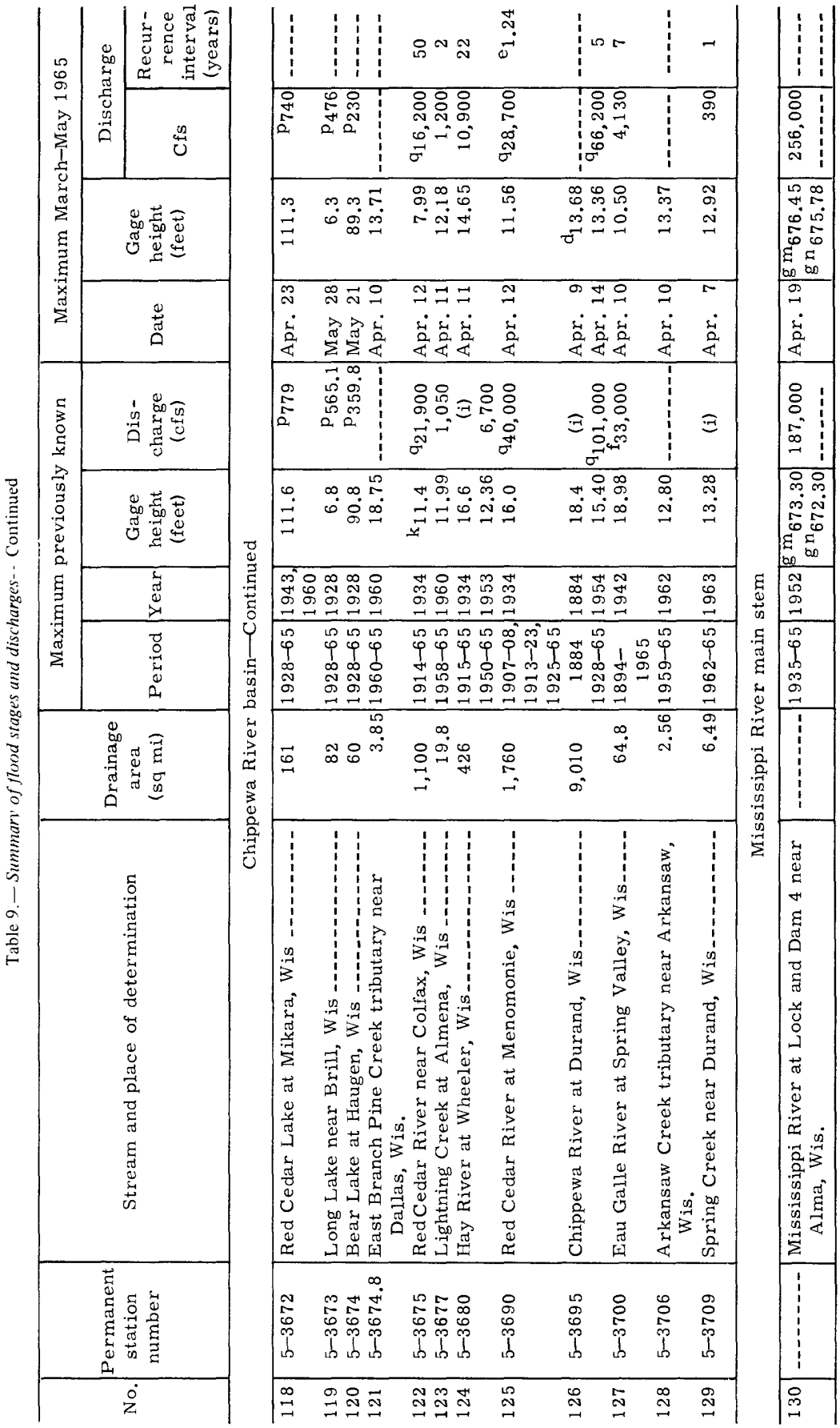


MARCH-MAY, UPPER MISSISSIPPI RIVER BASIN

A1 29

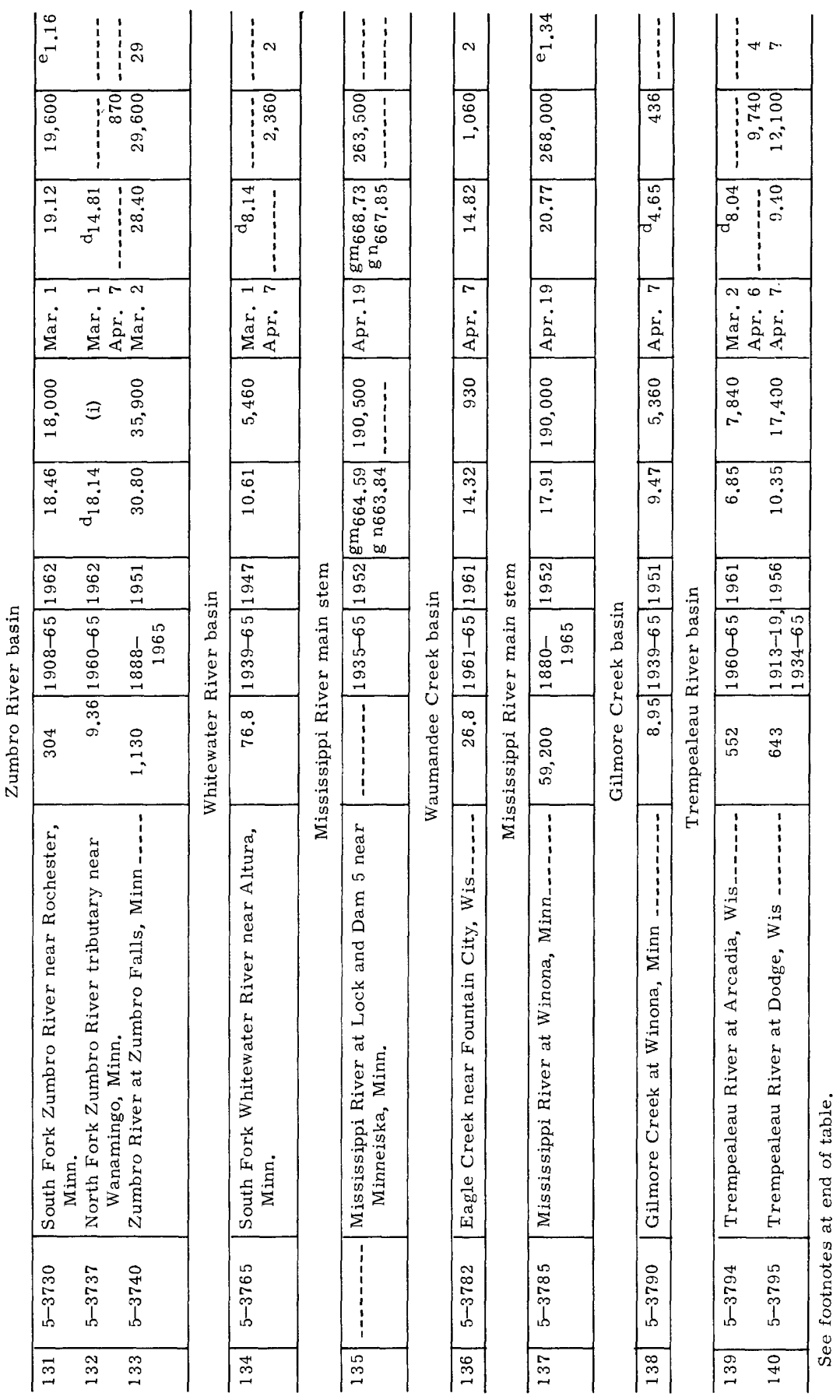


A130

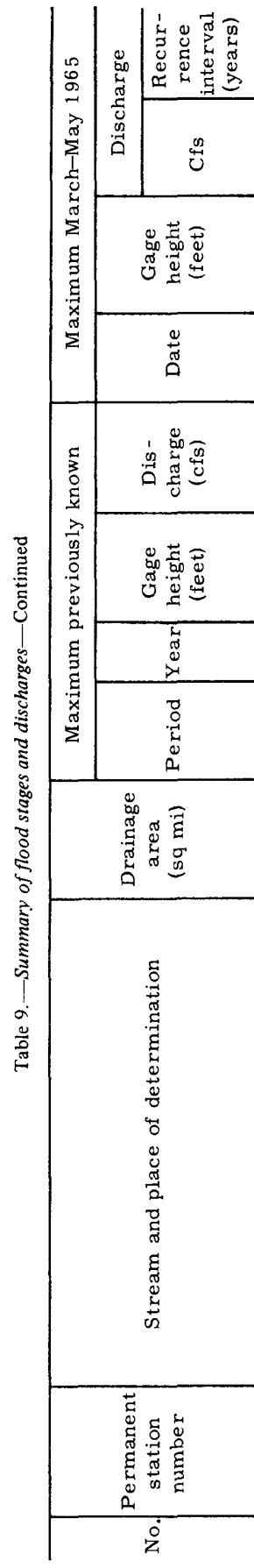

FLOODS OF 1965 IN THE UNITED STATES

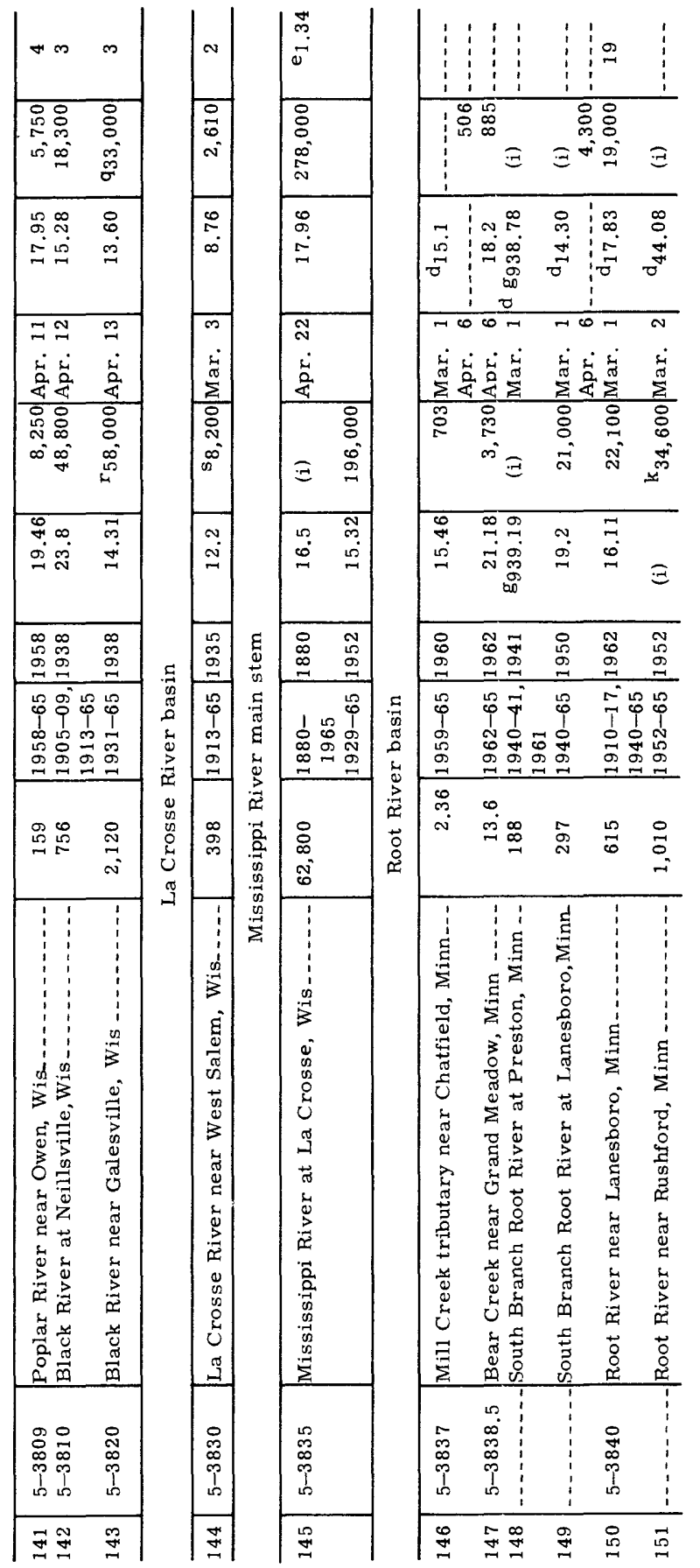


MARCH-MAY, UPPER MISSISSIPPI RIVER BASIN

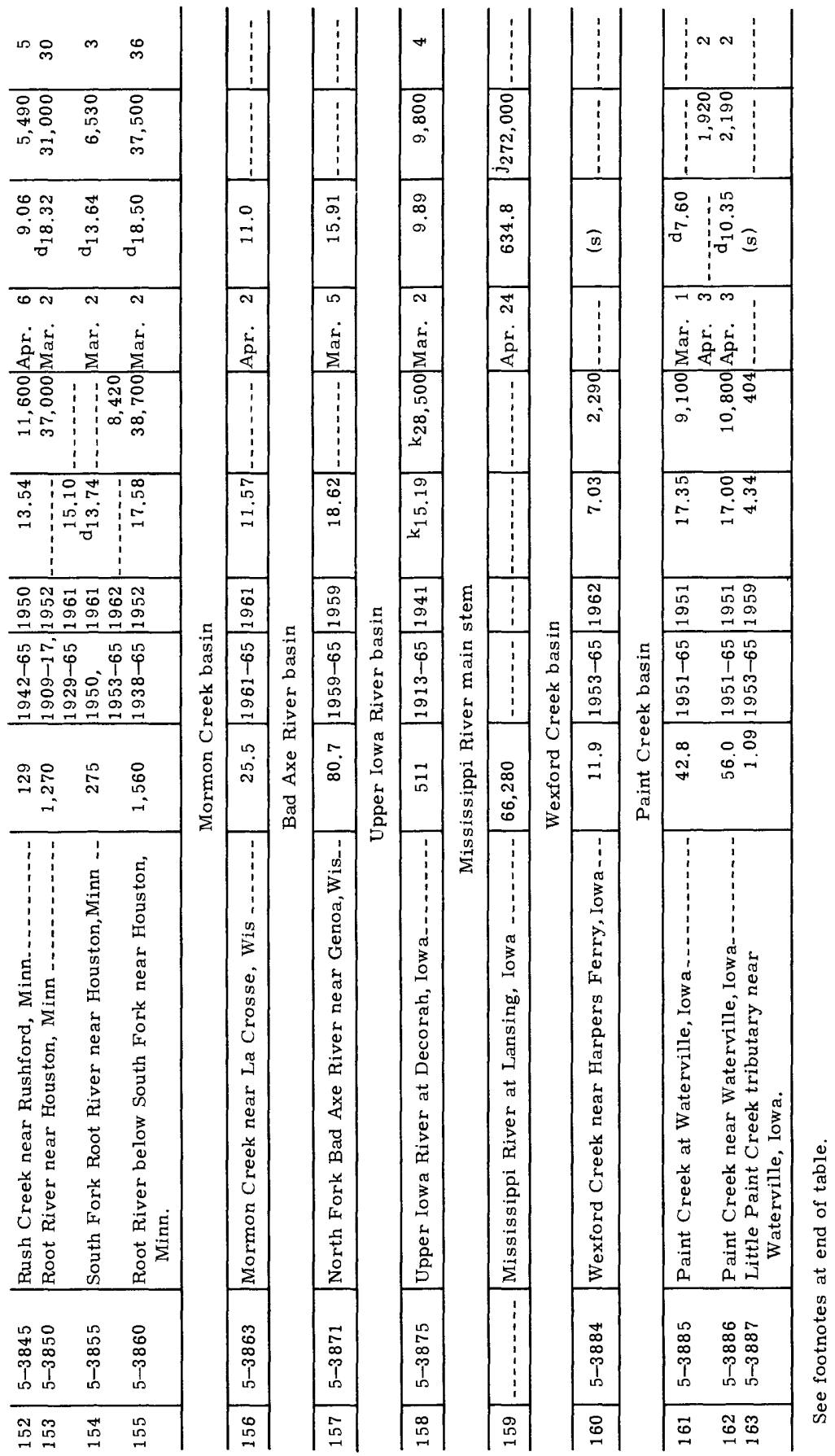




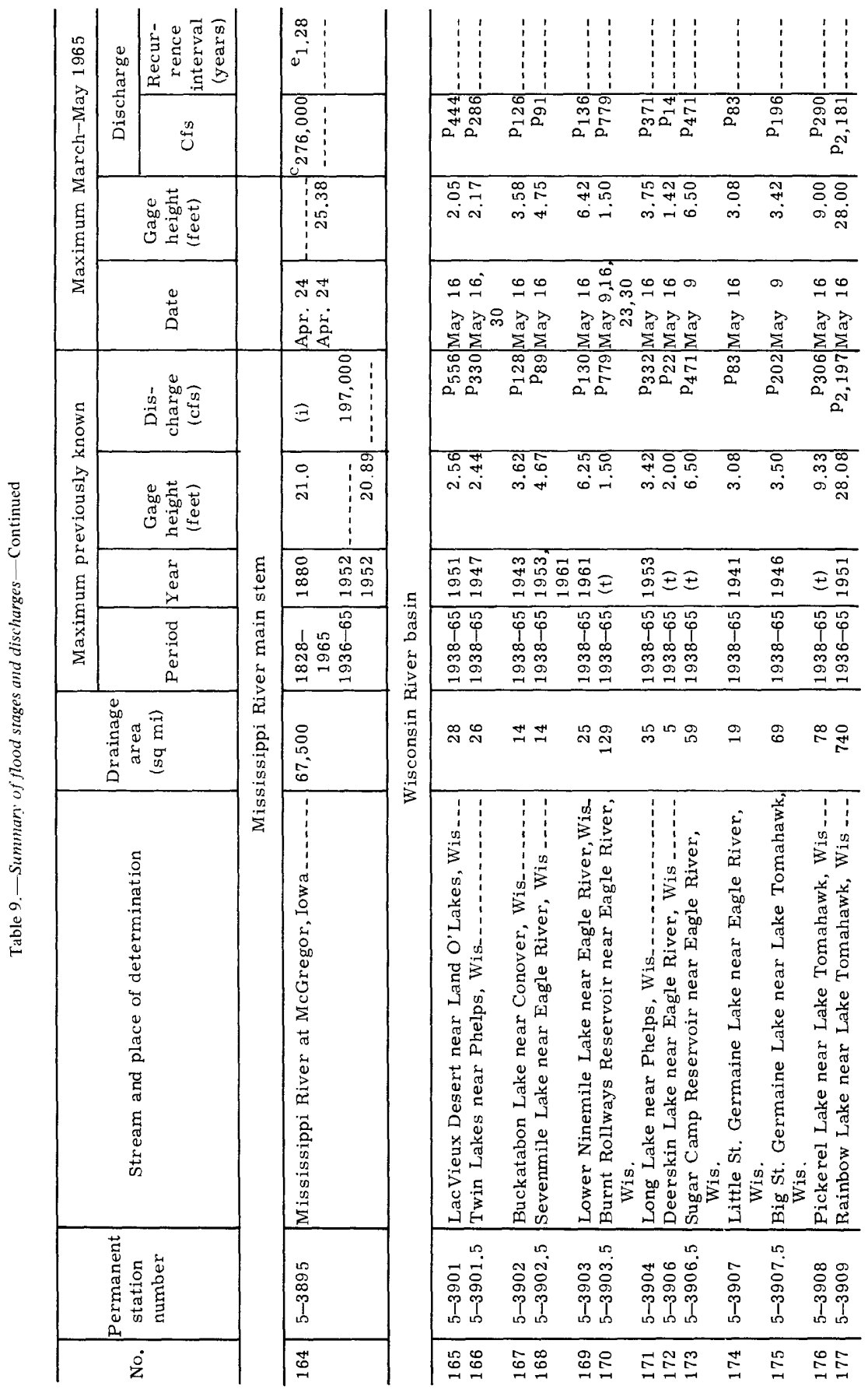




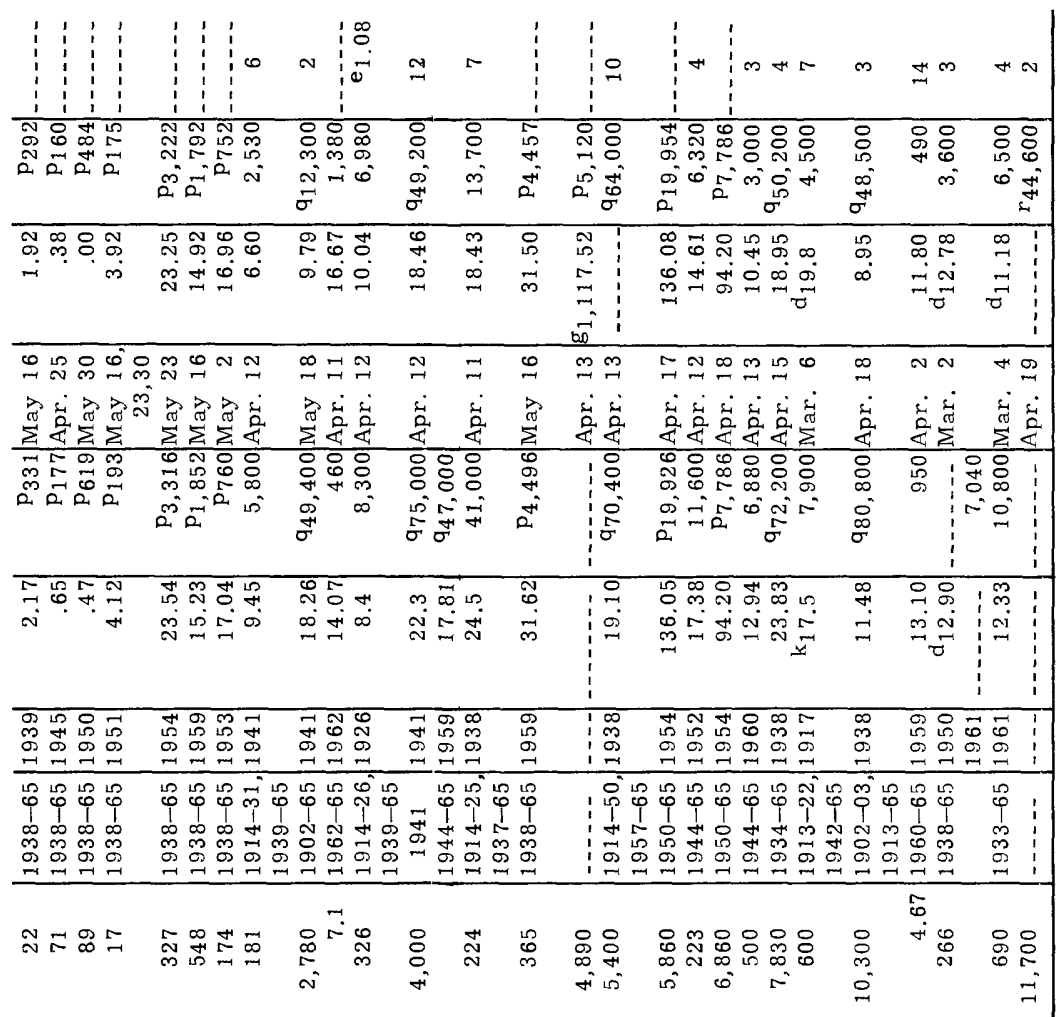

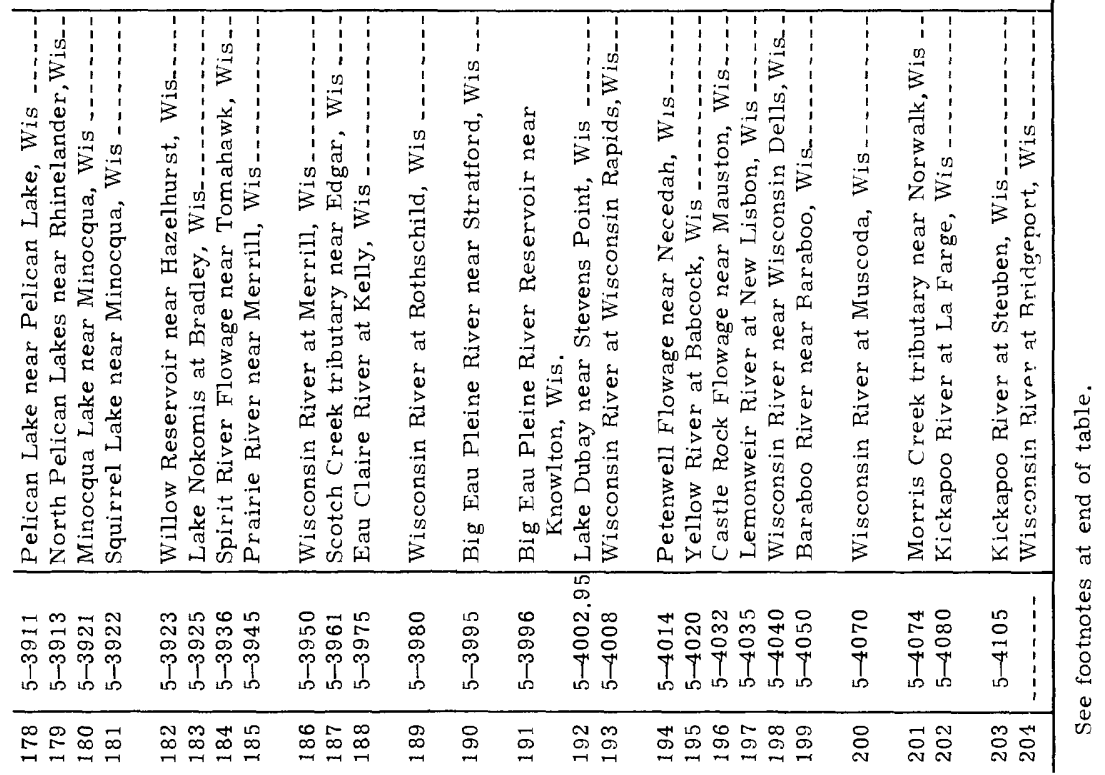




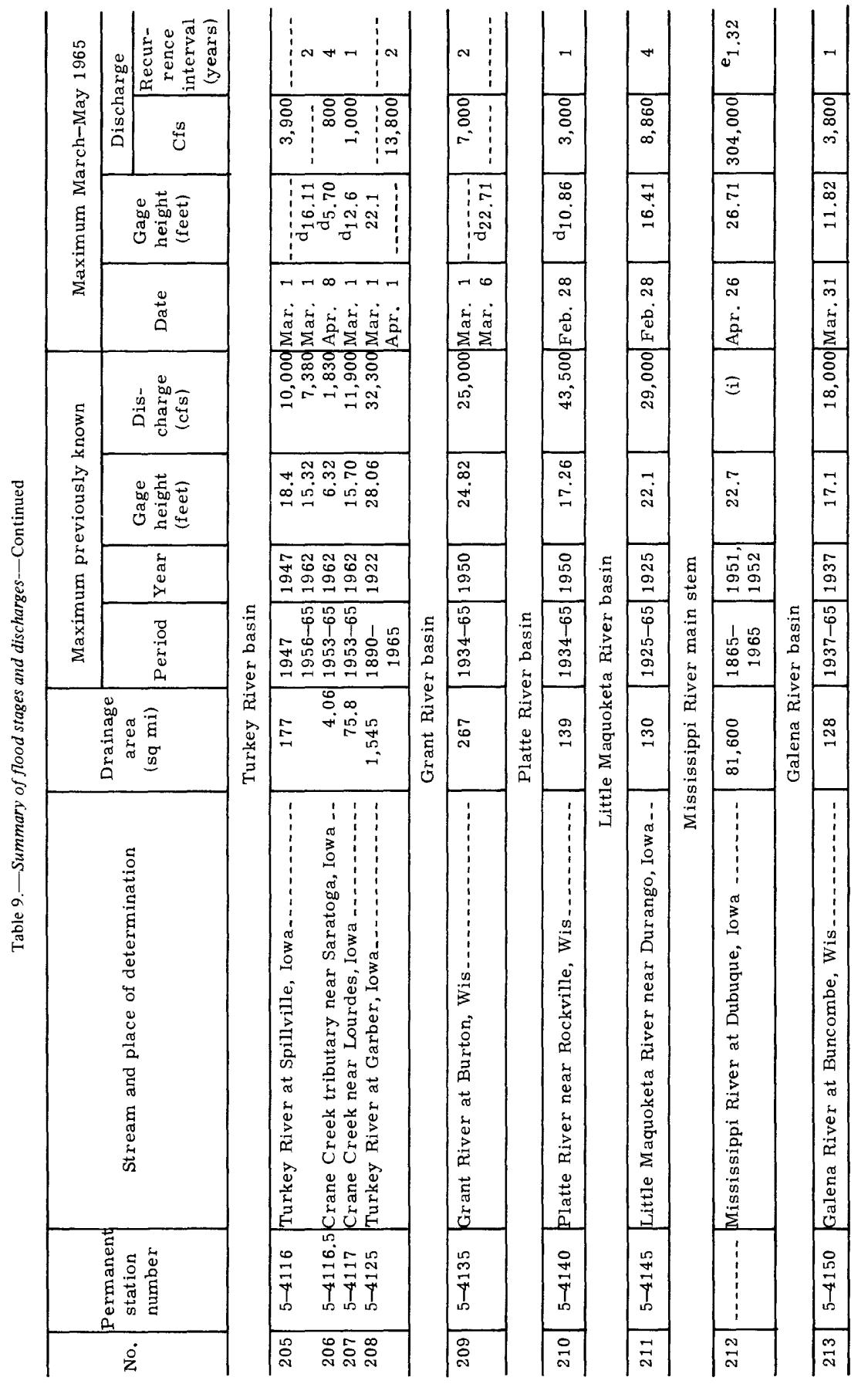


MARCH -MAY, UPPER MISSISSIPPI RIVER BASIN

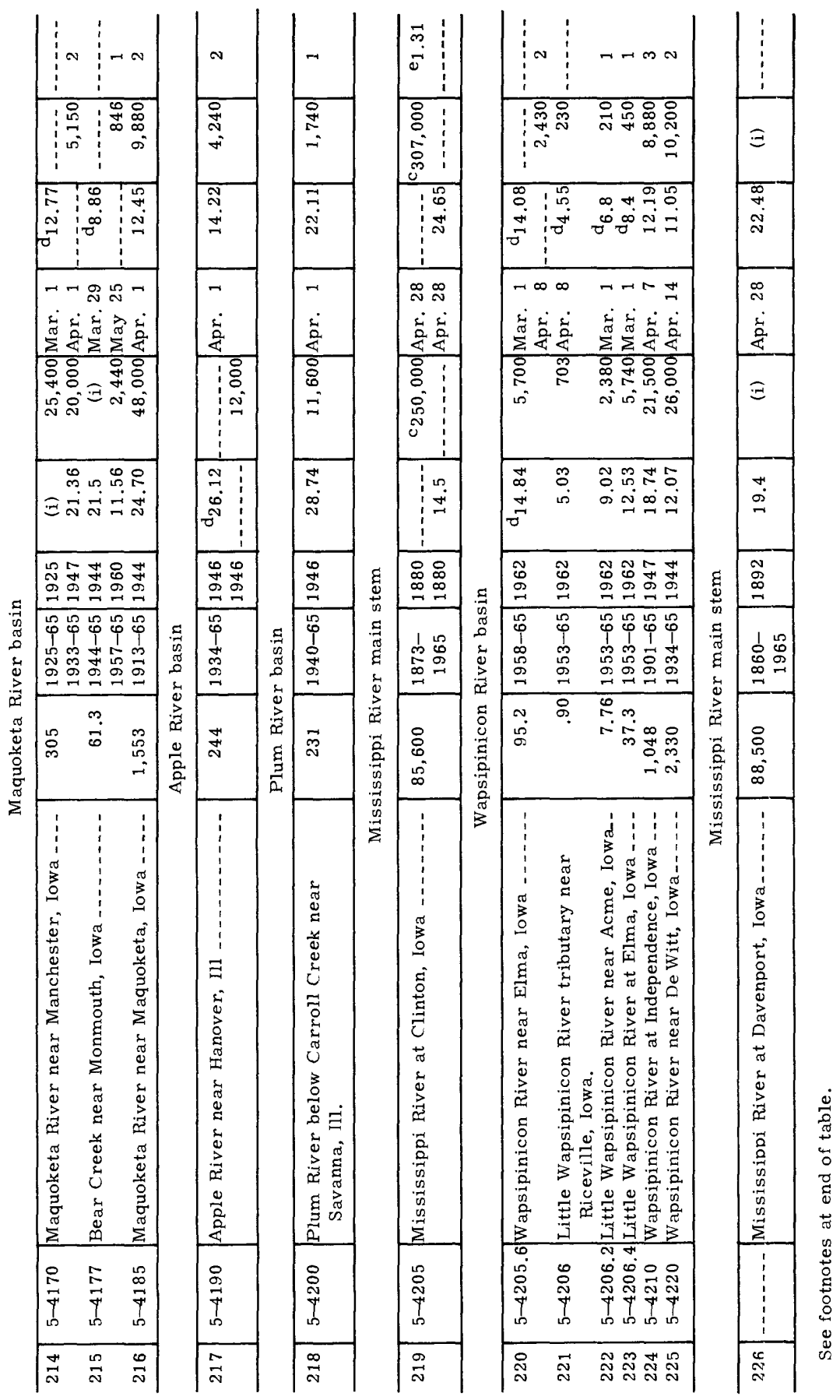




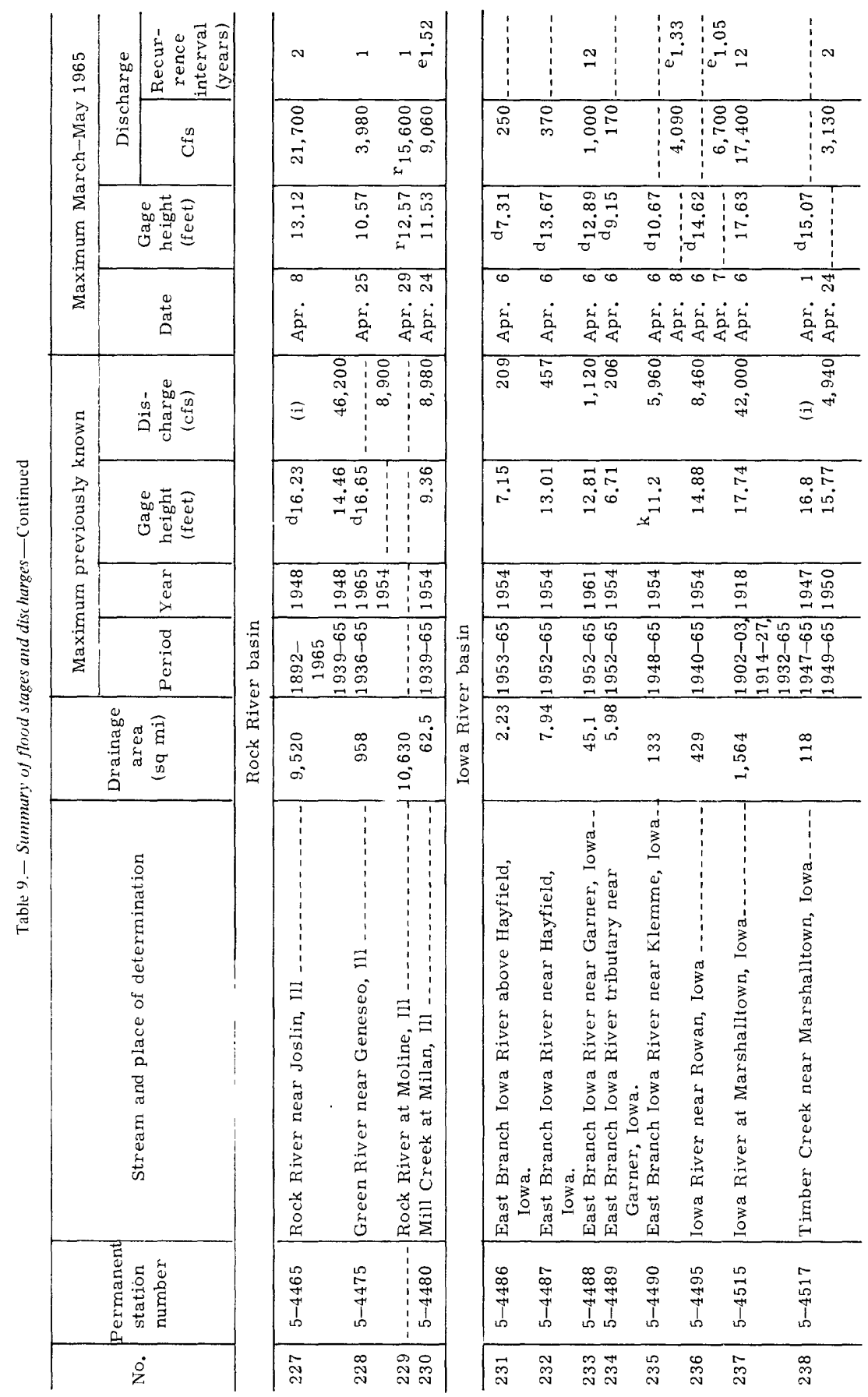




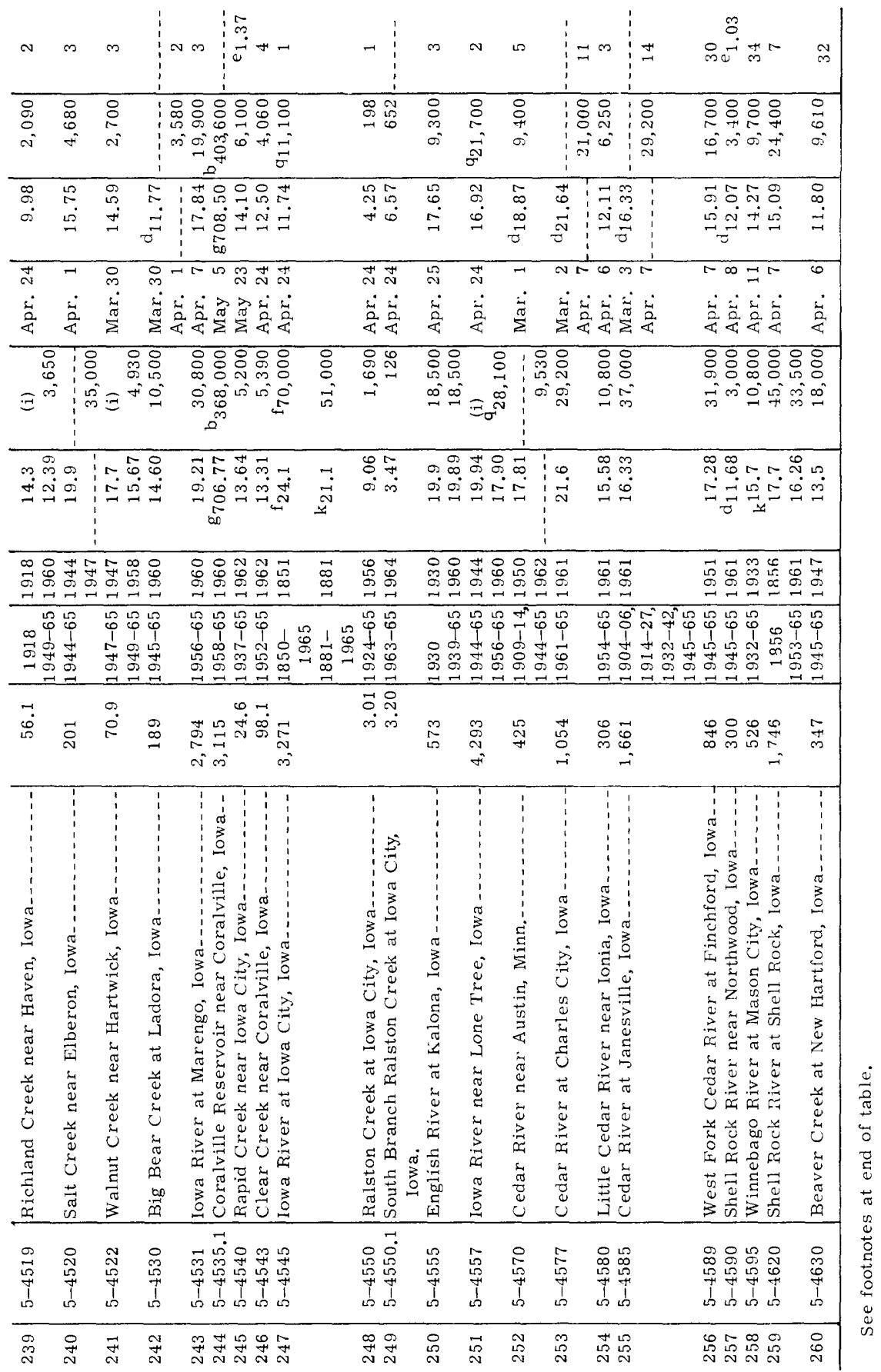




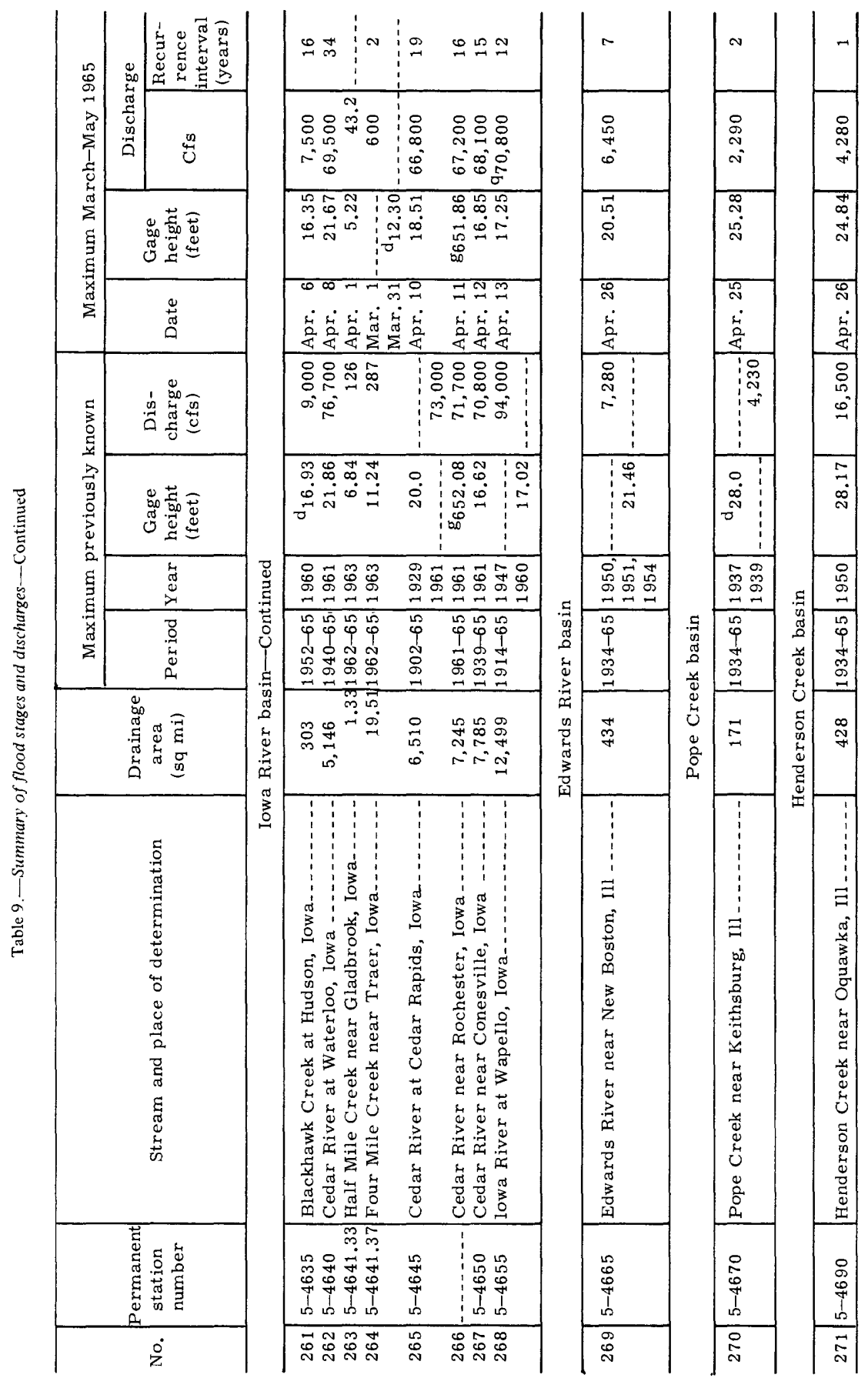




\begin{tabular}{|c|c|c|c|c|c|c|c|c|c|c|c|c|c|c|c|c|c|c|c|c|}
\hline $\begin{array}{l}1 \\
1 \\
1 \\
1\end{array}$ & & 10 & $n$ & L & $m$ & $\begin{array}{l}1 \\
\vdots \\
\vdots\end{array}$ & $N$ N & $N$ & $\mathrm{v}$ & $\left|\begin{array}{cc}N & 1 \\
- & 1 \\
0 & 1 \\
& 1\end{array}\right|$ & & $\begin{array}{l}1 \\
1 \\
1 \\
1 \\
1\end{array}$ & $\begin{array}{l}1 \\
1 \\
1\end{array}$ & $\begin{array}{ll}1 & 1 \\
1 & 1 \\
1 & 1 \\
1 & 1 \\
1 & 1\end{array}$ & $\begin{array}{lll}1 & \infty \\
1 & \vdots \\
1 & 0\end{array}$ & 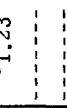 & $\begin{array}{l}0 \\
0 \\
0\end{array}$ & $\underset{0}{\infty}$ & $\stackrel{\infty}{\infty}$ & $\underset{\sim}{*}$ \\
\hline$\cong$ & & $\begin{array}{l}0 \\
0 \\
\text { N } \\
10\end{array}$ & \begin{tabular}{l}
0 \\
\multirow{2}{*}{} \\
$r$
\end{tabular} & $\begin{array}{l}0 \\
\dot{0} \\
\dot{q}\end{array}$ & $\begin{array}{l}\text { O } \\
\text { ㄱ. } \\
\text { I- }\end{array}$ & $\begin{array}{l}1 \\
1 \\
1 \\
1 \\
1 \\
1 \\
1 \\
1\end{array}$ & 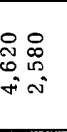 & $\begin{array}{l}8 \\
\text { ले } \\
\circ \\
\text { N }\end{array}$ & & 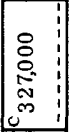 & & $\stackrel{8}{8}$ & $\stackrel{\infty}{m}$ & $\sum_{0}^{\infty}$ & 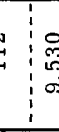 & $\begin{array}{l:l} & \\
0 & 0 \\
0 & 0 \\
0 & 0 \\
0 & -1 \\
& 0\end{array}$ & 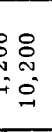 & $\begin{array}{l}8 \\
8 \\
0 \\
-1\end{array}$ & $\begin{array}{l}8 \\
0 \\
0 \\
-1\end{array}$ & $\begin{array}{l}8 \\
8 \\
\dot{8} \\
\pm \\
-1\end{array}$ \\
\hline $\begin{array}{l}0 \\
\dot{N}\end{array}$ & & $\begin{array}{l}m \\
\dot{q}\end{array}$ & $\begin{array}{l}\text { \% } \\
\text { in } \\
\text { vi }\end{array}$ & $\begin{array}{l}\text { N } \\
m \\
\dot{y} \\
-1\end{array}$ & $\begin{array}{l}\pi \\
\infty \\
\sigma^{\circ} \\
-\end{array}$ & & $\begin{array}{l}15 \\
10 \\
1 \\
1 \\
1\end{array}$ & \% & & \begin{tabular}{|l|l|} 
& + \\
1 & 0 \\
1 & 0 \\
1 &
\end{tabular} & & $\begin{array}{r}\overrightarrow{10} \\
\overrightarrow{1}\end{array}$ & $\begin{array}{l}\infty \\
\infty \\
\infty\end{array}$ & $\begin{array}{c:}\text { L0 } \\
0 \\
0 \\
0 \\
0 \\
0\end{array}$ & $\begin{array}{l}\infty \\
\infty \\
\infty \\
\infty \\
0 \\
0 \\
0\end{array}$ & 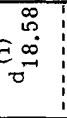 & $\begin{array}{l}\overrightarrow{1} \\
1 \\
1 \\
1\end{array}$ & $\begin{array}{l}m \\
\infty \\
0 \\
0 \\
\end{array}$ & $\begin{array}{c}\infty \\
0 \\
0 \\
m \\
m \\
-1 \\
-1\end{array}$ & $\begin{array}{l}0 \\
0 \\
\text { m }\end{array}$ \\
\hline 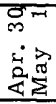 & & $\begin{array}{l}0 \\
\dot{a}\end{array}$ & $\begin{array}{l}0 \\
\dot{a} \\
0\end{array}$ & $\begin{array}{l}\omega \\
\dot{a} \\
\dot{\alpha}\end{array}$ & क & $\begin{array}{l}50 \\
+1 \\
\sum_{i}^{\circ}\end{array}$ & का & $\stackrel{\infty}{\pi}$ & & 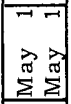 & & s. & $\begin{array}{l}0 \\
\dot{2} \\
\end{array}$ & $\begin{array}{l}\text { जL } \\
\text { si ci } \\
0 \\
0\end{array}$ & 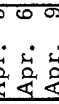 & 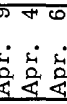 & 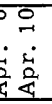 & दे & c. & $\begin{array}{l}\infty \\
\dot{a} \\
\vdots \\
\vdots\end{array}$ \\
\hline$\Xi$ & & $\begin{array}{l}10 \\
10 \\
10 \\
10 \\
1 \\
1\end{array}$ & 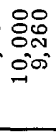 & $\begin{array}{r}0 \\
\infty \\
\infty \\
=0 \\
=\end{array}$ & $\stackrel{8}{8}$ & & 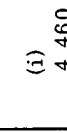 & $\begin{array}{l}0 \\
0 \\
0\end{array}$ & & $\begin{array}{l}8 \\
8 \\
0 \\
0 \\
0 \\
0\end{array}$ & & $\begin{array}{l}8 \\
0 \\
0 \\
-1\end{array}$ & 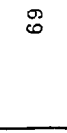 & $\begin{array}{l}0 \\
1=1 \\
\vdots\end{array}$ & $\begin{array}{l}0 \\
1 \\
0 \\
\infty\end{array}$ & $\begin{array}{l}10 \\
1 \\
1 \\
1 \\
1\end{array}$ & $\begin{array}{l}8 \\
0 \\
0 \\
0 \\
0 \\
0\end{array}$ & $\begin{array}{l}1 \\
\vdots \\
\vdots \\
\end{array}$ & $\begin{array}{r}1 \\
1 \\
1 \\
1 \\
\end{array}$ & $\begin{array}{l}8 \\
8 \\
0 \\
-1\end{array}$ \\
\hline $\begin{array}{l}0 \\
\infty \\
\infty\end{array}$ & & 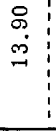 & 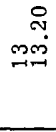 & $\begin{array}{l}50 \\
0=0\end{array}$ & $\begin{array}{l}\infty \\
\infty \\
\infty\end{array}$ & & 등 & 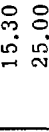 & & $\stackrel{9}{i}$ & & $\begin{array}{l}N \\
\infty\end{array}$ & $\stackrel{+}{\ddot{0}}$ & 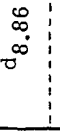 & $\begin{array}{l}\infty \\
i \\
i \\
i \\
i\end{array}$ & 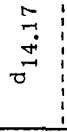 & $\begin{array}{l}1 \\
1 \\
1 \\
1 \\
1 \\
1 \\
1\end{array}$ & $\begin{array}{l}1 \\
\vdots \\
1 \\
\vdots \\
\vdots\end{array}$ & $\begin{array}{l}1 \\
\vdots \\
\vdots \\
1 \\
\end{array}$ & $\underset{\sim}{N}$ \\
\hline $\begin{array}{l}\overrightarrow{10} \\
\infty\end{array}$ & & 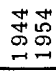 & $\begin{array}{l}+0 \\
+\infty \\
0 \\
\rightarrow-1\end{array}$ & 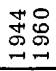 & $\underset{\sim}{\stackrel{4}{*}}$ & $\begin{array}{l}9 \\
\oplus \\
\oplus \\
1\end{array}$ & 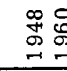 & $\begin{array}{ll}\circ & \circ \\
\circ & \circ \\
\circ & \circ \\
-1 & -1\end{array}$ & 它 & $\begin{array}{l}\vec{n} \\
\infty \\
\sim\end{array}$ & $\begin{array}{l}5 \\
\text { 至 } \\
0 \\
0\end{array}$ & 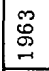 & $\begin{array}{l}N \\
\mathscr{\sigma} \\
\sim\end{array}$ & 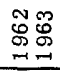 & $\begin{array}{l}m \\
\text { I } \\
0\end{array}$ & $\begin{array}{ll}N & \sim \\
0 & 0 \\
\sigma & 0\end{array}$ & $\begin{array}{ll}0 \\
0 \\
0\end{array}$ & $\begin{array}{l}1 \\
1 \\
1\end{array}$ & $\begin{array}{l}T \\
1 \\
1 \\
1\end{array}$ & $\begin{array}{l}5 \\
0\end{array}$ \\
\hline $\begin{array}{ll}1 & 1 \\
10 & \infty \\
\infty & \infty \\
\infty & \\
\end{array}$ & $\begin{array}{l}5 \\
5 \\
0 \\
0 \\
0 \\
4 \\
0 \\
5\end{array}$ & $\begin{array}{ll}N & 0 \\
N & 0 \\
1 & 0 \\
0 & 0 \\
0 & \infty \\
0 & 0\end{array}$ & $\begin{array}{c}n \\
+\infty \\
0 \\
0 \\
0 \\
\pi \\
\pi \\
\pi\end{array}$ & 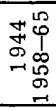 & $\begin{array}{l}10 \\
0 \\
j \\
0 \\
0\end{array}$ & $\begin{array}{l}0 \\
0 \\
1 \\
0 \\
0 \\
0 \\
0 \\
0\end{array}$ & 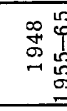 & 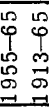 & 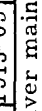 & 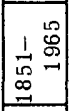 & 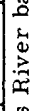 & $\begin{array}{l}10 \\
1 \\
0 \\
0 \\
0 \\
0\end{array}$ & $\begin{array}{l}10 \\
0 \\
0 \\
0 \\
\end{array}$ & $\begin{array}{l}1 \\
0 \\
0 \\
0 \\
0 \\
1\end{array}$ & 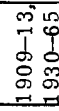 & $\begin{array}{ll}0 & 10 \\
0 & 0 \\
1 & 0 \\
0 & 0 \\
0 & 0 \\
0 & 0\end{array}$ & $\begin{array}{l}n \\
0 \\
0 \\
0 \\
0\end{array}$ & $\begin{array}{l}1 \\
\vdots \\
1\end{array}$ & $\begin{array}{l}i \\
\vdots \\
1 \\
\end{array}$ & $\begin{array}{l}n \\
1 \\
0 \\
0 \\
0\end{array}$ \\
\hline 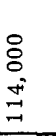 & 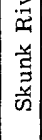 & $\frac{12}{m}$ & 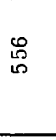 & $\underset{\sim}{\stackrel{D}{\sim}}$ & $\begin{array}{l}10 \\
0 \\
0 \\
-1 \\
-1\end{array}$ & $\begin{array}{l}\stackrel{\circ}{1} \\
\stackrel{1}{r}\end{array}$ & $\stackrel{\circ}{\circ}$ & $\begin{array}{l}\text { m } \\
\text { m } \\
+\end{array}$ & for & $\begin{array}{l}8 \\
8 \\
\circ \\
\infty \\
-1 \\
-1\end{array}$ & 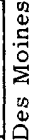 & $\begin{array}{l}3 \\
\vdots \\
\vdots \\
\vdots \\
1 \\
1\end{array}$ & $\underset{\sim}{\stackrel{\pi}{*}}$ & in & $\begin{array}{l}0 \\
\text { N } \\
-i\end{array}$ & i & $\underset{N}{N}$ & $\stackrel{0}{\infty}$ & $\begin{array}{l}\infty \\
0 \\
0 \\
0\end{array}$ & $\begin{array}{l}0 \\
\text { ஸ̃ } \\
\text { ஸ }\end{array}$ \\
\hline $\begin{array}{c}1 \\
1 \\
1 \\
1 \\
1 \\
0 \\
3 \\
0 \\
0 \\
0 \\
0 \\
0 \\
.5 \\
0 \\
0 \\
0 \\
0 \\
0 \\
0 \\
0 \\
0 \\
0 \\
0 \\
0 \\
0 \\
0 \\
0 \\
0 \\
0 \\
0 \\
0 \\
0 \\
0 \\
0 \\
0\end{array}$ & & 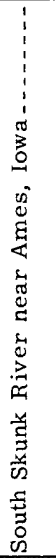 & 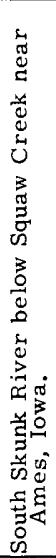 & 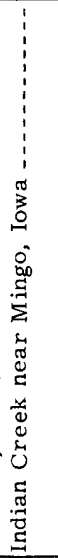 & 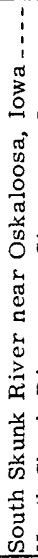 & 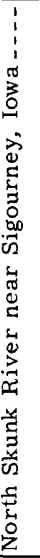 & 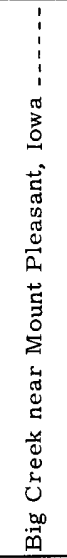 & ' & 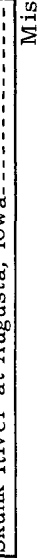 & 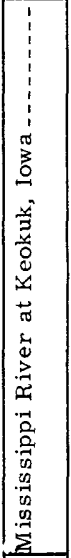 & & 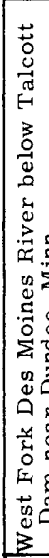 & 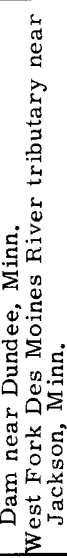 & 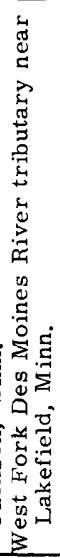 & 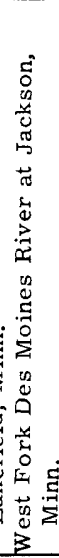 & 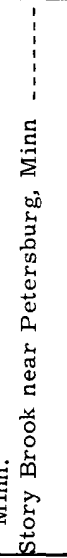 & 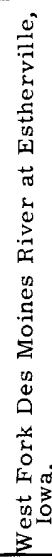 & 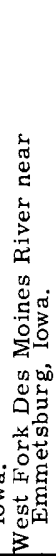 & 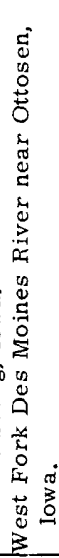 & 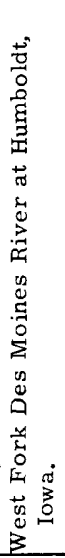 \\
\hline $\begin{array}{l}1 \\
\vdots \\
1 \\
1 \\
1 \\
\end{array}$ & & 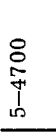 & \begin{tabular}{c}
0 \\
\multirow{2}{*}{+1} \\
1 \\
1
\end{tabular} & 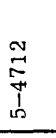 & 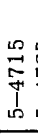 & 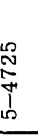 & $\sum_{n}^{\infty}$ & $\underset{\substack{+⿱ 亠 䒑}}{+}$ & & $\frac{1}{4}$ & & $\begin{array}{l}1 \\
1 \\
1 \\
1 \\
\end{array}$ & $\begin{array}{l}\infty \\
10 \\
i \\
1 \\
1 \\
1\end{array}$ & $\begin{array}{l}0 \\
10 \\
1 \\
1 \\
10\end{array}$ & $\begin{array}{l}0 \\
0 \\
2 \\
1 \\
1\end{array}$ & $\frac{\pi}{0}$ & 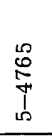 & $\begin{array}{l}1 \\
1 \\
1 \\
1 \\
1 \\
\end{array}$ & $\begin{array}{l}1 \\
1 \\
1 \\
1 \\
1\end{array}$ & 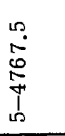 \\
\hline$\stackrel{N}{\stackrel{N}{N}}$ & & $\underset{n}{n}$ & $\underset{\pi}{\pi}$ & $\stackrel{10}{N}$ & $\underset{\sim}{\infty}$ & $\frac{N}{N}$ & $\underset{N}{\infty}$ & $\stackrel{\sigma}{N}$ & & $\begin{array}{l}0 \\
\infty \\
\sim\end{array}$ & & $\begin{array}{l}-1 \\
\infty \\
\mathbb{N}\end{array}$ & $\begin{array}{l}N \\
\infty \\
N\end{array}$ & $\begin{array}{l}m \\
\infty \\
N\end{array}$ & $\underset{N}{+}$ & $\begin{array}{l}\text { in } \\
\infty \\
\text { N }\end{array}$ & $\begin{array}{l}\infty \\
\infty \\
\sim\end{array}$ & $\underset{N}{\infty}$ & $\begin{array}{l}\infty \\
\infty \\
N\end{array}$ & $\begin{array}{l}\infty \\
\infty \\
\sim\end{array}$ \\
\hline
\end{tabular}




\begin{tabular}{|c|c|c|c|c|c|c|c|c|c|c|c|c|c|}
\hline $\begin{array}{l}2 \\
\stackrel{8}{9} \\
-1\end{array}$ & 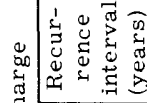 & & in & & & $\stackrel{2}{\Rightarrow}$ & $\vec{N}$ & డొ & & t & $\rightarrow$ & & $\stackrel{\infty}{\rightarrow}$ \\
\hline $\begin{array}{l}\text { ब. } \\
\sum_{1} \\
\text { d. } \\
0\end{array}$ & $\begin{array}{c}5 \\
0 \\
0 \\
0 \\
0\end{array}$ & & $\begin{array}{ll} & 8 \\
0 & \vdots \\
0 & +1 \\
\text { in }\end{array}$ & $\begin{array}{l}8 \\
8 \\
i \\
i n \\
\rightarrow\end{array}$ & & $\begin{array}{ll} & 0 \\
0 & 0 \\
0 & 0 \\
4 & 0 \\
0 & 0\end{array}$ & $\begin{array}{l}8 \\
\stackrel{8}{0} \\
\text { in } \\
-1\end{array}$ & $\begin{array}{l}88 \\
80 \\
0 \\
\text { in } \\
=\end{array}$ & & 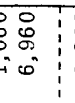 & \begin{tabular}{l|l} 
& 8 \\
& 8 \\
$\infty$ & 0 \\
$\infty$ &
\end{tabular} & $\begin{array}{l}8 \\
0 \\
\infty \\
0 \\
0 \\
0\end{array}$ & $\begin{array}{l}0 \\
8 \\
\infty \\
10 \\
10\end{array}$ \\
\hline 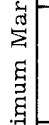 & 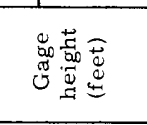 & & 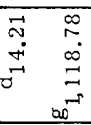 & $\begin{array}{l}m \\
\cdots \\
\sim \\
\sim\end{array}$ & 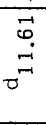 & 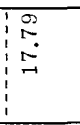 & $\begin{array}{l}5 \\
\text { in } \\
-1\end{array}$ & $\begin{array}{l}\sigma \\
\infty \\
\infty \\
\sim \\
\sim \\
\sim \\
\sim\end{array}$ & $\begin{array}{l}20 \\
0 \\
0 \\
0 \\
0\end{array}$ & 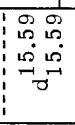 & 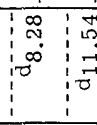 & 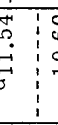 & $\begin{array}{l} \\
0 \\
0 \\
0 \\
-1\end{array}$ \\
\hline$\stackrel{\substack{m \\
\Sigma}}{2}$ & 雚 & & $\begin{array}{ll}\infty & 0 \\
\vdots & \dot{4} \\
\text { \& } & \text { \& }\end{array}$ & $\begin{array}{l}\sigma \\
\dot{a} \\
\dot{4}\end{array}$ & अ & $\begin{array}{l}\infty \\
\dot{c} \\
\dot{a} \\
\dot{a}\end{array}$ & $\begin{array}{l}0 \\
\dot{a}\end{array}$ & $\begin{array}{l}\circ 0 \\
\dot{0} \\
\text { \&. }\end{array}$ & 00 & 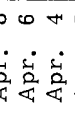 & 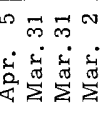 & 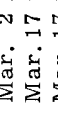 & \\
\hline $\begin{array}{l}5 \\
3 \\
0 \\
0\end{array}$ & 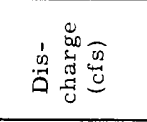 & & 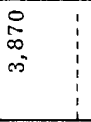 & $\begin{array}{l}8 \\
\vdots \\
\vdots \\
=\end{array}$ & $\begin{array}{l}8 \\
8 \\
0 \\
0\end{array}$ & $\begin{array}{l}8 \\
8 \\
0 \\
10 \\
0\end{array}$ & $\begin{array}{l}8 \\
8 \\
0 \\
i \\
i\end{array}$ & $\begin{array}{l}8: 8 \\
080 \\
50 \\
50\end{array}$ & & 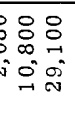 & $\begin{array}{l}\infty \\
\Rightarrow \quad \\
+ \\
+1\end{array}$ & 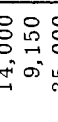 & \\
\hline $\begin{array}{l}2 \\
2 \\
0 \\
0 \\
0 \\
0 \\
0\end{array}$ & 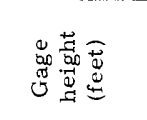 & $\mathbb{Q}$ & 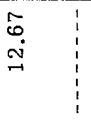 & $\begin{array}{l}\tilde{O} \\
\dot{H} \\
\text { D }\end{array}$ & $\stackrel{0}{\dot{0}}$ & $\begin{array}{l:l}\mathcal{N} & \\
0 & \\
\sigma & \\
- & \end{array}$ & $\vec{a}$ & 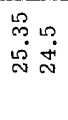 & $\underset{+}{\stackrel{g}{+}}$ & 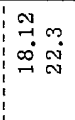 & $\begin{array}{ll}\infty & m \\
\infty & \stackrel{+}{\rightarrow}\end{array}$ & 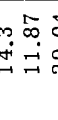 & \\
\hline $\begin{array}{l}\tilde{2} \\
\text { : } \\
\text { z్ }\end{array}$ & 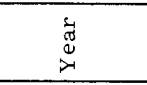 & 录 & 奇 & $\begin{array}{l}\text { 落 } \\
\stackrel{\rightarrow}{\rightarrow} \\
\end{array}$ & 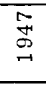 & 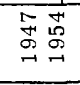 & $\begin{array}{l}\infty \\
\vec{\sigma} \\
\vec{\sim}\end{array}$ & 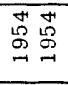 & & 䜤管 & 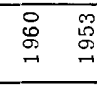 & 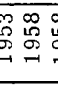 & \\
\hline 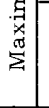 & $\begin{array}{l}\bar{g} \\
\stackrel{0}{\tilde{E}} \\
0\end{array}$ & 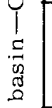 & 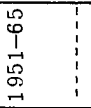 & 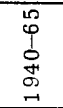 & 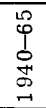 & 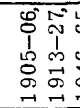 & $\begin{array}{l}1 \\
0 \\
\infty \\
\infty \\
-\end{array}$ & $\begin{array}{ll}0 & \\
0 & 1 \\
1 & 1 \\
0 & 0 \\
0 & \infty \\
-1 & \infty \\
-1\end{array}$ & \begin{aligned} & \multicolumn{1}{l}{} \\
& 0 \\
& 0 \\
& 0 \\
& 0 \\
& 0 \\
&\end{aligned} & 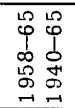 & 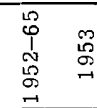 & 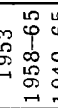 & \\
\hline & 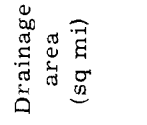 & 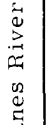 & $\underset{\substack{\infty \\
\infty}}{\mathfrak{\infty}}$ & $\begin{array}{l}\infty \\
0 \\
\infty \\
-\end{array}$ & 茼 & $\begin{array}{l}\stackrel{8}{\Phi} \\
\stackrel{+}{+}\end{array}$ & $\underset{\infty}{+}$ & 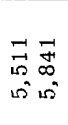 & $\begin{array}{l}0 \\
\dot{\infty}\end{array}$ & $\underset{\vec{H}}{\stackrel{m}{\vec{D}}} \stackrel{\overrightarrow{0}}{\overrightarrow{0}}$ & 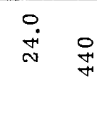 & 导 & $\begin{array}{l}\infty \\
\infty \\
\infty\end{array}$ \\
\hline & 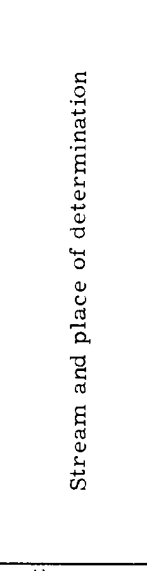 & 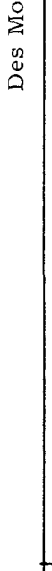 & 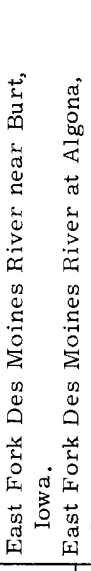 & 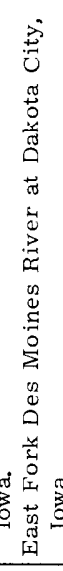 & 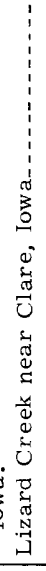 & 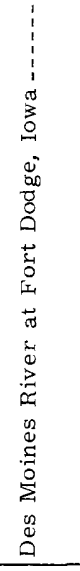 & 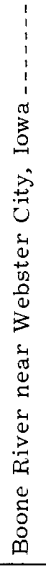 & 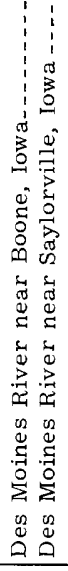 & 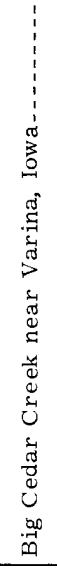 & 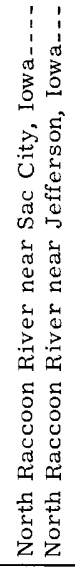 & 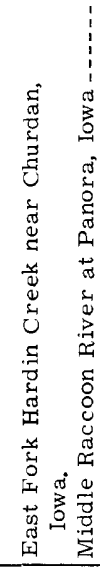 & 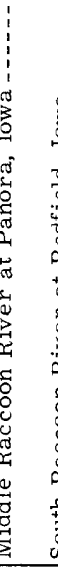 & 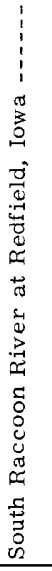 \\
\hline & 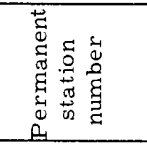 & & 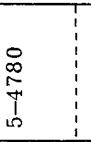 & $\begin{array}{c}8 \\
\stackrel{D}{9} \\
7 \\
1 \\
1\end{array}$ & $\begin{array}{l}8 \\
8 \\
\infty \\
1 \\
1 \\
1\end{array}$ & $\begin{array}{l}0 \\
0 \\
\infty \\
1 \\
1 \\
0\end{array}$ & 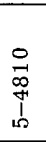 & $\begin{array}{ll}10 & 0 \\
0 & 0 \\
0 & 0 \\
1 & 0 \\
1 & 1 \\
0 & 1\end{array}$ & 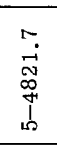 & 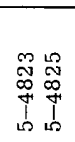 & $\begin{array}{cc}0 & 0 \\
0 & 0 \\
0 & 0 \\
0 & 0 \\
1 & 1 \\
1 & 1\end{array}$ & 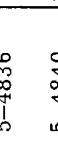 & 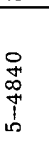 \\
\hline & $\dot{z}$ & & $\begin{array}{ll}\stackrel{\vec{D}}{\mathrm{~N}} & \overrightarrow{\mathrm{D}}\end{array}$ & N & $\begin{array}{l}\mathcal{M} \\
\stackrel{M}{\sigma} \\
\sim \\
N\end{array}$ & 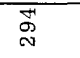 & 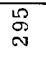 & 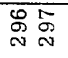 & 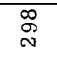 & 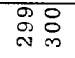 & $\overrightarrow{0} \quad \stackrel{N}{0}$ & ç & m \\
\hline
\end{tabular}




\begin{tabular}{|c|c|c|c|c|c|c|c|}
\hline ת & $\approx$ & $\mathscr{r}+$ & $N m$ & $\infty$ & $\infty$ & $\sigma$ & مמ \\
\hline $\begin{array}{l}8 \\
\text { D } \\
\text { N }\end{array}$ & $\begin{array}{l}8 \\
8 \\
10 \\
0 \\
0\end{array}$ & $\begin{array}{l}8 \% \\
80 \\
00 \\
\infty\end{array}$ & 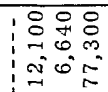 & $\begin{array}{l}8 \\
0 \\
0 \\
0 \\
0\end{array}$ & $\begin{array}{l}8 \\
8 \\
0 \\
0 \\
0\end{array}$ & $\begin{array}{l}0 \\
8 \\
\infty \\
\infty \\
\infty\end{array}$ & $\begin{array}{l}0 \\
0 \\
0 \\
0 \\
\overbrace{}^{2}\end{array}$ \\
\hline . & 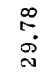 & 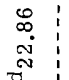 & 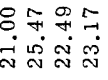 & $\begin{array}{l}\overrightarrow{0} \\
\text { ᄋ. } \\
\text { i }\end{array}$ & $\begin{array}{l}\infty \\
\infty \\
\infty \\
\sim \\
\sim\end{array}$ & $\begin{array}{l}\infty \\
\infty \\
0 \\
- \\
\sim\end{array}$ & 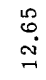 \\
\hline 6 & & $\stackrel{\infty}{=}$ & $0 \approx \approx \exists$ & $\stackrel{\infty}{\sim}$ & $\exists$ & $\cong$ & 0 \\
\hline 淧 & $\dot{\leftrightarrow}$ & 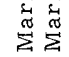 & 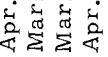 & $\sum_{\tilde{c}}^{\tilde{\sigma}}$ & 逢 & 安 & 客 \\
\hline $\begin{array}{l}\stackrel{8}{\text { D }} \\
\text { Fे }\end{array}$ & $\begin{array}{l}8 \\
\vdots \\
0 \\
5\end{array}$ & 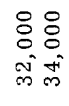 & 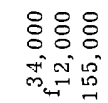 & $\begin{array}{l}8 \\
0 \\
0 \\
-1\end{array}$ & & 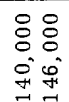 & $\begin{array}{l}8 \\
0 \\
\infty\end{array}$ \\
\hline 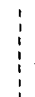 & in & 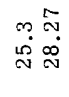 & 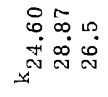 & & $\widetilde{a}$ & 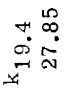 & : \\
\hline & & 筞鿖 & 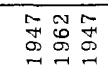 & & th & 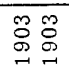 & 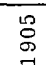 \\
\hline $\begin{array}{l}0 \\
0 \\
0 \\
5 \\
0 \\
-1 \\
-1\end{array}$ & $\begin{array}{l}1 \\
1 \\
\infty \\
\infty \\
\infty \\
\end{array}$ & $\begin{array}{ll}10 & 0 \\
0 & 0 \\
1 & 1 \\
0 & 0 \\
0 & 0 \\
0 & 5 \\
-1 & 7 \\
\end{array}$ & 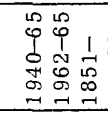 & $\begin{array}{l}10 \\
0 \\
0 \\
0 \\
1 \\
-1 \\
-1\end{array}$ & & $100^{\circ}$ & 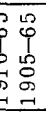 \\
\hline 萨 & $\begin{array}{l}\infty \\
\infty \\
\infty \\
\infty\end{array}$ & 㞼 品 & 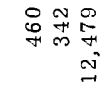 & $\underset{\infty}{\pi}$ & $\stackrel{m}{\rightarrow}$ & $\begin{array}{l}\infty \\
\tilde{0} \\
+ \\
-1\end{array}$ & $\stackrel{\text { : }}{\circ}$ \\
\hline
\end{tabular}
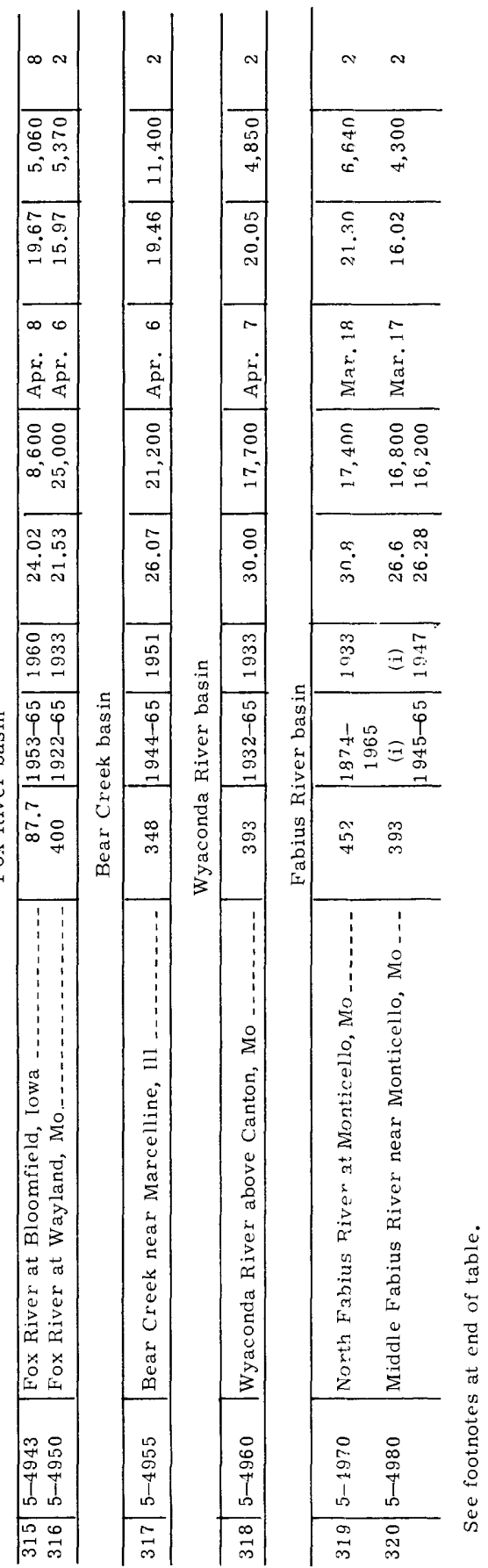


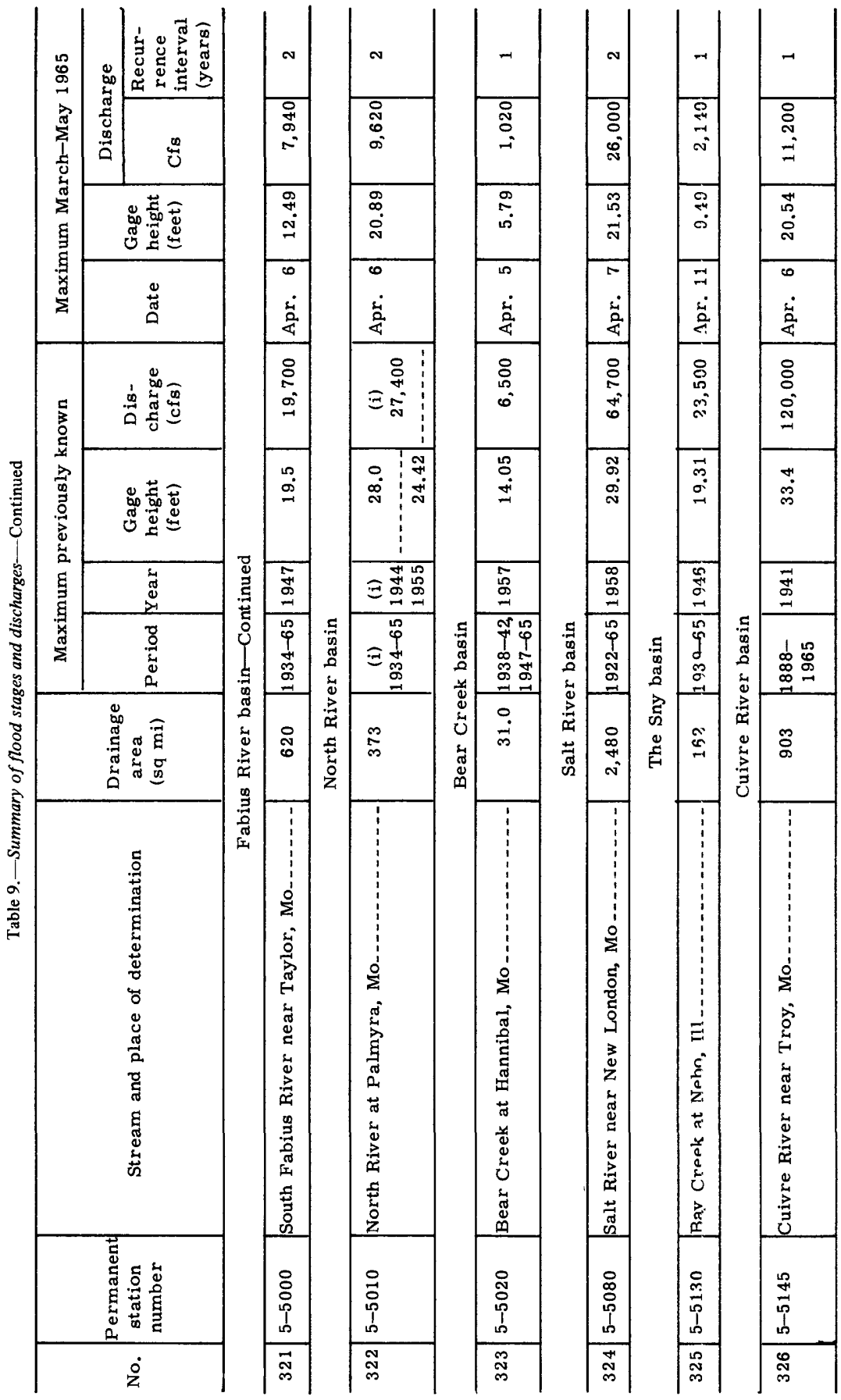


MARCH-MAY, UPPER MISSISSIPPI RIVER BASIN

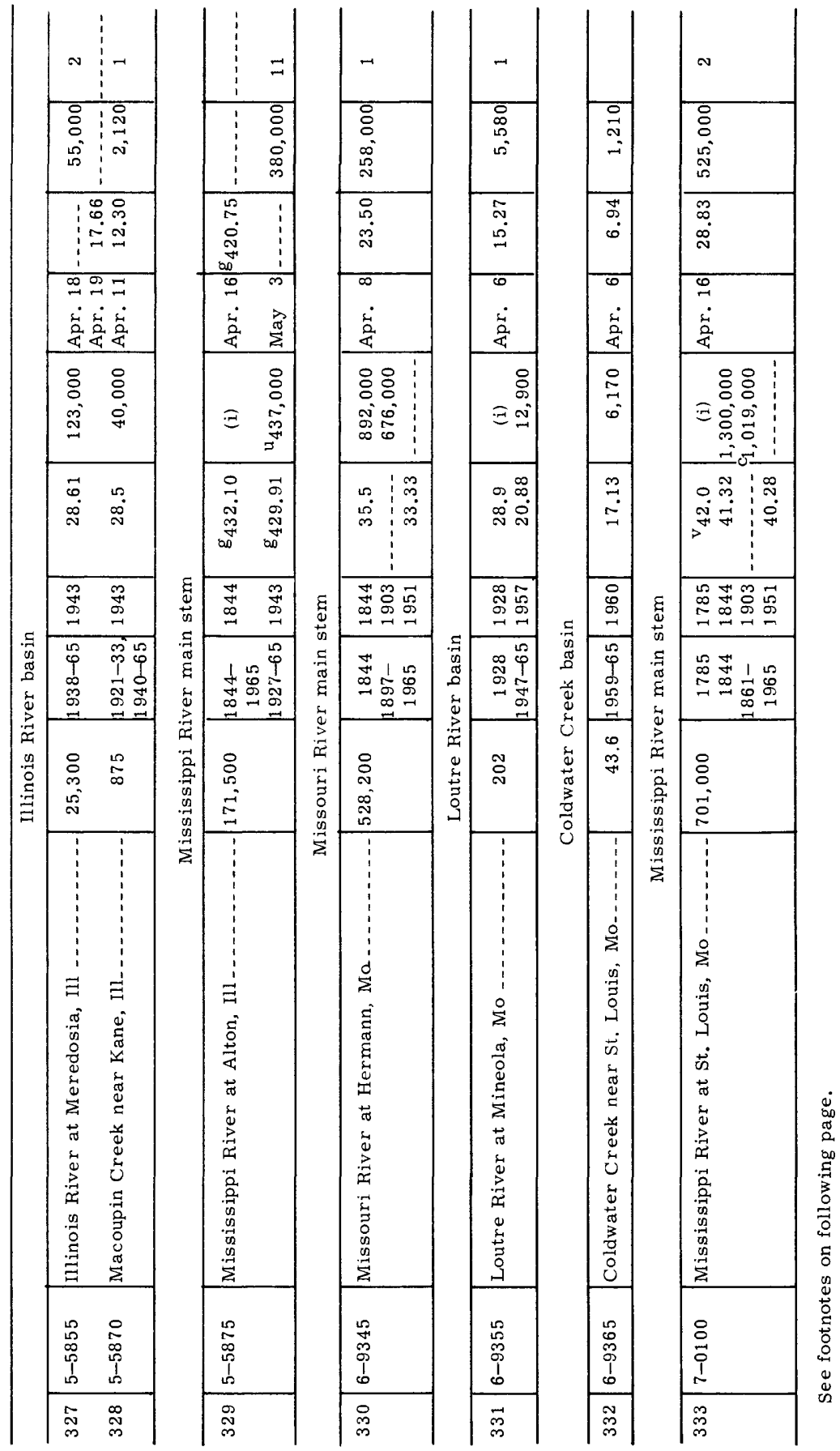




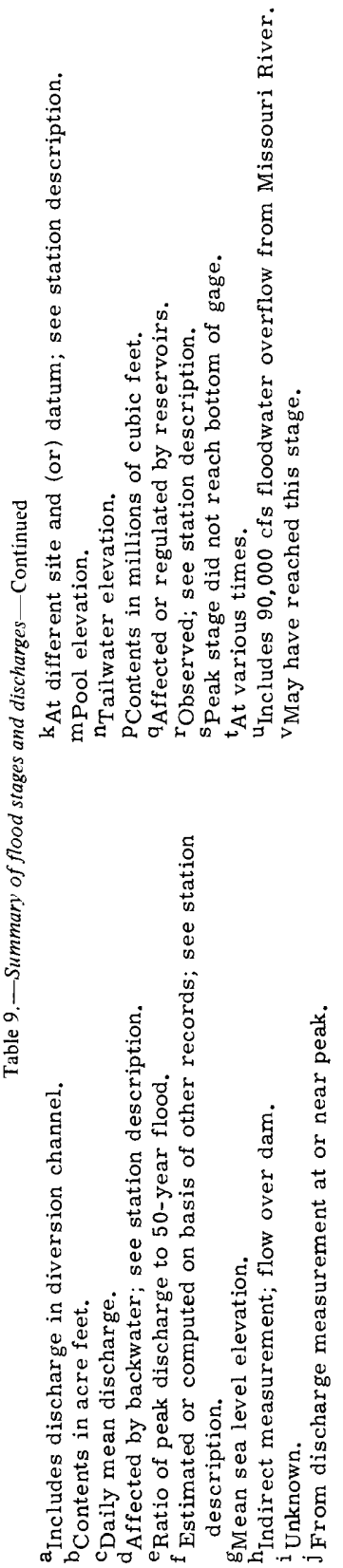




\section{STATION DATA}

\section{MISSISSIPPI RIVER MAIN STEM}

(1) 5-2205. Mississippi River below Sandy River, near Libby, Minn.

Location.-Lat $46^{\circ} 47^{\prime}$, long $93^{\circ} 20^{\prime}$, in sec.25, T.50 N., R.24 W., on right bank $600 \mathrm{ft}$ downstream from Sandy River, three-quarters of a mile northwest of Libby, and at mile 1,106 upstream from Ohio River.

Drainage area.:- $-5,060 \mathrm{sq} \mathrm{mi}$, approximately.

Gage-height record.-Water-stage recorder graph. Datum of gage is $1,204.55 \mathrm{ft}$ above mean sea level, adjustment of 1912 .

Discharge record.-Stage-discharge relation defined by current-meter measur ments. Backwater from ice Apr, 1-17.

Maxima.-April-May 1965: Discharge, 7,340 cfs 1330 hours Apr. 24 (gage height, $14.50 \mathrm{ft})$.

1930 to March 1965: Discharge, 16,000 cfs May 17, 1950 (gage height, $20.02 \mathrm{ft}$ ).

Remarks.-Flow regulated by powerplants and by Winnibigoshish, Leech, Pokegama, and Sandy Lakes.

Mcan discharge, in cubic feet per second, 1965

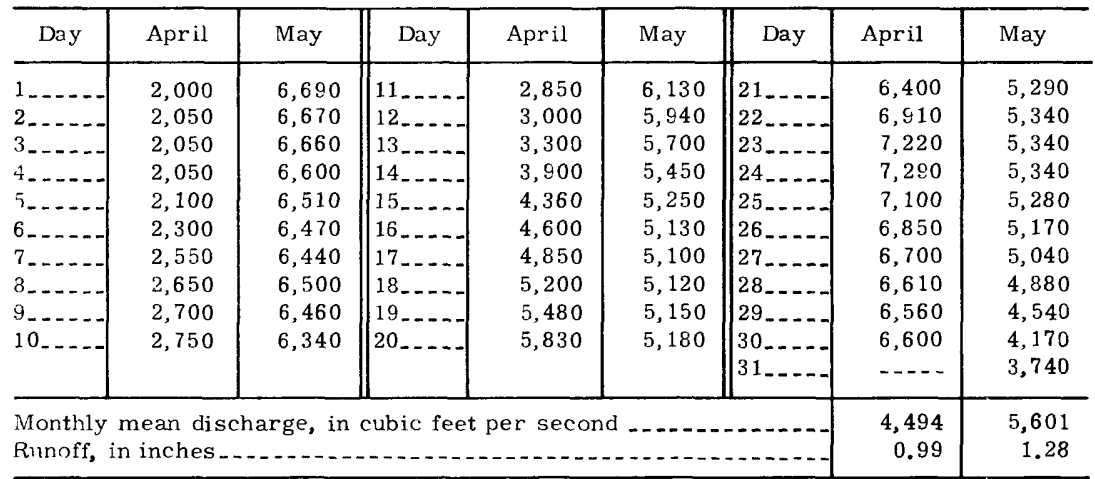


Gage height, in feet, and discharge, in cubic feet per second, at indicated time, 1965, of Mississippi River below Sandy River, near Libby, Minn.

\begin{tabular}{|c|c|c|c|c|c|c|c|c|c|c|c|}
\hline Date & Hour & $\begin{array}{c}\text { Gage } \\
\text { height }\end{array}$ & $\begin{array}{c}\text { Dis- } \\
\text { charge }\end{array}$ & Date & Hour & $\begin{array}{c}\text { Gage } \\
\text { height }\end{array}$ & $\begin{array}{c}\text { Dis- } \\
\text { charge }\end{array}$ & Date & Hour & $\begin{array}{c}\text { Gage } \\
\text { height }\end{array}$ & $\begin{array}{c}\text { Dis - } \\
\text { charge }\end{array}$ \\
\hline \multirow{4}{*}{ Apr. 12} & 0000 & 8.70 & & Apr. 17 & 2000 & 11.05 & $1 \ldots$ & Apr. 23 & 1200 & 14.36 & 7,230 \\
\hline & 1200 & 8.90 & $\ldots$ & & 2400 & 11.12 & - & & 2400 & 14.44 & 7,290 \\
\hline & 2400 & 9.23 & $\ldots$ & & & & & 24 & 1200 & 14.49 & 7,330 \\
\hline & & & & 18 & 1200 & 11.25 & -- & & 1330 & 14.50 & 7,340 \\
\hline \multirow[t]{2}{*}{13} & $\begin{array}{l}1200 \\
1400\end{array}$ & $\begin{array}{l}9.50 \\
9.50\end{array}$ & & & 2400 & 11.54 & $\ldots$ & & 2400 & 14.29 & 7,180 \\
\hline & 2400 & 10.06 & $\ldots$ & 19 & 1200 & 11.73 & & 25 & 1800 & 14.16 & 7,080 \\
\hline \multirow[t]{2}{*}{14} & 1200 & 10.67 & & & 2400 & 12.02 & & & 2400 & 13.98 & 6,960 \\
\hline & 2400 & 11.27 & & 20 & 1300 & 12.25 & 5,780 & 26 & 1200 & 13.82 & 6,840 \\
\hline \multirow[t]{2}{*}{15} & 1000 & 11.50 & & & 2400 & 12.85 & 6,170 & & 2400 & 13.69 & 6,750 \\
\hline & 2400 & 11.34 & 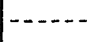 & & & & & 27 & 1200 & 13.60 & 6,690 \\
\hline \multirow[t]{3}{*}{16} & 1200 & 11.12 & & 21 & $\begin{array}{l}1300 \\
2400\end{array}$ & $\begin{array}{l}13.13 \\
13.70\end{array}$ & $\begin{array}{l}6,360 \\
6,760\end{array}$ & & 2400 & 13.55 & 6,660 \\
\hline & 2000 & 11.05 & & & & & 6890 & 28 & 1200 & 13,47 & 6,600 \\
\hline & 2400 & 11.02 & & 22 & $\begin{array}{l}1200 \\
1600\end{array}$ & $\begin{array}{l}13.88 \\
13.95\end{array}$ & $\begin{array}{l}6,890 \\
6,940\end{array}$ & 28 & 1600 & 13.43 & 6,570 \\
\hline 17 & 1200 & 10.94 & & & 2400 & 14.22 & 7,120 & & 2400 & 13.47 & 6,600 \\
\hline
\end{tabular}

(2) 5-2275. Mississippi River at Aitkin, Minn.

Location.-Lat $46^{\circ} 32^{\prime} 26^{\prime \prime}$, long $93^{\circ} 42^{\prime} 26^{\prime \prime}$, in W $\frac{1}{2}$ sec. 24, T.47 N., R. 27 W., at upstream side of highway bridge at north edge of Aitkin, 1 mile downstream from Mud River and at mile $1,055.9$ upstream from Ohio River.

Drainage area. $-6,140 \mathrm{sq} \mathrm{mi}$, approximately.

Gage-height record.-Water-stage recorder graph for river channel. Graph constructed on the basis of once or twice daily wire-weight gage readings for diversion channel for period Apr. 11 to May 17, once-daily wire-weight gage readings for remainder of the period. Datum of gage is $1,185.41 \mathrm{ft}$ above mean sea level datum of 1929 (levels by Corps of Engineers).

Discharge record.-Stage-discharge relation defined by current-meter measurements at both the river and diversion sites. Backwater from ice Apr. 1-18 in the river channel and Apr. 1-17 in the diversion channel.

Maxima.-April-May 1965: Discharge, 13,400 cfs 1000 hours Apr. 26. (Includes discharge in diversion channel).

River channel: Discharge, 7,540 cfs 1400-1700 hours Apr. 18 (gage haight, $14.50 \mathrm{ft}$ ). Diversion channel: Discharge, 5,870 cfs 1800 hours Apr. 25 to 0500 rours Apr. 26 (gage height $14.81 \mathrm{ft}$ ).

1945 to March 1965: Discharge, 20,000 cfs May 20, 1950 (gage height, $19.49 \mathrm{ft}$ ).

Remarks.- -Slight regulation by powerplants and by Winnibigoshish, Leech. Pokegama, and Sandy Lakes. Water diverted at medium and high stages into Aitkin diversion channel $6 \frac{1}{2}$ miles above station, bypasses station and returns to river $15 \frac{1}{2}$ miles below station. Diversion began Apr. 2, 1955. These records include flow in diversion channel. 
Mean discharge, in cubic feet per second, 1965, of Mississippi River at Aitkin, Minn.

\begin{tabular}{|c|c|c|c|c|c|c|c|c|}
\hline Day & April & May & Day & April & May & Day & April & May \\
\hline $\begin{array}{l}1 \ldots \\
2 \ldots \\
3 \ldots \\
4 \ldots \\
5 \ldots \\
6 \ldots \\
7 \ldots \\
8 \ldots \\
9 \ldots \\
10 \ldots\end{array}$ & $\begin{array}{l}2,180 \\
2,140 \\
2,140 \\
2,150 \\
2,300 \\
2,420 \\
2,650 \\
3,000 \\
3,100 \\
3,290\end{array}$ & $\begin{array}{r}11,600 \\
11,200 \\
10,800 \\
10,400 \\
10,000 \\
9,770 \\
9,600 \\
9,510 \\
9,430 \\
9,140\end{array}$ & $\begin{array}{l}11 \ldots \\
12 \ldots \\
13 \ldots \\
14 \ldots \\
15 \ldots \\
16 \ldots \\
17 \ldots \\
18 \ldots \\
19 \ldots \\
20 \ldots\end{array}$ & $\begin{array}{r}3,660 \\
4,080 \\
4,820 \\
6,100 \\
7,550 \\
9,350 \\
11,500 \\
13,000 \\
13,100 \\
13,000\end{array}$ & $\begin{array}{l}8,930 \\
8,660 \\
8,360 \\
7,980 \\
7,660 \\
7,290 \\
7,160 \\
7,110 \\
7,070 \\
7,050\end{array}$ & $\begin{array}{l}21 \ldots \ldots \\
22 \\
23 \\
23 \\
24 \ldots \ldots \\
25 \ldots \\
26 \ldots \ldots \\
27 \ldots \ldots \\
28 \ldots \ldots \\
29 \\
30 \ldots \ldots \\
31\end{array}$ & $\begin{array}{l}13,000 \\
13,000 \\
13,100 \\
13,200 \\
13,300 \\
13,300 \\
13,200 \\
12,900 \\
12,500 \\
12,100\end{array}$ & $\begin{array}{l}7,180 \\
7,390 \\
7,510 \\
\tau, 860 \\
\varepsilon, 080 \\
\varepsilon, 050 \\
\tau, 940 \\
\tau, 680 \\
\tau, 480 \\
\epsilon, 990 \\
\epsilon, 680\end{array}$ \\
\hline \multicolumn{7}{|c|}{$\begin{array}{l}\text { Monthly mean discharge, in cubic feet per second } \\
\text { Runoff, in inches }\end{array}$} & $\begin{array}{r}8,038 \\
1.46\end{array}$ & $\begin{array}{r}\varepsilon, 437 \\
1.58\end{array}$ \\
\hline
\end{tabular}

Gage height, in feet, and discharge, in cubic feet per second, at indicated time, 1965

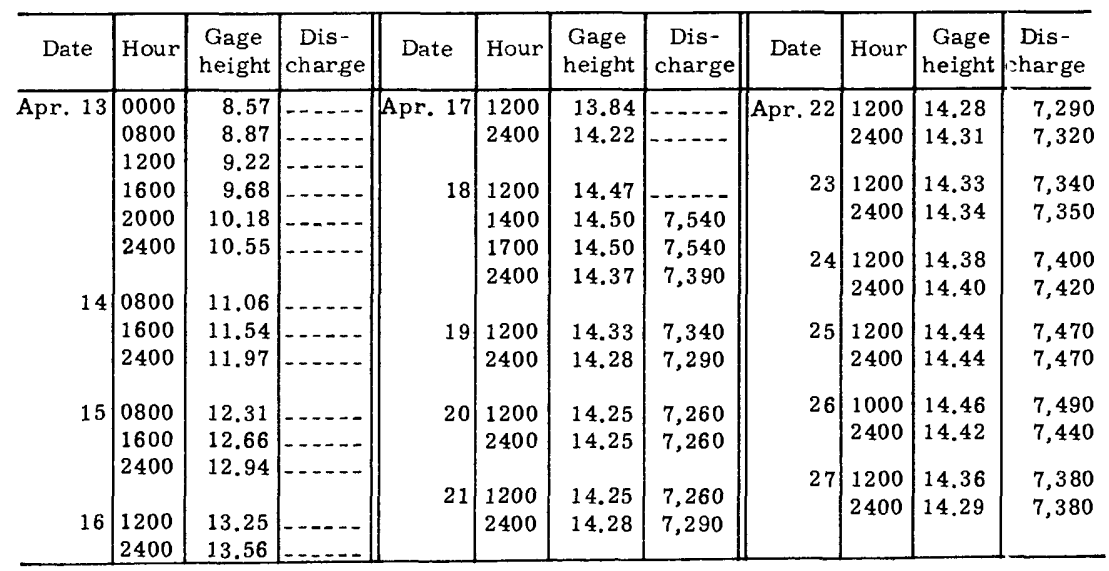


Gage height, in feet, and discharge, in cubic feet per second, at indicated time, 1965, of Mississippi River diversion near Aitkin, Minn.

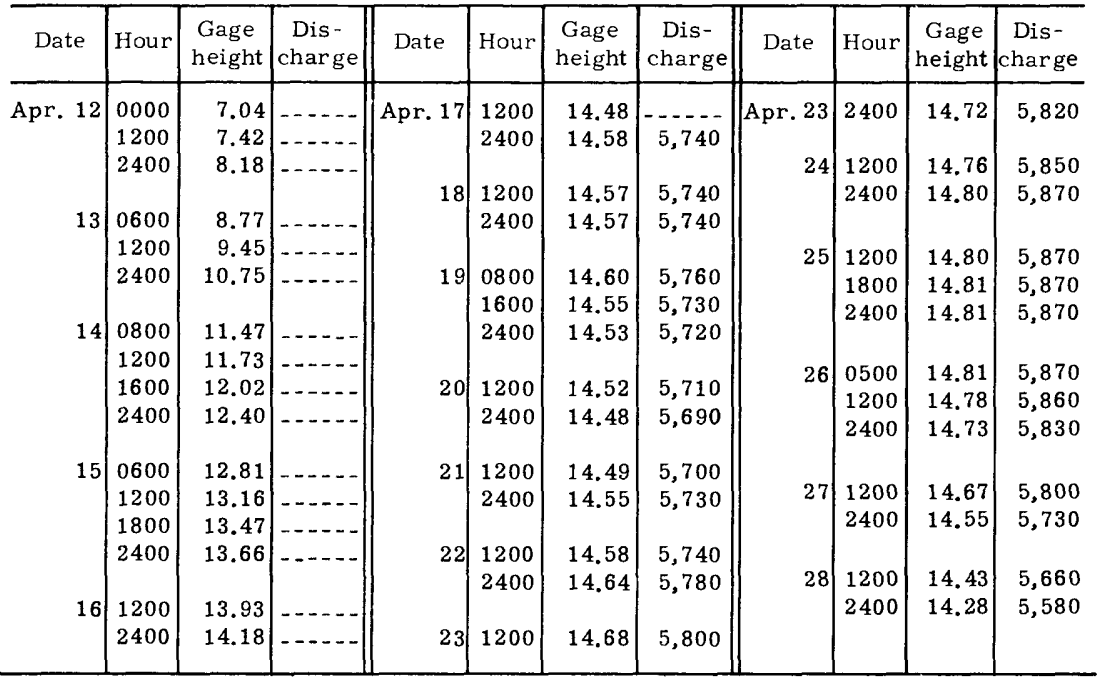

\section{PINE RIVER BASIN}

(3) 5-2305. Pine River Reservoir at Cross Lake, Minn.

Location.-Lat $46^{\circ} 40^{\prime} 09^{\prime \prime}$, long $94^{\circ} 06^{\prime} 44^{\prime \prime}$, in SW $\frac{1}{4} N W \frac{1}{4}$ sec. 21, T. 137 N., R.27 W., at dam on Pine River, at outlet of Cross Lake at village of Cross Lake.

Drainage area. -562 sq $\mathrm{mi}$.

Gage-height record.-Water-stage recorder graph. Datum of gage is $1,216.32 \mathrm{ft}$ above mean sea level, datum of 1929 (levels by Corps of Engineers).

Maxima.-March-May 1965: Contents, 100,800 acre-ft May 27, 28 (gage $\mathrm{f}$ eight, $13.18 \mathrm{ft}$ ). 1886 to February 1965: Contents, 173,600 acre-ft July 10, 1916 (gage height, 18.24 ft).

Remarks.- - Reservoir operations began in 1886. Capacity between gage heights $10.00 \mathrm{ft}$ and $18.50 \mathrm{ft}$ (maximum allowable range) is 118,700 acre-ft of which $53, \approx .80$ acre- $\mathrm{ft}$ is controlled storage between gage heights $10.00 \mathrm{ft}$ and $14.00 \mathrm{ft}$ (normal oparating range).

Cooperation.--Records furnished by Corps of Engineers in terms of cfs-days and converted to acre-feet by Geological Survey.

Gage height, in feet, and contents, in acre-ft

\begin{tabular}{|c|c|c|c|c|c|}
\hline Date & Gage height & Contents & Date & Gage height & Contents \\
\hline $\begin{array}{rr}\text { Feb. } 28 \\
\text { Mar. } 7 \\
14 \ldots \\
21 \\
28 \ldots \\
\\
\text { Apr. } 4 \ldots \\
11\end{array}$ & $\begin{array}{l}11.34 \\
11.42 \\
11.42 \\
11.36 \\
11.21 \\
11.12 \\
11.05\end{array}$ & $\begin{array}{l}76,150 \\
77,200 \\
77,200 \\
76,420 \\
74,460 \\
73,270 \\
72,360\end{array}$ & 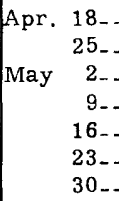 & $\begin{array}{l}11.68 \\
12.18 \\
12.62 \\
12.91 \\
12.89 \\
13.01 \\
13.13\end{array}$ & $\begin{array}{r}80,650 \\
87,270 \\
93,200 \\
97,110 \\
96,830 \\
98,480 \\
100,100\end{array}$ \\
\hline
\end{tabular}


(4) 5-2310. Pine River at Cross Lake Dam, at Cross Lake, Minn.

Location.-Lat $46^{\circ} 40^{\prime} 09^{\prime \prime}$, long $94^{\circ} 06^{\prime} 44^{\prime \prime}$, in SW $\frac{1}{4} \mathrm{NW} \frac{1}{4}$ sec. 21, T. 137 N., R. 27 W., at dam at outlet of Cross Lake at village of Cross Lake.

Drainage area.- $-562 \mathrm{sq} \mathrm{mi}$.

Gage-height record.-Water-stage recorder graph for headwater and twice daily readings on tailwater tape float gage. Datum of gages is $1,216.32 \mathrm{ft}$ above mean sea level, adjustment of 1929 .

Discharge record.-Discharge computed by Corps of Engineers with one check measurement made by Geological Survey.

Maxima.-April-May 1965: Daily discharge, 1,250 cfs Apr, 20.

$\overline{1886}$ to March 1965: Daily discharge, 2,250 cfs in June 1896 (does not include flow bypassing dam through crevasse).

Remarks.-Flow completely regulated by Pine River Reservoir.

Cooperation.-Computations of daily discharge furnished by Corps of Engineers; one discharge measurement made and records reviewed by Geological Survey.

Mean discharge, in cubic feet per second, 1965

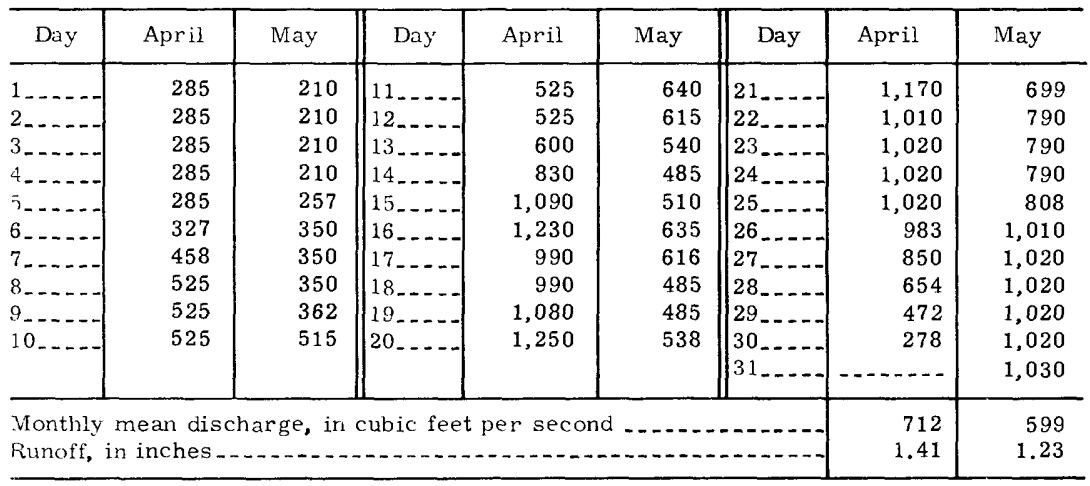

\section{CROW WING RIVER BASIN}

(5) 5-2440. Crow Wing River at Nimrod, Minn.

Location.-Lat $46^{\circ} 39^{\prime}$, long $94^{\circ} 53^{\prime}$, in sec.32, T.137 N., R.33 W., on right bank $200 \mathrm{ft}$ upstream from highway bridge, 0.2 mile north of Nimrod, and 0.7 mile upstream from Cat River.

Drainage area. - 1,010 sq mi, approximately.

Gage-height record.-Water-stage recorder graph. Datum of gage is 1,313.27 ft above mean sea level, datum of 1929 (levels by Wadena County Highway Department from Minnesota Highway Department bench mark).

Discharge record.-Stage-discharge relation defined by current-meter measurements. Backwater from ice Apr. 1-14.

Maxima.-April-May 1965: Discharge, 2,890 cfs 2230 hours Apr. 13 (gage heigh t., $7.57 \mathrm{ft}$, backwater from ice).

1910-14, 1930 to March 1965: Discharge 2, $750 \mathrm{cfs}$ May 23, 1962 (gage heigl , $6.04 \mathrm{ft}$ ); gage height, $7.64 \mathrm{ft}$ Apr. 20, 1950 (backwater from ice).

Remarks.-Flow affected by natural storage in many lakes. 
Mean discharge, in cubic feet per second, 1965, of Crow Wing River at Nimrod, Minn

\begin{tabular}{|c|c|c|c|c|c|c|c|c|}
\hline Day & April & May & Day & April & May & Day & April & May \\
\hline $\begin{array}{l}1 \ldots \ldots \\
2 \ldots \\
3 \\
4 \\
4 \\
5 \ldots \\
6 \ldots \\
7 \ldots \\
8 \ldots \\
9 \\
10 \ldots \ldots\end{array}$ & $\begin{array}{r}330 \\
340 \\
345 \\
360 \\
420 \\
550 \\
660 \\
780 \\
980 \\
1,200\end{array}$ & $\begin{array}{r}1,010 \\
980 \\
948 \\
909 \\
908 \\
972 \\
896 \\
870 \\
857 \\
838\end{array}$ & $\begin{array}{l}11 \ldots \\
12 \ldots \\
13 \ldots \\
14 \ldots \\
15 \ldots \\
16 \ldots \\
17 \ldots \\
18 \ldots \\
19 \ldots \\
20 \ldots\end{array}$ & $\begin{array}{l}1,600 \\
1,960 \\
2,600 \\
2,420 \\
2,360 \\
2,330 \\
2,580 \\
2,310 \\
2,020 \\
1,860\end{array}$ & $\begin{array}{r}831 \\
824 \\
818 \\
812 \\
954 \\
1,110 \\
1,110 \\
1,080 \\
1,040 \\
994\end{array}$ & $\begin{array}{l}21 \ldots \ldots \\
22 \ldots \ldots \\
23 \ldots \ldots \\
24 \ldots \ldots \\
25 \ldots \ldots \\
26 \ldots \ldots \\
27 \ldots \ldots \\
28 \ldots \\
29 \ldots \\
30 \ldots \ldots \\
31 \ldots \ldots\end{array}$ & $\begin{array}{r}1,730 \\
1,630 \\
1,540 \\
1,430 \\
1,980 \\
1,320 \\
1,240 \\
1,180 \\
1,110 \\
1,060\end{array}$ & $\begin{array}{r}987 \\
974 \\
1,010 \\
1,110 \\
1,160 \\
1,150 \\
1,100 \\
1,040 \\
994 \\
942 \\
909\end{array}$ \\
\hline \multicolumn{7}{|c|}{$\begin{array}{l}\text { Monthly mean discharge, in cubic feet per second } \\
\text { Runoff, in inches }\end{array}$} & $\begin{array}{r}1,388 \\
1.53\end{array}$ & $\begin{array}{r}972 \\
1.11\end{array}$ \\
\hline
\end{tabular}

Gage height, in feet, and discharge, in cubic feet per second, at indicated time, 1965

\begin{tabular}{|c|c|c|c|c|c|c|c|c|c|c|c|}
\hline Date & Hour & $\begin{array}{l}\text { Gage } \\
\text { height }\end{array}$ & $\begin{array}{c}\text { Dis- } \\
\text { charge }\end{array}$ & Date & Hour & $\begin{array}{l}\text { Gage } \\
\text { height }\end{array}$ & $\begin{array}{c}\text { Dis - } \\
\text { charge }\end{array}$ & Date & Hour & $\begin{array}{c}\text { Gage } \\
\text { height }\end{array}$ & $\begin{array}{c}\text { Dis- } \\
\text { charge }\end{array}$ \\
\hline 12 & $\begin{array}{l}0000 \\
1200 \\
2400 \\
0600 \\
1200 \\
1500 \\
1800 \\
2000 \\
2400 \\
0600 \\
0900 \\
1500 \\
1600 \\
1800 \\
2100\end{array}$ & $\begin{array}{l}5.80 \\
6.10 \\
6.48 \\
6.61 \\
6.72 \\
7.00 \\
7.28 \\
7.55 \\
7.45 \\
7.16 \\
7.05 \\
7.24 \\
7.14 \\
7.55 \\
7.20\end{array}$ & 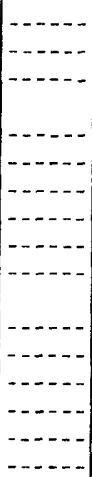 & Apr. 13 & $\begin{array}{l}2200 \\
2230 \\
2400 \\
0100 \\
0300 \\
0400 \\
0600 \\
0800 \\
1100 \\
1800 \\
2400 \\
0600 \\
1200 \\
1800 \\
2400\end{array}$ & $\begin{array}{l}7.45 \\
7.57 \\
7.35 \\
7.00 \\
6.69 \\
6.19 \\
5.87 \\
5.64 \\
5.63 \\
5.85 \\
5.79 \\
5.76 \\
5.66 \\
5.73 \\
5.68\end{array}$ & 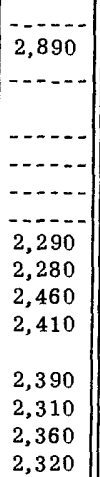 & Apr. 16 & $\begin{array}{l}1000 \\
1100 \\
1400 \\
1900 \\
2400 \\
0600 \\
1200 \\
1400 \\
2100 \\
2400 \\
0600 \\
1200 \\
1800 \\
2400\end{array}$ & $\begin{array}{l}5.52 \\
5.62 \\
5.61 \\
5.83 \\
5.91 \\
5.90 \\
5.78 \\
6.06 \\
6.27 \\
6.13 \\
5.80 \\
5.60 \\
5.48 \\
5.41\end{array}$ & $\begin{array}{l}2,200 \\
2,280 \\
2,270 \\
2,440 \\
2,510 \\
2,500 \\
2,400 \\
2,630 \\
2,800 \\
2,680 \\
2,420 \\
2,260 \\
2,160 \\
2,110\end{array}$ \\
\hline
\end{tabular}

(6) 5-2465. Gull Lake near Brainerd, Minn.

Location.-Lat $46^{\circ} 24^{\prime} 40^{\prime \prime}$, long $94^{\circ} 21^{\prime} 26^{\prime \prime}$, in $\mathrm{N} \frac{1}{2} \mathrm{sec} .20$, T.134 N., R.29 W., in pool of dam on Gull River, $800 \mathrm{ft}$ south of outlet of Gull Lake, a quarter of a mile upstream from Gull Lake Dam, and 8 miles northwest of Brainerd.

Drainage area.-287 sq $\mathrm{mi}$.

Gage-height record.-Water-stage recorder graph. Datum of gage is $1,188.14 \mathrm{ft}$ above mean sea level, adjustment of 1912 .

Maxima.-March-May 1965: Contents, 61,800 acre-ft May 26, 27 (gage həight, $6.31 \mathrm{ft}$ ). 1911 to February 1965: Contents, 74,800 acre-ft June 30, 1914 (gage height $7.30 \mathrm{ft}$ ).

Remarks.-Reservoir operations began in 1912. Capacity between gage heights $5.00 \mathrm{ft}$ and $7.00 \mathrm{ft}$ (maximum allowable range and normal operating range), 26,020 acre- $\mathrm{ft}$.

Cooperation.-Records furnished by Corps of Engineers, in terms of cfs-days and converted to acre-feet by Geological Survey. 
Gage height, in feet, and contents, in acre-ft, of Gull Lake near Brainerd, Minn.

\begin{tabular}{|c|c|c|c|c|c|}
\hline Date & Gage height & Contents & Date & Gage height & Contents \\
\hline $\begin{array}{lr}\text { Feb. } & 28 \\
\text { Mar. } & \\
& \\
14 & \\
& 21 \\
& 28 \\
\text { Apr. } & 4 \\
& 41 \\
& 11\end{array}$ & $\begin{array}{l}5.26 \\
5.31 \\
5.24 \\
5.13 \\
5.03 \\
4.99 \\
5.16\end{array}$ & $\begin{array}{l}48,180 \\
48,830 \\
47,920 \\
46,510 \\
45,200 \\
44,690 \\
46,890\end{array}$ & 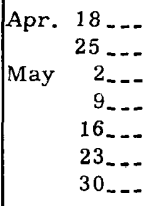 & $\begin{array}{l}6.14 \\
6.20 \\
6.05 \\
6.17 \\
6.11 \\
6.14 \\
6.21\end{array}$ & $\begin{array}{l}59,580 \\
60,360 \\
58,410 \\
59,960 \\
59,210 \\
59,580 \\
60,500\end{array}$ \\
\hline
\end{tabular}

(7) 5-2470. Gull River at Gull Lake Dam, near Brainerd, Minn.

Location.-Lat $46^{\circ} 24^{\prime} 40^{\prime \prime}$, long $94^{\circ} 21^{\prime} 12^{\prime \prime}$, in sec.20, T.134 N., R.29 W., in headwater and tailwater of dam at outlet of Gull Lake, 8 miles northwest of Brainerd.

Drainage area. -287 sq mi.

Gage-height record.-Water-stage recorder graph for headwater and twice-daily readings on tailwater staff gage. Datum of gages is $1,188.14 \mathrm{ft}$ above mean sea level, adjustment of 1912 .

Discharge record.-Discharge computed by Corps of Engineers with one check measurement made by Geological Survey.

Maxima.-April-May 1965: Daily discharge, 820 cfs May 27.

1911 to March 1965: Daily discharge, 1,120 cfs May 15, 1938.

Remarks.-Flow completely regulated by Gull Lake.

Cooperation.-Computations of daily discharge furnished by Corps of Engineers: one discharge measurement made and records reviewed by Geological Survey.

Mean discharge, in cubic feet per second, 1965

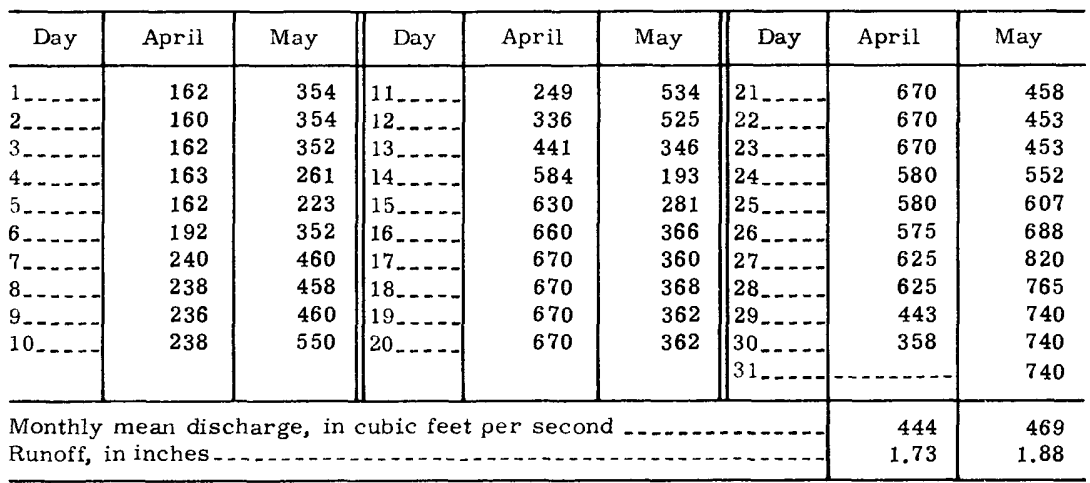




\section{MISSISSIPPI RIVER MAIN STEM}

(8) 5-2670. Mississippi River near Royalton, Minn.

Location.-Lat $45^{\circ} 51^{\prime} 40^{\prime \prime}$, long $94^{\circ} 21^{\prime} 30^{\prime \prime}$, in lot 2, sec.20, T.39 N., R.32 W., at plant of Minnesota Power and Light Co., 4 miles northwest of Royalton, and 4.5 miles downstream from Swan River, and at mile 956 upstream from Ohio River.

Drainage area.-11,600 sq mi, approximately.

Gage-height record.-Headwater and tailwater gages read hourly by comfany employees. Readings not reported to Geological Survey.

Discharge record.-Discharge computed by Minnesota Power and Light Company with two check measurements made by Geological Survey.

Maxima.-April-May 1965: Daily discharge, 37,700 cfs Apr. 16.

1924 to March 1965: Daily discharge, 29,400 cfs Apr. 13, 1952.

Remarks.-Flow partly regulated by powerplants, by Winnibigoshish, Leech, Pokegama, Sandy, and Gull Lakes and by Pine River Reservoir.

Cooperation.-Records collected by Minnesota Power and Light Co. under general supervision of Geological Survey, in connection with a Federal Power Commission project.

Mean discharge, in cubic feet per second, 1965

\begin{tabular}{|c|c|c|c|c|c|c|c|c|}
\hline Day & April & May & Day & April & May & Day & April & May \\
\hline 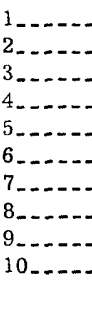 & $\begin{array}{l}3,240 \\
3,740 \\
3,150 \\
2,980 \\
3,070 \\
3,750 \\
4,180 \\
4,360 \\
5,070 \\
7,860\end{array}$ & $\begin{array}{l}21,600 \\
20,500 \\
19,900 \\
19,800 \\
18,700 \\
16,900 \\
16,700 \\
16,000 \\
17,200 \\
16,500\end{array}$ & $\mid \begin{array}{l}11 \ldots \\
12 \ldots \ldots \\
13 \ldots \ldots \\
14 \ldots \ldots \\
15 \ldots \ldots \\
16 \ldots \ldots \\
17 \ldots \ldots \\
18 \ldots \\
19 \ldots \\
20 \ldots\end{array}$ & $\begin{array}{l}11,300 \\
17,100 \\
24,100 \\
31,900 \\
37,400 \\
37,700 \\
36,300 \\
32,500 \\
32,200 \\
31,300\end{array}$ & $\begin{array}{l}16,500 \\
16,600 \\
15,500 \\
15,300 \\
15,000 \\
14,100 \\
14,400 \\
14,400 \\
14,200 \\
14,300\end{array}$ & $\begin{array}{l}21 \ldots \ldots \\
22 \ldots \ldots \\
23 \\
24 \\
25 \\
25 \\
26 \ldots \ldots \\
27 \ldots \ldots \\
28 \ldots \\
29 \\
29 \\
30 \\
31\end{array}$ & $\begin{array}{c}29,700 \\
28,200 \\
27,200 \\
26,500 \\
25,900 \\
25,400 \\
24,800 \\
24,700 \\
23,800 \\
23,300 \\
-\end{array}$ & $\begin{array}{l}15,100 \\
15,300 \\
15,100 \\
16,200 \\
17,800 \\
18,400 \\
18,500 \\
18,900 \\
18,400 \\
17,300 \\
16,500\end{array}$ \\
\hline \multicolumn{7}{|c|}{$\begin{array}{l}\text { Monthly mean discharge, in cubic feet per second } \\
\text { Runoff, in inches }\end{array}$} & $\begin{array}{r}19,730 \\
1.90\end{array}$ & $\begin{array}{r}16,810 \\
1.67\end{array}$ \\
\hline
\end{tabular}

\section{PLATTE RIVER BASIN}

(9) 5-2679. Hillman Creek near Pierz, Minn.

(Crest-stage station)

Location.-Lat $45^{\circ} 58^{\prime} 27^{\prime \prime}$, long $94^{\circ} 04^{\prime} 21^{\prime \prime}$, in $\mathrm{NE}_{\frac{1}{4}} \mathrm{SE} \frac{1}{4}$ sec.9, T.40 N., R.30 V., at bridge on county road, 1.1 miles upstream from mouth, and 1.5 miles east of Fierz.

Drainage area. $-52.6 \mathrm{sq} \mathrm{mi}$.

Gage-height record.-Crest stages only.

Discharge record.--Stage-discharge relation defined by current-meter measurements below $900 \mathrm{cfs}$ and extended above by logarithmic plotting.

Maxima.-April-May 1965: Discharge, 2,560 cfs Apr. 13 (gage height 14.68 ft).

1964 to March 1965: Discharge, 2,040 cfs May 7, 1964 (gage height, $14.55 \mathrm{ft}$ ). 


\section{SAUK RIVER BASIN}

(10) 5-2705. Sauk River near St. Cloud, Minn.

Location.-Lat $45^{\circ} 33^{\prime} 35^{\prime \prime}$, long $94^{\circ} 14^{\prime} 00^{\prime \prime}$, in $\mathrm{SE}_{\frac{1}{4}} \mathrm{SW} \frac{1}{4}$ sec.8, T.124 N., R.28 W., on right bank half a mile northwest of Waite Park, 3 miles west of St. Cloud, and 5 miles upstream from mouth.

Drainage area. $-925 \mathrm{sq} \mathrm{mi}$.

Gage-height record.-Water-stage recorder graph. Datum of gage is $1,034.95 \mathrm{ft}$ above mean sea level, datum of 1912.

Discharge record.-Stage-discharge relation defined by current-meter measurements. Backwater from ice Apr. 1-13.

Maxima.-April-May 1965: Discharge, 9,100 cfs 2215 hours Apr. 13 (gage height, $10.68 \mathrm{ft}$ ). $1909-13,1929$ to March 1965: Discharge, 5,580 cfs Apr. 13, 1951 (gage height, $7.89 \mathrm{ft}$ ).

Remarks.-Flow regulated by powerplants and reservoirs above station.

Mean discharge, in cubic feet per second, 1965

\begin{tabular}{|c|c|c|c|c|c|c|c|c|}
\hline Day & April & May & Day & April & May & Day & April & May \\
\hline & 53 & 1,530 & $11 \ldots$ & 2,500 & 1,000 & $21 \ldots$ & 3,610 & 895 \\
\hline & 55 & 1,440 & $12 \ldots$ & 4,500 & 1,030 & $22 \ldots \ldots$ & 3,210 & 865 \\
\hline & 65 & 1,340 & $13 \ldots$ & 7,500 & 970 & $23 \ldots$ & 2,840 & 930 \\
\hline $4 \ldots$ & 80 & 1,240 & $14 \ldots$ & 7,940 & 940 & $24 \ldots$ & 2,520 & 1,080 \\
\hline $5 \ldots$ & 100 & 732 & $15 \ldots$ & 7,440 & 960 & $25 \ldots$ & 2,360 & 1,200 \\
\hline $6 \ldots$ & 150 & 716 & $16 \ldots$ & 6,800 & 960 & $26 \ldots$ & 2,230 & 1,200 \\
\hline $7 \ldots$ & 250 & 870 & $17 \ldots$ & 6,040 & 920 & $27 \ldots$ & 2,040 & 1,220 \\
\hline & 450 & 855 & $18 \ldots$ & 5,270 & 920 & $28 \ldots$ & 1,910 & 1,200 \\
\hline & 800 & 915 & $19 \ldots$ & 4,610 & 870 & $29 \ldots$ & 1,770 & 1,160 \\
\hline 10. & 1,500 & 945 & 20 & 4,050 & 840 & $30 \ldots$ & 1,650 & 1,140 \\
\hline & & & & & & $31 \ldots$ & $\ldots$ & 1,140 \\
\hline \multicolumn{7}{|c|}{ Monthly mean discharge, in cubic feet per second } & 2,810 & 1,030 \\
\hline \multicolumn{7}{|c|}{ Runoff, in inches } & 3.39 & 1.29 \\
\hline
\end{tabular}


Gage height, in feet, and discharge, in cubic feet per second, at indicated time, 1965, of Sauk River near St. Cloud, Minn.

\begin{tabular}{|c|c|c|c|c|c|c|c|c|c|c|c|}
\hline Date & Hour & $\begin{array}{l}\text { Gage } \\
\text { height }\end{array}$ & $\begin{array}{c}\text { Dis- } \\
\text { charge }\end{array}$ & Date & Hour & $\begin{array}{c}\text { Gage } \\
\text { height }\end{array}$ & $\begin{array}{c}\text { Dis- } \\
\text { charge }\end{array}$ & Date & Hour & $\begin{array}{c}\text { Gage } \\
\text { height }\end{array}$ & $\begin{array}{c}\text { Dis- } \\
\text { charge }\end{array}$ \\
\hline pr. 9 & $\begin{array}{l}0000 \\
0600 \\
1200 \\
1800 \\
2400 \\
0600 \\
0800 \\
0900 \\
1000 \\
1100 \\
1200 \\
1300 \\
1400 \\
1600 \\
1800 \\
1900 \\
2100 \\
2300 \\
2400 \\
0800 \\
1200 \\
1600 \\
2400\end{array}$ & $\begin{array}{l}3.49 \\
3.68 \\
4.08 \\
4.27 \\
4.45 \\
4.54 \\
4.55 \\
4.83 \\
4.84 \\
4.89 \\
4.77 \\
4.80 \\
5.10 \\
5.01 \\
5.02 \\
4.90 \\
5.05 \\
5.25 \\
5.29 \\
5.89 \\
6.15 \\
6.94 \\
7.65\end{array}$ & 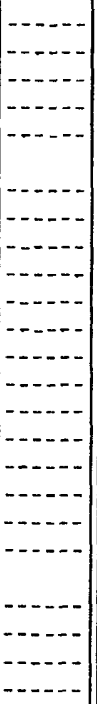 & Apr. 12 & $\begin{array}{l}0300 \\
0600 \\
0700 \\
0800 \\
0900 \\
1000 \\
1200 \\
1300 \\
1400 \\
1600 \\
1700 \\
1800 \\
2000 \\
2100 \\
2400 \\
0200 \\
0600 \\
1000 \\
1200 \\
1400 \\
1600 \\
1800 \\
2215\end{array}$ & $\begin{array}{r}7.85 \\
8.00 \\
8.05 \\
7.54 \\
7.50 \\
7.59 \\
7.93 \\
7.56 \\
7.67 \\
7.85 \\
7.96 \\
8.64 \\
8.70 \\
9.01 \\
9.05 \\
9.19 \\
9.19 \\
9.39 \\
9.77 \\
10.50 \\
10.42 \\
10.51 \\
10.68\end{array}$ & 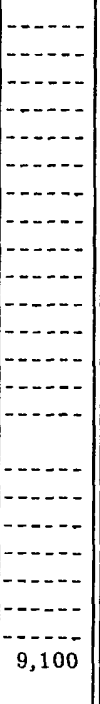 & Apr. 13 & $\begin{array}{l}2400 \\
0400 \\
0800 \\
1200 \\
2400 \\
1200 \\
2400 \\
1200 \\
2400 \\
1200 \\
2400 \\
1200 \\
2400 \\
1200 \\
2400 \\
1200 \\
2400\end{array}$ & $\begin{array}{r}10.48 \\
10.07 \\
9.97 \\
9.72 \\
9.59 \\
9.52 \\
9.32 \\
9.03 \\
8.75 \\
8.50 \\
8.21 \\
7.94 \\
7.66 \\
7.43 \\
7.21 \\
6.99 \\
6.79\end{array}$ & $\begin{array}{l}8,820 \\
8,250 \\
8,110 \\
7,760 \\
7,580 \\
7,480 \\
7,200 \\
6,790 \\
6,400 \\
6,050 \\
5,640 \\
5,270 \\
4,910 \\
4,610 \\
4,320 \\
4,040 \\
3,800\end{array}$ \\
\hline
\end{tabular}

JOHNSON CREEK BASIN

(11) 5-2720. Johnson Creek tributary near St. Augusta, Minz.

\section{(Crest-stage station)}

Location.-Lat $45^{\circ} 26^{\prime} 52^{\prime \prime}$, long $94^{\circ} 12^{\prime} 00^{\prime \prime}$, in NE $\frac{1}{4} \mathrm{SE} \frac{1}{4} \sec .21, \mathrm{~T} .123$ N., R.28 W., at culverts on county road, 0.7 mile upstream from mouth and 3.1 miles southwest of St. Augusta.

Gage-height record.-Crest stages only.

Discharge record.-Stage-discharge relation defined by current-meter measurements below $39 \mathrm{cfs}$ and by indirect measurement at $274 \mathrm{cfs}$.

Maxima.-April-May 1965: Discharge, 274 cfs Apr. 12 (gage height, $10.16 \mathrm{ft}$ ). $\overline{1964}$ to March 1965: Discharge, 39 cfs May 6, 1964 (gage height, 6.45 ft). 
(12) 5-2723, Johnson Creek near St. Augusta, Minn.

(Crest-stage station)

Location.-Lat $45^{\circ} 27^{\prime} 49^{\prime \prime}$, Iong $94^{\circ} 09^{\prime} 19^{\prime \prime}$, in NW $\frac{1}{4} \mathrm{SW} \frac{1}{4}$ sec.13, T.123 N., R.28 W., at bridge on County Highway $7,1.0$ mile south of St. Augusta, and 3.3 miles upstream from mouth.

Gage-height record.-Crest stages only.

Discharge record.-Stage-discharge relation defined by current-meter measurements.

Maxima.-April-May 1965: Discharge, 682 cfs Apr. 12 (gage height, $14.77 \mathrm{ft}$ ).

1964 to March 1965: Discharge, 227 cfs Apr. 13, 1964 (gage height, $12.85 \mathrm{ft}$ ).

\section{OTSEGO CREEK BASIN}

(13) 5-2737. Otsego Creek near Otsego, Minn.

(Crest-stage station)

Location.-Lat $45^{\circ} 17^{\prime} 19^{\prime \prime}$, Iong $93^{\circ} 38^{\prime} 59^{\prime \prime}$, in $\mathrm{SW}_{\frac{1}{4}} \mathrm{NE} \frac{1}{4}$ sec.13, T.121 N., R.24 W., at culvert on County Highway $39,1.3$ miles upstream from mouth, and 1.9 miles west of Otsego.

Gage-height record.-Crest-stages only.

Discharge record.-Stage-discharge relation defined by current-meter measurements below $20 \mathrm{cfs}$ and by flow-through-culvert computation at $202 \mathrm{cfs}$.

Maxima.-April-May 1965: Discharge, 202 cfs Apr. 11 (gage height, $7.48 \mathrm{ft}$ ); gage height, $8.19 \mathrm{ft}$ Apr. 9 (backwater from ice).

1964 to March 1965: Discharge, 23 cfs Apr, 6, 1964 (gage height, $4.39 \mathrm{ft}$, backwater from ice).

\section{ELK RIVER BASIN}

(14) 5-2742. Stony Brook tributary near Foley, Minn.

(Crest-stage station)

Location.-Lat $45^{\circ} 38^{\prime} 40^{\prime \prime}$, long $93^{\circ} 54^{\prime} 50^{\prime \prime}$, in NW $\frac{1}{4}$ sec.2, T.36 N., R.29 W., at culvert on State Highway 25, a quarter mile upstream from mouth, and $1 \frac{1}{2}$ miles south of Foley.

Drainage area. $-3.11 \mathrm{sq} \mathrm{mi}$.

Gage-height record.-Crest stages only.

Discharge record.--Stage-discharge relation defined by current-meter measurements below $51 \mathrm{cfs}$ and by indirect measurement at $132 \mathrm{cfs}$.

Maxima.-April-May 1965: Discharge, 83 cfs Apr, 12 (gage height, $11.26 \mathrm{ft}$, backwater from ice).

1960 to March 1965: Discharge, 132 cfs May 23, 1962 (gage height, $11.35 \mathrm{ft}$, from high-water profile). 
(15) 5-2747. St. Francis River at Santiago, Minn.

(Gaging station established June 1965)

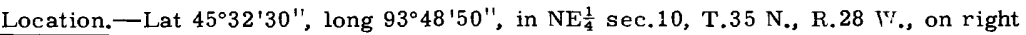
bank, 0.2 mile east of Santiago and 0.4 mile upstream from bridge on county road.

Gage-height record.-Floodmark and occasional gage readings on reference mark.

Discharge record.--Stage-discharge relation defined by current-meter measurements.

Maximum.-April-May 1965: Discharge, 2,940 cfs about 0915-1100 hours Apr. 14 (gage height, $12.17 \mathrm{ft}$ from floodmark).

(16) 5-2749. St. Francis River near Big Lake, Minn.

(Gaging station established, June 1965)

Location.-Lat $45^{\circ} 23^{\prime} 07^{\prime \prime}$, long $93^{\circ} 44^{\prime} 02^{\prime \prime}$, in NW $\frac{1}{4} \mathrm{NW} \frac{1}{4}$ sec.5, T.33 N., R.27 W., on right bank 3.6 miles north of Big Lake and 4 miles upstream from mouth.

Gage-height record.-Occasional gage readings on reference mark. Altitude of gage is $925 \mathrm{ft}$ (from topographic map).

Discharge record.-Stage-discharge relation defined by current-meter measurements.

Maximum.-April-May 1965: Discharge, 2,700 cfs 1340 hours Apr. 16 (gage height, $11.34 \mathrm{ft}$ from floodmark).

(17) 5-2750. Elk River near Big Lake, Minn.

Location.-Lat $45^{\circ} 20^{\prime}$, long $93^{\circ} 40^{\prime}$, in sec.23, T.33 N., R.27 W., on right bank at upstream side of highway bridge, 4 miles east of Big Lake and 4 miles downstream from St. Francis River.

Drainage area.-615 sq $\mathrm{mi}$.

Gage-height record.-Water stage recorder graph. Datum of gage is $8 \subseteq 9.60 \mathrm{ft}$ above mean sea level, datum of 1929 .

Discharge record.-Stage-discharge relation defined by current-meter measurements. Backwater from ice Apr. 1-14.

Maxima.-April-May 1965: Discharge, 7,360 cfs 1800 hours Apr. 16 (gage height, $10.86 \mathrm{ft})$.

1911-17, 1931 to March 1965: Discharge, 5,330 cfs Apr. 10, 1952 (gage height, $10.36 \mathrm{ft}$ ), from rating curve extended above $3,800 \mathrm{cfs}$.

Mean discharge, in cubic feet per second, 1965

\begin{tabular}{|c|c|c|c|c|c|c|c|c|}
\hline Day & April & May & Day & April & May & Day & Apnil & May \\
\hline $\begin{array}{l}1 \ldots \\
2 \ldots \\
3 \ldots \\
4 \ldots \\
5 \ldots \\
6 \ldots \\
7 \ldots \\
8 \ldots \\
9 \ldots \\
10_{-}\end{array}$ & $\begin{array}{r}83 \\
85 \\
89 \\
97 \\
113 \\
134 \\
156 \\
180 \\
230 \\
290\end{array}$ & $\begin{array}{r}941 \\
874 \\
804 \\
739 \\
689 \\
667 \\
753 \\
889 \\
1,000 \\
1,010\end{array}$ & $\begin{array}{l}11 \ldots \\
12 \\
13 \ldots \\
14 \ldots \\
15 \ldots \\
16 \ldots \\
17 \ldots \\
18 \ldots \\
19 \ldots \\
20 \ldots\end{array}$ & $\begin{array}{r}390 \\
569 \\
970 \\
2,690 \\
4,940 \\
7,170 \\
7,030 \\
5,900 \\
4,350 \\
3,260\end{array}$ & $\begin{array}{r}1,020 \\
1,020 \\
1,010 \\
945 \\
882 \\
826 \\
761 \\
717 \\
689 \\
678\end{array}$ & $\mid \begin{array}{l}21 \ldots \ldots \\
22 \\
23 \\
24 \ldots \\
25 \ldots \\
26 \ldots \\
26 \ldots \\
28 \ldots \\
28 \ldots \\
30 \ldots \\
31 \ldots\end{array}$ & $\begin{array}{r}2,590 \\
2,200 \\
1,890 \\
1,610 \\
1,460 \\
1,360 \\
1,230 \\
1,120 \\
1,050 \\
1,000\end{array}$ & $\begin{array}{r}674 \\
671 \\
671 \\
696 \\
735 \\
782 \\
830 \\
908 \\
996 \\
1,030 \\
1,070\end{array}$ \\
\hline \multicolumn{7}{|c|}{$\begin{array}{l}\text { Monthly mean discharge, in cubic feet per second } \\
\text { Runoff, in inches }\end{array}$} & $\begin{array}{r}1,808 \\
3.28\end{array}$ & $\begin{array}{r}838 \\
1.57\end{array}$ \\
\hline
\end{tabular}


Gage height, in feet, and discharge, in cubic feet per second, at indicated time, 1965, of Elk River near Big Lake. Minn.

\begin{tabular}{|c|c|c|c|c|c|c|c|c|c|c|c|}
\hline Date & Hour & $\begin{array}{l}\text { Gage } \\
\text { height }\end{array}$ & $\begin{array}{c}\text { Dis- } \\
\text { charge }\end{array}$ & Date & Hour & $\begin{array}{c}\text { Gage } \\
\text { height }\end{array}$ & $\begin{array}{c}\text { Dis- } \\
\text { charge }\end{array}$ & Date & Hour & $\begin{array}{c}\text { Gage } \\
\text { height }\end{array}$ & $\begin{array}{l}\text { Dis- } \\
\text { charge }\end{array}$ \\
\hline \multirow[t]{19}{*}{ Apr. 13} & 0000 & 4.56 & & \multirow[t]{24}{*}{ Apr. 15} & C600 & 8.79 & 4,220 & \multirow[t]{5}{*}{ Apr. 19 } & 0600 & 9.20 & 4,690 \\
\hline & 0400 & 4.62 & & & 0800 & 8.87 & 4,310 & & 1200 & 8.88 & 4,320 \\
\hline & 0600 & 4.61 & & & 1000 & 8.93 & 4,370 & & 1800 & 8.56 & 3,990 \\
\hline & 1000 & 4.52 & & & 1200 & 9.05 & 4,510 & & 2400 & 8.27 & 3,720 \\
\hline & 1100 & 4.57 & & & 1400 & 9.33 & 4,860 & & & & \\
\hline & 1200 & 4.61 & & & 1600 & 9.64 & 5,290 & \multirow[t]{3}{*}{20} & 1200 & 7.66 & 3,240 \\
\hline & 1300 & 4.57 & & & 1800 & 9.91 & 5,710 & & 2400 & 7.12 & 2,860 \\
\hline & 1400 & 4.60 & & & 2000 & 10.16 & 6,110 & & & & \\
\hline & 1500 & 4.82 & & & 2200 & 10.31 & 6,370 & \multirow[t]{3}{*}{21} & 1200 & 6.65 & 2,570 \\
\hline & 1600 & 5.05 & & & 2400 & 10.45 & 6,610 & & 2400 & 6.32 & 2,370 \\
\hline & 1700 & 5.16 & & & & & & & & & \\
\hline & 1800 & 5.36 & & & 0200 & 10.57 & 6,830 & \multirow[t]{3}{*}{22} & 1200 & 6.02 & 2,190 \\
\hline & 1900 & 5.36 & & & 0400 & 10.65 & 6,970 & & 2400 & 5.78 & 2,050 \\
\hline & 2000 & 5.51 & & & 0600 & 10.72 & 7,100 & & & & \\
\hline & 2100 & 5.74 & & & 0800 & 10.75 & 7,160 & \multirow[t]{3}{*}{23} & 1200 & 5.47 & 1,880 \\
\hline & 2200 & 5.93 & & & 1000 & 10.77 & 7,190 & & 2400 & 5.18 & 1,740 \\
\hline & 2300 & 6.29 & & & 1200 & 10,81 & 7,270 & & & & \\
\hline & 2400 & 6.24 & & & 1400 & 10.82 & 7,290 & \multirow[t]{5}{*}{24} & 0600 & 5.06 & 1,680 \\
\hline & & & & & 1600 & 10.84 & 7,330 & & 1200 & 4.90 & 1,600 \\
\hline \multirow[t]{13}{*}{14} & 0200 & 6.13 & & & 1800 & 10.86 & 7,360 & & 1800 & 4.80 & 1,550 \\
\hline & 0400 & 6.30 & & & 2000 & 10.84 & 7,330 & & 2400 & 4.71 & 1,500 \\
\hline & 0600 & 6.53 & & & 2200 & 10.83 & 7,310 & & & & \\
\hline & 0800 & 6.84 & & & 2400 & 10.82 & 7,290 & \multirow[t]{5}{*}{25} & 0600 & 4.63 & 1,460 \\
\hline & 1000 & 7.10 & & & & & & & 1200 & 4.62 & 1,460 \\
\hline & 1200 & 7.53 & & \multirow[t]{5}{*}{17} & 0600 & 10.77 & 7,190 & & 1800 & 4.62 & 1,460 \\
\hline & 1400 & 8.58 & & & 1200 & 10.69 & 7,040 & & 2400 & 4.59 & 1,440 \\
\hline & 1600 & 8.21 & & & 1800 & 10.62 & 6,920 & & & & \\
\hline & 1800 & 8.16 & & & 2400 & 10.46 & 6,630 & \multirow[t]{3}{*}{26} & 1200 & 4.41 & 1,360 \\
\hline & 2000 & 8.23 & & & & & & & 2400 & 4.24 & 1,300 \\
\hline & 2200 & 8.37 & & \multirow[t]{3}{*}{18} & 0600 & 10.28 & 6,320 & & & & \\
\hline & 2400 & 8.50 & 3,930 & & 1200 & 10.04 & 5,910 & \multirow[t]{4}{*}{27} & 1200 & 4.07 & 1,230 \\
\hline & & & & & 1800 & 9.80 & 5,530 & & 2400 & 3.92 & 1,170 \\
\hline \multirow[t]{2}{*}{15} & 0200 & 8.64 & 4,070 & & \multirow[t]{2}{*}{2400} & \multirow[t]{2}{*}{9.50} & \multirow[t]{2}{*}{5,090} & & & & \\
\hline & 0400 & 8.72 & 4,150 & & & & & & & & \\
\hline
\end{tabular}

\section{MISSISSIPPI RIVER MAIN STEM}

(18) 5-2755. Mississippi River at Elk River, Minn.

(Gaging station, discontinued 1956)

Location.-Lat $45^{\circ} 18^{\prime}$, long $93^{\circ} 34^{\prime}$, in $\mathrm{SE} \frac{1}{4}^{\prime}$ sec.34, T.33 N., R.26 W., fourth principaI meridian, on left banti in town of Elk River and at mile 884.6 above Ohio River.

Drainage area.-14,500 sq $\mathrm{mi}$, approximately.

Gage-height record.-Floodmark. Datum of gage is $847.92 \mathrm{ft}$ above mean sea lerel, adjustment of $1 \overline{912}$.

Discharge record.-Peak discharge computed on basis of records for Mississippi River near Anoka, Rum River near St. Francis, and Elk River near Big Lake and compared with discharge obtained from station record during the three years of high flow (1950-52) when the Mississippi River at Elk River gage was in operation.

Maxima.-April-May 1965: Discharge, 62,000 cfs Apr. 16 (gage height, $17.20 \mathrm{ft}$, from floodmark).

1915 to March 1965: Discharge, 49,200 cfs Apr. 12, 1952 (gage height, $14.49 \mathrm{ft}$ ). 


\section{CROW RIVER BASIN}

(19) 5-2760. North Fork Crow River near Regal, Minn.

(Gaging station, discontinued 1954)

Location.-Lat $45^{\circ} 22^{\prime} 55^{\prime \prime}$, long $94^{\circ} 47^{\prime} 40^{\prime \prime}$, in $\mathrm{S} \frac{1}{2}$ sec.11, T.122 N., R.33 W., on right bank $12 \mathrm{ft}$ upstream from highway bridge and 3 miles southeast of Regal.

Drainage area. -215 sq $\mathrm{mi}$.

Gage-height record.-Floodmark. Datum of gage is $1,195.92 \mathrm{ft}$ above mean sea level, datum of 1929 .

Discharge record.-Stage-discharge relation defined by two current-meter measurements below $900 \mathrm{cfs}$ and extended above on the basis of logarithmic plotting.

Maxima.-April-May 1965: Discharge, 2,630 cfs Apr. 9 (gage height, 6. 8 ft, from floodmark).

1943 to March 1965: Discharge, 2,120 cfs Apr. 11, 1952 (gage height, $6.11 \mathrm{ft}$ ); gage height, $6.73 \mathrm{ft}$ Apr. 6, 1951 (backwater from ice).

Remarks.-At high stages a portion of flow bypasses gage from a point about 1 mile upstream, and returns to the main channel at a point about two-thirds of a mile below gage. This flow is included in the station record.

(20) 5-2780. Middle Fork Crow River near Spicer, Minn

Location.—Lat $45^{\circ} 15^{\prime} 45^{\prime \prime}$, long $94^{\circ} 48^{\prime} 10^{\prime \prime}$, in $\mathrm{NE}_{\frac{1}{4}}$ sec. $27, \mathrm{~T} .121$ N., R.33 W., on right bank $75 \mathrm{ft}$ upstream from highway bridge, $1 \frac{1}{2}$ miles downstream from Lake Calhoun, 3 miles downstream from Green Lake, and 6.8 miles northeast of Spicor.

Drainage area. $-179 \mathrm{sq} \mathrm{mi}$.

Gage-height record.-Water-stage recorder graph. Datum of gage is $1,147.93 \mathrm{ft}$ above mean sea level, datum of 1929 (Kandiyohi County Highway Department bench mark).

Discharge record.-Stage-discharge relation defined by current-meter measurements. Backwater from ice Apr. 1-17.

Maxima.-Given in the following table.

April-May 1965:

April 9, 1630 hours

1949 to March 1965

June 29, $1953 \ldots \ldots$

June 25,1957

Discharge Gage height (cfs) (feet)

Remarks.-Flow affected by natural storage and some regulation from lekes above station.

\footnotetext{
${ }^{\mathrm{a} B a c k w a t e r}$ from ice.
} 
Mean discharge, in cubic feet per second, 1965, of Middle Fork Crow' River near Spicer, Minn.

\begin{tabular}{|c|c|c|c|c|c|c|c|c|}
\hline Day & April & May & Day & April & May & Day & April & May \\
\hline $\begin{array}{l}1 \\
2 \ldots \\
3 \ldots \\
4 \\
5 \ldots \\
6 \ldots \\
7 \ldots \\
8 \ldots \\
9 \\
10 \ldots\end{array}$ & $\begin{array}{r}1.0 \\
1.0 \\
1.0 \\
1.2 \\
1.5 \\
2.0 \\
3.0 \\
6.0 \\
55 \\
230\end{array}$ & $\begin{array}{l}270 \\
252 \\
224 \\
197 \\
198 \\
201 \\
206 \\
210 \\
218 \\
208\end{array}$ & $\mid \begin{array}{l}11 \ldots \\
12 \ldots \\
13 \\
14 \\
15 \\
16 \\
17 \\
18 \ldots \\
19 \\
20 \ldots\end{array}$ & $\begin{array}{l}260 \\
240 \\
220 \\
200 \\
190 \\
200 \\
203 \\
194 \\
175 \\
165\end{array}$ & $\begin{array}{l}207 \\
211 \\
217 \\
221 \\
227 \\
225 \\
209 \\
197 \\
227 \\
274\end{array}$ & $\begin{array}{l}21 \ldots \\
22 \\
23 \\
24 \\
25 \\
26 \ldots \\
26 \ldots \\
28 \ldots \\
29 \\
30 \ldots \\
31 \ldots\end{array}$ & $\begin{array}{r}166 \\
172 \\
178 \\
187 \\
198 \\
209 \\
243 \\
270 \\
281 \\
280 \\
----\end{array}$ & $\begin{array}{l}298 \\
302 \\
308 \\
322 \\
341 \\
341 \\
337 \\
324 \\
311 \\
302 \\
297\end{array}$ \\
\hline \multicolumn{7}{|c|}{$\begin{array}{l}\text { Monthly mean discharge, in cubic feet per second } \\
\text { Runoff, in inches }\end{array}$} & $\begin{array}{r}151 \\
0.94\end{array}$ & $\begin{array}{r}254 \\
1.64\end{array}$ \\
\hline
\end{tabular}

Gage herght, in feet, and discharge, in cubic feet per second, at indicated time, 1965

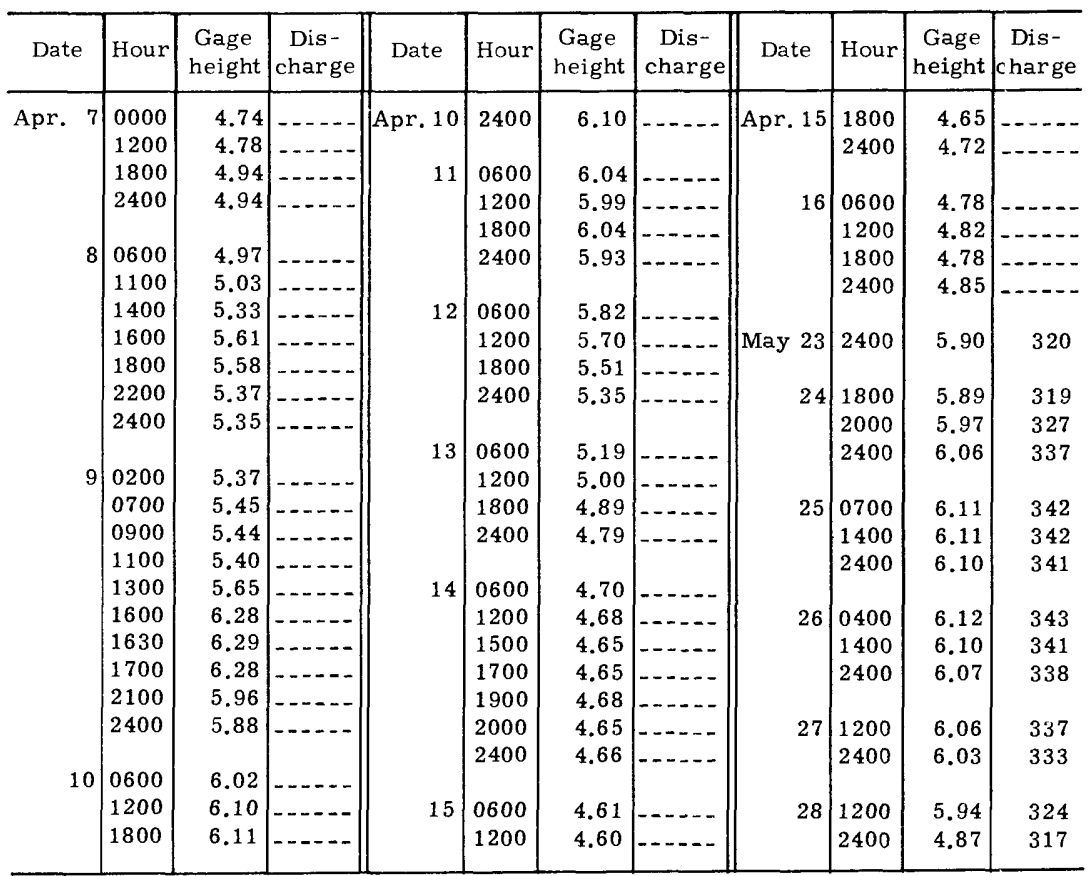


(21) 5-2785. South Fork Crow River at Cosmos, Minn.

(Gaging station, discontinued 1964)

Location.-Lat $44^{\circ} 56^{\prime} 05^{\prime \prime}$, long $94^{\circ} 40^{\prime} 20^{\prime \prime}$, in SW $\frac{1}{4}$ sec.14, T.117 N., R.32 W., on downstream side of bridge on State Highway 7, 1 mile east of Cosmos, $2 \frac{1}{4}$ miles upstream from small tributary, and $3 \frac{1}{4}$ miles west of Corvuso.

Drainage area. $-221 \mathrm{sq} \mathrm{mi}$.

Gage-height record.-Floodmark. Datum of gage is $1,079.09 \mathrm{ft}$ above mean sea level, datum of 1929 (levels by Corps of Engineers).

Discharge record.-Stage-discharge relation defined by current-meter measurements.

Maxima.-April-May 1965: Discharge, 2,130 cfs Apr. 11 (gage height, 13.05 ft, from floodmark).

1945 to March 1965: Discharge, 1,890 cfs June 17, 1957 (gage height, $12.62 \mathrm{ft}$ ).

(22) South Fork Crow River at Hutchinson, Minn.

(Miscellaneous site)

Location.-Lat $44^{\circ} 53^{\prime} 44^{\prime \prime}$, long $94^{\circ} 22^{\prime} 10^{\prime \prime}$, in SE $\frac{1}{4} \mathrm{SW} \frac{1}{4}$ sec.31, T.117 N., R.29 W., at dam just upstream from State Highways 15 and 22 in Hutchinson, and 6.2 miles upstream from McCuen Creek.

Drainage area.- $-462 \mathrm{sq} \mathrm{mi}$.

Discharge record.-Flow-over-dam measurement.

Maximum.-April-May 1965: Discharge, 4,670 cfs Apr. 11 (elevation, 1,044.10 ft above mean sea level, datum of 1929).

(23) 5-2787. Otter Creek near Lester Prairie, Minn.

(Crest-stage station)

Location.-Lat $44^{\circ} 54^{\prime} 23^{\prime \prime}$, long $94^{\circ} 04^{\prime} 24^{\prime \prime}$, in $\mathrm{SE}_{4}^{\frac{1}{4} \mathrm{SE}} \frac{1}{4}$ sec. $28, \mathrm{~T} .117$ N., R. 27 W., on right bank $33 \mathrm{ft}$ upstream from culvert on State Highway 7, 2.1 miles northwest of Lester Prairie, and 4.4 miles upstream from mouth.

Gage-height record.-Watcr-stage recorder graph.

Discharge record.-Stage-discharge relation defined by current-meter measurements.

Maxima.-April-May 1965: Discharge, 525 cfs Apr. 13 (gage height, $9.24 \mathrm{ft}$ ); gage height, $9.97 \mathrm{ft}$ Apr, 6 (backwater from ice).

1961 to March 1965: Discharge, 348 cfs May 22, 1962 (gage height, \&.66 ft); gage height, $8.73 \mathrm{ft}$ Mar. 28, 1962 (backwater from ice). 
(24) 5-2787.5 Otter Creek tributary near Lester Prairie, Minn.

\section{(Crest-stage station)}

Location.-Lat $44^{\circ} 53^{\prime} 34^{\prime \prime}$, long $94^{\circ} 04^{\prime} 24^{\prime \prime}$, in SE $\frac{1}{4} \mathrm{SE} \frac{1}{4}$ sec. 33, T. 117 N., R. 27 W., at culvert on County Highway 63,1.7 miles northwest of Lester Prairie, and 3.3 miles upstream from mouth.

Gage-height record.-Crest stages only.

Discharge record.-Stage-discharge relation defined by current-meter measurements below $49 \mathrm{cfs}$ and by indirect measurement at $79 \mathrm{cfs}$.

Maxima.-April-May 1965: Discharge, $87 \mathrm{cfs}$ Apr. 10 (gage height, $11.14 \mathrm{ft}$ ).

1962 to March 1965: Discharge, $79 \mathrm{cfs}$ May 23, 1962 (gage height, $10.83 \mathrm{ft}$ ).

(25) 5-2788.5 Buffalo Creek tributary near Brownton, Minn.

\section{(Crest-stage station)}

Location.-Lat $44^{\circ} 45^{\prime} 55^{\prime \prime}$, long $94^{\circ} 22^{\prime} 33^{\prime \prime}$, in NE $\frac{1}{4} \mathrm{SE} \frac{1}{4}$ sec.13, T.115 N., R.30 W., at culvert on State Highway 15, 0.6 mile upstream from mouth, and 2.6 miles northwest of Brownton.

Drainage area. $-9.45 \mathrm{sq} \mathrm{mi}$.

Gage-height record.-Crest stages only.

Discharge record.-Stage-discharge relation defined by current-meter measurements.

Maxima.-April-May 1965: Discharge, $124 \mathrm{cfs}$ Apr. 10; (gage height, $17.39 \mathrm{ft}$, backwater from ice); gage height, $17.42 \mathrm{ft} \mathrm{Apr.} 7$ (backwater from ice).

1961 to March 1965: Discharge, $70 \mathrm{cfs}$ Mar. 28, 1962 (gage height $16.16 \mathrm{ft}$, backwater from ice).

(26) 5-2790. South Fork Crow River near Mayer, Minn.

Location.-Lat $44^{\circ} 54^{\prime} 20^{\prime \prime}$, long $93^{\circ} 53^{\prime} 05^{\prime \prime}$, in SW $\frac{1}{4} \mathrm{SW} \frac{1}{4}$ sec. 30, T.117 N., R.25 W., near center of span on downstream side of bridge on State Highway 7, 1.3 miles north of Mayer, 4.3 miles southwest of Watertown, and 16 miles upstream from confluerce with North Fork.

Drainage area.- $-1,170 \mathrm{sq} \mathrm{mi}$, approximately.

Gage-height record.-Graph constructed on basis of once or twice-daily wire-weight gage readings and several engineers readings Apr. 1-30. For the period Apr. 11-18, gage readings obtained from temporary staff gage. Once-daily wire-weight gage readings, May 1-31. Datum of gage is $926.00 \mathrm{ft}$ above mean sea level (levels by Hennepin County Park Board Survey).

Discharge record.-Stage-discharge relation defined by current-meter measurements. Backwater from ice, Apr, 1-9.

Maxima.-April-May 1965: Discharge, 16,100 cfs 1530 hrs Apr. 13 (gage height, 19.23 ft, from floodmark).

1934 to March 1965: Discharge, 11,000 cfs Apr. 10, 1952 (gage height, $15.70 \mathrm{f}$ ). 
Mean discharge, in cubic feet per second, 1965, of South Fork Crow River near Mayer, Minn.

\begin{tabular}{|c|c|c|c|c|c|c|c|c|}
\hline Day & April & May & Day & April & May & Day & April & May \\
\hline $\begin{array}{l}1 \ldots \ldots \\
2 \ldots \ldots \\
3 \ldots \ldots \\
4 \ldots \ldots \\
5 \ldots \ldots \\
6 \ldots \ldots \\
7 \ldots \ldots \\
8 \ldots \ldots \\
9 \ldots \ldots \\
10 \ldots \ldots\end{array}$ & $\begin{array}{r}2.0 \\
2.4 \\
4.0 \\
9.5 \\
34 \\
68 \\
250 \\
1,160 \\
2,060 \\
5,910\end{array}$ & $\begin{array}{l}2,360 \\
2,120 \\
1,950 \\
1,760 \\
1,640 \\
1,510 \\
1,550 \\
1,670 \\
1,820 \\
1,950\end{array}$ & $\mid \begin{array}{l}11 \ldots \\
12 \ldots \ldots \\
13 \ldots \ldots \\
14 \ldots \ldots \\
15 \ldots \ldots \\
16 \ldots \ldots \\
17 \ldots \ldots \\
18 \ldots \ldots \\
19 \ldots \ldots \\
20 \ldots\end{array}$ & $\begin{array}{r}10,800 \\
14,500 \\
15,900 \\
15,200 \\
13,600 \\
11,400 \\
9,120 \\
7,390 \\
6,330 \\
5,510\end{array}$ & $\begin{array}{l}1,970 \\
1,760 \\
1,730 \\
1,600 \\
1,430 \\
1,310 \\
1,280 \\
1,190 \\
1,160 \\
1,160\end{array}$ & $\mid \begin{array}{l}21 \ldots \ldots \\
22 \ldots \ldots \\
23 \ldots \ldots \\
24 \ldots \ldots \\
25 \ldots \ldots \\
26 \ldots \ldots \\
26 \ldots \\
28 \ldots \\
29 \ldots \\
30 \ldots \\
31 \ldots\end{array}$ & $\begin{array}{l}5,160 \\
4,710 \\
4,060 \\
3,630 \\
3,330 \\
3,170 \\
2,990 \\
2,840 \\
2,710 \\
2,540 \\
\ldots \ldots\end{array}$ & $\begin{array}{l}1,160 \\
1,100 \\
1,060 \\
1,040 \\
1,070 \\
1,230 \\
1,320 \\
1,430 \\
1,630 \\
1,740 \\
1,700\end{array}$ \\
\hline \multicolumn{7}{|c|}{$\begin{array}{l}\text { Monthly mean discharge, in cubic feet per second } \\
\text { Runoff, in inches }\end{array}$} & $\begin{array}{r}5,146 \\
4.91\end{array}$ & $\begin{array}{r}1,529 \\
1.51\end{array}$ \\
\hline
\end{tabular}

Gage heıght, in feet, and discharge, in cubic feet per second, at indicated tıme, 1965

\begin{tabular}{|c|c|c|c|c|c|c|c|c|c|c|c|}
\hline Date & Hour & $\begin{array}{c}\text { Gage } \\
\text { height }\end{array}$ & $\begin{array}{c}\text { Dis } \\
\text { charge }\end{array}$ & Date & Hour & $\begin{array}{c}\text { Gage } \\
\text { height }\end{array}$ & $\begin{array}{c}\text { Dis- } \\
\text { charge }\end{array}$ & Date & Hour & $\begin{array}{c}\text { Gage } \\
\text { height }\end{array}$ & $\begin{array}{l}\text { Dis- } \\
\text { charge }\end{array}$ \\
\hline \multirow[t]{25}{*}{ Apr. } & 0000 & 3.04 & $---\infty$ & Apr. 9 & 1500 & 10.42 & $\ldots$ & Apr. 14 & 0600 & 19.01 & 15,600 \\
\hline & 0600 & 3.05 & $\ldots$ & & 1800 & 10.87 & $\ldots \ldots$ & & 1200 & 18.83 & 15,300 \\
\hline & 1200 & 3.25 & $\ldots$ & & 2100 & 11.25 & $-\cdots--$ & & 1800 & 18.64 & 14,900 \\
\hline & 1800 & 3.82 & $\ldots$ & & 2400 & 11.58 & 4,040 & & 2400 & 18.45 & 14,500 \\
\hline & 2400 & 4.10 & & & & & & & & & \\
\hline & & & & 10 & 0600 & 12.53 & 4,910 & 15 & 0600 & 18.23 & 14,100 \\
\hline & 0600 & 4.42 &.- & & 1200 & 13.42 & 5,800 & & 1200 & 17.99 & 13,600 \\
\hline & 1200 & 5.38 & $\ldots$ & & 1800 & 14.33 & 6,840 & & 1800 & 17.71 & 13,000 \\
\hline & 1800 & 6.24 & $\ldots$ & & 2400 & 15.19 & 8,140 & & 2400 & 17.43 & 12,500 \\
\hline & 2400 & 6.06 & $\ldots$ & & & & & & & & \\
\hline & & & & 11 & 0600 & 16.12 & 9,840 & 16 & 0600 & 17.20 & 12,000 \\
\hline & 0600 & 6.16 & $\ldots$ & & 1200 & 16.67 & 10,900 & & 1200 & 16.93 & 11,500 \\
\hline & 1200 & 6.98 & $\ldots$ & & 1800 & 17.14 & 11,900 & & 1800 & 16.62 & 10,800 \\
\hline & 1800 & 7.42 & $\ldots \ldots$ & & 2400 & 17.78 & 13,200 & & 2400 & 16.31 & 10,200 \\
\hline & 2400 & 7.81 & $-\ldots$ & & & & & & & & \\
\hline & & & & 12 & 0600 & 18.18 & 14,000 & 17 & 0600 & 16.00 & 9,600 \\
\hline & 0600 & 8.19 & $\ldots$ & & 1200 & 18.50 & 14,600 & & 1200 & 15.72 & 9,180 \\
\hline & 1200 & 8.54 & $\ldots$ & & 1800 & 18.74 & 15,100 & & 1800 & 15.46 & 8,630 \\
\hline & 1800 & 8.98 & $\ldots$ & & 2400 & 19.00 & 15,600 & & 2400 & 15.18 & 8,120 \\
\hline & 2400 & 9.67 & $\ldots$ & & & & & & & & \\
\hline & & & & 13 & 0600 & 19.06 & 15,900 & 18 & 0600 & 14.95 & 7,720 \\
\hline & 0300 & 9.86 & -- & & 1200 & 19.21 & 16,000 & & 1200 & 14.72 & 7,400 \\
\hline & 0600 & 10.03 & $\ldots$ & & 1530 & 19.23 & 16,100 & & 1800 & 14.48 & 7,020 \\
\hline & 0900 & 10.14 & $\ldots$ & & 1800 & 19.21 & 16,000 & & 2400 & 14.25 & 6,740 \\
\hline & 1200 & 10.15 & $-\cdots---$ & & 2400 & 19.13 & 15,900 & & & & \\
\hline
\end{tabular}


(27) 5-2790.3 South Fork Crow River tributary near Mayer, Minn.

(Crest-stage station)

Location.-Lat $44^{\circ} 54^{\prime} 21^{\prime \prime}$, long $93^{\circ} 53^{\prime} 51^{\prime \prime}$, in NW $\frac{1}{4} \mathrm{NE} \frac{1}{4}$ sec. 36, T.117 N., R.26 W., at culvert on State Highway 7, 0.7 mile above mouth, and 1.4 miles north of Mayer.

Gage-height record.-Crest stages only.

Discharge record.-Stage discharge relation defined by current-meter measurements below $22 \mathrm{cfs}$ and by flow-through-culvert computation at $243 \mathrm{cfs}$.

Maxima.-April--May 1965: Discharge, 306 cfs Apr. 8 (gage height, $9.06 \mathrm{ft}$, backwater from ice).

1962 to March 1965: Gage height, $6.56 \mathrm{ft}$ Mar. 28, 1962, backwater from ice (discharge not determined).

(28) 5-2800. Crow River at Rockford, Minn.

Location.-Lat $45^{\circ} 05^{\prime} 15^{\prime \prime}$, long $93^{\circ} 44^{\prime} 00^{\prime \prime}$, in sec.29, T.119 N., R. 24 W., on right bank at Rockford, $150 \mathrm{ft}$ downstream from bridge on State Highway 55 and 1 mile downstream from confluence of North and South Forks.

Drainage area.-2,520 sq $\mathrm{mi}$, approximately.

Gage-height record.-Water-stage recorder graph except Apr. 13-20 when graph was constructed on the basis of readings made on temporary staff gage. Datum of gage is $893.65 \mathrm{ft}$ above mean sea level, datum of 1912 .

Discharge record.-Stage-discharge relation defined by current-meter measurements. Backwater from ice Apr. 1-11.

Maxima.-April-May 1965: Discharge, 22,400 cfs 0100 hours Apr. 16 (gage heiqht, $19.27 \mathrm{ft}$, from floodmark).

1909-17, 1929 to March 1965: Discharge, 13,900 cfs Apr. 13, 1952 (gage height, $16.24 \mathrm{ft})$.

Mean discharge, in cubic feet per second, 1965

\begin{tabular}{|c|c|c|c|c|c|c|c|c|}
\hline Day & April & May & Day & April & May & Day & April & May \\
\hline $\begin{array}{l}1 \\
2 \\
3 \\
4 \\
5\end{array}$ & $\begin{array}{r}30 \\
30 \\
30 \\
31 \\
32 \\
50 \\
160 \\
450 \\
1,400 \\
3,100\end{array}$ & $\begin{array}{l}6,200 \\
5,700 \\
5,240 \\
4,810 \\
4,490 \\
4,230 \\
4,150 \\
4,280 \\
4,540 \\
4,600\end{array}$ & $\mid \begin{array}{l}11 \ldots \ldots \\
12 \ldots \ldots \\
13 \ldots \ldots \\
14 \ldots \ldots \\
15 \ldots \ldots \\
16 \ldots \ldots \\
17 \ldots \ldots \\
18 \ldots \ldots \\
19 \ldots \ldots \\
20 \ldots \ldots\end{array}$ & $\begin{array}{r}6,800 \\
11,900 \\
15,700 \\
19,500 \\
21,900 \\
22,100 \\
20,700 \\
18,900 \\
17,200 \\
15,300\end{array}$ & $\begin{array}{l}4,580 \\
4,450 \\
4,230 \\
3,990 \\
3,740 \\
3,510 \\
3,300 \\
3,200 \\
3,060 \\
2,990\end{array}$ & $\mid \begin{array}{l}21 \\
22 \ldots \ldots \\
23 \ldots \ldots \\
24 \ldots \ldots \\
25 \ldots \ldots \\
26 \ldots \ldots \\
27 \ldots \ldots \\
28 \ldots \ldots \\
29 \ldots \ldots \\
30 \ldots \ldots \\
31 \ldots \ldots\end{array}$ & $\begin{array}{r}13,700 \\
12,400 \\
11,300 \\
10,200 \\
9,370 \\
8,750 \\
8,190 \\
7,700 \\
7,180 \\
6,680 \\
\\
\end{array}$ & $\begin{array}{l}3,000 \\
2,930 \\
2,900 \\
2,870 \\
2,860 \\
2,890 \\
2,970 \\
3,060 \\
3,170 \\
3,300 \\
3,410\end{array}$ \\
\hline \multicolumn{7}{|c|}{$\begin{array}{l}\text { Monthly mean discharge, in cubic feet per second } \\
\text { Runoff, in inches }\end{array}$} & $\begin{array}{r}9,026 \\
4.00\end{array}$ & $\begin{array}{r}3,827 \\
1.75\end{array}$ \\
\hline
\end{tabular}


Gage height, in feet, and discharge, in cubic feet per second, at indicated time, 1965, of Crow River at Rockford, Minn.

\begin{tabular}{|c|c|c|c|c|c|c|c|c|c|c|c|}
\hline Date & Hour & $\begin{array}{c}\text { Gage } \\
\text { height }\end{array}$ & $\begin{array}{c}\text { Dis- } \\
\text { charge }\end{array}$ & Date & Hour & $\begin{array}{c}\text { Gage } \\
\text { height }\end{array}$ & $\begin{array}{c}\text { Dis- } \\
\text { charge }\end{array}$ & Date & Hour & $\begin{array}{c}\text { Gage } \\
\text { height }\end{array}$ & $\begin{array}{l}\text { Dis- } \\
\text { charge }\end{array}$ \\
\hline \multirow[t]{7}{*}{ Apr. 7} & 0000 & 2.65 & $\ldots-\cdots$ & Apr. 11 & 0600 & 11.26 & $-\cdots--$ & Apr. 17 & 1200 & 18.81 & 20,800 \\
\hline & 0200 & 2.66 & $\ldots$ & & 1200 & 12.15 & ----- & & 2400 & 18.48 & 19,700 \\
\hline & 0600 & 2.85 & & & 1800 & 13.11 & 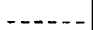 & & & & \\
\hline & 1200 & 3.36 & & & 2200 & 13.87 & ----- & 18 & 1200 & 18.19 & 18,900 \\
\hline & 1800 & 4.21 & & & 2400 & 13.43 & 10,100 & & 2400 & 17.85 & 18,000 \\
\hline & 2400 & 4.59 & & & & & & & & & \\
\hline & & & & 12 & 0600 & 14.28 & 11,200 & 19 & 1200 & 17.48 & 17,200 \\
\hline \multirow[t]{5}{*}{8} & 0600 & 4.75 & & & 1200 & 14.74 & 11,900 & & 2400 & 17.03 & 16,200 \\
\hline & 1200 & 4.87 & & & 1800 & 15.15 & 12,500 & & & & \\
\hline & 1800 & 5.19 & & & 2400 & 15.74 & 13,600 & 20 & 1200 & 16.60 & 15,300 \\
\hline & 2400 & 5.41 & & & & & & & 2400 & 16.20 & 14,500 \\
\hline & & & & 13 & 1200 & 16.83 & 15,800 & & & & \\
\hline \multirow[t]{5}{*}{9} & 0600 & 5.45 & & & 2400 & 17.69 & 17,700 & 21 & 1200 & 15.80 & 13,700 \\
\hline & 1200 & 5.69 & & & & & & & 2400 & 15.41 & 13,000 \\
\hline & 1800 & 6.65 & & 14 & 1200 & 18.41 & 19,500 & & & & \\
\hline & 2400 & 7.11 & & & 2400 & 18.91 & 21,100 & 22 & 1200 & 15.06 & 12,400 \\
\hline & & & & & & & & & 2400 & 14.75 & 11,900 \\
\hline \multirow[t]{5}{*}{10} & 0600 & 7.42 & & 15 & 1200 & 19.18 & 22,100 & & & & \\
\hline & 1200 & 7.95 & & & 2400 & 19.26 & 22,400 & 23 & 1200 & 14.35 & 11,300 \\
\hline & 1800 & 9.25 & & & & & & & 2400 & 13.94 & 10,700 \\
\hline & 2400 & 10.58 & & 16 & 0100 & 19.27 & 22,400 & & & & \\
\hline & & & & & 1200 & 19.19 & 22,100 & 24 & 1200 & 13.52 & 10,200 \\
\hline 11 & 0200 & 10.86 & --- & & 2400 & 19.05 & 21,600 & & 2400 & 13.13 & 9,720 \\
\hline
\end{tabular}

(29) 5-2803. School Lake Creek tributary near St. Michael, Minn.

(Crest-stage station)

Location.-Lat $45^{\circ} 12^{\prime} 09^{\prime \prime}$, long $93^{\circ} 41^{\prime} 31^{\prime \prime}$, in NW $\frac{1}{4} \mathrm{SE} \frac{1}{4}$ sec. 15, T.120 N., R. 24 W., at culvert on county road, 0.2 mile upstream from mouth, and 1.5 miles southwest of St. Michael.

Drainage area. $-2.04 \mathrm{sq} \mathrm{mi}$.

Gage-height record.-Crest stages only.

Discharge record.-Discharge obtained from indirect measurement.

Maxima.-April-May 1965: Discharge, 434 cfs Apr. 11 (gage height, 12.68 ft, from highwater profile).

1964 to March 1965: Gage height, $7.34 \mathrm{ft}$ May 6, 1964 (discharge not determined). 


\section{RUM RIVER BASIN}

(30) 5-2846. Robinson Brook near Onamia, Minn.

(Crest-stage station)

Location.-Lat $45^{\circ} 58^{\prime} 20^{\prime \prime}$, long $93^{\circ} 39^{\prime} 30^{\prime \prime}$, in NE $\frac{1}{4} \mathrm{SE} \frac{1}{4}$ sec. $11, \mathrm{~T} .40 \mathrm{~N}$., R.27 W., at culvert on U.S. Highway 169, a quarter mile upstream from mouth, and $6-3 / 4$ miles south of Onamia.

Drainage area. $-7.21 \mathrm{sq} \mathrm{mi}$.

Gage-height record.-Crest stages only.

Discharge record.-Stage-discharge relation defıned by current-meter measurements below $80 \mathrm{cfs}$ and by indirect measurement at $232 \mathrm{cfs}$.

Maxima.-April-May 1965: Discharge, 130 cfs Apr. 13 (gage height, 15.85 ft, be ckwater from ice).

1960 to March 1965: Discharge, 232 cfs June 8, 1963 (gage height, $16.75 \mathrm{ft}$ ).

(31) 5-2846.2 Rum River tributary near Onamia, Minn.

(Crest-stage station)

Location.-Lat $45^{\circ} 57^{\prime} 20^{\prime \prime}$, long $93^{\circ} 39^{\prime} 30^{\prime \prime}$, in $\mathrm{E}_{2}^{\frac{1}{2}}$ sec.14, T.40 N., R.27 W., at culvert on U.S. Highway 169 , a quarter mile upstream from mouth, and $7-3 / 4$ miles south of Onamia.

Drainage area. $-1.84 \mathrm{sq} \mathrm{mi}$.

Gage-height record.-Crest stages only.

Discharge record.-Stage-discharge relation defined by current-meter measurements below $24 \mathrm{cfs}$ and by indirect measurements at $80 \mathrm{cfs}$ and $172 \mathrm{cfs}$.

Maxima.-April-May 1965: Discharge, $96 \mathrm{cfs}$ Apr. 13 (gage height, $10.19 \mathrm{ft}$ ).

1960 to March 1965: Discharge, $172 \mathrm{cfs}$ June 8, 1963 (gage height, $13.37 \mathrm{ft}$, from high-water profile).

(32) 5-2847.5 Rum River at Spencer Brook, Minn.

(Gaging station, discontinued 1964)

Location,-Lat $45^{\circ} 31^{\prime} 45^{\prime \prime}$, long $93^{\circ} 26^{\prime} 21^{\prime \prime}$, in NW $\frac{1}{4} N E \frac{1}{4}$ sec. 15 , T.35 N., R. 25 W., near center of right span on downstream side of county highway bridge, $200 \mathrm{ft}$ downstream from Spencer Brook, $\frac{1}{2}$ mile north of Spencer Brook store, and $7 \frac{1}{2}$ miles soutr east of Princeton.

Gage-height record.-Floodmark, Datum of gage is $925.65 \mathrm{ft}$ above mean sea level, adjustment of 1912 (levels by Corps of Engineers).

Maxima.-April-May 1965: Discharge not determined, gage height, $15.36 \mathrm{ft}$ Apr. 14 (backwater from ice).

1960 to March 1965: Discharge, 7,350 cfs May 25, 1962 (gage height, $13.12 \mathrm{ft}$ ).

Remarks.-Occasional regulation by Ogechie (also controls Mille Lacs Lake) and Onamia Lakes.

Cooperation.-Floodmark furnished by Corps of Engineers. 
(33) Rum River at West Point, Minn.

(Miscellaneous site)

Location.-Lat $45^{\circ} 33^{\prime}$, long $93^{\circ} 24^{\prime}$, in $\mathrm{SE}_{\frac{1}{4}} \mathrm{SE} \frac{1}{4}$ sec. 36, T.36 N., R. 25 W., at bridge on State Highway 56 at West Point and 8 miles west of Cambridge.

Gage-height record.-Occasional chain gage readings during flood. Daturn of gage is $900 . \overline{34} \mathrm{ft}$ above mean sea level, adjustment of 1912 .

Discharge record.- Stage-discharge relation defined by current-meter measurements.

Maxima.-April-May 1965: Discharge, 10,800 cfs Apr. 16 (gage height, $29.38 \mathrm{ft}$, from floodmark).

1958 to March 1965: Discharge, 6,100 cfs May 26, 1962 (gage height, 27.90 ft).

Cooperation.-Gage height readings and previous maxima furnished by Corps of Engineers.

(34) 5-2849.2 Stanchfield Creek tributary near Day, Minn.

(Crest-stage station)

Location,-Lat $45^{\circ} 41^{\prime} 29^{\prime \prime}$, long $93^{\circ} 23^{\prime} 45^{\prime \prime}$, in NW $\frac{1}{4} \mathrm{SE} \frac{1}{4} \mathrm{sec} .13, \mathrm{~T} .37$ N., R.25 W., at culvert on County Highway 60, 0.5 mile upstream from mouth, and 1.5 miles southwest of Day.

Drainage area.-1.26 sq $\mathrm{mi}$.

Gage-height record.-Crest stages only.

Discharge record.- Stage-discharge relation defined by flow-through-culvert computations at $26 \mathrm{cfs}$ and $55 \mathrm{cfs}$.

Maxima.-April-May 1965: Discharge, 55 cfs Apr. 12 (gage height, 7.01 f1.); gage height, $7.39 \mathrm{ft}$ Apr. 10 (backwater from ice).

1961 to March 1965: Discharge, $70 \mathrm{cfs}$ May 23, 1962 (gage height, 8.52 ft).

(35) Rum River at Isanti, Minn.

(Miscellaneous site)

Location.-Lat $45^{\circ} 29^{\prime}$, long $93^{\circ} 16^{\prime}$, in W $\frac{1}{2}$ sec.30, T.35 N., R.23 W., at bridge on County Road 5, 0.8 mile west of Isanti.

Gage-height record.-Occasional chain gage readings during flood. Datum of gage is $891.53 \mathrm{ft}$ above mean sea level, adjustment of 1912 .

Discharge record.--Stage-discharge relation defined by current-meter measurements.

Maxima.-April-May 1965: Discharge, 9,400 cfs Apr. 19 (gage height, $16.63 \mathrm{ft}$, from floodmark).

1958 to March 1965: Discharge, 5,200 cfs May 29, 1962 (gage height, $12.30 \mathrm{ft}$ ).

Cooperation.-Gage height readings and previous maxima furnished by Corps of Engineers. 
(36) 5-2860. Rum River near St. Francis, Minn.

Location.-Lat $45^{\circ} 19^{\prime} 40^{\prime \prime}$, long $93^{\circ} 22^{\prime} 20^{\prime \prime}$, in SE $\frac{1}{4}$ sec. 19 , T.33 N., R. 24 W., on left bank at upstream side of highway bridge, 4 miles south of St. Francis and 15-3/4 miles upstream from mouth.

Drainage area.-1,360 sq $\mathrm{mi}$, approximately.

Gage height record.-Water-stage recorder graph. Datum of gage is $861.12 \mathrm{ft}$ above mean sea level, datum of 1912 .

Discharge record.-Stage-discharge relation defined by current-meter measurements. Backwater from ice Apr. 1-14.

Maxima.-April-May 1965: Discharge, 10,100 cfs 0030 hours Apr.20 (gage height, 11.57ft). 1929 to March 1965: Discharge 9,260 cfs Apr. 13, 1952 (gage height, $11.03 \mathrm{f}^{4}$ ).

Remarks.--Occasional regulation by Ogechie (also controls Mille Lacs Lake) and Onamia Lakes.

Mcan discharge, in cubic feet per second, 1965

\begin{tabular}{|c|c|c|c|c|c|c|c|c|}
\hline Day & April & May & Day & April & May & Day & April & May \\
\hline 1. & 129 & 2,700 & $11 \ldots$ & 500 & 2,200 & 21 & 9,180 & 1,420 \\
\hline 2 & 132 & 2,490 & $12 \ldots$ & 1,080 & 2,250 & 22 & 8,060 & 1,320 \\
\hline 3 & 136 & 2,260 & $13 \ldots$ & 1,600 & 2,250 & 23 & 7,290 & 1,220 \\
\hline 4 & 144 & 2,000 & 14. & 2,200 & 2,230 & 24 & 6,320 & 1,160 \\
\hline 5. & 160 & 1,760 & 15. & 2,740 & 2,150 & 25. & 5,480 & 1,190 \\
\hline $6 \ldots$ & 182 & 1,550 & 16. & 4,040 & 2,010 & 26. & 4,720 & 1,230 \\
\hline 7. & 202 & 1,520 & 17 & 6,710 & 1,800 & 27. & 4,130 & 1,310 \\
\hline 8 & 227 & 1,630 & $18 \ldots$ & 8,890 & 1,680 & $28 \ldots$ & 3,640 & 1,490 \\
\hline $9_{\ldots}$ & 268 & 1,830 & $19 \ldots$ & 9,900 & 1,570 & $29 \ldots$ & 3,250 & 1,710 \\
\hline $10 \ldots$ & 310 & 2,040 & $20 \ldots$ & 9,880 & 1,480 & $30 \ldots$ & 2,940 & 1,940 \\
\hline & & & & & & $31 \ldots$ & $\ldots$ & 2,130 \\
\hline \multirow{2}{*}{\multicolumn{8}{|c|}{$\begin{array}{l}\text { Monthly mean discharge, in cubic feet per second } \\
\text { Runoff, in inches }\end{array}$}} & 1,791 \\
\hline & & & & & & & & 1.52 \\
\hline
\end{tabular}

Gage height, in feet, and discharge, in cubic feet per second, at indicated time, 1965

\begin{tabular}{|c|c|c|c|c|c|c|c|c|c|c|c|}
\hline Date & Hour & $\begin{array}{l}\text { Gage } \\
\text { height }\end{array}$ & $\begin{array}{c}\text { Dis- } \\
\text { charge }\end{array}$ & Date & Hour & $\begin{array}{l}\text { Gage } \\
\text { height }\end{array}$ & $\begin{array}{c}\text { Dis- } \\
\text { charge }\end{array}$ & Date & Hour & $\begin{array}{c}\text { Gage } \\
\text { height }\end{array}$ & $\begin{array}{l}\text { Dis- } \\
\text { charge }\end{array}$ \\
\hline \multirow[t]{6}{*}{ pr. 12} & 0000 & 4.16 & & \multirow[t]{4}{*}{ Apr. 16} & 1200 & 6.84 & 3,890 & \multirow[t]{3}{*}{ Apr. 20} & 1800 & 11.36 & 9,760 \\
\hline & 0600 & 4.31 & .. & & 1800 & 7.39 & 4,550 & & 2400 & 11.27 & 9,720 \\
\hline & 1200 & 4.33 & & & \multirow[t]{2}{*}{2400} & 7.98 & 5,300 & & & & \\
\hline & 1800 & 4.46 & & & & & & \multirow[t]{5}{*}{21} & 0600 & 11.12 & 9,430 \\
\hline & 2400 & 4.61 & & 17 & 0600 & 8.56 & 6,070 & & 1200 & 10.96 & 9,200 \\
\hline & & & & & 1200 & 9.08 & 6,800 & & 1800 & 10.77 & 8,940 \\
\hline \multirow[t]{5}{*}{13} & 0600 & 4.94 & & & 1800 & 9.47 & 7,350 & & 2400 & 10.60 & 8,700 \\
\hline & 1200 & 4.80 & & & 2400 & 9.88 & 7,920 & & & & \\
\hline & 1800 & 5.37 & & & & & & \multirow[t]{5}{*}{22} & 0600 & 10.43 & 8,460 \\
\hline & 2400 & 5.40 & & 18 & 0600 & 10.45 & 8,490 & & 1200 & 10.28 & 8,250 \\
\hline & & & & & 1200 & 10.79 & 8,970 & & 1800 & 9.44 & 7,310 \\
\hline \multirow[t]{5}{*}{14} & 0600 & 5.35 & & & 1800 & 11.04 & 9,320 & & 2400 & 9.77 & 7,770 \\
\hline & 1200 & 5.43 & & & 2400 & 11.28 & 9,650 & & & & \\
\hline & 1800 & 5.50 & & & & & & \multirow[t]{3}{*}{23} & 1200 & 9.42 & 7,280 \\
\hline & 2400 & 5.59 & 2,490 & 19 & 0600 & 11.42 & 9,850 & & 2400 & 9.09 & 6,820 \\
\hline & & & & & 1200 & 11.49 & 9,950 & & & & \\
\hline \multirow[t]{5}{*}{15} & 0600 & 5.67 & 2,580 & & 1800 & 11.52 & 9,990 & \multirow[t]{3}{*}{24} & 1200 & 8.72 & 6,300 \\
\hline & 1200 & 5.78 & 2,700 & & 2400 & 11.56 & 10,000 & & 2400 & 8.39 & 5,840 \\
\hline & 1800 & 5.94 & 2,870 & & & & & & & & \\
\hline & 2400 & 6.20 & 3,160 & 20 & 0030 & 11.57 & 10,100 & \multirow[t]{3}{*}{25} & 1200 & 8.12 & 5,490 \\
\hline & & & & & 0600 & 11.52 & 9,990 & & 2400 & 7.83 & 5,110 \\
\hline 16 & 0600 & 6.48 & 3,470 & & 1200 & 11.45 & 9,890 & & & & \\
\hline
\end{tabular}




\section{MISSISSIPPI RIVER MAIN STEM}

(37) 5-2885. Mississippi River near Anoka, Minn.

Location.-Lat $45^{\circ} 07^{\prime} 36^{\prime \prime}$, long $93^{\circ} 17^{\prime} 48^{\prime \prime}$, in SW $\frac{1}{4}$ sec.12, T.119 N., R.21 V'., on right bank half a mile downstream from Coon Creek $1 \frac{1}{2}$ miles downstream from hydroelectric plant of Northern States Power Co. at Coon Rapids, $6 \frac{1}{2}$ miles downstream from Anoka, and at mile 864.8 upstream from Ohio River.

Drainage area.- $-19,100 \mathrm{sq} \mathrm{mi}$, approximately.

Gage-height record.-Digital recorder tape punched at 15 minute intervals except 0100 hours Apr. 16 to 1125 hours Apr. 19 when the graph was constructed on the basis of once or twice daily readings on the temporary staff gage. No digital re:ord 2000 hours May 17 to 1600 hours May 29. Datum of gage is $805.02 \mathrm{ft}$ above mean sea level, adjustment of 1912 .

Discharge record.-Stage-discharge relation defined by current-meter measurements. Backwater from ice Apr. 1-12. Discharge for the period May 17-29 was obtained from records for Coon Rapids Hydroelectric Plant which were adjusted for estimated flow from Coon Creek. Mean daily discharge computed from 96 punch-tape readings per day, Apr. 19 to May 16 and May 30,31 .

Maxima.-April-May 1965: Discharge, 91,000 cfs 0500 hours Apr. 17 (gage height $19.53 \mathrm{ft}$, from floodmark).

1931 to March 1965: Discharge, 75,900 cfs Apr. 14, 1952 (gage height, $17.51 \mathrm{ft}$ ).

Remarks.-Flow slightly regulated by six reservoirs on headwaters; total usable capacity, 1,640,600 acre-ft. Diurnal regulation caused by powerplant akove station.

Cooperation.-Coon Rapids Hydroelectric Plant records furnished by Northern States Power Company.

Mean discharge, in cubic feet per second, 1965

\begin{tabular}{|c|c|c|c|c|c|c|c|c|}
\hline Day & April & May & Day & April & May & Day & April & May \\
\hline 1 & 3,820 & 35,800 & $11_{-}$ & 15,900 & 28,400 & 21. & 70,500 & 23,500 \\
\hline 2 & 3,970 & 33,600 & 12 & 38,200 & 28,000 & $22 \ldots$ & 64,900 & 23,900 \\
\hline & 4,000 & 31,700 & $13 \ldots$ & 51,000 & 28,000 & $23 \ldots$ & 59,200 & 23,500 \\
\hline & 4,500 & 30,200 & $14_{-}$ & 68,600 & 26,600 & $24 \ldots \ldots$ & 54,300 & 24,300 \\
\hline & 4,300 & 29,600 & 15 & 79,800 & 26,300 & $25 \ldots$ & 51,000 & 26,000 \\
\hline & 4,350 & 27,700 & 16. & 89,200 & 25,600 & 26 & 48,100 & 27,900 \\
\hline & 5,080 & 27,200 & 17 & 90,300 & 24,200 & 27. & 44,900 & 28,600 \\
\hline & 6,020 & 27,200 & 18. & 86,400 & 24,500 & $28 \ldots$ & 42,400 & 28,700 \\
\hline $9 \ldots$ & 7,590 & 28,500 & 19. & 81,600 & 23,800 & 29. & 40,400 & 28,700 \\
\hline 10 & 10,300 & 28,700 & & 76,100 & 23,000 & $30 \ldots$ & 38,300 & 28,700 \\
\hline \multirow{2}{*}{\multicolumn{7}{|c|}{$\begin{array}{l}\text { Monthly mean discharge, in cubic feet per second } \\
\text { Runoff, in inches }\end{array}$}} & 41,500 & 27,470 \\
\hline & & & & & & & 2.42 & 1.66 \\
\hline
\end{tabular}


Gage height, in feet, and discharge, in cubic feet per second, at indicated time, 1965, of Mississippi Fiver near Anoka, Minn.

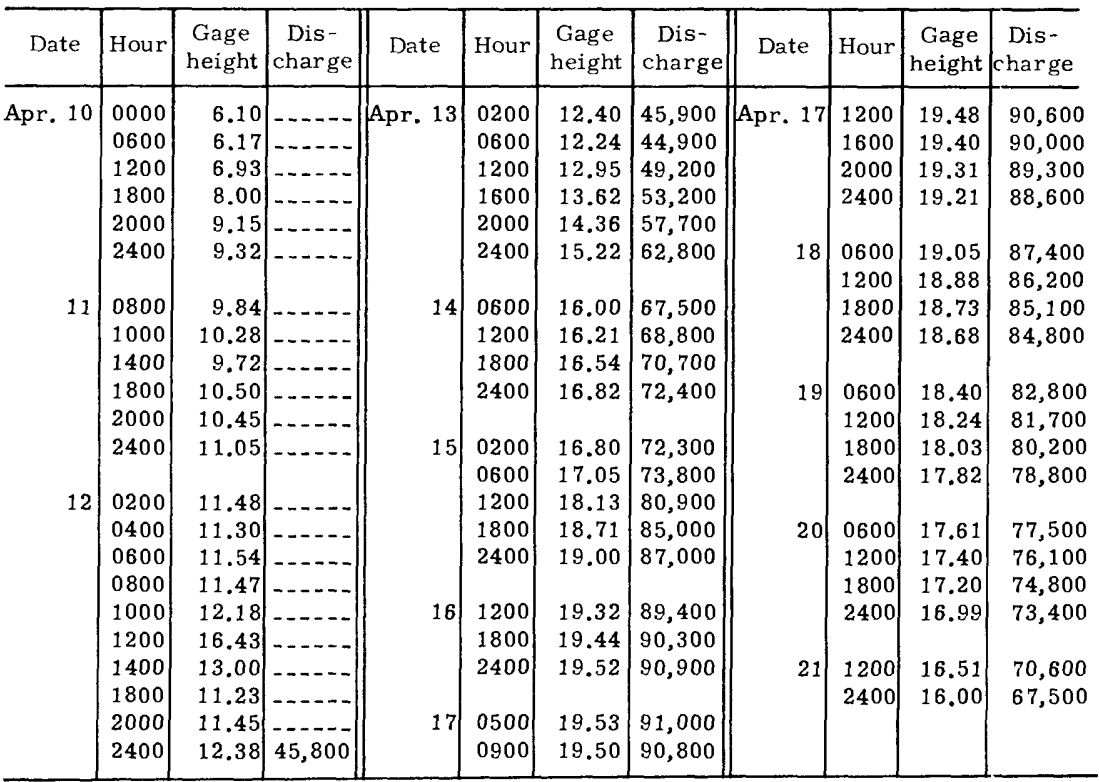

\section{BASSETT CREEK BASIN}

(38) Bassett Creek at County Highway 66, Golden Valley, Minn.

(Miscellaneous site)

Location,-Lat $45^{\circ} 00^{\prime} 05^{\prime \prime}$, long $93^{\circ} 21^{\prime} 16^{\prime \prime}$, in W $\frac{1}{2}$ sec. 28, T.118 N., R.21 W., at bridge on County Highway 66 in Golden Valley, Minn., and $\frac{1}{4}$ mile west of underpass on State Highway 100.

Discharge record.-Discharge obtained on the following days by current-meter measurements:

$\begin{array}{lc}\text { Date } & \text { Discharge, in cfs } \\ \text { Apr. } 3 & 5.92 \\ \text { Apr. } 6 & 67.0 \\ \text { May 10 } 10 & 79.5 \\ \text { June 1 } & 84.8\end{array}$

Maximum.-April-May 1965: Discharge, not determined, gage height, $4.73 \mathrm{ft}$ Aor. 6 (backwater from ice). 
(39) Bassett Creek at Fruen Mill Co., Minneapolis, Minn.

(Miscellaneous site)

Location.-Lat $44^{\circ} 58^{\prime} 45^{\prime \prime}$, long $93^{\circ} 18^{\prime} 52^{\prime \prime}$, in SE $\frac{1}{4}$ sec. 20, T. 29 N., R. 24 W., at Fruen Mill, Minneapolis, Minn., and $700 \mathrm{ft}$ downstream from Glenwood Ave.

Gage-height record.-Peak from graph based on once-daily staff gage readings. Datum of gage is $815.23 \mathrm{ft}$ above mean sea level, adjustment of 1929. (Levels by Corps of Engineers).

Discharge record.- Stage-discharge relation defined by current-meter measurements.

Maxima.-April-May 1965: Discharge, 360 cfs 0100 hours Apr. 11 (gage height, $2.57 \mathrm{ft}$ ). 1953-56, 1963 to March 1965: Discharge, $122 \mathrm{cfs}$ (current-meter measurement) May 1, 1954 (gage height, $1.78 \mathrm{ft}$ ).

Cooperation.-Gage-height record furnished by Corps of Engineers.

\section{MINNEHAHA CREEK BASIN}

(40) 5-2895. Minnehaha Creek at Minnetonka Mills, Minn

(Gaging station, discontinued 1964)

Location.-Lat $44^{\circ} 56^{\prime} 30^{\prime \prime}$, long $93^{\circ} 26^{\prime} 45^{\prime \prime}$, near center of $\mathrm{E} \frac{1}{2}$ sec. $15, \mathrm{~T} .117$ N., R.22 W., on left bank $40 \mathrm{ft}$ upstream from bridge on county highway at Minnetonka Mills, 2.2 miles downstream from outlet of Minnetonka Lake, and 2.9 miles northwest of Hopkins.

Drainage area.-130 sq mi.

Gage-height record.-Observed stage. Daturn of gage is $916.11 \mathrm{ft}$ above mean sea level, datum of 1929 .

Maxima.-April-May 1965: Discharge, 245 cfs (current-meter measurement made near peak) May 31 (gage height, $6.63 \mathrm{ft}$ ).

1953 to March 1965: Discharge, 235 cfs May 23, 1962 (gage height, $6.66 \mathrm{ft}$ ); gage height, $6.97 \mathrm{ft}$ Mar. 23, 1963 (backwater from ice).

Remarks.-Discharge affected by storage in Minnetonka Lake by fixed-crest dam.

(41) Minnehaha Creek at 50th Street, Edina, Minn.

(Miscellaneous site)

Location.-Lat $44^{\circ} 54^{\prime} 45^{\prime \prime}$, long $93^{\circ} 20^{\prime} 32^{\prime \prime}$, in NE $\frac{1}{4} \mathrm{SW} \frac{1}{4}$ sec. 18, T. 28 N., R. 24 W., at dam $150 \mathrm{ft}$ upstream from bridge on 50th Street at Edina.

Discharge record.--Stage-discharge relation defined by current-meter measurements.

Maximum.-April-May 1965: Discharge, $368 \mathrm{cfs}$ May 31, (elevation $885.90 \mathrm{ft}$ above mean sea level, datum of 1929 , from floodmark). 
(42) Minnehaha Creek at Minnehaha Ave., Minneapolis, Minn.

(Miscellaneous site)

Location.-Lat $44^{\circ} 54^{\prime} 56^{\prime \prime}$, long $93^{\circ} 12^{\prime} 44^{\prime \prime}$, in $\mathrm{SE}_{4}^{\frac{1}{4} \mathrm{NE}} \frac{1}{4}$ sec.18, T.28 N., R.23 W., at bridge on Minnehaha Ave. at Minneapolis, 0.1 mile above Minnehaha Falls 0.8 mile upstream from mouth.

Gage-height record.-Floodmark.

Discharge record.--Stage-discharge relation defined by current-meter measurements.

Maximum.-April-May 1965: Discharge, $500 \mathrm{cfs}$ May 31, (elevation, $804.43 \mathrm{ft}$ above mean sea level, datum of 1929).

\section{MINNESOTA RIVER BASIN}

(43) 5-2900. Little Minnesota River near Peever, S. Dak,

Location.-Lat $45^{\circ} 36^{\prime} 05^{\prime \prime}$, long $96^{\circ} 52^{\prime} 18^{\prime \prime}$, in SW $\frac{1}{4}$ sec. 13 , T.125 N., R.50 W., on right bank, 2 miles northwest of town of Browns Valley, Minn., $3 \frac{1}{4}$ miles upstream from proposed Lake Traverse diversion, 5.3 miles northeast of Peever, $7 \frac{1}{4}$ miles downstream from Jorgenson River, and 8 miles upstream from Big Stone Lake.

Drainage area.- $-447 \mathrm{sq} \mathrm{mi.}$

Gage-height record.-Water-stage recorder graph. Altitude of gage is 1,000 $\mathrm{ft}$ (from topographic map).

Discharge record.-Stage-discharge relation defined by current-meter measurements. Backwater from ice Apr. 1-11.

Maxima.-Given in the following table:

$\begin{array}{ccc}\text { April-May 1965 } & \begin{array}{c}\text { Discharge } \\ \text { (cfs) }\end{array} & \begin{array}{c}\text { Gage height } \\ \text { (feet) }\end{array} \\ \text { Apr. 12, 1400 hours } & 920 & 6.27 \\ \text { May 21, 1530 hours } & 2,880 & 9.48 \\ \text { 1939 to March 1965 } & & \\ \text { Apr. 8, 1952 } & 4,730 & \mathrm{a}_{13.16}^{12.16} \\ \text { Mar. 25, 1943 } & -\ldots .-- & \end{array}$

\footnotetext{
${ }^{\mathrm{a} B a c k w a t e r}$ from ice.
}

Mean discharge, in cubic feet per second, 1965

\begin{tabular}{|c|c|c|c|c|c|c|c|c|}
\hline Day & April & May & Day & April & May & Day & April & May \\
\hline $1 \ldots$ & 2.6 & 47 & $11 \ldots$ & 800 & 21 & $21 \ldots$ & 173 & 1,210 \\
\hline $2 \ldots$ & 3.3 & 42 & $12 \ldots$ & 881 & 19 & $22 \ldots$ & 161 & 634 \\
\hline $3 \ldots$. & 16 & 36 & $13 \ldots$ & 689 & 16 & $23 \ldots$ & 145 & 534 \\
\hline $4 \ldots$. & 116 & 32 & $14 \ldots$ & 541 & 16 & $24 \ldots$ & 125 & 819 \\
\hline $5 \ldots$. & 426 & 30 & $15 \ldots$ & 471 & 37 & $25 \ldots$ & 108 & 1,000 \\
\hline $6 \ldots . .$. & 250 & 29 & $16 \ldots$ & 391 & 145 & $26 \ldots$ & 94 & 750 \\
\hline $7 \ldots \ldots$ & 288 & 27 & $17 \ldots$ & 300 & 158 & $27 \ldots$ & 82 & 570 \\
\hline $8 \ldots \ldots$ & 624 & 23 & $18 \ldots$ & 243 & 154 & $28 \ldots$ & 71 & 451 \\
\hline $9 \ldots$ & 494 & 22 & $19 \ldots$ & 210 & 112 & $29 \ldots$ & 64 & 371 \\
\hline $10 \ldots$ & 480 & 20 & $20 \ldots$ & 190 & 93 & $30 \ldots$ & 55 & 326 \\
\hline & & & & & & $31 \ldots$ & 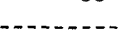 & 272 \\
\hline \multirow{2}{*}{\multicolumn{7}{|c|}{ Monthly mean discharge, in cubic feet per second }} & 283 & 259 \\
\hline & & & \multicolumn{4}{|c|}{ Runoff, in inches } & 0.71 & 0.67 \\
\hline
\end{tabular}


A 172

FLOODS OF 1965 IN THE UNITED STATES

wage height, in feet, and discharge, in cubic feet per second. at indicated time, 1965, of Little Minnesota River near Peever, S. Dak.

\begin{tabular}{|c|c|c|c|c|c|c|c|c|c|c|c|}
\hline Date & Hour & $\begin{array}{l}\text { Gage } \\
\text { height }\end{array}$ & $\begin{array}{c}\text { Dis- } \\
\text { charge }\end{array}$ & Date & Hour & $\begin{array}{c}\text { Gage } \\
\text { height }\end{array}$ & $\begin{array}{c}\text { Dis- } \\
\text { charge }\end{array}$ & Date & Hour & $\begin{array}{c}\text { Gage } \\
\text { height }\end{array}$ & $\begin{array}{l}\text { Dis- } \\
\text { charge }\end{array}$ \\
\hline pr. 1 & 0000 & 2.24 & & Apr. 8 & 1500 & 6.00 & - - - - & Apr. 15 & 1200 & 4.92 & 462 \\
\hline & 1200 & 2.23 & -- & & 1700 & 6.16 & $\ldots$ & & 2400 & 4.89 & 454 \\
\hline & 1800 & 2.58 & $--2--$ & & 2400 & 5.76 & ------ & & & & \\
\hline & 2400 & 2.85 & & & & & & 16 & 1200 & 4.64 & 389 \\
\hline & & & & 9 & 0600 & 5.32 & $\ldots$ & & 2400 & 4.42 & 332 \\
\hline 2 & 0600 & 2.67 & $-\cdots--$ & & 0900 & 5.25 & $\ldots$ & & & & \\
\hline & 1200 & 2.90 & $\ldots$ & & 1200 & 5.23 & - & May 19 & 2400 & 3.39 & 116 \\
\hline & 1800 & 3.23 & & & 1400 & 5.27 & $\ldots$ & & & & \\
\hline & 2200 & 3.43 & & & 1500 & 5.18 & $\ldots \ldots$ & 20 & 1200 & 3.34 & 91 \\
\hline & 2400 & 3.67 & $\ldots$ & & 1800 & 5.29 & $\ldots$ & & 2100 & 3.32 & 87 \\
\hline & & & & & 2400 & 5.13 & & & 2400 & 3.38 & 98 \\
\hline 3 & 0200 & 3.87 & & & & & & & & & \\
\hline & 0600 & 3.98 & & 10 & 0200 & 5.13 & -- & 21 & 0600 & 3.65 & 161 \\
\hline & 1500 & 4.41 & & & 0400 & 5.04 & $\ldots$ & & 0900 & 4.99 & 514 \\
\hline & 2200 & 4.42 & & & 0600 & 5.09 & $\ldots$ & & 1100 & 6.01 & 866 \\
\hline & 2400 & 4.46 & 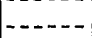 & & 0800 & 4.92 & - & & 1200 & 7.17 & 1,410 \\
\hline & & & & & 1000 & 5.05 & $\ldots-\ldots$ & & 1300 & 8.17 & 1,990 \\
\hline 4 & 0700 & 4.45 & $-\cdots$ & & 1200 & 5.18 & $\ldots-\ldots$ & & 1500 & 9.40 & 2,810 \\
\hline & 1000 & 4.56 & $-\ldots$ & & 1400 & 5.19 & - - - & & 1530 & 9.48 & 2,880 \\
\hline & 1100 & 4.48 & --- & & 1600 & 5.17 & $\ldots$ & & 1800 & 8.75 & 2,390 \\
\hline & 1800 & 5.06 & $-\ldots$ & & 1800 & 5.38 & -...- & & 2100 & 7.46 & 1,600 \\
\hline & 2400 & 5.49 & $\ldots$ & & 2200 & 5.72 & -..... & & 2400 & 6.57 & 1,150 \\
\hline & & & & & 2300 & 5.45 & $\ldots-\cdots$ & & & & \\
\hline 5 & 0800 & 5.53 & $-\ldots$ & & 2400 & 5.96 & $\ldots$ & 22 & 0600 & 5.73 & 773 \\
\hline & 1200 & 5.67 & & & & & & & 1200 & 5.13 & 574 \\
\hline & 2000 & 5.95 & & 11 & 0200 & 5.91 & $--\infty$ & & 1800 & 4.57 & 423 \\
\hline & 2400 & 5.73 & 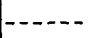 & & 0235 & 6.52 & $-\ldots$ & & 2400 & 4.43 & 386 \\
\hline & & & & & 0400 & 5.95 & $-\cdots-$ & & & & \\
\hline 6 & 1100 & 4.88 & - & & 0600 & 6.16 & $--n-n$ & 23 & 1200 & 4.89 & 509 \\
\hline & 1400 & 4.80 & $-\cdots$ & & 0800 & 5.99 & - & & 2400 & 5.60 & 731 \\
\hline & 2400 & 4.85 & 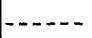 & & 1000 & 6.08 & $\ldots$ & & & & \\
\hline & & & & & 1400 & 6.14 & $\ldots$ & 24 & 1100 & 5.96 & 884 \\
\hline 7 & 0100 & 4.84 & & & 1600 & 6.16 & $-\cdots$ & & 2000 & 5.74 & 789 \\
\hline & 0700 & 4.41 & & & 2000 & 6.15 & $-\ldots$ & & 2400 & 5.85 & 834 \\
\hline & 0800 & 4.41 & $-\ldots$ & & 2400 & 6.12 & 852 & & & & \\
\hline & 0900 & 4.52 & $1-0-0$ & & & & & 25 & 0900 & 6.12 & 960 \\
\hline & 1100 & 4.59 & $-\ldots$ & 12 & 0600 & 6.17 & 874 & & 1300 & 6.44 & 1,100 \\
\hline & 1300 & 4.87 & $-\cdots$ & & 1200 & 6.25 & 910 & & 1600 & 6.55 & 1,160 \\
\hline & 1600 & 5.39 & $\ldots$ & & 1400 & 6.27 & 920 & & 2400 & 6.09 & 951 \\
\hline & 1800 & 5.66 & $--\infty+-$ & & 1800 & 6.21 & 892 & & & & \\
\hline & 1900 & 5.78 & $\ldots$ & & 2400 & 6.07 & 830 & 26 & 0600 & 5.67 & 769 \\
\hline & 2100 & 6.03 & $---n-$ & & & & & & 1200 & 5.55 & 724 \\
\hline & 2300 & 6.07 & $-\cdots$ & 13 & 0600 & 5.84 & 738 & & 1800 & 5.50 & 706 \\
\hline & 2400 & 5.96 & $\ldots$ & & 1200 & 5.64 & 669 & & 2400 & 5.34 & 649 \\
\hline & & & & & 2400 & 5.42 & 601 & & & & \\
\hline 8 & 0300 & 5.64 & ----- & & & & & 27 & 1200 & 5.06 & 565 \\
\hline & 0900 & 5.53 & $-\cdots---$ & 14 & 1200 & 5.17 & 528 & & 2400 & 4.83 & 501 \\
\hline & 1200 & 5.77 & $-\ldots$ & & 2400 & 5.09 & 506 & & & & \\
\hline
\end{tabular}


(44) 5-2910. Whetstone River near Big Stone City, S. Dak.

Location.-Lat $45^{\circ} 17^{\prime} 32^{\prime \prime}$, long $96^{\circ} 29^{\prime} 14^{\prime \prime}$, in $S E \frac{1}{4} N W \frac{1}{4}$ sec.18, T.121 N., R.46 W., on right bank $20 \mathrm{ft}$ downstream from highway bridge, $1 \frac{1}{2}$ miles west of Big Stone City, and $4 \frac{1}{2}$ miles upstream from Big Stone Lake.

Drainage area. $-389 \mathrm{sq} \mathrm{mi}$.

Gage-height record.-Water-stage recorder graph except Apr. 1-9. Graph was constructed on the basis of once or twice-daily wire-weight gage readings, Apr. 2-9. Datum of gage is $996.96 \mathrm{ft}$ above mean sea level, adjustment of 1912 .

Discharge record.--Stage-discharge relation defined by current-meter measurem ents. Discharge estimated Apr. 1 on the basis of two discharge measurements and weather records. Backwater from ice Apr. 2-7.

Maxima.-Given in the following table:

April-May 1965

Apr. 6, 1030 hours

May 24, 2300 hours

1910-12,1931 to March 1965

Apr. 8, 1952

Apr. 11, 1947

$a_{\text {Backwater from ice. }}$

$\begin{array}{cc}\begin{array}{c}\text { Discharge } \\ \text { (cfs) }\end{array} & \begin{array}{c}\text { Gage height } \\ \text { (feet) }\end{array} \\ 1,740 & \mathrm{a}_{8.57} \\ 1,100 & 6.63\end{array}$

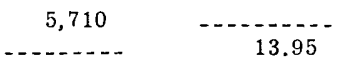

Maximum stage known, about $26 \mathrm{ft}$ in June 1919, from information by local resident.

Mcan discharge, in cubic feet per second, 1965

\begin{tabular}{|c|c|c|c|c|c|c|c|c|}
\hline Day & April & May & Day & April & May & Day & April & Nay \\
\hline $1 \ldots$ & 6 & 41 & $11 \ldots$ & 1,000 & 35 & $21 \ldots$ & 100 & 48 \\
\hline $2 \ldots \ldots$ & 27 & 38 & $12 \ldots$ & 905 & 33 & $22 \ldots$ & 92 & 50 \\
\hline $3 \ldots$ & 130 & 37 & $13 \ldots$ & 498 & 30 & $23 \ldots$ & 88 & 149 \\
\hline $4 \ldots \ldots$ & 427 & 35 & $14 \ldots$ & 354 & 31 & $24_{\ldots} \ldots$ & 78 & 683 \\
\hline $5 \ldots$ & 1,010 & 35 & $15 \ldots$ & 313 & 40 & $25 \ldots$ & 73 & 909 \\
\hline $6 \ldots$ & 1,680 & 38 & $16 \ldots$ & 281 & 177 & $26 \ldots$ & 65 & 608 \\
\hline $7 \ldots$ & 1,630 & 36 & $17 \ldots$ & 187 & 132 & $27 \ldots$ & 61 & 345 \\
\hline $8 \ldots$ & 1,460 & 33 & $18 \ldots$ & 130 & 79 & - & 54 & 174 \\
\hline $9 \ldots$ & 976 & 36 & 19. & 107 & 58 & $29 .$. & 50 & 126 \\
\hline 10. & 705 & 35 & $20 \ldots$ & 103 & 48 & $30 \ldots$ & 46 & 108 \\
\hline & & & & & & & $-\cdots$ & 100 \\
\hline \multirow{2}{*}{\multicolumn{7}{|c|}{$\begin{array}{l}\text { Monthly mean discharge, in cubic feet per second } \\
\text { Runoff, in inches }\end{array}$}} & 421 & 140 \\
\hline & & & & & & & 1.21 & 0.40 \\
\hline
\end{tabular}


Gage height, in feet, and discharge, in cubic feet per second, at indicated time, 1965, of Whetstone River near Big Stone City, S. Dak.

\begin{tabular}{|c|c|c|c|c|c|c|c|c|c|c|c|}
\hline Date & Hour & $\begin{array}{c}\text { Gage } \\
\text { height }\end{array}$ & $\begin{array}{c}\text { Dis- } \\
\text { charge }\end{array}$ & Date & Hour & $\begin{array}{l}\text { Gage } \\
\text { height }\end{array}$ & $\begin{array}{c}\text { Dis- } \\
\text { charge }\end{array}$ & Date & Hour & $\begin{array}{c}\text { Gage } \\
\text { height }\end{array}$ & $\begin{array}{c}\text { Dis- } \\
\text { charge }\end{array}$ \\
\hline \multirow[t]{4}{*}{ Apr. 2} & 0000 & 4.58 & & Apr. 10 & 1200 & 5.72 & 657 & May 23| & 10500 & 2.70 & 54 \\
\hline & 1200 & 4.68 & $\ldots$ & & 1500 & 5.57 & 610 & & 0600 & 3.04 & 82 \\
\hline & 2400 & 5.22 & 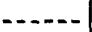 & & 1800 & 5.69 & 647 & & 0900 & 3.14 & 92 \\
\hline & & & & & 2400 & 6.22 & 824 & & 1200 & 3.38 & 124 \\
\hline \multirow[t]{5}{*}{3} & 0600 & 5.61 & $----n$ & & & & & & 1500 & 3.80 & 204 \\
\hline & 1200 & 5.65 & 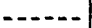 & 11 & 0600 & 6.71 & 1,000 & & $180 \mathrm{C}$ & 3.98 & 247 \\
\hline & 1800 & 5.51 & $-\cdots=-$ & & 0800 & 6.80 & 1,030 & & 2100 & 3.88 & 222 \\
\hline & 2400 & 5.37 & $-\cdots$ & & 1200 & 6.78 & 1,020 & & $240 C$ & 4.10 & 279 \\
\hline & & & & & 1600 & 6.73 & 1,000 & & & & \\
\hline \multirow[t]{5}{*}{4} & 0600 & 5.33 & $---n--$ & & 1800 & 6.81 & 1,030 & 24 & $040 \mathrm{C}$ & 4.35 & 350 \\
\hline & 1200 & 5.65 & - & & 2400 & 6.95 & 1,090 & & $080 \mathrm{C}$ & 4.65 & 439 \\
\hline & 1800 & 6.77 & $-\cdots-$ & & & & & & $120 \mathrm{C}$ & 5.38 & 660 \\
\hline & 2400 & 6.98 & 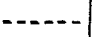 & 12 & 0600 & 6.88 & 1,080 & & 1600 & 6.08 & 898 \\
\hline & & & & & 1200 & 6.43 & 916 & & 2000 & 6.52 & 1,060 \\
\hline \multirow[t]{5}{*}{5} & 0600 & 6.92 & $\cdots-\cdots$ & & 1800 & 6.00 & 765 & & 2300 & 6.63 & 1,100 \\
\hline & 1200 & 7.11 & $-\cdots-n$ & & 2400 & 5.58 & 629 & & 2400 & 6.62 & 1,100 \\
\hline & 1800 & 7.65 & $-\ldots$ & & & & & & & & \\
\hline & 2400 & 8.17 & $=--.--$ & 13 & 1200 & 5.04 & 481 & 25 & 0400 & 6.54 & 1,070 \\
\hline & & & & & 2400 & 4.77 & 400 & & 0800 & 6.35 & 992 \\
\hline \multirow{6}{*}{6} & 0600 & 8.50 & 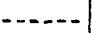 & & & & & & 1200 & 6.12 & 912 \\
\hline & 1030 & 8.57 & $\cdots$ & 14 & 1200 & 4.60 & 364 & & 1600 & 5.87 & 824 \\
\hline & 1200 & 8.56 & $=-\ldots$ & & 2400 & 4.34 & 290 & & 2000 & 5.67 & 755 \\
\hline & 1800 & 8.48 & $-\cdots-n$ & & & & & & 2400 & 5.51 & 701 \\
\hline & 2400 & 8.38 & $-\cdots$ & 15 & 0400 & 4.30 & 293 & & & & \\
\hline & & & & & 0800 & 4.35 & 307 & 26 & 0600 & 5.39 & 663 \\
\hline \multirow[t]{5}{*}{7} & 0600 & 8.32 & $=-\ldots$ & & 1200 & 4.39 & 318 & & 1200 & 5.23 & 613 \\
\hline & 1200 & 8.22 & ------ & & 1600 & 4.43 & 329 & & 1800 & 5.04 & 556 \\
\hline & 1800 & 8.08 & $-\cdots-\cdots$ & & 2000 & 4.43 & 329 & & 2400 & 4.84 & 496 \\
\hline & 2400 & 7.93 & 1,650 & & 2400 & 4.38 & 315 & & & & \\
\hline & & & & & & & & 27 & 0600 & 4.58 & 418 \\
\hline \multirow[t]{3}{*}{8} & 1200 & 7.66 & 1,500 & 16 & 1200 & 4.28 & 287 & & 1200 & 4.29 & 332 \\
\hline & 2400 & 7.18 & 1,170 & & 2400 & 4.08 & 234 & & 1800 & 4.07 & 271 \\
\hline & & & & & & & & & 2400 & 3.88 & 222 \\
\hline \multirow[t]{3}{*}{9} & 1200 & 6.67 & 972 & 17 & 1200 & 3.82 & 184 & & & & \\
\hline & 2400 & 6.15 & 790 & & 2400 & 3.62 & 147 & 28 & 1200 & 3.63 & 168 \\
\hline & & & & & & & & & 2400 & 3.46 & 137 \\
\hline 10 & 0600 & 5.95 & 731 & May 22 & 2400 & 2.68 & 52 & & & & \\
\hline
\end{tabular}

(45) 5-2915. Big Stone Lake at Ortonville, Minn.

Location.-Lat $45^{\circ} 18^{\prime} 18^{\prime \prime}$, long $96^{\circ} 26^{\prime} 57^{\prime \prime}$, in NW $\frac{1}{4} \mathrm{SW} \frac{1}{4}$ sec.9, T.121 N., R. 46 W., at powerplant intake at west edge of Ortonville, half a mile north of concrete dam at outlet, half a mile southwest of Ortonville.

Gage-height record.-Once-daily observer's readings on wire-weight gage. Datum of gage is $957.69 \mathrm{ft}$ above mean sea level, datum of 1929.

Maxima.--March-May 1965: Gage height, $9.30 \mathrm{ft}$ May 27.

1937 to February 1965: Gage height, $12.73 \mathrm{ft}$ April 17, 1952.

Remarks.--Reservoir is formed by natural lake with concrete dam at outlet. Fixed crest of dam is at elevation $963.64 \mathrm{ft}$, with one 5 -foot gate and two $2 \frac{1}{2}$-foot gates with lowest sill at elevation $958.40 \mathrm{ft}$ (all elevations are referred to datum of 1929).

Changes in gate openings are not made.

Gage height, in feet

\begin{tabular}{|c|c|c|c|c|}
\hline Feb. 28 & 6.17 & \multicolumn{2}{|c|}{ April 18} & 9.03 \\
\hline Mar. 7 & 6.10 & Apr & 25 & 8.86 \\
\hline Mar. 14 & 6.08 & May & 2 & 8.5 \\
\hline Mar. 21 & 6.20 & May & 9 & 8.0 \\
\hline Mar. 27 & 6.25 & May & 16 & 8.0 \\
\hline Apr. 5 & 6.80 & May & 23 & 7.9 \\
\hline Apr. 11 & 8.22 & May & 30 & 8.8 \\
\hline
\end{tabular}


(46) 5-2920. Minnesota River at Ortonville, Minn.

Location.-Lat $45^{\circ} 17^{\prime} 44^{\prime \prime}$, long $96^{\circ} 26^{\prime} 38^{\prime \prime}$, in NE $\frac{1}{4} N W \frac{1}{4}$ sec. $16, T .121$ N., R. 46 W., cn left bank $400 \mathrm{ft}$ downstream from bridge on U.S. Highway 12 and 1,300 ft downstrearz from dam at outlet of Big Stone Lake, at Ortonville.

Drainage area.-1, $160 \mathrm{sq} \mathrm{mi}$, approximately.

Gage-height record.-Water-stage recorder graph except Apr. 1, 2 when once-daily readings were made on outside staff gage. Datum of gage is $956.38 \mathrm{ft}$ above mean sea level, datum of 1929 .

Discharge record.-Stage-discharge relation defined by current-meter measurerents. Backwater from ice Apr. 1-7.

Maxima.-Given in the following table:

April-May 1965:

April 7, 1800 hours May 27, 1030 hours

1938 to March 1965

April 13, 1952

a Backwater from ice.

$\begin{array}{cc}\begin{array}{c}\text { Discharge } \\ \text { (cfs) }\end{array} & \begin{array}{c}\text { Gage height } \\ \text { (feet) }\end{array} \\ 870 & \mathrm{a}_{9.00} \\ 934 & 9.28\end{array}$

3,060

12.92

Remarks.-Flow affected by natural storage in Big Stone Lake above station.

Mean discharge, in cubic feet per second, 1965

\begin{tabular}{|c|c|c|c|c|c|c|c|c|}
\hline Day & April & May & Day & April & May & Day & April & May \\
\hline $1 \ldots$ & 6.9 & 476 & $11 \ldots$ & 500 & 298 & $21 \ldots \ldots$ & 590 & 381 \\
\hline $2 \ldots$ & 8.7 & 512 & $12 \ldots$ & 569 & 281 & $22 \ldots \ldots$ & 591 & 368 \\
\hline $3 \ldots$ & 10 & 457 & $13 \ldots$ & 600 & 281 & $23 \ldots$ & 591 & 401 \\
\hline $4 \ldots$ & 25 & 361 & $14 \ldots$ & 614 & 272 & $24 \ldots \ldots$ & 566 & 477 \\
\hline & 210 & 320 & $15 \ldots$ & 628 & 380 & $25 \ldots \ldots$ & 546 & 644 \\
\hline & 500 & 379 & $16 \ldots$ & 624 & 344 & $26 \ldots$ & 600 & 772 \\
\hline $7 \ldots$ & 600 & 373 & $17 \ldots$ & 621 & 269 & $27 \ldots$ & 642 & 853 \\
\hline & 725 & 372 & $18 \ldots$ & 618 & 397 & $28 \ldots$ & 548 & 717 \\
\hline 9 & 551 & 340 & 19 & 603 & 295 & $29 \ldots$ & 542 & 658 \\
\hline $10 \ldots$ & 411 & 408 & $20 \ldots$ & 584 & 230 & $30 \ldots \ldots$ & 490 & 658 \\
\hline \multirow{3}{*}{\multicolumn{7}{|c|}{$\begin{array}{l}\text { Monthly mean discharge, in cubic feet per second } \\
\text { Runoff, in inches }\end{array}$}} & & \\
\hline & & & & & & & 490 & 438 \\
\hline & & & & & & & 0.47 & 0.44 \\
\hline
\end{tabular}


Gage height, in feet, and discharge, in cubic feet per second, at indicated time, 1965, of Minnesota River at Ortonville, Minn.

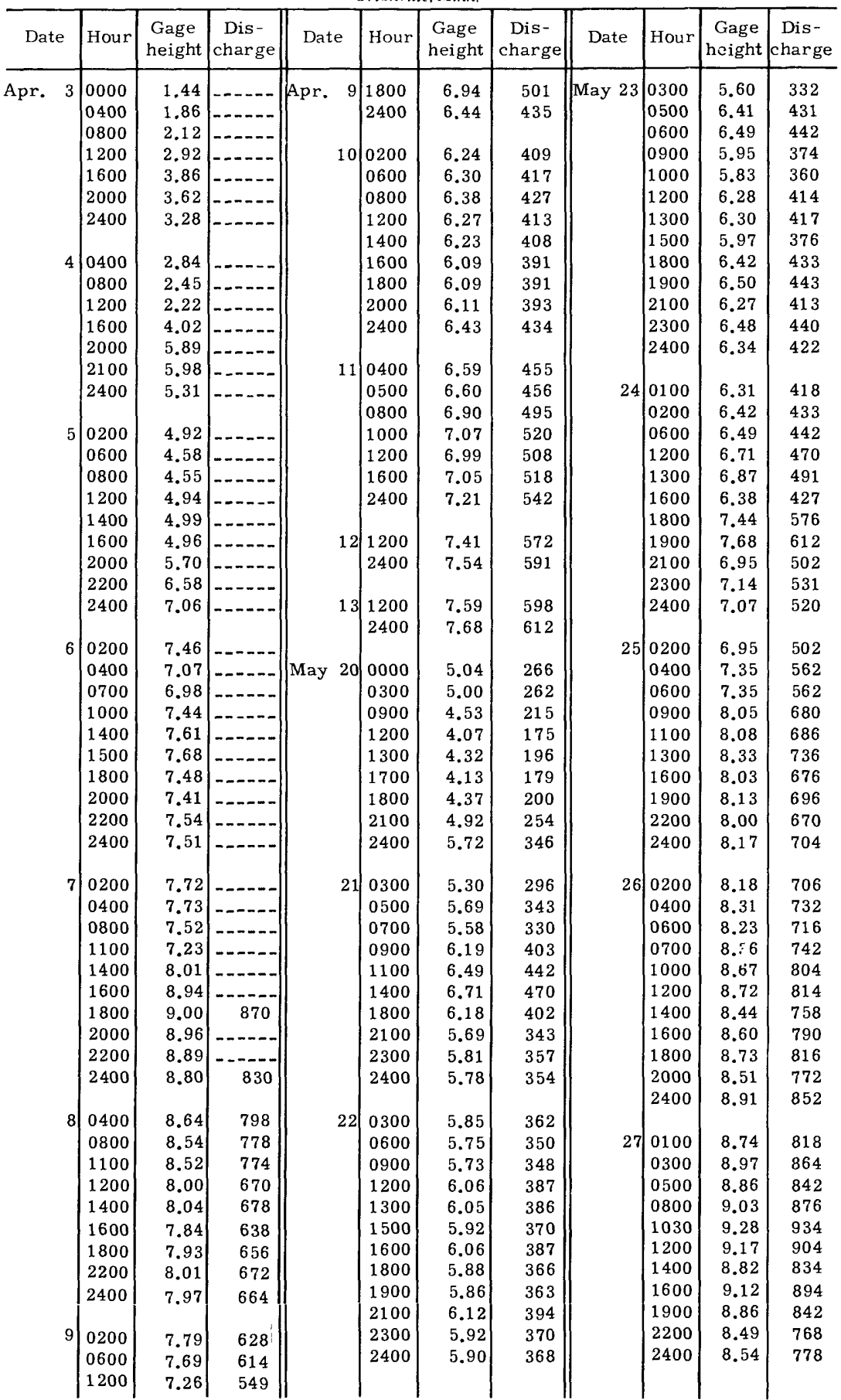


Gage height, in feet, and discharge, in cubic feet per second, at indicated time, 1965, of Minnesota River at Ortonville, Minn.-Continued

\begin{tabular}{|c|c|c|c|c|c|c|c|c|c|c|c|}
\hline Date & Hour & $\begin{array}{l}\text { Gage } \\
\text { height }\end{array}$ & $\begin{array}{c}\text { Dis- } \\
\text { charge }\end{array}$ & Date & Hour & $\begin{array}{l}\text { Gage } \\
\text { height }\end{array}$ & $\begin{array}{c}\text { Dis- } \\
\text { charge }\end{array}$ & Date & Hour & $\begin{array}{c}\text { Gage } \\
\text { height }\end{array}$ & $\begin{array}{c}\text { Dis- } \\
\text { charge }\end{array}$ \\
\hline May 28 & $\begin{array}{l}0300 \\
0500 \\
0700 \\
0900 \\
1100 \\
1300 \\
1800 \\
2400 \\
0300 \\
0600 \\
0700 \\
0800 \\
1100 \\
1300\end{array}$ & $\begin{array}{l}8.45 \\
8.29 \\
8.37 \\
8.30 \\
8.41 \\
8.23 \\
8.07 \\
7.91 \\
7.80 \\
7.81 \\
7.95 \\
7.80 \\
8.03 \\
7.91\end{array}$ & $\begin{array}{l}760 \\
728 \\
744 \\
730 \\
752 \\
716 \\
684 \\
652 \\
\\
630 \\
632 \\
660 \\
630 \\
676 \\
652\end{array}$ & May 29 & $\begin{array}{l}1500 \\
1800 \\
2000 \\
2400 \\
0100 \\
0300 \\
0500 \\
0900 \\
1100 \\
1400 \\
1700 \\
2000 \\
2200 \\
2300\end{array}$ & $\begin{array}{l}8.06 \\
8.02 \\
8.04 \\
7.99 \\
7.97 \\
8.00 \\
7.97 \\
8.03 \\
8.05 \\
7.97 \\
7.86 \\
7.88 \\
7.81 \\
7.74\end{array}$ & $\begin{array}{l}682 \\
674 \\
678 \\
668 \\
\\
664 \\
670 \\
664 \\
676 \\
680 \\
664 \\
642 \\
646 \\
632 \\
621\end{array}$ & May 30 & $\begin{array}{l}2400 \\
0200 \\
0500 \\
0600 \\
0800 \\
1000 \\
1300 \\
1500 \\
1700 \\
1900 \\
2100 \\
2300 \\
2400\end{array}$ & $\begin{array}{l}7.73 \\
7.81 \\
7.48 \\
7.44 \\
7.43 \\
7.91 \\
7.51 \\
7.35 \\
7.81 \\
7.69 \\
7.98 \\
7.83 \\
7.88\end{array}$ & $\begin{array}{l}620 \\
632 \\
582 \\
576 \\
574 \\
652 \\
586 \\
562 \\
632 \\
614 \\
666 \\
636 \\
646\end{array}$ \\
\hline
\end{tabular}

(47) 5-2930. Yellow Bank River near Odessa, Minn.

Location.-Lat $45^{\circ} 13^{\prime} 35^{\prime \prime}$, long $96^{\circ} 21^{\prime} 12^{\prime \prime}$, in $\operatorname{SE}_{\frac{1}{4}} \operatorname{SE} \frac{1}{4}$ Sec.1, T.120 N., R.46 W., or left bank $150 \mathrm{ft}$ downstream from highway bridge, $2 \frac{1}{2}$ miles southwest of Odessa, and $4 \frac{1}{2}$ miles upstream from mouth.

Drainage area. $-398 \mathrm{sq} \mathrm{mi}$.

Gage-height record.-Water-stage recorder graph. Datum of gage is $953.34 \mathrm{ft}$ above mean sea level, datum of 1929 .

Discharge record.-Stage-discharge relation defined by current-meter measurements. Backwater from ice Apr. 1-7.

Maxima.-Given in the following table:

April-May 1965

Apr. 8, 0800 hours

May 25, 1300 hours

1939 to March 1965

Apr. 4, 1952

Mar. 25, 1943

a Backwater from ice.

$\begin{array}{cc}\begin{array}{c}\text { Discharge } \\ \text { (cfs) }\end{array} & \begin{array}{c}\text { Gage height } \\ \text { (feet) }\end{array} \\ 3,540 & 13.46 \\ 806 & 6.37\end{array}$

6,260

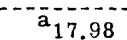


Mean discharge, in cubic feet per second, 1965, of Yellow Bank River near Odessa. Minn.

\begin{tabular}{|c|c|c|c|c|c|c|c|c|}
\hline Day & April & May & Day & April & May & Day & April & May \\
\hline $\begin{array}{l}1 \ldots \ldots \\
2 \ldots \ldots \\
3 \ldots \ldots \\
1 \\
5 \\
5 \\
6 \ldots \ldots \\
7 \ldots \ldots \\
8 \ldots \ldots \\
9 \ldots \ldots \\
10 \ldots \ldots\end{array}$ & $\begin{array}{r}28 \\
70 \\
150 \\
300 \\
650 \\
1,500 \\
2,580 \\
3,330 \\
2,170 \\
1,280\end{array}$ & $\begin{array}{l}81 \\
77 \\
73 \\
63 \\
65 \\
65 \\
64 \\
65 \\
71 \\
71\end{array}$ & $\begin{array}{l}11 \ldots \\
12 \ldots \\
13 \ldots \\
14 \\
15 \\
16 \ldots \\
17 \ldots \\
18 \ldots \\
19 \ldots \\
20 \ldots\end{array}$ & $\begin{array}{r}1,120 \\
1,210 \\
900 \\
625 \\
481 \\
381 \\
317 \\
268 \\
230 \\
207\end{array}$ & $\begin{array}{r}71 \\
77 \\
71 \\
63 \\
68 \\
79 \\
268 \\
225 \\
174 \\
149\end{array}$ & $\mid \begin{array}{l}21 \ldots \ldots \\
22 \ldots \ldots \\
23 \ldots \\
24 \ldots \\
25 \\
26 \ldots \\
27 \ldots \\
28 \ldots \\
29 \\
30 \ldots \\
31\end{array}$ & $\begin{array}{r}190 \\
174 \\
155 \\
140 \\
133 \\
126 \\
120 \\
115 \\
103 \\
90\end{array}$ & $\begin{array}{l}131 \\
115 \\
182 \\
304 \\
740 \\
585 \\
432 \\
318 \\
252 \\
216 \\
193\end{array}$ \\
\hline \multicolumn{7}{|c|}{$\begin{array}{l}\text { Monthly mean discharge, in cubic feet per second } \\
\text { Runoff, in inches }\end{array}$} & $\begin{array}{r}638 \\
1.79\end{array}$ & $\begin{array}{r}174 \\
0.51\end{array}$ \\
\hline
\end{tabular}

Gage height, in feet, and discharge, in cubic feet per second, at indicated time, 1965

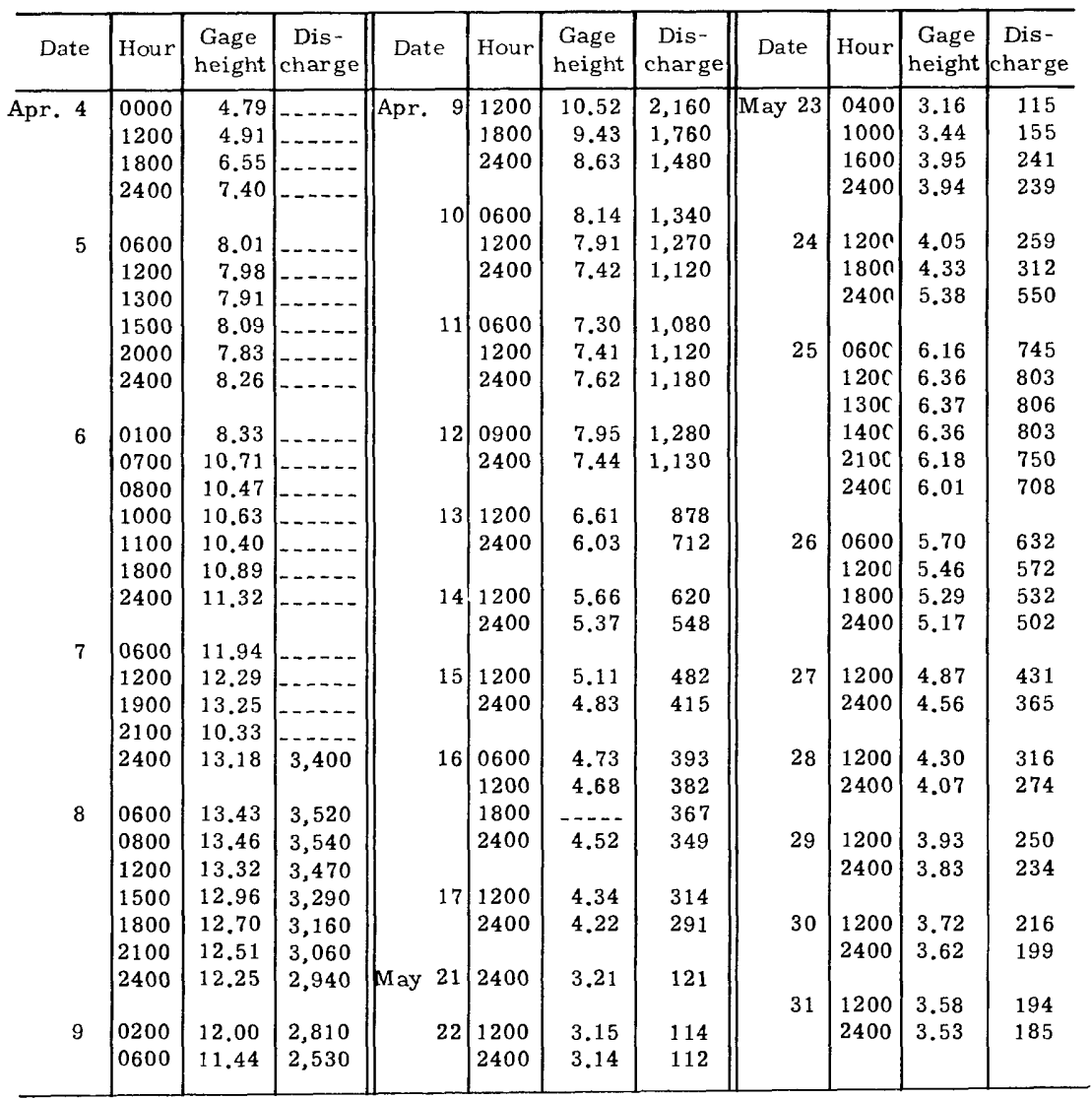


(48) 5-2940. Pomme de Terre River at Appleton, Minn.

Location.-Lat $45^{\circ} 12^{\prime} 10^{\prime \prime}$, long $96^{\circ} 01^{\prime} 20^{\prime \prime}$, in SW $\frac{1}{4} \mathrm{NW} \frac{1}{4}$ sec.14, T.120 N., R.43 W., on left bank at Appleton, $60 \mathrm{ft}$ upstream from bridge on U.S. Highway 59 and State Highway 119 and 8 miles upstream from mouth.

Drainage area.- $-905 \mathrm{sq} \mathrm{mi}$, approximately.

Gage-height record.-Water-stage recorder graph. Datum of gage is $978.00 \mathrm{ft}$ above mean sea level, datum of 1929 .

Discharge record.- Stage-discharge relation defined by current-meter measurements. Backwater from ice Apr. 1-8.

Maxima.-April-May 1965: Discharge, 2,310 cfs 2130 hours Apr. 9 (gage-height, $9.63 \mathrm{ft}$ ). 1931 to March 1965: Discharge, 5,050 cfs Apr. 8, 1952 (gage height, 10.13 f1, at site 4 miles upstream at datum $25.17 \mathrm{ft}$ higher).

Remarks.-Flow affected by lakes above station.

Mean discharge, in cubic feet per second, 1965

\begin{tabular}{|c|c|c|c|c|c|c|c|c|}
\hline Day & April & May & Day & April & May & Day & April & May \\
\hline $1 \ldots$ & 5.8 & 402 & $11 \ldots$ & 1,750 & 361 & $21 \ldots$ & 894 & 324 \\
\hline $2 \ldots$ & 6.4 & 384 & $12 \ldots$ & 1,660 & 338 & 22. & 826 & 333 \\
\hline & 7.3 & 374 & $13 \ldots$ & 1,880 & 315 & $23=$ & 772 & 370 \\
\hline $4 \ldots$ & 8.9 & 361 & $14 \ldots$ & 1,930 & 319 & $24 \ldots$ & 708 & 407 \\
\hline & 17 & 351 & $15 \ldots$ & 1,720 & 328 & 25 & 660 & 439 \\
\hline & 62 & 347 & $16 \ldots$ & 1,460 & 342 & 26 . & 612 & 444 \\
\hline & 230 & 342 & $17 \ldots$ & 1,290 & 333 & 27 & 564 & 439 \\
\hline & 1,940 & 338 & 18. & 1,180 & 310 & 28 & 526 & 425 \\
\hline & 2,170 & 351 & $19 \ldots$ & 1,080 & 301 & $29 \ldots$ & 478 & 430 \\
\hline $10 \ldots$ & 1,950 & 370 & $20 \ldots$ & 973 & 306 & $30 \ldots$ & 435 & 435 \\
\hline & & & & & & & -- & 435 \\
\hline \multicolumn{7}{|c|}{$\begin{array}{l}\text { Monthly mean discharge, in cubic feet per second } \\
\text { Runoff in inches }\end{array}$} & 927 & 366 \\
\hline \multicolumn{7}{|c|}{ Runoff, in inches } & 1.14 & 0.47 \\
\hline
\end{tabular}

Gage height, in feet, and discharge, in cubic feet per second, at indicated time, 1965

\begin{tabular}{|c|c|c|c|c|c|c|c|c|c|c|c|}
\hline Date & Hour & $\begin{array}{c}\text { Gage } \\
\text { height }\end{array}$ & $\begin{array}{c}\text { Dis- } \\
\text { charge }\end{array}$ & Date & Hour & $\begin{array}{l}\text { Gage } \\
\text { height }\end{array}$ & $\begin{array}{c}\text { Dis- } \\
\text { charge }\end{array}$ & Date & Hour & $\begin{array}{c}\text { Gage } \\
\text { height }\end{array}$ & $\begin{array}{l}\text { Dis- } \\
\text { charge }\end{array}$ \\
\hline \multirow[t]{10}{*}{ Apr. 5} & 0000 & 4.46 & $-\cdots-1$ & \multirow[t]{17}{*}{ Apr. 8} & 1400 & 9.40 & $\ldots$ & Apr. 10 & 1800 & 8.76 & 1,840 \\
\hline & 0600 & 4.46 & $\ldots$ & & 1800 & 9.38 & $\ldots . .$. & \multirow{8}{*}{$\left.\right|^{3}$} & 2000 & 8.79 & 1,860 \\
\hline & 1200 & 4.47 & $\ldots \ldots$ & & 2400 & 9.36 & 2,160 & & 2200 & 8.59 & 1,750 \\
\hline & 1500 & 4.49 & $\ldots$ & & 0200 & 9.29 & 2,130 & & 2300 & 8.93 & 1,930 \\
\hline & 1600 & 4.90 & $\ldots$ & & 0500 & 9.31 & 2,140 & & 2400 & 8.74 & 1,830 \\
\hline & 1700 & 4.83 & $\ldots$ & & 0600 & 9.28 & 2,120 & & & & \\
\hline & 1800 & 4.90 & $-\ldots$ & & 1000 & 9.17 & 2,060 & & 0600 & 8.60 & 1,750 \\
\hline & 2000 & 4.83 & $\ldots$ & & 1100 & 9.23 & 2,090 & & 1200 & 8.61 & 1,760 \\
\hline & 2200 & 4.89 & $-\ldots$ & & 1200 & 9.15 & 2,040 & & 2400 & 8.52 & 1,710 \\
\hline & 2400 & 4.93 & & & 1300 & 9.28 & 2,120 & \multirow[t]{2}{*}{12} & 1200 & 8.34 & 1,620 \\
\hline \multirow[t]{6}{*}{6} & 0600 & 4.78 & & & 1500 & 9.39 & 2,180 & & 2400 & 8.49 & 1,700 \\
\hline & 1200 & 4.99 & & & 1700 & 9.57 & 2,280 & \multirow{3}{*}{13} & & & \\
\hline & 1400 & 5.66 & & & 1800 & 9.54 & 2,260 & & 1200 & 8.88 & 1,910 \\
\hline & 1800 & 6.11 & $\ldots$ & & 1900 & 9.62 & 2,300 & & 2400 & 9.04 & 1,990 \\
\hline & 2000 & 6.35 & $\ldots$ & & 2130 & 9.63 & 2,310 & \multirow{3}{*}{14} & 1200 & & \\
\hline & 2400 & 6.04 & 政 & & 2200 & 9.61 & 2,300 & & $\begin{array}{l}1200 \\
2400\end{array}$ & $\begin{array}{l}8.94 \\
8.78\end{array}$ & 1,940 \\
\hline \multirow[t]{4}{*}{7} & 0600 & 5.60 & & & 2400 & 9.49 & 2,230 & & & & 1,000 \\
\hline & 1200 & 5.66 & & \multirow[t]{5}{*}{10} & 0200 & 9.41 & 2,190 & \multirow[t]{3}{*}{15} & 1200 & 8.57 & 1,730 \\
\hline & 1800 & 5.86 & & & 0600 & 9.14 & 2,050 & & 2400 & 8.29 & 1,580 \\
\hline & 2400 & 7.40 & $1-2=0$ & & 1000 & 9.93 & 1,930 & & & & \\
\hline \multirow[t]{2}{*}{8} & 0600 & 8.59 & & & 1200 & 8.88 & 1,910 & \multirow[t]{2}{*}{16} & 1200 & 8.03 & 1,450 \\
\hline & 1200 & 9.07 & & & 1400 & 8.78 & 1,850 & & 2400 & 7.84 & 1,350 \\
\hline
\end{tabular}


(49) 5-3000. Lac qui Parle River near Lac qui Parle, Minn.

Location.-Lat $45^{\circ} 00^{\prime}$, long $95^{\circ} 55^{\prime}$, in SW $\frac{1}{4} \mathrm{SW} \frac{1}{4}$ sec. $27, \mathrm{~T} .118$ N., R. 42 W., on right bank $40 \mathrm{ft}$ downstream from highway bridge and half a mile southwest of village of Lac qui Parle.

Drainage area. $-983 \mathrm{sq} \mathrm{mi.}$

Gage-height record.-Water-stage recorder graph except 1900 hours Apr. 8 to 1300 hours Apr. 9 when graph was constructed on the basis of engineer's gage reading on chain gage and floodmark. Datum of gage is $951.98 \mathrm{ft}$ above mean sea level (Minnesota Highway Department bench mark).

Discharge record.-Stage-discharge relation defined by current-meter m easurements. Backwater from ice Apr. 1-10.

Maxima.-Given in the following table:

April-May 1965

April 9, 0900 hours

May 26, 1200 hours

1910-14, 1931 to March 1965

Apr. 6, 1952

Mar. 24, 1948

$\mathrm{a}_{\text {Backwater from ice. }}$

$\begin{array}{cr}\begin{array}{c}\text { Discharge } \\ \text { (cfs) }\end{array} & \begin{array}{c}\text { Gage height } \\ \text { (feet) }\end{array} \\ 8,370 & \mathrm{a}_{19.37} \\ 1,410 & 5.69\end{array}$

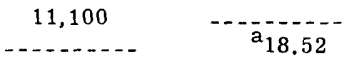

Mean discharge, in cubic feet per second, 1965

\begin{tabular}{|c|c|c|c|c|c|c|c|c|}
\hline Day & April & May & Day & April & May & Day & April & May \\
\hline $\begin{array}{l}1 \ldots \ldots \\
2 \ldots \ldots \\
3 \ldots \ldots \\
4 \ldots \ldots \\
5 \ldots \ldots \\
6 \ldots \ldots \\
7 \ldots \ldots \\
8 \ldots \ldots \\
9 \ldots \ldots \\
10 \ldots\end{array}$ & $\begin{array}{r}2.0 \\
10 \\
30 \\
75 \\
125 \\
445 \\
1,000 \\
5,600 \\
7,200 \\
5,800\end{array}$ & $\begin{array}{l}330 \\
305 \\
276 \\
256 \\
239 \\
229 \\
217 \\
215 \\
217 \\
206\end{array}$ & $\mid \begin{array}{l}11 \ldots \\
12 \ldots \ldots \\
13 \ldots \ldots \\
14 \ldots \ldots \\
15 \ldots \\
16 \ldots \\
17 \ldots \\
18 \ldots \\
19 \ldots \\
20 \ldots\end{array}$ & $\begin{array}{r}4,960 \\
4,220 \\
3,630 \\
2,850 \\
2,260 \\
1,850 \\
1,520 \\
1,250 \\
1,070 \\
922\end{array}$ & $\begin{array}{l}210 \\
215 \\
204 \\
210 \\
220 \\
220 \\
227 \\
232 \\
225 \\
217\end{array}$ & $\mid \begin{array}{l}21 \ldots \ldots \\
22 \\
23 \\
23 \\
24 \ldots \\
25 \ldots \\
26 \ldots \\
26 \ldots \\
27 \ldots \ldots \\
28 \ldots \ldots \\
29 \\
30 \ldots \ldots \\
31 \ldots \ldots\end{array}$ & $\begin{array}{r}737 \\
700 \\
610 \\
540 \\
498 \\
470 \\
454 \\
436 \\
407 \\
336 \\
\end{array}$ & $\begin{array}{r}263 \\
284 \\
796 \\
1,260 \\
1,340 \\
1,400 \\
1,300 \\
1,030 \\
792 \\
658 \\
566\end{array}$ \\
\hline $\begin{array}{l}\text { Monthl } \\
\text { Runoff }\end{array}$ & $\begin{array}{l}\text { an dis } \\
\text { inches }\end{array}$ & -.. - & & $\ldots$ & & & $\begin{array}{r}1,670 \\
1.90\end{array}$ & $\begin{array}{r}463 \\
0.54\end{array}$ \\
\hline
\end{tabular}


Gage height, in feet, and discharge, in cubic feet per second, at indicated time, 1965, of Lac qui Parle Kiver near Lac qui Parle, Minn.

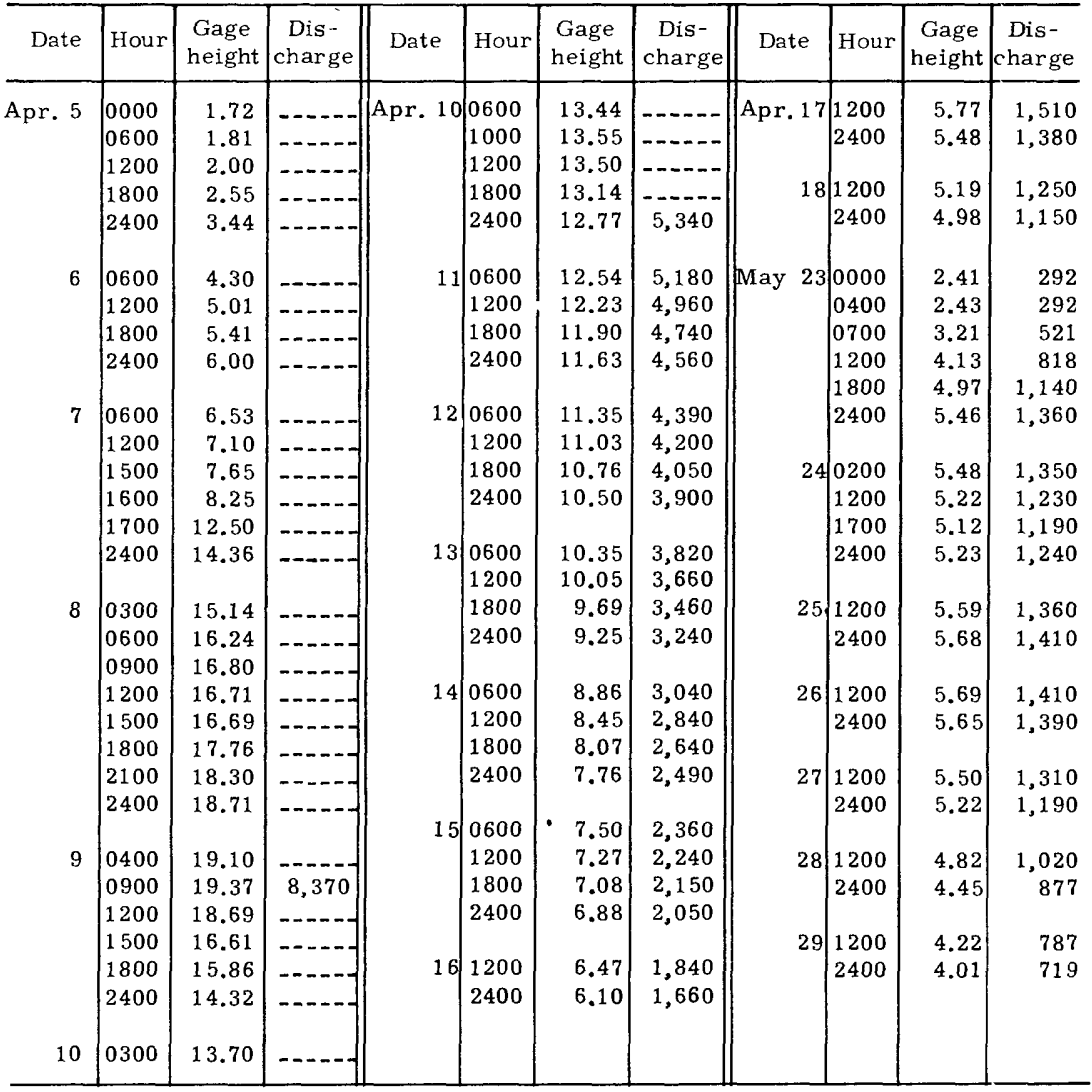

(50) 5-3010. Minnesota River near Lac qui Parle, Minn.

Location.-Lat $45^{\circ} 01^{\prime} 17^{\prime \prime}$, long $95^{\circ} 52^{\prime} 05^{\prime \prime}$, in NW $\frac{1}{4} \mathrm{NE} \frac{1}{4}$ sec. $24, \mathrm{~T} .118$ N., R.42 W., on left bank $200 \mathrm{ft}$ downstream from dam at Lac qui Parle Outlet, 2.4 miles northeast of village of Lac qui Parle, and 3.5 miles west of Watson.

Drainage area.-4,050 sq $\mathrm{mi}$, approximately.

Gage-height record.-Water-stage recorder graph. Datum of gage is $900.00 \mathrm{ft}$ above mean sea level, datum of 1929.

Discharge record.-Stage-discharge relation defined by current-meter measurements. Backwater from ice Apr. 1-8.

Maxima.-April-May 1965: Discharge, 10,700 cfs 0200 hours Apr. 14 (gage height., $36.17 \mathrm{ft}$ ).

1942 to March 1965: Discharge, 19,700 cfs Apr. 10, 1952 (gage height, $37.93 \mathrm{ft}$, from floodmark).

Remarks.-Part of flow from 2,050 square miles of Chippewa River basin at tirzes diverted into Minnesota River above station. Some regulation by Big Stone Lake since Apr. 17, 1927, Lac qui Parle since January, 1938, and Marsh Lake since Nov. 1, 1939. 
Mean discharge, in cubic feet per second, 1965, of Minnesota River near Lac qui Parle, Minn.

\begin{tabular}{|c|c|c|c|c|c|c|c|c|}
\hline Day & April & May & Day & April & May & Day & April & May \\
\hline $\begin{array}{l}1 \ldots \ldots \\
2 \ldots \ldots \\
3 \ldots \ldots \\
4 \\
4 \\
5\end{array} \ldots$ & $\begin{array}{r}108 \\
109 \\
110 \\
111 \\
112 \\
120 \\
380 \\
2,000 \\
3,110 \\
4,700\end{array}$ & $\begin{array}{l}3,320 \\
3,260 \\
3,190 \\
3,090 \\
3,000 \\
2,930 \\
2,830 \\
2,620 \\
2,500 \\
2,470\end{array}$ & $\begin{array}{l}11 \ldots \\
12 \ldots \ldots \\
13 \\
13_{\ldots} \ldots \\
15_{\ldots} \ldots \\
16 \ldots \\
17 \ldots \\
18 \ldots \\
19 \ldots \\
20 \ldots\end{array}$ & $\begin{array}{r}7,270 \\
9,560 \\
10,500 \\
10,600 \\
10,200 \\
9,640 \\
8,880 \\
7,940 \\
7,050 \\
6,300\end{array}$ & $\begin{array}{l}2,260 \\
1,970 \\
1,660 \\
1,500 \\
1,540 \\
1,540 \\
1,520 \\
1,650 \\
1,700 \\
1,680\end{array}$ & $\mid \begin{array}{l}21 \ldots \\
22 \ldots \\
23 \ldots \\
24 \ldots \\
25 \ldots \\
26 \ldots \\
26 \ldots \\
28 \ldots \\
29 \\
30 \ldots \\
31 \ldots\end{array}$ & $\begin{array}{l}5,930 \\
5,460 \\
5,070 \\
4,700 \\
4,390 \\
4,120 \\
3,920 \\
3,700 \\
3,540 \\
3,420 \\
-\end{array}$ & $\begin{array}{l}1,720 \\
1,710 \\
1,810 \\
1,920 \\
1,840 \\
1,970 \\
2,130 \\
2,060 \\
2,020 \\
2,120 \\
2,160\end{array}$ \\
\hline \multicolumn{7}{|c|}{$\begin{array}{l}\text { Monthly mean discharge, in cubic feet per second } \\
\text { Runoff, in inches }\end{array}$} & $\begin{array}{r}4,770 \\
1.31\end{array}$ & $\begin{array}{r}2,184 \\
0.62\end{array}$ \\
\hline
\end{tabular}

Gage height, in feet, and discharge, in cubic feet per second, at indicated time, 1965

\begin{tabular}{|c|c|c|c|c|c|c|c|c|c|c|c|}
\hline Date & Hour & $\begin{array}{l}\text { Gage } \\
\text { height }\end{array}$ & $\begin{array}{c}\text { Dis- } \\
\text { charge }\end{array}$ & Date & Hour & $\begin{array}{l}\text { Gage } \\
\text { height }\end{array}$ & $\begin{array}{c}\text { Dis- } \\
\text { charge }\end{array}$ & Date & Hour & $\begin{array}{c}\text { Gage } \\
\text { height }\end{array}$ & $\begin{array}{c}\text { Dis- } \\
\text { charge }\end{array}$ \\
\hline \multirow[t]{15}{*}{ Apr. } & 0000 & 30.10 & 2,640 & \multirow[t]{5}{*}{ Apr. 12} & 1200 & 35.90 & 9,680 & \multirow[t]{3}{*}{ Apr. 17} & \multirow{3}{*}{$\begin{array}{l}1200 \\
2400\end{array}$} & \multirow{3}{*}{$\begin{array}{l}35.67 \\
35.56\end{array}$} & \multirow{3}{*}{$\begin{array}{l}8,880 \\
8,490\end{array}$} \\
\hline & 0100 & 30.10 & 2,740 & & 2400 & 36.05 & 10,200 & & & & \\
\hline & 0800 & 30.49 & 2,900 & & & & & & & & \\
\hline & 1600 & 31.30 & 3,220 & & 1200 & 36.14 & 10,600 & \multirow[t]{2}{*}{18} & 1200 & 35.40 & 7,930 \\
\hline & 2400 & 32.32 & 3,680 & & 2400 & 36.16 & 10,700 & & 2400 & 35.25 & 7,430 \\
\hline & 0400 & 32.70 & 4,020 & \multirow[t]{7}{*}{14} & 0200 & 36.17 & 10,700 & \multirow[t]{5}{*}{19} & & 35.17 & 7,210 \\
\hline & 0600 & 32.77 & 4,080 & & 1200 & 36.15 & 10,600 & & 1200 & 35.16 & 7,180 \\
\hline & 0700 & 32.32 & 3,740 & & 2400 & 36.10 & 10,400 & & 1800 & 35.01 & 6,800 \\
\hline & 1100 & 33.30 & 4,540 & & & & & & 2400 & 34.91 & 6,600 \\
\hline & 1500 & 33.80 & 5,050 & & 1200 & 36.06 & 10,300 & & & & \\
\hline & 2400 & 34.54 & 6,000 & & 2400 & 35.97 & 9,920 & 20 & 1200 & 34.72 & 6,260 \\
\hline & & & & & & & & & 2400 & 34.61 & 6,100 \\
\hline & 0700 & 34.88 & 6,540 & 16 & 1200 & 35.90 & 9,680 & & & & \\
\hline & 1700 & 35.40 & 7,930 & & 2400 & 35.78 & 9,260 & & & & \\
\hline & 2400 & 35.62 & 8,700 & & & & & & & & \\
\hline
\end{tabular}

(51) 5-3045. Chippewa River near Milan, Minn.

Location.-Lat $45^{\circ} 06^{\prime} 39^{\prime \prime}$, long $95^{\circ} 47^{\prime} 57^{\prime \prime}$, in SE $\frac{1}{4} \mathrm{SE} \frac{1}{4}$ sec.16, T.119 N., R.41 W., on right bank $800 \mathrm{ft}$ upstream from bridge on State Highway $40,2.0$ miles upstr ?am from small tributary, and $5 \frac{1}{2}$ miles east of Milan.

Drainage area.-1,870 sq $\mathrm{mi}$, approximately.

Gage-height record.-Water-stage recorder graph. Datum of gage is $959.69 \mathrm{ft}$ above mean sea level, datum of 1929.

Discharge record.- Stage-discharge relation defined by current-meter measurements. Backwater from ice Apr. 1-10.

Maxima.-April-May 1965: Discharge, 6,770 cfs 1630 hours, Apr. 11 (gage-height, $11.93 \mathrm{ft}$ ) 1937 to March 1965: Discharge, 6,930 cfs Apr. 9, 1952 (gage-height, $12.12 \mathrm{ft}$ ), gageheight, $12.29 \mathrm{ft}$ Apr. 7, 1952 (backwater from ice).

Remarks.-Part of flow from 2,050 square miles of Chippewa River basin at times diverted into Minnesota River above station. 
Mean discharge, in cubic feet per second, 1965, of Chippewa River near Milan, Minn

\begin{tabular}{|c|c|c|c|c|c|c|c|c|}
\hline Day & April & May & Day & April & May & Day & April & May \\
\hline $\begin{array}{l}1 \\
2 \\
3 \\
4 \\
4 \\
5 \\
6 \\
7 \\
8\end{array}$ & $\begin{array}{r}3 \\
3 \\
4 \\
5 \\
11 \\
40 \\
500 \\
1,750 \\
3,100 \\
3,900\end{array}$ & $\begin{array}{r}1,150 \\
1,120 \\
1,080 \\
1,040 \\
1,030 \\
1,030 \\
1,010 \\
973 \\
1,040 \\
1,040\end{array}$ & $\begin{array}{l}11 \ldots \ldots \\
12 \ldots \\
13 \\
14 \\
15 \\
16 \ldots \\
17 \ldots \\
18 \ldots \\
19 \ldots \\
20 \ldots\end{array}$ & $\begin{array}{l}6,170 \\
6,270 \\
5,710 \\
5,160 \\
4,620 \\
3,920 \\
2,990 \\
2,450 \\
2,190 \\
2,020\end{array}$ & $\begin{array}{r}995 \\
951 \\
912 \\
902 \\
946 \\
1,160 \\
1,060 \\
968 \\
907 \\
885\end{array}$ & $\begin{array}{l}21 \ldots \ldots \\
22 \ldots \ldots \\
23 \ldots \ldots \\
24 \ldots \ldots \\
25 \ldots \ldots \\
26 \ldots \ldots \\
27 \ldots \ldots \\
28 \ldots \ldots \\
29 \ldots \ldots \\
30 \ldots \ldots \\
31 \ldots \ldots\end{array}$ & $\begin{array}{l}1,880 \\
1,730 \\
1,610 \\
1,510 \\
1,440 \\
1,390 \\
1,330 \\
1,270 \\
1,230 \\
1,190\end{array}$ & $\begin{array}{r}946 \\
940 \\
1,490 \\
2,930 \\
2,550 \\
2,120 \\
1,780 \\
1,570 \\
1,460 \\
1,380 \\
1,300\end{array}$ \\
\hline \multicolumn{7}{|c|}{$\begin{array}{l}\text { Monthly mean discharge, in cubic feet per second } \\
\text { Runoff, in inches }\end{array}$} & $\begin{array}{r}2,180 \\
1.30\end{array}$ & $\begin{array}{r}1,247 \\
0.77\end{array}$ \\
\hline
\end{tabular}

Gage hetght, in feet, and discharge, in cubic feet per second, at indicated time, 1965

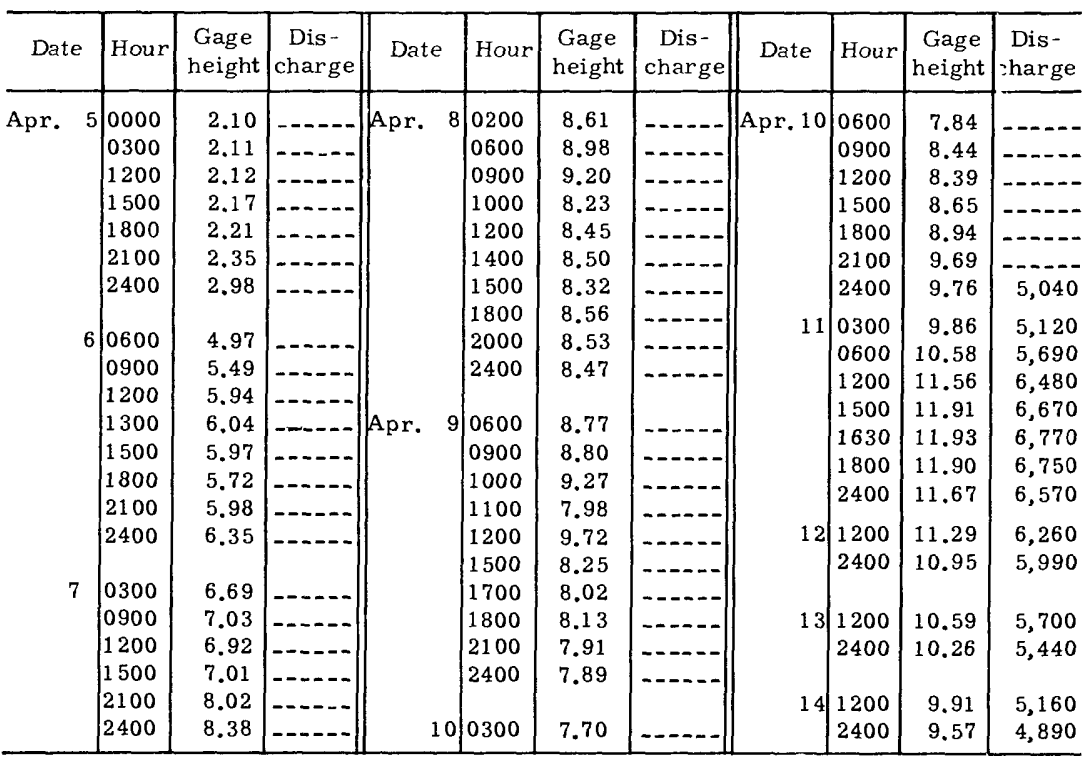


(52) Chippewa River diversion near Watson, Minn.

(Miscellaneous site)

Location.-Lat $45^{\circ} 01^{\prime}$, long $95^{\circ} 49^{\prime}$, on line between sections 15 and 16, T.118 N., R.41 W., about $1 \frac{l}{2}$ miles north on S.A.R. No.3 from Watson.

Gage-height record.-Once-daily staff reading on tailwater gage during flood only. Datum of gage is $900.00 \mathrm{ft}$ above mean sea level, adjustment of 1912 .

Discharge record.-Stage-discharge relation defined by seven current-meter measurements.

Maxima.-April-May 1965: Discharge, 3,430 cfs Apr. 12 (gage height, $43.80 \mathrm{ft}$ ).

1943 to March 1965: Discharge, 4,300 cfs Apr. 12, 1952, from discharge measurement at or near peak, gage height, $44.90 \mathrm{ft}$, Apr. 10, 1952, backwater from Lac qui Parle reservoir.

Cooperation: Maximum gage heights furnished by Corps of Engineers.

(53) Chippewa River below diversion dam, near Watson, Minn.

(Miscellaneous site)

Location.-Lat $45^{\circ} 01^{\prime}$, long $95^{\circ} 48^{\prime}$, on line between sec.15 and 22, T.118 N., R.41 W., $1 \frac{1}{2}$ miles northeast of Watson, 2.4 miles downstream from Dry Weather Creek, and 10 miles above mouth.

Gage-height record.-Once-daily staff gage reading on tailwater gage. Datum of gage is $900.00 \mathrm{ft}$ above mean sea level, adjustment of 1912 .

Discharge record.-Stage-discharge relation defined by three discharge measurements.

Maxima.-April-May 1965: Discharge, 3,160 cfs Apr. 12 (gage height, 44,25 ft).

1943 to March 1965: Discharge, 3,180 cfs Apr. 12, 1952 (gage height. $43.55 \mathrm{ft}$ ) from discharge measurement near peak.

Cooperation.-Peak stage furnished by Corps of Engineers.

\section{(54) 5-3110. Minnesota River at Montevideo, Minn.}

Location,-Lat $44^{\circ} 56^{\prime} 00^{\prime \prime}$, long $95^{\circ} 44^{\prime} 00^{\prime \prime}$, in NW $\frac{1}{4} \mathrm{NW} \frac{1}{4}$ sec.19, T.117 N., R.40 W., on right bank $100 \mathrm{ft}$ upstream from bridge on U.S. Highway 212, at Montevideo, and $400 \mathrm{ft}$ downstream from Chippewa River.

Drainage area. $-6,180 \mathrm{sq} \mathrm{mi}$, approximately.

Gage-height record.-Water-stage recorder graph. Datum of gage is $910.87 \mathrm{ft}$ above mean sea level, datum of 1912 .

Discharge record.-Stage-discharge relation defined by current-meter moasurements. Backwater from ice Apr. 1-10.

Maxima.-April-May 1965: Discharge, 12,900 cfs 0300 hours Apr. 14 (gage height, $16.64 \mathrm{ft}$ ).

1909 to March 1965: Discharge, 24,500 cfs Apr. 10, 1952 (gage height: $20.02 \mathrm{ft}$, from floodmark).

Remarks.-Flow regulated by Big Stone Lake since Apr. 17, 1937, Lac qui Parle since January 1938 and Marsh Lake since Nov. 1, 1939. 
Mean discharge, in cubic feet per second, 1965, of Minnesota River at Montevideo, Minn.

\begin{tabular}{|c|c|c|c|c|c|c|c|c|}
\hline Day & April & May & Day & April & May & Day & April & May \\
\hline 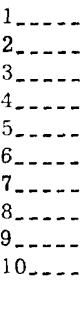 & $\begin{array}{r}111 \\
112 \\
114 \\
116 \\
123 \\
200 \\
800 \\
2,500 \\
4,000 \\
5,350\end{array}$ & $\begin{array}{l}4,460 \\
4,330 \\
4,220 \\
4,100 \\
3,990 \\
3,870 \\
3,760 \\
3,620 \\
3,620 \\
3,460\end{array}$ & $\begin{array}{l}11 \ldots \\
12 \ldots \\
13 \ldots \\
14 \ldots \\
15 \ldots \\
16 \ldots \\
17 \ldots \\
18 \ldots \\
19 \ldots \\
20 \ldots\end{array}$ & $\begin{array}{r}8,080 \\
10,600 \\
12,300 \\
12,600 \\
12,200 \\
11,600 \\
10,900 \\
10,000 \\
9,220 \\
8,460\end{array}$ & $\begin{array}{l}3,260 \\
2,930 \\
2,540 \\
2,400 \\
2,490 \\
2,710 \\
2,590 \\
2,620 \\
2,610 \\
2,570\end{array}$ & $\begin{array}{l}21 \ldots \\
22 \\
23 \ldots \\
24 \ldots \\
25 \ldots \\
26 \ldots \\
27 \ldots \\
28 \ldots \\
29 \ldots \\
30 \ldots \\
31 \ldots\end{array}$ & $\begin{array}{l}7,800 \\
7,170 \\
6,900 \\
6,500 \\
6,110 \\
5,700 \\
5,390 \\
5,100 \\
4,820 \\
4,620\end{array}$ & $\begin{array}{l}2,670 \\
2,660 \\
3,340 \\
4,880 \\
4,400 \\
4,100 \\
3,940 \\
3,640 \\
3,490 \\
3,520 \\
3,480\end{array}$ \\
\hline \multicolumn{7}{|c|}{$\begin{array}{l}\text { Monthly mean discharge, in cubic feet per second } \\
\text { Runoff, in inches }\end{array}$} & $\begin{array}{r}5,980 \\
1.08\end{array}$ & $\begin{array}{r}3,429 \\
0.64\end{array}$ \\
\hline
\end{tabular}

Gage height, in feet, and discharge, in cubic feet per second, at indicated time, 1965

\begin{tabular}{|c|c|c|c|c|c|c|c|c|c|c|c|}
\hline Date & Hour & $\begin{array}{c}\text { Gage } \\
\text { height }\end{array}$ & $\begin{array}{c}\text { Dis - } \\
\text { charge }\end{array}$ & Date & Hour & $\begin{array}{l}\text { Gage } \\
\text { height }\end{array}$ & $\begin{array}{c}\text { Dis- } \\
\text { charge }\end{array}$ & Date & Hour & $\begin{array}{c}\text { Gage } \\
\text { height }\end{array}$ & $\begin{array}{l}\text { Dis- } \\
\text { charge }\end{array}$ \\
\hline Apr. 5 & 0000 & 4.69 & ---- & Apr. 9 & 2400 & 13.50 & 4,600 & Apr. 15 & 2400 & 16.37 & 12,000 \\
\hline & 1200 & 4.67 & -- & 10 & 0600 & & 5.100 & & & & \\
\hline & 1500 & 4.69 & & 10 & $\begin{array}{l}0000 \\
1200\end{array}$ & $\begin{array}{l}14.09 \\
14.72\end{array}$ & $\begin{array}{l}5,100 \\
5,980\end{array}$ & 16 & 1200 & 16.26 & 11,600 \\
\hline & 2100 & 4.85 & - & & 1500 & 1502 & 6.520 & & 1500 & 16.24 & 11,500 \\
\hline & 2400 & 5.12 & - & & $\begin{array}{l}1500 \\
1800\end{array}$ & $\begin{array}{l}15.02 \\
15.21\end{array}$ & $\begin{array}{l}0,020 \\
6,920\end{array}$ & & 1800 & 16.27 & 11,600 \\
\hline & 0600 & & & & 2100 & 15.20 & 6,900 & & 2400 & 16.15 & 11,200 \\
\hline 6 & 0600 & 5.89 & & & 2400 & 15.17 & 6,830 & & & & \\
\hline & 1200 & 6.39 & -- & & & & & 17 & 0600 & 16.11 & 11,100 \\
\hline & 1800 & 6.72 & $-\cdots$ & 11 & 0600 & 15.32 & 7,190 & & 1200 & 16.06 & 10,900 \\
\hline & 2100 & 7.01 & $---\cdot-$ & & 1200 & 15.60 & 8,040 & & 1800 & 16.00 & 10,700 \\
\hline & 2400 & 7.13 & ----- & & 1800 & 15.85 & 8,940 & & 2400 & 15.94 & 10,500 \\
\hline & & & & & 2400 & 16.00 & 9,480 & & & & \\
\hline 7 & 0300 & 7.08 & -- & & & & & 18 & 0600 & 15.84 & 10,200 \\
\hline & 0600 & 6.92 & $--\cdots$ & 12 & 0600 & 16.16 & 10,100 & & 1200 & 15.80 & 10,000 \\
\hline & 0900 & 6.76 & $--\cdot-$ & & 1200 & 16.30 & 10,700 & & 1800 & 15.73 & 9,780 \\
\hline & 1200 & 6.73 & $\ldots-\cdots$ & & 1800 & 16.38 & 11,200 & & 2400 & 15.66 & 9,540 \\
\hline & 1500 & 7.09 & $\ldots$ & & 2400 & 16.48 & 11,700 & & & & \\
\hline & 1800 & 7.73 & ---- & & & & & 19 & 0600 & 15.60 & 9,340 \\
\hline & 2100 & 8.38 & $-\cdots--$ & 13 & 0600 & 16.54 & 12,100 & & 1200 & 15.58 & 9,270 \\
\hline & 2400 & 9.31 & & & 0900 & 16.59 & 12,300 & & 1800 & 15.52 & 9,070 \\
\hline & & & & & 1200 & 16.55 & 12,200 & & 2400 & 15.45 & 8,830 \\
\hline 8 & 0300 & 30.17 & - & & 1800 & 16.58 & 12,500 & & & & \\
\hline & 0600 & 10.70 & ---- & & 2400 & 16.59 & 12,700 & 20 & 0600 & 15.42 & 8,730 \\
\hline & 0900 & 11.07 & $\ldots$ & & & & & & 1200 & 15.32 & 8,400 \\
\hline & 1200 & 11.33 & & 14 & 0300 & 16.64 & 12,900 & & 1800 & 15.26 & 8,220 \\
\hline & 1800 & 11.83 & & & 0600 & 16.57 & 12,600 & & 2400 & 15.23 & 8,130 \\
\hline & 2400 & 12.33 & 3,900 & & 1200 & 16.59 & 12,700 & & & & \\
\hline & & & & & 1800 & 16.56 & 12,600 & 21 & 0600 & 15.17 & 7,960 \\
\hline 9 & 0600 & 12.68 & 4,110 & & 2400 & 16.50 & 12,400 & & 1200 & 15.12 & 7,820 \\
\hline & 1200 & 12.87 & 4,220 & & & & & & 1800 & 15.05 & 7,630 \\
\hline & 1800 & 13.01 & 4,310 & 15 & 1200 & 16.46 & 12,300 & & 2400 & 14.99 & 7,480 \\
\hline & 2100 & 13.15 & 4,390 & & & & & & & & \\
\hline
\end{tabular}


(55) 5-3114. South Branch Yellow Medicine River at Minneota, Minn.

Location.-Lat $44^{\circ} 33^{\prime} 50^{\prime \prime}$, long $95^{\circ} 59^{\prime} 50^{\prime \prime}$, in SE $\frac{1}{4}$ sec. 26 , T.113 N., R.43 W., on downstream side of bridge on State Highway $68,0.5$ mile northwest of Minneota, and 6 miles upstream from confluence with North Branch Yellow Medicine R ver.

Drainage area.-111 sq $\mathrm{mi}$, approximately.

Gage-height record.-Graph drawn on basis of once-daily wire-weight gage readings Apr. 1-18 and May 9-12. Once-daily gage readings all other periods. Datum of gage is $1,150.00 \mathrm{ft}$ above mean sea level, datum of 1929 .

Discharge record.-Stage-discharge relation defined by current-meter measurements. Backwater from ice Apr. 1-11.

Maxima.-April-May 1965: Discharge, 1,360 cfs 1300 hours Apr. 6 (gage height, $10.62 \mathrm{ft}$, backwater from ice).

1960 to March 1965: Discharge, 1,830 cfs Apr. 6, 1960 (gage height, $11.10 \mathrm{ft}$ ).

Mean discharge, in cubic feet per second, 1965

\begin{tabular}{|c|c|c|c|c|c|c|c|c|}
\hline Day & April & May & Day & April & May & Day & Apr ${ }^{\prime 1}$ & May \\
\hline $\begin{array}{l}1 \\
2 \\
2 \\
3 \\
4 \\
5 \\
6 \\
6 \\
7 \\
8 \\
9 \\
10\end{array}$ & $\begin{array}{r}0 \\
0 \\
0 \\
0 \\
100 \\
900 \\
860 \\
820 \\
770 \\
710\end{array}$ & $\begin{array}{r}43 \\
39 \\
40 \\
36 \\
36 \\
37 \\
39 \\
41 \\
136 \\
196\end{array}$ & $\begin{array}{l}11 \ldots \\
12 \\
13 \\
14 \\
15 \\
16 \\
17 \\
18 \\
19 \\
20\end{array}$ & $\begin{array}{r}620 \\
472 \\
329 \\
240 \\
172 \\
117 \\
113 \\
105 \\
81 \\
67\end{array}$ & $\begin{array}{r}137 \\
83 \\
64 \\
54 \\
64 \\
120 \\
86 \\
64 \\
54 \\
47\end{array}$ & $\begin{array}{l}21 \\
22 \\
23 \\
24 \\
25 \\
26 \\
27 \\
28 \\
28 \\
29 \\
30 \\
31\end{array}$ & $\begin{array}{r}62 \\
56 \\
53 \\
51 \\
6 气 \\
7 € \\
6 \varepsilon \\
5 € \\
4 £ \\
44 \\
-\end{array}$ & $\begin{array}{r}132 \\
102 \\
68 \\
98 \\
137 \\
123 \\
95 \\
75 \\
62 \\
60 \\
57\end{array}$ \\
\hline \multicolumn{7}{|c|}{ Monthly mean discharge, in cubic feet per second } & 235 & 78.2 \\
\hline
\end{tabular}

Gage height, in feet, and discharge, in cubic feet per second, at indicated time, 1965

\begin{tabular}{|c|c|c|c|c|c|c|c|c|c|c|c|}
\hline Date & Hour & $\begin{array}{l}\text { Gage } \\
\text { height }\end{array}$ & $\begin{array}{c}\text { Dis- } \\
\text { charge }\end{array}$ & Date & Hour & $\begin{array}{l}\text { Gage } \\
\text { height }\end{array}$ & $\begin{array}{c}\text { Dis- } \\
\text { charge }\end{array}$ & Date & Hour & $\begin{array}{c}\text { Gage } \\
\text { height }\end{array}$ & $\begin{array}{c}\text { Dis- } \\
\text { charge }\end{array}$ \\
\hline Apr. 6 & $\begin{array}{l}0000 \\
0300 \\
0400 \\
0500 \\
0700 \\
1300 \\
1800 \\
2400 \\
0600\end{array}$ & $\begin{array}{r}6.26 \\
7.21 \\
8.28 \\
9.00 \\
10.05 \\
10.62 \\
10.22 \\
9.65 \\
9.40\end{array}$ & 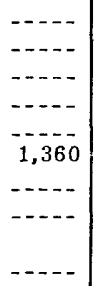 & Apr. 7 & $\begin{array}{l}1400 \\
2400 \\
0700 \\
1200 \\
2400 \\
0900 \\
2400\end{array}$ & $\begin{array}{l}9.64 \\
9.54 \\
9.53 \\
9.38 \\
9.34 \\
9.30 \\
9.15\end{array}$ & 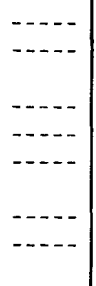 & Apr. 10 & $\begin{array}{l}1200 \\
2400 \\
0800 \\
2400 \\
2400\end{array}$ & $\begin{array}{l}9.10 \\
9.12 \\
9.25 \\
8.43 \\
7.52\end{array}$ & $\begin{array}{l} \\
- \\
372\end{array}$ \\
\hline
\end{tabular}


(56) 5-3135. Yellow Medicine River near Granite Falls, Minn.

Location.-Lat $44^{\circ} 43^{\prime}$, long $95^{\circ} 31^{\prime}$, in sec.35, T.115 N., R.39 W., on right bank $50 \mathrm{ft}$ downstream from highway bridge, 6 miles upstream from mouth, and 8 miles south of town of Granite Falls.

Drainage area. $-653 \mathrm{sq} \mathrm{mi}$.

Gage-height record.-Water-stage recorder graph except for period 1900 hours Apr. 9 to 1300 hours Apr. 11 when graph was drawn based on four chain gage readings.

Datum of gage is $971.59 \mathrm{ft}$ above mean sea level, datum of 1929 .

Discharge record.-Stage-discharge relation defined by current-meter measurements. Backwater from ice Apr. 1-9.

Maxima.-April-May 1965: Discharge, 6,820 cfs 0630 hours Apr. 10 (gage height, $\overline{9} .78 \mathrm{ft}$, from floodmark).

1931-38, 1939 to March 1965: Discharge, 11,800 cfs June 18, 1957 (gage height, $12.41 \mathrm{ft})$.

Flood in June, 1919 reached a stage of $17.5 \mathrm{ft}$, from information by local residents.

Mean discharge, in cubic feet per second, 1965

\begin{tabular}{|c|c|c|c|c|c|c|c|c|}
\hline Day & April & May & Day & April & May & Day & April & May \\
\hline $\begin{array}{l}1 \ldots \ldots \\
2 \ldots \\
3 \ldots \\
4 \ldots \\
5 \ldots \\
6 \ldots \\
7 \ldots \\
8 \ldots \\
9 \ldots \\
10 \ldots\end{array}$ & $\begin{array}{r}3.5 \\
3.5 \\
3.5 \\
4.0 \\
4.5 \\
20 \\
100 \\
400 \\
2,000 \\
6,140\end{array}$ & $\begin{array}{l}310 \\
274 \\
236 \\
219 \\
212 \\
195 \\
185 \\
192 \\
230 \\
326\end{array}$ & $\begin{array}{l}11 \ldots \ldots \\
12 \ldots \ldots \\
13 \ldots \ldots \\
14 \ldots \\
15 \ldots \ldots \\
16 \ldots \ldots \\
17 \ldots \ldots \\
18 \ldots \ldots \\
19 \ldots \ldots \\
20 \ldots \ldots\end{array}$ & $\begin{array}{l}5,120 \\
4,440 \\
3,700 \\
2,930 \\
2,400 \\
2,030 \\
1,740 \\
1,440 \\
1,190 \\
1,000\end{array}$ & $\begin{array}{l}565 \\
495 \\
410 \\
326 \\
297 \\
297 \\
352 \\
360 \\
310 \\
270\end{array}$ & $\mid \begin{array}{l}21 \\
22 \ldots \ldots \\
23 \ldots \ldots \\
24 \ldots \\
25 \ldots \ldots \\
26 \ldots \\
27 \ldots \\
28 \ldots \\
29 \ldots \\
30 \ldots \\
31 \ldots\end{array}$ & $\begin{array}{r}826 \\
700 \\
605 \\
535 \\
500 \\
490 \\
495 \\
476 \\
414 \\
356 \\
-\end{array}$ & $\begin{array}{r}289 \\
343 \\
712 \\
670 \\
1,060 \\
1,560 \\
1,750 \\
1,620 \\
1,280 \\
1,010 \\
838\end{array}$ \\
\hline \multicolumn{7}{|c|}{$\begin{array}{l}\text { Monthly mean discharge, in cubic feet per second } \\
\text { Runoff, in inches }\end{array}$} & $\begin{array}{r}1,336 \\
2.28\end{array}$ & $\begin{array}{r}555 \\
0.98\end{array}$ \\
\hline
\end{tabular}

Gage height, in feet, and discharge, in cubic feet per second, at indicated time, 1965

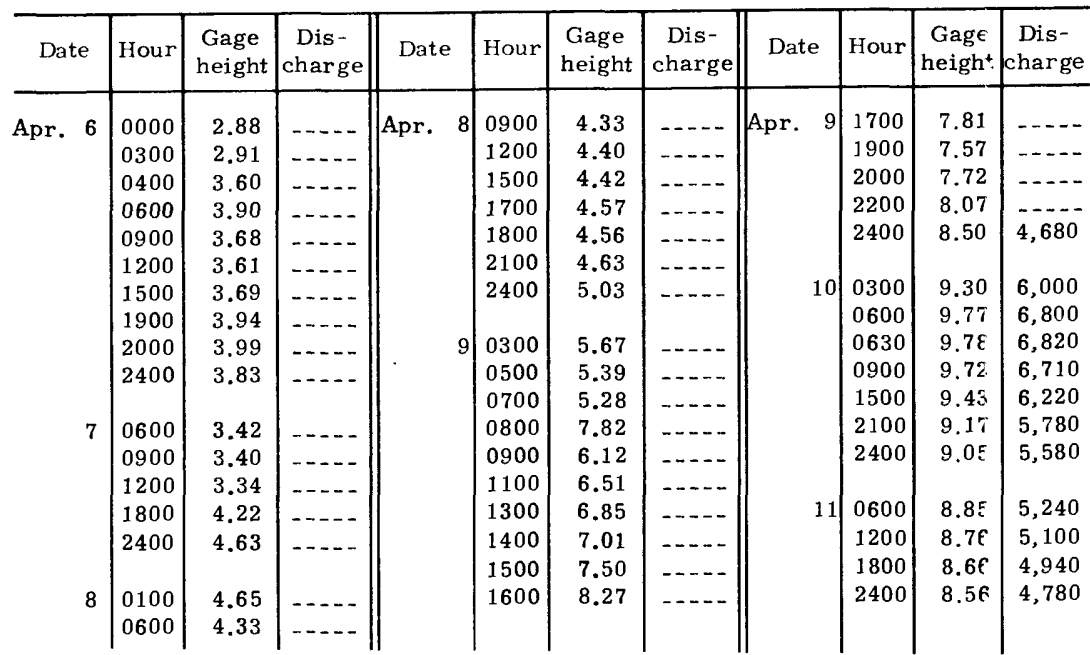


Gage height, in feet. and discharge, in cubic feet per second, at indicated time, 1965, of Yellow Medicine River near Granite Falls, Minn. - Continued

\begin{tabular}{|c|c|c|c|c|c|c|c|c|c|c|c|}
\hline Date & Hour & $\begin{array}{c}\text { Gage } \\
\text { height }\end{array}$ & $\begin{array}{c}\text { Dis- } \\
\text { charge }\end{array}$ & Date & Hour & $\begin{array}{c}\text { Gage } \\
\text { height }\end{array}$ & $\begin{array}{c}\text { Dis- } \\
\text { charge }\end{array}$ & Date & Hour & $\begin{array}{c}\text { Gage } \\
\text { height }\end{array}$ & $\begin{array}{c}\text { Dis- } \\
\text { charge }\end{array}$ \\
\hline Apr. 12 & $\begin{array}{l}1200 \\
2400 \\
1200 \\
2400\end{array}$ & $\begin{array}{l}8.34 \\
8.12 \\
7.83 \\
7.48\end{array}$ & $\begin{array}{l}4,440 \\
4,120 \\
3,710 \\
3,270\end{array}$ & Apr. 14 & $\begin{array}{l}1200 \\
2400 \\
1200\end{array}$ & $\begin{array}{l}7.16 \\
6.89 \\
6.61\end{array}$ & $\begin{array}{l}2,910 \\
2,640 \\
2,390\end{array}$ & Apr. 15 & $\begin{array}{l}2400 \\
1200 \\
2400\end{array}$ & $\begin{array}{l}6.37 \\
6.20 \\
5.98\end{array}$ & $\begin{array}{l}2,180 \\
2,040 \\
1,860\end{array}$ \\
\hline
\end{tabular}

(57) 5-3150. Redwood River at Marshall, Minn.

Location.-Lat $44^{\circ} 27^{\prime} 05^{\prime \prime}$, long $95^{\circ} 47^{\prime} 13^{\prime \prime}$, in $\mathrm{SE}_{\frac{1}{4}} \mathrm{NW} \frac{1}{4}$ sec.4, T.111 N., R.41 W., on upstream side of highway bridge on Fourth Street in Marshall and 10 miles upstream from Threemile Creek.

Drainage area. $-307 \mathrm{sq} \mathrm{mi}$.

Gage-height record.-Graph drawn on basis of two or more daily chain ge ge readings Apr. 4-18 on river channel. Graph drawn on basis of one or more daily readings on staff gage Apr. 4-17 on diversion channel. One or more daily readings all other periods. Datum of gage is $1,144.88 \mathrm{ft}$ above mean sea level, datum of 1929 .

Discharge record.-Stage-discharge relation defined by current-meter maasurements. Backwater from ice Apr. 1-11 on river, Apr. 1-7 on diversion channel.

Maxima.-April-May 1965: Discharge, 2,220 cfs 1500 hours Apr. 9 (includes flow in diversion).

River channel: Discharge, 1,150 cfs 1400 hours Apr. 11 (gage height, $5.70 \mathrm{ft}$, backwater from ice); gage height, $5.76 \mathrm{ft}, 1500$ hours Apr. 6 (from floodmark, backwater from ice).

Diversion channel: Discharge, 1,850 cfs 1500 hours Apr. 9 (gage height, $77.70 \mathrm{ft}$ ); gage height, $78.07 \mathrm{ft}, 1200$ hours Apr. 6 (backwater from ice).

1940 to March 1965: Discharge, $5,370 \mathrm{cfs}$ June 17, 1957 (gage height, $10.14 \mathrm{ft}$ ); gage height, $11.05 \mathrm{ft}$ Apr, 6, 1951 (from floodmark).

Remarks.-Water diverted at medium and high stages into diversion channel 3 miles above station, bypasses station and returns to river 1 mile below station.

Diversion began Mar. 18, 1964. These records include flow in diversion channel. Unknown amount of natural diversion into Cottonwood River basin at extremely high stages.

Mcan discharge, in cubic feet per second, 1965

\begin{tabular}{|c|c|c|c|c|c|c|c|c|}
\hline Day & April & May & Day & April & May & Day & April & May \\
\hline $\begin{array}{l}1 \ldots \ldots \\
2 \ldots \ldots \\
3 \ldots \ldots \\
4 \ldots \ldots \\
5 \ldots \ldots \\
6 \ldots \ldots \\
7 \ldots \ldots \\
8 \ldots \ldots \\
9 \ldots \ldots \\
10 \ldots\end{array}$ & $\begin{array}{r}3.0 \\
3.2 \\
3.3 \\
3.5 \\
138 \\
574 \\
758 \\
1,500 \\
1,880 \\
1,500\end{array}$ & $\begin{array}{l}143 \\
127 \\
115 \\
114 \\
115 \\
110 \\
104 \\
101 \\
174 \\
292\end{array}$ & $\mid \begin{array}{l}11 \ldots \\
12 \ldots \ldots \\
13 \ldots \ldots \\
14 \ldots \ldots \\
15 \ldots \\
16 \ldots \\
17 \ldots \\
18 \ldots \\
19_{\ldots} \ldots \\
20 \ldots\end{array}$ & $\begin{array}{r}1,560 \\
1,110 \\
979 \\
864 \\
761 \\
616 \\
534 \\
440 \\
373 \\
320\end{array}$ & $\begin{array}{l}301 \\
232 \\
186 \\
176 \\
242 \\
290 \\
285 \\
244 \\
212 \\
189\end{array}$ & $\begin{array}{l}21 \ldots \ldots \\
22 \ldots \ldots \\
23 \ldots \ldots \\
24 \ldots \ldots \\
25 \ldots \ldots \\
26 \ldots \ldots \\
27 \ldots \ldots \\
28 \ldots \ldots \\
29 \ldots \ldots \\
30 \ldots \ldots \\
31 \ldots \ldots\end{array}$ & $\begin{array}{l}285 \\
255 \\
228 \\
223 \\
246 \\
260 \\
235 \\
198 \\
176 \\
159 \\
\ldots-\ldots\end{array}$ & $\begin{array}{l}207 \\
196 \\
205 \\
200 \\
218 \\
239 \\
239 \\
193 \\
174 \\
159 \\
143\end{array}$ \\
\hline \multicolumn{7}{|c|}{$\begin{array}{l}\text { Monthly mean discharge, in cubic feet per second } \\
\text { Runoff, in inches. }\end{array}$} & $\begin{array}{r}540 \\
1.96\end{array}$ & $\begin{array}{r}191 \\
0.72\end{array}$ \\
\hline
\end{tabular}


MARCH-MAY, UPPER MISSISSIPPI RIVER BASIN

A189

Gage height. in feet, and discharge, in cubic feet per second, at indicated time, 1965, of Redwood River at Marshall, Minn.

\begin{tabular}{|c|c|c|c|c|c|c|c|c|c|c|c|}
\hline Date & Hour & $\begin{array}{l}\text { Gage } \\
\text { height }\end{array}$ & $\begin{array}{c}\text { Dis - } \\
\text { charge }\end{array}$ & Date & Hour & $\begin{array}{l}\text { Gage } \\
\text { height }\end{array}$ & $\begin{array}{c}\text { Dis- } \\
\text { charge }\end{array}$ & Date & Hour & $\begin{array}{c}\text { Gage } \\
\text { height }\end{array}$ & $\begin{array}{c}\text { Dis- } \\
\text { charge }\end{array}$ \\
\hline \multirow[t]{6}{*}{ Apr. 4} & 0000 & 2.07 & $\ldots$ & Apr. 8 & 0800 & 2.98 & & Apr. 12 & 0600 & 4.13 & 741 \\
\hline & 0600 & 2.29 & $\ldots$ & & 0900 & 3.30 & & & 1200 & 4.00 & 699 \\
\hline & 1200 & 2.78 & $\ldots$ & & 1000 & 4.50 & & & 2400 & 3.93 & 677 \\
\hline & 1600 & 3.50 & & & 1200 & 5.00 & & & & & \\
\hline & 2000 & 3.13 & $\ldots$ & & 1500 & 5.76 & & 13 & 1200 & 3.86 & 655 \\
\hline & 2400 & 3.01 & $\ldots$ & & $\begin{array}{l}1800 \\
2400\end{array}$ & $\begin{array}{l}4.90 \\
4.36\end{array}$ & & & 2400 & 3.73 & 615 \\
\hline \multirow[t]{4}{*}{5} & 0800 & 2.87 & & & & & & 14 & 1200 & 3.53 & 555 \\
\hline & 1000 & 2.78 & & 9 & 0600 & 4.31 & 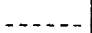 & & 2400 & 3.51 & 549 \\
\hline & 2400 & 2.75 & & & 1000 & 4.48 & -- & & & & \\
\hline & & & & & 1200 & 4.70 & $\ldots$ & 15 & 0800 & 3.51 & 549 \\
\hline \multirow[t]{8}{*}{6} & 0800 & 2.98 & $\ldots$ & & 1400 & 5.00 & $\ldots . .$. & & 2400 & 3.30 & 490 \\
\hline & 0900 & 3.30 & $\ldots$ & & 2400 & 4.67 & $\ldots$ & & & & \\
\hline & 1000 & 4.50 & $\ldots$ & & & & & 16 & 1200 & 3.22 & 469 \\
\hline & 1200 & 5.00 & $-\ldots$ & 10 & 0800 & 4.68 & 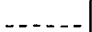 & & 2400 & 3.11 & 441 \\
\hline & 1500 & 5.76 & $\ldots$ & & 1800 & 4.46 & & & & & \\
\hline & 1800 & 4.90 & $\ldots$ & & 2400 & 4.41 & & 17 & 1000 & 3.16 & 454 \\
\hline & 2400 & 4.36 & $\ldots \ldots$ & & & & & & 1200 & 3.00 & 414 \\
\hline & & & & 11 & 0800 & 4.92 & $\ldots$ & & & & \\
\hline \multirow[t]{3}{*}{7} & 0800 & 4.53 & $\ldots$ & & 1400 & 5.70 & 1,150 & 18 & 1200 & 2.95 & 402 \\
\hline & 1400 & 5.04 & $-\ldots$ & & 2400 & 4.74 & 936 & & 2400 & 2.87 & 384 \\
\hline & 2400 & 4.56 & $-\cdots$ & & & & & & & & \\
\hline
\end{tabular}

Gage height, in feet, and discharge, in cubic feet per second, at indicated time, 1965, of Redwood River diversion channel at Marshall, Minn.

\begin{tabular}{|c|c|c|c|c|c|c|c|c|c|c|c|c|}
\hline Date & Hour & $\begin{array}{l}\text { Gage } \\
\text { height }\end{array}$ & $\begin{array}{c}\text { Dis- } \\
\text { charge }\end{array}$ & \multicolumn{2}{|c|}{ Date } & Hour & $\begin{array}{l}\text { Gage } \\
\text { height }\end{array}$ & $\begin{array}{c}\text { Dis- } \\
\text { charge }\end{array}$ & Date & Hour & $\begin{array}{c}\text { Gage } \\
\text { height }\end{array}$ & $\begin{array}{c}\text { Dis- } \\
\text { charge }\end{array}$ \\
\hline Apr. 5 & 0000 & 73.83 & $\ldots \ldots$ & Apr. & 9 & 0600 & 76.59 & 1,420 & Apr. 12 & 1000 & 73.26 & 321 \\
\hline & 0700 & 73.85 & $\ldots$ & & & 0800 & 76.90 & 1,540 & & 1600 & 73.21 & 306 \\
\hline & 1700 & 73.53 & $\ldots \ldots$ & & & 0900 & 76.77 & 1,490 & & 2400 & 73.23 & 321 \\
\hline & 2400 & 73.47 & $\ldots$ & & & 1200 & 77.08 & 1,610 & & & & \\
\hline & & & & & & 1500 & 77.70 & 1,850 & 13 & 1200 & 73.31 & 336 \\
\hline 6 & 0300 & 73.49 & - & & & 2400 & 76.72 & 1,470 & & 2400 & 73.27 & 324 \\
\hline & 0600 & 74.57 & $-\ldots$ & & & & & & & & & \\
\hline & 0800 & 77.12 & $\ldots \ldots$ & & 10 & 0600 & 75.95 & 1,180 & 14 & 1200 & 73.18 & 297 \\
\hline & 1200 & 78.07 & 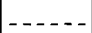 & & & 1400 & 75.60 & 1,060 & & 2400 & 73.03 & 267 \\
\hline & 1800 & 77.68 & - . - . - & & & 1800 & 75.13 & 896 & & & & \\
\hline & 2400 & 77.00 & 1,580 & & & 2400 & 74.96 & 836 & 15 & 1200 & 72.97 & 234 \\
\hline & & & & & & & & & & 2400 & 72.82 & 191 \\
\hline 7 & 0300 & 76.53 & 1,400 & & 11 & 0600 & 74.76 & 771 & & & & \\
\hline & 1200 & 76.64 & 1,440 & & & 1200 & 74.68 & 747 & 16 & 1200 & 72.63 & 145 \\
\hline & 2100 & 76.80 & 1,500 & & & 1800 & 74.64 & 735 & & 2400 & $72.5^{n}$ & 116 \\
\hline & 2400 & 76.70 & 1,460 & & & 2400 & 74.22 & 609 & & & & \\
\hline & & & & & & & & & 17 & 1200 & $72.4 n$ & 94 \\
\hline 8 & 1200 & 76.29 & 1,310 & & 12 & 0600 & 73.57 & 414 & & 2400 & 72.30 & 72 \\
\hline & 2400 & 76.12 & 1,240 & & & & & & & & & \\
\hline
\end{tabular}


(58) 5-3165. Redwood River near Redwood Falls, Minn.

Location.-Lat $44^{\circ} 31^{\prime} 25^{\prime \prime}$, long $95^{\circ} 10^{\prime} 20^{\prime \prime}$, in $\operatorname{SE} \frac{1}{4} \mathrm{NE} \frac{1}{4}$ sec.9, T.112 N., R.36 W., on right bank $20 \mathrm{ft}$ upstream from highway bridge, 3 miles west of town of Redwood Falls, and 8.5 miles upstream from mouth.

Drainage area. $-697 \mathrm{sq} \mathrm{mi}$.

Gage-height record.-Water-stage recorder graph. Datum of gage is $972.33 \mathrm{ft}$ above mean sea level, datum of 1929.

Discharge record.-Stage-discharge relation defined by current-meter measurements. Backwater from ice Apr. 1-10.

Maxima.-April-May 1965: Discharge, 7,050 cfs 0400 hours Apr. 9 (gage height, $13.32 \mathrm{ft}$, backwater from ice); gage height, $15.88 \mathrm{ft} 2400$ hours Apr. 8 (kackwater from ice).

1909-14, 1930 to March 1965: Discharge, 19,700 cfs June 18, 1957 (ge ge height, $15.92 \mathrm{ft}$, from floodmark).

Remarks.- Natural discharge affected by unknown amount of interbasin flow between Yellow Medicine, Redwood and Cottonwood River basins during extreme floods.

Mean discharge, in cubic feet per second, 1965

\begin{tabular}{|c|c|c|c|c|c|c|c|c|}
\hline Day & April & May & Day & April & May & Day & April & May \\
\hline $\begin{array}{l}1 \\
1 \\
2 \\
3 \\
4 \\
5 \\
6 \ldots \ldots \\
7 \\
8 \\
9 \\
9 \\
10\end{array}$ & $\begin{array}{r}2.0 \\
4.0 \\
9.0 \\
80 \\
300 \\
650 \\
1,000 \\
3,900 \\
6,600 \\
5,940\end{array}$ & $\begin{array}{l}336 \\
308 \\
276 \\
248 \\
230 \\
223 \\
220 \\
206 \\
223 \\
276\end{array}$ & $\mid \begin{array}{l}11 \ldots \ldots \\
12 \ldots \ldots \\
13 \ldots \ldots \\
14 \ldots \\
15 \ldots \\
16 \ldots \\
17 \ldots \\
18 \ldots \\
19 \ldots \\
20 \ldots\end{array}$ & $\begin{array}{r}5,270 \\
4,260 \\
3,210 \\
2,520 \\
2,120 \\
1,820 \\
1,540 \\
1,260 \\
992 \\
810\end{array}$ & $\begin{array}{l}375 \\
410 \\
370 \\
332 \\
340 \\
405 \\
430 \\
430 \\
385 \\
344\end{array}$ & $\mid \begin{array}{l}21 \\
22 \\
23 \\
23 \\
24 \ldots \\
25 \ldots \\
26 \ldots- \\
27 \ldots \\
28 \ldots \\
29-\ldots \\
30 \ldots \\
31\end{array}$ & $\begin{array}{l}708 \\
624 \\
552 \\
495 \\
495 \\
522 \\
510 \\
480 \\
430 \\
370\end{array}$ & $\begin{array}{l}328 \\
332 \\
320 \\
332 \\
365 \\
385 \\
410 \\
410 \\
370 \\
332 \\
304\end{array}$ \\
\hline \multicolumn{7}{|c|}{$\begin{array}{l}\text { Monthly mean discharge, in cubic feet per second } \\
\text { Runoff, in inches }\end{array}$} & $\begin{array}{r}1,580 \\
2.53\end{array}$ & $\begin{array}{r}331 \\
0.55\end{array}$ \\
\hline
\end{tabular}

Gage height, in feet, and discharge, in cubic feet per second, at indicated time, 1965

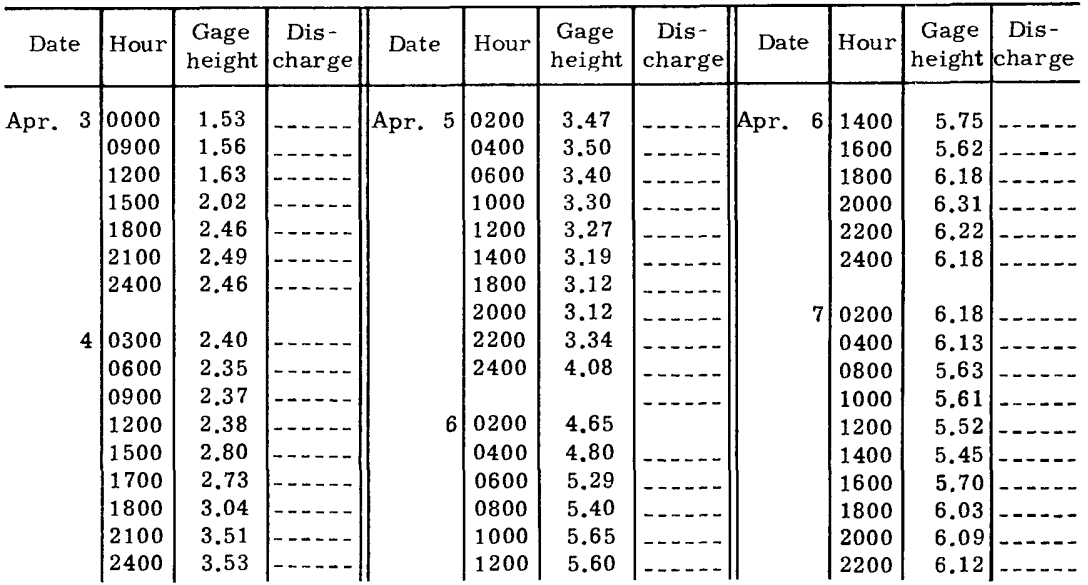


MARCH-MAY, UPPER MISSISSIPPI RIVER BASIN

Gage height, in feet, and discharge, in cubic feet per second, at indicated time, 1965, of Redwood River near Redwood Falls, Minn. - Continued

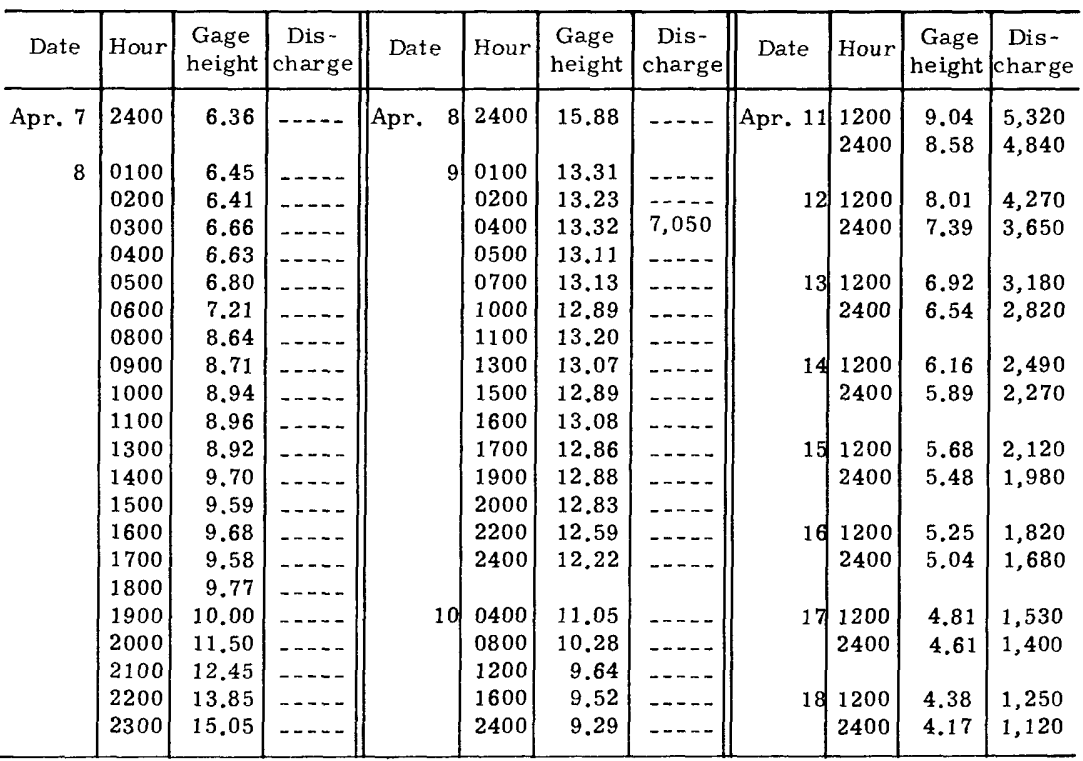

(59) 5-3167, Spring Creek near Sleepy Eye, Minn.

(Crest-stage station)

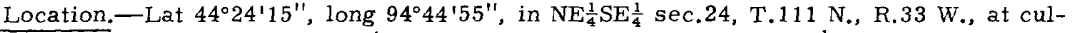
vert on county highway, $3-3 / 4$ miles upstream from mouth and $7 \frac{1}{2}$ miles north of Sleepy Eye.

Drainage area. $-30.0 \mathrm{sq} \mathrm{mi}$.

Gage-height record.-Crest stages only.

Discharge record.--Stage-discharge relation defined by current-meter measurements below $88 \mathrm{cfs}$ and by indirect measurements at $149 \mathrm{cfs}, 416 \mathrm{cfs}$, and $740 \mathrm{cfs}$.

Maxima.-April-May 1965: Discharge, 930 cfs Apr. 10 (gage height, $17.79 \mathrm{ft}$ ).

1959 to March 1965: Discharge, $680 \mathrm{cfs}$ July 7, 1962 (gage height, $15.89 \mathrm{ft}$ ). 


\section{(Crest-stage station)}

Location.-Lat $44^{\circ} 07^{\prime} 25^{\prime \prime}$, long $95^{\circ} 12^{\prime} 10^{\prime \prime}$, in $\mathrm{NE} \frac{1}{4} \mathrm{NE} \frac{1}{4}$ sec. $31, \mathrm{~T} .108$ N., R.36 W., on right bank $17 \mathrm{ft}$ upstream from culvert on County Highway 10, $4 \frac{1}{2}$ miles north of Jeffers.

Drainage area.- $-3.24 \mathrm{sq} \mathrm{mi}$.

Gage-height record.-Water-stage recorder graph.

Discharge record.- Stage-discharge relation defined by current-meter measurements below $25 \mathrm{cfs}$ and by indirect measurement at $508 \mathrm{cfs}$.

Maxima,-April-May 1965: Discharge, 400 cfs Apr, 6 (gage height, $10.64 \mathrm{ft}$, backwater from ice).

1961 to March 1965: Discharge, 508 cfs July 19, 1963 (gage height, $10.07 \mathrm{ft}$ ).

(61) 5-3170. Cottonwood River near New Ulm, Minn.

Location.-Lat $44^{\circ} 17^{\prime} 40^{\prime \prime}$, long $94^{\circ} 26^{\prime} 40^{\prime \prime}$, in $\mathrm{N}^{\frac{1}{2}}$ sec. 33 , T.110 N., R.30 W., on left bank $600 \mathrm{ft}$ upstream from highway bridge, 1.8 miles south of New Ulm, and 2 miles upstream from mouth.

Drainage area.--1,280 sq $\mathrm{mi}$, approximately.

Gage-height record.-Water-stage recorder graph except for the period 2200 hours Apr. 6 to 1700 hours Apr. 28 when graph was drawn on the basis of one or more daily readings on temporary staff gage or inside staff gage. Datum of gage is $799.09 \mathrm{ft}$ above mean sea level, datum of 1912 .

Discharge record.--Stage-discharge relation defined by current-meter measurements. Backwater from ice Apr. 1-9.

Maxima.-April-May 1965: Discharge 26,000 cfs 1615 hours Apr. 8 (gage height, $20.86 \mathrm{ft}$, backwater from ice).

1909-13, 1931 to March 1965: Discharge, 14,200 cfs Mar. 30, 1962 (gage height, $15.88 \mathrm{ft}$ ); gage-height, $16.94 \mathrm{ft}$ July 9, 1947 (from floodmark).

Remarks. - Some regulation by dam at Cottonwood Lake and several other small lakes above the station.

Mean discharge, in cubic feet per second, 1965

\begin{tabular}{|c|c|c|c|c|c|c|c|c|}
\hline Day & April & May & Day & April & May & Day & April & May \\
\hline $\begin{array}{l}1 \ldots \ldots \\
2 \ldots \ldots \\
3 \ldots \ldots \\
4 \ldots \ldots \\
5 \ldots \ldots \\
6 \ldots \ldots \\
7 \ldots \ldots \\
8 \ldots \ldots \\
9 \ldots \ldots \\
10 \ldots \ldots\end{array}$ & $\begin{array}{r}16 \\
17 \\
20 \\
80 \\
350 \\
1,640 \\
10,700 \\
23,000 \\
21,000 \\
15,900\end{array}$ & $\begin{array}{r}985 \\
904 \\
845 \\
778 \\
727 \\
698 \\
712 \\
1,160 \\
4,030 \\
3,800\end{array}$ & $\mid \begin{array}{l}11 \ldots \\
12 \ldots \\
13 \ldots \\
14 \ldots \\
15 \ldots \\
16 \ldots \\
17 \ldots \ldots \\
18 \ldots \\
19 \ldots \\
20 \ldots\end{array}$ & $\begin{array}{r}14,100 \\
11,700 \\
8,940 \\
7,010 \\
5,340 \\
4,300 \\
3,440 \\
2,890 \\
2,440 \\
2,130\end{array}$ & $\begin{array}{l}3,620 \\
2,460 \\
1,720 \\
1,500 \\
1,520 \\
2,280 \\
2,770 \\
2,850 \\
2,440 \\
1,970\end{array}$ & $\mid \begin{array}{l}21 \ldots \ldots \\
22 \ldots \ldots \\
23 \ldots \ldots \\
24 \ldots \ldots \\
25 \ldots \ldots \\
26 \ldots \ldots \\
26 \ldots \ldots \\
28 \ldots \ldots \\
29 \ldots \ldots \\
30 \ldots \ldots \\
31 \ldots \ldots\end{array}$ & $\begin{array}{l}1,82 \mathrm{C} \\
1,66 \mathrm{C} \\
1,33 \mathrm{C} \\
1,24 \mathrm{C} \\
1,34 \mathrm{C} \\
1,58 \mathrm{C} \\
1,46 \mathrm{C} \\
1,31 \mathrm{C} \\
1,17 \mathrm{C} \\
1,08 \mathrm{C} \\
-. .-\end{array}$ & $\begin{array}{l}1,540 \\
1,540 \\
1,620 \\
1,490 \\
2,210 \\
2,650 \\
2,370 \\
1,820 \\
1,400 \\
1,180 \\
1,100\end{array}$ \\
\hline \multicolumn{7}{|c|}{$\begin{array}{l}\text { Monthly mean discharge, in cubic feet per second } \\
\text { Runotf, in inches }\end{array}$} & $\begin{array}{r}4,967 \\
4.33\end{array}$ & $\begin{array}{r}1,829 \\
1.65\end{array}$ \\
\hline
\end{tabular}


Gage height, in feet, and discharge, in cubic feet per second, at indicated time, 1965, of Cottonwood

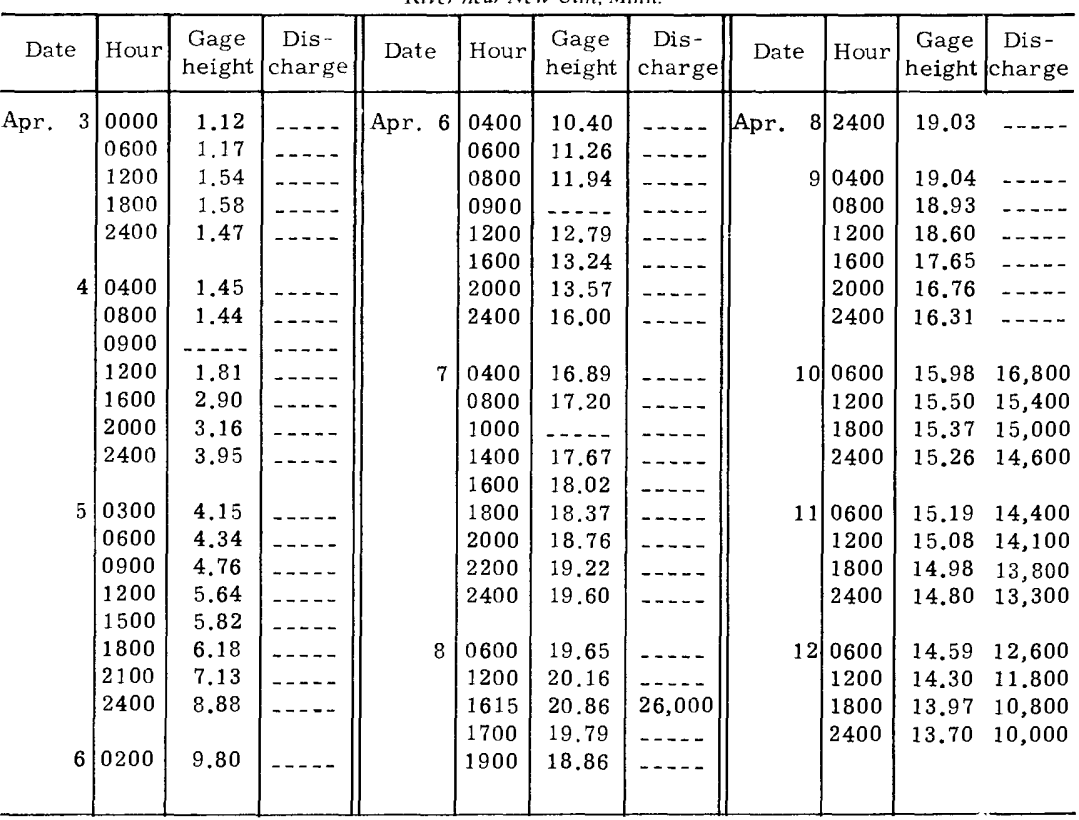

(62) 5-3180. East Branch Blue Earth River near Bricelyn, Minn.

Location.--Lat $43^{\circ} 37^{\prime} 50^{\prime \prime}$, long $93^{\circ} 47^{\prime} 25^{\prime \prime}$, in NE $\frac{1}{4} \mathrm{NE} \frac{1}{4} \sec .23, \mathrm{~T} .102$ N., R. 25 W., in center of span on downstream side of highway bridge, 2 miles upstream from Brush Creek, 3 miles downstream from South Walnut Lake, and 5 miles northeast of Bricelyn.

Drainage area. $-132 \mathrm{sq} \mathrm{mi}$.

Gage-height record.-Graph drawn on the basis of twice-daily wire-weight gage readings Apr. 3-10. Average of twice-daily readings used for periods Apr. 1-2 and Apr. 11 to May 31. Datum of gage is $1,131.86 \mathrm{ft}$ above mean sea level, datum of 1929 (Minnesota State Highway Department bench mark).

Discharge record.-Stage-discharge relation defined by current-meter measurements. Backwater from ice Apr. 1 to Apr. 6.

Maxima.-April-May 1965: Discharge, 1,260 cfs 2400 hours Apr. 6 (gage height, $11.70 \mathrm{ft}$ ).

1951 to March 1965: Discharge, 1,320 cfs Apr. 7, 1951 (gage height, $10.68 \mathrm{ft}$ ). 
Mean discharge, in cubic feet per second, 1965, of East Branch Blue Earth River near Bricelyn, Minn.

\begin{tabular}{|c|c|c|c|c|c|c|c|c|}
\hline Day & April & May & Day & April & May & Day & April & May \\
\hline $\begin{array}{l}1 \ldots \ldots \\
2 \ldots \ldots \\
3 \ldots \ldots \\
4 \ldots \\
5 \ldots \ldots \\
6 \ldots \ldots \\
7 \ldots \ldots \\
9 \\
10 \ldots \ldots\end{array}$ & $\begin{array}{r}10 \\
50 \\
100 \\
300 \\
500 \\
900 \\
1,240 \\
1,210 \\
1,160 \\
1,110\end{array}$ & $\begin{array}{l}242 \\
219 \\
196 \\
176 \\
159 \\
149 \\
141 \\
134 \\
121 \\
110\end{array}$ & $\begin{array}{l}11 \ldots \\
12 \ldots \\
13 \ldots \ldots \\
14 \ldots \ldots \\
15 \ldots \ldots \\
16 \ldots \ldots \\
17 \ldots \ldots \\
18 \ldots \ldots \\
19 \ldots \ldots \\
20 \ldots \ldots\end{array}$ & $\begin{array}{r}1,070 \\
994 \\
921 \\
840 \\
781 \\
714 \\
652 \\
586 \\
534 \\
490\end{array}$ & $\begin{array}{r}100 \\
90 \\
81 \\
72 \\
72 \\
92 \\
102 \\
110 \\
111 \\
108\end{array}$ & $\mid \begin{array}{l}21 \ldots \ldots \\
22 \ldots \ldots \\
23 \ldots \ldots \\
24 \ldots \ldots \\
25 \ldots \ldots \\
26 \ldots \ldots \\
26 \ldots \ldots \\
28 \ldots \ldots \\
29 \ldots \ldots \\
30 \ldots \ldots \\
31 \ldots \ldots\end{array}$ & $\begin{array}{l}44 \varepsilon \\
414 \\
384 \\
35 \subseteq \\
345 \\
343 \\
332 \\
316 \\
293 \\
269 \\
\end{array}$ & $\begin{array}{l}104 \\
100 \\
111 \\
125 \\
137 \\
167 \\
174 \\
178 \\
174 \\
171 \\
163\end{array}$ \\
\hline \multicolumn{7}{|c|}{$\begin{array}{l}\text { Monthly mean discharge, in cubic feet per second } \\
\text { Runoff, in inches. }\end{array}$} & $\begin{array}{r}589 \\
4.98\end{array}$ & $\begin{array}{r}135 \\
1.18\end{array}$ \\
\hline
\end{tabular}

Gage height, in feet, and discharge, in cubic feet per second, at indicated time, 1965

\begin{tabular}{|c|c|c|c|c|c|c|c|c|c|c|c|}
\hline Date & Hour & $\begin{array}{l}\text { Gage } \\
\text { height }\end{array}$ & $\begin{array}{c}\text { Dis- } \\
\text { charge }\end{array}$ & Date & Hour & $\begin{array}{l}\text { Gage } \\
\text { height }\end{array}$ & $\begin{array}{c}\text { Dis- } \\
\text { charge }\end{array}$ & Date & Hour & $\begin{array}{c}\text { Gage } \\
\text { height }\end{array}$ & $\begin{array}{c}\text { Dis- } \\
\text { charge }\end{array}$ \\
\hline Apr. 4 & 0000 & 8.74 & ---- & Apr. 6 & 1200 & 11.46 & $-n$ & Apr. 8 & 1200 & 11.54 & 1,210 \\
\hline & 0600 & 8.93 & $-\cdots$ & & 1800 & 11.64 & $\ldots$ & & 1800 & 11.52 & 1,210 \\
\hline & 1200 & 9.10 & ----0 & & 2400 & 11.70 & 1,260 & & 2400 & 11.50 & 1,200 \\
\hline & 1800 & 9.35 & $\ldots \ldots$ & & & & & & & & \\
\hline & 2400 & 9.66 &.--- & 7 & 0600 & 11.69 & 1,260 & 9 & 1200 & 11.37 & 1,160 \\
\hline & & & & & 1200 & 11.62 & 1,240 & & 2400 & 11.30 & 1,140 \\
\hline 5 & 0600 & 10.00 & & & 1800 & 11.56 & 1,220 & & & & \\
\hline & 1200 & 10.40 & -- & & 2400 & 11.53 & 1,210 & 10 & 1200 & 11.20 & 1,110 \\
\hline & 1800 & 10.75 & -- & & & & & & 2400 & 11.06 & 1,070 \\
\hline & 2400 & 11.00 & $--\cdots$ & 8 & 0600 & 11.54 & 1,210 & & & & \\
\hline 6 & 0600 & 11.20 & & & & & & & & & \\
\hline
\end{tabular}

(63) 5-3181. East Branch Blue Earth River tributary near Blue Earth, Minn.

(Crest-stage station)

Location.-Lat $43^{\circ} 37^{\prime} 10^{\prime \prime}$, long $94^{\circ} 01^{\prime} 00^{\prime \prime}$, in $\mathrm{W} \frac{1}{4} \mathrm{SE} \frac{1}{4}$ sec. $24, \mathrm{~T} .102$ N., R.27 W., at culvert on County Highway 13 , a quarter mile upstream from mouth, and $4 \frac{1}{4}$ miles east of Blue Earth.

Gage-height record.-Crest stages only.

Discharge record.-Stage-discharge relation defined by current-meter measurements below $63 \mathrm{cfs}$ and by indirect measurements at $233 \mathrm{cfs}$ and $406 \mathrm{cfs}$.

Maxima.-April-May 1965: Discharge, 187 cfs Apr. 6 (gage height, 6.54 f: backwater from debris).

1960 to March 1965: Discharge, $406 \mathrm{cfs}$ July 19, 1963 (gage height, $8.57 \mathrm{ft}$, from high-water profile). 
(64) 5-3183. North Fork Watonwan River near Delft, Minn.

(Crest-stage station)

Location.-Lat $44^{\circ} 00^{\prime} 00^{\prime \prime}$, long $95^{\circ} 07^{\prime} 20^{\prime \prime}$, in $\mathrm{E} \frac{1}{2} \mathrm{sec} .11, \mathrm{~T} .106$ N., R.36 W., at culvert on U.S. Highway $71,1-3 / 4$ miles northwest of Delft, and $5 \frac{1}{2}$ miles southeast of Jeffers.

Drainage area. $-13.1 \mathrm{sq} \mathrm{mi}$.

Gage-height record.-Crest stages only.

Discharge record.--Stage-discharge relation defined by current-meter measurements below $40 \mathrm{cfs}$ and by indirect measurement at $549 \mathrm{cfs}$.

Maxima.-April-May 1965: Discharge, 810 cfs Apr. 4 (gage height, 18.42 ft, bac'swater from ice).

1960 to March 1965: Discharge, 549 cfs Mar. 29, 1960 (gage height, $17.04 \mathrm{ft}$ ); gage height, 18.00 ft April 9, 1962 (backwater from ice).

(65) 5-3195. Watonwan River near Garden City, Minn.

(Gaging station, discontinued 1945)

Location (revised).-Lat $44^{\circ} 02^{\prime} 45^{\prime \prime}$, long $94^{\circ} 11^{\prime} 38^{\prime \prime}$, in SW $\frac{1}{4} \mathrm{NE} \frac{1}{4}$ sec. $28, T .107$ N., R. 28 W., on upstream side of highway bridge, 1.5 miles west of Garden City, 5 miles downstream from Perch Creek, and 7.3 miles upstream from mouth.

Drainage area. $-812 \mathrm{sq} \mathrm{mi}$.

Gage-height record.-Floodmark only. Altitude of gage is $907 \mathrm{ft}$ (from topographic map).

Discharge record.--Stage-discharge relation defined by current-meter measurements below 4,500 cfs and extended above by logarithmic plotting and one discharge measurement made in 1953 at site 2.6 miles downstream.

Maxima.-April-May 1965: Discharge, 19,000 cfs Apr. 7 (gage height, $18.89 \mathrm{ft}$, from floodmark).

1940-45, 1953 to March 1965: Discharge, 17,700 cfs June 9, 1953 (gage height, $18.6 \mathrm{ft}$ from floodmark).

(66) 5-3200. Blue Earth River near Rapidan, Minn.

Location.-Lat $44^{\circ} 05^{\prime} 44^{\prime \prime}$, long $94^{\circ} 06^{\prime} 33^{\prime \prime}$, in $\mathrm{SE} \frac{1}{4} \mathrm{SE} \frac{1}{4} \mathrm{sec} .6, \mathrm{~T} .107$ N., R. 27 W., on left bank 0.2 mile downstream from powerplant of Northern States Power Co., 2 miles west of Rapidan, $3 \frac{1}{2}$ miles downstream from Watonwan River, and 7-3/4 miles upstream from LeSueur River.

Drainage area.-2,430 sq $\mathrm{mi}$, approximately.

Gage-height record.-Water-stage recorder graph except 2200 hours Apr. 7 to 1200 hours Apr. 12 for which graph was reconstructed on basis of daily gage readings on the outside staff gage, and floodmark. Digital recorder tape punched at 15 minute intervals, May 13-31. Datum of gage is $808.80 \mathrm{ft}$ above mean sea level, datum of 1912.

Discharge record.-Stage-discharge relation defined by current-measurements. Backwater from ice Apr. 1-2.

Maxima.-April-May 1965: Discharge, 43,100 cfs 1220 hours Apr. 9 (gage height, $21.36 \mathrm{ft}$, from floodmark).

1909-10, 1939-45, 1949 to March 1965: Discharge, 26,100 cfs Apr. 8, 1951 (gage height, $14.97 \mathrm{ft}$ ) from rating curve extended above $16,000 \mathrm{cfs}$ by logarithmic flotting. 
Mean discharge, in cubic feet per second, 1965, of Blue Earth River near Rapidan, Minn.

\begin{tabular}{|c|c|c|c|c|c|c|c|c|}
\hline Day & April & May & Day & April & May & Day & April & May \\
\hline $\begin{array}{l}1 \ldots \ldots \\
2 \ldots \ldots \\
3 \ldots \ldots \\
4 \ldots \ldots \\
5 \ldots \ldots \\
6 \ldots \ldots \\
7 \ldots \ldots \\
8 \ldots \ldots \\
9 \ldots \ldots \\
10 \ldots\end{array}$ & $\begin{array}{r}230 \\
400 \\
1,360 \\
3,660 \\
8,380 \\
15,700 \\
28,500 \\
41,400 \\
42,500 \\
37,000\end{array}$ & $\begin{array}{l}4,260 \\
3,840 \\
3,520 \\
3,310 \\
3,170 \\
3,070 \\
3,350 \\
3,650 \\
3,530 \\
3,530\end{array}$ & $\mid \begin{array}{l}11 \ldots \\
12 \ldots \ldots \\
13 \ldots \ldots \\
14 \ldots \ldots \\
15 \ldots \ldots \\
16 \ldots \ldots \\
16_{\ldots} \ldots \\
18 \ldots \\
19 \ldots \\
20 \ldots\end{array}$ & $\begin{array}{r}31,600 \\
26,600 \\
21,700 \\
17,700 \\
14,700 \\
12,800 \\
11,300 \\
10,000 \\
8,820 \\
7,880\end{array}$ & $\begin{array}{l}3,650 \\
3,680 \\
3,440 \\
3,140 \\
2,960 \\
3,030 \\
3,370 \\
3,910 \\
4,080 \\
3,710\end{array}$ & $\begin{array}{l}21 \ldots . \\
22 \ldots- \\
23 \ldots \ldots \\
24 \ldots \ldots \\
25 \ldots \ldots \\
26 \ldots \ldots \\
27 \ldots \ldots \\
28 \ldots \ldots \\
29 \ldots \ldots \\
30 \ldots \ldots \\
31 \ldots \ldots\end{array}$ & $\begin{array}{l}7,100 \\
6,420 \\
5,720 \\
5,230 \\
4,940 \\
4,980 \\
5,170 \\
5,280 \\
5,180 \\
4,740 \\
-\end{array}$ & $\begin{array}{l}3,250 \\
2,930 \\
2,870 \\
3,160 \\
3,410 \\
3,960 \\
4,140 \\
4,370 \\
4,520 \\
4,160 \\
3,700\end{array}$ \\
\hline \multicolumn{7}{|c|}{$\begin{array}{l}\text { Monthly mean discharge, in cubic feet per second } \\
\text { Runotf, in inches }\end{array}$} & $\begin{array}{r}13,230 \\
6.08\end{array}$ & $\begin{array}{r}3,580 \\
1.70\end{array}$ \\
\hline
\end{tabular}

Gage height, in feet, and discharge, in cubic feet per second, at indicated time, 1965

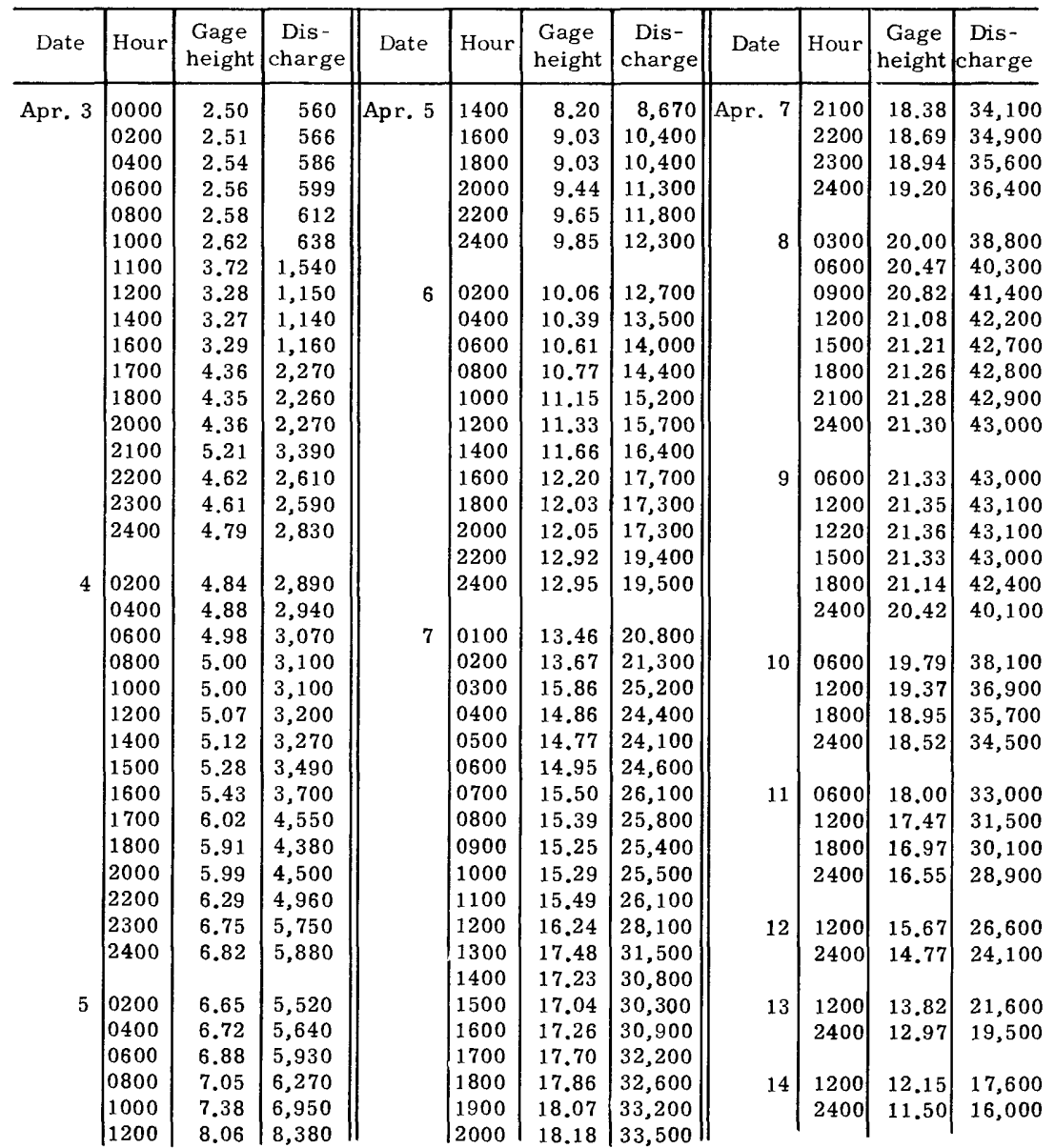


Gage height, in feet, and discharge, in cubic feet per second, at indicated time, 1965, of Blue Earth Ri'er near Rapidan, Minn.--Continued

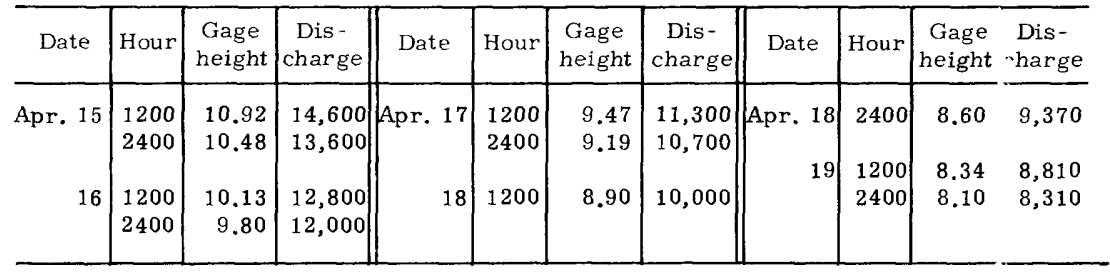

(67) 5-3203. Cobb River tributary near Mapleton, Minn.

(Crest-stage station)

Location.-Lat $44^{\circ} 01^{\prime} 05^{\prime \prime}$, long $93^{\circ} 57^{\prime} 30^{\prime \prime}$, in SW $\frac{1}{4} \mathrm{NE} \frac{1}{4}$ sec.4, T.106 N., R.26 W., at culvert on State Highway 22, 0.7 mile north of Beauford, 1.0 mile upstream from mouth, and 6.3 miles north of Mapleton.

Drainage area. $-7.25 \mathrm{sq} \mathrm{mi}$

Gage-height record.-Crest stages only.

Discharge record.-Stage-discharge relation defined by current-meter measurements below $100 \mathrm{cfs}$ and by indirect measurements at $258 \mathrm{cfs}, 359 \mathrm{cfs}$, and $526 \mathrm{cfs}$.

Maxima.-April-May 1965: Discharge, $293 \mathrm{cfs}$ Apr. 6 (gage height, $18.60 \mathrm{ft}$ ).

1959 to March 1965: Discharge, $526 \mathrm{cfs}$ May 21, 1960 (gage height, $22.24 \mathrm{f}$, from high-water profile).

(68) 5-3204. Maple River tributary near Mapleton, Minn.

(Crest-stage station)

Location.-Lat $43^{\circ} 55^{\prime} 20^{\prime \prime}$, long $94^{\circ} 01^{\prime} 20^{\prime \prime}$, in SW $\frac{1}{4}$ sec.1, T.105 N., R.27 W., at c'llvert on State Highway 30,1 mile upstream from mouth and $3 \frac{1}{4}$ miles west of Mapleton.

Drainage area. $-5.75 \mathrm{sq} \mathrm{mi}$.

Gage-height record.--Crest stages only.

Discharge record.- Stage-discharge relation defined by current-meter measurements below $145 \mathrm{cfs}$ and by indirect measurements at $273 \mathrm{cfs}$ and $445 \mathrm{cfs}$.

Maxima.-April-May 1965: Discharge, 285 cfs Apr. 6 (gage height, $21.11 \mathrm{ft}$, from floodmark, backwater from ice).

1959 to March 1965: Discharge, 445 cfs May 21, 1960 (gage height, $23.26 \mathrm{ft}$, from high-water profile). 
(69) 5-3205. Le Sueur River near Rapidan, Minn.

Location.—Lat $44^{\circ} 06^{\prime} 40^{\prime \prime}$, long $94^{\circ} 02^{\prime} 28^{\prime \prime}$, in SW $\frac{1}{4}$ sec.35, T.108 N., R.27 W., on right bank $600 \mathrm{ft}$ downstream from highway bridge, 1.8 miles northeast of Repidan, and 2.3 miles upstream from mouth.

Drainage area.-1,100 sq mi, approximately.

Gage-height record.-Water-stage recorder graph except for period 082C hours Apr. 6 to 0915 hours Apr. 11 when graph was constructed on the basis of two cr more daily gage readings on temporary staff gage, and flood mark. Datum of gage is $775.76 \mathrm{ft}$ above mean sea level, datum of 1929 .

Discharge record.- Stage-discharge relation defined by current-meter $r$ easurements. Backwater from ice Apr. 1-5.

Maxima.-April-May 1965: Discharge, 24,700 cfs 0500 hours Apr. 8 (gage height, $22.10 \mathrm{ft}$, from floodmark).

1939-45, 1949 to March 1965: Discharge, 21,200 cfs May 22, 1960 (gage height, $22.72 \mathrm{ft}$, from floodmark).

Mean discharge, in cubic feet per second, 1965

\begin{tabular}{|c|c|c|c|c|c|c|c|c|}
\hline Day & April & May & Day & April & May & Day & April & May \\
\hline $\begin{array}{l}1 \ldots \\
2 \ldots \\
3 \ldots \\
4 \ldots \\
5 \ldots \\
6 \ldots \\
7 \ldots \\
8 \ldots \\
9 \ldots \\
10 .\end{array}$ & $\begin{array}{r}150 \\
270 \\
600 \\
1,500 \\
4,700 \\
14,600 \\
20,200 \\
23,400 \\
20,000 \\
18,200\end{array}$ & $\begin{array}{l}2,030 \\
1,790 \\
1,640 \\
1,540 \\
1,470 \\
1,410 \\
1,460 \\
1,570 \\
1,540 \\
1,370\end{array}$ & $\begin{array}{l}11 \ldots \\
12 \ldots \\
13 \ldots \ldots \\
14 \ldots \ldots \\
15 \ldots \ldots \\
16 \ldots \ldots \\
17 \ldots \ldots \\
18 \ldots \ldots \\
19 \ldots \ldots \\
20 \ldots \ldots\end{array}$ & $\begin{array}{r}15,500 \\
12,100 \\
9,670 \\
7,490 \\
5,710 \\
4,660 \\
4,030 \\
3,540 \\
3,130 \\
2,770\end{array}$ & $\begin{array}{r}1,210 \\
1,070 \\
944 \\
854 \\
894 \\
1,320 \\
1,420 \\
1,640 \\
1,460 \\
1,400\end{array}$ & $\begin{array}{l}21 \ldots \ldots \\
22 \ldots \ldots \\
23 \\
24 \\
25 \\
26 \\
26 \\
27 \\
28 \\
29 \\
30 \\
31\end{array}$ & $\begin{array}{l}2,5 C 0 \\
2,2 \subseteq 0 \\
2,130 \\
2,050 \\
2,300 \\
2,8 \subset 0 \\
2,900 \\
2,850 \\
2,570 \\
2,290\end{array}$ & $\begin{array}{l}1,300 \\
1,190 \\
1,140 \\
1,180 \\
1,340 \\
1,470 \\
1,640 \\
1,820 \\
1,730 \\
1,540 \\
1,360\end{array}$ \\
\hline \multicolumn{7}{|c|}{$\begin{array}{l}\text { Monthly mean discharge, in cubic feet per second } \\
\text { Runoff, in inches }\end{array}$} & $\begin{array}{r}6,563 \\
6.66\end{array}$ & $\begin{array}{r}1,411 \\
1.48\end{array}$ \\
\hline
\end{tabular}

Gage height, in feet, and discharge, in cubic feet per second, at indicated time, 1965

\begin{tabular}{|c|c|c|c|c|c|c|c|c|c|c|c|}
\hline Date & Hour & $\begin{array}{l}\text { Gage } \\
\text { height }\end{array}$ & $\begin{array}{c}\text { Dis- } \\
\text { charge }\end{array}$ & Date & Hour & $\begin{array}{l}\text { Gage } \\
\text { height }\end{array}$ & $\begin{array}{c}\text { Dis- } \\
\text { charge }\end{array}$ & Date & Hour & $\begin{array}{l}\text { Gage } \\
\text { height }\end{array}$ & $\begin{array}{c}\text { Dis - } \\
\text { charge }\end{array}$ \\
\hline \multirow[t]{5}{*}{ Apr. 1} & 0000 & 4.40 & - & Apr. 4 & 0600 & 8.90 & -5 & Apr. 5 & 0500 & 8.67 & $\ldots$ \\
\hline & 1200 & 4.46 & -- & & 0800 & 9.04 & $\cdots$ & & 0700 & 8.77 & $\ldots$ \\
\hline & 1800 & 4.55 & & & 0900 & 8.99 & $-\ldots$ & & 0800 & 9.82 & $-\ldots$ \\
\hline & 2400 & 4.90 & 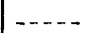 & & 1200 & 9.45 & & & 0900 & 9.20 & $\ldots$ \\
\hline & & & & & 1400 & 9.86 & & & 1000 & 9.32 & $-\ldots$ \\
\hline \multirow[t]{6}{*}{2} & 0000 & 5.24 & $-\cdots$ & & 1600 & 9.78 & $\ldots$ & & 1100 & 10.10 & --- \\
\hline & 0800 & 5.61 & $\ldots \ldots$ & & 1700 & 7.55 & $-\ldots$ & & 1200 & 9.67 & $\ldots-$ \\
\hline & 1200 & 5.84 & $\ldots$ & & 1800 & 7.42 & $\ldots$ & & 1300 & 10.49 & $\ldots$ \\
\hline & 1600 & 5.94 & --- & & 1900 & 8.11 & $\ldots$ & & 1500 & 10.34 & $\ldots$ \\
\hline & 2400 & 6.18 & $\ldots$ & & 2000 & 8.31 & 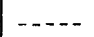 & & 1700 & 10.67 & $\ldots$. \\
\hline & & & & & 2100 & 8.54 & $\ldots$ & & 1800 & 11.85 & $\ldots$ \\
\hline \multirow[t]{5}{*}{3} & 0800 & 6.64 & $\cdots$ & & 2200 & 8.67 & $\ldots$ & & 1900 & 11.57 & $\ldots$. \\
\hline & 1600 & 6.94 & $\ldots \ldots$ & & 2300 & 9.26 & $\ldots$ & & 2200 & 12.37 & $\ldots$ \\
\hline & 2000 & 7.58 & $\ldots-\cdots$ & & 2400 & 8.96 & $\ldots$ & & 2400 & 13.36 & 9,440 \\
\hline & 2400 & 8.25 & $\cdots$ & & & & & & & & \\
\hline & & & & 5 & 0100 & 3.86 & $-\ldots$ & 6 & 0600 & 15.52 & 12,500 \\
\hline 4 & 0300 & 8.83 & $\cdots$ & & 0200 & 8.90 & $\ldots$ & & 0900 & 16.34 & 13,800 \\
\hline
\end{tabular}


Gage height, in feet, and discharge, in cubic feet per second, at indicated time, 1965, of Le Sueur Fiver near Rapidan, Minn. — Continued

\begin{tabular}{|c|c|c|c|c|c|c|c|c|c|c|c|}
\hline Date & Hour & $\begin{array}{l}\text { Gage } \\
\text { height }\end{array}$ & $\begin{array}{c}\text { Dis- } \\
\text { charge }\end{array}$ & Date & Hour & $\begin{array}{l}\text { Gage } \\
\text { height }\end{array}$ & $\begin{array}{c}\text { Dis- } \\
\text { charge }\end{array}$ & Date & Hour & $\begin{array}{c}\text { Gage } \\
\text { height }\end{array}$ & $\begin{array}{c}\text { Dis- } \\
\text { charge }\end{array}$ \\
\hline Apr. 6 & $\begin{array}{l}1200 \\
1500 \\
1800 \\
2100 \\
2400 \\
0600 \\
1500 \\
1800 \\
2400 \\
0500 \\
1200\end{array}$ & $\begin{array}{l}17.40 \\
17.87 \\
18.35 \\
18.68 \\
18.99 \\
\\
19.52 \\
20.07 \\
21.03 \\
21.61 \\
\\
22.10 \\
21.77\end{array}$ & $\begin{array}{l}15,500 \\
16,200 \\
17,000 \\
17,500 \\
18,000 \\
19,000 \\
20,000 \\
21,900 \\
23,300 \\
24,700 \\
23,800\end{array}$ & Apr. 8 & $\begin{array}{l}2400 \\
0600 \\
1000 \\
1800 \\
2400 \\
\\
0600 \\
0900 \\
1200 \\
1800 \\
2400\end{array}$ & $\begin{array}{l}20.93 \\
\\
20.56 \\
20.30 \\
19.41 \\
19.41 \\
\\
19.44 \\
19.38 \\
19.07 \\
18.78 \\
18.43\end{array}$ & $\begin{array}{l}21,700 \\
20,900 \\
20,400 \\
18,800 \\
18,800 \\
18,800 \\
18,700 \\
18,200 \\
17,700 \\
17,100\end{array}$ & Apr. 11 & $\begin{array}{l}0600 \\
1200 \\
1800 \\
2400 \\
0600 \\
1200 \\
1800 \\
2400 \\
2400 \\
2400\end{array}$ & $\begin{array}{l}17.98 \\
17.43 \\
16.95 \\
16.30 \\
15.63 \\
15.26 \\
14.84 \\
14.42 \\
12.64 \\
11.13\end{array}$ & $\begin{array}{r}16,400 \\
15,500 \\
14,800 \\
13,700 \\
12,600 \\
12,100 \\
11,500 \\
10,900 \\
8,440 \\
6,540\end{array}$ \\
\hline
\end{tabular}

(70) 5-3250. Minnesota River at Mankato, Minn.

Location.-Lat $44^{\circ} 10^{\prime} 10^{\prime \prime}$, long $94^{\circ} 00^{\prime} 15^{\prime \prime}$, in sec.7, T.108 N., R.26 W., on left bank at downstream side of Main Street Bridge in Mankato, 1.8 miles downstream from Blue Earth River and at mile 106.4.

Drainage area.-14,900 sq $\mathrm{mi}$, approximately.

Gage-height record.-Water-stage recorder graph except May 18. Datum of gage is $747.92 \mathrm{ft}$ above mean sea level, datum of 1929 .

Discharge record.-Stage-discharge relation defined by current-meter measurements.

Backwater from ice Apr. 1-4. Discharge for period of no gage-height record estimated on the basis of adjacent record.

Maxima.-April-May 1965: Discharge, 94,100 cfs 0100 hours Apr. 10 (ga ge height, $29.09 \mathrm{ft}$ ).

1903 to March 1965: Discharge, 66,600 cfs Apr, 9, 1951 (gage height, $26.20 \mathrm{ft}$ from floodmark).

Maximum stage known, $29.9 \mathrm{ft}$, Apr. 26, 1881, from floodmark, (discharge, 90,000 cfs).

Remarks.- Some diurnal fluctuation at low and medium stages caused by powerflants on Blue Earth River.

Mean discharge, in cublc feet per second, 1965

\begin{tabular}{|c|c|c|c|c|c|c|c|c|}
\hline Day & April & May & Day & April & May & Day & April & May \\
\hline $\begin{array}{l}1 \ldots \ldots \\
2 \ldots \ldots \\
3 \\
4 \\
4 \\
5 \\
6 \ldots \ldots \\
7 \\
8\end{array}$ & $\begin{array}{r}430 \\
900 \\
1,800 \\
5,900 \\
13,300 \\
30,000 \\
51,100 \\
79,000 \\
92,200 \\
92,700\end{array}$ & $\begin{array}{l}18,600 \\
17,300 \\
16,200 \\
15,200 \\
14,200 \\
13,500 \\
13,200 \\
13,600 \\
13,800 \\
15,000\end{array}$ & $\begin{array}{l}11 \ldots \\
12 \ldots \ldots \\
13 \ldots \ldots \\
14 \ldots \\
15 \ldots \ldots \\
16 \ldots \\
17 \ldots \ldots \\
18 \ldots \\
19 \ldots \\
20 \ldots\end{array}$ & $\begin{array}{l}91,600 \\
89,600 \\
83,200 \\
74,400 \\
66,000 \\
58,600 \\
52,800 \\
47,600 \\
42,800 \\
38,300\end{array}$ & $\begin{array}{l}16,000 \\
16,000 \\
14,700 \\
13,200 \\
12,400 \\
12,600 \\
12,900 \\
13,400 \\
14,000 \\
13,300\end{array}$ & $\begin{array}{l}21 \ldots \ldots \\
22 \ldots \ldots \\
23 \ldots \ldots \\
24 \ldots \\
25 \ldots \ldots \\
26 \ldots \ldots \\
26 \ldots \\
28 \ldots \\
29 \ldots \\
30 \ldots \\
31 \ldots\end{array}$ & $\begin{array}{l}34,700 \\
31,400 \\
28,400 \\
26,100 \\
24,600 \\
24,000 \\
23,400 \\
22,400 \\
21,300 \\
19,900 \\
\end{array}$ & $\begin{array}{l}12,300 \\
11,400 \\
11,000 \\
11,400 \\
12,100 \\
13,100 \\
13,900 \\
14,900 \\
16,000 \\
16,400 \\
15,800\end{array}$ \\
\hline \multicolumn{7}{|c|}{$\begin{array}{l}\text { Monthly mean discharge, in cubic feet per second } \\
\text { Runoff, in inches }\end{array}$} & $\begin{array}{r}42,280 \\
3.17\end{array}$ & $\begin{array}{r}14,110 \\
1.09\end{array}$ \\
\hline
\end{tabular}


Gage height, in feet, and discharge, in cubic feet per second, at indicated time, 1965, of Minnesota River

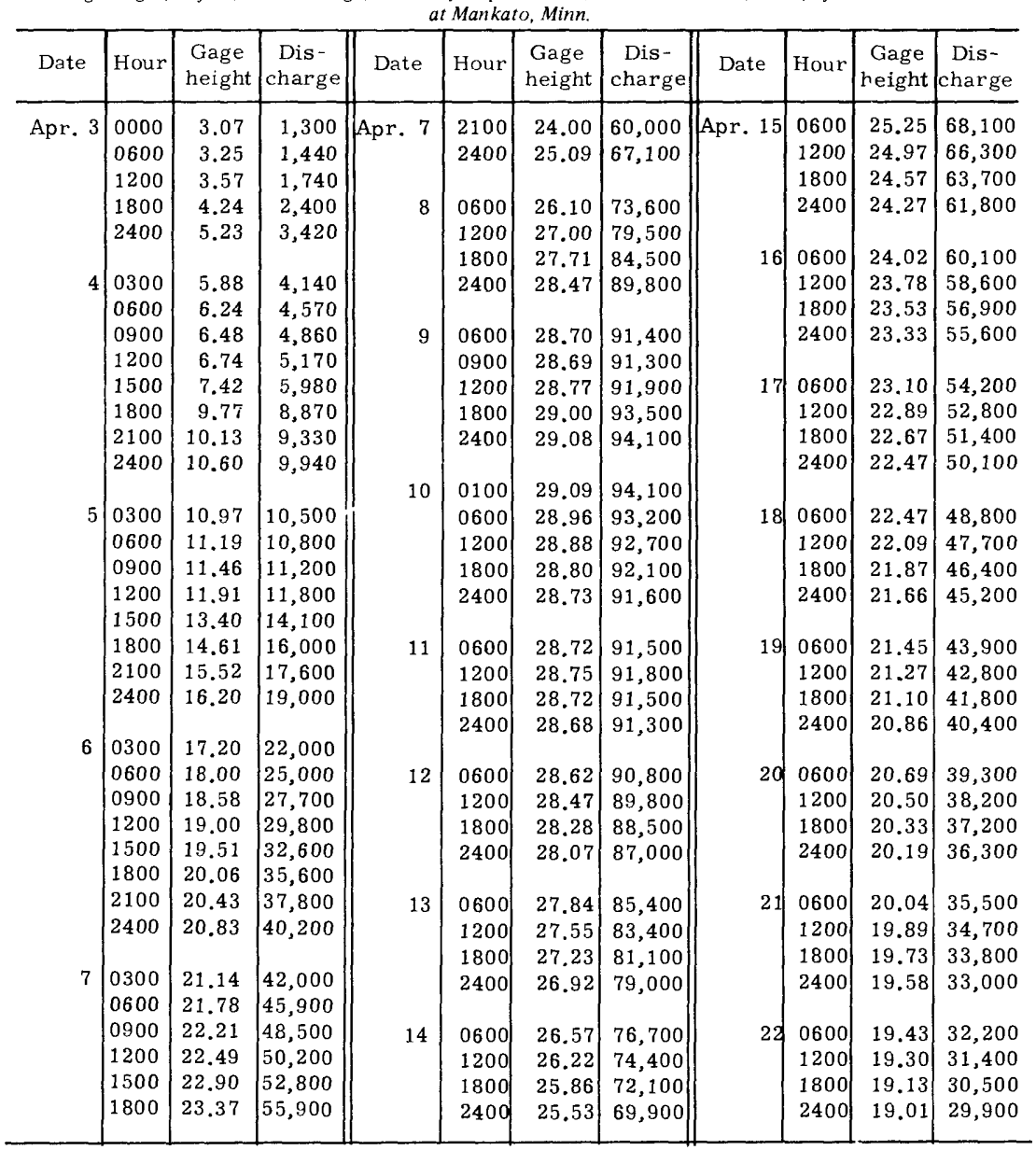

(71) 5-3262. Judicial Ditch No. 1-A near New Sweden, Minn.

(Miscellaneous site)

Location.-Lat $44^{\circ} 25^{\prime}$, long $94^{\circ} 15^{\prime}$, NW $\frac{1}{4} \mathrm{NW} \frac{1}{4}$ sec. 19, T.111 N., R. 28 W., on left bank, under bridge on Nicollet County Road No. 4. Two miles west of New Sweden and 4 miles southeast of Bernadette.

Gage-height record.-Occasional gage readings on staff gage during flood. Datum of gage is $962.07 \mathrm{ft}$ above mean sea level, adjustment of 1929 . (Levels by Minnesota Conservation Department, Division of Waters).

Discharge record.-Stage-discharge relation defined by current-meter measurements.

Maxima.-April-May 1965: Discharge, 1,170 cfs Apr. 8 (gage height, $13.35 \mathrm{ft}$ ).

$\overline{1} \overline{\mathrm{S}} \overline{2}$ to March 1965: Discharge not determined, gage height $8.70 \mathrm{ft}$ Mar. 28,1962 (backwater from ice).

Cooperation.-Gage heights furnished by Minnesota Conservation Department, Division of Waters. 
(72) 5-3300. Minnesota River near Carver, Minn.

Location.-Lat $44^{\circ} 43^{\prime} 28^{\prime \prime}$, long $93^{\circ} 37^{\prime} 58^{\prime \prime}$, in $\mathrm{NE}_{\frac{1}{4}} \mathrm{SW} \frac{1}{4}$ sec. 31 , T.115 N., R.23 W., on left bank $2 \frac{1}{2}$ miles south of Carver and at mile 36 upstream from Mississippi River. Auxiliary chain gage at same datum 2-3/4 miles upstream.

Drainage area. $-16,200 \mathrm{sq} \mathrm{mi}$, approximately.

Gage-height record.-Water-stage recorder graph except for the period 1000 hours Apr. 9 to 1400 hours Apr. 27 when graph was drawn on the basis of twice-daily gage readings on temporary staff gage. Auxiliary gage record obtained from a graph drawn on the basis of twice-daily gage readings. Datum of gages is $690.00 \mathrm{ft}$ above mean sea level, datum of 1929 .

Discharge record.-Fall-stage-discharge relation defined by current-meter measurements above stage of $7.0 \mathrm{ft}$. Below $7.0 \mathrm{ft}$, stage-discharge relation defined by currentmeter measurements. Backwater from ice Apr. 1-10. Fall used as a factor A pr. 11 to May 31.

Maxima.-April-May 1965: Discharge, 117,000 cfs 0600 hours Apr. 11 (gage height, $33.89 \mathrm{ft}$ ); gage height, $34.37 \mathrm{ft} 0100$ hours Apr. 12.

1934 to March 1965: Discharge, 64,100 cfs Apr. 11, 1951 (gage height, 27.71 ft); gage height, $28.31 \mathrm{ft}$ Apr. 16, 1952.

Cooperation,--Auxiliary gage readings furnished by Corps of Engineers.

Mean discharge, in cubic feet per second, 1965

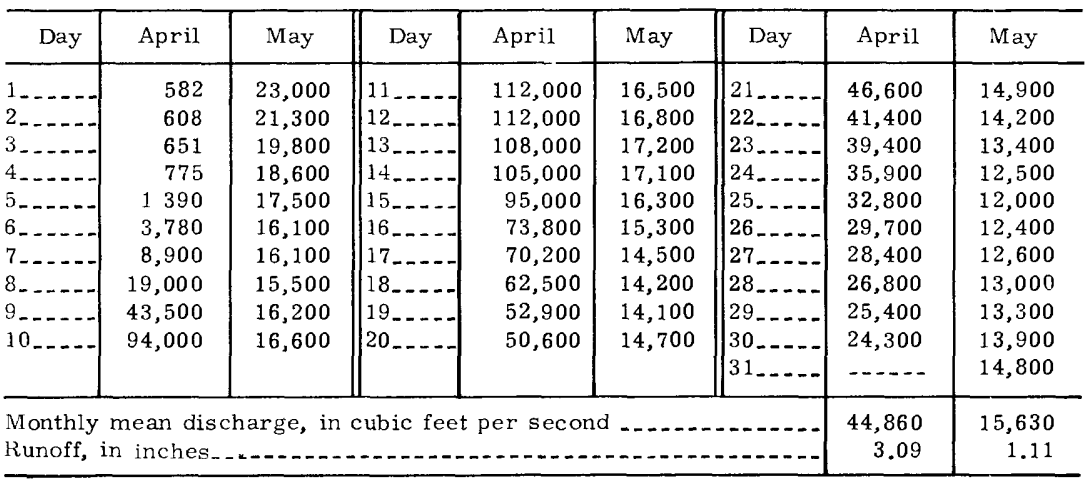

Gage height, in feet, and discharge, in cubic feet per second, at indicated time, 1965

\begin{tabular}{|c|c|c|c|c|c|c|c|c|c|c|c|}
\hline Date & Hour & $\begin{array}{c}\text { Gage } \\
\text { height }\end{array}$ & $\begin{array}{c}\text { Dis- } \\
\text { charge }\end{array}$ & Date & Hour & $\begin{array}{l}\text { Gage } \\
\text { height }\end{array}$ & $\begin{array}{c}\text { Dis- } \\
\text { charge }\end{array}$ & Date & Hour & $\begin{array}{c}\text { Gage } \\
\text { height }\end{array}$ & $\begin{array}{l}\text { Dis- } \\
\text { charge }\end{array}$ \\
\hline \multirow[t]{13}{*}{ Apr. } & 0000 & 4.66 & & \multirow[t]{13}{*}{ Apr. 4} & 0600 & 5.83 & & \multirow[t]{13}{*}{ Apr. 5} & 1200 & 11.80 & - \\
\hline & 1200 & 4.69 & & & 0900 & 6.00 & - & & 1400 & 12.32 & $\ldots$. \\
\hline & \multirow[t]{2}{*}{2400} & 4.78 & $\ldots$ & & 1200 & 6.23 & $\ldots .$. & & 1600 & 12.76 & $\ldots$ \\
\hline & & & & & 1500 & 6.54 & $\ldots$ & & 1800 & 13.24 & $\ldots$ \\
\hline & 1200 & 4.89 & $\ldots$ & & 1800 & 7.00 & $\ldots$ & & 2000 & 13.61 & $\ldots$ \\
\hline & 2400 & 5.05 & $\ldots$ & & 2100 & 7.62 & $\ldots$ & & 2200 & 13.99 & $\ldots$ \\
\hline & & & & & 2400 & 8.30 & $\ldots$ & & 2400 & 14.51 & $\ldots$ \\
\hline & 0600 & 5.13 & $\ldots$ & & & & & & & & \\
\hline & 1200 & 5.22 & $\ldots$ & & 0200 & 8.79 & $-\ldots$ & & 0200 & 14.79 & $\ldots$ \\
\hline & 1800 & 5.37 & $\ldots$ & & 0400 & 9.37 & $\ldots$ & & 0400 & 15.20 & $\ldots$ \\
\hline & 2400 & 5.56 & $\ldots$ & & 0600 & 9.96 & $\ldots$ & & 0600 & 15.62 & $\ldots$ \\
\hline & & & & & 0800 & 10.60 & $\ldots$ & & 0800 & 16.01 & $\ldots$ \\
\hline & 0300 & 5.69 & - & & 1000 & 11.22 & $\ldots$ & & 1000 & 16.34 & $\ldots$ \\
\hline
\end{tabular}


Gage height, in feet, and discharge, in cubic feet per second, at indicated time, 1965, of Minnesota River near Carver. Minn. - Continued

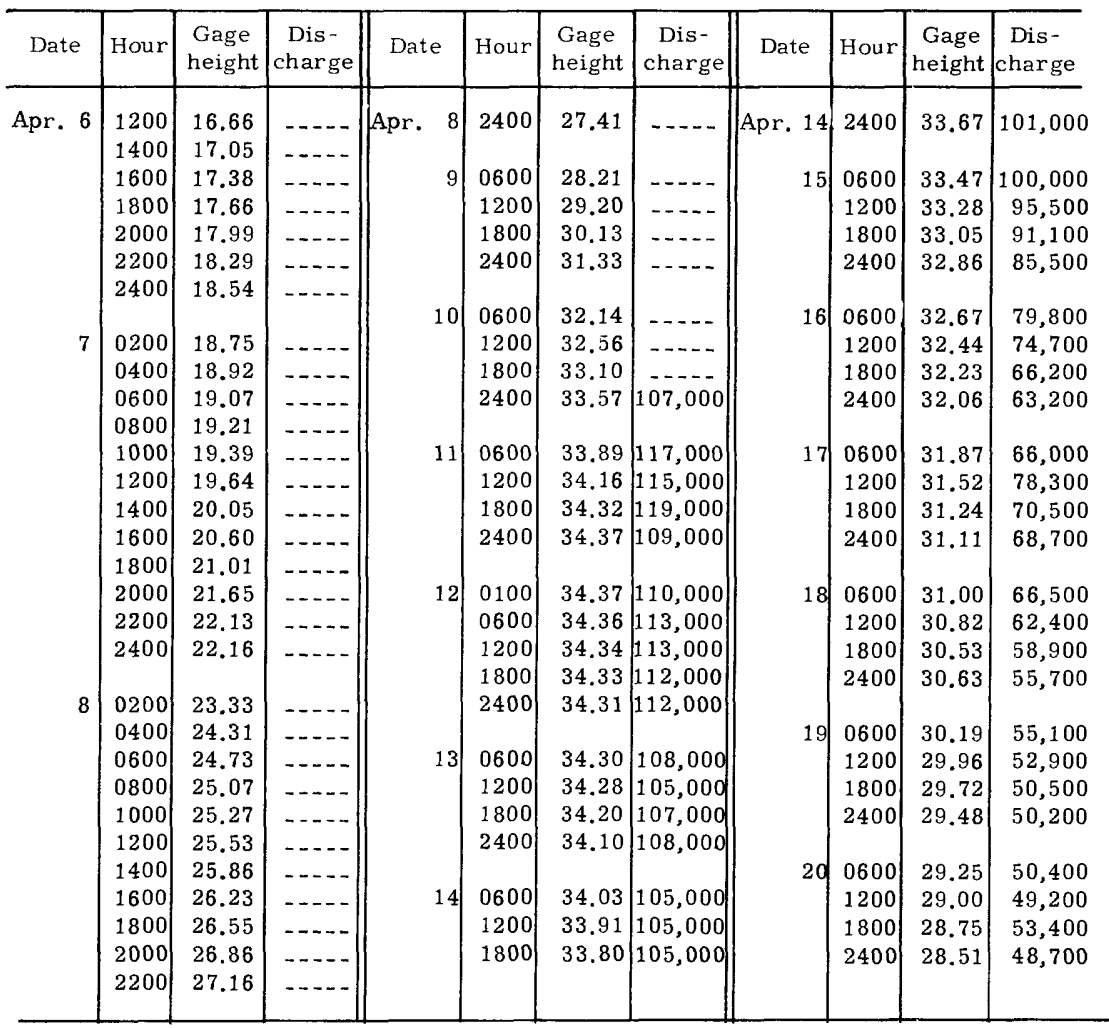

(73) 5-3301.5 Sand Creek tributary near Montgomery, Minn

(Crest-stage station)

Location.-Lat $44^{\circ} 25^{\prime} 40^{\prime \prime}$, long $93^{\circ} 30^{\prime} 30^{\prime \prime}$, in NE $\frac{1}{4}$ sec.18, T.111 N., R.22 W., at culvert on State Highway $21,3 \frac{1}{2}$ miles east of Montgomery.

Drainage area. $-0.29 \mathrm{sq} \mathrm{mi}$

Gage-height record.-Crest stages only.

Discharge record.--Stage-discharge relation defined by indirect measurement at 26 cfs and flow-through-culvert computations at $11 \mathrm{cfs}$ and $56 \mathrm{cfs}$.

Maxima.-April-May 1965: Discharge, 43 cfs Apr. 6 (gage height, $10.50 \mathrm{ft}$, backwater from ice).

1961 to March 1965: Discharge, $27 \mathrm{cfs}$ Sept. 8, 1964 (gage height, $8.75 \mathrm{ft}$ ); gage height, $10.35 \mathrm{ft}$ March 27, 1962 (backwater from ice). 
(74) 5-3302. Rice Lake tributary near Montgomery, Minn.

\section{(Crest-stage station)}

Location.-Lat $44^{\circ} 25^{\prime} 40^{\prime \prime}$, long $93^{\circ} 32^{\prime} 00^{\prime \prime}$, in $\mathrm{N} \frac{1}{2} \sec .13$, T.111 N., R.23 W., at culvert on State Highway $21,1 \frac{1}{4}$ miles upstream from Rice Lake and $2 \frac{1}{2}$ miles east of Montgomery.

Drainage area. $-2.49 \mathrm{sq} \mathrm{mi}$.

Gage-height record.-Crest stages only.

Discharge record.-Stage-discharge relation defined by current-meter measurements below $20 \mathrm{cfs}$ and by indirect measurement at $279 \mathrm{cfs}$.

Maxima.-April-May 1965: Discharge, 114 cfs Apr. 6 (gage height, $10.96 \mathrm{ft}$, backwater from ice).

1960 to March 1965: Discharge, $279 \mathrm{cfs}$ May 21, 1960 (gage height, $13.72 \mathrm{ft}$ ).

(75) 5-3303. Sand Creek near New Prague, Minn.

(Crest-stage station)

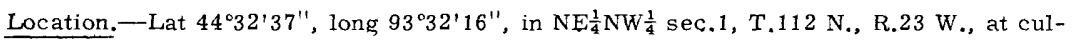
vert on State Highways 13 and 19, 1.9 miles east of New Prague.

Drainage area.- $-65 \mathrm{sq} \mathrm{mi}$, approximately.

Gage height record. -Crest stages only.

Discharge record.-Stage-discharge relation defined by current-meter measurements.

Maxima.-April-May 1965: Discharge, 1,070 cfs Apr. 8 (gage height, $14.79 \mathrm{ft}$ ).

1960 to March 1965: Discharge, 1,100 cfs May 21, 1960 (gage height, $14.84 \mathrm{ft}$ ).

(76) 5-3305.5 Raven Stream tributary near New Prague, Minn.

$$
\text { (Crest-stage station) }
$$

Location.-Lat $44^{\circ} 34^{\prime} 21^{\prime \prime}$, long $93^{\circ} 35^{\prime} 58^{\prime \prime}$, in NW $\frac{1}{4}$ sec. 28, T.113 N., R.23 W., on left bank $24 \mathrm{ft}$ upstream from culvert on county highway, 1.6 miles upstream from mouth, and 2.3 miles northwest of New Prague.

Drainage area.- -23 sq mi, approximately.

Gage-height record.-Water-stage recorder graph.

Discharge record.-Stage-discharge relation defined by current-meter measuræments below $227 \mathrm{cfs}$ and by indirect measurement at $929 \mathrm{cfs}$.

Maxima.-April-May 1965: Discharge, 505 efs Apr. 7 (gage height, $14.74 \mathrm{ft}$ ).

1960 to March 1965: Discharge, $929 \mathrm{cfs}$ May 21, 1960 (gage height, $17.34 \mathrm{f}^{4}$, from high-water profile). 
(77) Sand Creek at Jordan Minn.

(Miscellaneous site)

Location.--Lat $44^{\circ} 40^{\prime} 19^{\prime \prime}$, long $93^{\circ} 38^{\prime} 04^{\prime \prime}$, in NE⿺ $\frac{1}{4}$ sec.19, T.114 N., R.23 W., at bridge on U. S. Highway 169 at north edge of Jordan.

Drainage area. $-238 \mathrm{sq} \mathrm{mi}$.

Gage-height record.-Floodmarks. Datum of reference point is $758.942 \mathrm{ft}$ above mean sea level, adjustment of 1929. (Levels by Minnesota Highway Department).

Discharge record.-Stage-discharge relation defined by three current-meter measurements made during flood of May 1960.

Maxima.-April-May 1965: Discharge, 3,400 cfs Apr. 8 (gage height, $11.57 \mathrm{ft}$ ).

1960 to March 1965: Discharge, 8,650 cfs May 21, 1960 (gage height, $13.71 \mathrm{ft}$ ).

(78) 5-3309. Nine Mile Creek at Bloomington, Minn.

Location.-Lat $44^{\circ} 48^{\prime} 46^{\prime \prime}$, long $93^{\circ} 18^{\prime} 07^{\prime \prime}$, in NW $\frac{1}{4}$ sec. 21, T.27 N., R.24 V', on left bank between 105 th and 106th Streets in Bloomington, Minn., 1.2 miles downstream from bridge on Old Shakopee Road and 2.1 miles upstream from mouth.

Gage-height record.-Water-stage recorder graph. Altitude of gage is $731 \mathrm{ft}$ (from topographic map).

Discharge record.--Stage-discharge relation defined by current meter masurements.

Maxima.-April-May 1965: Discharge, 535 cfs 1200 hours Apr. 8 (gage height, $4.32 \mathrm{ft}$ ). 1963 to March 1965: Discharge, 199 cfs Aug. 29, 1964 (gage height, $3.63 \mathrm{ft}$ ).

Mean discharge, in cubic feet per second, 1965

\begin{tabular}{|c|c|c|c|c|c|c|c|c|}
\hline Day & April & May & Day & April & May & Day & April & May \\
\hline $\begin{array}{l}1 \ldots \ldots \\
2 \ldots \ldots \\
3 \ldots \ldots \\
4 \ldots \ldots \\
5 \ldots \ldots \\
6 \ldots \ldots \\
7 \ldots \ldots \\
8 \ldots \ldots \\
9 \ldots \ldots \\
10 \ldots\end{array}$ & $\begin{array}{l}7.2 \\
11 \\
20 \\
48 \\
71 \\
143 \\
309 \\
430 \\
411 \\
356\end{array}$ & $\begin{array}{l}40 \\
35 \\
33 \\
30 \\
28 \\
45 \\
61 \\
75 \\
96 \\
95\end{array}$ & $\begin{array}{l}11 \ldots \ldots \\
12 \ldots \ldots \\
13 \ldots \ldots \\
14 \ldots \ldots \\
15 \ldots \ldots \\
16 \ldots \ldots \\
17 \ldots \ldots \\
18 \ldots \ldots \\
16 \ldots \ldots \\
20 \ldots \ldots\end{array}$ & $\begin{array}{r}347 \\
329 \\
289 \\
247 \\
207 \\
173 \\
152 \\
134 \\
115 \\
96\end{array}$ & $\begin{array}{r}86 \\
65 \\
45 \\
38 \\
79 \\
107 \\
117 \\
113 \\
95 \\
81\end{array}$ & $\begin{array}{l}21 \ldots \\
22 \ldots \ldots \\
23 \ldots \ldots \\
24 \ldots \\
25 \ldots \\
26 \ldots \\
26 \ldots \\
28 \ldots \\
29 \ldots \\
30 \ldots \\
31 \ldots \\
\end{array}$ & $\begin{array}{l}80 \\
71 \\
61 \\
5 \Sigma \\
61 \\
65 \\
6 \varepsilon \\
65 \\
54 \\
48 \\
\end{array}$ & $\begin{array}{l}57 \\
39 \\
33 \\
32 \\
32 \\
28 \\
24 \\
22 \\
18 \\
16 \\
36\end{array}$ \\
\hline \multicolumn{7}{|c|}{$\begin{array}{l}\text { Monthly mean discharge, in cubic feet per second } \\
\text { Runoff, in inches }\end{array}$} & $\begin{array}{c}151 \\
-\end{array}$ & $\begin{array}{c}54.9 \\
-\end{array}$ \\
\hline
\end{tabular}


MARCH-MAY, UPPER MISSISSIPPI RIVER BASIN

Gage helght, in feet, and discharge, in cubic feet per second, at indicated time, 1965. of Nine Mile Creek at Bloomington, Minn

\begin{tabular}{|c|c|c|c|c|c|c|c|c|c|c|c|}
\hline Date & Hour & $\begin{array}{l}\text { Gage } \\
\text { height }\end{array}$ & $\begin{array}{c}\text { Dis - } \\
\text { charge }\end{array}$ & Date & Hour & $\begin{array}{l}\text { Gage } \\
\text { height }\end{array}$ & $\begin{array}{c}\text { Dis- } \\
\text { charge }\end{array}$ & Date & Hour & $\begin{array}{c}\text { Gage } \\
\text { height }\end{array}$ & $\begin{array}{c}\text { Dis- } \\
\text { charge }\end{array}$ \\
\hline \multirow[t]{25}{*}{ Apr. 2} & 0000 & 1.68 & 4.8 & \multirow[t]{7}{*}{ Apr. 6} & 0400 & 3.15 & 136 & \multirow[t]{25}{*}{ Apr. 9} & \multirow[t]{2}{*}{2400} & \multirow[t]{2}{*}{3.96} & \multirow[t]{2}{*}{359} \\
\hline & 1000 & 1.64 & 3.8 & & 0800 & 2.96 & 111 & & & & \\
\hline & 1200 & 1.82 & 9.9 & & 1200 & 3.16 & 137 & & 0700 & 3.86 & 324 \\
\hline & 1600 & 2.04 & 26 & & 1600 & 3.37 & 174 & & 0800 & 4.04 & 395 \\
\hline & 2000 & 1.91 & 16 & & 2000 & 3.34 & 169 & & 1200 & 3.95 & 356 \\
\hline & 2400 & 1.78 & 8.0 & & 2400 & 3.30 & 161 & & 2000 & 3.82 & 312 \\
\hline & & & & & & & & & 2200 & 4.20 & 475 \\
\hline & 0600 & 1.72 & 5.9 & \multirow[t]{7}{*}{7} & 0600 & 3.32 & 165 & & \multirow[t]{2}{*}{2400} & \multirow[t]{2}{*}{4.07} & \multirow[t]{2}{*}{410} \\
\hline & 1200 & 1.86 & 13 & & 1200 & 3.78 & 285 & & & & \\
\hline & 1500 & 1.95 & 19 & & 1600 & 4.25 & 500 & & 0600 & 3.88 & 330 \\
\hline & 1800 & 2.18 & 39 & & 1700 & 4.10 & 425 & & 1200 & 3.92 & 344 \\
\hline & 2000 & 2.23 & 43 & & 1900 & 4.18 & 465 & & 2400 & 3.90 & 336 \\
\hline & 2400 & 2.16 & 38 & & 2400 & 4.05 & 400 & & & & \\
\hline & & & & & & & & & 0800 & 3.88 & 330 \\
\hline & 0800 & 2.00 & 26 & \multirow[t]{6}{*}{8} & 0600 & 4.02 & 385 & & 1000 & 3.95 & 356 \\
\hline & 1200 & 2.17 & 40 & & 0800 & 3.99 & 371 & & 1200 & 3.86 & 324 \\
\hline & 1600 & 2.60 & 76 & & 1200 & 4.32 & 535 & & 1800 & 3.87 & 327 \\
\hline & 1900 & 2.57 & 73 & & 1800 & 4.11 & 430 & & 2400 & 3.87 & 309 \\
\hline & 2400 & 2.30 & 50 & & 2400 & 4.05 & 400 & & & & \\
\hline & & & & & & & & & 0600 & 3.75 & 293 \\
\hline & 0600 & 2.25 & 46 & \multirow[t]{5}{*}{9} & 0700 & 3.94 & 352 & & 1200 & 3.75 & 293 \\
\hline & 1200 & 2.54 & 71 & & 0900 & 4.09 & 420 & & 1800 & 3.74 & 290 \\
\hline & 1800 & 2.84 & 98 & & 1000 & 4.00 & 375 & & 1900 & 3.65 & 269 \\
\hline & 2200 & 2.74 & 88 & & 1300 & 4.27 & 510 & & 2400 & 3.64 & 267 \\
\hline & 2400 & 2.85 & 99 & & 1800 & 4.12 & 435 & & & & \\
\hline
\end{tabular}

(79) Minnesota River at Interstate Highway $35 \mathrm{~W}$ at Bloomington, Minn.

(Miscellaneous site)

Location.--Lat $44^{\circ} 47^{\prime} 30^{\prime \prime}$, long $93^{\circ} 17^{\prime} 19^{\prime \prime}$, on west line of sec. 27, T.27 N., R.24 V'., at bridge on Interstate Highway $35 \mathrm{~W}$ and 2.8 miles south from 90 th Street in Bloomington.

Gage-height record.-Elevations obtained during peak only. Datum of reference point is 753.31 ft above mean sea level, datum of 1929 (levels by Minnesota Highway Department).

Discharge record.-Peak discharge determined on the basis of one discharge masurement, comparison with discharge for Minnesota River near Carver and a plot of elevations obtained at site.

Maximum-April-May 1965: Discharge, about 120,000 cfs Apr, 15 (elevation, 718.20 ft). 
(80) 5-3310 Mississippi River at St. Paul, Minn.

Location.-Lat $44^{\circ} 56^{\prime} 40^{\prime \prime}$, long $93^{\circ} 05^{\prime} 20^{\prime \prime}$, in $\mathrm{SE} \frac{1}{4} \mathrm{NE} \frac{1}{4}^{2} \mathrm{sec} .6, \mathrm{~T} .28 \mathrm{~N} ., \mathrm{R} .22 \mathrm{~W}$., on left bank in St. Paul, $300 \mathrm{ft}$ upstream from Robert Street Bridge, 6 miles downstream from Minnesota River, and at mile 839.3 upstream from Ohio River. Auxiliary waterstage recorder in SE $\frac{1}{4}$ sec.22, T.28 N., R.22 W., in South St. Paul, 5.4 miles downstream at same datum.

Drainage area.- $-36,800 \mathrm{sq} \mathrm{mi}$, approximately.

Gage-height record.-Water-stage recorder graph except 1545 hours AF $* 11$ to 1630 hours Apr. 21 when graph was constructed on basis of several daily or hourly gage readings by Corps of Engineers made on temporary staff gage.

For auxiliary gage, water-stage recorder graph except 0600 hours Apr. 12 to 1330 hours Apr. 26 when several daily or bi-hourly gage readings by Corps of Engineers were made on temporary staff gage. Datum of gage is $684.16 \mathrm{ft}$ above mean sea level, datum of 1912 .

Discharge record.-Stage-fall-discharge relation affected by changes in slope and defined by current-meter measurements. Fall was a factor Apr. 5-7. Packwater from ice Apr. 1-4.

Maxima.-April-May 1965: Discharge, 171,000 cfs 1800 hours Apr. 16 (gage height, $26.01 \mathrm{ft}$ from graph based on gage readings).

1851 to March 1965: Discharge, 125,000 cfs Apr. 16, 1952 (gage height 22.02 ft).

Remarks.- - Slight regulation except during extreme floods by reservoirs on headwaters and by powerplants. Beginning June 20,1938, sewage from Minneapolis and St. Paul, which formerly entered above station, was diverted to a sewage-disposal plant, thence to river below station. Figures of daily discharge do not include this diversion.

Cooperation.- Gage-height record at South St. Paul furnished by Corps of Engineers. Diversion through sewage-disposal plant furnished by Minneapolis-St. Paul Sanitary District.

Mean discharge, in cubic feet per second, 1965

\begin{tabular}{|c|c|c|c|c|c|c|c|c|}
\hline Day & April & May & Day & April & May & Day & April & May \\
\hline & 4,070 & 66,100 & $11 \ldots$ & 91,300 & 41,700 & $21 \ldots$ & $138,0 ? 0$ & 39,700 \\
\hline $2 \ldots$ & 4,660 & 62,500 & $12=$ & 128,000 & 46,600 & $22 \ldots$ & $128,0 \div 0$ & 39,900 \\
\hline $3 \ldots$ & 5,040 & 58,900 & $13 \ldots$ & 147,000 & 46,200 & $23 \ldots$ & 118,070 & 39,600 \\
\hline $4 \ldots$ & 5,160 & 55,800 & $14 \ldots$ & 160,000 & 45,200 & $24 \ldots \ldots$ & 108,070 & 39,500 \\
\hline $5 \ldots$ & 9,060 & 53,500 & $15 \ldots$ & 166,000 & 45,200 & $25 \ldots$ & 99,370 & 40,100 \\
\hline & $\begin{array}{l}13,800 \\
17,400\end{array}$ & $\begin{array}{l}51,100 \\
49,800\end{array}$ & & $\begin{array}{l}171,000 \\
169,000\end{array}$ & $\begin{array}{l}44,800 \\
43,200\end{array}$ & $\mid \begin{array}{l}26 \\
27\end{array} \ldots$ & $\begin{array}{l}92,070 \\
84,300\end{array}$ & $\begin{array}{l}41,500 \\
42,400\end{array}$ \\
\hline $8 \ldots$ & 24,400 & 48,200 & $18 \ldots$ & 163,000 & 42,400 & 28 & 78,730 & 42,600 \\
\hline $9+-$ & 35,700 & 48,000 & 19. & 155,000 & 41,200 & 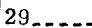 & 74,200 & 43,000 \\
\hline 10. & 53,500 & 47,400 & $20 \ldots$ & 147,000 & 40,400 & $30 \ldots$ & 70,000 & 43,000 \\
\hline & & & & & & $31 \ldots$ & $\ldots \ldots$ & 44,000 \\
\hline \multirow{2}{*}{\multicolumn{7}{|c|}{$\begin{array}{l}\text { Monthly mean discharge, in cubic feet per second* } \\
\text { Runoff, in inches* }\end{array}$}} & 89,130 & 46,780 \\
\hline & \multicolumn{6}{|c|}{ Runoff, in inches* } & 2.70 & 1.46 \\
\hline
\end{tabular}

* Adjusted for diversion 
Gage height, in feet, and discharge, in cubic feet per second, at indicated time, 1965, of Mississippi Rivir at St. Paul, Minn.

\begin{tabular}{|c|c|c|c|c|c|c|c|c|c|c|c|}
\hline Date & Hour & $\begin{array}{l}\text { Gage } \\
\text { height }\end{array}$ & $\begin{array}{c}\text { Dis- } \\
\text { charge }\end{array}$ & Date & Hour & $\begin{array}{l}\text { Gage } \\
\text { height }\end{array}$ & $\begin{array}{c}\text { Dis- } \\
\text { charge }\end{array}$ & Date & Hour & $\begin{array}{c}\text { Gage } \\
\text { height }\end{array}$ & $\begin{array}{c}\text { Dis- } \\
\text { charge }\end{array}$ \\
\hline \multirow[t]{6}{*}{ Apr. 5} & 0000 & 2.84 & 8,080 & Apr. 12 & 1500 & 22.21 & 133,000 & Apr. 21 & 1600 & 22.55 & 136,000 \\
\hline & 0600 & 2.78 & 8,120 & & 1800 & 22.58 & 136,000 & & 2000 & 22.39 & 134,000 \\
\hline & 1200 & 2.88 & 8,700 & & 2000 & 22.80 & 139,000 & & 2400 & 22.23 & 133,000 \\
\hline & 1800 & 3.12 & 9,680 & & 2400 & 23.01 & 141,000 & & & & \\
\hline & 2400 & 3.33 & 11,400 & & & & & 22 & 0200 & 22.15 & 132,000 \\
\hline & & & & 13 & 0200 & 23.18 & 112,000 & & 0400 & 22,06 & 131,000 \\
\hline \multirow[t]{5}{*}{6} & 0600 & 3.55 & 12,800 & & 0600 & 23.40 & 115,000 & & 0600 & 21.99 & 130,000 \\
\hline & 1200 & 3.74 & 13,800 & & 0800 & 23.54 & $1 \pm 7,000$ & & 0800 & 21.89 & 130,000 \\
\hline & 1800 & 3.72 & 15,100 & & 1200 & 23.71 & 148,000 & & 1000 & 21.81 & 129,000 \\
\hline & 2400 & 3.64 & 15,400 & & 1400 & 23.85 & 149,000 & & 1200 & 21.74 & 128,000 \\
\hline & & & & & 1800 & 23.96 & 150,000 & & 1400 & 21.65 & 127,000 \\
\hline \multirow[t]{5}{*}{7} & 0600 & 3.73 & 15,700 & & 2000 & 24.10 & 152,000 & & 1600 & 21.56 & 126,000 \\
\hline & 1200 & 3.92 & 16,400 & & 2400 & 24.20 & 153,000 & & 1800 & 21.47 & 125,000 \\
\hline & 1800 & 4.68 & 19,500 & & & & & & 2000 & 21.40 & 125,000 \\
\hline & 2400 & 4.98 & 20,800 & 14 & 0200 & 24.45 & 155,000 & & 2200 & 21.40 & 125,000 \\
\hline & & & & & 0600 & 24.61 & 157,000 & & 2400 & 21.31 & 124,000 \\
\hline \multirow[t]{9}{*}{8} & 0300 & 5.06 & 21,300 & & 0800 & 24.86 & $159,000 \mid$ & & & & \\
\hline & 0600 & 5.17 & 21,700 & & 1200 & 25.00 & 161,000 & 23 & 0400 & 21.10 & 122,000 \\
\hline & 0900 & 5.57 & 23,000 & & 1400 & 25.10 & 162,000 & & 0800 & 20.84 & 119,000 \\
\hline & 1200 & 6.02 & 24,400 & & 1800 & 25.22 & 163,000 & & 1200 & 20.68 & 118,000 \\
\hline & 1500 & 6.35 & 25,600 & & 2000 & 25.26 & 163,000 & & 1600 & 20.50 & 116,000 \\
\hline & 1800 & 6.65 & 26,700 & & 2400 & 25.34 & 164,000 & & 2000 & 20.32 & 114,000 \\
\hline & 2100 & 7.54 & 27,800 & & & & & & 2400 & 20.13 & 113,000 \\
\hline & 2400 & 7.24 & 29,000 & 15 & 0600 & 25.47 & 165,000 & & & & \\
\hline & & & & & 1200 & 25.55 & $166,000 \mid$ & 24 & 0400 & 19.95 & 111,000 \\
\hline \multirow[t]{9}{*}{9} & 0300 & 7.57 & 30,300 & & 1800 & 25.69 & 168,000 & & 0800 & 19.80 & 109,000 \\
\hline & 0600 & 7.90 & 31,600 & & 2400 & 25.85 & 170,000 & & 1200 & 19.60 & 108,000 \\
\hline & 0900 & 8.28 & 33,200 & & & & & & 1600 & 19.44 & 106,000 \\
\hline & 1200 & 8.76 & 35,300 & 16 & 0600 & 25.88 & 170,000 & & 2000 & 19.28 & 105,000 \\
\hline & 1500 & 9.36 & 37,800 & & 1200 & 25.96 & 171,000 & & 2400 & 19.12 & 103,000 \\
\hline & 1800 & 9.82 & 39,600 & & 1800 & 26.01 & 171,000 & & & & \\
\hline & 2100 & 10.17 & 41,600 & & 2400 & 25.94 & $171,000 \mid$ & 25 & 0400 & 18.93 & 102,000 \\
\hline & 2400 & 10.48 & 43,209 & & & & & & 0800 & 18.76 & 100,000 \\
\hline & & & & 17 & 0600 & 25.85 & 170,000 & & 1200 & 18.64 & 99,000 \\
\hline \multirow[t]{8}{*}{10} & 0300 & 10.79 & 44,800 & & 1200 & 25.80 & 169,000 & & 1600 & 18.54 & 98,100 \\
\hline & 0600 & 11.11 & 46,400 & & 1800 & 25.64 & $169,000 \mid$ & & 2000 & 18.44 & 97,200 \\
\hline & 0900 & 11.54 & 48,500 & & 2400 & 25.52 & 166,000 & & 2400 & 18.28 & 95,800 \\
\hline & 1200 & 12.32 & 52,400 & & & & & & & & \\
\hline & 1500 & 13.00 & 55,900 & 18 & 0600 & 25.41 & 165,000 & 26 & 0600 & 18.08 & 94,000 \\
\hline & 1800 & 13.65 & 59,500 & & 1200 & 25.20 & 163,000 & & 1200 & 17.86 & 92,000 \\
\hline & 2100 & 14.35 & 64,000 & & 1800 & 25.07 & 161,000 & & 1800 & 17.63 & 89,900 \\
\hline & 2400 & 15.20 & 70,200 & & 2400 & 24.84 & 160,000 & & 2400 & 17.42 & 88,000 \\
\hline \multirow[t]{9}{*}{11} & 0200 & 15.84 & 75,000 & 19 & 0600 & 24.70 & 158,000 & 27 & 0600 & 17.23 & 86,300 \\
\hline & 0600 & 16.56 & 80,600 & & 1200 & 24.47 & 155,000 & & 1200 & 16.94 & 83,800 \\
\hline & 0900 & 17.20 & 86,000 & & 1800 & 24.23 & 153,000 & & 1800 & 16.78 & 82,400 \\
\hline & 1200 & 17.87 & 92,100 & & 2400 & 24.08 & 151,000 & & 2400 & 16.64 & 81,200 \\
\hline & 1400 & 18.44 & 97,200 & & & & & & & & \\
\hline & 1800 & 18.98 & 102,000 & 20 & 0600 & 23.86 & 149,000 & 28 & 0600 & 16.46 & 79,800 \\
\hline & 2000 & 19.50 & 107,000 & & 1200 & 23.60 & 147,000 & & 1200 & 16.30 & 78,500 \\
\hline & 2400 & 19.98 & 111,000 & & 1800 & 23.39 & 144,000 & & 1800 & 16.18 & 77,600 \\
\hline & & & & & 2400 & 23.18 & 142,000 & & 2400 & 16.02 & 76,400 \\
\hline \multirow[t]{4}{*}{12} & 0200 & 20.44 & 116,000 & & & & & & & & \\
\hline & 0600 & 20.82 & 119,000 & 21 & 0400 & 23.02 & 141,000 & 29 & 0600 & 15.89 & 75,400 \\
\hline & 0800 & 21.25 & 123,000 & & 0800 & 22.86 & 139,000 & & 1200 & 15.73 & 74,200 \\
\hline & 1200 & 21.71 & 128,000 & & 1200 & 22.69 & 138,000 & & 1800 & 15.59 & 73,100 \\
\hline
\end{tabular}


Gage height, in feet, and discharge, in cubic feet per second, at indicated time, 1965, of Mississippi River at St. Paul, Minn. - Continued

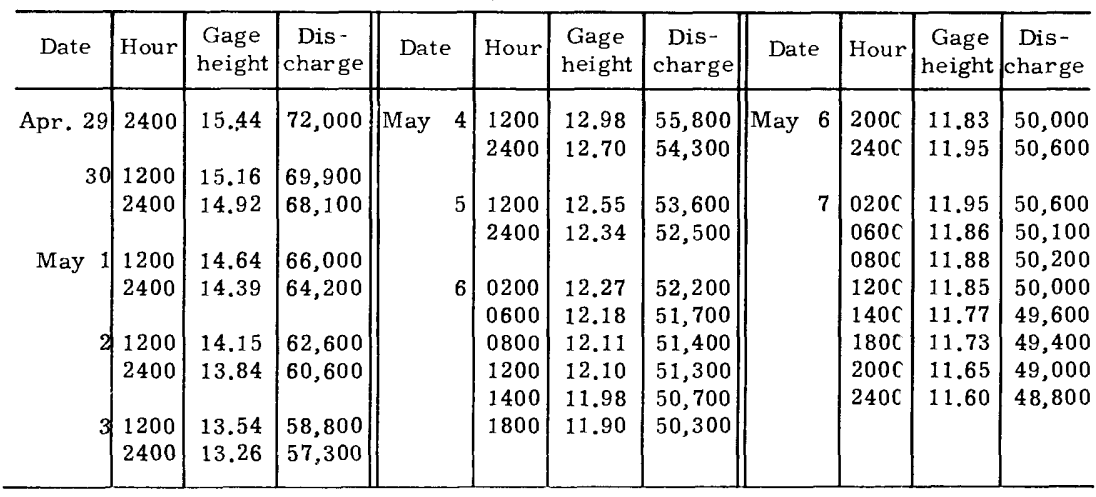

\section{ST. CROIX RIVER BASIN}

(81) 5-3325. Namekagon River near Trego, Wis.

Location.-Lat $45^{\circ} 56^{\prime} 50^{\prime \prime}$, long $91^{\circ} 53^{\prime} 15^{\prime \prime}$, in SW $\frac{1}{4}$ sec.17, T.40 N., R.12 VI., at powerplant of the Northern States Power Co., 4 miles downstream from Potato Creek and

5 miles northwest of Trego.

Drainage area.- $-503 \mathrm{sq} \mathrm{mi}$.

Gage-height record.- Headwater and tailwater gages read hourly.

Discharge record.--Discharge computed by Northern States Power Co. from its powerplant records on basis of ratings developed by Geological Survey.

Rating checked by current-meter measurement Apr. 16, 1965, at 1,660 cfs.

Maxima.-March-May 1965: Daily discharge, 1,640 cfs Apr. 13. 1927 to February 1965: Daily discharge, 5,200 cfs Sept. 2, 1941.

Remarks.-Discharge affected by operation of dam and powerplant.

Mean discharge, in cubic feet per second, 1965

\begin{tabular}{|c|c|c|c|c|c|c|c|c|c|c|c|}
\hline Day & March & April & May & Day & March & April & May & Day & March & April & May \\
\hline $\begin{array}{l}1 . . \\
2 \ldots \\
3 \ldots \\
4 \ldots \\
5 \\
6 \ldots \\
7 \\
7 \\
8 . \\
9 . \\
10 .\end{array}$ & $\begin{array}{l}359 \\
328 \\
347 \\
308 \\
304 \\
351 \\
352 \\
320 \\
327 \\
336\end{array}$ & $\begin{array}{l}281 \\
290 \\
307 \\
328 \\
334 \\
400 \\
436 \\
552 \\
503 \\
556\end{array}$ & $\begin{array}{r}825 \\
749 \\
712 \\
740 \\
684 \\
691 \\
1,040 \\
1,120 \\
1,130 \\
1,200\end{array}$ & 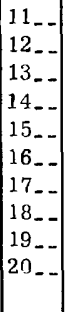 & $\begin{array}{l}323 \\
314 \\
308 \\
321 \\
317 \\
307 \\
282 \\
290 \\
288 \\
278\end{array}$ & $\begin{array}{r}650 \\
1,280 \\
1,640 \\
1,590 \\
1,490 \\
1,560 \\
1,500 \\
1,430 \\
1,350 \\
1,240\end{array}$ & $\begin{array}{r}1,100 \\
1,050 \\
957 \\
680 \\
855 \\
965 \\
956 \\
967 \\
990 \\
937\end{array}$ & $\begin{array}{l}21- \\
22_{-} \\
23_{-} \\
24_{-} \\
25_{-} \\
26_{-} \\
27- \\
28_{-} \\
29_{-} \\
30_{-} \\
31-\end{array}$ & $\begin{array}{l}278 \\
297 \\
287 \\
294 \\
303 \\
291 \\
299 \\
306 \\
284 \\
291 \\
337\end{array}$ & $\begin{array}{r}1,230 \\
949 \\
1,150 \\
1,230 \\
1,160 \\
1,110 \\
1,010 \\
935 \\
849 \\
830 \\
-\ldots--\end{array}$ & $\begin{array}{l}940 \\
903 \\
974 \\
956 \\
847 \\
854 \\
840 \\
807 \\
788 \\
810 \\
704\end{array}$ \\
\hline \multicolumn{9}{|c|}{$\begin{array}{l}\text { Monthly mean discharge, in cubic feet per second } \\
\text { Runoff, in inches }\end{array}$} & $\begin{array}{r}311 \\
0.71\end{array}$ & $\begin{array}{r}939 \\
2.08\end{array}$ & $\begin{array}{r}896 \\
2.05\end{array}$ \\
\hline
\end{tabular}


(82) 5-3331. Little Frog Creek near Minong, Wis.

(Crest-stage station)

Location.-Lat $46^{\circ} 05^{\prime} 48^{\prime \prime}$, long $91^{\circ} 46^{\prime} 39^{\prime \prime}$, in NW $\frac{1}{4}$ sec.29, T.42 N., R.11 W., at twin corrugated culverts on country road, 0.2 mile south of junction with State Highway 77 and 2.4 miles east of Minong.

Drainage area. $-13.6 \mathrm{sq} \mathrm{mi}$.

Gage-height record.-Crest stages only.

Discharge record.-Stage-discharge relation defined by current-meter measurements below $180 \mathrm{cfs}$.

Maxima.-March-May 1965: Discharge, $190 \mathrm{cfs}$ Apr. 15 (gage height, $14.31 \mathrm{ft}$ ).

1961 to February 1965: Discharge, about $230 \mathrm{cfs}$ May 15, 1961 (gage height, $15.06 \mathrm{ft}$ ).

(83) 5-3335. St. Croix River near Danbury, Wis.

Location.--Lat $46^{\circ} 04^{\prime} 30^{\prime \prime}$, long $92^{\circ} 14^{\prime} 50^{\prime \prime}$, in sec.33, T.42 N., R.15 W., on left bank at downstream side of bridge on State Highway 35, 3.5 miles downstream from Namekagon River, 10 miles northeast of Danbury, and at mile 129.2.

Drainage area. $-1,588 \mathrm{sq} \mathrm{mi}$.

Gage-height record.-Water-stage recorder graph. Datum of gage is $882.21 \mathrm{ft}$ above mean sea level, datum of 1929 .

Discharge record.--Stage-discharge relation defined by current-meter measurements. Backwater from ice Mar. 1 to Apr. 17

Maxima.-March-May 1965: Discharge, 6,460 cfs 1900-2400 hours Apr. 19 (gago height, $5.74 \mathrm{ft}$ ); gage height, $6.92 \mathrm{ft} 1430$ hours Apr. 15 (backwater from ice).

1914 to February 1965: Discharge, 10,200 cfs May 6, 1950 (gage height, $8.22 \mathrm{ft}$ ).

Mean discharge, in cubic feet per second, 1965

\begin{tabular}{|c|c|c|c|c|c|c|c|c|c|c|c|}
\hline Day & March & April & May & Day & March & April & May & Day & March & April & May \\
\hline $\begin{array}{l}1 \\
2 \\
2 \\
3 \\
4 \\
4 \\
5 \ldots- \\
6 \ldots- \\
7 \ldots- \\
8 \ldots- \\
9 \\
10 \\
10-\end{array}$ & $\begin{array}{l}720 \\
780 \\
840 \\
860 \\
880 \\
890 \\
880 \\
860 \\
840 \\
820\end{array}$ & $\begin{array}{r}940 \\
960 \\
1,000 \\
1,040 \\
1,100 \\
1,180 \\
1,280 \\
1,380 \\
1,400 \\
1,420\end{array}$ & $\begin{array}{l}2,180 \\
2,330 \\
2,210 \\
1,840 \\
1,630 \\
1,620 \\
2,180 \\
3,000 \\
3,640 \\
3,950\end{array}$ & 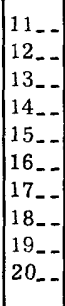 & $\begin{array}{l}800 \\
790 \\
780 \\
770 \\
760 \\
750 \\
740 \\
740 \\
750 \\
770\end{array}$ & $\begin{array}{l}1,800 \\
2,400 \\
3,800 \\
4,500 \\
5,000 \\
5,600 \\
6,000 \\
6,160 \\
6,360 \\
6,350\end{array}$ & $\begin{array}{l}3,930 \\
3,750 \\
3,430 \\
3,030 \\
2,450 \\
2,690 \\
2,800 \\
2,540 \\
2,480 \\
2,590\end{array}$ & $\begin{array}{l}21- \\
22- \\
23_{-} \\
24_{-} \\
25_{-} \\
26_{-} \\
27_{-} \\
28_{-} \\
29_{-} \\
30_{-}\end{array}$ & $\begin{array}{l}770 \\
780 \\
790 \\
800 \\
810 \\
820 \\
840 \\
860 \\
900 \\
930 \\
940\end{array}$ & $\begin{array}{l}5,940 \\
5,600 \\
5,070 \\
4,680 \\
4,120 \\
3,870 \\
3,680 \\
3,300 \\
2,720 \\
2,200\end{array}$ & $\begin{array}{l}2,620 \\
2,490 \\
2,440 \\
2,460 \\
2,640 \\
2,600 \\
2,450 \\
2,160 \\
1,930 \\
1,850 \\
1,800\end{array}$ \\
\hline \multicolumn{9}{|c|}{$\begin{array}{l}\text { Monthly mean discharge, in cubic feet per second } \\
\text { Runoff, in inches }\end{array}$} & $\begin{array}{r}815 \\
0.59\end{array}$ & $\begin{array}{r}3,362 \\
2.36\end{array}$ & $\begin{array}{r}2,571 \\
1.87\end{array}$ \\
\hline
\end{tabular}


Gage height, in feet. and discharge, in cubic feet per second, at indicated time, 1965, of St. Croix River near Danbury, Wis.

\begin{tabular}{|c|c|c|c|c|c|c|c|c|c|c|c|}
\hline Date & Hour & $\begin{array}{c}\text { Gage } \\
\text { height }\end{array}$ & $\begin{array}{c}\text { Dis- } \\
\text { charge }\end{array}$ & Date & Hour & $\begin{array}{c}\text { Gage } \\
\text { height }\end{array}$ & $\begin{array}{c}\text { Dis- } \\
\text { charge }\end{array}$ & Date & Hour & $\begin{array}{c}\text { Gage } \\
\text { height }\end{array}$ & $\begin{array}{c}\text { Dis- } \\
\text { charge }\end{array}$ \\
\hline Apr. 12 & $\begin{array}{l}0000 \\
0800 \\
1600 \\
2400 \\
0800 \\
1600 \\
2400 \\
0800 \\
1600 \\
2400 \\
0800 \\
1100 \\
1300\end{array}$ & $\begin{array}{l}4.20 \\
4.53 \\
4.68 \\
4.77 \\
5.01 \\
5.24 \\
5.66 \\
6.03 \\
6.26 \\
6.39 \\
6.49 \\
6.67 \\
6.50\end{array}$ & 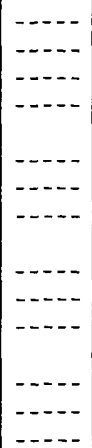 & Apr. 15 & $\begin{array}{l}1430 \\
1600 \\
1900 \\
2400 \\
0800 \\
1600 \\
2400 \\
1200 \\
2400 \\
0400 \\
0800 \\
1600\end{array}$ & $\begin{array}{l}6.92 \\
6.50 \\
6.15 \\
5.95 \\
5.91 \\
5.75 \\
5.59 \\
5.40 \\
5.48 \\
5.58 \\
5.48 \\
5.53\end{array}$ & 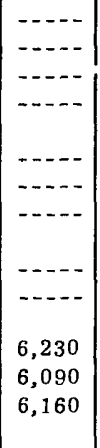 & $\begin{array}{r}\text { Apr. } 18 \\
19\end{array}$ & $\begin{array}{l}240\} \\
1207 \\
1907 \\
240 ? \\
1207 \\
2407 \\
1207 \\
2407 \\
1207 \\
240 ?\end{array}$ & $\begin{array}{l}5.60 \\
5.68 \\
5.74 \\
5.74 \\
5.69 \\
5.53 \\
5.34 \\
5.27 \\
5.12 \\
4.97\end{array}$ & $\begin{array}{l}6,260 \\
6,370 \\
6,460 \\
6,460 \\
6,390 \\
6,160 \\
5,900 \\
5,800 \\
5,600 \\
5,400\end{array}$ \\
\hline
\end{tabular}

(84) 5-3341. Sawyer Creek near Shell Lake, Wis.

\section{(Crest-stage station)}

Location.-Lat $45^{\circ} 46^{\prime} 08^{\prime \prime}$, long $91^{\circ} 54^{\prime} 40^{\prime \prime}$, in $\mathrm{SE} \frac{1}{4} \mathrm{sec} .13, \mathrm{~T} .38 \mathrm{~N} ., \mathrm{R} .13 \mathrm{~W}$. , at concrete box culvert on U.S. Highway $63,2.0$ miles north of Shell Lake and 4 miles south of junction with State Highway 70 in Spooner.

Drainage area. $-0.8 \mathrm{sq} \mathrm{mi}$ (approximately).

Gage-height record.-Crest stages only.

Maxima.-March-May 1965: Gage height, $13.11 \mathrm{ft}$ Apr. 11.

1960 to February 1965: Gage height, $11.61 \mathrm{ft}$ Apr. 26, 1960.

(85) 5-3353.8 Bashaw Brook near Shell Lake, Wis.

\section{(Crest-stage station)}

Location.-Lat $45^{\circ} 47^{\prime} 02^{\prime \prime}$, long $92^{\circ} 07^{\prime} 51^{\prime \prime}$, in SW $\frac{1}{4}$ sec.8, T.38 N., R.14 W., just upstream from twin concrete box culvert on country road 1.1 miles east from junction with County Trunk X, 2.2 miles south of State Highway 70, and 10.5 miles northwest of intersection of County Trunk B and U.S. Highway 63 in Shell Lake.

Drainage area.- $-28.2 \mathrm{sq} \mathrm{mi}$, of which $2.5 \mathrm{sq} \mathrm{mi}$ is non-contributing.

Gage-height record.-Water-stage recorder graph.

Discharge record.-Stage-discharge relation defined by current-meter measurements below $290 \mathrm{cfs}$.

Maxima.-March-May 1965: Discharge, $600 \mathrm{cfs} 1800$ hours Apr . 11 (gage height, $14.90 \mathrm{ft}$ ). 1959 to February 1965: Discharge, $172 \mathrm{cfs}$ Mar. 30, 1960 (gage height, $13.35 \mathrm{ft}$ ). 
(86) 5-3360. St. Croix River near Grantsburg, Wis.

Location.-Lat $45^{\circ} 55^{\prime} 25^{\prime \prime}$, long $92^{\circ} 38^{\prime} 20^{\prime \prime}$, near center of sec. 30, T.40 N., R.18 W., on left bank at Norway Point, 0.5 mile downstream from Sand Creek, 10 miles north of Grantsburg, and at mile 102.4.

Drainage area. $-2,820 \mathrm{sq} \mathrm{mi}$, approximately.

Gage-height record.-Water-stage recorder graph. Datum of gage is $848.98 \mathrm{ft}$ abore mean sea level, adjustment of 1912 (levels by Northern States Power Co.).

Discharge record.-Stage-discharge relation defined by current-meter measurements. Backwater from ice Mar. 1 to Apr. 17.

Maxima.-March-May 1965: Discharge, 16,800 cfs 0200 hours Apr. 18 (gage height, $12.64 \mathrm{ft}$; gage height, $13.01 \mathrm{ft} 1300$ hours Apr. 16 (backwater from ice). 1923 to February 1965: Discharge, 26,300 cfs May 7, 1950 (gage height, $15.06 \mathrm{ft}$ ).

Mean discharge, in cubic feet per second, 1965

\begin{tabular}{|c|c|c|c|c|c|c|c|c|c|c|c|}
\hline Day & March & April & May & Day & March & April & May & Day & March & April & May \\
\hline $\begin{array}{l}1 \\
2 \\
2 \\
3 \\
3 \\
4 \\
5 \\
5 \\
6 \\
7--- \\
7--- \\
8 \ldots- \\
9--- \\
10--\end{array}$ & $\begin{array}{l}1,120 \\
1,180 \\
1,240 \\
1,300 \\
1,340 \\
1,360 \\
1,380 \\
1,380 \\
1,360 \\
1,360\end{array}$ & $\begin{array}{l}1,420 \\
1,460 \\
1,500 \\
1,600 \\
1,700 \\
1,760 \\
1,800 \\
2,000 \\
2,200 \\
2,300\end{array}$ & $\begin{array}{l}4,830 \\
4,490 \\
4,340 \\
4,010 \\
3,370 \\
3,830 \\
5,080 \\
6,310 \\
7,100 \\
7,790\end{array}$ & \begin{tabular}{|l|}
$11 \ldots-$ \\
$12 \ldots--$ \\
$13 \ldots-$ \\
$14 \ldots$ \\
$15 \ldots$ \\
$16 \ldots$ \\
$17--$ \\
$18 \ldots$ \\
$19 .-$ \\
$20 \ldots$
\end{tabular} & $\begin{array}{l}1,340 \\
1,340 \\
1,320 \\
1,300 \\
1,300 \\
1,280 \\
1,280 \\
1,260 \\
1,260 \\
1,260\end{array}$ & $\begin{array}{r}2,600 \\
3,800 \\
5,400 \\
9,000 \\
11,600 \\
12,000 \\
14,000 \\
16,400 \\
15,700 \\
15,700\end{array}$ & $\begin{array}{l}8,300 \\
8,020 \\
7,290 \\
6,460 \\
5,620 \\
4,950 \\
4,640 \\
4,840 \\
4,730 \\
4,590\end{array}$ & $\begin{array}{l}21- \\
22- \\
23- \\
24- \\
25_{-} \\
26_{-} \\
27-- \\
28- \\
29_{-} \\
30_{-} \\
31_{-}\end{array}$ & $\begin{array}{l}1,260 \\
1,260 \\
1,260 \\
1,260 \\
1,280 \\
1,300 \\
1,320 \\
1,340 \\
1,360 \\
1,380 \\
1,400\end{array}$ & $\begin{array}{r}15,200 \\
14,200 \\
12,900 \\
11,500 \\
10,200 \\
9,140 \\
8,170 \\
7,350 \\
6,610 \\
5,680 \\
-.-.-\end{array}$ & $\begin{array}{l}4,550 \\
4,480 \\
4,170 \\
4,070 \\
4,390 \\
4,600 \\
4,530 \\
4,220 \\
3,690 \\
3,260 \\
3,120\end{array}$ \\
\hline \multicolumn{9}{|c|}{$\begin{array}{l}\text { Monthly mean discharge, in cubic feet per second } \\
\text { Runoff, in inches }\end{array}$} & $\begin{array}{r}1,303 \\
0.53\end{array}$ & $\begin{array}{r}7,496 \\
2.97\end{array}$ & $\begin{array}{r}5,022 \\
2.05\end{array}$ \\
\hline
\end{tabular}

Gage height, in feet, and discharge, in cubic feet per second, at indicated time, 1965

\begin{tabular}{|c|c|c|c|c|c|c|c|c|c|c|c|}
\hline Date & Hour & $\begin{array}{l}\text { Gage } \\
\text { height }\end{array}$ & $\begin{array}{c}\text { Dis- } \\
\text { charge }\end{array}$ & Date & Hour & $\begin{array}{c}\text { Gage } \\
\text { height }\end{array}$ & $\begin{array}{c}\text { Dis - } \\
\text { charge }\end{array}$ & Date & Hour & $\begin{array}{c}\text { Gage } \\
\text { height }\end{array}$ & $\begin{array}{l}\text { Dis- } \\
\text { charge }\end{array}$ \\
\hline \multirow[t]{6}{*}{ Apr. 12} & 0000 & 8.37 & $\cdots$ & Apr. 15 & 1200 & 12.23 & --- & Apr. 18 & 2400 & 12.32 & 15,900 \\
\hline & 0600 & 8.56 & $\ldots$ & & 1800 & 12.47 & $\ldots-$ & & & & \\
\hline & 1200 & 8.72 & $\ldots$ & & 2400 & 12.65 & $\ldots$ & 19 & 1200 & 12.25 & 15,600 \\
\hline & 1800 & 8.94 & $\ldots$ & & & & & & 2400 & 12,26 & 15,700 \\
\hline & 2400 & 9.20 & $\ldots$. & 16 & 0600 & 12.78 & & & & & \\
\hline & & & & & 1200 & 12.96 & & 20 & 1200 & 12.28 & 15,700 \\
\hline \multirow[t]{5}{*}{13} & 0600 & 9.39 & -. & & 1300 & 13.01 & & & 2400 & 12.24 & 15,600 \\
\hline & 1200 & 9.59 & $-\cdots$ & & 1800 & 12.80 & & & & & \\
\hline & 1800 & 9.89 & $\ldots \ldots$ & & 2400 & 12.93 & & 21 & 2400 & 11.99 & 14,900 \\
\hline & 2400 & 10.21 & $\cdots$ & & & & & & & & \\
\hline & & & & 17 & 0600 & 12.60 & & 22 & 2400 & 11.56 & 13,600 \\
\hline \multirow[t]{5}{*}{14} & 0600 & 10.52 & & & 1200 & 12.46 & & & & & \\
\hline & 1200 & 10.90 & & & 1800 & 12.56 & & 23 & 2400 & 11.04 & 12,200 \\
\hline & 1800 & 11.27 & $\ldots$ & & 2400 & 12.62 & & & & & \\
\hline & 2400 & 11.65 & $\ldots$ & & & & & 24 & 2400 & 10.49 & 10,800 \\
\hline & & & & 18 & 0200 & 12.64 & 16,800 & & & & \\
\hline 15 & 0600 & 11.91 & $\cdots$ & & 1200 & 12.49 & 16,400 & 25 & 2400 & 10.01 & 9,690 \\
\hline
\end{tabular}


(87) 5-3362. Glaisby Brook near Kettle River, Minn.

Location.-Lat $46^{\circ} 27^{\prime} 19^{\prime \prime}$, long $92^{\circ} 51^{\prime} 34^{\prime \prime}$, in $\mathrm{SE} \frac{1}{4} \mathrm{NW} \frac{1}{4} \mathrm{sec} .22$, T.46 N., R.20 W., on left bank 20 ft upstream from Bridge No. 2468 on State Highways 27 and $73,1.0$ mile upstream from mouth, and 2.4 miles south of Kettle River.

Gage-height record.-Water-stage recorder graph. Altitude of gage is 1,105 ft (from topographic map).

Discharge record.- Stage-discharge relation defined by current-meter measurements below $308 \mathrm{cfs}$ and extended to peak stage by logarithmic plotting. Baskwater from ice Apr. 1-15.

Maxima.-April-May 1965: Discharge, $813 \mathrm{cfs}$ at 2200 hours Apr. 18 (gage height, $8.42 \mathrm{ft}$ ).

1959 to March 1965: Discharge, $476 \mathrm{cfs}$ May 23, 1962 (gage height: $6.17 \mathrm{ft}$ ).

Mcan discharge, in cubic feet per second, 1965

\begin{tabular}{|c|c|c|c|c|c|c|c|c|}
\hline Day & April & May & Day & April & May & Day & April & May \\
\hline $\begin{array}{l}1 \ldots \ldots \\
2 \ldots \ldots \\
3 \ldots \ldots \\
4 \ldots \ldots \\
5 \ldots \ldots \\
6 \ldots \ldots \\
7 \ldots \ldots \\
8 \ldots \ldots \\
9 \ldots \ldots \\
10 \ldots\end{array}$ & $\begin{array}{l}1.3 \\
1.4 \\
1.6 \\
1.8 \\
2.5 \\
5.0 \\
5.6 \\
7.0 \\
10 \\
20\end{array}$ & $\begin{array}{l}58 \\
53 \\
50 \\
45 \\
43 \\
48 \\
52 \\
52 \\
50 \\
47\end{array}$ & $\mid \begin{array}{l}11 \ldots \\
12 \ldots \\
13 \ldots \ldots \\
14 \ldots \\
15 \ldots \ldots \\
16 \ldots \ldots \\
17 \ldots \ldots \\
18 \ldots \\
19 \ldots \\
20 \ldots\end{array}$ & $\begin{array}{r}30 \\
70 \\
140 \\
250 \\
400 \\
488 \\
461 \\
660 \\
693 \\
502\end{array}$ & $\begin{array}{l}44 \\
37 \\
32 \\
26 \\
26 \\
26 \\
26 \\
36 \\
40 \\
38\end{array}$ & $\begin{array}{l}21 \ldots \ldots \\
22 \ldots \ldots \\
23 \\
24 \ldots \\
25 \ldots \\
26 \ldots \ldots \\
26 \ldots \ldots \\
28 \ldots \ldots \\
28 \ldots \ldots \\
30 \ldots \ldots \\
31 \ldots \ldots\end{array}$ & $\begin{array}{r}376 \\
322 \\
264 \\
198 \\
141 \\
111 \\
92 \\
80 \\
71 \\
62 \\
\end{array}$ & $\begin{array}{l}44 \\
52 \\
56 \\
57 \\
57 \\
53 \\
47 \\
39 \\
32 \\
27 \\
25\end{array}$ \\
\hline \multicolumn{7}{|c|}{ Monthly mean discharge, in cubic feet per second } & 182 & 42.5 \\
\hline
\end{tabular}

Gage height, in feet, and discharge, in cubic feet per second, at indicated time, 1965

\begin{tabular}{|c|c|c|c|c|c|c|c|c|c|c|c|}
\hline Date & Hour & $\begin{array}{l}\text { Gage } \\
\text { height }\end{array}$ & $\begin{array}{c}\text { Dis- } \\
\text { charge }\end{array}$ & Date & Hour & $\begin{array}{c}\text { Gage } \\
\text { height }\end{array}$ & $\begin{array}{c}\text { Dis- } \\
\text { charge }\end{array}$ & Date & Hour & $\begin{array}{c}\text { Gage } \\
\text { height }\end{array}$ & $\begin{array}{l}\text { Dis- } \\
\text { charge }\end{array}$ \\
\hline \multirow[t]{9}{*}{ Apr. 12} & 0000 & 3.22 & $\ldots$ & Apr. 15 & 2200 & 6.74 & 710 & Apr.19 & 1000 & 7.63 & 690 \\
\hline & 0100 & 3.29 & $\ldots$ & & 2400 & 6.60 & 536 & & 1700 & 7.37 & 650 \\
\hline & 1100 & 3.16 & $-\ldots$ & & & & & & 240 ? & 7.06 & 604 \\
\hline & 1500 & 3.37 & $\ldots$ & 16 & 0400 & 6.19 & 479 & & & & \\
\hline & 1800 & 3.73 & $\ldots$ & & 0900 & 5.96 & 450 & 20 & $060 ?$ & 6.85 & 572 \\
\hline & 2000 & 4.06 & $\ldots$ & & 1400 & 6.34 & 500 & & $130 \pi$ & 6.22 & 483 \\
\hline & 2100 & 3.91 & $\ldots$ & & 1600 & 6.27 & 490 & & $240^{\circ}$ & 5.46 & 393 \\
\hline & 2400 & 4.02 & $\ldots$ & & 2000 & 6.43 & 512 & & & & \\
\hline & & & & & 2400 & 6.19 & 479 & 21 & $060^{\circ}$ & 5.48 & 395 \\
\hline \multirow[t]{9}{*}{13} & 0300 & 4.06 & $\ldots$ & & & & & & 240 & 5.12 & 344 \\
\hline & 0900 & 3.99 & $\ldots$ & 17 & 0500 & 5.69 & 420 & & & & \\
\hline & 1200 & 3.88 & $\ldots$ & & 0900 & 5.59 & 409 & 22 & $120 r$ & 4.97 & 322 \\
\hline & 1500 & 4.08 & $\ldots$ & & 1200 & 5.97 & 451 & & $240 C$ & 4.85 & 298 \\
\hline & 1600 & 4.06 & - . & & 1500 & 5.88 & 441 & & & & \\
\hline & 1800 & 4.80 & $\ldots$ & & 1700 & 6.12 & 470 & 23 & 1206 & 4.67 & 262 \\
\hline & 2000 & 5.28 & --- & & 2400 & 6.78 & 562 & & 2400 & 4.53 & 234 \\
\hline & 2400 & 5.20 & 368 & & & & & & & & \\
\hline & & & & 18 & 0600 & 6.96 & 589 & 24 & 1200 & 4.34 & 196 \\
\hline \multirow[t]{4}{*}{14} & 0900 & 4.85 & 298 & & 0900 & 6.65 & 543 & & 2400 & 4.18 & 165 \\
\hline & 1700 & 5.36 & 402 & & 1300 & 7.32 & 643 & & & & \\
\hline & 2400 & 5.69 & 472 & & 1700 & 8.03 & 751 & 25 & 1200 & 4.02 & 138 \\
\hline & & & & & 2200 & 8.42 & 813 & & $240 \mathrm{C}$ & 3.92 & 124 \\
\hline \multirow[t]{4}{*}{15} & 0300 & 5.71 & 476 & & 2400 & 8.37 & 805 & & & & \\
\hline & 1000 & 5.67 & 467 & & & & & 26 & 1200 & 3.81 & 110 \\
\hline & 1400 & 6.25 & 595 & 19 & 0500 & 8.11 & 764 & & 2400 & 3.73 & 101 \\
\hline & 1700 & 6.60 & 677 & & & & & & & & \\
\hline
\end{tabular}


(88) 5-3365.5 Wolf Creek tributary near Sandstone, Minn.

(Crest-stage station)

Location.-Lat $46^{\circ} 09^{\prime} 45^{\prime \prime}$, long $92^{\circ} 51^{\prime} 58^{\prime \prime}$, in $\mathrm{NE} \frac{1}{4} \mathrm{SE} \frac{1}{4} \mathrm{sec} .33, \mathrm{~T} .43$ N., R.20 W., at culvert on U.S. Highway $61,0.2$ mile upstream from mouth and 2.2 miles north of Sandstone.

Gage-height record.-Crest stages only.

Discharge record.- Stage-discharge relation defined by current-meter measurements below $33 \mathrm{cfs}$ and by indirect measurements at $121 \mathrm{cfs}$ and $200 \mathrm{cfs}$.

Maxima.-April-May 1965: Discharge, $200 \mathrm{cfs}$ Apr. 15 (gage height, $19.20 \mathrm{ft}$ ).

1960 to March 1965: Discharge, $121 \mathrm{cfs}$ May 23, 1962 (gage height, $17.80 \mathrm{ft}$ ).

(89) 5-3385. Snake River near Pine City, Minn.

Location.-Lat $45^{\circ} 50^{\prime} 30^{\prime \prime}$, long $92^{\circ} 56^{\prime} 00^{\prime \prime}$, in $\mathrm{SE} \frac{1}{4} \mathrm{NW} \frac{1}{4} \mathrm{sec} .26, \mathrm{~T} .39 \mathrm{~N}$., R.21 W., on left bank at site of former powerplant and dam, half a mile downstream from Cross Lake and $1 \frac{1}{2}$ miles northeast of Pine City.

Drainage area. $-958 \mathrm{sq} \mathrm{mi}$.

Gage-height record.-Water-stage recorder graph. Datum of gage is $919.00 \mathrm{ft}$ above mean sea level, datum of 1929 .

Discharge record.-Stage-discharge relation defined by current-meter measurements. Backwater from ice Apr. 1-11.

Maxima.-April-May 1965: Discharge, 11,500 cfs 0800 hours Apr. 18 (gage height, $9.56 \mathrm{ft})$.

1913-17, 1951 to March 1965: Discharge, 7,730 cfs May 28, 1962; gage height, $8.30 \mathrm{ft}$ Apr. 12, 13, 1952.

A discharge measurement of $12,500 \mathrm{cfs}$ was made May 9, 1950.

Mean discharge, in cubic feet per second, 1965

\begin{tabular}{|c|c|c|c|c|c|c|c|c|}
\hline Day & April & May & Day & April & May & Day & April & May \\
\hline $\begin{array}{l}1 \ldots \ldots \\
2 \ldots \ldots \\
3 \ldots \ldots \\
4 \ldots \\
5 \ldots \ldots \\
6 \ldots \ldots \\
7 \ldots \ldots \\
8 \ldots \ldots \\
9 \ldots \ldots \\
10 \ldots \ldots\end{array}$ & $\begin{array}{r}60 \\
60 \\
65 \\
65 \\
65 \\
80 \\
95 \\
120 \\
160 \\
250\end{array}$ & $\begin{array}{l}3,740 \\
3,190 \\
2,720 \\
2,330 \\
2,060 \\
1,940 \\
2,000 \\
1,980 \\
2,270 \\
2,420\end{array}$ & $\mid \begin{array}{l}11 \ldots \\
12 \ldots \\
13 \ldots \ldots \\
14 \ldots \\
15 \ldots \\
16_{\ldots} \ldots \\
17 \ldots \\
18 \ldots \\
19 \ldots \\
20 \ldots\end{array}$ & $\begin{array}{r}630 \\
1,350 \\
2,460 \\
4,400 \\
6,680 \\
9,620 \\
10,900 \\
11,300 \\
11,200 \\
10,900\end{array}$ & $\begin{array}{l}2,460 \\
2,420 \\
2,310 \\
2,150 \\
1,980 \\
1,810 \\
1,620 \\
1,620 \\
1,500 \\
1,390\end{array}$ & $\mid \begin{array}{l}21 \ldots \\
22 \ldots \ldots \\
23 \ldots \ldots \\
24 \ldots \\
25 \ldots \\
26 \ldots \\
27 \ldots \ldots \\
28 \ldots \\
29 \\
29 \\
30 \ldots \\
31 \ldots \\
\ldots\end{array}$ & $\begin{array}{r}10,700 \\
10,500 \\
10,200 \\
9,550 \\
8,660 \\
7,670 \\
6,580 \\
5,680 \\
4,940 \\
4,320 \\
-\end{array}$ & $\begin{array}{l}1,300 \\
1,160 \\
1,090 \\
1,110 \\
1,210 \\
1,370 \\
1,530 \\
1,590 \\
1,600 \\
1,540 \\
1,410\end{array}$ \\
\hline Runoff, & & & & & & & $\begin{array}{r}4,975 \\
5.79\end{array}$ & $\begin{array}{l}1,897 \\
2,28\end{array}$ \\
\hline
\end{tabular}


Gage height, in feet, and discharge, in cubic feet per second, at indicated time, 1965, of Snake River near

\begin{tabular}{|c|c|c|c|c|c|c|c|c|c|c|c|}
\hline \multicolumn{12}{|c|}{ Pine City, Minn. } \\
\hline Date & Hour & $\begin{array}{l}\text { Gage } \\
\text { height }\end{array}$ & $\begin{array}{c}\text { Dis- } \\
\text { charge }\end{array}$ & Date & Hour & $\begin{array}{c}\text { Gage } \\
\text { height }\end{array}$ & $\begin{array}{c}\text { Dis- } \\
\text { charge }\end{array}$ & Date & Hour & $\begin{array}{c}\text { Gage } \\
\text { height }\end{array}$ & $\begin{array}{c}\text { Dis - } \\
\text { charge }\end{array}$ \\
\hline \multirow[t]{6}{*}{ Apr. 10} & 0000 & 3.43 & $\ldots$ & Apr...15 & 2000 & 8.09 & 7,640 & Apr. 2 & 231200 & 9.09 & 10,200 \\
\hline & 0600 & 3.46 & --- & & 2400 & 8.34 & 8,280 & & 2400 & 8.98 & 9,950 \\
\hline & 1200 & 3.48 & $---n$ & & & & & & & & \\
\hline & 1800 & 3.55 & & 16 & 0400 & 8.56 & 8,860 & & 241200 & 8.84 & 9,580 \\
\hline & 2400 & 3.66 & - & & 0800 & 8.74 & 9,320 & & 2400 & 8.65 & 9,090 \\
\hline & & & & & 1200 & 8.91 & 9,770 & & & & \\
\hline \multirow[t]{5}{*}{11} & 0600 & 3.78 & & & 1600 & 9.03 & 10,100 & & 551200 & 8.49 & 8,670 \\
\hline & 1200 & 3.88 & & & 2000 & 9.11 & 10,300 & & 2400 & 8.31 & 8,210 \\
\hline & 1800 & 4.07 & & & 2400 & 9.19 & 10,500 & & & & \\
\hline & 2400 & 4.21 & & & & & & & 61200 & 8.10 & 7,670 \\
\hline & & & & 17 & 0600 & 9.28 & 10,700 & & 2400 & 7.88 & 7,120 \\
\hline \multirow[t]{5}{*}{12} & 0600 & 4.34 & 1,090 & & 1200 & 9.36 & 10,900 & & & & \\
\hline & 1200 & 4.46 & 1,220 & & 1800 & 9.39 & 11,000 & & 71200 & 7.65 & 6,560 \\
\hline & 1800 & 4.82 & 1,650 & & 2400 & 9.45 & 11,200 & & $24 \cap 0$ & 7.45 & 6,090 \\
\hline & 2400 & 5.05 & 1,930 & & & & & & & & \\
\hline & & & & 18 & 0600 & 9.50 & 11,300 & 28 & $82^{n} 0$ & 7.26 & 5,680 \\
\hline \multirow[t]{7}{*}{13} & 0400 & 5.13 & 2,030 & & 0800 & 9.56 & 11,500 & & $24 \cap 0$ & 7.05 & 5,260 \\
\hline & 0800 & 5.21 & 2,130 & & 1200 & 9.51 & 11,300 & & & & \\
\hline & 1200 & 5.35 & 2,310 & & 1800 & 9.52 & 11,400 & 29 & $91^{2} 0$ & 6.88 & 4,920 \\
\hline & 1600 & 5.57 & 2,610 & & 2400 & 9.51 & 11,300 & & 2490 & 6.74 & 4,660 \\
\hline & 2000 & 5.84 & 3,020 & & & & & & & & \\
\hline & 2400 & 6.06 & 3,400 & 19 & 0600 & 9.50 & 11,300 & & 301220 & 6.56 & 4,310 \\
\hline & & & & & 1200 & 9.48 & 11,200 & & 2400 & 6.40 & 4,010 \\
\hline \multirow[t]{7}{*}{14} & 0400 & 6.25 & 3,740 & & 1800 & 9.44 & 11,100 & & & & \\
\hline & 0800 & 6.42 & 4,050 & & 2400 & 9.41 & 11,100 & May 1 & $1 \longdiv { 1 2 \} 0 }$ & 6.25 & 3,740 \\
\hline & 1200 & 6.60 & 4,390 & & & & & & 2400 & 6.09 & 3,450 \\
\hline & 1600 & 6.78 & 4,730 & 20 & 1200 & 9.34 & 10,900 & & & & \\
\hline & 2000 & 6.95 & 5,060 & & 2400 & 9.29 & 10,800 & 2 & 21270 & 5.94 & 3,190 \\
\hline & 2400 & 7.14 & 5,440 & & & & & & 2400 & 5.78 & 2,930 \\
\hline & & & & 21 & 1200 & 9.26 & 10,700 & & & & \\
\hline \multirow[t]{4}{*}{15} & 0400 & 7.32 & 5,800 & & 2400 & 9.24 & 10,600 & 3 & \begin{tabular}{l|l}
3 & 1290
\end{tabular} & 5.64 & 2,720 \\
\hline & 0800 & 7.50 & 6,200 & & & & & & 2400 & 5.50 & 2,510 \\
\hline & 1200 & 7.68 & 6,630 & 22 & 1200 & 9.21 & 10,500 & & & & \\
\hline & 1600 & 7.87 & 7,100 & & 2400 & 9.20 & 10,500 & & & & \\
\hline
\end{tabular}

(90) 5-3395. St. Croix River near Rush City, Minn.

(Gaging station, discontinued 1961)

Location.-Lat $45^{\circ} 42^{\prime} 15^{\prime \prime}$, long $92^{\circ} 52^{\prime} 20^{\prime \prime}$, in SW $\frac{1}{4}$ sec.8, T.37 N., R.20 W., on right bank $200 \mathrm{ft}$ upstream from old site of Northern Pacific Railway bridge, 5 miles east of Rush City, 10 miles downstream from Snake River, and at mile 80.6.

Drainage area. $-5,120 \mathrm{sq} \mathrm{mi}$.

Gage-height record.-Crest stages only. Datum of gage is $772.47 \mathrm{ft}$ above mean sea level, datum of 1929 .

Maxima.-March-May 1965: Gage height, $15.67 \mathrm{ft}$ about Apr. 18 (probable backwater from ice).

1923 to February 1965: Discharge, 60,000 cfs May 8, 1950 (gage height, $19.04 \mathrm{ft}$ from floodmarks). 
(91) 5-3400. Sunrise River near Stacy, Minn.

Location.-Lat $45^{\circ} 24^{\prime} 30^{\prime \prime}$, long $92^{\circ} 55^{\prime} 55^{\prime \prime}$, in NW $\frac{1}{4} \mathrm{NW} \frac{1}{4}$ sec.26, T.34 N., R.21 W., on right bank on upstream side of highway bridge, $2 \frac{1}{2}$ miles northeast of Stacy, $2 \frac{1}{2}$ miles upstream from Minnesota Division of Game and Fish dam and 3 miles downstream from West Branch Sunrise River.

Drainage area. $-167 \mathrm{sq} \mathrm{mi}$.

Gage-height record.-Water-stage recorder graph. Altitude of gage is $865 \mathrm{ft}$ (from topographic map).

Discharge.- Stage-discharge relation defined by current-meter measurements. Back-

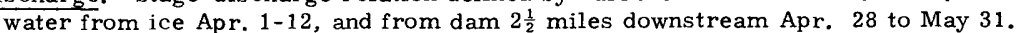

Maxima.-April-May 1965: Discharge, $684 \mathrm{cfs} 0900$ hours Apr. 15 (gage height, $8.11 \mathrm{ft}$ ); gage height, $8.20 \mathrm{ft} 0700$ hours May 11 (backwater from dam).

1949 to March 1965: Discharge, 806 cfs Apr. 12, 1952 (gage height, $7.88 \mathrm{ft}$ ).

Remarks.- Subject to backwater from Minnesota Division of Game and Fish dam approximately $2 \frac{1}{2}$ miles downstream. At high stages a small part of flow discharges into the Rum River and Coon Creek basins from West Arm of Coon Lake and South Coon Lake, respectively.

Mean discharge, in cubic feet per second, 1965

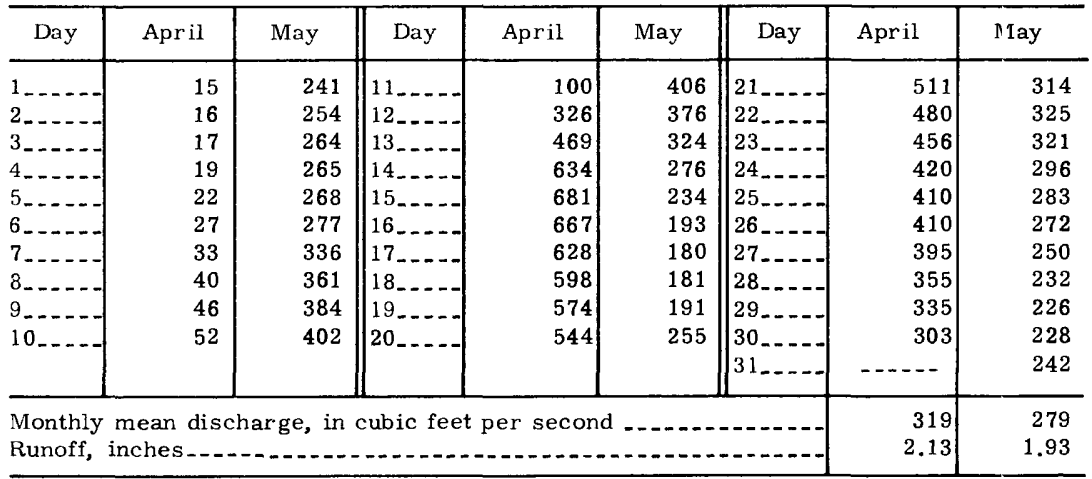

Gage height, in feet, and discharge, in cubic feet per second, at indicated time, 1965

\begin{tabular}{|c|c|c|c|c|c|c|c|c|c|c|c|}
\hline Date & Hour & $\begin{array}{c}\text { Gage } \\
\text { height }\end{array}$ & $\begin{array}{c}\text { Dis - } \\
\text { charge }\end{array}$ & Date & Hour & $\begin{array}{c}\text { Gage } \\
\text { height }\end{array}$ & $\begin{array}{c}\text { Dis- } \\
\text { charge }\end{array}$ & Date & Hour & $\begin{array}{c}\text { Gage } \\
\text { height }\end{array}$ & $\begin{array}{l}\text { Dis- } \\
\text { harge }\end{array}$ \\
\hline Apr. 9 & 0000 & 5.44 & $-\cdots$ & Apr. 13 & 1200 & 7.42 & 465 & Apr. 17 & 2400 & 7.91 & 614 \\
\hline & 0900 & 5.28 & $\ldots$ & & 2400 & 7.75 & 558 & & & & \\
\hline & 2000 & 5.68 & $-\ldots$ & & & & & 18 & 1200 & 7.86 & 596 \\
\hline & 2400 & 5.70 & $\ldots$ & 14 & 1200 & 8.01 & 648 & & 2400 & 7.83 & 586 \\
\hline & & & & & 2400 & 8.10 & 680 & & & & \\
\hline 10 & 1200 & 5.82 & $\ldots$ & & & & & 19 & 1200 & 7.80 & 575 \\
\hline & 2400 & 6.05 & -- & 15 & $\begin{array}{l}0900 \\
2400\end{array}$ & $\begin{array}{l}8.11 \\
8.09\end{array}$ & $\begin{array}{l}684 \\
676\end{array}$ & & 2400 & 7.76 & 561 \\
\hline 11 & 1200 & 6.29 & $-\cdots$ & & & & & 20 & 1200 & 7.71 & 544 \\
\hline & 2400 & 6.53 & $-\ldots$ & 16 & $\begin{array}{l}1200 \\
2400\end{array}$ & $\begin{array}{l}8.07 \\
8.02\end{array}$ & $\begin{array}{l}670 \\
652\end{array}$ & & 2400 & 7.66 & 528 \\
\hline 12 & 1200 & 6.80 & $\cdots$ & & & & & 21 & 1200 & 7.60 & 5.10 \\
\hline & 2400 & 7.10 & $\ldots$ & 17. & 1200 & 7.94 & 624 & & 2400 & 7.55 & 4.98 \\
\hline
\end{tabular}


(92) 5-3405. St. Croix River at St. Croix Falls, Wis.

Location.-Lat $45^{\circ} 24^{\prime} 30^{\prime \prime}$, long $92^{\circ} 38^{\prime} 45^{\prime \prime}$, in NW $\frac{1}{4}$ sec. 30 , T.34 N., R.18 W., on left bank $1,800 \mathrm{ft}$ downstream from powerplant of Northern States Power Co., in St. Croix Falls, and at mile 52.2.

Drainage area. $-5,930 \mathrm{sq} \mathrm{mi}$, approximately.

Gage-height record.-Digital recorder tape punched at fifteen-minute intervals except 1300 hours Apr. 15 to 1900 hours Apr. 23 for which graph was reconstructed on basis of high-water mark in gage house and twice-daily outside gage reacings. Datum of gage is $690.47 \mathrm{ft}$ above mean sea level, adjustment of 1912 .

Discharge record.- Stage-discharge relation defined by current-meter measurements. Mean daily discharges computed from 96 punch-tape recordings per day except Apr. 15-23. Backwater from ice Mar. 30, 31.

Maxima.-March-May 1965: Discharge, 45,700 cfs 1200 hours Apr. 18 (gage height, $20.98 \mathrm{ft}$ ).

1902 to February 1965: Discharge, 54,900 cfs May 8, 1950 (gage height, $25.19 \mathrm{ft}$ ).

Remarks.-Flow affected by powerplant upstream.

Mean discharge, in cubic feet per second, 1965

\begin{tabular}{|c|c|c|c|c|c|c|c|c|c|c|c|}
\hline Day & March & April & May & Day & March & April & May & Day & March & April & May \\
\hline & 2,100 & 1,890 & 15,200 & $11 \ldots$ & 2,100 & 11,400 & 17,400 & 21. & 1,870 & 42,900 & 9,110 \\
\hline 2. & 1,550 & 2,130 & 13,800 & $12 \ldots$ & 2,060 & 17,100 & 17,200 & 22 & 1,870 & 41,100 & 8,970 \\
\hline & 1,800 & 1,760 & 12,100 & $13 \ldots$ & 2,000 & 19,300 & 15,900 & $23 \ldots$ & 1,930 & 38,400 & 8,760 \\
\hline & 1,750 & 1,840 & 9,620 & $14 \ldots$ & 1,900 & 25,000 & 14,200 & 24 & 1,910 & 35,500 & 8,570 \\
\hline & 2,060 & 2,090 & 8,960 & $15 \ldots$ & 2,020 & 34,400 & 12,900 & $25 \ldots$ & 1,850 & 32,400 & 8,980 \\
\hline & 2,040 & 4,030 & 9,830 & $16 \ldots$ & 2,050 & 40,500 & 11,700 & 26. & 1,960 & 29,700 & 9,430 \\
\hline & 2,020 & 3,370 & 14,200 & $17 \ldots$ & 2,000 & 43,500 & 10,200 & $27 \ldots$ & 1,850 & 26,300 & 9,640 \\
\hline & 2,140 & 4,250 & 16,000 & $18 \ldots$ & 1,910 & 45,100 & 10,600 & $28_{\ldots}$ & 2,080 & 23,100 & 9,160 \\
\hline & 2,040 & 4,710 & 16,500 & $19 \ldots$ & 2,140 & 44,400 & 10,500 & $\mid 29_{\ldots}$ & 2,000 & 20,300 & 8,510 \\
\hline & 2,080 & 7,030 & 17,500 & $20 \ldots$ & 1,850 & 43,400 & 9,600 & 30. & 1,880 & 17,800 & 7,780 \\
\hline & & & & & & & & & 1,860 & & 7,300 \\
\hline \multirow{2}{*}{\multicolumn{9}{|c|}{$\begin{array}{l}\text { Monthly mean discharge, in cubic feet per second } \\
\text { Runoff, in inches }\end{array}$}} & 1,957 & 22,160 & 11,620 \\
\hline & & & & & & & & & 0.38 & 4.17 & 2.26 \\
\hline
\end{tabular}

Gage height, in feet, and discharge, in cubic feet per second, at indicated time, 1965

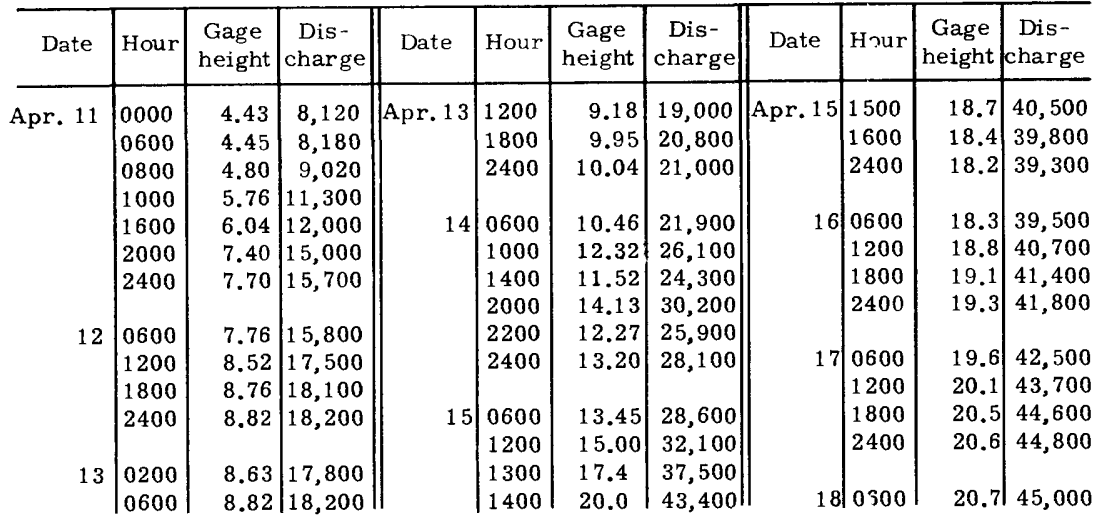


MARCH-MAY, UPPER MISSISSIPPI RIVER BASIN

Gage height, in feet, and discharge, in cubic feet per second, at indicated time, 1965, of St C'roix River at St Croix Falls, Wis - Continued

\begin{tabular}{|c|c|c|c|c|c|c|c|c|c|c|c|c|}
\hline Date & Hour & $\begin{array}{c}\text { Gage } \\
\text { height }\end{array}$ & $\begin{array}{c}\text { Dis- } \\
\text { charge }\end{array}$ & Date & Hour & $\begin{array}{l}\text { Gage } \\
\text { height }\end{array}$ & $\begin{array}{c}\text { Dis- } \\
\text { charge }\end{array}$ & \multicolumn{2}{|c|}{ Date } & Hour & $\begin{array}{c}\text { Gage } \\
\text { height }\end{array}$ & $\begin{array}{l}\text { Dis- } \\
\text { charge }\end{array}$ \\
\hline \multirow[t]{6}{*}{ Apr. 18} & 1000 & 20.9 & 45,500 & Apr. 25 & 1200 & 15.01 & 32,100 & May & 8 & 2400 & 7.81 & 15,900 \\
\hline & 1200 & 20.98 & 45,700 & & 1800 & 14.84 & 31,800 & & & & & \\
\hline & 1400 & 20.9 & 45,500 & & 2400 & 14.56 & 31,100 & & 9 & 0600 & 8.09 & 16,600 \\
\hline & 1800 & 20.7 & 45,000 & & & & & & & 1200 & 7.85 & 16,000 \\
\hline & 2400 & 20.5 & 44,600 & 26 & 1200 & 13.92 & 29,700 & & & 1400 & 8.14 & 16,700 \\
\hline & & & & & 2400 & 13.21 & 28,100 & & & 2400 & 8.27 & 16,900 \\
\hline \multirow[t]{5}{*}{19} & 0600 & 20.4 & 44,400 & & & & & & & & & \\
\hline & 1200 & 20.4 & 44,400 & 27 & 1200 & 12.44 & 26,400 & & 10 & 0600 & 8.40 & 17,300 \\
\hline & 1800 & 20.4 & 44,400 & & 2400 & 11.66 & 24,600 & & & 1800 & 8.58 & 17,700 \\
\hline & 2400 & 20.3 & 44,100 & & & & & & & 2400 & $8.56^{\circ}$ & 17,600 \\
\hline & & & & 28 & 1200 & 11.01 & 23,100 & & & & & \\
\hline \multirow[t]{3}{*}{20} & 0600 & 20.0 & 43,400 & & 2400 & 10.38 & 21,700 & & 11 & 0600 & 8.55 & 17,600 \\
\hline & 2400 & 19.9 & 43,200 & & & & & & & 0800 & 8.28 & 17,000 \\
\hline & & & & 29 & 1200 & 9.73 & 20,300 & & & 1200 & 8.42 & 17,300 \\
\hline \multirow[t]{4}{*}{21} & 1200 & 19.8 & 43,000 & & 2400 & 9.16 & 19,000 & & & 2400 & 8.46 & 17,400 \\
\hline & 1800 & 19.7 & 42,700 & & & & & & & & & \\
\hline & 2400 & 19.5 & 42,300 & 30 & 1200 & 8.64 & 17,800 & & 12 & 1200 & 8.40 & 17,300 \\
\hline & & & & & 2400 & 8.06 & 16,500 & & & 2400 & 8.18 & 16,800 \\
\hline \multirow[t]{3}{*}{22} & 1200 & 19.0 & 41,100 & & & & & & & & & \\
\hline & 2400 & 18.4 & 39,800 & May & 1200 & 7.49 & 15,200 & & 13 & 0600 & 8.09 & 16,600 \\
\hline & & & & & 2400 & 7.00 & 14,100 & & & 0800 & 7.39 & 15,000 \\
\hline \multirow[t]{4}{*}{23} & 1200 & 17.8 & 38,400 & & & & & & & 1200 & 7.92 & 16,200 \\
\hline & 1800 & 17.5 & 37,700 & 6 & 2400 & 6.39 & 12,800 & & & 1800 & 7.75 & 15,800 \\
\hline & 2400 & 17.3 & 37,300 & & & & & & & 2000 & 7.44 & 15,100 \\
\hline & & & & 7 & 0400 & 6.22 & 12,300 & & & 2400 & 7.44 & 15,10 \\
\hline \multirow[t]{4}{*}{24} & 0600 & 17.04 & 36,700 & & 1200 & 7.08 & 14,300 & & & & & \\
\hline & 1200 & 16.32 & 35,100 & & 1400 & 7.64 & 15,600 & & 14 & 0600 & 7.34 & 14,900 \\
\hline & 2400 & 15.78 & 33,900 & & 2400 & 7.63 & 15,600 & & & 1200 & 6.91 & 13,90 \\
\hline & & & & & & & & & & 1800 & 6.83 & 13,800 \\
\hline \multirow[t]{2}{*}{25} & 0800 & 15.33 & 32,900 & 8 & 0200 & 8.14 & 16,700 & & & 2400 & 6.77 & 13,600 \\
\hline & 1000 & 14.74 & 31,500 & & 1200 & 7.76 & 15,800 & & & & & \\
\hline
\end{tabular}

(93) 5-3415. Apple River near Somerset, Wis.

Location.-Lat $45^{\circ} 09^{\prime} 30^{\prime \prime}$, long $92^{\circ} 43^{\prime} 00^{\prime \prime}$, in sec.21, T.31 N., R.19 W., at powerp'ant of Northern States Power Co., 3.5 miles downstream from Somerset.

Drainage area. $-555 \mathrm{sq} \mathrm{mi}$.

Gage-height record.-Headwater and tailwater gages read hourly.

Discharge record.-Discharge computed by Northern States Power Co. from its powerplant records on bas is of ratings developed by Geological Survey.

Maxima.-March-May 1965: Daily discharge, 2,510 cfs Apr. 13.

1901 to February 1965: Daily discharge, 2,460 cfs June 17, 1943.

Remarks.-Flow affected by many powerplants upstream.

Cooperation.--Records of daily discharge furnished by Northern States Power Co. 
Mean discharge, in cubic feet per second, 1965, of Apple River near Somersot, Wis.

\begin{tabular}{|c|c|c|c|c|c|c|c|c|c|c|c|}
\hline Day & March & April & May & Day & March & April & May & Day & March & April & May \\
\hline $\begin{array}{l}2 . \\
3 . \\
4 . \\
5 . \\
6 . \\
7 . \\
8 . \\
9 . \\
10\end{array}$ & $\begin{array}{l}160 \\
255 \\
310 \\
325 \\
323 \\
310 \\
325 \\
235 \\
265 \\
315\end{array}$ & $\begin{array}{r}290 \\
288 \\
341 \\
445 \\
470 \\
395 \\
740 \\
1,060 \\
1,220 \\
1,470\end{array}$ & $\begin{array}{l}967 \\
665 \\
455 \\
320 \\
565 \\
530 \\
537 \\
644 \\
710 \\
870\end{array}$ & $\begin{array}{l}11-- \\
12_{--} \\
13_{--} \\
14_{--} \\
15_{--} \\
16_{--} \\
17_{--} \\
18_{--} \\
19_{--} \\
20_{--}\end{array}$ & $\begin{array}{l}295 \\
287 \\
320 \\
370 \\
310 \\
300 \\
260 \\
275 \\
267 \\
260\end{array}$ & $\begin{array}{l}1,590 \\
2,040 \\
2,510 \\
2,500 \\
2,470 \\
2,450 \\
2,480 \\
2,480 \\
2,480 \\
1,540\end{array}$ & $\begin{array}{l}645 \\
650 \\
635 \\
521 \\
387 \\
530 \\
685 \\
470 \\
490 \\
505\end{array}$ & 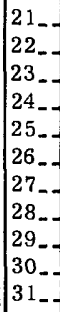 & $\begin{array}{l}325 \\
225 \\
280 \\
265 \\
305 \\
266 \\
300 \\
295 \\
195 \\
255 \\
285\end{array}$ & $\begin{array}{r}1,280 \\
1,250 \\
1,260 \\
1,120 \\
1,020 \\
1,050 \\
1,040 \\
1,040 \\
740 \\
984 \\
----\end{array}$ & $\begin{array}{l}478 \\
650 \\
690 \\
485 \\
340 \\
475 \\
635 \\
635 \\
616 \\
518 \\
360\end{array}$ \\
\hline \multicolumn{9}{|c|}{$\begin{array}{l}\text { Monthly mean discharge, in cubic feet per second } \\
\text { Runoff, in inches }\end{array}$} & $\begin{array}{r}283 \\
0.59\end{array}$ & $\begin{array}{r}1,335 \\
2.68\end{array}$ & $\begin{array}{r}570 \\
1.18\end{array}$ \\
\hline
\end{tabular}

(94) 5-3419. Kinnickinnic River tributary at River Falls, Wis.

(Crest-stage station)

Location.-Lat $44^{\circ} 49^{\prime} 57^{\prime \prime}$, long $92^{\circ} 38^{\prime} 23^{\prime \prime}$, in NE $\frac{1}{4}$ sec.14, T.27 N., R.19 W., at bridge on County Trunk FF, 0.5 mile west of intersection with State Highway 29, and 1.6 miles southwest of intersection of State Highways 29 and 35 in River Falls.

Drainage area. $-7.26 \mathrm{sq} \mathrm{mi}$.

Gage-height record.-Crest stages only.

Maxima.-March-May 1965: Gage height, $12.14 \mathrm{ft}$ Apr. 8.

1959 to February 1965: Gage height $11.98 \mathrm{ft}$ July 8, 1959.

(95) St. Croix River at Prescott, Wis.

(Miscellaneous site)

Location.-Lat $44^{\circ} 44^{\prime} 56^{\prime \prime}$, long $92^{\circ} 48^{\prime} 12^{\prime \prime}$, in sec.9, T.26 N., R.20 W., at bridge on U.S. Highway 10,600 ft upstream from Mississippi River, and $500 \mathrm{ft}$ north of Chicago, Burlington and Quincy Railroad bridge in Prescott.

Gage-height record.-Floodmark and gage heights are those for Mississippi River at Prescott, Wis. Datum of gage is $600.00 \mathrm{ft}$ above mean sea level, adjustment of 1912 (levels by Corps of Engineers).

Discharge record.-Discharge obtained on the following days by current-meter measurement.

Date

Apr. 14, 1965

Apr. 17

Apr. 26

May 7
Discharge, in cfs

$$
\begin{aligned}
& 12,900 \\
& 43,700 \\
& 36,600
\end{aligned}
$$$$
12,600
$$ 


\section{MISSISSIPPI RIVER MAIN STEM}

(96) 5-3445. Mississippi River at Prescott, Wis.

Location.-Lat $44^{\circ} 44^{\prime} 45^{\prime \prime}$, long $92^{\circ} 48^{\prime} 00^{\prime \prime}$, in sec.9, T.26 N., R.20 W., on left ban'r at Prescott, $200 \mathrm{ft}$ downstream from St. Croix River, $300 \mathrm{ft}$ south of Chicago, Burlington and Quincy Railroad bridge, $800 \mathrm{ft}$ south of bridge on U.S. Highway 10, and at mile 811.4 upstream from Ohio River. Auxiliary water-stage recorder 10.7 miles downstream from base gage.

Drainage area.- $-44,800 \mathrm{sq} \mathrm{mi}$, approximately.

Gage-height record.-Water-stage recorder graph except 1810 hours Apr. 14 to 1730 hours Apr. 24 when graph was constructed on bas is of several gage readings $\mathrm{k}$ y engineers and twice-daily gage readings by Corps of Engineers on temporary staf ${ }^{f}$ gage. $^{2}$

For auxiliary gage, water-stage recorder graph except 1600 hours Apr. 12 to 1730 hours Apr. 14 and 2130 hours Apr. 15 to 1700 hours Apr. 26. Datum of gage is 600.00 $\mathrm{ft}$ above mean sea level, datum of 1912 (levels by Corps of Engineers).

Discharge record.-Stage-fall-discharge relation affected by changes in slope and defined by current-meter measurements. Fall was a factor for computing the discharge Apr. 5, 6. For the period Apr. 1-4, flow was computed on the basis of discharge for Lock and Dam No, 3 and adjusted for storage. These records furnished by Corps of Engineers.

Maxima.-April-May 1965: Discharge, 228,000 cfs 0600 hours Apr. 18 (gage height, $93.11 \mathrm{ft}$, from graph based on gage readings).

1928 to March 1965: Discharge, 155,000 cfs Apr. 16-18, 1952 (gage height, 89.03 ft).

Remarks.- Some regulation by reservoirs, navigation dams, and powerplants at low and medium stages.

Mean discharge, in cubic feet per second, 1965

\begin{tabular}{|c|c|c|c|c|c|c|c|c|}
\hline Day & April & May & Day & April & May & Day & April & May \\
\hline $\begin{array}{l}1 \ldots . \\
2 \ldots \\
3 \ldots \\
4 \ldots \\
5 \ldots \\
6 \ldots \\
7 \ldots \\
8 \ldots \\
9 \ldots \\
10 .\end{array}$ & $\begin{array}{r}9,100 \\
8,670 \\
10,000 \\
11,800 \\
16,600 \\
22,600 \\
28,600 \\
36,200 \\
43,300 \\
55,700\end{array}$ & $\begin{array}{l}91,500 \\
84,300 \\
78,400 \\
73,400 \\
68,700 \\
65,700 \\
64,900 \\
65,500 \\
64,900 \\
65,400\end{array}$ & $\begin{array}{l}11 \ldots \\
12 \ldots \\
13 \ldots \\
14 \ldots \\
15 \ldots \\
16 \ldots \\
17 \ldots \\
18 \ldots \\
19 \ldots \\
20 \ldots\end{array}$ & $\begin{array}{r}75,900 \\
108,000 \\
143,000 \\
172,000 \\
199,000 \\
214,000 \\
223,000 \\
226,000 \\
222,000 \\
214,000\end{array}$ & $\begin{array}{l}65,400 \\
65,400 \\
64,800 \\
63,900 \\
62,600 \\
61,700 \\
59,700 \\
58,400 \\
57,200 \\
56,200\end{array}$ & $\begin{array}{l}21 \ldots \ldots \\
22 \ldots \ldots \\
23 \ldots \ldots \\
24 \ldots- \\
25 \ldots- \\
26 \ldots- \\
27 \ldots- \\
28 \ldots- \\
29-\ldots \\
30 \ldots- \\
31\end{array}$ & $\begin{array}{r}206,000 \\
192,000 \\
180,000 \\
167,000 \\
154,000 \\
140,000 \\
128,000 \\
116,000 \\
107,000 \\
99,100\end{array}$ & $\begin{array}{l}55,200 \\
54,200 \\
53,500 \\
53,100 \\
52,500 \\
53,500 \\
53,700 \\
54,500 \\
54,600 \\
54,500 \\
54,500\end{array}$ \\
\hline \multicolumn{7}{|c|}{$\begin{array}{l}\text { Monthly mean discharge, in cubic feet per second } \\
\text { Runoff, in inches }\end{array}$} & $\begin{array}{r}117,600 \\
2.93\end{array}$ & $\begin{array}{r}62,320 \\
1.60\end{array}$ \\
\hline
\end{tabular}


Gage height, in feet, and discharge, in cubic feet per second, at indicated time, 1965, of Mississippi River at Prescott, Wis.

\begin{tabular}{|c|c|c|c|c|c|c|c|c|c|c|c|}
\hline Date & Hour & $\begin{array}{c}\text { Gage } \\
\text { height }\end{array}$ & $\begin{array}{c}\text { Dis- } \\
\text { charge }\end{array}$ & Date & Hour & $\begin{array}{l}\text { Gage } \\
\text { height }\end{array}$ & $\begin{array}{c}\text { Dis- } \\
\text { charge }\end{array}$ & Date & Hoir & $\begin{array}{c}\text { Gage } \\
\text { height }\end{array}$ & $\begin{array}{c}\text { Dis- } \\
\text { charge }\end{array}$ \\
\hline \multirow[t]{18}{*}{ Apr. 5} & 0000 & 75.05 & & Apr. 12 & 0600 & 85.65 & 99,100 & Apr. 21 & 1200 & 92.00 & 207,000 \\
\hline & 0300 & 75.06 & -..... & & 1200 & 86.27 & 108,000 & & 1800 & 91.76 & 202,000 \\
\hline & 0600 & 75.04 & ---- & & 1800 & 86.87 & 117,000 & & 2400 & 91.58 & 199,000 \\
\hline & 0900 & 75.02 &...--- & & 2400 & 87.47 & 127,000 & & & & \\
\hline & 1200 & 74.95 & - & & & & & 22 & 1200 & 91.20 & 192,000 \\
\hline & 1500 & 74.93 & $\ldots$ & 13 & 0600 & 88.02 & 135,000 & & 2400 & 90.87 & 186,000 \\
\hline & 1800 & 75.01 & $-\ldots$ & & 1200 & 88.51 & 144,000 & & & & \\
\hline & 2100 & 75.16 & ---- & & 1800 & 88.95 & 151,000 & 23 & 1200 & 90.50 & 179,000 \\
\hline & 2400 & 75.29 & 18,000 & & 2400 & 89.30 & 157,000 & & 2400 & 90.22 & 174,000 \\
\hline & 0300 & 75.39 & 19,800 & 14 & 0600 & 89.72 & 165,000 & 24 & 0600 & 90.05 & 171,000 \\
\hline & 0600 & 75.54 & 20,400 & & 1200 & 90.10 & 172,000 & & 1200 & 89.85 & 167,000 \\
\hline & 0900 & 75.77 & 21,600 & & 1800 & 90.47 & 178,000 & & 1800 & 89.60 & 163,000 \\
\hline & 1200 & 75.94 & 22,400 & & 2400 & 90.84 & 185,000 & & 2400 & 89.42 & 160,000 \\
\hline & 1500 & 76.20 & 23,700 & & & & & & & & \\
\hline & 1800 & 76.55 & 25,400 & 15 & 0600 & 91.19 & 192,000 & 25 & 1230 & 89.09 & 154,000 \\
\hline & 2100 & 76.65 & 26,000 & & 1200 & 91.59 & 199,000 & & 2400 & 88.70 & 147,000 \\
\hline & 2400 & 76.76 & 26,500 & & 1800 & 92.04 & 208,000 & & & & \\
\hline & & & & & 2400 & 92.27 & 212,000 & 26 & 1270 & 88.32 & 140,000 \\
\hline \multirow[t]{9}{*}{7} & 0300 & 76.79 & 26,600 & & & & & & 2400 & 87.91 & 134,000 \\
\hline & 0600 & 76.85 & 27,000 & 16 & 0300 & 92.27 & 212,000 & & & & \\
\hline & 0900 & 76.88 & 27,100 & & 0600 & 92.24 & 212,000 & 27 & 1230 & 87.61 & 129,000 \\
\hline & 1200 & 76.94 & 27,400 & & 0900 & 92.26 & 212,000 & & 2490 & 87.21 & 122,000 \\
\hline & 1500 & 77.18 & 28,600 & & 1200 & 92.34 & 213,000 & & & & \\
\hline & 1800 & 77.54 & 30,400 & & 1500 & 92.41 & 215,000 & 28 & 1230 & 86.83 & 116,000 \\
\hline & 2100 & 77.84 & 31,900 & & 1800 & 92.48 & 216,000 & & 2400 & 86.52 & 112,000 \\
\hline & 2400 & 78.02 & 32,800 & & 2100 & 92.53 & 217,000 & & & & \\
\hline & & & & & 2400 & 92.59 & 218,000 & 29 & 1230 & 86.20 & 107,000 \\
\hline \multirow[t]{5}{*}{8} & 0600 & 78.40 & 34,700 & & & & & & 2400 & 85.94 & 103,000 \\
\hline & 1200 & 78.61 & 35,800 & 17 & 0600 & 92.71 & 220,000 & & & & \\
\hline & 1800 & 79.06 & 38,100 & & 1200 & 92.84 & 223,000 & 30 & 1230 & 85.65 & 99,100 \\
\hline & 2400 & 79.33 & 39,600 & & 1800 & 92.94 & 225,000 & & 2400 & 85.37 & 95,200 \\
\hline & & & & & 2400 & 93.05 & 227,000 & & & & \\
\hline \multirow[t]{9}{*}{9} & 0300 & 79.42 & 40,100 & & & & & May & 1270 & 85.11 & 91,500 \\
\hline & 0600 & 79.59 & 41,000 & 18 & 0600 & 93.11 & 228,000 & & 2400 & 84.83 & 87,700 \\
\hline & 0900 & 79.70 & 41,600 & & 1200 & 93.07 & 227,000 & & & & \\
\hline & 1200 & 79.81 & 42,300 & & 1800 & 92.97 & 225,000 & 2 & 1270 & 84.58 & 84,100 \\
\hline & 1500 & 80.02 & 43,500 & & 2400 & 92.90 & 224,000 & & 2400 & 84.37 & 81,400 \\
\hline & 1800 & 80.38 & 45,800 & & & & & & & & \\
\hline & 2100 & 80.64 & 47,600 & 19 & 0600 & 92.86 & 223,000 & 3 & $12 ? 0$ & 84.12 & 78,300 \\
\hline & 2400 & 80.85 & 49,000 & & 1200 & 92.83 & 223,000 & & 2490 & 83.87 & 75,600 \\
\hline & & & & & 1800 & 92.77 & 222,000 & & & & \\
\hline \multirow[t]{5}{*}{10} & 0600 & 81.24 & 51,900 & & 2400 & 92.66 & 220,000 & 4 & 1270 & 83.65 & 73,400 \\
\hline & 1200 & 81.65 & 55,100 & & & & & & 2470 & 83.45 & 71,400 \\
\hline & 1800 & 82.15 & 59,200 & 20 & 0600 & 92.51 & 217,000 & & & & \\
\hline & 2400 & 82.71 & 64,100 & & 1200 & 92.33 & 213,000 & 5 & $06: 0$ & 83.34 & 70,300 \\
\hline & & & & & 1800 & 92.18 & 210,000 & & 1220 & 83.16 & 68,500 \\
\hline \multirow[t]{4}{*}{11} & 0600 & 83.26 & 69,500 & & 2400 & 92.10 & 209,000 & & $18 ? 0$ & 83.00 & 66,900 \\
\hline & 1200 & 83.82 & 75,100 & & & & & & 2400 & 82.98 & 66,700 \\
\hline & 1800 & 84.40 & 81,800 & 21 & 0400 & 92.10 & $209,00 \mathrm{~d}$ & & & & \\
\hline & 2400 & 85.04 & 90,600 & & 1000 & 92.00 & 207,000 & & & & \\
\hline
\end{tabular}




\section{VERMILLION RIVER BASIN}

(97) 5-3459. Vermillion River tributary near Hastings, Minn.

$$
\text { (Crest-stage station) }
$$

Location.-Lat $44^{\circ} 43^{\prime} 10^{\prime \prime}$, long $92^{\circ} 56^{\prime} 03^{\prime \prime}$, in $\mathrm{NE} \frac{1}{4} \mathrm{SE} \frac{1}{4}$ sec. $35, \mathrm{~T} .115 \mathrm{~N}$., R.18 W. $\varepsilon \mathrm{t}$ culvert on county highway, 2.0 miles upstream from mouth, and 4.1 miles west of Hastings.

Drainage area. $-14.3 \mathrm{sq} \mathrm{mi}$.

Gage-height record.-Crest stages only.

Discharge record.-Stage-discharge relation defined by current-meter measurement at $182 \mathrm{cfs}$ and by indirect measurement at $544 \mathrm{cfs}$.

Maxima.-March-May 1965: Discharge, 544 cfs Apr. 6 (gage height, $21.95 \mathrm{ft}$, from high-water profile).

1960 to February 1965: Gage height, 18.50 ft Mar. 30, 1962, backwater from ice (discharge not determined).

\section{TRIMBELLE CREEK BASIN}

(98) 5-3466. Little Trimbelle Creek near Bay City, Wis.

(Crest-stage station)

Location.-Lat $44^{\circ} 38^{\prime} 01^{\prime \prime}$, long $92^{\circ} 34^{\prime} 05^{\prime \prime}$, in $\mathrm{S} \frac{1}{2}$ sec. $21, \mathrm{~T} .25 \mathrm{~N} ., \mathrm{R} .18 \mathrm{~W}$. , at $\mathrm{k}$ ridge on County Trunk K, 0.9 mile north of intersection with County Trunk E, and 7.0 miles northwest of Bay City.

Drainage area.-19.9 sq $\mathrm{mi}$.

Gage-height record.-Crest stages only.

Discharge record.-Stage-discharge relation defined by current-meter measurements below 1030 cfs.

Maxima.-March-May 1965: Discharge, 1700 cfs Apr. 7 (gage height 12.74).

1961 to February 1965; Discharge, $810 \mathrm{cfs}$ Mar. 28, 1962 (gage height $11.6 \mathrm{ft}$ ).

\section{MISSISSIPPI RIVER MAIN STEM}

(99) Mississippi River at Lock and Dam 3 near Red Wing, Minn.

(Miscellaneous site)

Location.-Lat $44^{\circ} 37^{\prime}$, long $92^{\circ} 37^{\prime}$, on east and west section line between sections 4 and 9, T.11 3 N., R.15 W., at Lock and Dam 3, 13 miles northwest of Red Wing, and at river mile 797.06 above Ohio River.

Gage-height record.-Peak stages from pool and tailwater water-stage recorder graphs.

Discharge record.-Corps of Engineers stage-discharge relation extended on the basis of Geological Survey current-meter measurement.

Maxima.-April-May 1965: Discharge, 228,000 cfs 2400 hours Apr. 18 (elevations, $688.22 \mathrm{ft}$ pool, $687.93 \mathrm{ft}$, tailwater).

1938 to March 1965: Discharge, $151,000 \mathrm{cfs}$ Apr. 17, 1952 (elevations, $684.68 \mathrm{ft}$ pool, $684.12 \mathrm{ft}$ tailwater).

Cooperation.--Records furnished by Corps of Engineers. 


\title{
CANNON RIVER BASIN
}

\author{
(100) 5-3551.5. Pine Creek near Cannon Falls, Minn.
}

\section{(Crest-stage station)}

Location.-Lat $44^{\circ} 32^{\prime} 27^{\prime \prime}$, long $92^{\circ} 53^{\prime} 40^{\prime \prime}$, in NE $\frac{1}{4} \mathrm{NE} \frac{1}{4}$ sec.6, T.112 N., R.17 W., at culvert on State Highway 20, 2.0 miles upstream from mouth and 2.1 miles north of Cannon Falls.

Drainage area.-20.2 sq mi.

Gage-height record.-Crest stages only.

Discharge record.-Stage-discharge relation defined by current-meter measurements below $244 \mathrm{cfs}$ and by indirect measurement at $844 \mathrm{cfs}$.

Maxima.-March-May 1965: Discharge, 844 cfs Apr. 8 (gage height, 7.54 ft from highwater profile).

1960 to February 1965: Discharge, 133 cfs May 12, 1962 (gage height, $2.78 \mathrm{ft}$ ).

(101) 5-3552. Cannon River at Welch, Minn.

Location.-Lat $44^{\circ} 33^{\prime} 50^{\prime \prime}$, long $92^{\circ} 43^{\prime} 55^{\prime \prime}$, in NW $\frac{1}{4} \mathrm{SW} \frac{1}{4}$ sec. $27, \mathrm{~T} .113 \mathrm{~N}$., R.16 W., on right bank 0.3 mile downstream from highway bridge at Welch and 1.8 miles upstream from Belle Creek.

Drainage area. $-1,320 \mathrm{sq} \mathrm{mi}$, approximately.

Gage-height record.-Digital recorder tape punched at 15 minute interrals except 0630 hours Mar. 1 to 2400 hours Mar. 6 (for this period the auxiliary recorder graph was reconstructed on the basis of flood mark and adjacent record) and 0830 hours Mar. 19 to 1000 hours Mar. 26. Datum of gage is $699.16 \mathrm{ft}$ above mean sea level, datum of 1929 .

Discharge record.-Stage-discharge relation defined by current-meter measurements. Backwater from ice Mar. 1 to Apr. 4 (no gage-height record 0830 ho'srs Mar. 19 to 1000 hours Mar. 26). Mean daily discharges computed from 96 punch-tape readings per day Apr. 5 to May 31.

Maxima.-Given in the following table.

March-May 1965:

Discharge (cfs)

7,900

Apr. 8, 0245 hrs

15,800

February 1965

Apr. 2, 1952

abackwater from ice.
Gage height (feet)

$a_{14.00}$

14.01

12.00

Remarks.-Diurnal fluctuation caused by powerplants above station. 
Mean discharge, in cubic feet per second, 1965, of Cannon River at Welch, Minn.

\begin{tabular}{|c|c|c|c|c|c|c|c|c|c|c|c|}
\hline Day & March & April & May & Day & March & April & May & Day & March & April & May \\
\hline & 3,500 & 360 & 2,030 & $11 \ldots$ & 720 & 17,500 & 1,470 & 21. & 250 & 3,570 & 1,660 \\
\hline & 4,800 & 250 & 1,890 & $12 \ldots$ & 720 & 13,500 & 1,180 & $22 \ldots$ & 430 & 3,190 & 1,480 \\
\hline & 3,400 & 500 & 1,790 & $13 \ldots$ & 710 & 11,100 & 896 & $23 \ldots$ & 450 & 2,980 & 1,340 \\
\hline & 2,000 & 2,500 & 1,710 & $14 \ldots$ & 700 & 9,480 & 860 & 24 & 440 & 2,800 & 1,280 \\
\hline & 1,300 & 7,340 & 1,700 & $15 \ldots$ & 700 & 7,800 & 814 & 25. & 430 & 2,760 & 1,290 \\
\hline & 950 & 19,800 & 1,700 & $16 \ldots$ & 700 & 6,620 & 1,570 & $26 \ldots$ & 410 & 2,890 & 1,340 \\
\hline & 940 & 27,200 & 1,780 & $17 \ldots$ & 690 & 5,790 & 2,500 & $27 \ldots$ & 250 & 2,960 & 1,360 \\
\hline & 980 & 28,700 & 1,980 & $\mid 18 \ldots$ & 680 & 5,190 & 2,370 & 28 & 200 & 2,720 & 1,270 \\
\hline 9 & 940 & 23,800 & 1,760 & $19_{-}$ & 650 & 4,590 & 2,080 & 29 & 350 & 2,470 & 1,200 \\
\hline 10 & 750 & 22,600 & 1,610 & $20 \ldots$ & 470 & 4,030 & 1,750 & 30. & 360 & 2,210 & 1,120 \\
\hline & & & & & & & & & 360 & $\ldots$ & 1,050 \\
\hline \multicolumn{9}{|c|}{ Monthly mean discharge, in cubic feet per second . . } & 975 & 8,240 & 1,543 \\
\hline & 0.85 & 6.96 & 1.35 \\
\hline
\end{tabular}

Gage height, in feet, and discharge, in cubtc feet per second, at indicated time, 1965

\begin{tabular}{|c|c|c|c|c|c|c|c|c|c|c|c|}
\hline Date & Hour & $\begin{array}{c}\text { Gage } \\
\text { height }\end{array}$ & $\begin{array}{c}\text { Dis - } \\
\text { charge }\end{array}$ & Date & Hour & $\begin{array}{l}\text { Gage } \\
\text { height }\end{array}$ & $\begin{array}{c}\text { Dis- } \\
\text { charge }\end{array}$ & Date & Hour & $\begin{array}{c}\text { Gage } \\
\text { height }\end{array}$ & $\begin{array}{c}\text { Dis- } \\
\text { charge }\end{array}$ \\
\hline \multirow[t]{6}{*}{ Feb. 28} & 0000 & 3.10 & $\ldots$ & Mar. & 52400 & 5.87 & - & Apr. 6 & 1000 & $12.5^{4}$ & 20,300 \\
\hline & 0600 & 2.28 & $\ldots$ & & & & & & 1200 & 12.45 & 19,500 \\
\hline & 1200 & 2.14 & - & 6 & 60600 & 5.40 & -.... & & 1800 & 12.43 & 19,300 \\
\hline & 2000 & 4.06 & $\ldots-\cdots$ & & 1200 & 5.00 & $-\cdots$ & & 2000 & 12.84 & 23,200 \\
\hline & 2400 & 5.55 &.---- & & 1500 & 4.22 & & & 2200 & 12.97 & 24,700 \\
\hline & & & & & 1800 & 3.79 & & & 2400 & 13.32 & 28,500 \\
\hline \multirow[t]{12}{*}{ Mar. 1} & 0200 & 6.28 & - & & 2400 & 3.53 & & & & & \\
\hline & 0600 & 7.86 & $=-$ & & & & & 7 & 0200 & 13.35 & 28,900 \\
\hline & 0800 & 9.30 & $\ldots$ & Apr. & 20000 & 3.34 & 881 & & 1200 & $12.6 \varepsilon$ & 21,600 \\
\hline & 1000 & 11.40 & $\ldots$ & & 0400 & 3.08 & 715 & & 1600 & 13.16 & 26,800 \\
\hline & 1100 & 13.35 & $\ldots$ & & 1200 & 2.10 & 250 & & 2400 & 13.81 & 33,900 \\
\hline & 1200 & 13.86 & $\ldots$ & & 1600 & 3.59 & 1,040 & & & & \\
\hline & 1300 & 14.00 & 7,900 & & 2400 & 3.51 & 992 & 8 & 0200 & $13.8 \mathrm{E}$ & 84,500 \\
\hline & 1600 & 13.20 & $\ldots$ & & & & & & 0245 & 14.01 & 36,100 \\
\hline & 1800 & 12.57 & - - - - & & 30600 & 2.73 & 517 & & 0600 & 13.66 & 32,300 \\
\hline & 1900 & 12.55 &.----- & & 1200 & 2.64 & 472 & & 0800 & 13.32 & 28,500 \\
\hline & 2400 & 12.76 &..---- & & 1800 & 3.97 & 1,290 & & 1200 & 13.11 & 26,200 \\
\hline & & & & & 2400 & 4.48 & 1,650 & & 1600 & $12.9 \mathrm{c}$ & 24,900 \\
\hline \multirow[t]{6}{*}{2} & 0600 & 12.07 & --- & & & & & & 2400 & 13.20 & 27,200 \\
\hline & 0900 & 12.06 & & & $4 \mid 0600$ & 5.07 & 2,090 & & & & \\
\hline & 1200 & 12.08 & $\ldots$ & & 1400 & 5.80 & 2,720 & 9 & 0600 & 12.93 & 24,200 \\
\hline & 1800 & 12.05 & -- & & 1600 & 6.72 & 3,590 & & 1200 & 12.60 & 20,800 \\
\hline & 2400 & 11.86 & $\ldots$ & & 1800 & 7.44 & 4,340 & & 2400 & 13.08 & 25,900 \\
\hline & & & & & 2100 & 7.78 & 4,740 & & & & \\
\hline \multirow[t]{3}{*}{3} & 1200 & 11.29 & $\ldots-.-$. & & 2400 & 7.69 & 4,630 & 10 & 0600 & 12.94 & 24,300 \\
\hline & 2400 & 10.73 & $\ldots-. .--$ & & & & & & 1400 & 12.60 & 20,800 \\
\hline & & & & & $5 \longdiv { 0 2 0 0 }$ & 7.68 & 3,880 & & 2400 & 12.79 & 22,700 \\
\hline \multirow[t]{3}{*}{4} & 1200 & 10.17 & $\ldots$ & & 0800 & 8.38 & 4,980 & & & & \\
\hline & 2400 & 9.67 & $\ldots-.-1$ & & 1400 & 9.76 & 7,780 & 11 & 0100 & 12.58 & 20,600 \\
\hline & & & & & 2000 & 10.84 & 10,800 & & 0600 & 12.38 & 18,800 \\
\hline \multirow[t]{3}{*}{5} & 1200 & 9.12 & $-\ldots .-$. & & 2400 & 11.56 & 13,600 & & 1200 & 12.15 & 17,200 \\
\hline & 1500 & 8.54 &.- & & & & & & 2400 & 11.81 & 15,100 \\
\hline & 1800 & 7.37 & $\ldots-$ & & 0600 & 12.30 & 18,200 & & & & \\
\hline
\end{tabular}




\section{CHIPPEWA RIVER BASIN}

(102) 5-3554. Moose Lake near Winter, Wis.

Location.—Lat $46^{\circ} 02^{\prime} 00^{\prime \prime}$, long $91^{\circ} 04^{\prime} 30^{\prime \prime}$, in $\mathrm{NE} \frac{1}{4}$ sec. $14, \mathrm{~T} .41$ N., R.6 W., on West Fork Chippewa River, 15 miles north of Winter.

Drainage area.- $-225 \mathrm{sq} \mathrm{mi}$.

Gage-height record.-Staff gage. Datum of gage is mean sea level (Northern States Power Co. benchmark).

Maxima.-March-May 1965: Contents at end of week, 407 million cubic feet May 28 (elevation $1374.10 \mathrm{ft}$ ).

1926 to February 1965: Contents on last day of month, 520 millior cubic feet Aug. 31, 1941 (elevation, $1375.6 \mathrm{ft}$ ).

Remarks.-Dam was completed and storage began in 1893. Usable capacity in winter, 400 million cubic feet between elevations $1367.0 \mathrm{ft}$ and $1374.0 \mathrm{ft}$; in summer, no usable capacity (limits established by Northern States Power Co.).

Cooperation.-Elevations and capacity table furnished by Northern States Power Co.

Elevation, in feet, and contents, in millions of cubic feet, 1965

\begin{tabular}{|c|c|c|c|c|c|}
\hline Date & Elevation & Contents & Date & Elevation & Contents \\
\hline Feb. $26 \ldots$ & 1367.10 & 5 & Apr. 16. & 1370.60 & 180 \\
\hline Mar. 5n.. & 1367.10 & 5 & Apr. 23. & 1372.00 & 265 \\
\hline Mar.12.... & 1367.20 & 10 & Apr. $30_{-}$ & 1373.70 & 379 \\
\hline Mar.19.... & 1367.20 & 10 & May & 1373.90 & 393 \\
\hline Mar. 26.... & 1367.20 & 10 & May 14- & 1373.90 & 393 \\
\hline Apr. 2- & 1367.20 & 10 & May 21. & 1373.85 & 390 \\
\hline Apr. $9 \ldots$ & 1367.50 & 25 & May 28_ & 1374.10 & 407 \\
\hline
\end{tabular}

(103) 5-3556. Lake Chippewa near Winter, Wis.

Location.-Lat $45^{\circ} 53^{\prime} 20^{\prime \prime}$, long $91^{\circ} 04^{\prime} 40^{\prime \prime}$, in $\mathrm{SE} \frac{1}{4}$ sec.2, T.39 N., R.6 W., on Chippewa River just downstream from mouth of East Fork Chippewa River and 5.5 miles northwest of Winter.

Drainage area.- $-775 \mathrm{sq} \mathrm{mi}$.

Gage-height record.-Chain gage. Datum of gage is mean sea level (Northern States Power Co, bench mark).

Maxima.-March-May 1965: Contents observed, 10,000 million cubic feet May 19-20 (elevation, $1313.00 \mathrm{ft}$ ).

1923 to February 1965: Contents observed, 11,000 million cubic feet Sept. 18, 19, 1926 (elevation, $1314.4 \mathrm{ft}$ ).

Remarks.-Dam was completed and storage began in 1923. Usable capacity, 10,000 million cubic feet between elevations $1287.0 \mathrm{ft}$ and $1313.0 \mathrm{ft}$ (limits stipulated by Public Service Commission of Wisconsin).

Cooperation.-Elevations and capacity table furnished by Northern States Power Co. 
Elevation, in feet, and contents, in millions of cubic feet, 1965, of Lake Chippewa near Winter, $V^{\prime}$ is.

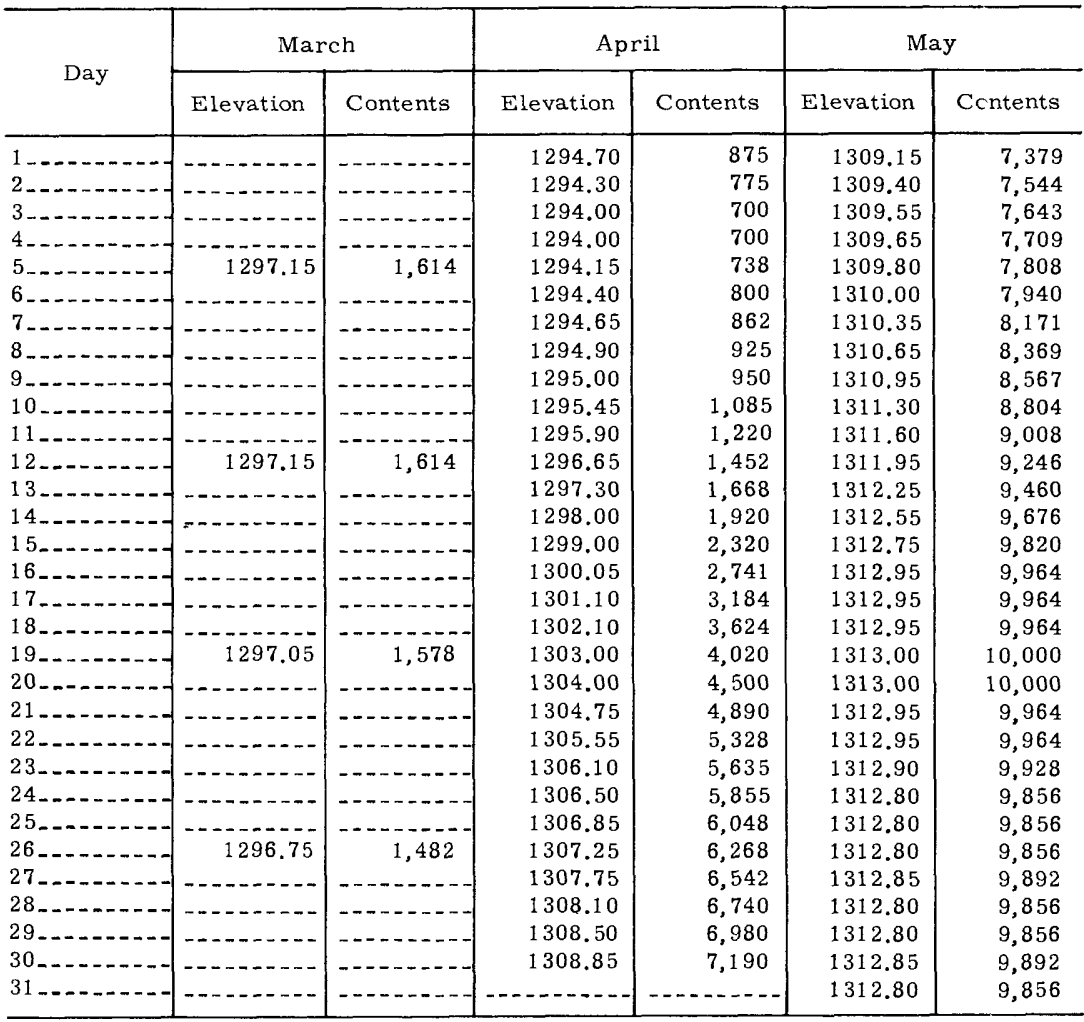

(104) 5-3560. Chippewa River at Bishops Bridge, near Winter, Wis.

Location.-Lat $45^{\circ} 50^{\prime} 55^{\prime \prime}$, long $91^{\circ} 04^{\prime} 45^{\prime \prime}$, in sec. 23 , T. 39 N., R. 6 W., on right bank $15 \mathrm{ft}$ upstream from highway bridge, 3.2 miles downstream from Lake Chippewa Dam, and 3.7 miles northwest of Winter.

Drainage area. $-787 \mathrm{sq} \mathrm{mi}$.

Gage-height record.-Water-stage recorder graph. Altitude of gage is $1,270 \mathrm{ft}$ (from Lake Chippewa datum).

Discharge record.-Stage-discharge relation defined by current meter measurements. Backwater from ice March 20-24.

Maxima.-March-May 1965: Discharge, 2,890 cfs, 2300 hours May 19 (gage height, $7.35 \mathrm{ft})$.

1912 to February 1965: Discharge, 7,520 cfs Sept. 4, 5, 1941 (gage height, $11.05 \mathrm{ft}$ ).

Remarks,-Flow completely regulated by Moose Lake and Lake Chippewa. 
Mean discharge, in cubic feet per second, 1965, of Chippewa River at Bishops Bridge, near Winter, Wis.

\begin{tabular}{|c|c|c|c|c|c|c|c|c|c|c|c|}
\hline Day & March & April & May & Day & March & April & May & Day & March & April & May \\
\hline & 564 & 874 & 108 & $11 \ldots$ & 457 & 195 & 141 & $21 \ldots$ & 448 & 185 & 2,870 \\
\hline 2. & 253 & 731 & 108 & $12 \ldots$ & 457 & 214 & 124 & 22 & 440 & 161 & 2,850 \\
\hline 3. & 253 & 260 & 108 & $13 \ldots$ & 452 & 223 & 173 & $23=$ & 440 & 147 & 2,440 \\
\hline 4. & 343 & 246 & 103 & $14 \ldots$ & 452 & 256 & 792 & $24 \ldots$ & 440 & 136 & 1,740 \\
\hline 5. & 457 & 246 & 106 & $15 \ldots$ & 452 & 297 & 1,480 & $25=$ & 440 & 127 & 1,600 \\
\hline 6. & 457 & 253 & 116 & $16 \ldots$ & 452 & 267 & 2,430 & 26 & 641 & 122 & 1,600 \\
\hline 7. & 457 & 253 & 133 & $17=$ & 446 & 250 & 2,430 & 27. & 917 & 116 & 1,600 \\
\hline 8. & 457 & 253 & 192 & $18 \ldots$ & 452 & 250 & 2,430 & $28 \ldots$ & 934 & 113 & 1,600 \\
\hline 9. & 457 & 223 & 188 & 19. & 452 & 250 & 2,710 & 29. & 908 & 111 & 1,600 \\
\hline 10 & 457 & 144 & 153 & $20 \ldots$ & 450 & 220 & 2,880 & $30 \ldots$ & 892 & 108 & 1,590 \\
\hline & & & & & & & & $31 \ldots$ & 883 & 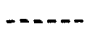 & 1,590 \\
\hline \multicolumn{9}{|c|}{ Monthly mean discharge, in cubic feet per second } & 518 & 241 & 1,225 \\
\hline
\end{tabular}

(105) 5-3565. Chippewa River near Bruce, Wis.

Location.-Lat $45^{\circ} 27^{\prime} 05^{\prime \prime}$, long $91^{\circ} 15^{\prime} 40^{\prime \prime}$, in $\mathrm{SE} \frac{1}{4}$ sec.5, T. 34 N., R. 7 W., on right bank 1 mile east of Bruce and 1 mile downstream from Thornapple River.

Drainage area.-1,630 sq $\mathrm{mi}$, approximately.

Gage-height record.-Digital recorder tape punched at sixty-minute irtervals and crest-stage indicator. Datum of gage $1,059.62 \mathrm{ft}$ above mean sea level, datum of 1929.

Discharge record.-Stage-discharge relation defined by current-meter measurements below 20,000 cfs. Mean daily discharges computed from 24 punch-tape recordings per day. Backwater from ice Mar. 1 to Apr. 16.

Maxima.-March-May 1965: Discharge, 12,300 cfs 1630 hours Apr. 15 (gage height $15.19 \mathrm{ft}$, backwater from ice).

1913 to February 1965: Discharge, $25,800 \mathrm{cfs}$ Sept. 1, 1941 (gage height, $20.46 \mathrm{ft}$, from floodmarks).

Remarks.-Flow from 48 percent of the drainage area regulated by Moose Lake and Lake Chippewa.

Mean discharge, in cublic feet per second, 1965

\begin{tabular}{|c|c|c|c|c|c|c|c|c|c|c|c|}
\hline Day & March & April & May & Day & March & April & May & Day & March & April & May \\
\hline & 1,000 & 1,420 & 1,610 & $11 \ldots$ & 880 & 4,000 & 3,900 & 21. & 780 & 6,330 & 4,360 \\
\hline 2. & 900 & 1,450 & 1,460 & $12_{\ldots}$ & 860 & 7,000 & 2,950 & 22. & 800 & 5,930 & 4,060 \\
\hline 3. & 640 & 1,300 & 1,360 & $13 \ldots$ & 880 & 9,000 & 2,210 & 23 & 800 & 5,340 & 3,840 \\
\hline . & 620 & 800 & 1,280 & $14 \ldots$ & 860 & 11,000 & 1,810 & 24 & 770 & 4,740 & 3,050 \\
\hline 5 & 700 & 720 & 1,130 & $15 \ldots$ & 860 & 11,700 & 2,520 & 25 & 760 & 3,870 & 2,540 \\
\hline 6. & 920 & 820 & 1,170 & $16 .-$ & 840 & 11,200 & 4,300 & 26 & 770 & 3,300 & 3,040 \\
\hline 7. & 900 & 1,000 & 1,710 & $17--$ & 860 & 10,100 & 5,330 & 27 & 1,100 & 2,990 & 3,520 \\
\hline 8 & 920 & 1,300 & 3,010 & $18 .-$ & 840 & 8,650 & 5,090 & 28 & 1,400 & 2,550 & 3,160 \\
\hline 9 & 920 & 1,500 & 5,310 & $19 .-$ & 800 & 7,360 & 4,840 & 29 & 1,450 & 2,150 & 2,870 \\
\hline \multirow[t]{2}{*}{$10_{-}$} & 880 & 2,200 & 5,170 & $20 .-$ & 820 & 6,700 & 4,700 & 30. & 1,400 & 1,850 & 2,610 \\
\hline & & & & & & & & 31 & 1,450 & $\begin{array}{l}1,000 \\
-\ldots .-1 .\end{array}$ & 2,520 \\
\hline \multicolumn{9}{|c|}{ Monthly mean discharge, in cubic feet per second.............. } & 915 & 4,609 & 3,111 \\
\hline
\end{tabular}


MARCH-MAY, UPPER MISSISSIPPI RIVER BASIN

Gage height, in feet, and discharge, in cubic feet per second, at indicated time, 1965, of Chippewa River

\begin{tabular}{|c|c|c|c|c|c|c|c|c|c|c|c|}
\hline Date & Hour & $\begin{array}{c}\text { Gage } \\
\text { height }\end{array}$ & $\begin{array}{c}\text { Dis - } \\
\text { charge }\end{array}$ & Date & Hour & $\begin{array}{c}\text { Gage } \\
\text { height }\end{array}$ & $\begin{array}{c}\text { Dis- } \\
\text { charge }\end{array}$ & Date & Hour & $\begin{array}{c}\text { Gage } \\
\text { height }\end{array}$ & $\begin{array}{c}\text { Dis- } \\
\text { charge }\end{array}$ \\
\hline \multirow[t]{5}{*}{ Apr. 10} & 0000 & 5.54 & $\ldots$ & Apr. 19 & 0800 & 9.35 & 7,540 & May 16 & 1000 & 6.16 & 4,110 \\
\hline & 0800 & 5.74 & - & & 1600 & 9,08 & 7,240 & & 1800 & 7.07 & 5,030 \\
\hline & 1600 & 6.01 & -.. & & 2400 & 8.87 & 7,010 & & 2400 & 7.32 & 5,300 \\
\hline & 2400 & 6.59 & - & & 200 & & & & 10900 & & $=350$ \\
\hline & tong & 79 & & 20 & 1200 & 8.78 & 6,910 & 17 & 0800 & 7.36 & 5,350 \\
\hline \multirow{4}{*}{11} & $\begin{array}{l}0600 \\
1200\end{array}$ & $\begin{array}{l}7.23 \\
7.88\end{array}$ & -- & & 2400 & 8.49 & 6,590 & & $\begin{array}{l}1400 \\
2400\end{array}$ & $\begin{array}{l}7.38 \\
7.26\end{array}$ & $\begin{array}{l}5,370 \\
5,240\end{array}$ \\
\hline & 1800 & 8.85 & & 21 & 1200 & 8.28 & 6,360 & & & & \\
\hline & 2400 & 10.01 & - & & 2400 & 7.99 & 6,040 & 18 & 0800 & 7.14 & 5,100 \\
\hline & & & & & & & & & 1600 & 7.13 & 5,090 \\
\hline \multirow[t]{5}{*}{12} & 0200 & 10.39 & & 22 & 0800 & 8.02 & 6,070 & & 2400 & 7.05 & 5,000 \\
\hline & 0800 & 11.26 & & & 1600 & 7.86 & 5,900 & & & & \\
\hline & 1600 & 11.94 & & & 2400 & 7.66 & 5,680 & 19 & 1200 & 6.88 & 4,830 \\
\hline & 2400 & 12.30 & $-\infty$ & & & & & & 2400 & 6.83 & 4,780 \\
\hline & & & & 23 & 1200 & 7.36 & 5,350 & & & & \\
\hline \multirow[t]{4}{*}{13} & 1200 & 12.37 & & & 2400 & 7.04 & 4,990 & 20 & 1200 & 6.79 & 4,740 \\
\hline & 1800 & 13.18 & & & & & & & 2400 & 6.56 & 4,510 \\
\hline & 2400 & 13.53 & & May 7 & 2400 & 4.04 & 2,230 & & & & \\
\hline & & & & & & & & 21 & 1200 & 6.47 & 4,420 \\
\hline \multirow[t]{4}{*}{14} & 0600 & 14.09 & $\ldots$ & 8 & 0800 & 4.29 & 2,430 & & 2400 & 6.21 & 4,160 \\
\hline & 1200 & 14.42 & & & 1600 & 5.20 & 3,220 & & & & \\
\hline & 1800 & 14.32 & & & 2000 & 5.86 & 3,810 & 22 & 1200 & 6.13 & 4,080 \\
\hline & 2400 & 14.38 & & & 2400 & 6.41 & 4,360 & & 2400 & 6.00 & 3,950 \\
\hline \multirow[t]{7}{*}{15} & 0600 & 14.61 & & 9 & 0600 & 7.01 & 4,960 & 23 & 1200 & 5.93 & 3,880 \\
\hline & 1200 & 14.83 & & & 1400 & 7.57 & 5,580 & & 2400 & 5.56 & 3,540 \\
\hline & 1600 & 15.11 & $-----\infty$ & & 2000 & 7.67 & 5,690 & & & & \\
\hline & 1630 & 15.19 & 12,300 & & 2400 & 7.63 & 5,640 & 24 & 1200 & 5.02 & 3,060 \\
\hline & 2000 & 15.05 & -- & & & & & & 2400 & 4.57 & 2,660 \\
\hline & 2400 & 14.66 & & 10 & 0800 & 7.43 & 5,420 & & & & \\
\hline & & & & & 1600 & 7.07 & 5,030 & 25 & 0800 & 4.44 & 2,550 \\
\hline \multirow[t]{5}{*}{16} & 0600 & 14.21 & - & & 2400 & 6.59 & 4,540 & & 1800 & 4.36 & 2,490 \\
\hline & 1200 & 13.96 & ----- & & & & & & 2400 & 4.45 & 2,560 \\
\hline & 1800 & 13.07 & & 11 & 0800 & 6.14 & 4,090 & & & & \\
\hline & 2400 & 12.41 & 11,600 & & 1600 & 5.77 & 3,730 & 26 & 0800 & $4.7 \%$ & 2,830 \\
\hline & & & & & 2400 & 5.38 & 3,380 & & 1600 & 5.19 & 3,210 \\
\hline \multirow[t]{5}{*}{17} & 0600 & 11.95 & 10,900 & & & & & & 2400 & 5.49 & 3,480 \\
\hline & 1200 & 11.36 & 10,100 & 12 & 0800 & 5.08 & 3,110 & & & & \\
\hline & 1800 & 10.72 & 9,240 & & 1600 & 4.76 & 2,820 & 27 & 0800 & $5.6 \mathrm{C}$ & 3,580 \\
\hline & 2400 & 11.38 & 10,100 & & 2400 & 4.45 & 2,560 & & 1600 & $5.5^{4}$ & 3,530 \\
\hline & & & & & & & & & 2400 & 5.39 & 3,390 \\
\hline \multirow[t]{3}{*}{18} & 0800 & 10.49 & 8,940 & 15 & 2400 & 4.89 & 2,940 & & & & \\
\hline & 1600 & 9.92 & 8,200 & & & & & 28 & 1200 & 5.13 & 3,160 \\
\hline & 2400 & 9.55 & 7,760 & 16 & 0400 & 5.18 & 3,200 & & 2400 & 4.96 & 3,000 \\
\hline
\end{tabular}


(106) 5-3573. Rest Lake near Manitowish, Wis.

Location.-Lat $46^{\circ} 08^{\prime} 15^{\prime \prime}$, long $89^{\circ} 53^{\prime} 05^{\prime \prime}$, in NW $\frac{1}{4}$ sec.9, T.42 N., R.5 E., on Manitowish River, 3.7 miles upstream from Circle Lily Creek and 6.2 miles east. of Manitowish.

Drainage area. $-243 \mathrm{sq} \mathrm{mi}$.

Gage-height record.-Staff gage. Altitude of gage is 1,490 ft (by U.S. Geological Survey bench mark near lake).

Maxima.-March-May 1965: Contents at end of week, 1,010 million cul ic feet May 21, 28 (gage height, $108.5 \mathrm{ft}$ ).

1926 to February 1965: Contents on last of month, 1,054 million cubic feet Aug. 31 , 1941 (gage height, $108.7 \mathrm{ft}$ ).

Remarks.-Reservoir operation began in 1887. Reservoir consists of 9 lakes. Usable capacity July 1 to August 31,260 million cubic feet between gage heights $107.25 \mathrm{ft}$ and $108.50 \mathrm{ft}$, and September 1 to $J$ une 30,660 million cubic feet between gage heights $105.00 \mathrm{ft}$ and $108.50 \mathrm{ft}$ (limits stipulated by Public Service Commission of Wisconsin).

Cooperation.-Gage heights and capacity table furnished by Northern States Power Co.

Gage height, in feet and inches, and contents, in millons of cubic feet, 1965

\begin{tabular}{|c|c|c|c|c|c|c|c|}
\hline \multirow{2}{*}{ Date } & \multicolumn{2}{|c|}{ Gage height } & \multirow{2}{*}{ Contents } & \multirow{2}{*}{ Date } & \multicolumn{2}{|c|}{ Gage height } & \multirow{2}{*}{ Contents } \\
\hline & Feet & Inches & & & Feet & Inches & \\
\hline Feb. $26 \ldots$ & 105 & 0 & 350 & Apr. $16 \ldots$ & 105 & 10 & 491 \\
\hline Mar. $5 \ldots$ & 105 & 0 & 350 & $23 \ldots$ & 106 & 5 & 595 \\
\hline $12 \ldots$ & 105 & 0 & 350 & $30 \ldots$ & 106 & 3 & 565 \\
\hline $19 \ldots$ & 105 & 0 & 350 & $7 \ldots$ & 106 & 9 & 655 \\
\hline $26 \ldots$ & 105 & 0 & 350 & $14 \ldots$ & 108 & 3 & 955 \\
\hline Apr. $\quad 2 \ldots$ & 105 & 0 & 350 & $21 \ldots$ & 108 & 6 & 1,010 \\
\hline $9 \ldots$ & 105 & 0 & 350 & $28 \ldots$ & 108 & 6 & 1,010 \\
\hline
\end{tabular}

(107) 5-3574. Flambeau Flowage near Mercer, Wis.

Location.-Lat $46^{\circ} 04^{\prime} 15^{\prime \prime}$, long $90^{\circ} 13^{\prime} 30^{\prime \prime}$, in SE $\frac{1}{4}$ sec. 34, T. 42 N., R.2 E., on North Fork Flambeau River, 10.2 miles southwest of Mercer.

Drainage area. $-666 \mathrm{sq} \mathrm{mi}$.

Gage-height record.- Staff gage. Datum of gage is mean sea level (Northern States Power Co. bench mark).

Maxima.-March-May 1965: Daily contents observed, 6,052 million cuk ic feet May $11-15$ (elevation $1572.20 \mathrm{ft}$ ).

1926 to February 1965: Contents observed, 6,620 million cubic feet Oct. 16, 1927 (elevation, $1,572.95 \mathrm{ft}$ ).

Remarks. -Dam was completed in 1926. Usable capacity 5,895 million cubic feet between elevations $1,552 \mathrm{ft}$ and $1,572 \mathrm{ft}$ (limits stipulated by Public Service Commission of Wisconsin).

Cooperation.-Elevations and capacity table furnished by Northern States Power Co. 
Elevation, in feet, and contents, in millions of cubic feet, 1965, of Flambeau Flowage near Mercer, Wis.

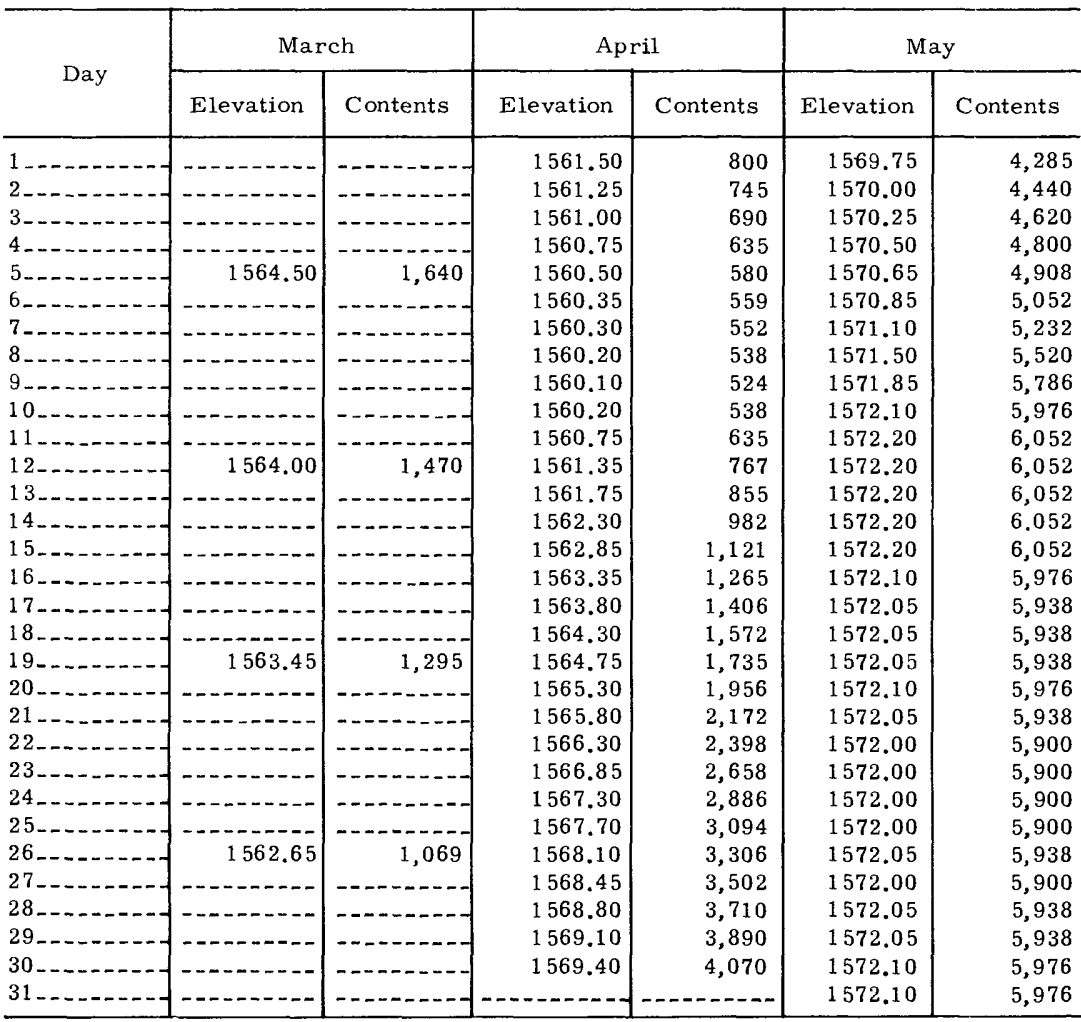

(108) 5-3585. Flambeau River at Babbs Island, near Winter, Wis.

Location.-Lat $45^{\circ} 46^{\prime} 10^{\prime \prime}$, long $90^{\circ} 45^{\prime} 45^{\prime \prime}$, in SE $\frac{1}{4}$ sec.17, T.38 N., R.3 W., on right bank 3.6 miles upstream from Connors Creek, 11.5 miles upstream from South Fork Flambeau River, 13 miles east of Winter, and at mile 61.9.

Drainage area. $-1,000 \mathrm{sq} \mathrm{mi}$.

Gage-height record.-Water-stage recorder graph. Altitude of gage is 1,330 f: (from river profile map).

Discharge record.-Stage-discharge relation defined by current-meter measurements below 7,000 cfs. Backwater from ice Mar. 1 to Apr. 14.

Maxima.-March-May 1965: Discharge, 5,360 cfs 0400-1000 hours Apr. 17 (gage height, $6.03 \mathrm{ft})$.

1929 to February 1965: Discharge, 9,440 cfs June 25, 1946 (gage height, $8.45 \mathrm{ft}$ ).

Remarks.-Flow regulated by Rest Lake and Flambeau Flowage. 
Mean discharge, in cubic feet per second, 1965. of Flambeau River at Babbs Island, near Winter, Wis.

\begin{tabular}{|c|c|c|c|c|c|c|c|c|c|c|c|}
\hline Day & March & April & May & Day & March & April & May & Day & March & April & May \\
\hline $1-$ & 680 & 870 & 1,210 & $11 \ldots$ & 640 & 1,700 & 3,750 & 21. & 640 & 2,730 & 3,150 \\
\hline 2. & 720 & 900 & 1,230 & $12 \ldots$ & 640 & 2,300 & 3,880 & $22_{-}$ & 500 & 2,840 & 3,070 \\
\hline 3 & 720 & 960 & 1,030 & $13 \ldots$ & 640 & 2,600 & 3,260 & 23 & 700 & 2,730 & 2,800 \\
\hline 4. & 680 & 1,060 & 939 & 14 & 640 & 3,000 & 3,330 & $24_{\ldots}$ & 680 & 2,600 & 2,540 \\
\hline 5. & 680 & 1,020 & 890 & $15 \ldots$ & 640 & 3,870 & 3,340 & 25. & 660 & 2,450 & 2,170 \\
\hline 6. & 680 & 960 & 856 & $16 \ldots$ & 640 & 4,910 & 4,410 & $26_{-}$ & 700 & 1,980 & 1,750 \\
\hline 7. & 720 & 900 & 1,030 & $17 .-$ & 640 & 5,270 & 4,880 & $27-$ & 780 & 1,800 & 1,690 \\
\hline 8 & 520 & 960 & 1,510 & $18 \ldots$ & 660 & 4,580 & 4,100 & 28. & 800 & 1,560 & 1,720 \\
\hline 3 & 660 & 1,060 & 2,900 & $19 \ldots$ & 660 & 3,050 & 3,600 & $\mid 29$ & 800 & 1,440 & 1,400 \\
\hline \multirow[t]{2}{*}{$10--$} & 660 & 1,300 & 3,880 & $20 \ldots$ & 640 & 2,650 & 3,220 & 30. & 820 & 1,200 & 1,510 \\
\hline & & & & & & & & 31 & 820 & $-\cdots$ & 1,460 \\
\hline \multicolumn{9}{|c|}{ Monthly mean discharge, in cubic feet per second. } & 679 & 2,175 & 2,468 \\
\hline
\end{tabular}

Gage height, in feet, and discharge, in cubic feet per second, at indicated time, 1965

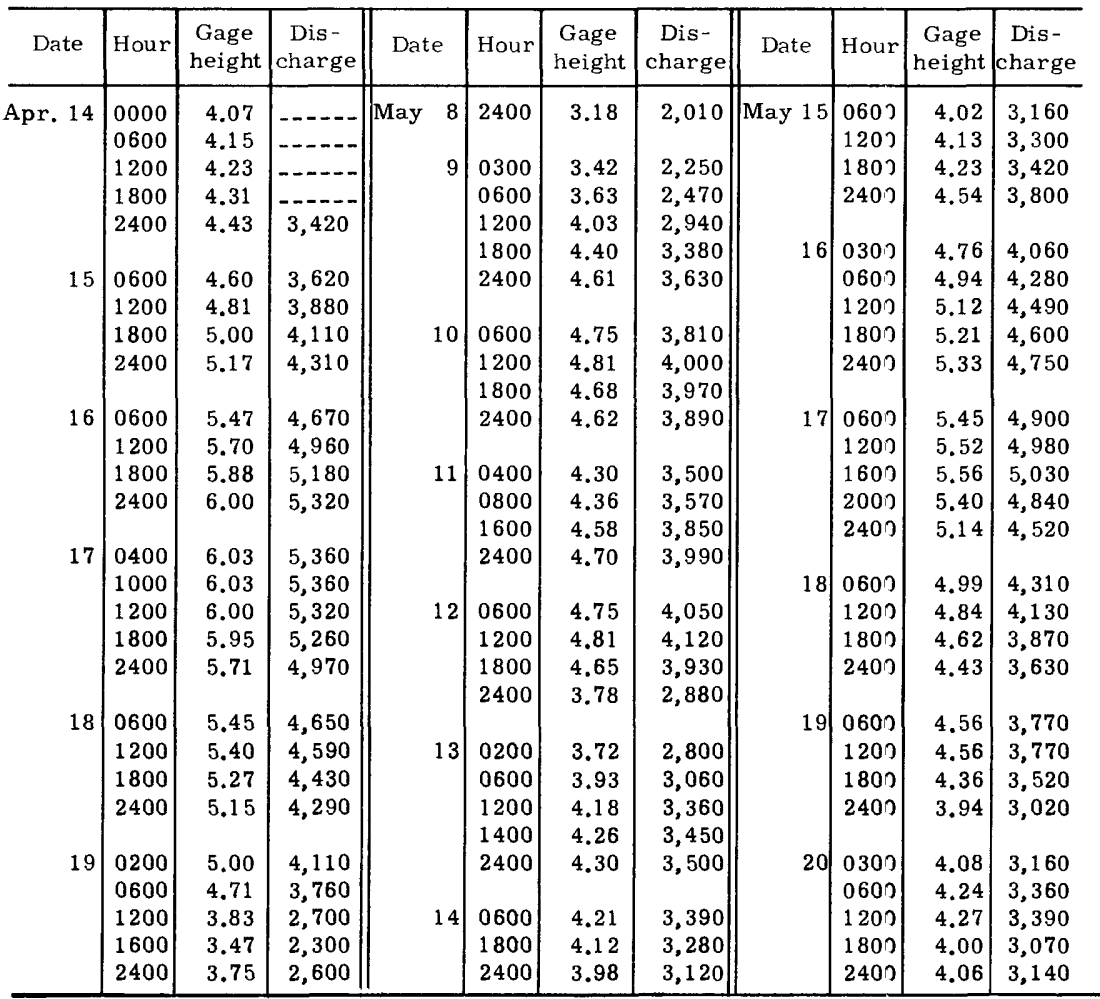


(109) 5-3595 South Fork Flambeau River near Phillips, Wis.

Location.-Lat $45^{\circ} 42^{\prime} 15^{\prime \prime}$, long $90^{\circ} 36^{\prime} 55^{\prime \prime}$, in NW $\frac{1}{4} \mathrm{SW} \frac{1}{4}$ sec.10, T.37 N., R.2 W., on downstream side of bridge on left span, 0.4 mile downstream from Big Elk River and 12 miles west of Phillips.

Drainage area. $-615 \mathrm{sq} \mathrm{mi}$.

Gage-height record.-Graph drawn on basis of crest-stage gage reading and once-daily wire-weight gage readings, except May 24-31. Altitude of gage is $1,360 \mathrm{ft}$ (by barometer).

Discharge record.--Stage-discharge relation defined by current-meter measurements. Discharge for period of no gage-height record May 24-31 was estimated on basis of weather records and records for nearby stations. Backwater from ice Mar, 1 to Apr. 13.

Maxima.-March-May 1965: Discharge, 5,690 cfs 0200 hours Apr. 19 (gage height, $11 . \overline{64} \mathrm{ft}$ ).

1929 to February 1965: Discharge, 10,200 cfs June 18, 1943 (gage height, $14.32 \mathrm{ft}$ ).

Mean discharge, in cubic feet per second, 1965

\begin{tabular}{|c|c|c|c|c|c|c|c|c|c|c|c|}
\hline Day & March & April & May & Day & March & April & May & Day & March & April & May \\
\hline 2 & 240 & 300 & 1,940 & $11 \ldots$ & 270 & 700 & 3,490 & 21 & 260 & 4,300 & 2,560 \\
\hline 2 & 250 & 310 & 1,770 & $12--$ & 280 & 1,000 & 3,510 & 22 & 270 & 4,140 & 1,980 \\
\hline 3. & 270 & 330 & 1,720 & $13 \ldots$ & 290 & 1,700 & 3,610 & 23. & 270 & 4,090 & 1,940 \\
\hline & 280 & 350 & 1,700 & $14 \ldots$ & 300 & 2,910 & 3,640 & 24 & 270 & 3,920 & 1,900 \\
\hline 5. & 300 & 380 & 1,620 & $15 \ldots$ & 310 & 3,690 & 3,910 & 25. & 280 & 3,700 & 1,800 \\
\hline 6. & 300 & 400 & 1,750 & $16 \ldots$ & 300 & 4,290 & 3,820 & 26. & 280 & 3,410 & 1,900 \\
\hline 7. & 310 & 410 & 2,280 & $17 \ldots$ & 290 & 4,340 & 4,080 & 27. & 270 & 3,010 & 1,900 \\
\hline & 300 & 430 & 2,600 & $18 \ldots$ & 270 & 4,800 & 3,930 & 28. & 270 & 2,660 & 1,800 \\
\hline$\dot{y}$ & 280 & 450 & 3,020 & $19 \ldots$ & 260 & 5,150 & 3,740 & 29. & 270 & 2,440 & 1,700 \\
\hline \multirow[t]{2}{*}{$10-$} & 270 & 540 & 3,390 & 20 & 250 & 4,590 & 3,620 & 30 & 270 & 2,320 & 1,600 \\
\hline & & & & & & & & 31 & 290 & $-\ldots$ & 1,600 \\
\hline \multicolumn{9}{|c|}{ Monthly mean discharge, in cubic feet per second } & 278 & 2,369 & 2,575 \\
\hline \multicolumn{9}{|c|}{ Runnfi, in inches $\ldots \ldots \ldots$} & 0.52 & 4.30 & 4.83 \\
\hline
\end{tabular}

Gage hetght, in feet, and discharge, in cubic feet per second, at indicated time, 1965

\begin{tabular}{|c|c|c|c|c|c|c|c|c|c|c|c|}
\hline Date & Hour & $\begin{array}{c}\text { Gage } \\
\text { height }\end{array}$ & $\begin{array}{c}\text { Dis- } \\
\text { charge }\end{array}$ & Date & Hour & $\begin{array}{l}\text { Gage } \\
\text { height }\end{array}$ & $\begin{array}{c}\text { Dis- } \\
\text { charge }\end{array}$ & Date & Hour & $\begin{array}{c}\text { Gage } \\
\text { heigh } t\end{array}$ & $\begin{array}{l}\text { Dis- } \\
\text { charge }\end{array}$ \\
\hline \multirow[t]{6}{*}{ Apr. 11} & 0000 & 6.37 & & \multirow[t]{4}{*}{ Apr. 14} & 1200 & 9.36 & 3,000 & \multirow[t]{5}{*}{ Apr. 18} & 0600 & 10.74 & 4,510 \\
\hline & 0400 & 6.48 & & & 1800 & 9.46 & 3,100 & & 1200 & 10.86 & 4,650 \\
\hline & 1200 & 6.74 & $\ldots$ & & 2400 & 9.50 & 3,140 & & 1800 & 11.14 & 4,990 \\
\hline & 1800 & 6.94 & $\ldots \ldots$ & & & & & & 2400 & 11.58 & 5,600 \\
\hline & \multirow[t]{2}{*}{2400} & 7.04 & $-\ldots$ & 15 & 0400 & 9.60 & 3,250 & & & & \\
\hline & & & & & 0800 & 9.74 & 3,400 & \multirow[t]{6}{*}{19} & 0200 & $11 . € 4$ & 5,690 \\
\hline \multirow[t]{5}{*}{12} & 0600 & 7.12 & $\ldots---$ & & 1200 & 9.96 & 3,650 & & 0800 & 11.48 & 5,450 \\
\hline & 1200 & 7.16 & $-\ldots--$ & & 1800 & 10.36 & 4,090 & & 1200 & 11.24 & 5,120 \\
\hline & 1800 & 7.30 & $\ldots \ldots$ & & 2400 & 10.54 & 4,280 & & 1800 & $10 . \subseteq 6$ & 4,770 \\
\hline & 2400 & 7.47 & $\ldots$ & \multirow{5}{*}{16} & & & & & 2400 & 10.84 & 4,630 \\
\hline & & & & & 0600 & 10.60 & 4,350 & & & & \\
\hline \multirow[t]{6}{*}{13} & 0400 & 7.66 & & & 1200 & 10.56 & 4,310 & \multirow[t]{3}{*}{20} & 1200 & $10 . \varepsilon 6$ & 4,650 \\
\hline & 0800 & 7.86 & & & 2400 & 10.46 & 4,200 & & 2400 & 10.66 & 4,420 \\
\hline & 1200 & 8.08 & & & & & & & & & \\
\hline & 1800 & 8.42 & 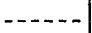 & \multirow[t]{4}{*}{17} & 0600 & 10.52 & 4,260 & \multirow[t]{4}{*}{21} & 1200 & 10.56 & 4,310 \\
\hline & 2400 & 8.76 & 2,430 & & 1200 & 10.60 & 4,350 & & 2400 & 10.44 & 4,170 \\
\hline & & & & & 1800 & 10.66 & 4,420 & & & & \\
\hline $14 \mid$ & $|0400|$ & 9.00 & 2,650 & & 2400 & 10.70 & 4,460 & & & & \\
\hline
\end{tabular}


Gage height, in feet, and discharge, in cubic feet per second, at indicated time, 1965, of South Fork Flambeau Rlver near Phillips, $W_{i s}$ Continued

\begin{tabular}{|c|c|c|c|c|c|c|c|c|c|c|c|}
\hline Date & Hour & $\begin{array}{c}\text { Gage } \\
\text { height }\end{array}$ & $\begin{array}{c}\text { Dis- } \\
\text { charge }\end{array}$ & Date & Hour & $\begin{array}{c}\text { Gage } \\
\text { height }\end{array}$ & $\begin{array}{c}\text { Dis- } \\
\text { charge }\end{array}$ & Date & Hoir & $\begin{array}{c}\text { Gage } \\
\text { height }\end{array}$ & $\begin{array}{c}\text { Dis- } \\
\text { charge }\end{array}$ \\
\hline Apr. 22 & $\begin{array}{l}1200 \\
2400\end{array}$ & $\begin{array}{l}10.40 \\
10.40\end{array}$ & $\begin{array}{l}4,130 \\
4,130\end{array}$ & Apr. 23 & $\begin{array}{l}1200 \\
2400\end{array}$ & $\begin{array}{l}10.38 \\
10.28\end{array}$ & $\begin{array}{l}4,110 \\
4,000\end{array}$ & Apr. 24 & $\begin{array}{l}1200 \\
2400\end{array}$ & $\begin{array}{l}10.22 \\
10.14\end{array}$ & $\begin{array}{l}3,930 \\
3,840\end{array}$ \\
\hline
\end{tabular}

(110) 5-3605 Flambeau River near Bruce, Wis.

Location.-Lat $45^{\circ} 22^{\prime} 20^{\prime \prime}$, long $91^{\circ} 12^{\prime} 35^{\prime \prime}$, in lot 7 of NW $\frac{1}{4}$ sec. 2 . T.33 N., R.7 W., on right bank 2.5 miles downstream from Thornapple powerplant, 6 miles upstream from mouth, and 7 miles southeast of Bruce.

Drainage area. $-1,897 \mathrm{sq} \mathrm{mi}$.

Gage-height record.-Digital recorder tape punched at sixty-minute intervals. Altitude of gage 1,060 ft (by river survey, WSP 417).

Discharge record.-Stage-discharge relation defined by current-meter measurements below 11,000 cfs. Mean daily discharges computed from 24 punch-tase recordings per day. Backwater from ice March 1 to April 12.

Maxima.-March-May 1965: Discharge, 10,500 cfs 1000 hours Apr. 20 (gage height, $8.19 \mathrm{ft}$ )

1951 to February 1965: Discharge, 17,400 cfs May 1, 1954 (gage haight, $10.90 \mathrm{ft}$ ).

Remarks.-Flow regulated by several powerplants above stations and by Rest Lake and Flambeau Flowage.

Mean discharge, in cubic feet per second, 1965

\begin{tabular}{|c|c|c|c|c|c|c|c|c|c|c|c|}
\hline Day & March & April & May & Day & March & April & May & Day & March & April & May \\
\hline & 1,100 & 1,500 & 4,230 & 11. & 1,020 & 3,200 & 7,760 & 21 & 1,600 & 8,240 & 5,530 \\
\hline & 1,080 & 1,700 & 3,930 & $12_{-}$ & 1,060 & 3,800 & 7,730 & 22 & 1,620 & 8,120 & 5,420 \\
\hline & 1,000 & 1,680 & 3,920 & 13. & 980 & 4,660 & 6,550 & 23 & 1,640 & 8,110 & 4,850 \\
\hline & 1,040 & 1,840 & 3,350 & 14. & 1,040 & 5,840 & 6,210 & 24 & 1,690 & 7,990 & 4,350 \\
\hline & 1,120 & 2,000 & 3,440 & 15. & 940 & 6,520 & 5,450 & 25. & 1,680 & 7,510 & 4,160 \\
\hline & 1,080 & 1,900 & 3,050 & 16. & 1,200 & 10,000 & 7,670 & 26 & 1,600 & 6,700 & 4,130 \\
\hline & 1,060 & 1,860 & 2,730 & 17. & 1,500 & 9,980 & 9,360 & $27_{-}$ & 1,560 & 5,700 & 4,070 \\
\hline & 1,080 & 1,940 & 4,010 & $18 \ldots$ & 1,540 & 10,100 & 9,410 & $28 \ldots$ & 1,660 & 5,080 & 3,210 \\
\hline & 1,060 & 2,100 & 7,660 & 19 & 1,540 & 10,100 & 8,110 & 29 & 1,700 & 4,800 & 2,740 \\
\hline 10 & 1,000 & 2,800 & 8,270 & 20 & 1,620 & 9,580 & 6,950 & $30_{-}$ & 1,660 & 4,430 & 2,650 \\
\hline & & & & & & & & 31 & 1,640 & -1 & 2,610 \\
\hline \multicolumn{9}{|c|}{ Monthly mean discharge, in cubic feet per second } & 1,326 & 5,326 & 5,275 \\
\hline
\end{tabular}

Gage hetght, in feet, and discharge, in cubic feet per second, at indicated time, 1965

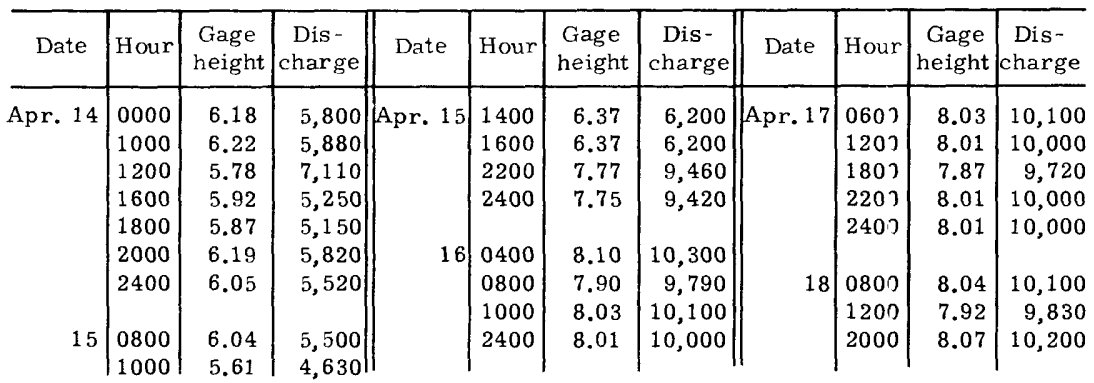


MARCH-MAY, UPPER MISSISSIPPI RIVER BASIN

Gage height. in feet, and discharge, in cubic feet per second, at indicated time, 1965, of Flambeau River near

\begin{tabular}{|c|c|c|c|c|c|c|c|c|c|c|c|c|}
\hline Date & Hour & $\begin{array}{c}\text { Gage } \\
\text { height }\end{array}$ & $\begin{array}{c}\text { Dis- } \\
\text { charge }\end{array}$ & Date & & Hour & $\begin{array}{l}\text { Gage } \\
\text { height }\end{array}$ & $\begin{array}{c}\text { Dis- } \\
\text { charge }\end{array}$ & Date & Hour & $\begin{array}{c}\text { Gage } \\
\text { height }\end{array}$ & $\begin{array}{c}\text { Dis- } \\
\text { charge }\end{array}$ \\
\hline \multirow[t]{2}{*}{ Apr. 18} & 2400 & 8.04 & 10,100 & Apr. 2 & 27 & 1000 & 6.74 & 7,020 & May 14 & 1000 & 6.37 & 6,200 \\
\hline & & & & & & 1200 & 5.31 & 4,040 & & 1200 & 6.49 & 6,470 \\
\hline \multirow[t]{3}{*}{19} & 0800 & 8.01 & 10,000 & & & 1400 & 5.35 & 4,120 & & 1600 & 6.48 & 6,450 \\
\hline & 1600 & 8.05 & 10,200 & & & 1600 & 6.00 & 5,420 & & 2000 & 6.30 & 6,050 \\
\hline & 2400 & 8.13 & 10,400 & & & 2400 & 5.95 & 5,320 & & 2400 & 6.25 & 5,940 \\
\hline \multirow[t]{7}{*}{20} & 0800 & 8.16 & 10,400 & May & 7 & 2400 & 4.57 & 2,740 & 15 & 1000 & 6.21 & 5,880 \\
\hline & 1000 & 8.19 & 10,500 & & & & & & & 1200 & 6.11 & 5,650 \\
\hline & 1200 & 8.09 & 10,300 & & 8 & 0800 & 4.53 & 2,680 & & 1400 & 5.12 & 3,700 \\
\hline & 1400 & 7.52 & 9,340 & & & 1200 & 3.88 & 1,770 & & 1800 & 5.86 & 4,630 \\
\hline & 2000 & 7.26 & 8,220 & & & 1400 & 5.43 & 4,270 & & 2000 & 6.08 & 5,590 \\
\hline & 2400 & 7.23 & 8,150 & & & 1800 & 6.23 & 5,900 & & 2400 & 6.29 & 6,030 \\
\hline & & & & & & 2400 & 6.42 & 6,310 & & & & \\
\hline \multirow[t]{4}{*}{21} & 0800 & 7.28 & 8,270 & & & & & & 16 & 0600 & 6.38 & 6,230 \\
\hline & 1600 & 7.27 & 8,220 & & 9 & 0800 & 6.44 & 6,360 & & 0800 & 6.52 & 6,530 \\
\hline & 2400 & 7.25 & 8,200 & & & 1200 & 6.67 & 6,860 & & 1000 & 7.52 & 8,850 \\
\hline & & & & & & 1600 & 7.24 & 8,180 & & 1200 & 7.32 & 8,370 \\
\hline \multirow[t]{7}{*}{22} & 0800 & 7.26 & 8,220 & & & 1800 & 7.91 & 9,810 & & 1800 & 7.36 & 8,460 \\
\hline & 1000 & 6.85 & 7,260 & & & 2000 & 7.91 & 9,810 & & 2400 & 7.29 & 8,300 \\
\hline & 1200 & 7.16 & 7,980 & & & 2400 & 7.63 & 9,120 & & & & \\
\hline & 1400 & 7.35 & 8,440 & & & & & & 17 & 0800 & 7.29 & 8,300 \\
\hline & 1600 & 7.25 & 8,200 & & 10 & 0800 & 7.59 & 9,020 & & 1000 & 7.76 & 9,440 \\
\hline & 2400 & 7.20 & 8,080 & & & 1200 & 7.25 & 8,200 & & 1200 & 8.05 & 10,200 \\
\hline & & & & & & 1400 & 6.13 & 5,690 & & 1800 & 7.99 & 9,980 \\
\hline \multirow[t]{5}{*}{23} & 0800 & 7.21 & 8,100 & & & 1600 & 7.26 & 8,220 & & 2400 & 7.98 & 9,960 \\
\hline & 1000 & 7.09 & 7,820 & & & 2000 & 7.15 & 7,960 & & & & \\
\hline & 1200 & 7.28 & 8,270 & & & 2400 & 7.17 & 8,010 & 18 & 0800 & 8.00 & 10,000 \\
\hline & 2400 & 7.22 & 8,130 & & 11 & 0800 & 7.23 & 8,150 & & 1200 & 7.99 & 9,980 \\
\hline & & & & & & 1200 & 7.21 & 8,100 & & 1800 & 7.48 & 8,750 \\
\hline \multirow[t]{5}{*}{24} & 0800 & 7.22 & 8,130 & & & 1600 & 7.15 & 7,960 & & 2200 & 7.22 & 8,130 \\
\hline & 1400 & 7.23 & 8,150 & & & 1800 & 6.49 & 6,470 & & 2400 & 7.23 & 8,150 \\
\hline & 1800 & 7.08 & 7,790 & & & 2000 & 6.58 & 6,670 & & & & \\
\hline & 2400 & 7.03 & 7,680 & & & 2200 & 6.99 & 7,590 & 19 & 0600 & 7.25 & 8,200 \\
\hline & & & & & & 2400 & 6.99 & 7,590 & & 1200 & 7.17 & 8,010 \\
\hline \multirow[t]{6}{*}{25} & 0800 & 7.03 & 7,680 & & & 2400 & 0.00 & 1,000 & & 2400 & $7.2 \mathrm{C}$ & 8,080 \\
\hline & 1600 & 7.00 & 7,610 & & 12 & 0800 & 7.04 & 7,700 & & & & \\
\hline & 1800 & 6.67 & 6,860 & & & 1000 & 7.22 & 8,130 & 20 & 0800 & $7.2 \mathrm{C}$ & 8,080 \\
\hline & 2000 & 6.84 & 7,240 & & & 1400 & 7.15 & 7,960 & & 1000 & 6.26 & 5,970 \\
\hline & 2400 & 6.88 & 7,330 & & & 1800 & 6.94 & 7,470 & & 1200 & 6.22 & 5,880 \\
\hline & & & & & & 2000 & 6.93 & 7,450 & & 1400 & $6.4 \mathrm{C}$ & 6,470 \\
\hline \multirow[t]{6}{*}{26} & 0800 & 6.88 & 7,330 & & & 2400 & 7.02 & 7,660 & & 1800 & 6.51 & 6,510 \\
\hline & 1200 & 6.53 & 6,560 & & & & & & & 2400 & 6.44 & 6,360 \\
\hline & 1600 & 6.49 & 6,470 & & 13 & 0600 & 7.03 & 7,680 & & & & \\
\hline & 1800 & 6.21 & 5,860 & & & 1000 & 6.97 & 7,310 & 21 & 1000 & 6.41 & 6,290 \\
\hline & 2400 & 6.34 & 6,140 & & & 1200 & 6.36 & 6,180 & & 1200 & $5.9 \varepsilon$ & 5,380 \\
\hline & & & & & & 1400 & 5.72 & 4,850 & & 1600 & 5.61 & 4,630 \\
\hline \multirow[t]{2}{*}{27} & 0600 & 6.42 & 6,310 & & & 1800 & 6.25 & 5,940 & & 2000 & $5.62^{1}$ & 4,650 \\
\hline & 0800 & 6.93 & 7,450 & & & 2400 & 6.30 & 6,050 & & 2400 & $5.7 !$ & 4,990 \\
\hline
\end{tabular}


(111) 5-3620. Jump River at Sheldon, Wis.

Location.-Lat $45^{\circ} 18^{\prime} 30^{\prime \prime}$, long $90^{\circ} 57^{\prime} 20^{\prime \prime}$, in sec.26, T.33 N., R.5 W., on right bank just downstream from highway bridge in Sheldon, 1,500 ft upstream from Shoulder Creek and 11 miles upstream from mouth.

Drainage area. $-574 \mathrm{sq} \mathrm{mi}$.

Gage-height record.-Water-stage recorder graph. Datum of gage is $1,092.75 \mathrm{ft}$ above mean sea level, datum of 1929.

Discharge record.-Stage-discharge relation defined by current-meter measurements below $13,000 \mathrm{cfs}$ and by contracted-opening measurement at $46,000 \mathrm{cfs}$. Backwater from ice Mar. 1 to Apr. 11.

Maxima.-March-May 1965: Discharge, 11,400 cfs 2000 hours Apr. 13 (gage height, $11.59 \mathrm{ft}$ ).

1915 to February 1965: Discharge, 46,000 cfs Aug. 31, 1941 (gage height, $18.8 \mathrm{ft}$, from floodmarks).

Mean discharge, in cubic feet per second, 1965

\begin{tabular}{|c|c|c|c|c|c|c|c|c|c|c|c|}
\hline Day & March & April & May & Day & March & April & May & Day & March & April & May \\
\hline & 50 & 62 & 1,720 & $11 \ldots$ & 180 & 4,000 & 2,240 & 21. & 72 & 4,200 & 1,250 \\
\hline 2 & 60 & 66 & 1,470 & $12 \ldots$ & 150 & 9,670 & 1,600 & $22_{-}$ & 70 & 4,120 & 1,150 \\
\hline 3 & 120 & 68 & 1,300 & $13 \ldots$ & 120 & 8,940 & 1,150 & 23 & 68 & 3,790 & 706 \\
\hline 4. & 110 & 70 & 1,160 & $14 \ldots$ & 100 & 9,090 & 859 & $24 \ldots$ & 66 & 3,290 & 546 \\
\hline 5 & 130 & 74 & 1,010 & $15 \ldots$ & 92 & 9,570 & 785 & 25 & 64 & 2,720 & 472 \\
\hline 6. & 260 & 100 & 973 & $16 \ldots$ & 88 & 7,960 & 3,700 & $26 \ldots$ & 63 & 2,680 & 505 \\
\hline & 230 & 200 & 1,260 & $17 \ldots$ & 84 & 6,260 & 5,970 & 27. & 62 & 3,440 & 1,370 \\
\hline 8_ & 160 & 300 & 1,560 & $18 \ldots$ & 82 & 5,560 & 4,540 & 28 & 62 & 2,800 & 1,290 \\
\hline 9. & 280 & 700 & 2,220 & $19 \ldots$ & 78 & 4,920 & 2,930 & 29 & 61 & 2,560 & 1,040 \\
\hline & 240 & 1,400 & 2,730 & $20 \ldots$ & 76 & 4,350 & 1,870 & 30. & 60 & 2,040 & 805 \\
\hline & & & & & & & & 31 & 60 & $\ldots$ & 631 \\
\hline \multirow{2}{*}{\multicolumn{9}{|c|}{ Monthly mean discharge, in cubic feet per second }} & 110 & 3,500 & 1,639 \\
\hline & & & & & & & & 0.22 & 6.80 & 3.29 \\
\hline
\end{tabular}

Gage height, in feet, and discharge, in cubic feet per second, at indicated time, 1965

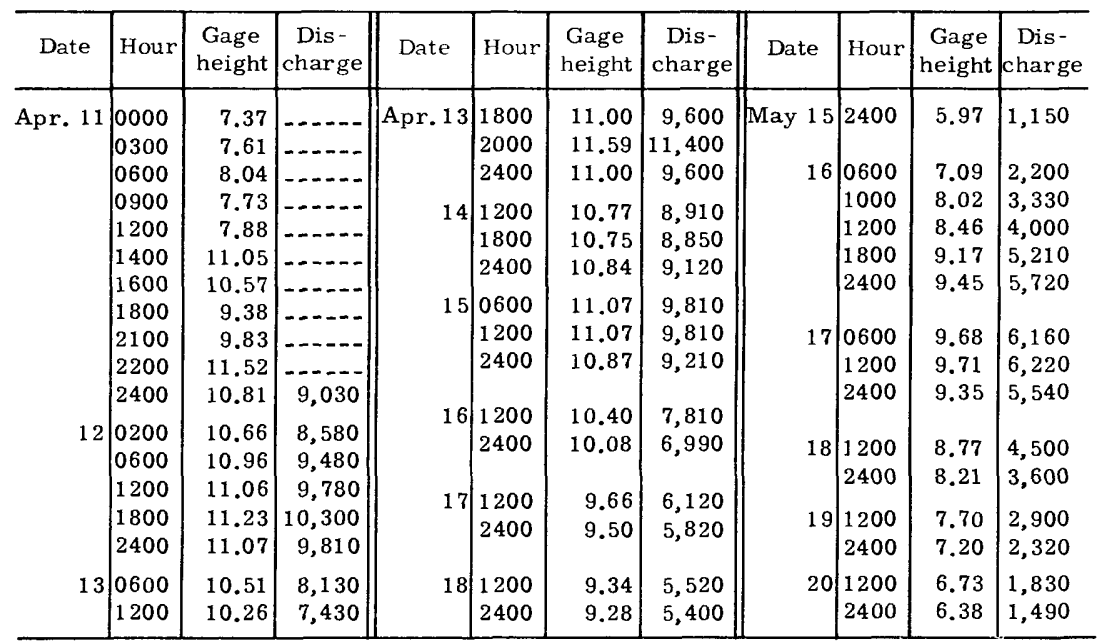


(112) 5-3640. Yellow River at Cadott, Wis.

(Gaging station; crest-stage station beginning 1962)

Location.-Lat $44^{\circ} 57^{\prime} 10^{\prime \prime}$, long $91^{\circ} 09^{\prime} 00^{\prime \prime}$, in NE $\frac{1}{4} \mathrm{sec} .31, \mathrm{~T} .29 \mathrm{~N}$., R.6 W., at bridge on State Highway 27 in Cadott, $1,000 \mathrm{ft}$ upstream from Svetlik Dam, and 6.0 miles upstream from Drywood Creek.

Drainage area. $-351 \mathrm{sq} \mathrm{mi}$.

Gage-height record.-Crest stages only.

Discharge record.-Stage-discharge relation defined by current-meter measuroments below $15,600 \mathrm{cfs}$.

Maxima.-March-May 1965: Discharge, about 11,000 cfs Apr. 11 (gage height, $15.6 \mathrm{ft}$, ice affected).

1942 to February 19, 1965: Discharge, 15,600 cfs June 28, 1943 (gage height $12.15 \mathrm{ft}$ at site 1,400 $\mathrm{ft}$ downstream at different datum).

(113) 5-3642. Lake Wissota near Chippewa Falls, Wis.

Location.-Lat $44^{\circ} 56^{\prime} 20^{\prime \prime}$, long $91^{\circ} 20^{\prime} 25^{\prime \prime}$, in NW $\frac{1}{4}$ sec. 3, T. 28 N., R.8 W., on Chippewa River, 2.0 miles east of city limits of Chippewa Falls.

Drainage area. $-5,548 \mathrm{sq} \mathrm{mi}$.

Gage-height record.-Float gage. Datum of gage is mean sea level (Northern States Power Co, bench mark).

Maxima.--March-May 1965: Daily contents observed, 4,221 million cubic feet Apr. 16 (elevation $898.80 \mathrm{ft}$ ).

1934 to February 1965: Contents observed, 4,854 million cubic feet Aug. 31, 1941 (elevation, $901.12 \mathrm{ft}$ ).

Remarks.-Dam was completed in 1917. This powerplant service pond has a capacity of 3,547 million cubic feet at normal operating elevation of $898.0 \mathrm{ft}$. Maximum elevation allowable is $902.0 \mathrm{ft}$. Usable contents below elevation $898.0 \mathrm{ft}$ allowed by express permission of the Public Service Commission of Wisconsin in anticipation of increased runoff.

Cooperation.-Elevations and capacity table furnished by Northern States Power Co. 
Elevation, in feet, and contents, in millions of cubic feet, 1965, of Lake Wissota near Chippewa Falls. Wis.

\begin{tabular}{|c|c|c|c|c|c|c|}
\hline \multirow{2}{*}{ Day } & \multicolumn{2}{|c|}{ March } & \multicolumn{2}{|c|}{ April } & \multicolumn{2}{|c|}{ May } \\
\hline & Elevation & Contents & Elevation & Contents & Elevation & Contents \\
\hline 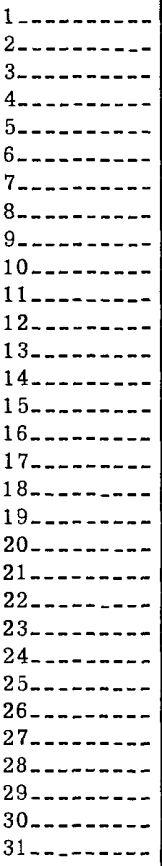 & 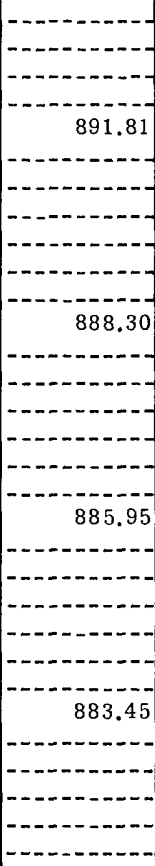 & 2,379 & $\begin{array}{l}882.34 \\
882.49 \\
882.86 \\
883.49 \\
883.81 \\
883.68 \\
883.65 \\
883.92 \\
884.14 \\
886.16 \\
890.54 \\
896.11 \\
896.61 \\
898.69 \\
898.65 \\
898.80 \\
898.65 \\
898.32 \\
898.07 \\
898.22 \\
897.97 \\
897.98 \\
897.87 \\
898.05 \\
897.77 \\
897.90 \\
898.04 \\
897.95 \\
898.30 \\
898.00 \\
-2---\end{array}$ & $\begin{array}{r}77 \\
111 \\
195 \\
340 \\
414 \\
384 \\
377 \\
440 \\
491 \\
971 \\
2,055 \\
3,500 \\
3,633 \\
4,191 \\
4,180 \\
4,221 \\
4,180 \\
4,091 \\
4,024 \\
4,064 \\
3,997 \\
3,999 \\
3,970 \\
4,018 \\
3,943 \\
3,978 \\
4,016 \\
3,991 \\
4,086 \\
4,005 \\
------\end{array}$ & (1988 & 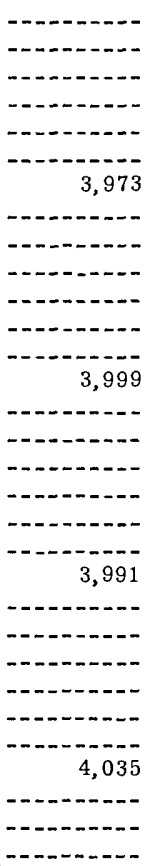 \\
\hline
\end{tabular}

(114) 5-3655. Chippewa River at Chippewa Falls, Wis.

Location.-Lat $44^{\circ} 55^{\prime} 35^{\prime \prime}$, long $91^{\circ} 24^{\prime} 40^{\prime \prime}$, in lot 1 , sec.12, T.28 N., R.9 W., on right bank at Chippewa Falls, 1 mile downstream from Duncan Creek.

Drainage area. $-5,600 \mathrm{sq} \mathrm{mi}$, approximately.

Gage-height record.-Digital recorder tape punched at one-hour intervals. Datum of gage is $798.46 \mathrm{ft}$ above mean sea level, datum of 1929 .

Discharge record.-Stage-discharge relation defined by current-meter measurements. Mean daily discharges computed from 24 punch-tape recordings per day.

Maxima.-March-May 1965: Discharge, 44,700 cfs 2200 hours Apr. 16 (gage-height, $16.47 \mathrm{ft}$ ).

1888 to February 1965: Discharge, 102,000 cfs Sept. 1, 1941 (gage height, $24.8 \mathrm{ft}$ ). Maximum stage known, $26.94 \mathrm{ft}$ Sept. 10, 1884, site and datum in use to June 1932.

Remarks.-Flow regulated by many powerplants above station, and by five reservoirs, Moose Lake, Lake Chippewa, Rest Lake, Flambeau Flowage, and Lake Wissota. 
Mean discharge, in cubic feet per second, 1965, of Chippewa River at Chippewa Falls, Wis.

\begin{tabular}{|c|c|c|c|c|c|c|c|c|c|c|c|}
\hline Day & March & April & May & Day & March & April & May & Day & March & April & May \\
\hline & 4,420 & 3,210 & 11,200 & $11 \ldots$ & 4,790 & 12,300 & 20,300 & 21 & 2,900 & 27,500 & 15,200 \\
\hline 2 & 4,530 & 2,260 & 9,870 & $12=$ & 4,960 & 16,400 & 16,300 & 22 & 4,230 & 25,500 & 12,600 \\
\hline 3. & 6,450 & 1,340 & 8,430 & $13=$ & 4,640 & 36,200 & 13,800 & 23 & 4,180 & 24,200 & 11,800 \\
\hline 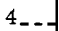 & 6,080 & 1,710 & 9,010 & $14=$ & 3,010 & 40,100 & 11,200 & 24 & 3,770 & 22,900 & 9,950 \\
\hline 5 & 5,390 & 3,970 & 8,400 & & 3,630 & 41,800 & 9,890 & 2 & 4,080 & 21,500 & 9,950 \\
\hline 6. & 5,940 & 6,050 & 8,260 & $16 \ldots$ & 3,610 & 43,900 & 19,400 & & 4,190 & 17,300 & 10,100 \\
\hline 7 & 5,770 & 8,240 & 8,350 & 17. & 3,300 & 43,000 & 27,800 & 2 & 2,750 & 22,900 & 9,990 \\
\hline 8. & 5,880 & 10,200 & 8,940 & $18 \ldots$ & 3,620 & 39,200 & 28,100 & 28. & 1,700 & 15,200 & 10,300 \\
\hline & 5,790 & 9,850 & 14,800 & $19_{-}$ & 4,390 & 33,100 & 20,900 & 29. & 3,440 & 15,800 & 10,000 \\
\hline & 5,150 & 11,100 & 21,400 & 20 & 3,060 & 31,200 & 17,400 & 30. & 4,210 & 13,900 & 8,880 \\
\hline & & & & & & & & & 4,270 & & 8,260 \\
\hline \multicolumn{9}{|c|}{ Monthly mean discharge, in cubic feet per second } & 4,327 & 20,060 & 13,250 \\
\hline
\end{tabular}

Gage height, in feet, and discharge, in cubic feet per second, at indicated time, 1965

\begin{tabular}{|c|c|c|c|c|c|c|c|c|c|c|c|}
\hline Date & Hour & $\begin{array}{l}\text { Gage } \\
\text { height }\end{array}$ & $\begin{array}{c}\text { Dis- } \\
\text { charge }\end{array}$ & Date & Hour & $\begin{array}{l}\text { Gage } \\
\text { height }\end{array}$ & $\begin{array}{c}\text { Dis- } \\
\text { charge }\end{array}$ & Date & Hour & $\begin{array}{c}\text { Gag? } \\
\text { height }\end{array}$ & $\begin{array}{c}\text { Dis- } \\
\text { charge }\end{array}$ \\
\hline \multirow[t]{9}{*}{ Apr. 12} & 0000 & 8.45 & 12,500 & \multirow[t]{6}{*}{ Apr. 19} & 0600 & 14.74 & 36,000 & \multirow[t]{9}{*}{ Apr. 26} & 1800 & 9.33 & 15,000 \\
\hline & 0600 & 8.46 & 12,500 & & 1200 & 14.18 & 33,300 & & 2200 & 9.49 & 15,500 \\
\hline & 1200 & 8.12 & 11,500 & & 1600 & 13.77 & 31,600 & & 2400 & 9.61 & 15,900 \\
\hline & 1600 & 8.16 & 11,600 & & 1800 & 12.99 & 28,500 & & & & \\
\hline & 1800 & 9.00 & 14,000 & & 2400 & 13.64 & 31,100 & & 0800 & 10.55 & 19,100 \\
\hline & 2000 & 10.41 & 18,600 & & & & & & 1000 & 12.76 & 27,500 \\
\hline & 2200 & 14.30 & 33,800 & \multirow[t]{3}{*}{20} & 0600 & 13.81 & 31,700 & & 1800 & 12.24 & 25,500 \\
\hline & 2400 & 14.85 & 36,600 & & 1200 & 13.75 & 31,500 & & 2400 & 11.13 & 21,200 \\
\hline & & & & & 1800 & 13.70 & 31,300 & & & & \\
\hline \multirow[t]{5}{*}{13} & 0600 & 14.92 & 36,900 & \multirow{5}{*}{21} & 2400 & 13.29 & 29,700 & \multirow[t]{5}{*}{28} & 0200 & 9.39 & 15,100 \\
\hline & 1200 & 14.92 & 36,900 & & & & & & 0800 & $9.0 ?$ & 14,300 \\
\hline & 1800 & 14.59 & 35,200 & & 0600 & 13.28 & 29,600 & & 1000 & $9.2 ?$ & 14,700 \\
\hline & 2400 & 14.52 & 34,900 & & 1200 & 12.97 & 28,400 & & 2400 & 9.55 & 15,700 \\
\hline & & & & & 1800 & 12.10 & 24,900 & & & & \\
\hline \multirow[t]{6}{*}{14} & 0600 & 14.96 & 37,100 & \multirow{6}{*}{22} & 2400 & 12.03 & 24,600 & \multirow[t]{2}{*}{ May 8} & 2400 & 7.59 & 10,100 \\
\hline & 0800 & 15.95 & 42,000 & & & & & & & & \\
\hline & 1600 & 15.85 & 41,600 & & 0600 & 12.46 & 26,300 & 9 & 0600 & 7.52 & 9,900 \\
\hline & 2000 & 15.73 & 41,000 & & 1200 & 12.36 & 25,900 & & 0800 & 8.07 & 11,200 \\
\hline & 2400 & 15.80 & 41,300 & & 1800 & 12.13 & 25,000 & & 1000 & 9.10 & 14,300 \\
\hline & & & & & 2400 & 12.08 & 24,800 & & 1200 & 8.87 & 13,600 \\
\hline \multirow[t]{5}{*}{15} & 0600 & 15.65 & 40,600 & \multirow{5}{*}{23} & & & & & 1600 & 9.19 & 14,600 \\
\hline & 1200 & 15.59 & 40,200 & & 0600 & 12.22 & 25,400 & & 1800 & 10.95 & 20,500 \\
\hline & 1800 & 16.25 & 43,600 & & 1200 & 12.27 & 25,600 & & 2400 & 11.13 & 21,200 \\
\hline & 2400 & 16.36 & 44,100 & & 1800 & 11.14 & 21,200 & & & & \\
\hline & & & & & 2400 & 11.45 & 22,300 & 10 & 0600 & 10.90 & 20,400 \\
\hline \multirow[t]{6}{*}{16} & 0600 & 16.19 & 43,200 & \multirow{6}{*}{24} & & & & & 1400 & 11.51 & 22,500 \\
\hline & 1200 & 16.29 & 43,800 & & 0600 & 11.71 & 23,300 & & 2200 & 11.19 & 21,400 \\
\hline & 1800 & 16.35 & 44,000 & & 1200 & 11.61 & 22,900 & & 2400 & 10.91 & 20,400 \\
\hline & 2200 & 16.47 & 44,700 & & 1800 & 11.53 & 22,600 & & & & \\
\hline & 2400 & 16.42 & 44,400 & & 2400 & 11.37 & 22,000 & 11 & 0600 & 11.03 & 20,800 \\
\hline & & & & & & & & & 1000 & 10.79 & 20,000 \\
\hline \multirow[t]{5}{*}{17} & 0600 & 16.32 & 43,900 & \multirow[t]{5}{*}{25} & 0200 & 11.45 & 22,300 & & 1600 & 11.C 4 & 20,800 \\
\hline & 1200 & 16.21 & 43,400 & & 1200 & 11.29 & 21,700 & & 2400 & $10 . \varepsilon 6$ & 19,500 \\
\hline & 1800 & 16.01 & 42,400 & & 2000 & 10.95 & 20,500 & & & & \\
\hline & 2400 & 15.79 & 41,200 & & 2400 & 11.06 & 20,900 & 12 & 0600 & $10 . \Sigma 3$ & 18,400 \\
\hline & & & & & & & & & 1000 & $8 . \varepsilon 9$ & 13,700 \\
\hline \multirow[t]{4}{*}{18} & 0600 & 15.55 & 40,000 & \multirow[t]{4}{*}{26} & 0200 & 11.14 & 21,200 & & 1800 & 9.74 & 16,300 \\
\hline & 1200 & 15.49 & 39,800 & & 0800 & 10.94 & 20,500 & & 2400 & 9. 67 & 16,100 \\
\hline & 1800 & 15.27 & 38,600 & & 1000 & 9.75 & 16,400 & & & & \\
\hline & 2400 & 15.03 & $|37,400|$ & & 1200 & 9.091 & 14,300 & 13 & 0800 & 9.62 & 15,900 \\
\hline
\end{tabular}


Gage height, in feet, and discharge, in cubic feet per second, at indicated time, 1965, of Chippewa River at Chippewa Falls, Wis. --Continued

\begin{tabular}{|c|c|c|c|c|c|c|c|c|c|c|c|}
\hline Date & Hour & $\begin{array}{l}\text { Gage } \\
\text { height }\end{array}$ & $\begin{array}{c}\text { Dis- } \\
\text { charge }\end{array}$ & Date & Hour & $\begin{array}{c}\text { Gage } \\
\text { height }\end{array}$ & $\begin{array}{c}\text { Dis- } \\
\text { charge }\end{array}$ & Date & Hour & $\begin{array}{c}\text { Gage } \\
\text { height }\end{array}$ & $\begin{array}{c}\text { Dis- } \\
\text { charge }\end{array}$ \\
\hline May 13 & $\begin{array}{l}1000 \\
1200 \\
2400 \\
2400 \\
0200 \\
0400 \\
0600 \\
1200 \\
2000 \\
2400 \\
0200 \\
0600\end{array}$ & $\begin{array}{r}8.62 \\
8.38 \\
8.60 \\
7.61 \\
\\
8.33 \\
10.43 \\
11.29 \\
10.95 \\
10.72 \\
11.33 \\
12.93 \\
12.77\end{array}$ & $\begin{array}{l}12,900 \\
12,300 \\
12,900 \\
10,100 \\
12,100 \\
18,700 \\
21,700 \\
20,500 \\
19,700 \\
21,900 \\
28,200 \\
27,600\end{array}$ & Iay 17 & $\begin{array}{l}1200 \\
1800 \\
2200 \\
2400 \\
0200 \\
1200 \\
1800 \\
2400 \\
0200 \\
0800 \\
1400 \\
1600 \\
1800\end{array}$ & $\begin{array}{r}12.81 \\
12.80 \\
12.87 \\
13.37 \\
13.45 \\
13.13 \\
12.34 \\
12.29 \\
12.14 \\
11.75 \\
11.28 \\
10.69 \\
9.67\end{array}$ & $\begin{array}{l}27,700 \\
27,700 \\
28,000 \\
30,000 \\
30,300 \\
29,000 \\
25,900 \\
25,700 \\
25,100 \\
23,500 \\
21,700 \\
19,600 \\
16,100\end{array}$ & May 19 & $\begin{array}{l}2000 \\
2400 \\
0600 \\
1000 \\
1400 \\
2000 \\
2400 \\
0600 \\
1200 \\
1800 \\
2000 \\
2200 \\
2400\end{array}$ & $\begin{array}{r}9.62 \\
9.90 \\
10.15 \\
10.20 \\
10.23 \\
9.90 \\
9.88 \\
\\
9.86 \\
9.63 \\
8.80 \\
8.98 \\
8.80 \\
8.99\end{array}$ & $\begin{array}{l}15,900 \\
16,900 \\
17,700 \\
17,900 \\
18,000 \\
16,900 \\
16,800 \\
16,700 \\
15,900 \\
13,400 \\
14,000 \\
13,400 \\
14,000\end{array}$ \\
\hline
\end{tabular}

(115) 5-3665. Eau Claire River near Fall Creek, Wis.

(Gaging station; crest-stage station beginning 1958)

Location.-Lat $44^{\circ} 48^{\prime} 35^{\prime \prime}$, long $91^{\circ} 16^{\prime} 50^{\prime \prime}$, in NW $\frac{1}{4}$ sec.19, T.27 N., R.7 W., $500 \mathrm{ft}$ east of County Trunk K, 0.7 mile downstream from Beaver Creek, 1.3 miles ipstream from Big Falls, and 3.2 miles north of Fall Creek.

Drainage area. $-758 \mathrm{sq} \mathrm{mi}$.

Gage-height record.-Crest stages only. Altitude of gage is $830 \mathrm{ft}$ (from topographic map).

Discharge record.-Stage-discharge relation defined by current-meter measurements below $11,600 \mathrm{cfs}$ and by slope-area measurement at 16,600 cfs.

Maxima.-March-May 1965: Discharge, 10,600 cfs Apr. 11 (gage height, $12.6 \mathrm{ft}$, from floodmarks).

1943-1955, 1958 to February 1965: Discharge, 17,200 cfs May 30, 1955 (gage height, $16.11 \mathrm{ft})$.

(116) 5-3670.3. Willow Creek near Eau Claire, Wis.

(Crest-stage station)

Location.-Lat $44^{\circ} 44^{\prime} 11^{\prime \prime}$, long $91^{\circ} 26^{\prime} 48^{\prime \prime}$, on common boundary of secs.14 and 15, T.26 N., R.9 W., at box culvert on State Highway 93, 0.5 mile upstream from Lowe Creek, and 4 miles south of intersection of U.S. Highways 12 and 53 in Eau Claire.

Drainage area. $-4.38 \mathrm{sq} \mathrm{mi}$.

Gage-height record.-Crest stages only.

Discharge record.-Stage-discharge relation defined by current-meter measurement at $171 \mathrm{cfs}$.

Maxima.-March-May 1965: Discharge, $180 \mathrm{cfs}$ Apr. 9 (gage height $12.7 \mathrm{ft}$ ). 1958 to February 1965: Gage height, $14.12 \mathrm{ft}$ July 8, 1959. 
(117) 5-3671. Birch Lake at Birchwood, Wis.

Location.-Lat $45^{\circ} 39^{\prime} 35^{\prime \prime}$, long $91^{\circ} 33^{\prime} 25^{\prime \prime}$, in W $\frac{1}{2}$ sec. 25 , T.37 N., R.10 W., on Red Cedar River at Birchwood, Wis.

Drainage area. -68 sq mi, approximately.

Gage-height record.-Staff gage. Altitude of gage is $1,090 \mathrm{ft}$ (from nearby bench mark).

Maxima.-March-May 1965: Contents at end of week, 824 million cubic feet Apr. 23 (gage height, $118.0 \mathrm{ft}$ ).

1928 to February 1965: Contents on last day of month, 1,061 million cubic feet June 30, 1943 (gage height, $119.4 \mathrm{ft}$ ).

Remarks. - Reservoir operation began in 1882. Reservoir includes Lake Chetac and Birch Lake. Usable capacity in summer, 669.7 million cubic feet between gage height 114.0 and $118.5 \mathrm{ft}$, and winter, 908.3 million cubic feet between gage height $112.0 \mathrm{ft}$ and $118.5 \mathrm{ft}$ (1imits stipulated by Public Service Commission of Wisconsin).

Cooperation.-Gage heights and capacity table furnished by Northern States Power Co.

Gage height. in feet and inches, and contents, in millions of cubic feet, 1965

\begin{tabular}{|c|c|c|c|c|c|c|c|}
\hline \multirow{2}{*}{ Date } & \multicolumn{2}{|c|}{ Gage height } & \multirow{2}{*}{ Contents } & \multirow{2}{*}{ Date } & \multicolumn{2}{|c|}{ Gage height } & \multirow{2}{*}{ Contents } \\
\hline & Feet & Inches & & & Feet & Inches & \\
\hline Feb. $26 \ldots$ & 116 & 8 & 618 & Apr. $16 \ldots$ & 117 & 1 & 679 \\
\hline Mar. $5 \ldots$. & 116 & 7 & 602 & $23 \ldots$ & 118 & 0 & 824 \\
\hline $12 \ldots$ & 116 & 6 & 587 & $30 \ldots$ & 117 & 11 & 808 \\
\hline $19 \ldots$ & 116 & 5 & 572 & May $7 \ldots$ & 117 & 8 & 776 \\
\hline $26 \ldots$ & 116 & 4 & 557 & $14 \ldots$ & 117 & 10 & 792 \\
\hline Apr. $2 \ldots$ & 116 & 1 & 527 & $21 \ldots$ & 117 & 8 & 776 \\
\hline $9 \ldots$ & 116 & 0 & 512 & $28 \ldots \ldots$ & 117 & 6 & 743 \\
\hline
\end{tabular}

(118) 5-3672. Red Cedar Lake at Mikana, Wis.

Location. -Lat $45^{\circ} 35^{\prime} 20^{\prime \prime}$, long $91^{\circ} 36^{\prime} 05^{\prime \prime}$, on line between secs.21 and 22, T.36 N., R.10 W., on Red Cedar River at south edge of Mikana.

Drainage area. $-161 \mathrm{sq} \mathrm{mi}$.

Gage-height record.-Staff gage. Altitude of gage is $1,080 \mathrm{ft}$ (Northern States Power Co).

Maxima.-March-May 1965: Contents at end of week, 740 million cubic feet Apr. 23 (gage height, $111.3 \mathrm{ft}$ ).

1928 to February 1965: Contents on last day of month, 779.0 million cubic feet, June 30, 1943, and Aug. 31, 1960 (gage height, $111.6 \mathrm{ft}$ ).

Remarks.-Reservoir operation began in 1882, Reservoir includes Balsam, Hemlock and Red Cedar Lakes. Usable capacity is 577.4 million cubic feet between gage height $105.0 \mathrm{ft}$ and $110.0 \mathrm{ft}$ (limits established by Northern States Power Cc.).

Cooperation.-Gage heights and capacity table furnished by Northern States Fower Co. 
Gage height, in feet and inches, and contents, in millions of cubic feet, 1965, of Red Cedar Lake at Mikana, Wis.

\begin{tabular}{|c|c|c|c|c|c|c|c|}
\hline \multirow{2}{*}{ Date } & \multicolumn{2}{|c|}{ Gage height } & \multirow{2}{*}{ Contents } & \multirow{2}{*}{ Date } & \multicolumn{2}{|c|}{ Gage height } & \multirow{2}{*}{ Contents } \\
\hline & Feet & Inches & & & Feet & Inches & \\
\hline Feb, 26_. & 109 & 6 & 516 & Apr. 16... & 109 & 5 & 503 \\
\hline Mar. 5. & 109 & 5 & 503 & $23 \ldots$ & 111 & 4 & 740 \\
\hline 12 & 109 & 5 & 503 & $30 \ldots-\ldots$ & 111 & 0 & 702 \\
\hline 19. & 109 & 1 & 466 & May $7 \ldots$ & 110 & 7 & 652 \\
\hline 26 & 108 & 11 & 442 & $14 \ldots$ & 110 & 11 & 690 \\
\hline \multirow[t]{2}{*}{ Apr. } & 108 & 6 & 394 & $21 \ldots \ldots$ & 110 & 7 & 652 \\
\hline & 108 & 2 & 358 & $28 \ldots \ldots$ & 110 & 6 & 640 \\
\hline
\end{tabular}

(119) 5-3673. Long Lake near Brill, Wis.

Location.-Lat $45^{\circ} 40^{\prime} 05^{\prime \prime}$, long $91^{\circ} 40^{\prime} 50^{\prime \prime}$, in SW $\frac{1}{4}$ sec. 24 , T.37 N., R.11 W., on Brill River 1 mile east of Nobleton and 5.4 miles north of Brill.

Drainage area. -82 sq mi, approximately.

Gage-height record.- Staff gage. Altitude of gage is 1,210 ft (from nearby bench marks).

Maxima.- March-May 1965: Contents at end of week, 476 million cubic feet May 28 (gage height $6.3 \mathrm{ft}$ ).

1928 to February 1965: Contents on last day of month, 565.1 million cubic feet July 31,1928 (gage height, $6.8 \mathrm{ft}$ ).

Remarks.-Reservoir operation began in 1883. Usable capacity June 15 to September 5 , 385.7 million cubic feet between gage height $5.0 \mathrm{ft}$ and $7.2 \mathrm{ft}$ and September 6 to June 14, 636.8 million cubic feet between gage height $3.5 \mathrm{ft}$ and $7.2 \mathrm{ft}$ (1imits stipulated by Public Service Commission of Wisconsin).

Cooperation.-Gage heights and capacity table furnished by Northern States Power Co.

Gage height, in feet and inches, and contents, in millions of cubic feet, 1965

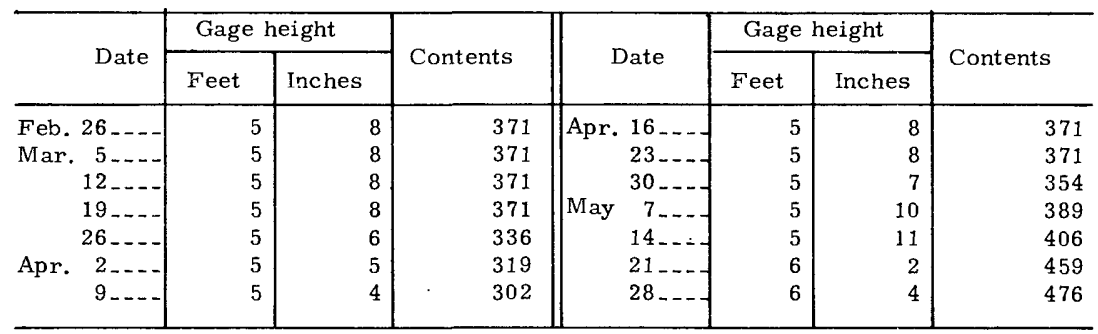


(120) 5-3674. Bear Lake at Haugen, Wis.

Location.-Lat $45^{\circ} 36^{\prime} 30^{\prime \prime}$, long $91^{\circ} 46^{\prime} 25^{\prime \prime}$, in NE $\frac{1}{4}$ sec. 18, T.36 N., R.11 W., on Rear Creek at Haugen.

Drainage area. $-60 \mathrm{sq} \mathrm{mi}$, approximately.

Gage-height record.- Staff gage. Altitude of gage is $1,140 \mathrm{ft}$ (from nearby bench marks).

Maxima.-March-May 1965: Contents at end of week, 230 million cubic feet May 21 (gage height $89.3 \mathrm{ft}$ ).

1928 to February 1965: Contents on last day of month, 359.8 million cubic feet Apr. 30, 1928 (gage height, $90.8 \mathrm{ft}$ ).

Remarks.-Reservoir operation began in 1880. Reservoir includes Kekegoma and Bear Lakes. Usable capacity 413.3 million cubic feet between gage height $87.0 \mathrm{ft}$ and $91.8 \mathrm{ft}$ (limits stipulated by Public Service Commission of Wisconsin).

Cooperation.-Gage heights and capacity table furnished by Northern States Power Co.

Gage height, in feet and inches, and contents, in millions of cubic feet, 1965

\begin{tabular}{|c|c|c|c|c|c|c|c|}
\hline \multirow{2}{*}{ Date } & \multicolumn{2}{|c|}{ Gage height } & \multirow{2}{*}{ Contents } & \multirow{2}{*}{ Date } & \multicolumn{2}{|c|}{ Gage height } & \multirow{2}{*}{ Contents } \\
\hline & Feet & Inches & & & Feet & Inches & \\
\hline Feb. 26 . & 88 & 8 & 182 & Apr. $16 \ldots$ & 89 & 1 & 213 \\
\hline Mar. 5. & 88 & 10 & 190 & $23-\ldots$ & 88 & 8 & 182 \\
\hline 12 & 88 & 8 & 182 & $30 \ldots$ & 88 & 7 & 175 \\
\hline 19 & 88 & 8 & 182 & May 7... & 89 & 0 & 205 \\
\hline 26 & 88 & 5 & 160 & $14 \ldots$ & 89 & 0 & 205 \\
\hline Apr. 2 & 88 & 2 & 145 & $21 \ldots$ & 89 & 4 & 230 \\
\hline 9 & 88 & 0 & 130 & $28 \ldots$ & 89 & 2 & 222 \\
\hline
\end{tabular}

(121) 5-3674.8. East Branch Pine Creek tributary near Dallas, Wis.

(Crest-stage station)

Location.-Lat $45^{\circ} 16^{\prime} 50^{\prime \prime}$, long $91^{\circ} 48^{\prime} 30^{\prime \prime}$, in SW $\frac{1}{4}$ sec.1, T.32 N., R.12 W., at crlvert on County Trunk $0,1.5$ miles north of Dallas.

Drainage area. $-3.85 \mathrm{sq} \mathrm{mi}$

Gage-height record.-Crest stages only.

Maxima.-March-May 1965: Gage height, $13.71 \mathrm{ft}$ Apr. 10.

1960 to February 1965: Gage height, $18.75 \mathrm{ft} \mathrm{Aug.} \mathrm{28,} 1960$. 
(122) 5-3675. Red Cedar River near Colfax, Wis.

(Gaging station, crest-stage station beginning 1962)

Location.-Lat $45^{\circ} 03^{\prime} 50^{\prime \prime}$, long $91^{\circ} 42^{\prime} 45^{\prime \prime}$, in SW $\frac{1}{4}$ sec.22, T.30 N., R.11 W., 3.2 miles downstream from Trout Creek and 4.7 miles north of Colfax.

Drainage area.-1,100 sq $\mathrm{mi}$ (approximately).

Gage-height record.-Crest stages only. Altitude of gage is $940 \mathrm{ft}$ (from topographic map).

Discharge record.- - Stage-discharge relation defined by current-meter measurements below $15,800 \mathrm{cfs}$.

Maxima.-March-May 1965: Discharge, 16,200 cfs Apr. 12, 1965 (gage height $7.99 \mathrm{ft}$ ). 1914 to February 1965: Discharge, 21,900 cfs Apr. 3, 1934 (gage height, $11.4 \mathrm{ft}$ at site 1 mile downstream at different datum), from rating curve extended above 4,900 cfs.

(123) 5-3677. Lightning Creek at Almena, Wis.

(Crest-stage station)

Location.-Lat $45^{\circ} 25^{\prime} 17^{\prime \prime}$, long $90^{\circ} 01^{\prime} 57^{\prime \prime}$, in NW $\frac{1}{4}$ sec. 19, T.34 N., R.13 $\mathrm{V}$., at bridge on County Trunk $P$ at the north edge of Almena, $0.8 \mathrm{mi}$ north from intersection with U.S. Highway 8.

Drainage area. $-19.8 \mathrm{sq} \mathrm{mi}$.

Gage-height record.-Crest stages only.

Discharge record.- Stage-discharge relation defined by current-meter measurements below 334 cfs.

Maxima.-March-May 1965: Discharge, about 1,200 cfs Apr. 11 (gage height, $12.18 \mathrm{ft}$ ). 1958 to February 1965: Discharge, about 1,050 cfs Mar. 27, 1960 (gige height, $11.99 \mathrm{ft})$.

(124) 5-3680. Hay River at Wheeler, Wis.

Location.-Lat $45^{\circ} 02^{\prime} 50^{\prime \prime}$, long $91^{\circ} 54^{\prime} 40^{\prime \prime}$, in SW $\frac{1}{4}$ sec. 25 , T.30 N., R.13 W., on right bank $25 \mathrm{ft}$ downstream from highway bridge in Wheeler, 1.8 miles upstream from Otter Creek, and 2.4 miles downstream from South Fork Hay River.

Drainage area. $-426 \mathrm{sq} \mathrm{mi}$.

Gage-height record.-Water-stage recorder graph except Mar. 13-20. Datum of gage is $893.66 \mathrm{ft}$ above mean sea level, datum of 1929 .

Discharge record.- Stage-discharge relation defined by current-meter measurements below 6,000 cfs and extended above by logarithmic plotting supported by inflow-outflow study at powerplant service pond downstream. Backwater from ice Mar. 1 to Apr. 4.

Maxima.-March-May 1965: Discharge, 10,900 c fs 0700 hours Apr. 11 (gage height, $14.65 \mathrm{ft}$ ).

1950 to February 1965: Discharge, 6,700 cfs Mar. 22, 1953 (gage h?ight, $12.36 \mathrm{ft}$ ). Maximum stage known since at least $1915,16.6 \mathrm{ft}$ in April 1934, frcm floodmarks. 
Mean discharge, in cubic feet per second, 1965, of Hay River at Wheeler, Wis.

\begin{tabular}{|c|c|c|c|c|c|c|c|c|c|c|c|}
\hline Day & March & April & May & Day & March & April & May & Day & March & April & May \\
\hline $\begin{array}{l}1 \ldots- \\
2 \ldots--1 \\
3 \ldots \\
4 \ldots- \\
5 \ldots \\
6 \ldots- \\
7 \ldots \\
8 \ldots \\
9 \\
1\end{array}$ & $\begin{array}{r}300 \\
500 \\
1,000 \\
900 \\
780 \\
700 \\
620 \\
570 \\
520 \\
480\end{array}$ & $\begin{array}{r}176 \\
180 \\
186 \\
200 \\
232 \\
895 \\
3,930 \\
4,990 \\
3,460 \\
5,740\end{array}$ & $\begin{array}{l}376 \\
379 \\
366 \\
349 \\
334 \\
332 \\
334 \\
485 \\
776 \\
469\end{array}$ & \begin{tabular}{|l|}
$11_{--}$ \\
$12_{-}$ \\
$13_{--}$ \\
$14_{-}$ \\
$15_{-}$ \\
$16_{-}$ \\
$17_{--}$ \\
$18_{--}$ \\
$19_{--}$ \\
$20_{--}$
\end{tabular} & $\begin{array}{l}440 \\
360 \\
320 \\
290 \\
270 \\
240 \\
220 \\
210 \\
200 \\
190\end{array}$ & $\begin{array}{r}9,490 \\
7,720 \\
5,470 \\
3,950 \\
2,950 \\
2,340 \\
1,520 \\
1,100 \\
881 \\
722\end{array}$ & $\begin{array}{l}376 \\
342 \\
310 \\
293 \\
281 \\
334 \\
349 \\
314 \\
293 \\
267\end{array}$ & $\begin{array}{l}21- \\
22- \\
23- \\
24_{-} \\
25_{-} \\
26- \\
27- \\
28- \\
29_{-} \\
30_{-} \\
31-\end{array}$ & $\begin{array}{l}180 \\
170 \\
166 \\
164 \\
164 \\
166 \\
164 \\
162 \\
166 \\
170 \\
172\end{array}$ & $\begin{array}{r}660 \\
595 \\
558 \\
528 \\
505 \\
573 \\
627 \\
549 \\
466 \\
424 \\
- \\
---\end{array}$ & $\begin{array}{l}255 \\
258 \\
251 \\
249 \\
247 \\
281 \\
293 \\
255 \\
240 \\
232 \\
228\end{array}$ \\
\hline $\begin{array}{l}\text { Mion } \\
\text { Run }\end{array}$ & $\begin{array}{l}\text { Iy mea } \\
\text {, in in }\end{array}$ & disc & 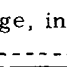 & $\mathrm{Ab}$ & $-\cdots$ & 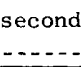 & & & $\begin{array}{r}353 \\
0.96\end{array}$ & $\begin{array}{r}2,054 \\
5.38\end{array}$ & $\begin{array}{r}327 \\
0.89\end{array}$ \\
\hline
\end{tabular}

(125) 5-3690. Red Cedar River at Menomonie, Wis.

Location.-Lat $44^{\circ} 53^{\prime} 00^{\prime \prime}$, long $91^{\circ} 55^{\prime} 55^{\prime \prime}$, in NW $\frac{1}{4}$ sec.26, T.28 N., R.13 W., on right bank at Menomonie, $900 \mathrm{ft}$ downstream from powerplant of Northern States Power Co., and 1,000 ft downstream from Wilson Creek.

Drainage area.-1,760 sq $\mathrm{mi}$, approximately.

Gage-height record.-Digital recorder tape punched at fifteen-minute intervals. Datum of gage is $780 \mathrm{ft}$ above mean sea level (Northern States Power Co. bench mark).

Discharge record.-Stage-discharge relation defined by current-meter measurements below 19,000 cfs and extended above partly on basis of computed flow over Cedar Falls Dam 6 miles upstream. Mean daily discharges computed from 96 purch-tape recordings per day.

Maxima.-March-May 1965: Discharge, 28,700 cfs 0600 hours Apr. 12 (gage height, $11.56 \mathrm{ft}$ ).

1907-8, 1913-23, 1925 to February 1965: Discharge, 40,000 cfs Apr. 4, 1934 (gage height, 16.0 ft, from floodmarks).

Remarks.-Flow regulated by powerplants at Menomonie and Cedar Falls anc' by Birch, Cedar, Long, and Bear Lakes.

Mean discharge, in cubic feet per second, 1965

\begin{tabular}{|c|c|c|c|c|c|c|c|c|c|c|c|}
\hline Day & March & April & May & Day & March & April & May & Day & March & April & May \\
\hline & 1,240 & 1,010 & 1,870 & 11. & 1,250 & 22,200 & 2,080 & 21. & 777 & 3,000 & 1,420 \\
\hline 2. & 2,220 & 1,380 & 1,500 & 12. & 1,170 & 27,900 & 2,080 & 22 & 962 & 2,660 & 1,340 \\
\hline 3 & 1,920 & 1,430 & 1,510 & 13. & 1,080 & 22,600 & 2,110 & 23. & 856 & 2,580 & 1,370 \\
\hline 4. & 2,190 & 1,370 & 1,180 & 14 & 1,100 & 16,000 & 1,610 & 24 & 855 & 2,520 & 1,320 \\
\hline 5 & 2,020 & 1,730 & 677 & 15. & 852 & 11,200 & 1,920 & 25. & 892 & 2,620 & 1,350 \\
\hline 6 & 1,840 & 3,280 & 1,020 & 16. & 1,210 & 8,290 & 1,840 & $26 \ldots$ & 949 & 2,540 & 1,520 \\
\hline 7. & 1,580 & 6,250 & 1,360 & 17. & 1,000 & 6,300 & 1,550 & 27. & 873 & 2,540 & 1,680 \\
\hline 8. & 1,820 & 11,200 & 1,620 & 18 & 1,010 & 4,560 & 1,620 & $28 \ldots$ & 786 & 2,560 & 1,790 \\
\hline 9 & 1,560 & 11,500 & 2,400 & 19. & 802 & 4,270 & 1,610 & 29. & 1,100 & 2,420 & 1,700 \\
\hline 10 & 1,510 & 13,300 & 2,650 & 20 & 884 & 3,300 & 1,430 & 30 & 1,010 & 2,050 & 1,340 \\
\hline & & & & & & & & & 1,090 & & 1,290 \\
\hline \multicolumn{9}{|c|}{ Monthly mean discharge, in cubic feet per second } & 1,239 & 6,819 & 1,605 \\
\hline
\end{tabular}


Gage height, in feet, and discharge, in cubic feet per second, at indicated time. 1965, of Red Cedar River at Menomonie, Wis.

\begin{tabular}{|c|c|c|c|c|c|c|c|c|c|c|c|}
\hline Date & Hour & $\begin{array}{l}\text { Gage } \\
\text { height }\end{array}$ & $\begin{array}{c}\text { Dis - } \\
\text { charge }\end{array}$ & Date & Hour & $\begin{array}{l}\text { Gage } \\
\text { height }\end{array}$ & $\begin{array}{c}\text { Dis- } \\
\text { charge }\end{array}$ & Date & Hour & $\begin{array}{c}\text { Gage } \\
\text { height }\end{array}$ & $\begin{array}{c}\text { Dis- } \\
\text { charge }\end{array}$ \\
\hline \multirow[t]{7}{*}{ Apr. 6} & 0000 & 3.26 & 2,380 & Apr. 10 & 2000 & 7.70 & 14,400 & Apr. 15 & 0200 & 7.41 & 13,300 \\
\hline & 0200 & 3.24 & 2,350 & & 2200 & 8.31 & 16,600 & & 0400 & 7.00 & 11,800 \\
\hline & 0400 & 3.52 & 2,770 & & 2400 & 7.84 & 14,900 & & 0800 & 6.96 & 11,700 \\
\hline & 1200 & 3.67 & 3,010 & & & & & & 1000 & 6.20 & 9,050 \\
\hline & 1600 & 4.23 & 3,980 & 11 & 0200 & 9.79 & 22,100 & & $120 \mathrm{C}^{1}$ & 6.96 & 11,700 \\
\hline & 2400 & 4.20 & 3,920 & & 0400 & 8.24 & 16,400 & & 1600 & 6.42 & 9,770 \\
\hline & & & & & 0600 & 9.34 & 20,500 & & $180 \mathrm{C}$ & 6.62 & 10,400 \\
\hline \multirow[t]{10}{*}{7} & 0200 & 4.32 & 4,150 & & 1200 & 9.27 & 20,200 & & $200 \mathrm{C}$ & 6.62 & 10,400 \\
\hline & 0800 & 4.51 & 4,540 & & 1400 & 10.18 & 23,600 & & $240 \mathrm{C}$ & 6.40 & 9,700 \\
\hline & 1000 & 5.33 & 6,510 & & 1600 & 10.14 & 23,400 & & & & \\
\hline & 1400 & 5.34 & 6,540 & & 1800 & 10.50 & 24,800 & 16 & $020 \mathrm{C}$ & 6.20 & 9,050 \\
\hline & 1600 & 4.84 & 5,270 & & 2000 & 11.10 & 27,000 & & $060 \mathrm{C}$ & 6.18 & 8,990 \\
\hline & 1800 & 5.67 & 7,460 & & 2200 & 11.23 & 27,500 & & $080 \mathrm{C}$ & 5.83 & 7,930 \\
\hline & 2000 & 5.86 & 8,010 & & 2400 & 11.06 & 26,800 & & $240 \mathrm{C}$ & 5.85 & 7,980 \\
\hline & 2200 & 6.54 & 10,200 & & & & & & & & \\
\hline & 2400 & 6.72 & 10,800 & 12 & 0200 & 11.26 & 27,600 & 17 & 0600 & 5.84 & 7,960 \\
\hline & & & & & 0400 & 11.39 & 28,000 & & 0800 & 5.15 & 6,030 \\
\hline \multirow[t]{9}{*}{8.} & 0200 & 6.59 & 10,300 & & 0600 & 11.56 & 28,700 & & 1600 & 5.19 & 6,130 \\
\hline & 0800 & 6.59 & 10,300 & & 1200 & 11.25 & 27,500 & & 1800 & 4.86 & 5,320 \\
\hline & 1000 & 6.94 & 11,600 & & 1400 & 11.41 & 28,100 & & 2400 & 4.65 & 4,840 \\
\hline & 1200 & 5.98 & 8,370 & & 2000 & 11.45 & 28,300 & & & & \\
\hline & 1400 & 6.78 & 11,000 & & 2400 & 11.25 & 27,500 & 18 & 0200 & 4.72 & 5,000 \\
\hline & 1600 & 7.39 & 13,200 & & & & & & 1400 & 4.63 & 4,800 \\
\hline & 2000 & 7.38 & 13,200 & 13 & 0800 & 10.96 & 26,500 & & 1600 & 4.59 & 4,710 \\
\hline & 2400 & 7.06 & 12,000 & & 1000 & 9.82 & 22,200 & & $1800^{\prime}$ & 4.34 & 4,190 \\
\hline & & & & & 1200 & 9.91 & 22,600 & & 2000 & 4.64 & 4,820 \\
\hline \multirow[t]{7}{*}{9} & 0200 & 7.05 & 12,000 & & 1400 & 8.34 & 16,800 & & 2200 & 4.23 & 3,980 \\
\hline & 0600 & 6.90 & 11,400 & & 1600 & 9.01 & 19,200 & & 2400 & 4.34 & 4,190 \\
\hline & 0800 & 6.49 & 10,000 & & 2400 & 9.05 & 19,400 & & & & \\
\hline & 1400 & 6.49 & 10,000 & & & & & 19 & 0800 & 4.33 & 4,170 \\
\hline & 1600 & 7.36 & 13,100 & 14 & 0400 & 9.00 & 19,200 & & 1000 & 4.71 & 4,970 \\
\hline & 2400 & 7.33 & 13,000 & & 0600 & 8.62 & 17,800 & & 1400 & 4.70 & 4,950 \\
\hline & & & & & 0800 & 8.66 & 17,900 & & 1600 & 4.26 & 4,030 \\
\hline \multirow[t]{6}{*}{10} & 0200 & 7.32 & 13,000 & & 1000 & 8.01 & 15,500 & & 2400 & 4.27 & 4,050 \\
\hline & 0800 & 7.05 & 12,000 & & 1200 & 7.51 & 13,700 & & & & \\
\hline & 1200 & 7.04 & 11,900 & & 1400 & 8.04 & 15,600 & 20 & 0600 & 4.25 & 4,100 \\
\hline & 1400 & 7.29 & 12,900 & & 1600 & 8.03 & 15,600 & & 0800 & 3.37 & 2,540 \\
\hline & 1600 & 7.30 & 12,900 & & 2200 & 7.36 & 13,100 & & 1000 & 3.61 & 2,920 \\
\hline & 1800 & 7.57 & 13,900 & & 2400 & 7.40 & 13,300 & & 2400 & 3.82 & 3,250 \\
\hline
\end{tabular}


(126) 5-3695. Chippewa River at Durand, Wis.

Location.-Lat $44^{\circ} 37^{\prime} 45^{\prime \prime}$, long $91^{\circ} 58^{\prime} 10^{\prime \prime}$, in SW $\frac{1}{4}$ sec. 21 , T.25 N., R.13 W., on laft bank at Durand, $75 \mathrm{ft}$ downstream from highway bridge and 9.5 miles downstream from Red Cedar River.

Drainage area. $-9,010 \mathrm{sq} \mathrm{mi}$, approximately.

Gage-height record.-Digital recorder tape punched at sixty-minute intervals. Datum of gage is $694.59 \mathrm{ft}$ above mean sea level, datum of 1929 .

Discharge record.-Stage-discharge relation defined by current-meter measurements below 93,000 cfs. Mean daily discharges computed from 24 punch-tape reccrdings per day. Backwater from ice Mar. 1 to Apr. 10.

Maxima.-March-May 1965: Discharge, 66,200 cfs 1200 hours Apr. 14 (gage height, $13.36 \mathrm{ft}$ ); gage height, $13.68 \mathrm{ft}$, probably Apr. 9 (result of ice jam).

1928 to February 1965: Discharge, 101,000 cfs May 3, 1954 (gage height, $15.40 \mathrm{ft}$ ). Maximum stage known, $18.4 \mathrm{ft}$ Sept. 12,1884

Remarks.-Flow partly regulated by powerplants, Moose Lake, Lake Chippewa, Rest Lake, Flambeau Flowage, Lake Wissota, and Birch, Cedar, Bear, and Long Lakes on upper Red Cedar River.

Nean discharge, in cuble feet per second, 1965

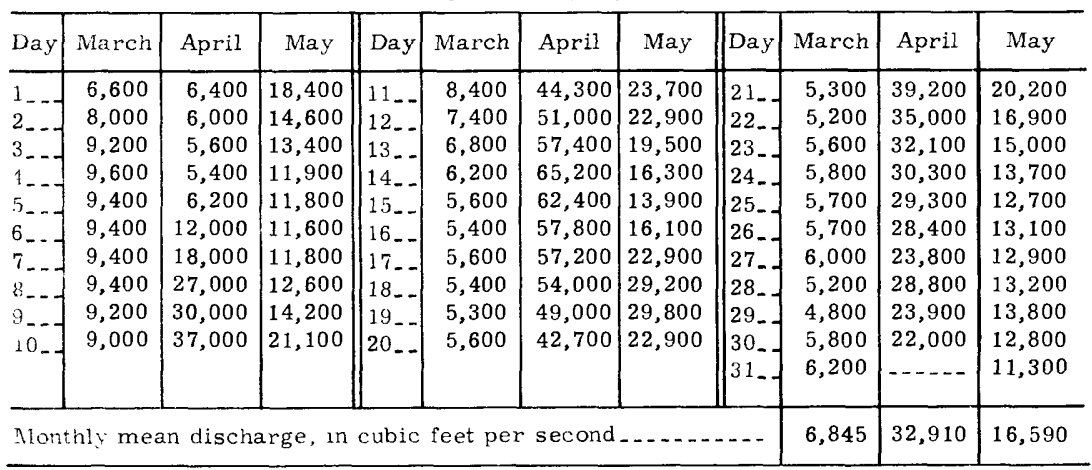

Gage height, in feet, and discharge, in cubic feet per second, at indicated time, 1965

\begin{tabular}{|c|c|c|c|c|c|c|c|c|c|c|c|}
\hline Date & Hour & $\begin{array}{c}\text { Gase } \\
\text { height }\end{array}$ & $\begin{array}{c}\text { Dis- } \\
\text { charge }\end{array}$ & Date & Hour & $\begin{array}{l}\text { Gage } \\
\text { height }\end{array}$ & $\begin{array}{c}\text { Dis- } \\
\text { charge }\end{array}$ & Date & Hour & $\begin{array}{l}\text { Gago } \\
\text { height }\end{array}$ & $\begin{array}{c}\text { Dis- } \\
\text { charge }\end{array}$ \\
\hline \multirow[t]{6}{*}{ Apr. 6} & 0000 & 6.33 & & Apr. 9 & 0600 & 12.70 & & \multirow[t]{6}{*}{ Apr. 11} & \multirow[t]{2}{*}{2400} & \multirow[t]{2}{*}{11.83} & \multirow[t]{2}{*}{47,800} \\
\hline & 0600 & 7.14 & & & 1400 & 12.17 & & & & & \\
\hline & 1200 & 8.07 & & & 1800 & 12.66 & & & 0600 & 12.05 & 49,500 \\
\hline & 1800 & 8.88 & & & 2200 & 13.11 & & & 1200 & 12.25 & 51,600 \\
\hline & 2400 & 9.44 & & & 2400 & 12.00 & $\ldots$ & & 1800 & 12.33 & 52,500 \\
\hline & & & & & & & & & 2400 & $12.3^{\circ}$ & 52,800 \\
\hline \multirow[t]{5}{*}{7} & 0600 & 9.81 & & 10 & 0200 & 11.55 & $\ldots$ & \multirow{5}{*}{13} & & & \\
\hline & 1200 & 10.07 & & & 0600 & 11.14 & $\ldots$ & & 1200 & 12.65 & 56,400 \\
\hline & 1800 & 10.54 & & & 1200 & 11.01 & $\ldots$ & & 1800 & 12.98 & 60,700 \\
\hline & 2400 & 11.13 & & & 1800 & 10.94 & $\ldots$ & & 2400 & 13.19 & 63,700 \\
\hline & & & & & 2400 & 11.00 & 40,700 & & & & \\
\hline \multirow[t]{4}{*}{8} & 0600 & 11.95 & & & & & & \multirow[t]{4}{*}{14} & 0600 & 13.29 & 65,200 \\
\hline & 1200 & 12.44 & & 11 & 0600 & 11.18 & 42,000 & & 1200 & 13.36 & 66,200 \\
\hline & 1600 & 12.65 & $\ldots$ & & 1200 & 11.46 & 44,200 & & 1800 & 13.31 & 65,400 \\
\hline & 2400 & 12.54 & $\ldots$ & & 1800 & 11.69 & $|46,100|$ & & 2400 & 13.20 & 63,800 \\
\hline
\end{tabular}


Gage height, in feet, and discharge, in cubic feet per second, at indicated time, 1965. of Cnippewa River at Durand, Wis. - Continued

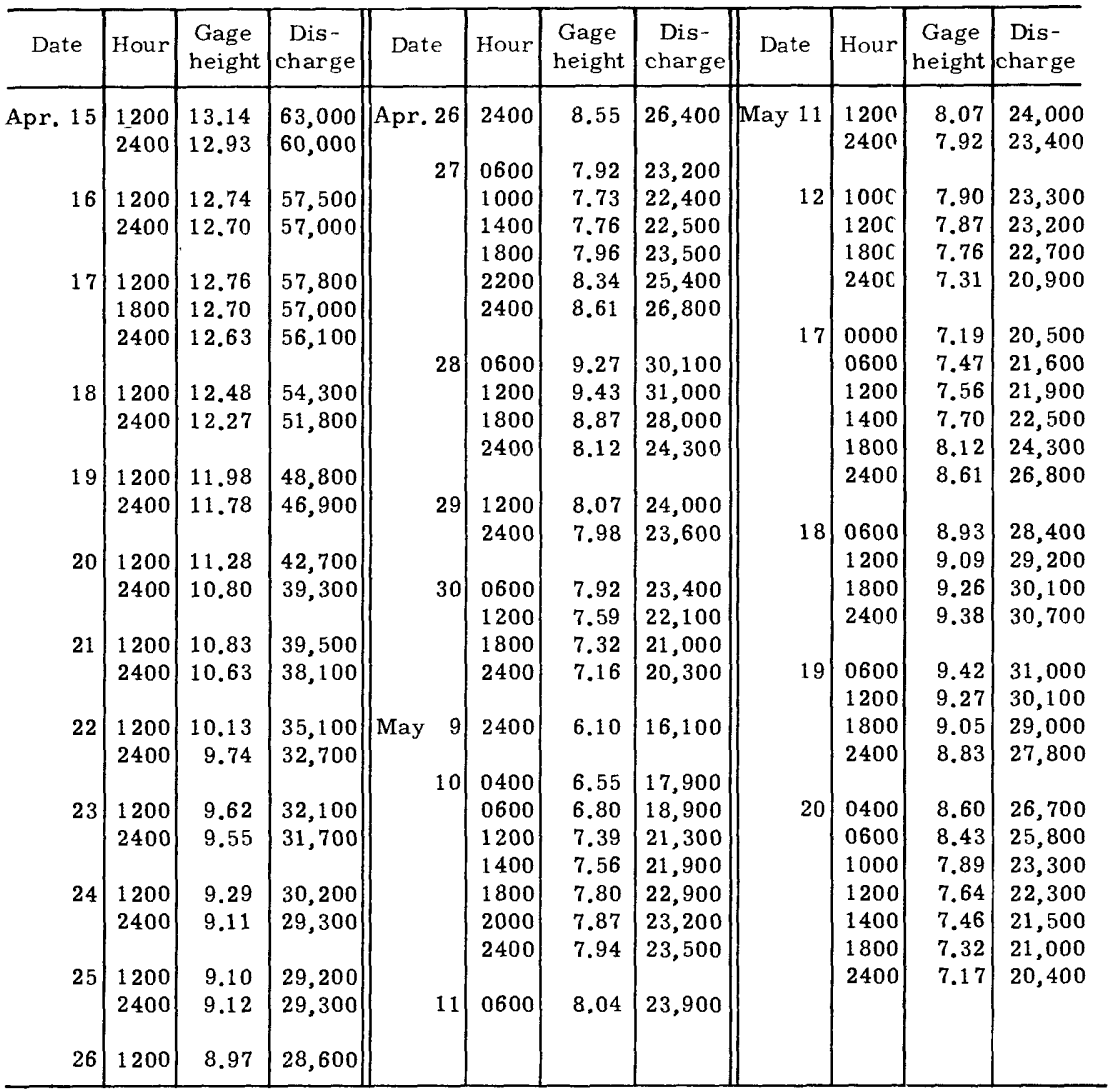

(127) 5-3700. Eau Galle River at Spring Valley, Wis.

Location.-Lat $44^{\circ} 51^{\prime} 00^{\prime \prime}$, long $92^{\circ} 14^{\prime} 15^{\prime \prime}$, between secs. 5 and 6, T.27 N., R.15 W., on downstream side near center of bridge at Spring Valley, 0.1 mile upstream from Mines Creek, 0.5 mile downstream from Lousy Creek, and at mile 29.96.

Drainage area. $-64.8 \mathrm{sq} \mathrm{mi}$.

Gage-height record.-Wire-weight gage read twice daily. Graph drawn on basis of numerous wire-weight gage readings daily and crest data during rises. Datum of gage is $910.45 \mathrm{ft}$ above mean sea level, datum of 1929 (Corps of Engine ers bench mark).

Discharge record.-Stage-discharge relation defined by current-meter measurements below 4,700 cfs and a slope-area measurement at 4,700 cfs. Backwater from ice Mar. 9 to Apr. 2.

Maxima.-March-May 1965: Discharge, 4,130 cfs at 2000 hours Apr. 10 (gage height $10.50 \mathrm{ft}$ ).

1944 to February 1965: Discharge, 7,000 cfs Apr. 15, 1954 (gage height, $11.50 \mathrm{ft}$ ).

Maximum stage known since at least $1894,18.98 \mathrm{ft}$, Sept. 18, 1942, from floodmarks (discharge, 33,000 cfs, estimated by Corps of Engineers on basis of slope-area measurement by Geological Survey of peak discharge of $39,000 \mathrm{cfs}$ at Elmwood, drainage area, $91.9 \mathrm{sq} \mathrm{mi})$. 
Mean discharge, in cubic feet per second, 1965, of Eau Galle River at Spring Valley, Wis.

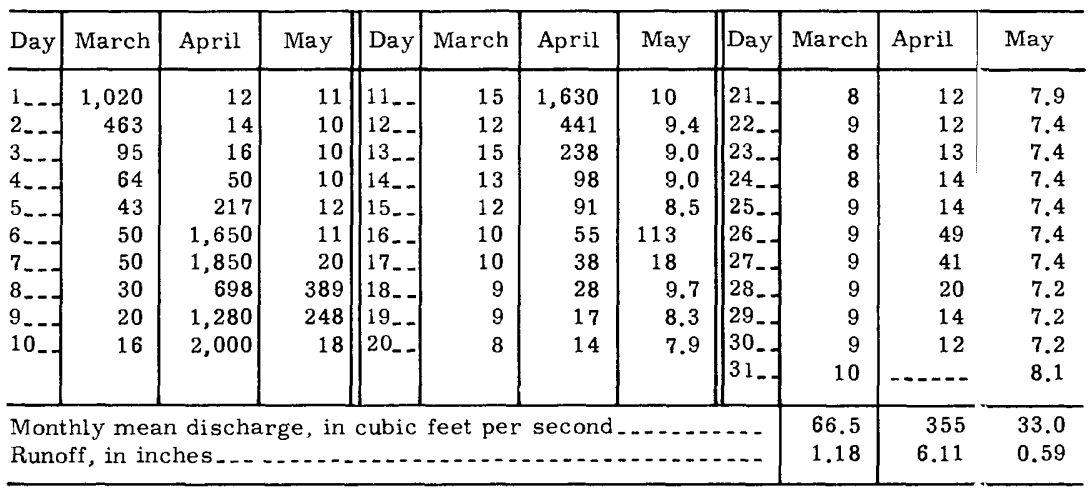

Gage height, in feet, and discharge, in cubic feet per second, at indicated time, 1965

\begin{tabular}{|c|c|c|c|c|c|c|c|c|c|c|c|}
\hline Date & Hour & $\begin{array}{l}\text { Gage } \\
\text { height }\end{array}$ & $\begin{array}{c}\text { Dis- } \\
\text { charge }\end{array}$ & Date & Hour & $\begin{array}{l}\text { Gage } \\
\text { height }\end{array}$ & $\begin{array}{c}\text { Dis- } \\
\text { charge }\end{array}$ & Date & Hour & $\begin{array}{l}\text { Gage } \\
\text { height }\end{array}$ & $\begin{array}{c}\text { Dis- } \\
\text { charge }\end{array}$ \\
\hline \multirow[t]{16}{*}{ Mar. 1} & 0000 & 2.30 & 50 & Apr. 6 & 1800 & 8.15 & 2,400 & Apr. 10 & 0800 & 5.10 & 680 \\
\hline & 0200 & 2.55 & 66 & & 2000 & 7.90 & 2,230 & & 1200 & 6.10 & 1,080 \\
\hline & 0600 & 3.40 & 179 & & 2400 & 7.40 & 1,890 & & 1600 & $9 . \mathrm{CO}$ & 3,000 \\
\hline & 0800 & 3.92 & 305 & & & & & & 1800 & $10 . C 0$ & 3,720 \\
\hline & 1200 & 5.30 & 855 & 7 & 0600 & 6.95 & 1,620 & & 2000 & 10.50 & 4,130 \\
\hline & 1600 & 6.95 & 1,880 & & 1200 & 6.70 & 1,470 & & 2200 & $9 . \subseteq 0$ & 3,640 \\
\hline & 1800 & 7.30 & 2,160 & & 1600 & 7.05 & 1,680 & & 2400 & 9.20 & 3,140 \\
\hline & 2000 & 7.20 & 2,080 & & 1800 & 8.00 & 2,300 & & & & \\
\hline & 2400 & 6.60 & 1,600 & & 2000 & 8.80 & 2,860 & 11 & 0400 & 7.70 & 2,090 \\
\hline & & & & & 2200 & 8.00 & 2,300 & & 0800 & 6.30 & 1,230 \\
\hline & 0400 & 5.30 & 855 & & 2400 & 7.15 & 1,740 & & 1200 & 5.90 & 1,030 \\
\hline & 0800 & 4.32 & 428 & & & & & & 1400 & 6.50 & 1,350 \\
\hline & 1200 & 3.85 & 298 & 8 & 0400 & 6.00 & 1,080 & & 1800 & 7.40 & 1,890 \\
\hline & 1800 & 3.30 & 160 & & 0800 & 4.90 & 600 & & 2000 & 7.00 & 1,650 \\
\hline & 2400 & 3.00 & 112 & & 1200 & 4.45 & 438 & & 2400 & 5.50 & 845 \\
\hline & & & & & 1600 & 4.60 & 490 & & & & \\
\hline \multirow[t]{7}{*}{ Apr. 4} & 2400 & 2.30 & 50 & & 2000 & 4.80 & 560 & 12 & 0600 & 4.00 & 300 \\
\hline & & & & & 2400 & 4.00 & 300 & & 1200 & 3.80 & 250 \\
\hline & 1200 & 2.52 & 60 & & & & & & 1800 & 4.60 & 490 \\
\hline & 1600 & 3.75 & 240 & 9 & 0600 & 3.35 & 160 & & 2000 & 4.85 & 580 \\
\hline & 2000 & 4.76 & 546 & & 1000 & 4.00 & 300 & & 2400 & 4.70 & 525 \\
\hline & 2400 & 5.00 & 640 & & 1400 & 6.00 & 1,080 & & & & \\
\hline & & & & & 1800 & 9.60 & 3,420 & 13 & 0600 & 3.10 & 114 \\
\hline \multirow[t]{5}{*}{6} & 0200 & 4.82 & 568 & & 2000 & 9.55 & 3,380 & & 1200 & 2.85 & 77 \\
\hline & 0400 & 5.10 & 680 & & 2200 & 8.40 & 2,580 & & 1600 & 3.60 & 210 \\
\hline & 0800 & 6.90 & 1,590 & & 2400 & 6.50 & 1,350 & & 2000 & 4.40 & 420 \\
\hline & 1200 & 7.40 & 1,890 & & & & & & 2400 & 3.90 & 275 \\
\hline & 1600 & 7.90 & 2,230 & 10 & 0400 & 5.30 & 760 & & & & \\
\hline
\end{tabular}


(128) 5-3706. Arkansaw Creek Tributary near Arkansaw, Wis.

\section{(Crest-stage station)}

Location.-Lat $44^{\circ} 38^{\prime} 31^{\prime \prime}$, long $92^{\circ} 03^{\prime} 09^{\prime \prime}$, in SW $\frac{1}{4}$ sec.14, T.25 N., R.14 W., at box culvert on U.S. Highway 10, 0.6 mile east of intersection with County Trunk Z, 1.2 miles northwest of Arkansaw, and 4 miles west of Chippewa River bridge in Durand.

Drainage area. $-2.56 \mathrm{sq} \mathrm{mi}$.

Gage-height record.-Crest stages only.

Maxima.--March-May 1965: Gage height, $13.37 \mathrm{ft}$ Apr. 10.

1959 to February 1965: Gage height, $12.80 \mathrm{ft}$ Mar. 28, 1962.

(129) 5-3709. Spring Creek near Durand, Wis.

\section{(Crest-stage station)}

Location.-Lat $44^{\circ} 34^{\prime} 13^{\prime \prime}$, long $91^{\circ} 57^{\prime} 48^{\prime \prime}$, in $\mathrm{S}_{2}^{\frac{1}{2}}$ sec.9, T.24 N., R.13 W., at bridge on country road approximately 0.2 mile upstream from County Trunk AA crossing and 4 miles south of Chippewa River Bridge in Durand.

Drainage area. $-6.49 \mathrm{sq} \mathrm{mi}$.

Gage-height record.-Crest stages only.

Discharge record.--Stage-discharge relation defined by current-meter measurements below 335 cfs.

Maxima.-March-May 1965: Discharge, $390 \mathrm{cfs}$ Apr. 7 (gage height, $12.92 \mathrm{ft}$ ). 1962 to February 1965: Gage height, $13.28 \mathrm{ft}$ July 17, 1963.

\section{MISSISSIPPI RIVER MAIN STEM}

(130) Mississippi River at Lock and Dam 4 near Alma, Wis.

(Miscellaneous site)

Location.-Lat $44^{\circ} 20^{\prime}$, long $91^{\circ} 56^{\prime}$, in sec.2, T.21 N., R.13 W., at Lock and Dam 4 in Alma, and at river mile 752.87 above Ohio River.

Gage-height record.-Peak stages from pool and tailwater water-stage recorder graphs.

Discharge record.-Corps of Engineers stage-discharge relation extended on the basis of three Geological Survey current-meter measurements.

Maxima.-April-May 1965: Discharge, 256,000 cfs 1200 hours Apr. 19 (elevations, $676.45 \mathrm{ft}$ pool, $675.78 \mathrm{ft}$, tailwater).

1935 to March 1965: Discharge, 187,000 cfs Apr. 19, 1952 (elevations, $673.30 \mathrm{ft}$ pool, $672.30 \mathrm{ft}$ tailwater).

Cooperation.-Records furnished by Corps of Engineers. 


\section{ZUMBRO RIVER BASIN}

(131) 5-3730. South Fork Zumbro River near Rochester, Minn.

Location.-Lat $44^{\circ} 04^{\prime} 00^{\prime \prime}$, long $92^{\circ} 27^{\prime} 55^{\prime \prime}$, in $\mathrm{SE}_{4}^{\frac{1}{4}}$ sec.14, T.107 N., R.14 W., on left bank $30 \mathrm{ft}$ upstream from ford, a quarter of a mile downstream from sewage plant, 1.6 miles north of Rochester, 2 miles downstream from Cascade Creek, and $2 \frac{1}{2}$ miles downstream from Silver Lake Dam.

Drainage area. $-304 \mathrm{sq} \mathrm{mi}$.

Gage-height.-Water-stage recorder graph except Mar. 1-3, 10-11 and 19-25. Graph was reconstructed Mar. 1-3, on the basis of adjacent record and floodmark. Datum of gage is $949.56 \mathrm{ft}$ above mean sea level, datum of 1929 .

Discharge record.- Stage-discharge relation defined by current-meter measurements. Discharge Mar. 10-11 and 19-25 was estimated on the basis of one dischargo measurement and weather records.

Maxima.-Given in the following table.

March-May 1965:

Mar. 1, 1900 hours

Discharge

(cfs)

19,600

8,010

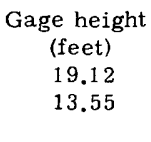

1952 to February 1965:

Mar. 29, 1962

18,000

18.46

Previous maximum stage known since at least 1908, about $17.5 \mathrm{ft}$, July 21, 1951 , from information by sewage plant superintendant.

Mean discharge, in cubic feet per second, 1965

\begin{tabular}{|c|c|c|c|c|c|c|c|c|c|c|c|}
\hline Day & March & April & May & Day & March & April & May & Day & March & April & May \\
\hline 2 & 10,900 & 1,040 & 133 & $11 \ldots$ & 82 & 1,030 & 108 & $21-$ & 46 & 129 & 85 \\
\hline 2 & 2,320 & 1,590 & 125 & $12 \ldots$ & 79 & 1,150 & 102 & $22=$ & 46 & 120 & 78 \\
\hline 3. & 502 & 2,020 & 129 & 13 & 75 & 406 & 98 & $23 \ldots$ & 47 & 120 & 76 \\
\hline 4 & 275 & 5,020 & 119 & $14 \ldots$ & 75 & 280 & 95 & $24-$ & 49 & 127 & 80 \\
\hline 5. & 205 & 4,540 & 170 & $15 \ldots$ & 71 & 245 & 113 & $25 \ldots$ & 50 & 250 & 113 \\
\hline 6. & 172 & 6,430 & 225 & $16 \ldots$ & 66 & 200 & 155 & $26-\mid$ & 46 & 377 & 334 \\
\hline 7. & 145 & 3,240 & 178 & 17. & 66 & 188 & 125 & $27-$ & 46 & 268 & 296 \\
\hline 8. & 119 & 4,100 & 145 & 18. & 52 & 185 & 136 & 28. & 42 & 202 & 155 \\
\hline 9. & 100 & 2,790 & 129 & 19 & 47 & 170 & 98 & 29 & 44 & 172 & 110 \\
\hline 10. & 92 & 1,400 & 113 & $20 \ldots$ & 47 & 141 & 89 & $30-1$ & 53 & 149 & 98 \\
\hline & & & & & & & & & 119 & $\ldots$ & 94 \\
\hline \multirow{2}{*}{\multicolumn{9}{|c|}{$\begin{array}{l}\text { Monthly mean discharge, in cubic feet per second } \\
\text { Runoff, in inches }\end{array}$}} & 519 & 1,269 & 132 \\
\hline & & & & & & & & & 1.97 & 4.66 & 0.502 \\
\hline
\end{tabular}


A250

FLOODS OF 1965 IN THE UNITED STATES

Gage height, in feet, and discharge, in cubic feet per second, at indicated time, 1965, of South Fork Zumbro

River near Rochester, Minn.

\begin{tabular}{|c|c|c|c|c|c|c|c|c|c|c|c|}
\hline Date & Hour & $\begin{array}{c}\text { Gage } \\
\text { height }\end{array}$ & $\begin{array}{c}\text { Dis- } \\
\text { charge }\end{array}$ & Date & Hour & $\begin{array}{c}\text { Gage } \\
\text { height }\end{array}$ & $\begin{array}{c}\text { Dis- } \\
\text { charge }\end{array}$ & Date & Hour & $\begin{array}{c}\text { Gage } \\
\text { height }\end{array}$ & $\begin{array}{l}\text { Dis- } \\
\text { charge }\end{array}$ \\
\hline \multirow[t]{14}{*}{ Feb. } & 0000 & 1.94 & 22 & Mar. 31 & 0800 & 2.81 & 42 & Apr. 6 & 2400 & 12.57 & 6,080 \\
\hline & 0600 & 1.88 & 19 & & 1400 & 3.12 & 80 & & & & \\
\hline & 1200 & 1.98 & 23 & & 1800 & 3.66 & 203 & 7 & 0490 & 11.38 & 4,110 \\
\hline & 1400 & 2.00 & 24 & & 2200 & 3.65 & 213 & & $08 \cap 0$ & 9.12 & 2,440 \\
\hline & 1800 & 2.60 & 82 & & 2400 & 4.13 & 460 & & 1200 & 8.14 & 1,930 \\
\hline & 2400 & 2.27 & 41 & & & & & & 1600 & 9.00 & 2,340 \\
\hline & & & & Apr. 1 & 0600 & 4.55 & 616 & & 1700 & 8.28 & 2,000 \\
\hline & 0500 & 2.10 & 27 & & 1200 & 5.33 & 802 & & 2030 & 10.42 & 3,400 \\
\hline & 1000 & 2.27 & 38 & & 1600 & 6.24 & 1,220 & & 2230 & 11.32 & 4,050 \\
\hline & 1200 & 2.48 & 60 & & 2000 & 7.20 & 1,740 & & 2490 & 12.40 & 5,310 \\
\hline & 1600 & 4.06 & 433 & & 2400 & 7.80 & 2,070 & & & & \\
\hline & 1900 & 6.15 & 934 & & & & & 8 & 0490 & 11.59 & 4,340 \\
\hline & 2400 & 7.75 & 1,390 & 2 & 0400 & 7.14 & 1,710 & & 0830 & 10.07 & 3,120 \\
\hline & & & & & 0800 & 6.28 & 1,240 & & 1100 & 9.16 & 2,470 \\
\hline \multirow[t]{31}{*}{ Mar. } & 0200 & 9.70 & 2,390 & & 1100 & 5.99 & 1,100 & & 1600 & 10.25 & 3,260 \\
\hline & 0400 & 11.25 & 4,020 & & 1800 & 7.16 & 1,720 & & 1830 & 11.30 & 4,030 \\
\hline & 0800 & 13.25 & 7,590 & & 2200 & 7.66 & 1,990 & & 2030 & 12.25 & 5,140 \\
\hline & 1000 & 15.00 & 10,600 & & 2400 & 7.37 & 1,830 & & 2330 & 13.27 & 7,160 \\
\hline & 1200 & 16.20 & 13,000 & & & & & & 2400 & 13.23 & 7,120 \\
\hline & 1400 & 17.00 & 14,700 & 3 & 0900 & 6.79 & 1,510 & & & & \\
\hline & 1600 & 17.85 & 16,600 & & 1500 & 7.19 & 1,730 & 9 & 0490 & 11.84 & 4,640 \\
\hline & 1800 & 18.60 & 18,300 & & 2000 & 8.34 & 2,450 & & 06120 & 10.74 & 3,660 \\
\hline & 1900 & 19.12 & 19,600 & & 2300 & 10.03 & 3,830 & & 0800 & 9.50 & 2,740 \\
\hline & 2000 & 18.30 & 17,600 & & 2400 & 9.94 & 3,840 & & 1200 & 8.13 & 1,920 \\
\hline & 2200 & 16.25 & 13,200 & & & & & & 1500 & 7.72 & 1,810 \\
\hline & 2400 & 14.60 & 9,890 & 4 & 0600 & 10.73 & 4,630 & & 1630 & 5.88 & 942 \\
\hline & & & & & 0900 & 10.37 & 4,450 & & 1800 & 6.94 & 1,430 \\
\hline & 0200 & 12.90 & 7,030 & & 1000 & 11.56 & 5,380 & & 2400 & 8.09 & 1,900 \\
\hline & 0400 & 11.50 & 4,250 & & 1400 & 11.25 & 4,980 & & & & \\
\hline & 0800 & 9.15 & 2,170 & & 1700 & 12.02 & 5,660 & 10 & 0100 & 8.11 & 1,910 \\
\hline & 1000 & 7.05 & 1,030 & & 2100 & 11.58 & 5,180 & & 0600 & 7.24 & 1,540 \\
\hline & 1300 & 6.61 & 953 & & 2400 & 13.18 & 6,940 & & 1600 & 6.50 & 1,150 \\
\hline & 1800 & 5.85 & 778 & & & & & & 2400 & .74 & 1,270 \\
\hline & 2400 & 5.30 & 640 & 5 & 0100 & 13.22 & 7,360 & & & & \\
\hline & & & & & 0500 & 12.63 & 6,160 & 11 & 0600 & 6.25 & 1,080 \\
\hline & 0600 & 5.05 & 580 & & 1000 & 11.39 & 4,120 & & 1200 & 5.80 & 870 \\
\hline & 1800 & 4.54 & 430 & & 1200 & 10.76 & 3,630 & & 1600 & 5.53 & 762 \\
\hline & 2400 & 4.24 & 346 & & 1600 & 9.98 & 3,040 & & 2400 & 6.89 & 1,400 \\
\hline & & & & & 1800 & 10.39 & 3,330 & & & & \\
\hline & 2400 & 2.82 & 43 & & 2400 & 11.17 & 3,900 & 12 & 0500 & 8.15 & 1,930 \\
\hline & & & & & & & & & 1200 & 6.06 & 980 \\
\hline & 0800 & 2.75 & 37 & 6 & 0600 & 12.02 & 4,890 & & 2400 & 4.91 & 518 \\
\hline & 1400 & 2.84 & 45 & & 1130 & 13.55 & 8,010 & & & & \\
\hline & 1800 & 3.18 & 89 & & 1400 & 13.46 & 7,870 & 13 & 1200 & 4.45 & 395 \\
\hline & 2400 & 2.97 & 60 & & 1800 & 13.38 & 7,740 & & 2400 & 4.19 & 317 \\
\hline
\end{tabular}


(132) 5-3737. North Fork Zumbro River tributary near Wanamingo, Minn.

(Crest-stage station)

Location.-Lat $44^{\circ} 17^{\prime} 10^{\prime \prime}$, long $92^{\circ} 52^{\prime} 20^{\prime \prime}$, in $\mathrm{SE} \frac{1}{4} \mathrm{SE} \frac{1}{4}$ sec.32, T.110 N., R.17 W., at culvert on County Highway $1,3 \frac{1}{4}$ miles upstream from mouth, and $4 \frac{1}{4}$ miles southwest of Wanamingo.

Drainage area. $-9.36 \mathrm{sq} \mathrm{mi}$.

Gage-height record.-Crest stages only.

Discharge record.-Stage-discharge relation defined by indirect measurements at $607 \mathrm{cfs}$ and $1,310 \mathrm{cfs}$.

Maxima.-March-May 1965: Discharge, $870 \mathrm{cfs}$ Apr. 7 (gage height, $13.00 \mathrm{ft}$, backwater from ice); gage height, $14.81 \mathrm{ft}$ Mar. 1 (backwater from ice).

1960 to February 1965: Gage height, $18.14 \mathrm{ft}$ Mar. 28, 1962, backwater from ice (discharge not determined).

(133) 5-3740. Zumbro River at Zumbro Falls, Minn.

Location.-Lat $44^{\circ} 17^{\prime} 12^{\prime \prime}$, long $92^{\circ} 25^{\prime} 26^{\prime \prime}$, in sec.36, T.110 N., R.14 W., on left bank in Zumbro Falls, 1,000 ft downstream from Spring Creek, 0.7 mile upstream from highway bridge on U.S. Highway 63 , and 6.3 miles downstream from North Branch.

Drainage area. $-1,130 \mathrm{sq} \mathrm{mi}$, approximately.

Gage-height record.-Digital recorder tape punched at 15 minute intervals except 0500 hours Mar. 1 to 1200 hours Mar. 29 when record was obtained from the auxiliary record graph. For the period Mar. 1-3 the graph was constructed on the basis of floodmark and partial gage height record. Datum of gage is $811.26 \mathrm{ft}$ above moan sea level, datum of 1929 .

Discharge record.- Stage-discharge relation defined by current-meter measurements. Mean daily discharge computed from 96 punch-tape readings per day, Mar. 4 and Mar. 30 to May 31 .

Maxima.-Given in the following table.

March-May 1965:

Mar. 2, 0500 hours

Apr. 7, 0200 hours

1909-17, 1929 to February 1965:

July 22, 1951
Discharge

(cfs)

29,600

26,800

35,900
Gage height

(feet)

28.40

27.26

30.80

Flood of April 1888 reached a stage of abour $30.5 \mathrm{ftat}$ present site or $29.7 \mathrm{ftat}$ original site. Flood in 1859 is known to have exceeded that of 1888 (gage height not determined).

Remarks.-Diurnal fluctuation at low and medium flows caused by powerplant above station. 
Mean discharge, in cubic feet per second, 1965, of Zumbro River at Zumbro Falls, Minn.

\begin{tabular}{|c|c|c|c|c|c|c|c|c|c|c|c|}
\hline Day & March & April & May & Day & March & April & May & Day & March & April & May \\
\hline 1 & 12,600 & 767 & 375 & $11 \ldots$ & 542 & 8,500 & 599 & 21. & 109 & 1,020 & 677 \\
\hline 2 & 22,800 & 1,650 & 217 & $12 \ldots$ & 574 & 5,650 & 593 & 22 & 250 & 1,000 & 316 \\
\hline 3 & 4,020 & 3,470 & 512 & 13 & 282 & 3,110 & 523 & 23 & 245 & 1,000 & 201 \\
\hline & 1.550 & 10,300 & 677 & 14 & 152 & 2.030 & 413 & 24 & 158 & 761 & 501 \\
\hline & 1,130 & 18,200 & 665 & $15 \ldots$ & 289 & 1,620 & 228 & 25 & 227 & 779 & 569 \\
\hline 6. & 1,020 & 21,600 & 665 & $16 \ldots$ & 514 & 1,360 & 599 & 26 & 217 & 1,150 & 653 \\
\hline 7. & 992 & 23,100 & 884 & $17 .-$ & 394 & 1,230 & 1,280 & $27 \ldots$ & 187 & 1,190 & 635 \\
\hline & 946 & 19,700 & 653 & $18 \ldots$ & 264 & 1,160 & 871 & 28 & 95 & 1,050 & 653 \\
\hline u. & 533 & 17,100 & 325 & $19 \ldots$ & 374 & 1,120 & 803 & 29. & 297 & 982 & 308 \\
\hline 10. & 462 & 10,300 & 501 & $20 \ldots$ & 211 & 1,050 & 707 & $30_{-}$ & 512 & 949 & 192 \\
\hline & & & & & & & & 31 & 527 & & 177 \\
\hline \multirow{2}{*}{\multicolumn{9}{|c|}{ Monthly mean discharge, in cubic feet per second }} & 1,693 & 5,430 & 547 \\
\hline & & & & & & & & Runoif, in inches & 1.73 & 5.36 & 0.56 \\
\hline
\end{tabular}

Gage height, in feet, and discharge, in cubic feet per second, at indicated time, 1965

\begin{tabular}{|c|c|c|c|c|c|c|c|c|c|c|c|}
\hline Date & Hour & $\begin{array}{l}\text { Gage } \\
\text { height }\end{array}$ & $\begin{array}{c}\text { Dis - } \\
\text { charge }\end{array}$ & Date & Hour & $\begin{array}{c}\text { Gage } \\
\text { height }\end{array}$ & $\begin{array}{c}\text { Dis- } \\
\text { charge }\end{array}$ & Date & Hour & $\begin{array}{c}\text { Gage } \\
\text { height }\end{array}$ & $\begin{array}{c}\text { Dis- } \\
\text { charge }\end{array}$ \\
\hline \multirow[t]{6}{*}{ Feb. 28} & 0000 & 6.51 & 97 & Mar. 4 & 2400 & 8.81 & 1,250 & Apr. 8 & $0 € \cap 0$ & 23.42 & 18,300 \\
\hline & 1000 & 6.48 & 91 & & & & & & 0900 & 23.32 & 18,100 \\
\hline & 2000 & 6.93 & 220 & Apr. 1 & 0000 & 8.09 & 788 & & 1400 & 24.19 & 19,900 \\
\hline & 2200 & 7.44 & 435 & & 1000 & 6.94 & 216 & & 1800 & 24.98 & 21,600 \\
\hline & 2400 & 8.10 & 794 & & 1400 & 8.15 & 813 & & 2400 & 24.19 & 19,900 \\
\hline & & & & & 1800 & 8.84 & 1,260 & & & & \\
\hline \multirow[t]{13}{*}{ Mar. 1} & 0200 & 9.54 & 1,760 & & 2400 & 9.00 & 1,370 & 9 & 0600 & 23.32 & 18,100 \\
\hline & 0400 & 11.12 & 2,950 & & & & & & 1200 & 23.01 & 17,600 \\
\hline & 0600 & 13.46 & 4,860 & 2 & 1000 & 8.73 & 1,790 & & 1800 & 22.22 & 16,200 \\
\hline & 0800 & 15.60 & 7,060 & & 1600 & 9.64 & 1,810 & & 2400 & 20.24 & 13,200 \\
\hline & 1000 & 17.35 & 9,260 & & 2000 & 10.52 & 2,460 & & & & \\
\hline & 1200 & 18.85 & 11,200 & & 2400 & 10.15 & 2,190 & 10 & 0600 & 18.42 & 10,600 \\
\hline & 1400 & 21.20 & 14,600 & & & & & & 1200 & 17.63 & 9,620 \\
\hline & 1600 & 23.32 & 18,100 & 3 & 0200 & 9.92 & 2,020 & & 1800 & 17.72 & 9,740 \\
\hline & 1800 & 24.50 & 20,600 & & 0800 & 11.06 & 2,890 & & 2300 & 17.96 & 10,000 \\
\hline & 2000 & 25.35 & 22,400 & & 1600 & 11.89 & 3,550 & & 2400 & 17.92 & 10,000 \\
\hline & 2200 & 26.17 & 24,200 & & 2000 & 13.31 & 4,720 & & & & \\
\hline & 2400 & 27.18 & 26,600 & & 2400 & 15.06 & 6,440 & 11 & 0300 & 17.79 & 9,830 \\
\hline & & & & & & & & & 0600 & 17.26 & 9,140 \\
\hline \multirow[t]{11}{*}{2} & 0200 & 27.75 & 28,000 & 4 & 0400 & 16.11 & 7,670 & & 1200 & 16.59 & 8,270 \\
\hline & 0500 & 28.40 & 29,600 & & 0800 & 16.77 & 8,490 & & 1900 & 15.99 & 7,530 \\
\hline & 0900 & 27.84 & 28,200 & & 1400 & 17.71 & 9,710 & & 2000 & 15.98 & 7,520 \\
\hline & 1200 & 26.87 & 25,800 & & 1800 & 19.89 & 12,700 & & 2400 & 16.51 & 8,160 \\
\hline & 1400 & 25.85 & 23,500 & & 2000 & 20.94 & 14,200 & & & & \\
\hline & 1600 & 24.50 & 20,600 & & 2400 & 21.90 & 15,700 & 12 & 0.400 & 15.41 & 6,840 \\
\hline & 1800 & 23.00 & 17,600 & & & & & & 1100 & 14.17 & 5,530 \\
\hline & 2000 & 21.40 & 14,900 & 5 & 0600 & 22.39 & 16,500 & & 1500 & 13.99 & 5,350 \\
\hline & 2200 & 19.82 & 12,600 & & 1000 & 22.79 & 17,200 & & 1800 & 13.54 & 4,940 \\
\hline & 2400 & 18.00 & 10,100 & & 1600 & 23.95 & 19,400 & & 2400 & 12.62 & 4,390 \\
\hline & & & & & 2200 & 24.48 & 20,500 & & & & \\
\hline \multirow[t]{12}{*}{3} & 0200 & 16.68 & 8,380 & & 2400 & 24.37 & 20,300 & 13 & 0500 & 11.83 & 3,510 \\
\hline & 0400 & 15.35 & 6,780 & & & & & & 1200 & 11.14 & 2,960 \\
\hline & 0600 & 14.00 & 5,360 & 6 & 0600 & 23.90 & 19,300 & & 1800 & 10.79 & 2,680 \\
\hline & 0800 & 12.35 & 3,930 & & 1200 & 24.36 & 20,300 & & 2400 & 10.42 & 2,400 \\
\hline & 1000 & 11.23 & 3,030 & & 1800 & 25.94 & 23,700 & & & & \\
\hline & 1200 & 10.98 & 2,830 & & 2400 & 27.13 & 26,400 & 14 & 1200 & 9.93 & 2,040 \\
\hline & 1800 & 10.37 & 2,370 & & & & & & 2400 & 9.54 & 1,760 \\
\hline & 2400 & 9.78 & 1,930 & 7 & 0200 & 27.26 & 26,800 & & & & \\
\hline & & & & & 0600 & 26.77 & 25,600 & 15 & 1200 & 9.36 & 1,630 \\
\hline & 0600 & 9.50 & 1,730 & & 1200 & 25.00 & 21,600 & & 2400 & 9.17 & 1,500 \\
\hline & 1200 & 9.20 & 1,520 & & 1800 & 25.19 & 22,000 & & & & \\
\hline & 1800 & 8.98 & 1,370 & & 2400 & 24.17 & 19,900 & & & & \\
\hline
\end{tabular}




\section{WHITEWATER RIVER BASIN}

(134) 5-3765. South Fork Whitewater River near Altura, Minn.

Location.-Lat $44^{\circ} 04^{\prime} 10^{\prime \prime}$, long $91^{\circ} 58^{\prime} 49^{\prime \prime}$, in $\mathrm{SE} \frac{1}{4}$ sec.14, T.107 N., R.10 W., on left bank $500 \mathrm{ft}$ upstream from highway bridge, 1.4 miles upstream from small tributary entering from the west, 2 miles west of Altura, and 2.4 miles upstream from Keefer Creek.

Drainage area. $-76.8 \mathrm{sq} \mathrm{mi}$.

Gage-height record.-Water-stage recorder graph except 1930 hours Feb. 28 to 0500 hours Mar. 2 when graph was constructed on the basis of floodmarks and adjazent record. Datum of gage is $761.80 \mathrm{ft}$ above mean sea level, adjustment of 1912 (levels by Corps of Engineers).

Discharge record.-Stage-discharge relation defined by current-meter measure ments. Backwater from ice Feb. 28, Mar. 1, 12, 17-24.

Maxima.-Given in following table.

March-May 1965:

Discharge

(cfs)

1,300

2,360

5,460
Gage height

(feet)

a 8.14

7.23

10.61

1939 to February 1965 :

Aug. 31, 1947

aBackwater from ice.

Mean discharge, in cubic feet per second, 1965

\begin{tabular}{|c|c|c|c|c|c|c|c|c|c|c|c|}
\hline Day & March & April & May & Day & March & April & May & Day & March & April & May \\
\hline 1. & 700 & 217 & 15 & $11 \ldots$ & 11 & 298 & 18 & 21 & 10 & 17 & 14 \\
\hline 2. & 274 & 385 & 14 & $12_{--}$ & 10 & 290 & 17 & 22. & 10 & 16 & 13 \\
\hline 3. & 32 & 392 & 15 & $13=$ & 12 & 54 & 16 & 23. & 10 & 16 & 14 \\
\hline 4. & 22 & 994 & 14 & $14_{--}$ & 12 & 38 & 14 & 24 & 11 & 16 & 14 \\
\hline 5. & 18 & 968 & 16 & 15. & 11 & 32 & 14 & 25. & 11 & 19 & 14 \\
\hline 6. & 15 & 1,500 & 34 & $16_{--}$ & 11 & 26 & 22 & 26. & 11 & 29 & 17 \\
\hline 7. & 15 & 1,100 & 32 & $17_{-}$ & 11 & 23 & 21 & 27. & 11 & 27 & 19 \\
\hline 8. & 12 & 1,140 & 24 & $18=-$ & 10 & 22 & 18 & 28 & 10 & 21 & 16 \\
\hline 9. & 12 & 492 & 22 & 19. & 10 & 21 & 16 & $29_{2}$ & 11 & 19 & 14 \\
\hline 10 & 11 & 228 & 19 & $20--$ & 10 & 18 & 14 & $30_{-}$ & 11 & 17 & 14 \\
\hline & & & & & & & & & 13 & $-\infty--\infty$ & 14 \\
\hline \multirow{2}{*}{\multicolumn{9}{|c|}{$\begin{array}{l}\text { Monthly mean discharge, in cubic feet per second } \\
\text { Runoff, in inches }\end{array}$}} & 43.2 & 281 & 17.4 \\
\hline & & & & & & & & & 0.65 & 4.08 & 0.26 \\
\hline
\end{tabular}

Gage height, in feet, and discharge, in cubic feet per second, at indicated time, 1965

\begin{tabular}{|c|c|c|c|c|c|c|c|c|c|c|c|}
\hline Date & Hour & $\begin{array}{c}\text { Gage } \\
\text { height }\end{array}$ & $\begin{array}{c}\text { Dis - } \\
\text { charge }\end{array}$ & Date & Hour & $\begin{array}{l}\text { Gage } \\
\text { height }\end{array}$ & $\begin{array}{c}\text { Dis- } \\
\text { charge }\end{array}$ & Date & Hour & $\begin{array}{c}\text { Gage } \\
\text { height. }\end{array}$ & $\begin{array}{c}\text { Dis- } \\
\text { charge }\end{array}$ \\
\hline \multirow[t]{9}{*}{ Feb. 28} & 0000 & 1.21 & & \multirow[t]{10}{*}{ Mar. 1} & 1200 & 8.09 & $1-$ & \multirow[t]{4}{*}{ Mar. 2} & 1700 & 1.74 & 120 \\
\hline & 0600 & 1.16 & - & & 1300 & 8.14 & 1,300 & & 2000 & 1.48 & 86 \\
\hline & 1200 & 1.12 & 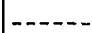 & & 1400 & 8.13 & $\ldots-\cdots$ & & 2400 & 1.21 & 59 \\
\hline & 00 & 1.16 & & & 1800 & 6.88 & 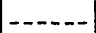 & & & & \\
\hline & 1700 & 2.31 &.- & & 2400 & 4.70 & 820 & \multirow[t]{6}{*}{ Apr. 1} & 0000 & .76 & 25 \\
\hline & 1900 & 2.72 & & & & & & & 0200 & 1.02 & 43 \\
\hline & 2100 & 3.85 & $\ldots$ & & 0400 & 3.61 & 565 & & 0300 & .98 & 40 \\
\hline & 2400 & 4.65 & $\ldots$ & & 0600 & 3.11 & 411 & & 0400 & 1.12 & 51 \\
\hline & & & & & 0900 & 2.38 & 233 & & 0500 & 1.20 & 58 \\
\hline Mar. & 0 & 6.65 & & & 1400 & 1.79 & 128 & & 0600 & 1.70 & 114 \\
\hline
\end{tabular}


Gage height, in feet, and discharge, in cubic feet per second, at indicated time, 1965, of South Fork Whit'wat'r Rwer near Altura, Minn. - Continued

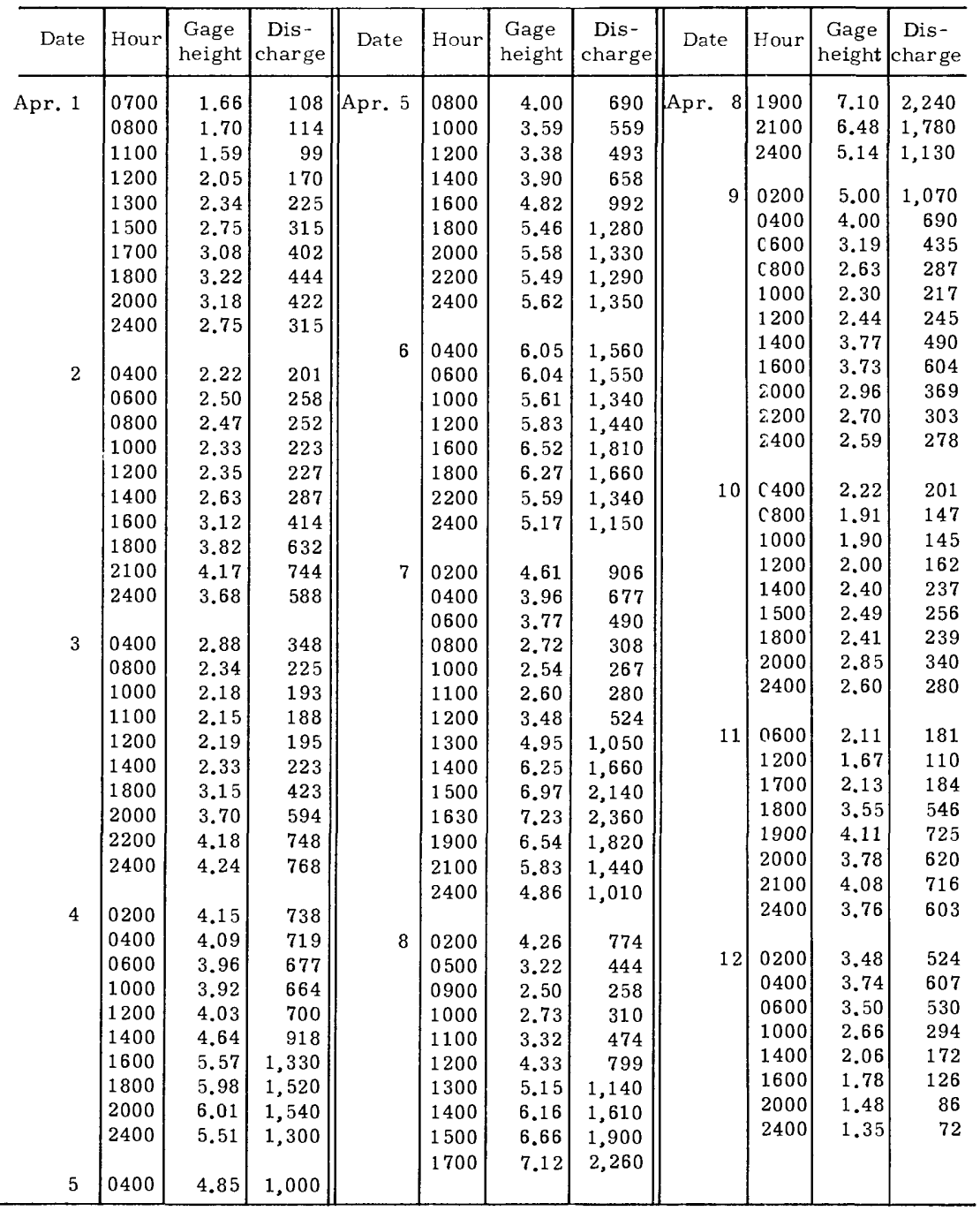




\section{MISSISSIPPI RIVER MAIN STEM}

(135) Mississippi River at Lock and Dam 5 near Minneiska, Minn.

\section{(Miscellaneous site)}

Location.-Lat $44^{\circ} 10^{\prime}$, long $91^{\circ} 50^{\prime}$, in sec.7, T.108 N., R.8 W., at Lock and Dam 5, $3 \frac{1}{2}$ miles southeast of Minneiska, and at river mile 738.27 above Ohio River.

Gage-height record.-Peak stages from pool and tailwater water-stage recorder graphs. Datum of gages is $600.00 \mathrm{ft}$ above mean sea level, adjustment of 1912 .

Discharge record.-Corps of Engineers stage-discharge relation extended on the basis of two Geological Survey current-meter measurements.

Maxima.-April-May 1965: Discharge, 263,000 cfs 1600 hours Apr. 19 (elevations, $668.73 \mathrm{ft}$, pool, $667.85 \mathrm{ft}$ tailwater).

1935 to March 1965: Discharge, 190,500 efs Apr. 20, 1952 (elevations, $664.59 \mathrm{ft}$ pool, $663.84 \mathrm{ft}$ tailwater).

Cooperation.-Records furnished by Corps of Engineers.

\section{WAUMANDEE CREEK BASIN}

(136) 5-3782. Eagle Creek near Fountain City, Wis.

(Crest-stage station)

Location.-Lat $44^{\circ} 09^{\prime} 49^{\prime \prime}$, long $91^{\circ} 42^{\prime} 28^{\prime \prime}$, in SW $\frac{1}{4}$ sec. 33, T.20 N., R.11 W., at britge on County Trunk G, 2.5 miles north of Fountain City.

Drainage area.-26.8 sq mi.

Gage-height record.-Crest stages only.

Discharge record.- Stage-discharge relation defined by current-meter measurements below $431 \mathrm{cfs}$ and by contracted-opening measurement at $930 \mathrm{cfs}$.

Maxima.-March-May 1965: Discharge, 1,060 cfs Apr, 7 (gage height, $14.82 \mathrm{ft}$ ). 1961 to February 1965: Discharge, $930 \mathrm{cfs}$ Mar. 25, 1961 (gage height, $14.32 \mathrm{ft}$ ). 
(137) 5-3785. Mississippi River at Winona, Minn.

Location.-Lat $44^{\circ} 03^{\prime} 20^{\prime \prime}$, long $91^{\circ} 38^{\prime} 15^{\prime \prime}$, in sec. 23 , T.107 N., R. 7 W., on right bank at Winona pumping station in Winona, $9 \frac{1}{2}$ miles upstream from Trempealeau River and at mile 725.7 upstream from the Ohio River. Auxiliary water-stage recorder at navigation dam $5 \mathrm{~A}$ in sec.9, T.107 N., R.7 W., 2.7 miles upstream.

Drainage area. $-59,200 \mathrm{sq}$ mi. approximately.

Gage-height record.-Water-stage recorder graph except 1200 hours Apr. 15 to 1600 hours Apr. 28 when graph was constructed on basis of several gage readings by engineers and twice-daily gage readings by Corps of Engineers on temporary staff gage and floodmark.

For auxiliary gage, water-stage recorder graph.

Datum of base gage is $639.64 \mathrm{ft}$ above mean sea level, datum of 1929 , and for the auxiliary gage, $600.00 \mathrm{ft}$ above mean sea level, datum of 1912 .

Discharge record.-Stage-fall-discharge relation affected by changes in slope and defined by current-meter measurements. Fall was a factor in computing discharge Apr. 4, 5 . Backwater from ice Apr. 1-4.

Maxima.-April-May 1965: Discharge, 268,000 cfs 2400 hours Apr. 19 (gage height, $20.77 \mathrm{ft}$, from floodmark).

1880 to March 1965: Discharge, 190,000 cfs Apr. 20, 1952 (gage height, 17.91).

Remarks.- - Some regulation by reservoirs, navigation dams, and powerplants at low and medium stages. Flood flow not materially affected by artificial storage.

Cooperation.-Gage height record at dam 5A furnished by Corps of Ergineers.

Mean discharge, in cubic feet per second, 1965

\begin{tabular}{|c|c|c|c|c|c|c|c|c|}
\hline Day & April & May & Day & April & May & Day & April & May \\
\hline & 14,400 & 154,000 & 11 & 112,000 & 87,600 & $21 \ldots \ldots$ & 257,000 & 93,200 \\
\hline & 21,300 & 144,000 & $12 \ldots$ & 121,000 & 91,100 & $22 \ldots$ & 250,000 & 89,600 \\
\hline & 27,200 & 135,000 & 13 & 131,000 & 93,700 & $23 \ldots \ldots$ & 238,000 & 84,300 \\
\hline & 35,600 & 124,000 & 14 & 150,000 & 94,800 & $24 \ldots$ & 227,000 & 79,600 \\
\hline & 53,200 & 115,000 & 15 & 178,000 & 94,200 & $25 \ldots$ & 216,000 & 75,700 \\
\hline & 66,800 & 107,000 & 16 & 210,000 & 92,500 & $26 \ldots$ & 205,000 & 74,300 \\
\hline & 77,300 & 99,800 & $17 \ldots$ & 215,000 & 89,200 & $27 \ldots \ldots$ & 195,000 & 73,300 \\
\hline & 87,600 & 94,900 & $18 \ldots$ & 238,000 & 89,200 & $28 \ldots$ & 184,000 & 71,800 \\
\hline & 98,400 & 91,800 & 19. & 263,000 & 91,200 & $29 \ldots$ & 173,000 & 69,900 \\
\hline \multirow[t]{2}{*}{$10 \ldots$} & 104,000 & 88,700 & 20 & 264,000 & 93,200 & $30 \ldots$ & 164,000 & 69,500 \\
\hline & & & & & & $31 \ldots$ & $---1--$ & 69,900 \\
\hline \multicolumn{7}{|c|}{ Monthly mean discharge, in cubic feet per second } & 152,600 & 94,260 \\
\hline \multicolumn{7}{|c|}{ Runoff, in inches } & 2.88 & 1.84 \\
\hline
\end{tabular}


Gage height. in feet, and discharge, in cubic feet per second, at indicated time, 1965, of Mississippi River at

\begin{tabular}{|c|c|c|c|c|c|c|c|c|c|c|c|}
\hline Date & Hour & $\begin{array}{l}\text { Gage } \\
\text { height }\end{array}$ & $\begin{array}{c}\text { Dis- } \\
\text { charge }\end{array}$ & Date & Hour & $\begin{array}{l}\text { Gage } \\
\text { height }\end{array}$ & $\begin{array}{c}\text { Dis- } \\
\text { charge }\end{array}$ & Date & Hour & $\begin{array}{c}\text { Gage } \\
\text { height }\end{array}$ & $\begin{array}{c}\text { Dis- } \\
\text { charge }\end{array}$ \\
\hline \multirow[t]{4}{*}{ Apr. 14} & 0000 & 15.31 & 138,000 & \multirow[t]{3}{*}{ Apr. 17} & 1800 & 19.20 & 212,000 & \multirow[t]{3}{*}{ Apr. 20} & 1800 & 20.62 & 262,000 \\
\hline & 1200 & 15.95 & 149,000 & & 2400 & 19.47 & 219,000 & & 2400 & 20.57 & 261,000 \\
\hline & 2400 & 16.72 & 163,000 & & & & & & & & \\
\hline & & & & \multirow[t]{4}{*}{18} & 0600 & 19.82 & 229,000 & \multirow[t]{3}{*}{21} & 1200 & 20.42 & 257,000 \\
\hline \multirow[t]{3}{*}{15} & 1200 & 17.62 & $178,000 \mid$ & & 1200 & 20.12 & 239,000 & & 2400 & 20.30 & 254,000 \\
\hline & 2400 & 18.41 & 194,000 & & 1800 & 20.33 & 247,000 & & & & \\
\hline & & & & & 2400 & 20.48 & 254,000 & 22 & 1200 & 20.19 & 251,000 \\
\hline \multirow[t]{5}{*}{16} & 0600 & 18.85 & 204,000 & \multirow{5}{*}{19} & & & & \multirow{5}{*}{23} & 2400 & 19.84 & 242,000 \\
\hline & 1200 & 19.14 & 210,000 & & 0600 & 20.60 & 260,000 & & & & \\
\hline & 1800 & 19.39 & 217,000 & & 1200 & 20.67 & 264,000 & & 1200 & 19.68 & 238,000 \\
\hline & 2400 & 19.76 & 227,000 & & 1800 & 20.70 & 265,000 & & 2400 & 19.44 & 232,000 \\
\hline & & & & & 2400 & 20.77 & 268,000 & & & & \\
\hline \multirow[t]{3}{*}{17} & 0200 & 19.81 & 228,000 & \multirow{3}{*}{20} & & & & \multirow[t]{3}{*}{24} & 1200 & 19.20 & 227,000 \\
\hline & 0600 & 19.24 & 213,000 & & 0600 & 20.70 & 265,000 & & 2400 & 18.92 & 221,000 \\
\hline & 1200 & 19.10 & 210,000 & & 1200 & 20.67 & 264,000 & & & & \\
\hline
\end{tabular}

\section{GILMORE CREEK BASIN}

(138) 5-3790. Gilmore Creek at Winona, Minn.

(Crest-stage station)

Location.--Lat $44^{\circ} 02^{\prime} 40^{\prime \prime}$, long $91^{\circ} 41^{\prime} 25^{\prime \prime}, \mathrm{N} \frac{1}{2}$ sec.29, T.107 N., R.7 W., on left bank at west edge of Winona, $1,500 \mathrm{ft}$ upstream from bridge on U.S. Highway 14 , and $2 \frac{1}{4}$ miles upstream from Lake Winona.

Drainage area. $-8.95 \mathrm{sq} \mathrm{mi}$.

Gage-height record.-Crest stages only.

Discharge record.--Stage-discharge relation defined by current-meter measurements below $380 \mathrm{cfs}$.

Maxima.-March-May 1965: Discharge, 436 cfs Apr. 7 (gage height, $4.65 \mathrm{ft}$, bac'rwater from debris).

1939 to February 1965: Discharge, 5,360 cfs July 21, 1951 (gage height, 9.47 ft), from rating curve extended above $260 \mathrm{cfs}$ on basis of slope-area measurement at $2,200 \mathrm{cfs}$ and logarithmic plotting.

\section{TREMPEALEAU RIVER BASIN}

(139) 5-3794. Trempealeau River at Arcadia, Wis.

Location.-Lat $44^{\circ} 15^{\prime} 15^{\prime \prime}$, long $91^{\circ} 30^{\prime} 25^{\prime \prime}$, in SW $\frac{1}{4}$ sec. 32 , T.21 N., R.9 W., near rirht bank on downstream side of bridge on State Highways 93 and 95 in Arcadia, half a mile downstream from Turton Creek.

Drainage area. $-552 \mathrm{sq} \mathrm{mi}$.

Gage-height record.-Wire-weight gage read twice daily. Graph drawn on basis of numerous wire-weight gage readings daily during rises. Datum of gage is $719.61 \mathrm{ft}$ above mean sea level, datum of 1929 .

Discharge record.- Stage-discharge relation defined by current-meter measurements below 7,200 cfs and extended by logarithmic plotting. Backwater from ice Mar. 1 to Apr. 5.

Maxima.-March-May 1965: Discharge, 9,740 cfs at 2200 hours Apr. 6 (gage height, $7.15 \mathrm{ft}$ ); gage height, $8.04 \mathrm{ft} 1600$ hours Mar. 2 (backwater from ice).

1960 to February 1965: Discharge, 7,840 cfs Mar. 26, 1961 (gage height, 6. $85 \mathrm{ft}$ ). 
Mean discharge, in cubic feet per second, 1965, of Trempealeau Rner at Arcadia, Wis.

\begin{tabular}{|c|c|c|c|c|c|c|c|c|c|c|c|}
\hline Day & March & April & May & Day & March & April & May & Day & March & April & May \\
\hline 1 & 700 & 270 & 384 & $11 \ldots$ & 1,000 & 2,850 & 299 & 21 & 280 & 513 & 247 \\
\hline 2 & 2,000 & 500 & 375 & $12_{-}$ & 780 & 2,860 & 285 & 22 & 270 & 484 & 238 \\
\hline 3 & 3,000 & 800 & 372 & $13 \ldots$ & 660 & 1,860 & 272 & 23. & 260 & 477 & 238 \\
\hline 4. & 2,000 & 1,800 & 378 & $14_{-}$ & 560 & 1,240 & 264 & 24 & 250 & 474 & 234 \\
\hline 5. & 1,600 & 4,000 & 375 & $15 \ldots$ & 470 & 888 & 252 & 25 & 240 & 536 & 234 \\
\hline 6. & 1,400 & 7,400 & 494 & $16 \ldots$ & 430 & 724 & 272 & 26 & 240 & 968 & 339 \\
\hline 7 & 1,800 & 8,010 & 399 & $17 \ldots$ & 390 & 662 & 272 & 27 & 240 & 830 & 436 \\
\hline 8 & 2,300 & 6,470 & 351 & $18=$ & 350 & 696 & 321 & 28. & 240 & 675 & 412 \\
\hline 9 & 1,900 & 3,990 & 327 & $19_{-}$ & 310 & 635 & 304 & 29. & 230 & 510 & 321 \\
\hline 10 & 1,250 & 3,030 & 316 & $20 \ldots$ & 290 & 560 & 272 & & 230 & 436 & 285 \\
\hline & & & & & & & & 31 & 240 & $\ldots$ & 267 \\
\hline \multirow{2}{*}{\multicolumn{9}{|c|}{$\begin{array}{l}\text { Monthly mean discharge, in cubic feet per second } \\
\text { Runoff, in inches }\end{array}$}} & 836 & 1,839 & 317 \\
\hline & & & & & & & & & 1.75 & 3.72 & 0.66 \\
\hline
\end{tabular}

Gage height, in feet, and discharge, in cubic feet per second, at indicated time, 1965

\begin{tabular}{|c|c|c|c|c|c|c|c|c|c|c|c|}
\hline Date & Hour & $\begin{array}{c}\text { Gage } \\
\text { height }\end{array}$ & $\begin{array}{c}\text { Dis- } \\
\text { charge }\end{array}$ & Date & Hour & $\begin{array}{l}\text { Gage } \\
\text { height }\end{array}$ & $\begin{array}{c}\text { Dis- } \\
\text { charge }\end{array}$ & Date & Hour & $\begin{array}{c}\text { Gage } \\
\text { height }\end{array}$ & $\begin{array}{l}\text { Dis- } \\
\text { charge }\end{array}$ \\
\hline \multirow[t]{9}{*}{ Mar. I } & 0000 & 2.60 & -...- & \multirow{8}{*}{ Apr. 4} & 0200 & 5.10 & $\ldots$ & \multirow[t]{4}{*}{ Apr. 8} & 1600 & 6.48 & 6,500 \\
\hline & 0400 & 3.60 & $\ldots$ & & 0600 & 5.34 & $\ldots$ & & 2000 & 6.18 & 5,410 \\
\hline & 0800 & 4.40 & $\ldots$ & & 1000 & 5.38 & $\ldots$ & & 2400 & 5.95 & 4,700 \\
\hline & 1200 & 5.15 & $\ldots$ & & 1400 & 5.41 & $\ldots$ & & & & \\
\hline & 1600 & 5.85 & $-\cdots$ & & 1600 & 5.68 & $\ldots-$ & \multirow[t]{4}{*}{ Apr. 9} & 0600 & 5.70 & 4,040 \\
\hline & 1800 & 6.35 & $\ldots$ & & 2000 & 5.82 & $\ldots-$ & & 1800 & 5.62 & 3,850 \\
\hline & 2000 & 6.70 & $\ldots$ & & 2400 & 5.81 & $\ldots-$ & & 2400 & 5.50 & 3,580 \\
\hline & 2400 & 6.85 & & & & & & & & & \\
\hline & & & & \multirow[t]{4}{*}{ Apr. 5} & 0800 & 5.78 & $\ldots-$ & \multirow[t]{4}{*}{ Apr. 10} & 0600 & 5.35 & 3,290 \\
\hline \multirow[t]{8}{*}{ Mar. 2} & 0400 & 6.75 & - & & 1600 & 5.93 & $\ldots \ldots$ & & 1200 & 5.18 & 3,010 \\
\hline & 0800 & 6.70 & $\ldots$ & & 2400 & 6.03 & 4,940 & & 1800 & 4.90 & 2,620 \\
\hline & 1200 & 6.95 & $\ldots$ & & & & & & 2400 & 5.05 & 2,820 \\
\hline & 1600 & 8.04 & $\ldots$ & \multirow[t]{7}{*}{ Apr. 6} & 0400 & 6.14 & 5,280 & \multirow{5}{*}{ Apr. 11} & 0600 & 4.96 & 2,700 \\
\hline & 1700 & 6.90 & $\ldots$ & & 0800 & 6.52 & 6,670 & & 1200 & 4.85 & 2,560 \\
\hline & 2000 & 6.60 & $\ldots$. & & 1200 & 6.75 & 7,700 & & 1800 & 5.15 & 2,960 \\
\hline & 2400 & 6.40 & $\ldots$. & & 1600 & 6.83 & 8,090 & & 2200 & 5.42 & 3,420 \\
\hline & & & & & 2000 & 7.10 & 9,470 & & 2400 & 5.32 & 3,240 \\
\hline \multirow[t]{2}{*}{ Mar. 3} & 1200 & 5.90 & $\ldots$ & & 2200 & 7.15 & 9,740 & \multirow{4}{*}{ Apr. 12} & 0600 & 510 & 890 \\
\hline & 2400 & 5.50 & $\ldots$ & & 2400 & 7.12 & 9,580 & & 1200 & 5.00 & $\begin{array}{l}2,890 \\
2,750\end{array}$ \\
\hline \multirow[t]{8}{*}{ Apr. 3} & 0000 & 3.20 & $\ldots$ & Apr. 7 & 0400 & 6.95 & 8,700 & & 1800 & 5.12 & 2,920 \\
\hline & 0200 & 3.60 & $\ldots$. & & 1000 & 6.56 & 6,840 & & 2400 & 4.80 & 2,500 \\
\hline & 0600 & 3.99 & $\ldots$ & & 1600 & 6.75 & 7,700 & \multirow[t]{3}{*}{ Apr. 13} & 1200 & 3.95 & 1,760 \\
\hline & 1200 & 3.80 & & & 2200 & 6.93 & 8,600 & & 2400 & 3.42 & 1,430 \\
\hline & 1400 & 3.76 & & & 2400 & 6.80 & 7,940 & & & & \\
\hline & 1800 & 4.13 & & & & & & Apr. 14 & 1200 & 3.08 & 1,250 \\
\hline & 2200 & 4.52 & $\ldots-\ldots$ & Apr. 8 & 0600 & 6.65 & 7,240 & & 2400 & 2.64 & 1,040 \\
\hline & 2400 & 4.80 & $\ldots$. & & 1200 & 6.40 & 6,180 & & & & \\
\hline
\end{tabular}


(140) 5-3795. Trempealeau River at Dodge, Wis.

Location.--Lat $44^{\circ} 07^{\prime} 55^{\prime \prime}$, long $91^{\circ} 33^{\prime} 10^{\prime \prime}$, in sec.10, T.19 N., R.10 W, near left bank on downstream side of highway bridge in Dodge, 9 miles upstream from mouth.

Drainage area. $-643 \mathrm{sq} \mathrm{mi}$.

Gage-height record.-Wire-weight gage read twice daily. Graph drawn on basis of wire-weight gage readings and supplemented by crest gage readings during rises. Datum of gage is $663.42 \mathrm{ft}$ above mean sea level, datum of 1929 .

Discharge record.-Stage-discharge relation defined by current-meter measur ments below $15,000 \mathrm{cfs}$. Backwater from ice Mar. 1 to Apr. 6.

Maxima.-March to May 1965: Discharge, 12,100 cfs 2200 hours Apr. 7 (gage height, $9.40 \mathrm{ft}$ ).

1913-19, 1934 to February 1965: Discharge, 17,400 cfs Apr. 4, 1956 (gage height, $10.35 \mathrm{ft})$.

Mean discharge, in cubic feet per second, 1965

\begin{tabular}{|c|c|c|c|c|c|c|c|c|c|c|c|}
\hline Day & March & April & May & Day & March & April & May & Day & March & April & May \\
\hline & 600 & 260 & 520 & $11 \ldots$ & 1,400 & 3,670 & 388 & 21. & 290 & 626 & 327 \\
\hline & 900 & 400 & 489 & $12 \ldots$ & 1,000 & 3,460 & 361 & 22 & 280 & 595 & 306 \\
\hline & 1,800 & 600 & 487 & $13--$ & 800 & 2,840 & 346 & $23 \ldots$ & 270 & 564 & 293 \\
\hline & 4,000 & 1,000 & 505 & $14_{--}$ & 600 & 1,940 & 331 & $24_{-}$ & 260 & 583 & 285 \\
\hline 5. & 2,400 & 2,000 & 505 & $15 \ldots$ & 500 & 1,300 & 325 & $25 \ldots$ & 260 & 660 & 278 \\
\hline 6 & 1,800 & 5,000 & 549 & $16 \ldots$ & 450 & 957 & 357 & $26 \ldots$ & 250 & 1,010 & 361 \\
\hline 7. & 1,600 & 9,970 & 559 & $17--$ & 400 & 796 & 356 & $27 \ldots$ & 250 & 1,030 & 507 \\
\hline 8. & 1,500 & 9,930 & 485 & $18 \ldots$ & 360 & 820 & 400 & $28 \ldots$ & 250 & 826 & 498 \\
\hline & 2,700 & 6,470 & 428 & $19_{--}$ & 330 & 809 & 404 & 29. & 240 & 675 & 458 \\
\hline & 2,400 & 4,280 & 400 & $20 \ldots$ & 310 & 743 & 359 & $30 \ldots$ & 230 & 571 & 361 \\
\hline & & & & & & & & & 240 & -.... & 323 \\
\hline \multirow{2}{*}{\multicolumn{9}{|c|}{ Monthly mean discharge, in cubic feet per second }} & 925 & 2,146 & 405 \\
\hline \multicolumn{4}{|c|}{ Runoff, in inches } & & & & & & 1.66 & 3.72 & 0.73 \\
\hline
\end{tabular}

Gage height, in feet, and discharge, in cubic feet per second, at indicated time, 1965

\begin{tabular}{|c|c|c|c|c|c|c|c|c|c|c|c|}
\hline Date & Hour & $\begin{array}{c}\text { Gage } \\
\text { height }\end{array}$ & $\begin{array}{c}\text { Dis - } \\
\text { charge }\end{array}$ & Date & Hour & $\begin{array}{c}\text { Gage } \\
\text { height }\end{array}$ & $\begin{array}{c}\text { Dis- } \\
\text { charge }\end{array}$ & Date & Hour & $\begin{array}{c}\text { Gage } \\
\text { height }\end{array}$ & $\begin{array}{c}\text { Dis- } \\
\text { charge }\end{array}$ \\
\hline ar. 1 & $\begin{array}{l}0000 \\
0600 \\
1200 \\
1800 \\
2400 \\
\\
0600 \\
1200 \\
1800 \\
2400 \\
0600 \\
1200 \\
1600 \\
1800 \\
2200\end{array}$ & $\begin{array}{l}1.80 \\
2.20 \\
3.10 \\
4.10 \\
4.32 \\
4.28 \\
4.28 \\
4.40 \\
4.70 \\
\\
5.10 \\
6.00 \\
7.10 \\
7.70 \\
8.10\end{array}$ & 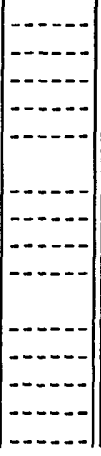 & Mar. 3 & $\begin{array}{l}2400 \\
0600 \\
1200 \\
1800 \\
2400 \\
0600 \\
1600 \\
2000 \\
2400 \\
0600 \\
0800 \\
1800 \\
2400\end{array}$ & $\begin{array}{l}8.15 \\
8.10 \\
8.00 \\
7.80 \\
7.15 \\
6.85 \\
7.25 \\
7.20 \\
6.80 \\
6.30 \\
6.25 \\
6.50 \\
5.70\end{array}$ & 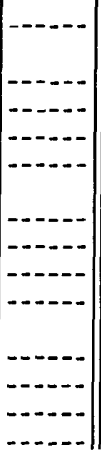 & Mar. 7 & $\begin{array}{l}0600 \\
1600 \\
2400 \\
0600 \\
2400 \\
\\
1200 \\
1600 \\
1800 \\
2400 \\
\\
0600 \\
1200 \\
1600 \\
2400\end{array}$ & $\begin{array}{l}5.30 \\
5.80 \\
5.25 \\
5.10 \\
6.03 \\
6.95 \\
7.23 \\
7.23 \\
6.84 \\
6.3 ? \\
6.63 \\
6.83 \\
6.2 ?\end{array}$ & 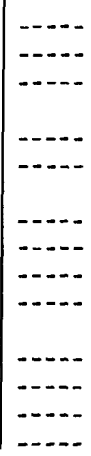 \\
\hline
\end{tabular}


Gage height, in feet, and discharge, in cubic feet per second, at indicated time, 1965. of Trempealeau River at

\begin{tabular}{|c|c|c|c|c|c|c|c|c|c|c|c|}
\hline Date & Hour & $\begin{array}{l}\text { Gage } \\
\text { height }\end{array}$ & $\begin{array}{c}\text { Dis- } \\
\text { charge }\end{array}$ & Date & Hour & $\begin{array}{c}\text { Gage } \\
\text { height }\end{array}$ & $\mid \begin{array}{c}\text { Dis- } \\
\text { charge }\end{array}$ & Date & Ho'sr & $\begin{array}{c}\text { Gage } \\
\text { height }\end{array}$ & $\begin{array}{c}\text { Dis - } \\
\text { charge }\end{array}$ \\
\hline \multirow[t]{4}{*}{ Mar. 11} & $\begin{array}{l}0600 \\
1200\end{array}$ & $\begin{array}{l}5.55 \\
5.30\end{array}$ & $\cdots$ & Apr. 5 & 1800 & 7.42 & $\ldots$ & Apr. 9 & 2490 & 7.82 & 4,910 \\
\hline & 1800 & 5.10 & & 6 & 2400 & 8.45 & 7,480 & 10 & 0600 & 7.70 & 4,570 \\
\hline & 2400 & 4.95 & & & & & & & 1200 & 7.60 & 4,330 \\
\hline & & & & 7 & 0600 & 8.80 & 9,100 & & 1800 & 7.36 & 3,880 \\
\hline \multirow[t]{5}{*}{ Apr. 3} & 0000 & 2.98 & $-n$ & & 1200 & 8.92 & 9,700 & & 2400 & 7.28 & 3,750 \\
\hline & 0600 & 3.03 & & & 1800 & 9.20 & 11,100 & 11 & 1200 & 7.20 & 3,640 \\
\hline & 1200 & 3.38 & $\ldots$ & & 2200 & 9.40 & 12,100 & & 2400 & 7.20 & 3,640 \\
\hline & $\begin{array}{l}1800 \\
2400\end{array}$ & $\begin{array}{l}3.95 \\
4.34\end{array}$ & & & 2400 & 9.38 & 12,000 & .12 & 1200 & 7.08 & 3,490 \\
\hline & 2400 & 4.34 & & 8 & 0600 & 9.00 & 10,100 & & 2400 & 6.86 & 3,230 \\
\hline \multirow[t]{4}{*}{4} & 0600 & 4.70 & $-\ldots$. & & 1200 & 8.90 & 9,600 & 13 & 1200 & & \\
\hline & 1200 & 4.95 & $\ldots$ & & 1800 & 8.92 & 9,700 & 13. & 12400 & 6.50 & 2,850 \\
\hline & 1800 & 5.28 & & & 2400 & 8.70 & 8,620 & & & & 2,420 \\
\hline & 2400 & 5.73 & $\ldots$ & 9 & 0600 & 8.42 & 7,350 & 14 & 1200 & 5.26 & 1,900 \\
\hline \multirow[t]{2}{*}{5} & 0600 & 6.31 & & & 1200 & 8.18 & 6,290 & & 2400 & 4.60 & 1,530 \\
\hline & 1200 & 6.92 & $\ldots$ & & 1800 & 7.98 & 5,470 & & & & \\
\hline
\end{tabular}

BLACK RIVER BASIN

(141) 5-3809. Poplar River near Owen, Wis.

(Crest-stage station)

Location.-Lat $44^{\circ} 53^{\prime} 10^{\prime \prime}$, long $90^{\circ} 34^{\prime} 17^{\prime \prime}$, in NW $\frac{1}{4}$ sec. 25, T.28 N., R.2 W., at bridge on County Trunk N., 1.3 miles east of Longwood, and 4.2 miles south of Owen.

Drainage area. $-159 \mathrm{sq} \mathrm{mi}$.

Gage-height record.-Water-stage recorder graph.

Discharge record.-Stage-discharge relation defined by current-meter measurements below $7,160 \mathrm{cfs}$.

Maxima.-March-May 1965: Discharge, 5,750 cfs 1500 hours Apr. 11 (gage height, $17.95 \mathrm{ft}$ ).

1958 to February 1965: Discharge, 8,250 cfs June 5, 1958 (gage height, $19.46 \mathrm{ft}$ ).

(142) 5-3810. Black River at Neillsville, Wis.

Location.-Lat $44^{\circ} 33^{\prime} 35^{\prime \prime}$, long $90^{\circ} 36^{\prime} 50^{\prime \prime}$, in sec.15, T.24 N., R.2 W., or right bank at downstream side of bridge on U.S. Highway 10 in Neillsville, 1 mile downstream from O'Neill Creek and 2.6 miles upstream from Cunningham Creek.

Drainage area.-756 sq $\mathrm{mi}$.

Gage-height record.-Digital recorder tape punched at sixty-minute intervals. Datum of gage is $962.77 \mathrm{ft}$ above mean sea level, datum of 1929 .

Discharge record.-Stage-discharge relation defined by current-meter measurements below 40,000 cfs. Mean daily discharges computed from 24 punch-tare recordings per day. Backwater from ice Mar. 1-9, 11-22, Mar. 26 to Apr. 4.

Maxima.-March-May 1965: Discharge, 18,300 cfs 0400 hours Apr. 12 (gage height, $15.28 \mathrm{ft}$.

1905-09, 1913 to February 1965: Discharge, 48,800 cfs Sept. 10, 1938 (gage height, $23.8 \mathrm{ft}$ ). 
Mean discharge, in cubic feet per second, 1965, of Black River at Neillsville, Wis.

\begin{tabular}{|c|c|c|c|c|c|c|c|c|c|c|c|}
\hline Day & March & April & May & Day & March & April & May & Day & March & April & May \\
\hline $\begin{array}{l}1 . \\
2 \\
3 \\
3 \\
4 \\
5 \\
6 \\
6 \\
7 \\
8 \\
9 \\
9 \\
{ }_{-}\end{array}$ & $\begin{array}{r}400 \\
2,500 \\
2,000 \\
1,550 \\
1,600 \\
1,650 \\
1,750 \\
1,800 \\
2,000 \\
1,390\end{array}$ & $\begin{array}{r}76 \\
80 \\
105 \\
130 \\
464 \\
1,880 \\
4,300 \\
5,520 \\
5,460 \\
7,820\end{array}$ & $\begin{array}{r}1,260 \\
1,030 \\
981 \\
1,010 \\
898 \\
987 \\
1,420 \\
1,470 \\
1,360 \\
1,720\end{array}$ & 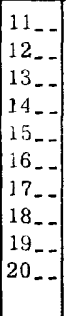 & $\begin{array}{l}880 \\
660 \\
500 \\
350 \\
240 \\
200 \\
160 \\
145 \\
135 \\
125\end{array}$ & $\begin{array}{r}15,200 \\
16,900 \\
11,600 \\
8,520 \\
6,810 \\
5,450 \\
4,060 \\
3,790 \\
3,640 \\
3,080\end{array}$ & $\begin{array}{r}1,430 \\
1,010 \\
764 \\
584 \\
463 \\
588 \\
1,220 \\
1,330 \\
1,260 \\
1,010\end{array}$ & $\begin{array}{l}21_{-} \\
22_{-} \\
23_{-} \\
24_{-} \\
25_{-} \\
26_{-} \\
27- \\
28_{-} \\
29_{-} \\
30_{-} \\
31_{-}\end{array}-$ & $\begin{array}{r}125 \\
135 \\
159 \\
132 \\
110 \\
92 \\
84 \\
76 \\
74 \\
73 \\
74\end{array}$ & $\begin{array}{l}2,690 \\
2,560 \\
2,460 \\
2,140 \\
2,560 \\
5,200 \\
6,180 \\
4,050 \\
2,520 \\
1,700 \\
-----\end{array}$ & $\begin{array}{r}735 \\
527 \\
398 \\
321 \\
282 \\
1,550 \\
2,260 \\
1,730 \\
1,080 \\
729 \\
518\end{array}$ \\
\hline \multicolumn{9}{|c|}{$\begin{array}{l}\text { Monthly mean discharge, in cubic feet per second } \\
\text { Runoff, in inches }\end{array}$} & $\begin{array}{r}683 \\
1.04\end{array}$ & $\begin{array}{r}4,565 \\
6.74\end{array}$ & $\begin{array}{r}1,030 \\
1.57\end{array}$ \\
\hline
\end{tabular}

Gage height, in feet, and discharge, in cubic feet per second, at indicated time, 1965

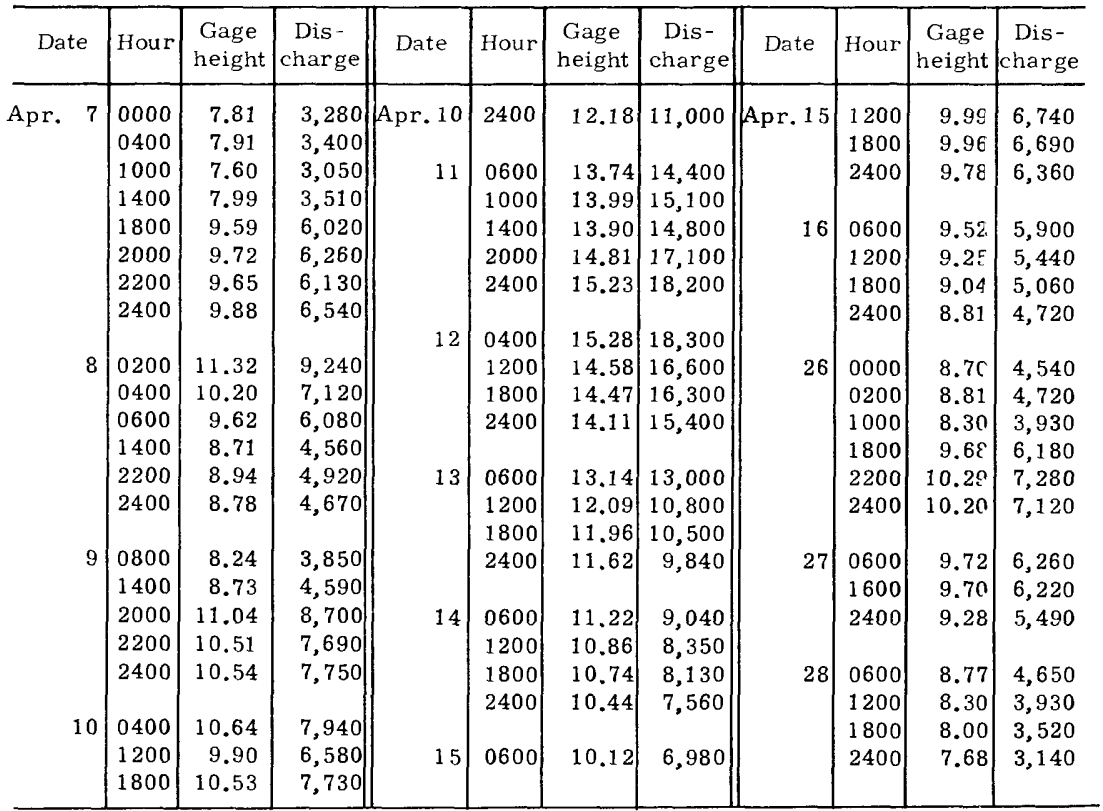


(143) 5-3820. Black River near Galesville, Wis.

Location.-Lat $44^{\circ} 03^{\prime} 45^{\prime \prime}$, long $91^{\circ} 17^{\prime} 30^{\prime \prime}$, in sec. 2 , T.18 N., R.8 W., on left bank $30 \mathrm{ft}$ downstream from bridge on U.S. Highway 53, 4.5 miles southeast of Galesville, and 5 miles downstream from Fleming Creek.

Drainage area. $-2,120 \mathrm{sq} \mathrm{mi}$, approximately.

Gage-height record.-Water-stage recorder graph except Mar. 26, Mey 3-6 for which graph was reconstructed on basis of once-daily wire-weight gage readings and recorded range lines. Datum of gage is $658.43 \mathrm{ft}$ above mean sea level, unadjusted.

Discharge record.- Stage-discharge relation defined by current meter measurements. Backwater from ice Mar. 1 to Apr. 5.

Maxima.-March-May 1965: Discharge, 33,000 cfs 1600 hours Apr. 13 (gage height, $13.60 \mathrm{ft}$ ).

1931 to February 1965: Discharge observed, 58,000 cfs Sept. 11, 1938 (gage height, $14.31 \mathrm{ft})$.

Remarks.-Flow regulated at Ilatfield Dam powerplant where drainage area is 1,290 sq $\mathrm{mi}$ and storage capacity is 272 million cubic feet.

Mean discharge, in cubic feet per second, 1965

\begin{tabular}{|c|c|c|c|c|c|c|c|c|c|c|c|}
\hline Day & March & April & May & Day & March & April & May & Day & March & April & May \\
\hline & 700 & 1,900 & 6,540 & 11. & 4,700 & 14,500 & 3,930 & 21. & 1,900 & 7,140 & 3,320 \\
\hline 2 & 1,600 & 2,200 & 4,580 & 12 & 3,900 & 22,200 & 3,770 & 22. & 1,750 & 5,950 & 2,840 \\
\hline 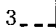 & 2,000 & 2,600 & 3,800 & 13. & 3,400 & 31,800 & 3,000 & $123-$ & 1,650 & 5,360 & 2,320 \\
\hline & 3,000 & 3,200 & 3,470 & 14. & 3,000 & 26,300 & 2,560 & 24. & 1,550 & 5,330 & 2,050 \\
\hline & 4,500 & 3,600 & 3,910 & 15. & 2,600 & 20,000 & 2,350 & 25. & 1,450 & 5,330 & 2,050 \\
\hline & 4,600 & 3,860 & 3,760 & 16. & 2,300 & 15,400 & 2,260 & 26 & 1,350 & 5,580 & 3,190 \\
\hline & 4,700 & 5,110 & 3,470 & 17. & 2,000 & 12,500 & 2,210 & 27. & 1,250 & 6,670 & 3,400 \\
\hline & 4,800 & 6,860 & 3,650 & 18 & 1,800 & 10,200 & 2,270 & 28. & 1,200 & 9,690 & 5,230 \\
\hline & 5,100 & 10,300 & 3,850 & 19. & 2,000 & 8,370 & 3,120 & 29. & 1,150 & 12,000 & 6,080 \\
\hline \multirow[t]{2}{*}{10} & 6,000 & 14,200 & 3,850 & 20. & 2,050 & 7,650 & 3,640 & 30. & 1,100 & 9,640 & 5,420 \\
\hline & & & & & & & & 31 & 1,500 & & 3,500 \\
\hline \multicolumn{9}{|c|}{ Monthly mean discharge, in cubic feet per second } & 2,600 & 9,848 & 3,529 \\
\hline \multicolumn{9}{|c|}{ Runoff, in inches } & 1.41 & 5.18 & 1.92 \\
\hline
\end{tabular}

Gage height, in feet, and discharge, in cubic feet per second, at indicated time, 1965

\begin{tabular}{|c|c|c|c|c|c|c|c|c|c|c|c|}
\hline Date & Hour & $\begin{array}{l}\text { Gage } \\
\text { height }\end{array}$ & $\begin{array}{c}\text { Dis- } \\
\text { charge }\end{array}$ & Date & Hour & $\begin{array}{c}\text { Gage } \\
\text { height }\end{array}$ & $\begin{array}{c}\text { Dis- } \\
\text { charge }\end{array}$ & Date & Hour & $\begin{array}{c}\text { Gage } \\
\text { height }\end{array}$ & $\begin{array}{c}\text { Dis- } \\
\text { charge }\end{array}$ \\
\hline \multirow[t]{5}{*}{ Apr. 9} & 0000 & 8.29 & 8,470 & \multirow[t]{4}{*}{ Apr. 12 } & 1200 & 12.29 & 21,100 & \multirow[t]{4}{*}{ Apr. 15} & 1220 & 12.08 & 19,900 \\
\hline & 1200 & 9.05 & 9,800 & & 1800 & 12.87 & 25,700 & & 1820 & 11.82 & 18,600 \\
\hline & 2000 & 9.96 & 11,900 & & 2400 & 13.23 & 29,300 & & 2490 & 11.57 & 17,400 \\
\hline & 2400 & 10.45 & 13,400 & & & & & & & & \\
\hline & & & & \multirow[t]{6}{*}{13} & 0600 & 13.44 & 31,400 & \multirow[t]{5}{*}{16} & 0630 & 11.31 & 16,200 \\
\hline \multirow[t]{5}{*}{10} & 0600 & 10.81 & 14,400 & & 1200 & 13.55 & 32,500 & & 1230 & 11.08 & 15,300 \\
\hline & 1200 & 10.86 & 14,600 & & 1600 & 13.60 & 33,000 & & 1870 & 10.80 & 14,400 \\
\hline & 1800 & 10.75 & 14,200 & & 2000 & 13.52 & 32,200 & & 2490 & 10.61 & 13,800 \\
\hline & 2400 & 10.63 & 13,900 & & 2400 & 13.39 & 30,900 & & & & \\
\hline & & & & & & & & \multirow[t]{5}{*}{17} & 0600 & 10.40 & 13,200 \\
\hline \multirow[t]{5}{*}{11} & 0600 & 10.62 & 13,900 & 14 & 0600 & 13.12 & 28,200 & & 1250 & 10.18 & 12,500 \\
\hline & 1200 & 10.65 & 14,000 & & 1200 & 12.91 & 26,100 & & 1800 & 9.95 & 11,800 \\
\hline & 1800 & 10.90 & 14,700 & & 1800 & 12.69 & 24,100 & & 2490 & 9.72 & 11,200 \\
\hline & 2400 & 11.47 & 16,900 & & 2400 & 12.53 & 22,800 & & & & \\
\hline & 0600 & 11.84 & 18,700 & 15 & 0600 & 12.30 & 21,200 & 26 & 2400 & 6.37 & 5,810 \\
\hline
\end{tabular}


Gage height, in feet, and discharge, in cubic feet per second, at indicated time, 1965, of Black Riler near Galesville, Wis. - Continued

\begin{tabular}{|c|c|c|c|c|c|c|c|c|c|c|c|}
\hline Date & Hour & $\begin{array}{c}\text { Gage } \\
\text { height }\end{array}$ & $\begin{array}{c}\text { Dis- } \\
\text { charge }\end{array}$ & Date & Hour & $\begin{array}{l}\text { Gage } \\
\text { height }\end{array}$ & $\begin{array}{c}\text { Dis- } \\
\text { charge }\end{array}$ & Date & Hour & $\begin{array}{c}\text { Gage } \\
\text { height }\end{array}$ & $\begin{array}{c}\text { Dis- } \\
\text { charge }\end{array}$ \\
\hline Apr. 27 & $\begin{array}{l}1200 \\
2400\end{array}$ & $\begin{array}{l}6.92 \\
7.88\end{array}$ & $\begin{array}{l}6,530 \\
7,800\end{array}$ & & 2400 & 9.92 & 11,800 & & 2400 & 9.69 & 11,200 \\
\hline Apr. 28 & $\begin{array}{l}1200 \\
1800\end{array}$ & $\begin{array}{l}8.93 \\
9.50\end{array}$ & $\begin{array}{r}9,570 \\
10,700\end{array}$ & Apr. 29 & $\begin{array}{l}0900 \\
1800\end{array}$ & $\begin{array}{r}10.18 \\
9.95\end{array}$ & $\begin{array}{l}12,500 \\
11,800\end{array}$ & Apr. 30 & $\begin{array}{l}1200 \\
2400\end{array}$ & $\begin{array}{l}8.94 \\
8.13\end{array}$ & $\begin{array}{l}9,590 \\
8,200\end{array}$ \\
\hline
\end{tabular}

\section{LA CROSSE RIVER BASIN}

(144) 5-3830. La Crosse River near West Salem, Wis.

Location.-Lat $43^{\circ} 54^{\prime} 05^{\prime \prime}$, long $91^{\circ} 07^{\prime} 05^{\prime \prime}$, is $\mathrm{SE}_{4}^{\frac{1}{4}} \mathrm{sec} .32$, T.17 N., R. 6 W., on left, bank $30 \mathrm{ft}$ upstream from highway bridge, 2 miles west of West Salem, and 6 miles downstream from Dutch Creek.

Drainage area. $-398 \mathrm{sq} \mathrm{mi}$.

Gage-height record.-Digital recorder tape punched at sixty-minute intervals except Mar. 8-18, Mar, 28 to May 31 for which graph was reconstructed on basis of twicedaily or more chain-gage readings. Datum of gage is $668.0 \mathrm{ft}$ above mean sea level, adjustment of 1912 .

Discharge record.- Stage-discharge relation defined by current-meter measur?ments below 3,500 cfs. Mean daily discharges computed from 24 punch-tape recordings per day except Mar, 8-18, 28-31. Backwater from ice Mar, 9-30.

Maxima.-March-May 1965: Discharge, 2,610 cfs 0130 hours Mar. 3 (gage height, $8.76 \mathrm{ft}$ ).

1913 to February 1965: Discharge observed, 8,200 cfs Aug. 6, 1935 (gage height, $12.2 \mathrm{ft}$ ).

Remarks.- Minor regulation by powerplant several miles upstream,

Mean discharge, in cubic feet per second, 1965

\begin{tabular}{|c|c|c|c|c|c|c|c|c|c|c|c|}
\hline Day & March & April & May & Day & March & April & May & Day & March & April & May \\
\hline & 905 & 576 & 308 & $11 \ldots$ & 280 & 1,130 & 300 & 21 & 130 & 353 & 298 \\
\hline & 2,080 & 970 & 300 & $12 \ldots$ & 230 & 1,900 & 280 & 22 & 150 & 294 & 302 \\
\hline & 2,440 & 960 & 288 & $13 \ldots$ & 210 & 1,220 & 274 & 23 & 180 & 298 & 310 \\
\hline 4 & 1,140 & 1,190 & 302 & $14_{\ldots}$ & 200 & 813 & 266 & $\mid 24_{-}$ & 250 & 306 & 302 \\
\hline 5 & 1,450 & 1,060 & 304 & $15 \ldots$ & 190 & 511 & 264 & 25 & 290 & 362 & 298 \\
\hline 6. & 2,200 & 1,290 & 306 & $16 \ldots$ & 190 & 310 & 206 & 26 & 290 & 576 & 452 \\
\hline & 1,920 & 1,360 & 314 & $17 \ldots$ & 190 & 374 & 310 & 27 & 290 & 571 & 718 \\
\hline 8 & 810 & 1,440 & 308 & $18=$ & 180 & 397 & 385 & 28 & 280 & 369 & 837 \\
\hline 9 & 600 & 1,790 & 308 & 19. & 190 & 392 & 329 & 29 . & 270 & 452 & 528 \\
\hline & 350 & 1,590 & 300 & $20 \ldots$ & 160 & 438 & 318 & $30_{-}$ & 260 & 318 & 320 \\
\hline & & & & & & & & 31. & 290 & & 322 \\
\hline \multirow{2}{*}{\multicolumn{9}{|c|}{$\begin{array}{l}\text { Monthly mean discharge, in cubic feet per second } \\
\text { Runoff, in inches }\end{array}$}} & 600 & 787 & 344 \\
\hline & & & & & & & & & .1 .74 & 2.21 & 1.00 \\
\hline
\end{tabular}


Gage height, in feet, and discharge, in cubic feet per second, at indicated time, 1965, of La Crosse River near West Salem, Wis.

\begin{tabular}{|c|c|c|c|c|c|c|c|c|c|c|c|}
\hline Date & Hour & $\begin{array}{c}\text { Gage } \\
\text { height }\end{array}$ & $\begin{array}{c}\text { Dis- } \\
\text { charge }\end{array}$ & Date & Hour & $\begin{array}{c}\text { Gage } \\
\text { height }\end{array}$ & $\begin{array}{c}\text { Dis- } \\
\text { charge }\end{array}$ & Date & Hour & $\begin{array}{c}\text { Gage } \\
\text { neight }\end{array}$ & $\begin{array}{c}\text { Dis- } \\
\text { charge }\end{array}$ \\
\hline \multirow[t]{10}{*}{ Mar. 1} & 0000 & 3.06 & 427 & Mar. 6 & 2400 & 8.37 & 2,310 & Apr. 6 & 2400 & 6.95 & 1,550 \\
\hline & 0800 & 3.31 & 484 & & & & & & & & \\
\hline & 1000 & 3.63 & 561 & 7 & 1200 & 8.16 & 2,180 & 7 & 1200 & 6.73 & 1,420 \\
\hline & 1200 & 4.35 & 740 & & 1800 & 6.46 & 1,390 & & 2407 & 5.75 & 1,060 \\
\hline & 1600 & 5.89 & 1,200 & & 2400 & 6.06 & 1,250 & & & & \\
\hline & 1800 & 6.39 & 1,370 & & & & & 8 & 0400 & 5.66 & 1,040 \\
\hline & 2000 & 6.47 & 1,400 & 31 & 2400 & 3.15 & 332 & & 1007 & 6.29 & 1,260 \\
\hline & 2200 & 6.89 & 1,570 & & & & & & $120 ?$ & 6.92 & 1,540 \\
\hline & 2400 & 6.86 & 1,560 & Apr. 1 & 0600 & 3.17 & 337 & & $180 ?$ & 7.47 & 1,840 \\
\hline & & & & & 1200 & 3.67 & 475 & & $240 ?$ & 7.27 & 1,700 \\
\hline \multirow[t]{5}{*}{2} & 0800 & 6.73 & 1,500 & & 1800 & 4.96 & 82.8 & & & & \\
\hline & 1000 & 7.58 & 1,890 & & 2400 & 5.53 & 999 & 9 & 060 ? & 6.91 & 1,530 \\
\hline & 1400 & 8.53 & 2,420 & & & & & & 140 ? & 7.71 & 1,960 \\
\hline & 2400 & 8.75 & 2,600 & 2 & 0800 & 5.76 & 1,070 & & 2402 & 7.56 & 1,880 \\
\hline & & & & & 1400 & 4.99 & 837 & & & & \\
\hline \multirow[t]{4}{*}{3} & 0131 & 8.76 & 2,610 & & 1800 & 5.42 & 966 & 10 & 0802 & 7.88 & 2,040 \\
\hline & 1200 & 8.64 & 2,510 & & 2400 & 5.28 & 924 & & 1207 & 6.67 & 1,440 \\
\hline & 2400 & 8.34 & 2,290 & & & & & & $180)$ & 6.19 & 1,260 \\
\hline & & & & 3 & 0800 & 4.95 & 825 & & 2403 & 6.07 & 1,190 \\
\hline \multirow[t]{9}{*}{4} & $0400^{\circ}$ & 7.88 & 2,040 & & 1200 & 5.88 & 1,100 & & & & \\
\hline & 0800 & 7.59 & 1,900 & & 2400 & 5.31 & 933 & 11 & $120)$ & 5.84 & 1,120 \\
\hline & 1000 & 5.26 & 1,010 & & & & & & $180)$ & 5.77 & 1,100 \\
\hline & 1200 & 3.46 & 520 & 4 & 0600 & 5.11 & 873 & & 240 ) & 5.84 & 1,120 \\
\hline & 1400 & 2.98 & 408 & & 1200 & 6.37 & 1,290 & & & & \\
\hline & 1800 & 3.78 & 597 & & 1600 & 6.70 & 1,410 & 12 & 040? & 6.05 & 1,180 \\
\hline & $200 \mathrm{C}$ & 4.29 & 723 & & 2400 & 6.63 & 1,380 & & 0600 & 6.77 & 1,480 \\
\hline & 2400 & 4.07 & 668 & & & & & & 0800 & 7.89 & 2,040 \\
\hline & & & & 5 & 0800 & 6.32 & 1,270 & & 1400 & 8.67 & 2,540 \\
\hline \multirow[t]{7}{*}{5} & 0400 & 4.20 & 700 & & 1200 & 5.47 & 981 & & 2000 & 7.70 & 1,950 \\
\hline & 1000 & 5.57 & 1,100 & & 1600 & 4.97 & 831 & & 2400 & 7.57 & 1,880 \\
\hline & 1400 & 6.72 & 1,500 & & 2400 & 5.04 & 852 & & & & \\
\hline & 1600 & 8.38 & 2,320 & & & & & 13 & 0800 & 7.55 & 1,860 \\
\hline & 2200 & 8.00 & 2,100 & 6 & 0800 & 5.37 & 951 & & 1000 & 6.57 & 1,360 \\
\hline & 2400 & 8.12 & 2,160 & & 1000 & 6.32 & 1,270 & & 1200 & 4.05 & 590 \\
\hline & & & & & 1200 & 6.90 & 1,530 & & 1800 & 4.88 & 804 \\
\hline 6 & 1200 & 8.17 & 2,180 & & 1800 & 7.01 & 1,580 & & 2400 & 5.07 & 861 \\
\hline
\end{tabular}

\section{MISSISSIPPI RIVER MAIN STEM}

(145) 5-3835. Mississippi River at La Crosse, Wis.

(Gaging station, discontinued 1955)

Location,-Lat $43^{\circ} 48^{\prime} 45^{\prime \prime}$, long $91^{\circ} 15^{\prime} 25^{\prime \prime}$, in sec.31, T.16 N., R.7 W., on left bank $1,300 \mathrm{ft}$ upstream from highway bridge at La Crosse, 0.4 mile downstream from La Crosse River, and at mile 697.8 above Ohio River.

Drainage area.- $-62,800 \mathrm{sq} \mathrm{mi}$, approximately.

Gage-height record.-Floodmark and gage reading on and near peak. Datum of gage is $626.32 \mathrm{ft}$ above mean sea level, adjustment of 1912 .

Discharge record.-Stage-discharge relation defined by current-meter measurements.

Maxima.-April-May 1965: Discharge, 278,000 cfs 0915-1410 hours Apr. 22 (gage height $17.96 \mathrm{ft}$, from floodmark).

1929 to March 1965: Discharge, 196,000 cfs Apr. 20, 1952 (gage height, $15.32 \mathrm{ft}$ ). Maximum-stage known, $16.5 \mathrm{ft} \mathrm{J}$ une 19, 1880, present datum, from floodmark. 


\section{ROOT RIVER BASIN}

(146) 5-3837. Mill Creek tributary near Chatfield, Minn.

\section{(Crest-stage station)}

Location.-Lat $43^{\circ} 53^{\prime} 57^{\prime \prime}$, long $92^{\circ} 14^{\prime} 16^{\prime \prime}$, in SW $\frac{1}{4} \mathrm{NW} \frac{1}{4} \mathrm{sec} .14, \mathrm{~T} .105 \mathrm{~N}$., R.12 W., at culvert on county highway, 0.8 mile upstream from Mill Creek and 4.5 miles northwest of Chatfield.

Drainage area. $-2.36 \mathrm{sq} \mathrm{mi}$.

Gage-height record.-Crest stages only.

Discharge record.- Stage-discharge relation defined by indirect measurements at $402 \mathrm{cfs}$ and 702 cfs.

Maxima.-March-May 1965: Discharge, $506 \mathrm{cfs}$ Apr. 6 (gage height, $14.20 \mathrm{ft}$, from floodmark); gage height, $15.1 \mathrm{ft}$ Mar. 1 (Backwater from ice).

1959 to February 1965: Discharge, 703 cfs July 2, 1960 (gage height, 15.46 ft, from high-water profile).

(147) 5-3838.5 Bear Creek near Grand Meadow, Minn.

(Crest-stage station)

Location.-Lat $43^{\circ} 43^{\prime} 25^{\prime \prime}$, long $92^{\circ} 35^{\prime} 20^{\prime \prime}$, in $N E \frac{1}{4} \mathrm{SE} \frac{1}{4} \mathrm{sec} .14, \mathrm{~T} .103 \mathrm{~N}$., R. 15 W., on Ieft bank 42 ft upstream from bridge on county highway, $1 \frac{1}{2}$ miles northwest of Grand Meadow, and 4 miles upstream from North Fork Bear Creek.

Drainage area. $-13.6 \mathrm{sq} \mathrm{mi}$.

Gage-height record.-Crest stages only.

Discharge record.-Stage-discharge relation defined by current-meter measurements below $320 \mathrm{cfs}$ and by indirect measurement at $3,730 \mathrm{cfs}$.

Maxima.--March-May 1965: Discharge, 885 cfs Apr. 6 (gage height, $18.2 \mathrm{ft}$, from floodmark).

1962 to February 1965: Discharge, 3,730 cfs March 28, 1962 (gage height, $21.18 \mathrm{ft}$, from high-water profilel. 
Location.-Lat $43^{\circ} 40^{\prime}$, long $92^{\circ} 05^{\prime}$, in sec.6, T.102 N., R.10 W., on upstream side of bridge on County Highway 12.

Drainage area.-188 sq mi. (from Corps of Engineers).

Gage-height record.-Occasional readings made from reference point during flood. Datum of reference point is $944.86 \mathrm{ft}$ above mean sea level, datum of 1929 (levels by Corps of Engineers).

Discharge record.-Discharge obtained on the following days by current-meter measurements.

$\begin{array}{lr}\text { Date } & \text { Discharg } \\ \text { Mar. 3 } & 182 \\ \text { Apr. 2 } & 530 \\ \text { Apr. 3 } & 441 \\ \text { Apr. 4 } & 1,340\end{array}$

Maxima.-March-May 1965: Discharge unknown, elevation, $938.78 \mathrm{ft}$ Mar. I (backwater from ice, from floodmark).

1940-41, 1961: Discharge unknown, elevation, $939.19 \mathrm{ft}$ May 29, 1941, datum of gage $928.10 \mathrm{ft}$ mean sea level, adjustment of 1912 .

Cooperation.--Peak elevations furnished by Corps of Engineers.

(149) South Branch Root River at Lanesboro, Minn.

(Gaging station, discontinued 1942, miscellaneous site)

Location.-Lat $43^{\circ} 44^{\prime}$, long $91^{\circ} 58^{\prime}$, in sec.13, T.103 N., R.10 W., on downstream side of bridge in Lanesboro.

Drainage area. $-297 \mathrm{sq} \mathrm{mi}$.

Gage-height record.-Occasional gage-height readings on chain gage during flood. Datum of gage is $816.00 \mathrm{ft}$ above mean sea level, adjustment of 1912 .

Discharge record.- Stage-discharge relation defined by three current-meter measurements.

Maxima.-Given in the following table.

March-May 1965:
Mar. 1
Apr. 6
Mar. 26, 1950 to February 1965
a Backwater from ice
Cooperation.-Peak stages furnished by Corps of Engineers.


(150) 5-3840. Root River near Lanesboro, Minn.

Location.-Lat $43^{\circ} 44^{\prime} 58^{\prime \prime}$, long $91^{\circ} 58^{\prime} 43^{\prime \prime}$, in sec.1, T.103 N., R.10 W., on left bank half a mile upstream from highway bridge, $1 \frac{1}{4}$ miles upstream from South Branch, and $2 \frac{1}{2}$ miles northeast of Lanesboro.

Drainage area.-615 sq mi.

Gage-height record.-Water-stage recorder graph except 0900 hours Mar. 1 to 1740 hours Mar. 3 when graph was constructed on the basis of floodmark and adjacent record. Datum of gage is $791.84 \mathrm{ft}$ above mean sea level, adjustment of 1912 .

Discharge record.-Stage-discharge relation defined by current-meter meas drements. Backwater from ice Mar. 1-31, Apr. 5-7.

Maxima.-Given in the following table.

March-May 1965 :

Mar. 1, 1900 hours

Apr. 6, 2300 hours

Apr. 9, 0200 hours

$1910-17,1940$ to February 1965 :

Mar. 29, 1962

${ }^{a}$ Backwater from ice.
Discharge (cfs)

Gage height (feet)

\section{9,000 \\ 14,000}

22,100 $a_{17.83}$

$\mathrm{a}_{13.33}$

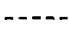

16.11

Remarks.-Diurnal fluctuation at times during medium and low flow caused by powerplant above station.

Mean discharge, in cubic feet per second, 1965

\begin{tabular}{|c|c|c|c|c|c|c|c|c|c|c|c|}
\hline Day & March & April & May & Day & March & April & May & Day & March & April & May \\
\hline & 8,000 & 1,610 & 283 & $11 \ldots$ & 205 & 3,730 & 274 & 21 & 117 & 310 & 196 \\
\hline 2. & 3,500 & 2,360 & 260 & $12 \ldots$ & 190 & 7,990 & 245 & 22. & 115 & 304 & 180 \\
\hline 3 & 955 & 2,420 & 245 & $13 \ldots$ & 170 & 1,660 & 223 & 23. & 114 & 289 & 178 \\
\hline 4. & 650 & 5,920 & 248 & $14 \ldots$ & 160 & 798 & 210 & 24. & 113 & 277 & 169 \\
\hline 5. & 410 & 8,950 & 254 & $15 \ldots$ & 145 & 618 & 199 & 25. & 112 & 274 & 169 \\
\hline 6. & 381 & 11,400 & 662 & $16 \ldots$ & 135 & 514 & 232 & 26. & 111 & 320 & 205 \\
\hline 7. & 329 & 10,500 & 722 & $17 \ldots$ & 130 & 446 & 374 & 27. & 111 & 442 & 432 \\
\hline 8. & 296 & 10,100 & 470 & 18 & 120 & 390 & 310 & 28. & 111 & 414 & 482 \\
\hline 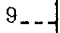 & 255 & 10,100 & 353 & $19=$ & 119 & 353 & 257 & 29 & 116 & 229 & 307 \\
\hline & 230 & 3,930 & 301 & $20 \ldots$ & 118 & 320 & 218 & 30. & 140 & 310 & 248 \\
\hline & & & & & & & & & 200 & $-\cdots---$ & 218 \\
\hline \multirow{2}{*}{\multicolumn{9}{|c|}{$\begin{array}{l}\text { Monthly mean discharge, in cubic feet per second } \\
\text { Runoff, in inches }\end{array}$}} & 576 & 2,913 & 294 \\
\hline & & & & & & & & & 1.08 & 5.28 & 0.55 \\
\hline
\end{tabular}

Gage height, in feet, and discharge, in cubic feet per second, at indtcated time, 1965

\begin{tabular}{|c|c|c|c|c|c|c|c|c|c|c|c|}
\hline Date & Hour & $\begin{array}{l}\text { Gage } \\
\text { height }\end{array}$ & $\begin{array}{c}\text { Dis- } \\
\text { charge }\end{array}$ & Date & Hour & $\begin{array}{l}\text { Gage } \\
\text { height }\end{array}$ & $\begin{array}{c}\text { Dis- } \\
\text { charge }\end{array}$ & Date & Hour & $\begin{array}{c}\text { Gage } \\
\text { height }\end{array}$ & $\begin{array}{c}\text { Dis- } \\
\text { charge }\end{array}$ \\
\hline \multirow[t]{6}{*}{ Feb. 28} & 0000 & 2.02 & & \multirow[t]{9}{*}{ Mar. 1} & 0400 & .73 & & \multirow[t]{9}{*}{ Mar. 1} & 1300 & 13.65 & \\
\hline & 0600 & 1.85 & -- & & 0500 & 6.64 & & & 1400 & 15.00 & $1-2$ \\
\hline & 1200 & 1.86 & $\ldots$ & & 0600 & 6.50 & & & 1500 & 16.02 & \\
\hline & 1800 & 3.22 & $\ldots$ & & 0700 & 5.79 & $-m$ & & 1600 & 16.86 & \\
\hline & 2400 & 4.41 & $\ldots$ & & 0800 & 7.02 & $1-$ & & 1700 & 17.50 & 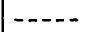 \\
\hline & & & & & 0900 & 8.35 & & & 1800 & 17.72 & $\ldots$ \\
\hline \multirow[t]{3}{*}{ Mar. 1} & 0100 & 4.50 & -... & & 1000 & 9.58 & & & 1900 & 17.83 & 19,000 \\
\hline & 0200 & 4.75 & $\ldots$ & & 1100 & 10.83 & -- & & 2000 & 17.82 & $\ldots$ \\
\hline & 0300 & 5.54 & 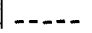 & & 1200 & 12.15 & -- & & 2100 & 17.70 & $\ldots-n$ \\
\hline
\end{tabular}


FLOODS OF 1965 IN THE UNITED STATES

Gage height, in feet, and discharge, in cubic feet per second, at indicated time, 1965, of Root River near Lanesboro, Minn. - Continued

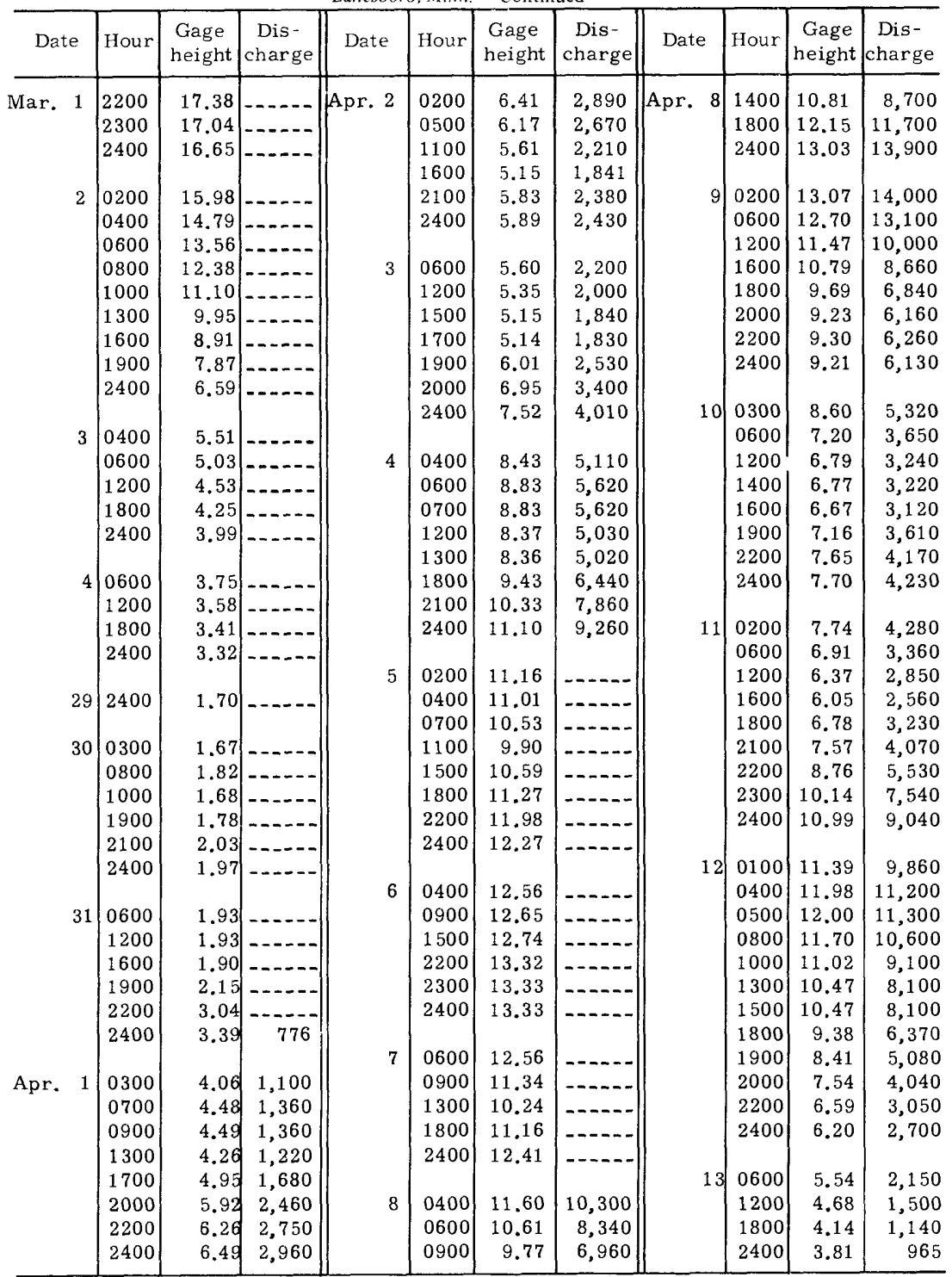


(151) Root River near Rushford, Minn.

(Miscellaneous site)

Location.-Lat $43^{\circ} 47^{\prime} 52^{\prime \prime}$, long $91^{\circ} 48^{\prime} 16^{\prime \prime}$, SW $\frac{1}{4} \mathrm{NW} \frac{1}{4} \mathrm{sec} .21, \mathrm{~T} .104$ N., R.8 W., at bridge on U.S. Highway 16, 2.8 miles west of Rushford, Minn.

Drainage area. $-1,0 \div 5 \mathrm{sq} \mathrm{mi}$.

Gage-height record.-Occasional readings made from chain gage during the flood. Datum of gage is $700.00 \mathrm{ft}$ above mean sea level, adjustment of 1912. (Levels by Corps of Engineers).

Discharge record.- - Stage-discharge relation defined by current-meter measurements.

Maxima.-Given in the following table.

March-May 1965:
Mar. 2

Cooperation: Peak stages furnished by Corps of Engineers.

(152) 5-3845. Rush Creek near Rushford, Minn.

Location.-Lat $43^{\circ} 50^{\prime} 00^{\prime \prime}$, long $91^{\circ} 46^{\prime} 40^{\prime \prime}$, on line between secs.3 and 10, T.104 N., R.8 W., on downstream side near center of span of highway bridge, $1 \frac{1}{2}$ miles northwest of Rushford and 3 miles upstream from mouth.

Drainage area. $-129 \mathrm{sq} \mathrm{mi}$.

Gage-height record.-Graph drawn on the basis of once or twice-daily wire-weight gage readings and floodmark Mar. 1, 2 and twice-daily observer's and many enzineers' wire-weight gage readings Mar. 31 to Apr. 11. Average of twice-daily wirə-weight gage readings for remainder of period except Mar. 3 when one reading is avallable. Datum of gage is $735.00 \mathrm{ft}$ above mean sea level, adjustment of 1912 .

Discharge record.- -Stage-discharge relation defined by current-meter measurements. Backwater from ice Mar. 20, 21, 24.

Maxima.-Given in following table.

\begin{tabular}{|c|c|c|}
\hline & $\begin{array}{c}\text { Discharge } \\
\text { (cfs) }\end{array}$ & $\begin{array}{c}\text { Gage-height } \\
\text { (feet) }\end{array}$ \\
\hline \multicolumn{3}{|l|}{ March-May 1965: } \\
\hline Mar. 1, 2100 hours & 3,130 & 8.75 \\
\hline Apr. 6, 0500 hours & 5,490 & 9.06 \\
\hline \multicolumn{3}{|l|}{1942 to February $1965:$} \\
\hline Mar. 26, 1950 & $a_{11,600}$ & 13.54 \\
\hline
\end{tabular}

${ }^{\mathrm{a}}$ From rating curve extended above $1,400 \mathrm{cfs}$ on basis of contracted-opening measurements at gage heights 11.0 and $13.5 \mathrm{ft}$. 
Mean discharge, in cubic feet per second. 1965, of Rush Creek near Rushford, Minn

\begin{tabular}{|c|c|c|c|c|c|c|c|c|c|c|c|}
\hline Day & March & April & May & Day & March & April & May & Day & March & April & May \\
\hline 10 & 1,600 & 1,020 & 37 & $11 \ldots$ & 46 & 242 & 37 & $21 \ldots$ & 37 & 40 & 36 \\
\hline 2. & 699 & 404 & 37 & $12 \ldots$ & 42 & 217 & 36 & 22 & 34 & 39 & 36 \\
\hline 3. & 76 & 268 & 38 & $13 \ldots$ & 46 & 108 & 36 & 23. & 34 & 39 & 36 \\
\hline 1. & 68 & 1,330 & 38 & $14 \ldots$ & 44 & 51 & 36 & 24 & 36 & 40 & 36 \\
\hline 5 & 59 & 1,640 & 39 & $15 \ldots$ & 44 & 48 & 37 & $25 \ldots$ & 32 & 44 & 37 \\
\hline $6 \ldots$ & 65 & 3,770 & 37 & $16 \ldots$ & 44 & 44 & 37 & 26 & 34 & 42 & 42 \\
\hline 7. & 87 & 1,700 & 36 & $17 .-$ & 44 & 44 & 37 & $27 \ldots$ & 37 & 41 & 38 \\
\hline 8 & 58 & 1,950 & 36 & $18 \ldots$ & 42 & 44 & 37 & $28 \ldots$ & 36 & 40 & 37 \\
\hline 9. & 54 & 815 & 39 & 19 & 42 & 42 & 37 & 29. & 40 & 39 & 36 \\
\hline 10. & 49 & 212 & 38 & $20 \ldots$ & 40 & 41 & 35 & $30 \ldots$ & 44 & 38 & 36 \\
\hline & & & & & & & & 31 & 88 & $\ldots$ & 37 \\
\hline \multirow{2}{*}{\multicolumn{9}{|c|}{ MTonthly mean discharge, in cubic feet per second }} & 119 & 480 & 37.0 \\
\hline & & & & & & & & & 1.07 & 4.15 & 0.33 \\
\hline
\end{tabular}

Gage herght, in feet, and discharge, in cubic feet per second, at indicatcd tumo, 1965

\begin{tabular}{|c|c|c|c|c|c|c|c|c|c|c|c|}
\hline Date & Hour & $\begin{array}{l}\text { Gage } \\
\text { height }\end{array}$ & $\begin{array}{c}\text { Dis- } \\
\text { charge }\end{array}$ & Date & Hour & $\begin{array}{c}\text { Gage } \\
\text { height }\end{array}$ & $\begin{array}{c}\text { Dis- } \\
\text { charge }\end{array}$ & Date & Hour & $\begin{array}{c}\text { Gage } \\
\text { height }\end{array}$ & $\begin{array}{c}\text { Dis- } \\
\text { charge }\end{array}$ \\
\hline \multirow[t]{10}{*}{ Feb. 28} & 0000 & 1.83 & 44 & Apr. 2 & 0300 & 3.96 & 554 & Apr. 7 & 0400 & 4.41 & 786 \\
\hline & 0900 & 1.88 & 46 & & 0600 & 3.57 & 421 & & 0800 & 3.49 & 397 \\
\hline & 1000 & 2.00 & 50 & & 1200 & 2.95 & 254 & & 1200 & 5.31 & 1,380 \\
\hline & 1100 & 2.23 & 58 & & 2100 & 3.47 & 391 & & 1400 & 6.25 & 2,160 \\
\hline & 1200 & 2.54 & 72 & & 2400 & 3.43 & 385 & & 1800 & 7.74 & 3,680 \\
\hline & 1300 & 2.87 & 91 & & & & & & 2000 & 6.82 & 2,680 \\
\hline & 1500 & 3.39 & 126 & 3 & 0600 & 3.09 & 300 & & 2400 & 5.76 & 1,740 \\
\hline & 1800 & 4.01 & 200 & & 1200 & 2.55 & 194 & & & & \\
\hline & 2400 & 4.96 & 367 & & 1800 & 2.75 & 223 & 8 & 0400 & 4.33 & 738 \\
\hline & & & & & 2400 & 3.17 & 313 & & 0900 & 2.85 & 233 \\
\hline \multirow[t]{6}{*}{ Mar. 1} & 0600 & 5.84 & 611 & & & & & & 1200 & 4.40 & 780 \\
\hline & 1200 & 6.94 & 1,320 & 4 & 0600 & 4.15 & 640 & & 1400 & 6.25 & 2,160 \\
\hline & 1800 & 8.42 & 2,800 & & 1200 & 5.27 & 1,350 & & 1600 & 7.50 & 3,400 \\
\hline & 2100 & 8.75 & 3,130 & & 1600 & 5.70 & 1,680 & & 2000 & 8.20 & 4,260 \\
\hline & 2400 & 8.15 & 2,530 & & 2000 & 6.57 & 2,450 & & 2400 & 6.95 & 2,800 \\
\hline & & & & & 2400 & 5.99 & 1,930 & & & & \\
\hline \multirow[t]{6}{*}{2} & 0600 & 6.23 & 1,030 & & & & & 9 & 0200 & 6.09 & 2,020 \\
\hline & 1200 & 4.35 & 380 & 5 & 0400 & 5.10 & 1,230 & & 0400 & 5.20 & 1,300 \\
\hline & 1500 & 3.36 & 188 & & 0800 & 4.25 & 692 & & 0800 & 3.45 & 385 \\
\hline & 1800 & 2.49 & 100 & & 1200 & 3.87 & 520 & & 1000 & 2.71 & 206 \\
\hline & 2400 & 2.37 & 92 & & 1400 & 4.45 & 810 & & 1400 & 3.30 & 343 \\
\hline & & & & & 1600 & 5.35 & 1,410 & & 1800 & 4.51 & 846 \\
\hline \multirow[t]{2}{*}{30} & 2400 & 1.55 & 48 & & 1800 & 6.31 & 2,220 & & 2400 & 3.77 & 484 \\
\hline & & & & & 2000 & 7.08 & 2,930 & & & & \\
\hline \multirow[t]{5}{*}{31} & 1200 & 1.64 & 55 & & 2400 & 8.23 & 4,310 & 10 & 0400 & 2.93 & 250 \\
\hline & 1800 & 1.98 & 92 & & & & & & 0800 & 2.06 & 107 \\
\hline & 2100 & 2.45 & 162 & 6 & 0500 & 9.06 & 5,490 & & 1800 & 2.81 & 225 \\
\hline & 2400 & 3.11 & 292 & & 0800 & 8.80 & 5,110 & & 2400 & 2.58 & 182 \\
\hline & & & & & 1000 & 8.43 & 4,590 & & & & \\
\hline \multirow{5}{*}{ Apr. 1} & 0300 & 3.74 & 474 & & 1400 & 7.55 & 3,460 & 11 & 0800 & 2.23 & 127 \\
\hline & 0600 & 4.40 & 780 & & 1800 & 6.78 & 2,640 & & 1400 & 2.87 & 237 \\
\hline & 1200 & 5.19 & 1,290 & & 2000 & 6.30 & 2,210 & & 1800 & 3.47 & 391 \\
\hline & 1800 & 5.40 & 1,450 & & 2400 & 5.36 & 1,420 & & 2400 & 3.33 & 351 \\
\hline & 2400 & 4.57 & 882 & & & & & & & & \\
\hline
\end{tabular}


(153) 5-3850. Root River near Houston, Minn.

Location.-Lat $43^{\circ} 46^{\prime} 05^{\prime \prime}$, long $91^{\circ} 35^{\prime} 1^{\prime \prime}$, in sec.32, T.104 N., R. 6 W., on right bank 1 mile west of Houston and $2 \frac{1}{2}$ miles upstream from South Fork.

Drainage area.- $-1,270 \mathrm{sq} \mathrm{mi}$, approximately.

Gage-height record.-Water-stage recorder graph except 0600 hours Mar. 1 to 1100 hours Mar. 3 and 0100 hours May 27 to 2400 hours May 31. The graph was constructed on the basis of floodmark and twice or more daily readings made from reference point, 0600 hours Mar. 1 to 1100 hours Mar. 3. Datum of gage is $671.86 \mathrm{ft}$ above mean sea level, datum of 1929 .

Discharge record.- - Stage-discharge relation defined by current-meter measurements. Backwater from ice Mar. 1-31. Discharge May 27-31 estimated on basis of weather records and records for station Root River near Lanesboro.

Maxima.-Given in the following table.

March-May 1965:

$\begin{array}{cc}\begin{array}{c}\text { Discharge } \\ \text { (cfs) }\end{array} & \begin{array}{c}\text { Gago height } \\ \text { (feet) }\end{array} \\ 31,000 & \mathrm{a}_{18.32} \\ 19,800 & 12.37 \\ & \\ 37,000 & 13.90 \\ \text { Unknown } & 15.10\end{array}$

Mar. 2, 1200 hours

Apr. 7, 0200 hours

$1909-17,1929$ to February 1965 :

Apr. 1, 1952

Unknown

15.10

${ }^{\text {B }}$ Backwater from ice.

Mean discharge, in cubic feet per second, 1965

\begin{tabular}{|c|c|c|c|c|c|c|c|c|c|c|c|}
\hline Day & March & Apri1 & May & Day & March & April & May & Day & March & April & May \\
\hline 1. & 7,400 & 2,590 & 673 & $11 \ldots$ & 468 & 6,600 & 626 & 21. & 340 & 964 & 454 \\
\hline 2 & 20,000 & 4,290 & 641 & $12 \ldots$ & 449 & 10,000 & 561 & 22. & 330 & 892. & 414 \\
\hline 3 & 7,600 & 3,460 & 600 & $13 \ldots$ & 433 & 5,660 & 528 & 23. & 330 & $83 \varepsilon$ & 394 \\
\hline & 1,900 & 7,950 & 595 & $14 \ldots$ & 418 & 2,460 & 504 & 24 . & 330 & 790 & 390 \\
\hline 5 & 1,200 & 11,500 & 590 & $15 \ldots$ & 402 & 1,900 & 490 & 25. & 325 & 796 & 394 \\
\hline 6. & 700 & 16,300 & 646 & 16 & 387 & 1,610 & 490 & 26. & 325 & 802. & 410 \\
\hline 7. & 600 & 18,800 & 1,280 & $17 \ldots$ & 375 & 1,420 & 504 & 27 & 325 & 844 & 500 \\
\hline 8. & 578 & 16,300 & 1,030 & $18 \ldots$ & 367 & 1,270 & 561 & 28. & 325 & 880 & 779 \\
\hline 9. & 517 & 17,100 & 784 & $19 \ldots$ & 361 & 1,140 & 585 & $29_{-}$ & 330 & 802 & 561 \\
\hline 10 & 494 & 9,170 & 700 & 20 & 350 & 1,040 & 504 & $30 \ldots$ & 390 & 727 & 405 \\
\hline & & & & & & & & 31. & 500 & -n- & 472 \\
\hline \multirow{2}{*}{\multicolumn{9}{|c|}{$\begin{array}{l}\text { Monthly mean discharge, in cubic feet per second } \\
\text { Runoff, in inches }\end{array}$}} & 1,576 & 4,963 & 586 \\
\hline & & & & & & & & & 1.43 & 4.36 & 0.53 \\
\hline
\end{tabular}

Gage height, in feet, and discharge, in cubic feet per second, at indicated time, 1965

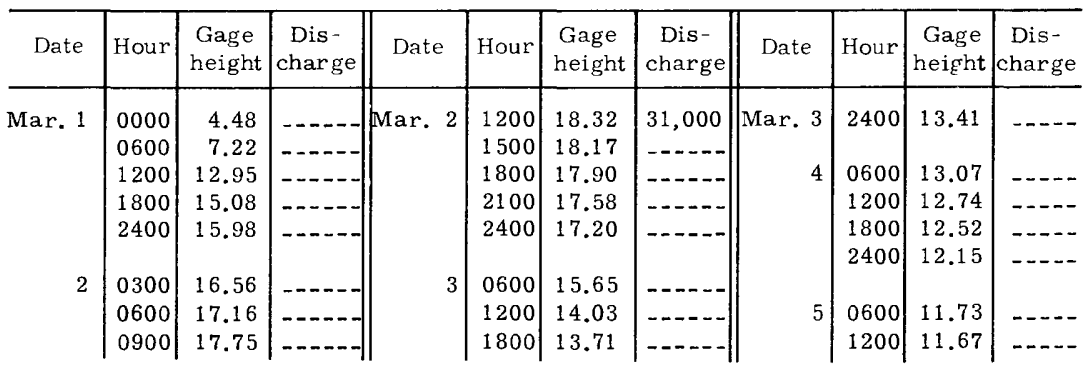


FLOODS OF 1965 IN THE UNITED STATES

Gage height, in feet, and discharge, in cubic feet per second, at indicated time, 1965, of Root River near Houston, Minn. — Continued

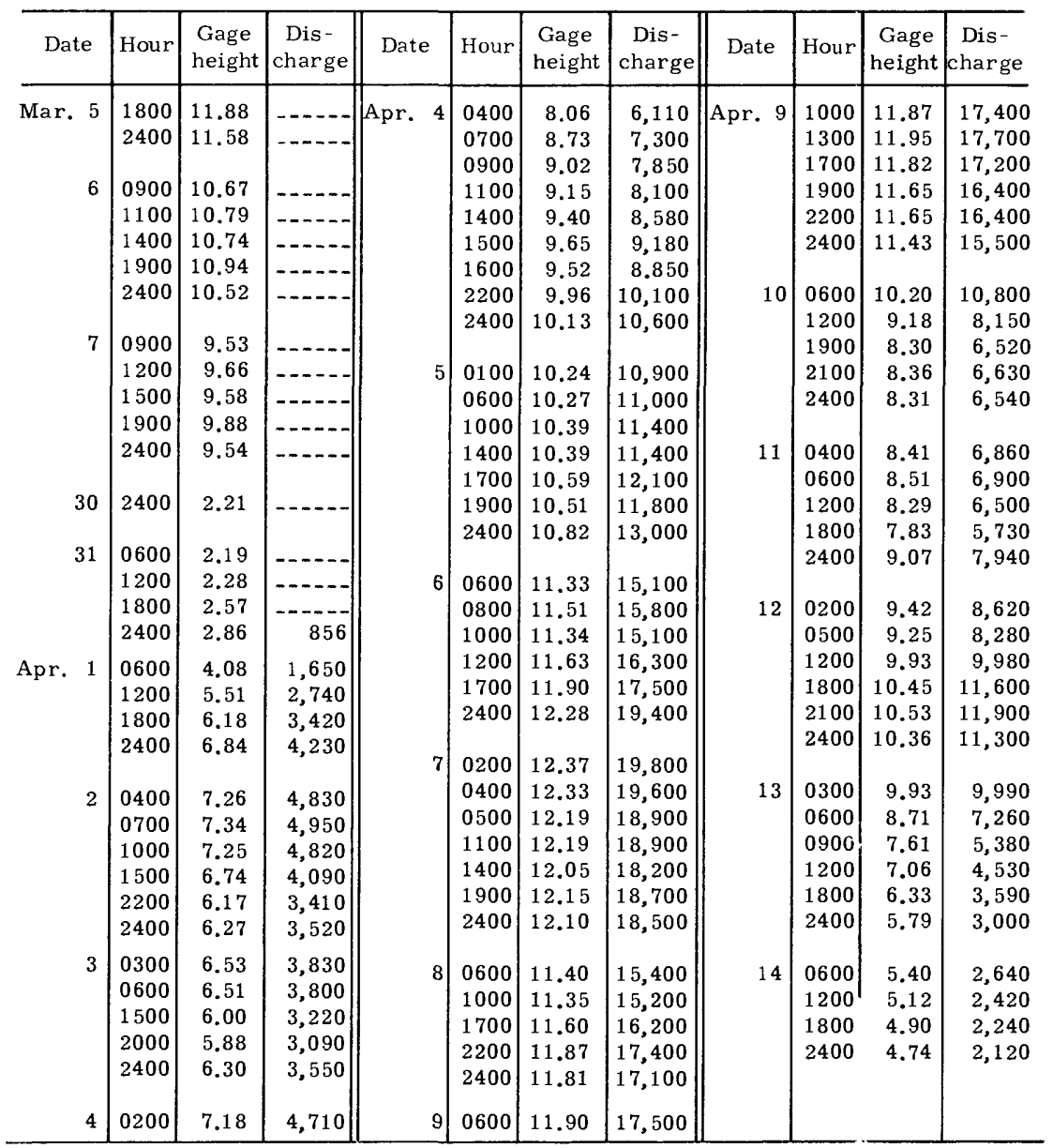


(154) 5-3855. South Fork Root River near Houston, Minn.

Location.-Lat $43^{\circ} 44^{\prime}$, long $91^{\circ} 34$, in NE $\frac{1}{4} \mathrm{SW} \frac{1}{4}$ sec. 9 , T.103 N., R.6 W., on left bank $50 \mathrm{ft}$ downstream from bridge on State Highway 76, half a mile upstream from Badger Creek and $1 \frac{1}{2}$ miles south of Houston.

Drainage area. $-275 \mathrm{sq} \mathrm{mi}$.

Gage-height record.-Water-stage recorder graph except 2230 hours Feb. 28 to 1550 hours Mar. 1, 2230 hours Mar. 2 to 1230 hours Mar. 3 and 0100 hours Apr. 17 to 2400 hours Apr. 19 when graph was constructed on the basis of adjacent record. Datum of gage is $680.41 \mathrm{ft}$ above mean sea level, datum of 1929 .

Discharge record. $\rightarrow$ Stage-discharge relation defined by current-meter measurements. No gage height record Apr. 17-19, discharge estimated on the basis of adjacent record and weather records. Backwater from ice Mar. 1-28, 30, 31.

Maxima.-Given in following table.

March-May 1965 :

Mar. 2, 0400 hours

Apr 6, 0930 hours

1953 to February 1965 :

Mar. 29, 1962

Mar. 26, 1961

Discharge

(cfs)

6,530

4,170

8,420

-..--

\section{Gage-height}

(feet)

ล 13.64

11.86

13.35

Q 3.74

${ }^{\mathrm{a}}$ Backwater from ice.

Mean discharge, in cubic feet per second, 1965

\begin{tabular}{|c|c|c|c|c|c|c|c|c|c|c|c|}
\hline Day & March & April & May & Day & March & April & May & Day & March & April & May \\
\hline$\lambda_{3}$ & 3,900 & 1,090 & 111 & $11 \ldots$ & 100 & 982 & 97 & 21. & 105 & 105 & 80 \\
\hline 2 & 2,700 & 978 & 106 & $12 \ldots$ & 100 & 920 & 96 & 22 & 100 & 102 & 80 \\
\hline 3. & 300 & 658 & 102 & $13 \ldots$ & 98 & 307 & 95 & 23 & 98 & 101 & 85 \\
\hline 1. & 145 & 2,320 & 100 & $14 \ldots$ & 97 & 218 & 94 & 24 & 97 & 105 & 82 \\
\hline 5 & 140 & 2,250 & 105 & $15 \ldots$ & 96 & 195 & 122 & 25 & 96 & 122 & 91 \\
\hline 6. & 130 & 3,770 & 103 & $16 \ldots$ & 92 & 179 & 166 & 26. & 95 & 130 & 868 \\
\hline 7. & 125 & 2,260 & 101 & $17 .-$ & 95 & 151 & 97 & 27 & 95 & 125 & 253 \\
\hline$\because$. & 120 & 2,500 & 103 & $18 \ldots$ & 105 & 133 & 94 & 28 & 94 & 126 & 146 \\
\hline 9 & 110 & 1,910 & 106 & $19 \ldots$ & 110 & 118 & 80 & 29 & 92 & 117 & 123 \\
\hline \multirow[t]{2}{*}{$10_{-}$} & 105 & 783 & 97 & $20 \ldots$ & 109 & 109 & 80 & $30 \ldots$ & 94 & 114 & 108 \\
\hline & & & & & & & & 31. & 146 & $\ldots-\ldots$ & 111 \\
\hline \multicolumn{9}{|c|}{ 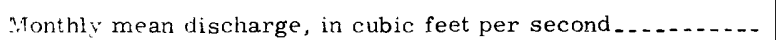 } & 319 & 766 & 132 \\
\hline \multicolumn{9}{|c|}{;unıf: in inches } & 1.34 & 3.11 & 0.55 \\
\hline
\end{tabular}


Gage height, in feet, and discharge, in cubic feet per second, at indicated time, 1965, of South Fork Root River near Houston, Minn.

\begin{tabular}{|c|c|c|c|c|c|c|c|c|c|c|c|}
\hline Date & Hour & $\begin{array}{l}\text { Gage } \\
\text { height }\end{array}$ & $\begin{array}{c}\text { Dis- } \\
\text { charge }\end{array}$ & Date & Hour & $\begin{array}{c}\text { Gage } \\
\text { height }\end{array}$ & $\begin{array}{c}\text { Dis- } \\
\text { charge }\end{array}$ & Date & Hour & $\begin{array}{c}\text { Gage } \\
\text { height }\end{array}$ & $\begin{array}{l}\text { Dis- } \\
\text { charge }\end{array}$ \\
\hline \multirow[t]{8}{*}{ Feb. 28} & 0000 & 3.81 & -.. & Mar. 8 & 0200 & 7.13 & & Apr. 5 & 1200 & 9.33 & 1,490 \\
\hline & 1400 & 3.91 & & & 0400 & 6.53 & & & 1500 & 8.28 & 1,240 \\
\hline & 2000 & 4.98 & $\ldots$ & & 0800 & 5.91 & $\ldots$ & & 1800 & 9.46 & 1,530 \\
\hline & 2100 & 5.73 & $-\ldots$ & & 1200 & 5.56 & -..- & & 2100 & 10.60 & 2,110 \\
\hline & 2200 & 7.66 & $\ldots$ & & 1800 & 5.15 & $\ldots$ & & 2400 & 11.44 & 3,290 \\
\hline & 2300 & 8.80 & $\ldots$ & & 2400 & 4.30 & & & & & \\
\hline & 2400 & 9.95 & $\ldots$ & & & & & 6 & 0600 & 11.80 & 4,030 \\
\hline & & & & 9 & 0600 & 3.76 & & & 0930 & 11.86 & 4,170 \\
\hline \multirow[t]{14}{*}{ Mar. 1} & 0200 & 10.70 & -- & & 1200 & 3.43 & $\ldots$ & & 1800 & 11.60 & 3,600 \\
\hline & 0400 & 11.75 & $\ldots$ & & 1800 & 3.12 & & & 2400 & 11.52 & 3,440 \\
\hline & 0800 & 12.34 & -- & & 2400 & 2.94 & & & & & \\
\hline & 1200 & 12.65 & -- & & & & & 7 & 0600 & 11.20 & 2,860 \\
\hline & 1800 & 13.04 &.- & 30 & 2400 & 2.38 & & & 0800 & 10.54 & 2,060 \\
\hline & 2400 & 13.55 & --- & & & & & & 1000 & 9.53 & 1,550 \\
\hline & 0400 & 13.64 & 6,530 & 31 & 1400 & 2.02 & & & 1200 & 8.54 & 1,300 \\
\hline & 0800 & 13.30 & - & & 1800 & 2.30 & $\ldots$ & & 1300 & 8.12 & 1,210 \\
\hline & 1000 & 12.68 & & & 2100 & 3.04 & $\ldots \ldots$ & & 1500 & 8.62 & 1,310 \\
\hline & 1200 & 12.02 & $\ldots$ & & 2400 & 4.16 & 449 & & 1700 & 10.00 & 1,720 \\
\hline & 1600 & 11.24 & $\ldots$ & & & & & & 2000 & 10.81 & 2,330 \\
\hline & 2000 & 10.16 & & Apr. 1 & 0400 & 5.25 & 645 & & 2400 & 11.42 & 3,250 \\
\hline & 2400 & 9.16 & & & 0600 & 6.58 & 897 & & & & \\
\hline & & & & & 0800 & 7.65 & 1,110 & 8 & 0500 & 11.47 & 3,340 \\
\hline \multirow[t]{6}{*}{3} & 0400 & 8.50 & $\cdots$ & & 1200 & 8.25 & 1,230 & & 0800 & 10.59 & 2,110 \\
\hline & 0800 & 7.98 & $\ldots$ & & 1800 & 8.54 & 1,300 & & 1000 & 9.27 & 1,480 \\
\hline & 1200 & 7.57 & - & & 2400 & 9.14 & 1,440 & & 1200 & 8.03 & 1,190 \\
\hline & 1800 & 7.28 & & & & & & & 1300 & 7.76 & 1,130 \\
\hline & 2400 & 7.04 & & 2 & 0100 & 9.31 & 1,490 & & 1500 & 9.05 & 1,420 \\
\hline & & & & & 0500 & 8.80 & 1,360 & & 1700 & 10.40 & 1,940 \\
\hline \multirow[t]{3}{*}{4} & 1200 & 6.48 & -1. & & 0800 & 7.78 & 1,130 & & 1900 & 11.09 & 2,700 \\
\hline & 2400 & 6.29 & $\ldots$ & & 1000 & 7.06 & 989 & & 2200 & 11.71 & 3,830 \\
\hline & & & & & 1200 & 6.41 & 865 & & 2400 & 11.67 & 3,750 \\
\hline \multirow[t]{6}{*}{5} & 1200 & 6.34 & $\ldots$ & & 1800 & 5.32 & 658 & & & & \\
\hline & 1400 & 7.00 & $\cdots$ & & 2400 & 5.51 & 916 & 9 & 0200 & 11.62 & 3,640 \\
\hline & 1800 & 8.00 & -- & & & & & & 0500 & 11.67 & 3,750 \\
\hline & 2000 & 8.41 & $\ldots$ & 3 & 0300 & 5.36 & 665 & & 0800 & 10.98 & 2,540 \\
\hline & 2400 & 8.14 & $\ldots$ & & 0700 & 5.93 & 774 & & 0900 & 10.15 & 1,780 \\
\hline & & & & & 1200 & 5.02 & 604 & & 1000 & 9.52 & 1,550 \\
\hline \multirow[t]{8}{*}{6} & 0400 & 7.36 & $\ldots$ & & 1800 & 4.26 & 467 & & 1100 & 8.70 & 1,330 \\
\hline & 0700 & 6.99 & $\ldots$ & & 2200 & 5.62 & 715 & & 1200 & 7.94 & 1,170 \\
\hline & 1000 & 6.73 & $-\cdots$ & & 2400 & 7.87 & 1,150 & & 1300 & 7.22 & 1,020 \\
\hline & 1400 & 6.99 & & & & & & & 1400 & 6.79 & 937 \\
\hline & 1800 & 7.77 & & 4 & 0100 & 8.97 & 1,400 & & 1700 & 6.38 & 859 \\
\hline & 2200 & 8.44 & & & 0200 & 9.71 & 1,610 & & 2000 & 6.97 & 971 \\
\hline & 2400 & 8.19 & -- & & 0400 & 10.34 & 1,900 & & 2400 & 7.14 & 1,000 \\
\hline & & & & & 0600 & 10.77 & 2,290 & & & & \\
\hline \multirow[t]{7}{*}{7} & 0400 & 7.47 & $\cdots$ & & 1000 & 11.20 & 2,860 & 10 & 0400 & 6.77 & 914 \\
\hline & 0800 & 6.81 & -- & & 1400 & 10.88 & 2,420 & & 0800 & 6.12 & 810 \\
\hline & 1400 & 6.43 & $\ldots$ & & 1900 & 10.54 & 2,060 & & 1200 & 5.27 & 649 \\
\hline & 1800 & 7.25 & --- & & 2400 & 11.40 & 3,210 & & 1600 & 4.86 & 575 \\
\hline & 2000 & 7.97 & 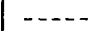 & & & & & & 2000 & 5.66 & 722 \\
\hline & 2200 & 8.54 & $\ldots$ & 5 & 0200 & 11.45 & 3,300 & & 2200 & 6.53 & 888 \\
\hline & 2400 & 7.97 & $\ldots$ & & 0800 & 11.00 & 2,570 & & 2400 & 7.38 & 1,050 \\
\hline
\end{tabular}


MARCH—MAY, UPPER MISSISSIPPI RIVER BASIN

Gage height, in feet, and discharge, in cubic feet per second, at indicated time, 1965, of South Fork Root

River near Houston, Minn. - Continued

\begin{tabular}{|c|c|c|c|c|c|c|c|c|c|c|c|}
\hline Date & Hour & $\begin{array}{c}\text { Gage } \\
\text { height }\end{array}$ & $\begin{array}{c}\text { Dis- } \\
\text { charge }\end{array}$ & Date & Hour & $\begin{array}{c}\text { Gage } \\
\text { height }\end{array}$ & $\begin{array}{c}\text { Dis- } \\
\text { charge }\end{array}$ & Date & Hour & $\begin{array}{c}\text { Gage } \\
\text { height }\end{array}$ & $\begin{array}{c}\text { Dis- } \\
\text { charge }\end{array}$ \\
\hline \multirow[t]{7}{*}{ Apr. 11} & 0200 & 7.62 & 1,100 & \multirow[t]{7}{*}{ Apr. 11} & 2100 & 9.14 & 1,440 & \multirow[t]{7}{*}{ Apr. 12} & 1200 & 6.15 & 816 \\
\hline & 0600 & 6.95 & 968 & & 2300 & 9.67 & 1,600 & & 1400 & 5.63 & 717 \\
\hline & 1200 & 5.99 & 785 & & 2400 & 9.63 & 1,480 & & 1800 & 4.66 & 539 \\
\hline & 1600 & 5.17 & 631 & & & & & & 2400 & $3 . \Omega 1$ & 405 \\
\hline & 1800 & 5.88 & 764 & & 0600 & 8.96 & 1,390 & & & & \\
\hline & 1900 & 6.76 & 931 & & 0800 & 8.12 & 1,200 & & 1200 & 3.22 & 290 \\
\hline & 2000 & 8.03 & 1,190 & & 1000 & 7.06 & 989 & & 2400 & 2.22 & 242 \\
\hline
\end{tabular}

(155) 5-3860. Root River below South Fork near Houston, Minn.

(Gaging station, discontinued 1961)

Location.-Lat $43^{\circ} 46^{\prime} 13^{\prime \prime}$, long $91^{\circ} 32^{\prime} 03^{\prime \prime}$, in $\mathrm{SE} \frac{1}{4} \mathrm{NE} \frac{1}{4}$ sec.34, T.104 N., R.6 W., on right bank $600 \mathrm{ft}$ downstream from South Fork and $1 \frac{1}{2}$ miles northeast of Houston.

Drainage area. $-1,560 \mathrm{sq} \mathrm{mi}$, approximately.

Gage-height record.-Peak stages only from chain gage readings. Datum of gage is $660.00 \mathrm{ft}$ above mean sea level, adjustment of 1912 .

Discharge record.-Peak discharge obtained by summation of peaks for Root River and South Fork Root River near Houston.

Maxima.-Given in the following table.

March-May 1965:

Mar. 2

Apr. 7 -

1938 to February 1965 :

Apr. 1, 1952

${ }^{\text {a }}$ Backwater from ice.

$\begin{array}{cc}\begin{array}{c}\text { Discharge } \\ \text { (cfs) }\end{array} & \begin{array}{c}\text { Gage height } \\ \text { (feet) }\end{array} \\ 37,500 & a_{18.50} \\ 24,000 & 16.50 \\ 38,700 & 17.58\end{array}$

17.58

Cooperation.--Peak stages furnished by Corps of Engineers.

\section{MORMON CREEK BASIN}

(156) 5-3863. Mormon Creek near La Crosse, Wis.

(Crest-stage station)

Location.-Lat $43^{\circ} 46^{\prime} 00^{\prime \prime}$, long $91^{\circ} 08^{\prime} 27^{\prime \prime}$, in NE $\frac{1}{4}$ sec.19, T.1 5 N., R.6 W., at bridge on country road 0.2 mile north of intersection with U.S. Highways 14 and 61 , and 6.2 miles southeast from junction of U.S. Highways 14 and 61 and State Highway 33 in La Crosse.

Drainage area. $-25.5 \mathrm{sq} \mathrm{mi}$.

Gage-height record.-Crest stages only.

Maxima.-March-May 1965: Gage height, $11.0 \mathrm{ft}$ April 2, 1965.

1961 to February 1965: Gage height, $11.57 \mathrm{ft}$ Mar. 25, 1961. 


\section{BAD AXE RIVER BASIN}

(157) 5-3871. North Fork Bad Axe River near Genoa, Wis.

(Crest-stage station)

Location.-Lat $43^{\circ} 33^{\prime} 10^{\prime \prime}$, long $91^{\circ} 08^{\prime} 58^{\prime \prime}$, in SW $\frac{1}{4}$ sec. 36, T.13 N., R. 7 W., at bridge on State Highway 56, 4.1 miles southeast from Genoa, and 13.1 miles west of Viroqua.

Drainage area. $-80.7 \mathrm{sq} \mathrm{mi}$.

Gage-height record.-Water-stage recorder graph.

Maxima.-March-May 1965: Gage height, $15.91 \mathrm{ft}$ Mar. 5.

1959 to February 1965: Gage height, $18.62 \mathrm{ft}$ Aug. 29, 1959.

\section{UPPER IOWA RIVER BASIN}

(158) 5-3875. Upper Iowa River at Decorah, Iowa

Location.-Lat $43^{\circ} 18^{\prime} 20^{\prime \prime}$, long $91^{\circ} 48^{\prime} 05^{\prime \prime}$, in NE $\frac{1}{4} \mathrm{SW} \frac{1}{4}$ sec.16, T.98 N., R.8 W., on right bank 1, $200 \mathrm{ft}$ upstream from bridge on State Highway 52, 1,500 ft downstream from Dry Run cutoff, and 3 miles upstream from Trout Run.

Drainage area.- $-511 \mathrm{sq} \mathrm{mi}$.

Gage-height record.-Water-stage recorder graph. Datum of gage is $85 \mathrm{C} .00 \mathrm{ft}$ above mean sea level, datum of 1929.

Discharge record.-Stage-discharge relation defined by current-meter reasurements below $20,000 \mathrm{cfs}$.

Maxima.-March-May 1965: Discharge, 9,800 cfs 0300 hours Mar. 2 (gage height, $9.89 \mathrm{ft}$ ).

1913 to February 1965: Discharge, 28,500 cfs May 29, 1941 (gage height, $15.19 \mathrm{ft}$ ), at site of former gaging station near Decorah, 4 miles downstream.

Mean discharge, in cubic feet per second, 1965

\begin{tabular}{|c|c|c|c|c|c|c|c|c|c|c|c|}
\hline Day & March & April & May & Day & March & April & May & Day & March & April & May \\
\hline & 5,700 & 2,140 & 243 & $11 \ldots$ & 182 & 3,010 & 206 & $21 \ldots$ & 118 & 260 & 163 \\
\hline 2 & 8,350 & 2,420 & 226 & $12 \ldots$ & 166 & 3,440 & 189 & $22 \ldots$ & 106 & 238 & 151 \\
\hline 3. & 1,770 & 2,460 & 214 & $13--$ & 163 & 2,360 & 176 & $23 \ldots$ & 121 & 234 & 151 \\
\hline & 648 & 4,490 & 200 & 14 & 151 & 832 & 170 & $24 \ldots$ & 111 & 226 & 142 \\
\hline 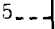 & 462 & 5,460 & 196 & $15--$ & 148 & 620 & 163 & 25 & 127 & 275 & 190 \\
\hline . & 402 & 7,910 & 192 & $16--$ & 136 & 501 & 200 & $26 \ldots$ & 104 & 355 & 2,090 \\
\hline & 402 & 7,470 & 617 & $17=$ & 136 & 432 & 170 & 27. & 98 & 420 & 522 \\
\hline & 320 & 6,430 & 456 & $18 .-$ & 94 & 366 & 238 & $28 \ldots$ & 89 & 372 & 390 \\
\hline 9. & 243 & 5,240 & 285 & $19--$ & 118 & 315 & 203 & 29 & 86 & 300 & 315 \\
\hline 10. & 203 & 2,880 & 230 & $20 \ldots$ & 121 & 285 & 176 & $30_{\ldots}$ & 98 & 265 & 275 \\
\hline & & & & & & & & & 533 & $-\cdots$ & 243 \\
\hline \multirow{3}{*}{\multicolumn{9}{|c|}{$\begin{array}{l}\text { Monthly mean discharge, in cubic feet per second } \\
\text { Runoff, in inches } \\
\text { Runoff, in acre-feet }\end{array}$}} & 694 & 2,067 & 303 \\
\hline & & & & & & & & & 1.57 & 4.51 & 0.68 \\
\hline & & & & & & & & & 42,660 & 123,000 & 18,610 \\
\hline
\end{tabular}


MARCH-MAY, UPPER MISSISSIPPI RIVER BASIN

Gage height, in feet, and discharge, in cubic feet per second, at indicated time, 1965, of Upper Iova River at

\begin{tabular}{|c|c|c|c|c|c|c|c|c|c|c|c|}
\hline Date & Hour & $\begin{array}{l}\text { Gage } \\
\text { height }\end{array}$ & $\begin{array}{c}\text { Dis- } \\
\text { charge }\end{array}$ & Date & Hour & $\begin{array}{l}\text { Gage } \\
\text { height }\end{array}$ & $\begin{array}{c}\text { Dis- } \\
\text { charge }\end{array}$ & Date & Hour & $\begin{array}{c}\text { Gaoge } \\
\text { height }\end{array}$ & $\begin{array}{c}\text { Dis- } \\
\text { charge }\end{array}$ \\
\hline \multirow[t]{2}{*}{ Feb. 28} & 2400 & 5.84 & 1,220 & Mar. 1 & 1800 & 9.25 & 7,760 & \multirow[t]{11}{*}{ Mar. 2} & 2100 & 9.20 & 7,620 \\
\hline & & & & & 1900 & 9.17 & 7,530 & & 2300 & 9.29 & 7,880 \\
\hline \multirow[t]{9}{*}{ Mar. 1} & 0200 & 5.82 & 1,220 & \multirow{9}{*}{2} & 2100 & 9.28 & 7,850 & & 2400 & 8.95 & 6,900 \\
\hline & 0400 & 6.10 & 1,490 & & 2300 & 9.65 & 9,000 & & & & \\
\hline & 0530 & 6.60 & 2,100 & & 2400 & 9.51 & 8,560 & & 0200 & 7.50 & 3,590 \\
\hline & 0700 & 7.00 & 2,720 & & & & & & 0400 & 6.87 & 2,500 \\
\hline & 0800 & 8.83 & 6,580 & & 0300 & 9.89 & 9,800 & & 0700 & 6.30 & 1,720 \\
\hline & 0900 & 8.03 & 4,640 & & 0900 & 9.48 & 8,470 & & 1200 & 5.84 & 1,220 \\
\hline & 1100 & 8.90 & 6,770 & & 1300 & 9.25 & $7 ; 760$ & & 1800 & 5.61 & 1,010 \\
\hline & 1300 & 9.60 & 8,840 & & 1800 & 9.32 & 7,970 & & 2400 & 5.35 & 800 \\
\hline & 1500 & 9.16 & 7,500 & & & & & & & & \\
\hline
\end{tabular}

\section{MISSISSIPPI RIVER MAIN STEM}

(159) Mississippi River at Lansing, Iowa

(Miscellaneous site)

Location.-NE $\frac{1}{4}$ sec.29, T.99 N., R.3 W., at bridge on U.S. Highway 9 at Lansing.

Drainage area. $-66,280 \mathrm{sq} \mathrm{mi}$.

Discharge record.-Discharge obtained by current-meter measurement near peak.

Maximum.-March-May 1965: Discharge, 272,000 cfs Apr. 24, (gage height, $634.8 \mathrm{ft}$ ).

\section{WEXFORD CREEK BASIN}

(160) 5-3884. Wexford Creek near Harpers Ferry, Iowa

$$
\text { (Crest-stage station) }
$$

Location.- $-\mathrm{SE} \frac{1}{4}$ sec. 25 , T.98 N., R. 3 W., at bridge, 5 miles north of Harpers Ferry.

Drainage area. $-11.9 \mathrm{sq} \mathrm{mi}$.

Gage-height record.-Crest stages only.

Discharge record.-Stage-discharge relation defined by step-backwater corputations and current-meter measurement at $575 \mathrm{cfs}$.

Maxima.-March-May 1965: Discharge, peak stage did not reach bottom of gage. 1953 to February 1965: Discharge, 2,290 cfs July 2, 1962 (gage height, $7.03 \mathrm{ft}$ ). 


\section{PAINT CREEK BASIN}

(161) 5-3885. Paint Creek at Waterville, Iowa

Location.-Lat $43^{\circ} 12^{\prime} 35^{\prime \prime}$, long $91^{\circ} 18^{\prime} 20^{\prime \prime}$, in NW $\frac{1}{4} \mathrm{NW} \frac{1}{4}$ sec.22, T.97 N., R.4 W., on right bank $20 \mathrm{ft}$ downstream from bridge on State Highway 373 and 0.5 mile northwest of Waterville.

Drainage area. $-42.8 \mathrm{sq} \mathrm{mi}$.

Gage-height record.-Water-stage recorder graph.

Discharge record.- Stage-discharge relation defined by current-meter measurements below $3,000 \mathrm{cfs}$, extended to $9,100 \mathrm{cfs}$ by logarithmic plotting and computed results of indirect measurement further downstream. Backwater from ice Mar. 1-3, 12-26, 30,31 .

Maxima.-March-May 1965: Discharge, 1,920 cfs 2130 hours Apr. 3; gage-height, $7.60 \mathrm{ft} 1700$ hours Mar. 1 (backwater from ice).

1951 to February 1965: Discharge, 9,100 cfs August 1951 (gage height, $17.35 \mathrm{ft}$ ). A stage higher than that of August 1951 may have occurred during the spring of 1949.

Mean discharge, in cubic feet per second, 1965

\begin{tabular}{|c|c|c|c|c|c|c|c|c|c|c|c|}
\hline Day & March & April & May & Day & March & April & May & Day & March & April & May \\
\hline 2 & 900 & 534 & 4.9 & $11 \ldots$ & 7.6 & 78 & 3.4 & 21. & 2.7 & 4.7 & 3.8 \\
\hline 2. & 50 & 88 & 4.5 & $12 \ldots$ & 4.0 & 31 & 3.4 & $22 \ldots$ & 2.6 & 4.5 & 3 . \\
\hline 3. & 15 & 342 & 4.0 & $13_{-}$ & 3.1 & 12 & 3.4 & 23 & 2.5 & 4.2 & 3. \\
\hline & 6.0 & 393 & 3.9 & $14 \ldots$ & 6.0 & 8.9 & 3.4 & $24 \ldots$ & 2.5 & 4.9 & 3.5 \\
\hline 5. & 118 & 337 & 4.0 & $15 \ldots$ & 8.0 & 8.1 & 3.6 & 25 & 2.5 & 7.4 & 9.6 \\
\hline 6. & 77 & 314 & 4.0 & $16 \ldots$ & 5.0 & 6.4 & 18 & $26_{-}$ & 2.5 & 9.6 & 420 \\
\hline & 145 & 150 & 3.8 & $17 \ldots$ & 4.0 & 6.0 & 4.5 & 27 & 2.6 & 6.4 & 18 \\
\hline 8. & 79 & 186 & 3.6 & $18 \ldots$ & 3.3 & 5.6 & 14 & $28_{-}$ & 2.7 & 7.4 & 11 \\
\hline & 33 & 40 & 3.9 & 19. & 3.0 & 5.1 & 5.6 & $29_{-}$ & 2.8 & 5.8 & 8. \\
\hline 10 & 15 & 31 & 3.5 & $20 \ldots$ & 2.8 & 4.9 & 4.2 & 30. & 35 & 5.2 & 7.4 \\
\hline & & & & & & & & & 350 & ------ & 6.4 \\
\hline \multirow{2}{*}{\multicolumn{9}{|c|}{$\begin{array}{l}\text { Monthly mean discharge, in cubic feet per second } \\
\text { Runoff in inches }\end{array}$}} & 61.1 & 88.0 & 19.3 \\
\hline & & & & & & & & & 1.65 & 2.29 & 0.5 \\
\hline \multicolumn{9}{|c|}{ e-feet } & 3,760 & 5,240 & 1,190 \\
\hline
\end{tabular}

Gage height, in feet, and discharge, it cubic feet per second, at indicated time, 1965

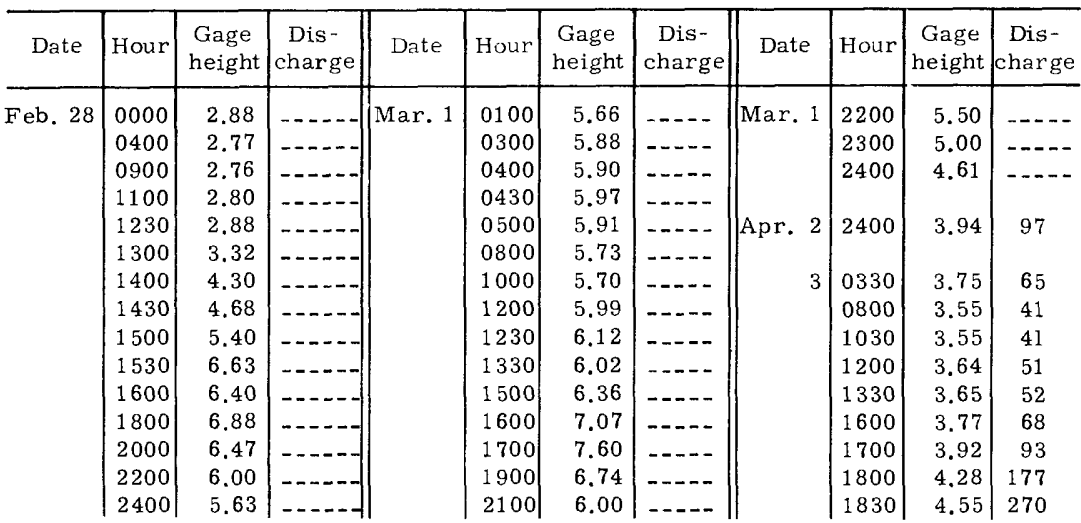


Gage height, in feet, and discharge, in cubic feet per second, at indicated time, 1965, of Paint Creek at Waterville, lowa-Continued

\begin{tabular}{|c|c|c|c|c|c|c|c|c|c|c|c|}
\hline Date & Hour & $\begin{array}{c}\text { Gage } \\
\text { height }\end{array}$ & $\begin{array}{c}\text { Dis- } \\
\text { charge }\end{array}$ & Date & Hour & $\begin{array}{l}\text { Gage } \\
\text { height }\end{array}$ & $\begin{array}{c}\text { Dis- } \\
\text { charge }\end{array}$ & Date & Hour & $\begin{array}{c}\text { Gage } \\
\text { height }\end{array}$ & $\begin{array}{c}\text { Dis- } \\
\text { charge }\end{array}$ \\
\hline Apr. 3 & $\begin{array}{l}1900 \\
1930 \\
2000 \\
2030 \\
2100 \\
2130 \\
2300 \\
2400 \\
0130\end{array}$ & $\begin{array}{l}4.88 \\
5.30 \\
5.98 \\
6.40 \\
6.90 \\
7.32 \\
6.95 \\
6.54 \\
6.00\end{array}$ & $\begin{array}{r}438 \\
690 \\
1,010 \\
1,190 \\
1,500 \\
1,920 \\
1,550 \\
1,260 \\
1,020\end{array}$ & Apr. 4 & $\begin{array}{l}0200 \\
0300 \\
0400 \\
0500 \\
0600 \\
0730 \\
0900 \\
1100 \\
1230\end{array}$ & $\begin{array}{l}5.64 \\
5.20 \\
4.87 \\
4.63 \\
4.43 \\
4.25 \\
4.12 \\
4.04 \\
4.15\end{array}$ & $\begin{array}{l}870 \\
630 \\
432 \\
305 \\
226 \\
169 \\
136 \\
118 \\
143\end{array}$ & Apr. 4 & $\begin{array}{l}1330 \\
1400 \\
1500 \\
1600 \\
1730 \\
2000 \\
2100 \\
2230 \\
2400\end{array}$ & $\begin{array}{l}4.36 \\
4.50 \\
4.85 \\
5.05 \\
5.13 \\
4.88 \\
4.68 \\
4.48 \\
4.28\end{array}$ & $\begin{array}{l}202 \\
250 \\
420 \\
540 \\
588 \\
438 \\
330 \\
243 \\
177\end{array}$ \\
\hline
\end{tabular}

(162) 5-3886. Paint Creek near Waterville, Iowa

(Crest-stage station)

Location.-Near center sec.36, T.97 N., R.4 W., at bridge 3 miles southeast of Waterville.

Drainage area. $-56.0 \mathrm{sq} \mathrm{mi}$.

Gage-height record.-Crest stages only.

Discharge record.--Stage-discharge relation defined by current-meter measurements below $1,500 \mathrm{cfs}$ and by slope-area measurements at 3,040 cfs and 10,800 cfs.

Maxima.-March-May 1965: Discharge, about 2,190 cfs Apr. 3 (gage height, $10.35 \mathrm{ft}$, backwater from ice).

1953 to February 1965: Discharge, 4,870 cfs Mar. 27, 1960 (gage height, $13.81 \mathrm{ft}$ )

Remarks.-Flood of Aug. 5, 1951 reached a stage of $17.00 \mathrm{ft}$ (discharge, 10,800 cfs).

(163) 5-3887. Little Paint Creek tributary near Waterville, Iowa

\section{(Crest-stage station)}

Location.- - SW $\frac{1}{4}$ sec.1, T.97 N., R.4 W., at culvert, $3 \frac{1}{2}$ miles northeast of Waterville.

Drainage area. $-1.09 \mathrm{sq} \mathrm{mi}$.

Gage-height record.-Crest stages only.

Discharge record.--Stage-discharge relation defined by current-meter measurements below $180 \mathrm{cfs}$ and step-backwater computations.

Maxima.-March-May 1965: Discharge, peak stage did not reach bottom of gage.

1953 to February 1965: Discharge, 404 cfs June 28, 1959 (gage height, 4.34 ft). 
(164) 5-3895. Mississippi River at McGregor, Iowa

Location.-Lat $43^{\circ} 01^{\prime} 30^{\prime \prime}$, long $91^{\circ} 10^{\prime} 20^{\prime \prime}$, in $\mathrm{SE} \frac{1}{4} \mathrm{SE} \frac{1}{4} \sec .22$, T.95 N., R.3 W'., on right bank in city park at north end of Main Street in McGregor, 2.6 miles upstream from Wiscons in River, 4.3 miles downstream from Yellow River, and at mile 633.4 from

Ohio River. Auxiliary gage located at site 14.1 miles upstream in tailwater of Dam 9.

Drainage area.- $-67,500 \mathrm{sq} \mathrm{mi}$, approximately.

Gage-height record.-Water-stage recorder graph, except Mar. 22-25. Datum of gage is $605.30 \mathrm{ft}$ above mean sea level, adjustment of 1912 , and auxiliary ge ge is $600.00 \mathrm{ft}$.

Discharge record.-Stage-discharge relation defined by current-meter measurements below 276,000 cfs and is affected by backwater from Wisconsin River and Dam 10. Fall is used as a factor. Backwater from ice Mar. 1-31.

Maxima.-March-May 1965: Daily discharge, 276,000 cfs Apr. 24. Gage height, 25.38 ft 0400 hrs Apr. 24

1936 to February 1965: Daily discharge, 197,500 cfs Apr. 22, 1952. Gage height, $20.89 \mathrm{ft}$ Apr. 23, 1952.

Maximum stage known since at least 1828, that of Apr. 24, 1965.

Remarks. - Flow regulated by reservoirs and navigation dams.

Mean gage height, in feet, and discharge, in cubic feet per second, 1965

\begin{tabular}{|c|c|c|c|c|c|c|}
\hline \multirow{2}{*}{ Day } & \multicolumn{2}{|c|}{ March } & \multicolumn{2}{|c|}{ April } & \multicolumn{2}{|c|}{ May } \\
\hline & Gage height & Discharge & Gage height & Discharge & Gage height & Discharge \\
\hline $1 \ldots-n-n$ & 7.47 & 24,700 & 8.11 & 23,100 & 22.08 & 203,000 \\
\hline $2 \ldots \ldots \ldots-\ldots$ & 8.82 & 41,000 & 8.49 & 33,200 & 21.42 & 191,000 \\
\hline $3 \ldots \ldots \ldots$ & 10.08 & 56,900 & 8.85 & 39,500 & 20.70 & 181,000 \\
\hline 4 & 11.29 & 63,300 & 9.30 & 46,800 & 19.91 & 169,000 \\
\hline $5 \ldots$ & 12.53 & 59,800 & 9.74 & 54,600 & 18.94 & 159,000 \\
\hline $6 \ldots$ & 13.35 & 59,300 & 10.39 & 64,600 & 18.08 & 150,000 \\
\hline 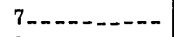 & 13.48 & 56,200 & 11.24 & 74,400 & 17.38 & 139,000 \\
\hline $8+-10-10-1$ & 13.40 & 52,500 & 12.22 & 87,000 & 16.76 & 129,000 \\
\hline 9 & 13.15 & 47,700 & 13.48 & 109,000 & 16.10 & 123,000 \\
\hline $10 \ldots$ & 12.81 & 43,000 & 14.94 & 121,000 & 15.38 & 117,000 \\
\hline $11 \ldots \ldots$ & 12.35 & 39,000 & 16.03 & 132,000 & 14.70 & 110,000 \\
\hline $12 \ldots \ldots$ & 11.70 & 35,400 & 16.78 & 140,000 & 14.17 & 106,000 \\
\hline $13 \ldots \ldots$ & 11.01 & 33,600 & 17.25 & 146,000 & 13.82 & 101,000 \\
\hline $14 \ldots \ldots$ & 10.49 & 31,400 & 17.72 & 153,000 & 13.62 & 98,800 \\
\hline $15 \ldots \ldots$ & 9.88 & 23,500 & 18.33 & 165,000 & 13.55 & 99,000 \\
\hline $16 \ldots \ldots$ & 9.18 & 23,000 & 19.02 & 173,000 & 13.58 & 101,000 \\
\hline $17 \ldots \ldots$ & 8.75 & 19,100 & 19.88 & 185,000 & 13.55 & 99,000 \\
\hline $18 \ldots$ & 8.87 & 18,100 & 20.80 & 196,000 & 13.50 & 100,000 \\
\hline $19 \ldots \ldots$ & 8.70 & 18,700 & 21.92 & 211,000 & 13.42 & 97,200 \\
\hline $20 \ldots \ldots$ & 8.51 & 19,100 & 23.27 & 238,000 & 13.37 & 96,100 \\
\hline $21 \ldots \ldots$ & 8.27 & 16,200 & 24.31 & 256,000 & 13.45 & 95,900 \\
\hline $22 \ldots \ldots \ldots$ & 7.95 & 16,300 & 24.98 & 268,000 & 13.62 & 95,500 \\
\hline $23 \ldots \ldots$ & 7.94 & 16,700 & 25.32 & 275,000 & 13.78 & 96,000 \\
\hline $24 \ldots \ldots \ldots$ & 7.94 & 16,700 & 25.36 & 276,000 & 13.82 & 94,900 \\
\hline $25 \ldots \ldots$ & 7.94 & 16,600 & 25.21 & 270,000 & 13.70 & 96,300 \\
\hline $26 \ldots \ldots$ & 7.98 & 16,400 & 24.88 & 264,000 & 13.58 & 95,500 \\
\hline $27 \ldots \ldots$ & 8.05 & 16,500 & 24.39 & 255,000 & 13.42 & 96,100 \\
\hline $28 \ldots$ & 8.08 & 17,200 & 23.89 & 242,000 & 13.18 & 93,400 \\
\hline $29 \ldots \ldots$ & 8.11 & 18,000 & 23.27 & 231,000 & $12 . \varepsilon 3$ & 89,000 \\
\hline $30 \ldots$ & 8.02 & 16,100 & 22.71 & 216,000 & 12.67 & 87,100 \\
\hline $31 \ldots$ & 7.84 & 17,100 & $-\cdots-\cdot-\cdot-\cdot$ & $-\cdots \cdots$ & 12.60 & 84,300 \\
\hline \multirow{3}{*}{\multicolumn{2}{|c|}{$\begin{array}{l}\text { Mean } \\
\text { Inches } \\
\text { Acre-feet }\end{array}$}} & 30,620 & \multicolumn{2}{|r|}{164,800} & \multicolumn{2}{|r|}{115,900} \\
\hline & & 0.52 & \multicolumn{2}{|r|}{2.72} & \multirow{2}{*}{\multicolumn{2}{|c|}{$\begin{array}{r}1.98 \\
7,127,000\end{array}$}} \\
\hline & & $1,883,000$ & & $9,809,000$ & & \\
\hline
\end{tabular}


Anmual maximum stages and mean daily discharges, for indicated years, of Mississippi River at McGregor, Iowa

\begin{tabular}{|c|c|c|c|c|c|c|c|}
\hline Year & Date & $\begin{array}{l}\text { Gage } \\
\text { height } \\
\text { (feet) }\end{array}$ & $\begin{array}{c}\text { Discharge } \\
\text { (cfs) }\end{array}$ & Year & Date & $\begin{array}{l}\text { Gage } \\
\text { height } \\
\text { (feet) }\end{array}$ & $\begin{array}{c}\text { Discharge } \\
\text { (cfs) }\end{array}$ \\
\hline 1826 & May & $\mathrm{a}_{26.0}$ & ------- & 1948. & Apr. 11 & 12.81 & 84,000 \\
\hline $1880^{-}$ & $J$ une 22 & 21.5 & 196,000 & 1949 & Apr. 4,13 & 11.42 & 73,100 \\
\hline 1881 & Oct. 21 & ${ }^{2} 19.0$ & $---n--n$ & 1950 & May 17 & 15.26 & 123,000 \\
\hline 1888 & May 13 & $a_{20.1}$ & $--n---n$ & 1951 & Apr. 22 & 20.83 & 185,700 \\
\hline 1916 & May 1 & 18.3 & --n-n-n & 1952 & Apr. 22 & 20.89 & 197,500 \\
\hline $1920_{-}$ & Apr. 4,5 & 19.6 & $----n---$ & 1953 & July 3 & $\mathrm{~d}_{12.90}$ & 86,200 \\
\hline 1922 & Apr. 19 & $\mathrm{a}_{19.4}$ & $--n-1$ & 1954 & May 9 & 18.69 & 165,500 \\
\hline 1937. & May 6,9 & 16.68 & 54,700 & 1955 & Apr. 14 & 12.20 & 73,700 \\
\hline 1938 & May 27 & 23.75 & 101,400 & 1956 & Apr. 15,16 & 14.88 & 105,000 \\
\hline 1939. & Apr. 6 & 15.76 & 96,900 & 1957 & July 8 & 12.80 & 95,800 \\
\hline 1940 . & Apr. 19 & 10.50 & 52,100 & 1958. & Apr. 14 & 8.98 & 55,800 \\
\hline 1941 & Apr. 20 & 14.66 & 102,800 & 1959 & Apr. 3 & 12.32 & 72,300 \\
\hline 1942 . & June 7 & 17.38 & 113,800 & 1960 & $J$ une 4,5 & 16.08 & 83,100 \\
\hline 1943 . & $\mathrm{J}$ une 28 & 16.89 & 124,600 & 1961 & Mar. 29,30 & 15.47 & 114,000 \\
\hline $1944 \ldots$ & $\mathrm{J}$ une 24 & 16.17 & 122,500 & 1962 & Apr. $18-20$ & 15.12 & 104,000 \\
\hline 1945. & Mar. 29 & 16.80 & 127,700 & 1963 & Apr. 1 & 11.84 & 72,000 \\
\hline $1946 \ldots$ & Mar. 29 & 15.84 & 101,200 & 1964. & May 19 & 11.56 & 75,600 \\
\hline $1947 \ldots$ & Apr. 21 & 13.70 & 85,500 & 1965 & Apr. 24 & 25.38 & 276,000 \\
\hline
\end{tabular}

${ }^{2}$ At Prairie du Chien, Wisconsin. Zero of gage at elevation $605.4 \mathrm{ft}$ above mean sea level, adjustment of 1912 .

${ }^{b}$ Occurred Sept. $18,1938$.

Cocurred June 17, 1940.

Occurred Mar. 31, 1953.

e Occurred May 14, 15, 1960. 


\section{WISCONSIN RIVER BASIN}

(165) 5-3901. Lac Vieux Desert near Land O'Lakes, Wis.

Location.—Lat $46^{\circ} 07^{\prime} 20^{\prime \prime}$, long $89^{\circ} 09^{\prime} 10^{\prime \prime}$, in $\mathrm{SE}_{\frac{1}{4}} \mathrm{NE}^{\frac{1}{4}}$ sec.17, T.42 N., R.11 E., head of Wisconsin River, 5 miles southeast of village of Land O'Lakes.

Drainage area. -28 sq mi, approximately.

Gage-height record.-Staff gage. Datum of gage is $1,679.53 \mathrm{ft}$ above mean sea level, datum of 1929 .

Maxima.-March to May 1965: Contents at end of week, 444 million cubic feet May 16 (gage height, 2 ft 0.5 in).

1938 to February 1965: Contents on last day of month, 556 million cubic feet Apr. 30, 1951 (gage height, $2 \mathrm{ft} 6-3 / 4 \mathrm{in}$ ).

Remarks.--Reservoir operation began in 1908. Usable capacity 652 million cubic feet between gage heights $-0 \mathrm{ft} 2$ in and $3 \mathrm{ft} 0$ in (limits stipulated by Public Service Commission of Wisconsin).

Cooperation.-Gage heights and capacity tables furnished by Wisconsin Valley Improvement Co.

Gage height, in feet and inches, and contents, in millions of cubic feet, 1965

\begin{tabular}{|c|c|c|c|c|c|c|c|}
\hline \multirow{2}{*}{ Date } & \multicolumn{2}{|c|}{ Gage height } & \multirow{2}{*}{ Contents } & \multirow{2}{*}{ Date } & \multicolumn{2}{|c|}{ Gage height } & \multirow{2}{*}{ Contents } \\
\hline & Feet & Inches & & & Feet & Inches & \\
\hline $\begin{array}{lr}\text { Feb. } & 28 \ldots \\
\text { Mar. } & 7 \ldots \\
& 14 \\
21 & \\
& 28 \\
& \\
\text { Apr. } & 4 \\
& 11\end{array}$ & $\begin{array}{r}-0 \\
-0 \\
-0 \\
-0 \\
-0 \\
0 \\
0\end{array}$ & $\begin{array}{l}2 \\
2 \\
2 \\
2 \\
2 \\
.75 \\
1.5\end{array}$ & $\begin{array}{r}0 \\
0 \\
0 \\
0 \\
0 \\
44 \\
56\end{array}$ & \begin{tabular}{|rr} 
Apr. 18 & $\ldots$ \\
& 25 \\
May & $2 \ldots$ \\
& 9 \\
& $16 \ldots$ \\
& $23 \ldots$ \\
& $30 \ldots$
\end{tabular} & $\begin{array}{l}0 \\
0 \\
1 \\
1 \\
2 \\
2 \\
1\end{array}$ & $\begin{array}{c}4 \\
8 \\
1 \\
7.5 \\
.5 \\
0 \\
10.5\end{array}$ & $\begin{array}{r}96 \\
161 \\
246 \\
356 \\
444 \\
435 \\
408\end{array}$ \\
\hline
\end{tabular}

(166) 5-3901.5 Twin Lakes near Phelps, Wis.

Location.-Lat $46^{\circ} 01^{\prime} 20^{\prime \prime}$, long $89^{\circ} 10^{\prime} 10^{\prime \prime}$, in SW $\frac{1}{4} \mathrm{NE}_{\frac{1}{4}}$ Sec.19, T.41 N., R.11 E., on Twin River, 5 miles southwest of Phelps.

Drainage area.- $-26 \mathrm{sq} \mathrm{mi,} \mathrm{approximately.}$

Gage-height record.- - Staff gage. Altitude of gage is $1,640 \mathrm{ft}$ (from river-profile map).

Maxima.--March to May 1965: Contents at end of week, 286 million cubic feet May 16, 30 (gage height, $2 \mathrm{ft} 2$ in).

1938 to February 1965: Contents on last day of month, 330 million cubic feet April 30, 1947 (gage height, 2 ft $5 \frac{1}{4}$ in).

Remarks.- - Reservoir operation began in 1908. Reservoir includes Nortt and South Twin Lakes. Usable capacity Oct. 1 to May 31,313 million cubic feet between gage heights $0 \mathrm{ft} 4$ in and $2 \mathrm{ft} 4 \mathrm{in}$, and June 1 to Sept. 30, 261 million cubic feet between gage heights $0 \mathrm{ft} 8$ in and $2 \mathrm{ft} 4$ in (limits stipulated by Public Service Commission of Wisconsin).

Cooperation.-Gage heights and capacity tables furnished by Wisconsin Valley Improvement Co. 
Gage height, in feet and inches, and contents, in millions of cubic feet, 1965, of Twin Lakes near Phelps, Wis.

\begin{tabular}{|c|c|c|c|c|c|c|c|}
\hline \multirow{2}{*}{ Date } & \multicolumn{2}{|c|}{ Gage height } & \multirow{2}{*}{ Contents } & \multirow{2}{*}{ Date } & \multicolumn{2}{|c|}{ Gage height } & \multirow{2}{*}{ Contents } \\
\hline & Feet & Inches & & & Feet & Inches & \\
\hline Feb. 28_.. & 0 & 4 & 0 & Apr. $18 \ldots$ & 0 & 8 & 52 \\
\hline Mar. $\eta_{\ldots}$ & 0 & 4 & 0 & $25 \ldots$ & 0 & 11 & 91 \\
\hline $14 \ldots$ & 0 & 4 & 0 & May $\quad 2 \ldots$ & 1 & 2.5 & 136 \\
\hline $21 \ldots$ & 0 & 4 & 0 & $9 \ldots$ & 1 & 7 & 195 \\
\hline $28 \ldots$ & 0 & 4 & 0 & $16 \ldots$ & 2 & 2 & 286 \\
\hline Apr. $\quad 4 \ldots$ & 0 & 5 & 13 & $23 \ldots$ & 2 & 1.5 & 280 \\
\hline $11 \ldots$ & 0 & 7 & 39 & $30 \ldots$ & 2 & 2 & 286 \\
\hline
\end{tabular}

(167) 5-3902. Buckatabon Lake near Conover, Wis.

Location.-Lat $46^{\circ} 01^{\prime} 15^{\prime \prime}$, long $89^{\circ} 18^{\prime} 35^{\prime \prime}$, in $\mathrm{SE} \frac{1}{4} \mathrm{NE} \frac{1}{4} \sec .24, \mathrm{~T} .41$ N., R.9 E., on Buckatabon Creek, 3.3 miles southwest of Conover.

Drainage area.-14 sq $\mathrm{mi}$, approximately.

Gage-height record.- Staff gage. Datum of gage is $1,637.85 \mathrm{ft}$ above mean sea level (levels by Wisconsin Valley Improvement Co.).

Maxima.-March to May 1965: Contents at end of week, 126 million cubic feet May 16 (gage height, 3 ft 7 in).

1938 to February 1965: Contents on last day of month, 128 million cubic feet

Sept. 30, 1943 (gage height, $3 \mathrm{ft} 7 \frac{1}{2} \mathrm{in}$ ).

Remarks.--Reservoir operation began in 1908. Reservoir includes Upper and Lower Buckatabon Lakes. Usable capacity 130 million cubic feet between gage heights $0 \mathrm{ft}$ 6 in and $3 \mathrm{ft} 8$ in (limits stipulated by Public Service Commission of Wisconsir).

Cooperation.--Gage heights and capacity tables furnished by Wisconsin Valley Inprovement Co.

Gage height, in feet and inches, and contents, in millions of cubic feet, 1965

\begin{tabular}{|c|c|c|c|c|c|c|c|}
\hline \multirow{2}{*}{ Date } & \multicolumn{2}{|c|}{ Gage height } & \multirow{2}{*}{ Contents } & \multirow{2}{*}{ Date } & \multicolumn{2}{|c|}{ Gage height } & \multirow{2}{*}{ Contents } \\
\hline & Feet & Inches & & & Feet & Inches & \\
\hline Feb. 28_.. & 0 & 6 & 0 & Apr. $18 \ldots$ & 1 & 10 & 49 \\
\hline Mar. $7 \ldots$ & 0 & 6.5 & 2 & $25 \ldots$ & 2 & 4 & 70 \\
\hline 14 & 0 & 6.5 & 2 & May 2... & 2 & 8 & 84 \\
\hline $21 \ldots$ & 0 & 6.5 & 2 & $9 \ldots$ & 3 & 2.5 & 108 \\
\hline $28 \ldots$ & 0 & 6.5 & 2 & $16 \ldots$ & 3 & 7 & 126 \\
\hline Apr. $\quad 4 \ldots$ & 0 & 11.5 & 16 & $23 \ldots$ & 3 & 4.5 & 116 \\
\hline $11 \ldots$ & 1 & 4 & 30 & $30 \ldots$ & 3 & 4.5 & 116 \\
\hline
\end{tabular}


(168) 5-3902.5 Sevenmile Lake near Eagle River, Wis.

Location.-Lat $45^{\circ} 52^{\prime} 30^{\prime \prime}$, long $89^{\circ} 04^{\prime} 10^{\prime \prime}$, in $\mathrm{SE}_{\frac{1}{4}} \mathrm{NE} \frac{1}{4} \sec .11$, T.39 N., R.11 E., on Sevenmile Creek, 9.1 miles southeast of village of Eagle River

Drainage area.-14 sq mi, approximately.

Gage-height record.-Staff gage. Datum of gage is 1,646.30 ft above mean sea level (levels by Wisconsin Valley Improvement Co.).

Maxima.- March to May 1965: Contents at end of week, 91 million cubic feet May 16 (gage height, $4 \mathrm{ft} 9 \mathrm{in}$ ).

1938 to February 1965: Contents on last day of month, 89 million cubic feet June 30 and Aug. 31, 1953 and June 30 and July 31, 1961 (gage height, 4 ft 8 in).

Remarks.-Reservoir operation began in 1908. Usable capacity 93 million cubic feet between gage heights $0 \mathrm{ft} 6$ in and $4 \mathrm{ft} 10$ in (limits stipulated by Public Service Commission of Wisconsin).

Cooperation.-Gage heights and capacity tables furnished by Wisconsin Valley Improvement Company.

Gage herght, in feet and inches, and contents, in millions of cubic feet. 1965

\begin{tabular}{|c|c|c|c|c|c|c|c|}
\hline \multirow{2}{*}{ Date } & \multicolumn{2}{|c|}{ Gage height } & \multirow{2}{*}{ Contents } & \multirow{2}{*}{ Date } & \multicolumn{2}{|c|}{ Gage height } & \multirow{2}{*}{ Contents } \\
\hline & Feet & Inches & & & Feet & Inches & \\
\hline Feb, 28... & 0 & 6 & 0 & Apr. $18 \ldots$ & 2 & 4 & 37 \\
\hline Mar. $7 \ldots$ & 0 & 6 & 0 & $25 \ldots$ & 3 & 0 & 52 \\
\hline 14 & 0 & 6 & 0 & May 2_.- & 3 & 8 & 66 \\
\hline $21 \ldots$ & 0 & 6 & 0 & $9 \ldots$ & 4 & 7 & 87 \\
\hline $28 \ldots$ & 0 & 6 & 0 & $16 \ldots$ & 4 & 9 & 91 \\
\hline Apr. $4 \ldots$ & 1 & 2 & 12 & $23 \ldots$ & 4 & 7 & 87 \\
\hline $11 \ldots$ & 1 & 6 & 20 & $30 \ldots$ & 4 & 7 & 87 \\
\hline
\end{tabular}

(169) 5-3903. Lower Ninemile Lake near Eagle River, Wis.

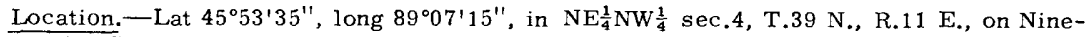
mile Creek, 6.6 miles southeast of village of Eagle River.

Drainage area. $-25 \mathrm{sq} \mathrm{mi}$, approximately.

Gage-height record.-Staff gage. Datum of gage is $1,638.27 \mathrm{ft}$ above mean sea level (levels of Wiscons in Valley Improvement Co.).

Maxima.--March to May 1965: Contents at end of week, 136 million cubic feet May 16 (gage height, $6 \mathrm{ft} 5 \mathrm{in}$ ).

1938 to February 1965: Contents on last day of month,130 million cubic feet July 31 , 1961 (gage height, $6 \mathrm{ft} 3 \mathrm{in}$ ).

Remarks. - Reservoir operation began in 1908. Usable capacity 121 million cubic feet between gage heights $1 \mathrm{ft} 5$ in and $6 \mathrm{ft} 0$ in (limits stipulated by Public Service Commission of Wisconsin).

Cooperation.-Gage heights and capacity tables furnished by Wisconsin Valley Improvement $\mathrm{Co}$. 
Gage height, in feet and inches, and contents, in millions of cubic feet. 1965, of Lower Nincmile Lake near Eagle River Wis.

\begin{tabular}{|c|c|c|c|c|c|c|c|}
\hline \multirow{2}{*}{ Date } & \multicolumn{2}{|c|}{ Gage height } & \multirow{2}{*}{ Contents } & \multirow{2}{*}{ Date } & \multicolumn{2}{|c|}{ Gage height } & \multirow{2}{*}{ Contents } \\
\hline & Feet & Inches & & & Feet & Inches & \\
\hline Feb. 28 & 1 & 5 & 0 & Apr. $18 \ldots$ & 4 & 10 & 80 \\
\hline Mar. $7 .$. & 1 & 5 & 0 & $25 \ldots$ & 5 & 6 & 103 \\
\hline $14 \ldots$ & 1 & 5 & 0 & May & 5 & 11 & 118 \\
\hline $21 \ldots$ & 1 & 5 & 0 & $9 \ldots$ & 6 & 4 & 133 \\
\hline $28 \ldots$ & 1 & 5 & 0 & $16 \ldots$ & 6 & 5 & 136 \\
\hline Apr. 4 & 3 & 1 & 32 & $23_{-}-$ & 6 & 3 & 130 \\
\hline $11 \ldots$ & 3 & 9 & 48 & $30 \ldots$ & 6 & 3 & 130 \\
\hline
\end{tabular}

(170) 5-3903.5 Burnt Rollways Reservoir near Eagle River, Wis.

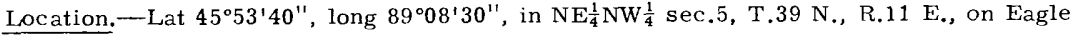
River, 5.3 miles southeast of village of Eagle River.

Drainage area.-129 sq mi, approximately.

Gage-height record.-Staff gage. Altitude of gage is 1,620 ft (from river-profile map).

Maxima.-March to May 1965: Contents at end of week, 779 million cubic feet May 9, $16,23,30$ (gage height, $1 \mathrm{ft} 6 \mathrm{in}$ ).

1938 to February 1965: Contents on last day of month, 779 million cubic fest at times (gage height, $1 \mathrm{ft} 6 \mathrm{in}$ ).

Remarks.--Reservoir operation began in 1908. Reservoir includes Whitefish, Rig, Dog, Deer, Mud, Big Stone, Moccasin, Spirit, Medicine, Little Fork, Fourmile, Big Fork, Island, Round, Town Line, Range Line, Planting Ground, and Long Lakes. Us able capacity Nov. 1 to Apr. 30, 779 million cubic feet between gage heights $-1 \mathrm{ft} 3$ in and $1 \mathrm{ft} 6$ in and May 1 to Oct. 31, 471 million cubic feet between gage heights $0 \mathrm{ft} 0$ in and $1 \mathrm{ft} 6$ in (limits stipulated by Public Service Commission of Wisconsin).

Cooperation.-Gage heights and capacity tables furnished by Wisconsin Valley Improvement Co.

Gage height, in fect and inches, and contents, in millions of cubic feet, 1965

\begin{tabular}{|c|c|c|c|c|c|c|c|}
\hline \multirow{2}{*}{ Date } & \multicolumn{2}{|c|}{ Gage height } & \multirow{2}{*}{ Contents } & \multirow{2}{*}{ Date } & \multicolumn{2}{|c|}{ Gage height } & \multirow{2}{*}{ Contents } \\
\hline & Feet & Inches & & & Feet & Inches & \\
\hline Feb. 28_.- & -1 & 2 & 19 & Apr. $18 \ldots$ & 0 & 1 & 333 \\
\hline Mar. 7... & -1 & 1 & 39 & $25 \ldots$ & 0 & 11.5 & 604 \\
\hline 14 & -1 & 0 & 58 & May & 1 & 5.5 & 766 \\
\hline $21 \ldots$ & -0 & 11.5 & 68 & $9 \ldots$ & 1 & 6 & 779 \\
\hline $28 \ldots$ & -0 & 9.5 & 107 & $16 \ldots$ & 1 & 6 & 779 \\
\hline Apr. $\quad 4 \ldots$ & -0 & 7.5 & 148 & $23-\ldots$ & 1 & 6 & 779 \\
\hline $11 \ldots$ & -0 & 5 & 200 & $30 \ldots$ & 1 & 6 & 779 \\
\hline
\end{tabular}


(171) 5-3904. Long Lake near Phelps, Wis.

Location.-Lat $46^{\circ} 02^{\prime} 45^{\prime \prime}$, long $89^{\circ} 02^{\prime} 35^{\prime \prime}$, in NW $\frac{1}{4} \mathrm{SE} \frac{1}{4}$ sec.7, T.41 N., K.12 E., on Deerskin River, 2.5 miles southeast of Phelps.

Drainage area.- -35 sq mi, approximately.

Gage-height record.-Staff gage. Datum of gage is $1,695.14 \mathrm{ft}$ above mean sea level (levels by Wisconsin Valley Improvement Co.).

Maxima.--March to May 1965: Contents at end of week, $371 \mathrm{million}$ cubic feet May 16 (gage height $3 \mathrm{ft} 9 \mathrm{in}$ ).

1938 to February 1965: Contents on last day of month, 332 million cubic feet June 30,1953 (gage height, $3 \mathrm{ft} 5 \mathrm{in}$ ).

Remarks.- -Reservoir operations began in 1908. Usable capacity Oct. 1 to May 31 , $400 \mathrm{million}$ cubic feet between gage heights $0 \mathrm{ft} 4$ in and $4 \mathrm{ft} 0$ in and June 1 to Sept. 30, 332 million cubic feet between gage heights $1 \mathrm{ft} 0$ in and $4 \mathrm{ft} 0$ in (Iimits stipulated by Public Service Commission of Wisconsin).

Cooperation.-Gage heights and capacity tables furnished by Wisconsin Valley Improvement Co.

Gage height, in feet and inches, and contents, in millons of cubic feet, 1965

\begin{tabular}{|c|c|c|c|c|c|c|c|}
\hline \multirow{2}{*}{ Date } & \multicolumn{2}{|c|}{ Gage height } & \multirow{2}{*}{ Contents } & \multirow{2}{*}{ Date } & \multicolumn{2}{|c|}{ Gage heigl ${ }^{\dagger}$} & \multirow{2}{*}{ Contents } \\
\hline & Feet & Inches & & & Feet & Inches & \\
\hline Feb. $28 \ldots$ & 0 & 4 & 0 & Apr. $18 \ldots$ & 1 & 0.5 & 72 \\
\hline Mar. $7 . .-$ & 0 & 4 & 0 & $25 \ldots$ & 2 & 3.5 & 206 \\
\hline 14 & 0 & 4 & 0 & May & 2 & 9.5 & 261 \\
\hline $21 \ldots$ & 0 & 4 & 0 & $9 .$. & 3 & 4 & 323 \\
\hline $28 \ldots$ & 0 & 4 & 0 & $16 \ldots$ & 3 & 9 & 371 \\
\hline Apr. 4 & 0 & 5 & 8 & $23 \ldots$ & 3 & 5 & 332 \\
\hline $11 \ldots$ & 0 & 7 & 25 & $30 \ldots$ & 3 & 3 & 313 \\
\hline
\end{tabular}

(172) 5-3906. Deerskin Lake near Eagle River, Wis.

Location.-Lat $45^{\circ} 59^{\prime} 05^{\prime \prime}$, long $89^{\circ} 09^{\prime} 40^{\prime \prime}$, in SE⿺ $\frac{1}{4} \mathrm{sec}, 31$, T.41 N., R.11 E., on Deerskin Creek, 6.3 miles northeast of village of Eagle River.

Drainage area. $-5 \mathrm{sq} \mathrm{mi}$, approximately.

Gage-height record.-Staff gage. Datum of gage is $1,640.16 \mathrm{ft}$ above rean sea level (levels by Wisconsin Valley Improvement Co.).

Maxima.-March to May 1965: Contents at end of week, 14 million cubic feet May 16 (gage height, 1 ft 5 in).

1938 to February 1965: Contents on last day of month, 22 million, cubic feet Oct. 31, 1938, May 31, and June 30, 1939, Apr. 30 and May 31, 1941, Sept. 30, 1959 (gage height, 2 ft 0 in).

Remarks. - The lake has been used as a reservoir since 1908. Usable capacity 22 million cubic feet between gage height $0 \mathrm{ft} 4$ in and $2 \mathrm{ft} 0$ in (limits stipulated by Public Service Commission of Wisconsin).

Cooperation,-Gage heights and capacity tabIes furnished by Wisconsin Valley Improvement Co. 
Gage height, in feet and inches, and contents, in millions of cubic feet, 1965, of Deerskin Lake near Eagle River, Wis.

\begin{tabular}{|c|c|c|c|c|c|c|c|}
\hline \multirow{2}{*}{ Date } & \multicolumn{2}{|c|}{ Gage height } & \multirow{2}{*}{ Contents } & \multirow{2}{*}{ Date } & \multicolumn{2}{|c|}{ Gage height } & \multirow{2}{*}{ Contents } \\
\hline & Feet & Inches & & & Feet & Inches & \\
\hline Feb. 28_.. & 0 & 4 & 0 & Apr. $18 \ldots$ & 0 & 8 & 4 \\
\hline Mar. $7 \ldots$ & 0 & 4 & 0 & $25 \ldots$ & 0 & 9 & 5 \\
\hline $14 \ldots$ & 0 & 4 & 0 & May 2... & 0 & 11 & 7 \\
\hline $21 \ldots$ & 0 & 4 & 0 & $9 \ldots$ & 1 & 0 & 8 \\
\hline $28 \ldots$ & 0 & 4 & 0 & $16 \ldots$ & 1 & 5 & 14 \\
\hline Apr. $\quad 4 \ldots$ & 0 & 4 & 0 & $23 \ldots$ & 1 & 3.5 & 12 \\
\hline $11 \ldots$ & 0 & 7.5 & 4 & $30 \ldots$ & 1 & 3 & 11 \\
\hline
\end{tabular}

(173) 5-3906.5 Sugar Camp Reservoir near Eagle River, Wis.

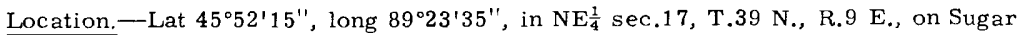
Camp Creek, 7.6 miles southwest of village of Eagle River.

Drainage area. $-59 \mathrm{sq} \mathrm{mi}$, approximately.

Gage-height record.- Staff gage. Datum of gage is $1,591.94 \mathrm{ft}$ above mean sea level (levels by Wiscons in Valley Improvement Co.).

Maxima.-March to May 1965: Contents at end of week, 471 million cubic feet May 9 (gage height, $6 \mathrm{ft} 6$ in).

1938 to February 1965: Contents on last day of month, 471 million cubic feet Apr. 30, 1939, Sept. 30, Oct. 31, Nov. 30, Dec. 31, 1941, Sept. 30 and Nov. 30, 1942 (gage height $6 \mathrm{ft} 6 \mathrm{in}$ ).

Remarks.--Reservoir operation began in 1908. Reservoir includes Chain, Echo, Stone, Sand, and Dam Lakes. Usable capacity Sept, 15 to May 31, 471 million cubic feet between gage height $1 \mathrm{ft} 0$ in and $6 \mathrm{ft} 6$ in and June 1 to Sept. 14, 180 million cubic feet between gage height $4 \mathrm{ft} 0$ in and $6 \mathrm{ft} 0$ in (limits stipulated by Public Service Commission of Wisconsin).

Cooperation.-Gage heights and capacity tables furnished by Wisconsin Valley Improvement Co.

Gage height, in feet and inches, and contents, in millions of cubic feet, 1965

\begin{tabular}{|c|c|c|c|c|c|c|c|}
\hline \multirow{2}{*}{ Date } & \multicolumn{2}{|c|}{ Gage height } & \multirow{2}{*}{ Contents } & \multirow{2}{*}{ Date } & \multicolumn{2}{|c|}{ Gage height } & \multirow{2}{*}{ Contents } \\
\hline & Feet & Inches & & & Feet & Inches & \\
\hline Feb. 28_.. & 1 & 9 & 56 & Apr. $18 \ldots$ & 3 & 5 & 193 \\
\hline Mar. 7_.. & 1 & 9 & 56 & $25 \ldots$ & 5 & 2 & 345 \\
\hline 14 & 1 & 9 & 56 & May $2 \ldots$ & 6 & 2 & 439 \\
\hline $21 \ldots$ & 1 & 9 & 56 & $9 \ldots$ & 6 & 6 & 471 \\
\hline $28 \ldots$ & 1 & 9 & 56 & $16 \ldots$ & 6 & 4 & 455 \\
\hline Apr. $4 \ldots$ & 1 & 9 & 56 & $23 \ldots$ & 6 & 1 & 431 \\
\hline $11 \ldots$ & 2 & 4 & 102 & $30 \ldots$ & 6 & 0 & 423 \\
\hline
\end{tabular}


(174) 5-3907. Little St. Germain Lake near Eagle River, Wis.

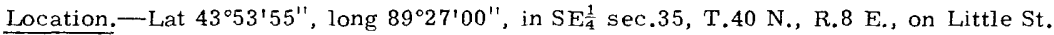
Germain Creek, 9.6 miles west of village of Eagle River.

Drainage area.--19 sq mi, approximately.

Gage-height record.-Staff gage. Datum of gage is $1,611.54 \mathrm{ft}$ above mean sea level (levels by Wisconsin Valley Improvement Co.).

Maxima.- - March to May 1965: Contents at end of week, 83 million cubic feet May 16 (gage height, 3 ft 1 in).

1938 to February 1965: Contents on last day of month, 83 million cubic feet Aug. 31, 1941 (gage height 3 ft 1 in).

Remarks. - The lake has been used as a reservoir since 1908. Usable capacity 79 million cubic feet between gage height $1 \mathrm{ft} 2$ in and $3 \mathrm{ft} 0$ in (limits stipulated by Public Service Commission of Wisconsin).

Cooperation.-Gage heights and capacity tables furnished by Wisconsin Valley Improvement Co.

Gage height, in feet and inches, and contents, in millons of cublc feet, 1965

\begin{tabular}{|c|c|c|c|c|c|c|c|}
\hline \multirow{2}{*}{ Date } & \multicolumn{2}{|c|}{ Gage height } & \multirow{2}{*}{ Contents } & \multirow{2}{*}{ Date } & \multicolumn{2}{|c|}{ Gage height } & \multirow{2}{*}{ Contents } \\
\hline & Feet & Inches & & & Feet & Inches & \\
\hline Feb. 28... & 1 & 3 & 4 & Apr. $18 \ldots$ & 1 & 10 & 29 \\
\hline Mar. 7 & 1 & 3.5 & 6 & $25 \ldots$ & 2 & 2 & 43 \\
\hline 14 & 1 & 3 & 4 & May 2... & 2 & 5.5 & 56 \\
\hline $21 \ldots$ & 1 & 3 & 4 & $9 \ldots$ & 2 & 11.5 & 77 \\
\hline $28 \ldots$ & 1 & 2.5 & 2 & $16 \ldots$ & 3 & 1 & 83 \\
\hline Apr. $4 \ldots$ & 1 & 4 & 7 & $23 \ldots$ & 2 & 11.5 & 77 \\
\hline $11 \ldots$ & 1 & 7 & 18 & $30 \ldots$ & 2 & 11 & 75 \\
\hline
\end{tabular}

(175) 5-3907.5 Big St. Germain Lake near Lake Tomahawk, Wis.

Location.-Lat $45^{\circ} 55^{\prime} 10^{\prime \prime}$, long $89^{\circ} 31^{\prime} 55^{\prime \prime}$, in $\mathrm{SE} \frac{1}{4} \mathrm{sec} .30$, T.40 N., R.8 E., on St. Germain River, 7.8 miles northeast of village of Lake Tomahawk.

Drainage area.-69 sq mi, approximately.

Gage-height record.-Staff gage. Datum of gage is $1,588.32 \mathrm{ft}$ above mean sea level (levels by Public Service Commission of Wisconsin).

Maxima.-March to May 1965: Contents at end of week, 196 million cubic feet May 9 (gage height 3 ft 5 in).

1938 to February 1965: Contents on last day of month, 202 million cubic feet Dec, 31, 1946 (gage height, 3 ft 6 in).

Remarks. - The lake has been used as a reservoir since 1908. Reservoir includes Lake Content, and Big St. Germain and Fawn Lakes. Usable capacity, Sert. 15 to May 31, 202 million cubic feet between gage height $0 \mathrm{ft} 6$ in and $3 \mathrm{ft} 6$ in and June 1 to Sept. 14, 91 million cubic feet between gage height $1 \mathrm{ft} 8$ in and $3 \mathrm{ft} 0$ in (limits stipulated by Public Service Commission of Wisconsin.

Cooperation.-Gage heights and capacity tables furnished by Wisconsin Valley Improvement Co. 
Gage height, in feet and inches, and contents, in millions of cubic fect, 1965, of Big St. Germain Lake niar Lake Tomahawk, Wis.

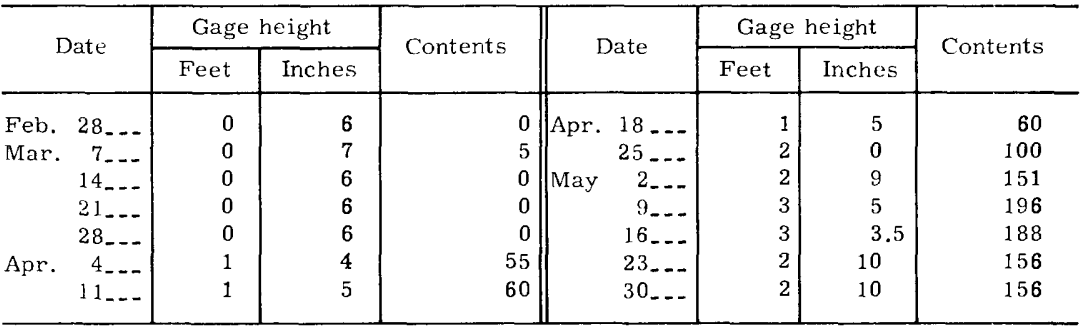

(176) 5-3908. Pickerel Lake near Lake Tomahawk, Wis.

Location,-Lat $45^{\circ} 52^{\prime} 20^{\prime \prime}$, Iong $89^{\circ} 31^{\prime} 45^{\prime \prime}$, in NE⿺ $\frac{1}{4}$ sec. 18 T.39 N., R. 8 E., on St. Germain River 5 miles northeast of village of Lake Tomahawk.

Drainage area. $-78 \mathrm{sq} \mathrm{mi}$, approximately.

Gage-height record.-Staff gage. Datum of gage is $1,582.00 \mathrm{ft}$ above mean sea level (levels by Wiscons in Valley Improvement Co.).

Maxima.-March to May 1965: Contents at end of week, 290 million cubic feet May 16 (gage height, $9 \mathrm{ft} 0 \mathrm{in}$ ).

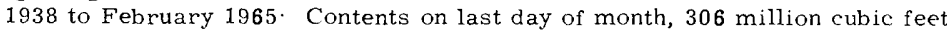
Dec. 31, 1938, Apr. 30, 1947, Sept. 30, 1959 (gage height, 9 ft 4 in).

Remarks.-The dam was built in 1935. Usable capacity Sept. 15 to May 31,338 million cubic feet between gage height $0 \mathrm{ft} 0$ in and $10 \mathrm{ft} 0$ in and June 1 to Sept. 14, 93 million cubic feet between gage height $8 \mathrm{ft} 0$ in and $10 \mathrm{ft} 0$ in (levels stipulated by Public Service Commission of Wisconsin).

Cooperation.-Gage heights and capacity tables furnished by Wisconsin Valley Improvement $\mathrm{Co}$.

Gage height, in fect and inches, and contents, in milhons of cubic feet, 1965

\begin{tabular}{|c|c|c|c|c|c|c|c|}
\hline \multirow{2}{*}{ Date } & \multicolumn{2}{|c|}{ Gase height } & \multirow{2}{*}{ Conterits } & \multirow{2}{*}{ Date } & \multicolumn{2}{|c|}{ Gage height } & \multirow{2}{*}{ Conterits } \\
\hline & Fet't & Inches & & & Feet & Inches & \\
\hline Feb. $28 \ldots$ & 5 & 4 & 141 & Apr. $18 \ldots$ & 6 & 0 & 165 \\
\hline Mar. $\quad 7 \ldots$ & 4 & 6 & 111 & $25 \ldots$ & 7 & 1 & 207 \\
\hline $14 \ldots$ & 2 & 6 & 52 & May $2 \ldots$ & 7 & 6 & 222 \\
\hline $31 \ldots$ & 0 & 11 & 16 & $9_{-} \ldots$ & 8 & 7 & 270 \\
\hline $28 \ldots$ & 0 & 9 & 13 & $16 \ldots$ & 9 & 0 & 290 \\
\hline Anr. $4 \ldots$ & 2 & 5 & 50 & $23 \ldots$ & 8 & 6 & 266 \\
\hline $11 \ldots$ & 4 & 4 & 105 & $30 \ldots$ & 8 & 6 & 266 \\
\hline
\end{tabular}


(177) 5-3909. Rainbow Lake near Lake Tomahawk, Wis.

Location.-Lat $45^{\circ} 50^{\prime} 00^{\prime \prime}$, long $89^{\circ} 32^{\prime} 40^{\prime \prime}$, in SW $\frac{1}{4}$ sec.30, T.39 N., R. 8 E., on Wisconsin River, 2.7 miles northeast of village of Lake Tomahawk.

Drainage area. $-740 \mathrm{sq} \mathrm{mi,} \mathrm{approximately.}$

Gage-height record.-Float-tape gage. Datum of gage is 1,570.00 ft above mean sea level (levels by Wisconsin Valley Improvement $\mathrm{Co}$.).

Maxima.-March to May 1965: Contents at end of week, 2,181 million cubic feet May 16 (gage height, $28 \mathrm{ft} 0 \mathrm{in}$ ).

1936 to February 1965: Contents on last day of month, 2,197 million cubic feet Apr. 30, 1951 (gage height, $28 \mathrm{ft} 1 \mathrm{in}$ ).

Remarks.-Dam was completed November 1935. Usable capacity was 1,639 million cubic feet between gage height $6 \mathrm{ft} 0$ in and $25 \mathrm{ft} 0$ in until August 1949 when it was increased to 2,181 million cubic feet between gage height $6 \mathrm{ft} 0$ in ard $28 \mathrm{ft} 0$ in (limits stipulated by Public Service Commission of Wisconsin).

Cooperation.-Gage heights and capacity tables furnished by Wisconsin Valley Improvement Co.

Gage height, in feet and inches, and contents, in millions of cubic feet, 1965

\begin{tabular}{|c|c|c|c|c|c|c|c|}
\hline \multirow{2}{*}{ Date } & \multicolumn{2}{|c|}{ Gage height } & \multirow{2}{*}{ Contents } & \multirow{2}{*}{ Date } & \multicolumn{2}{|c|}{ Gage height } & \multirow{2}{*}{ Contents } \\
\hline & Feet & Inches & & & Feet & Inch es & \\
\hline Feb. 28_.- & 17 & 4 & 633 & Apr. $18 \ldots$ & 16 & 2 & 530 \\
\hline Mar. 7 & 16 & 1 & 523 & $25 \ldots$ & 21 & 5 & 1,097 \\
\hline 14 & 14 & 6.5 & 404 & May $2 \ldots$ & 25 & 7 & 1,737 \\
\hline $21 \ldots$ & 11 & 6.5 & 213 & $9 \ldots$ & 27 & 9 & 2,133 \\
\hline $28 \ldots$ & 10 & 9.5 & 177 & $16 \ldots$ & 28 & 0 & 2,181 \\
\hline Apr. 4 & 6 & 4 & 8 & $23 \ldots$ & 27 & 6.5 & 2,093 \\
\hline $11 \ldots$ & 8 & 8 & 84 & $30 \ldots$ & 27 & 6 & 2,085 \\
\hline
\end{tabular}

(178) 5-3911. Pelican Lake near Pelican Lake, Wis.

Location.-Lat $45^{\circ} 31^{\prime} 45^{\prime \prime}$, long $89^{\circ} 12^{\prime} 20^{\prime \prime}$, in $S_{2}^{\frac{1}{2}}$ sec.11, T.35 N., R.10 E., on Pelican River, 2.8 miles northwest of village of Pelican Lake.

Drainage area.- $-22 \mathrm{sq} \mathrm{mi,} \mathrm{approximately.}$

Gage-height record-Staff gage. Datum of gage is $1,589.98 \mathrm{ft}$ mean sea level (levels by Wisconsin Valley Improvement Co.).

Maxima.-March to May 1965: Contents at end of week, 292 million cubic feet May 16 (gage height, $1 \mathrm{ft} 11 \mathrm{in}$ ).

1938 to February 1965: Contents on last day of month, 331 million cubic feet June 30,1939 (gage height, 2 ft 2 in).

Remarks.-Reservoir operation began in 1909. No usable capacity Apr. 1 to Oct. 31. Usable capacity Nov. 1 to Mar. 31, 305 million cubic feet between gage heights $0 \mathrm{ft}$ 0 in and $2 \mathrm{ft} 0$ in (limits stipulated by Public Service Commission of Wisconsin).

Cooperation.- Gage heights and capacity table furnished by Wisconsin Valley Improvement Co. 
Gage height, in feet and inches, and contents, in millions of cubic feet, 1965, of Pelican Lake near Pelican Lake, Wis.

\begin{tabular}{|c|c|c|c|c|c|c|c|}
\hline \multirow{2}{*}{ Date } & \multicolumn{2}{|c|}{ Gage height } & \multirow{2}{*}{ Contents } & \multirow{2}{*}{ Date } & \multicolumn{2}{|c|}{ Gage height } & \multirow{2}{*}{ Contents } \\
\hline & Feet & Inches & & & Feet & Inches & \\
\hline Feb. 28_.. & 0 & 1 & 13 & Apr. $18 \ldots$ & 0 & 10 & 127 \\
\hline Mar. $7 \ldots$ & 0 & 1 & 13 & $25 \ldots$ & 1 & 3 & 190 \\
\hline $14 \ldots$ & 0 & 0 & 0 & May & 1 & 6.5 & 234 \\
\hline $21 \ldots$ & 0 & 0 & 0 & $9_{\ldots}$ & 1 & 8 & 254 \\
\hline $28 \ldots$ & 0 & 0 & 0 & $16 \ldots$ & 1 & 11 & 292 \\
\hline Apr. 4 & 0 & 1.5 & 19 & $23_{\ldots} \ldots$ & 1 & 9.5 & 273 \\
\hline $11 \ldots$ & 0 & 5 & 63 & $30 \ldots$ & 1 & 8.5 & 260 \\
\hline
\end{tabular}

(179) 5-3913. North Pelican Lakes near Rhinelander, Wis.

Location.-Lat $45^{\circ} 38^{\prime} 05^{\prime \prime}$, long $89^{\circ} 14^{\prime} 35^{\prime \prime}$, in $\mathrm{SE}_{\frac{1}{4}}$ sec.4, T.36 N., R.10 E., on Ncrth Branch Pelican River, 8 miles east of city limits of Rhinelander.

Drainage area.- $-71 \mathrm{sq} \mathrm{mi,} \mathrm{approximately.}$

Gage-height record.-Staff gage. Datum of gage is $1,569.10 \mathrm{ft}$ above mean sea level (levels by Wisconsin Valley Improvement Co.).

Maxima.-March to May 1965: Contents at end of week, 160 million cubic feet Apr. 25 (gage height, $0 \mathrm{ft} 4.5 \mathrm{in}$ ).

1938 to February 1965: Contents on last day of month 177 million cubic feet Mar. 31, 1945 (gage height, 0 ft 7-3/4 in).

Remarks.-The lakes have been used as a reservoir since 1908. Reservoir includes: Moen, Second, Third, Fourth and Fifth Lakes. Usable capacity, June 1 to Sext. 15, 19 million cubic feet between gage heights $-0 \mathrm{ft} 4$ in and $0 \mathrm{ft} 0$ in; Sept. 16 to May 31, 218 million cubic feet between gage heights $1 \mathrm{ft} 4$ in and $-2 \mathrm{ft} 6$ in (limits stibulated by Public Service Commission of Wisconsin).

Cooperation.-Gage heights and capacity tables furnished by Wisconsin Valley Improvement Co.

Gage height, in feet and inches, and contents, in millions of cubic feet, 1965

\begin{tabular}{|c|c|c|c|c|c|c|c|}
\hline \multirow{2}{*}{ Date } & \multicolumn{2}{|c|}{ Gage height } & \multirow{2}{*}{ Contents } & \multirow{2}{*}{ Date } & \multicolumn{2}{|c|}{ Gage height } & \multirow{2}{*}{ Contents } \\
\hline & Feet & Inches & & & Feet & Inches & \\
\hline Feb. 28. & -2 & 0 & 27 & Apr. $18 \ldots$ & -0 & 4 & 120 \\
\hline Mar. 7 & -1 & 11 & 31 & $25 \ldots$ & 0 & 4.5 & 160 \\
\hline 14 & -1 & 11 & 31 & $2 \ldots$ & 0 & 2.5 & 151 \\
\hline $21 \ldots$ & -1 & 11 & 31 & $9 \ldots$ & 0 & 4 & 158 \\
\hline $28 \ldots$ & -1 & 11 & 31 & $16 \ldots$ & 0 & 3 & 153 \\
\hline Apr. $\quad 4 \ldots$ & -1 & 10 & 36 & $23 \ldots$ & 0 & 1.5 & 146 \\
\hline $11 \ldots$ & -1 & 7.5 & 47 & $30 \ldots$ & -0 & 1 & 134 \\
\hline
\end{tabular}


(180) 5-3921. Minocqua Lake near Minocqua, Wis.

Location.--Lat $45^{\circ} 52^{\prime} 35^{\prime \prime}$, long $89^{\circ} 43^{\prime} 40^{\prime \prime}$, on line between secs.10 and 15, T.39 N., R. 6 E., on Tomahawk River, 1 mile west of Minocqua.

Drainage area. $-89 \mathrm{sq} \mathrm{mi,} \mathrm{approximately.}$

Gage-height record.--Staff gage. Datum of gage is $1,584.56 \mathrm{ft}$ above moan sea level (levels by Wiscons in Valley Improvement Co.).

Maxima.--March to May 1965: Contents at end of week, 484 million cubic feet May 30 (gage height, 0 ft 0 in).

1938 to February 1965: Contents on last day of month, 619 million cubic feet July 31,1950 , (gage height, 0 ft $5-5 / 8$ in).

Remarks.-Reservoir operation began in 1910. Reservoir includes Little Tomahawk, Tomahawk, Mid, Minocqua, Baker and Kewasokogan Lakes. Usable capacity Oct. 1 to May 31, 628 million cubic feet between gage heights $-1 \mathrm{ft} 10$ in and $0 \mathrm{ft} 6$ in, and June 1, to Sept. 30, 283 million cubic feet between gage heights $-0 \mathrm{ft} 6$ in and $0 \mathrm{ft} 6 \mathrm{in}$, (limits stipulated by Public Service Commission of Wisconsin).

Cooperation.-Gage heights and capacity tables furnished by Wisconsin Valley Improvement Co.

Gage height, in feet and inches, and contents, in millions of cubic feet, 1965

\begin{tabular}{|c|c|c|c|c|c|c|c|}
\hline \multirow{2}{*}{ Date } & \multicolumn{2}{|c|}{ Gage height } & \multirow{2}{*}{ Contents } & \multirow{2}{*}{ Date } & \multicolumn{2}{|c|}{ Gage height } & \multirow{2}{*}{ Contents } \\
\hline & Feet & Inches & & & Feet & Inchas & \\
\hline Feb. $28 \ldots$ & -1 & 10 & 0 & Apr. $18 \ldots$ & -1 & 3.5 & 136 \\
\hline Mar. 7..- & -1 & 10 & 0 & $25 \ldots$ & -1 & 1 & 189 \\
\hline 14 & -1 & 10 & 0 & May 2... & -0 & 11 & 233 \\
\hline $21 \ldots$ & -1 & 10 & 0 & $9 \ldots$ & -0 & 6 & 345 \\
\hline $28 \ldots$ & -1 & 10 & 0 & $16 \ldots$ & -0 & 3.5 & 402 \\
\hline A.pr. 4 & -1 & 8 & 41 & $23 \ldots$ & -0 & 1.5 & 448 \\
\hline $11 \ldots$ & -1 & 6 & 83 & $30 \ldots$ & 0 & 0 & 484 \\
\hline
\end{tabular}

(181) 5-3922. Squirrel Lake near Minocqua, Wis.

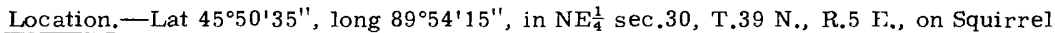
River, 9.4 miles west of Minocqua.

Drainage area.- $-17 \mathrm{sq}$ mi., approximately.

Gage-height record.-Staff gage. Datum of gage is $1,560.93 \mathrm{ft}$ above moan sea level (levels by Wisconsin Valley Improvement Co.).

Maxima.-March to May 1965: Contents at end of week, 175 million cubic feet May 16, 23,30 (gage height, $3 \mathrm{ft} 11$ in.).

1938 to February 1965: Contents on last day of month, 193 million cubic feet Apr. 30, 1951 (gage height, $4 \mathrm{ft} 1-\frac{1}{2}$ in).

Remarks.-Reservoir operation began in 1908. Usable capacity Oct, 1 to May 31,182 million cubic feet between gage heights $1 \mathrm{ft} 7$ in and $4 \mathrm{ft} 0 \mathrm{in}$; and June 1 to Sept. 30 , 165 million cubic feet between gage heights $1 \mathrm{ft} 10$ in and $4 \mathrm{ft} 0$ in (lirnits stipulated by Public Service Commission of Wisconsin).

Cooperation.-Gage heights and capacity tables furnished by Wisconsin Valley Improvement Co. 
Gage height, in feet and inches, and contents, in millions of cubic feet, 1965, of Squirrel Lake near Minocqua. Wis.

\begin{tabular}{|c|c|c|c|c|c|c|c|}
\hline \multirow{2}{*}{ Date } & \multicolumn{2}{|c|}{ Gage height } & \multirow{2}{*}{ Contents } & \multirow{2}{*}{ Date } & \multicolumn{2}{|c|}{ Gage height } & \multirow{2}{*}{ Contents } \\
\hline & Feet & Inches & & & Feet & Inches & \\
\hline $\begin{array}{lr}\text { Feb. } & 28 \\
\text { Mar. } & 7 \\
& 14 \\
& 21 \\
& 28 \\
\text { Apr. } & 4 \\
& 11\end{array}$ & $\begin{array}{l}1 \\
1 \\
1 \\
1 \\
1 \\
1 \\
2\end{array}$ & $\begin{array}{l}7 \\
7 \\
7 \\
8 \\
8 \\
9 \\
2\end{array}$ & $\begin{array}{r}0 \\
0 \\
0 \\
5 \\
5 \\
11 \\
41\end{array}$ & Apr. $18 \ldots$ & $\begin{array}{l}2 \\
3 \\
3 \\
3 \\
3 \\
3 \\
3 \\
3\end{array}$ & $\begin{array}{r}9 \\
3 \\
5 \\
7 \\
11 \\
11 \\
11\end{array}$ & $\begin{array}{r}83 \\
122 \\
135 \\
148 \\
175 \\
175 \\
175\end{array}$ \\
\hline
\end{tabular}

(182) 5-3923. Willow Reservoir near Hazelhurst, Wis.

Location.-Lat $45^{\circ} 42^{\prime} 45^{\prime \prime}$, long $89^{\circ} 50^{\prime} 40^{\prime \prime}$, in NE $\frac{1}{4}$ sec.10, T.37 N., R.5 E., on Tomahawk River, 8.8 miles southwest of Hazelhurst.

Drainage area.- $-327 \mathrm{sq} \mathrm{mi}$, approximately.

Gage-height record.-Staff gage. Datum of gage is $1505.87 \mathrm{ft}$ above mean sea level (levels by Wisconsin Valley Improvement Co.).

Maxima.-March to May 1965: Contents at end of week, 3,222 million cubic fe :t May 23 (gage height, $23 \mathrm{ft} 3 \mathrm{in}$ ).

1938 to February 1965: Contents on last day of month, 3,316 million cubic feet June 30,1954 (gage height, $23 \mathrm{ft} 6-\frac{1}{2}$ in).

Remarks.-Reservoir operation began in 1927. Usable capacity, 3,302 million cubic feet between gage heights $5 \mathrm{ft} 0$ in and $23 \mathrm{ft} 6$ in (limits stipulated by Public Service Commission of Wisconsin).

Cooperation.--Gage heights and capacity tables furnished by Wisconsin Valley Improvement Co.

Gage height, in feet and inches, and contents, in millions of cubic feet, 1965

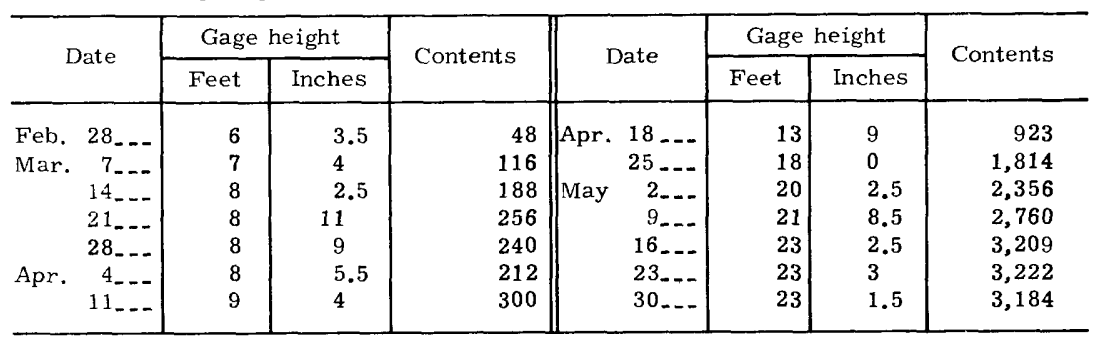


(183) 5-3925. Lake Nokomis at Bradley, Wis.

Location.-Lat $45^{\circ} 32^{\prime} 20^{\prime \prime}$, long $89^{\circ} 44^{\prime} 45^{\prime \prime}$, in NW $\frac{1}{4}$ sec.9, T.35 N., R. 6 E., on Tomahawk River, 0.4 mile east of $\mathrm{Br}$ adley.

Drainage area. -548 sq $\mathrm{mi}$.

Gage-height record.-Staff gage. Datum of gage is 1,448.24 ft above mean sea level, datum of 1929 .

Maxima.--March to May 1965: Contents at end of week, 1,792 million cubic feet May 16 (gage height, $14 \mathrm{ft} 11 \mathrm{in}$ ).

1938 to February 1965: Contents on last day of month, 1,852 million cubic feet Sept. 30, 1959 (gage height, $15 \mathrm{ft} 2-3 / 4$ in).

Remarks.- Reservoir operation began in 1912. Us able capacity, 1,808 million cubic feet between gage heights $0 \mathrm{ft} 0$ in and $15 \mathrm{ft} 0$ in (limits stipulated by Public Service Commission of Wisconsin).

Cooperation.-Gage heights and capacity tables furnished by Wisconsin Valley Improvement $\mathrm{Co}$.

Gage height, in feet and inches, and contents, in millions of cubic feet, 1965

\begin{tabular}{|c|c|c|c|c|c|c|c|}
\hline \multirow{2}{*}{ Date } & \multicolumn{2}{|c|}{ Gage height } & \multirow{2}{*}{ Contents } & \multirow{2}{*}{ Date } & \multicolumn{2}{|c|}{ Gage height } & \multirow{2}{*}{ Contents } \\
\hline & Feet & Inches & & & Feet & Inches & \\
\hline Feb. $28 \ldots$ & 9 & 2 & 775 & Apr. $18 \ldots$ & 8 & 6 & 671 \\
\hline Mar. 7 & 7 & 9.5 & 568 & $25 \ldots$ & 12 & 1 & 1,265 \\
\hline 14 & 6 & 0 & 326 & 2 & 14 & 3 & 1,664 \\
\hline $21 \ldots$ & 3 & 5 & 93 & $9 \ldots$ & 14 & 9 & 1,760 \\
\hline 28 & 2 & 7.5 & 52 & $16 \ldots$ & 14 & 11 & 1,792 \\
\hline Apr. 4 & 2 & 5 & 44 & $23 \ldots$ & 14 & 10.5 & 1,784 \\
\hline 11 & 3 & 11 & 127 & $30 \ldots$ & 14 & 6 & 1,712 \\
\hline
\end{tabular}

(184) 5-3936. Spirit River Flowage near Tomahawk, Wis.

Location.-Lat $45^{\circ} 26^{\prime} 20^{\prime \prime}$, long $89^{\circ} 44^{\prime} 30^{\prime \prime}$, in $\mathrm{SE} \frac{1}{4}$ sec.9, T.34 N., R.6 E., on Spirit River, 2 miles south of Tomahawk.

Drainage area. $-174 \mathrm{sq} \mathrm{mi}$.

Gage-height record.-Staff gage. Datum of gage is $1,420.53 \mathrm{ft}$ above mean sea level, datum of 1929 .

Maxima.--March to May 1965: Contents at end of week, 752 million cubic feet May 2 (gage height, $16 \mathrm{ft} 11.5 \mathrm{in}$ ).

1938 to February 1965: Contents on last day of month, 760 million cubic feet, June 30, 1953 (gage height, $17 \mathrm{ft} \frac{1}{2} \mathrm{in}$ ).

Remarks.- Reservoir operation began in 1923. Usable capacity, 756 million cubic feet between gage heights, $0 \mathrm{ft} 0$ in and $17 \mathrm{ft} 0$ in (limits stipulated by Public Service Commission of Wisconsin).

Cooperation.--Gage heights and capacity tables furnished by Wisconsin Valley Improvement Co. 
Gage height, in feet and inches, and contents, in millions of cubic feet, 1965, of Spirit River Flowage near Tomahawk, Wis.

\begin{tabular}{|c|c|c|c|c|c|c|c|}
\hline \multirow{2}{*}{ Date } & \multicolumn{2}{|c|}{ Gage height } & \multirow{2}{*}{ Contents } & \multirow{2}{*}{ Date } & \multicolumn{2}{|c|}{ Gage height } & \multirow{2}{*}{ Contents } \\
\hline & Feet & Inches & & & Feet & Inches & \\
\hline Feb. 28_.. & 3 & 3 & 31 & Apr. $18 \ldots$ & 16 & 5.5 & 707 \\
\hline Mar. 7 & 5 & 3 & 74 & 25 & 15 & $11^{\circ}$ & 659 \\
\hline $14 \ldots$ & 6 & 5.5 & 108 & May 2... & 16 & 11.5 & 752 \\
\hline $21 \ldots$ & 6 & 11 & 125 & $9 \ldots$ & 16 & 7.5 & 722 \\
\hline $28 \ldots$ & 6 & 7.5 & 114 & $16 \ldots$ & 16 & 10 & 741 \\
\hline Apr. $\quad 4 \ldots-$ & 0 & 0 & 0 & $23 \ldots$ & 16 & 6.5 & 714 \\
\hline $11 \ldots$ & 3 & 11 & 43 & $30 \ldots$ & 16 & 6 & 711 \\
\hline
\end{tabular}

(185) 5-3945. Prairie River near Merrill, Wis.

Location.-Lat $45^{\circ} 14^{\prime} 10^{\prime \prime}$, long $89^{\circ} 38^{\prime} 50^{\prime \prime}$, on line between secs.20 and $29, \mathrm{~T} .32 \mathrm{~N}$,

R. 7 E., near center of span on downstream side of highway bridge, 1.5 miles upstream from Meadow Creek, 4.5 miles northeast of Merrill, and 8 miles upstream from mouth.

Drainage area. $-181 \mathrm{sq} \mathrm{mi}$.

Gage-height record.-Graph drawn on basis of once-daily chain-gage readings and flood mark. Altitude of gage is 1,300 ft (from topographic map).

Discharge record.-Stage-discharge relation defined by current-meter measurements below 2,600 cfs. Backwater from ice Mar. 1-4, Mar. 10 to Apr. 5.

Maxima.-March-May 1965: Discharge, 2,530 cfs 0600 hours Apr. 12 (gage height, $6.60 \mathrm{ft}$ ).

1914-31, 1939 to February 1965: Discharge, 5,800 cfs Aug. 31, 1941 (gage height, $9.45 \mathrm{ft}$, from floodmarks).

Mean discharge, in cubic feet per second, 1965

\begin{tabular}{|c|c|c|c|c|c|c|c|c|c|c|c|}
\hline Day & March & April & May & Day & March & April & May & Day & March & April & May \\
\hline 1 & 72 & 72 & 570 & $11 \ldots$ & 96 & 1,500 & 398 & 21. & 78 & 960 & 324 \\
\hline 2 & 76 & 72 & 528 & $12_{-}$ & 91 & 2,020 & 350 & 22. & 76 & 877 & 264 \\
\hline 3. & 81 & 74 & 513 & $13 \ldots$ & 90 & 1,260 & 312 & 23. & 76 & 754 & 226 \\
\hline$\dot{t}_{-}$ & 83 & 76 & 461 & $14=-$ & 90 & 1,140 & 264 & 24. & 76 & 728 & 166 \\
\hline 5. & 86 & 78 & 412 & $15 \ldots$ & 91 & 1,140 & 312 & 25 & 76 & 695 & 170 \\
\hline 6. & 98 & 82 & 454 & $16 \ldots$ & 90 & 1,060 & 600 & 26. & 75 & 720 & 252 \\
\hline 7. & 147 & 95 & 520 & $17 \ldots$ & 86 & 1,000 & 833 & 27. & 74 & 877 & 247 \\
\hline 8. & 126 & 183 & 574 & $18=-$ & 82 & 998 & 768 & $28 \ldots$ & 73 & 970 & 247 \\
\hline 9. & 119 & 611 & 574 & $19 \ldots$ & 80 & 928 & 478 & $29 \ldots$ & 72 & 800 & 211 \\
\hline \multirow[t]{2}{*}{$10--$} & 106 & 1,110 & 454 & $20 \ldots$ & 78 & 897 & 370 & $30 \ldots$ & 72 & 634 & 196 \\
\hline & & & & & & & & $31_{-}$ & 72 & ----- & 204 \\
\hline & & & & & & & & & 86.7 & 747 & 395 \\
\hline Run & $f$, in & 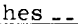 & & & & & & & 0.55 & 4.60 & 2.52 \\
\hline
\end{tabular}


Gage height, in feet, and discharge, in cubic feet per second, at indicated time. 1965, of Prairie River near Merrill, Wis.

\begin{tabular}{|c|c|c|c|c|c|c|c|c|c|c|c|}
\hline Date & Hour & $\begin{array}{c}\text { Gage } \\
\text { height }\end{array}$ & $\begin{array}{c}\text { Dis - } \\
\text { charge }\end{array}$ & Date & Hour & $\begin{array}{c}\text { Gage } \\
\text { height }\end{array}$ & $\begin{array}{c}\text { Dis- } \\
\text { charge }\end{array}$ & Date & Hour & $\begin{array}{c}\text { Gage } \\
\text { height }\end{array}$ & $\begin{array}{c}\text { Dis- } \\
\text { charge }\end{array}$ \\
\hline Apr. 9 & 0000 & 2.60 & 272 & Apr. 12 & 2400 & 5.16 & 1,440 & May 15 & 2407 & 3.13 & 447 \\
\hline & 0600 & 3.11 & 440 & & & & & & & & \\
\hline & 1200 & 3.59 & 622 & 13 & 0600 & 4.85 & 1,250 & 16 & $120 ?$ & 3.57 & 614 \\
\hline & 1800 & 3.96 & 776 & & 1200 & 4.83 & 1,240 & & $240 ?$ & 3.84 & 724 \\
\hline & 2400 & 4.29 & 939 & & 1800 & 4.85 & 1,250 & & & & \\
\hline & & & & & 2400 & 4.76 & 1,200 & 17 & $120 ?$ & 4.13 & 857 \\
\hline 10 & 1200 & 4.65 & 1,130 & & & & & & $180 \uparrow$ & 4.19 & 887 \\
\hline & 2400 & 4.87 & 1,260 & 14 & 1200 & 4.62 & 1,120 & & 2403 & 4.16 & 872 \\
\hline & & & & & 2400 & 4.66 & 1,140 & & & & \\
\hline 11 & 1200 & 5.07 & 1,380 & & & & & 18 & 1203 & 3.98 & 785 \\
\hline & 1800 & 5.40 & 1,590 & 15 & 1200 & 4.67 & 1,140 & & 2407 & 3.61 & 630 \\
\hline & 2400 & 6.12 & 2,130 & & 2400 & 4.62 & 1,120 & & & & \\
\hline & & & & & & & & 19 & 1203 & 3.13 & 447 \\
\hline 12 & 0600 & 6.60 & 2,530 & 16 & 1200 & 4.50 & 1,050 & & 1801 & 3.04 & 415 \\
\hline & 1200 & 6.06 & 2,080 & & 2400 & 4.43 & 1,010 & & 2400 & 2.98 & 394 \\
\hline & 1800 & 5.52 & 1,670 & & & & & & & & \\
\hline
\end{tabular}

(186) 5-3950. Wisconsin River at Merrill, Wis.

Location.--Lat $45^{\circ} 10^{\prime} 40^{\prime \prime}$, long $89^{\circ} 40^{\prime} 45^{\prime \prime}$, on line between secs.12 and 13, T.31 N., R.6 E., on left bank $300 \mathrm{ft}$ downstream from highway bridge at east end of Merrill and 0.5 mile downstream from Prairie River.

Drainage area. $-2,780 \mathrm{sq} \mathrm{mi}$, approximately.

Gage-height record.-Digital recorder tape punched at fifteen-minute irtervals. Datum of gage is $1,228.85 \mathrm{ft}$ above mean sea level, datum of 1929 .

Discharge record.--Stage-discharge relation defined by current-meter measurements below $20,000 \mathrm{cfs}$. Mean daily discharges computed from 96 punch tap recordings per day. Backwater from ice Mar. 1 to Apr. 4.

Maxima.-March-May 1965: Discharge, 12,300 cfs 1300 hours May 18 (gage height, $9.79 \mathrm{ft}$ ).

1902 to February 1965: Discharge, 49,400 cfs Aug. 31, 1941 (gage height, $18.26 \mathrm{ft}$ ).

Remarks.-Flow regulated by 20 reservoirs and 9 powerplants above station.

Mean discharge, in cubic feet per second, 1965

\begin{tabular}{|c|c|c|c|c|c|c|c|c|c|c|c|}
\hline Day & March & April & May & Day & March & April & May & Day & March & April & May \\
\hline & 1,700 & 1,500 & 4,610 & _ & 1,800 & 7,860 & 7,040 & 21 & 1,300 & 9,630 & 6,680 \\
\hline$a$. & 1,900 & 1,500 & 4,290 & 12. & 1,700 & 9,140 & 6,430 & 22. & 1,400 & 9,380 & 6,870 \\
\hline 3. & 2,100 & 1,500 & 4,890 & 13 & 1,700 & 7,760 & 5,560 & 23. & 1,300 & 8,760 & 6,290 \\
\hline 4. & 2,100 & 1,400 & 5,410 & 14 & 1,600 & 9,380 & 5,250 & 24 & 1,500 & 8,050 & 6,010 \\
\hline 5. & 2,000 & 1,360 & 4,460 & $15_{-}$ & 1,500 & 9,970 & 4,710 & 25. & 1,600 & 7,240 & 4,890 \\
\hline 6. & 1,900 & 1,470 & 4,600 & $16 \ldots$ & 1,700 & 10,300 & 8,040 & 26. & 1,500 & 6,320 & 4,370 \\
\hline 7. & 1,900 & 1,580 & 5,910 & 17. & 1,700 & 10,500 & 8,570 & 27. & 1,300 & 7,360 & 5,900 \\
\hline 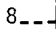 & 1,900 & 1,600 & 6,380 & 18 & 1,500 & 10,500 & 10,200 & 28. & 1,500 & 7,580 & 6,160 \\
\hline & 2,000 & 2,100 & 7,350 & $19 \ldots$ & 1,500 & 9,950 & 8,280 & 29. & 1,600 & 6,650 & 4,740 \\
\hline & 1,900 & 3,250 & 7,490 & 20 & 1,400 & 9,650 & 7,260 & 30 & 1,500 & 5,390 & 3,670 \\
\hline & & & & & & & & & 1,400 & & 3,420 \\
\hline \multicolumn{9}{|c|}{ Monthly mean discharge, in cubic feet per second } & 1,658 & 6,288 & 5,991 \\
\hline
\end{tabular}


MARCH-MAY, UPPER MISSISSIPPI RIVER BASIN

Gage height, in feet, and discharge, in cubic feet per second, at indicated time, 1965, of Wisconsin $R$ :'er at Merrill, Wis.

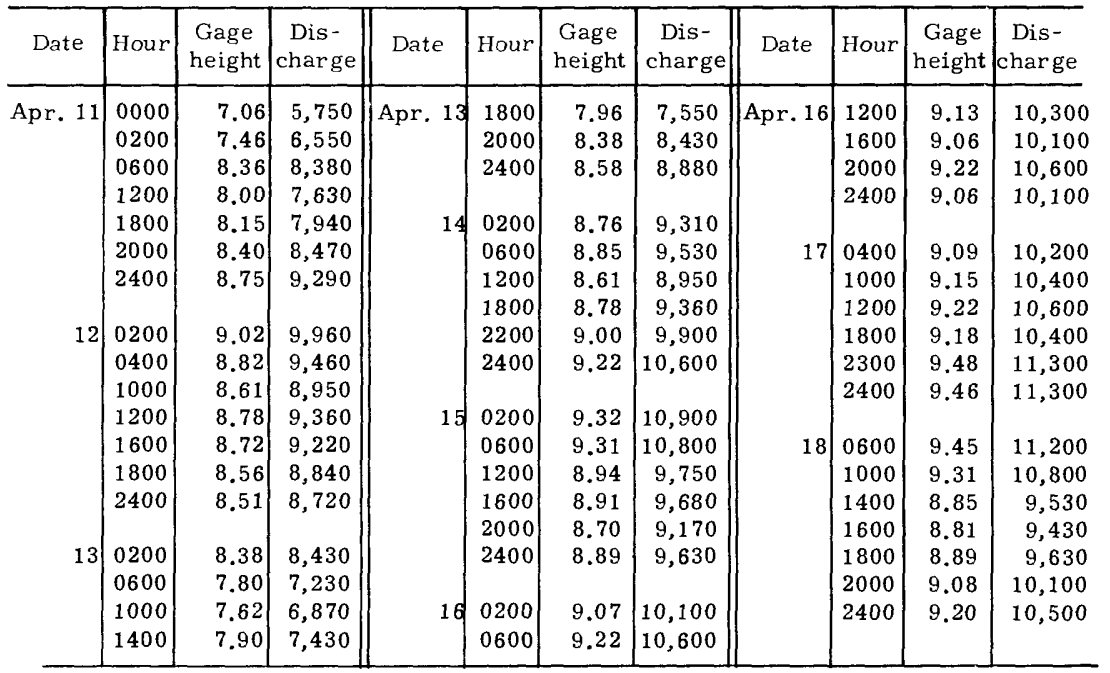

(187) 5-3961. Scotch Creek tributary near Edgar, Wis.

(Crest-stage station)

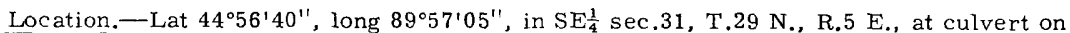
State Highway 29, 0.6 mile east of intersection with County Trunk H, and 1.5 miles northeast of Edgar.

Drainage area. $-7.1 \mathrm{sq} \mathrm{mi}$.

Gage-height record.-Crest stages only.

Discharge record.--Stage-discharge relation defined by current-meter measurements below $161 \mathrm{cfs}$ and by culvert flow measurement at $1380 \mathrm{cfs}$.

Maxima.-March-May 1965: Discharge, 1,380 cfs Apr. 11 (gage height, 16.67 ft).

1962 to February 1965: Discharge, $460 \mathrm{cfs}$ Sept. 13, 1962 (gage height, $14.07 \mathrm{ft}$ ). 
(188) 5-3975. Eau Claire River at Kelly, Wis.

Location.-Lat $44^{\circ} 55^{\prime} 05^{\prime \prime}$, long $89^{\circ} 33^{\prime} 00^{\prime \prime}$, on line between secs. 9 and 10, T.28 N., R. 8 E., on right bank $50 \mathrm{ft}$ downstream from highway bridge, 0.7 mile northeast of Kelly, 1.3 miles upstream from Big Sandy Creek, 4.5 miles upstrearn from mouth, and 5 miles southeast of Wausau.

Drainage area. $-326 \mathrm{sq} \mathrm{mi}$.

Gage-height record.-Digital recorder tape punched at sixty-minute intervals. Datum of gage is $1,177.88 \mathrm{ft}$ above mean sea level, datum of 1929 .

Discharge record.--Stage-discharge relation defined by current-meter measurements below 6,400 cfs. Mean daily discharges computed from 24 punch-tap recordings per day. Backwater from ice Mar. 1 to Apr. 9.

Maxima.-March-May 1965: Discharge, 6,980 cfs 2000 hours Apr. 12 (gage height, $10.04 \mathrm{ft}$ ).

1914-26, 1939 to February 1965: Discharge, 8,300 cfs Aug. 21, 1926 (gage height, $8.4 \mathrm{ft}$ from graph based on gage readings).

Mean discharge, in cubic feet per second, 1965

\begin{tabular}{|c|c|c|c|c|c|c|c|c|c|c|c|}
\hline Day & March & April & May & Day & March & April & May & Day & March & April & May \\
\hline 1. & 58 & 73 & 717 & $11 \ldots$ & 280 & 3,150 & 576 & 21 & 96 & 1,160 & 432 \\
\hline 2. & 120 & 71 & 652 & $12 \ldots$ & 210 & 6,420 & 474 & 22. & 92 & 1,130 & 357 \\
\hline 3 & 200 & 70 & 628 & $13 \ldots$ & 170 & 5,410 & 380 & 23 & 90 & 1,060 & 310 \\
\hline & 180 & 70 & 706 & $14 \ldots$ & 150 & 4,040 & 315 & 24 & 86 & 949 & 263 \\
\hline 5 & 180 & 80 & 621 & $15_{-}$ & 146 & 3,340 & 376 & 25 & 81 & 861 & 252 \\
\hline $6 \ldots$ & 300 & 100 & 535 & $16 \ldots$ & 134 & 2,520 & 2,030 & 26 & 78 & 1,040 & 414 \\
\hline 7. & 260 & 120 & 497 & $17 \ldots$ & 128 & 1,960 & 1,610 & 27 & 76 & 1,370 & 503 \\
\hline $8, \ldots$ & 310 & 250 & 483 & $18 \ldots$ & 130 & 1,780 & 1,320 & 28 & 74 & 1,300 & 386 \\
\hline $9 \ldots$ & 330 & 600 & 538 & $19_{--}$ & 108 & 1,490 & 899 & 29 & 73 & 1,100 & 311 \\
\hline \multirow[t]{2}{*}{10.} & \multirow[t]{2}{*}{320} & \multirow[t]{2}{*}{1,980} & \multirow[t]{2}{*}{615} & \multirow[t]{2}{*}{$20 \ldots$} & \multirow[t]{2}{*}{102} & \multirow[t]{2}{*}{1,310} & \multirow[t]{2}{*}{582} & 30. & 73 & 844 & 261 \\
\hline & & & & & & & & 31. & 73 & & 228 \\
\hline & & & & his & & & & & 152 & 1,522 & 5.89 \\
\hline Run & if $\mathrm{f}$ in & ies - - & . & & 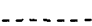 & . & 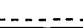 & & 0.54 & 5.21 & 2.08 \\
\hline
\end{tabular}

Gage height, in feet, and discharge, in cubic feet per second, at indicated tim?, 1965

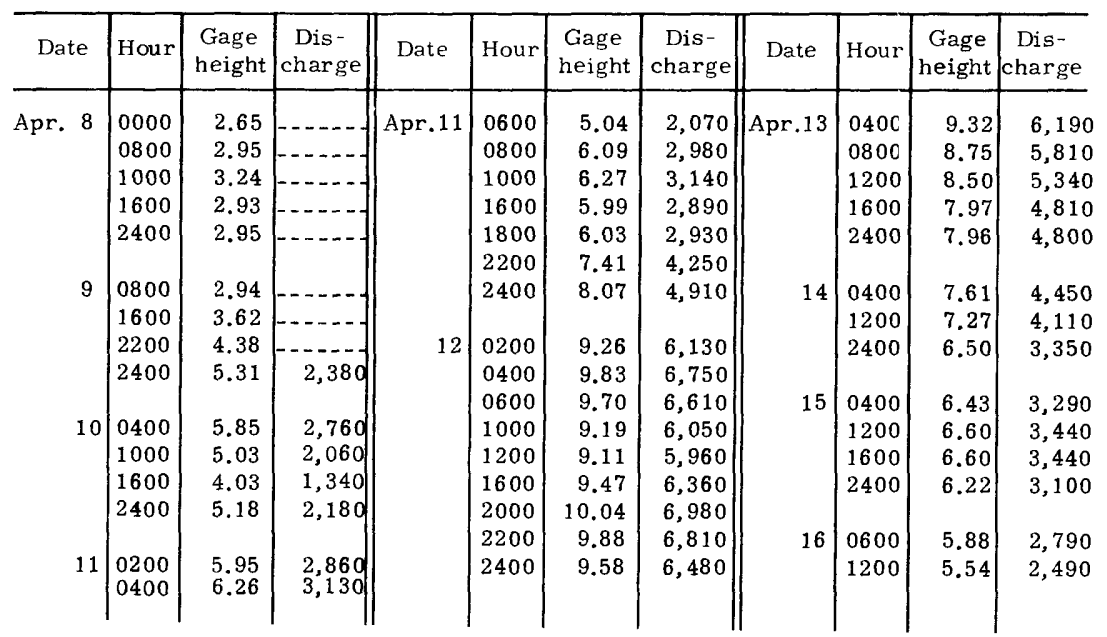


Gage height, in feet, and discharge, in cubic feet per second, at indicated time, 1965, of Eau Claire River at Kelly, Wis,-Continued

\begin{tabular}{|c|c|c|c|c|c|c|c|c|c|c|c|}
\hline Date & Hour & $\begin{array}{c}\text { Gage } \\
\text { height }\end{array}$ & $\begin{array}{c}\text { Dis - } \\
\text { charge }\end{array}$ & Date & Hour & $\begin{array}{c}\text { Gage } \\
\text { height }\end{array}$ & $\begin{array}{c}\text { Dis- } \\
\text { charge }\end{array}$ & Date & Hour & $\begin{array}{c}\text { Gage } \\
\text { height }\end{array}$ & $\begin{array}{c}\text { Dis- } \\
\text { charge }\end{array}$ \\
\hline Apr. 16 & $\begin{array}{l}1800 \\
2400 \\
0800 \\
1600 \\
2400 \\
1200 \\
2400 \\
0800 \\
1600 \\
2400\end{array}$ & $\begin{array}{l}5.32 \\
5.10 \\
4.93 \\
4.82 \\
4.80 \\
4.72 \\
4.44 \\
4.31 \\
4.19 \\
4.22\end{array}$ & $\begin{array}{l}2,300 \\
2,120 \\
1,980 \\
1,900 \\
1,930 \\
1,820 \\
1,610 \\
1,520 \\
1,430 \\
1,450\end{array}$ & $\begin{array}{r}\text { May } 15 \\
16\end{array}$ & $\begin{array}{l}0600 \\
1600 \\
2400 \\
2400 \\
0400 \\
0600 \\
1000 \\
1400 \\
1800 \\
2400\end{array}$ & $\begin{array}{l}4.15 \\
3.86 \\
3.77 \\
2.80 \\
\\
3.99 \\
4.49 \\
5.16 \\
5.56 \\
5.53 \\
5.15\end{array}$ & $\begin{array}{r}1,410 \\
1,240 \\
1,180 \\
674 \\
1,310 \\
1,640 \\
2,170 \\
2,500 \\
2,480 \\
2,160\end{array}$ & May 17 & $\begin{array}{l}0600 \\
1400 \\
1800 \\
2400 \\
0800 \\
1600 \\
2400 \\
1200 \\
1800 \\
2400\end{array}$ & $\begin{array}{l}4.68 \\
4.28 \\
4.16 \\
4.09 \\
4.10 \\
4.00 \\
3.71 \\
3.25 \\
3.03 \\
2.86\end{array}$ & $\begin{array}{r}1,780 \\
1,500 \\
1,420 \\
1,370 \\
1,380 \\
1,320 \\
1,150 \\
\\
904 \\
791 \\
705\end{array}$ \\
\hline
\end{tabular}

(189) 5-3980. Wisconsin River at Rothschild, Wis.

Location.-Lat $44^{\circ} 53^{\prime} 10^{\prime \prime}$, long $89^{\circ} 37^{\prime} 50^{\prime \prime}$, in sec.26, T.28 N., R.7 E., on left bank at Rothschild, 0.5 mile downstream from Rothschild Dam, 2 miles downstream from Eau Claire River, 5 miles upstream from Black Creek, and 5.5 miles south of bridge on State Highway 29 in Wausau.

Drainage area. $-4,000 \mathrm{sq} \mathrm{mi}$. approximately.

Gage-height record.-Digital recorder tape punched at sixty-minute intervals. Datum of gage is $1,135.86 \mathrm{ft}$ above mean sea level, datum of 1929. Auxiliary digital recorder tape punched at sixty-minute intervals in Mosinee pond 8 miles downstream.

Discharge record.-Stage-discharge relation defined by current meter measurements. Fall used as a factor for discharges below 7,000 cfs. Mean daily discharges computed from 24 punch-tape recordings per day.

Maxima.-March-May 1965: Discharge, 49,200 cfs 1600 hours Apr. 12 (gage height $18.46 \mathrm{ft}$ ).

1944 to February 1965: Discharge, 47,000 cfs Sept. 27, 1959 (gage height $17.81 \mathrm{ft}$ ).

Flood of Sept. 1, 1941, reached a stage of $22.3 \mathrm{ft}$, from tailwater data at F othschild Dam (discharge, $75,000 \mathrm{cfs}$, from rating curve extended above $45,000 \mathrm{cfs}$ by logarithmic plotting.)

Remarks.-Flow regulated by 20 reservoirs and 12 powerplants above station

Mean discharge, th cubic feet per second, 1965

\begin{tabular}{l|r|r|r||r|r|r|r||r|r|r|r}
\hline Day & March & April & May & Day & March & April & May & Day & March & April & May \\
\hline $1 .--$ & 2,010 & 1,720 & 7,380 & $11--$ & 2,940 & 21,700 & 9,190 & $21-$ & 1,350 & 14,400 & 7,480 \\
$2 .--$ & 2,640 & 1,880 & 6,980 & $12--$ & 2,240 & 44,300 & 7,480 & $22-$ & 1,930 & 14,600 & 7,700 \\
$3--$ & 4,030 & 1,610 & 6,860 & $13--$ & 1,980 & 30,200 & 6,930 & $23-$ & 2,030 & 12,700 & 7,150 \\
$4---$ & 4,020 & 1,800 & 7,680 & $14--$ & 1,790 & 24,200 & 6,270 & $24--$ & 1,750 & 11,700 & 6,750 \\
$5---$ & 3,640 & 1,780 & 6,840 & $15--$ & 1,950 & 23,800 & 6,200 & $25-$ & 2,000 & 10,400 & 6,370 \\
$6 .--$ & 3,830 & 1,930 & 6,500 & $16--$ & 1,930 & 20,400 & 12,600 & $26--$ & 2,010 & 10,300 & 6,280 \\
$7---$ & 3,830 & 3,410 & 7,150 & $17--$ & 2,000 & 17,900 & 14,900 & $27--$ & 1,540 & 15,800 & 7,510 \\
$8---$ & 3,670 & 5,170 & 7,670 & $18--$ & 1,910 & 18,400 & 13,300 & $28--$ & 1,330 & 13,900 & 7,470 \\
$9---$ & 4,110 & 6,210 & 9,170 & $19--$ & 2,000 & 16,600 & 11,700 & $29--$ & 1,950 & 10,600 & 6,190 \\
$10--$ & 3,510 & 7,730 & 10,000 & $20--$ & 1,600 & 15,400 & 9,150 & $30--$ & 1,980 & 8,210 & 5,170 \\
&
\end{tabular}


Gage height, in feet, and discharge, in cubic feet per second, at indicated time, 1965, of Wisconsin River at Rothschild, Wis.

\begin{tabular}{|c|c|c|c|c|c|c|c|c|c|c|c|}
\hline Date & Hour & $\begin{array}{c}\text { Gage } \\
\text { height }\end{array}$ & $\begin{array}{c}\text { Dis- } \\
\text { charge }\end{array}$ & Date & Hour & $\begin{array}{l}\text { Gage } \\
\text { height }\end{array}$ & $\begin{array}{c}\text { Dis- } \\
\text { charge }\end{array}$ & Date & Hour & $\begin{array}{l}\text { Gage } \\
\text { height }\end{array}$ & $\begin{array}{l}\text { Dis- } \\
\text { charge }\end{array}$ \\
\hline \multirow[t]{10}{*}{ Apr. 11} & 0000 & 6.64 & 7,730 & \multirow[t]{4}{*}{ Apr. 12} & 2000 & 18.05 & 47,200 & \multirow[t]{3}{*}{ Apr. 14} & 2000 & 12.41 & 23,100 \\
\hline & 1000 & 6.64 & 7,730 & & 2200 & 17.44 & 44,200 & & \multirow[t]{2}{*}{2400} & \multirow[t]{2}{*}{12.64} & \multirow[t]{2}{*}{23,900} \\
\hline & 1100 & 13.13 & 25,700 & & 2400 & 16.92 & 41,700 & & & & \\
\hline & 1200 & 13.69 & 27,800 & & & & & \multirow[t]{7}{*}{15} & 0400 & 12.53 & 23,500 \\
\hline & 1400 & 14.39 & 30,600 & \multirow[t]{6}{*}{13} & 0200 & 16.49 & 39,700 & & 0800 & 12.68 & 24,100 \\
\hline & 1600 & 14.90 & 32,700 & & 0400 & 15.93 & 37,200 & & 1200 & 12.70 & 24,100 \\
\hline & 1800 & 15.23 & 34,100 & & 0600 & 15.20 & 34,000 & & 1600 & 12.80 & 24,500 \\
\hline & 2000 & 15.51 & 35,300 & & 0800 & 14.75 & 32,000 & & 2000 & 12.61 & 23,800 \\
\hline & 2400 & 15.65 & 35,900 & & 1000 & 14.17 & 29,700 & & 2400 & 12.29 & 22,700 \\
\hline & & & & & 1200 & 13.84 & 28,400 & & & & \\
\hline \multirow[t]{8}{*}{12} & 0200 & 15.84 & 36,800 & \multirow{8}{*}{14} & 1600 & 13.03 & 25,300 & \multirow[t]{8}{*}{16} & 0400 & 11.98 & 21,600 \\
\hline & 0400 & 16.26 & 38,700 & & 2000 & 13.20 & 25,900 & & 0800 & 11.89 & 21,300 \\
\hline & 0600 & 16.97 & 41,900 & & 2400 & 12.76 & 24,300 & & 1200 & 11.55 & 20,200 \\
\hline & 0800 & 17.32 & 43,600 & & & & & & 1400 & 12.31 & 22,800 \\
\hline & 1000 & 17.59 & 44,900 & & 0400 & 12.97 & 25,100 & & 1600 & 11.70 & 20,700 \\
\hline & 1200 & 18.20 & 47,900 & & 0800 & 12.81 & 24,500 & & 1800 & 11.31 & 19,400 \\
\hline & 1400 & 18.44 & 49,100 & & 1200 & 12.63 & 23,900 & & 2000 & 10.92 & 18,100 \\
\hline & 1600 & 18.46 & 49,200 & & 1600 & 12.70 & 24,100 & & 2400 & 10.98 & 18,300 \\
\hline
\end{tabular}

(190) 5-3995. Big Eau Pleine River near Stratford, Wis.

Location.-Lat $44^{\circ} 49^{\prime} 15^{\prime \prime}$, long $90^{\circ} 04^{\prime} 35^{\prime \prime}$, on line between sec.13, T. $27 \mathrm{~N}^{+}$, R. $3 \mathrm{E}$,, and sec.18, T. 27 N., R.4 E., on left bank $15 \mathrm{ft}$ upstream from bridge on State Highway 97 , 1 mile north of Stratford, and 1.4 miles downstream from small tributary.

Drainage area. -224 sq $\mathrm{mi}$.

Gage-height record.-Water-stage recorder graph except Mar. 10-14, 21-29 when intake was frozen. Datum of gage is $1,154.24 \mathrm{ft}$ above mean sea level, datur of 1929 .

Discharge record.-Stage-discharge relation defined by current-meter measurements below 24,000 cfs. Backwater from ice Mar. 1 to Apr. 9. Rate of change in stage used is a factor Apr. 10-15, 26, 27, May 16, 26.

Maxima.-March-May 1965: Discharge, 13,700 cfs 0500 hours Apr. 11 (gage height, $18.43)$

1914-25, 1937 to February 1965: Discharge, 41,000 cfs Sept. 9, 1938 (gage height, $24.5 \mathrm{ft}$, from floodmarks).

Flood of June 5, 1914, reached a stage of $20.7 \mathrm{ft}$, from floodmarks (discharge, $40,000 \mathrm{cfs}$ ), former site and datum.

Mean discharge, in cubli feet per second, 1965

\begin{tabular}{|c|c|c|c|c|c|c|c|c|c|c|c|}
\hline Day & March & April & May & Day & March & April & May & Day & March & April & May \\
\hline 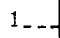 & 40 & 17 & 195 & $11 \ldots$ & 115 & 11,100 & 176 & 21. & 28 & 604 & 115 \\
\hline 2 & 150 & 18 & 182 & $12 \ldots$ & 94 & 6,160 & 118 & 22. & 26 & 598 & 85 \\
\hline 3. & 350 & 18 & 220 & $13 \ldots$ & 80 & 2,090 & 90 & 23. & 24 & 488 & 67 \\
\hline & 250 & 19 & 258 & 14 & 70 & 1,710 & 72 & 24 & 22 & 384 & 55 \\
\hline & 200 & 20 & 173 & $15 \ldots$ & 58 & 1,690 & 74 & 25. & 21 & 511 & 55 \\
\hline 6. & 280 & 50 & 267 & $16 \ldots$ & 50 & 984 & 1,520 & 26 & 20 & 2,320 & 977 \\
\hline 7. & 180 & 400 & 292 & $17 \ldots$ & 44 & 818 & 716 & 27 & 19 & 2,200 & 886 \\
\hline 8. & 260 & 1,400 & 183 & $18 \ldots$ & 39 & 1,140 & 477 & - & 18 & 660 & 318 \\
\hline 9 & 240 & 2,300 & 260 & $19 \ldots$ & 34 & 836 & 358 & 29 & 18 & 346 & 173 \\
\hline 10 & 160 & 5,670 & 328 & $20 \ldots$ & 31 & 651 & 180 & $30_{-}$ & 17 & 228 & 115 \\
\hline & & & & & & & & 31. & 17 & 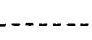 & 84 \\
\hline \multirow{2}{*}{\multicolumn{9}{|c|}{$\begin{array}{l}\text { Monthly mean discharge, in cubic feet per second } \\
\text { Runoff, in inches }\end{array}$}} & 95.3 & 1,514 & 293 \\
\hline & & & & & & & & & 0.49 & 7.54 & 1.51 \\
\hline
\end{tabular}


Gage height, in feet, and discharge, in cubic feet per second, at indicated time, 1965, of Big Eau Pleine River near Stratford, Wis.

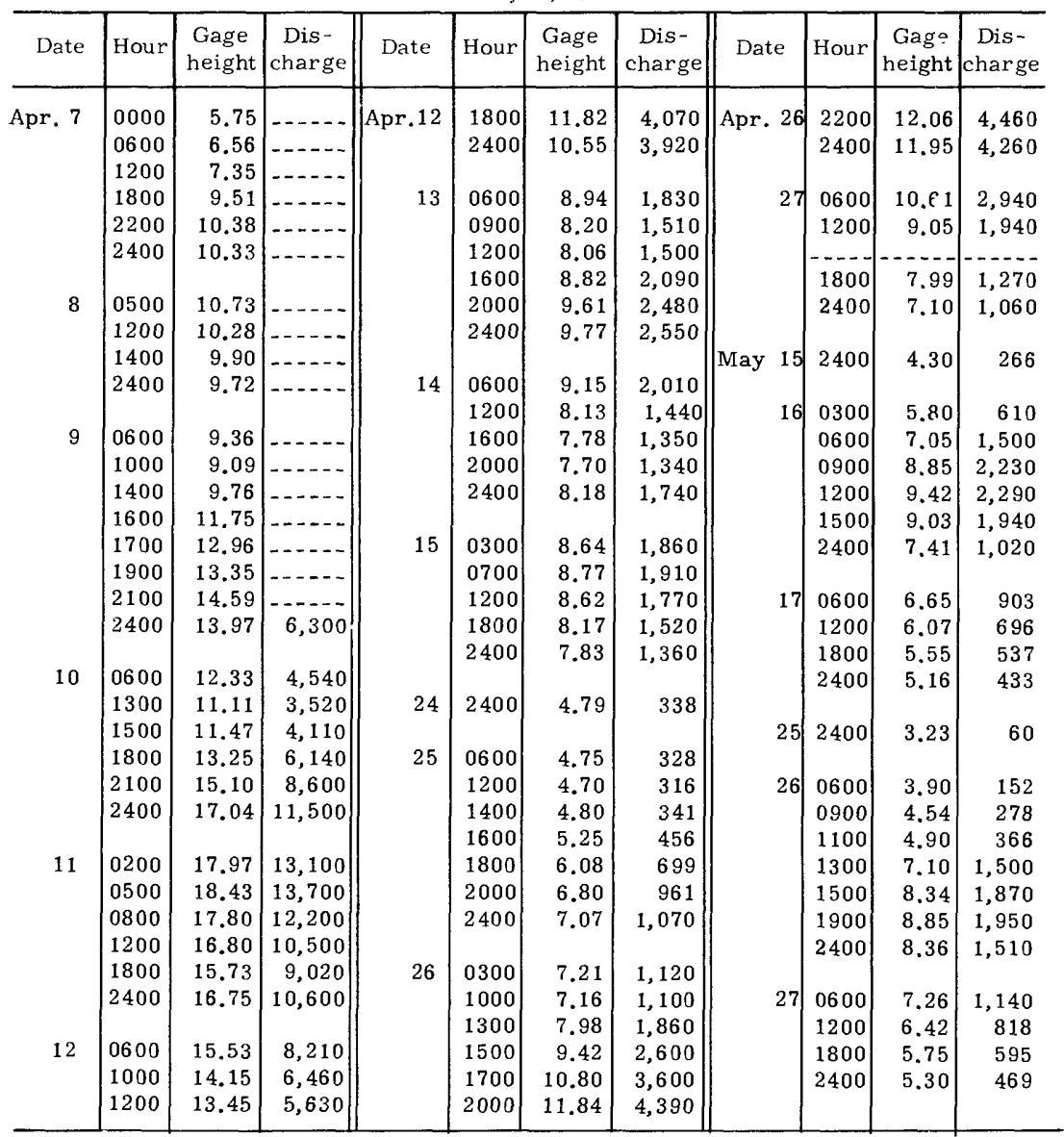

(191) 5-3996. Big Eau Pleine Reservoir near Knowlton, Wis.

Location.--Lat $44^{\circ} 43^{\prime} 50^{\prime \prime}$, long $89^{\circ} 45^{\prime} 35^{\prime \prime}$, in SW $\frac{1}{4}$ sec.14, T.26 N., R.6 E., on Rig Eau Pleine River, 4 miles south of Moon and 4.0 miles west of Knowlton.

Drainage area. $-365 \mathrm{sq} \mathrm{mi}$, approximately.

Gage-height record.-Float gage. Datum of gage is $1,115.00 \mathrm{ft}$ above mean s€a level (levels by Wisconsin Valley Improvement Co.).

Maxima.-March to May 1965: Contents at end of week, 4,457 million cubic feet May 16

(gage height, $31 \mathrm{ft} 6$ in).

1938 to February 1965: Contents observed, 4,496 million cubic feet Oct. 25, 1959

(gage height, $31 \mathrm{ft} 7 \frac{1}{2} \mathrm{in}$ ).

Remarks.- Reservoir operation began March 1937. Usable capacity, 4,457 million cubic feet between gage heights $0 \mathrm{ft} 0$ in and $31 \mathrm{ft} 6$ in (limits stipulated by Public Service Commission of Wisconsin).

Cooperation.-Gage heights and capacity tables furnished by Wisconsin Valley Improvement Co. 
Gage height, in feet and inches, and contents, in millions of cubic feet, 1965, of Big Eau Pleine Reservair near Knowlton, Wis.

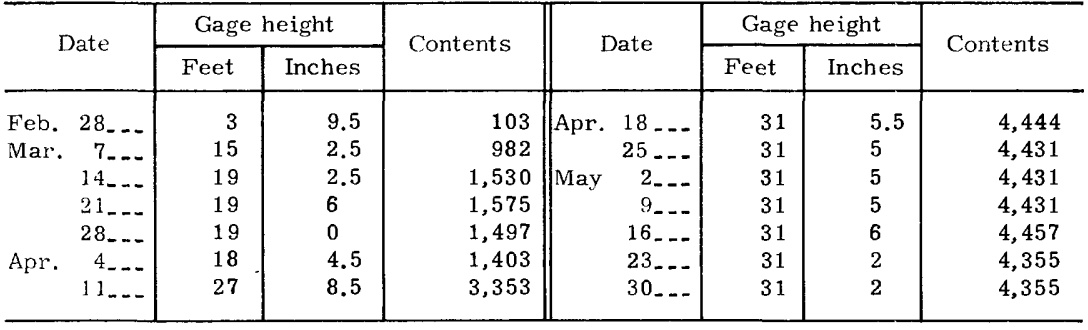

(192) 5-4002.95 Lake Dubay near Stevens Point, Wis.

Location.-Lat $44^{\circ} 39^{\prime} 50^{\prime \prime}$, long $89^{\circ} 39^{\prime} 05^{\prime \prime}$, in sec.10, T.25 N., R.7 E., $1 \frac{1}{2}$ miles downstream from Little Eau Pleine River and $10 \frac{1}{2}$ miles northwest of Stevens Point.

Drainage area.-4,890 sq $\mathrm{mi}$, approximately.

Gage-height record.-Staff gage and recording headwater gage. Datum of gage is mean sea level (power company levels).

Maximum.-March-May 1965: Daily contents, 5,120 million cubic feet Apr. 13 (elevation, $1,117.52 \mathrm{ft}$ ).

Remarks.-Usable capacity, 2,117 million cubic feet between elevations $1,110 \mathrm{ft}$ and $1,117 \mathrm{ft}$ (limits stipulated by Public Service Commission of Wisconsin).

Cooperation.-Elevations and capacity table furnished by Wisconsin Valley Improvement Co.

Elevation, in feet, and contents, in millions of cubic feet, 1965

\begin{tabular}{|c|c|c|c|c|c|c|}
\hline \multirow{2}{*}{ Day } & \multicolumn{2}{|c|}{ March } & \multicolumn{2}{|c|}{ April } & \multicolumn{2}{|c|}{ May } \\
\hline & Elevation & Contents & Elevation & Contents & Elevation & Contents \\
\hline $1 \ldots \ldots$ & 1113.31 & 3,693 & 1110.13 & 2,838 & 1116.60 & 4,774 \\
\hline $2 \ldots \ldots \ldots$ & 1113.51 & 3,753 & 1110.08 & 2,825 & 1116.88 & 4,878 \\
\hline $3 \ldots \ldots \ldots$ & 1114.19 & 3,957 & 1110.08 & 2,825 & 1116.95 & 4,903 \\
\hline $4 \ldots$ & 1115.11 & 4,242 & 1109.99 & 2,803 & 1116.56 & 4,759 \\
\hline $5 \ldots \ldots$ & 1115.77 & 4,468 & 1110.08 & 2,825 & 1116.16 & 4,611 \\
\hline $6 \ldots$ & 1116.09 & 4,585 & 1109.64 & 2,719 & 1115.45 & 4,355 \\
\hline $7 \ldots \ldots$ & 1116.15 & 4,608 & 1110.00 & 2,805 & 1115.93 & 4,526 \\
\hline $8 \ldots$ & 1116.14 & 4,604 & 1110.68 & 2,975 & 1116.58 & 4,767 \\
\hline $9 \ldots \ldots$ & 1116.11 & 4,593 & 1112.04 & 3,330 & 1116.58 & 4,767 \\
\hline $10 \ldots$ & 1115.99 & 4,548 & 1114.11 & 3,933 & 1116.50 & 4,737 \\
\hline $11 \ldots \ldots$ & 1115.78 & 4,472 & 1116.23 & 4,637 & 1116.44 & 4,715 \\
\hline $12 \ldots \ldots$ & 1115.73 & 4,454 & 1117.20 & 4,998 & 1116.40 & 4,700 \\
\hline $13 \ldots \ldots$ & 1115.20 & 4,271 & 1117.52 & 5,120 & 1116.18 & 4,619 \\
\hline $14 \ldots \ldots$ & 1114.65 & 4,098 & 1116.75 & 4,880 & 1116.51 & 4,741 \\
\hline $15 \ldots \ldots$ & 1114.30 & 3,990 & 1116.87 & 4,854 & 1116.97 & 4,911 \\
\hline $16 \ldots$ & 1114.20 & 3,960 & 1116.43 & 4,711 & 1116.88 & 4,878 \\
\hline $17 \ldots$ & 1113.73 & 3,819 & 1115.12 & 4,245 & 1116.67 & 4,800 \\
\hline $18 \ldots \ldots$ & 1113.11 & 3,633 & 1114.23 & 3,969 & 1116.25 & 4,644 \\
\hline - & 1112.68 & 3,507 & 1116.23 & 4,637 & 1115.57 & 4,396 \\
\hline - $20 \ldots$ & 1112.47 & 3,448 & 1116.68 & 4,804 & 1115.60 & 4,407 \\
\hline $21 \ldots$ & 1111.88 & 3,287 & 1116.65 & 4,792 & 1115.98 & 4,545 \\
\hline $22 \ldots \ldots$ & 1111.52 & 3,191 & 1116.45 & 4,718 & 1116.58 & 4,767 \\
\hline $23 \ldots$ & 1111.08 & 3,077 & 1116.26 & 4,648 & 1116.95 & 4,903 \\
\hline $24 \ldots$ & 1110.75 & 2,992 & 1116.14 & 4,604 & 1116.70 & 4,811 \\
\hline
\end{tabular}


Elevation, in feet, and contents, in millions of cubic feet, 1965, of Lake Dubay near Stevens Point, Wis. -.C.Continued

\begin{tabular}{|c|c|c|c|c|c|c|}
\hline \multirow{2}{*}{ Day } & \multicolumn{2}{|c|}{ March } & \multicolumn{2}{|c|}{ April } & \multicolumn{2}{|l|}{ May } \\
\hline & Elevation & Contents & Elevation & Contents & Elevation & Contents \\
\hline $25 \ldots \ldots$ & 1110.29 & 2,877 & 1116.13 & 4,600 & 1116.73 & 4,822 \\
\hline $26 \ldots \ldots$ & 1110.40 & 2,905 & 1116.15 & 4,607 & 1116.73 & 4,822 \\
\hline $27 \ldots \ldots$ & 1110.24 & 2,865 & 1116.47 & 4,726 & 1116.69 & 4,807 \\
\hline $28 \ldots \ldots$ & 1109.92 & 2,786 & 1116.45 & 4,718 & 1116.82 & 4,855 \\
\hline $29 \ldots$ & 1110,12 & 2,835 & 1115.45 & 4,355 & 1116.53 & 4,748 \\
\hline$\ldots$ & 1110.08 & 2,825 & 1115.50 & 4,372 & 1116.43 & 4,711 \\
\hline $31 \ldots \ldots-\ldots$ & 1110.14 & 2,840 & -------- & -------- & 1116.55 & 4,756 \\
\hline
\end{tabular}

(193) 5-4008. Wisconsin River at Wisconsin Rapids, Wis.

Location.-Lat $44^{\circ} 22^{\prime} 05^{\prime \prime}$, long $89^{\circ} 51^{\prime} 30^{\prime \prime}$, in SW $\frac{1}{4}$ sec. 24, T.22 N., R. 5 E., at Centralia powerplant of Nekoosa-Edwards Paper Co., 1.6 miles downstream from Chinago and Northwestern Railway bridge in Wiscons in Rapids.

Drainage area.- $-400 \mathrm{sq} \mathrm{mi}$, approximately.

Gage-height record.-Headwater and tailwater recording gages and generatior data read hourly and entered in daily log sheet by company employees. Elevation of powerplant pond is $980 \mathrm{ft}$ and datum of powerplant gages is $887.83 \mathrm{ft}$ above mean sea level (levels by Wisconsin Valley Improvement Co.).

Discharge record.-Dis charge computed hourly from powerplant records, using 0600 hours to 0600 hours basis for daily discharge. Powerplant rating checked by several recent high-water current-meter measurements, two near 47,000 cfs.

Maxima.-March-May 1965: Discharge, 64,000 cfs 2300 hours Apr. 13.

1914-50, 1957 to February 1965: Discharge, 70,400 cfs at site 7.0 miles downstream, Sept. 12, 1938 (gage height, $19.10 \mathrm{ft}$, datum then in use), from rating curve extended above $58,000 \mathrm{cfs}$.

Remarks.-Discharge computed from powerplant records on basis of load-discharge rating of hydroelectric units as developed by Geological Survey and taintor-gate rating and spillway ratings based on theoretical formulas and discharge measurements. Flow regulated by 21 reservoirs and many powerplants above station.

Mean discharge, in cubic feet per second, 1965

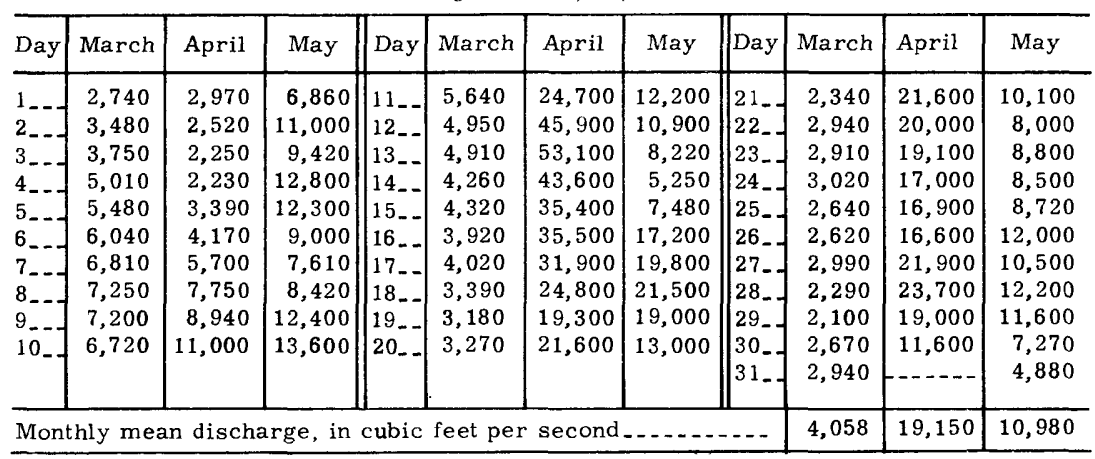


Gage height, in feet, and discharge, in cubic feet per second, at indicated time, 1965, of Wi-consin River at Wisconsin Rapids, Wis.

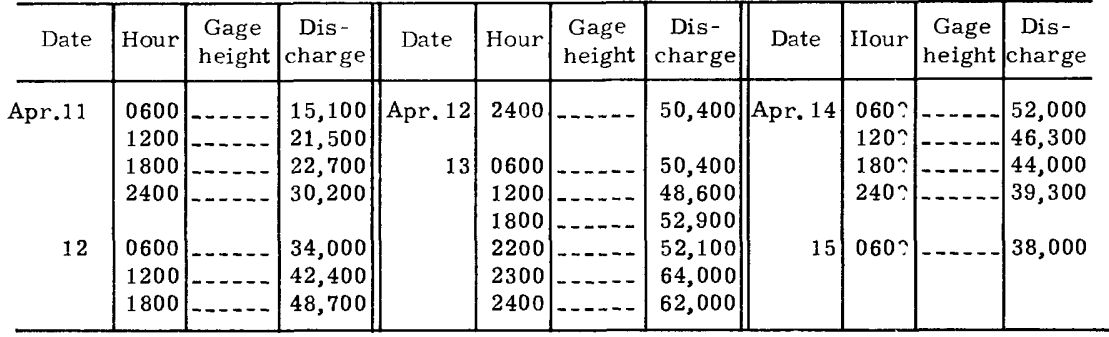

(194) 5-4014. Petenwell Flowage near Necedah, Wis.

Location.-Lat $44^{\circ} 03^{\prime} 25^{\prime \prime}$, long $90^{\circ} 01^{\prime} 15^{\prime \prime}$, in $\mathrm{SE}_{\frac{1}{4}} \mathrm{sec} .4, \mathrm{~T} .18$ N., R.4 E., on Wisconsin River, 0.7 mile upstream from bridge on State Highway 21 , and 3.5 miles northeast of Necedah.

Drainage area. $-5,860 \mathrm{sq} \mathrm{mi}$, approximately.

Gage-height record.-Staff gage and recording headwater gage. Datum of gage is $790.2 \mathrm{ft}$ above mean sea level (levels by Wisconsin River Power Co.).

Maxima.-March-May 1965: Daily contents, 19,954 million cubic feet Apr. 17 (gage height, $136.08 \mathrm{ft}$ ).

1950 to February 1965: Contents on last day of month, 19,926 million cubic feet Oct. 31, 1954 (gage height, $136.05 \mathrm{ft}$ ).

Remarks.-Dam was completed in 1949. Reservoir operation began in April 1950. Limits of operation are stipulated by Public Service Commission of Wisconsin as follows: maximum gage height Mar. 16 to June $15,136.00 \mathrm{ft}(19,880$ million cubic feet $)$ and June 16 to Mar. 15, $135.00 \mathrm{ft}(18,980 \mathrm{mcf})$; minimum gage height Apr. 16 to Dec. 31 , $134.00 \mathrm{ft}(18,090 \mathrm{mcf})$ and Jan. 1 to Apr. $15,130.00 \mathrm{ft}(14,690 \mathrm{mcf})$.

Cooperation.-Gage heights and capacity table furnished by Wisconsin Valley Improvement Co.

Gage height, in feet, and contents, in millions of cubic feet, 1965

\begin{tabular}{|c|c|c|c|c|c|c|}
\hline \multirow{2}{*}{ Day } & \multicolumn{2}{|c|}{ March } & \multicolumn{2}{|c|}{ April } & \multicolumn{2}{|l|}{ May } \\
\hline & Elevation & Contents & Elevation & Contents & Elevation & Contents \\
\hline $1 \ldots$ & 133.87 & 17,976 & 130.04 & 14,723 & 135.93 & 19,817 \\
\hline $2 \ldots \ldots \ldots$ & 133.81 & 17,923 & 130.05 & 14,731 & 135.85 & 19,745 \\
\hline $3 \ldots \ldots$ & 133.64 & 17,773 & 130.05 & 14,731 & 136.03 & 19,907 \\
\hline $4 \ldots \ldots$ & 133.50 & 17,650 & 130.10 & 14,772 & 135.52 & 19,448 \\
\hline $5 \ldots \ldots$ & 133.39 & 17,553 & 130.12 & 14,788 & 135.48 & 19,412 \\
\hline $6 \ldots \ldots-n$ & 133.32 & 17,492 & 130.16 & 14,821 & 135.52 & 19,448 \\
\hline $7 \ldots \ldots$ & 133.34 & 17,509 & 130.18 & 14,838 & 135.63 & 19,547 \\
\hline $8 \ldots \ldots$ & 133.92 & 18,020 & 130.34 & 14,965 & 135.62 & 19,538 \\
\hline $9 \ldots$ & 134.06 & 18,143 & 130.50 & 15,100 & 135.68 & 19,592 \\
\hline $10 \ldots$ & 134.08 & 18,160 & 130.73 & 15,293 & 135.94 & 19,826 \\
\hline $11 \ldots \ldots$ & 134.02 & 18,108 & 131.20 & 15,688 & 136.02 & 19,898 \\
\hline $12 \ldots$ & 133.95 & 18,046 & 132.42 & 16,713 & 135.89 & 19,781 \\
\hline 13 & 133.73 & 17,852 & 134.26 & 18,319 & 136.05 & 19,926 \\
\hline $14 \ldots \ldots$ & 133.54 & 17,685 & 135.55 & 19,475 & 136.00 & 19,880 \\
\hline 15 & 133.32 & 17,492 & 135.92 & 19,808 & 135.80 & 19,700 \\
\hline $16 \ldots$ & 133.10 & 17,298 & 135.77 & 19,673 & 135.82 & 19,718 \\
\hline $17 \ldots$ & 132.88 & 17,107 & 136.08 & 19,954 & 135.91 & 19,799 \\
\hline $18 \ldots$ & 132.61 & 16,875 & 135.78 & 19,682 & 135.80 & 19,700 \\
\hline
\end{tabular}


Gage height, in feet, and contents, in millions of cubic feet, 1965, of Petenwell Flowage near Necedah, Wis. - Continued

\begin{tabular}{|c|c|c|c|c|c|c|}
\hline \multirow{2}{*}{ Day } & \multicolumn{2}{|c|}{ March } & \multicolumn{2}{|c|}{ April } & \multicolumn{2}{|l|}{ May } \\
\hline & Elevation & Contents & Elevation & Contents & Elevation & Contents \\
\hline $19 \ldots-----$ & 132.28 & 16,595 & 135.75 & 19,655 & 135.95 & 19,835 \\
\hline $20 \ldots \ldots-\ldots$ & 132.10 & 16,444 & 135.70 & 19,610 & 135.88 & 19,772 \\
\hline $21 \ldots \ldots$ & 131.92 & 16,293 & 135.95 & 19,835 & 135.69 & 19,601 \\
\hline $22 \ldots \ldots-\cdots$ & 131.74 & 16,142 & 136.03 & 19,907 & 135.73 & 19,637 \\
\hline $23 \ldots \ldots$ & 131.44 & 15,890 & 136.04 & 19,916 & 135.72 & 19,628 \\
\hline $24 \ldots \ldots \ldots \ldots$ & 131.15 & 15,646 & 135.95 & 19,835 & 135.76 & 19,664 \\
\hline $25 \ldots \ldots$ & 130.87 & 15,411 & 135.80 & 19,700 & 135.85 & 19,745 \\
\hline $26 \ldots \ldots-\cdots$ & 130.66 & 15,234 & 135.69 & 19,601 & 136.00 & 19,880 \\
\hline $27 \ldots \ldots$ & 130.46 & 15,067 & 135.60 & 19,520 & 135.76 & 19,664 \\
\hline $28 \ldots \ldots \ldots$ & 130.30 & 14,936 & 135.66 & 19,574 & 135.51 & 19,439 \\
\hline $29 \ldots \ldots \ldots$ & 130.11 & 14,780 & 135.87 & 19,763 & 135.69 & 19,601 \\
\hline $30 \ldots \ldots$ & 130.00 & 14,690 & 135.85 & 19,745 & 135.87 & 19,763 \\
\hline $31--\ldots---$ & 129.97 & 14,665 & & $-\ldots-n-\ldots$ & 135.79 & 19,691 \\
\hline
\end{tabular}

(195) 5-4020. Yellow River at Babcock, Wis.

Location.-Lat $44^{\circ} 18^{\prime} 05^{\prime \prime}$, long $90^{\circ} 07^{\prime} 15^{\prime \prime}$, in NW $\frac{1}{4}$ sec.14, T.21 N., R.3 E., on right bank at downstream side of highway bridge at Babcock, 1.9 miles upstream from Hemlock Creek.

Drainage area. -223 sq mi.

Gage-height record.-Digital recorder tape punched at sixty-minute intervals except 0100 hours Mar. 3 to 1700 hours Mar. 10. Datum of gage is $954.75 \mathrm{ft}$ above mean sea level, datum of 1929 .

Discharge record.-Stage-discharge relation defined by current-meter meast rements. Mean daily discharges computed from 24 punch-tape recordings per day except 0100 hours Mar. 3 to 1700 hours Mar. 10. Backwater from ice Mar. 1 to Apr. 9.

Maxima.-March-May 1965: Discharge, 6,320 cfs 0800 hours Apr. 12 (gage h?ight, $14.61 \mathrm{ft}$ ).

1944 to February 1965: Discharge, 11,600 cfs Apr. 2, 1952 (gage height, 17, $38 \mathrm{ft}$ ).

Mean discharge, in cubic feet per second, 1965

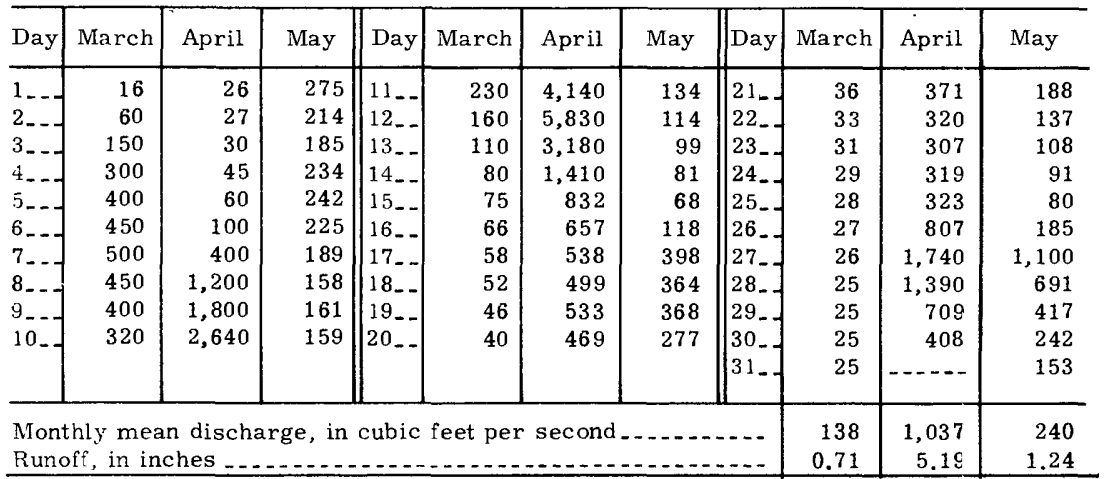


Gage height, in feet, and discharge, in cubic feet per second, at indicated time, 1965, of Yellow River at Babcock, Wis.

\begin{tabular}{|c|c|c|c|c|c|c|c|c|c|c|c|}
\hline Date & Hour & $\begin{array}{c}\text { Gage } \\
\text { height }\end{array}$ & $\begin{array}{c}\text { Dis- } \\
\text { charge }\end{array}$ & Date & Hour & $\begin{array}{c}\text { Gage } \\
\text { height }\end{array}$ & $\begin{array}{c}\text { Dis- } \\
\text { charge }\end{array}$ & Date & Hour & $\begin{array}{c}\text { Gage } \\
\text { height }\end{array}$ & $\begin{array}{c}\text { Dis- } \\
\text { charge }\end{array}$ \\
\hline \multirow[t]{8}{*}{ Apr. 6} & 0000 & 2.50 & $\ldots \ldots$ & Apr. 11 & 1200 & 12.38 & 3,700 & Apr. 26 & 1400 & 7.35 & 773 \\
\hline & 1200 & 2.48 & $-\ldots$ & & 1400 & 13.04 & 4,300 & & $160 \mathrm{c}$ & 8.02 & 956 \\
\hline & 1400 & 2.24 & $\ldots \ldots$ & & 1600 & $13.6 \mathrm{I}$ & 4,890 & & $180 \mathrm{C}$ & 8.59 & 1,170 \\
\hline & 1600 & 2.56 & 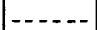 & & 1800 & 14.04 & 5,460 & & $240 \mathrm{C}$ & 9.31 & 1,500 \\
\hline & 1800 & 3.50 & $---\cdot$ & & 2400 & 14.43 & 6,040 & & & & \\
\hline & 2000 & 4.07 & ------ & & & & & 27 & $060 \mathrm{C}$ & 9.58 & 1,640 \\
\hline & 2400 & 4.84 & $1----$ & 12 & 0400 & 14.56 & 6,240 & & $120 \mathrm{C}$ & 9.90 & 1,820 \\
\hline & & & & & 0600 & 14.60 & 6,300 & & $160 \mathrm{C}$ & 9.97 & 1,860 \\
\hline \multirow[t]{7}{*}{7} & 0400 & 5.31 & $\ldots$ & & 0800 & 14.61 & 6,320 & & $200 c$ & 9.90 & 1,820 \\
\hline & 0800 & 5.90 & $\ldots$ & & 1200 & 14.51 & 6,160 & & $240 \mathrm{C}$ & 9.77 & 1,740 \\
\hline & 1200 & 6.65 & $---\cdot-$ & & 1600 & 14.26 & 5,790 & & & & \\
\hline & 1600 & 7.50 & $-\ldots$ & & 2000 & 13.88 & 5,230 & 28 & $060 \mathrm{C}$ & 9.50 & 1,600 \\
\hline & 2000 & 8.45 & $-\cdots$ & & 2400 & 13.38 & 4,640 & & 1200 & 9.15 & 1,420 \\
\hline & 2400 & 9.13 & $\ldots$ & & & & & & 1800 & 8.70 & 1,210 \\
\hline & & & & 13 & 0400 & 12.85 & 4,120 & & 2400 & 8.16 & 998 \\
\hline \multirow[t]{7}{*}{8} & 0400 & 9.59 & - & & 0800 & 12.33 & 3,660 & & & & \\
\hline & 0800 & 10.37 & - - - & & 1200 & 11.79 & 3,170 & 29 & 0600 & 7.59 & 833 \\
\hline & 1200 & 11.32 & 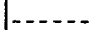 & & 1800 & 11.00 & 2,550 & & 1200 & 7.01 & 695 \\
\hline & 1600 & 11.88 & $\ldots$ & & 2400 & 10.25 & 2,030 & & 1800 & 6.47 & 593 \\
\hline & 1800 & 11.93 &..--- & & & & & & 2400 & 5.96 & 521 \\
\hline & 2400 & 11.79 & $---\cdots$ & 14 & 0400 & 9.80 & 1,760 & & & & \\
\hline & & & & & 0800 & 9.41 & 1,560 & May 26 & 2400 & 5.63 & 478 \\
\hline \multirow[t]{6}{*}{9} & 0800 & 11.35 & -...- & & 1200 & 9.10 & 1,400 & & & & \\
\hline & 1000 & 11.48 & $-\cdots$ & & 1800 & 8.65 & 1,190 & 27 & 0200 & 6.41 & 584 \\
\hline & 1400 & 11.77 & $\ldots$ & & 2400 & 8.22 & 1,020 & & 0600 & 7.98 & 944 \\
\hline & 1800 & 11.65 & $-\ldots$ & & & & & & 1000 & 8.94 & 1,320 \\
\hline & 2400 & 11.31 & 2,790 & 15 & 0600 & 7.86 & 908 & & 1200 & 9.05 & 1,380 \\
\hline & & & & & 1200 & 7.54 & 820 & & 1600 & 8.92 & 1,310 \\
\hline \multirow[t]{6}{*}{10} & 0600 & 11.00 & 2,550 & & 1800 & 7.25 & 750 & & 2000 & 8.57 & 1,160 \\
\hline & 1000 & 10.81 & 2,420 & & 2400 & 7.10 & 715 & & 2400 & 8.15 & 995 \\
\hline & 1200 & 10.95 & 2,520 & & & & & & & & \\
\hline & 2000 & 11.28 & 2,760 & 25 & 2400 & 4.64 & 349 & 28 & 0400 & 7.70 & 863 \\
\hline & 2400 & 11.32 & 2,800 & & & & & & 0800 & 7.26 & 752 \\
\hline & & & & 26 & 0600 & 5.19 & 421 & & 1200 & 6.85 & 662 \\
\hline \multirow[t]{2}{*}{11} & 0400 & 11.28 & 2,760 & & 1000 & 6.03 & 530 & & 1800 & 6.40 & 582 \\
\hline & 0800 & 11.41 & 2,870 & & 1200 & 6.68 & 629 & & 2400 & 6.03 & 530 \\
\hline
\end{tabular}

(196) 5-4032. Castle Rock Flowage near Mauston, Wis.

Location.-Lat $43^{\circ} 51^{\prime} 50^{\prime \prime}$, long $89^{\circ} 57^{\prime} 35^{\prime \prime}$, in sec.13, T.16 N., R.4 E., $4 \frac{1}{2}$ miles upstream from Duck Creek, 2 miles south of Germantown, and 7 miles northeast of Mauston.

Drainage area. $-6,860 \mathrm{sq} \mathrm{mi}$ approximately.

Gage-height record.- Staff gage and recording headwater gage. Datum of gage is $790.2 \mathrm{ft}$ above mean sea level (levels by Wisconsin River Power Co.).

Maxima.-March-May 1965: Daily contents, 7,786 million cubic feet Apr. 18 (gage height, $94.20 \mathrm{ft}$ ).

1950 to February 1965: Contents on last day of month, 7,786 million cubic feet June 30,1954 (gage height, $94.20 \mathrm{ft}$ ).

Remarks.-Dam was completed in 1950. Reservoir operation began in May 1950. Limits of operation are stipulated by Public Service Commission of Wisconsin as follows: Maximum gage height Mar. 16 to June $15,94.00 \mathrm{ft}$. $(7,630 \mathrm{million}$ cubic feet $)$ and June 16 to Mar. $15,93.00 \mathrm{ft}(6,880 \mathrm{mcf})$; minimum gage height May 1 to Jan. 31 , $92.00 \mathrm{ft}$. $(6,180 \mathrm{mcf})$ and Feb. 1 to Apr. $30,87,00 \mathrm{ft}(3,360 \mathrm{mcf})$.

Cooperation.-Gage heights and capacity table furnished by Wisconsin Valley Improvement Co. 
Gage height, in feet, and contents, in millions of cubic feet, 1965, of Castle Rock Flowage near Mauston, Wis.

\begin{tabular}{|c|c|c|c|c|c|c|}
\hline \multirow{2}{*}{ Day } & \multicolumn{2}{|c|}{ March } & \multicolumn{2}{|c|}{ April } & \multicolumn{2}{|c|}{ May } \\
\hline & Gage height & Contents & Gage height & Contents & Gage height & Contents \\
\hline $\begin{array}{l}1 \\
2 \\
3 \\
3 \\
4 \\
5 \\
6 \\
7 \\
8\end{array}$ & $\begin{array}{l}90.28 \\
90.52 \\
90.83 \\
90.97 \\
91.10 \\
91.19 \\
91.31 \\
90.65 \\
90.57 \\
90.72 \\
90.83 \\
90.87 \\
90.87 \\
90.87 \\
90.65 \\
90.47 \\
90.25 \\
90.10 \\
89.90 \\
89.50 \\
89.12 \\
88.65 \\
88.35 \\
88.07 \\
87.78 \\
87.63 \\
87.37 \\
87.20 \\
87.04 \\
87.06 \\
87.14 \\
\end{array}$ & $\begin{array}{l}5,088 \\
5,232 \\
5,425 \\
5,511 \\
5,594 \\
5,652 \\
5,728 \\
5,313 \\
5,263 \\
5,356 \\
5,425 \\
5,449 \\
5,449 \\
5,449 \\
5,313 \\
5,202 \\
5,070 \\
4,980 \\
4,862 \\
4,630 \\
4,417 \\
4,161 \\
4,002 \\
3,856 \\
3,714 \\
3,642 \\
3,523 \\
3,448 \\
3,378 \\
3,386 \\
3,422\end{array}$ & \begin{tabular}{|}
87.14 \\
87.11 \\
87.07 \\
87.25 \\
87.35 \\
87.48 \\
87.63 \\
87.80 \\
88.10 \\
88.55 \\
88.87 \\
89.80 \\
91.00 \\
93.60 \\
93.67 \\
93.83 \\
93.78 \\
94.20 \\
93.85 \\
93.83 \\
93.75 \\
93.84 \\
93.94 \\
93.97 \\
93.90 \\
93.71 \\
93.56 \\
93.47 \\
93.85 \\
93.93 \\
-----
\end{tabular} & $\begin{array}{r}3,422 \\
3,408 \\
3,391 \\
3,470 \\
3,514 \\
3,571 \\
3,642 \\
3,724 \\
3,872 \\
4,107 \\
4,203 \\
4,804 \\
5,530 \\
7,326 \\
7,379 \\
7,501 \\
7,463 \\
7,786 \\
7,516 \\
7,501 \\
7,440 \\
7,508 \\
7,584 \\
7,607 \\
7,554 \\
7,410 \\
7,296 \\
7,228 \\
7,516 \\
7,577 \\
----\end{array}$ & $\begin{array}{l}93.97 \\
94.03 \\
94.03 \\
93.68 \\
93.50 \\
93.48 \\
93.68 \\
93.85 \\
93.93 \\
94.00 \\
93.85 \\
93.85 \\
93.98 \\
94.00 \\
93.98 \\
94.07 \\
93.68 \\
93.58 \\
93.62 \\
93.90 \\
93.78 \\
93.78 \\
93.82 \\
93.77 \\
93.85 \\
93.90 \\
93.87 \\
93.70 \\
93.38 \\
93.60 \\
93.89\end{array}$ & $\begin{array}{l}7,607 \\
7,653 \\
7,653 \\
7,387 \\
7,250 \\
7,235 \\
7,387 \\
7,516 \\
7,577 \\
7,630 \\
7,516 \\
7,516 \\
7,615 \\
7,630 \\
7,615 \\
7,685 \\
7,387 \\
7,311 \\
7,341 \\
7,554 \\
7,463 \\
7,463 \\
7,493 \\
7,455 \\
7,516 \\
7,554 \\
7,531 \\
7,402 \\
7,161 \\
7,326 \\
7,546\end{array}$ \\
\hline
\end{tabular}

(197) 5-4035. Lemonweir River at New Lisbon, Wis.

Location.-Lat $43^{\circ} 52^{\prime} 50^{\prime \prime}$, long $90^{\circ} 09^{\prime} 40^{\prime \prime}$, in sec.8, T.16 N., R.3 E., near center span on downstream side of bridge on State Highway 80 in New Lisbon, $200 \mathrm{ft}$ downstream from recreation dam and 1 mile upstream from Webster Creek.

Drainage area. $-500 \mathrm{sq} \mathrm{mi}$, approximately.

Gage-height record.-Wire-weight gage read twice daily. Graph drawn on basis of twice-daily wire-weight gage readings during rises. Datum of gage is $867.05 \mathrm{ft}$ above mean sea level, datum of 1929 .

Discharge record.-Stage-discharge relation defined by current-meter measurements below 5,500 cfs. Backwater from ice Mar. 17 to Apr. 2.

Maxima.-March-May 1965: Discharge, 3,000 cfs 1200 hours Apr. 13 (gage height, $10.45 \mathrm{ft}$ ).

1944 to February 1965: Discharge, 6,880 cfs May 8, 1960 (gage height, $12.94 \mathrm{ft}$ ). 
Mean discharge, in cubic feet per second, 1965, of Lemonweir River at New Lisbon, Wis.

\begin{tabular}{|c|c|c|c|c|c|c|c|c|c|c|c|}
\hline Day & March & April & May & Day & March & April & May & Day & March & April & May \\
\hline 1. & 363 & 180 & 1,080 & $11 \ldots$ & 940 & 2,120 & 573 & 21 & 180 & 1,150 & 623 \\
\hline 2. & 1,100 & 300 & 937 & $12 \ldots$ & 703 & 2,580 & 498 & 22. & 180 & 1,070 & 644 \\
\hline 3. & 1,400 & 445 & 802 & $13 \ldots$ & 573 & 2,950 & 398 & 23. & 170 & 1,020 & 581 \\
\hline 4 & 1,030 & 622 & 673 & $14 \ldots$ & 423 & 2,710 & 370 & 2 & 160 & 988 & 514 \\
\hline 5 & 1,120 & 863 & 621 & $15 \ldots$ & 357 & 2,340 & 312 & 2 & 150 & 943 & 461 \\
\hline 6 & 1,640 & 1,120 & 621 & $16 \ldots$ & 317 & 1,980 & 299 & $26 \ldots$ & 140 & 1,000 & 730 \\
\hline 7. & 1,660 & 1,450 & 634 & 17. & 280 & 1,680 & 362 & 27. & 140 & 1,080 & 1,230 \\
\hline 8 & 1,390 & 1,810 & 644 & $18 \ldots$ & 230 & 1,480 & 488 & 28 & 130 & 1,140 & 1,870 \\
\hline 9 & 1,340 & 2,100 & 649 & 19. & 210 & 1,360 & 641 & 29. & 130 & 1,210 & 2,170 \\
\hline 10 & 1,280 & 2,130 & 632 & 20 & 190 & 1,260 & 661 & 30 & 140 & 1,190 & 1,920 \\
\hline & & & & & & & & & 150 & $-\cdots$ & 1,580 \\
\hline \multicolumn{9}{|c|}{ Monthly mean discharge, in cubic feet per second } & 588 & 1,409 & 781 \\
\hline \multicolumn{9}{|c|}{ Runoff, in inches } & 1.35 & 3.14 & 1.80 \\
\hline
\end{tabular}

Gage height, in feet, and discharge, in cubic feet per second, at indicated time, 1965

\begin{tabular}{|c|c|c|c|c|c|c|c|c|c|c|c|}
\hline Date & Hour & $\begin{array}{l}\text { Gage } \\
\text { height }\end{array}$ & $\begin{array}{c}\text { Dis- } \\
\text { charge }\end{array}$ & Date & Hour & $\begin{array}{l}\text { Gage } \\
\text { height }\end{array}$ & $\begin{array}{c}\text { Dis- } \\
\text { charge }\end{array}$ & Date & Hour & $\begin{array}{l}\text { Gage } \\
\text { height }\end{array}$ & $\begin{array}{c}\text { Dis- } \\
\text { charge }\end{array}$ \\
\hline \multirow{5}{*}{ Apr. 6} & 0000 & 6.55 & 985 & \multirow{5}{*}{ Apr. 10} & 0600 & 9.36 & 2,190 & \multirow[t]{3}{*}{ Apr. 14} & 1600 & 10.03 & 2,640 \\
\hline & 0800 & 6.85 & 1,080 & & 1200 & 9.27 & 2,130 & & 2400 & 9.85 & 2,520 \\
\hline & 1600 & 7.10 & 1,150 & & 1800 & 9.19 & 2,080 & & & & \\
\hline & \multirow[t]{2}{*}{2400} & \multirow[t]{2}{*}{7.40} & \multirow[t]{2}{*}{1,270} & & 2400 & 9.17 & 2,070 & 15 & 0800 & 9.70 & 2,410 \\
\hline & & & & & & & & & 1600 & 9.50 & 2,270 \\
\hline \multirow[t]{4}{*}{7} & 0800 & 7.71 & 1,390 & \multirow[t]{4}{*}{11} & 1200 & 9.22 & 2,100 & & 2400 & 9.30 & 2,150 \\
\hline & 1600 & 8.00 & 1,510 & & 1800 & 9.27 & 2,130 & & & & \\
\hline & 2400 & 8.32 & 1,640 & & 2400 & 9.43 & 2,230 & 16 & 0800 & 9.13 & 2,050 \\
\hline & & & & & & & & & 1600 & 8.90 & 1,920 \\
\hline \multirow[t]{4}{*}{8} & 0600 & 8.52 & 1,730 & \multirow[t]{4}{*}{12} & 0600 & 9.65 & 2,380 & \multirow{4}{*}{17} & 2400 & 8.70 & 1,820 \\
\hline & 1200 & 8.70 & 1,820 & & 1200 & 9.95 & 2,580 & & & & \\
\hline & 1800 & 8.85 & 1,900 & & 1800 & 10.21 & 2,790 & & 0800 & 8.52 & 1,730 \\
\hline & 2400 & 9.00 & 1,970 & & 2400 & 10.33 & 2,890 & & $\begin{array}{l}1600 \\
2400\end{array}$ & $\begin{array}{l}8.30 \\
8.13\end{array}$ & $\begin{array}{l}1,630 \\
1,560\end{array}$ \\
\hline \multirow[t]{4}{*}{9} & 0600 & 9.14 & 2,050 & \multirow[t]{4}{*}{13} & 0600 & 10.42 & 2,970 & \multirow{4}{*}{18} & 0800 & 799 & \\
\hline & 1200 & 9.23 & 2,110 & & 1200 & 10.45 & 3,000 & & 1600 & 7.86 & $\begin{array}{l}1,010 \\
1,450\end{array}$ \\
\hline & 1800 & 9.30 & 2,150 & & 1800 & 10.40 & 2,950 & & 2400 & 7.79 & 1,430 \\
\hline & 2400 & 9.36 & 2,190 & & 2400 & 10.33 & 2,890 & & 1200 & 7.63 & 1,360 \\
\hline 10 & 0200 & 9.37 & 2,190 & 14 & 0800 & 10.20 & 2,780 & 19 & 2400 & 7.50 & 1,310 \\
\hline
\end{tabular}


(198) 5-4040. Wisconsin River near Wisconsin Dells, Wis.

Location.-Lat $43^{\circ} 36^{\prime} 20^{\prime \prime}$, long $89^{\circ} 45^{\prime} 25^{\prime \prime}$, in extreme western part of sec.14, T.13 N., R.6 E., on right bank 0.5 mile downstream from Dell Creek and 3 miles downstream from Wisconsin Dells.

Drainage area.-7,830 sq $\mathrm{mi}$, approximately.

Gage-height record.-Digital recorder tape punched at sixty-minute intervals. Altitude of gage is $810 \mathrm{ft}$ (from topographic map).

Discharge record.--Stage-discharge relation defined by current-meter measirements. Mean daily discharges computed from 24 punch-tape recordings per day.

Maxima.-March-May 1965: Discharge, 50,200 cfs 2000 hours Apr. 15 (gage height, $18.95 \mathrm{ft}$ ).

1934 to February 1965: Discharge, 72,200 cfs Sept. 14, 1938 (gage height, $23.83 \mathrm{ft}$ ).

Remarks.-Flow regulated by 23 reservoirs and many powerplants above station. In 1938, when the maximum of record occurred, there were 21 reservoirs above station, the two large reservoirs Petenwell and Castle Rock not yet in existence.

Mean discharge, in cubic feet per second, 1965

\begin{tabular}{|c|c|c|c|c|c|c|c|c|c|c|c|}
\hline & March & April & May & Day & March & April & May & Day & March & April & May \\
\hline & 5,200 & 4,460 & 16,800 & 1 1.. & 9,370 & 10,400 & & 12 & 8,940 & 00 & 300 \\
\hline & 5,400 & 4,430 & 12,600 & $12 \ldots$ & 9,820 & 11,800 & 15,100 & 2 & 650 & 22 & 200 \\
\hline & 6,000 & 3,930 & 13,300 & $13 \ldots$ & 9,760 & 19,800 & 11,500 & 23. & 530 & 22 & 10,700 \\
\hline & 7,400 & 3,670 & 20,700 & $14_{-}$ & 9,830 & 29,800 & 11,100 & 24. & 500 & 23 & 10,500 \\
\hline & 9,100 & 4,080 & 18,100 & $15 \ldots$ & 9,860 & 46,300 & 10 , & 25. & 8,590 & 23 & 10,300 \\
\hline & 9,440 & 5,220 & 14,100 & $16 \ldots$ & 9,740 & 46,600 & 10,300 & 26. & 7,770 & 00 & 11,000 \\
\hline & 8,820 & 6,720 & 10,900 & $17 \ldots$ & 9,660 & 40,500 & 18 & 27. & 7,240 & 22 & 17,300 \\
\hline & 7,680 & 7,460 & 10,000 & $18 \ldots$ & 9,410 & 200 & 21 & 28. & 120 & 22 & 18,100 \\
\hline & 8,200 & 8,360 & 10,400 & $19 \ldots$ & 9,340 & 33,600 & 22 & 29 & 5,940 & 22 , & 14,600 \\
\hline & 8,740 & 8,940 & 11,000 & $20 \ldots$ & 9,220 & 25,600 & 20,700 & 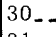 & 4,310 & 23,807 & 11,200 \\
\hline & & & & & & & & & 4,130 & $\ldots$ & 11,600 \\
\hline \multicolumn{9}{|c|}{ Ionthly mean discharge, in cubic } & 8,120 & 19,697 & 14,21 \\
\hline
\end{tabular}

Gage height, in feet, and discharge, in cubic feet per second, at indicated time, 1965

\begin{tabular}{|c|c|c|c|c|c|c|c|c|c|c|c|}
\hline Date & Hour & $\begin{array}{l}\text { Gage } \\
\text { height }\end{array}$ & $\begin{array}{c}\text { Dis - } \\
\text { charge }\end{array}$ & Date & Hour & $\begin{array}{l}\text { Gage } \\
\text { height }\end{array}$ & $\begin{array}{l}\text { Dis- } \\
\text { charge }\end{array}$ & Date & Hour & $\begin{array}{c}\text { Gage } \\
\text { height }\end{array}$ & $\begin{array}{c}\text { Dis- } \\
\text { charge }\end{array}$ \\
\hline \multirow[t]{7}{*}{ Apr. 13} & 0000 & 10.16 & 3,900 & \multirow[t]{6}{*}{ Apr.15 } & 1000 & 18.19 & 45,500 & \multirow[t]{4}{*}{ Apr.18 } & 1200 & 16.85 & 38,200 \\
\hline & 0200 & 10.64 & 15,300 & & 1400 & 18.61 & 48,100 & & 1800 & 16.50 & 36,600 \\
\hline & 0800 & 11.44 & 17,800 & & 1600 & 18.83 & 49,400 & & 400 & 16.57 & 36,900 \\
\hline & 1400 & 12.10 & 19,900 & & 2000 & 18.95 & 50,200 & & & & \\
\hline & 1800 & 12.98 & 23,000 & & 2400 & 18.87 & 49 ? & \multirow[t]{5}{*}{19} & 0800 & 16.29 & 35,600 \\
\hline & 2400 & 13.67 & 25,400 & & 0800 & 18.55 & 47 & & 1000 & 15.89 & 33,900 \\
\hline & & & & & 1200 & 18.36 & & & 1400 & 15.59 & 32,700 \\
\hline \multirow[t]{8}{*}{14} & 0400 & 14.05 & 26,800 & & 1600 & $\begin{array}{l}18.30 \\
18.22\end{array}$ & $\begin{array}{l}40,500 \\
45,700\end{array}$ & & 2400 & 14.87 & 29,900 \\
\hline & 1000 & 14.38 & 28,000 & & & & & & & & \\
\hline & 1200 & 14.73 & 29,400 & & & & & \multirow[t]{9}{*}{20} & 0600 & 14.47 & 28,400 \\
\hline & 1800 & 15.35 & 31,800 & & 28 & 1 & 44 & & 0800 & 14.09 & 27,000 \\
\hline & 2000 & 15.40 & 32,000 & 17 & 0600 & 17.67 & 42,500 & & 1000 & 13.75 & 25,700 \\
\hline & 2200 & 16.14 & 35,000 & & 1200 & 17.26 & 40,300 & & 1200 & 13.61 & 25,200 \\
\hline & 2400 & 16.63 & 37,200 & & 1800 & 17.02 & 39,000 & & 1400 & 13.23 & 23,800 \\
\hline & & & & & 2200 & 16.76 & 37,800 & & 1600 & 12.96 & 22,900 \\
\hline \multirow[t]{3}{*}{15} & 0200 & 17.20 & 39,900 & \multirow[b]{3}{*}{18} & 2400 & 16.81 & 38,000 & & 2000 & 13.02 & 23,100 \\
\hline & 0400 & 17.47 & 41,400 & & & & & & 2400 & 13.38 & 24,400 \\
\hline & 0800 & 17.92 & 43,900 & & 0600 & 16.67 & 37,300 & & & & \\
\hline
\end{tabular}


(199) 5-4050. Baraboo River near Baraboo, Wis.

Location.-Lat $43^{\circ} 28^{\prime} 55^{\prime \prime}$, long $89^{\circ} 38^{\prime} 00^{\prime \prime}$, in NW $\frac{1}{4}$ sec. 35, T. 12 N., R.7 E., on left bank $50 \mathrm{ft}$ downstream from highway bridge, 0.3 mile downstream from Rowley Creek and 4 miles east of Baraboo.

Drainage area.- $-600 \mathrm{sq} \mathrm{mi}$.

Gage-height record.-Water-stage recorder graph, except Mar. 2-9 and crest-stage gage. Datum of gage is $788.21 \mathrm{ft}$ above mean sea level datum of 1929 .

Discharge record.--Stage-discharge relation defined by current-meter measurements below $5,600 \mathrm{cfs}$. Backwater from ice Mar. 1-22.

Maxima.-March-May 1965: Discharge, about 4,500 cfs time unknown Mar. 6 (19.8 ft, backwater from ice).

1913-22, 1942 to February 1965: Discharge observed, 7,900 cfs Mar. 26, 1917 (gage height, $17.5 \mathrm{ft}$, estimated, at site 2.3 miles upstream at datum $7.6 \mathrm{ft}$ higher).

Mean discharge, in cubic feet per second, 1965

\begin{tabular}{|c|c|c|c|c|c|c|c|c|c|c|c|}
\hline Day & March & April & May & Day & March & April & May & Day & March & April & May \\
\hline & 600 & 718 & 302 & $\mid 11$ - & 1,600 & 2,740 & 159 & 21. & 123 & 308 & 195 \\
\hline 2. & 1,890 & 994 & 250 & $12 \ldots$ & 1,300 & 2,420 & 183 & 22. & 126 & 275 & 178 \\
\hline 3. & 3,000 & 1,200 & 235 & $13 \ldots$ & 700 & 2,040 & 173 & 23 & 125 & 270 & 164 \\
\hline 4. & 4,000 & 1,400 & 190 & 14 & 300 & 1,620 & 164 & 24 & 176 & 260 & 134 \\
\hline 5. & 4,200 & 1,750 & 104 & $15 \ldots$ & 220 & 1,320 & 143 & 25 & 154 & 332 & 190 \\
\hline 6. & 4,400 & 2,290 & 188 & $16 \ldots$ & 180 & 970 & 123 & 26. & 136 & 627 & 220 \\
\hline 7. & 4,200 & 2,720 & 238 & $17 \ldots$ & 155 & 523 & 111 & $27-$ & 138 & 671 & 181 \\
\hline 8. & 3,700 & 3,070 & 242 & $18 \ldots$ & 140 & 408 & 104 & 28 & 127 & 640 & 320 \\
\hline 9 & 3,080 & 3,260 & 238 & 19 & 130 & 362 & 98 & 29. & 138 & 533 & 440 \\
\hline \multirow[t]{2}{*}{$10 \ldots$} & 2,400 & 3,060 & 215 & $20 \ldots$ & 125 & 332 & 169 & $30_{2}$ & 164 & 380 & 410 \\
\hline & & & & & & & & 3 & 355 & & 282 \\
\hline \multicolumn{9}{|c|}{ Monthly mean discharge, in cubic feet per second } & 1,228 & 1,250 & 205 \\
\hline \multicolumn{9}{|c|}{ Runoff, in inches } & 2.36 & 2.32 & 0.39 \\
\hline
\end{tabular}

Gage height, in feet, and discharge, in cubic feet per second, at indicated time, 1965

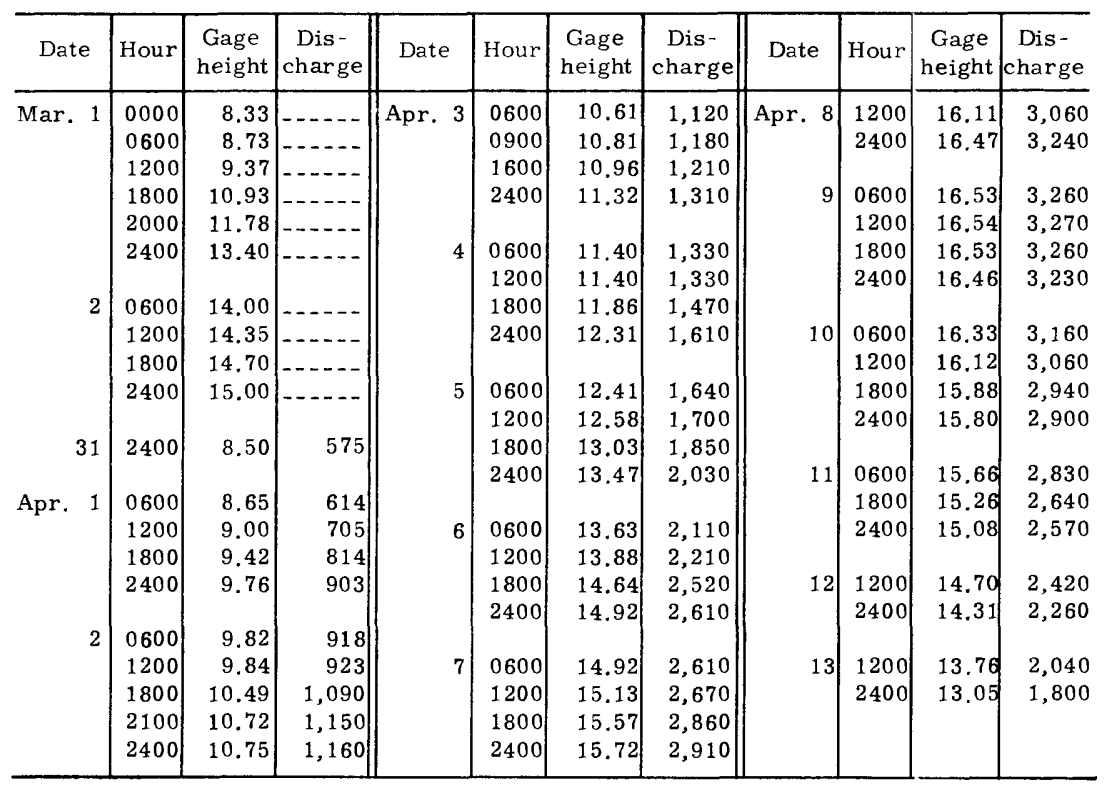


(200) 5-4070. Wisconsin River at Muscoda, Wis.

Location.-Lat $43^{\circ} 12^{\prime} 00^{\prime \prime}$, long $90^{\circ} 26^{\prime} 25^{\prime \prime}$, in sec.1, T.8 N., R.1 W., on left bank at bridge on State Highway 80, 0.5 mile upstream from Eagle Mill Creek and 1 mile north of Muscoda.

Drainage area.-10,300 $\mathrm{sq} \mathrm{mi}$, approximately.

Gage-height record.-Digital recorder tape punched at sixty-minute intervals. Datum of gage is $667.05 \mathrm{ft}$ above mean sea level, datum of 1929 .

Discharge record.-Stage-discharge relation defined by current-meter measurements. Mean daily discharges computed from 24 punch-tape recordings per day. Packwater from ice Mar. 1 to Apr. 7.

Maxima.-March-May 1965: Discharge, 48,500 cfs 2300 hours Apr. 18 (gage height, $8.95 \mathrm{ft})$.

1902-03, 1913 to February 1965: Discharge, 80,800 cfs Sept. 16, 1938 (gage height, $11.48 \mathrm{ft}$ ).

Remarks.-Flow regulated by 23 reservoirs and many powerplants above station. In 1938, when the maximum of record occurred, there were 21 reservoirs abcve station, the two large reservoirs, Petenwell and Castle Rock not yet in existence.

Mean discharge, in cubic feet per second, 1965

\begin{tabular}{|c|c|c|c|c|c|c|c|c|c|c|c|}
\hline Day & March & April & May & Day & March & April & May & Day & March & April & May \\
\hline & 8,000 & 8,400 & 23,200 & $1{ }^{1}$ & 14,000 & 14,600 & 11,800 & 21. & 10,000 & 38,000 & 20,600 \\
\hline & 12,000 & 8,200 & 23,300 & 12 & 13,000 & 15,000 & 12,400 & $22_{2}$ & 11,000 & 32,400 & 20,300 \\
\hline 3. & 16,000 & 7,800 & 18,800 & 13 & 14,000 & 14,600 & 13,800 & 23 & 12,000 & 27,100 & 18,800 \\
\hline & 14,000 & 7,800 & 14,900 & 14 & 14,000 & 15,400 & 14,100 & 24 & 12,000 & 24,600 & 14,800 \\
\hline & 13,000 & 7,600 & 16,200 & 15. & 13,000 & 20,400 & 13,400 & 25 & 12,000 & 25,100 & 13,500 \\
\hline 6. & 14,000 & 9,000 & 20,400 & 16. & 12,000 & 25,200 & 12,600 & 26 & 11,000 & 24,600 & 12,400 \\
\hline & 15,000 & 10,800 & 19,100 & 17 & 11,000 & 32,000 & 11,900 & $27_{-}$ & 11,000 & 25,500 & 12,500 \\
\hline & 15,000 & 12,800 & 15,500 & 18 & 10,000 & 45,900 & 11,800 & 28. & 11,000 & 25,300 & 12,200 \\
\hline 9. & 15,000 & 13,900 & 13,500 & 19 & 10,000 & 46,900 & 15,700 & 29. & 10,000 & $25,30 \mathrm{c}$ & 15,800 \\
\hline \multirow[t]{2}{*}{$10_{-1}$} & 14,000 & 14,400 & 11,300 & 20 & 10,000 & 41,800 & 19,500 & 30 & 9,200 & $24,00 \mathrm{C}$ & 16,600 \\
\hline & & & & & & & & & 8,800 & $\ldots$ & 14,700 \\
\hline \multicolumn{9}{|c|}{ Monthly mean discharge, in cubic feet per second } & 12,100 & 21,480 & 15,660 \\
\hline
\end{tabular}

Gage height, in feet, and discharge, in cubic feet per second, at indicated time, 1965

\begin{tabular}{|c|c|c|c|c|c|c|c|c|c|c|c|}
\hline Date & Hour & $\begin{array}{l}\text { Gage } \\
\text { height }\end{array}$ & $\begin{array}{c}\text { Dis - } \\
\text { charge }\end{array}$ & Dạte & Hour & $\begin{array}{c}\text { Gage } \\
\text { height }\end{array}$ & $\begin{array}{c}\text { Dis- } \\
\text { charge }\end{array}$ & Date & Hour & $\begin{array}{c}\text { Gage } \\
\text { height }\end{array}$ & $\begin{array}{l}\text { Dis- } \\
\text { charge }\end{array}$ \\
\hline \multirow[t]{6}{*}{ Apr.14 } & 0000 & 3.85 & 14,300 & \multirow[t]{3}{*}{ Apr. 17} & 1800 & 7.53 & 35,000 & \multirow[t]{3}{*}{ Apr. 20} & 1800 & 8.20 & 41,000 \\
\hline & 0600 & 3.84 & 14,300 & & 2400 & 8.14 & 40,400 & & 2400 & 8.16 & 40,600 \\
\hline & 1200 & 4.06 & 15,100 & & & & & & & & \\
\hline & 2000 & 4.44 & 16,700 & \multirow[t]{7}{*}{18} & 0600 & 8.53 & 44,300 & \multirow[t]{5}{*}{21} & 0600 & 8.07 & 39,700 \\
\hline & 2400 & 4.64 & 17,700 & & 1200 & 8.77 & 46,700 & & 1200 & 7.88 & 38,000 \\
\hline & & & & & 1600 & 8.89 & 47,900 & & 1800 & 7.71 & 36,500 \\
\hline \multirow[t]{5}{*}{15} & 0600 & 4.93 & 19,200 & & 2000 & 8.91 & 48,100 & & 2400 & 7.54 & 35,100 \\
\hline & 1200 & 5.20 & 20,500 & & 2300 & 8.95 & 48,500 & & & & \\
\hline & 1800 & 5.44 & 21,700 & & 2400 & 8.94 & 48,400 & 22 & 0600 & 7.39 & 33,900 \\
\hline & 2400 & 5.67 & 23,000 & & & & & & 1200 & 7.20 & 32,600 \\
\hline & & & & \multirow[t]{5}{*}{19} & 0600 & 8.92 & 48,200 & & 1800 & 6.97 & 31,000 \\
\hline \multirow[t]{5}{*}{16} & $n 600$ & 5.86 & 24,200 & & 1200 & 8.85 & 47,500 & & 2400 & 6.73 & 29,400 \\
\hline & 1200 & 6.03 & 25,200 & & 1800 & 8.67 & 45,700 & & & & \\
\hline & 1800 & 6.19 & 26,100 & & 2400 & 8.50 & 44,000 & 23 & 0600 & 6.52 & 28,100 \\
\hline & 2400 & 6.37 & 27,200 & & & & & & 1200 & 6.33 & 27,000 \\
\hline & & & & \multirow[t]{3}{*}{20} & 0600 & 8.35 & 42,500 & & 1800 & 6.19 & 26,100 \\
\hline \multirow[t]{2}{*}{17} & 0600 & 6.60 & 28,600 & & 1200 & 8.25 & 41,500 & & 2400 & 6.05 & 25,300 \\
\hline & 1200 & 6.94 & 30,800 & & & & & & & & \\
\hline
\end{tabular}


(201) 5-4074. Morris Creek tributary near Norwalk, W

$$
\text { (Crest-stage station) }
$$

Location.-Lat $43^{\circ} 51^{\prime} 10^{\prime \prime}$, long $90^{\circ} 37^{\prime} 32^{\prime \prime}$, in NW $\frac{1}{4}$ sec. 21, T.16 N., R. 2 W., at bridge on County Trunk T, 1.2 miles north of intersection with State Highway 71, and 2 miles north of Norwalk.

Drainage area. $-4.67 \mathrm{sq} \mathrm{mi}$.

Gage-height record.-Crest stages only.

Discharge record.-Stage-discharge relation defined by current-meter measurements below $617 \mathrm{cfs}$.

Maxima.-March-May 1965: Discharge, $490 \mathrm{cfs}$ Apr. 2 (gage height, $11.80 \mathrm{ft}$ ). 1960 to February 1965: Discharge, 950 cfs Oct, 24, 1959 (gage height, $13.10 \mathrm{ft}$ ).

(202) 5-4080. Kickapoo River at La Farge, Wis.

Location.-Lat $43^{\circ} 34^{\prime} 30^{\prime \prime}$, long $90^{\circ} 38^{\prime} 35^{\prime \prime}$, on east-west quarter section l'ne in $\mathrm{W} \frac{1}{2}$ sec.29, T.13 N., R.2 W., on left bank $10 \mathrm{ft}$ upstream from bridge on St?te Highway 82, in La Farge, 0.3 mile upstream from Otter Creek, and 1 mile downstream from powerplant.

Drainage area.- $-266 \mathrm{sq} \mathrm{mi}$.

Gage-height record.-Digital recorder tape punched at fifteen-minute intervals. Datum of gage is $782.00 \mathrm{ft}$ above mean sea level, adjustment of 1912 .

Discharge record.-Stage-discharge relation defined by current-meter measurements below 5,700 cfs. Mean daily discharges computed from 96 punch-tape recordings per day. Rate of change in stage used as factor Apr. 11, 12, May 26, 27. Backwater from ice Mar. 1 to Apr. 4.

Maxima.-March-May 1965: Discharge,about 3,600 cfs 1415 hours Mar. 2 (gage-height, $12.78 \mathrm{ft}$., backwater from ice).

1938 to February 1965: Discharge, 7,040 cfs Mar. 26, 1961 (gage height, 12.70 ft); gage height, $12.90 \mathrm{ft}$ Mar. 8, 1950 (backwater from ice).

Remarks.-Considerable diurnal fluctuation during low-water periods caused by operation of powerplant, 1 mile upstream.

Mean discharge, in cubic feet per second, 1965

\begin{tabular}{|c|c|c|c|c|c|c|c|c|c|c|c|}
\hline Day & March & April & May & Day & March & April & May & Day & March & April & May \\
\hline $1-$ & 900 & 500 & 129 & $11 \ldots$ & 250 & 2,260 & 103 & 21 & 100 & 120 & 126 \\
\hline $2 \ldots$ & 2,500 & 560 & 133 & $12 \ldots$ & 210 & 1,970 & 104 & $22=$ & 96 & 154 & 338 \\
\hline 3. & 1,800 & 700 & 124 & $13 \ldots$ & 180 & 332 & 93 & 23 & 92 & 159 & 149 \\
\hline $4 \ldots$ & 400 & 1,400 & 116 & $14 \ldots$ & 160 & 238 & 91 & 24 & 88 & 171 & 134 \\
\hline 5. & 1,000 & 1,690 & 157 & $15 \ldots$ & 150 & 336 & 86 & $25 \ldots$ & 84 & 229 & 141 \\
\hline 6 & 2,200 & 2,330 & 153 & $16_{-}$ & 140 & 239 & 227 & $26 \ldots$ & 80 & 322 & 973 \\
\hline 7. & 700 & 1,890 & 129 & 17. & 130 & 206 & 152 & $27-$ & 78 & 214 & 576 \\
\hline 8 & 1,000 & 1,620 & 128 & $18 \ldots$ & 120 & 223 & 188 & 28 & 80 & 177 & 239 \\
\hline 9 & 660 & 2,350 & 138 & $19 \ldots$ & 110 & 188 & 183 & 29 & 88 & 141 & 178 \\
\hline \multirow[t]{2}{*}{$10 \ldots$} & 340 & 652 & 116 & $20 \ldots$ & 104 & 170 & 117 & $30_{-}$ & 130 & 137 & 157 \\
\hline & & & & & & & & $31-$ & 350 & & 145 \\
\hline \multicolumn{9}{|c|}{ Monthly mean discharge, in cubic feet per second } & 462 & 723 & 188 \\
\hline \multicolumn{9}{|c|}{ Runoff, in inches. } & 2.00 & 3.03 & 0.81 \\
\hline
\end{tabular}


MARCH-MAY, UPPER MISSISSIPPI RIVER BASIN

Gage height, in feet, and discharge, in cubic feet per second, at indicated time, 1965, of Kickapoo River at

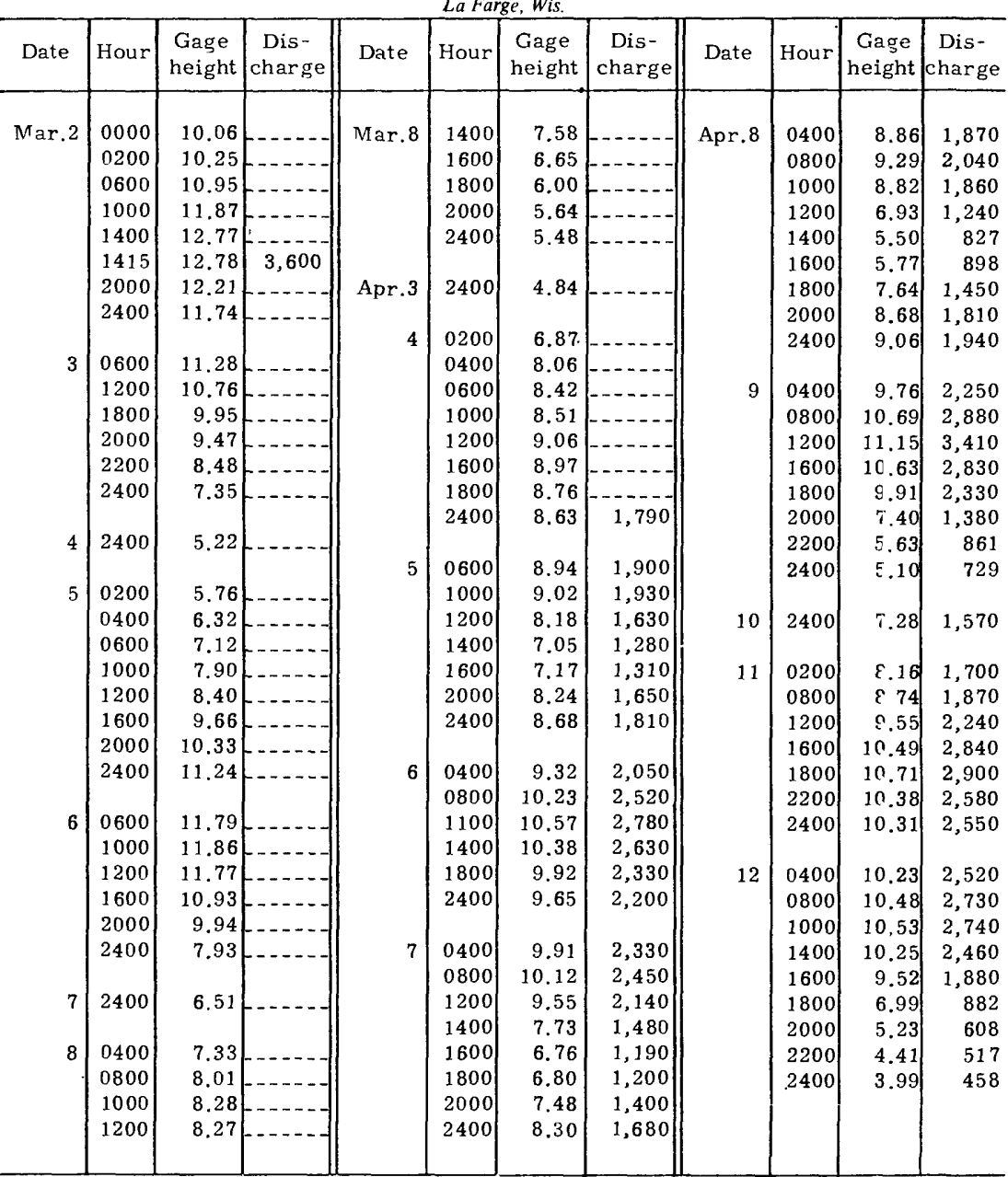

(203) 5-4105. Kickapoo River at Steuben, Wis.

Location.-Lat $43^{\circ} 11^{\prime} 25^{\prime \prime}$, long $90^{\circ} 52^{\prime} 30^{\prime \prime}$, in NW $\frac{1}{4}$ sec. 8, T. 8 N., R.4 W., on right bank $0.8 \mathrm{mile}$ upstream from Duffy Creek, 1 mile northwest of Steuben and 14 miles upstream from mouth.

Drainage area.-690 sq $\mathrm{mi}$.

Gage-height record.-Water-stage recorder graph. Datum of gage is $657.82 \mathrm{ft}$ above mean sea level, adjustment of 1912 .

Discharge record.--Stage-discharge relation defined by current-meter measurements. Backwater from ice March 1-8, 17, 18, 20-26.

Maxima.-March-May 1965: Discharge, 6,500 cfs 0700 hours Mar. 4 (gage height $11.18 \mathrm{ft}$, backwater from ice).

1933 to February 1965: Discharge, 10,800 cfs Mar, 28, 1961 (gage height, $12.33 \mathrm{ft}$ ). 
Mean discharge, in cubic feet per second, 1965, of Kickapoo River at Steuben, Wis.

\begin{tabular}{|c|c|c|c|c|c|c|c|c|c|c|c|}
\hline Day & March & April & May & Day & March & April & May & Day & March & April & May \\
\hline & 800 & 1,250 & 466 & $11 \ldots$ & 1,020 & 2,700 & 423 & $21 \ldots$ & 300 & 507 & 410 \\
\hline 2. & 2,000 & 1,510 & 447 & $12 \ldots$ & 505 & 2,540 & 393 & $22 \ldots$ & 300 & 488 & 378 \\
\hline 3. & 5,000 & 1,710 & 429 & $13 \ldots$ & 452 & 3,180 & 370 & $23 \ldots$ & 290 & 458 & 470 \\
\hline & 5,500 & 2,040 & 420 & $14_{-}$ & 402 & 2,660 & 364 & $24 \ldots$ & 280 & 486 & 471 \\
\hline U & 3,900 & 2,470 & 430 & $15 \ldots$ & 386 & 2,030 & 354 & $25 \ldots$ & 270 & 557 & 417 \\
\hline 6. & 3,700 & 2,740 & 631 & $16 \ldots$ & 339 & 1,090 & 366 & $26 \ldots$ & 270 & 683 & 773 \\
\hline - & 3,200 & 2,820 & 539 & $17 \ldots$ & 340 & 786 & 444 & 27. & 270 & 721 & 873 \\
\hline 8. & 3,600 & 2,820 & 476 & $18 \ldots$ & 310 & 643 & 483 & $28 \ldots$ & 263 & 634 & 1,020 \\
\hline $9_{-}$ & 2,670 & 2,800 & 459 & $19 \ldots$ & 295 & 592 & 450 & 29. & 272 & 544 & 922 \\
\hline 10 & 2,140 & 2,700 & 446 & $20 \ldots$ & 300 & 537 & 468 & $30_{-}$ & 304 & 493 & 609 \\
\hline & & & & & & & & & 660 & & 499 \\
\hline \multirow{2}{*}{\multicolumn{9}{|c|}{ Monthly mean discharge, in cubic feet per second }} & 1,301 & 1,506 & 506 \\
\hline & & & & & & \multicolumn{3}{|c|}{ Runoff, in inches } & 2.17 & 2.44 & 0.85 \\
\hline
\end{tabular}

Gage height, in feet, and discharge, in cubic feet per second, at indicatcd time, 1965

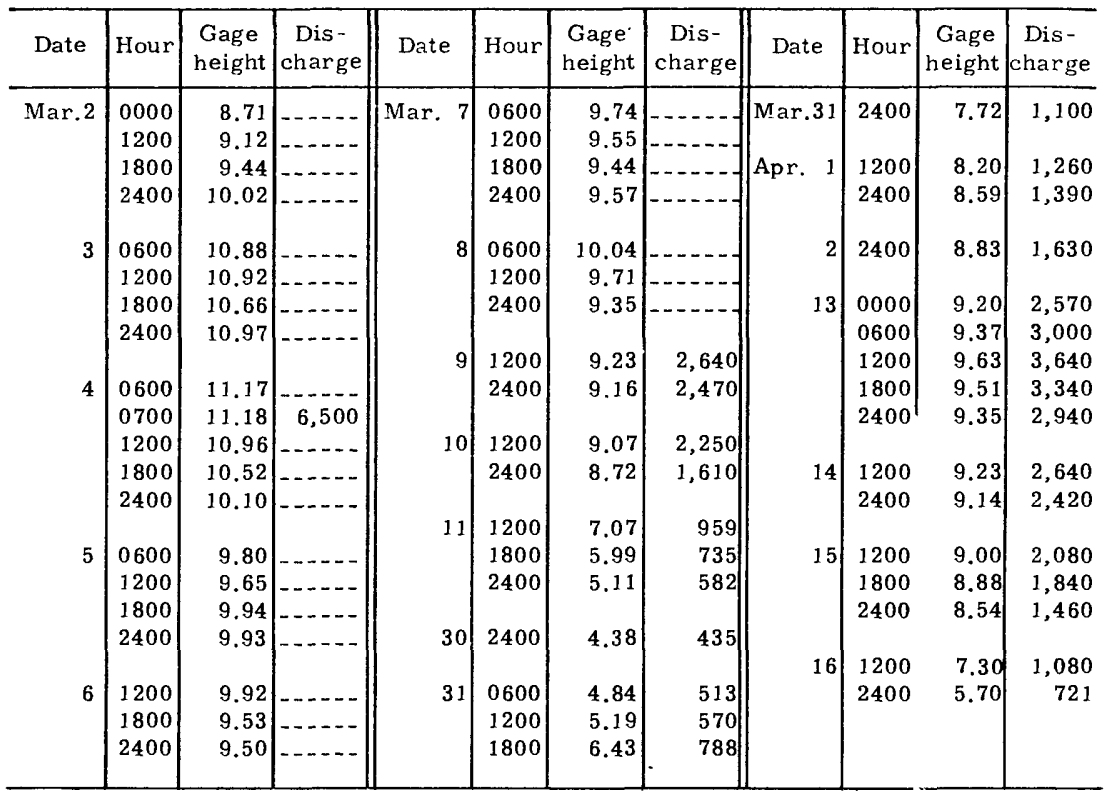

(204) Wisconsin River at Bridgeport, Wis.

(Miscellaneous site)

Location.-SW $\frac{1}{4} \sec .11$, T. 6 N., R. 6 W., at bridge on U.S. Highway 18 at Bridgeport.

Drainage area.- $-11,700 \mathrm{sq} \mathrm{mi}$.

Discharge record.--Discharge obtained on the following days by current-meter measurements.

Date

Apr. 15

Apr. 19

Apr. 24

May 1
Discharge, in cfs

20,100

44,600

32,500

26,300 


\section{TURKEY RIVER BASIN}

(205) 5-4116. Turkey River at Spillville, Iowa

Location.-Lat $43^{\circ} 12^{\prime} 30^{\prime \prime}$, long $91^{\circ} 57^{\prime} 00^{\prime \prime}$, in SW $\frac{1}{4} \mathrm{NE} \frac{1}{4}$ sec. $19, \mathrm{~T} .97$ N., R.9 W., on right bank $60 \mathrm{ft}$ downstream from county highway bridge, at north edge of Spillville, $150 \mathrm{ft}$ downstream from old mill dam, and 3,000 ft upstream from Wonder Creek.

Drainage area. $-177 \mathrm{sq} \mathrm{mi}$.

Gage-height record.-Water-stage recorder graph except Mar. 3-10, Apr. 14-20, May 27-31. Datum of gage is $1,034.77 \mathrm{ft}$ above mean sea level, datum of $192 \mathrm{~s}$.

Discharge record.-Stage-discharge relation defined by current-meter measurements below $2,410 \mathrm{cfs}$, extended to $3,500 \mathrm{cfs}$ by logarithmic plotting. Backwater from ice Mar. 1 to Apr. 4.

Maxima.-March-May 1965: Discharge, about 3,900 cfs Mar. ; gage height, $16.11 \mathrm{ft}$ 0800 hrs Mar. 1 (backwater from ice).

1956 to February 1965: Discharge, 7,380 cfs Mar. 29, 1962 (gage height, $15.32 \mathrm{ft}$ ).

Flood in June 1947 reached a stage of $18.4 \mathrm{ft}$, from floodmark (discharge about $10,000 \mathrm{cfs}$ ).

Mean discharge, in cubic feet per second, 1965

\begin{tabular}{|c|c|c|c|c|c|c|c|c|c|c|c|}
\hline Day & March & April & May & Day & March & April & May & Day & March & April & May \\
\hline & 2,150 & 1,480 & 92 & $11 \ldots$ & 45 & 699 & 57 & 21 & 20 & 83 & 40 \\
\hline 2. & 1,690 & 1,700 & 77 & 12 & 40 & 927 & 54 & $22 \ldots$ & 18 & 80 & 40 \\
\hline 3 & 450 & 1,380 & 73 & 13 & 36 & 261 & 53 & 23 & 16 & 77 & 41 \\
\hline $1_{-}$ & 190 & 1,700 & 68 & $14 \ldots$ & 33 & 210 & 51 & 24. & 15 & 79 & 40 \\
\hline 5. & 118 & 2,420 & 68 & $15 \ldots$ & 32 & 174 & 52 & $25=$ & 14 & 110 & 59 \\
\hline 6. & 92 & 2,440 & 67 & 16. & 32 & 148 & 65 & $26 \ldots$ & 14 & 202 & 967 \\
\hline 7. & 91 & 1,460 & 68 & 17. & 32 & 128 & 59 & 27. & 13 & 173 & 400 \\
\hline 8 & 76 & 1,260 & 69 & $18 \ldots$ & 30 & 112 & 54 & $28 \ldots$ & 13 & 144 & 200 \\
\hline $9_{-}$ & 60 & 1,330 & 64 & $19-$ & 25 & 100 & 47 & |29- & 13 & 120 & 142 \\
\hline $10_{\ldots}$ & 50 & 445 & 61 & 20 & 22 & 90 & 43 & $30 \ldots$ & 13 & 104 & 122 \\
\hline & & & & & & & & & 60 & & 106 \\
\hline \multirow{3}{*}{\multicolumn{9}{|c|}{$\begin{array}{l}\text { Monthly mean discharge, in cubic feet per second } \\
\text { Runoff, in inches } \\
\text { Runoff, in acre-feet }\end{array}$}} & 178 & 655 & 110 \\
\hline & & & & & & & & & 1.16 & 4.13 & 0.71 \\
\hline & & & & & & & & & 10,920 & 38,950 & 6,740 \\
\hline
\end{tabular}

Gage height, in feet, and discharge, in cubic feet per second, at indicated time, 1965

\begin{tabular}{|c|c|c|c|c|c|c|c|c|c|c|c|}
\hline Date & Hour & $\begin{array}{c}\text { Gage } \\
\text { height }\end{array}$ & $\begin{array}{c}\text { Dis- } \\
\text { charge }\end{array}$ & Date & Hour & $\begin{array}{c}\text { Gage } \\
\text { height }\end{array}$ & $\begin{array}{c}\text { Dis- } \\
\text { charge }\end{array}$ & Date & Hour & $\begin{array}{c}\text { Gage } \\
\text { height }\end{array}$ & $\begin{array}{c}\text { Dis- } \\
\text { charge }\end{array}$ \\
\hline Mar. 1 & $\begin{array}{l}0000 \\
0600 \\
0700 \\
0800 \\
0900 \\
1000 \\
1100 \\
1600 \\
1800 \\
2400\end{array}$ & $\begin{array}{l}14.20 \\
14.75 \\
15.29 \\
16.11 \\
12.90 \\
12.76 \\
12.62 \\
14.14 \\
14.29 \\
13.06\end{array}$ & & $\begin{array}{r}\text { Apr. } 7 \\
8\end{array}$ & $\begin{array}{l}2400 \\
0400 \\
0600 \\
1000 \\
1600 \\
1800 \\
2000 \\
2300 \\
2400\end{array}$ & $\begin{array}{r}7.51 \\
6.62 \\
6.42 \\
6.84 \\
7.66 \\
8.27 \\
10.03 \\
11.68 \\
11.58\end{array}$ & $\begin{array}{r}1,090 \\
790 \\
737 \\
866 \\
1,130 \\
1,340 \\
2,080 \\
2,810 \\
2,750\end{array}$ & Apr. 9 & $\begin{array}{l}0200 \\
0600 \\
1200 \\
1600 \\
1700 \\
1800 \\
2000 \\
2400\end{array}$ & $\begin{array}{r}10.02 \\
8.58 \\
8.96 \\
7.81 \\
7.01 \\
6.42 \\
6.28 \\
5.85\end{array}$ & $\begin{array}{r}2,010 \\
1,440 \\
1,580 \\
1,170 \\
914 \\
737 \\
688 \\
572\end{array}$ \\
\hline
\end{tabular}


(Crest-stage station)

Location.-Near SE corner of sec.21, T.99 N., R.13 W., at bridge on State Highway 9, 1 mile east of Saratoga.

Drainage area. $-4.06 \mathrm{sq} \mathrm{mi}$.

Gage-height record.-Crest stages only.

Discharge record.-Stage-discharge relation defined by current-meter measurements below $355 \mathrm{cfs}$ and by contracted-opening measurement at $1,830 \mathrm{cfs}$.

Maxima.-March-May 1965: Discharge, about $800 \mathrm{cfs}$ Apr. 8 (gage height, $5.70 \mathrm{ft}$, backwater from ice).

1953 to February 1965: Discharge, 1,830 cfs Aug. 31, 1962 (gage height, $6.32 \mathrm{ft}$ ).

(207) 5-4117. Crane Creek near Lourdes, Iowa

(Crest-stage station)

Location.- $\mathrm{NW} \frac{1}{4}$ sec.6, T.97 N., R.12 W., at bridge on State Highway 272, 1 mile southwest of Lourdes.

Drainage area. $-75.8 \mathrm{sq} \mathrm{mi}$.

Gage-height record.-Crest stages only.

Discharge record.-Stage-discharge relation defined by current-meter measurements below $3,340 \mathrm{cfs}$ and by contracted-opening measurement at $11,900 \mathrm{c} s$.

Maxima.-March-May 1965: Discharge, about 1,000 cfs Mar. 1 (gage height, $12.6 \mathrm{ft}$, backwater from icel.

1953 to February 1965: Discharge, 11,900 cfs Aug. 31, 1962 (gage height, $15.70 \mathrm{ft}$ ).

(206) 5-4125. Turkey River at Garber, Iowa

Location.-Lat $42^{\circ} 44^{\prime} 20^{\prime \prime}$, long $91^{\circ} 15^{\prime} 45^{\prime \prime}$, in $\mathrm{NE} \frac{1}{4} \mathrm{SW} \frac{1}{4} \sec .36, \mathrm{~T} .92 \mathrm{~N}$., R.4 W., on left bank $10 \mathrm{ft}$ downstream from highway bridge at Garber, $800 \mathrm{ft}$ upstream from Wayman Creek, 2,000 ft downstream from Elk Creek, and 1 mile downstrean from Volga River.

Drainage area. $-1,545 \mathrm{sq} \mathrm{mi}$.

Gage-height record.-Water-stage recorder graph except Feb. 27-Mar. 5, for which graph was reconstructed on basis of twice daily wire-weight gage readings. Datum of gage is $634.46 \mathrm{ft}$ above mean sea level, datum of 1929 .

Discharge record.-Stage-discharge relation defined by current-meter measurements below 25,000 cfs. Backwater from ice Mar. 1-9, 19-28.

Maxima.-March-May 1965: Discharge, 13,800 cfs 1800 hours Apr. 1; gage height observed, $22.10 \mathrm{ft} 0800-0900$ hours Mar. 1 .

1913-16, 1919-27, 1929-30, 1932 to February 1965: Discharge,32,300 cfs Feb. 23, 1922 (gage height, $28.06 \mathrm{ft}$, from floodmark).

Maximum stage known since about 1890, that of Feb, 23, 1922. 
Mean discharge, in cubic feet per second, 1965, of Turkey River at Garber, Iowa

\begin{tabular}{|c|c|c|c|c|c|c|c|c|c|c|c|}
\hline Day & March & April & May & Day & March & April & May & Day & March & April & May \\
\hline & 10,000 & 12,600 & 660 & $11 \ldots$ & 875 & 4,080 & 407 & 21. & 290 & 730 & 327 \\
\hline 2. & 7,000 & 8,460 & 602 & 12 & 695 & 3,780 & 384 & 22 & 270 & 630 & 327 \\
\hline 3. & 5,000 & 6,240 & 556 & 13. & 805 & 3,910 & 362 & 23. & 260 & 597 & 341 \\
\hline & 3,500 & 8,020 & 522 & 14 & 925 & 2,720 & 344 & 24. & 250 & 602 & 317 \\
\hline 5 & 4,200 & 9,820 & 496 & $15 \ldots$ & 1,020 & 1,540 & 334 & 25 & 240 & 700 & 310 \\
\hline 6 & 3,600 & 10,000 & 475 & $16 \ldots$ & 800 & 1,220 & 358 & 26 & 230 & 850 & 3,760 \\
\hline 7. & 3,200 & 9,390 & 458 & $17 \ldots$ & 670 & 1,070 & 334 & 27 & 230 & 1,000 & 3,830 \\
\hline 8 & 3,800 & 6,960 & 458 & $18 \ldots$ & 348 & 935 & 358 & $28 \ldots$ & 225 & 1,020 & 2,250 \\
\hline & 1,500 & 6,230 & 450 & $19_{-}$ & 330 & 830 & 410 & $29 \ldots$ & 221 & 850 & 1,310 \\
\hline 10 & 1,140 & 5,830 & 434 & $20=$ & 310 & 755 & 366 & $30_{-}$ & 448 & 750 & 996 \\
\hline & & & & & & & & & 4,130 & & 855 \\
\hline \multicolumn{9}{|c|}{ Monthly mean discharge, in cubic feet per second } & 1,823 & 3,761 & 755 \\
\hline \multicolumn{9}{|c|}{ Runoff, in inches } & 1.36 & 2.72 & 0.56 \\
\hline \multicolumn{9}{|c|}{ Runoff, in acre-feet } & 112,100 & 223,800 & 46,400 \\
\hline
\end{tabular}

Gage height, in feet, and discharge, in cubic feet per second, at indicated time, 1965

\begin{tabular}{|c|c|c|c|c|c|c|c|c|c|c|c|}
\hline Date & Hour & $\begin{array}{c}\text { Gage } \\
\text { height }\end{array}$ & $\begin{array}{c}\text { Dis - } \\
\text { charge }\end{array}$ & Date & Hour & $\begin{array}{l}\text { Gage } \\
\text { height }\end{array}$ & $\begin{array}{c}\text { Dis- } \\
\text { charge }\end{array}$ & Date & Hour & $\begin{array}{c}\text { Gage } \\
\text { height }\end{array}$ & $\begin{array}{c}\text { Dis - } \\
\text { charge }\end{array}$ \\
\hline \multirow[t]{11}{*}{ Feb. 28} & 0000 & 9.16 & 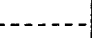 & \multirow[t]{20}{*}{ Mar. 2} & 0300 & 19.75 & & \multirow[t]{3}{*}{ Mar. 31} & 2000 & 15.31 & 8,590 \\
\hline & 0300 & 10.30 & & & 0730 & 19.44 & & & 2400 & 16.93 & 10,700 \\
\hline & 0500 & 11.50 & & & 1530 & 19.36 & & & & & \\
\hline & 0730 & 11.72 & & & 2400 & 19.27 & & \multirow[t]{17}{*}{ Apr. 1} & 0600 & 18.00 & 12,200 \\
\hline & 1200 & 11.61 & & & & & & & 1200 & 18.39 & 12,700 \\
\hline & 1600 & 11.50 & & & 0830 & 19.17 & & & 1800 & 19.15 & 13,800 \\
\hline & 1700 & 15.84 & & & 1300 & 19.10 & & & 1900 & 19.14 & 13,700 \\
\hline & 1800 & 16.60 & & & 1500 & 18.95 & & & 2100 & 18.87 & 13,400 \\
\hline & 2000 & 18.00 & & & 1700 & 17.97 & & & 2400 & 17.78 & 11,900 \\
\hline & 2400 & 20.50 & & & 1800 & 17.25 & & & & & \\
\hline & & & & & 2000 & 15.40 & & & 0300 & 16.54 & 10,200 \\
\hline \multirow[t]{9}{*}{ Mar. 1} & 0300 & 21.60 & & & 2200 & 14.06 & & & 0600 & 15.54 & 8,880 \\
\hline & 0600 & 22.07 & & & 2400 & 13.26 & & & 0900 & 15.04 & 8,250 \\
\hline & 0800 & 22.10 & & & & & & & 1200 & 14.77 & 7,920 \\
\hline & 0900 & 22.10 & & & 0000 & 7.82 & 1,410 & & 1500 & 14.55 & 7,660 \\
\hline & 1230 & 22.00 & & & 0600 & 8.57 & 1,910 & & 1800 & 14.49 & 7,590 \\
\hline & 1530 & 22.02 & & & 0900 & 9.26 & 2,430 & & 2200 & 14.46 & 7,550 \\
\hline & 1800 & 21.90 & & & 1100 & 9.36 & 2,510 & & 2400 & 14.29 & 7,350 \\
\hline & 2100 & 21.25 & & & 1400 & 9.46 & 2,590 & & & & \\
\hline & 2400 & 20.50 & & & 1800 & 13.00 & 5,910 & & & & \\
\hline
\end{tabular}




\section{GRANT RIVER BASIN}

(209) 5-4135. Grant River at Burton, Wis.

Location,-Lat $42^{\circ} 43^{\prime} 10^{\prime \prime}$, long $90^{\circ} 49^{\prime} 10^{\prime \prime}$, in sec. 23 , T.3 N., R.4 W., on right bank at downstream side of highway bridge at Burton, 6 miles northwest of Potosi and 9.5 miles upstream from mouth.

Drainage area.- $-267 \mathrm{sq} \mathrm{mi}$.

Gage-height record.-Water-stage recorder graph. Datum of gage is $606.89 \mathrm{ft}$ above mean sea level, adjustment of 1912 .

Discharge record.- Stage-discharge relation defined by current-meter measurements below 17,500 cfs and by slope-area measurements at 25,000 cfs. Backwater from ice Feb. 26 to Mar. 9, Mar. 14 to Apr. 1, and backwater from Mississippi River Apr. 15 to May 3.

Maxima.-March-May 1965: Discharge, about 7,000 cfs 0800 hours Mar. 1 (gage height, $22.05 \mathrm{ft}$, backwater from ice); gage height, $22.71 \mathrm{ft} 0200$ hours Mar. 6, backwater from ice.

1934 to February 1965: Discharge, 25,000 cfs July 16, 1950 (gage height $24.82 \mathrm{ft}$ ).

Mean discharge, in cubic feet per second, 1965

\begin{tabular}{|c|c|c|c|c|c|c|c|c|c|c|c|}
\hline Day & March & April & May & Day & March & April & May & Day & March & April & May \\
\hline . & 5,000 & 3,000 & 100 & $11 \ldots$ & 305 & 239 & 74 & $21 \ldots$ & 66 & 76 & 57 \\
\hline 2 & 1,700 & 607 & 92 & $12_{-}$ & 229 & 177 & 74 & $22 \ldots$ & $6^{4}$ & 80 & 80 \\
\hline 3. & 900 & 258 & 92 & $13 \ldots$ & 136 & 134 & 70 & $23_{-}$ & 62 & 94 & 78 \\
\hline 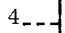 & 200 & 721 & 107 & $14_{-}$ & 115 & 126 & 69 & 24 & 62 & 130 & 70 \\
\hline 5. & 1,000 & 541 & 96 & $15 \ldots$ & 110 & 120 & 70 & 25 & 62 & 175 & 69 \\
\hline 6. & 2,000 & 596 & 98 & $16 \ldots$ & 97 & 115 & 84 & $26 \ldots$ & 62 & 160 & 106 \\
\hline 7. & 1,700 & 246 & 89 & $17 \ldots$ & 85 & 105 & 81 & 27 & 66 & 150 & 167 \\
\hline 8. & 1,200 & 203 & 87 & $18 \ldots$ & 80 & 95 & 69 & 28 & 70 & 130 & 93 \\
\hline 9. & 800 & 195 & 88 & 19 & 75 & 90 & 63 & |29_. & 75 & 120 & 81 \\
\hline 10 & 453 & 140 & 81 & 20 & 70 & 80 & 58 & 30. & 120 & 110 & 80 \\
\hline & & & & & & & & $\mid 31 \ldots$ & 1,700 & & 76 \\
\hline \multirow{2}{*}{\multicolumn{9}{|c|}{$\begin{array}{l}\text { Monthly mean discharge, in cubic feet per second } \\
\text { Runoff, in inches }\end{array}$}} & 602 & 300 & 83.8 \\
\hline & & & & & & & & & 2.60 & 1.26 & 0.36 \\
\hline
\end{tabular}


Gage height, in feet, and discharge, in cubic feet per second, at indicated time, 1965, of Grant River at Burton, Wis.

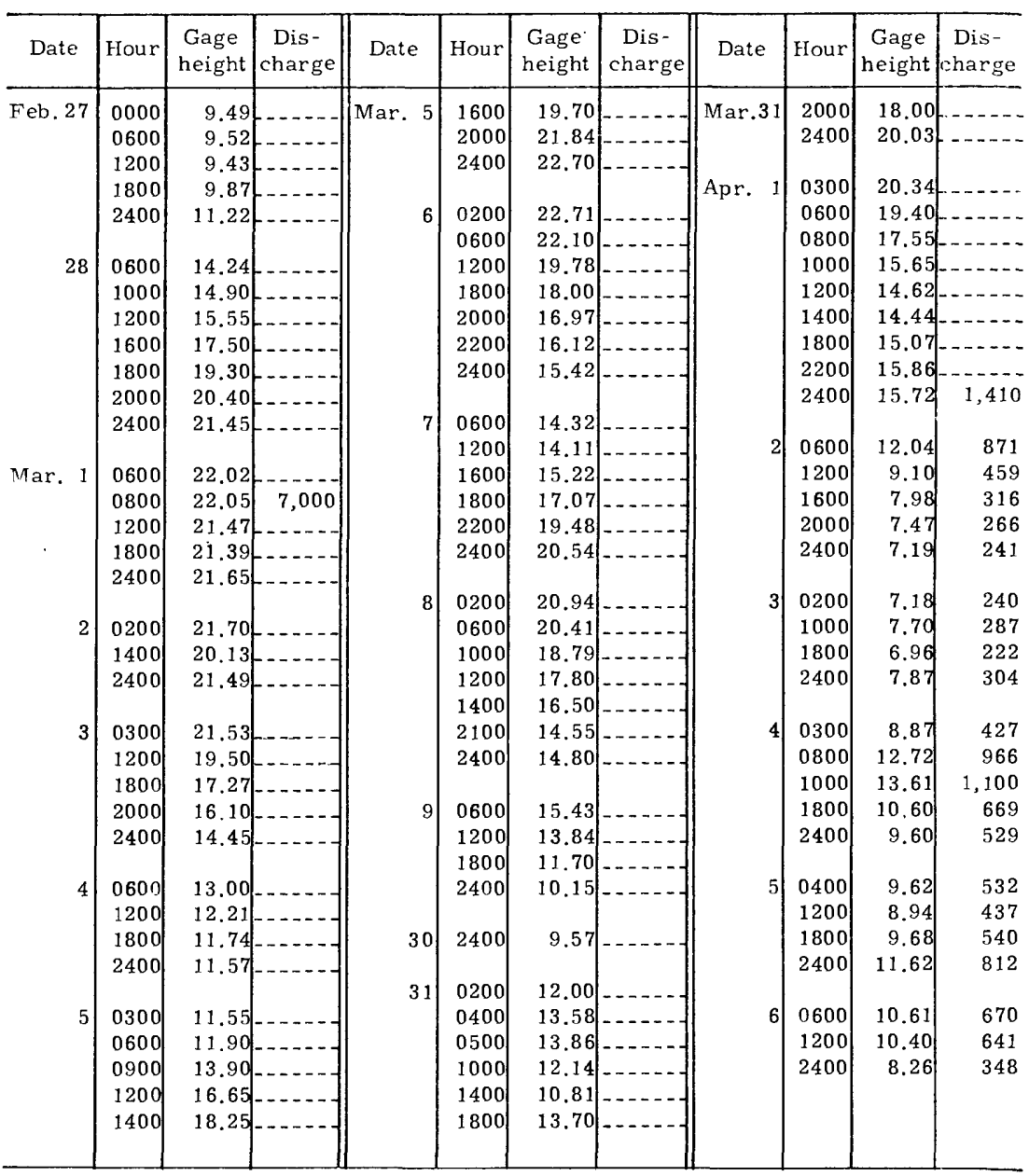




\section{PLATTE RIVER BASIN}

(210) 5-4140. Platte River near Rockville, Wis.

Location.-Lat $42^{\circ} 43^{\prime} 55^{\prime \prime}$, Iong $90^{\circ} 38^{\prime} 25^{\prime \prime}$, in SW $\frac{1}{4}$ sec. 17, T.3 N., R. 2 \%., on right bank just downstream from highway bridge, 0.8 mile upstream from BIalely Branch, 2.2 miles east of Rockville, 4.5 miles northeast of Potosi, and 15.2 miles upstream from mouth.

Drainage area. -139 sq $\mathrm{mi}$.

Gage-height record.--Water-stage recorder graph except parts of Feb. 28 to Mar. 2 for which graph was reconstructed on basis of available recorder record, floodmark and numerous float-tape gage readings. Datum of gage is $642.95 \mathrm{ft}$ above mean sea Ievel, adjustment of 1912 .

Discharge record.-Stage-discharge relation defined by current-meter measurements below $7000 \mathrm{cfs}$, and by slope-area measurement at 43,500 cfs. Backwater from ice Feb. 27 to Mar. 2, Mar. 19-28.

Maxima.-February-May 1965: Discharge, about 3000 cfs 2200 hours Feb. 28 (gage height, $10.86 \mathrm{ft}$, backwater from ice).

1934 to January 1965: Discharge, 43,500 cfs July 16, 1950 (gage height $17.26 \mathrm{ft}$ ).

Mean discharge, in cubic feet per second, 1965

\begin{tabular}{|c|c|c|c|c|c|c|c|c|c|c|c|}
\hline Day & March & April & May & Day & March & April & May & Day & Marcl & April & May \\
\hline 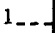 & 1,600 & 1,080 & 60 & $11 \ldots$ & 61 & 191 & 47 & 21 & 45 & 54 & 40 \\
\hline 2 & 960 & 336 & 57 & $12 \ldots$ & 52 & 125 & 45 & 22 & 45 & 52 & 47 \\
\hline 3. & 387 & 240 & 54 & $13 \ldots$ & 55 & 84 & 44 & 23 & 43 & 51 & 47 \\
\hline 4. & 82 & 467 & 52 & 14 & 54 & 75 & 42 & 24 & 43 & 56 & 43 \\
\hline 5. & 828 & 474 & 132 & $15 \ldots$ & 69 & 76 & 44 & 25. & 42 & 92 & 42 \\
\hline 6. & 468 & 748 & 72 & $16 \ldots$ & 56 & 69 & 50 & 26 & 42 & 110 & 47 \\
\hline 7. & 549 & 207 & 59 & $17 \ldots$ & 66 & 66 & 47 & 27. & 43 & 81 & 77 \\
\hline 8. & 413 & 177 & 62 & $18 \ldots$ & 52 & 63 & 45 & 28 & 46 & 85 & 55 \\
\hline 9. & 224 & 139 & 55 & $19 \ldots$ & 47 & 64 & 42 & 29 & 50 & 72 & 48 \\
\hline 10 & 72 & 92 & 50 & 20 & 45 & 57 & 40 & 30 & 115 & 65 & 46 \\
\hline & & & & & & & & & 968 & & 45 \\
\hline \multirow{2}{*}{\multicolumn{9}{|c|}{$\begin{array}{l}\text { Monthly mean discharge, in cubic feet per second } \\
\text { Runoff, in inches }\end{array}$}} & 246 & 185 & 52.8 \\
\hline & & & & & & & & & 2.04 & 1.48 & 0.44 \\
\hline
\end{tabular}

Gage height, in feet, and discharge, in cubic feet per second, at indicated time, 1965

\begin{tabular}{|c|c|c|c|c|c|c|c|c|c|c|c|}
\hline Date & Hour & $\begin{array}{c}\text { Gage } \\
\text { height }\end{array}$ & $\begin{array}{c}\text { Dis- } \\
\text { charge }\end{array}$ & Date & Hour & $\begin{array}{c}\text { Gage } \\
\text { height }\end{array}$ & $\begin{array}{c}\text { Dis- } \\
\text { charge }\end{array}$ & Date & Hour & $\begin{array}{c}\text { Gage } \\
\text { height }\end{array}$ & $\begin{array}{c}\text { Dis- } \\
\text { charge }\end{array}$ \\
\hline \multirow[t]{12}{*}{ Feb. 28} & 0000 & 4.80 & & Mar. 1 & 1800 & 9.9 & & Mar. 3 & 2400 & 3.94 & 118 \\
\hline & 0600 & 4.87 & & & 2400 & 10.4 & & & & & \\
\hline & 1100 & 5.15 & & & & & & 4 & 0600 & 3.73 & 89 \\
\hline & 1200 & 5.11 & & 2 & 0400 & 10.6 & & & 1200 & 3.61 & 76 \\
\hline & 1400 & 5.24 & & & 0900 & 10.3 & & & 1800 & 3.53 & 69 \\
\hline & 1500 & 5.60 & & & 1200 & 8.9 & & & 2400 & 3.54 & 70 \\
\hline & 1600 & 8.0 & & & 1500 & 7.9 & & & & & \\
\hline & 1800 & 10.0 & & & 1800 & 7.2 & & 5 & 0300 & 3.66 & 81 \\
\hline & 2000 & 10.7 & & & 2100 & 8.3 & $\ldots$ & & 0600 & 4.16 & 157 \\
\hline & 2200 & 10,86 & 3,000 & & 2400 & 7.40 & 1,200 & & 0900 & 5.05 & 364 \\
\hline & 2400 & 10.8 & & & & & & & 1200 & 6.56 & 821 \\
\hline & & & & 3 & 0600 & 5.40 & 462 & & 1800 & 8.3 & 1,660 \\
\hline \multirow[t]{2}{*}{ Mar. 1} & 0600 & 10.0 & & & 1200 & 4.64 & 261 & & 2000 & 8.4 & 1,730 \\
\hline & 1200 & 9.8 & & & 1800 & 4.21 & 167 & & 2400 & 7.43 & 1,220 \\
\hline
\end{tabular}


Gage height, in feet, and discharge, in cubic feet per second, at indicated time, 1965, of Platte River rear Rockville, Wis.--Continued

\begin{tabular}{|c|c|c|c|c|c|c|c|c|c|c|c|}
\hline Date & Hour & $\begin{array}{c}\text { Gage } \\
\text { height }\end{array}$ & $\begin{array}{c}\text { Dis- } \\
\text { charge }\end{array}$ & Date & Hour & $\begin{array}{c}\text { Gage' } \\
\text { height }\end{array}$ & $\begin{array}{c}\text { Dis- } \\
\text { charge }\end{array}$ & Date & Hour & $\begin{array}{c}\text { Gage } \\
\text { height }\end{array}$ & $\begin{array}{c}\text { Dis- } \\
\text { charge }\end{array}$ \\
\hline ar. 6 & $\begin{array}{l}0600 \\
1200 \\
1600 \\
2000 \\
2400 \\
0300 \\
1300 \\
1600 \\
1800 \\
2100 \\
2400 \\
0300 \\
0600 \\
1300 \\
1800 \\
2300 \\
2400 \\
0600 \\
1200 \\
1800 \\
2400 \\
0000\end{array}$ & $\begin{array}{l}5.68 \\
4.89 \\
4.64 \\
4.72 \\
4.68 \\
4.65 \\
4.07 \\
5.40 \\
7.15 \\
8.1 \\
7.20 \\
5.66 \\
4.97 \\
4.23 \\
5.10 \\
5.90 \\
5.82 \\
4.76 \\
4.18 \\
3.87 \\
3.73 \\
3.32\end{array}$ & $\begin{array}{r}544 \\
322 \\
261 \\
280 \\
270 \\
\\
263 \\
141 \\
462 \\
1,080 \\
1,550 \\
1,100 \\
\\
538 \\
343 \\
171 \\
378 \\
610 \\
586 \\
\\
290 \\
161 \\
107 \\
89 \\
\\
54\end{array}$ & Mar. 30 & $\begin{array}{l}1200 \\
1500 \\
1800 \\
2200 \\
2300 \\
2400 \\
0300 \\
1300 \\
1600 \\
1800 \\
2200 \\
2400 \\
0600 \\
1000 \\
1500 \\
1900 \\
2400 \\
0600 \\
1200 \\
1800 \\
2400 \\
0300\end{array}$ & $\begin{array}{l}3.35 \\
3.45 \\
3.90 \\
5.05 \\
5.00 \\
5.08 \\
5.43 \\
4.70 \\
6.60 \\
8.40 \\
9.30 \\
8.80 \\
6.25 \\
5.83 \\
7.20 \\
7.86 \\
6.54 \\
5.13 \\
4.52 \\
4.30 \\
4.67 \\
5.22\end{array}$ & $\begin{array}{r}56 \\
64 \\
111 \\
364 \\
351 \\
373 \\
471 \\
275 \\
835 \\
1,730 \\
2,810 \\
2,110 \\
\\
715 \\
589 \\
1,100 \\
1,430 \\
814 \\
\\
386 \\
233 \\
185 \\
268 \\
411\end{array}$ & Apr. 3 & $\begin{array}{l}1200 \\
1800 \\
2100 \\
2400 \\
0500 \\
1600 \\
2400 \\
0900 \\
1500 \\
2000 \\
2200 \\
2400 \\
0300 \\
0500 \\
0700 \\
1200 \\
1800 \\
2100 \\
2400 \\
0600 \\
1200 \\
1800 \\
2400\end{array}$ & $\begin{array}{l}4.22 \\
4.07 \\
4.30 \\
4.77 \\
5.75 \\
5.12 \\
5.80 \\
4.72 \\
5.28 \\
6.09 \\
5.93 \\
6.37 \\
7.62 \\
7.57 \\
7.59 \\
5.68 \\
5.13 \\
5.09 \\
5.09 \\
4.57 \\
4.22 \\
4.12 \\
4.17\end{array}$ & $\begin{array}{r}169 \\
141 \\
185 \\
292 \\
\\
565 \\
383 \\
580 \\
280 \\
427 \\
667 \\
619 \\
755 \\
1,310 \\
1,290 \\
1,300 \\
544 \\
386 \\
375 \\
374 \\
\\
244 \\
169 \\
150 \\
158\end{array}$ \\
\hline
\end{tabular}

\section{LITTLE MAQUOKETA RIVER BASIN}

(211) 5-4145. Little Maquoketa River near Durango, Iowa.

Location.-Lat $42^{\circ} 33^{\prime} 25^{\prime \prime}$, long $90^{\circ} 44^{\prime} 45^{\prime \prime}$, in NW $\frac{1}{4} \mathrm{NE}^{\frac{1}{4}}$ sec.5, T.89 N., R.2 E., on laft bank $10 \mathrm{ft}$ upstream from highway bridge, $1 \frac{1}{2}$ miles east of Durango, 5 miles ncrthwest of Dubuque, and 7.5 miles upstream from mouth.

Drainage area. $-130 \mathrm{sq} \mathrm{mi}$.

Gage-height record.-Water-stage recorder graph. Datum of gage is $612.03 \mathrm{ft}$ above mean sea level, datum of 1929.

Discharge record.-Stage-discharge relation defined by current-meter measurements below 6,300 cfs, extended to $25,000 \mathrm{cfs}$ by logarithmic plotting and results of th ree slope-area measurements. Backwater from ice Mar. 9-11, 18-29.

Maxima.-March-May 1965: Discharge, 8,860 cfs 1830 hours Feb. 28 (gage height, $16.41 \mathrm{ft}$ ).

1925 to February 1965: Discharge, about 29,000 cfs June 15, 1925 (gage heiqht, $22.1 \mathrm{ft}$. 
Mean discharge, in cubic feet per second, 1965, of Little Maquoketa River near Durango, Iow'a

\begin{tabular}{|c|c|c|c|c|c|c|c|c|c|c|c|}
\hline Day & March & April & May & Day & March & April & May & Day & Marcr & April & May \\
\hline 1 & 1,100 & 1,220 & 54 & $11 \ldots$ & 56 & 354 & 29 & 21 & 23 & 43 & 20 \\
\hline 2 & 996 & 283 & 48 & $12 \ldots$ & 47 & 127 & 28 & 22 & 22 & 40 & 21 \\
\hline 3. & 339 & 326 & 42 & 13 & 91 & 81 & 25 & 23 & 21 & 37 & 39 \\
\hline 4 & 68 & 566 & 39 & 14. & 139 & 70 & 24 & 24 & $2 \vartheta$ & 53 & 28 \\
\hline 5 & 358 & 811 & 43 & $15 \ldots$ & 122 & 73 & 25 & 25 & 27 & 186 & 23 \\
\hline 6. & 261 & 734 & 41 & $16 \ldots$ & 94 & 62 & 37 & 26. & 19 & 134 & 45 \\
\hline 7. & 271 & 177 & 36 & 17 & 62 & 68 & 31 & 27. & 19 & 89 & 113 \\
\hline 8 & 260 & 170 & 32 & $18=$ & 33 & 58 & 27 & 28. & 21 & 95 & 46 \\
\hline & 100 & 113 & 35 & $19=$ & 28 & 50 & 24 & $29 \ldots$ & 25 & 74 & 36 \\
\hline 10 & 68 & 102 & 33 & $20 \ldots$ & 25 & 46 & 22 & $30 \ldots$ & 425 & 63 & 34 \\
\hline \multirow{3}{*}{\multicolumn{9}{|c|}{$\begin{array}{l}\text { Monthl } y^{\prime} \text { mean discharge, in cubic feet per second } \\
\text { Runoff. in inches } \\
\text { Runoff, in acre-feet }\end{array}$}} & 215 & 210 & 35.8 \\
\hline & & & & & & & & & 1.91 & 1.80 & 0.32 \\
\hline & & & & & & & & & 13,220 & 12,510 & 2,200 \\
\hline
\end{tabular}

Gage height, in fect, and desclearge, in , ubic jeat per second, at indeated time, 1965

\begin{tabular}{|c|c|c|c|c|c|c|c|c|c|c|c|}
\hline Date & IIour & $\begin{array}{l}\text { Gage } \\
\text { height }\end{array}$ & $\begin{array}{c}\text { Dis - } \\
\text { charge }\end{array}$ & Date & Ilour & $\begin{array}{l}\text { Gage } \\
\text { height }\end{array}$ & $\begin{array}{c}\text { Dis- } \\
\text { charge }\end{array}$ & Date & Hour & $\begin{array}{l}\text { Gage } \\
\text { height }\end{array}$ & $\begin{array}{l}\text { Dis- } \\
\text { charge }\end{array}$ \\
\hline \multirow[t]{11}{*}{ Feb. 27} & 0000 & 2.93 & 24 & F'eb. 28 & 1930 & 14.65 & 6,490 & \multirow[t]{11}{*}{ Mar.31 } & 1230 & 4.54 & 292 \\
\hline & 1400 & 2.92 & 23 & & 2000 & 13.05 & 4,800 & & 1400 & 4.98 & 397 \\
\hline & 1600 & 2.98 & 28 & & 2030 & 11.60 & 3,560 & & 1500 & 7.00 & 1,050 \\
\hline & 1700 & 3.12 & 42 & & 2100 & 10.80 & 3,000 & & 1600 & 8.10 & 1,520 \\
\hline & 1800 & 3.97 & 170 & & 2200 & 9.58 & 2,270 & & 1700 & 10.20 & 2,650 \\
\hline & 1930 & 4.69 & 322 & & 2300 & 8.60 & 1,740 & & 1900 & 12.34 & 4,180 \\
\hline & 2200 & 4.11 & 197 & & 2400 & 7.85 & 1,390 & & 2000 & 12.52 & 4,340 \\
\hline & 2300 & 5.65 & 579 & & & & & & 2100 & 12.35 & 4,180 \\
\hline & 2330 & 7.00 & 1,040 & \multirow[t]{13}{*}{ Mar.30 } & 0000 & 3.14 & 47 & & 2200 & 11.10 & 3,220 \\
\hline & 2400 & 8.50 & 1,690 & & 0200 & 3.41 & 82 & & 2400 & 8.70 & 1,800 \\
\hline & & & & & 0500 & 3.48 & 92 & & & & \\
\hline 28 & 0030 & 9.41 & 2,160 & & 1000 & 3.40 & 81 & \multirow[t]{12}{*}{ Apr. 1} & $0 € 00$ & 6.83 & 982 \\
\hline & 0200 & 8.23 & 1,560 & & 1500 & 3.69 & 124 & & $0 \in 00$ & 6.40 & 827 \\
\hline & 0600 & 7.00 & 1,040 & & 1600 & 3.97 & 173 & & 1000 & 6.90 & 1,010 \\
\hline & 1100 & 6.18 & 748 & & 1700 & 4.80 & 353 & & 1400 & 7.23 & 1,140 \\
\hline & 1400 & 7.00 & 1,040 & & 1800 & 5.70 & 601 & & 1700 & 8.59 & 1,740 \\
\hline & 1500 & 8.55 & 1,720 & & 1900 & 6.60 & 895 & & $1 \varepsilon 00$ & 8.72 & 1,810 \\
\hline & 1600 & 10.30 & 2,700 & & 2000 & 7.95 & 1,450 & & $1 \subseteq 00$ & 8.72 & 1,810 \\
\hline & 1630 & 1]. 50 & 3,490 & & 2130 & 8.35 & 1,630 & & $2 C 00$ & 8.45 & 1,680 \\
\hline & 1700 & 13.80 & 4,950 & & 2400 & 7.76 & 1,360 & & 2300 & 6.78 & 962 \\
\hline & 1800 & 15.85 & 8,010 & & & & & & 2400 & 6.27 & 784 \\
\hline & 1830 & 16.41 & 8,860 & \multirow[t]{2}{*}{31} & 0500 & 5.67 & 592 & & & & \\
\hline & 1900 & 15.95 & 8,150 & & 1000 & 4.67 & 322 & & & & \\
\hline
\end{tabular}




\section{MISSISSIPPI RIVER MAIN STEM}

(212) Mississippi River at Dubuque, Iowa

(Miscellaneous site)

Location. - Lat $42^{\circ} 29^{\prime}$, long $90^{\circ} 38^{\prime}$, on right bank at the foot of Fourth Street in Dubuque, adjacent to and on the downstream side of the right abutment of the Illinois Central Railroad Bridge and at mile 579,9 above the Ohio River.

Drainage area. $-81,600 \mathrm{sq} \mathrm{mi}$, approximately.

Gage-height record.-Water-stage recorder graph. Datum of gage is $584.95 \mathrm{ft}$ above mean sea level, datum of 1929 .

Discharge record.-Stage-discharge relation defined by current-meter measurements and analysis of gate operation procedure at Dam 12. Relation only applicable for standard gate operation procedures and discharges above $100,000 \mathrm{cfs}$.

Maxima.-April-May 1965: Discharge, 304,000 cfs 0030 hrs Apr. 26 (gage height, $26.71 \mathrm{ft}$ ).

1865 to February 1965: Gage height, 22.7 ft Apr. 22, 1951 and Apr. 25, 1952.

Remarks -Current-meter measurements for 1944-65 and annual maximum gaṛe heights prior to 1965 furnished by Corps of Engineers. Gage-height record furnisher by U.S. Weather Bureau.

Mean gage height, in feet, and discharge, in cubic feet per second, 1965

\begin{tabular}{|c|c|c|c|c|c|c|c|c|c|}
\hline \multirow[b]{2}{*}{ Day } & \multicolumn{2}{|c|}{ April } & \multicolumn{2}{|c|}{ May } & & \multicolumn{2}{|c|}{ April } & \multicolumn{2}{|c|}{$\overline{\mathrm{M}} \varepsilon \mathrm{y}$} \\
\hline & $\begin{array}{c}\text { Gage } \\
\text { height }\end{array}$ & Discharge & $\begin{array}{l}\text { Gage } \\
\text { height }\end{array}$ & Dis charge & Day & $\begin{array}{c}\text { Gage } \\
\text { height }\end{array}$ & Discharge & $\begin{array}{c}\text { Gage } \\
\text { height }\end{array}$ & Dis charge \\
\hline 1 - & -- & - & 24.59 & 266,000 & $16 \ldots$ & 19.29 & 178,000 & 14.67 & 111,000 \\
\hline 2 & $\ldots$ & $\ldots$ & 23.96 & 254,000 & $17 \ldots$ & 20.04 & 190,000 & 14.57 & 110,000 \\
\hline $3 \ldots$ & $\ldots$ & $\ldots$ & 23.34 & 244,000 & 18 & 20.84 & 202,000 & 14.55 & 110,000 \\
\hline $4 \ldots$ & & & 22.66 & 232,000 & $19 \ldots$ & 21.78 & 217,000 & 14.47 & 109,000 \\
\hline $5 \ldots$ & - & - & 21.90 & 219,000 & $20=$ & 22.88 & 236,000 & 14.43 & 108,000 \\
\hline $6 \ldots$ & & & 21.06 & 206,000 & $21 \ldots$ & 24.12 & 257,000 & 14.40 & 108,000 \\
\hline 7. & & & 20.17 & 192,000 & $22 \ldots$ & 25.18 & 276,000 & 14.48 & 109,000 \\
\hline 8 & & & 19.37 & 180,000 & $23 \ldots$ & 25.95 & 290,000 & 14.61 & 110,000 \\
\hline 9 & - & -1 & 18.61 & 168,000 & $24--$ & 26.42 & 299,000 & 14.82 & 113,000 \\
\hline 10. & 14.70 & 111,000 & 17.87 & 157,000 & $25 \ldots$ & 26.67 & 304,000 & 14.97 & 115,000 \\
\hline $11 \ldots$ & 15.97 & 129,000 & 17.07 & 145,000 & 26 & 26.64 & 303,000 & 14.91 & 114,000 \\
\hline $12 \ldots$ & 16.70 & 140,000 & 16.25 & 133,000 & $27 \ldots$ & 26.40 & 299,000 & 15.00 & 115,000 \\
\hline 13 & 17.46 & 151,000 & 15.42 & 121,000 & $28 \ldots$ & 26.08 & 293,000 & 14.60 & 110,000 \\
\hline $14 \ldots$ & 18.12 & 161,000 & 14.97 & 115,000 & $29 \ldots$ & 25.64 & 285,000 & 14.26 & 106,000 \\
\hline & 18.72 & 170,000 & 14.73 & 112,000 & $30 \ldots$ & 25.12 & 275,000 & $\ldots \ldots$ & - \\
\hline
\end{tabular}

Annual maximum gage heights for indicated years

\begin{tabular}{|c|c|c|c|c|c|}
\hline Year & Date & $\begin{array}{l}\text { Gage height } \\
\text { (feet) }\end{array}$ & Year & Date & $\begin{array}{l}\text { Gage height } \\
\text { (feet) }\end{array}$ \\
\hline $1866 \ldots$ & May & 20.17 & $\mid 1881 \ldots \ldots$ & Oct. 24,25 & 20.2 \\
\hline $1869 \ldots$ & $\ldots$ & 15.02 & $1882 \ldots \ldots$ & Apr. 20 & 15.7 \\
\hline $1870 \ldots$ & Apr. 20 & 21.83 & $1883 \ldots$ & Apr. 27-29 & 15.7 \\
\hline $1874 \ldots$ & May $13-17$ & 10.4 & $1884 \ldots \ldots$ & Oct. 16 & 13.6 \\
\hline $1875 \ldots$ & Apr. 26 & 15.5 & $1885 \ldots \ldots$ & May 6, 7 & 11.5 \\
\hline $1876 \ldots$ & May 27-29 & 15.4 & $1886 \ldots$ & Apr. 29 & 14.4 \\
\hline $1877 \ldots$ & July $18-19$ & 7.7 & $1887 \ldots \ldots$ & Apr. $25-27$ & 14.0 \\
\hline $1878 \ldots$ & Aug. 1 & 5.4 & $1888 \ldots$ & May 12,13 & 21.4 \\
\hline $1879 \ldots$ & May 30 & 9.7 & $1889 \ldots \ldots$ & Apr. 1-3, May 30 & 6.4 \\
\hline $1880 \ldots$ & June 23,24 & 21.7 & & & \\
\hline
\end{tabular}


Annual maximum gage height for indicated years of Mississippi River at Dubuque, Iov'a - Continued

\begin{tabular}{|c|c|c|c|c|c|}
\hline Year & Date & $\begin{array}{l}\text { Gage height } \\
\text { (feet) }\end{array}$ & Year & Date & $\begin{array}{c}\text { Gage height } \\
\text { (feet) }\end{array}$ \\
\hline 1890 & June 26 & 13.2 & $1928 \ldots \ldots$ & Apr. 7, 8 & 14.0 \\
\hline $1891 \ldots$ & Apr. 30, May 1, 2 & 12.9 & $\mid 1929 \ldots \ldots$ & Apr. 17 & 17.0 \\
\hline $1892--$ & June 24 & 17.6 & $1930 \ldots \ldots$ & June 26,27 & 11.0 \\
\hline 1893 & May $11-14$ & 15.7 & $1931 \ldots \ldots$ & Dec. 3-4 & 7.6 \\
\hline 1894 & May 29 & 15.5 & $\mid 1932 \ldots \ldots$ & Apr. 20,21 & 13.1 \\
\hline $1895_{--}$ & June 24-26 & 6.3 & $\mid 1933 \ldots \ldots$ & Apr. 11 & 11.9 \\
\hline 1896 & May 29 & 13.9 & $\mid 1934 \ldots \ldots$ & Apr. 17 & 12.0 \\
\hline 1897 & Apr. 15 & 17.9 & $1935 \ldots \ldots$ & Apr. 3-5 & 16.2 \\
\hline $1898 \ldots$ & June $21-23$ & 9.4 & $\mid 1936 \ldots \ldots$ & Apr. 5 & 16.8 \\
\hline $1899 \ldots$ & June 22-44 & 14.8 & $1937 \ldots \ldots$ & Mar. 8 & 12.2 \\
\hline $1900 \ldots$ & Oct. 17 & 14.6 & $1938 \ldots \ldots$ & Sept. 21 & 20.6 \\
\hline $1901 \ldots$ & Apr. 20 & 11.0 & $\mid 1939 \ldots \ldots$ & Apr. 6 & 17.5 \\
\hline $1902 \ldots$ & May 24 & 12.6 & $1940 \ldots \ldots$ & June 18 & 11.4 \\
\hline $1903 \ldots$ & Sept. 27 & 17.4 & $1941 \ldots \ldots$ & Apr. 23 & 16.4 \\
\hline $1904 \ldots$ & June 8, 9 & 11.7 & $1942 \ldots \ldots$ & June 12 & 19.3 \\
\hline 1905 & June 18-19 & 17.4 & $1943 \ldots \ldots$ & June 29 & 18.9 \\
\hline 1906 & Apr. 21-25 & 16.6 & $\mid$ & June 27 & 19.0 \\
\hline 1907 & Apr. 9,10 & 16.1 & $1945 \ldots \ldots$ & Mar. 29, 30 & 18.9 \\
\hline $1908 \ldots$ & July 19 & 14.9 & $\mid 1946 \ldots \ldots$ & Mar. 27 & 17.8 \\
\hline $1909 \ldots$ & Apr. 20 & 13.2 & $1947 \ldots \ldots$ & June 14 & 15.5 \\
\hline $1910_{\ldots} \ldots$ & Apr. 2, 3 & 9.1 & $\mid 1948 \ldots \ldots$ & Mar. 31, Apr. 1 & 14.2 \\
\hline $1911 \ldots$ & Oct. 19 & 14.6 & $1949 \ldots \ldots$ & Apr. $4-11,15$ & 12.4 \\
\hline $1912 \ldots$ & Apr. 4 & 12.2 & $1950 \ldots \ldots$ & May 20 & 16.7 \\
\hline $1913 \ldots$ & Mar. 25 & 12.6 & $1951 \ldots \ldots$ & Apr. 22 & 22.7 \\
\hline $1914 \ldots$ & July 12 & 14.4 & $1952 \ldots \ldots$ & Apr. 25 & 22.7 \\
\hline $1915 \ldots$ & Apr. 21, 22 & 12.1 & $1953 \ldots \ldots$ & Apr. 1, 2, 4 & 14.0 \\
\hline $1916 \ldots$ & May 3 & 19.8 & $1954 \ldots \ldots$ & May 12 & 20.2 \\
\hline $1917 \ldots$ & Apr. 18, 19 & 15.5 & $\mid 1955 \ldots \ldots$ & Apr. 14,15 & 13.3 \\
\hline $1918 \ldots$ & June 11,12 & 13.6 & $1956 \ldots \ldots$ & Apr. 19 & 16.0 \\
\hline $1919 \ldots$ & Apr. 22,23 & 16.6 & $1957 \ldots \ldots$ & July 13 & 13.7 \\
\hline $1920 \ldots$ & Apr. 7 & 21.0 & $1958 \ldots \ldots$ & Apr. 16,17 & 10.0 \\
\hline $1921 \ldots$ & May 10 & 10.7 & $1959 \ldots \ldots$ & Apr. 2 & 14.3 \\
\hline $1922 \ldots$ & Apr. 21 & 21.0 & $\mid 1960 \ldots-\ldots$ & May 17 & 17.5 \\
\hline $1923 \ldots$ & May 3, 4 & 12.4 & $\mid 1961 \ldots \ldots$ & Mar. 31 & 16.7 \\
\hline $1924=-$ & May 8 & 11.7 & $\mid 1962 \ldots \ldots$ & Apr. 19 & 16.3 \\
\hline $1925 \ldots$ & June 25 & 11.1 & $1963 \ldots \ldots$ & Apr. 2-4 & 12.5 \\
\hline 1926 & Oct. 5 & 11.3 & |1964_.... & May $18-21$ & 12.20 \\
\hline 1927 & Mar. 28 & 15.6 & | & Apr. 26 & 26.71 \\
\hline
\end{tabular}




\section{GALENA RIVER BASIN}

(213) 5-4150. Galena River at Buncombe, Wis.

Location.-Lat $42^{\circ} 30^{\prime} 50^{\prime \prime}$, long $90^{\circ} 22^{\prime} 40^{\prime \prime}$, near center of sec.33, T.1 N., R.1 E., on left bank at Buncombe, 0.6 mile upstream from Coon Branch, 1.5 miles upstream from Scrabble Branch, 2 miles upstream from Wisconsin-Illinois State line, and 3.5 miles southeast of Hazel Green.

Drainage area.- $-128 \mathrm{sq} \mathrm{mi}$.

Gage-height record.-Water-stage recorder graph. Datum of gage is $682.77 \mathrm{ft}$ : bove mean sea level, adjustment of 1912 (Corps of Engineers Bench mark).

Discharge record.--Stage-discharge relation defined by current-meter measurements below $8,000 \mathrm{cfs}$ and by slope-area measurement at $12,400 \mathrm{cfs}$ and extended above for 1937 peak by logarithmic plotting. Backwater from ice Mar. 19-27.

Maxima.-March-May 1965: Discharge, 3,800 cfs 2000 hours Mar. 31 (gage height, $11 . \overline{82 \mathrm{f}} \mathrm{t}$ ).

1939 to February 1965: Discharge, 12,400 cfs Feb. 20, 1953 (gage height,15.68 ft).

Flood in February 1937 reached a stage of about $17.1 \mathrm{ft}$, from information by local resident (discharge, $18,000 \mathrm{cfs}$ ).

Mean discharge, in cubic feet per second, 1965

\begin{tabular}{|c|c|c|c|c|c|c|c|c|c|c|c|}
\hline Day & March & April & May & Day & March & April & May & Day & March & April & May \\
\hline 1 & 890 & 919 & 37 & $11 \ldots$ & 43 & 98 & 25 & 21 & 24 & 31 & 19 \\
\hline 2 & 910 & 210 & 35 & 12 & 36 & 65 & 24 & 22 & 23 & 30 & 20 \\
\hline 3 & 359 & 118 & 34 & 13 & 35 & 48 & 23 & 23 & 22 & 29 & 28 \\
\hline 4 & 65 & 140 & 33 & 14 & 39 & 43 & 23 & 24 & 22 & 35 & 26 \\
\hline 5 & 178 & 330 & 40 & $15 \ldots$ & 42 & 47 & 23 & 25. & 22 & 64 & 22 \\
\hline 6 & 265 & 279 & 34 & $16 \ldots$ & 40 & 43 & 24 & 26 & 22 & 75 & 31 \\
\hline 7. & 192 & 98 & 32 & $17 .-$ & 47 & 47 & 24 & $27-$ & 23 & 53 & 114 \\
\hline 8 & 310 & 66 & 31 & $18 \ldots$ & 30 & 42 & 22 & 28 & 25 & 53 & 37 \\
\hline 9 & 163 & 64 & 28 & 19 & 26 & 38 & 20 & 29 & 29 & 45 & 26 \\
\hline 10 & 54 & 54 & 26 & $20 \ldots$ & 25 & 31 & 18 & $30_{-}$ & 137 & 41 & 25 \\
\hline & & & & & & & & $31_{-}$ & 1,370 & 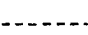 & 23 \\
\hline \multicolumn{9}{|c|}{ Monthly mean discharge, in cubic feet per second } & 176 & 108 & 29.9 \\
\hline \multicolumn{9}{|c|}{ Runoff, in inches } & 1.58 & 0.94 & 0.27 \\
\hline
\end{tabular}

Ginge herght, in fect, and discharge, in cubic fect per second, at indicated tine, 1965

\begin{tabular}{|c|c|c|c|c|c|c|c|c|c|c|c|}
\hline Date & Hour & $\begin{array}{c}\text { Gage } \\
\text { height }\end{array}$ & $\begin{array}{c}\text { Dis- } \\
\text { chargc }\end{array}$ & Date & Hour & $\begin{array}{c}\text { Gagc } \\
\text { height }\end{array}$ & $\begin{array}{c}\text { Dis- } \\
\text { charge }\end{array}$ & Date & Hlour & $\begin{array}{c}\text { Gage } \\
\text { height }\end{array}$ & $\begin{array}{c}\text { Dis- } \\
\text { charge }\end{array}$ \\
\hline \multirow[t]{12}{*}{ Feb. 28} & 0000 & 3.54 & 110 & Mar. 1 & 1200 & 5.40 & 587 & Mar. 3 & 0800 & 4.80 & 422 \\
\hline & 0400 & 3.62 & 123 & & 1600 & 5.75 & 710 & & 1200 & 4.21 & 238 \\
\hline & 1800 & 3.44 & 96 & & 2000 & 6.88 & 1,150 & & 2400 & $3.4 i$ & 94 \\
\hline & 1200 & 3.24 & 68 & & 2200 & 7.35 & 1,350 & & & & \\
\hline & 1400 & 4.30 & 260 & & 2400 & 7.28 & 1,320 & 29 & 2400 & $2.9 \AA$ & 39 \\
\hline & 1600 & 6.00 & 803 & & & & & & & & \\
\hline & 1800 & 6.60 & 1,040 & 2 & 0400 & 6.49 & 1,000 & 30 & 0600 & $2.7 \varepsilon$ & 28 \\
\hline & 2000 & 9.20 & 2,260 & & 0800 & 6.43 & 972 & & 1200 & 2.92 & 39 \\
\hline & 2100 & 10.20 & 2,770 & & 1200 & 6.80 & 1,120 & & 1400 & 3.04 & 49 \\
\hline & 2200 & 9.60 & 2,460 & & 1600 & 5.80 & 728 & & 1600 & 4.00 & 192 \\
\hline & 2400 & 7.80 & 1,560 & & 2000 & 5.57 & 646 & & 2000 & $4.4 \mathrm{f}$ & 300 \\
\hline & & & & & 2400 & 5.63 & 667 & & 2400 & 5.08 & 482 \\
\hline \multirow[t]{2}{*}{ Mar.1 } & 0400 & 5.95 & 784 & & & & & & & & \\
\hline & 0800 & 5.47 & 611 & 3 & 0400 & 5.95 & 784 & 31 & 0200 & $5.2 \varepsilon$ & 547 \\
\hline
\end{tabular}


Gage height, in feet, and discharge, in cubic feet per second, at indicated time, 1965 of Galena River at Buncombe. Wis-Continued

\begin{tabular}{|c|c|c|c|c|c|c|c|c|c|c|c|}
\hline Date & IIour & $\begin{array}{l}\text { Gage } \\
\text { height }\end{array}$ & $\begin{array}{c}\text { Dis- } \\
\text { chirgc }\end{array}$ & Date & Hout & $\begin{array}{l}\text { Gage } \\
\text { height }\end{array}$ & $\begin{array}{c}\text { Dis- } \\
\text { charge }\end{array}$ & Date & Horr & $\begin{array}{c}\text { Gage } \\
\text { height }\end{array}$ & $\begin{array}{c}\text { Dis- } \\
\text { charge }\end{array}$ \\
\hline \multirow[t]{8}{*}{ Mar. 31} & 0800 & 4.60 & 338 & \multirow[t]{3}{*}{ Mar. 31} & 2300 & 9.70 & 2,510 & \multirow[t]{8}{*}{ Apr. 1} & 2020 & 6.60 & 1,040 \\
\hline & 1200 & 4.49 & 307 & & 2400 & 8.20 & 1,760 & & 2490 & 5.22 & 527 \\
\hline & 1400 & 5.90 & 765 & & & & & & & & \\
\hline & 1600 & 8.10 & 1,710 & \multirow[t]{5}{*}{ Apr. 1} & 0200 & 6.65 & 1,060 & & 0600 & 4.10 & 214 \\
\hline & 1700 & 10.10 & 2,660 & & 0400 & 5.85 & 746 & & 1230 & 3.70 & 136 \\
\hline & 1800 & 11.30 & 3,380 & & 0800 & 5.44 & 601 & & 1800 & 3.50 & 104 \\
\hline & 2000 & 11.82 & 3,800 & & 1200 & 6.06 & 826 & & 2230 & 4.13 & 221 \\
\hline & 2200 & 10.90 & 3,160 & & 1800 & 7.27 & 1,310 & & 2400 & 3.96 & 184 \\
\hline
\end{tabular}

\section{MAQUOKETA RIVER BASIN}

(214) 5-4170. Maquoketa River near Manchester, lowa

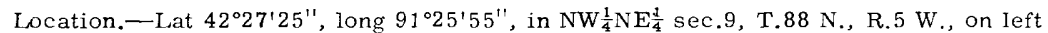
bank 2 miles southeast of Manchester and 4.7 miles downstream from Honey and Prairie Creeks.

Drainage area. $-305 \mathrm{sq} \mathrm{mi}$.

Gage-height record.-Water-stage recorder graph. Datum of gage $895.06 \mathrm{ft}$ above mean sea level, adjustment of 1912 .

Discharge record.- Stage-discharge relation defined by current-meter measurements below $9,700 \mathrm{cfs}$ and extended to $20,000 \mathrm{cfs}$ by velocity-area studies. Backwater from ice Mar. 1, 6-8, 19-27.

Maxima.-March-May 1965: Discharge, 5,150 cfs 0330 hours Apr. 1; gage height, $12.77 \mathrm{ft} 0430$ hours Mar. 1 (backwater from ice).

1933 to February 1965: Discharge, 20,000 cfs June 13, 1947 (gag€ height, $21.36 \mathrm{ft}$, from floodmarks).

Maximum flood known at Manchester June 15, 1925, stage unknown, discharge $25,400 \mathrm{cfs}$.

Mean discharge, in cubic feet per second, 1965

\begin{tabular}{|c|c|c|c|c|c|c|c|c|c|c|c|}
\hline Day & March & April & May & Day & March & April & May & Day & March & April & May \\
\hline & 3,500 & 4,530 & 112 & $11 \ldots$ & 220 & 311 & 98 & 21 & 80 & 123 & 61 \\
\hline 2 & 2,430 & 1,420 & 116 & $12 \ldots$ & 130 & 280 & 94 & $22 \ldots$ & 75 & 84 & 43 \\
\hline 3 & 529 & 447 & 132 & $13 \ldots$ & 186 & 200 & 94 & 23. & 72 & 108 & 90 \\
\hline & 218 & 565 & 110 & $14 \ldots$ & 293 & 207 & 86 & 24 & 70 & 93 & 164 \\
\hline & 456 & 821 & 110 & $15 \ldots$ & 316 & 155 & 61 & $25 \ldots$ & 67 & 204 & 127 \\
\hline 6. & 900 & 1,160 & 109 & 16 & 267 & 169 & 61 & $26 \ldots$ & 66 & 311 & 576 \\
\hline & 1,000 & 542 & 106 & $17 .$. & 153 & 142 & 101 & 27 & 66 & 255 & 756 \\
\hline & 1,100 & 340 & 91 & $18 \ldots$ & 103 & 147 & 95 & 28 & 65 & 232 & 380 \\
\hline & 600 & 302 & 77 & $19 \ldots$ & 92 & 155 & 87 & 29 & 67 & 221 & 206 \\
\hline & 251 & 251 & 128 & $20--$ & 84 & 139 & 82 & 30 & 119 & 157 & 194 \\
\hline & & & & & & & & 31 & 1,550 & & 196 \\
\hline \multicolumn{9}{|c|}{ Monthly mean discharge, in cubic feet per second } & 488 & 469 & 153 \\
\hline \multicolumn{9}{|c|}{ Runiff, in inches } & 1.84 & 1.72 & 0.58 \\
\hline \multicolumn{9}{|c|}{ Runoff, in acre-feet } & 30,000 & 27,910 & 9,410 \\
\hline
\end{tabular}


Gage height, in feet, and discharge, in cubic feet per second, at indicated time, 1965, of Maquoketa River nr. Manchester, Iowa

\begin{tabular}{|c|c|c|c|c|c|c|c|c|c|c|c|}
\hline Date & Hour & $\begin{array}{c}\text { Gage } \\
\text { height }\end{array}$ & $\begin{array}{c}\text { Dis- } \\
\text { charge }\end{array}$ & Date & Hour & $\begin{array}{l}\text { Gage } \\
\text { height }\end{array}$ & $\begin{array}{c}\text { Dis- } \\
\text { charge }\end{array}$ & Date & Hour & $\begin{array}{c}\text { Gage } \\
\text { height }\end{array}$ & $\begin{array}{c}\text { Dis- } \\
\text { charge }\end{array}$ \\
\hline Mar. 1 & $\begin{array}{l}0000 \\
0200 \\
0330 \\
0400 \\
0430 \\
0600 \\
0730 \\
1100 \\
1300 \\
1600 \\
2000 \\
2400 \\
\\
0400 \\
0800 \\
1200 \\
2000 \\
2400\end{array}$ & $\begin{array}{r}11.26 \\
11.93 \\
11.31 \\
11.80 \\
12.77 \\
11.18 \\
10.68 \\
10.44 \\
10.38 \\
10.68 \\
10.66 \\
10.43 \\
10.02 \\
9.48 \\
8.83 \\
7.33 \\
6.85\end{array}$ & 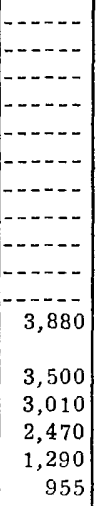 & Apr. 1 & $\begin{array}{l}0000 \\
0600 \\
0900 \\
1200 \\
1400 \\
1500 \\
1600 \\
1800 \\
2000 \\
2400 \\
0200 \\
0330 \\
0500 \\
0800 \\
1200 \\
1400 \\
1600\end{array}$ & $\begin{array}{r}5.77 \\
5.87 \\
5.89 \\
6.10 \\
6.60 \\
7.10 \\
8.00 \\
9.51 \\
10.41 \\
11.35 \\
\\
11.61 \\
11.64 \\
11.62 \\
11.50 \\
11.39 \\
11.25 \\
11.00\end{array}$ & $\begin{array}{r}306 \\
355 \\
365 \\
480 \\
780 \\
1,130 \\
1,810 \\
3,040 \\
3,860 \\
4,840 \\
\\
5,120 \\
5,150 \\
5,130 \\
5,000 \\
4,880 \\
4,720 \\
4,450\end{array}$ & Apr. 1 & $\begin{array}{l}1900 \\
2400 \\
0600 \\
1000 \\
1400 \\
1700 \\
2000 \\
2200 \\
2400 \\
1000 \\
1600 \\
2000 \\
2400\end{array}$ & $\begin{array}{r}10.45 \\
9.36 \\
8.13 \\
7.55 \\
7.01 \\
6.72 \\
6.50 \\
6.39 \\
6.31 \\
\\
6.00 \\
5.91 \\
5.92 \\
6.08\end{array}$ & $\begin{array}{r}3,900 \\
2,900 \\
1,910 \\
1,450 \\
1,070 \\
864 \\
720 \\
654 \\
606 \\
\\
420 \\
375 \\
380 \\
468\end{array}$ \\
\hline
\end{tabular}

(215) 5-4177. Bear Creek near Monmouth, Iowa

Location.-Lat $42^{\circ} 02^{\prime} 30^{\prime \prime}$, Iong $90^{\circ} 53^{\prime} 00^{\prime \prime}$, in $\mathrm{NE} \frac{1}{4} \mathrm{SE} \frac{1}{4} \mathrm{sec} .31, \mathrm{~T} .84 \mathrm{~N} ., \mathrm{R} .1 \mathrm{E}$., on right bank $15 \mathrm{ft}$ downstream from highway bridge, 1.6 miles upstream from Rat Run, 2.8 miles south of Monmouth, and 8.2 miles upstream from mouth.

Drainage area. $-61.3 \mathrm{sq} \mathrm{mi}$.

Gage-height record.-Water-stage recorder graph, except Apr. 13 to May 17 . Oncedaily wire-weight gage readings used for this period. Datum of gage is.728.80 ft above mean sea level, datum of 1929 .

Discharge record.- Stage-discharge relation defined by current-meter measurements below $6,000 \mathrm{cfs}$. Backwater from ice Mar. 1-7, 10, 11, 16-31.

Maxima.-March-May 1965: Discharge, 846 cfs 2130 hours May 25; gage height, $8.86 \mathrm{ft} 2000$ hours Mar. 29 (backwater from ice).

1957 to February 1965: Discharge, 2,440 cfs Jan. 12, 1960 (gage height, $11.56 \mathrm{ft}$ ).

Flood in June 1944 reached a stage of about $21.5 \mathrm{ft}$, from floodmark (discharge not determined).

Mean discharge, in cublc feet per second, 1965

\begin{tabular}{|c|c|c|c|c|c|c|c|c|c|c|c|}
\hline Day & March & April & May & Day & March & April & May & Day & March & April & May \\
\hline & 100 & 210 & 20 & $11 \ldots$ & 23 & 55 & 13 & 21. & 20 & 13 & 7.9 \\
\hline 2. & 85 & 100 & 18 & $12 \ldots$ & 29 & 35 & 12 & 22 & 15 & 13 & 7.9 \\
\hline 3. & 70 & 65 & 16 & $13 \ldots$ & 45 & 22 & 12 & 23 & 13 & 13 & 15 \\
\hline 4 & 25 & 128 & 16 & $14 \ldots$ & 81 & 20 & 12 & 24 & 12 & 26 & 13 \\
\hline 5. & 22 & 275 & 16 & $15 \ldots$ & 96 & 22 & 12 & 25 & 11 & 48 & 131 \\
\hline 6. & 60 & 268 & 15 & - _ 16 & 110 & 16 & 19 & 26. & 11 & 38 & 94 \\
\hline $\bar{t}_{-}$ & 80 & 88 & 14 & $17 \ldots$ & 90 & 16 & 14 & 27. & 11 & 30 & 192 \\
\hline & 146 & 51 & 14 & $18 \ldots$ & 60 & 14 & 10 & 28. & 14 & 30 & 37 \\
\hline 9. & 128 & 37 & 14 & - 19 & 40 & 13 & 9.3 & 29. & 170 & 24 & 30 \\
\hline 10. & 33 & 26 & 14 & $20 \ldots$ & 25 & 13 & 8.6 & $30_{n}$ & 350 & 21 & 27 \\
\hline & & & & & & & & 31 & 270 & $----n$ & 24 \\
\hline \multicolumn{9}{|c|}{ Monthly mean discharge, in cubic feet per second } & 724 & 57.7 & 27.7 \\
\hline \multicolumn{9}{|c|}{ Runoft, in inches } & 1.36 & 1.05 & 0.52 \\
\hline \multicolumn{9}{|c|}{ Runoff, in acre-feet } & 4,450 & 3,430 & 1,700 \\
\hline
\end{tabular}


Gage height, in feet, and discharge, in cubic feet per second, at indicated timt, 1965, of Bear Creek near Monmouth, Iowa

\begin{tabular}{|c|c|c|c|c|c|c|c|c|c|c|c|}
\hline Date & Hour & $\begin{array}{c}\text { Gage } \\
\text { height }\end{array}$ & $\begin{array}{c}\text { Dis- } \\
\text { charge }\end{array}$ & Date & Hour & $\begin{array}{l}\text { Gage } \\
\text { height }\end{array}$ & $\begin{array}{c}\text { Dis- } \\
\text { charge }\end{array}$ & Date & Hour & $\begin{array}{c}\text { Gage } \\
\text { height }\end{array}$ & $\begin{array}{c}\text { Dis- } \\
\text { charge }\end{array}$ \\
\hline 30 & $\begin{array}{l}0000 \\
1100 \\
1200 \\
1400 \\
1600 \\
1800 \\
1900 \\
2000 \\
2100 \\
2300 \\
2400 \\
0400 \\
1200 \\
1500 \\
1900 \\
2100 \\
2130 \\
2300 \\
2400 \\
0600 \\
0930 \\
1230 \\
1700 \\
1930 \\
2130 \\
2400\end{array}$ & $\begin{array}{l}5.31 \\
5.39 \\
5.44 \\
5.69 \\
6.37 \\
7.06 \\
7.64 \\
8.86 \\
7.69 \\
7.90 \\
7.87 \\
7.01 \\
6.11 \\
6.47 \\
7.29 \\
8.09 \\
8.42 \\
8.06 \\
8.08 \\
6.71 \\
6.12 \\
6.05 \\
6.53 \\
6.67 \\
7.02 \\
7.10\end{array}$ & 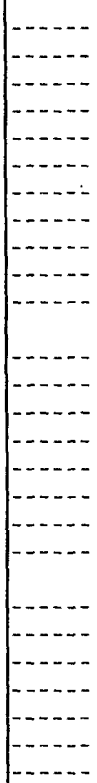 & 列 & $\begin{array}{l}0000 \\
0700 \\
0800 \\
0900 \\
1100 \\
1300 \\
1500 \\
1930 \\
2100 \\
2200 \\
2400 \\
0100 \\
0300 \\
0700 \\
0900 \\
1000 \\
1100 \\
1200 \\
1400 \\
1600 \\
1700 \\
1900 \\
2400 \\
2400 \\
1630\end{array}$ & $\begin{array}{l}5.86 \\
5.79 \\
6.23 \\
6.31 \\
6.92 \\
6.84 \\
7.19 \\
7.22 \\
7.00 \\
6.99 \\
7.04 \\
7.06 \\
7.07 \\
7.12 \\
7.13 \\
7.12 \\
6.79 \\
6.55 \\
6.29 \\
6.12 \\
6.08 \\
6.05 \\
5.99\end{array}$ & $\begin{array}{r}88 \\
75 \\
164 \\
182 \\
351 \\
327 \\
439 \\
450 \\
375 \\
372 \\
387 \\
\\
394 \\
397 \\
414 \\
418 \\
414 \\
312 \\
244 \\
178 \\
140 \\
132 \\
126 \\
114 \\
\\
11 \\
10\end{array}$ & May 25 & $\begin{array}{l}1830 \\
1900 \\
1930 \\
2000 \\
2030 \\
2100 \\
2130 \\
2200 \\
2400 \\
0100 \\
02.30 \\
0600 \\
1200 \\
1800 \\
2130 \\
2400 \\
0230 \\
03 ? 0 \\
0330 \\
06 ? 0 \\
09 ? 0 \\
1490 \\
2490\end{array}$ & $\begin{array}{l}5.40 \\
5.38 \\
6.15 \\
7.40 \\
8.02 \\
8.20 \\
8.23 \\
8.11 \\
7.05 \\
6.52 \\
6.05 \\
5.67 \\
5.56 \\
5.65 \\
5.90 \\
6.75 \\
7.48 \\
7.50 \\
7.47 \\
6.55 \\
6.17 \\
5.88 \\
5.65\end{array}$ & $\begin{array}{r}21 \\
20 \\
148 \\
516 \\
752 \\
832 \\
846 \\
792 \\
394 \\
\\
239 \\
128 \\
57 \\
41 \\
54 \\
98 \\
303 \\
\\
548 \\
554 \\
544 \\
250 \\
155 \\
96 \\
55\end{array}$ \\
\hline
\end{tabular}

(216) 5-4185. Maquoketa River near Maquoketa, Iowe

Location.-Lat $42^{\circ} 05^{\prime} 05^{\prime \prime}$, long $90^{\circ} 37^{\prime} 55^{\prime \prime}$, in SW $\frac{1}{4} \mathrm{NE} \frac{1}{4}$ sec. 17, T. 84 N., R.3 E., on right bank $500 \mathrm{ft}$ upstream from bridge on State Highway 62, 1,200 ft upstream from Prairie Creek, 2.0 miles northeast of Maquoketa, and 2.2 miles downstream from North Fork, and 28 miles upstream from mouth.

Drainage area. $-1,553 \mathrm{sq} \mathrm{mi}$.

Gage-height record.-Water-stage recorder graph, except May 12-24, where graph was reconstructed with the use of wire-weight readings and recorder record. Datum of gage is $636.52 \mathrm{ft}$ above mean sea level, adjustment of 1912 .

Discharge record. - Stage-discharge relation defined by current-meter measurements below 45,000 cfs. Backwater from ice Mar. 1-4, 18-29.

Maxima.-March-May 1965: Discharge, 9,880 cfs $1100 \mathrm{hrs} \mathrm{Apr.} 1$ (gage height, $12.45 \mathrm{ft}$ ). 1913 to February 1965: Discharge, 48,000 cfs June 27, 1944 (gage height, 24.70 ft). 
Mean discharge, in cubic feet per second, 1965, of Maquoketa River near Maquoketa, lowa

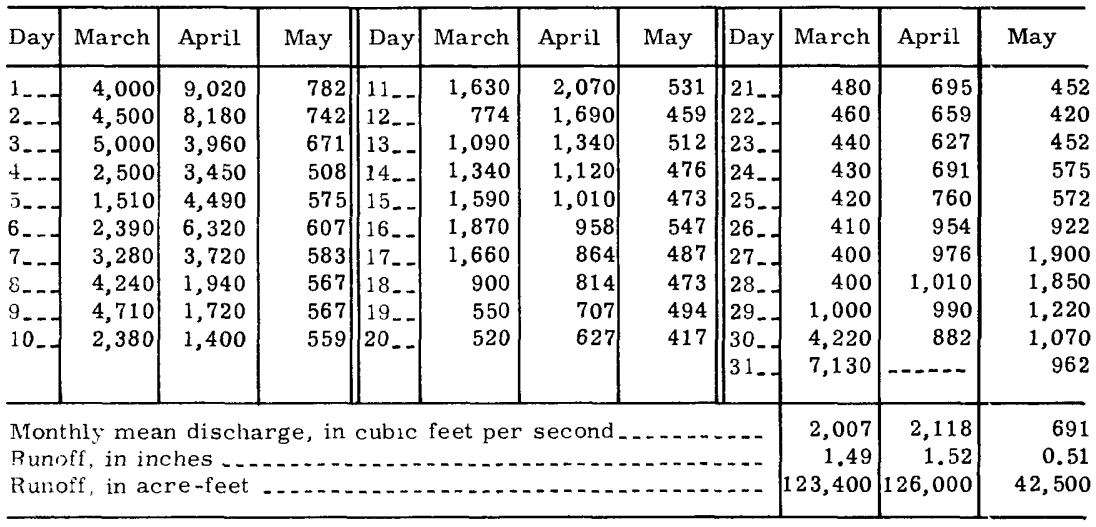

Gage height, in fiet, and discharge, in cubic feet per second, at indicated time, 1965

\begin{tabular}{|c|c|c|c|c|c|c|c|c|c|c|c|}
\hline Date & Hour & $\begin{array}{c}\text { Gage } \\
\text { height }\end{array}$ & $\begin{array}{c}\text { Dis - } \\
\text { charge }\end{array}$ & Date & Hour & $\begin{array}{c}\text { Gage } \\
\text { height }\end{array}$ & $\begin{array}{c}\text { Dis- } \\
\text { charge }\end{array}$ & Date & Hour & $\begin{array}{c}\text { Gage } \\
\text { height }\end{array}$ & $\begin{array}{c}\text { Dis- } \\
\text { charge }\end{array}$ \\
\hline \multirow[t]{8}{*}{ Apr. } & 0000 & 10.79 & 7,870 & \multirow[t]{8}{*}{ Apr. 2} & 1600 & 11.12 & 8,240 & \multirow[t]{8}{*}{ Apr. 3} & 1100 & 5.51 & 2,910 \\
\hline & 0400 & 11.73 & 8,980 & & 2400 & 10.16 & 7,180 & & 1200 & 5.91 & 3,190 \\
\hline & 0800 & 12.19 & 9,550 & & & & & & 1500 & 5.61 & 2,980 \\
\hline & 1100 & 12.45 & 9,880 & & 0200 & 9.62 & 6,580 & & 1800 & 5.51 & 2,910 \\
\hline & 2400 & 10.97 & 8,070 & & 0400 & 8.86 & 5,760 & & 2000 & 5.35 & 2,800 \\
\hline & & & & & 0800 & 7.44 & 4,400 & & 2200 & 5.51 & 2,910 \\
\hline & 0100 & 11.56 & 8,770 & & 0900 & 6.48 & 3,630 & & 2400 & 5.90 & 3,180 \\
\hline & 0900 & 11.18 & 8,320 & & 1000 & 5.18 & 2,680 & & & & \\
\hline
\end{tabular}

\section{APPLE RIVER BASIN}

(217) 5-4190. Apple River near Hanover, I11.

Location.-Lat $42^{\circ} 15^{\prime} 05^{\prime \prime}$, long $90^{\circ} 17^{\prime} 10^{\prime \prime}$, in $\mathrm{NE} \frac{1}{4} \mathrm{NW} \frac{1}{4}$ sec.16, T.26 N., R.2 E., on right bank 0.3 mile southwest of Hanover and 12 miles upstream from mouth.

Drainage area. $-244 \mathrm{sq} \mathrm{mi}$.

Gage-height record.-Digital recorder tape punched at 15-minute intervals. P-ior to Apr. 7,1965 , water-stage recorder graph. Datum of gage is $591.00 \mathrm{ft}$ above mean sea level, adjustment of 1912 .

Discharge record.-Stage-discharge relation defined by current-meter measurements. Discharge for period of backwater from Mississippi River, Apr, 17 to May 10, estimated on basis of two discharge measurements and records for nearby stations. Mean daily discharge computed from 96 punch-tape recordings per day begirning Apr. 7, 1965.

Maxima.-April-May 1965: Discharge, 4, 240 cfs 0400 hours Apr. 1 (gage height, $14.22 \mathrm{ft}$ ).

1934 to March 1965: Discharge, 12,000 cfs Jan. 5, 1946; gage height, $26.12 \mathrm{ft}$ Jan. 5, 1946 (ice jam). 
Mean dischurge, in cubic feet per second. 1965, of Apple River near Hanover. Ill

\begin{tabular}{|c|c|c|c|c|c|c|c|c|}
\hline Day & April & May & Day & April & May & Day & Aoril & May \\
\hline $\begin{array}{l}1 \\
2 \\
3 \\
4 \\
4 \\
5 \\
6 \\
7\end{array}$ & $\begin{array}{r}2,650 \\
817 \\
330 \\
521 \\
919 \\
1,100 \\
240 \\
174 \\
167 \\
130\end{array}$ & $\begin{array}{r}80 \\
78 \\
76 \\
76 \\
90 \\
100 \\
80 \\
90 \\
85 \\
80\end{array}$ & $\begin{array}{l}11 \ldots \\
12 \ldots \\
13 \ldots \\
14 \ldots \\
15 \ldots \\
16 \ldots \\
17 \ldots \\
18 \ldots \\
19 \ldots \\
20 \ldots\end{array}$ & $\begin{array}{r}585 \\
432 \\
170 \\
138 \\
150 \\
143 \\
120 \\
110 \\
100 \\
90\end{array}$ & $\begin{array}{l}66 \\
58 \\
56 \\
53 \\
52 \\
59 \\
60 \\
60 \\
59 \\
52\end{array}$ & $\begin{array}{l}21 \ldots \ldots \\
22 \ldots \ldots \\
23 \ldots \ldots \\
24 \ldots \ldots \\
25 \ldots \ldots \\
26 \ldots \ldots \\
26 \ldots \\
28 \ldots \ldots \\
29 \ldots \ldots \\
30 \ldots \ldots \\
31 \ldots \ldots\end{array}$ & $\begin{array}{r}85 \\
80 \\
75 \\
80 \\
140 \\
150 \\
120 \\
100 \\
90 \\
85\end{array}$ & $\begin{array}{l}47 \\
50 \\
59 \\
59 \\
53 \\
55 \\
77 \\
71 \\
55 \\
52 \\
50\end{array}$ \\
\hline \multicolumn{7}{|c|}{$\begin{array}{l}\text { Monthly mean discharge, in cubic feet per second } \\
\text { Runoff, in inches }\end{array}$} & $\begin{array}{r}336 \\
1.54\end{array}$ & $\begin{array}{l}65.7 \\
0.31\end{array}$ \\
\hline
\end{tabular}

Gage height, in feet, and discharge, in cubic feet per second, at indicated time, 1965

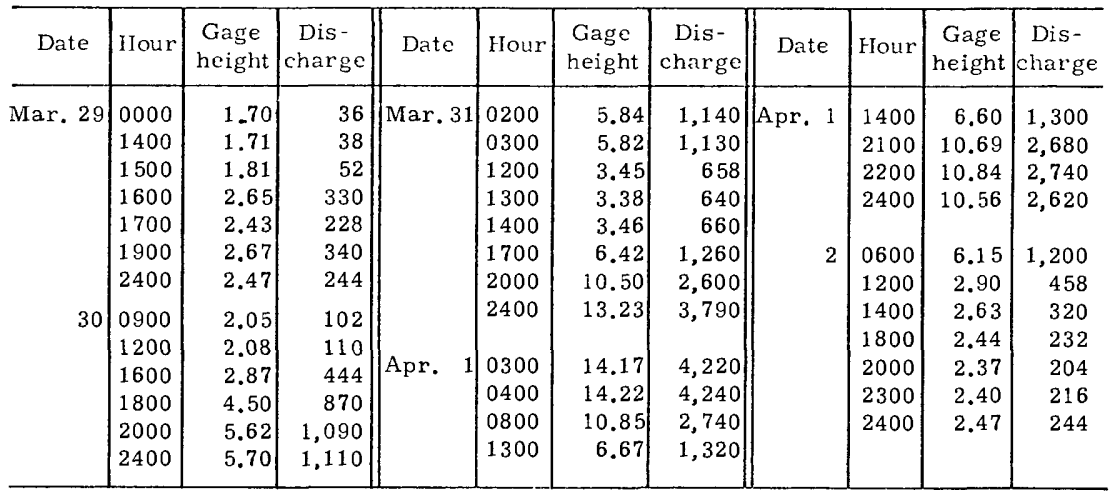




\section{PLUM RIVER BASIN}

(218) 5-4200. Plum River below Carroll Creek, near Savanna, Ill.

Location.-Lat $42^{\circ} 06^{\prime} 50^{\prime \prime}$, long $90^{\circ} 05^{\prime} 35^{\prime \prime}$, in NW $\frac{1}{4} \mathrm{SW} \frac{1}{4}$ sec. 31 , T.25 N., R.4 E., on left bank 0.7 mile upstream from Camp Creek, 2.6 miles downstream from Carroll Creek, 3.5 miles northeast of Savanna, and 13 miles upstream from mouth.

Drainage area. $-231 \mathrm{sq} \mathrm{mi}$.

Gage-height record.-Digital recorder tape punched at 15-minute intervals. Prior to May 11, 1965, water-stage recorder graph. Datum of gage is $580.00 \mathrm{ft}$ abore mean sea level, adjustment of 1912 .

Discharge record.-Stage-discharge relation defined by current-meter measurements. Discharge for period of backwater from Mississippi River Apr. 18 to May 10 estimated on basis of two discharge measurements and records for nearby stations. Mean daily discharge computed from 96 punch-tape recordings per day beginning May 11, 1965 .

Maxima.-April-May 1965: Discharge, 1,740 cfs 1330 hours Apr. 1 (gage height, $22.11 \mathrm{ft}$ ).

1940 to March 1965: Discharge, 11,600 cfs Jan. 6, 1946 (gage height, $28.74 \mathrm{ft}$ ).

Mcan discharge, in cubic feet per second, 1965

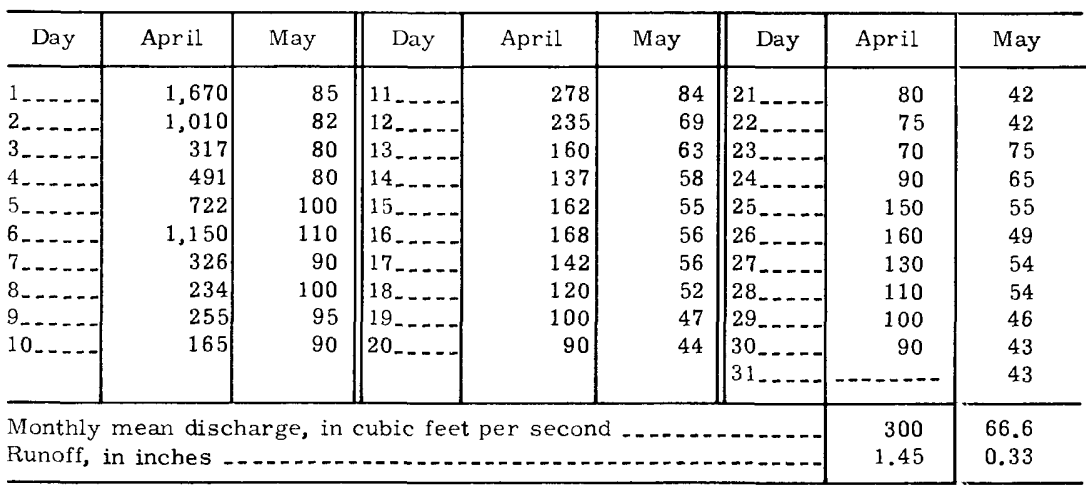

Gage height, in feet, and discharge, in cubic feet per second, at indicated time, 1965

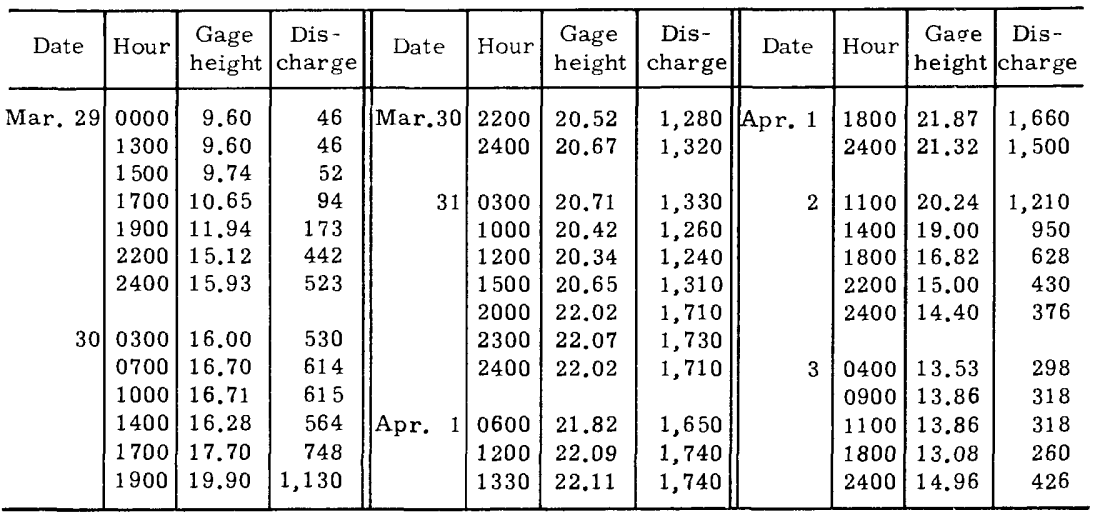




\section{MISSISSIPPI RIVER MAIN STEM}

(219) 5-4205. Mississippi River at Clinton, Iowa

Location.-Lat $41^{\circ} 46^{\prime} 50^{\prime \prime}$, long $90^{\circ} 15^{\prime} 07^{\prime \prime}$, in NW $\frac{1}{4}$ sec.34, T.81 N., R.6 E., on right bank at foot of Eighth Avenue in Camanche, 5.0 miles upstream from Wapsioinicon River, 6.4 miles downstream from Clinton, 10.6 miles downstream from Dam 13 , and at mile 511.8 upstream from Ohio River.

Drainage area.-85,600 sq mi, approximately, at Fulton-Lyons Bridge (formerly U.S. Highway 30) where discharge measurements are made.

Gage-height record.-Water-stage recorder graph. Datum of gage is $562.68 \mathrm{ft}$ above mean sea level, datum of 1929.

Discharge record.-Stage-discharge relation defined by current-meter measurements below 307,000 cfs. Backwater from ice Mar, 1-6, 9-27.

Maxima.-March-May 1965: Daily discharge, 307,000 cfs Apr. 28; gage height, $24.65 \mathrm{ft}$ 0600 hrs Apr. 28.

1873 to February 1965: Daily discharge, 250,000 cfs June 25, 1880; gage height, $14.5 \mathrm{ft}$ June 25,1880 , at site 14.8 miles downstream at Le Claire, at datum $0.07 \mathrm{ft}$ lower.

Maximum stage known since at least 1828 that of Apr. 28, 1965.

Cooperation--Two discharge measurements furnished by Corps of Engineers.

Mean gage height, in feet, and discharge, in cubic feet per second, 1965

\begin{tabular}{|c|c|c|c|c|c|c|}
\hline \multirow{2}{*}{ Day } & \multicolumn{2}{|c|}{ March } & \multicolumn{2}{|c|}{ April } & \multicolumn{2}{|c|}{ May } \\
\hline & Gage height & Discharge & Gage height & Discharge & Gage height & Discharge \\
\hline $1 \ldots \ldots \ldots$ & 9.30 & 28,000 & 11.22 & 60,800 & 23.90 & 284,000 \\
\hline $2 \ldots \ldots-n$ & 9.93 & 40,000 & 11.95 & 71,600 & 23.53 & 275,000 \\
\hline $3 \ldots-\ldots$ & 10.36 & 50,000 & 11.99 & 72,900 & 23.10 & 264,000 \\
\hline $4 \ldots \ldots$ & 11.04 & 56,000 & 11.68 & 69,300 & 22.66 & 252,000 \\
\hline $5 \ldots-\ldots-$ & 11.18 & 60,000 & 11.93 & 74,300 & 22.13 & 240,000 \\
\hline $6 \ldots \ldots$ & 10.96 & 58,000 & 13.00 & 88,800 & 21.57 & 228,000 \\
\hline $7 \ldots \ldots$ & 10.97 & 62,800 & 13.33 & 91,700 & 20.93 & 216,000 \\
\hline $8 \ldots \ldots \ldots$ & 11.65 & 73,200 & 13.41 & 91,900 & 20.31 & 205,000 \\
\hline $9 \ldots \ldots$ & 12.68 & 82,000 & 13.51 & 93,400 & 19.63 & 192,000 \\
\hline $10 \ldots \ldots$ & 12.96 & 84,000 & 13.83 & 95,900 & 18.92 & 180,000 \\
\hline $11 \ldots \ldots$ & 12.66 & 75,000 & 14.67 & 112,000 & 18.21 & 170,000 \\
\hline $12 \ldots \ldots$ & 12.07 & 70,000 & 15.22 & 125,000 & 17.52 & 157,000 \\
\hline $13 \ldots \ldots$ & 11.87 & 66,000 & 15.81 & 134,000 & 16.83 & 147,000 \\
\hline $14 \ldots \ldots$ & 11.67 & 62,000 & 16.59 & 143,000 & 16.12 & 135,000 \\
\hline $15 \ldots \ldots$ & 11.26 & 56,000 & 17.13 & 155,000 & 15.54 & 126,000 \\
\hline $16 \ldots$ & 11.11 & 51,000 & 17.61 & 162,000 & 15.20 & 121,000 \\
\hline $17 \ldots$ & 10.69 & 47,000 & 17.97 & 169,000 & 14.92 & 117,000 \\
\hline $18 \ldots$ & 10.85 & 43,000 & 18.37 & 176,000 & 14.61 & 112,000 \\
\hline $19 \ldots$ & 10.30 & 30,500 & 18.89 & 186,000 & 14.47 & 111,000 \\
\hline $20 \ldots \ldots$ & 10.44 & 33,000 & 19.47 & 195,000 & 14.42 & 110,000 \\
\hline $21 \ldots \ldots$ & 10.70 & 36,000 & 20.18 & 209,000 & 14.41 & 110,000 \\
\hline $22 \ldots \ldots \ldots$ & 10.58 & 34,000 & 21.10 & 226,000 & 14.45 & 110,000 \\
\hline $23 \ldots \ldots$ & 10.32 & 32,000 & 22.03 & 245,000 & 14.47 & 111,000 \\
\hline $24 \ldots \ldots \ldots$ & 10.15 & 31,000 & 22.82 & 263,000 & 14.51 & 112,000 \\
\hline $25 \ldots \ldots$ & 10.12 & 30,000 & 23.63 & 280,000 & 14.59 & 113,000 \\
\hline $26 \ldots \ldots$ & 10.16 & 31,000 & 24.24 & 300,000 & 14.75 & 116,000 \\
\hline $27 \ldots \ldots$ & 10.14 & 32,000 & 24.55 & 305,000 & 14.94 & 118,000 \\
\hline $28 \ldots \ldots$ & 9.98 & 32,900 & 24.63 & 307,000 & 14.96 & 118,000 \\
\hline $29 \ldots \ldots$ & 9.76 & 34,400 & 24.49 & 300,000 & 14.90 & 116,000 \\
\hline $30 \ldots \ldots$ & 10.01 & 40,800 & 24.26 & 294,000 & 14.63 & 112,000 \\
\hline $31 \ldots \ldots$ & 10.47 & 48,200 & $-\ldots \ldots \ldots$ & $\ldots \ldots$ & 14.30 & 107,000 \\
\hline \multicolumn{2}{|c|}{ Mean } & 48,700 & & 169,900 & \multicolumn{2}{|r|}{157,600} \\
\hline \multirow{2}{*}{\multicolumn{2}{|c|}{$\begin{array}{l}\text { Inches } \\
\text { Acre-feet }\end{array}$}} & 0.66 & & 2.21 & \multirow{2}{*}{\multicolumn{2}{|c|}{$\begin{array}{r}2.12 \\
9,689,000\end{array}$}} \\
\hline & & $2,995,000$ & & $10,110,000$ & & \\
\hline
\end{tabular}




\section{WAPSIPINICON RIVER BASIN}

(220) 5-4205.6 Wapsipinicon River near Elma, Iowa

Location.-Lat $43^{\circ} 14^{\prime} 35^{\prime \prime}$, long $92^{\circ} 31^{\prime} 50^{\prime \prime}$, in NW $\frac{1}{4} \mathrm{NW} \frac{1}{4}$ sec.8, T.97 N., R.14 W., on right bank $10 \mathrm{ft}$ downstream from county highway bridge, 0.2 mile downstream from unnamed creek, and 4.9 miles west of Elma.

Drainage area. $-95.2 \mathrm{sq} \mathrm{mi}$.

Gage-height record.-Water-stage recorder graph except March 3, 4, 9, 10, 21-25, May 8-20. Datum of gage is $1,131.46 \mathrm{ft}$ above mean sea level, datum of 1929 .

Discharge record.--Stage-discharge relation defined by current-meter measurements below 4,190 cfs, extended to $5,500 \mathrm{cfs}$ by logarithmic plotting. Backwater from ice Mar. 1 to Apr. 7.

Maxima.-March-May 1965: Discharge, 2,430 cfs 1600 hours Apr. 8 (gage h?ight, $13.19 \mathrm{ft}$; gage height, $14.08 \mathrm{ft} 1800$ hours Mar. 1, backwater from ice.

1958 to February 1965: Discharge, about 5,700 cfs Mar. 29, 1962 (gage height, $14.84 \mathrm{ft}$, backwate $\mathrm{r}$ from ice).

Mean discharge, in cubic feet per second, 1965

\begin{tabular}{|c|c|c|c|c|c|c|c|c|c|c|c|}
\hline Day & March & April & May & Day & March & April & May & Day & March & April & May \\
\hline 1 . & 600 & 370 & 24 & $11 \ldots$ & 19 & 857 & 21 & 21. & 9.2 & 23 & 16 \\
\hline 2. & 560 & 410 & 22 & $12 \ldots$ & 18 & 1,220 & 19 & 22 & 8.4 & 23 & 15 \\
\hline 3. & 300 & 540 & 20 & $13 \ldots$ & 17 & 385 & 18 & $23 \ldots$ & 7.8 & 27 & 15 \\
\hline 4 & 130 & 920 & 18 & $14 \ldots$ & 16 & 100 & 17 & 24 & 7.2 & 31 & 15 \\
\hline 5 & 68 & 1,700 & 18 & $15 \ldots$ & 15 & 82 & 20 & $25 \ldots$ & 7.0 & 73 & 17 \\
\hline $6 \ldots$ & 43 & 1,980 & 108 & $16_{-}$ & 15 & 62 & 32 & $26 \ldots$ & 6.8 & 105 & 49 \\
\hline 72 & 34 & 1,760 & 164 & $17 .-$ & 14 & 50 & 37 & 27. & 6.6 & $6 ?$ & 41 \\
\hline 8 & 28 & 1,640 & 74 & $18 \ldots$ & 12 & 42 & 30 & 28 & 6.4 & 41 & 29 \\
\hline 9. & 24 & 1,480 & 34 & $19 \ldots$ & 11 & 36 & 22 & $29=$ & 6.6 & 32 & 23 \\
\hline 10 & 21 & 686 & 26 & $20 \ldots$ & 10 & 30 & 18 & 30. & 8.0 & 27 & 20 \\
\hline & & & & & & & & & 150 & & 18 \\
\hline \multicolumn{9}{|c|}{ Monthly mean discharge, in cubic feet per second } & 70.3 & 493 & 32.3 \\
\hline \multicolumn{9}{|c|}{ Runoff, in inches } & 0.85 & $5.7 ?$ & 0.39 \\
\hline \multicolumn{9}{|c|}{ Runoif, in acre-feet } & 4,320 & 29,36 ? & 1,980 \\
\hline
\end{tabular}


Gage height, in feet, and discharge, in cubic feet per second, at indicated time, 1965, of Wapipinicon River

\begin{tabular}{|c|c|c|c|c|c|c|c|c|c|c|c|}
\hline Date & Hour & $\begin{array}{c}\text { Gage } \\
\text { height }\end{array}$ & $\begin{array}{c}\text { Dis- } \\
\text { charge }\end{array}$ & Date & Hour & $\begin{array}{c}\text { Gage } \\
\text { height }\end{array}$ & $\begin{array}{c}\text { Dis- } \\
\text { charge }\end{array}$ & Date & Hour & $\begin{array}{l}\text { Gage } \\
\text { height }\end{array}$ & $\begin{array}{c}\text { Dis- } \\
\text { charge }\end{array}$ \\
\hline \multirow[t]{7}{*}{ Feb. 28} & 0000 & 6.55 & --- & Apr. 8 & 1300 & 12.84 & 1,800 & Apr. 11 & 1700 & 12.23 & 1,230 \\
\hline & 1100 & 6.63 & $-\cdots$ & & 1400 & 13.02 & 2,120 & & 1900 & 12.54 & 1,460 \\
\hline & 1400 & 7.42 & $\cdots$ & & 1600 & 13.19 & 2,430 & & 2400 & 12.18 & 1,200 \\
\hline & 1600 & 8.90 & $-\cdots$ & & 2100 & 12.87 & 1,850 & & & & \\
\hline & 1800 & 9.97 & $\ldots-n$ & & 2400 & 12.64 & 1,550 & 12 & 0400 & 12.00 & 1,100 \\
\hline & 2400 & 11.63 & $\ldots \ldots$ & & & & & & 0600 & 11.89 & 1,040 \\
\hline & & & & 9 & 0400 & 12.63 & 1,540 & & 1000 & 12.07 & 1,140 \\
\hline \multirow[t]{4}{*}{ Mar. 1} & 0600 & 12.92 & $-\cdots$ & & 0800 & 12.78 & 1,710 & & 1400 & 12.45 & 1,380 \\
\hline & 1800 & 14.08 & $\ldots-$. & & 1000 & 12.89 & 1,880 & & 1600 & 12.58 & 1,460 \\
\hline & 2400 & 13.87 & $-\cdots$ & & 1200 & 12.70 & 1,610 & & 2200 & 12.19 & 1,200 \\
\hline & & & & & 1600 & 12.57 & 1,480 & & 2400 & 11.92 & 1,060 \\
\hline \multirow[t]{4}{*}{2} & 0600 & 13.54 &.- & & 1800 & 12.33 & 1,290 & & & & \\
\hline & 1200 & 13.18 & $-\cdots$ & & 2200 & 12.00 & 1,100 & 13 & 0200 & 11.57 & 910 \\
\hline & 2400 & 12.65 & $-\cdots$ & & 2400 & 11.76 & 984 & & 0400 & 11.05 & 752 \\
\hline & & & & & & & & & 0500 & 10.69 & 678 \\
\hline \multirow[t]{6}{*}{ Apr. 6} & 0000 & 13.43 & & 10 & 0400 & 11.27 & 811 & & 0600 & 10.28 & 596 \\
\hline & 0400 & 13.36 & & & 0800 & 10.74 & 688 & & 0700 & 9.83 & 514 \\
\hline & 1300 & 13.66 & $---n$ & & 1400 & 10.24 & 588 & & 0800 & 9.32 & 438 \\
\hline & 1600 & 13.63 & $\ldots$ & & 1800 & 10.27 & 594 & & 0900 & 8.83 & 369 \\
\hline & 2400 & 13.31 & - & & 2400 & 10.46 & 632 & & 1000 & 8.48 & 328 \\
\hline & & & & & & & & & 1100 & 7.93 & 269 \\
\hline \multirow[t]{2}{*}{7} & 2400 & 12.39 & 1,330 & 11 & 1200 & 10.37 & 614 & & 1300 & 7.33 & 219 \\
\hline & & & & & 1300 & 10.38 & 616 & & 1600 & 6.71 & 166 \\
\hline \multirow[t]{3}{*}{8} & 0600 & 12.19 & 1,200 & & 1500 & 10.93 & 726 & & 2000 & 6.32 & 136 \\
\hline & 1100 & 12.43 & 1,360 & & 1600 & 11.29 & 817 & & 2400 & 6.07 & 122 \\
\hline & 1200 & 12.64 & 1,550 & & & & & & & & \\
\hline
\end{tabular}

(221) 5-4206. Little Wapsipinicon River tributary near Riceville, Iowa

(Crest-stage station)

Location.-Near south quarter-corner of sec.27, T.99 N., R.14 W., at culvert 3.5 miles east of Riceville and 0.4 mile south of State Highway 9.

Drainage area. $-0.90 \mathrm{sq} \mathrm{mi}$.

Gage-height record.-Crest stages only.

Discharge record.-Stage-discharge relation defined by current-meter measurements below $117 \mathrm{cfs}$ and by flow-through-culvert measurement of $703 \mathrm{cfs}$.

Maxima.-March-May 1965: Discharge, about $230 \mathrm{cfs}$ Apr. 8 (gage height, $4.55 \mathrm{ft}$, backwater from ice).

1953 to February 1965: Discharge, 703 cfs Aug. 31, 1962 (gage heigrt, $5.03 \mathrm{ft}$ ). 
(222) 5-4206.2 Little Wapsipinicon River near Acme, Iowa

(Crest-stage station)

Location.-Sec.10, T.98 N., R.14 W., at bridge on County Road D, 1 mile north of Acme.

Drainage area. $-7.76 \mathrm{sq} \mathrm{mi}$.

Gage-height record.-Crest stages only.

Discharge record. - Stage-discharge relation defined by current-meter measurements below $861 \mathrm{cfs}$ and by contracted-opening measurement at $2,380 \mathrm{cfs}$.

Maxima.-March-May 1965: Discharge, about $210 \mathrm{cfs}$ Mar. 1 (gage height, $6.8 \mathrm{ft}$, backwater from ice).

1953 to February 1965: Discharge, 2,380 cfs Aug. 31, 1962 (gage height, $9.02 \mathrm{ft}$ ).

(223) 5-4206.4 Little Wapsipinicon River at Elma, Iowa

(Crest-stage station)

Location.- -NW $\frac{1}{4}$ sec.12, T.97 N., R.14 W., at bridge, on County Road A near west city limits of Elma.

Drainage area. $-37.3 \mathrm{sq} \mathrm{mi}$.

Gage-height record.-Crest stages and occasional low-flow stages.

Discharge record.- - Stage-discharge relation defined by current-meter meas'drements and contracted opening measurement at $5,740 \mathrm{cfs}$.

Maxima.-March-May 1965: Discharge, about $450 \mathrm{cfs}$ Mar. 1 (gage height, $8.4 \mathrm{ft}$, backwater from ice).

1953 to February 1965: Discharge, 5,740 cfs Aug. 31, 1962 (gage height, $12.53 \mathrm{ft}$ ).

(224) 5-4210. Wapsipinicon River at Independence, Iowa

Location.-Lat $42^{\circ} 27^{\prime} 50^{\prime \prime}$, long $91^{\circ} 53^{\prime} 40^{\prime \prime}$, in $\mathrm{SE} \frac{1}{4}$ sec. $4, \mathrm{~T} .88$ N., R9 W., on right bank at Sixth Street in Independence, $1,800 \mathrm{ft}$ downstream from dam at abandoned hydroelectric plant, 4-3/4 miles downstream from Otter Creek, and $10 \frac{1}{4}$ miles u’stream from Pine Creek.

Drainage area.- $-1,048 \mathrm{sq} \mathrm{mi}$.

Gage-height record.-Water-stage recorder graph. Datum of gage is $882.85 \mathrm{ft}$ above mean sea level, datum of 1929.

Discharge record.-Stage-discharge relation defined by current-meter meas ırements below 20,600 cfs. Backwater from ice Mar. 20-30.

Maxima.-March-May 1965: Discharge, 8,880 cfs 1400 hours Apr. 7 (gage height, $12.19 \mathrm{ft})$.

1933 to February 1965: Discharge, 21,500 cfs J une 14, 1947 (gage height, $18.74 \mathrm{ft}$ ).

Maximum stage since at least 1901, that of June 14, 1947. 
Mean discharge, in cubic feet per second, 1965, of Wapsipinicon River at Independer ce, Iowa

\begin{tabular}{|c|c|c|c|c|c|c|c|c|c|c|c|}
\hline Day & March & April & May & Day & March & April & May & Day & March & April & May \\
\hline $\begin{array}{l}1 . \\
2 . \\
3 . \\
4 . \\
5 . \\
6 . \\
7 . \\
8 . \\
9 . \\
10\end{array}$ & $\begin{array}{l}2,380 \\
3,790 \\
2,780 \\
1,670 \\
3,920 \\
5,010 \\
4,240 \\
4,300 \\
3,030 \\
1,910\end{array}$ & $\begin{array}{l}4,400 \\
6,170 \\
3,840 \\
3,750 \\
5,160 \\
7,200 \\
8,540 \\
8,420 \\
7,390 \\
5,990\end{array}$ & $\begin{array}{l}735 \\
609 \\
532 \\
467 \\
435 \\
405 \\
360 \\
375 \\
360 \\
360\end{array}$ & 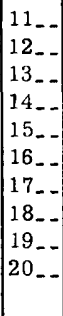 & $\begin{array}{r}1,430 \\
1,050 \\
789 \\
753 \\
807 \\
780 \\
600 \\
375 \\
284 \\
230\end{array}$ & $\begin{array}{r}5,230 \\
5,420 \\
4,170 \\
3,310 \\
3,370 \\
3,160 \\
2,380 \\
1,840 \\
1,220 \\
870\end{array}$ & $\begin{array}{l}382 \\
339 \\
290 \\
266 \\
272 \\
278 \\
236 \\
260 \\
311 \\
318\end{array}$ & $\mid \begin{array}{l}21 \\
22 \\
23 \\
24 \ldots \\
25 \ldots \\
26 \\
27 \\
28 \ldots \\
29 \\
30 \\
31\end{array}$ & $\begin{array}{l}200 \\
180 \\
170 \\
160 \\
150 \\
145 \\
140 \\
135 \\
130 \\
150 \\
723\end{array}$ & $\begin{array}{r}744 \\
654 \\
574 \\
558 \\
672 \\
870 \\
910 \\
900 \\
920 \\
861 \\
-\end{array}$ & $\begin{array}{r}290 \\
304 \\
412 \\
360 \\
325 \\
829 \\
1,190 \\
1,090 \\
1,140 \\
1,300 \\
1,570\end{array}$ \\
\hline \multicolumn{9}{|c|}{$\begin{array}{l}\text { Monthly mean discharge, in cubic feet per second } \\
\text { Runoff, in inches } \\
\text { Runoff, in acre-feet }\end{array}$} & $\begin{array}{r}1,368 \\
1,51 \\
84,120\end{array}$ & $\begin{array}{r}3,316 \\
3.53 \\
197,300\end{array}$ & $\begin{array}{r}529 \\
0.58 \\
32,530\end{array}$ \\
\hline
\end{tabular}

Gage height, in feet, and discharge, in cubic feet per second, at indicated time, 1965

\begin{tabular}{|c|c|c|c|c|c|c|c|c|c|c|c|}
\hline Date & Hour & $\begin{array}{c}\text { Gage } \\
\text { height }\end{array}$ & $\begin{array}{c}\text { Dis - } \\
\text { charge }\end{array}$ & Date & Hour & $\begin{array}{l}\text { Gage } \\
\text { height }\end{array}$ & $\begin{array}{c}\text { Dis- } \\
\text { charge }\end{array}$ & Date & Hour & $\begin{array}{c}\text { Gage } \\
\text { height }\end{array}$ & $\begin{array}{c}\text { Dis- } \\
\text { charge }\end{array}$ \\
\hline Apr. 4 & $\begin{array}{l}0000 \\
0600 \\
1200 \\
1800 \\
2400 \\
1200 \\
1800 \\
2400 \\
0600 \\
1200 \\
1800\end{array}$ & $\begin{array}{l}7.54 \\
7.68 \\
8.00 \\
8.31 \\
8.59 \\
\\
9.24 \\
9.45 \\
9.94 \\
10.48 \\
10.82 \\
11.53\end{array}$ & $\begin{array}{l}3,140 \\
3,330 \\
3,740 \\
4,120 \\
4,460 \\
5,200 \\
5,420 \\
5,950 \\
6,580 \\
6,990 \\
7,940\end{array}$ & Apr. 6 & $\begin{array}{l}2100 \\
2400 \\
0300 \\
0800 \\
1100 \\
1200 \\
1300 \\
1400 \\
1700 \\
1900 \\
2400\end{array}$ & $\begin{array}{l}11.83 \\
11.90 \\
11.87 \\
11.70 \\
11.72 \\
11.76 \\
12.14 \\
12.19 \\
12.12 \\
12.15 \\
12.09\end{array}$ & $\begin{array}{l}8,360 \\
8,460 \\
8,420 \\
8,180 \\
8,210 \\
8,260 \\
8,810 \\
8,880 \\
8,780 \\
8,820 \\
8,740\end{array}$ & Apr. 8 & $\begin{array}{l}0600 \\
1200 \\
2400 \\
0600 \\
1200 \\
1800 \\
2400 \\
1200 \\
2400\end{array}$ & $\begin{array}{r}12.05 \\
11.86 \\
11.59 \\
11.41 \\
11.14 \\
10.86 \\
10.56 \\
9.93 \\
9.44\end{array}$ & $\begin{array}{l}8,680 \\
8,400 \\
8,030 \\
7,770 \\
7,400 \\
7,040 \\
6,680 \\
5,940 \\
5,410\end{array}$ \\
\hline
\end{tabular}

(225) 5-4220. Wapsipinicon River near De Witt, Iowa

Location.-Lat $41^{\circ} 45^{\prime} 55^{\prime \prime}$, long $90^{\circ} 32^{\prime} 00^{\prime \prime}$, in SW $\frac{1}{4} N E \frac{1}{4}$ sec.6, T.80 N., R.4 E., on left bank $15 \mathrm{ft}$ downstream from bridge on U.S. Highway 61,3 miles south of De Witt, 6.2 miles upstream from Brophy Creek, and 18.2 miles upstream from mouth.

Drainage area.-2,330 sq $\mathrm{mi}$ (includes that of Silver Creek).

Gage-height record.-Water-stage recorder graph except Mar. 20-24. D-tum of gage is $598.81 \mathrm{ft}$ above mean sea level, datum of 1929 .

Discharge record. - Stage-discharge relation defined by current-meter measurements below $25,000 \mathrm{cfs}$. Backwater from ice Mar. 1-19.

Maxima.-March-May 1965: Discharge, 10,200 cfs 2230 hours Apr. 14 (gage height, $11.05 \mathrm{ft})$.

1934 to February 1965: Discharge, 26,000 cfs June 27, 1944 (gage height, $12.07 \mathrm{ft}$ ).

Cooperation.-Two discharge measurements furnished by Corps of Engineers. 
Mean discharge, in cubic feet per second, 1965, of Wapsipinicon River near De Witt, Iown

\begin{tabular}{|c|c|c|c|c|c|c|c|c|c|c|c|}
\hline Day & March & April & May & Day & March & April & May & Day & March & April & May \\
\hline $\begin{array}{l}1 \\
2 \\
2 \\
3 \ldots \\
4 \ldots- \\
5 \\
6 \ldots- \\
6 \ldots- \\
7 \ldots- \\
8 \ldots- \\
9 \ldots- \\
10\end{array}$ & $\begin{array}{l}1,400 \\
1,500 \\
1,600 \\
2,000 \\
2,400 \\
2,700 \\
3,100 \\
3,500 \\
4,000 \\
4,500\end{array}$ & $\begin{array}{l}4,170 \\
3,470 \\
3,590 \\
4,430 \\
5,470 \\
7,480 \\
8,750 \\
8,900 \\
8,650 \\
8,160\end{array}$ & $\begin{array}{l}2,010 \\
1,850 \\
1,740 \\
1,590 \\
1,560 \\
1,400 \\
1,320 \\
1,290 \\
1,480 \\
1,340\end{array}$ & 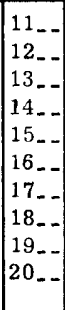 & $\begin{array}{l}5,000 \\
5,600 \\
6,400 \\
6,800 \\
6,000 \\
4,800 \\
5,200 \\
3,500 \\
1,900 \\
1,600\end{array}$ & $\begin{array}{l}8,400 \\
9,200 \\
9,800 \\
9,980 \\
9,700 \\
8,690 \\
7,640 \\
6,420 \\
4,990 \\
4,320\end{array}$ & \begin{tabular}{r|}
1,210 \\
1,110 \\
1,040 \\
979 \\
946 \\
891 \\
918 \\
852 \\
798 \\
750
\end{tabular} & $\begin{array}{l}21- \\
22- \\
23- \\
24_{-} \\
25_{-} \\
26 \ldots \\
22_{-} \\
28_{-} \\
29_{-} \\
30_{-} \\
31_{-}\end{array}$ & $\begin{array}{l}1,400 \\
1,300 \\
1,250 \\
1,200 \\
1,160 \\
1,080 \\
1,050 \\
1,020 \\
1,700 \\
4,480 \\
4,480\end{array}$ & \begin{tabular}{|r|}
3,437 \\
2,687 \\
2,210 \\
2,277 \\
2,877 \\
2,707 \\
2,410 \\
2,607 \\
2,557 \\
2,237 \\
.---
\end{tabular} & $\begin{array}{r}705 \\
680 \\
814 \\
962 \\
858 \\
918 \\
1,280 \\
1,760 \\
1,890 \\
2,050 \\
1,910\end{array}$ \\
\hline \multicolumn{9}{|c|}{$\begin{array}{l}\text { Monthly mean discharge, in cubic feet per second } \\
\text { Runoff, in inches } \\
\text { Runoff, in acre-feet }\end{array}$} & $\begin{array}{r}3,020 \\
1.49 \\
185,700\end{array}$ & $\begin{array}{r}5,607 \\
2.67 \\
333,50^{-}\end{array}$ & $\begin{array}{r}1,255 \\
0.62 \\
77,160\end{array}$ \\
\hline
\end{tabular}

Gage height, in feet, and discharge, in cubic feet per second, at indicated time, 1965

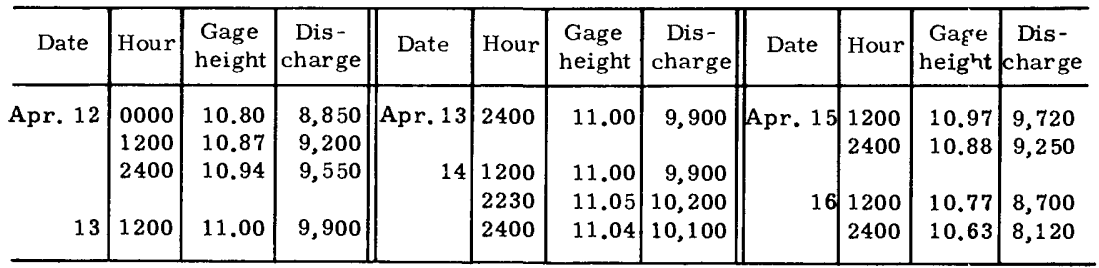

\section{MISSISSIPPI RIVER MAIN STEM}

(226) Mississippi River at Davenport, Iowa

(Miscellane ous site)

Location.-Lat $41^{\circ} 31^{\prime} 10^{\prime \prime}$, long $90^{\circ} 33^{\prime} 55^{\prime \prime}$, in center wall of Lock 15 about $15 \mathrm{ft}$ upstream from pivot pier of Government bridge at Davenport, $500 \mathrm{ft}$ upstream from $\Gamma \mathrm{zm} 15$, and at mile $\mathbf{4 8 2 . 9}$ upstream from Ohio River.

Drainage area. $-88,500 \mathrm{sq} \mathrm{mi}$, approximately.

Gage-height record.-Peak stages only from water-stage recorder and staff gage readings. Datum of gage is $542.50 \mathrm{ft}$ above mean sea level, adjustment of 1912 , and prior to Sept. 2, 1864, at datum $0.6 \mathrm{ft}$ higher. Prior to Sept. 6, 1872, several staff gages at old bridge site 2,300 ft upstream, and Sept. 7, 1872, to Feb. 8, 1933, staff gage at site $15 \mathrm{ft}$ downstream on pier of Government bridge.

Maxima.-March-May 1965: Gage height, $22.48 \mathrm{ft}$ Apr. 28.

1860 to February 1965: Gage height, $19.4 \mathrm{ft}$ J une 27, 1892.

Remarks.-Stage records furnished by Corps of Engineers. 
Annual maximum gage heights, for indicated y'ears, of Mississippi River at Dal'enport, Iowa

\begin{tabular}{|c|c|c|c|c|c|}
\hline Year & Date & $\begin{array}{l}\text { Gage } \\
\text { height } \\
\text { (feet) }\end{array}$ & Year & Date & $\begin{array}{c}\text { Gage } \\
\text { height } \\
\text { (feet) }\end{array}$ \\
\hline $1860 \ldots$ & June 21 & 12.6 & $1890 \ldots$ & July 1 & 11.9 \\
\hline $1861 \ldots$ & Apr. $27-29$ & 14.2 & $1891 \ldots$ & May 3-5 & 10.3 \\
\hline $1862 \ldots$ & May 4-7 & 15.2 & $1892 \ldots$ & June 27 & 19.4 \\
\hline $1863 \ldots$ & May $1-5$ & 8.0 & $1893 \ldots$ & Mar. 13 & 15.1 \\
\hline $1864 \ldots$ & Feb. 27 & 7.7 & $1894 \ldots$ & May 31 & 12.3 \\
\hline $1865 \ldots$ & Mar. 4 & 11.1 & $1895 \ldots$ & Mar. 4 & 8.9 \\
\hline $1866 \ldots$ & May 4 & 15.7 & $1896 \ldots$ & May 28-30 & 12.0 \\
\hline $1867 \ldots$ & June 27,28 & 14.3 & $1897 \ldots$ & Apr. 17 & 15.1 \\
\hline 1868 & Mar. 10 & *22.0 & $1898 \ldots$ & Feb. 14 & 9.9 \\
\hline $1869 \ldots$ & Oct. $9-10$ & 12.9 & $1899 \ldots$ & J une 25,26 & 11.85 \\
\hline $1870 \ldots$ & Apr. 25 & 16.8 & $1900 \ldots$ & Mar. 13 & 13.1 \\
\hline $1871 \ldots$ & May 14-16 & 12.8 & $1901 \ldots$ & Mar. 16 & 9.6 \\
\hline $1872 \ldots$ & May 28 & 9.1 & $1902 \ldots$ & May 29 & 10.5 \\
\hline $1873 \ldots$ & May 8 & 12.9 & $1903 \ldots$ & Oct. 1 & 13.65 \\
\hline $1874 \ldots$ & Mar. 9 & 15.6 & $1904 \ldots$ & Mar. 22 & 13.2 \\
\hline $1875 \ldots \ldots$ & Apr. 29 & 12.9 & $1905 \ldots$ & $J$ une 21 & 14.3 \\
\hline $1876 \ldots$ & Apr. 16 & 13.8 & $1906 \ldots$ & Apr. $22-26$ & 13.9 \\
\hline $1877 \ldots$ & Apr. 4,5 & 9.9 & $1907 \ldots$ & Apr. 13 & 13.6 \\
\hline $1878 \ldots$ & July 17 & 7.1 & $1908 \ldots$ & July 21 & 12.25 \\
\hline $1879 \ldots$ & $J$ une 1 & 7.8 & $1909 \ldots$ & Dec. 25 & 13.7 \\
\hline $1880 \ldots$ & June 26 & 18.4 & $1910 \ldots$ & Jan. 1 & 11.9 \\
\hline $1881 \ldots$ & Oct. 27 & 17.7 & $1911 \ldots$ & Oct. 22,23 & 11.2 \\
\hline $1882 \ldots$ & Apr. 23 & 14.1 & $1912 \ldots$ & Mar. 30 & 12.7 \\
\hline $1883 \ldots$ & May 2 & 13.3 & 1913 & Mar. 28,29 & 12.8 \\
\hline $1884 \ldots$ & Mar. 28, 29 & 11.9 & $1914 \ldots$ & July 14,15 & 10.65 \\
\hline $1885 \ldots$ & May 8 & 10.1 & $1915 \ldots$ & June $3-5,7$ & 9.5 \\
\hline $1886 \ldots$ & May $1-3$ & 12.5 & $1916 \ldots$ & May 5 & 15.9 \\
\hline $1887 \ldots$ & Feb. 12 & 12.9 & $1917 \ldots$ & Apr. 21 & 12.35 \\
\hline $1888 \ldots$ & May 16 & 18.6 & $1918 \ldots$ & June 14 & 10.35 \\
\hline $1889 \ldots$ & Mar. 12 & 7.8 & $1919 \ldots$ & Apr. 25 & 13.7 \\
\hline
\end{tabular}

*Ice gorge-raised $5 \mathrm{ft}$ in one day.

\section{ROCK RIVER BASIN}

(227) 5-4465. Rock River near Joslin, Ill.

Location.-Lat $41^{\circ} 33^{\prime} 35^{\prime \prime}$, long $90^{\circ} 10^{\prime} 55^{\prime \prime}$, in NE $\frac{1}{4}$ sec. 18, T.18 N., R. 3 E., on right bank at downstream side of bridge on State Highway 92, 1.8 miles east of Jcslin, 12 miles downstream from Rock Creek, and 27 miles upstream from mouth.

Drainage area.- $-9,520 \mathrm{sq} \mathrm{mi}$, approximately.

Gage-height record.-Digital recorder tape punched at 15-minute intervals, except May 24, 28-31. Datum of gage is $564.06 \mathrm{ft}$ above mean sea level, datum of 1929 (levels by Corps of Engineers).

Discharge record.-Stage-discharge relation defined by current-water measurements. Discharge for periods of no gage-height record computed from record : for nearby stations. Mean daily discharge computed from 96 punch-tape recordirgs per day.

Maxima.-April-May 1965: Discharge, 21,700 cfs 0945 hours Apr. 8 (gage height, $13.12 \mathrm{ft}$ ).

1939 to March 1965: Discharge, 46,200 cfs Mar. 22, 1948 (gage height, $14.46 \mathrm{ft}$ ); gage height, $16.23 \mathrm{ft}$ Mar. 3, 1948 (backwater from ice).

Stage known since 1892, that of Mar. 3, 1948 . 
Mean discharge, in cubic feet per second, 1965, of Rock River near Joslin. Ill

\begin{tabular}{|c|c|c|c|c|c|c|c|c|}
\hline Day & April & May & Day & April & May & Day & April & May \\
\hline $\begin{array}{l}1 \ldots \\
2 \ldots \\
3 \\
4 \\
4 \\
5 \\
6 \ldots \\
7 \\
7 \ldots \\
8 \\
9 \\
10\end{array}$ & $\begin{array}{l}12,300 \\
16,900 \\
19,800 \\
19,800 \\
19,400 \\
19,800 \\
21,200 \\
21,500 \\
20,600 \\
19,200\end{array}$ & $\begin{array}{r}10,200 \\
9,240 \\
8,570 \\
8,010 \\
7,700 \\
8,100 \\
9,420 \\
9,660 \\
10,300 \\
9,640\end{array}$ & $\begin{array}{l}11 \ldots \ldots \\
12 \ldots \ldots \\
13 \ldots \ldots \\
14 \ldots \ldots \\
15 \ldots \ldots \\
16 \ldots \ldots \\
17 \ldots \ldots \\
18 \ldots \ldots \\
19_{\ldots} \ldots \\
20 \ldots \ldots\end{array}$ & $\begin{array}{l}18,300 \\
17,600 \\
17,200 \\
16,600 \\
16,300 \\
15,900 \\
15,300 \\
14,200 \\
12,900 \\
12,000\end{array}$ & $\begin{array}{l}9,140 \\
8,790 \\
8,000 \\
6,970 \\
5,800 \\
5,430 \\
4,890 \\
3,380 \\
3,520 \\
4,090\end{array}$ & $\begin{array}{l}21 \ldots \\
22 \ldots \ldots \\
23 \ldots \\
24 \ldots \\
25 \ldots \\
26 \ldots \\
26 \ldots \\
28 \ldots \\
29 \ldots \\
30 \ldots \\
31 \ldots\end{array}$ & $\begin{array}{r}11,100 \\
10,300 \\
9,720 \\
9,580 \\
10,100 \\
10,700 \\
11,200 \\
11,600 \\
11,500 \\
10,700 \\
-\end{array}$ & $\begin{array}{l}3,730 \\
3,690 \\
3,320 \\
3,200 \\
3,460 \\
3,740 \\
3,790 \\
3,700 \\
3,400 \\
3,200 \\
3,400\end{array}$ \\
\hline \multicolumn{7}{|c|}{$\begin{array}{l}\text { Monthly mean discharge, ir cubic feet per second } \\
\text { Runoff, in inches }\end{array}$} & $\begin{array}{r}15,110 \\
1.77\end{array}$ & $\begin{array}{r}6,112 \\
0.74\end{array}$ \\
\hline
\end{tabular}

(228) 5-4475. Green River near Geneseo, Ill.

Location.-Lat $41^{\circ} 29^{\prime} 20^{\prime \prime}$, long $90^{\circ} 09^{\prime} 30^{\prime \prime}$, in $\mathrm{NE} \frac{1}{4} \mathrm{SW} \frac{1}{4}$ sec.4, T.17 N., R.3 E., on right bank at upstream side of bridge on State Highway 82, 1.4 miles upstream from Geneseo Creek and 2.4 miles north of Geneseo.

Drainage area. $-958 \mathrm{sq} \mathrm{mi}$.

Gage-height record.-Digital recorder tape punched at 15-minute intervals. Prior to Apr. 28, 1965, water-stage recorder graph. Datum of gage is $580.66 \mathrm{ft}$ above mean sea level, datum of 1929 (levels by Corps of Engineers).

Discharge record.-Stage-discharge relation defined by current-meter measurements. Mean daily discharge computed from 96 punch-tape recordings per day beginning Apr. 28, 1965.

Maxima.-April-May 1965: Discharge, 3,980 cfs 2200 hours Apr, 25 (gage hoight, $10.57 \mathrm{ft}$ ).

1936 to March 1965: Discharge,8,900 cfs Oct. 11, 1954 (gage height, $14.69 \mathrm{ft}$ ); gage height, $16.65 \mathrm{ft} \mathrm{Jan.} \mathrm{24,} 1965$ (ice jam).

Mean discharge, in cubic feet per second, 1965

\begin{tabular}{|c|c|c|c|c|c|c|c|c|}
\hline Day & April & May & Day & April & May & Day & April & May \\
\hline 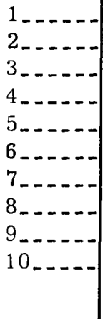 & $\begin{array}{r}1,280 \\
1,190 \\
1,020 \\
924 \\
1,120 \\
2,900 \\
3,190 \\
2,410 \\
2,110 \\
1,840\end{array}$ & $\begin{array}{r}1,320 \\
1,160 \\
1,000 \\
911 \\
960 \\
1,160 \\
1,350 \\
1,660 \\
1,960 \\
1,540\end{array}$ & $\mid \begin{array}{l}11 \ldots \ldots \\
12 \\
13 \\
14 \ldots \\
15 \\
16 \ldots \\
17 \\
18 \ldots \\
18 \ldots \\
19 \ldots \\
20 \ldots\end{array}$ & $\begin{array}{r}1,850 \\
1,660 \\
1,300 \\
1,150 \\
1,750 \\
1,890 \\
1,560 \\
1,260 \\
1,080 \\
956\end{array}$ & $\begin{array}{r}1,220 \\
1,040 \\
900 \\
780 \\
688 \\
644 \\
622 \\
584 \\
549 \\
514\end{array}$ & $\begin{array}{l}21 \ldots \\
22 \\
23 \\
24 \\
25 \\
26 \\
26 \\
28 \\
28 \\
29 \\
30 \\
31\end{array}$ & $\begin{array}{r}876 \\
812 \\
764 \\
2,230 \\
3,380 \\
3,150 \\
2,280 \\
2,080 \\
1,930 \\
1,590 \\
-\end{array}$ & $\begin{array}{l}493 \\
482 \\
451 \\
434 \\
430 \\
448 \\
476 \\
454 \\
427 \\
412 \\
391\end{array}$ \\
\hline \multicolumn{7}{|c|}{$\begin{array}{l}\text { Monthly mean discharge, in cubic feet per second } \\
\text { Runoff, in inches }\end{array}$} & $\begin{array}{r}1,718 \\
2.00\end{array}$ & $\begin{array}{r}821 \\
0.99\end{array}$ \\
\hline
\end{tabular}


Gage height, in feet, and discharge, in cubic feet per second, at indicated time, 1965, of Green River near Geneseo, Ill.

\begin{tabular}{|c|c|c|c|c|c|c|c|c|c|c|c|}
\hline Date & Hour & $\begin{array}{c}\text { Gage } \\
\text { height }\end{array}$ & $\begin{array}{c}\text { Dis - } \\
\text { charge }\end{array}$ & Date & Hour & $\begin{array}{l}\text { Gage } \\
\text { height }\end{array}$ & $\begin{array}{c}\text { Dis- } \\
\text { charge }\end{array}$ & Date & Hour & $\begin{array}{c}\text { Gage } \\
\text { reight }\end{array}$ & $\begin{array}{c}\text { Dis- } \\
\text { charge }\end{array}$ \\
\hline Apr. 24 & $\begin{array}{l}0000 \\
0200 \\
0400 \\
0500 \\
0800 \\
1100 \\
1200 \\
1900 \\
2100 \\
2400 \\
0400 \\
0600 \\
0900 \\
1300 \\
1600 \\
1900\end{array}$ & $\begin{array}{r}4.25 \\
4.27 \\
4.38 \\
4.71 \\
6.70 \\
8.90 \\
9.18 \\
9.41 \\
9.42 \\
9.31 \\
\\
8.96 \\
8.97 \\
9.23 \\
10.11 \\
10.38 \\
10.54\end{array}$ & $\begin{array}{r}780 \\
788 \\
832 \\
964 \\
1,760 \\
2,720 \\
2,890 \\
3,050 \\
3,050 \\
2,980 \\
\\
2,760 \\
2,760 \\
2,920 \\
3,590 \\
3,810 \\
3,960\end{array}$ & Apr. 25 & $\begin{array}{l}2200 \\
2400 \\
0200 \\
0900 \\
1400 \\
1900 \\
2400 \\
1200 \\
2400 \\
1200 \\
2400 \\
1200\end{array}$ & $\begin{array}{r}10.57 \\
10.54 \\
10.45 \\
9.73 \\
9.22 \\
8.86 \\
8.60 \\
7.92 \\
7.42 \\
7.44 \\
7.53 \\
7.15\end{array}$ & $\begin{array}{l}3,980 \\
3,960 \\
3,880 \\
3,280 \\
2,910 \\
2,700 \\
2,550 \\
2,250 \\
2,050 \\
2,060 \\
2,090 \\
1,940\end{array}$ & $\begin{array}{r}\text { Apr. 29 } \\
30\end{array}$ & $\begin{array}{l}2400 \\
1200 \\
2400 \\
1200 \\
2400 \\
1200 \\
2400 \\
1200 \\
2400 \\
1200 \\
2400\end{array}$ & $\begin{array}{l}6.66 \\
\\
6.24 \\
5.95 \\
5.59 \\
5.43 \\
5.17 \\
5.02 \\
4.76 \\
4.68 \\
4.56 \\
4.54\end{array}$ & $\begin{array}{r}1,740 \\
1,580 \\
1,460 \\
1,320 \\
1,250 \\
1,150 \\
1,090 \\
\\
984 \\
952 \\
\\
904 \\
896\end{array}$ \\
\hline
\end{tabular}

(229) Rock River at Moline, Ill.

(Miscellaneous site)

Location.-SE $\frac{1}{4}$ sec.16, T.17 N., R.1 W., at bridge on U.S. Highway 150 at Moline.

Drainage area. $-10,630 \mathrm{sq} \mathrm{mi}$.

Discharge record.-Discharge obtained on the following days by current-meter measurements. Stage-discharge relation affected by backwater from $M$ ississippi River.

Date

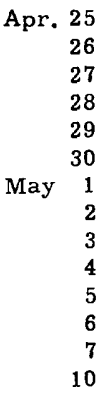

\section{Gage height}

(ft)

$$
\begin{array}{r}
11.16 \\
11.84 \\
12.24 \\
12.53 \\
12.57 \\
12.39 \\
12.07 \\
11.69 \\
11.12 \\
10.58 \\
10.09 \\
9.60 \\
9.34 \\
9.54
\end{array}
$$

Discharge

13,600

14,400

14,400

14,400

15,600

13,600

12,400

11,600

10,200

9,550

9,350

8,870

10,800

12,300

Cooperation.-Data furnished by Corps of Engineers. 
(230) 5-4480. Mill Creek at Milan, Ill.

Location.-Lat $41^{\circ} 26^{\prime} 35^{\prime \prime}$, long $90^{\circ} 33^{\prime} 15^{\prime \prime}$, in SW $\frac{1}{4} \mathrm{SE} \frac{1}{4}$ sec. 24, T.1 7 N., R.2 W., on left bank at upstream side of Knoxville Road Bridge, 1 mile southeast of Milar.

Drainage area. $-62.5 \mathrm{sq} \mathrm{mi}$.

Gage-height record.-Digital recorder tape punched at 15-minute intervals. Prior to Apr. 27, 1965, water-stage recorder graph except Apr. 9-25, for which graph was drawn on basis of once-daily wire-weight gage readings. Peak on Apr. 24 vas determined from high-water mark. Datum of gage is $566.23 \mathrm{ft}$ above mean sea level, datum of 1929 (levels by Corps of Engineers).

Discharge record.-Stage-discharge relation defined by current-meter measurements. Mean daily discharge computed from 96 punch-tape recordings per day beg:nning Apr. 27, 1965.

Maxima.-April-May 1965: Discharge, 9,060 cfs 0900 hours Apr. 24 (gage height, $11.53 \mathrm{ft}$, from high-water mark).

1939 to March 1965: Discharge, 8,980 cfs Oct. 10, 1954 (gage height, 9.39 ft).

Mean discharge, in cubic feet per second, 1965

\begin{tabular}{|c|c|c|c|c|c|c|c|c|}
\hline Day & April & May & Day & April & May & Day & April & May \\
\hline $\begin{array}{l}1 \\
2 \\
3 \\
4 \\
5 \\
6 \\
7 \\
8\end{array}$ & $\begin{array}{c}14 \\
11 \\
9.0 \\
15 \\
253 \\
1,170 \\
89 \\
112 \\
112 \\
91\end{array}$ & $\begin{array}{l}99 \\
81 \\
67 \\
65 \\
66 \\
56 \\
55 \\
97 \\
58 \\
45\end{array}$ & $\begin{array}{l}11 \ldots \\
12 \ldots \\
13 \\
14 \ldots \\
15 \ldots \\
16 \ldots \\
17 \ldots \\
18 \ldots \\
19 \ldots \\
20 \ldots \\
20 \ldots\end{array}$ & $\begin{array}{r}220 \\
102 \\
43 \\
74 \\
766 \\
56 \\
250 \\
96 \\
79 \\
64 \\
57\end{array}$ & $\begin{array}{l}40 \\
37 \\
34 \\
32 \\
30 \\
30 \\
29 \\
27 \\
25 \\
23\end{array}$ & $\begin{array}{l}21 \ldots \ldots \\
22 \ldots \ldots \\
23 \ldots \ldots \\
24 \ldots \ldots \\
25 \ldots \ldots \\
26 \ldots \ldots \\
27 \ldots \ldots \\
28 \ldots \ldots \\
29 \ldots \ldots \\
30 \ldots \ldots \\
31 \ldots \ldots\end{array}$ & $\begin{array}{r}51 \\
45 \\
41 \\
4,470 \\
977 \\
316 \\
210 \\
227 \\
153 \\
121 \\
\end{array}$ & $\begin{array}{l}24 \\
24 \\
23 \\
21 \\
22 \\
29 \\
29 \\
20 \\
19 \\
22 \\
20\end{array}$ \\
\hline \multicolumn{7}{|c|}{$\begin{array}{l}\text { Monthly mean discharge, in cubic feet per second } \\
\text { Runoff, in inches }\end{array}$} & $\begin{array}{r}335 \\
5.97\end{array}$ & $\begin{array}{l}40.3 \\
0.74\end{array}$ \\
\hline
\end{tabular}

Gage height, in feet, and discharge, in cubic feet per second, at indicated time, 1965

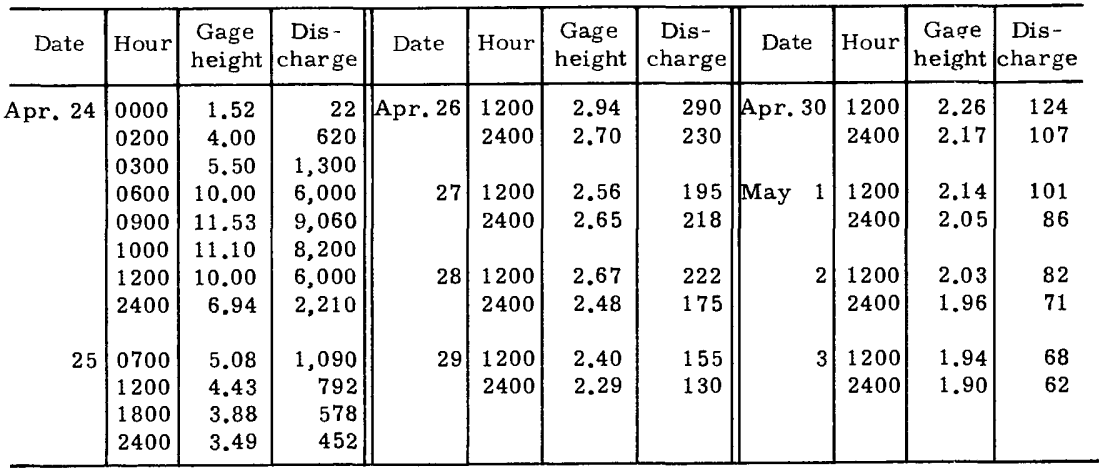




\section{IOWA RIVER BASIN}

(231) 5-4486. East Branch Iowa River above Hayfield, Iowa

$$
\text { (Crest-stage station) }
$$

Location.-Near south quarter corner sec.4, T.96 N., R.24 W., at bridge $1 \frac{1}{2}$ miles southeast of Hayfield.

Drainage area.- $-2.23 \mathrm{sq} \mathrm{mi}$.

Gage-height record.-Crest stages only.

Discharge record.-Stage-discharge relation defined by current-meter measurements below $141 \mathrm{cfs}$ and extended above by logarithmic plotting.

Maxima.-March-May 1965: Discharge, about $250 \mathrm{cfs}$ Apr. 6 (gage heigrt, $7.31 \mathrm{ft}$, backwater from ice).

1953 to February 1965: Discharge, 209 cfs June 18, 1954 (gage height, $7.15 \mathrm{ft}$ ).

(232) 5-4487. East Branch Iowa River near Hayfield, Iowa

$$
\text { (Crest-stage station) }
$$

Location.--NW $\frac{1}{4}$ sec. 35, T.97 N., R.24 W., at bridge, 2 miles east of Hayfield.

Drainage area. $-7.94 \mathrm{sq} \mathrm{mi}$.

Gage-height record.-Crest stages only.

Discharge record.-Stage-discharge relation defined by current-meter reasurements below $436 \mathrm{cfs}$ and extended above by logarithmic plotting.

Maxima.-March-May 1965: Discharge, about $370 \mathrm{cfs}$ Apr. 6 (gage height, $13.67 \mathrm{ft}$, backwater from ice).

1952 to February 1965: Discharge, 457 cfs June 18, 1954 (gage height, $13.01 \mathrm{ft}$ ).

(233) 5-4488. East Branch Iowa River near Garner, Iowa

\section{(Crest-stage station)}

Location.-Near center of sec.25, T.96 N., R.24 W., at bridge on U.S. Highway 18, 1.2 miles west of Garner.

Drainage area. $-45.1 \mathrm{sq} \mathrm{mi}$.

Gage-height record, - Crest stages only.

Discharge record.-Stage-discharge relation defined by current-meter msasurements.

Maxima.-March-May 1965: Discharge, about 1,000 cfs Apr. 6 (gage height, $12.89 \mathrm{ft}$, backwater from ice).

1952 to February 1965: Discharge, 1,120 cfs Mar. 26, 1961 (gage height, $12.81 \mathrm{ft}$ ). 
Location.-Near center sec.27, T.96 N., R.24 W., at culvert on U.S. Highway 18, 2.1 miles west of Garner.

Drainage area. $-5.98 \mathrm{sq} \mathrm{mi}$.

Gage-height record.-Crest stages only.

Discharge record. - Stage-discharge relation defined by current-meter measurements.

Maxima.-March-May 1965: Discharge, about 170 cfs Apr. 6 (gage height, $9.15 \mathrm{ft}$, backwater from ice).

1952 to February 1965: Discharge, 206 cfs June 17, 1954 (gage height, $6.71 \mathrm{ft}$ ).

(235) 5-4490. East Branch Iowa River near Klemme, Iowa

Location.-Lat $43^{\circ} 00^{\prime} 30^{\prime \prime}$, long $93^{\circ} 37^{\prime} 35^{\prime \prime}$, in NE $\frac{1}{4} \mathrm{NW} \frac{1}{4}$ sec.36, T.95 N., R.24 W., on left bank $15 \mathrm{ft}$ downstream from county highway bridge, 1.0 mile west of Klemme, and 15.4 miles upstream from confluence with West Branch Iowa River.

Drainage area. $-133 \mathrm{sq} \mathrm{mi}$.

Gage-height record.-Water-stage recorder graph except Mar. 2-14, 18-28. Datum of gage is $1,179.02 \mathrm{ft}$ above mean sea level, datum of 1929. Prior to Oct, 1, 1955, wireweight gage at site 0.6 mile upstream at datum $1.11 \mathrm{ft}$ higher.

Discharge record. - Stage-discharge relation defined by current-meter measurements below 2,740 cfs, extended to $4,090 \mathrm{cfs}$ by logarithmic plotting. Backwater from ice Mar. 1 to Apr. 7.

Maxima.-March-May 1965: Discharge, 4,090 cfs 2200 hours Apr. 8 (gage height, $9.94 \mathrm{ft}$ ); gage height, $10.67 \mathrm{ft} 1030$ hours Apr. 6 (backwater from ice).

1948 to February 1965: Discharge, 5,960 cfs J une 19, 1954 (gage height, $11.2 \mathrm{ft}$, from floodmark at site 0.6 mile upstream at datum $1.11 \mathrm{ft}$ higher).

Mean discharge, in cubic feet per second, 1965

\begin{tabular}{|c|c|c|c|c|c|c|c|c|c|c|c|}
\hline Day & March & April & May & Day & March & April & May & Day & March & April & May \\
\hline- & 108 & 190 & 125 & $11 \ldots$ & 47 & 1,000 & 67 & 21. & 15 & 200 & 86 \\
\hline 2 & 240 & 475 & 114 & $12 \ldots$ & 42 & 1,060 & 61 & 22 & 13 & 180 & 79 \\
\hline 3 & 90 & 500 & 104 & $13_{-}$ & 37 & 763 & 57 & $23 \ldots$ & 11 & 164 & 132 \\
\hline$t_{2}$ & 76 & 550 & 92 & $14 \ldots$ & 33 & 635 & 66 & $24_{-}$ & 10 & 154 & 254 \\
\hline $5 \ldots$ & 72 & 1,220 & 94 & $15 \ldots$ & 30 & 514 & 100 & $25_{-}$ & 9.6 & 174 & 231 \\
\hline $6 \ldots$ & 68 & 2,400 & 139 & $16 \ldots$ & 27 & 419 & 209 & 26 _ & 9.2 & 241 & 453 \\
\hline $7 \ldots$ & 65 & 2,500 & 135 & $17 \ldots$ & 26 & 341 & 181 & 27 & 9.2 & 208 & 500 \\
\hline 8 & 60 & 3,170 & 114 & $18 \ldots$ & 24 & 299 & 136 & 28 & 9.0 & 175 & 313 \\
\hline $9_{-}$ & 56 & 2,340 & 94 & $19 \ldots$ & 20 & 257 & 106 & 29 & 9.0 & 157 & 227 \\
\hline \multirow[t]{2}{*}{$10 \ldots$} & 52 & 1,190 & 77 & $20_{-}$ & 17 & 228 & 89 & $30_{-}$ & 18 & 141 & 230 \\
\hline & & & & & & & & & 80 & ----- & 201 \\
\hline \multicolumn{9}{|c|}{ Monthly mean discharge, in cubic feet per second } & 44.6 & 728 & 157 \\
\hline \multicolumn{9}{|c|}{ Runoff, in inches } & 0.39 & 6.11 & 1.36 \\
\hline \multicolumn{9}{|c|}{ Runoff, in acre-feet } & 2,740 & 43,330 & 9,650 \\
\hline
\end{tabular}


Gage height, in feet, and discharge, in cubic feet per second, at indicated time, 1965, of East Branch Iowa River near Klemme, Iowa

\begin{tabular}{|c|c|c|c|c|c|c|c|c|c|c|c|}
\hline Date & Hour & $\begin{array}{c}\text { Gage } \\
\text { height }\end{array}$ & $\begin{array}{c}\text { Dis- } \\
\text { charge }\end{array}$ & Date & Hour & $\begin{array}{l}\text { Gage } \\
\text { height }\end{array}$ & $\begin{array}{c}\text { Dis- } \\
\text { charge }\end{array}$ & Date & Hour & $\begin{array}{c}\text { Gage } \\
\text { height }\end{array}$ & $\begin{array}{l}\text { Dis- } \\
\text { charge }\end{array}$ \\
\hline \multirow[t]{6}{*}{ Apr. 6} & 0000 & 10.03 & & \multirow[t]{6}{*}{ Apr. 8} & 1200 & 9.59 & 3,040 & \multirow[t]{7}{*}{ Apr. 9} & 0600 & 9.70 & 3,420 \\
\hline & 0500 & 10.08 & & & 1600 & 9.62 & 3,140 & & 0800 & 9.53 & 2,830 \\
\hline & 1030 & 10.67 & & & 1800 & 9.74 & 3,560 & & 1000 & 9.35 & 2,320 \\
\hline & 1800 & 10.55 & & & 2200 & 9.94 & 4,090 & & 1200 & 9.15 & 2,000 \\
\hline & 2400 & 10.19 & & & 2400 & 9.92 & 4,020 & & 1400 & 8.91 & 1,690 \\
\hline & & & & & & & & & 1800 & 8.73 & 1,450 \\
\hline 7 & 2400 & 9.45 & 2,570 & 9 & 0400 & 9.78 & 3,530 & & 2400 & 8.61 & 1,320 \\
\hline
\end{tabular}

(236) 5-4495. Iowa River near Rowan, Iowa

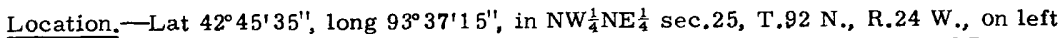
bank, $10 \mathrm{ft}$ downstream from county highway bridge, 3.8 miles northwest of Rowan, 9.4 miles downstream from confluence of East and West Branches, and at mile 316.4.

Drainage area. $-429 \mathrm{sq} \mathrm{mi}$.

Gage-height record.-Water-stage recorder graph except Mar. 2-14, 21-30. Graph was reconstructed on basis of daily wire-weight gage readings Mar. 6, 21, 26, 28. Datum of gage is $1,143.35 \mathrm{ft}$ above mean sea level, datum of 1929 .

Discharge record. $\rightarrow$ Stage-discharge relation defined by current-meter measurements below $8,300 \mathrm{cfs}$. Backwater from ice Mar. 1 to Apr. 7.

Maxima.-March-May 1965: Discharge, about 6,700 cfs Apr. 7; gage height, $14.62 \mathrm{ft}$ 1800 hours Apr. 6 (backwater from ice).

1940 to February 1965: Discharge, 8,460 cfs J une 21, 1954 (gage height, $14.88 \mathrm{ft}$ ).

Mean discharge, in cubic feet per second, 1965

\begin{tabular}{|c|c|c|c|c|c|c|c|c|c|c|c|}
\hline Day & March & April & May & Day & March & April & May & Day & March & Anril & May \\
\hline & 510 & 800 & 485 & $11 \ldots$ & 200 & 4,300 & 258 & $21 \ldots$ & 64 & 914 & 200 \\
\hline & 1,000 & 1,700 & 434 & $12 \ldots$ & 180 & 4,430 & 236 & $22 \ldots$ & 52 & 745 & 202 \\
\hline 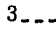 & 380 & 1,900 & 387 & 13. & 158 & 3,880 & 210 & $23 \ldots$ & 48 & 641 & 202 \\
\hline & 310 & 2,500 & 346 & $14 \ldots$ & 140 & 3,170 & 200 & $24_{-}$ & 43 & 578 & 268 \\
\hline . & 290 & 4,200 & 312 & $15 \ldots$ & 130 & 2,700 & 206 & $25 \ldots$ & 40 & 622 & 431 \\
\hline 6. & 270 & 5,800 & 373 & $16 \ldots$ & 118 & 2,320 & 299 & $26 \ldots$ & 37 & 764 & 749 \\
\hline & 250 & 6,000 & 418 & $17=$ & 110 & 1,970 & 390 & $27 \ldots$ & 35 & 828 & 992 \\
\hline 8. & 240 & 5,790 & 404 & $18 \ldots$ & 102 & 1,620 & 353 & $28 \ldots$ & 35 & 722 & 1,020 \\
\hline & 220 & 5,480 & 346 & 19. & 95 & 1,320 & 282 & 29. & 37 & 595 & 892 \\
\hline 10 & 210 & 5,230 & 294 & $20 \ldots$ & 82 & 1,100 & 217 & 30 & 54 & 539 & 736 \\
\hline & & & & & & & & & 300 & $-\cdots-n$ & 663 \\
\hline \multicolumn{9}{|c|}{ Monthly mean discharge, in cubic feet per second } & 185 & 2,439 & 413 \\
\hline \multicolumn{9}{|c|}{ Runoff, in inches } & 0.50 & 6.34 & 1.11 \\
\hline \multicolumn{9}{|c|}{ Runoff, in acre-feet } & 11,390 & 145,100 & 25,400 \\
\hline
\end{tabular}


MARCH-MAY, UPPER MISSISSIPPI RIVER BASIN

Gage height, in feet, and discharge, in cubic feet per second, at indicated time, 1965, of Iowa River near Rowan, Iowa

\begin{tabular}{|c|c|c|c|c|c|c|c|c|c|c|c|}
\hline Date & Hour & $\begin{array}{c}\text { Gage } \\
\text { height }\end{array}$ & $\begin{array}{c}\text { Dis- } \\
\text { charge }\end{array}$ & Date & Hour & $\begin{array}{c}\text { Gage } \\
\text { height }\end{array}$ & $\begin{array}{c}\text { Dis- } \\
\text { charge }\end{array}$ & Date & Hour & $\begin{array}{c}\text { Gage } \\
\text { height }\end{array}$ & $\begin{array}{c}\text { Dis- } \\
\text { charge }\end{array}$ \\
\hline \multirow[t]{6}{*}{ Apr. 6} & 0000 & 13.93 & & Apr. 7 & 2400 & 13.27 & 6,210 & Apr. 10 & 1400 & 12.57 & 5,230 \\
\hline & 0200 & 14.12 & & & & & & & 1800 & 12.40 & 4,990 \\
\hline & 1200 & 14.53 & & 8 & 0800 & 13.08 & 5,940 & & 2400 & 12.15 & 4,640 \\
\hline & 1400 & 14.58 & & & 1800 & 12.77 & 5,510 & & & & \\
\hline & 1800 & 14.62 & & & 2200 & 12.79 & 5,540 & 11 & 1200 & 11.80 & 4,130 \\
\hline & 2400 & 14.55 & & & 2400 & 12.75 & 5,480 & & 2400 & 11.82 & 4,310 \\
\hline \multirow[t]{4}{*}{7} & 0600 & 14.34 & & 9 & 2400 & 12.76 & 5,490 & 12 & 0800 & 12.10 & 4,570 \\
\hline & 1200 & 14.18 & & & & & & & 1400 & 12.04 & 4,490 \\
\hline & 1400 & 13.80 & & 10 & 0800 & 12.76 & 5,490 & & 2400 & 11.88 & 4,250 \\
\hline & 1800 & 13.43 & & & & & & & & & \\
\hline
\end{tabular}

(237) 5-4515. Iowa River at Marshalltown, Iowa

Location.-Lat $42^{\circ} 04^{\prime} 05^{\prime \prime}$, long $92^{\circ} 54^{\prime} 05^{\prime \prime}$, in NW $\frac{1}{4} S W \frac{1}{4}$ sec.24, T.84 N., R.18 W., on right bank in city park in Marshalltown, $300 \mathrm{ft}$ upstream from Burnett Creek, 0.2 mile downstream from bridge on State Highway 14, 2.0 miles upstream from Linn Creek, and at mile 222.6 .

Drainage area.-1,564 sq $\mathrm{mi}$, including that of Burnett Creek.

Gage-height record.-Water-stage recorder graph. Datum of gage $853.10 \mathrm{ft}$ above mean sea level, datum 1929.

Discharge record.-Stage-discharge relation defined by current-meter measurements below $21,000 \mathrm{cfs}$ and extended to $42,000 \mathrm{cfs}$ from velocity-area study. Backwater from ice Mar. 1-30.

Maxima.-March-May 1965: Discharge, 17,400 cfs 1600 hours Apr. 6 (gage height $17.63 \mathrm{ft}$ ).

1902-3, 1914-27, 1932 to February 1965: Discharge, 42,000 cfs June 4, 1918 (gage height, $17.74 \mathrm{ft}$, from floodmarks).

Mean discharge, in cubic feet per second, 1965

\begin{tabular}{|c|c|c|c|c|c|c|c|c|c|c|c|}
\hline Day & March & April & May & Day & March & April & May & Day & March & April & May \\
\hline & 4,000 & 9,520 & 1,550 & 10 & 1,500 & 10,600 & 820 & L & 480 & $2,68 n$ & 584 \\
\hline & 8,200 & 9,340 & 1,370 & 12. & 1,200 & 8,800 & 748 & $22_{-}$ & 400 & $2,33 n$ & 571 \\
\hline & 5,600 & 7,080 & 1,200 & $13_{--}$ & 1,050 & 8,020 & 680 & 3. & 330 & $2,00 ?$ & 694 \\
\hline & 3,700 & 7,640 & 1,190 & $14 \ldots$ & 900 & 7,400 & 610 & 4 & 310 & 1,890 & 694 \\
\hline & 2,600 & 11,400 & 1,060 & $15 \ldots$ & 840 & 7,070 & 571 & $25 \ldots$ & 300 & 2,000 & 788 \\
\hline & 1,900 & 16,900 & 959 & $16 \ldots$ & 840 & 6,540 & 554 & $26 \ldots$ & 300 & 2,150 & 2,470 \\
\hline 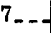 & 2,000 & 14,800 & 874 & $17 \ldots$ & 880 & 5,840 & 530 & $27 \ldots$ & 300 & 2,060 & 4,660 \\
\hline 8. & 2,100 & 13,400 & 951 & $18 \ldots$ & 800 & 5,060 & 571 & 28 & 300 & 1,960 & 4,110 \\
\hline & 1,900 & 13,300 & 974 & 19. & 700 & 4,270 & 640 & $29 \ldots$ & 320 & $1,84 n$ & 3,430 \\
\hline & 1,700 & 12,900 & 986 & $20 \ldots$ & 580 & 3,360 & 640 & $30_{-}$ & 1,100 & 1,720 & 2,970 \\
\hline & & & & & & & & & 4,000 & & 2,660 \\
\hline \multirow{3}{*}{\multicolumn{9}{|c|}{$\begin{array}{l}\text { Monthly mean discharge, in cubic feet per second } \\
\text { Runoff, in inches } \\
\text { Runoff, in acre-feet }\end{array}$}} & 1,649 & $6,7 \subseteq 6$ & 1,326 \\
\hline & & & & & & & & & 1.22 & 4.85 & 0.98 \\
\hline & & & & & & & & & 101,400 & $404,4 \mathrm{CO}$ & 81,540 \\
\hline
\end{tabular}


Gage height, in feet, and discharge, in cubic feet per second, at indicated time. 1965, of Iowa River at

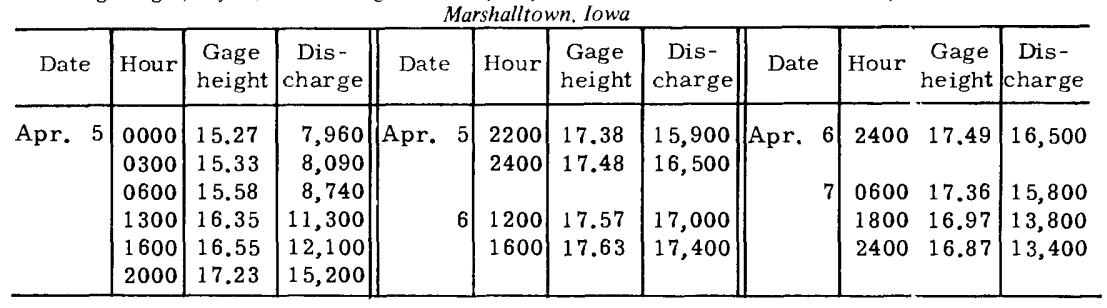

\section{(238) 5-4517. Timber Creek near Marshalltown, Iowa}

Location.-Lat $42^{\circ} 00^{\prime} 25^{\prime \prime}$, long $92^{\circ} 51^{\prime} 30^{\prime \prime}$, in $\mathrm{SE}_{4} \frac{1}{4} \mathrm{SW} \frac{1}{4} \mathrm{sec} .8, \mathrm{~T} .83 \mathrm{~N} ., \mathrm{R} .17 \mathrm{~W}$. , on left bank $20 \mathrm{ft}$ downstream from bridge on U.S. Highway $30,2.7$ miles upstream from mouth and 3.0 miles southeast of Marshalltown.

Drainage area. $-118 \mathrm{sq} \mathrm{mi}$.

Gage-height record.-Water-stage recorder graph, except Mar. 1-5, 17, 18, 30, 31 . Daily wire-weight gage readings available for this period. Datum of gage is $849.44 \mathrm{ft}$ above sea level, datum of 1929 .

Discharge record.-Stage-discharge relation defined by current-meter measurements below 3,100 cfs. Backwater from ice and debris Mar. 1 to Apr. 16.

Maxima.-March-May 1965: Discharge, 3,130 cfs 1100 hours Apr. 24; gage-height, $15.07 \mathrm{ft} 0200$ hours Apr. 1 (backwater from ice).

1949 to February 1965: Discharge, 4,940 cfs June 18, 1950 (gage height $15.77 \mathrm{ft}$ ). Flood in June 1947 reached a stage of $16.8 \mathrm{ft}$ (discharge not determined).

Cooperation.-One discharge measurement furnished by Corps of Engine?rs.

Mean discharge, in cubic feet per second, 1965

\begin{tabular}{|c|c|c|c|c|c|c|c|c|c|c|c|}
\hline Day & March & April & May & Day & March & April & May & Day & March & A pril & May \\
\hline & 1,100 & 1,300 & 110 & $11 \ldots$ & 19 & 150 & 49 & 21 & 39 & 45 & 32 \\
\hline- & 400 & 500 & 96 & $12 \ldots$ & 18 & 105 & 49 & 22 & 31 & 42 & 105 \\
\hline S & 200 & 370 & 84 & $13 \ldots$ & 25 & 92 & 45 & $23 \ldots$ & 26 & 40 & 124 \\
\hline & 100 & 500 & 77 & 14. & 45 & 84 & 43 & $24=$ & 23 & 1,360 & 66 \\
\hline . & 50 & 1,100 & 75 & $15 \ldots$ & 100 & 76 & 44 & 25 & 21 & 649 & 68 \\
\hline 6. & 30 & 600 & 70 & $16 \ldots$ & 400 & 70 & 43 & $26 \ldots$ & 20 & 310 & 524 \\
\hline 7. & 25 & 200 & 63 & 17. & 1,000 & 65 & 40 & 27 & 19 & 221 & 188 \\
\hline & 22 & 160 & 64 & $18 \ldots$ & 500 & 57 & 37 & 28 & 30 & 191 & 127 \\
\hline & 21 & 140 & 65 & 19. & 66 & 51 & 33 & 29 & 230 & 149 & 119 \\
\hline 10 & 20 & 130 & 52 & $20 \ldots$ & 46 & 48 & 33 & $30 \ldots$ & 900 & 127 & 117 \\
\hline & & & & & & & & 31 & 1,500 & - & 106 \\
\hline \multicolumn{9}{|c|}{ Monthly mean discharge, in cubic feet per second } & 227 & 298 & 886 \\
\hline \multicolumn{9}{|c|}{ Runoff, in inches } & 2.21 & 2.82 & 0.87 \\
\hline \multicolumn{9}{|c|}{ Runoff, in acre-feet } & 13,940 & 17,720 & 5,450 \\
\hline
\end{tabular}


Gage height, in feet, and discharge, in cubic feet per second, at indicated time, 1965, of Timber Creek near

\begin{tabular}{|c|c|c|c|c|c|c|c|c|c|c|c|}
\hline Date & Hour & $\begin{array}{c}\text { Gage } \\
\text { height }\end{array}$ & $\begin{array}{c}\text { Dis- } \\
\text { charge }\end{array}$ & Date & Hour & $\begin{array}{c}\text { Gage } \\
\text { height }\end{array}$ & $\begin{array}{c}\text { Dis- } \\
\text { charge }\end{array}$ & Date & Hour & $\begin{array}{c}\text { Gage } \\
\text { height }\end{array}$ & $\begin{array}{c}\text { Dis- } \\
\text { :harge }\end{array}$ \\
\hline \multirow[t]{9}{*}{ Mar. 31} & 0000 & 13.40 & & Apr. 1 & 1530 & 12.27 & $-\infty$ & \multirow[t]{12}{*}{ Apr. 24} & 1200 & 13.80 & 2,690 \\
\hline & 0900 & 13.88 & & & 1700 & 12.45 & $\ldots$ & & 1500 & 12.55 & 1,820 \\
\hline & 1100 & 13.91 & & & 2100 & 12.39 & & & 1700 & 9.70 & 925 \\
\hline & 1500 & 13.69 & & & 2400 & 12.08 & & & 1900 & 7.80 & 590 \\
\hline & 1800 & 14.05 & & & & & & & 2200 & 6.89 & 463 \\
\hline & 2000 & 14.76 & & 24 & 0000 & 3.10 & 56 & & 2400 & 7.09 & 490 \\
\hline & 2200 & 14.90 & & & 0200 & 6.00 & 347 & & & & \\
\hline & 2400 & 14.93 & & & 0300 & 9.30 & 845 & & 1200 & 8.55 & 708 \\
\hline & & & & & 0500 & 10.81 & 1,190 & & 1700 & 9.38 & 853 \\
\hline \multirow[t]{3}{*}{ Apr. 1} & 0200 & 15.07 & & & 0700 & 11.56 & 1,410 & & 2100 & 7.92 & 596 \\
\hline & 0600 & 14.16 & & & 0900 & 13.72 & 2,630 & & 2400 & 7.00 & 461 \\
\hline & 1200 & 13.08 & & & 1100 & 14.31 & 3,130 & & & & \\
\hline
\end{tabular}

(239) 5-4519. Richland Creek near Haven, Iowa

Location.-Lat $41^{\circ} 53^{\prime} 55^{\prime \prime}$, long $92^{\circ} 28^{\prime} 35^{\prime \prime}$, in $\mathrm{SE} \frac{1}{4} \mathrm{NE} \frac{1}{4}$ sec. $21, \mathrm{~T} .82 \mathrm{~N} ., \mathrm{R} .14 \mathrm{~W}$., on right bank $5 \mathrm{ft}$ upstream from highway bridge, 0.5 mile north of Haven, and 3.0 miles upstream from mouth.

\section{Drainage area.- $-56.1 \mathrm{sq} \mathrm{mi}$.}

Gage-height record.-Water-stage recorder graph, except April 26-28, May 27-30. Daily wire-weight gage readings were available for those periods. Datum of gage is $798.69 \mathrm{ft}$ above mean sea level, datum of 1929 .

Discharge record.-Stage-discharge relation defined by current-meter measurements below $2,200 \mathrm{cfs}$ and extended to $3,560 \mathrm{cfs}$ on basis of logarithmic plotting. Backwater from ice Mar. 2-16, 19-23, 28, 29.

Maxima.-March-May 1965: Discharge, 2,090 cfs 1200 hours Apr. 24 (gage height, $9.98 \mathrm{ft}$ ).

1949 to February 1965: Discharge, 3,650 cfs Mar. 30, 1960 (gage height, $12.39 \mathrm{ft}$ ).

Flood in $J$ une 1918 reached a stage of $14.3 \mathrm{ft}$ (discharge not determined).

Cooperation.-Two discharge measurements furnished by Corps of Engineers.

Mean discharge, in cubic feet per second, 1965

\begin{tabular}{|c|c|c|c|c|c|c|c|c|c|c|c|}
\hline Day & March & April & May & Day & March & April & May & Day & March & April & May \\
\hline $\begin{array}{l}1 \\
2 \ldots- \\
2 \ldots- \\
3 \\
4 \ldots- \\
5 \ldots- \\
6 \ldots- \\
7 \ldots- \\
8 \ldots- \\
9 \ldots-- \\
10\end{array}$ & $\begin{array}{r}764 \\
150 \\
40 \\
20 \\
23 \\
13 \\
10 \\
9.0 \\
8.3 \\
7.5\end{array}$ & $\begin{array}{r}588 \\
86 \\
66 \\
84 \\
776 \\
268 \\
33 \\
63 \\
34 \\
29\end{array}$ & $\begin{array}{l}58 \\
50 \\
44 \\
61 \\
52 \\
40 \\
34 \\
34 \\
33 \\
27\end{array}$ & $\begin{array}{l}11 \ldots-- \\
12_{--} \\
13_{-} \\
14_{-} \\
15_{-} \\
16_{-} \\
17_{-} \\
18_{--} \\
19_{--} \\
20_{--}\end{array}$ & $\begin{array}{r}7.0 \\
11 \\
35 \\
60 \\
120 \\
180 \\
611 \\
170 \\
25 \\
15\end{array}$ & \begin{tabular}{l|l|}
41 & \\
27 & \\
21 & \\
20 & \\
19 \\
16 \\
15 \\
14 \\
13 \\
12
\end{tabular} & $\begin{array}{l}26 \\
24 \\
22 \\
22 \\
24 \\
24 \\
21 \\
19 \\
17 \\
17\end{array}$ & $\begin{array}{l}21-- \\
22-- \\
23-- \\
24-- \\
25-- \\
26-- \\
27-- \\
28-- \\
29_{-} \\
30_{-} \\
31--\end{array}$ & $\begin{array}{c}11 \\
9.4 \\
8.0 \\
7.3 \\
7.3 \\
7.3 \\
13 \\
54 \\
170 \\
610 \\
871\end{array}$ & $\begin{array}{r}12 \\
10 \\
10 \\
956 \\
413 \\
144 \\
101 \\
90 \\
78 \\
66 \\
\end{array}$ & $\begin{array}{r}17 \\
21 \\
25 \\
19 \\
19 \\
270 \\
46 \\
34 \\
29 \\
28 \\
121\end{array}$ \\
\hline \multicolumn{9}{|c|}{$\begin{array}{l}\text { Monthly mean discharge, in cubic feet per second } \\
\text { Runoff, in inches } \\
\text { Runoff, in acre -feet }\end{array}$} & $\begin{array}{r}131 \\
2.68 \\
8,030\end{array}$ & $\begin{array}{r}137 \\
2.72 \\
8,140\end{array}$ & $\begin{array}{r}41.2 \\
0.85 \\
2.530\end{array}$ \\
\hline
\end{tabular}


Gage height, in feet, and discharge, in cubic feet per second, at indicated time, 1965, of Richland Creek near Haven, lowa

\begin{tabular}{|c|c|c|c|c|c|c|c|c|c|c|c|}
\hline Date & Hour & $\begin{array}{c}\text { Gage } \\
\text { height }\end{array}$ & $\begin{array}{c}\text { Dis - } \\
\text { charge }\end{array}$ & Date & Hour & $\begin{array}{l}\text { Gage } \\
\text { height }\end{array}$ & $\begin{array}{c}\text { Dis- } \\
\text { charge }\end{array}$ & Date & Hour & $\begin{array}{c}\text { Gage } \\
\text { height }\end{array}$ & $\begin{array}{c}\text { Dis- } \\
\text { charge }\end{array}$ \\
\hline pr. 24 & $\begin{array}{l}0000 \\
0030 \\
0100 \\
0130 \\
0200 \\
0230 \\
0400 \\
0600 \\
0800 \\
0900 \\
1000 \\
1100 \\
1200 \\
1300\end{array}$ & $\begin{array}{l}1.70 \\
2.30 \\
4.00 \\
5.80 \\
6.78 \\
6.92 \\
6.73 \\
6.95 \\
7.18 \\
7.68 \\
8.70 \\
9.60 \\
9.98 \\
9.82\end{array}$ & $\begin{array}{r}91 \\
173 \\
460 \\
768 \\
1,030 \\
1,080 \\
1,020 \\
1,080 \\
1,150 \\
1,300 \\
1,640 \\
1,960 \\
2,090 \\
2,040\end{array}$ & Apr. 24 & $\begin{array}{l}1400 \\
1500 \\
1600 \\
1700 \\
1900 \\
2100 \\
2300 \\
2400 \\
0100 \\
0200 \\
0300 \\
0400 \\
0500\end{array}$ & $\begin{array}{l}8.90 \\
7.15 \\
4.90 \\
3.64 \\
2.99 \\
2.95 \\
3.44 \\
3.40 \\
\\
3.50 \\
3.89 \\
4.23 \\
4.28 \\
4.06\end{array}$ & $\begin{array}{r}1,720 \\
1,140 \\
604 \\
399 \\
288 \\
282 \\
365 \\
358 \\
\\
375 \\
441 \\
497 \\
505 \\
470\end{array}$ & Apr. 25 & $\begin{array}{l}0200 \\
0300 \\
1000 \\
1300 \\
1500 \\
1600 \\
1800 \\
2100 \\
2400 \\
0500 \\
1200 \\
2100\end{array}$ & $\begin{array}{l}3.20 \\
3.08 \\
3.25 \\
4.17 \\
4.64 \\
4.60 \\
4.00 \\
3.06 \\
2.72 \\
2.28 \\
1.98 \\
1.77\end{array}$ & $\begin{array}{r}324 \\
304 \\
332 \\
487 \\
562 \\
556 \\
460 \\
300 \\
242 \\
\\
170 \\
125 \\
99\end{array}$ \\
\hline
\end{tabular}

(240) 5-4520. Salt Creek near Elberon, Iowa

Location.-Lat $41^{\circ} 57^{\prime} 45^{\prime \prime}$, long $92^{\circ} 18^{\prime} 55^{\prime \prime}$, in NW $\frac{1}{4} \mathrm{NW} \frac{1}{4}$ sec.36, T.83 N., R.13 W., near center of span on downstream side of bridge on U.S. Highway 30,1.2 miles northwest of Irving, 2.5 miles south of Elberon, and 9.0 miles upstream from mouth.

\section{Drainage area. $-201 \mathrm{sq} \mathrm{mi}$.}

Gage-height record.-Water-stage recorder graph, except Mar. 3-5, 19-21. Daily wire-weight readings were available. Datum of gage is $781.58 \mathrm{ft}$ at ove mean sea level (Iowa Highway Commission bench mark).

Discharge record.-Stage-discharge relation defined by current-meter measurements below $17,000 \mathrm{cfs}$ and extended by logarithmic plotting to $35,000 \mathrm{cfs}$. Backwater from ice Mar. 1-31.

Maxima.-March-May 1965: Discharge, 4,680 cfs 0300 hours Apr. 1 (gage height, $15.75 \mathrm{ft})$.

1945 to February 1965: Discharge, $35,000 \mathrm{cfs}$ June 13, 1947 (gag o height, $17.6 \mathrm{ft}$ ).

Flood of June 16,1944, reached a stage of $19.9 \mathrm{ft}$, from floodmark (discharge, $30,000 \mathrm{cfs}$, estimated).

Cooperation.-One discharge measurement furnished by Corps of Engineers.

Mean discharge, in cubic feet per second, 1965

\begin{tabular}{|c|c|c|c|c|c|c|c|c|c|c|c|}
\hline Day & March & April & May & Day & March & April & May & Day & March & April & May \\
\hline 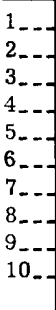 & $\begin{array}{r}2,100 \\
1,000 \\
400 \\
180 \\
90 \\
56 \\
48 \\
42 \\
39 \\
36\end{array}$ & $\begin{array}{r}3,420 \\
1,280 \\
482 \\
762 \\
2,320 \\
2,450 \\
343 \\
322 \\
253 \\
197\end{array}$ & $\begin{array}{r}114 \\
101 \\
79 \\
196 \\
212 \\
140 \\
113 \\
104 \\
97 \\
79\end{array}$ & \begin{tabular}{|l|}
$11 .-$ \\
$12 \ldots-$ \\
$13 \ldots--$ \\
$14 \ldots-$ \\
$15 \ldots$ \\
$16 \ldots-$ \\
$17 .-$ \\
$18=-$ \\
$19 .-$ \\
$20 \ldots-$
\end{tabular} & $\begin{array}{r}34 \\
34 \\
41 \\
90 \\
240 \\
600 \\
700 \\
240 \\
150 \\
85\end{array}$ & $\begin{array}{r}246 \\
183 \\
147 \\
135 \\
129 \\
112 \\
103 \\
94 \\
86 \\
81\end{array}$ & $\begin{array}{l}72 \\
67 \\
61 \\
59 \\
60 \\
65 \\
56 \\
50 \\
42 \\
40\end{array}$ & $\begin{array}{ll}21 \\
22- \\
23- \\
24_{-} \\
25_{-} \\
26_{-} \\
27- \\
28_{-} \\
29_{-} \\
30_{-} \\
31_{-}\end{array}$ & $\begin{array}{r}62 \\
50 \\
43 \\
37 \\
35 \\
33 \\
31 \\
52 \\
210 \\
900 \\
2,250\end{array}$ & $\begin{array}{r}75 \\
69 \\
65 \\
171 \\
329 \\
281 \\
201 \\
178 \\
151 \\
131 \\
\\
\end{array}$ & $\begin{array}{r}38 \\
55 \\
117 \\
82 \\
75 \\
1,380 \\
822 \\
262 \\
219 \\
210 \\
680\end{array}$ \\
\hline $\begin{array}{l}\text { Mor } \\
\text { Run } \\
\text { Run }\end{array}$ & $\begin{array}{l}\text { ly mea } \\
\mathrm{f} \text {, in in } \\
\mathrm{f} \text {, in ac }\end{array}$ & $\begin{array}{l}\text { disch } \\
\text { es - } \\
\text {-feet }\end{array}$ & e, i & 01 & eet $p$ & con & & - & $\begin{array}{r}320 \\
1.83 \\
19,650\end{array}$ & $\begin{array}{r}493 \\
2.74 \\
29,350\end{array}$ & $\begin{array}{r}185 \\
1.06 \\
11,400\end{array}$ \\
\hline
\end{tabular}


Gage height, in feet, and discharge, in cubic feet per second, at indicated time, 1965, of Salt Creek rear

\begin{tabular}{|c|c|c|c|c|c|c|c|c|c|c|c|}
\hline Date & Hour & $\begin{array}{l}\text { Gage } \\
\text { height }\end{array}$ & $\begin{array}{c}\text { Dis- } \\
\text { charge }\end{array}$ & Date & Hour & $\begin{array}{c}n, \text { lowa } \\
\text { Gage } \\
\text { height }\end{array}$ & $\begin{array}{c}\text { Dis- } \\
\text { charge }\end{array}$ & Date & Hour & $\begin{array}{c}\text { Gage } \\
\text { height }\end{array}$ & $\begin{array}{c}\text { Dis- } \\
\text { charge }\end{array}$ \\
\hline Mar. 29 & $\begin{array}{l}0000 \\
0200 \\
1200 \\
1400 \\
1600 \\
1700 \\
1800 \\
1900 \\
2100 \\
2400 \\
0400 \\
0800 \\
1300 \\
1400\end{array}$ & $\begin{array}{r}6.13 \\
6.17 \\
5.93 \\
6.06 \\
6.80 \\
7.90 \\
9.40 \\
10.55 \\
11.37 \\
11.12 \\
\\
10.90 \\
11.05 \\
11.09 \\
11.48\end{array}$ & |- & \begin{tabular}{|ll} 
& \\
& \\
Apr. & 1
\end{tabular} & $\begin{array}{l}1500 \\
1600 \\
1800 \\
2000 \\
2200 \\
2400 \\
\\
0700 \\
1400 \\
2000 \\
2400 \\
0300 \\
1200\end{array}$ & $\begin{array}{l}12.42 \\
13.20 \\
13.74 \\
13.86 \\
13.83 \\
13.91 \\
\\
14.62 \\
14.15 \\
14.35 \\
15.06 \\
15.75 \\
14.73\end{array}$ & 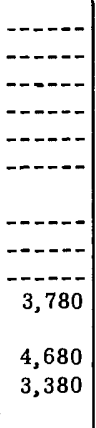 & Apr. 1 & $\begin{array}{l}1800 \\
2400 \\
0600 \\
0800 \\
1000 \\
1100 \\
1200 \\
1300 \\
1400 \\
1600 \\
1800 \\
2300 \\
2400\end{array}$ & $\begin{array}{r}14.13 \\
13.83 \\
\\
13.77 \\
13.64 \\
12.83 \\
12.20 \\
11.43 \\
10.80 \\
10.25 \\
9.55 \\
9.20 \\
9.02 \\
9.03\end{array}$ & $\begin{array}{r}2,660 \\
2,310 \\
\\
2,250 \\
2,100 \\
1,470 \\
1,150 \\
928 \\
776 \\
665 \\
548 \\
492 \\
463 \\
465\end{array}$ \\
\hline
\end{tabular}

(241) 5-4522. Walnut Creek near Hartwick, Iowa

Location.-Lat $41^{\circ} 50^{\prime} 10^{\prime \prime}$, long $92^{\circ} 23^{\prime} 20^{\prime \prime}$, in SE $\frac{1}{4} \mathrm{SW} \frac{1}{4}$ sec.8, T.81 N., R.13 W., on left bank 5 ft upstream from highway bridge, 1.2 miles downstream from North Walnut Creek, 4.0 miles northwest of Hartwick, and 6.5 miles upstream from mouth.

Drainage area. $-70.9 \mathrm{sq} \mathrm{mi}$.

Gage-height record.-Water-stage recorder graph, except Mar. 18-21, Apr. 7-23, May 2-15, 27-31. Datum of gage $786.59 \mathrm{ft}$ above mean sea level, datum of 1929 .

Discharge record. - Stage-discharge relation defined by current-meter measurements below 2,600 cfs and extended to $4,930 \mathrm{cfs}$ on basis of contracted-opening and f'owover-embankment measurement of peak flow.

Maxima.-March-May 1965: Discharge, 2,700 cfs 1930 hours Mar, 30 (gage height, $14.59 \mathrm{ft})$.

1949 to February 1965: Discharge, 4,930 cfs Sept. 3, 1958 (gage height, $15.67 \mathrm{ft}$ ). Flood in June 1947, reached a stage $17.7 \mathrm{ft}$ (discharge not determined).

Cooperation.-Two discharge measurements furnished by Corps of Engineers.

Mean discharge, in cubic feet per second, 1965

\begin{tabular}{|c|c|c|c|c|c|c|c|c|c|c|c|}
\hline Day & March & April & May & Day & March & April & May & Day & March & April & May \\
\hline $\begin{array}{l}1 \ldots \\
2 \ldots \\
3 \ldots \\
4 \\
5 \\
5 \\
6 \\
7 \\
7 \\
8 \\
9 \\
10\end{array}$ & $\begin{array}{r}320 \\
100 \\
75 \\
50 \\
33 \\
20 \\
16 \\
14 \\
13 \\
12\end{array}$ & $\begin{array}{r}423 \\
105 \\
109 \\
104 \\
977 \\
246 \\
53 \\
89 \\
58 \\
46\end{array}$ & $\begin{array}{l}73 \\
66 \\
64 \\
59 \\
58 \\
53 \\
48 \\
42 \\
43 \\
39\end{array}$ & $\begin{array}{l}11 \ldots- \\
12_{--} \\
13_{--} \\
14 \ldots- \\
15_{--} \\
16_{--} \\
17 \ldots- \\
18_{--} \\
19_{--} \\
20_{--}\end{array}$ & $\begin{array}{r}11 \\
17 \\
24 \\
45 \\
86 \\
170 \\
800 \\
140 \\
50 \\
27\end{array}$ & $\begin{array}{l}65 \\
47 \\
35 \\
30 \\
26 \\
23 \\
21 \\
18 \\
17 \\
15\end{array}$ & $\begin{array}{l}36 \\
34 \\
31 \\
31 \\
31 \\
25 \\
26 \\
22 \\
18 \\
18\end{array}$ & \begin{tabular}{l|}
$21_{-}-$ \\
$22--$ \\
$23-$ \\
$24_{-}-$ \\
$25_{-}-$ \\
$26_{-}-$ \\
$27--$ \\
$28_{-}-$ \\
$29_{-}$ \\
$30_{-}$ \\
$31_{-}$
\end{tabular} & $\begin{array}{c}20 \\
16 \\
13 \\
11 \\
10 \\
9.6 \\
16 \\
43 \\
150 \\
845 \\
916\end{array}$ & $\begin{array}{r}14 \\
13 \\
12 \\
798 \\
478 \\
199 \\
159 \\
131 \\
99 \\
86 \\
--\end{array}$ & $\begin{array}{r}17 \\
17 \\
21 \\
19 \\
19 \\
115 \\
40 \\
38 \\
36 \\
36 \\
32\end{array}$ \\
\hline \multicolumn{9}{|c|}{$\begin{array}{l}\text { Monthly mean discharge, in cubic feet per second } \\
\text { Runoff, in inches } \\
\text { Runoff, in acre-feet }\end{array}$} & $\begin{array}{r}131 \\
2.14 \\
8,080\end{array}$ & $\begin{array}{r}150 \\
2.36 \\
8,920\end{array}$ & $\begin{array}{r}38.9 \\
0.63 \\
2.390\end{array}$ \\
\hline
\end{tabular}


Gage height, in feet, and discharge, in cubic feet per second, at indicated time, 1965, of Walmut Creek near Hartwick. lowa

\begin{tabular}{|c|c|c|c|c|c|c|c|c|c|c|c|}
\hline Date & Hour & $\begin{array}{c}\text { Gage } \\
\text { height }\end{array}$ & $\begin{array}{c}\text { Dis- } \\
\text { charge }\end{array}$ & Date & Hour & $\begin{array}{c}\text { Gage } \\
\text { height }\end{array}$ & $\begin{array}{c}\text { Dis- } \\
\text { charge }\end{array}$ & Date & Hour & $\begin{array}{c}\text { Gage } \\
\text { height }\end{array}$ & $\begin{array}{c}\text { Dis- } \\
\text { charge }\end{array}$ \\
\hline \multirow[t]{13}{*}{ Mar. 30} & 0000 & 6.25 & 250 & \multirow[t]{16}{*}{ Mar. 31 } & 0500 & 6.95 & 318 & \multirow[t]{16}{*}{ Apr. 1} & 0100 & 11.10 & 925 \\
\hline & 0800 & 6.25 & 250 & & 0700 & 6.10 & 237 & & 02.00 & 10.00 & 700 \\
\hline & 1230 & 6.05 & 232 & & 1030 & 5.52 & 189 & & 0100 & 8.40 & 478 \\
\hline & 1400 & 6.45 & 268 & & 1200 & 5.75 & 207 & & $0=00$ & 7.74 & 401 \\
\hline & $15 \cup 0$ & 8.50 & 490 & & 1300 & 6.40 & 264 & & 1000 & 6.78 & 301 \\
\hline & 1600 & 11.30 & 975 & & 1400 & 8.70 & 516 & & 1200 & 6.54 & 277 \\
\hline & 1700 & 12.70 & 1,360 & & 1500 & 11.50 & 1,020 & & 1230 & 6.54 & 277 \\
\hline & 1800 & 14.00 & 1,900 & & 1700 & 13.70 & 1,730 & & 1400 & 6.75 & 298 \\
\hline & 1930 & 14.59 & 2,700 & & 1800 & 14.12 & 2,020 & & 1500 & 7.03 & 326 \\
\hline & 2100 & 14.38 & 2,370 & & 1900 & 14.22 & 2,150 & & 1700 & 7.78 & 406 \\
\hline & 2200 & 14.12 & 2,020 & & 2000 & 14.19 & 2,110 & & 1800 & 7.98 & 428 \\
\hline & 2400 & 12.95 & 1,440 & & 2100 & 13.98 & 1,890 & & 1900 & 8.02 & 432 \\
\hline & & & & & 2200 & 13.60 & 1,690 & & 2000 & 7.83 & 411 \\
\hline \multirow[t]{3}{*}{ Mar. 31} & 0100 & 11.30 & 975 & & 2300 & 13.11 & 1,490 & & 2200 & 7.12 & 335 \\
\hline & 0200 & 9.75 & 662 & & 2400 & 12.28 & 1,260 & & 2400 & 6.10 & 237 \\
\hline & 0300 & 8.50 & 490 & & & & & & & & \\
\hline
\end{tabular}

(242) 5-4530. Big Bear Creek at Ladora, Iowa

Location.-Lat $41^{\circ} 45^{\prime} 00^{\prime \prime}$, long $92^{\circ} 11^{\prime} 00^{\prime \prime}$, in SW $\frac{1}{4} \mathrm{SW} \frac{1}{4}$ sec.7, T.80 N., R.11 W., on left bank $10 \mathrm{ft}$ downstream from highway bridge, a quarter of a mile south of Ladora, and $2 \frac{1}{2}$ miles upstream from Little Bear Creek.

Drainage area. $-189 \mathrm{sq} \mathrm{mi}$.

Gage-height record.-Water-stage recorder graph. Datum of gage is $754.94 \mathrm{ft}$ above mean sea level, datum of 1929.

Discharge record. - Stage-discharge relation defined by current-meter measurements below 8,000 cfs. Backwater from ice Mar. 1-30.

Maxima.-March-May 1965: Discharge, 3,580 cfs 0100 hours Apr. 1 (gage height, $11.34 \mathrm{ft}$ ); gage height, $11.77 \mathrm{ft} 2200$ hours Mar. 30 (backwater from ice).

1945 to February 1965: Discharge, 10,500 cfs Mar. 30, 1960 (gage height, $14.60 \mathrm{ft}$ ).

Cooperation.-One discharge measurement furnished by Corps of Engineers.

Mean discharge, in cubli feet per second, 1965

\begin{tabular}{|c|c|c|c|c|c|c|c|c|c|c|c|}
\hline Day & March & Apri1 & May & Day & March & April & May & Day & March & April & May \\
\hline & 1,100 & 1,590 & 180 & $11 \ldots$ & 56 & 240 & 73 & 21 & 60 & 48 & 45 \\
\hline $2 \ldots$ & 600 & 421 & 153 & $12 \ldots$ & 95 & 137 & 72 & 22. & 50 & 44 & 44 \\
\hline $3 \ldots$ & 370 & 250 & 133 & $13 \ldots$ & 150 & 104 & 66 & 23 & 40 & 43 & 82 \\
\hline 4. & 150 & 324 & 123 & $14 \ldots$ & 240 & 94 & 65 & 24 & 33 & 1,110 & 58 \\
\hline $5 \ldots$ & 100 & 1,730 & 127 & $15 \ldots$ & 430 & 84 & 72 & 25 & 30 & 1,240 & 54 \\
\hline 6 & 84 & 1,160 & 112 & $16 \ldots$ & 580 & 72 & 68 & $26 \ldots$ & 29 & 594 & 110 \\
\hline 7. & 72 & 214 & 99 & $17 \ldots$ & 1,600 & 67 & 57 & $27-$ & 60 & 361 & 100 \\
\hline 3. & 66 & 226 & 96 & $18 \ldots$ & 300 & 61 & 54 & $28-1$ & 400 & 310 & 64 \\
\hline $9_{-}$ & 62 & 204 & 100 & $19=$ & 150 & 55 & 48 & $29_{-}$ & 640 & 248 & 63 \\
\hline \multirow[t]{2}{*}{$10_{-}-1$} & 58 & 183 & 81 & $20 \ldots$ & 90 & 53 & 45 & $30 \ldots$ & 1,650 & 210 & 67 \\
\hline & & & & & & & & 31 & 2,210 & $-\ldots$ & 126 \\
\hline \multicolumn{9}{|c|}{ Monthly mean discharge, in cubic feet per second } & 373 & 383 & 85.1 \\
\hline \multicolumn{9}{|c|}{ Runoff, in inches } & 2.27 & 2.26 & 0.52 \\
\hline \multicolumn{9}{|c|}{ Runoff, in acre-feet } & 22,920 & 22,760 & 5,230 \\
\hline
\end{tabular}


MARCH $\longrightarrow$ MAY, UPPER MISSISSIPPI RIVER BASIN

Gage height, in feet, and discharge, in cubic feet per second, at indicated time, 1965, of Big Bear Creek at Ladora, lowa

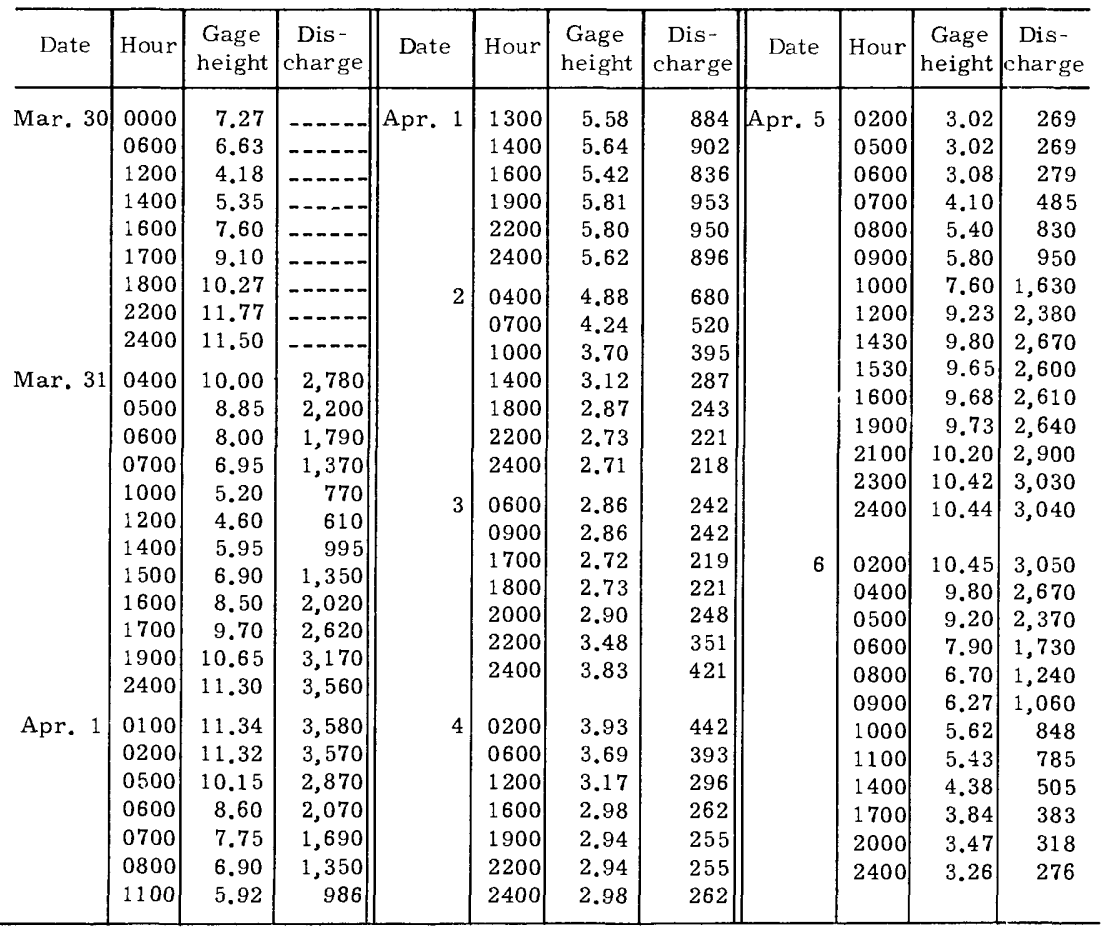

\section{(243) 5-4531. Iowa River at Marengo, Iowa}

Location.-Lat $41^{\circ} 48^{\prime} 35^{\prime \prime}$, long $92^{\circ} 04^{\prime} 20^{\prime \prime}$, in NW $\frac{1}{4} \mathrm{SE} \frac{1}{4}$ sec.24, T.81 N., R.11 W., on right bank at downstream side of abandoned county highway bridge, 0.7 mile downstream from Bear Creek, 0.8 mile north of Marengo, and 4.9 miles upstream from Hilton Creek, and at mile 139.4 .

Drainage area. $-2,794 \mathrm{sq} \mathrm{mi}$.

Gage-height record.-Water-stage recorder graph, except Mar. 3, 4, 18. Datum of gage is $720.52 \mathrm{ft}$ above mean sea level, datum of 1929 .

Discharge record.-Stage-discharge relation defined by current-meter measurements below 31,000 cfs. Backwater from ice Mar. 1-31.

Maxima.-March-May 1965: Discharge, 19,900 cfs 1000 hours Apr. 7 (gage height, $17.84 \mathrm{ft})$.

1956 to February 1965: Discharge, 30,800 cfs Mar. 31, 1960 (gage height, $19.21 \mathrm{ft}$ ).

Cooperation.-Six discharge measurements furnished by Corps of Engineers. 
Mean discharge, in cubic feet per second, 1965, of Iowa River at Marengc, Iowa

\begin{tabular}{|c|c|c|c|c|c|c|c|c|c|c|c|}
\hline Day & March & April & May & Day & March & April & May & Day & March & April & May \\
\hline 3 & $\begin{array}{l}3,300 \\
5,000 \\
4,800 \\
4,500 \\
4,200 \\
4,000 \\
4,300 \\
4,700 \\
5,000 \\
3,500\end{array}$ & $\begin{array}{l}12,200 \\
13,100 \\
12,200 \\
14,100 \\
15,000 \\
17,500 \\
19,300 \\
19,300 \\
18,700 \\
16,400\end{array}$ & $\begin{array}{l}3,100 \\
2,810 \\
2,480 \\
2,280 \\
2,460 \\
2,350 \\
1,990 \\
1,840 \\
1,770 \\
1,690\end{array}$ & $\mid \begin{array}{l}11 \ldots \\
12 \ldots \\
13 \ldots \\
14 \ldots \\
15 \ldots \\
16 \ldots \\
17 \ldots \\
18 \ldots \\
19 \ldots \\
20 \ldots\end{array}$ & $\begin{array}{l}2,500 \\
2,100 \\
2,500 \\
2,100 \\
2,300 \\
3,000 \\
5,000 \\
6,200 \\
5,000 \\
3,600\end{array}$ & $\begin{array}{r}15,800 \\
15,200 \\
14,100 \\
12,000 \\
10,700 \\
9,910 \\
9,170 \\
8,430 \\
7,720 \\
7,080\end{array}$ & $\begin{array}{l}1,650 \\
1,580 \\
1,420 \\
1,360 \\
1,320 \\
1,280 \\
1,200 \\
1,120 \\
1,040 \\
1,020\end{array}$ & $\begin{array}{l}21 .- \\
22-- \\
23-- \\
24-- \\
25-- \\
26 .- \\
27-- \\
28- \\
29-- \\
30- \\
31--\end{array}$ & $\begin{array}{r}3,000 \\
2,5 C 0 \\
2,2 C 0 \\
1,9 C 0 \\
1,8 C 0 \\
1,7 C 0 \\
1,6 C 0 \\
2,3 C 0 \\
3,5 C 0 \\
6,0<0 \\
10,0 C 0\end{array}$ & $\begin{array}{r}6,460 \\
5,830 \\
4,680 \\
6,770 \\
7,540 \\
7,030 \\
6,240 \\
5,430 \\
4,200 \\
3,490 \\
-\end{array}$ & $\begin{array}{l}1,040 \\
1,040 \\
1,360 \\
1,460 \\
1,370 \\
1,870 \\
3,680 \\
3,970 \\
3,920 \\
4,010 \\
4,140\end{array}$ \\
\hline \multicolumn{9}{|c|}{$\begin{array}{l}\text { Monthly mean discharge, in cubic feet per second } \\
\text { Runoff, in inches } \\
\text { Runoff, in acre-feet }\end{array}$} & $\begin{array}{r}3,6 \varepsilon 1 \\
1.52 \\
226,3 \subset 0\end{array}$ & $\begin{array}{r}10,850 \\
4.33 \\
645,800\end{array}$ & $\begin{array}{r}2,052 \\
0.85 \\
126,200\end{array}$ \\
\hline
\end{tabular}

Gage hetght, in feet, and discharge, in cubic feet per second, at indicated time, 1965

\begin{tabular}{c|c|c|c||r|r|r|r||r|r|r|r}
\hline Date & Hour & $\begin{array}{c}\text { Gage } \\
\text { height }\end{array}$ & $\begin{array}{c}\text { Dis- } \\
\text { charge }\end{array}$ & Date & Hour & $\begin{array}{c}\text { Gage } \\
\text { height }\end{array}$ & $\begin{array}{c}\text { Dis- } \\
\text { charge }\end{array}$ & Date & Hour & $\begin{array}{c}\text { Gage } \\
\text { height }\end{array}$ & $\begin{array}{c}\text { Dis- } \\
\text { charge }\end{array}$ \\
\hline Apr. 3 & 0000 & 15.75 & 11,200 & Apr. 4 & 1200 & 16.57 & 14,200 & Apr. 6 & 0100 & 17.68 & 19,000 \\
& 0300 & 15.68 & 11,000 & & 1800 & 16.54 & 14,100 & & 1500 & 17.17 & 16,200 \\
& 0600 & 15.72 & 11,200 & & 2400 & 16.48 & 13,800 & & 2400 & 17.53 & 18,100 \\
& 1200 & 15.93 & 11,800 & & & & & & & & \\
& 1800 & 16.30 & 13,300 & 5 & 1000 & 16.56 & 13,500 & 7 & 1000 & 17.84 & 19,900 \\
& 2400 & 16.48 & 14,000 & & 2400 & 17.60 & 18,800 & & 2400 & 17.67 & 18,900 \\
\hline
\end{tabular}

(244) 5-4535.1 Coralville Reservoir near Coralville, Iowa

Location.-Lat $41^{\circ} 43^{\prime} 20^{\prime \prime}$, long $91^{\circ} 31^{\prime} 30^{\prime \prime}$, in SW $\frac{1}{4} \mathrm{NE} \frac{1}{4}$ sec.22, T.80 N., R. 6 W., at outlet works at left end of Coralville Dam, 4.0 miles northeast of Coralville and at mile 83.3.

Drainage area.- $-3,115 \mathrm{sq} \mathrm{mi}$.

Gage-height record.-Water-stage recorder graph. Datum of gage is at mean sea level, datum of 1929 (levels by Corps of Engineers).

Maxima.-March-May 1965: Contents, 403,600 acre-ft 1200 hours May 5 (elevation, $708.50 \mathrm{ft}$.

1958 to February 1965: Contents, 368,000 acre-ft Apr. 8, 1960 (elevation, $706.77 \mathrm{ft}$ ).

Remarks.-Reservoir is formed by earth-fill rock-faced dam; comp'eted in 1957. Storage began in September 1958. Capacity, 489,000 acre-ft between elevations 650 (sill of outlet conduit) and $712 \mathrm{ft}$ (crest of spillway). No dead storc ge. Figures given herein represent total contents based on a flat pool condition. Water is stored for flood control and conservation. Records are furnished by Corps of Engineers. 
Elevation, in feet, and contents, in acre-feet, 1965, of Coralville Reservoir near Coralville, Iowa

\begin{tabular}{|c|c|c|c|c|c|c|c|}
\hline Date & Time & Elevation & Contents & Date & Time & Elevation & Contents \\
\hline Jan. $31 \ldots$ & 2400 & 673.92 & 27,600 & Apr. $30 \ldots$ & 2400 & 707.33 & 379,400 \\
\hline Feb. $28 \ldots$ & 2400 & 671.12 & 19,500 & May $5 \ldots$ & 1200 & 708.50 & 403,600 \\
\hline Mar. $31 \ldots$ & 2400 & 669.08 & 15,300 & May $31 \ldots$ & 2400 & 699.58 & 237,300 \\
\hline
\end{tabular}

(245) 5-4540. Rapid Creek near Iowa City, Iowa

Location.-Lat $41^{\circ} 42^{\prime} 00^{\prime \prime}$, long $91^{\circ} 29^{\prime} 05^{\prime \prime}$, in $\mathrm{NE}_{4} \frac{1}{4} \mathrm{NE} \frac{1}{4}$ sec.36, T.80 N., R.6 W., on left bank $80 \mathrm{ft}$ upstream from bridge on State Highway 1 (formerly 261), 3 miles northeast of lowa City, and 4.0 miles upstream from mouth.

Drainage area. $-24.6 \mathrm{sq} \mathrm{mi}$.

Gage-height record.-Water-stage recorder graph. Datum of gage is $673.72 \mathrm{ft}$ ahove mean sea level, datum of 1929 .

Discharge record.-Stage-discharge relation defined by current-meter measurements below 2,500 cfs and extended to $6,1000 \mathrm{cfs}$ on basis of contracted opening measurement of peak flow. Backwater from ice Mar. 18-27.

Maxima.-March-May 1965: Discharge, 6,100 cfs 0800 hours May 23 (gage height, $14.10 \mathrm{ft}$ ).

1937 to February 1965: Discharge, 5, 200 cfs July 14, 1962 (gage height, 13.64 ft).

Mean discharge, in cublc feet per second, 1965

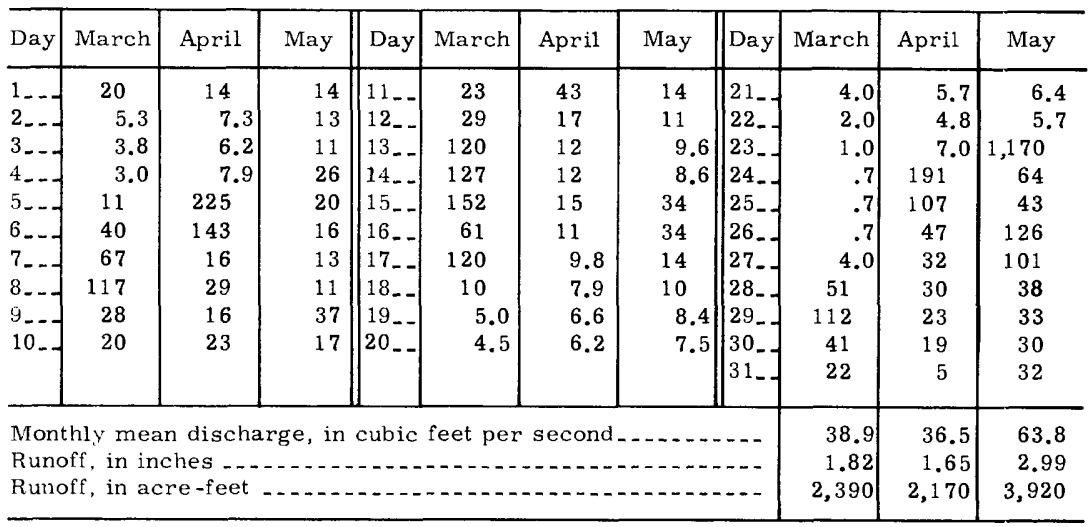


Gage height, in feet, and discharge, in cubic feet per second, at indicated time, 1965, of Rapid Creek near

\begin{tabular}{|c|c|c|c|c|c|c|c|c|c|c|c|}
\hline Date & Hour & $\begin{array}{c}\text { Gage } \\
\text { height }\end{array}$ & $\begin{array}{c}\text { Dis - } \\
\text { charge }\end{array}$ & Date & Hour & $\begin{array}{l}\text { Gage } \\
\text { height }\end{array}$ & $\begin{array}{c}\text { Dis- } \\
\text { charge }\end{array}$ & Date & Hcur & $\begin{array}{c}\text { Gage } \\
\text { height }\end{array}$ & $\begin{array}{c}\text { Dis- } \\
\text { charge }\end{array}$ \\
\hline Apr. 5 & $\begin{array}{l}0000 \\
0500 \\
0700 \\
0800 \\
0900 \\
1100 \\
1300 \\
1500 \\
1900 \\
2000 \\
2030 \\
2100 \\
2200 \\
2400 \\
0200\end{array}$ & $\begin{array}{l}3.52 \\
3.51 \\
3.89 \\
5.03 \\
6.24 \\
7.32 \\
7.35 \\
5.89 \\
5.05 \\
5.87 \\
7.03 \\
8.87 \\
9.67 \\
9.80 \\
9.48\end{array}$ & $\begin{array}{r}7.0 \\
6.8 \\
17.5 \\
104 \\
276 \\
401 \\
403 \\
224 \\
106 \\
220 \\
373 \\
543 \\
688 \\
725 \\
641\end{array}$ & Apr. 6 & $\begin{array}{l}0300 \\
0330 \\
0400 \\
0500 \\
0800 \\
1300 \\
2400 \\
2400 \\
0100 \\
0200 \\
0300 \\
0400\end{array}$ & $\begin{array}{l}8.52 \\
7.23 \\
6.25 \\
5.33 \\
4.65 \\
4.33 \\
4.03 \\
\\
3.43 \\
3.53 \\
4.00 \\
6.00 \\
8.18\end{array}$ & $\begin{array}{c}500 \\
393 \\
278 \\
140 \\
64.5 \\
39.1 \\
22.9 \\
\\
5.3 \\
\\
7.3 \\
21.6 \\
240 \\
466\end{array}$ & May 23 & $\begin{array}{l}0500 \\
0 € 00 \\
0700 \\
0 \varepsilon 00 \\
0 £ 00 \\
1000 \\
1100 \\
1200 \\
1 乏 00 \\
1400 \\
1500 \\
1600 \\
1900 \\
2 \Delta 00\end{array}$ & $\begin{array}{r}11.15 \\
11.88 \\
13.50 \\
14.10 \\
13.36 \\
12.29 \\
11.59 \\
10.75 \\
9.30 \\
7.77 \\
6.75 \\
5.85 \\
5.26 \\
4.95\end{array}$ & $\begin{array}{r}1,410 \\
2,130 \\
4,900 \\
6,100 \\
4,620 \\
2,680 \\
1,820 \\
1,110 \\
605 \\
433 \\
342 \\
218 \\
131 \\
94.5\end{array}$ \\
\hline
\end{tabular}

\section{(246) 5-4543. Clear Creek near Coralville, Iowa}

Location.-Lat $41^{\circ} 40^{\prime} 35^{\prime \prime}$, long $91^{\circ} 35^{\prime} 55^{\prime \prime}$, in NW $\frac{1}{4} \mathrm{SW} \frac{1}{4}$ sec.6, T.79 N., R.6 W., on left bank about $50 \mathrm{ft}$ upstream from highway bridge, 1.2 miles west of Coralville, and 2.2 miles upstream from mouth.

Drainage area. $-98.1 \mathrm{sq} \mathrm{mi}$.

Gage-height record.-Water-stage recorder graph, except Mar. 19-24, Apr. 1-4, 9, 10, 12-23, Apr. 29 to May 5, May 29-31. Datum of gage is $648.43 \mathrm{ft}$ above mean sea level, datum of 1929 (levels by Corps of Engineers).

Discharge record.-Stage-discharge relation defined by current-meter measurements below 5,600 cfs. Backwater from ice Mar. 1-15, 18-28.

Maxima.-March-May 1965: Discharge, 4,060 cfs 2400 hours Apr. 24 (gage height, $12.50 \mathrm{ft}$ ).

1952 to February 1965: Discharge, 5,390 cfs May 29, 1962 (gage height, $13.31 \mathrm{ft}$ ).

Cooperation.-Two discharge measurements furnished by Corps of Engineers.

Mean discharge, itl cubic feet per second, 1965

\begin{tabular}{|c|c|c|c|c|c|c|c|c|c|c|c|}
\hline Day & March & April & May & Day & March & April & May & Day & March & April & May \\
\hline 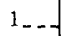 & 280 & 95 & 115 & $11 \ldots$ & 85 & 237 & 60 & $21 \ldots$ & 42 & 30 & 30 \\
\hline 2. & 200 & 85 & 110 & $12 \ldots$ & 60 & 125 & 54 & $22 \ldots$ & 36 & 27 & 28 \\
\hline 3. & 80 & 80 & 105 & $13 \ldots$ & 110 & 95 & 48 & $23 \ldots$ & 31 & 25 & 500 \\
\hline 4 & 55 & 75 & 131 & $14 \ldots$ & 150 & 75 & 45 & $24 \ldots$ & 27 & 1,520 & 109 \\
\hline 5. & 38 & 616 & 120 & $15 \ldots$ & 240 & 60 & 77 & $25 \ldots$ & 25 & 1,990 & 83 \\
\hline 6. & 100 & 1,180 & 89 & $16 \ldots$ & 396 & 54 & 96 & 26. & 24 & 483 & 158 \\
\hline 7. & 150 & 153 & 76 & $17 .-$ & 552 & 46 & 51 & 27. & 27 & 229 & 419 \\
\hline 8 & 210 & 181 & 67 & $18 \ldots$ & 200 & 40 & 44 & $28 \ldots$ & 150 & 193 & 113 \\
\hline 9 & 160 & 125 & 152 & 19. & 74 & 37 & 36 & $29 \ldots$ & 415 & 140 & 92 \\
\hline $10_{2}$ & 110 & 120 & 74 & $20 \ldots$ & 54 & 33 & 32 & 30. & 309 & 120 & 85 \\
\hline & & & & & & & & 31. & 173 & $\ldots-. .-$ & 82 \\
\hline \multirow{3}{*}{\multicolumn{9}{|c|}{$\begin{array}{l}\text { Monthly mean discharge, in cubic feet per second } \\
\text { Runoff, in inches } \\
\text { Runoff, in acre-feet }\end{array}$}} & 147 & 276 & 106 \\
\hline & & & & & & & & & 1.73 & 3.13 & 1.24 \\
\hline & & & & & & & & & 9,050 & 16,400 & 6,510 \\
\hline
\end{tabular}


Gage height, in feet, and discharge, in cubic feet per second, at indicated time, 1965, of Clear Creek near

\begin{tabular}{|c|c|c|c|c|c|c|c|c|c|c|c|}
\hline \multicolumn{12}{|c|}{ Coralville, lowa } \\
\hline Date & Hour & $\begin{array}{l}\text { Gage } \\
\text { height }\end{array}$ & $\begin{array}{c}\text { Dis - } \\
\text { charge }\end{array}$ & Date & Hour & $\begin{array}{l}\text { Gage } \\
\text { height }\end{array}$ & $\begin{array}{c}\text { Dis - } \\
\text { charge }\end{array}$ & Date & Hour & $\begin{array}{c}\text { Gage } \\
\text { height }\end{array}$ & $\begin{array}{c}\text { Dis- } \\
\text { charge }\end{array}$ \\
\hline \multirow[t]{11}{*}{ Apr. 24} & 0000 & 3.15 & 64 & \multirow[t]{11}{*}{ Apr. 25} & 0100 & 12.45 & 3,960 & \multirow[t]{11}{*}{ Apr. 26} & 0300 & $8.3 \varepsilon$ & 812 \\
\hline & 0200 & 4.27 & 147 & & 0300 & 12.12 & 3,280 & & 0500 & 7.42 & 630 \\
\hline & 0400 & 5.15 & 249 & & 0800 & 11.40 & 2,040 & & 0700 & 6.82 & 518 \\
\hline & 0600 & 5.87 & 358 & & 0900 & 11.31 & 1,900 & & 0900 & $6.4 \mathrm{C}$ & 444 \\
\hline & 0800 & 7.65 & 669 & & 1000 & 11.26 & 1,840 & & 1000 & $6.2^{\circ}$ & 426 \\
\hline & 1000 & 9.10 & 968 & & 1300 & 10.99 & 1,510 & & 1200 & 6.14 & 400 \\
\hline & 1200 & 10.30 & 1,240 & & 2000 & 10.36 & 1,260 & & 1500 & 5.84 & 351 \\
\hline & 1400 & 11.12 & 1,600 & & 2100 & 10.28 & 1,240 & & 1900 & 5.55 & 309 \\
\hline & 1800 & 11.57 & 2,260 & & 2200 & 10.15 & 1,200 & & 2400 & 5.35 & 281 \\
\hline & 2200 & 12.37 & 3,790 & & 2400 & 9.68 & 1,090 & & & & \\
\hline & 2400 & 12.50 & 4,060 & & & & & & & & \\
\hline
\end{tabular}

(247) 5-4545. Iowa River at Iowa City, Iowa

Location.-Lat $41^{\circ} 39^{\prime} 25^{\prime \prime}$, long $91^{\circ} 32^{\prime} 25^{\prime \prime}$, in $\mathrm{SE} \frac{1}{4} \mathrm{SE} \frac{1}{4}$ sec.9, T.79 N., R.6 W., on right bank $25 \mathrm{ft}$ downstream from Hydraulics Laboratory of State University of Iowa in Iowa City, $175 \mathrm{ft}$ downstream from University Dam, 0.9 mile upstream from Ralston Creek, 3.6 miles downstream from Clear Creek, and at mile 74.2.

Drainage area. $-3,271 \mathrm{sq} \mathrm{mi}$.

Gage-height record.-Water-stage recorder graph Mar. 1 to Apr. 21 and digital recorder tape punched at 15 minute intervals Apr. 22 to May 31. Datum of gage is $39.00 \mathrm{ft}$ above Iowa City datum, and $627.27 \mathrm{ft}$ above mean sea level, datum of 1929 .

Discharge record.-Stage-discharge relation defined by current-meter measurements.

Maxima.--March-May 1965: Discharge, 11,100 cfs 0430 hours Apr. 24 (gage həight, $11.74 \mathrm{ft}$.

1903 to February 1965: Discharge, 42,500 cfs June 8, 1918 (gage height, 19.6 ft., from graph based on gage readings, at site $2,600 \mathrm{ft}$ downstream at datum $0.2 \mathrm{ft}$ higher).

Flood of July 17, 1881, reached a stage of $21.1 \mathrm{ft}$, from flood marks of site 2,600 ft downstream at datum $0.2 \mathrm{ft}$ higher, (discharge, 51,000 cfs). Maximum stage known since at least 1850 , about $3 \mathrm{ft}$ higher than that of July 17,1881 , occurred in June 1851 (discharge, 70,000 cfs estimated).

Remarks: Flow regulated by Coralville Reservoir (capacity, 489,000 acre-ft) since Sept. 17, 1958.

Mean discharge, in cubic feet per second, 1965

\begin{tabular}{|c|c|c|c|c|c|c|c|c|c|c|c|}
\hline Day & March & April & May & Day & March & April & May & Day & March & April & May \\
\hline & 2,540 & 4,860 & 281 & $11 \ldots$ & 4,210 & 3,660 & 5,830 & 21. & 3,380 & 9,820 & 5,880 \\
\hline$z_{-}$ & 3,680 & 5,260 & 258 & $12 \ldots$ & 5,240 & 1,350 & 5,800 & 22. & 3,710 & 9,810 & 5,970 \\
\hline & 4,150 & 6,730 & 235 & $13_{-}$ & 5,690 & 1,260 & 5,830 & 23 & 3,430 & 9,760 & 6,640 \\
\hline & 4,180 & 8,380 & 289 & 14 & 5,630 & 3,820 & 5,900 & 24 & 2,050 & 6,080 & 4,270 \\
\hline J. & 3,460 & 9,360 & 1,380 & $15 \ldots$ & 5,460 & 9,470 & 5,990 & 25. & 1,360 & 2,850 & 6,060 \\
\hline 6. & 2,540 & 9,400 & 4,060 & $16 \ldots$ & 5,390 & 9,880 & 6,050 & 26. & 1,260 & 857 & 6,200 \\
\hline 7. & 1,470 & 7,630 & 5,830 & $17 \ldots$ & 5,550 & 9,900 & 5,860 & 27. & 1,140 & 462 & 6,710 \\
\hline 8. & 1,750 & 8,310 & 5,860 & $18 \ldots$ & 4,560 & 9,900 & 5,810 & 28. & 1,360 & 412 & 6,090 \\
\hline 9 & 1,540 & 9,230 & 6,070 & $19 \ldots$ & 3,230 & 9,890 & 5,760 & 29. & 2,300 & 349 & 6,040 \\
\hline 10. & 2,150 & 8,890 & 5,890 & 20 & 3,300 & 9,830 & 5,800 & $30-$ & 4,620 & 305 & 6,040 \\
\hline & & & & & & & & 3 & 4,700 & $\ldots$ & 6,000 \\
\hline \multirow{2}{*}{\multicolumn{9}{|c|}{$\begin{array}{l}\text { Monthly mean discharge, in cubic feet per second } \\
\text { Runoff, in acre-feet }\end{array}$}} & 3,388 & 6,257 & 4,990 \\
\hline & & & & & & & & & 208,300 & 372,300 & 306,800 \\
\hline
\end{tabular}


Gage height, in feet, and discharge, in cubic feet per second, at indicated time, 1965, of Iowa River at Iowa City, lowa

\begin{tabular}{|c|c|c|c|c|c|c|c|c|c|c|c|}
\hline Date & Hour & $\begin{array}{c}\text { Gage } \\
\text { height }\end{array}$ & $\begin{array}{c}\text { Dis- } \\
\text { charge }\end{array}$ & Date & Hour & $\begin{array}{c}\text { Gage } \\
\text { height }\end{array}$ & $\begin{array}{c}\text { Dis- } \\
\text { charge }\end{array}$ & Date & Hour & $\begin{array}{c}\text { Gage } \\
\text { height }\end{array}$ & $\begin{array}{c}\text { Dis- } \\
\text { charge }\end{array}$ \\
\hline Apr. 24 & $\begin{array}{l}0000 \\
0430 \\
0700\end{array}$ & $\begin{array}{r}10.64 \\
11.74 \\
9.70\end{array}$ & $\begin{array}{r}9,860 \\
11,100 \\
8,770\end{array}$ & Apr. 24 & $\begin{array}{l}0900 \\
1200 \\
1800\end{array}$ & $\begin{array}{l}7.31 \\
5.12 \\
4.10\end{array}$ & $\begin{array}{l}6,200 \\
4,170 \\
3,320\end{array}$ & Apr. 24 & $\begin{array}{l}2000 \\
2400\end{array}$ & $\begin{array}{l}4.10 \\
4.62\end{array}$ & $\begin{array}{l}3,320 \\
3,740\end{array}$ \\
\hline
\end{tabular}

(248) 5-4550. Ralston Creek at Iowa City, Iowa

Location.-Lat $41^{\circ} 39^{\prime} 50^{\prime \prime}$, long $91^{\circ} 30^{\prime} 45^{\prime \prime}$, in $\mathrm{SE} \frac{1}{4} \mathrm{NW} \frac{1}{4}$ sec.11, T.79 N., P.6 W., on left bank $10 \mathrm{ft}$ upstream from bridge on Rochester Avenue (formerly State Highway 1), near east edge of Iowa City, and 2.2 miles upstream from mouth.

Drainage area. $-3.01 \mathrm{sq} \mathrm{mi}$.

Gage-height record.-Water-stage recorder graph except Apr, 9-12. Datum of gage is $662.53 \mathrm{ft}$ above mean sea level, datum of 1929 (State University of Ic wa bench mark).

Discharge record.-Stage-discharge relation defined by current-meter measurements. Backwater from ice Mar. 1-29.

Maxima.-March-May 1965: Discharge, 198 cfs 0330 hours Apr. 24 (gage height, $4.25 \mathrm{ft}$ ).

1924 to February 1965: Discharge, 1,690 cfs July 18, 1956 (gage l eight, $9.06 \mathrm{ft}$ ).

Mean discharge, in cubic feet per second, 1965

\begin{tabular}{|c|c|c|c|c|c|c|c|c|c|c|c|}
\hline Day & March & April & May & Day & March & April & May & Day & March & April & May \\
\hline $\begin{array}{l}1 \ldots \\
2 \ldots- \\
3 \ldots \\
4 \ldots \\
5 \ldots \\
6 \ldots \\
7 \ldots \\
8 \ldots \\
9 \ldots \\
10 .\end{array}$ & $\begin{array}{l}1.9 \\
.70 \\
.28 \\
.20 \\
3.7 \\
6.0 \\
5.0 \\
6.0 \\
2.5 \\
3.0\end{array}$ & $\begin{array}{c}1.5 \\
.76 \\
.79 \\
.99 \\
20 \\
5.1 \\
1.8 \\
4.8 \\
1.9 \\
2.2\end{array}$ & $\begin{array}{l}1.7 \\
1.3 \\
1.1 \\
5.6 \\
3.1 \\
1.9 \\
1.5 \\
1.3 \\
6.5 \\
1.9\end{array}$ & $\begin{array}{l}11 .- \\
12 \_- \\
13 .- \\
14 \ldots-- \\
15_{--} \\
16_{--} \\
17-- \\
18_{--} \\
19-- \\
20--\end{array}$ & $\begin{array}{l}2.0 \\
2.0 \\
2.0 \\
2.0 \\
2.6 \\
3.0 \\
4.4 \\
1.5 \\
.39 \\
.30\end{array}$ & $\begin{array}{l}3.0 \\
1.5 \\
1.2 \\
2.4 \\
3.1 \\
1.6 \\
1.3 \\
1.0 \\
.89 \\
.76\end{array}$ & $\begin{array}{l}1.4 \\
1.1 \\
.96 \\
.85 \\
8.0 \\
2.8 \\
1.5 \\
1.1 \\
.82 \\
.73\end{array}$ & $\begin{array}{l}21-- \\
22-- \\
23-- \\
24- \\
25-- \\
26-- \\
27-- \\
28 .- \\
29_{-}- \\
30_{-} \\
31_{-}-\end{array}$ & $\begin{array}{r}0.20 \\
.16 \\
.13 \\
.10 \\
.09 \\
.10 \\
.34 \\
3.0 \\
6.9 \\
4.9 \\
3.1\end{array}$ & $\begin{array}{c}0.67 \\
.59 \\
.70 \\
52 \\
14 \\
5.6 \\
4.5 \\
3.7 \\
2.7 \\
2.1 \\
- \\
\end{array}$ & $\begin{array}{c}0.67 \\
.64 \\
12 \\
2.4 \\
1.7 \\
14 \\
5.6 \\
3.3 \\
3.2 \\
2.5 \\
4.6\end{array}$ \\
\hline \multicolumn{9}{|c|}{$\begin{array}{l}\text { Monthly mean discharge, in cubic feet per second } \\
\text { Runoff, in inches } \\
\text { Runoff, in acre-feet }\end{array}$} & $\begin{array}{r}2.21 \\
0.85 \\
136\end{array}$ & $\begin{array}{r}4.77 \\
1.77 \\
284\end{array}$ & $\begin{array}{r}3.09 \\
1.18 \\
190\end{array}$ \\
\hline
\end{tabular}


Gage height, in feet, and discharge, in cubic feet per second, at indicated time 1965, of Ralston Croek at

\begin{tabular}{|c|c|c|c|c|c|c|c|c|c|c|c|}
\hline Date & Hour & $\begin{array}{l}\text { Gage } \\
\text { height }\end{array}$ & $\begin{array}{c}\text { Dis- } \\
\text { charge }\end{array}$ & Date & Hour & $\begin{array}{l}\text { Gage } \\
\text { height }\end{array}$ & $\begin{array}{c}\text { Dis- } \\
\text { charge }\end{array}$ & Date & Hour & $\begin{array}{c}\text { Gage } \\
\text { height }\end{array}$ & $\begin{array}{l}\text { Dis- } \\
\text { charge }\end{array}$ \\
\hline Apr. 23 & $\begin{array}{l}0000 \\
0900 \\
1600 \\
1700 \\
1900 \\
2200 \\
2300 \\
2400\end{array}$ & $\begin{array}{l}1.90 \\
1.94 \\
1.91 \\
2.13 \\
1.91 \\
1.91 \\
2.18 \\
2.36\end{array}$ & $\begin{array}{r}0.5 \\
.6 \\
.6 \\
1.3 \\
.6 \\
.6 \\
1.5 \\
2.5\end{array}$ & Apr. 24 & $\begin{array}{l}0100 \\
0200 \\
0300 \\
0330 \\
0500 \\
0800 \\
1100\end{array}$ & $\begin{array}{l}3.10 \\
3.56 \\
4.15 \\
4.25 \\
3.57 \\
3.00 \\
2.87\end{array}$ & $\begin{array}{c}35.0 \\
94.8 \\
182 \\
198 \\
96.1 \\
24.0 \\
14.9\end{array}$ & Apr. 24 & $\begin{array}{l}1200 \\
1330 \\
1500 \\
1800 \\
2100 \\
2400\end{array}$ & $\begin{array}{l}3.05 \\
3.63 \\
3.33 \\
2.94 \\
2.83 \\
2.90\end{array}$ & $\begin{array}{l}29.5 \\
104 \\
64.9 \\
19.5 \\
12.9 \\
16.5\end{array}$ \\
\hline
\end{tabular}

(249) 5-4550.1 South Branch Ralston Creek at Iowa City, Iowa

Location.-Lat $41^{\circ} 38^{\prime} 50^{\prime \prime}$, long $91^{\circ} 30^{\prime} 30^{\prime \prime}$, in SW $\frac{1}{4} \mathrm{NE} \frac{1}{4}$ sec.14, T.79 N., R.6 W., on right bank $60 \mathrm{ft}$ downstream from Muscatine Avenue bridge in Iowa City and $1.3 \mathrm{miles}$ upstream from confluence with Ralston Creek.

Drainage area.- $-3.20 \mathrm{sq} \mathrm{mi}$, approximately.

Gage-height record.-Water-stage recorder graph, except Mar. 1-15. Several outside staff gage readings used for this period. Datum of gage is $678.03 \mathrm{ft}$ above mean sea level, datum of 1929 .

Discharge record.- Stage-discharge relation defined by current-meter measurements below $220 \mathrm{cfs}$ and by culvert computation at $780 \mathrm{cfs}$. Backwater from ice Mar. 1, 13, $16-25,28-30$.

Maxima.-March-May 1965: Discharge, 652 cfs 0330 hours Apr. 24 (gage height, $6.57 \mathrm{ft}$ ).

1963 to February 1965: Discharge, 126 cfs June 22, 1964 (gage height, $3.47 \mathrm{ft}$ ).

Mean discharge, in cubtc feet per second, 1965

\begin{tabular}{|c|c|c|c|c|c|c|c|c|c|c|c|}
\hline Day & March & April & May & Day & March & April & May & Day & March & April & May \\
\hline 1. & 1.0 & 1.6 & 2.3 & $11 \ldots$ & 2.0 & 7.4 & 2.0 & 21. & 0.4 & 1.1 & 1.0 \\
\hline 2 & .5 & .9 & 1.8 & $12 \ldots$ & 2.0 & 3.4 & 1.6 & $22 \ldots$ & .3 & .9 & .8 \\
\hline 3. & .3 & .8 & 1.8 & $13 \ldots$ & 2.0 & 2.4 & 1.5 & $23 \ldots$ & .3 & .9 & 25 \\
\hline 4. & .2 & .9 & 13 & $14 \ldots$ & 2.0 & 3.2 & 1.3 & $24 \ldots$ & .2 & 122 & 3.0 \\
\hline 5. & 4.0 & 41 & 6.7 & $15 \ldots$ & 2.0 & 6.7 & 10 & $25 \ldots$ & .2 & 24 & 2.1 \\
\hline 6. & 6.0 & 8.4 & 3.9 & $16 \ldots$ & 2.3 & 3.0 & 2.3 & 26 & .4 & 9.9 & 30 \\
\hline 7. & 5.0 & 2.4 & 3.0 & $17_{-}$ & 4.0 & 2.4 & 1.6 & 27 & .6 & 7.5 & 7.9 \\
\hline 8. & 6.0 & 8.1 & 2.5 & 18. & 2.0 & 1.6 & 1.3 & 28 & 2.0 & 5.8 & 5.0 \\
\hline & 3.0 & 2.6 & 8.6 & $19_{-}$ & 1.0 & 1.5 & 1.1 & $29_{\ldots}$ & 7.0 & 3.9 & 4.6 \\
\hline 10 & 2.5 & 10 & 2.5 & 20 & .6 & 1.3 & 1.1 & $30 \ldots$ & 5.0 & 3.0 & 3.3 \\
\hline & & & & & & & & & 2.0 & $\ldots$ & 3.6 \\
\hline \multicolumn{9}{|c|}{ Monthly mean discharge, in cubic feet per second } & 2.15 & 9.62 & 5.04 \\
\hline \multicolumn{9}{|c|}{ Runoff, in inches } & 0.78 & 3.35 & 1.82 \\
\hline \multicolumn{9}{|c|}{ Runoff, in acre-feet } & 132 & 572 & 310 \\
\hline
\end{tabular}


Gage height, in feet, and discharge, in cubic feet per second, at indicated time 1965, of South Branch Ralston Creek at Iow'a City, Iowa

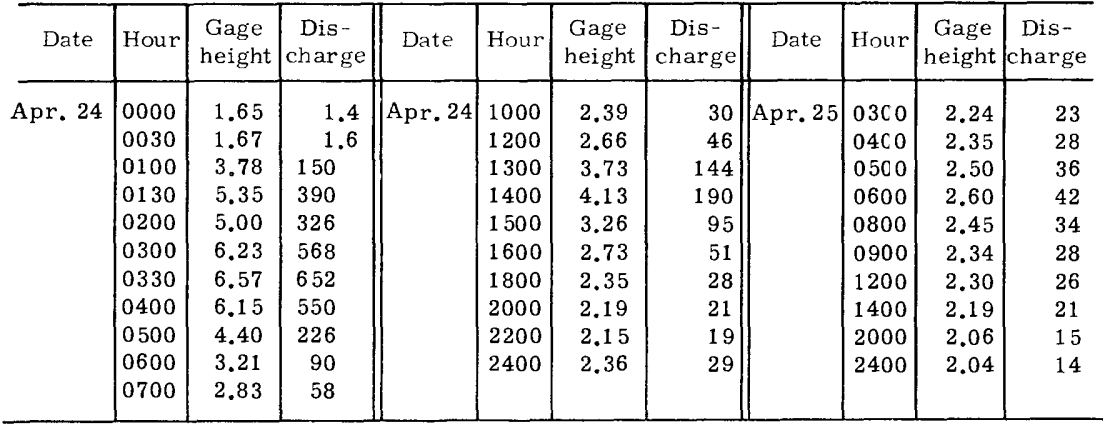

(250) 5-4555. English River at Kalona, Iowa

Location.-Lat $41^{\circ} 28^{\prime} 10^{\prime \prime}$, long $91^{\circ} 43^{\prime} 00^{\prime \prime}$, in $\mathrm{SE} \frac{1}{4} \mathrm{SE} \frac{1}{4}$ sec. 13, T.77 N., R. 8 W., on right bank $30 \mathrm{ft}$ upstream from bridge on State Highway 1, 1 mile south of Kalona, 4.5 miles downstream from Smith Creek, and 14.5 miles upstream from mouth.

\section{Drainage area. $-573 \mathrm{sq} \mathrm{mi}$.}

Gage-height record.-Water-stage recorder graph. Datum of gage is $633.45 \mathrm{ft}$ above mean sea level, datum of 1929 (levels by Corps of Engineers).

Discharge record. $\rightarrow$ Stage-discharge relation defined by current-meter measurements below 17,700 cfs. Backwater from ice Mar. 1, 4-14, 21-27.

Maxima.-March-May 1965: Discharge, 9,300 cfs 1230 hours Apr. 25 (gage height, $17.65 \mathrm{ft}$ )

1939 to February 1965: Discharge, 18,500 cfs Mar. 31, 1960 (gage height, $19.89 \mathrm{ft}$ ).

Maximum stage known, $19.9 \mathrm{ft}$ in June 1930 from floodmarks (discharge 18,500 cfs).

Cooperation.--One discharge measurement furnished by Corps of Engineers.

Mean discharge, in cublc feet per second, 1965

\begin{tabular}{|c|c|c|c|c|c|c|c|c|c|c|c|}
\hline Day & March & April & May & Day & March & April & May & Day & March & April & May \\
\hline & 980 & 2,650 & 552 & $11 \ldots$ & 220 & 834 & 183 & 21. & 300 & 136 & 100 \\
\hline $2 \ldots$ & 1,450 & 1,230 & 450 & $12_{--}$ & 210 & 730 & 158 & 22 & 200 & 124 & 95 \\
\hline 3 & 708 & 418 & 365 & $13 \ldots$ & 330 & 353 & 149 & 23. & 160 & 212 & 456 \\
\hline 4. & 450 & 333 & 313 & $14_{-}$ & 540 & 279 & 136 & 24 & 140 & 6,200 & 436 \\
\hline 5. & 300 & 1,030 & 294 & $15 \ldots$ & 1,140 & 391 & 156 & 25 & 125 & 8,700 & 201 \\
\hline 6. & 200 & 4,020 & 296 & $16 \ldots$ & 2,230 & 296 & 322 & 26. & 110 & 6,710 & 192 \\
\hline 7. & 310 & 2,900 & 256 & $17=$ & 4,480 & 237 & 254 & 27. & 100 & 3,200 & 846 \\
\hline 8. & 450 & 752 & 225 & $18--$ & 5,370 & 201 & 158 & 28. & 446 & 1,270 & 363 \\
\hline 9 & 280 & 708 & 256 & $19_{--}$ & 2,670 & 169 & 129 & 29. & 1,880 & 914 & 217 \\
\hline \multirow[t]{2}{*}{$10_{-}-1$} & 240 & 462 & 223 & $20--$ & 702 & 145 & 112 & 30 & 2,070 & 690 & 199 \\
\hline & & & & & & & & 31. & 2,470 & $-\ldots-n$ & 397 \\
\hline \multicolumn{9}{|c|}{ Monthly mean discharge, in cubic feet per second.... } & 1,008 & 1,543 & 274 \\
\hline \multicolumn{9}{|c|}{ Runoff, in inches } & 2.03 & 3.00 & 0.55 \\
\hline \multicolumn{9}{|c|}{ Ruboff, in acre-feet } & 62,010 & 91,820 & 16,840 \\
\hline
\end{tabular}


MARCH-MAY, UPPER MISSISSIPPI RIVER BASIN

Gage height. in feet, and discharge, in cubic feet per second, at indicated time 1965, of English Ri"er at Kalona, Lowa

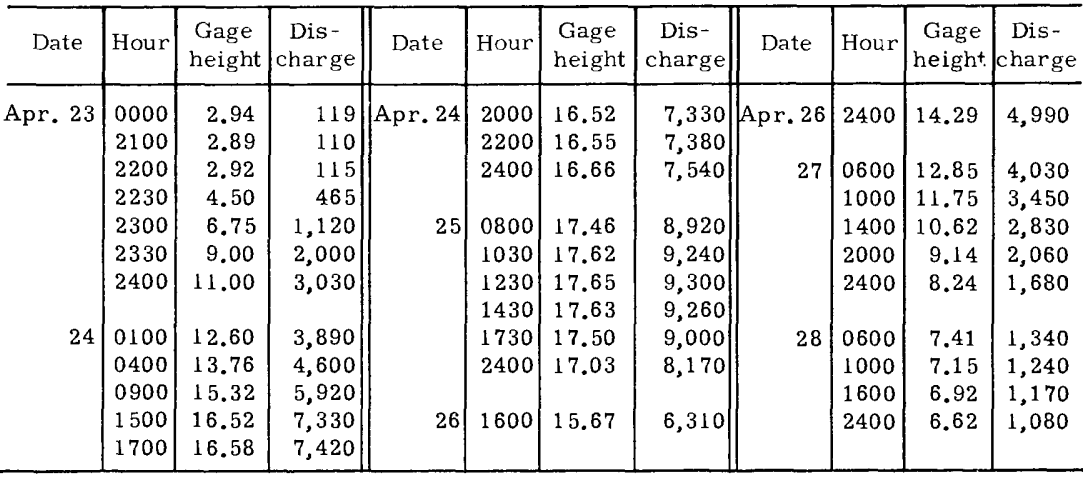

(251) 5-4557. Iowa River near Lone Tree, Iowa

Location.-Lat $41^{\circ} 25^{\prime} 35^{\prime \prime}$, long $91^{\circ} 28^{\prime} 20^{\prime \prime}$, in NW $\frac{1}{4} \mathrm{NE} \frac{1}{4}$ sec.6, T.76 N., R.5 W., on left bank $10 \mathrm{ft}$ downstream from county highway bridge, 5 miles southwest of Lone Tree, and 6 miles downstream from English River and at mile 47.2.

Drainage area.-4,293 sq $\mathrm{mi}$.

Gage-height record.-Water-stage recorder graph. Datum of gage is $588.16 \mathrm{ft}$ above mean sea level, datum of 1929.

Discharge record.-Stage-discharge relation defined by current-meter measurements below 31,200 cfs. Backwater from ice Mar. 7-9, 26, 27.

Maxima.-March-May 1965: Discharge, 21,700 cfs 2400 hours Apr. 24 (gage height, $16.92 \mathrm{ft}$ ).

1956 to February 1965: Discharge, 28,100 cfs Apr. 1, 1960 (gage height, $17.90 \mathrm{ft}$ ). Flood of May 25, 1944, reached a stage of $19.94 \mathrm{ft}$, from information by Corps of Engineers.

Remarks.-Flow regulated by Coralville Reservoir (capacity 489,000 acre-ft) since Sept. 17, 1958.

Cooperation.-Three discharge measurements furnished by Corps of Engineers.

Mean discharge, in cuble feet per second, 1965

\begin{tabular}{|c|c|c|c|c|c|c|c|c|c|c|c|}
\hline Day & March & April & May & Day & March & April & May & Day & March & April & May \\
\hline & 2,820 & 8,070 & 1,920 & $11 \ldots$ & 4,240 & 9,320 & 6,160 & 21 & 4,420 & 10,000 & 6,260 \\
\hline 2 & 5,640 & 7,710 & 1,600 & 12. & 5,320 & 4,280 & 6,100 & $22 \ldots$ & 4,280 & 9,950 & 6,310 \\
\hline 3 & 5,830 & 6,830 & 1,340 & $13_{-}$ & 6,530 & 2,930 & 6,040 & 23. & 4,590 & 9,960 & 7,340 \\
\hline & 4,050 & 7,710 & 1,140 & $14 \ldots$ & 7,730 & 2,810 & 6,130 & 24 & 3,300 & 17,700 & 6,710 \\
\hline & 3,840 & 8,810 & 1,260 & $15 \ldots$ & 8,080 & 7,790 & 6,180 & 25 & 2,310 & 20,600 & 6,300 \\
\hline & 3,960 & 12,100 & 3,350 & $16 \ldots$ & 8,950 & 9,370 & 6,670 & $26 \ldots$ & 1,600 & 17,300 & 6,840 \\
\hline & 1,800 & 15,000 & 5,650 & $17 \ldots$ & 10,400 & 9,980 & 6,580 & $27 \ldots$ & 1,300 & 10,500 & 8,530 \\
\hline & 2,100 & 12,500 & 6,100 & $18 \ldots$ & 11,600 & 10,100 & 6,360 & 28. & 2,170 & 4,330 & 7,970 \\
\hline & 1,850 & 9,950 & 6,540 & $19 \ldots$ & 9,920 & 10,100 & 6,230 & 29 & 4,580 & 2,970 & 6,890 \\
\hline & 2,500 & 10,000 & 6,400 & $20 \ldots$ & 5,810 & 10,100 & 6,220 & 30 & 7,560 & 2,370 & 6,790 \\
\hline & & & & & & & & & 7,680 & $-\cdots$ & 6,700 \\
\hline \multicolumn{9}{|c|}{ Monthly mean discharge, in cubic feet per second } & 5,057 & 9,371 & 5,633 \\
\hline \multicolumn{9}{|c|}{ Runoff, in inches } & 1.36 & 2.44 & 1.51 \\
\hline \multicolumn{9}{|c|}{ Runoff, in acre-feet $\ldots \ldots$} & 310,900 & $557,60 \mathrm{c}$ & 346,300 \\
\hline
\end{tabular}


Gage height, in feet, and discharge, in cubic feet per second, at indicated time, 1965, of Iowa River near

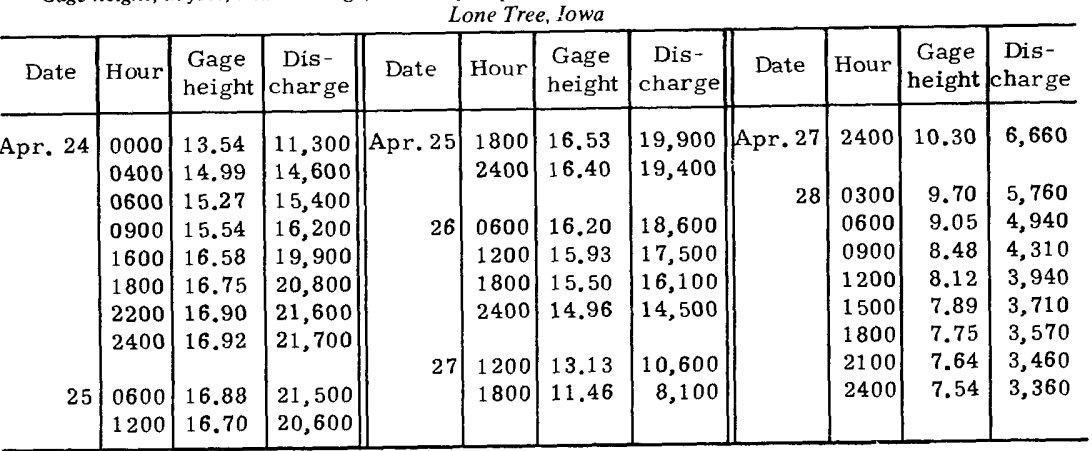

(252) 5-4570. Cedar River near Austin, Minn.

Location.-Lat $43^{\circ} 38^{\prime} 10^{\prime \prime}$, long $92^{\circ} 58^{\prime} 20^{\prime \prime}$, in $\mathrm{NE} \frac{1}{4} \mathrm{SE} \frac{1}{4} \sec .15, \mathrm{~T} .102 \mathrm{~N}$., R.18 W., on left bank $200 \mathrm{ft}$ upstream from abandoned powerhouse, $500 \mathrm{ft}$ downstream from highway bridge, 1.1 miles downstream from Turtle Creek, and 1.1 miles south of Austin.

Drainage area. $-425 \mathrm{sq} \mathrm{mi}$.

Discharge record.-Stage-discharge relation defined by current-meter measurements. Backwater from ice Mar. 1, 2, 17-19.

Maxima.-Given in the following table.

$\begin{array}{cc}\begin{array}{c}\text { Discharge } \\ (\mathrm{cfs})\end{array} & \begin{array}{c}\text { Gage height } \\ (\mathrm{ft})\end{array} \\ & \mathrm{a}_{18.87} \\ 9,400 & 16.21 \\ 8,410 & \\ 9,530 & -17.81\end{array}$

March-May 1965 :

Mar. 1, 2130 hour

Apr. 6, 1730 hours

$1909-14,1944$ to February 1965 :

Mar. 29, 1962

Mar. 26, $1950 \ldots \ldots$

17.81

${ }^{\text {a }}$ Backwater from ice.

Mean discharge, in cubic feet per second, 1965

\begin{tabular}{|c|c|c|c|c|c|c|c|c|c|c|c|}
\hline Day & March & April & May & Day & March & April & May & Day & March & April & May \\
\hline & 4,620 & 203 & 229 & $11 \ldots$ & 132 & 3,770 & 162 & 21. & 57 & 419 & 126 \\
\hline 2 & 3,480 & 561 & 212 & $12 \ldots$ & 114 & 3,460 & 151 & 22 & 60 & 370 & 119 \\
\hline 3. & 796 & 782 & 209 & $13 \ldots$ & 104 & 2,340 & 140 & 23 & 60 & 342 & 119 \\
\hline 4 & 452 & 2,730 & 215 & $14 \ldots$ & 97 & 1,780 & 134 & $24 \ldots$ & 62 & 335 & 126 \\
\hline 5. & 323 & 6,410 & 220 & $15 \ldots$ & 95 & 1,400 & 148 & 25. & 60 & 356 & 162 \\
\hline 6. & 264 & 7,920 & 276 & $16 \ldots$ & 91 & 1,080 & 164 & $26 \ldots$ & 60 & 475 & 489 \\
\hline 7. & 223 & 5,760 & 258 & 17 & 85 & 841 & 181 & $27 \ldots$ & 60 & 482 & 405 \\
\hline 8 & 195 & 6,080 & 232 & $18 \ldots$ & 72 & 681 & 170 & 28 & 58 & 356 & 255 \\
\hline $9_{-}$ & 164 & 5,470 & 201 & $19 \ldots$ & 60 & 573 & 151 & $29 \ldots$ & 63 & 288 & 198 \\
\hline 10 & 148 & 4,280 & 176 & $20 \ldots$ & 57 & 475 & 134 & $30_{-}$ & 72 & 252 & 164 \\
\hline & & & & & & & & 31. & 91 &.---- & 142 \\
\hline \multirow{2}{*}{\multicolumn{9}{|c|}{$\begin{array}{l}\text { Monthly mean discharge, in cubic feet per second } \\
\text { Runoff in acre-feet }\end{array}$}} & 396 & 2,009 & 196 \\
\hline & & & \multicolumn{6}{|c|}{ Runoff, in acre-feet } & 1.07 & 5.27 & 0.53 \\
\hline
\end{tabular}


MARCH-MAY, UPPER MISSISSIPPI RIVER BASIN

Gage height, in feet, and discharge, in cubic feet per second, at indicated time, 1965, of Cedar River near

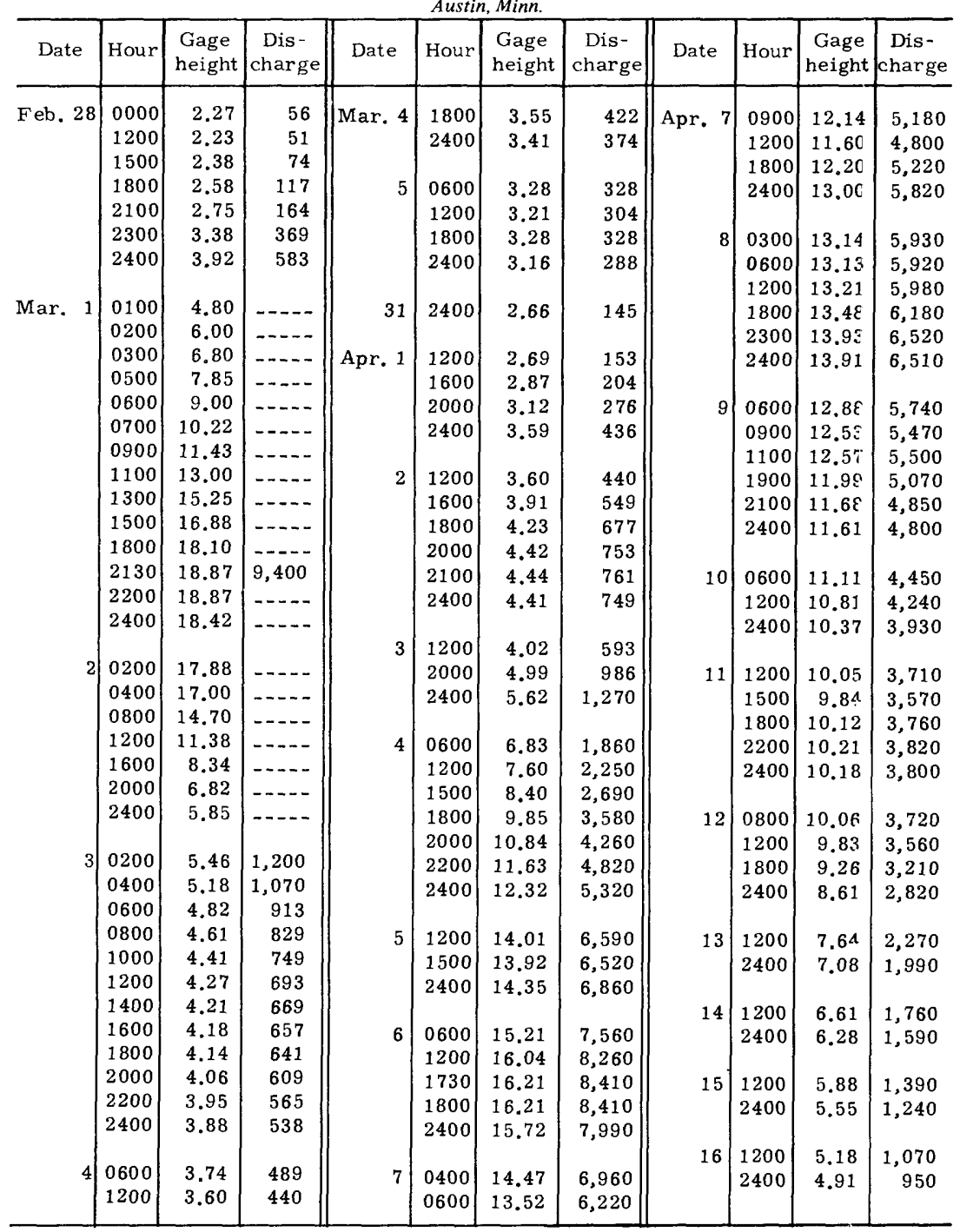


(253) 5-4577. Cedar River at Charles City, Iowa

Location,-Lat $43^{\circ} 03^{\prime} 45^{\prime \prime}$, long $92^{\circ} 40^{\prime} 25^{\prime \prime}$, in $\mathrm{SE}_{\frac{1}{4}} \mathrm{NE} \frac{1}{4}, \mathrm{sec}, 12$, T.95 N., P.16 W., on right bank $500 \mathrm{ft}$ downstream from bridge on U.S. Highway 18 (St. Mary's Street) in Charles City, 13 miles upstream from Little Cedar River, and at mile 253.1 above mouth of Iowa River (U.S. Geological Survey river profile).

Drainage area.-1,054 sq $\mathrm{mi}$.

Gage-height record.-Water-stage recorder graph except Mar. 11-15. Datum of gage is $973.02 \mathrm{ft}$ above mean sea level, datum of 1929 .

Discharge record.-Stage-discharge relation defined by current-meter measurements below 29,200 cfs. Backwater from ice Mar. 1 to Apr. 5.

Maxima.-March-May 1965: Discharge, 21,000 cfs 0100 hours Apr. 7 (gage height, $19.14 \mathrm{ft}$ ); gage height, $21.64 \mathrm{ft} 0730$ hours Mar. 2.

1964 to February 1965: Discharge, about 1,500 cfs Feb. 28, 1965.

Flood of Mar. 27, 1961 reached a stage of $21.6 \mathrm{ft}$ from floodmarks $(29,200 \mathrm{cfs})$.

Mean discharge, in cubic fect per second, 1965

\begin{tabular}{|c|c|c|c|c|c|c|c|c|c|c|c|}
\hline Day & March & April & May & Day & March & April & May & Day & March & April & May \\
\hline 1. & 3,000 & 1,800 & 599 & $11 \ldots$ & 560 & 8,830 & 469 & 21. & 218 & 937 & 370 \\
\hline 2. & 14,000 & 3,500 & 547 & $12 \ldots$ & 480 & 11,400 & 424 & 22. & 206 & 833 & 346 \\
\hline 3. & 9,400 & 3,000 & 502 & $13 \ldots$ & 430 & 6,970 & 400 & 23. & 196 & 748 & 334 \\
\hline 4 & 5,200 & 6,200 & 456 & $14 \ldots$ & 380 & 3,910 & 370 & 24 & 188 & 710 & 334 \\
\hline 5. & 3,100 & 12,000 & 492 & $15 \ldots$ & 370 & 2,860 & 388 & 25 & 182 & 762 & 365 \\
\hline 6. & 2,100 & 19,800 & 813 & $16 \ldots$ & 370 & 2,270 & 400 & 26. & 178 & 836 & 577 \\
\hline $7_{-}$ & 1,500 & 19,600 & 936 & 17. & 350 & 1,820 & 512 & 27. & 170 & 955 & 975 \\
\hline 8 & 1,060 & 16,500 & 833 & $18 \ldots$ & 300 & 1,450 & 528 & 28 & 168 & 944 & 1,010 \\
\hline 9 & 840 & 15,200 & 651 & $19_{-}$ & 260 & 1,220 & 450 & 29 & 164 & 807 & 687 \\
\hline 10 & 670 & 10,300 & 540 & $20--$ & 236 & 1,070 & 400 & $30_{-}$ & 168 & 677 & 547 \\
\hline & & & & & & & & 31 & 500 & 8. & 462 \\
\hline \multirow{3}{*}{\multicolumn{9}{|c|}{$\begin{array}{l}\text { Monthly mean discharge, in cubic feet per second } \\
\text { Runoff, in inches } \\
\text { Runoff, in acre feet }\end{array}$}} & 1,514 & 5,264 & 539 \\
\hline & & & & & & & & & 1.66 & 5.57 & 0.59 \\
\hline & & & & & & & & & 93,110 & 313,200 & 33,160 \\
\hline
\end{tabular}


Gage height. in feet, and discharge, in cubic feet per second, at indicated time, 1965, of Cedar Ruver at Charles City. Iowa

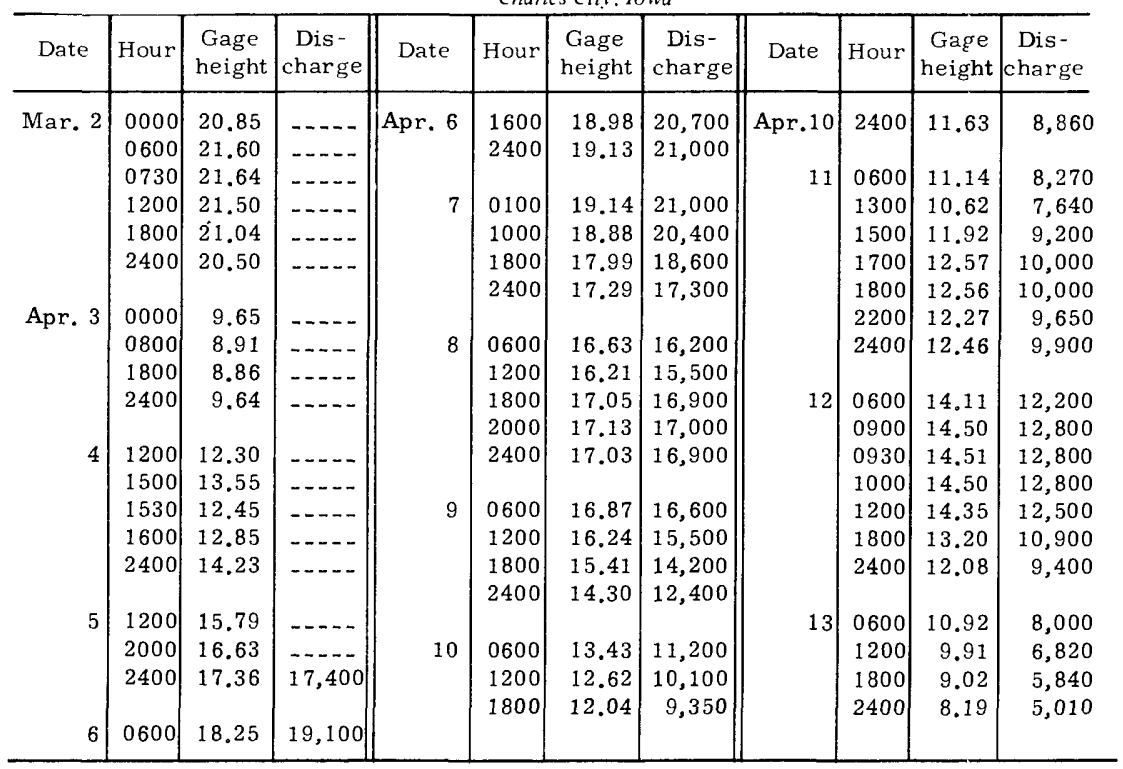

(254) 5-4580. Little Cedar River near Ionia, Iowa

Location.-Lat $43^{\circ} 02^{\prime} 00^{\prime \prime}$, long $92^{\circ} 30^{\prime} 10^{\prime \prime}$, in SW $\frac{1}{4} \mathrm{NE}^{\frac{1}{4}} \mathrm{sec} .21, \mathrm{~T} .95 \mathrm{~N}$., R.14 W., on left bank $12 \mathrm{ft}$ downstream from county highway bridge, 2.5 miles west of Ionia, and 7.0 miles upstream from mouth.

Drainage area.-306 sq $\mathrm{mi}$.

Gage-height record.-Water-stage recorder graph except Mar. 3, 4. Datum of gage is $973.35 \mathrm{ft}$ above mean sea level, datum of 1929 .

Discharge record.--Stage-discharge relation defined by current-meter measurements below $10,200 \mathrm{cfs}$. Backwater from ice Mar. 1, 31 .

Maxima.-March-May 1965: Discharge, 6,250 cfs 2200 hours Apr. 6 (gage height, $12.11 \mathrm{ft}$ ).

1954 to February 1965: Discharge, 10,800 cfs Mar. 27, 1961 (gage height, $15.58 \mathrm{ft}$ ).

Flood of June 22, 1954, reached a stage of $11.37 \mathrm{ft}$ (discharge, 4,600 cfs). 
Mean discharge, in cubic feet per second, 1965, of Little Cedar River near Ionia, Iowa

\begin{tabular}{|c|c|c|c|c|c|c|c|c|c|c|c|}
\hline Day & March & April & May & Day & March & April & May & Day & March & April & May \\
\hline 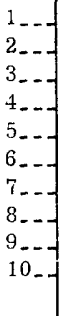 & $\begin{array}{r}2,000 \\
4,870 \\
1,300 \\
440 \\
281 \\
213 \\
160 \\
152 \\
122 \\
105\end{array}$ & $\begin{array}{l}1,270 \\
1,400 \\
1,540 \\
2,020 \\
3,870 \\
5,530 \\
5,500 \\
4,270 \\
4,250 \\
2,840\end{array}$ & $\begin{array}{r}112 \\
102 \\
95 \\
88 \\
84 \\
115 \\
287 \\
180 \\
142 \\
112\end{array}$ & $\left|\begin{array}{l}11 \ldots- \\
12_{-} \\
13_{-} \\
14_{-} \\
15_{-} \\
16_{-} \\
17_{--} \\
18_{--} \\
19_{--} \\
20_{-}\end{array}\right|$ & $\begin{array}{l}97 \\
88 \\
79 \\
70 \\
70 \\
69 \\
42 \\
40 \\
46 \\
45\end{array}$ & $\begin{array}{r}2,190 \\
3,210 \\
2,660 \\
581 \\
364 \\
272 \\
210 \\
178 \\
163 \\
151\end{array}$ & $\begin{array}{r}97 \\
84 \\
79 \\
74 \\
78 \\
146 \\
159 \\
123 \\
104 \\
88\end{array}$ & $\left.\begin{array}{l}21- \\
22_{-} \\
23_{-} \\
24_{-} \\
25_{-} \\
26_{-} \\
27- \\
28_{-} \\
29_{-} \\
30_{-} \\
31\end{array}\right]$ & $\begin{array}{r}41 \\
37 \\
35 \\
33 \\
32 \\
29 \\
29 \\
29 \\
29 \\
32 \\
300\end{array}$ & $\begin{array}{r}129 \\
117 \\
113 \\
112 \\
163 \\
239 \\
207 \\
170 \\
151 \\
121 \\
- \\
--\end{array}$ & $\begin{array}{r}76 \\
70 \\
65 \\
63 \\
153 \\
442 \\
210 \\
175 \\
149 \\
121 \\
112\end{array}$ \\
\hline \multicolumn{9}{|c|}{$\begin{array}{l}\text { Monthly mean discharge, in cubic feet per second } \\
\text { Runoff, in inches } \\
\text { Runoff, in acre-feet }\end{array}$} & $\begin{array}{r}352 \\
1.33 \\
21,650\end{array}$ & $\begin{array}{r}1,466 \\
5.35 \\
87,250\end{array}$ & $\begin{array}{r}129 \\
0.48 \\
7,400\end{array}$ \\
\hline
\end{tabular}

Gage height, in feet, and discharge, in cubic feet per second, at indicated time, 1965

\begin{tabular}{|c|c|c|c|c|c|c|c|c|c|c|c|}
\hline Date & Hour & $\begin{array}{l}\text { Gage } \\
\text { height }\end{array}$ & $\begin{array}{c}\text { Dis - } \\
\text { charge }\end{array}$ & Date & Hour & $\begin{array}{l}\text { Gage } \\
\text { height }\end{array}$ & $\begin{array}{c}\text { Dis- } \\
\text { charge }\end{array}$ & Date & Hour & $\begin{array}{c}\text { Gage } \\
\text { height }\end{array}$ & $\begin{array}{c}\text { Dis- } \\
\text { charge }\end{array}$ \\
\hline \multirow[t]{8}{*}{ Apr. 3} & 0000 & 7.02 & 1,670 & \multirow[t]{8}{*}{ Apr. 7} & 0600 & 11.57 & 5,650 & \multirow[t]{17}{*}{ Apr. 11} & 1200 & 7.00 & 1,660 \\
\hline & 0600 & 6.98 & 1,650 & & 1800 & 11.34 & 5,390 & & 1400 & 7.15 & 1,750 \\
\hline & 2000 & 6.57 & 1,370 & & 2400 & 10.69 & 4,690 & & 1800 & 8.28 & 2,580 \\
\hline & 2400 & 6.79 & 1,530 & & 1200 & 9.4 & 3,530 & & 2000 & 9.22 & 3,360 \\
\hline & 0200 & 7.07 & 1,700 & & 1400 & 9.58 & 3,670 & & 2200 & 9.79 & 3,860 \\
\hline & 1400 & 7.35 & 1,880 & & 1800 & 10.42 & 4,430 & & 2400 & 9.67 & 3,750 \\
\hline & 2000 & 8.26 & 2,570 & & 2100 & 11.18 & 5,220 & & & & \\
\hline & 2400 & 8.43 & 2,700 & & 2400 & 10.89 & 4,900 & & 0600 & 8.70 & 2,920 \\
\hline \multirow[t]{5}{*}{5} & 0400 & 8.64 & 2,870 & \multirow[t]{4}{*}{9} & 0600 & 10.30 & 4,320 & & \multirow[t]{2}{*}{2400} & \multirow[t]{2}{*}{9.59} & \multirow[t]{2}{*}{3,680} \\
\hline & 1000 & 9.74 & 3,820 & & 1800 & 10.05 & 4,100 & & & & \\
\hline & 1600 & 10.46 & 4,460 & & 2400 & 9.74 & 3,820 & & 0200 & 9.58 & 3,670 \\
\hline & 2400 & 10.77 & 4,770 & & & & & & 0800 & 9.10 & 3,260 \\
\hline & & & & 10 & 0600 & 9.13 & $3,28 d$ & & 1400 & 8.44 & 2,710 \\
\hline \multirow[t]{4}{*}{6} & 1600 & 11.66 & 5,750 & & 1600 & 8.26 & $2,57 \mathrm{~d}$ & & 1800 & 7.53 & 2,000 \\
\hline & 2000 & 12.03 & 6,160 & & 2400 & 7.30 & 1,850 & & 2000 & 6.70 & 1,480 \\
\hline & 2200 & 12.11 & 6,250 & & & & & & \multirow[t]{2}{*}{2400} & \multirow[t]{2}{*}{5.81} & \multirow[t]{2}{*}{928} \\
\hline & 2400 & 12.06 & 6,190 & 11 & 0600 & 6.82 & $1,55 \mathrm{~d}$ & & & & \\
\hline
\end{tabular}

(255) 5-4585. Cedar River at Janesville, Iowa

Location.-Lat $42^{\circ} 39^{\prime} 00^{\prime \prime}$, long $92^{\circ} 27^{\prime} 50^{\prime \prime}$, in NE $\frac{1}{4} \mathrm{SW} \frac{1}{4}$ sec.35, T.91 N., R.14 W., on left bank $300 \mathrm{ft}$ downstream from county highway bridge at Janesville 3.3 miles upstream from West Fork Cedar River, and at mile 207.7 above the mouth of Iowa River (U.S. Geological Survey river profiles).

Drainage area.- $-1,661 \mathrm{sq} \mathrm{mi}$.

Gage-height record.-Water-stage recorder graph except Mar. 3 for which graph was reconstructed from wire-weight gage readings. Datum of gage is $868.26 \mathrm{ft}$ above mean sea level, datum of 1929.

Discharge record.-Stage-discharge relation defined by current-meter reasurements below 36,200 cfs. Backwater from ice Mar. 1-8, Mar. 18 to Apr. 5.

Maxima.-March-May 1965: Discharge, 29,200 cfs 2400 hours Apr. 7 (gage height, $14.33 \mathrm{ft}$ ); gage height, $16.33 \mathrm{ft} 1700$ hours Mar. 3 (backwater from ice).

1904-06, 1914-27, 1932-42, 1945 to February 1965: Discharge, 37,020 cfs Mar. 28, 1961 (gage height, $16.33 \mathrm{ft}$ ).

FIood of Mar. 17, 1945, reached a stage of $16.2 \mathrm{ft}$,from floodmark at site $300 \mathrm{ft}$ upstream (discharge, $34,300 \mathrm{cfs}$ Mar. 16,1929, reached a stage of abo'st $16 \mathrm{ft}$ from information by City of Waterloo (discharge not determined). 
Mean discharge, in cubic feet per second, 1965, of Cedar River at Janesville, Iowa

\begin{tabular}{|c|c|c|c|c|c|c|c|c|c|c|c|}
\hline Day & March & April & May & Day & March & April & May & Day & March & April & May \\
\hline & 840 & 5,200 & 1,040 & $11 \ldots$ & 1,490 & 14,700 & 835 & & 520 & 1,520 & 556 \\
\hline 2 & 2,800 & 5,000 & 890 & $12--$ & 1,220 & 11,000 & 754 & 22 & 490 & $1,34 \mathrm{C}$ & 548 \\
\hline 3 & 13,000 & 4,700 & 890 & $13 \ldots$ & 1,030 & 13,400 & 684 & & 460 & $1,21 \mathrm{C}$ & 525 \\
\hline & 11,000 & 6,000 & 808 & $14 \ldots$ & 880 & 10,700 & 660 & 2 & 470 & $1,12 \mathrm{C}$ & 564 \\
\hline & 8,200 & 8,800 & 772 & 15 & 910 & 5,930 & 620 & 25 & 440 & $1,15 \mathrm{C}$ & 510 \\
\hline & 6,000 & 17,700 & 745 & $16 \ldots$ & 781 & 4,170 & 612 & 2 & 410 & $1,33 C$ & 878 \\
\hline & 4,200 & 25,400 & 910 & $17 \ldots$ & 696 & 3,320 & 620 & 2 & 310 & $1,31 \mathrm{C}$ & 1,290 \\
\hline & 3,000 & 27,400 & 1,150 & $18 \ldots$ & 530 & 2,660 & 803 & - & 234 & $1,32 C$ & 1,130 \\
\hline & 2,340 & 22,100 & 1,120 & - & 560 & 2,200 & 688 & & 310 & $1,29 \mathrm{C}$ & 1,270 \\
\hline & 1,800 & 20,400 & 1,000 & $20 \ldots$ & 560 & 1,750 & 503 & & 480 & $1,15 \mathrm{C}$ & 1,100 \\
\hline & & & & & & & & & 1,300 & & 980 \\
\hline \multirow{3}{*}{\multicolumn{9}{|c|}{$\begin{array}{l}\text { Monthly mean discharge, in cubic feet per second } \\
\text { Runoff, in inches } \\
\text { Runoff, in acre-feet }\end{array}$}} & 2,170 & $7,50 \mathrm{C}$ & 821 \\
\hline & & & & & & & & & 1.51 & 5.04 & 0.57 \\
\hline & & & & & & & & & 133,400 & $446,80 \mathrm{C}$ & 50,490 \\
\hline
\end{tabular}

Gage height, in feet, and discharge, in cubic feet per second, at indicated time, 1965

\begin{tabular}{|c|c|c|c|c|c|c|c|c|c|c|c|}
\hline Date & Hour & $\begin{array}{c}\text { Gage } \\
\text { height }\end{array}$ & $\begin{array}{c}\text { Dis- } \\
\text { charge }\end{array}$ & Date & Hour & $\begin{array}{c}\text { Gage } \\
\text { height }\end{array}$ & $\begin{array}{c}\text { Dis- } \\
\text { charge }\end{array}$ & Date & Hour & $\begin{array}{l}\text { Gago } \\
\text { heigl }\end{array}$ & $\begin{array}{c}\text { Dis- } \\
\text { charge }\end{array}$ \\
\hline \multirow[t]{8}{*}{ Mar. 3} & 0000 & 12.32 & $\ldots$ & Apr. 7 & 2400 & 14.33 & 29,200 & Apr. 11 & 1800 & 10.32 & 12,800 \\
\hline & 0400 & 13.55 & 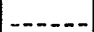 & & & & & & 2400 & 9.80 & 11,500 \\
\hline & 1200 & 15.27 & 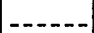 & 8 & 0600 & 14.25 & 28,800 & & & & \\
\hline & 1600 & 16.20 & $\ldots$ & & 1600 & 13.92 & 26,800 & 12 & 0600 & 9.33 & 10,600 \\
\hline & 1700 & 16.33 & $\ldots$ & & 2400 & 13.35 & 24,300 & & 1000 & 9.25 & 10,300 \\
\hline & 2000 & 15.77 & $\ldots$ & & & & & & 1400 & 9.37 & 10.600 \\
\hline & 2400 & 14.78 & $\ldots$ & 9 & 0600 & 12.93 & 22,400 & & 2400 & 10.14 & 12,400 \\
\hline & & & & & 1200 & 12.71 & 21,400 & & & & \\
\hline \multirow[t]{5}{*}{ Apr. 5} & 0000 & 10.33 & 13,000 & & 2400 & 12.80 & 21,800 & 13 & 1200 & 10.63 & 13,800 \\
\hline & 0600 & 11.08 & 15,400 & & & & & & 1600 & 10.63 & 13,900 \\
\hline & 1200 & 11.81 & 17,900 & 10 & 0600 & 12.72 & 21,400 & & 2400 & 10.48 & 13,400 \\
\hline & 2400 & 12.84 & 22,000 & & 1200 & 12.55 & 20,700 & & & & \\
\hline & & & & & 1800 & 12.30 & 19,600 & 14 & 0600 & $10.0 ?$ & 12,300 \\
\hline \multirow[t]{3}{*}{7} & 0600 & 13.21 & 23,600 & & 2400 & 11.88 & 18,200 & & 1200 & 9.42 & 10,700 \\
\hline & 1200 & 13.34 & 24,200 & & & & & & 1800 & 8.66 & 9,130 \\
\hline & 1800 & 14.10 & 28,000 & 11 & 0900 & 11.11 & 15,500 & & 2400 & 7.86 & 7,690 \\
\hline
\end{tabular}

(256) 5-4589. West Fork Cedar River at Finchford, Iowa

Location.-Lat $42^{\circ} 37^{\prime} 50^{\prime \prime}$, long $92^{\circ} 32^{\prime} 25^{\prime \prime}$, in SW $\frac{1}{4} \mathrm{SE} \frac{1}{4} \mathrm{sec} .6, \mathrm{~T} .90 \mathrm{~N}$, , R.14 W., on left bank $100 \mathrm{ft}$ downstream from county highway bridge in Finchford, and 3.2 miles upstream from Shell Rock River.

Drainage area. $-846 \mathrm{sq} \mathrm{mi}$.

Gage-height record.-Water-stage recorder graph. Datum of gage is $867.06 \mathrm{f}^{+}$above mean sea level, datum of 1929 .

Discharge record.- Stage-discharge relation defined by current meter measurements below $22,600 \mathrm{cfs}$, extended to 31,900 by logarithmic plotting and one indirect measurement of peak flow. Backwater from ice Mar. 1 to Apr. 4.

Maxima.-March-May 1965: Discharge, 16,700 cfs 0930 hours Apr. 7 (gage height, $15.91 \mathrm{f} t$ ).

1945 to February 1965: Discharge, 31,900 cfs June 27, 1951 (gage height, $17.28 \mathrm{ft}$, from floodmarks). 
Mean discharge, in cubic feet per second, 1965. of West Fork Cedar River at Finchford, lowa

\begin{tabular}{|c|c|c|c|c|c|c|c|c|c|c|c|}
\hline Day & March & April & May & Day & March & April & May & Day & March & April & May \\
\hline 1 & 700 & 900 & 942 & $11 \ldots$ & 370 & 5,440 & 446 & 21 & 160 & 1,220 & 273 \\
\hline 2 & 1,700 & 2,750 & 830 & 12 & 330 & 4,220 & 400 & 22. & 150 & 1,080 & 268 \\
\hline & 3,010 & 3,010 & 740 & $13 \ldots$ & 310 & 3,680 & 370 & 23 & 142 & 946 & 283 \\
\hline & 3,500 & 7,400 & 666 & $14 \ldots$ & 300 & 3,910 & 351 & 24 & 140 & 856 & 341 \\
\hline & 1,720 & 9,400 & 606 & 15. & 286 & 3,180 & 337 & 25 & 138 & 866 & 432 \\
\hline & 1,060 & 13,800 & 568 & $16 \ldots$ & 280 & 2,560 & 324 & 26 & 134 & 1,030 & 1,050 \\
\hline 7 & 760 & 16,300 & 539 & 17. & 250 & 2,130 & 322 & 27. & 132 & 1,300 & 1,810 \\
\hline 8. & 560 & 13,700 & 539 & 18. & 212 & 1,840 & 322 & 28 & 130 & 1,490 & 2,500 \\
\hline & 480 & 9,170 & 536 & $19 \ldots$ & 192 & 1,680 & 314 & 29 & 130 & 1,290 & 2,650 \\
\hline & 415 & 7,410 & 500 & $20_{-}$ & 174 & 1,440 & 296 & $30_{-}$ & 132 & 1,090 & 2,070 \\
\hline & & & & & & & & & 150 & $\ldots$ & 1,660 \\
\hline \multirow{3}{*}{\multicolumn{9}{|c|}{$\begin{array}{l}\text { Monthly mean discharge, in cubtc feet per second } \\
\text { Rumoff, in inches } \\
\text { Runoff, in acre-feet }\end{array}$}} & 585 & 70 & 751 \\
\hline & & & & & & & & & 0.80 & 9 & 1.02 \\
\hline & & & & & & & & & 35,990 & 248,100 & 46,190 \\
\hline
\end{tabular}

Gage height, in feet, and discharge, in cubic feet per second, at indicated time, 1965

\begin{tabular}{r|r|r|r|r|r|r|r||r|r|r|r}
\hline Date & Hour & $\begin{array}{c}\text { Gage } \\
\text { height }\end{array}$ & $\begin{array}{c}\text { Dis- } \\
\text { charge }\end{array}$ & Date & Hour & $\begin{array}{c}\text { Gage } \\
\text { height }\end{array}$ & $\begin{array}{c}\text { Dis- } \\
\text { charge }\end{array}$ & Date & Hour $\begin{array}{c}\text { Gage } \\
\text { height }\end{array}$ & $\begin{array}{c}\text { Dis- } \\
\text { charge }\end{array}$ \\
\hline Apr. 5 & 0000 & 13.81 & 10,200 & Apr. 6 & 1600 & 15.30 & 14,900 & Apr. 7 & 2400 & 15.52 & 16,000 \\
& 0800 & 13.80 & 8,800 & & 2400 & 15.47 & 15,800 & & & & \\
& 2400 & 14.33 & 10,600 & & & & & 0800 & 15.29 & 14,900 \\
6 & 0800 & 14.93 & 13,200 & & 1800 & 15.61 & 16,400 & & 2400 & 14.31 & 10,500 \\
\hline
\end{tabular}

(257) 5-4590. Shell Rock River near Northwood, Iowa

Location.-Lat $43^{\circ} 24^{\prime} 50^{\prime \prime}$, long $93^{\circ} 13^{\prime} 10^{\prime \prime}$, in NW $\frac{1}{4} N W \frac{1}{4}$ sec.9, T.99 N., R.20 W., on right bank $50 \mathrm{ft}$ downstream from county highway bridge, 2 miles south of Northwood, and 4.1 miles upstream from Elk Creek.

Drainage area. $-300 \mathrm{sq} \mathrm{mi}$.

Gage-height record.-Water-stage recorder graph except Mar. 2-28, Apr. 4. Datum of gage is 1,176.48 ft above mean sea level, datum of 1929 .

Discharge record. - Stage-discharge relation defined by current-meter measurements below $2,430 \mathrm{cfs}$, extended to 3,200 by logarithmic plotting. Backwater from ice Mar. 1 to Apr. 10.

Maxima.-March-May 1965: Discharge, 3,400 cfs 2000 hours Apr. 8 (gage height, $12.07 \mathrm{ft}$, backwater from ice).

1945 to February 1965: Discharge, about 3,000 cfs Mar. 2ङ, 1961 (ge.ge height, $11.68 \mathrm{ft}$, backwater from ice). 
Mean discharge, in cubic feet per second, 1965, of Shell Rock River near Northwood, Iowa

\begin{tabular}{|c|c|c|c|c|c|c|c|c|c|c|c|}
\hline Day & March & April & May & Day & March & April & May & Day & March & April & May \\
\hline 1. & 640 & 148 & 546 & $11 \ldots$ & 262 & 2,960 & 257 & 21 & 82 & $1,26 n$ & 170 \\
\hline 2. & 520 & 158 & 488 & $12_{-}$ & 240 & 3,100 & 220 & 22 & 72 & $1,14 n$ & 170 \\
\hline 3 & 450 & 178 & 431 & $13 \ldots$ & 210 & 2,820 & 198 & 23 & 64 & $1,04 n$ & 166 \\
\hline 4 & 420 & 465 & 388 & $14 \ldots$ & 180 & 2,600 & 185 & 24. & 56 & 959 & 159 \\
\hline 5 & 390 & 1,300 & 416 & $15_{-}$ & 170 & 2,400 & 183 & 25 & 54 & $90 ?$ & 157 \\
\hline 6 & 380 & 2,000 & 431 & $16 \ldots$ & 160 & 2,190 & 218 & 26. & 52 & 890 & 251 \\
\hline 7 & 360 & 2,800 & 394 & $17 \ldots$ & 150 & 1,990 & 233 & 27. & 52 & 818 & 293 \\
\hline 8 & 340 & 3,200 & 361 & $18 \ldots$ & 140 & 1,750 & 205 & 28. & 50 & 742 & 283 \\
\hline 9 & 310 & 3,100 & 319 & ـ & 120 & 1,570 & 200 & 29. & 51 & 674 & 259 \\
\hline 1 & 290 & 3,000 & 278 & $20 \ldots$ & 100 & 1,410 & 187 & & 58 & 609 & 231 \\
\hline & & & & & & & & & & ------ & 214 \\
\hline \multirow{3}{*}{\multicolumn{9}{|c|}{$\begin{array}{l}\text { Monthly mean discharge, in cubic feet per second } \\
\text { Runnff, in inches } \\
\text { Runoff, in acre-feet }\end{array}$}} & 210 & 1,603 & 274 \\
\hline & & & & & & & & & 0.81 & 5.97 & 1.05 \\
\hline & & & & & & & & & 12,920 & 95,56 ? & 16,840 \\
\hline
\end{tabular}

Gage height, in feet, and discharge, in cubic feet per second, at indicated tume, 1965

\begin{tabular}{|c|c|c|c|c|c|c|c|c|c|c|c|}
\hline Date & Hour & $\begin{array}{l}\text { Gage } \\
\text { height }\end{array}$ & $\begin{array}{c}\text { Dis- } \\
\text { charge }\end{array}$ & Date & Hour & $\begin{array}{c}\text { Gage } \\
\text { height }\end{array}$ & $\begin{array}{c}\text { Dis- } \\
\text { charge }\end{array}$ & Date & Hour & $\begin{array}{l}\text { Gage } \\
\text { height }\end{array}$ & $\begin{array}{c}\text { Dis- } \\
\text { charge }\end{array}$ \\
\hline \multirow[t]{9}{*}{ Apr. } & 0000 & 10.00 & $-\infty-0$ & Apr. 8 & 2400 & 11.90 & & \multirow[t]{9}{*}{ Apr. 11} & \multirow[t]{2}{*}{2400} & \multirow[t]{2}{*}{10.90} & 3,170 \\
\hline & 1200 & 10.64 & --- & \multirow{3}{*}{9} & & & & & & & \\
\hline & 2400 & 11.26 & --- & & 1600 & 11.64 & - & & $\begin{array}{l}00 \\
00\end{array}$ & 10.92 & \multirow{2}{*}{$\begin{array}{l}3,190 \\
3,120\end{array}$} \\
\hline & & & & & 2000 & 11.37 & & & 00 & 10.84 & \\
\hline & 0600 & 11.25 & -- & \multirow{3}{*}{10} & 2400 & 11.21 & $-\ldots$ & & \multirow[t]{2}{*}{2400} & 10.62 & \multirow{3}{*}{$\begin{array}{l}2,950 \\
2,800 \\
2,710\end{array}$} \\
\hline & 1000 & 11.04 & - & & 1200 & 10.86 & & & & & \\
\hline & 2400 & 11.80 & & & 2400 & 10.63 & 2,950 & & \multirow{3}{*}{2400} & \multirow{3}{*}{$\begin{array}{l}10.42 \\
10.28\end{array}$} & \\
\hline & 1200 & 11.85 & -- & \multirow[t]{2}{*}{11} & 1400 & 10.52 & 2,870 & & & & \\
\hline & 2000 & 12.07 & 3,400 & & & & & & & & \\
\hline
\end{tabular}

(258) 5-4595. Winnebago River at Mason City, Iowa

Location.-Lat $43^{\circ} 10^{\prime} 00^{\prime \prime}$, long $93^{\circ} 11^{\prime} 40^{\prime \prime}$, in $\mathrm{NE} \frac{1}{4} \mathrm{NW} \frac{1}{4}$ sec.3, T.96 N., R.20 W., on right bank $650 \mathrm{ft}$ upstream from Thirteenth Street Bridge in Mason City, and 1.0 mile upstream from Willow Creek.

Drainage area. $-526 \mathrm{sq} \mathrm{mi}$.

Gage-height record.-Water-stage recorder graph. Datum of gage is $1,069.59 \mathrm{ft}$ above mean sea level, datum of 1929 .

Discharge record.-Stage-discharge relation defined by current-meter measurements below $10,600 \mathrm{cfs}$.

Maxima.-March-May 1965: Discharge, 9,700 cfs 2130 hours Apr. 11 (gage height, $14.27 \mathrm{ft}$ ).

1932 to February 1965: Discharge, 10,800 cfs Mar. 30, 1933 (gage height, $15.7 \mathrm{ft}$ ). 
Mean discharge, in cubic feet per second, 1965, of Winnebago River at Mason City, Iowa

\begin{tabular}{|c|c|c|c|c|c|c|c|c|c|c|c|}
\hline Day & March & April & May & Day & March & April & May & Day & March & April & May \\
\hline & 3,920 & 504 & 882 & $11 \ldots$ & 218 & 6,220 & 801 & $21 \ldots$ & 54 & 1,240 & 440 \\
\hline 2. & 1,210 & 992 & 796 & $12 \ldots$ & 182 & 5,880 & 662 & 22. & 53 & 1,120 & 435 \\
\hline 3 & 465 & 1,240 & 729 & $13 \ldots$ & 152 & 3,640 & 518 & 23 & 49 & 1,020 & 535 \\
\hline 20 & 440 & 2,560 & 644 & $14 \ldots$ & 124 & 2,820 & 468 & 24 & 49 & 1,000 & 796 \\
\hline & 420 & 4,840 & 1,760 & - 15 & 114 & 2,410 & 477 & 25 & 49 & 1,110 & 763 \\
\hline . & 390 & 7,920 & 1,830 & $16 \ldots$ & 106 & 2,090 & 792 & 26. & 53 & 1,300 & 1,360 \\
\hline & 360 & 7,340 & 1,570 & 17. & 92 & 1,890 & 756 & 27. & 53 & 1,160 & 1,470 \\
\hline & 320 & 7,750 & 1,510 & $18 \ldots$ & 80 & 1,720 & 634 & 28 & 54 & 1,110 & 1,230 \\
\hline & 290 & 6,690 & 1,260 & $19 \ldots$ & 66 & 1,550 & 508 & 29. & 57 & 1,020 & 1,030 \\
\hline 10 & 254 & 5,900 & 1,000 & 20 & 56 & 1,390 & 435 & 30. & 63 & 960 & 976 \\
\hline & & & & & & & & & 90 & . & 860 \\
\hline \multirow{3}{*}{\multicolumn{9}{|c|}{$\begin{array}{l}\text { Monthly mean discharge, in cubic feet per second } \\
\text { Runoff, in inches } \\
\text { Runoff, in acre-feet }\end{array}$}} & 319 & 2,880 & 901 \\
\hline & & & & & & & & & 0.70 & 6.11 & 1.97 \\
\hline & & & & & & & & & 19,600 & 171,300 & 55,390 \\
\hline
\end{tabular}

Gage height, in feet, and discharge, in cubic feet per second, at indicated time, 1965

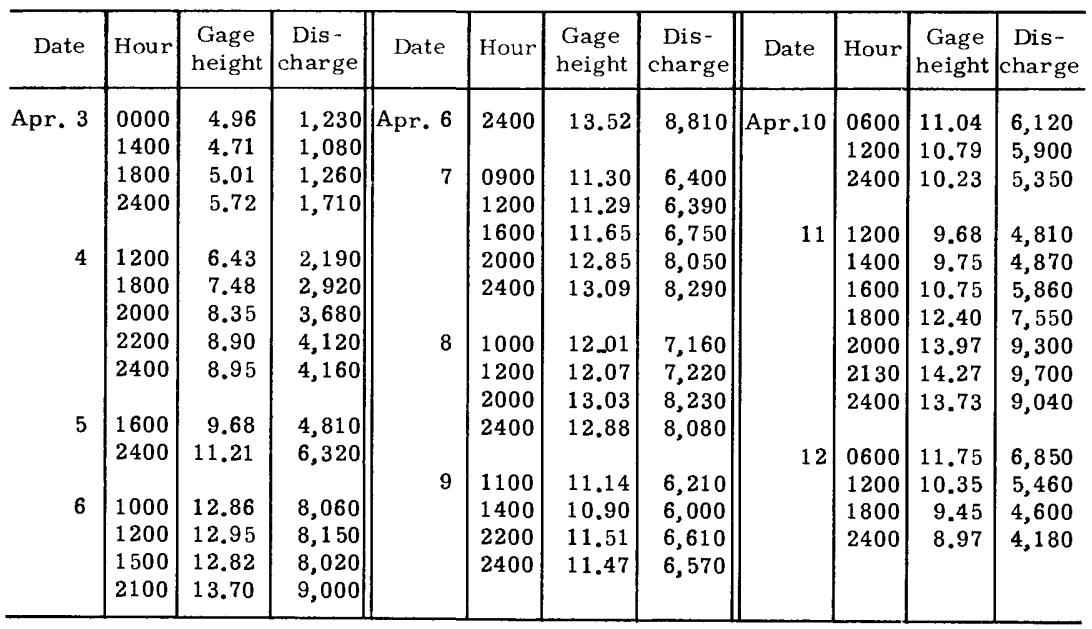

(259) 5-4620. Shell Rock River at Shell Rock, Iowa

Location.-Lat $42^{\circ} 42^{\prime} 50^{\prime \prime}$, long $92^{\circ} 34^{\prime} 55^{\prime \prime}$, in NW $\frac{1}{4} \mathrm{NE} \frac{1}{4}$ sec.11, T.91 N., R.15 W., on right bank $400 \mathrm{ft}$ upstream from bridge on State Highway 3 in Shell Rock, and 11 miles upstream from mouth.

Drainage area.- $-1,746 \mathrm{sq} \mathrm{mi}$.

Gage-height record.-Water-stage recorder graph. Datum of gage is $8 ? 5.34 \mathrm{ft}$ above mean sea level, datum of 1929.

Discharge record.-Stage-discharge relation defined by current-meter measurements below 32,900 cfs. Backwater from ice Mar. 1-3, Apr. 1.

Maxima.-March-May 1965: Discharge, 24,400 cfs 1000 hours Apr. 7 (gage height, $15.09 \mathrm{ft})$.

1953 to February 1965: Discharge, 33,500 cfs Mar. 28, 1961 (gage height, 16.26 ft).

Flood in 1856 reached a stage of $17.7 \mathrm{ft}$ at bridge $400 \mathrm{ft}$ downstream, from information furnished by Corps of Engineers (discharge, about $45,000 \mathrm{cfs}$ ). 
Mean discharge, in cubic feet per second, 1965, of Shell Rock River at Shell Rock, Iowa

\begin{tabular}{|c|c|c|c|c|c|c|c|c|c|c|c|}
\hline Day & March & April & May & Day & March & April & May & Day & March & April & May \\
\hline & 4,950 & 6,000 & 2,200 & & 835 & 13,100 & 1,650 & $181-6$ & 390 & 3,490 & 1,160 \\
\hline & 13,900 & 8,850 & 1,990 & 12. & 817 & 13,500 & 1,530 & 22 & 395 & 3,190 & 1,130 \\
\hline & 8,000 & 6,380 & 1,870 & $13 \ldots$ & 790 & 16,000 & 1,380 & 23 & 372 & 2,880 & 1,080 \\
\hline & 2,900 & 6,620 & 1,630 & $14 \ldots$ & 782 & 9,930 & 1,250 & $24=-$ & 337 & 2,780 & 1,200 \\
\hline & 1,410 & 12,200 & 1,530 & $15 \ldots$ & 726 & 7,880 & 1,240 & 25 & 321 & 2,880 & 1,490 \\
\hline & 1,210 & 19,100 & 2,640 & $16 \ldots$ & 662 & 6,820 & 1,250 & 26 & 317 & 3,140 & 2,880 \\
\hline & 1,240 & 23,400 & 2,860 & 17. & 897 & 6,050 & 1,470 & 27. & 309 & 3,230 & 2,780 \\
\hline & 1,080 & 19,400 & 2,520 & $18_{\ldots}$ & 705 & 5,210 & 1,470 & 28. & 305 & 2,820 & 2,680 \\
\hline & 1,010 & 20,800 & 2,380 & 19. & 504 & 4,540 & 1,280 & 29. & 309 & 2,640 & 2,340 \\
\hline & 926 & 17,100 & 1,970 & 20. & 396 & 3,870 & 1,240 & $30 \ldots$ & 329 & 2,400 & 2,140 \\
\hline & & & & & & & & & 941 & & 2,060 \\
\hline \multicolumn{9}{|c|}{ Monthly mean discharge, in cubic feet per second } & 1,550 & 8,540 & 1,816 \\
\hline \multicolumn{9}{|c|}{ Runoff, in inches } & 1.02 & 5.46 & 1.20 \\
\hline \multicolumn{9}{|c|}{ Runoff, in acre - feet } & 95,340 & 508,200 & 111,700 \\
\hline
\end{tabular}

Gage height, in feet, and discharge, in cubic feet per second, at indicated time, 1965

\begin{tabular}{|c|c|c|c|c|c|c|c|c|c|c|c|}
\hline Date & Hour & $\begin{array}{c}\text { Gage } \\
\text { height }\end{array}$ & $\begin{array}{c}\text { Dis- } \\
\text { charge }\end{array}$ & Date & Hour & $\begin{array}{c}\text { Gage } \\
\text { height }\end{array}$ & $\begin{array}{c}\text { Dis- } \\
\text { charge }\end{array}$ & Date & Hour & $\begin{array}{c}\text { Gag? } \\
\text { heigl }\end{array}$ & $\begin{array}{l}\text { Dis- } \\
\text { charge }\end{array}$ \\
\hline \multirow[t]{4}{*}{ Apr. 4} & 1200 & 11.04 & 6,050 & Apr. 7 & 1400 & 15.04 & 24,000 & Apr. 12 & 0800 & 12.72 & 12,000 \\
\hline & 1800 & 11.37 & 7,140 & & 2400 & 14.85 & 22,600 & & 1200 & 12.83 & 12,400 \\
\hline & 2400 & 11.91 & 8,990 & & & & & & 1600 & 13.14 & 13,700 \\
\hline & & & & 8 & 1200 & 14.20 & 18,600 & & 2400 & 14.05 & 17,800 \\
\hline \multirow[t]{5}{*}{5} & 0600 & 12.35 & 10,600 & & 1800 & 14.05 & 17,800 & & & & \\
\hline & 1200 & 12.70 & 11,900 & & 2400 & 14.15 & 18,400 & 13 & 0400 & 14.18 & 18,500 \\
\hline & 1800 & 13.17 & 13,800 & & & & & & 0800 & 14.07 & 18,000 \\
\hline & 2400 & 13.76 & 16,400 & 9 & 1200 & 14.66 & 21,400 & & 1200 & $13.7^{\circ}$ & 16,500 \\
\hline & & & & & 1800 & 14.76 & 22,000 & & 1800 & 13.21 & 14,000 \\
\hline \multirow[t]{4}{*}{6} & 0600 & 14.10 & $18,1,00$ & & 2400 & 14.63 & 21,200 & & 2400 & 12.66 & 12,000 \\
\hline & 1800 & 14.45 & 20,000 & & & & & & & & \\
\hline & 2400 & 14.73 & 21,800 & 10 & 1200 & 13.82 & 16,700 & 14 & 0600 & 12.25 & 10,600 \\
\hline & & & & & 2400 & 13.19 & 13,900 & & 1200 & $11.9^{\circ}$ & 9,710 \\
\hline \multirow[t]{2}{*}{7} & 0600 & 14.94 & 23,300 & & & & & & 2400 & 11.59 & 8,570 \\
\hline & 1000 & 15.09 & 24,400 & 11 & 2400 & 12.80 & 12,300 & & & & \\
\hline
\end{tabular}

(260) 5-4630. Beaver Creek at New Hartford, Iowa

Location.-Lat $42^{\circ} 34^{\prime} 20^{\prime \prime}$, long $92^{\circ} 36^{\prime} 55^{\prime \prime}$, in $\mathrm{SE} \frac{1}{4} \mathrm{SE} \frac{1}{4}$ sec. 28, T.90 N., R.15 W., on downstream side of center bridge pier on county highway bridge, a quarter of a mile north of New Hartford, and 8 miles upstream from mouth.

Drainage area. $-347 \mathrm{sq} \mathrm{mi}$.

Gage-height record.-Water-stage recorder graph except for Mar. 10-16, 21-30. Graph was reconstructed on basis of once-daily wire weight gage readings Mar. 14-16, 21-30. Datum of gage is $881.30 \mathrm{ft}$ above mean sea level, datum of 1929 .

Discharge record. - Stage-discharge relation defined by current-meter measurements below 10,600 cfs. Backwater from ice Mar. 1 to Apr. 5.

Maxima.-March-May 1965: Discharge, 9,610 cfs 1100 hours Apr. 6 (gage height, $11.80 \mathrm{ft}$ ).

1945 to February 1965: Discharge, 18,000 cfs June 13, 1947 (gage height, $13.5 \mathrm{ft}$, from graph based on gage readings), from rating curve extended above 7,300 cfs by logarithmic plotting.

Cooperation.-One discharge measurement furnished by Corps of Engineers. 
Mean discharge, in cubic feet per second, 1965, of Beaver Creek at New Hartford, Iowa

\begin{tabular}{|c|c|c|c|c|c|c|c|c|c|c|c|}
\hline Day & March & April & May & Day & March & April & May & Day & March & A pril & May \\
\hline & 600 & 600 & 195 & $11 \ldots$ & 104 & 935 & 93 & 21. & 46 & 202 & 62 \\
\hline$a_{-}$ & 2,000 & 4,100 & 173 & $12_{-}$ & 94 & 811 & 87 & $22_{-}$ & 43 & 186 & 66 \\
\hline 3. & 1,000 & 3,500 & 152 & $13 \ldots$ & 88 & 531 & 82 & 23. & 41 & 174 & 86 \\
\hline 4 & 670 & 3,200 & 137 & 14. & 84 & 422 & 79 & 24 & 39 & 172 & 172 \\
\hline $5 \ldots$ & 370 & 6,000 & 129 & $15 \ldots$ & 80 & 396 & 78 & 25 & 37 & 236 & 189 \\
\hline $6 \ldots$ & 272 & 8,520 & 124 & $16 \ldots$ & 78 & 357 & 78 & 26 & 36 & 391 & 1,190 \\
\hline 7. & 200 & 4,600 & 117 & $17 \ldots$ & 72 & 308 & 75 & 27 & 36 & 359 & 3,980 \\
\hline 8. & 166 & 2,810 & 112 & $18 \ldots$ & 64 & 271 & 71 & $28 \ldots$ & 35 & 304 & 1,680 \\
\hline 9. & 132 & 2,000 & 111 & $19=$ & 57 & 240 & 65 & $29 \ldots$ & 35 & 259 & 797 \\
\hline 10 & 114 & 1,080 & 101 & $20 \ldots$ & 51 & 219 & 62 & $30_{\ldots}$ & 35 & 220 & 704 \\
\hline & & & & & & & & $31_{-}$ & 76 & $-\cdots---$ & 596 \\
\hline \multirow{3}{*}{\multicolumn{9}{|c|}{$\begin{array}{l}\text { Monthly mean discharge, in cubic feet per second } \\
\text { Runoff, in inches } \\
\text { Rumoff, in acre }\end{array}$}} & 218 & 1,447 & 376 \\
\hline & & & & & & & & & 0.72 & 4.65 & 1.25 \\
\hline & & & & & & & & & 13,400 & 86,090 & 23,090 \\
\hline
\end{tabular}

Gage height, in feet, and discharge, in cubic feet per second, at indicated time, 1965

\begin{tabular}{|c|c|c|c|c|c|c|c|c|c|c|c|}
\hline Date & Hour & $\begin{array}{c}\text { Gage } \\
\text { height }\end{array}$ & $\begin{array}{c}\text { Dis- } \\
\text { charge }\end{array}$ & Date & Hour & $\begin{array}{c}\text { Gage } \\
\text { height }\end{array}$ & $\begin{array}{c}\text { Dis- } \\
\text { charge }\end{array}$ & Date & Hour & $\begin{array}{l}\text { Gage } \\
\text { height }\end{array}$ & $\begin{array}{l}\text { Dis- } \\
\text { charge }\end{array}$ \\
\hline Apr. 2 & $\begin{array}{l}0000 \\
0200 \\
0400 \\
0600 \\
0800 \\
1200 \\
1800 \\
2400 \\
0800 \\
1600 \\
2400 \\
1200\end{array}$ & $\begin{array}{r}9.73 \\
10.14 \\
10.58 \\
11.22 \\
11.66 \\
11.79 \\
11.42 \\
10.92 \\
\\
10.30 \\
9.88 \\
9.73 \\
9.79\end{array}$ & 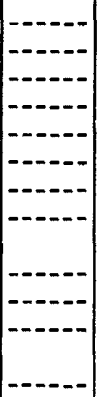 & Apr. 4 & $\begin{array}{l}1800 \\
2400 \\
0800 \\
1800 \\
2400 \\
\\
1100 \\
1600 \\
2400 \\
\\
0600 \\
1000 \\
1200\end{array}$ & $\begin{array}{r}9.92 \\
10.17 \\
10.83 \\
11.25 \\
11.38 \\
11.80 \\
11.62 \\
11.03 \\
10.51 \\
10.32 \\
10.10\end{array}$ & \begin{tabular}{l} 
\\
\hdashline... \\
\hdashline \\
\hdashline, 740 \\
\\
9,610 \\
9,000 \\
6,610 \\
5,330 \\
4,910 \\
4,450
\end{tabular} & Apr. 7 & $\begin{array}{l}1800 \\
2400 \\
1800 \\
2400 \\
\\
1200 \\
1800 \\
2400 \\
\\
0600 \\
1200 \\
1800 \\
2400\end{array}$ & $\begin{array}{l}9.62 \\
9.43 \\
\\
8.90 \\
8.87 \\
\\
8.53 \\
8.27 \\
7.84 \\
\\
7.50 \\
7.13 \\
6.83 \\
6.78\end{array}$ & $\begin{array}{r}3,600 \\
3,300 \\
\\
2,520 \\
2,480 \\
\\
2,050 \\
1,760 \\
1,410 \\
\\
1,190 \\
1,040 \\
942 \\
922\end{array}$ \\
\hline
\end{tabular}

(261) 5-4635. Blackhawk Creek at Hudson, Iowa

Location.-Lat $42^{\circ} 24^{\prime} 30^{\prime \prime}$, long $92^{\circ} 27^{\prime} 45^{\prime \prime}$, in SW $\frac{1}{4} \mathrm{NE} \frac{1}{4}$, sec. $27, \mathrm{~T} .88 \mathrm{~N}, \mathrm{R} .14 \mathrm{~W}$, on left bank $35 \mathrm{ft}$ downstream from bridge on State Highway 58, and 0.2 mile northwest of Chicago Great Western Railway tracks in the west edge of Hudson.

Drainage area. -303 sq $\mathrm{mi}$.

Gage-height record.-Water-stage recorder except Mar. 3, Apr. 6-19, May 5-22, for which graph was reconstructed on basis of daily wire-weight gage readings. Datum of gage is $865.03 \mathrm{ft}$ above mean sea level, datum of 1929 .

Discharge record.-Stage-discharge relation defined by current-meter reasurements below 7,720 cfs. Backwater from ice Mar. 1-5, Mar. 16 to April 1.

Maxima.-March-May 1965: Discharge, 7,500 cfs 0900 hours Apr. 6 (gage height, $16.35 \mathrm{ft}$ ).

1952 to February 1965: Discharge, about 9,000 cfs Mar. 31, 1960 (gage height, $16.93 \mathrm{ft}$, backwater from ice). 
Mean discharge, in cubic feet per second, 1965, of Blackhawk Creek at Hudson. Iowa

\begin{tabular}{|c|c|c|c|c|c|c|c|c|c|c|c|}
\hline Day & March & April & May & Day & March & April & May & Day & March & April & May \\
\hline $\begin{array}{l}1- \\
2- \\
3- \\
1 \\
5 \\
6- \\
6- \\
7- \\
8- \\
9\end{array}$ & $\begin{array}{r}1,080 \\
1,700 \\
1,140 \\
700 \\
415 \\
297 \\
222 \\
190 \\
156 \\
142\end{array}$ & $\begin{array}{r}4,000 \\
3,090 \\
1,700 \\
2,080 \\
3,650 \\
5,880 \\
2,040 \\
654 \\
549 \\
330\end{array}$ & $\begin{array}{r}160 \\
141 \\
122 \\
110 \\
105 \\
96 \\
88 \\
83 \\
78 \\
69\end{array}$ & 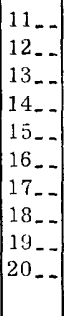 & $\begin{array}{r}123 \\
111 \\
99 \\
101 \\
147 \\
154 \\
154 \\
118 \\
86 \\
60\end{array}$ & $\begin{array}{r}458 \\
366 \\
241 \\
194 \\
182 \\
171 \\
139 \\
135 \\
121 \\
99\end{array}$ & $\begin{array}{l}61 \\
58 \\
53 \\
51 \\
49 \\
47 \\
45 \\
42 \\
39 \\
35\end{array}$ & 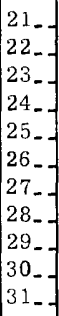 & $\begin{array}{r}45 \\
40 \\
34 \\
32 \\
30 \\
29 \\
28 \\
27 \\
27 \\
40 \\
250\end{array}$ & $\begin{array}{r}\subsetneq 2 \\
\& 4 \\
79 \\
\varepsilon 7 \\
1 \varepsilon 5 \\
345 \\
290 \\
244 \\
215 \\
184 \\
- \\
---\end{array}$ & $\begin{array}{r}33 \\
38 \\
140 \\
283 \\
222 \\
309 \\
461 \\
297 \\
233 \\
227 \\
222\end{array}$ \\
\hline \multicolumn{9}{|c|}{$\begin{array}{l}\text { Monthly mean discharge, in cubic feet per second } \\
\text { Runoff. in inches } \\
\text { Runoff, in acre-feet }\end{array}$} & $\begin{array}{r}251 \\
0.95 \\
15,430\end{array}$ & $\begin{array}{r}929 \\
3.42 \\
55,310\end{array}$ & $\begin{array}{r}129 \\
0.49 \\
7,930\end{array}$ \\
\hline
\end{tabular}

Gage height, in feet, and discharge, in cubic feet per second, at indicated time, 1965

\begin{tabular}{|c|c|c|c|c|c|c|c|c|c|c|c|}
\hline Date & Hour & $\begin{array}{c}\text { Gage } \\
\text { height }\end{array}$ & $\begin{array}{c}\text { Dis- } \\
\text { charge }\end{array}$ & Date & Hour & $\begin{array}{c}\text { Gage } \\
\text { height }\end{array}$ & $\begin{array}{c}\text { Dis- } \\
\text { charge }\end{array}$ & Date & Hour & $\begin{array}{c}\text { Gage } \\
\text { height }\end{array}$ & $\begin{array}{l}\text { Dis- } \\
\text { charge }\end{array}$ \\
\hline Apr. 1 & $\begin{array}{l}0000 \\
0100 \\
0200 \\
0300 \\
0400 \\
0500 \\
0700 \\
1100 \\
1200 \\
1300 \\
1800 \\
2400 \\
0600 \\
1000 \\
1400 \\
1800 \\
2100 \\
2400 \\
0400\end{array}$ & $\begin{array}{l}13.41 \\
14.00 \\
14.72 \\
15.24 \\
15.20 \\
15.37 \\
15.68 \\
16.00 \\
16.01 \\
15.98 \\
15.70 \\
15.57 \\
15.33 \\
15.14 \\
15.15 \\
14.96 \\
14.63 \\
14.24 \\
14.18\end{array}$ & \begin{tabular}{r}
$-\cdots$ \\
$-\cdots$ \\
\hdashline-1 \\
\hdashline, 40 \\
3,490 \\
4,340 \\
5,600 \\
5,640 \\
5,520 \\
4,400 \\
4,010 \\
3,320 \\
3,040 \\
3,050 \\
2,860 \\
2,560 \\
2,270 \\
2,240
\end{tabular} & Apr. 3 & $\begin{array}{l}0800 \\
1700 \\
2400 \\
\\
0500 \\
1000 \\
1300 \\
1800 \\
2400 \\
\\
0400 \\
0800 \\
1200 \\
1500 \\
1900 \\
2400\end{array}$ & $\begin{array}{l}13.00 \\
12.67 \\
12.77 \\
12.87 \\
13.37 \\
14.27 \\
14.72 \\
14.63 \\
14.65 \\
14.94 \\
15.22 \\
15.49 \\
15.79 \\
16.04\end{array}$ & $\begin{array}{l}1,590 \\
1,470 \\
1,500 \\
1,540 \\
1,780 \\
2,290 \\
2,640 \\
2,560 \\
2,580 \\
2,840 \\
3,160 \\
3,780 \\
4,760 \\
6,000 \\
7,200 \\
7,500\end{array}$ & Apr. 6 & $\begin{array}{l}1200 \\
1600 \\
1900 \\
2200 \\
2400 \\
\\
0200 \\
0800 \\
1400 \\
1900 \\
2000 \\
2200 \\
2400 \\
\\
0300 \\
0600 \\
1200 \\
1800 \\
2400\end{array}$ & $\begin{array}{r}16.28 \\
15.94 \\
15.65 \\
15.35 \\
15.14 \\
\\
14.92 \\
14.32 \\
13.73 \\
12.98 \\
12.36 \\
11.48 \\
10.84 \\
1 C .14 \\
\subseteq .74 \\
\subseteq .42 \\
\subseteq .31 \\
\subseteq .36\end{array}$ & $\begin{array}{r}7,150 \\
5,360 \\
4,250 \\
3,450 \\
3,040 \\
\\
2,820 \\
2,320 \\
1,960 \\
1,580 \\
1,380 \\
1,100 \\
935\end{array}$ \\
\hline
\end{tabular}


(262) 5-4640. Cedar River at Waterloo, Iowa

Location.-Lat $42^{\circ} 29^{\prime} 40^{\prime \prime}$, long $92^{\circ} 20^{\prime} 00^{\prime \prime}$, in NW $\frac{1}{4} N W \frac{1}{4} \sec .25, T .89$ N., R.13 W., on left bank at foot of East Seventh Street, 0.3 mile upstream from Eleventh Avenue Bridge in Waterloo, 1 mile downstream from Blackhawk Creek, and at mile 187.9 above the mouth of Iowa River (U.S. Geological Survey river profile).

Drainage area. $-5,146 \mathrm{sq} \mathrm{mi}$.

Gage-height record.-Water-stage recorder graph. Datum of gage is $824.09 \mathrm{ft}$ above mean sea level, datum of 1929 .

Discharge record.-Stage-discharge relation defined by current-meter measurements below 76,400 cfs. Backwater from ice Mar. 1.

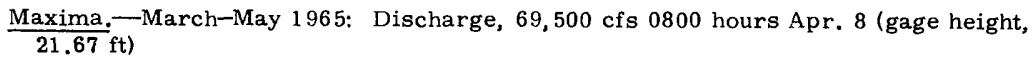

1940 to February 1965: Discharge, 76,700 cfs Mar. 29, 1961 (gage height, 21.86 ft).

Flood of Mar. 16, 1929, reached a stage of about $20 \mathrm{ft}$, determined by Corps of Engineers, from information by city of Waterloo (discharge, $65,000 \mathrm{cfs}$ ). Flood of Apr. 2, 1933, reached a stage about $0.5 \mathrm{ft}$ lower than Mar. 16, 1929, from information by city of Waterloo (discharge, $61,000 \mathrm{cfs}$ ).

Mean discharge, in cubic feet per second, 1965

\begin{tabular}{|c|c|c|c|c|c|c|c|c|c|c|c|}
\hline Day & March & April & iMay & Day & March & April & May & Day & March & April & May \\
\hline & 5,000 & 11,200 & 5,030 & $11 \ldots$ & 3,090 & 47,200 & 3,750 & 21. & 1,350 & 7,500 & 2,260 \\
\hline & 12,300 & 19,800 & 4,520 & 12 & 2,680 & 35,200 & 3,420 & 22 & 1,240 & 6,730 & 2,480 \\
\hline 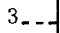 & 24,200 & 21,300 & 4,360 & $13 \ldots$ & 2,480 & 34,100 & 2,890 & 23. & 1,180 & 6,060 & 2,370 \\
\hline-3 & 30,700 & 21,800 & 4,060 & $14 \ldots$ & 2,370 & 34,900 & 2,730 & 24 & 1,110 & 5,670 & 2,730 \\
\hline & 21,800 & 29,800 & 3,720 & $15--$ & 2,370 & 23,600 & 2,630 & 25 & 1,150 & 5,610 & 3,030 \\
\hline - & 11,800 & 51,700 & 3,650 & $16 \ldots$ & 2,530 & 16,500 & 2,560 & $26 \ldots$ & 1,090 & 6,090 & 4,110 \\
\hline & 7,490 & 65,800 & 4,580 & $\left|77_{-}\right|$ & 2,190 & 13,500 & 2,730 & 27. & 1,020 & 6,600 & 7,760 \\
\hline & 5,320 & 68,000 & 4,810 & $18_{-}$ & 1,380 & 11,600 & 2,860 & 28 & 960 & 6,570 & 9,070 \\
\hline 9 & 4,140 & 60,200 & 4,580 & $\left|19_{-}\right|$ & 1,400 & 10,100 & 2,930 & 29. & 990 & 6,150 & 7,840 \\
\hline \multirow[t]{2}{*}{$10_{-}-$} & 3,470 & 55,400 & 4,390 & $20 \ldots$ & 1,440 & 8,740 & 2,510 & $30_{-}$ & 1,340 & 5,480 & 6,850 \\
\hline & & & & & & & & & 2,780 & - & 5,960 \\
\hline \multicolumn{9}{|c|}{ Monthly mean discharge, in cubic feet per second } & 5,237 & 23,430 & 4,102 \\
\hline \multicolumn{9}{|c|}{ Runoff, in inches } & 1.17 & 5.08 & 0.92 \\
\hline \multicolumn{9}{|c|}{ Runoff, in acre-feet } & 322,000 & $1,3^{\urcorner} 4,000$ & 252,200 \\
\hline
\end{tabular}


Gage height, in feet, and discharge, in cubic feet per second, at indicated time, 1965, of Cedar River at Waterloo, Iowa

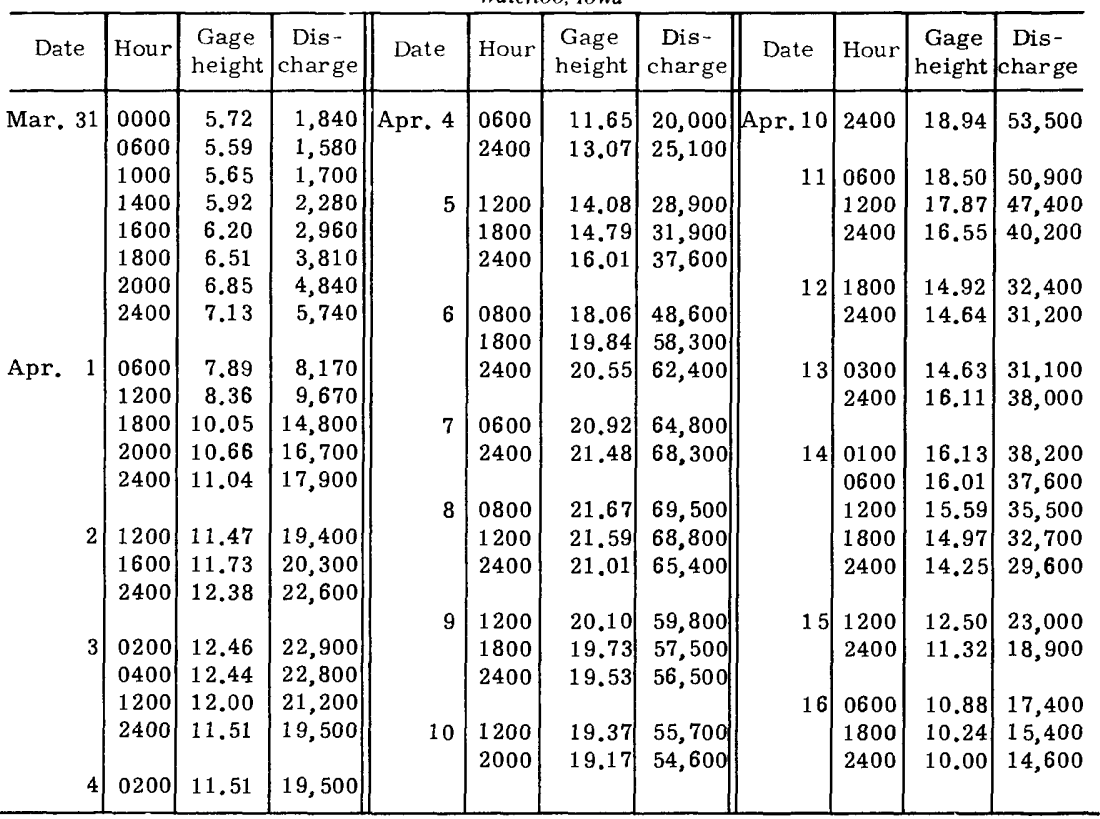

(263) 5-4641.33 Half Mile Creek near Gladbrook, Iowa

Location.-Lat $42^{\circ} 12^{\prime} 40^{\prime \prime}$, 1ong $92^{\circ} 36^{\prime} 40^{\prime \prime}$, in SW $\frac{1}{4} \mathrm{SW} \frac{1}{4}$ sec.33, T.86 N., R.15 W., on right bank $10 \mathrm{ft}$ downstream from county highway bridge, $3 / 4$ mile upstream from mouth, and 7 miles northeast of Gladbrook.

Drainage area. $-1.33 \mathrm{sq} \mathrm{mi}$.

Gage-height record.-Digital recorder tape punched at 5 minute intervals except Mar. 1-9. Datum of gage is $948.16 \mathrm{ft}$ above mean sea level, datum of $192 \varepsilon$.

Discharge record.-Stage-discharge relation defined by current-meter measurements below $96.0 \mathrm{cfs}$. Discharge for period of no gage-height record estimated on basis of weather records and records for nearby stations.

Maxima.-March-May 1965: Discharge, 43.2 cfs 1825 hours Apr. 1 (gage height, $5.22 \mathrm{ft}$.

1962 to February 1965: Discharge, 126 cfs July 18, 1963 (gage height, $6.84 \mathrm{ft}$ ). 
Mean discharge, in cubic feet per second, 1965, of Half Mile Creek near Gladbrook, lowa

\begin{tabular}{|c|c|c|c|c|c|c|c|c|c|c|c|}
\hline Day & March & April & May & Day & March & April & May & Day & March & April & May \\
\hline $\begin{array}{l}1 \ldots \\
2 \ldots \\
3 \ldots \\
4 \ldots \\
5 \ldots \\
6 \ldots \\
7 \ldots \\
8 \ldots \\
9 \ldots \\
10 .\end{array}$ & $\begin{array}{r}29.0 \\
16.0 \\
9.00 \\
4.50 \\
2.30 \\
1.00 \\
.40 \\
.20 \\
.12 \\
.10\end{array}$ & $\begin{array}{c}40.8 \\
21.2 \\
11.4 \\
16.0 \\
23.0 \\
27.6 \\
11.4 \\
15.3 \\
11.4 \\
6.95\end{array}$ & $\begin{array}{r}0.34 \\
.30 \\
.28 \\
.29 \\
.35 \\
.32 \\
.27 \\
.27 \\
.22 \\
.19\end{array}$ & $\begin{array}{l}11 \ldots \\
12 \ldots \\
13 \ldots \\
14-- \\
15 \ldots \\
16 \ldots \\
17 \ldots \\
18-- \\
19- \\
20 \ldots\end{array}$ & $\begin{array}{r}0.12 \\
.34 \\
.52 \\
1.05 \\
1.61 \\
2.07 \\
1.07 \\
.45 \\
.15 \\
.12\end{array}$ & $\begin{array}{r}2.50 \\
1.10 \\
.60 \\
.40 \\
.26 \\
.22 \\
.22 \\
.20 \\
.18 \\
.18\end{array}$ & $\begin{array}{r}0.18 \\
.16 \\
.15 \\
.15 \\
.20 \\
.17 \\
.14 \\
.12 \\
.11 \\
.10\end{array}$ & $\mid \begin{array}{l}21 \\
22 \\
23- \\
22_{-} \\
25_{-} \\
26_{-} \\
27- \\
28- \\
29_{-} \\
30_{-} \\
31 .\end{array}$ & $\begin{array}{r}0.12 \\
.13 \\
.11 \\
.09 \\
.08 \\
.08 \\
.08 \\
.08 \\
.13 \\
8.15 \\
29.2\end{array}$ & $\begin{array}{r}0.18 \\
.17 \\
.18 \\
.60 \\
2.10 \\
.97 \\
.73 \\
.60 \\
.47 \\
.40 \\
-.--\end{array}$ & $\begin{array}{r}0.10 \\
.37 \\
.31 \\
.26 \\
.27 \\
4.36 \\
.85 \\
.62 \\
.71 \\
.66 \\
.56\end{array}$ \\
\hline $\begin{array}{l}\text { Mont } \\
\text { Bunn } \\
\text { Runo }\end{array}$ & $\begin{array}{l}\text { thly mea } \\
\text { off, in in } \\
\text { ff, in ac }\end{array}$ & $\begin{array}{l}\text { disch } \\
\text { hes -- } \\
\text { e-feet }\end{array}$ & e, ir & i & eet $p$ & 800 & $\cdots$ & -- & $\begin{array}{r}3.50 \\
3.03 \\
215\end{array}$ & $\begin{array}{r}6.58 \\
5.52 \\
391\end{array}$ & $\begin{array}{r}0.432 \\
0.37 \\
27\end{array}$ \\
\hline
\end{tabular}

Gage hesght, in feet, and discharge, in cubic feet per second, at indicated time, 1965

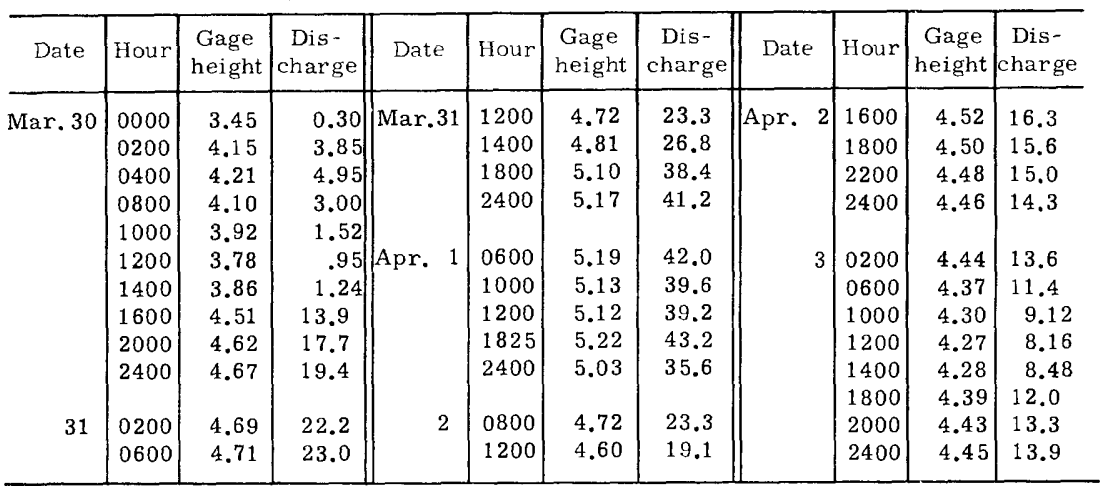

(264) 5-4641.37 Four Mile Creek near Traer, Iowa

Location.-Lat $42^{\circ} 12^{\prime} 05^{\prime \prime}$, long $92^{\circ} 33^{\prime} 45^{\prime \prime}$, near center of sec.2, T.85 N., R.15 W., on left bank $10 \mathrm{ft}$ downstream from county highway bridge, 2 miles upstreanı from mouth, and 5 miles west of Traer.

\section{Drainage area.-19.51 sq $\mathrm{mi}$.}

Gage-height record.-Water-stage recorder graph, except Mar. 2-10, Apr. 1, Apr. 7 to May 4. Datum of gage is $905.87 \mathrm{ft}$ above mean sea level, datum of 1929 .

Discharge record.-Stage-discharge relation defined by current-meter measurements below $600 \mathrm{cfs}$, Backwater from ice Mar. 2 to Apr. 4.

Maxima.--March-May 1965: Discharge, $600 \mathrm{cfs} 1600$ hours Mar. 1 (gage height, $11.91 \mathrm{ft}$ ); gage height, $12.30 \mathrm{ft} 1930$ hours Mar. 31 (backwater from ice).

1962 to February 1965: Discharge, 287 cfs July 18, 1963 (gage height, $11.24 \mathrm{ft}$ ). 
Mean discharge, in cubic feet per second, 1965, of Four Mile Creek near Traer, Iowa

\begin{tabular}{|c|c|c|c|c|c|c|c|c|c|c|c|}
\hline Day & March & April & May & Day & March & April & May & Day & March & April & May \\
\hline $\begin{array}{l}1 . \\
2 . \\
3 . \\
4 . \\
5 . \\
6 . \\
7 . \\
8 . \\
9 . \\
10\end{array}$ & $\begin{array}{r}472 \\
250 \\
140 \\
66 \\
40 \\
14 \\
5.0 \\
1.7 \\
1.0 \\
.8\end{array}$ & $\begin{array}{r}280 \\
150 \\
180 \\
230 \\
473 \\
179 \\
20 \\
32 \\
11 \\
13\end{array}$ & $\begin{array}{l}4.8 \\
4.5 \\
4.1 \\
4.5 \\
5.5 \\
4.8 \\
4.1 \\
3.9 \\
3.4 \\
2.9\end{array}$ & $\mid \begin{array}{l}11_{-} \\
12_{-} \\
13_{-} \\
14_{-} \\
15_{-} \\
16_{--} \\
17 \ldots- \\
18_{--} \\
19_{--} \\
20_{--}\end{array}$ & $\begin{array}{l}0.7 \\
1.1 \\
3.0 \\
8.0 \\
22 \\
24 \\
10 \\
6.0 \\
2.7 \\
1.5\end{array}$ & $\begin{array}{c}14 \\
7.0 \\
5.6 \\
5.4 \\
5.6 \\
4.5 \\
4.0 \\
3.5 \\
3.5 \\
3.2\end{array}$ & $\begin{array}{l}2.8 \\
2.6 \\
2.5 \\
2.4 \\
2.7 \\
2.4 \\
2.2 \\
1.9 \\
1.8 \\
1.7\end{array}$ & 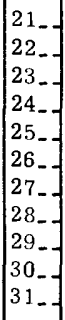 & $\begin{array}{r}0.9 \\
.7 \\
.6 \\
.5 \\
.5 \\
.4 \\
.5 \\
.7 \\
1.5 \\
50 \\
200\end{array}$ & $\begin{array}{r}3.1 \\
3.1 \\
3.4 \\
6.0 \\
16 \\
15 \\
11 \\
8.0 \\
6.6 \\
5.4 \\
- \\
-\end{array}$ & $\begin{array}{c}1.7 \\
3.6 \\
5.2 \\
4.4 \\
5.1 \\
40 \\
16 \\
10 \\
9.6 \\
9.6 \\
8.4\end{array}$ \\
\hline \multicolumn{9}{|c|}{$\begin{array}{l}\text { Monthly mean discharge, in cubic feet per second } \\
\text { Runoff, in inches } \\
\text { Runoff, in acre-feet }\end{array}$} & $\begin{array}{r}42.8 \\
2.53 \\
2,630\end{array}$ & $\begin{array}{r}56.7 \\
3.24 \\
3,330\end{array}$ & $\begin{array}{r}5.78 \\
0.34 \\
355\end{array}$ \\
\hline
\end{tabular}

Gage herght, in feet, and discharae, in cubic feet per second, at indicated time, 1965

\begin{tabular}{|c|c|c|c|c|c|c|c|c|c|c|c|}
\hline Date & Hour & $\begin{array}{c}\text { Gage } \\
\text { height }\end{array}$ & $\begin{array}{c}\text { Dis - } \\
\text { charge }\end{array}$ & Date & Hour & $\begin{array}{c}\text { Gage } \\
\text { height }\end{array}$ & $\begin{array}{c}\text { Dis- } \\
\text { charge }\end{array}$ & Date & Hour & $\begin{array}{c}\text { Gage } \\
\text { height }\end{array}$ & $\begin{array}{c}\text { Dis- } \\
\text { charge }\end{array}$ \\
\hline Mar. 1 & $\begin{array}{l}0000 \\
0400 \\
0700 \\
0800 \\
0900 \\
1000 \\
1200 \\
1400 \\
1600 \\
1800 \\
2000 \\
2400 \\
2400 \\
0400 \\
0800\end{array}$ & $\begin{array}{l}11.47 \\
11.16 \\
10.99 \\
11.00 \\
11.54 \\
11.78 \\
11.86 \\
11.88 \\
11.91 \\
11.85 \\
11.76 \\
11.52 \\
8.26 \\
8.72 \\
9.00\end{array}$ & $\begin{array}{r}416 \\
343 \\
318 \\
319 \\
439 \\
536 \\
575 \\
585 \\
600 \\
570 \\
527 \\
432 \\
\\
-\ldots- \\
-\ldots-\end{array}$ & 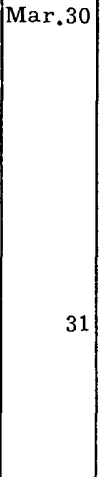 & $\begin{array}{l}1100 \\
1200 \\
1300 \\
1500 \\
1600 \\
1700 \\
1800 \\
1900 \\
2200 \\
2400 \\
0400 \\
0700 \\
0800 \\
1100 \\
1200\end{array}$ & $\begin{array}{r}9.18 \\
9.14 \\
9.48 \\
10.00 \\
10.94 \\
11.54 \\
11.65 \\
11.66 \\
11.61 \\
11.55 \\
11.23 \\
11.00 \\
10.99 \\
10.70 \\
10.70\end{array}$ & $\begin{array}{l}-1 \\
- \\
- \\
- \\
-\end{array}$ & \begin{tabular}{|l} 
Mar. 31 \\
\\
Apr. 1
\end{tabular} & $\begin{array}{l}1300 \\
1400 \\
1500 \\
1700 \\
1800 \\
1900 \\
1930 \\
2000 \\
2400 \\
0400 \\
0800 \\
1200 \\
1400 \\
2000 \\
2400\end{array}$ & $\begin{array}{l}11.10 \\
11.63 \\
11.70 \\
12.00 \\
12.21 \\
12.29 \\
12.30 \\
12.25 \\
12.17 \\
12.05 \\
11.89 \\
11.63 \\
11.60 \\
11.74 \\
11.72\end{array}$ & - \\
\hline
\end{tabular}

(265) 5-4645. Cedar River at Cedar Rapids, Iowa

Location.-Lat $41^{\circ} 58^{\prime} 20^{\prime \prime}$, long $91^{\circ} 40^{\prime} 05^{\prime \prime}$, in SE $\frac{1}{4} N W \frac{1}{4}$ sec. $28, T .83$ N., R.7 W., on right bank $500 \mathrm{ft}$ upstream from Eighth Avenue Bridge in Cedar Rapids, 2.7 miles upstream from Prairie Creek, and at mile 112.7 above mouth of Iowa River.

Drainage area.-6, $-610 \mathrm{sq} \mathrm{mi}$.

Gage-height record.-Water-stage recorder graph, except Apr. 12, 13 and May 26. Datum of gage is $700.47 \mathrm{ft}$ above mean sea level, datum of 1929 .

Discharge record.- - Stage-discharge relation defined by current-meter measurements below 72,000 cfs. Backwater from ice Mar. 2-6, 19-31.

Maxima.-March-May 1965: Discharge, 66,800 efs 1730 hours Apr. 10 (gage height, $18.51 \mathrm{ft}$ ).

1902 to February 1965: Discharge, 73,000 cfs Mar. 31, 1961; gage height, $20.0 \mathrm{ft}$ Mar. 18, 1929.

Flood in June 1851 reached a stage of about $20 \mathrm{ft}$ (discharge, 65,000 cfs. estimated). 
Mean discharge, in cubic feet per second, 1965, of Cedar River at Cedar Rapids, Iowa

\begin{tabular}{|c|c|c|c|c|c|c|c|c|c|c|c|}
\hline Day & March & April & May & Day & March & April & May & Day & March & April & May \\
\hline $\begin{array}{l}1 \ldots . \\
2 \ldots \\
3 \ldots \\
4 \ldots \\
5 \ldots \\
6 \ldots \\
7 \ldots \\
8 \ldots \\
9 \ldots \\
10 \ldots\end{array}$ & $\begin{array}{r}4,440 \\
7,000 \\
7,500 \\
8,000 \\
10,000 \\
24,000 \\
27,200 \\
19,800 \\
14,800 \\
10,500\end{array}$ & $\begin{array}{r}9,240 \\
11,600 \\
14,300 \\
20,000 \\
23,300 \\
28,500 \\
35,100 \\
49,100 \\
61,200 \\
66,100\end{array}$ & $\begin{array}{l}7,080 \\
6,280 \\
5,380 \\
5,240 \\
5,070 \\
4,650 \\
4,350 \\
4,710 \\
5,280 \\
5,070\end{array}$ & $\begin{array}{l}11 \ldots- \\
12 \ldots- \\
13-- \\
14 \ldots \\
15-- \\
16 \_- \\
17-- \\
18 \ldots \\
19-- \\
20 \ldots\end{array}$ & $\begin{array}{l}7,480 \\
5,620 \\
5,620 \\
5,040 \\
5,730 \\
6,200 \\
6,080 \\
5,180 \\
1,700 \\
1,500\end{array}$ & $\begin{array}{l}62,100 \\
55,000 \\
45,000 \\
36,400 \\
32,400 \\
33,000 \\
25,600 \\
18,300 \\
14,600 \\
12,500\end{array}$ & $\begin{array}{l}4,890 \\
4,500 \\
4,050 \\
3,680 \\
3,580 \\
3,360 \\
3,230 \\
3,160 \\
3,260 \\
3,330\end{array}$ & $\mid \begin{array}{l}21 . \\
22 . \\
23 . \\
24 . \\
25- \\
26 \ldots \\
27- \\
28 \ldots \\
29- \\
30 . \\
31_{-}\end{array}$ & $\begin{array}{l}1,700 \\
1,900 \\
1,800 \\
1,700 \\
1,900 \\
2,100 \\
2,200 \\
2,000 \\
2,200 \\
4,000 \\
6,700\end{array}$ & $\begin{array}{r}10,900 \\
9,520 \\
8,400 \\
7,880 \\
7,880 \\
7,600 \\
7,440 \\
7,680 \\
7,800 \\
7,600 \\
\end{array}$ & $\begin{array}{l}3,130 \\
3,060 \\
3,100 \\
3,180 \\
3,180 \\
4,000 \\
5,340 \\
6,800 \\
8,440 \\
9,040 \\
8,560\end{array}$ \\
\hline \multicolumn{9}{|c|}{$\begin{array}{l}\text { Monthly mean discharge, in cubic feet per second } \\
\text { Runoff, in inches } \\
\text { Runoff, in acre -feet }\end{array}$} & $\begin{array}{r}6,825 \\
1.21 \\
419,700\end{array}$ & $\begin{array}{r}24,530 \\
4.20 \\
1,460,000\end{array}$ & $\begin{array}{r}4,774 \\
0.85 \\
293,500\end{array}$ \\
\hline
\end{tabular}

Gage height, in feet, and discharge, in cubic feet per second, at indicated time, 1965

\begin{tabular}{|c|c|c|c|c|c|c|c|c|c|c|c|}
\hline Date & Hour & $\begin{array}{c}\text { Gage } \\
\text { height }\end{array}$ & $\begin{array}{c}\text { Dis - } \\
\text { charge }\end{array}$ & Date & Hour & $\begin{array}{c}\text { Gage } \\
\text { height }\end{array}$ & $\begin{array}{c}\text { Dis- } \\
\text { charge }\end{array}$ & Date & Hour & $\begin{array}{c}\text { Gage } \\
\text { height }\end{array}$ & $\begin{array}{c}\text { Dis- } \\
\text { charge }\end{array}$ \\
\hline Apr. 6 & $\begin{array}{l}0000 \\
0600 \\
1200 \\
1800 \\
2400 \\
\\
0600 \\
1200 \\
1800\end{array}$ & $\begin{array}{r}9.67 \\
10.10 \\
10.43 \\
10.63 \\
10.88 \\
\\
11.30 \\
11.68 \\
12.38\end{array}$ & $\begin{array}{l}25,600 \\
27,400 \\
28,800 \\
29,700 \\
30,800 \\
32,600 \\
34,300 \\
37,400\end{array}$ & $\begin{array}{r}\text { Apr. } 7 \\
8\end{array}$ & $\begin{array}{l}2400 \\
0600 \\
1200 \\
1800 \\
2400 \\
0600\end{array}$ & $\begin{array}{l}13.22 \\
14.22 \\
15.00 \\
15.88 \\
16.57 \\
17.08\end{array}$ & $\begin{array}{l}41,100 \\
45,600 \\
49,100 \\
53,100 \\
56,400 \\
59,000\end{array}$ & Apr. 9 & $\begin{array}{l}1200 \\
1800 \\
2400 \\
0600 \\
1200 \\
1730 \\
2400\end{array}$ & $\begin{array}{l}17.53 \\
17.93 \\
18.14 \\
18.33 \\
18.44 \\
18.51 \\
18.33\end{array}$ & $\begin{array}{l}61,500 \\
63,600 \\
64,800 \\
65,800 \\
66,400 \\
66,800 \\
65,800\end{array}$ \\
\hline
\end{tabular}

(266) Cedar River near Rochester, Iowa

(Miscellaneous site)

Location. $-\mathrm{SE} \frac{1}{4}$ sec.13, T.79 N., R.3 W., at Interstate Highway I-80 bridg?, 1.5 miles south of Rochester.

Drainage area.- $-7,245 \mathrm{sq} \mathrm{mi}$.

Discharge record.-Stage-discharge relation defined by current-meter measurements.

Maxima.-March-May 1965: Discharge, 67,200 cfs Apr. 11 (elevation $651.86 \mathrm{ft}$ above mean sea level).

1961 to March 1965: Discharge 71,700 cfs Apr. 1, 1961 (elevation, $652.08 \mathrm{ft}$ above mean sea leve1). 
(267) 5-4650. Cedar River near Conesville, Iowa

Location.-Lat $41^{\circ} 24^{\prime} 30^{\prime \prime}$, long $91^{\circ} 17^{\prime} 15^{\prime \prime}$, in SW $\frac{1}{4} \mathrm{SW} \frac{1}{4}$ sec.2, T.76 N., R.4 W., on right bank $10 \mathrm{ft}$ downstream from highway bridge, 3.4 miles northeast of Conesville, 5.2 miles downstream from Wapsinonoc Creek, 10.7 miles upstream from mouth, and at mile 39.8 upstream from mouth of Iowa River. (U.S. Geological Survey river profile).

Drainage area.- $-7,785 \mathrm{sq} \mathrm{mi}$.

Gage-height record.-Water-stage recorder graph, except Mar. 3-5, 21-28. Wireweight gage readings were available. Datum of gage is $581.95 \mathrm{ft}$ above meen sea level, datum of 1929 .

Discharge record.-Stage-discharge relation defined by current-meter meas irements below 70,000 cfs. Backwater from ice Mar, 1-7, 14-29.

Maxima.-March-May 1965: Discharge, 68,100 cfs 2100 hours Apr. 12 (gage height, $16.85 \mathrm{ft}$ ).

1939 to February 1965: Discharge, 70,800 cfs Apr. 2, 1961 (gage height, $16.62 \mathrm{ft}$ ).

Flood in March 1929 reached a stage of $15.8 \mathrm{ft}$, from information by local residents to Corps of Engineers.

Cooperation,-One discharge measurement furnished by Corps of Engineers.

Mean discharge, in cubic feet per second, 1965

\begin{tabular}{|c|c|c|c|c|c|c|c|c|c|c|c|}
\hline Day & March & April & May & Day & March & April & May & Day & March & April & May \\
\hline 10 & $\begin{array}{r}3,300 \\
4,000 \\
4,200 \\
4,500 \\
4,700 \\
8,400 \\
13,600 \\
26,100 \\
34,000 \\
27,000\end{array}$ & $\begin{array}{r}9,840 \\
11,200 \\
12,500 \\
13,700 \\
16,400 \\
22,200 \\
28,900 \\
30,800 \\
35,200 \\
46,400\end{array}$ & $\begin{array}{r}10,000 \\
9,200 \\
8,320 \\
7,610 \\
7,450 \\
7,050 \\
6,620 \\
6,310 \\
6,940 \\
7,240\end{array}$ & $\begin{array}{l}11 \ldots \\
12 \ldots \\
13 \ldots \\
14 \ldots \\
15 \ldots \\
16 \ldots \\
17 \ldots \\
18 \ldots \\
19 \ldots \\
20 \ldots\end{array}$ & $\begin{array}{r}18,700 \\
11,100 \\
8,080 \\
8,790 \\
8,500 \\
9,400 \\
11,200 \\
11,200 \\
5,600 \\
3,300\end{array}$ & $\begin{array}{l}58,900 \\
66,700 \\
65,400 \\
57,200 \\
48,700 \\
39,300 \\
34,500 \\
32,800 \\
27,600 \\
21,700\end{array}$ & $\begin{array}{l}6,820 \\
6,460 \\
6,100 \\
5,590 \\
5,160 \\
4,940 \\
4,750 \\
4,430 \\
4,250 \\
4,170\end{array}$ & $\begin{array}{l}21 \\
22 \\
23 \\
24 \\
25 \\
25 \\
26 . \\
27- \\
28 . \\
28_{-} \\
30_{-} \\
31\end{array}$ & $\begin{array}{l}2,600 \\
2,550 \\
2,600 \\
2,800 \\
2,900 \\
2,800 \\
2,700 \\
2,700 \\
3,500 \\
7,600 \\
8,080\end{array}$ & $\begin{array}{l}18,000 \\
15,400 \\
13,200 \\
17,600 \\
16,500 \\
13,500 \\
12,000 \\
11,400 \\
10,900 \\
10,500\end{array}$ & $\begin{array}{l}4,180 \\
4,150 \\
5,430 \\
6,370 \\
4,700 \\
4,480 \\
6,350 \\
7,310 \\
7,510 \\
8,700 \\
9,920\end{array}$ \\
\hline \multicolumn{9}{|c|}{$\begin{array}{l}\text { Monthly mean discharge, in cubic feet per second } \\
\text { Runoff, in inches } \\
\text { Runoff, il acre-feet }\end{array}$} & $\begin{array}{r}8,597 \\
1.27 \\
528,600\end{array}$ & $\begin{array}{r}27,300 \\
3.91 \\
1,624,000\end{array}$ & $\begin{array}{r}6,404 \\
0.95 \\
393,700\end{array}$ \\
\hline
\end{tabular}

Gage height, in feet, and discharge, in cubic feet per second, at indicated time, 1965

\begin{tabular}{r|c|c|c||r|r|r|r||r|r|r|r}
\hline Date & Hour & $\begin{array}{c}\text { Gage } \\
\text { height }\end{array}$ & $\begin{array}{c}\text { Dis- } \\
\text { charge }\end{array}$ & Date & Hour & $\begin{array}{c}\text { Gage } \\
\text { height }\end{array}$ & $\begin{array}{c}\text { Dis- } \\
\text { charge }\end{array}$ & Date & Hour & $\begin{array}{c}\text { Gage } \\
\text { height }\end{array}$ & $\begin{array}{c}\text { Dis- } \\
\text { charge }\end{array}$ \\
\hline Apr. 10 & 0000 & 14.87 & 39,400 & Apr.11 & 1600 & 16.24 & 58,100 & Apr. 12 & 2400 & 16.84 & 67,900 \\
& 1200 & 15.38 & 45,900 & & 2400 & 16.58 & 63,500 & & & & \\
& 2400 & 15.98 & 54,200 & & & & & 13 & 0600 & 16.78 & 66,900 \\
& & & 12 & 0600 & 16.72 & 65,800 & & 1200 & 16.73 & 66,000 \\
& 0900 & 16.36 & 60,000 & & 1200 & 16.80 & 67,200 & & 1800 & 16.62 & 64,100 \\
& 1000 & 16.38 & 60,300 & & 1800 & 16.84 & 67,900 & & 2400 & 16.47 & 61,700 \\
& 1400 & 16.24 & 58,100 & & 2100 & 16.85 & 68,100 & & & & \\
\hline
\end{tabular}


(268) 5-4655. Iowa River at Wapello, Iowa

Location.--Lat $41^{\circ} 10^{\prime} 40^{\prime \prime}$, long $91^{\circ} 10^{\prime} 55^{\prime \prime}$, in NW $\frac{1}{4} \mathrm{SE} \frac{1}{4}$ sec. 27, T.74 N., R.3 W., on right bank $30 \mathrm{ft}$ downstream from bridge on State Highway 99 at east edge of Wapello, 13.0 miles downstream from Cedar River, and at mile 16.0 (revised).

Drainage area. $-12,499 \mathrm{sq} \mathrm{mi}$.

Gage-height record.-Water-stage recorder graph, except May 6-9, for which graph was reconstructed on basis of daily wire-weight gage readings. Datum of g7ge is $548.98 \mathrm{ft}$ above mean sea level, adjustment of 1912 .

Discharge record.- Stage-discharge relation defined by current-meter measurements below 70,000 cfs. Backwater from ice March 1-31. Backwater from Mississippi River April 29 to May 9.

Maxima.-March-May 1965: Discharge, 70,800 cfs 0900 hours Apr. 13 (Eage height, $17.25 \mathrm{ft}$ ).

1914 to February 1965: Discharge, 94,000 cfs June 18, 1947 (gage height, $16.14 \mathrm{ft}$ ); gage height, $17.02 \mathrm{ft} \mathrm{Apr.} \mathrm{5,1960,} \mathrm{before} \mathrm{levee} \mathrm{broke} \mathrm{downstream} \mathrm{from} \mathrm{gage.}$

Remarks.-High flows regulated by Coralville Flood Control Reservoir (capacity 489,000 acre-ft) since Sept. 17, 1958.

Cooperation.-One discharge measurement furnished by Corps of Engineers.

Mean discharge, in cubic feet per second, 1965

\begin{tabular}{|c|c|c|c|c|c|c|c|c|c|c|c|}
\hline Day & March & April & May & Day & March & April & May & Day & March & April & May \\
\hline 10. & $\begin{array}{r}5,600 \\
5,400 \\
8,000 \\
11,000 \\
14,000 \\
13,000 \\
12,000 \\
11,000 \\
17,000 \\
33,000\end{array}$ & $\begin{array}{l}16,900 \\
19,000 \\
19,000 \\
20,200 \\
22,600 \\
28,200 \\
36,000 \\
45,100 \\
47,400 \\
51,100\end{array}$ & $\begin{array}{r}11,300 \\
10,400 \\
9,400 \\
8,530 \\
8,150 \\
8,790 \\
10,500 \\
11,100 \\
13,800 \\
14,000\end{array}$ & $\begin{array}{l}11 \ldots- \\
12 \ldots \\
13 \ldots \\
14 \ldots- \\
15 \ldots- \\
16 \ldots \\
17 \ldots \\
18 \ldots \\
19 \ldots \\
20 \ldots-\end{array}$ & $\begin{array}{l}27,000 \\
18,000 \\
15,000 \\
14,000 \\
14,000 \\
15,000 \\
19,000 \\
20,000 \\
15,000 \\
10,000\end{array}$ & $\begin{array}{l}63,700 \\
68,200 \\
70,300 \\
67,300 \\
63,400 \\
58,300 \\
50,500 \\
46,600 \\
44,800 \\
39,000\end{array}$ & $\begin{array}{r}13,200 \\
12,600 \\
12,100 \\
11,600 \\
11,200 \\
10,900 \\
11,100 \\
10,500 \\
10,100 \\
9,860\end{array}$ & $\mid \begin{array}{l}21 \\
22- \\
23- \\
24_{-} \\
25- \\
26_{-} \\
27- \\
28 \\
29 \\
30_{-} \\
31\end{array}$ & $\begin{array}{r}8,000 \\
6,500 \\
5,800 \\
5,200 \\
4,800 \\
4,500 \\
4,300 \\
4,100 \\
6,000 \\
11,000 \\
15,000\end{array}$ & $\begin{array}{r}32,700 \\
27,700 \\
24,200 \\
24,800 \\
35,000 \\
37,200 \\
30,900 \\
21,400 \\
15,000 \\
12,700 \\
\end{array}$ & $\begin{array}{r}9,840 \\
9,910 \\
10,100 \\
14,100 \\
10,400 \\
10,700 \\
11,800 \\
15,200 \\
14,000 \\
14,200 \\
15,400\end{array}$ \\
\hline \multicolumn{9}{|c|}{$\begin{array}{l}\text { Monthly mean discharge, in cubic feet per second } \\
\text { Runnf, in inches } \\
\text { Runoff, in acre-feet }\end{array}$} & $\begin{array}{r}12,010 \\
1.11 \\
738,200\end{array}$ & $\begin{array}{r}37,970 \\
3.39 \\
2,260,000\end{array}$ & $\begin{array}{r}11,440 \\
1.06 \\
703,700\end{array}$ \\
\hline
\end{tabular}

Gage height, in feet, and discharge, in cubic feet per second, at indicated time, 1965

\begin{tabular}{r|r|c|c||r|r|r|r||r|r|r|r}
\hline Date & Hour & $\begin{array}{c}\text { Gage } \\
\text { height }\end{array}$ & $\begin{array}{c}\text { Dis - } \\
\text { charge }\end{array}$ & Date & Hour & $\begin{array}{c}\text { Gage } \\
\text { height }\end{array}$ & $\begin{array}{c}\text { Dis- } \\
\text { charge }\end{array}$ & Date & Hour & $\begin{array}{c}\text { Gage } \\
\text { height }\end{array}$ & $\begin{array}{c}\text { Dis - } \\
\text { charge }\end{array}$ \\
\hline Apr. 11 & 0000 & 15.18 & 56,700 & Apr.12 & 1500 & 16.95 & 68,600 & Apr.14 & 1200 & 16.77 & 67,400 \\
& 0600 & 15.72 & 60,100 & & 1800 & 17.05 & 69,400 & & 1800 & 16.57 & 65,900 \\
& 1200 & 16.26 & 63,800 & & 2400 & 17.16 & 70,100 & & 2400 & 16.46 & 65,200 \\
& 1800 & 16.87 & 68,100 & & & & & & & & \\
& 2100 & 17.00 & 69,000 & 13 & 0900 & 17.25 & 70,800 & 15 & 0600 & 16.28 & 64,000 \\
& 2400 & 16.87 & 68,100 & & 1800 & 17.17 & 70,200 & & 1200 & 16.18 & 63,300 \\
& & & & & 2400 & 17.08 & 69,600 & & 1800 & 16.09 & 62,600 \\
& 0600 & 16.65 & 66,600 & & & & & & 2400 & 15.96 & 61,900 \\
& 0900 & 16.68 & 66,800 & 14 & 0600 & 16.95 & 68,600 & & & & \\
\hline
\end{tabular}




\section{EDWARDS RIVER BASIN}

(269) 5-4665. Edwards River near New Boston, Ill.

Location.-Lat $41^{\circ} 11^{\prime} 15^{\prime \prime}$, long $90^{\circ} 58^{\prime} 05^{\prime \prime}$, at quarter corner between secs.21 and 28 , T.14 N., R. 5 W., on left bank at downstream side of bridge on State Highway 17, 1.5 miles northeast of New Boston and 5 miles upstream from mouth.

Drainage area. $-434 \mathrm{sq} \mathrm{mi}$.

Gage-height record.-Digital recorder tape punched at 15-minute intervals. Prior to Apr. 7, 1965, water-stage recorder graph. No gage-height record Apr. 1, 2, May 9, 13-31. Datum of gage is $529.92 \mathrm{ft}$ above mean sea level, adjustment of 1912 (levels by Corps of Engineers).

Discharge record.- Stage-discharge relation defined by current-meter measurements. Discharge for periods of no gage-height record computed from records for n?arby stations. Mean daily discharge computed from 96 punch-tape recordings per day beginning Apr. 7, 1965.

Maxima.-April-May 1965: Discharge, 6,450 cfs 1030 hours Apr. 26 (gage height, $20.51 \mathrm{ft}$.

1934 to March 1965: Discharge, 7,280 cfs Apr. 26, 1950, May 12, 1951; gage height, $21.46 \mathrm{ft}$ Oct. $13,1954$.

Mcan discharge, in cubic feet per second, 1965

\begin{tabular}{|c|c|c|c|c|c|c|c|c|}
\hline Day & April & May & Day & April & May & Day & April & May \\
\hline $\begin{array}{l}1 \ldots \ldots \\
2 \ldots \ldots \\
3 \ldots \ldots \\
4 \ldots \ldots \\
5 \ldots \ldots \\
6 \ldots \ldots \\
7 \\
8 \ldots \ldots \\
9 \\
10 \ldots \ldots\end{array}$ & $\begin{array}{r}600 \\
450 \\
345 \\
323 \\
302 \\
342 \\
767 \\
2,740 \\
1,320 \\
783\end{array}$ & $\begin{array}{r}941 \\
821 \\
724 \\
674 \\
681 \\
566 \\
511 \\
781 \\
1,200 \\
758\end{array}$ & $\mid \begin{array}{l}11 \ldots \\
12 \ldots \\
13 \ldots \\
14 \ldots \\
15 \\
16 \ldots \\
16_{\ldots} \ldots \\
18 \ldots \\
19 \ldots \\
20 \ldots\end{array}$ & $\begin{array}{l}709 \\
804 \\
542 \\
432 \\
483 \\
582 \\
644 \\
625 \\
472 \\
380\end{array}$ & $\begin{array}{l}528 \\
398 \\
300 \\
260 \\
230 \\
210 \\
190 \\
170 \\
150 \\
140\end{array}$ & $\begin{array}{l}21 \ldots \ldots \\
22 \ldots \ldots \\
23 \ldots \ldots \\
24 \ldots \ldots \\
25 \ldots \ldots \\
26 \ldots \ldots \\
26 \ldots \ldots \\
28 \ldots \ldots \\
29 \\
30 \ldots \\
31 \ldots \\
\end{array}$ & $\begin{array}{r}357 \\
348 \\
347 \\
407 \\
607 \\
5,930 \\
4,430 \\
2,860 \\
1,620 \\
1,170 \\
\end{array}$ & $\begin{array}{l}130 \\
250 \\
210 \\
180 \\
150 \\
250 \\
400 \\
300 \\
200 \\
160 \\
140\end{array}$ \\
\hline \multicolumn{7}{|c|}{$\begin{array}{l}\text { Monthly mean discharge, in cubic feet per second } \\
\text { Runoff, in inches }\end{array}$} & $\begin{array}{r}1,057 \\
2.72\end{array}$ & $\begin{array}{r}407 \\
1.08\end{array}$ \\
\hline
\end{tabular}

Gage height, in feet, and discharge, in cubic feet per second, at indicated time, 1965

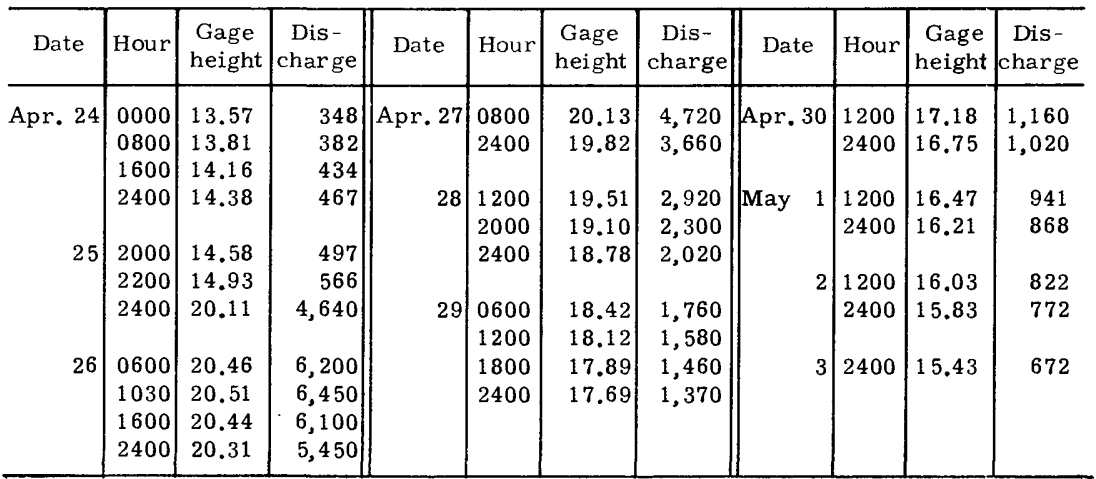




\section{POPE CREEK BASIN}

(270) 5-4670. Pope Creek near Keithsburg, Ill.

Location.-Lat $41^{\circ} 07^{\prime} 45^{\prime \prime}$, long $90^{\circ} 55^{\prime} 10^{\prime \prime}$, in $\mathrm{SE} \frac{1}{4}$ sec.11, T.13 N., R. 5 W., near center of span on downstream side of highway bridge, 2 miles northeast of $\mathrm{F}$ eithsburg and 3.3 miles upstream from mouth.

Drainage area. $-171 \mathrm{sq} \mathrm{mi}$.

Gage-height record.-Graph based on twice-daily readings of wire-weight gage, except May 10,11. Datum of gage is $524.07 \mathrm{ft}$ above mean sea level, adjustment of 1912 (levels by Corps of Engineers).

Discharge record.-Stage-discharge relation defined by current-meter measurements. Discharge for period of backwater from Mississippi River Apr. 26 tc May 7, estimated on basis of one discharge measurement and records for nearby stations. Discharge for period of no gage-height record estimated on basis of records for nearby stations.

Maxima.-April-May 1965: Discharge, 2,290 cfs 1300 hours Apr. 25 (gage height, $25.28 \mathrm{ft}$ ).

1934 to March 1965: Discharge, 4,230 cfs Mar. 12, 1939 (gage height, $26.24 \mathrm{ft}$ ); gage height observed, $28.0 \mathrm{ft}$ Feb. 21, 1937 (backwater from ice).

Mean discharge, in cubic feet per second, 1965

\begin{tabular}{|c|c|c|c|c|c|c|c|c|}
\hline Day & April & May & Day & April & May & Day & April & May \\
\hline $\begin{array}{l}1 \\
2 \\
3 \\
4\end{array}$ & $\begin{array}{r}108 \\
73 \\
57 \\
53 \\
105 \\
1,110 \\
848 \\
352 \\
338 \\
232\end{array}$ & $\begin{array}{l}190 \\
160 \\
140 \\
120 \\
140 \\
120 \\
105 \\
338 \\
249 \\
180\end{array}$ & $\begin{array}{l}11 \ldots \\
12 \ldots \ldots \\
13 \ldots \ldots \\
14 \ldots \ldots \\
15 \ldots \ldots \\
16 \ldots \ldots \\
17 \ldots \ldots \\
18 \ldots \\
19 \ldots \\
20 \ldots\end{array}$ & $\begin{array}{l}426 \\
285 \\
181 \\
148 \\
420 \\
317 \\
225 \\
172 \\
134 \\
114\end{array}$ & $\begin{array}{r}150 \\
135 \\
120 \\
106 \\
97 \\
90 \\
81 \\
73 \\
64 \\
57\end{array}$ & $\begin{array}{l}21 \ldots \ldots \\
22 \\
23 \\
24 \\
24 \\
25 \ldots \\
26 \ldots \ldots \\
26 \ldots \ldots \\
28 \ldots \ldots \\
28 \ldots \ldots \\
30 \ldots \ldots \\
31\end{array}$ & $\begin{array}{r}102 \\
94 \\
92 \\
1,070 \\
1,980 \\
1,000 \\
550 \\
400 \\
300 \\
240 \\
\end{array}$ & $\begin{array}{l}54 \\
49 \\
65 \\
48 \\
47 \\
57 \\
68 \\
73 \\
50 \\
41 \\
41\end{array}$ \\
\hline \multicolumn{7}{|c|}{$\begin{array}{l}\text { Monthly mean discharge, in cubic feet per second } \\
\text { Runoff, in inches }\end{array}$} & $\begin{array}{r}384 \\
2.51\end{array}$ & $\begin{array}{r}107 \\
0.72\end{array}$ \\
\hline
\end{tabular}

\section{HENDERSON CREEK BASIN}

(271) 5-4690. Henderson Creek near Oquawka, Ill.

Location.-Lat $41^{\circ} 00^{\prime} 05^{\prime \prime}$, long $90^{\circ} 51^{\prime} 15^{\prime \prime}$, in NE $\frac{1}{4} \mathrm{SW} \frac{1}{4}$ sec. $28, \mathrm{~T} .12 \mathrm{~N} ., \mathrm{R} .4$ W., on left bank at downstream side of bridge on State Highway 94,1 mile south of Bald Bluff, 6.5 miles northeast of Oquawka, and 22 miles upstream from mouth.

\section{Drainage area. $-428 \mathrm{sq} \mathrm{mi}$.}

Gage-height record.-Digital recorder tape punched at 15-minute intervals. Prior to Apr. 27, 1965, water-stage recorder graph. Datum of gage is $541.67 \mathrm{ft}$ above mean sea level, adjustment of 1912 (levels by Corps of Engineers).

Discharge record.-Stage-discharge relation defined by current-meter measurements. Mean daily discharge computed from 96 punched-tape recordings per day beginning Apr. 27, 1965.

Maxima.-April-May 1965: Discharge, 4,280 cfs 0500 hours Apr. 26 (gage height, $24.84 \mathrm{ft}$ ).

1934 to March 1965: Discharge, 16,500 cfs Apr. 25, 1950 (gage height, $28.17 \mathrm{ft}$ ). 
Mean discharge, in cubic feet per second, 1965, of Henderson Creek near Oquawka, Ill.

\begin{tabular}{|c|c|c|c|c|c|c|c|c|}
\hline Day & April & May & Day & April & May & Day & April & May \\
\hline $\begin{array}{l}1 \ldots \ldots \\
2 \ldots \\
3 \\
4 \\
4 \\
5 \\
6 \ldots \\
7 \ldots \\
8 \ldots \\
9 \\
10 \ldots\end{array}$ & $\begin{array}{r}364 \\
288 \\
221 \\
215 \\
526 \\
2,500 \\
2,140 \\
1,130 \\
1,540 \\
990\end{array}$ & $\begin{array}{r}635 \\
536 \\
460 \\
637 \\
1,950 \\
1,180 \\
620 \\
832 \\
1,610 \\
933\end{array}$ & $\begin{array}{l}11 \ldots \\
12 \ldots \\
13 \ldots \\
14 \ldots \\
15 \ldots \\
16 \ldots \\
17 \ldots \\
18 \ldots \\
19 \ldots \\
20 \ldots\end{array}$ & $\begin{array}{r}1,660 \\
1,510 \\
798 \\
620 \\
984 \\
966 \\
721 \\
581 \\
481 \\
420\end{array}$ & $\begin{array}{l}569 \\
474 \\
409 \\
361 \\
322 \\
296 \\
267 \\
241 \\
223 \\
202\end{array}$ & $\begin{array}{l}21 \ldots \\
22 \ldots \\
23 \ldots \\
24 \ldots \\
25 \ldots \\
26 \ldots \\
26 \ldots \\
28 \ldots \\
29 \ldots \\
30 \ldots \\
31 \ldots\end{array}$ & $\begin{array}{r}378 \\
330 \\
308 \\
962 \\
2,970 \\
3,760 \\
2,030 \\
1,180 \\
915 \\
747\end{array}$ & $\begin{array}{l}191 \\
356 \\
317 \\
206 \\
222 \\
540 \\
582 \\
324 \\
226 \\
212 \\
199\end{array}$ \\
\hline \multicolumn{7}{|c|}{$\begin{array}{l}\text { Wonthly mean discharge, in cubic feet per second } \\
\text { Runoff, in inches }\end{array}$} & $\begin{array}{r}1,074 \\
2.80\end{array}$ & $\begin{array}{r}520 \\
1.40\end{array}$ \\
\hline
\end{tabular}

Gage height, in feet, and discharge, in cubic feet per second, at indicated time, 1965

\begin{tabular}{|c|c|c|c|c|c|c|c|c|c|c|c|}
\hline Date & Hour & $\begin{array}{c}\text { Gage } \\
\text { height }\end{array}$ & $\begin{array}{c}\text { Dis- } \\
\text { charge }\end{array}$ & Date & Hour & $\begin{array}{l}\text { Gage } \\
\text { height }\end{array}$ & $\begin{array}{c}\text { Dis- } \\
\text { charge }\end{array}$ & Date & Hour & $\begin{array}{c}\text { Gage } \\
\text { height }\end{array}$ & $\begin{array}{c}\text { Dis- } \\
\text { charge }\end{array}$ \\
\hline Apr. 24 & $\begin{array}{l}0000 \\
0400 \\
0600 \\
0900 \\
1000 \\
1400 \\
1500 \\
1600 \\
1700 \\
2000 \\
2400 \\
0200 \\
0800 \\
1800 \\
2000 \\
2200 \\
2400\end{array}$ & $\begin{array}{l}15.83 \\
15.85 \\
16.10 \\
16.82 \\
17.18 \\
20.98 \\
21.53 \\
21.89 \\
22.15 \\
22.67 \\
22.97 \\
23.07 \\
23.73 \\
24.56 \\
24.67 \\
24.75 \\
24.80\end{array}$ & $\begin{array}{r}302 \\
305 \\
340 \\
449 \\
507 \\
1,200 \\
1,330 \\
1,440 \\
1,520 \\
1,730 \\
1,850 \\
1,900 \\
2,390 \\
3,720 \\
3,940 \\
4,100 \\
4,200\end{array}$ & Apr. 26 & $\begin{array}{l}0300 \\
0500 \\
0800 \\
1200 \\
2000 \\
2400 \\
0800 \\
1200 \\
1800 \\
2400 \\
0600 \\
1200 \\
1800 \\
2400\end{array}$ & $\begin{array}{l}24.83 \\
24.84 \\
24.79 \\
24.65 \\
24.25 \\
24.10 \\
23.89 \\
23.20 \\
22.54 \\
21.81 \\
21.18 \\
20.80 \\
20.53 \\
20.18\end{array}$ & $\begin{array}{l}4,260 \\
4,280 \\
4,180 \\
3,900 \\
3,130 \\
2,870 \\
2,580 \\
1,960 \\
1,680 \\
1,410 \\
1,240 \\
1,160 \\
1,110 \\
1,040\end{array}$ & Apr. 29 & $\begin{array}{l}0800 \\
1600 \\
2400 \\
0800 \\
1600 \\
2400 \\
1200 \\
2400 \\
1200 \\
2400 \\
1200 \\
2400\end{array}$ & $\begin{array}{l}19.74 \\
19.3 \mathrm{C} \\
18.9 \mathrm{C} \\
18.7 \mathrm{C} \\
18.4 \mathrm{C} \\
18.2 \mathrm{E} \\
17.9 \mathrm{~s} \\
17.64 \\
17.3 \mathrm{E} \\
17.1 \mathrm{C} \\
16.8 \mathrm{C} \\
16.7 \mathrm{C}\end{array}$ & $\begin{array}{l}953 \\
874 \\
818 \\
\\
766 \\
724 \\
688 \\
\\
634 \\
585 \\
\\
537 \\
494 \\
460 \\
430\end{array}$ \\
\hline
\end{tabular}

\section{MISSISSIPPI RIVER MAIN STEM}

(272) Mississippi River at Burlington, Iowa

\section{(Miscellaneous site)}

Location.-Lat $40^{\circ} 48^{\prime} 10^{\prime \prime}$, long $91^{\circ} 05^{\prime} 30^{\prime \prime}$, on pivot pier of Chicago Burlington and Quincy Railroad bridge at Burlington, and at mile 403.1 upstream from Ohio River.

Drainage area. $-114,000 \mathrm{sq} \mathrm{mi}$, approximately.

Gage-height record.-Peak stages only from staff gage readings. Datum of gage is $511.45 \mathrm{ft}$ above mean sea level, adjustment of 1912 .

Maxima.-March-May 1965: Gage height, 21.0 ft Apr. 30, May 1.

1851 to February 1965: Gage height, $18.9 \mathrm{ft}$ in J une 1851.

Remarks. - Stage records furnished by Corps of Engineers. 
Annual maximum gage heights, for indicated years, of Mississippi River at Burlington, lowa

\begin{tabular}{|c|c|c|c|c|c|}
\hline Year & Date & $\begin{array}{l}\text { Gage } \\
\text { height } \\
\text { (feet) }\end{array}$ & Year & Date & $\begin{array}{c}\text { Gage } \\
\text { height } \\
\text { (feet) }\end{array}$ \\
\hline 1851 & June & 18.9 & 1919 & May 8 & 13.79 \\
\hline 1869 & Oct. 12,13 & 11.8 & 1920 & Apr. 11 & 14.79 \\
\hline $1870 \ldots \ldots$ & Apr. 26-28 & 15.0 & 1921 & May 13 & 9.42 \\
\hline $1871 \ldots$ & May 21 & 12.1 & 1922 & Apr. 24, 25 & 15.42 \\
\hline $1872 \ldots$ & June 7,8 & 9.6 & 1923 & Apr. 10 & 11.18 \\
\hline $1873 \ldots \ldots$ & J une $18-20$ & 11.2 & 1924 & Aug. 25 & 12.16 \\
\hline $1874 \ldots$ & Mar. 27 & 7.7 & 1925 & June 23 & 9.4 \\
\hline $1879 \ldots \ldots$ & July 15 & 7.07 & 1926 & Oct. $6-9$ & 11.53 \\
\hline $1880_{-}$ & J une 28 & 15.8 & 1927 & Apr. 3 & 12.88 \\
\hline 1881 & Oct. 31 & 16.53 & 1928 & Dec, 28 & 14.09 \\
\hline 1882 & Apr. 25 & 13.3 & 1929 & Mar. 23 & 15.82 \\
\hline $1883 \ldots \ldots$ & May 5 & 11.8 & 1930 & J une 18 & 11.8 \\
\hline $1884 \ldots$ & Mar. 30,31 & 13.8 & $1931 \ldots$ & Dec. 3 & 9.5 \\
\hline $1885_{2}$ & May 10 & 9.78 & 1932 & Mar. 9 & 11.4 \\
\hline 1886 & May 7 & 12.0 & 1933 & Apr. 10 & 12.4 \\
\hline $1887 \ldots \ldots$ & Feb. 14 & 9.78 & $1934 \ldots \ldots$ & Dec. 12 & 11.5 \\
\hline $1888 \ldots$ & May 18,19 & 17.55 & 1935 & Apr. 11 & 11.7 \\
\hline $1889^{-}$ & May 24,25 & 6.00 & 1936 & Apr. 10 & 12.6 \\
\hline $1890 \ldots$ & June 30 & 11.62 & $1937 \ldots$ & Mar. $10-12$ & 13.8 \\
\hline $1891 \ldots$ & May 5, 6 & 9.70 & 1938. & Sept. 25,26 & 15.0 \\
\hline $1892 \ldots \ldots$ & June $8-30$ & 17.5 & 1939 & Apr. 11,12 & 13.1 \\
\hline $1893 \ldots$ & May 16 & 12.47 & 1940 & Apr. 19, 20 & 9.3 \\
\hline $1894 \ldots$ & June 3,4 & 10.4 & $1941 \ldots$ & Apr. 27,28 & 13.0 \\
\hline 1895 & $\operatorname{Mar} .2,3$ & 5.3 & $1942 \ldots$ & June 16,17 & 15.5 \\
\hline 1896 & June 3 & 10.25 & $1943 \ldots$ & Apr. 18,19 & 14.1 \\
\hline 1897 & Apr. 27-29 & 13.2 & $1944 \ldots \ldots$ & May 27 & 17.1 \\
\hline 1898 & Mar. 15 & 6.8 & $1945 \ldots \ldots$ & Apr. 1, 2 & 15.4 \\
\hline $1899 \ldots$ & $\mathrm{J}$ une $28-30$ & 10.3 & $1946 \ldots$ & Jan. 11 & 17.1 \\
\hline $1900-\ldots-n$ & Oet. 23,24 & 9.0 & $1947 \ldots$ & June 20,21 & 17.0 \\
\hline $1901 \ldots$ & Mar. 24 & 9.4 & $1948-\ldots$ & Mar. 23 & 16.4 \\
\hline $1902 \ldots-\ldots$ & July 21 & 10.8 & $1949 \ldots \ldots$ & Mar. 12 & 12.2 \\
\hline $1903 \ldots \ldots$ & June 5,6 & 14.9 & $1950 \ldots$ & Apr. 26 & 13.8 \\
\hline $1904 \ldots$ & Mar. 29 & 10.8 & $1951 \ldots$ & Apr. 29 & 18.1 \\
\hline $1905 \ldots \ldots$ & June 25 & 12.2 & $1952 \ldots-\ldots$ & Apr. 28 & 17.85 \\
\hline $1906 \ldots$ & Apr. 24-26 & 12.3 & $1953 \ldots$ & Apr. $3-6$ & 12.3 \\
\hline $1907 \ldots-\ldots$ & Apr. 15,16 & 11.4 & $1954 \ldots \ldots$ & May 16-18 & 14.8 \\
\hline $1908 \ldots-\ldots$ & $\mathrm{J}$ une 9 & 11.25 & $1955 \ldots$ & Apr. 27 & 13.2 \\
\hline $1909 \ldots$ & May 5,6 & 11.7 & $1956 \ldots$ & Apr. 21 & 12.2 \\
\hline $1910 \ldots$ & Mar. 22 & 8.35 & $1957 \ldots$ & July 15 & 11.1 \\
\hline $1911 \ldots-\ldots$ & Feb. 20 & 10.2 & $1958 \ldots \ldots$ & $\mathrm{J}$ une 13 & 9.8 \\
\hline $1912 \ldots$ & Apr. 5,6 & 13.35 & $1959 \ldots$ & Apr. 5, 6 & 14.5 \\
\hline $1913 \ldots$ & Mar. 29, 30 & 11.7 & $1960 \ldots \ldots$ & Apr. 4 & 18.7 \\
\hline $1914 \ldots \ldots$ & J une 23 & 9.32 & $1961 \ldots \ldots$ & Apr. 5 & 16.3 \\
\hline $1915 \ldots$ & J une 7,8 & 10.4 & $1962 \ldots \ldots$ & Apr. 7 & 16.7 \\
\hline $1916 \ldots$ & May 9 & 14.2 & $1963 \ldots$ & Mar. 22 & 12.1 \\
\hline $1917 \ldots \ldots$ & J une 17 & 11.62 & $1964 \ldots \ldots$ & May 21-23 & 10.4 \\
\hline $1918 \ldots \ldots-\ldots$ & June 12 & 12.95 & $1965 \ldots \ldots$ & Apr. 30, Mey 1 & 21.0 \\
\hline
\end{tabular}




\section{SKUNK RIVER BASIN}

(273) 5-4700. South Skunk River near Ames, Iowa

Location.-Lat $42^{\circ} 04^{\prime} 05^{\prime \prime}$, long $93^{\circ} 37^{\prime} 05^{\prime \prime}$, in $\mathrm{NW} \frac{1}{4} \mathrm{SW} \frac{1}{4}$ sec. $23, \mathrm{~T} .84 \mathrm{~N}$., R.24 W., on left bank 2.5 miles north of Ames, 3.5 miles downstream from Keigley Branch, 5.2 miles upstream from Squaw Creek, and at mile 228.1.

Drainage area. $-315 \mathrm{sq} \mathrm{mi}$.

Gage-height record.-Water-stage recorder graph except Mar. 1 to Apr. 5. Graph reconstructed from daily wire-weight gage readings Mar. 30, Apr. 1. Datum of gage is $893.61 \mathrm{ft}$ above sea level, datum of 1929 (Iowa Highway Commission bench mark).

Discharge record.-Stage-discharge relation defined by current-meter measurements below 8,630 cfs. Discharge Apr. 2-5 estimated on basis of weather records and records from nearby stations. Backwater from ice Mar. 1-31, Apr. 7-9.

Maxima.-March-May 1965: Discharge, 5,260 cfs 0530 hours Apr. 6 (gage height, $9.43 \mathrm{ft})$.

1920-27, 1932 to February 1965: Discharge, 8,630 cfs June 10, 1954; gage height, $13.90 \mathrm{ft}$ May 20, 1944 .

Mean discharge, in cubic feet per second, 1965

\begin{tabular}{|c|c|c|c|c|c|c|c|c|c|c|c|}
\hline Day & March & April & May & Day & March & April & May & Day & March & April & May \\
\hline 1 & 500 & 3,090 & 152 & $11 \ldots$ & 51 & 1,390 & 77 & $21 \ldots$ & 74 & $150^{\circ}$ & 35 \\
\hline 2. & 1,200 & 2,600 & 130 & $12 \ldots$ & 54 & 1,040 & 68 & 22 & 58 & 125 & 60 \\
\hline 3. & 400 & 2,100 & 111 & $13 \ldots$ & 65 & 773 & 60 & 23 & 47 & 115 & 284 \\
\hline 4. & 180 & 2,400 & 100 & $14 \ldots$ & 90 & 630 & 55 & $24 \ldots$ & 37 & 182 & 216 \\
\hline 5. & 120 & 3,400 & 91 & $15 \ldots$ & 140 & 540 & 53 & 25 & 34 & 309 & 214 \\
\hline 6. & 100 & 5,150 & 83 & $16 \ldots$ & 210 & 412 & 50 & 26 & 32 & 469 & 1,860 \\
\hline 1 & 82 & 3,400 & 77 & 17. & 500 & 323 & 49 & $27 \ldots$ & 30 & 345 & 2,270 \\
\hline 8. & 72 & 2,500 & 96 & $18 \ldots$ & 200 & 257 & 46 & $28 \ldots$ & 28 & 276 & 1,060 \\
\hline 9 & 63 & 2,000 & 118 & $19 \ldots$ & 124 & 209 & 39 & 29 & 26 & 219 & 681 \\
\hline 10 & 57 & 1,480 & 91 & $20 \ldots$ & 92 & 177 & 34 & $30_{-}$ & 110 & 180 & 596 \\
\hline & & & & & & & & & 200 & $-\cdots$ & 468 \\
\hline \multirow{3}{*}{\multicolumn{9}{|c|}{$\begin{array}{l}\text { Monthly mean discharge, in cubic feet per second } \\
\text { Runnff, in inches } \\
\text { Runoff, in acre -feet }\end{array}$}} & 193 & 1,208 & 301 \\
\hline & & & & & & & & & 0.71 & 4.28 & 1.10 \\
\hline & & & & & & & & & 11,850 & 71,880 & 18,490 \\
\hline
\end{tabular}

Gage height, in feet, and discharge, in cubic feet per second, at indlcated time, 1965

\begin{tabular}{c|c|r|r||r|r|r|r||r|r|r|r}
\hline Date & Hour & $\begin{array}{c}\text { Gage } \\
\text { height }\end{array}$ & $\begin{array}{c}\text { Dis - } \\
\text { charge }\end{array}$ & Date & Hour & $\begin{array}{c}\text { Gage } \\
\text { height }\end{array}$ & $\begin{array}{c}\text { Dis- } \\
\text { charge }\end{array}$ & Date & Hour & $\begin{array}{c}\text { Gage } \\
\text { height }\end{array}$ & $\begin{array}{c}\text { Dis - } \\
\text { charge }\end{array}$ \\
\hline Apr. 6 & 0000 & 9.17 & 5,020 & Apr.6 & 1800 & 9.35 & 5,190 & Apr. 7 & 0600 & 8.60 & 3,940 \\
& 0200 & 9.34 & 5,180 & & 2000 & 9.28 & 5,120 & & 1200 & 7.12 & 3,190 \\
& 0530 & 9.43 & 5,260 & & 2400 & 8.88 & 4,740 & & 2400 & 6.68 & $-\cdots$ \\
& 1200 & 9.34 & 5,180 & & & & & & & & \\
\hline
\end{tabular}


(274) 5-4710. South Skunk River below Squaw Creek near Ames Iowa

Location.-Lat $42^{\circ} 00^{\prime} 30^{\prime \prime}$, long $93^{\circ} 35^{\prime} 40^{\prime \prime}$, in NE $\frac{1}{4} \mathrm{NW} \frac{1}{4}$ sec. $13, \mathrm{~T} .83 \mathrm{~N}$., R.24 W., on right bank $15 \mathrm{ft}$ downstream from county highway bridge, a quarter of a mila downstream from Squaw Creek, a quarter of a mile upstream from bridge on U.S. Highway 30 , 2 miles southeast of Ames, and at mile 222.6.

Drainage area. -556 sq $\mathrm{mi}$.

Gage-height record.-Water-stage recorder graph except Mar. 3, 28, 29. Datum of gage is $867.10 \mathrm{ft}$ above mean sea level, datum of 1929 .

Discharge record.-Stage-discharge relation defined by current-meter measurements below $8,460 \mathrm{cfs}$, extended to $9,260 \mathrm{cfs}$ by logarithmic plotting. Backweter from ice Mar. 1, 4-1 5, 19-30.

Maxima.-March-May 1965: Discharge, 7,340 cfs 0400 hours Apr. 6 (gag̣e height, $12.59 \mathrm{ft}$ ).

1952 to February 1965: Discharge, 9,260 cfs Mar. 30, 1960 (gage height, $13.20 \mathrm{ft}$ )

Flood of May 19, 1944, reached a stage of $13 \mathrm{ft}$, from floodmarks (discharge, about $10,000 \mathrm{cfs})$.

Mean discharge, in cubic feet per second, 1965

\begin{tabular}{|c|c|c|c|c|c|c|c|c|c|c|c|}
\hline Day & March & April & May & Day & March & April & May & Day & March & April & May \\
\hline 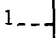 & 5,000 & 5,910 & 338 & $11 \ldots$ & 114 & 2,170 & 121 & 21. & 160 & 236 & 49 \\
\hline 2 & 2,690 & 4,480 & 276 & $12 \ldots$ & 120 & 1,610 & 110 & 22 & 138 & 201 & 82 \\
\hline 3 & 800 & 3,540 & 231 & $13 \ldots$ & 150 & 1,180 & 98 & 23 & 120 & 196 & 328 \\
\hline 4 & 280 & 4,530 & 201 & 14 & 200 & 939 & 87 & $24_{-}$ & 110 & 477 & 306 \\
\hline 5. & 240 & 6,080 & 192 & $15 \ldots$ & 370 & 815 & 83 & 25 & 100 & 891 & 295 \\
\hline 6. & 210 & 7,020 & 173 & $16 \ldots$ & 610 & 651 & 72 & 26. & 94 & 1,050 & 3,120 \\
\hline 7. & 180 & 5,450 & 158 & $17 \ldots$ & 1,010 & 518 & 70 & 27. & 92 & 758 & 3,420 \\
\hline 8. & 160 & 4,330 & 161 & 18_- & 476 & 406 & 62 & 28 & 90 & 592 & 1,640 \\
\hline$y$. & 146 & 3,260 & 182 & $19 \ldots$ & 240 & 335 & 50 & $29 \ldots$ & 90 & 470 & 1,120 \\
\hline 10 & 128 & 2,340 & 149 & $20 \ldots$ & 190 & 276 & 44 & 30. & 500 & 393 & 970 \\
\hline & & & & & & & & 31. & 2,460 & 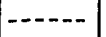 & 790 \\
\hline \multirow{3}{*}{\multicolumn{9}{|c|}{$\begin{array}{l}\text { Nonthly mean discharge, in cubic feet per second } \\
\text { Runoff, in inches } \\
\text { Funofi, in acre-feet }\end{array}$}} & 557 & 2,037 & 483 \\
\hline & & & & & & & & & 1.16 & -4.09 & 1.00 \\
\hline & & & & & & & & & 34,250 & 121,200 & 29,710 \\
\hline
\end{tabular}

Gage height, in feet, and discharge, in cubic feet per second, at indicated time, 1965

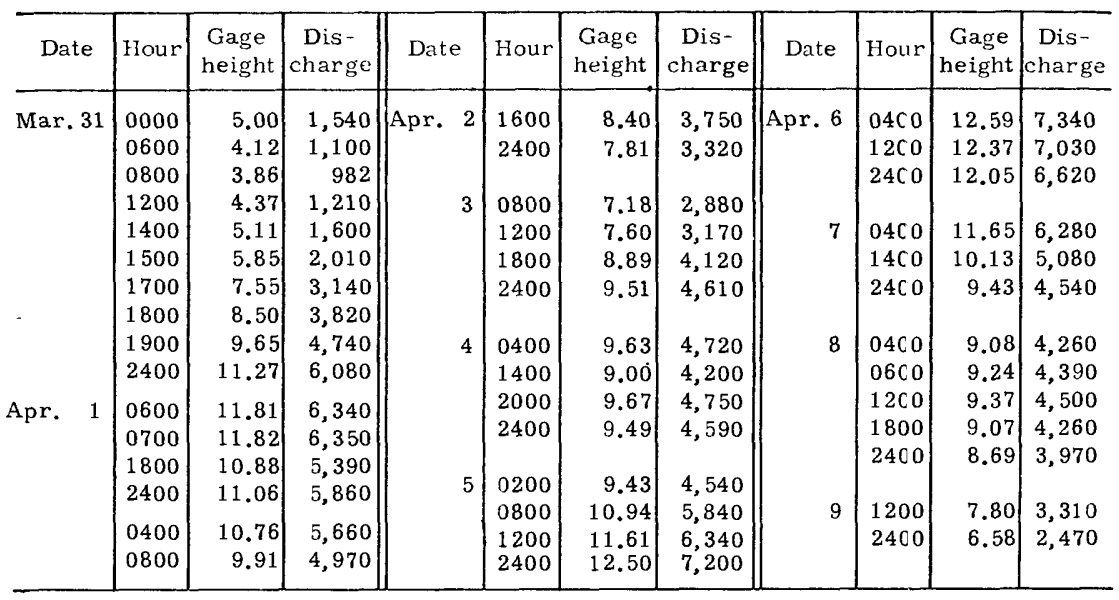


(275) 5-4712. Indian Creek near Mingo, Iowa

Location.-Lat $41^{\circ} 48^{\prime} 20^{\prime \prime}$, long $93^{\circ} 18^{\prime} 25^{\prime \prime}$, in NW $\frac{1}{4} N W \frac{1}{4}$ sec. $28, T .81$ N., R.21 W.r on right bank $30 \mathrm{ft}$ downstream from bridge on State Highway $117,0.7$ mile downstream from Wolf Creek, 2.2 miles upstream from Byers Branch, and 3.3/4 miles northwest of Mingo.

Drainage area.-276 sq $\mathrm{mi}$.

Gage-height record.-Water-stage recorder graph except Mar. 2-10, 18, 19, May 8-25, 29-31. Daily wire-weight readings are available for most of the se periods.

Discharge record.-Stage-discharge relation defined by current-meter measurements below 5,100 cfs. Backwater from ice Mar. 1-9, 17-27.

Maxima.-March-May 1965: Discharge, 4,610 cfs 0230 hours Apr. 6 (gage height, $14.32 \mathrm{ft}$ ).

1958 to February 1965: Discharge, 5,860 cfs May 7, 1960 (gage height, $15.07 \mathrm{ft}$ ).

Flood of May 20, 1944, reached a stage of $21.4 \mathrm{ft}$, from information by local residents (discharge not determined).

Mean discharge, in cubic feet per second, 1965

\begin{tabular}{|c|c|c|c|c|c|c|c|c|c|c|c|}
\hline Day & March & April & May & Day & March & April & May & Day & March & April & May \\
\hline 1 & 1,400 & 3,380 & 197 & $11 \ldots$ & 59 & 580 & 100 & 21. & 110 & 86 & 58 \\
\hline 2 & 500 & 2,260 & 178 & $12 \ldots$ & 53 & 416 & 92 & 22 & 90 & 74 & 87 \\
\hline 3. & 130 & 1,000 & 146 & $13_{-}$ & 71 & 320 & 87 & 23. & 80 & 95 & 132 \\
\hline & 120 & 1,360 & 134 & $14 \ldots$ & 146 & 285 & 77 & 24 & 70 & 789 & 214 \\
\hline 5 & 140 & 3,030 & 132 & $15 \ldots$ & 383 & 234 & 74 & 25 & 65 & 851 & 240 \\
\hline 6. & 125 & 4,080 & 121 & $16 \ldots$ & 551 & 189 & 71 & 26 & 60 & 712 & 407 \\
\hline 7. & 130 & 1,160 & 115 & 17 & 2,330 & 163 & 68 & 27. & 70 & 454 & 503 \\
\hline ? & 100 & 990 & 118 & 18 & 500 & 133 & 63 & 28. & 194 & 352 & 287 \\
\hline 9 & 110 & 752 & 115 & 19 & 200 & 110 & 58 & $29_{-}$ & 330 & 283 & 222 \\
\hline 10 & 92 & 562 & 104 & $20 \ldots$ & 150 & 99 & 57 & $\left.30_{-}\right]$ & 793 & 232 & 216 \\
\hline & & & & & & & & 31. & 1,850 & - & 216 \\
\hline \multirow{3}{*}{\multicolumn{9}{|c|}{$\begin{array}{l}\text { Nonthl, mean discharge, in cubic feet per second } \\
\text { Rivoff, in inches } \\
\text { Kinotf, in acre -feet }\end{array}$}} & 354 & 834 & 151 \\
\hline & & & & & & & & & 1.48 & 3.37 & 0.63 \\
\hline & & & & & & & & & 21,760 & 49,650 & 9,300 \\
\hline
\end{tabular}


Gage height, in feet, and discharge, in cubic feet per second, at indicated time, 1965, of Indian Creek near

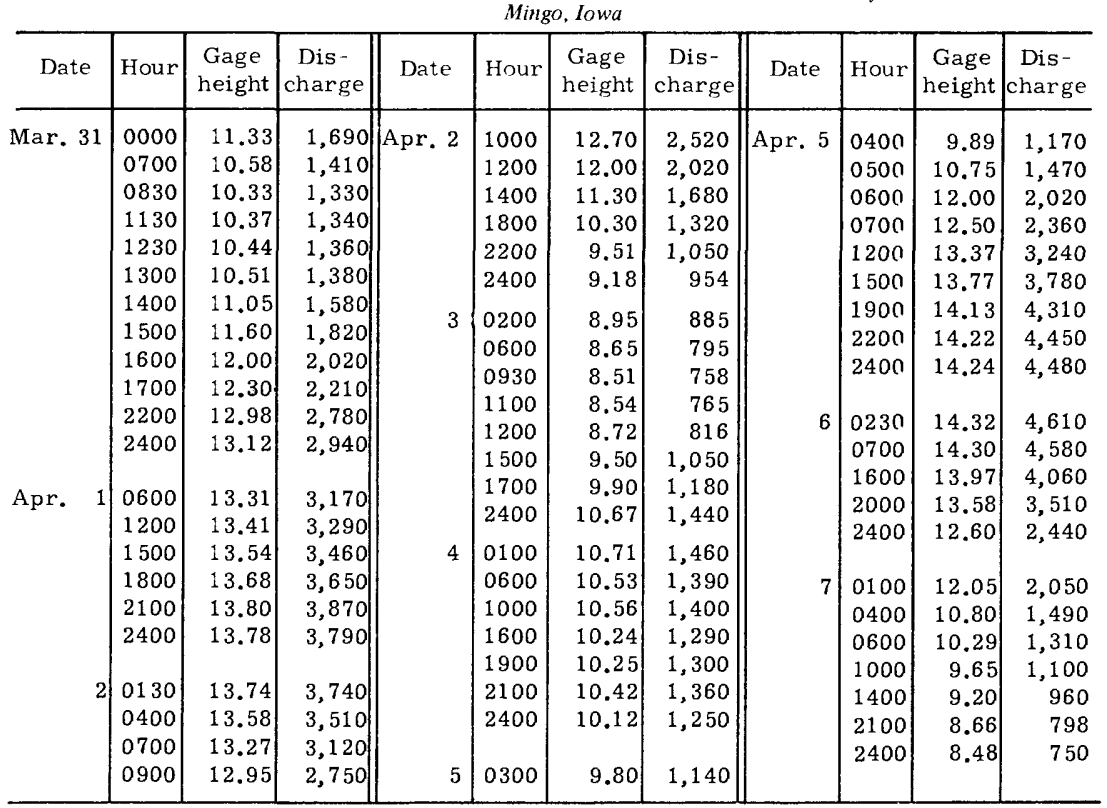


(276) 5-4715. South Skunk River near Oskaloosa, Iowa

Location.-Lat $41^{\circ} 21^{\prime} 15^{\prime \prime}$, long $92^{\circ} 39^{\prime} 30^{\prime \prime}$, in NW $\frac{1}{4} \mathrm{SW} \frac{1}{4}$ sec.25, T.76 N., R.16 W., or right bank $300 \mathrm{ft}$ upstream from bridge on U.S. Highway 63 and 4 miles north of Oskaloosa, and 147.3 miles upstream from mouth.

Drainage area. $-1,635 \mathrm{sq} \mathrm{mi}$.

Gage-height record.-Water-stage recorder graph, except Mar. 19-27, Apr. 1-5. Daily wire-weight gage readings used Mar. 20 and Apr. 1-5. Datum of gage is $685.5 \mathrm{C} \mathrm{ft}$ above mean sea level, datum of 1929 .

Discharge record.-Stage-discharge relation defined by current-meter measurements below 18,000 cfs and on basis of velocity-area study at 37,000 cfs. Backwater from ice Mar. 1 to Apr. 2.

Maxima.-March-May 1965: Discharge, 11,200 cfs 0500 hours Apr. 9 (gage heigrt, $19.87 \mathrm{ft}$ ).

1945 to February 1965: Discharge, 20,000 cfs June 15, 1947 (gage height, $21.26 \mathrm{ft}$ from floodmarks).

Flood in May 1944 reached a stage of $25.8 \mathrm{ft}$, from floodmarks (discharge, 37,000 cfs).

Mean discharge, in cubic feet per second, 1965

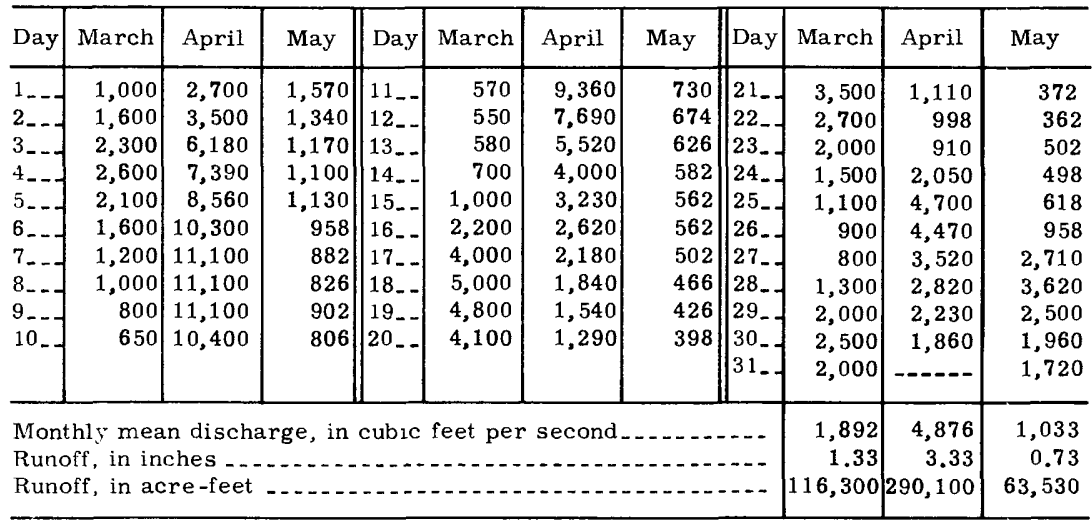

Gage heıght, in feet, and discharge, in cubic feet per second, at indicated time, 1965

\begin{tabular}{c|c|c|c||r|r|r|r||r|r|r|r}
\hline Date & Hour & $\begin{array}{c}\text { Gage } \\
\text { height }\end{array}$ & $\begin{array}{c}\text { Dis- } \\
\text { charge }\end{array}$ & Date & Hour & $\begin{array}{c}\text { Gage } \\
\text { height }\end{array}$ & $\begin{array}{c}\text { Dis- } \\
\text { charge }\end{array}$ & Date & Hour & $\begin{array}{c}\text { Gage } \\
\text { height }\end{array}$ & $\begin{array}{c}\text { Dis- } \\
\text { charge }\end{array}$ \\
\hline Apr.5 5 & 0000 & 17.52 & 7,530 & Apr. 6 & 2400 & 19.74 & 11,000 & Apr. 8 & 2400 & 19.85 & 11,200 \\
& 0600 & 17.73 & 7,820 & 7 & 0600 & 19.83 & 11,200 & & & & \\
& 1200 & 18.18 & 8,470 & & 1200 & 19.80 & 11,100 & & 0500 & 19.87 & 11,200 \\
& 1800 & 18.73 & 9,320 & & 1800 & 19.78 & 11,100 & & 1200 & 19.82 & 11,100 \\
& 2400 & 19.00 & 9,750 & & 2400 & 19.68 & 10,900 & & 2400 & 19.62 & 10,800 \\
& 1200 & 19.35 & 10,300 & 8 & 1200 & 19.79 & 11,100 & & & & \\
\hline
\end{tabular}


(277) 5-4725. North Skunk River near Sigourney, Ioশa

Location.-Lat $41^{\circ} 18^{\prime} 05^{\prime \prime}$, long $92^{\circ} 12^{\prime} 10^{\prime \prime}$, in $\mathrm{NE} \frac{1}{4} \mathrm{SE} \frac{1}{4} \sec .14, \mathrm{~T} .75 \mathrm{~N}$, , F.12 W., on right bank $20 \mathrm{ft}$ downstream from bridge on State Highway $149,2 \frac{1}{2}$ miles south of Sigourney, and 16.2 miles upstream from mouth.

Drainage area. -730 sq $\mathrm{mi}$.

Gage-height record.-Water-stage recorder graph except Mar. 19 to Apr. 4. Datum of gage is $651.53 \mathrm{ft}$ above mean sea level, datum of 1929 .

Discharge record.-Stage-discharge relation defined by current-meter measurements below $19,500 \mathrm{cfs}$ and by indirect measurement at 27,500 cfs. Backwater from ice Mar. 1-31.

Maxima.-March-May 1965: Discharge, 4,620 cfs 1430, hours April 8; (gage height, $17.55 \mathrm{ft}$ ); gage height, $18.14 \mathrm{ft} 2000$ hours Mar. 17, (backwater from ice).

1945 to February 1965: Discharge, 27,500 cfs Mar. 31, 1960 (gage height, $25.33 \mathrm{ft}$ ).

Flood in May 1944 reached a stage of $22.8 \mathrm{ft}$, from floodmark (discharge, 14,500 cfs).

Mean discharge, in cubic feet per second, 1965

\begin{tabular}{|c|c|c|c|c|c|c|c|c|c|c|c|}
\hline Day & March & April & May & Day & March & April & May & Day & March & April & May \\
\hline 1. & 800 & 1,100 & 781 & $11 \ldots$ & 270 & 1,650 & 327 & 21 & $1,50 \mathrm{C}$ & 222 & 156 \\
\hline 2. & 1,200 & 900 & 652 & $12 \ldots$ & 250 & 1,370 & 273 & 22. & $1,20 \mathrm{C}$ & 202 & 146 \\
\hline 3. & 1,300 & 900 & 544 & 13 & 300 & 752 & 248 & 23 & $90 \mathrm{C}$ & 188 & 142 \\
\hline$x_{-1}$ & 800 & 1,200 & 470 & $14 \ldots$ & 500 & 561 & 231 & $24 \ldots$ & $70 \mathrm{C}$ & 557 & 301 \\
\hline 5. & 500 & 1,950 & 448 & $15 \ldots$ & 1,000 & 664 & 234 & $25 \ldots$ & $55 \mathrm{C}$ & 2,640 & 271 \\
\hline 6. & 400 & 3,230 & 512 & $16 \ldots$ & 2,300 & 460 & 342 & $26 \ldots$ & $46 C$ & 3,100 & 234 \\
\hline 7. & 450 & 2,940 & 440 & $17 \ldots$ & 3,000 & 382 & 257 & $27 \ldots$ & $41 \mathrm{C}$ & 4,080 & 455 \\
\hline 8. & 500 & 4,290 & 384 & $18 \ldots$ & 2,700 & 320 & 219 & 28 & $37 \mathrm{C}$ & 3,400 & 838 \\
\hline & 400 & 2,650 & 353 & $19 \ldots$ & 2,300 & 279 & 192 & 29. & $70 \mathrm{C}$ & 1,460 & 410 \\
\hline 10 & 330 & 1,100 & 372 & $20 \ldots$ & 1,800 & 245 & 170 & $30_{-}$ & $1,50 \mathrm{C}$ & 975 & 322 \\
\hline & & & & & & & & 31. & $1,30 \mathrm{C}$ & -..... & 311 \\
\hline \multicolumn{9}{|c|}{ Monthly mean discharge, in cubic feet per second } & $99 c^{1}$ & 1,459 & 356 \\
\hline \multicolumn{9}{|c|}{ Runoff, in inches } & 1.56 & 2.23 & 0.56 \\
\hline \multicolumn{9}{|c|}{ Runoff, in acre-feet } & $60,87 \mathrm{C}$ & 86,810 & 21,890 \\
\hline
\end{tabular}

Guge height, in feet, and discharge, in cubic feet per second, at indicated time, 1965

\begin{tabular}{|c|c|c|c|c|c|c|c|c|c|c|c|}
\hline Date & Hour & $\begin{array}{c}\text { Gage } \\
\text { height }\end{array}$ & $\begin{array}{c}\text { Dis - } \\
\text { charge }\end{array}$ & Date & Hour & $\begin{array}{c}\text { Gage } \\
\text { height }\end{array}$ & $\begin{array}{c}\text { Dis- } \\
\text { charge }\end{array}$ & Date & Hour & $\begin{array}{c}\text { Gage } \\
\text { height }\end{array}$ & $\begin{array}{c}\text { Dis - } \\
\text { charge }\end{array}$ \\
\hline Mar. 16 & $\begin{array}{l}0000 \\
0600 \\
0700 \\
1200 \\
1600 \\
1800 \\
2000 \\
2400 \\
0300 \\
0500 \\
0700 \\
1000 \\
1300 \\
1600\end{array}$ & $\begin{array}{l}12.97 \\
13.25 \\
13.26 \\
13.11 \\
14.05 \\
14.70 \\
15.11 \\
15.43 \\
16.13 \\
16.59 \\
16.50 \\
17.05 \\
17.80 \\
18.05\end{array}$ & 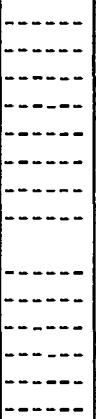 & 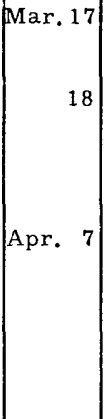 & $\begin{array}{l}2000 \\
2400 \\
0300 \\
0900 \\
1500 \\
2400 \\
\\
0000 \\
0300 \\
0600 \\
1200 \\
1800 \\
2400\end{array}$ & $\begin{array}{l}18.14 \\
18.04 \\
17.90 \\
17.07 \\
16.29 \\
15.98 \\
14.05 \\
14.05 \\
14.15 \\
14.51 \\
14.98 \\
15.86\end{array}$ & 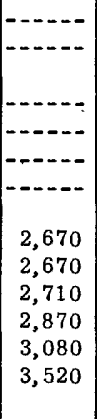 & Apr. 8 & $\begin{array}{l}0600 \\
1200 \\
1430 \\
1800 \\
2400 \\
\\
0600 \\
1200 \\
1800 \\
2400 \\
1200 \\
2400 \\
2400\end{array}$ & $\begin{array}{l}16.86 \\
17.48 \\
17.55 \\
17.48 \\
17.13 \\
16.22 \\
13.55 \\
10.70 \\
10.01 \\
\\
9.01 \\
8.49 \\
8.70\end{array}$ & $\begin{array}{r}4,110 \\
4,560 \\
4,620 \\
4,560 \\
4,300 \\
\\
3,710 \\
2,490 \\
1,560 \\
1,350 \\
\\
1,070 \\
932 \\
983\end{array}$ \\
\hline
\end{tabular}


(278) 5-4735. Big Creek near Mount Pleasant, Iowa

Location.-Lat $41^{\circ} 00^{\prime} 50^{\prime \prime}$, long $91^{\circ} 34^{\prime} 45^{\prime \prime}$, in NW $\frac{1}{4} \mathrm{NW} \frac{1}{4}$ sec.29, T.72 N., R.6 W., cn left bank $12 \mathrm{ft}$ downstream from highway bridge, $100 \mathrm{ft}$ downstream from Lynn Crsek, 0.7 mile downstream from Brandywine Creek, and 3.4 miles northwest of Mount Pleasant.

\section{Drainage area. $-106 \mathrm{sq} \mathrm{mi}$.}

Gage-height record.-Water-stage recorder graph. Datum of gage is $630.53 \mathrm{ft}$ above mean sea level, datum of 1929 .

Discharge record.-Stage-discharge relation defined by current-meter measurements below $4,100 \mathrm{cfs}$ and by contracted-opening measurement at $6,160 \mathrm{cfs}$. Backwater from ice Mar. 1, 5-12, 15, 16, 18-28.

Maxima.-March-May 1965: Discharge, 2,580 cfs 1330 hours Mar. 17 (gage height, $12.07 \mathrm{ft}$ ).

1955 to February 1965: Discharge, 4,460 cfs Mar. 29, 1960 (gage height, $15.30 \mathrm{ft}$ ).

Flood of Aug. 3, 1948, reached a stage of about $27 \mathrm{ft}$, from floodmarks by lccal residents (discharge not determined).

Mean discharge, in cubic feet per second, 1965

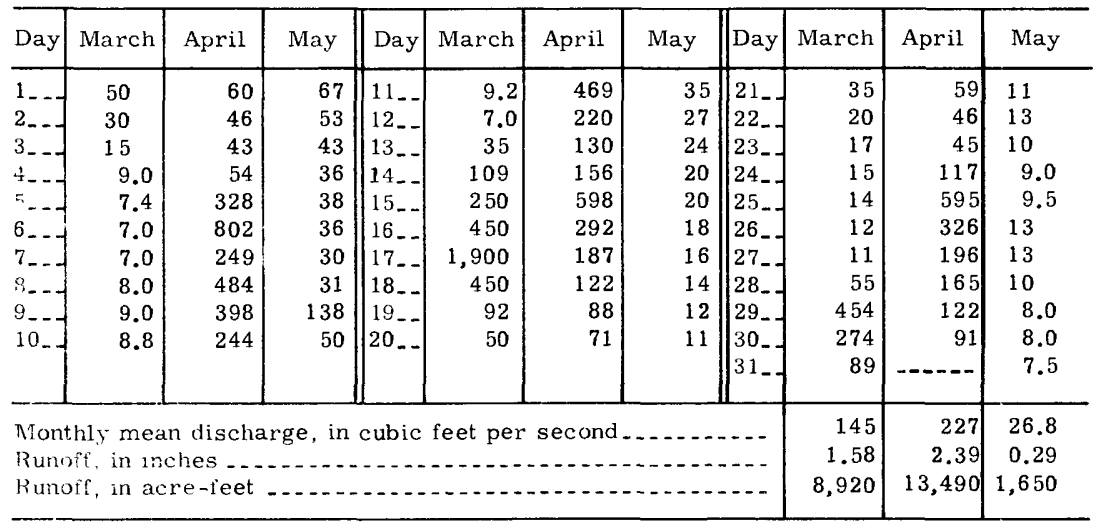

Gage height, in fect, and discharge, in cubic feet per second, at indicated time, 1965

\begin{tabular}{|c|c|c|c|c|c|c|c|c|c|c|c|}
\hline Date & Hour & $\begin{array}{c}\text { Gage } \\
\text { height }\end{array}$ & $\begin{array}{c}\text { Dis - } \\
\text { charge }\end{array}$ & Date & Hour & $\begin{array}{l}\text { Gage } \\
\text { height }\end{array}$ & $\begin{array}{c}\text { Dis- } \\
\text { charge }\end{array}$ & Date & Hour & $\begin{array}{c}\text { Gage } \\
\text { height }\end{array}$ & $\begin{array}{c}\text { Dis- } \\
\text { charge }\end{array}$ \\
\hline Mar. 17 & $\begin{array}{l}0000 \\
0200 \\
1330 \\
2000\end{array}$ & $\begin{array}{r}7.02 \\
8.06 \\
12.07 \\
10.06\end{array}$ & $\begin{array}{r}895 \\
1,350 \\
2,580 \\
1,820\end{array}$ & Mar. 17 & $\begin{array}{l}2400 \\
0400 \\
1100\end{array}$ & $\begin{array}{l}8.82 \\
6.95 \\
4.88\end{array}$ & $\begin{array}{r}1,410 \\
878 \\
428\end{array}$ & Mar. 18 & $\begin{array}{l}1500 \\
1700 \\
2000 \\
2400\end{array}$ & $\begin{array}{l}4.92 \\
4.85 \\
4.11 \\
3.45\end{array}$ & - \\
\hline
\end{tabular}


(279) 5-4740. Skunk River at Augusta, Iowa

Location.-Lat $40^{\circ} 45^{\prime} 10^{\prime \prime}$, long $91^{\circ} 16^{\prime} 30^{\prime \prime}$, in NE $\frac{1}{4} \mathrm{NE} \frac{1}{4}$ sec.26, T.69 N., R.4 W., on left bank $300 \mathrm{ft}$ upstream from bridge on State Highway 394 at Augusta, 2 miles upstream from Long Creek, at mile 12.5.

Drainage area. $-4,303 \mathrm{sq} \mathrm{mi}$.

Gage-height record.-Water-stage recorder graph, except Mar. 19-2C, for which graph was reconstructed on basis of daily wire-weight gage readings. Datum of gage is $521.24 \mathrm{ft}$ above mean sea level, datum of 1929 .

Discharge record.-Stage-discharge relation defined by current-meter measurements below 50,000 cfs. Backwater from ice Mar. 1-18, 22-26. Backwater from Mississippi River Apr. 14-30, May 1-14.

Maxima.-March-May 1965: Discharge, 20,300 cfs 0230 hours Mar. 18 (gage height, $16.99 \mathrm{ft}$ ).

1913, 1914 to February 1965: Discharge, 51,000 cfs Apr. 3, 1960 (gage height, $25,00 \mathrm{ft}$ ).

Flood of June 1, 1903, reached a stage of about $21 \mathrm{ft}$ (discharge, about 45,000 cfs).

Cooperation.-One discharge measurement furnished by Corps of Engineers.

Mean discharge, in cubic feet per second, 1965

\begin{tabular}{|c|c|c|c|c|c|c|c|c|c|c|c|}
\hline Day & March & April & May & Day & March & April & May & Day & March & April & May \\
\hline & 3,000 & 40 & 8,000 & 1 & 1,7 & 18,200 & 0 & 21 & 9,250 & 3,200 & 1,080 \\
\hline & 5,000 & 5,990 & 7,000 & $12 \ldots$ & 1,600 & 19,100 & 3,100 & $22 \ldots$ & 7,400 & 2,800 & 979 \\
\hline & 7,000 & 5,900 & 5,900 & $13 \ldots$ & 1,500 & 17,300 & 2,800 & 23 & 6,000 & 2,800 & 907 \\
\hline & 6,500 & 6,380 & 5,200 & $14 \ldots$ & 2,200 & 13,200 & 2,500 & $24 \ldots$ & 4,500 & 3,000 & 840 \\
\hline & 6,000 & 8,010 & 4,800 & $15 \ldots$ & 3,500 & 10,000 & 2,170 & 25 & 3,500 & 6,000 & 808 \\
\hline & 3,500 & 14,800 & 4,500 & $16 \ldots$ & 5,000 & 7,100 & 1,820 & 26 & 2,800 & 9,500 & 970 \\
\hline 7. & 3,000 & 15,800 & 4,300 & 17. & 16,000 & 6,100 & 1,560 & 27 & 2,410 & 9,100 & 1,130 \\
\hline 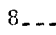 & 2,500 & 17,100 & 4,000 & $18 \ldots$ & 19,000 & 5,000 & 1,520 & $28 \ldots$ & 2,000 & 8,200 & 1,660 \\
\hline & 2,200 & 17,700 & 3,900 & $19 \ldots$ & 17,400 & 4,200 & 1,360 & 29. & 3,820 & 8,500 & 3,350 \\
\hline & 2,000 & 16,800 & 3,800 & $20 \ldots$ & 14,000 & 3,700 & 1,180 & $30_{-}$ & 8,890 & 8,900 & 4,570 \\
\hline & & & & & & & & 31 & 7,190 & $\cdots-\cdots$ & 4,210 \\
\hline \multirow{3}{*}{\multicolumn{9}{|c|}{$\begin{array}{l}\text { Monthly mean discharge, in cubic feet per second } \\
\text { Runoff, in inches } \\
\text { Runoff, in acre-feet }\end{array}$}} & 5,818 & 9,351 & 3,013 \\
\hline & & & & & & & & & 1.56 & 2.42 & 0.81 \\
\hline & & & & & & & & & 357,700 & 556,400 & 185,300 \\
\hline
\end{tabular}

Gage height, in fect, and discharge, in cubic feet per second, at indicated time, 1965

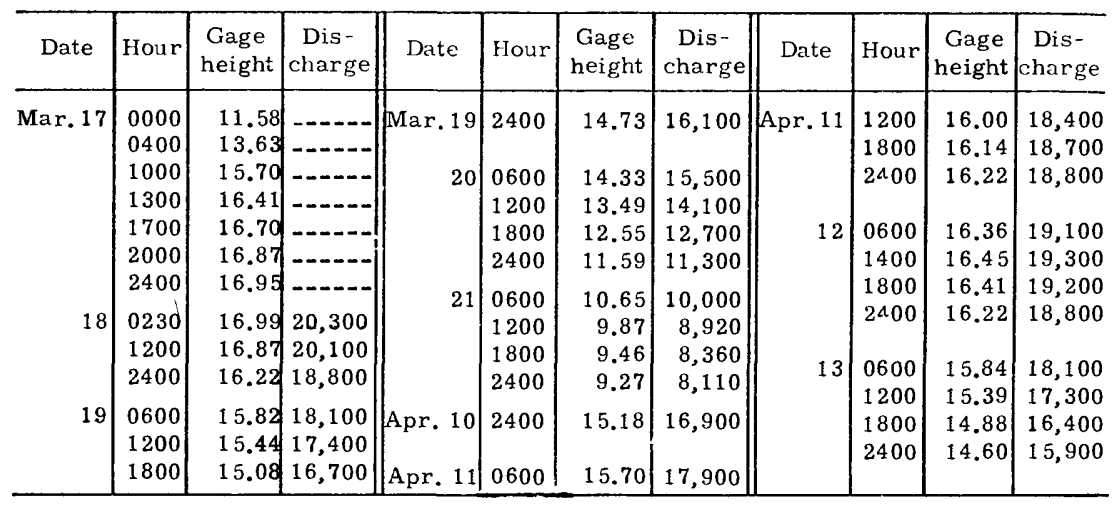




\section{MISSISSIPPI RIVER MAIN STEM}

(280) 5-4745. Mississippi River at Keokuk, Iowa

Location.-Lat $40^{\circ} 23^{\prime} 35^{\prime \prime}$, long $91^{\circ} 22^{\prime} 25^{\prime \prime}$, in SE $\frac{1}{4} \mathrm{SW} \frac{1}{4}$ sec. 30 , T.65 N., R.4 W., near right bank in tailwater at downstream end of new lock below dam and powerplant of Union Electric Co. at Keokuk, 2.8 miles upstream from Des Moines River and at mile 364.2 upstream from Ohio River.

Drainage area.-119,000 sq mi, approximately.

Gage-height record.-Water-stage recorder. Datum of gage is $477.41 \mathrm{ft}$ above mean sea level, datum of 1929 (levels by Corps of Engineers); $477.83 \mathrm{ft}$ above mean sea level, adjustment of $1912 ; 477.34 \mathrm{ft}$ above mean gulf level; and $484.65 \mathrm{ft}$ above Memphis datum. Jan. 1, 1878, to May 1913, staff gage at Galland (formerly Ne shville), 8 miles upstream; zero of gage was set to low-water mark of 1864 , or $497.94 \mathrm{ft}$ above mean sea level, adjustment of 1912 .

Discharge record.-Records furnished by Union Electric Company except for period of Apr. 21 to May 11 which were computed by the U.S. Geological Survey on basis of backwater rating of the tailwater gage. Furnished records computed from records of turbine operation at power plant and openings of spillway gages in dam.

Maxima.-April-May 1965: Daily discharge, 327,000 cfs May 1; gage height, $22.14 \mathrm{ft}$, May 1.

1878 to February 1965: Daily discharge, 314,000 cfs May 18, 1888 (gage height, $12.0 \mathrm{ft}$, site and datum then in use; $19.6 \mathrm{ft}$ present site and datum).

Flood of $J$ une 6,1851 , reached a stage of $21.0 \mathrm{ft}$, present site and datum; estimated as $13.5 \mathrm{ft}$ at Galland (discharge, $360,000 \mathrm{cfs}$ ).

Remarks.-Discharge records furnished by Union Electric Company except for period Apr. 21 to May 11. Annual peak stages furnished by Corps of Engineers. 
Mean gage height, in feet, and discharge, in cubic feet per second, 1965, of Mississippi River at Keokuk. Iowa

\begin{tabular}{|c|c|c|c|c|c|c|}
\hline \multirow{2}{*}{ Day } & \multicolumn{2}{|c|}{ March } & \multicolumn{2}{|c|}{ April } & \multicolumn{2}{|c|}{ May } \\
\hline & Gage height & Discharge & Gage height & Discharge & Gage height & Discharge \\
\hline $\begin{array}{l}2 \\
3 \\
4 \\
5 \\
6 \\
7 \\
8 \\
9 \\
10 \\
11 \\
12 \\
133 \\
14 \\
15 \\
16 \\
17 \\
18 \\
19 \\
20 \\
21 \\
22 \\
23 \\
24 \\
25 \\
26 \\
21 \\
28 \\
29 \\
30 \\
31\end{array}$ & 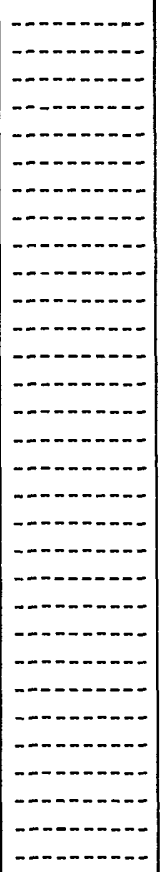 & $\begin{array}{r}48,900 \\
60,600 \\
64,400 \\
77,300 \\
77,300 \\
108,800 \\
116,600 \\
115,000 \\
117,700 \\
123,300 \\
136,300 \\
138,800 \\
126,900 \\
116,100 \\
116,000 \\
121,700 \\
130,100 \\
134,000 \\
110,800 \\
76,200 \\
60,500 \\
62,300 \\
62,600 \\
54,200 \\
48,200 \\
46,500 \\
44,600 \\
50,000 \\
56,200 \\
75,000 \\
81,700\end{array}$ & $\begin{array}{r}- \\
- \\
-\end{array}$ & $\begin{array}{r}82,700 \\
97,600 \\
111,600 \\
122,200 \\
133,200 \\
146,200 \\
157,600 \\
173,600 \\
184,500 \\
191,000 \\
191,500 \\
200,000 \\
213,900 \\
223,100 \\
235,700 \\
244,700 \\
250,500 \\
247,200 \\
239,300 \\
234,400 \\
234,000 \\
238,000 \\
239,000 \\
246,000 \\
267,000 \\
292,000 \\
305,000 \\
300,000 \\
302,000 \\
321,000 \\
---\cdots\end{array}$ & 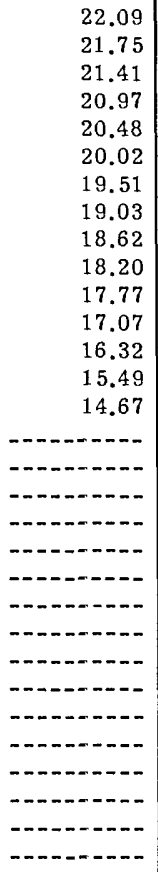 & $\begin{array}{l}327,000 \\
318,000 \\
310,000 \\
298,000 \\
286,000 \\
279,000 \\
269,000 \\
260,000 \\
253,000 \\
246,000 \\
238,000 \\
223,400 \\
211,600 \\
198,200 \\
185,400 \\
174,400 \\
161,900 \\
151,500 \\
142,400 \\
134,600 \\
129,900 \\
128,100 \\
126,200 \\
125,400 \\
129,000 \\
126,900 \\
128,100 \\
130,000 \\
133,200 \\
135,800 \\
136,000\end{array}$ \\
\hline $\begin{array}{l}\text { Mean } \\
\text { Inches - } \\
\text { Acre-feet }\end{array}$ & - & $\begin{array}{rr} & 89,000 \\
- & 0.86 \\
5,472,000\end{array}$ & & $\begin{array}{r}214,200 \\
2.01 \\
12,740,000\end{array}$ & & $\begin{array}{r}196,600 \\
1.91 \\
12,090,000\end{array}$ \\
\hline
\end{tabular}


Annual naximum stages and mean daily discharges, for indicated vears of Mississippi River at Keakuk, low'a

\begin{tabular}{|c|c|c|c|c|c|c|c|}
\hline Year & Date & $\begin{array}{c}\text { Gage } \\
\text { height } \\
\text { (feet) }\end{array}$ & $\begin{array}{c}\text { Discharge } \\
\text { (cfs) }\end{array}$ & Year & Date & $\begin{array}{l}\text { Gage } \\
\text { height } \\
\text { (feet) }\end{array}$ & $\begin{array}{c}\text { Discharge } \\
\text { (cfs) }\end{array}$ \\
\hline 1851 & J une 6 & 21.0 & 360,000 & 1 & May 9 & $-m-\cdots$ & 213,000 \\
\hline & May 8 & 14.6 & $-----n----$ & $1917---\mid$ & J une 17 & --- & 163,000 \\
\hline & July 16 & 14.7 & & $1918 \ldots--$ & J une 12 & 16.7 & 192,000 \\
\hline $1870_{-}$ & Apr. 27 & 16.4 & ----------- & $1919 \ldots$ & May 8 & 17.15 & 205,000 \\
\hline 1871 . & May & 12.2 & $--2-0-n-n$ & 1920 & Apr. $10-11$ & $-\infty-n$ & 230,000 \\
\hline 1872 & $J$ une 7 & 12.1 & & 1921 . & May $12-13$ & ----- & 108,000 \\
\hline 1873 & $J$ une $14-15$ & 12.5 & 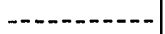 & 1922 & Apr. 24,25 & 17.45 & 240,000 \\
\hline 1874 & Mar. 12 & 8.8 & & 1923 & Apr. 9,10 & 12.0 & 148,000 \\
\hline 1875 & July 9 & 12.9 & & 1924 & Aug. 24, 25 & ----- & 160,000 \\
\hline 1876 & Apr. 17 & 16.5 & & 1925 & J une 23 & 10.3 & 112,000 \\
\hline 1877 & Apr. $9-10$ & 11.6 & & 1926 - & Sept. 28 & $-\ldots-n$ & 146,000 \\
\hline 187 & $J$ une 11 & $-----n$ & 150,000 & 1927 & Apr. 3 & $-\cdots--$ & 175,000 \\
\hline 187 & J une 2,3 & 8.45 & 110,000 & $1928 \ldots$ & Apr. 12 & $-\cdots-n$ & 150,000 \\
\hline $1880_{-}$ & June 29 & 17.5 & 271,000 & 1929 & Mar. 23 & 19.3 & 247,000 \\
\hline 1881 - & Apr. 23,24 & $---n--$ & 241,000 & 1930 & June 18 & 13.9 & 163,000 \\
\hline 1882 & Oct. 31,1881 & 18.9 & 293,000 & 1931 - & July 4 & $----n$ & 52,500 \\
\hline 1883 & May 18 & 15.4 & 201,000 & 1932 & Apr. 24, 25 & 10.2 & 106,000 \\
\hline 1884 & Apr. 1 & 16.7 & 236,000 & 19 & Apr. 9 & 14.5 & 160,000 \\
\hline 188 & Oct. $9,10,1884$ & $-\cdots-\cdots$ & 170,000 & $4 \ldots$ & Apr. 22 & $---n--$ & 83,500 \\
\hline 18 & May 6 & 15.95 & 212,000 & 193 & Apr. 11,12 & ------ & 138,000 \\
\hline 18 & May 4 & $-----n$ & 156,000 & 1936 & Apr. 9,10 & 12.3 & 148,000 \\
\hline 18 & May 18 & 19.6 & 314,000 & $7---$ & Mar. 10 & 16.1 & 190,000 \\
\hline 188 & Apr. 20 & -- & 84,200 & $1938 \ldots$ & Sept. 26 & 16.4 & 193,800 \\
\hline & June 8,18 & & & & Oct. 1,1938 & $---m-n$ & 159,100 \\
\hline & July 1 & 12.6 & 178 & $1940_{-} \ldots$ & Apr. 19 & 6.4 & 81,700 \\
\hline & May 3 & $-m--n$ & 141,000 & $1-\ldots$ & Apr. 27 & 12.3 & 154,400 \\
\hline & J une 29 & 19.25 & 306,000 & $1942 \ldots--$ & June 16 & 15.80 & 200,900 \\
\hline 18 & May $15-17$ & 14.85 & 203,000 & $1943 \ldots$ & Apr. 18 & ------ & 174,000 \\
\hline 18 & June 4 & 11.3 & 158,000 & $1944 \ldots$ & May 27,28 & 20.85 & 256,000 \\
\hline 1 & Mar. 11 & ---- & 59,200 & $5---$ & Mar. 26 & 16.80 & 203,300 \\
\hline & $J$ une 3 & & 161,000 & 19 & Jan. 11 & 16.95 & 223,300 \\
\hline & Apr. 28, 29 & 18.4 & 230,000 & $7---$ & June 21 & 20.2 & 245,700 \\
\hline 189 & Mar. 20 & 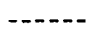 & 108,000 & $1948 \ldots--$ & Mar. 23 & 18.9 & 233,600 \\
\hline 189 & $J$ une 29 & $----n$ & 159,000 & $1949-\ldots$ & Mar. 12 & 13.3 & 150,700 \\
\hline 1 & Apr. 5, 6 & ---- & 124,000 & $1950 \ldots--$ & Apr. 25,26 & ----- & 175,900 \\
\hline & Mar. 24-26 & & 150,000 & 19 & Apr. 29 & ------ & 265,100 \\
\hline & July 21,22 & 1 & 181,000 & --- & Apr. 27 & ----- & 253,800 \\
\hline 190 & J une 6 & 19.6 & 270,000 & $1953---$ & Apr. 1,2 & 12.5 & 137,200 \\
\hline 190 & Oct. 7, 1903 & ------ & 186,000 & $1954 \ldots$ & May 17 & $---n$ & 181,400 \\
\hline 190 & June 10 & 18.5 & 212,000 & & Apr. 25 & 12.7 & 156,600 \\
\hline & Apr. 26-28 & 14.0 & 192,000 & 19 & Apr. 22 & 10.2 & 131,500 \\
\hline 15 & Apr. 17-18 & ----- & 178,000 & 195 & July 15 & 8.70 & 106,000 \\
\hline 19 & June 9 & -- & 178,000 & |1958_- & June 13 & 8.29 & 99,000 \\
\hline 19 & May 5-7 & & 181,000 & $\mid 1959 \ldots$ & Apr. 5 & 14.17 & 182,000 \\
\hline 19 & Mar. 20-23 & 10.1 & 124,000 & $1960-\ldots$ & Apr. 4 & 21.83 & 289,500 \\
\hline & Feb. 21 & 12.3 & 156,000 & $1961 \ldots$ & Apr. 5 & 17.11 & 208,400 \\
\hline & Apr. 6,7 & 17.7 & 220,000 & $1962 \ldots--$ & Apr. 7 & 18.58 & 224,100 \\
\hline & Mar. 29 & & 169,000 & $1963 \ldots$ & Mar. 22 & 10.76 & 128,700 \\
\hline & June 24 & 11.2 & 122,000 & $1964 \ldots-$ & May 21 & 7.96 & 96,400 \\
\hline 191 & Feb. 28 & & 142,000 & $1965 \ldots--$ & May 1 & 22.14 & 327,000 \\
\hline
\end{tabular}

Note.-Annual peak stages referenced to tailwater gage, datum 477.83 feet, adjustment of 1912 . 


\section{DES MOINES RIVER BASIN}

(281) West Fork Des Moines River below Talcott Dam near Dundee, Minn.

(Miscellaneous site)

Location.--Lat $43^{\circ} 53^{\prime} 10^{\prime \prime}$, long $95^{\circ} 26^{\prime} 11^{\prime \prime}$, near center of sec.20, T.105 N., R.38 W., at outlet of Talcott Lake, $3 \frac{1}{4}$ miles northeast of Dundee.

Gage-height record.-Peak from graph based on twice-daily tailwater staff gage readings, Apr. 6-8. Datum of gage is $1,395.29 \mathrm{ft}$ above mean sea level, adjustment of 1929 (levels by Minnesota Conservation Department Division of Waters).

Discharge record.-Stage-discharge relation defined by current-meter measurements.

Maxima.-April-May 1965: Discharge, 4,700 cfs 0600 hours Apr. 7 (gage height, $11.51 \mathrm{ft}$ ).

1963 to March 1965: Discharge, 1,000 cfs Aug. 4, 1963 (gage height, $8.22 \mathrm{ft}$ ).

Cooperation.-Gage heights furnished by Minnesota Conservation Department, Division of Waters.

(282) 5-4758. West Fork Des Moines River tributary near Jackson, Minn.

\section{(Crest-stage station)}

Location.-Lat $43^{\circ} 41^{\prime} 40^{\prime \prime}$, long $95^{\circ} 01^{\prime} 30^{\prime \prime}$, in NW $\frac{1}{4} \mathrm{SE} \frac{1}{4}$, sec. $27, \mathrm{~T} .103 \mathrm{~N}$., R.35 W., at culvert on county road three-quarters of a mile upstream from mouth, and $5 \frac{1}{2}$ miles north of Jackson.

Drainage area. $-1.42 \mathrm{sq} \mathrm{mi}$.

Gage-height record.-Crest stages only.

Discharge record.-Stage-discharge relation defined by current-meter measurements below $6 \mathrm{cfs}$ and by indirect measurement at $49 \mathrm{cfs}$.

Maxima.-April-May 1965: Discharge, 38 cfs Apr. 5 (gage height, 17.86 ft, backwater from ice).

1960 to March 1965: Discharge, $69 \mathrm{cfs}$ Mar. 28, 1962 (gage height, $16.34 \mathrm{ft}$, backwater from ice).

(283) 5-4759. West Fork Des Moines River tributary near Lakefield, Minn.

$$
\text { (Crest-stage station) }
$$

Location.-Lat $43^{\circ} 40^{\prime} 30^{\prime \prime}$, long $95^{\circ} 03^{\prime} 20^{\prime \prime}$, in $\operatorname{SE} \frac{1}{4} \mathrm{SE} \frac{1}{4}$ sec.32, T.103 N., P.35 W., at culvert on County Highway 19, $1 \frac{1}{2}$ miles upstream from mouth, and 5-3/4 miles east of Lakefield.

Drainage area. $-4.52 \mathrm{sq} \mathrm{mi}$.

Gage-height record.-Crest stages only.

Discharge record.-Stage-discharge relation defined by current-meter measurements below $47 \mathrm{cfs}$ and by indirect measurement at $119 \mathrm{cfs}$.

Maxima.-April-May 1965: Discharge, 112 cfs Apr. 5 (gage height, $10.46 \mathrm{ft}$, backwater from ice); gage height, $10.65 \mathrm{ft}$ April 1 (backwater from ice).

1960 to March 1965: Discharge, 119 cfs June 9, 1963 (gage height, $7.00 \mathrm{ft}$ ); gage height, $8.86 \mathrm{ft}$ Mar. 28, 1962 (backwater from ice). 
(284) 5-4760. West Fork Des Moines River at Jackson, Minn.

Location.-Lat $43^{\circ} 37^{\prime} 10^{\prime \prime}$, long $94^{\circ} 59^{\prime} 10^{\prime \prime}$, in SE $\frac{1}{4} \mathrm{SW} \frac{1}{4}$ sec. $24, \mathrm{~T} .102 \mathrm{~N}$, R.35 W., on right bank in Jackson, $200 \mathrm{ft}$ downstream from dam at powerplant.

Drainage area. $-1,220 \mathrm{sq} \mathrm{mi}$, approximately.

Gage-height record.-Water-stage recorder graph except 0100 hours Apr. 6 to May 31 . Graph was constructed on the basis of six or more daily gage readings, on powerplant gage above dam, 0100 hours Apr. 6 to Apr. 27 and average of twice-daily gage readings on the outside staff gage Apr. 28 to May 31 . Datum of gage is $1,287.75 \mathrm{ft}$ above mean sea level, datum of 1929 .

Discharge record.-Stage-discharge relation defined by current-measurements. Backwater from ice Apr. 1-9. Discharge for the period Apr. 6-14 was obtained from powerplant gage stage-discharge relation.

Maxima.-April-May 1965: Discharge, 9,530 cfs 2200 hours Apr. 9 (gage height, $18.38 \mathrm{ft}$, from powerplant gage); gage height, $18.62 \mathrm{ft} 1500$ hours Apr. 6, from floodmark (backwater from ice).

1909-13, 1930 to March 1965: Discharge, 8,360 cfs June 8, 1953 (gage height, $17.43 \mathrm{ft}$, from floodmark), from rating curve extended above $3,500 \mathrm{cfs}$ on basis of contracted-opening measurement of peak flow.

Remarks.- Some regulation by Yankton, Long, Shetek, and Heron Lakes above station.

Mcan discharge, in cubic feet per second, 1965

\begin{tabular}{|c|c|c|c|c|c|c|c|c|}
\hline Day & April & May & Day & April & May & Day & April & May \\
\hline & 77 & 1,590 & 11 & 8,330 & 1,500 & 21. & 2,760 & 1,780 \\
\hline 2 & 124 & 1,440 & 12 & 7,110 & 1,480 & 22. & 2,320 & 1,730 \\
\hline $3 \ldots$ & 295 & 1,440 & 13 & 5,980 & 1,510 & 23 & 2,220 & 1,660 \\
\hline & 645 & 1,390 & $14 \ldots$ & 5,360 & 1,550 & $24 \ldots$ & 2,150 & 1,590 \\
\hline $5 \ldots$ & 1,530 & 1,290 & $15 \ldots$ & 4,750 & 1,620 & $25 \ldots$ & 2,150 & 1,780 \\
\hline $6 .-$ & 5,300 & 1,170 & $16 \ldots$ & 4,310 & 1,640 & $26 \ldots$ & 2,160 & 1,850 \\
\hline & 5,600 & 1,170 & $17 \ldots$ & 3,860 & 1,590 & 27. & 2,140 & 1,810 \\
\hline & 5,100 & 1,270 & 18. & 3,550 & 1,590 & $28 \ldots$ & 2,040 & 1,730 \\
\hline 9 & 8,000 & 1,500 & 19. & 3,300 & 1,590 & $29 \ldots$ & 1,770 & 1,730 \\
\hline 10. & 9,100 & 1,840 & 20. & 3,070 & 1,640 & $30 \ldots$ & 1,750 & 1,690 \\
\hline & & & & & & $31 \ldots$ & - & 1,690 \\
\hline \multirow{2}{*}{\multicolumn{7}{|c|}{$\begin{array}{l}\text { Monthly mean discharge, in cubic feet per second } \\
\text { Runoff, in inches }\end{array}$}} & 3,561 & 1,576 \\
\hline & & & & & & & 3.26 & 1.49 \\
\hline
\end{tabular}

Gage height, in feet, and discharge, in cubic feet per second, at indicated time, 1965

\begin{tabular}{|c|c|c|c|c|c|c|c|c|c|c|c|}
\hline Date & Hour & $\begin{array}{l}\text { Gage } \\
\text { height }\end{array}$ & $\begin{array}{c}\text { Dis- } \\
\text { charge }\end{array}$ & Date & Hour & $\begin{array}{l}\text { Gage } \\
\text { height }\end{array}$ & $\begin{array}{c}\text { Dis - } \\
\text { charge }\end{array}$ & Date & Hour & $\begin{array}{l}\text { Gago } \\
\text { heigl }\end{array}$ & $\begin{array}{l}\text { Dis- } \\
\text { charge }\end{array}$ \\
\hline \multirow[t]{6}{*}{ Mar. 31} & 0000 & 4.27 & -.-- & \multirow[t]{12}{*}{ Apr. 2} & 0600 & 8.83 & --0 & \multirow[t]{12}{*}{ Apr. 3} & 2200 & 10.45 & \\
\hline & 1200 & 4.37 &.--- & & 1200 & 8.28 & & & 2300 & 11.22 & \\
\hline & 1300 & 4.30 & $---n$ & & 1600 & 8.09 & & & 2400 & 10.97 & \\
\hline & 2100 & 6.79 & -... & & 2400 & 8.21 & & & & & \\
\hline & 2400 & 7.10 & $-\ldots$ & & & & & & 0100 & $10.8 ?$ & \\
\hline & & & & & 0900 & 8.10 & & & 0500 & 10.61 & \\
\hline \multirow{6}{*}{ Apr. 1} & 0500 & 7.20 & ---- & & 1200 & 8.10 & & & 0600 & 10.93 & \\
\hline & 1200 & 7.08 & -- & & 1500 & 9.83 & & & 1200 & 10.77 & \\
\hline & 1500 & 7.89 & $-\ldots$ & & 1700 & 11.09 & - & & 1800 & 11.34 & \\
\hline & 1800 & 8.87 & $\ldots$ & & 1800 & 10.98 & - & & 2400 & 11.56 & \\
\hline & 2100 & 9.17 & --- & & 1900 & 10.99 & & & & & \\
\hline & 2400 & 9.15 & & & 2100 & 10.55 & & & 0100 & 11.59 & \\
\hline
\end{tabular}


Gage height and discharge at indicated time, 1965, of West Fork Des Moines Rlver at Jackson, Minn. - Continued

\begin{tabular}{|c|c|c|c|c|c|c|c|c|c|c|c|}
\hline Date & Hour & $\begin{array}{c}\text { Gage } \\
\text { height }\end{array}$ & $\begin{array}{c}\text { Dis- } \\
\text { charge }\end{array}$ & Date & Hour & $\begin{array}{c}\text { Gage } \\
\text { height }\end{array}$ & $\begin{array}{c}\text { Dis- } \\
\text { charge }\end{array}$ & Date & Hour & $\begin{array}{c}\text { Gage } \\
\text { height }\end{array}$ & $\begin{array}{c}\text { Dis- } \\
\text { charge }\end{array}$ \\
\hline pr. 5 & $\begin{array}{l}1200 \\
1700 \\
1800 \\
2100 \\
2400 \\
0600 \\
0900 \\
1200 \\
1500 \\
1800 \\
2100 \\
2400 \\
\\
0300 \\
0700 \\
0800 \\
1200 \\
1500\end{array}$ & $\begin{array}{l}11.61 \\
11.79 \\
13.15 \\
13.74 \\
14.72 \\
\\
17.41 \\
18.07 \\
18.52 \\
18.96 \\
18.54 \\
18.64 \\
18.46 \\
\\
18.64 \\
18.67 \\
18.59 \\
18.67 \\
18.55\end{array}$ & 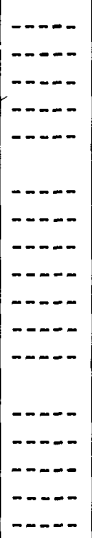 & Apr. 7 & $\begin{array}{l}1800 \\
2100 \\
2400 \\
0300 \\
0600 \\
0800 \\
1200 \\
1400 \\
1700 \\
1800 \\
2000 \\
2100 \\
2400 \\
\\
0600 \\
1000 \\
1300 \\
1500\end{array}$ & $\begin{array}{l}18.46 \\
18.38 \\
18.21 \\
18.17 \\
18.04 \\
17.96 \\
17.71 \\
17.71 \\
17.46 \\
17.62 \\
17.88 \\
17.71 \\
17.79 \\
18.04 \\
18.04 \\
17.79 \\
18.04\end{array}$ & $\begin{array}{l} \\
-\end{array}$ & Apr. 9 & $\begin{array}{l}1800 \\
2200 \\
2400 \\
0600 \\
0800 \\
1200 \\
1300 \\
1800 \\
2400\end{array}$ & $\begin{array}{l}18.29 \\
18.38 \\
18.29 \\
18.21 \\
18.25 \\
18.29 \\
18.26 \\
18.18 \\
18.12 \\
17.72 \\
17.12 \\
16.78 \\
16.58\end{array}$ & \begin{tabular}{l}
9,530 \\
\hdashline 9,070 \\
9,170 \\
9,270 \\
9,200 \\
8,990 \\
8,830 \\
7,828 \\
6,390 \\
5,580 \\
5,140
\end{tabular} \\
\hline
\end{tabular}

Note.-Gage-heights Apr. 6-14 are from gage at power plant above dam.

(285) 5-4761. Story Brook near Petersburg, Minn.

(Crest-stage station)

Location.-Lat $43^{\circ} 32^{\prime} 20^{\prime \prime}$, long $94^{\circ} 59^{\prime} 40^{\prime \prime}$, in SW $\frac{1}{4} \mathrm{NW} \frac{1}{4}$ sec. 24 , T.101 N., R.35 W., at bridge on U.S. Highway 71, 3 miles upstream from mouth, and 4 miles west of Petersburg.

Gage-height record.-Crest stages only.

Discharge record.-Stage-discharge relation defined by current-meter measurements below $252 \mathrm{cfs}$ and by indirect measurement at $2,110 \mathrm{cfs}$.

Maxima.-April-May 1965: Discharge, 1,200 cfs Apr. 6 (gage height, 12.75 ft, backwater from ice); gage height, $18.58 \mathrm{ft} \mathrm{Apr.} 4$ (backwater from ice).

1960 to March 1965: Discharge, 2,110 cfs July 4, 1962 (gage height, $12.77 \mathrm{ft}$ ); gage height, $14.17 \mathrm{ft}$ Mar. 28, 1962 (backwater from ice).

(286) 5-4765. West Fork Des Moines River at Estherville, Iowa

Location.-Lat $43^{\circ} 24^{\prime} 00^{\prime \prime}$, long $94^{\circ} 50^{\prime} 40^{\prime \prime}$, in SW $\frac{1}{4} \mathrm{SE} \frac{1}{4}$ sec.10, T.99 N., R.34 W., on right bank in city park, 1,200 ft downstream from bridge on State Highway 9 at Estherville, 2.5 miles upstream from Brown Creek and at mile 404.4 upstream from mouth of Des Moines River (U.S. Geological Survey river profile).

Drainage area. $-1,372 \mathrm{sq} \mathrm{mi}$.

Gage-height record.-Water-stage recorder graph. Datum of gage is $1,247.55 \mathrm{ft}$ above mean sea level, datum of 1929 .

Discharge record.-Stage-discharge relation defined by current-meter measurements below $10,600 \mathrm{cfs}$. Backwater from ice Mar. 17-25, Apr. 4-8.

Maxima.-March-May 1965: Discharge, 10,200 cfs 1900 hours Apr. 10 (gage height, $15.61 \mathrm{ft}$ ).

1951 to February 1965: Discharge, 10,800 cfs June 8, 1953 (gage height, $15.53 \mathrm{ft}$ ). 
Mean discharge, in cubic feet per second. 1965, of West Fork Des Moines River at Estherville, Iowa

\begin{tabular}{|c|c|c|c|c|c|c|c|c|c|c|c|}
\hline Day & March & April & May & Day & March & April & May & Day & March & April & May \\
\hline $\begin{array}{l}1_{-} \\
2_{-} \\
3 . \\
1_{-} \\
5 . \\
6- \\
7_{-} \\
\varepsilon_{-} \\
{ }_{-}\end{array}$ & $\begin{array}{r}108 \\
56 \\
50 \\
49 \\
48 \\
45 \\
44 \\
43 \\
40 \\
37\end{array}$ & $\begin{array}{r}490 \\
1,120 \\
1,340 \\
1,660 \\
3,000 \\
5,700 \\
7,200 \\
7,600 \\
7,860 \\
9,660\end{array}$ & $\begin{array}{l}1,810 \\
1,670 \\
1,540 \\
1,490 \\
1,660 \\
1,400 \\
1,390 \\
1,790 \\
1,760 \\
1,900\end{array}$ & $\begin{array}{l}11 \ldots \\
12_{-}- \\
13_{--} \\
14_{---} \\
15_{--} \\
16_{--} \\
17_{--} \\
18_{--} \\
19_{--} \\
20_{--}\end{array}$ & $\begin{array}{l}36 \\
35 \\
35 \\
39 \\
42 \\
46 \\
48 \\
33 \\
23 \\
27\end{array}$ & $\begin{array}{l}9,490 \\
8,300 \\
7,000 \\
6,060 \\
5,530 \\
4,980 \\
4,380 \\
3,960 \\
3,680 \\
3,380\end{array}$ & $\begin{array}{l}2,090 \\
1,790 \\
1,620 \\
1,670 \\
1,880 \\
2,070 \\
1,930 \\
1,800 \\
1,730 \\
1,730\end{array}$ & $\begin{array}{l}21_{-} \\
23- \\
24_{-} \\
25_{-} \\
26_{-} \\
27_{-} \\
28_{-} \\
29_{-} \\
30_{-} \\
31-\end{array}-$ & $\begin{array}{l}31 \\
35 \\
38 \\
38 \\
36 \\
35 \\
33 \\
31 \\
30 \\
33 \\
93\end{array}$ & $\begin{array}{r}3,100 \\
2,760 \\
2,490 \\
2,360 \\
2,340 \\
2,390 \\
2,410 \\
2,320 \\
2,140 \\
1,940 \\
-----\end{array}$ & $\begin{array}{l}1,960 \\
2,110 \\
1,940 \\
1,800 \\
1,980 \\
2,180 \\
2,160 \\
2,010 \\
1,880 \\
1,860 \\
1,850\end{array}$ \\
\hline \multicolumn{9}{|c|}{$\begin{array}{l}\text { Monthl } \text { mean discharge, in cubic feet per second } \\
\text { Runoff. in inches } \\
\text { Rumofi, in acre-feet }\end{array}$} & $\begin{array}{r}42.5 \\
0.04 \\
2,610\end{array}$ & $\begin{array}{r}4,221 \\
3.43 \\
251,200\end{array}$ & $\begin{array}{r}1,821 \\
1.53 \\
112,000\end{array}$ \\
\hline
\end{tabular}

Gage height, in feet, and discharge, in cubic feet per second, at indicated time, 1965

\begin{tabular}{|c|c|c|c|c|c|c|c|c|c|c|c|}
\hline Date & Hour & $\begin{array}{l}\text { Gage } \\
\text { height }\end{array}$ & $\begin{array}{c}\text { Dis- } \\
\text { charge }\end{array}$ & Date & Hour & $\begin{array}{c}\text { Gage } \\
\text { height }\end{array}$ & $\begin{array}{c}\text { Dis- } \\
\text { charge }\end{array}$ & Date & Hour & $\begin{array}{c}\text { Gage } \\
\text { height }\end{array}$ & $\begin{array}{c}\text { Dis- } \\
\text { charge }\end{array}$ \\
\hline Apr. 9 & 0000 & 13.84 & 7,440 & \multirow[t]{4}{*}{ Apr. 10} & 1800 & 15.55 & 10,100 & \multirow[t]{7}{*}{ Apr. 11} & \multirow[t]{2}{*}{2400} & \multirow[t]{2}{*}{$14.7 £$} & \multirow[t]{2}{*}{8,910} \\
\hline & 1000 & 13.85 & 7,450 & & 1900 & 15.61 & 10,200 & & & & \\
\hline & 2000 & 14.55 & 8,500 & & 2400 & 15.56 & 10,100 & & 0600 & 14.62 & 8,620 \\
\hline & 2400 & 14.67 & 8,710 & & & & & & 1200 & 14.41 & 8,270 \\
\hline & & & & \multirow[t]{3}{*}{11} & 0600 & 15.38 & 9,870 & & 1800 & 14.21 & 8,030 \\
\hline \multirow[t]{2}{*}{10} & 0400 & 14.87 & 8,960 & & \multirow[t]{2}{*}{1800} & \multirow[t]{2}{*}{14.95} & \multirow[t]{2}{*}{9,100} & & \multirow[t]{2}{*}{2400} & \multirow[t]{2}{*}{$13.9=$} & \multirow[t]{2}{*}{7,610} \\
\hline & 0800 & 15.22 & 9,590 & & & & & & & & \\
\hline
\end{tabular}

(287) West Fork Des Moines River at Emmetsburg, Iowa

(Miscellaneous site)

Location.-NW $\frac{1}{4}$ sec. 26, T.96 N., R.33 W., at bridge on county road about 1 mile west of Emmetsburg.

Drainage area. $-1,672 \mathrm{sq} \mathrm{mi}$.

Discharge record. - Stage-discharge relation defined by current-meter measurements below $9,780 \mathrm{cfs}$ and extended above by logarithmic plotting.

Maximum.-March-May 1965: Discharge, 12,000 cfs Apr. 11 (elevation, 1,206.83 ft above mean sea level).

(288) West Fork Des Moines River near Ottosen, Iowa

(Miscellaneous site)

Location.-SE $\frac{1}{4}$ sec.1, T.93 N., R.31 W., at county line on State Highway 44,3 miles west of Ottosen.

Drainage area.- $-2,018 \mathrm{sq} \mathrm{mi}$.

Discharge record.- - Stage-discharge relation defined by current-meter measurements below $13,200 \mathrm{cfs}$ and extended above by logarithmic plotting.

Maxima.-March-May 1965 : Discharge, 14,000 cfs Apr. 12 (elevation, 1,136.68 ft above mean sea level). 
(289) 5-4767.5 West Fork Des Moines River at Humboldt, Iowa

Location.-Lat $42^{\circ} 43^{\prime} 10^{\prime \prime}$, long $94^{\circ} 13^{\prime} 10^{\prime \prime}$, in SE $\frac{1}{4} \mathrm{SW} \frac{1}{4}$ sec.1, T.91 N., R.29 W., on First Avenue bridge in city of Humboldt, about 700 feet below dam, 3.9 miles upstream from confluence with East Fork Des Moines River, and at mile 334.3 upstream from mouth of Des Moines River(U.S. Geological Survey river profile).

Drainage area. $-2,256 \mathrm{sq} \mathrm{mi}$.

Gage-height record.-Graph constructed from daily wire-weight gage readings. Datum of gage is $1,053.54 \mathrm{ft}$ above mean sea level, datum of 1929 .

Discharge record.--Stage-discharge relation defined by current-meter measurements below 14,400 cfs. Backwater from ice Mar. 19-26.

Maxima.-March-May 1965: Discharge, 14,400 cfs 1400 hours Apr. 8 (gage height, $13.90 \mathrm{ft}$ ).

October 1964 to February 1965: Discharge, 1,360 cfs Oct, 1, 1964 (gage height, $5.11 \mathrm{ft}$ ).

Flood of June 23, 1947, reached a stage of $12.2 \mathrm{ft}$ (discharge, 11,000 cfs) at present site and datum.

Nean discharge, in cubic feet per second, 1965

\begin{tabular}{|c|c|c|c|c|c|c|c|c|c|c|c|}
\hline Day & March & April & May & Day & March & April & May & Day & March & April & May \\
\hline 1 - & 948 & 1,520 & 3,610 & $11 \ldots$ & 217 & 12,700 & 3,100 & 21 & 270 & 6,370 & 2,740 \\
\hline 2. & 885 & 2,150 & 3,340 & $12 \ldots$ & 217 & 12,200 & 3,010 & 22 & 242 & 5,740 & 2,660 \\
\hline 3. & 579 & 2,920 & 3,040 & $13 \ldots$ & 245 & 13,400 & 2,850 & 23 & 224 & 5,210 & 2,680 \\
\hline 4 & 462 & 4,600 & 2,830 & $14 \ldots$ & 290 & 12,800 & 2,730 & $24 \ldots$ & 212 & 4,910 & 2,860 \\
\hline 5 & 335 & 7,330 & 2,600 & $15 \ldots$ & 432 & 11,700 & 2,610 & $25 \ldots$ & 208 & 4,670 & 3,140 \\
\hline 西 & 276 & 9,340 & 2,510 & $16 \ldots$ & 596 & 10,500 & 2,790 & $26 \ldots$ & 206 & 4,460 & 3,440 \\
\hline 7. & 233 & 12,500 & 2,540 & 17. & 634 & 9,260 & 3,160 & 27. & 205 & 4,410 & 3,360 \\
\hline & 241 & 14,300 & 2,560 & $18 \ldots$ & 484 & 8,420 & 3,280 & 28 & 205 & 4,160 & 3,560 \\
\hline & 245 & 14,000 & 2,560 & $19 \ldots$ & 400 & 7,650 & 3,170 & 29 & 213 & 3,920 & 3,530 \\
\hline & 229 & 13,700 & 2,860 & $20 \ldots$ & 310 & 6,980 & 2,950 & $30 \ldots$ & 240 & 3,780 & 3,360 \\
\hline & & & & & & & & & 478 & 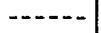 & 3,170 \\
\hline \multirow{3}{*}{\multicolumn{9}{|c|}{$\begin{array}{l}\text { Monthly mean discharge, in cubic feet per second } \\
\text { Runoff, in inches } \\
\text { Ruiroff, in acre-feet }\end{array}$}} & 354 & 7,853 & 2,987 \\
\hline & & & & & & & & & 0.18 & 3.88 & 1.53 \\
\hline & & & & & & & & & 21,740 & 467,300 & 183,700 \\
\hline
\end{tabular}

Gage hetght, in feet, and discharge, in cubic feet per second, at indicated time, 1965

\begin{tabular}{|c|c|c|c|c|c|c|c|c|c|c|c|}
\hline Date & Hour & $\begin{array}{c}\text { Gage } \\
\text { height }\end{array}$ & $\begin{array}{c}\text { Dis- } \\
\text { charge }\end{array}$ & Date & Hour & $\begin{array}{c}\text { Gage } \\
\text { height }\end{array}$ & $\begin{array}{c}\text { Dis- } \\
\text { charge }\end{array}$ & Date & Hour & $\begin{array}{c}\text { Gage } \\
\text { height }\end{array}$ & $\begin{array}{c}\text { Dis- } \\
\text { charge }\end{array}$ \\
\hline \multirow[t]{4}{*}{ Mar. 30} & 0000 & 3.33 & 217 & Apr. 2 & 2400 & 6.35 & 2,470 & Apr. 6 & 2407 & 11.85 & 10,800 \\
\hline & 1200 & 3.37 & 233 & & & & & & & & \\
\hline & 2400 & 3.47 & 276 & 3 & 1200 & 6.73 & 2,850 & 7 & 0607 & 12.52 & 12,000 \\
\hline & & & & & 2400 & 7.33 & 3,490 & & 1007 & 12.59 & 12,100 \\
\hline \multirow[t]{6}{*}{31} & 1000 & 3.60 & 335 & & & & & & 2400 & 13.64 & 14,000 \\
\hline & 1400 & 3.87 & 478 & 4 & 1200 & 8.24 & 4,570 & & & & \\
\hline & 1800 & 4.09 & 609 & & 2400 & 9.06 & 5,760 & 8 & 1200 & 13.88 & 14,400 \\
\hline & 2200 & 4.40 & 820 & & & & & & 140 ? & 13.90 & 14,400 \\
\hline & 2400 & 4.56 & 932 & 5 & 1200 & 9.90 & 7,200 & & 1807 & 13.89 & 14,400 \\
\hline & & & & & 1600 & 10.15 & 7,650 & & 2403 & 13.76 & 14,200 \\
\hline \multirow[t]{7}{*}{ Apr. 1} & 0400 & 4.99 & 1,260 & & 1800 & 11.10 & 9,360 & & & & \\
\hline & 0600 & 5.12 & 1,370 & & 2000 & 10.60 & 8,460 & 9 & 0807 & 13.60 & 13,900 \\
\hline & 1200 & 5.38 & 1,570 & & 2400 & 10.55 & 8,370 & & 2400 & 13.65 & 14,000 \\
\hline & 1800 & 5.56 & 1,730 & & & & & & & & \\
\hline & 2400 & 5.71 & 1,870 & 6 & 0400 & 10.59 & 8,440 & 10 & 080 ? & 13.62 & 13,900 \\
\hline & & & & & 1200 & 10.96 & 9,110 & & 1200 & 13.55 & 13,800 \\
\hline & 1200 & 6.00 & 2,130 & & 1800 & 11.52 & 10,100 & & 2400 & 13.23 & 13,200 \\
\hline
\end{tabular}


(290) 5-4780. East Fork Des Moines River near Burt, Iowa

Location.-Lat $43^{\circ} 12^{\prime} 35^{\prime \prime}$, long $94^{\circ} 10^{\prime} 40^{\prime \prime}$, in NW $\frac{1}{4} \mathrm{NE} \frac{1}{4}$ sec.20, T.97 N., R.28 W., on right bank $30 \mathrm{ft}$ downstream from highway bridge, 0.8 mile upstream from Buffalo Creek, 2.5 miles northeast of Burt, 5.3 miles downstream from Mud Creek, and at mile 389.7 upstream from mouth of Des Moines River (U.S. Geological Survey river profile).

Drainage area. $-462 \mathrm{sq} \mathrm{mi}$.

Gage-height record.-Water-stage recorder graph except Mar. 1-1 5, 18-30. Datum of gage is $1,114.42 \mathrm{ft}$ (revised) above mean sea level, datum of 1929 .

Discharge record.-Stage-discharge relation defined by current-meter measurements below 3,180 cfs, extended to $5,000 \mathrm{cfs}$ by logarithmic plotting and velocity-area study. Backwater from ice Mar. 1 to Apr. 9.

Maxima.-March-May 1965: Discharge, 5,000 cfs 2100 hours Apr. 6 (gage height, $14.21 \mathrm{ft}$, backwater from ice).

1951 to February 1965: Discharge, 3,870 cfs June 21, 1954 (gage height, $12.67 \mathrm{ft}$ ).

Mean discharge, in cubic fect per second, 1965

\begin{tabular}{|c|c|c|c|c|c|c|c|c|c|c|c|}
\hline Day & March & April & May & Day & March & April & May & Day & March & April & May \\
\hline & 52 & 400 & 1,000 & $11 \ldots$ & 42 & 3,110 & 1,110 & $21 \ldots$ & 34 & 1,550 & 682 \\
\hline 2 & 60 & 470 & 960 & $12 \ldots$ & 43 & 2,920 & 1,020 & $22_{-}$ & 32 & 1,450 & 650 \\
\hline $3 \ldots$ & 50 & 660 & 888 & $13 \ldots$ & 44 & 2,680 & 920 & $23 \ldots$ & 30 & 1,340 & 625 \\
\hline & 47 & 1,100 & 828 & $14 \ldots$ & 46 & 2,480 & 844 & $24 \ldots$ & 29 & 1,270 & 610 \\
\hline 5. & 44 & 2,000 & 858 & $15 \ldots$ & 47 & 2,340 & 814 & $25 \ldots$ & 27 & 1,250 & 625 \\
\hline 6. & 42 & 3,900 & 1,110 & $16 \ldots$ & 48 & 2,190 & 883 & $26 \ldots$ & 26 & 1,310 & 744 \\
\hline $7 \ldots$ & 40 & 3,600 & 1,180 & $17 .-$ & 47 & 2,090 & 876 & 27. & 26 & 1,260 & 884 \\
\hline $8 \ldots$ & 40 & 3,900 & 1,230 & $18 \ldots$ & 45 & 1,980 & 837 & 28 & 25 & 1,230 & 848 \\
\hline 9 & 40 & 4,500 & 1,120 & 19 & 42 & 1,820 & 828 & $29_{-}$ & 25 & 1,170 & 807 \\
\hline \multirow[t]{2}{*}{10} & 41 & 3,490 & 1,040 & $20 \ldots$ & 37 & 1,700 & 762 & 30. & 27 & 1,090 & 741 \\
\hline & & & & & & & & 31. & 100 & - & 688 \\
\hline \multicolumn{9}{|c|}{ 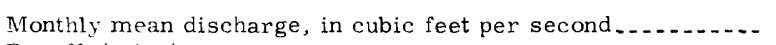 } & 41.2 & 2,008 & 871 \\
\hline \multicolumn{9}{|c|}{ Runnff, in inches } & 0.10 & 4.85 & 2.17 \\
\hline \multicolumn{9}{|c|}{ Runoff, in acre-feet } & 2,530 & 119,500 & 53,580 \\
\hline
\end{tabular}

Gage heıght, in feet, and discharge, in cubic feet per second, at indicated time, 1965

\begin{tabular}{|c|c|c|c|c|c|c|c|c|c|c|c|}
\hline Date & Hour & $\begin{array}{c}\text { Gage } \\
\text { height }\end{array}$ & $\begin{array}{c}\text { Dis - } \\
\text { charge }\end{array}$ & Date & Hour & $\begin{array}{l}\text { Gage } \\
\text { height }\end{array}$ & $\begin{array}{c}\text { Dis- } \\
\text { charge }\end{array}$ & Date & Hour & $\begin{array}{c}\text { Gage } \\
\text { heigh t. }\end{array}$ & $\begin{array}{l}\text { Dis- } \\
\text { charge }\end{array}$ \\
\hline April 4 & $\begin{array}{l}0000 \\
0800 \\
1200 \\
1800 \\
2200 \\
2400 \\
0200 \\
0600 \\
1800 \\
2400 \\
1200\end{array}$ & $\begin{array}{l}11.80 \\
11.83 \\
11.87 \\
12.04 \\
12.19 \\
12.32 \\
12.45 \\
12.97 \\
13.33 \\
13.53 \\
13.90\end{array}$ & - & Apr. 6 & $\begin{array}{l}1600 \\
2100 \\
2400 \\
1000 \\
2400 \\
\\
1800 \\
2000 \\
2400\end{array}$ & $\begin{array}{l}14.13 \\
14.21 \\
14.19 \\
13.80 \\
13.64 \\
\\
13.96 \\
13.95 \\
13.84\end{array}$ & 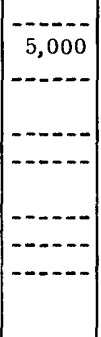 & Apr. 9 & $\begin{array}{l}0800 \\
1200 \\
1800 \\
2400 \\
\\
1200 \\
2400 \\
1200 \\
2400\end{array}$ & $\begin{array}{l}13.46 \\
13.42 \\
13.23 \\
12.85 \\
\\
12.74 \\
12.54 \\
12.49 \\
12.23\end{array}$ & $\begin{array}{l} \\
3,700 \\
3,500 \\
3,280 \\
3,100 \\
2,960\end{array}$ \\
\hline
\end{tabular}


(Miscellaneous site)

Location.-NW $\frac{1}{4} \sec .2$, T.95 N., R.29 W., at bridge on U.S. Highway 169 at north edge of Algona.

Drainage area.- $-882 \mathrm{sq} \mathrm{mi}$.

Discharge record.-Stage-discharge relation defined by current-meter measurements below $10,700 \mathrm{cfs}$ and extended above by logarithmic plotting.

Maximum.-March-May 1965: Discharge, 11,400 cfs, Apr. 9 (elevation, 1,118.78 ft above mean sea level).

(292) 5-4790. East Fork Des Moines River at Dakota City, Iowa

Location.-Lat $42^{\circ} 43^{\prime} 25^{\prime \prime}$, long $94^{\circ} 11^{\prime} 30^{\prime \prime}$, in NW $\frac{1}{4} \mathrm{SE} \frac{1}{4}$ sec.6, T.91 N., R.28 W., on right bank $50 \mathrm{ft}$ upstream from old mill dam, in city park at east edge of Lakota City, $500 \mathrm{ft}$ upstream from county highway bridge, 0.6 mile downstream from bridge on State Highway 3, 3.4 miles (revised) upstream from confluence with West Fort Des Moines River, and at mile 333.8 upstream from mouth of Des Moines River (U.S. Geological Survey river profile).

Drainage area. $-1,308 \mathrm{sq} \mathrm{mi}$.

Gage-height record.-Water-stage recorder graph except Mar. 2, 3. Datum of gage is $1,038.71 \mathrm{ft}$ above mean sea level, datum of 1929 .

Discharge record.-Stage-discharge relation defined by current-meter measurements below $17,300 \mathrm{cfs}$. Backwater from ice Mar. 1 to Apr. 6.

Maxima.-March-May 1965: Discharge, 15,700 cfs 0500 hours Apr. 9 (gage height, $23.13 \mathrm{ft}$ ).

1940 to February 1965: Discharge, $18,800 \mathrm{cfs} J$ une 21, 1954 (gage height, $16.95 \mathrm{ft}$, from floodmark, at site 8 miles upstream near Hardy at different datum).

Mean discharge, in cubic feet per second, 1965

\begin{tabular}{|c|c|c|c|c|c|c|c|c|c|c|c|}
\hline Day & March & April & May & Day & March & April & May & Day & March & April & May \\
\hline & 1,420 & 1,020 & 2,420 & $11 \ldots$ & 420 & 10,600 & 1,770 & 21. & 320 & 3,380 & 1,720 \\
\hline & 1,300 & 1,520 & 2,250 & $12 \ldots$ & 400 & 8,520 & 1,760 & 22 & 270 & 3,090 & 1,630 \\
\hline & 1,120 & 3,000 & 2,070 & $13 \ldots$ & 390 & 7,460 & 1,690 & 23. & 240 & 2,850 & 1,550 \\
\hline & 960 & 4,700 & 1,910 & $14 \ldots$ & 380 & 6,660 & 1,650 & 24. & 220 & 2,650 & 1,470 \\
\hline & 840 & 7,000 & 1,780 & $15 \ldots$ & 390 & 6,000 & 1,720 & 25. & 190 & 2,560 & 2,120 \\
\hline & 740 & 10,800 & 1,680 & $16 \ldots$ & 440 & 5,410 & 1,890 & 26 & 172 & 2,570 & 2,120 \\
\hline & 630 & 14,400 & 1,570 & $17 \ldots$ & 770 & 4,930 & 1,960 & 27. & 158 & 2,580 & 2,250 \\
\hline & 560 & 15,400 & 1,540 & $18 \ldots$ & 610 & 4,450 & 1,880 & 28. & 142 & 2,550 & 1,990 \\
\hline & 490 & 15,400 & 1,600 & $19 \ldots$ & 500 & 4,050 & 1,810 & 29. & 136 & 2,560 & 1,900 \\
\hline 10 & 450 & 13,200 & 1,710 & $20 \ldots$ & 390 & 3,700 & 1,780 & 30. & 130 & 2,540 & 1,970 \\
\hline & & & & & & & & 31 & 170 & $-\ldots$ & 1,970 \\
\hline \multirow{3}{*}{\multicolumn{9}{|c|}{$\begin{array}{l}\text { Tonthly mean discharge, in cubic feet per second } \\
\text { Runoff, in inches } \\
\text { Runoff, in acre-feet }\end{array}$}} & 495 & 5,852 & 1,822 \\
\hline & & & & & & & & & 0.44 & 4.99 & 1.61 \\
\hline & & & & & & & & & 30,440 & 348,200 & 112,000 \\
\hline
\end{tabular}


Gage height. in feet, and discharge, in cubic feet per second, at indicated time, 1965, of East Fork Des Moines River at Dakota Citl, Iowa

\begin{tabular}{c|c|c|c||r|r|r|r||r|r|r|r}
\hline Date & Hour & $\begin{array}{c}\text { Gage } \\
\text { height }\end{array}$ & $\begin{array}{c}\text { Dis- } \\
\text { charge }\end{array}$ & Date & Hour & $\begin{array}{c}\text { Gage } \\
\text { height }\end{array}$ & $\begin{array}{c}\text { Dis- } \\
\text { charge }\end{array}$ & Date & Hour & $\begin{array}{c}\text { Gage } \\
\text { height }\end{array}$ & $\begin{array}{c}\text { Dis- } \\
\text { charge }\end{array}$ \\
\hline Apr. 7 & 0000 & 21.85 & 13,600 & Apr. 9 & 1200 & 23.03 & 15,600 & Apr. 11 & 2400 & 19.08 & 9,410 \\
& 0400 & 22.08 & 13,900 & & 2400 & 22.42 & 14,500 & & & & \\
& 0800 & 21.80 & 13,500 & & & & & 12 & 0800 & 18.55 & 8,720 \\
& 1200 & 22.38 & 14,400 & 10 & 0800 & 21.88 & 13,600 & & 1600 & 18.18 & 8,230 \\
& 2000 & 22.88 & 15,300 & & 1600 & 21.35 & 12,800 & & 2400 & 17.85 & 7,820 \\
& 2400 & 22.98 & 15,500 & & 2400 & 20.81 & 11,900 & & & & \\
8 & 1200 & 22.88 & 15,300 & & & & & 13 & 0800 & 17.63 & 7,560 \\
& 2400 & 23.07 & 15,600 & & 0800 & 20,21 & 11,000 & & 1600 & 17.46 & 7,370 \\
& 0500 & 23.13 & 15,700 & & 1600 & 19.65 & 10,200 & & 2400 & 17.21 & 7,090 \\
\hline
\end{tabular}

(293) 5-4800. Lizard Creek near Clare, Iowa

Location.-Lat $42^{\circ} 32^{\prime} 40^{\prime \prime}$, long $94^{\circ} 20^{\prime} 45^{\prime \prime}$, in NE $\frac{1}{4} \mathrm{NE} \frac{1}{4}$ sec.11, T.89 N., R.30 W., on right bank $20 \mathrm{ft}$ downstream from county highway bridge, 3 miles south of Clare, 8 miles northwest of Fort Dodge, and 8.9 miles upstream from South Lizard Creek.

Drainage area. $-257 \mathrm{sq} \mathrm{mi}$.

Gage-height record.-Water-stage recorder graph except Mar. 3-7, 18, 19. Datum of gage is 1,079.30 ft above mean sea level, datum of 1929 .

Discharge record.- Stage-discharge relation defined by current-meter measurements below 5,270 cfs, extended to $10,000 \mathrm{cfs}$ by logarithmic plotting. Backwater from ice Mar. 1 to Apr. 6.

Maxima.-March-May 1965: Discharge, 4,670 cfs 1530 hours Apr. 7; gage height, $11.61 \mathrm{ft} 1300$ hours Apr. 4 (backwater from ice).

1940 to February 1965: Discharge, $10,000 \mathrm{cfs}$ J une 23, 1947 (gage height, $16.0 \mathrm{ft}$, from floodmark).

Cooperation.-One discharge measurement furnished by the Corps of Engineers.

Nean discharge, in cubtc feet per second, 1965

\begin{tabular}{|c|c|c|c|c|c|c|c|c|c|c|c|}
\hline Lay & March & April & May & Day & March & April & May & Day & March & April & May \\
\hline $1-$ & 700 & 74 & 143 & $11 \ldots$ & 38 & 985 & 123 & $21 \ldots$ & 34 & 223 & 128 \\
\hline 2 & 370 & 210 & 128 & 12 & 42 & 845 & 104 & 22 & 27 & 199 & 138 \\
\hline 3. & 130 & 480 & 106 & $13 \ldots$ & 90 & 696 & 91 & $23 \ldots$ & 24 & 173 & 151 \\
\hline- & 60 & 1,100 & 93 & $14 \ldots$ & 210 & 588 & 84 & $24 \ldots$ & 22 & 154 & 196 \\
\hline & 47 & 1,800 & 89 & $15 \ldots$ & 510 & 488 & 93 & $25 \ldots$ & 21 & 161 & 284 \\
\hline$\theta$. & 41 & 2,500 & 84 & $16 \ldots$ & 680 & 390 & 363 & $26 \ldots$ & 20 & 238 & 814 \\
\hline 7. & 37 & 3,680 & 78 & $17 .-$ & 640 & 330 & 321 & 27. & 20 & 264 & 578 \\
\hline $3_{3}$ & 36 & 2,450 & 80 & $18 .-$ & 250 & 280 & 244 & 28. & 19 & 223 & 388 \\
\hline $9_{2}$ & 36 & 1,800 & 154 & $19 \ldots$ & 120 & 241 & 182 & $29 \ldots$ & 19 & 190 & 305 \\
\hline 10 & 36 & 1,130 & 151 & $20 .-$ & 62 & 214 & 138 & $30 \ldots$ & 20 & 165 & 277 \\
\hline & & & & & & & & 31. & 29 & ------ & 253 \\
\hline \multirow{3}{*}{\multicolumn{9}{|c|}{$\begin{array}{l}\text { Monthl, mean discharge, in cublc feet per second... } \\
\text { Puncifi, in mehes } \\
\text { liunoff, wh acre-feet }\end{array}$}} & 142 & 742 & 205 \\
\hline & & & & & & & & & 0.64 & 3.22 & 0.92 \\
\hline & & & & & & & & & 8,710 & 44,170 & 12,620 \\
\hline
\end{tabular}


Gage height, in feet, and discharge, in cubic feet per second, at indicated time, 1965, of Lizard Creek near Clare Iowa

\begin{tabular}{|c|c|c|c|c|c|c|c|c|c|c|c|}
\hline Date & Hour & $\begin{array}{c}\text { Gage } \\
\text { height }\end{array}$ & $\begin{array}{c}\text { Dis- } \\
\text { charge }\end{array}$ & Date & Hour & $\begin{array}{l}\text { Gage } \\
\text { height }\end{array}$ & $\begin{array}{c}\text { Dis- } \\
\text { charge }\end{array}$ & Date & Hcur & $\begin{array}{c}\text { Gage } \\
\text { height }\end{array}$ & $\begin{array}{c}\text { Dis- } \\
\text { charge }\end{array}$ \\
\hline Apr. 3 & $\begin{array}{l}0000 \\
0800 \\
1200 \\
1400 \\
1600 \\
2400 \\
1300 \\
1400 \\
1500 \\
2400 \\
1200\end{array}$ & $\begin{array}{r}11.02 \\
10.68 \\
10.85 \\
11.10 \\
11.24 \\
11.36 \\
11.61 \\
7.28 \\
8.45 \\
9.28 \\
9.20\end{array}$ & $\begin{array}{l}-- \\
-- \\
-- \\
--\end{array}$ & Apr. 5 & $\begin{array}{l}1200 \\
1400 \\
1600 \\
2400 \\
0600\end{array}$ & $\begin{array}{l}8.39 \\
8.98 \\
8.81 \\
9.10\end{array}$ & $\begin{array}{l}2,440 \\
2,420 \\
2,860 \\
2,730 \\
2,960 \\
3,090\end{array}$ & Apr. 7 & $\begin{array}{l}1200 \\
1530 \\
2400 \\
0400 \\
1800 \\
2400 \\
0600 \\
1800 \\
2400\end{array}$ & $\begin{array}{r}10.55 \\
11.10 \\
9.23 \\
8.68 \\
8.11 \\
8.07 \\
7.91 \\
7.06 \\
6.62\end{array}$ & $\begin{array}{l}4,180 \\
4,670 \\
3,060 \\
2,630 \\
2,230 \\
2,200 \\
2,090 \\
1,550 \\
1,280\end{array}$ \\
\hline
\end{tabular}

(294) 5-4805. Des Moines River at Fort Dodge, Iowa

Location.-Lat $42^{\circ} 30^{\prime} 25^{\prime \prime}$, long $94^{\circ} 12^{\prime} 00^{\prime \prime}$, in NW $\frac{1}{4} \mathrm{SW} \frac{1}{4}$ sec.19, T.89 N., F .28 W., on right bank $400 \mathrm{ft}$ upstream from Soldier Creek, 1,800 ft downstream from Illinois Central Railroad bridge in Fort Dodge, 2,000 ft downstream from Lizard Creek, and at mile 314.6 .

Drainage area.-4,190 sq $\mathrm{mi}$.

Gage-height record.-Water-stage recorder graph. Datum of gage is $869.38 \mathrm{ft}$ above mean sea level, datum of 1929.

Discharge record.-Stage-discharge relation defined by current-meter measurements below $35,600 \mathrm{cfs}$. Backwater from ice Mar. 1 to Apr. 5.

Maxima.-March-May 1965: Discharge, 35,600 cfs 0630 hours Apr. 8 (gage height, $17.79 \mathrm{ft})$.

1905-06, 1913-27, 1946 to February 1965: Discharge, 35,400 cfs June 21,1954; gage height, $19.62 \mathrm{ft}$, from floodmark, June 23, 1947.

Cooperation.-One discharge measurement furnished by the Corps of Engineers.

Mean discharge, in cubic feet per second, 1965

\begin{tabular}{|c|c|c|c|c|c|c|c|c|c|c|c|}
\hline Day & March & April & May & Day & March & April & May & Day & March & April & May \\
\hline & 3,420 & 3,300 & 6,770 & $11 \ldots$ & 860 & 26,200 & 5,380 & 21. & 890 & 11,300 & 4,980 \\
\hline & 2,780 & 7,000 & 6,260 & $12 \ldots$ & 850 & 22,800 & 5,350 & 22. & 740 & 10,400 & 4,870 \\
\hline & 2,220 & 9,600 & 5,750 & $13 \ldots$ & 900 & 21,900 & 5,020 & 23. & 500 & 9,620 & 4,800 \\
\hline & 1,920 & 12,000 & 5,290 & $14 \ldots$ & 1,160 & 20,900 & 4,800 & 24 & 505 & 8,860 & 4,960 \\
\hline & 1,700 & 17,000 & 5,020 & $15 \ldots$ & 1,900 & 19,000 & 4,810 & 25. & 500 & 8,560 & 5,920 \\
\hline & 1,500 & 23,500 & 4,890 & $16 \ldots$ & 2,400 & 17,200 & 5,320 & 26 & 500 & 8,470 & 9,920 \\
\hline & 1,360 & 30,600 & 4,650 & $17 \ldots$ & 2,220 & 15,500 & 5,830 & 27 & 470 & 8,220 & 8,420 \\
\hline & 1,210 & 35,100 & 4,560 & $18 \ldots$ & 1,820 & 14,200 & 5,840 & 28 & 460 & 7,760 & 7,340 \\
\hline & 1,080 & 34,000 & 4,560 & $19 \ldots$ & 1,340 & 13,100 & 5,600 & 29 & 455 & 7,460 & 6,740 \\
\hline & 920 & 30,900 & 5,000 & $20 \ldots$ & 1,090 & 12,200 & 5,310 & 30 & 510 & 7,180 & 6,470 \\
\hline & & & & & & & & & 1,300 & & 6,130 \\
\hline \multicolumn{9}{|c|}{ 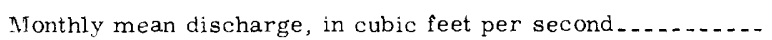 } & 1,274 & 15,790 & 5,695 \\
\hline \multicolumn{9}{|c|}{ Runoff, in inches } & 0.35 & 4.21 & 1.57 \\
\hline \multicolumn{9}{|c|}{ Runoif, in acre-feet } & 78,310 & 939,800 & 350,200 \\
\hline
\end{tabular}


Gage height, in feet, and discharge, in cubic feet per second, at indicated time, 1965, of Des Moines River at Fort Dodge, Iowa

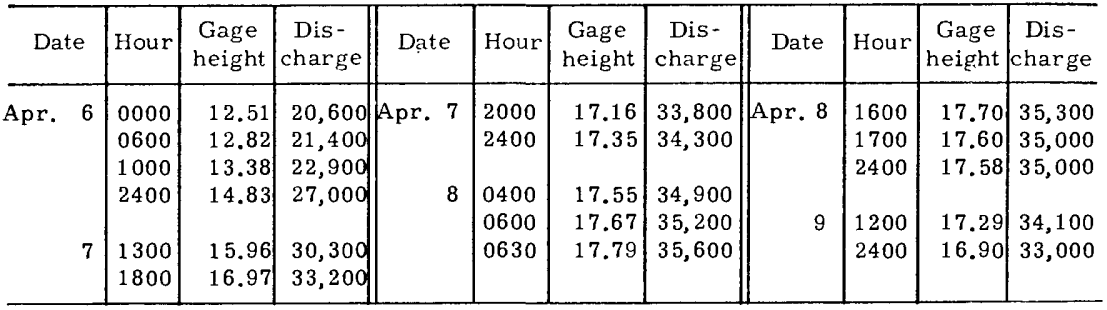

(295) 5-4810. Boone River near Webster City, Iowa

Location.-Lat $42^{\circ} 26^{\prime} 00^{\prime \prime}$, long $93^{\circ} 48^{\prime} 15^{\prime \prime}$, in NW $\frac{1}{4} \mathrm{SE} \frac{1}{4}$ sec.18, T.88 N., R.25 W., on right bank $10 \mathrm{ft}$ upstream from bridge on State Highway 60,2 miles south of Webster City, and 4.5 miles downstream from White Fox Creek.

Drainage area. $-844 \mathrm{sq} \mathrm{mi}$.

Gage-height record.-Water-stage recorder graph except Mar. 1-1 5, 19-25. Graph was reconstructed from daily wire-weight gage readings Mar. 3, 15. Datum of gage is $989.57 \mathrm{ft}$ above mean sea level, datum of 1929 .

Discharge record.- Stage-discharge relation defined by current-meter meas'drements below $19,600 \mathrm{cfs}$, extended to $21,500 \mathrm{cfs}$ by logarithmic plotting. Backwater from ice Mar. 1, 18-25.

Maxima.-March-May 1965: Discharge 15,200 cfs 2200 hours Apr. 6 (gage həight, $15.91 \mathrm{ft})$.

1896 to February 1965: Discharge, 21,500 cfs J une 10, 1918 (gage height, $19.1 \mathrm{ft}$ ).

Mean discharge, in cubic feet per second, 1965

\begin{tabular}{|c|c|c|c|c|c|c|c|c|c|c|c|}
\hline Day & March & April & May & Day & March & April & May & Day & March & April & May \\
\hline & 4,500 & 2,630 & 780 & $11 \ldots$ & 250 & 7,190 & 510 & 21 & 164 & $93 ?$ & 478 \\
\hline 2. & 3,100 & 3,110 & 680 & $12 \ldots$ & 220 & 5,780 & 438 & 22. & 140 & 803 & 510 \\
\hline 3. & 2,320 & 3,230 & 596 & $13 \ldots$ & 200 & 4,630 & 383 & 23. & 120 & 725 & 568 \\
\hline & 1,600 & 4,940 & 528 & $14 \ldots$ & 200 & 3,760 & 362 & 24. & 100 & 655 & 609 \\
\hline & 1,160 & 8,220 & 478 & 15 & 209 & 3,050 & 362 & 25 & 90 & $75 ?$ & 956 \\
\hline & 840 & 13,800 & 609 & $16 \ldots$ & 204 & 2,520 & 644 & 26. & 80 & 1,463 & 3,920 \\
\hline & 650 & 14,400 & 1,050 & $17 \ldots$ & 190 & 2,100 & 1,060 & 27. & 74 & 1,660 & 3,930 \\
\hline 8. & 520 & 13,600 & 965 & $18 \ldots$ & 400 & 1,770 & 890 & 28. & 74 & 1,403 & 3,120 \\
\hline & 400 & 11,400 & 768 & $19 \ldots$ & 270 & 1,410 & 690 & 29. & 74 & 1,123 & 2,340 \\
\hline 10 & 306 & 10,100 & 609 & $20 \ldots$ & 200 & 1,160 & 551 & 30. & 91 & 905 & 2,000 \\
\hline & & & & & & & & & 669 & & 1,680 \\
\hline \multicolumn{9}{|c|}{ Monthly mean discharge, in cubic feet per second } & 626 & 4,307 & 1,067 \\
\hline \multicolumn{9}{|c|}{ Runoff, in inches } & 0.86 & 5.69 & 1.46 \\
\hline \multicolumn{9}{|c|}{ Runoff, in acre-feet } & 38,510 & 256,300 & 65,580 \\
\hline
\end{tabular}


Gage height, in feet, and discharge, in cubic feet per second, at indicated time, 1965, of Boone Riler near Webster City, Iowa

\begin{tabular}{|c|c|c|c|c|c|c|c|c|c|c|c|}
\hline Date & Hour & $\begin{array}{c}\text { Gage } \\
\text { height }\end{array}$ & $\begin{array}{c}\text { Dis- } \\
\text { charge }\end{array}$ & Date & Hour & $\begin{array}{l}\text { Gage } \\
\text { height }\end{array}$ & $\begin{array}{c}\text { Dis- } \\
\text { charge }\end{array}$ & Date & Hour & $\begin{array}{c}\text { Gage } \\
\text { height }\end{array}$ & $\begin{array}{c}\text { Dis- } \\
\text { charge }\end{array}$ \\
\hline \multirow[t]{5}{*}{ Mar. 30} & 000 & 1.93 & 75.9 & \multirow[t]{5}{*}{ Apr. 1} & 1300 & 6.20 & 2,540 & \multirow[t]{4}{*}{ Apr. 5} & $040 \mathrm{C}$ & 10.37 & 6,580 \\
\hline & 1400 & 1.93 & 75.9 & & 1700 & 6.87 & 3,130 & & $160 \mathrm{C}$ & 12.02 & 8,830 \\
\hline & 1800 & 2.10 & 114 & & 2200 & 7.36 & 3,540 & & 2400 & 13.37 & 10,900 \\
\hline & 2200 & 2.14 & 124 & & 2400 & 7.39 & 3,570 & & & & \\
\hline & 2400 & 2.24 & 148 & & & & & \multirow[t]{5}{*}{6} & 0600 & 14.64 & 13,000 \\
\hline \multirow[t]{10}{*}{31} & 1100 & 2.33 & 170 & \multirow[t]{2}{*}{2} & 0700 & 6.86 & 3,120 & & $120 \mathrm{C}$ & 15.35 & 14,200 \\
\hline & 1200 & 2.47 & 206 & & 2400 & 6.61 & 2,900 & & $180 \mathrm{C}$ & 15.83 & 15,100 \\
\hline & 1300 & 2.71 & 278 & \multirow[t]{4}{*}{3} & 0800 & 6.49 & 2,790 & & 2200 & 15.91 & 15,200 \\
\hline & 1400 & 3.06 & 398 & & 1200 & 6.76 & 3,030 & & 2400 & 15.89 & 15,200 \\
\hline & 1500 & 3.43 & 550 & & 2400 & 8.07 & 4,150 & \multirow{5}{*}{7} & & & \\
\hline & 1600 & 3.84 & 755 & & 0 & & & & 1000 & 15.20 & 14,000 \\
\hline & 1700 & 4.45 & 1,160 & 4 & 0600 & 8.07 & & & 1200 & 15.15 & 13,900 \\
\hline & 1800 & 4.82 & 1,430 & & 1000 & 8.22 & 4,280 & & 2400 & 15.60 & 14,700 \\
\hline & & 5.32 & 1,820 & & 1200 & 8.40 & 4,440 & & & & \\
\hline & & 5.34 & 1,830 & & 2000 & 10.12 & 6,260 & 8 & 1200 & 14.95 & 13,500 \\
\hline Apr. 1 & 1000 & 5.94 & 2,330 & & & 1 & 6,320 & & 2400 & 14.37 & 12,500 \\
\hline
\end{tabular}

(296) 5-4815. Des Moines River near Boone, Iowa

Location.-Lat $42^{\circ} 04^{\prime} 40^{\prime \prime}$, long $93^{\circ} 55^{\prime} 55^{\prime \prime}$, in NE $\frac{1}{4} \mathrm{NE} \frac{1}{4}$ sec. 24, T. 84 N., R. 27 W., on left bank $30 \mathrm{ft}$ upstream from Boone Water Department dam, 2 miles nort'west of Boone, 2.2 miles upstream from Bluff Creek, and at mile 258.8.

Drainage area.- $-5,511 \mathrm{sq} \mathrm{mi}$.

Gage-height record.-Water-stage recorder graph except Mar. 2-4, 9, 10, 19, Apr. 7-12, for which graph was reconstructed from daily (during March) and hourly (during April) outside staff gage readings. Datum of gage is $872.16 \mathrm{ft}$ (revised) above mean sea level, datum of 1929 (levels by Corps of Engineers).

Discharge record.-Stage-discharge relation defined by current-meter measurements below 57,400 cfs. Backwater from ice Mar. 1 to Apr. 5.

Maxima.-March-May 1965: Discharge, 51,600 cfs 0400 hours Apr. 9 (gage height, $22.89 \mathrm{ft}$ ).

1920 to February 1965: Discharge, 57,400 cfs June 22, 1954 (gage height, $25.35 \mathrm{ft}$, from graph based on hourly gage readings).

Mean discharge, in cubic feet per second, 1965

\begin{tabular}{|c|c|c|c|c|c|c|c|c|c|c|c|}
\hline Day & March & April & May & Day & March & April & May & Day & March & April & May \\
\hline $\begin{array}{l}2 . \\
3 . \\
4 . \\
5 .\end{array}$ & $\begin{array}{l}1,600 \\
6,000 \\
7,500 \\
6,000 \\
4,300 \\
3,300 \\
2,700 \\
2,300 \\
1,940 \\
1,720\end{array}$ & $\begin{array}{r}4,110 \\
11,000 \\
13,000 \\
19,000 \\
21,000 \\
34,900 \\
42,400 \\
49,300 \\
50,800 \\
47,900\end{array}$ & $\begin{array}{l}7,950 \\
7,430 \\
6,870 \\
6,320 \\
5,820 \\
5,640 \\
5,700 \\
5,920 \\
5,620 \\
5,470\end{array}$ & $\begin{array}{l}11 \ldots \\
12 \ldots \\
13 \ldots \\
14 \ldots \\
15 \ldots \\
16 \ldots \\
17- \\
18 \ldots \\
19- \\
20 \ldots\end{array}$ & $\begin{array}{l}1,580 \\
1,420 \\
1,320 \\
1,280 \\
1,760 \\
2,600 \\
3,000 \\
2,800 \\
2,480 \\
2,100\end{array}$ & $\begin{array}{l}43,400 \\
36,800 \\
31,500 \\
28,500 \\
26,200 \\
23,000 \\
20,300 \\
18,000 \\
16,000 \\
14,400\end{array}$ & $\begin{array}{l}5,720 \\
5,890 \\
5,720 \\
5,380 \\
5,210 \\
5,330 \\
6,200 \\
6,730 \\
6,510 \\
6,130\end{array}$ & $\mid \begin{array}{l}21 \\
22 \\
23 \\
23_{-} \\
24_{-} \\
25_{-} \\
26- \\
27_{-} \\
28- \\
29- \\
30_{-} \\
31_{-}\end{array}$ & $\begin{array}{r}1,640 \\
1,300 \\
1,100 \\
960 \\
840 \\
740 \\
660 \\
620 \\
660 \\
760 \\
1,600\end{array}$ & $\begin{array}{r}12,800 \\
11,600 \\
10,500 \\
9,810 \\
9,420 \\
9,600 \\
9,860 \\
9,540 \\
8,890 \\
8,400 \\
\end{array}$ & $\begin{array}{r}5,720 \\
5,620 \\
5,680 \\
5,620 \\
5,820 \\
9,620 \\
15,000 \\
13,100 \\
10,500 \\
9,270 \\
8,560\end{array}$ \\
\hline \multicolumn{9}{|c|}{$\begin{array}{l}\text { Monthly mean discharge, in cubic feet per second } \\
\text { Runinf, in inches } \\
\text { Runoff, in acre-feet }\end{array}$} & $\begin{array}{r}2,212 \\
0,46 \\
136,000\end{array}$ & $\begin{array}{r}21,730 \\
4.40 \\
1,293,000\end{array}$ & $\begin{array}{r}6,970 \\
1.46 \\
428,600\end{array}$ \\
\hline
\end{tabular}


Gage height, in feet, and discharge. in cubic feet per second, at indicated time, 1965. of Des Moines River near Boone, lowa

\begin{tabular}{|c|c|c|c|c|c|c|c|c|c|c|c|}
\hline Date & Hour & $\begin{array}{c}\text { Gage } \\
\text { height }\end{array}$ & $\begin{array}{c}\text { Dis- } \\
\text { charge }\end{array}$ & Date & Hour & $\begin{array}{c}\text { Gage } \\
\text { height }\end{array}$ & $\begin{array}{c}\text { Dis- } \\
\text { charge }\end{array}$ & Date & Hour & $\begin{array}{l}\text { Gage } \\
\text { height }\end{array}$ & $\begin{array}{l}\text { Dis- } \\
\text { charge }\end{array}$ \\
\hline \multirow[t]{6}{*}{ Apr. 6} & 0000 & 15.82 & 28,600 & \multirow[t]{9}{*}{ Apr. 8} & 0800 & 22.13 & 48,700 & \multirow{5}{*}{$\begin{array}{r}\text { Apr. } 9 \\
10\end{array}$} & 2400 & 22.37 & 49,600 \\
\hline & 0600 & 16.89 & 31,600 & & 1600 & 22.62 & 50,600 & & & \multirow{2}{*}{22.07} & \multirow{4}{*}{$\begin{array}{l}48,500 \\
47,600 \\
46,200\end{array}$} \\
\hline & 1200 & 18.14 & 35,200 & & 2000 & 22.79 & 51,200 & & \multirow{3}{*}{$\begin{array}{l}0800 \\
1400 \\
2400\end{array}$} & & \\
\hline & 1800 & 19.14 & 38,500 & & \multirow[t]{2}{*}{2400} & \multirow[t]{2}{*}{22.87} & \multirow[t]{2}{*}{51,500} & & & 45 & \\
\hline & 2400 & 19.57 & 39,900 & & & & & & & & \\
\hline & & & & & 0400 & 22.89 & 51,600 & \multirow[t]{4}{*}{11} & 0800 & 20.97 & 44,400 \\
\hline 7 & 1000 & 19.95 & 41,100 & & 0800 & 22.84 & 51,400 & & 1600 & 20.42 & 42,600 \\
\hline & 1800 & 20.89 & 44,200 & & 1600 & 22.66 & 50,700 & & 2400 & 19.67 & 40,200 \\
\hline & 2400 & 21.45 & 46,200 & & & & & & & & \\
\hline
\end{tabular}

(297) 5-4816.50 Des Moines River near Saylorville, Iowa

Location.-Lat $41^{\circ} 41^{\prime} 50^{\prime \prime}$, long $93^{\circ} 40^{\prime} 05^{\prime \prime}$, near center of sec.5,T.79 N., R.24 W., near center of span on downstream side of highway bridge, 2.0 miles west of Say! orville, 2.0 miles downstream from Rock Creek, 2.2 miles upstream from Beaver Creek, and at mile 211.4 .

Drainage area. $-5,841 \mathrm{sq} \mathrm{mi}$.

Gage-height record.-Wire-weight gage read once daily. Datum of gage is $787.54 \mathrm{ft}$ above mean sea level, datum of 1929.

Discharge record.-Stage-discharge relation defined by current-meter measurements below 44,600 cfs. Backwater from ice Mar. 1 to Apr. 3.

Maxima.-March-May 1965: Discharge, 47,400 cfs 1700 hours Apr. 10 (gage height, $24.02 \mathrm{ft}$ ).

1893 to February 1965: Discharge, 60,000 cfs J une 24, 1954 (gage height, $24.5 \mathrm{ft}$ ).

Cooperation.-Three discharge measurements furnished by Corps of Engineers.

Mean discharge, in cubie feet per second, 1965

\begin{tabular}{|c|c|c|c|c|c|c|c|c|c|c|c|}
\hline Day & March & April & May & Day & March & April & May & Day & March & April & May \\
\hline 6. & $\begin{array}{l}2,500 \\
5,150 \\
6,700 \\
7,400 \\
5,450 \\
4,200 \\
3,520 \\
3,070 \\
2,590 \\
2,200\end{array}$ & $\begin{array}{r}3,310 \\
5,200 \\
10,000 \\
15,200 \\
19,400 \\
28,600 \\
35,600 \\
45,300 \\
45,600 \\
47,100\end{array}$ & $\begin{array}{r}10,000 \\
9,330 \\
8,560 \\
7,790 \\
7,130 \\
6,520 \\
6,410 \\
6,640 \\
6,590 \\
6,110\end{array}$ & 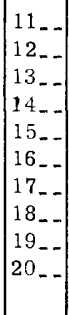 & $\begin{array}{l}1,920 \\
1,830 \\
1,890 \\
2,200 \\
2,740 \\
3,800 \\
5,200 \\
4,580 \\
3,600 \\
2,700\end{array}$ & $\begin{array}{l}46,100 \\
42,200 \\
36,900 \\
32,600 \\
30,000 \\
28,200 \\
25,600 \\
22,900 \\
21,000 \\
19,100\end{array}$ & $\begin{array}{l}5,990 \\
6,250 \\
6,370 \\
6,060 \\
5,750 \\
5,580 \\
5,820 \\
6,820 \\
7,200 \\
6,880\end{array}$ & $\mid \begin{array}{l}21 \\
22 \\
22 \\
23- \\
24_{-} \\
25_{-} \\
26_{-} \\
27- \\
28_{-} \\
29_{-} \\
30_{-} \\
31_{-}\end{array}$ & $\begin{array}{r}2,100 \\
1,500 \\
1,200 \\
1,000 \\
900 \\
800 \\
740 \\
740 \\
900 \\
1,680 \\
2,470\end{array}$ & $\begin{array}{r}17,300 \\
15,500 \\
14,400 \\
14,000 \\
13,000 \\
12,600 \\
12,100 \\
12,200 \\
11,700 \\
10,700 \\
\end{array}$ & $\begin{array}{r}7,920 \\
6,030 \\
6,160 \\
6,040 \\
6,030 \\
7,580 \\
11,000 \\
15,700 \\
17,700 \\
12,900 \\
10,800\end{array}$ \\
\hline \multicolumn{9}{|c|}{$\begin{array}{l}\text { Monthly mean discharge, in cubic feet per second. } \\
\text { Runf, in inches } \\
\text { Rumoff, m acre-feet }\end{array}$} & $\begin{array}{r}2,815 \\
0.56 \\
173,100\end{array}$ & $\begin{array}{r}23,110 \\
4.41 \\
1,375,000\end{array}$ & $\begin{array}{r}7,925 \\
1.56 \\
487,300\end{array}$ \\
\hline
\end{tabular}


Gage height, in feet, and discharge, in cubic feet per second, at indicated time, 1965, of Des Moines River near Savlorville, Iowa

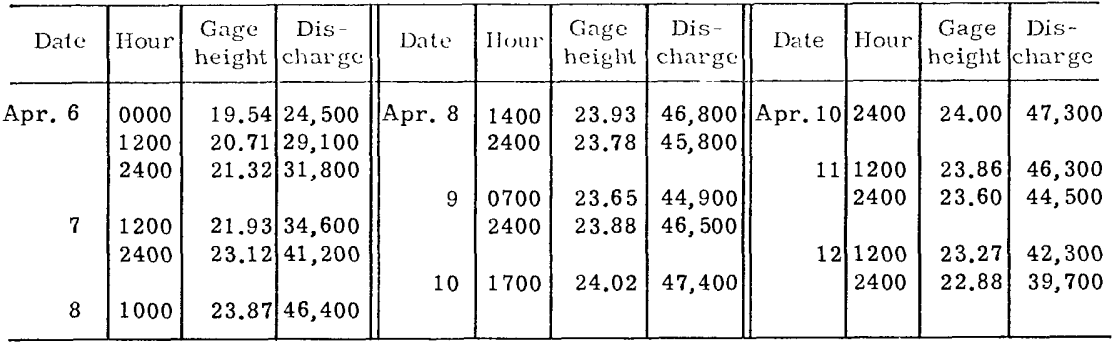

(298) 5-4821.7 Big Cedar Creek near Varina, Iowa

Location.-Lat $42^{\circ} 41^{\prime} 21^{\prime \prime}$, long $94^{\circ} 47^{\prime} 55^{\prime \prime}$, in NE $\frac{1}{4} \mathrm{NE} \frac{1}{4}$ sec.24, T.91 N., R.34 W., on left bank $5 \mathrm{ft}$ downstream from county highway bridge, 3.1 miles upstream from Drainage Ditch 74 , and 3.3 miles northeast of Varina.

Drainage area. $-80.0 \mathrm{sq} \mathrm{mi}$.

Gage-height record.-Water-stage recorder graph except Mar. 1-12, 18-29. Datum of gage is $1,225.12 \mathrm{ft}$ above mean sea level, datum of 1929 .

Discharge record.- - Stage-discharge relation defined by current-meter measurements below 2,070 cfs. Backwater from ice Mar. 1 to Apr. 8 .

Maxima.-March-May 1965: Discharge, 1,060 cfs 1200 hours Apr. 8 (gage height, $10.17 \mathrm{ft}$ ); gage height $15.05 \mathrm{ft} 2200$ hours Apr. 6 (backwater from ice).

1959 to February 1965: Discharge, 2,080 cfs Aug. 31, 1962 (gage height, $13.68 \mathrm{ft}$ ); gage height, $14.49 \mathrm{ft}$ Mar. 29, 1962 (ice jam).

Mean discharge, in cubic feet per second, 1965

\begin{tabular}{|c|c|c|c|c|c|c|c|c|c|c|c|}
\hline Day & March & April & May & Day & March & April & May & Day & March & April & May \\
\hline 1 & 56 & 47 & 42 & $11 \ldots$ & 34 & 389 & 59 & 21 & 37 & 72 & 63 \\
\hline 2 & 62 & 110 & 38 & $12 \ldots$ & 38 & 337 & 48 & 22 & 31 & 59 & 101 \\
\hline 3. & 50 & 250 & 32 & $13=$ & 60 & 291 & 41 & $23-$ & 27 & 50 & 196 \\
\hline 4 & 38 & 490 & 28 & $14 \ldots$ & 130 & 255 & 37 & $24 \ldots$ & 25 & 43 & 151 \\
\hline $5 \ldots$ & 34 & 730 & 28 & $15 \ldots$ & 150 & 213 & 119 & $25-$ & 22 & 61 & 178 \\
\hline $6 \ldots$ & 32 & 910 & 26 & $16 \ldots$ & 160 & 170 & 234 & 26 - & 21 & 108 & 224 \\
\hline 7. & 30 & 860 & 24 & $17 \ldots$ & 110 & 143 & 153 & $27-$ & 20 & 86 & 179 \\
\hline 8. & 30 & 940 & 178 & $18 \ldots$ & 84 & 118 & 105 & 28 & 20 & 70 & 121 \\
\hline $9_{-}$ & 30 & 606 & 130 & $19 \ldots$ & 66 & 99 & 78 & $29-$ & 20 & 58 & 103 \\
\hline 10. & 32 & 432 & 83 & $20 \ldots$ & 50 & 86 & 64 & $30 \ldots$ & 22 & 49 & 102 \\
\hline & & & & & & & & $31-1$ & 26 & $---\cdots$ & 91 \\
\hline \multirow{3}{*}{\multicolumn{9}{|c|}{$\begin{array}{l}\text { Monthly mean discharge, in cubic feet per second. } \\
\text { Runoff, in inches } \\
\text { Rumoff, in acre-feet }\end{array}$}} & 49.9 & 271 & 98.6 \\
\hline & & & & & & & & & 0.72 & 3.78 & 1.42 \\
\hline & & & & & & & & & 3,070 & 16,130 & 6,060 \\
\hline
\end{tabular}


Gage height, in feet, and discharge, in cubic feet per second, at indicated time, 1965, of Big Cedar Creek near Varina. Iowa

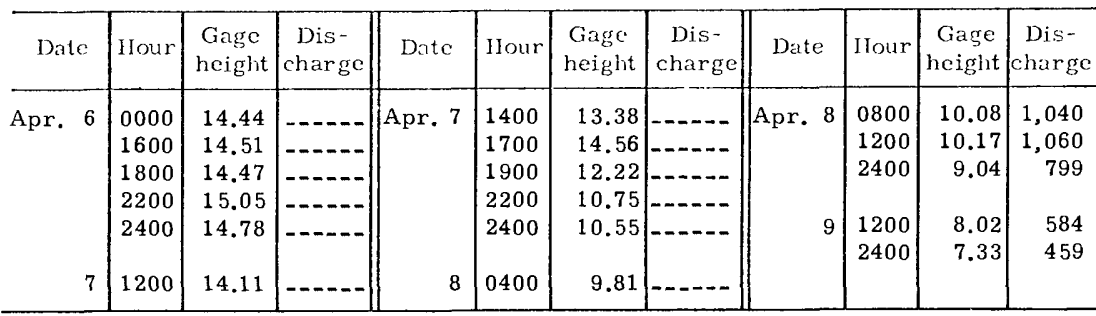

(299) 5-4823. North Raccoon River near Sac City, Iowa

Location.-Lat $42^{\circ} 20^{\prime} 20^{\prime \prime}$, long $94^{\circ} 59^{\prime} 10^{\prime \prime}$, in NE $\frac{1}{4} \mathrm{NW} \frac{1}{4}$ sec.24, T.87 N., R.36 W., on right bank $15 \mathrm{ft}$ downstream from county highway bridge, 0.2 mile upstream from Indian Creek, and 4.5 miles south of Sac City.

Drainage area. $-713 \mathrm{sq} \mathrm{mi}$.

Gage-height record.-Water-stage recorder graph except Mar. 2, 4-10, 13-30. Graph was reconstructed from daily wire-weight gage readings Mar. 5, 18, 19, 22, 24, 25, 27,30 . Datum of gage is $1,144.60 \mathrm{ft}$ above mean sea level, datum of 1929 (lovels by Iowa Natural Resources Council).

Discharge record.- Stage-discharge relation defined by current-meter measurements below 10,600 cfs. Backwater from ice Mar. 1 to Apr. 5.

Maxima.-March-May 1965: Discharge, 6,960 cfs 2400 hours Apr. 6 (gage height, $15.59 \mathrm{ft})$.

1958 to February 1965: Discharge, 10,800 cfs Sept. 1, 1962 (gage height, $18.12 \mathrm{ft}$ ).

Flood of $J$ une 21, 1954, reached a stage of $15.61 \mathrm{ft}$, from floodmark (discharge, $7,000 \mathrm{cfs}$ ).

Mean $d_{l}$ scharge, in cublc feet per second, 1965

\begin{tabular}{|c|c|c|c|c|c|c|c|c|c|c|c|}
\hline Day & March & April & May & Day & March & April & May & Day & March & April & May \\
\hline & 1,000 & 1,200 & 279 & $11 \ldots$ & 120 & 3,130 & 384 & $21 \ldots$ & 268 & 461 & 363 \\
\hline 2 & 600 & 3,100 & 244 & $12 \ldots$ & 144 & 2,460 & 313 & 22 & 226 & 413 & 386 \\
\hline 3. & 390 & 2,900 & 220 & $13 \ldots$ & 190 & 1,870 & 270 & $23 \ldots$ & 192 & 370 & 524 \\
\hline & 200 & 3,400 & 199 & $14 \ldots$ & 280 & 1,500 & 241 & $24_{\ldots}$ & 168 & 325 & 735 \\
\hline 5. & 150 & 4,800 & 183 & |15 & 370 & 1,260 & 362 & $25_{\ldots}$ & 138 & 325 & 857 \\
\hline $6 \ldots$ & 132 & 6,630 & 177 & $16 \ldots$ & 490 & 1,020 & 1,030 & $26 \ldots$ & 130 & 418 & 1,470 \\
\hline 7 & 120 & 6,190 & 165 & $17 \ldots$ & 620 & 860 & 917 & 27 . & 130 & 510 & 1,370 \\
\hline $8_{-}$ & 112 & 5,610 & 460 & $18 \ldots$ & 880 & 710 & 665 & $28 \ldots$ & 138 & 425 & 958 \\
\hline $9 \ldots$ & 108 & 4,670 & 766 & $19 \ldots$ & 580 & 597 & 483 & $29_{2}$ & 152 & 363 & 717 \\
\hline 10 & 106 & 3,730 & 524 & $20 \ldots$ & 320 & 518 & 391 & $30 \ldots$ & 252 & 319 & 644 \\
\hline & & & & & & & & 31 & 540 & $\ldots$ & 583 \\
\hline \multirow{3}{*}{\multicolumn{9}{|c|}{$\begin{array}{l}\text { Monthly mean discharge, in cubic feet per second } \\
\text { Runoff, in inches } \\
\text { Runoff, in aere-feet }\end{array}$}} & 298 & 2,003 & 545 \\
\hline & & & & & & & & & 0.48 & 3.13 & 0.88 \\
\hline & & & & & & & & & 18,340 & 119,200 & 33,480 \\
\hline
\end{tabular}


Gage height, in feet, and discharge, in cubic feet per second, at indicated time, 1965, of North Raccoon River near Sac City. Iowa

\begin{tabular}{|c|c|c|c|c|c|c|c|c|c|c|c|}
\hline Date & Hour & $\begin{array}{l}\text { Gage } \\
\text { height }\end{array}$ & $\begin{array}{c}\text { Dis- } \\
\text { charge }\end{array}$ & Date & llour & $\begin{array}{l}\text { Gage } \\
\text { height }\end{array}$ & $\begin{array}{c}\text { Dis- } \\
\text { charge }\end{array}$ & Date & Jour & $\begin{array}{l}\text { Gage } \\
\text { height }\end{array}$ & $\begin{array}{l}\text { Dis- } \\
\text { charge }\end{array}$ \\
\hline Apr. 5 & 0000 & 14.37 & $\ldots$ & Apr. 6 & 1800 & 15.47 & 6,790 & Apr. 8 & 1200 & 14.64 & 5,660 \\
\hline & 0200 & 13.90 & ----- & & 2400 & 15.59 & 6,960 & & 1600 & 14.46 & 5,440 \\
\hline & 0400 & 13.89 & ----- & & & & & & 2400 & 14.31 & 5,260 \\
\hline & 1200 & 14.13 & $-\ldots$ & 7 & 1600 & 14.71 & 5,740 & & & & \\
\hline & 1800 & 14.69 & ----- & & 2200 & 14.87 & 5,950 & 9 & 2200 & 13.19 & 4,180 \\
\hline & 2400 & 14.86 & 5,940 & & 2400 & 14.86 & 5,940 & & 2400 & 13.11 & 4,100 \\
\hline 6 & 1000 & 15.47 & 6,790 & & & & & & & & \\
\hline
\end{tabular}

\section{(300) 5-4825. North Raccoon River near Jefferson, Iowa}

Location.-Lat $41^{\circ} 59^{\prime} 20^{\prime \prime}$, long $94^{\circ} 22^{\prime} 30^{\prime \prime}$, in SW $\frac{1}{4} \mathrm{NW} \frac{1}{4}$ sec.20, T.83 N., R.30 W., on right bank $50 \mathrm{ft}$ downstream from bridge on State Highway 17, 2 miles soutr of Jefferson, and 4.2 miles upstream from Hardin Creek.

Drainage area. $-1,619 \mathrm{sq} \mathrm{mi}$.

Gage-height record.-Water-stage recorder graph except Mar. 1 to Apr. 1, for which graph was reconstructed on basis of daily wire-weight gage readings. Datum of gage is $967.09 \mathrm{ft}$ above mean sea level, datum of 1929 .

Discharge record.- Stage-discharge relation defined by current-meter measurements below 28,400 cfs. Backwater from ice Mar. 1 to Apr. 5.

Maxima.-March-May 1965: Discharge, 9,890 cfs 2330 hours Apr. 5 (gø ge height, $15.29 \mathrm{ft}$ ); gage height, $15.59 \mathrm{ft} 2200$ hours Apr. 4 (backwater from ice).

1940 to February 1965: Discharge, 29,100 cfs June 23, 1947 (gage $\mathrm{k}$ eight, $22.3 \mathrm{ft}$ ).

Mean discharge, in cubic feet per second, 1965

\begin{tabular}{|c|c|c|c|c|c|c|c|c|c|c|c|}
\hline Day & March & April & May & Day & March & April & May & Day & March & April & May \\
\hline & 800 & 3,700 & 826 & $11 \ldots$ & 260 & 7,300 & 922 & $21_{-}$ & 650 & 1,050 & 761 \\
\hline 2. & 3,500 & 3,100 & 728 & $12 \ldots$ & 310 & 5,850 & 741 & $22_{-}$ & 540 & 935 & 746 \\
\hline 3. & 1,600 & 2,800 & 646 & $13 \ldots$ & 460 & 4,460 & 618 & 23. & 460 & 836 & 1,300 \\
\hline & 720 & 5,200 & 588 & $14 \ldots$ & 700 & 3,290 & 550 & $24 \ldots$ & 370 & 839 & 1,440 \\
\hline 5. & 360 & 7,400 & 530 & $15 \ldots$ & 1,100 & 2,590 & 581 & $25_{-}$ & 310 & 1,010 & 1,590 \\
\hline . & 305 & 9,030 & 519 & $16 \ldots$ & 1,800 & 2,130 & 822 & $26 \ldots$ & 305 & 1,220 & 3,890 \\
\hline . & 280 & 8,380 & 511 & $17_{-}$ & 3,000 & 1,790 & 1,310 & 27. & 305 & 1,230 & 8,400 \\
\hline 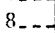 & 270 & 9,240 & 511 & $18 \ldots$ & 2,300 & 1,560 & 1,400 & 28. & 310 & 1,240 & 7,300 \\
\hline & 258 & 9,320 & 550 & 19. & 900 & 1,340 & 1,130 & 29. & 340 & 1,100 & 4,110 \\
\hline 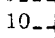 & 250 & 8,520 & 1,010 & $20 \ldots$ & 760 & 1,200 & 897 & $30 \ldots$ & 580 & 945 & 2,800 \\
\hline & & & & & & & & 31 & 1,300 & - & 2,270 \\
\hline \multicolumn{9}{|c|}{ Monthly mean discharge, in cubic feet per second } & 819 & 3,620 & 1,613 \\
\hline \multicolumn{9}{|c|}{ Runoff, in inches } & 0.58 & 2.49 & 1.15 \\
\hline \multicolumn{9}{|c|}{ liunoff, in acre-feet } & 50,390 & 215,400 & 99,170 \\
\hline
\end{tabular}


Gage height, in feet, and discharge, in cubic feet per second, at indicated time 1965, of North Racioon River near Jefferson, lowa

\begin{tabular}{|c|c|c|c|c|c|c|c|c|c|c|c|}
\hline Date & Ilonx & $\begin{array}{c}\text { Gage } \\
\text { hoight }\end{array}$ & $\begin{array}{c}\text { Dis - } \\
\text { charge }\end{array}$ & Date & Inour & $\begin{array}{c}\text { Gavge } \\
\text { height }\end{array}$ & $\begin{array}{c}\text { Dis- } \\
\text { charge }\end{array}$ & Date & Hour & $\begin{array}{l}\text { Gage } \\
\text { height }\end{array}$ & $\begin{array}{l}\text { Dis- } \\
\text { charge }\end{array}$ \\
\hline \multirow[t]{6}{*}{$\overline{\mathrm{Apr} .4}$} & 0000 & 13.45 & $-\ldots$ & \multirow[t]{6}{*}{ Apr. 8} & 2000 & 15.12 & 9,600 & \multirow[t]{3}{*}{ May 26} & 1800 & 12.02 & 5,170 \\
\hline & 1900 & 14.03 & - & & 2400 & 15.15 & 9,660 & & 2400 & 12.97 & 6,360 \\
\hline & 2000 & 15.10 & 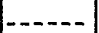 & & & & & & & & \\
\hline & 2200 & 15.59 & 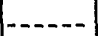 & & 0600 & 15.11 & 9,590 & \multirow[t]{7}{*}{27} & 1000 & 14.47 & 8,550 \\
\hline & 2400 & 15.56 & $\ldots$ & & 2400 & 14.65 & 8,840 & & 1200 & 14.56 & 8,700 \\
\hline & & & & & & & & & 1400 & 14.79 & 9,060 \\
\hline \multirow[t]{4}{*}{5} & 0600 & 15.12 & $\cdots$ & \multirow[t]{4}{*}{10} & 1200 & 14.50 & 8,600 & & 1600 & 14.89 & 9,220 \\
\hline & 1200 & 14.75 & 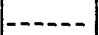 & & 2000 & 14.31 & 8,300 & & 1900 & 14.94 & 9,300 \\
\hline & 2330 & 15.29 & 9,890 & & 2100 & 14.15 & 8,040 & & 2100 & 14.91 & 9,260 \\
\hline & 2400 & 15.28 & 9,880 & & 2400 & 14.08 & 7,930 & & 2400 & 14.80 & 9,080 \\
\hline \multirow[t]{5}{*}{6} & 0600 & 15.08 & 9,540 & \multirow[t]{9}{*}{ May 25} & 2400 & 7.41 & 1,720 & \multirow[t]{4}{*}{28} & 0600 & 14.37 & 8,390 \\
\hline & 1200 & 14.83 & 9,130 & & & & & & 1200 & $1 £ .65$ & 7,310 \\
\hline & 1800 & 14.37 & 8,390 & & 0200 & 7.45 & 1,750 & & 2400 & 12.14 & 5,320 \\
\hline & 2400 & 14.28 & 8,250 & & 0400 & 7.66 & 1,840 & & & & \\
\hline & & & & & 0600 & 8.56 & 2,320 & 29 & 1000 & 11.02 & 4,170 \\
\hline \multirow[t]{3}{*}{7} & 0400 & 14.23 & 8,170 & & 0800 & 9.66 & 3,040 & & 1400 & 10.64 & 3,830 \\
\hline & 2400 & 14.54 & 8,660 & & 1000 & 10.46 & 3,670 & & 1800 & 10.31 & 3,550 \\
\hline & & & & & 1200 & 10.95 & 4,100 & & 2400 & 8.92 & 3,240 \\
\hline 8 & 1200 & 14.93 & 9,290 & & & & & & & & \\
\hline
\end{tabular}

(301) 5-4830. East Fork Hardin Creek near Churdan, Iowa

Location.-Lat $42^{\circ} 06^{\prime} 25^{\prime \prime}$, long $94^{\circ} 22^{\prime} 10^{\prime \prime}$, in SE $\frac{1}{4} \mathrm{SW} \frac{1}{4} \sec .5$, T.84 N., R.30 W., on left bank $35 \mathrm{ft}$ upstream from county highway bridge, 4.4 miles upstream from mouth, and 6.5 miles southeast of Churdan.

\section{Drainage area. $-24.0 \mathrm{sq} \mathrm{mi}$.}

Gage-height record.-Water-stage recorder graph. Datum of gage is $1,050.9$ ) $\mathrm{ft}$ above mean sea level, datum of 1929 .

Discharge record.-Stage-discharge relation defined by current-meter measurements below $200 \mathrm{cfs}$, extended to $413 \mathrm{cfs}$ by logarithmic plotting. Backwater from ice Mar. 1 to Apr. 4.

Maxima.-March-May 1965: Discharge, about $300 \mathrm{cfs}$ Mar. 31; gage height, $8.28 \mathrm{ft}$ 1430 hours Mar. 31 (backwater from ice).

1952 to February 1965: Discharge, $413 \mathrm{cfs}$ May 5, 1960 (gage height, $8.92 \mathrm{ft}$ ).

Mean discharge, in cubic feet per second, 1965

\begin{tabular}{|c|c|c|c|c|c|c|c|c|c|c|c|}
\hline Day & March & April & May & Day & March & April & May & Day & March & April & May \\
\hline $\begin{array}{l}1 \\
2 \\
2 \\
3 \\
4 \\
4 \\
5 \\
6 \ldots- \\
6 \ldots- \\
7 \ldots- \\
3 \ldots- \\
9 \ldots- \\
10 \ldots-\end{array}$ & $\begin{array}{c}25 \\
14 \\
8.8 \\
6.8 \\
5.8 \\
5.8 \\
7.0 \\
9.0 \\
12 \\
18\end{array}$ & $\begin{array}{r}150 \\
80 \\
100 \\
126 \\
179 \\
156 \\
136 \\
134 \\
113 \\
97\end{array}$ & \begin{tabular}{r|}
11 \\
9.5 \\
8.7 \\
7.6 \\
8.0 \\
7.3 \\
6.0 \\
7.3 \\
7.0 \\
6.0
\end{tabular} & $\begin{array}{l}11 \ldots- \\
12_{-} \\
13_{-} \\
14_{-} \\
15_{\ldots} \\
16_{-} \\
17 \ldots \\
18_{-} \\
19_{-} \\
20_{-}\end{array}$ & $\begin{array}{l}27 \\
76 \\
58 \\
50 \\
44 \\
39 \\
36 \\
32 \\
27 \\
21\end{array}$ & \begin{tabular}{c|}
70 \\
42 \\
26 \\
19 \\
15 \\
13 \\
10 \\
8.0 \\
8.0 \\
7.0
\end{tabular} & $\begin{array}{l}5.5 \\
5.5 \\
5.0 \\
5.0 \\
5.2 \\
5.0 \\
4.5 \\
4.5 \\
4.0 \\
4.2\end{array}$ & $\begin{array}{l}21 \\
22_{-} \\
23 \ldots \\
24_{-} \\
25_{-} \\
26 \ldots \\
26_{-} \\
28_{-} \\
29_{-} \\
30_{-} \\
31_{-}\end{array}$ & $\begin{array}{r}19 \\
16 \\
15 \\
13 \\
12 \\
11 \\
11 \\
12 \\
15 \\
22 \\
160\end{array}$ & $\begin{array}{c}5.8 \\
5.8 \\
5.3 \\
25 \\
59 \\
53 \\
29 \\
22 \\
17 \\
13 \\
\end{array}$ & $\begin{array}{c}4.5 \\
5.0 \\
6.5 \\
7.6 \\
8.5 \\
29 \\
17 \\
11 \\
8.7 \\
8.0 \\
7.3\end{array}$ \\
\hline $\begin{array}{l}\text { Nont } \\
\text { Runn } \\
\text { Ruino }\end{array}$ & $\begin{array}{l}\text { hly mea } \\
\text { ff in ind } \\
f f \text { in ac }\end{array}$ & $\begin{array}{l}\text { discha } \\
\text { hes --- } \\
\text { ce-feet }\end{array}$ & e, in & $\mathrm{bi}$ & et & con & 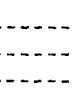 & & $\begin{array}{r}26.7 \\
1.28 \\
1,640\end{array}$ & $\begin{array}{r}57.5 \\
2.67 \\
3,420\end{array}$ & $\begin{array}{l}7.74 \\
0.37 \\
476\end{array}$ \\
\hline
\end{tabular}


Gage height, in feet, and discharge, in cubic feet per second, at indicated time, 1965, of East Fork Hardin Creek near Churdan, Iowa

\begin{tabular}{|c|c|c|c|c|c|c|c|c|c|c|c|}
\hline Date & IJour & $\begin{array}{c}\text { Gage } \\
\text { height }\end{array}$ & $\begin{array}{c}\text { Dis- } \\
\text { charge }\end{array}$ & Date & 1 Iour & $\begin{array}{l}\text { Gage } \\
\text { height }\end{array}$ & $\begin{array}{c}\text { Dis- } \\
\text { charge }\end{array}$ & Date & Ilour & $\begin{array}{c}\text { Gage } \\
\text { height }\end{array}$ & $\begin{array}{c}\text { Dis- } \\
\text { charge }\end{array}$ \\
\hline Mar. 31 & $\begin{array}{l}0000 \\
0700 \\
1100 \\
1430 \\
2400\end{array}$ & $\begin{array}{l}6.27 \\
6.06 \\
6.37 \\
8.28 \\
6.73\end{array}$ & 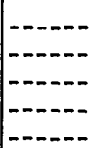 & Apr. 4 & $\begin{array}{l}2400 \\
0200 \\
0600\end{array}$ & $\begin{array}{l}4.62 \\
4.91 \\
6.05\end{array}$ & $\begin{array}{l}120 \\
138 \\
208\end{array}$ & Apr. 5 & $\begin{array}{l}0700 \\
1200 \\
2200 \\
2400\end{array}$ & $\begin{array}{l}6.13 \\
5.75 \\
5.45 \\
5.47\end{array}$ & $\begin{array}{l}214 \\
188 \\
170 \\
171\end{array}$ \\
\hline
\end{tabular}

(302) 5-4836. Middle Raccoon River at Panora, Iowa

Location.-Lat $41^{\circ} 41^{\prime} 15^{\prime \prime}$, long $94^{\circ} 22^{\prime} 15^{\prime \prime}$, in NE $\frac{1}{4} N W \frac{1}{4}$ sec.5, T.79 N., R.3? W., on left bank $15 \mathrm{ft}$ downstream from county highway bridge, 0.2 mile southwest of Panora, and 1.5 miles upstream from Andy's Branch.

Drainage area. $-440 \mathrm{sq} \mathrm{mi}$.

Gage-height record.-Water-stage recorder graph except Mar. 3. Datum of gage is $991.20 \mathrm{ft}$ above mean sea level, datum of 1929 .

Discharge record.-Stage-discharge relation defined by current-meter measurements below $4,440 \mathrm{cfs}$, extended to $9,150 \mathrm{cfs}$ by logarithmic plotting. Backwater from ice Mar. $1-3,13-16,19-23$.

Maxima.-March-May 1965: Discharge, 6,890 cfs 0330 hours Mar. 17; gage height, $11.54 \mathrm{ft} 0530$ hours Mar. 2 (backwater from ice).

1953 to February 1965: Discharge, about 14,000 cfs June 10, 1953 (gage height, $14.3 \mathrm{ft}$ ).

Mean discharge, in cubli feet per second, 1965

\begin{tabular}{|c|c|c|c|c|c|c|c|c|c|c|c|}
\hline Day & March & April & May & Day & March & April & May & Day & March & April & May \\
\hline & 3,800 & 3,190 & 201 & $11 \ldots$ & 304 & 733 & 122 & 21. & 280 & 162 & 106 \\
\hline $2_{-}$ & 3,800 & 3,190 & 174 & 12. & 631 & 502 & 117 & 22 & 200 & 155 & 137 \\
\hline 3. & 1,990 & 1,510 & 155 & 13 & 1,300 & 383 & 114 & 23. & 134 & 142 & 152 \\
\hline & 1,000 & 1,860 & 145 & $14 \ldots$ & 1,420 & 325 & 106 & 24 & 119 & 156 & 264 \\
\hline 5 & 525 & 3,250 & 135 & D. & 1,540 & 320 & 109 & 25. & 116 & 282 & 236 \\
\hline 6. & 366 & 2,400 & 125 & 16 & 1,610 & 285 & 154 & 26 & 111 & 404 & 959 \\
\hline 7 & 315 & 1,220 & 122 & $17 \ldots$ & 3,630 & 245 & 145 & 27. & 105 & 417 & 2,540 \\
\hline 8 & 315 & 1,050 & 119 & 18 & 984 & 214 & 122 & 28 & 183 & 325 & 1,280 \\
\hline 9 & 281 & 881 & 180 & 19 & 380 & 185 & 112 & $29_{-}$ & 355 & 275 & 644 \\
\hline 10 & 256 & 752 & 145 & $20 \ldots$ & 320 & 166 & 106 & $30_{-}$ & 743 & 231 & 584 \\
\hline & & & & & & & & & 1,950 & $--n-\infty$ & 473 \\
\hline \multicolumn{9}{|c|}{ Monthly mean discharge, in cubic feet per second } & 938 & 840 & 325 \\
\hline \multicolumn{9}{|c|}{ Runoff, in inches } & 2.46 & 2.13 & 0.85 \\
\hline \multicolumn{9}{|c|}{ Runoff, in acre-feet } & 57,650 & 50,000 & 20,000 \\
\hline
\end{tabular}


Gage height, in feet, and discharge, in cubic feet per second, at indicated time, 1965, of Middle Raccoon River at Panora, lowa

\begin{tabular}{|c|c|c|c|c|c|c|c|c|c|c|c|}
\hline Date & Ilour & $\begin{array}{l}\text { Gage } \\
\text { height }\end{array}$ & $\begin{array}{c}\text { Dis- } \\
\text { charge }\end{array}$ & Date & Hour & $\begin{array}{l}\text { Gage } \\
\text { heigit }\end{array}$ & $\begin{array}{c}\text { Dis- } \\
\text { charge }\end{array}$ & Date & Hour & $\begin{array}{c}\text { Gage } \\
\text { height }\end{array}$ & $\begin{array}{c}\text { Dis- } \\
\text { charge }\end{array}$ \\
\hline ar. 1 & $\begin{array}{l}0000 \\
0400 \\
0600 \\
1200 \\
2400 \\
0530 \\
0600 \\
0700 \\
1100 \\
1300 \\
2000 \\
2400 \\
0000 \\
0100 \\
0330 \\
0700 \\
0900 \\
1300 \\
1800 \\
2400 \\
0400\end{array}$ & $\begin{array}{r}7.60 \\
8.75 \\
9.24 \\
9.69 \\
11.12 \\
11.54 \\
10.24 \\
9.61 \\
9.80 \\
10.18 \\
9.72 \\
8.61 \\
8.34 \\
9.14 \\
10.87 \\
9.11 \\
8.42 \\
8.01 \\
7.77 \\
7.30 \\
6.66\end{array}$ & 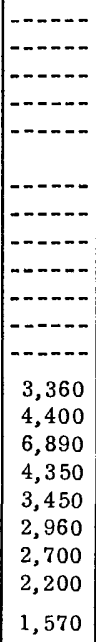 & $\mid \begin{array}{l}\text { Mar. 18 } \\
\text { Apr. } 3\end{array}$ & $\begin{array}{l}0800 \\
1400 \\
1800 \\
2400 \\
2400 \\
0600 \\
1400 \\
1800 \\
2400 \\
0200 \\
0400 \\
0500 \\
0800 \\
1200 \\
1600 \\
2000 \\
2400 \\
0600 \\
1200\end{array}$ & $\begin{array}{l}6.07 \\
5.51 \\
5.33 \\
5.27 \\
6.41 \\
6.62 \\
7.12 \\
7.34 \\
7.22\end{array}$ & $\begin{array}{r}1,090 \\
662 \\
536 \\
496 \\
1,360 \\
1,540 \\
2,020 \\
2,240 \\
2,120 \\
2,040 \\
2,380 \\
3,230 \\
4,690 \\
3,250 \\
3,330 \\
3,190 \\
3,180 \\
2,940 \\
2,330\end{array}$ & $\begin{array}{l}\text { Apr. } 6 \\
\text { May } 25\end{array}$ & \begin{tabular}{|l}
2400 \\
2400 \\
0600 \\
1000 \\
1200 \\
1300 \\
1400 \\
1600 \\
2000 \\
2400 \\
1600 \\
2200 \\
2400 \\
0200 \\
0600 \\
1000 \\
1600 \\
2400
\end{tabular} & $\begin{array}{l}6.67 \\
4.89 \\
5.05 \\
5.09 \\
5.21 \\
5.66 \\
6.12 \\
6.58 \\
6.95 \\
7.18 \\
7.81 \\
7.90 \\
7.80\end{array}$ & $\begin{array}{r}1,580 \\
280 \\
\\
362 \\
384 \\
456 \\
775 \\
1,130 \\
1,500 \\
1,850 \\
2,080 \\
\\
2,740 \\
2,840 \\
2,730\end{array}$ \\
\hline
\end{tabular}

(303) 5-4840. South Raccoon River at Redfield, Iowa

Location.-Lat $41^{\circ} 34^{\prime} 45^{\prime \prime}$, long $94^{\circ} 11^{\prime} 00^{\prime \prime}$, in SW $\frac{1}{4} \mathrm{SW} \frac{1}{4}$ sec.3, T.78 N., R.29 W., on left bank $10 \mathrm{ft}$ upstream from county highway bridge at Redfield, 0.8 mile downstream from bridge on State Highway 90, 1 mile downstream from Middle Raccoon River, and 15.6 miles upstream from mouth.

Drainage area.- $-988 \mathrm{sq} \mathrm{mi}$.

Gage-height record.-Water-stage recorder graph except Mar. 3-13, 18-31, A pr. 10-15, 18-24, where graph was reconstructed from daily wire-weight gage reading $s$ except Mar. 5, 6. Datum of gage is $896.43 \mathrm{ft}$ above mean sea level, datum of 1929 .

Discharge record. - Stage-discharge relation defined by current-meter measurements below 35,000 cfs. Backwater from ice Mar, 25-27.

Maxima.-March-May 1965: Discharge, 15,800 cfs 1300 hours Mar. 17 (gage height, $19.60 \mathrm{ft}$ ).

1940 to February 1965: Discharge, 35,000 cfs July 2, 1958 (gage height, $29.04 \mathrm{ft}$, from floodmark). 
Mean discharge, in cubic feet per second, 1965, of South Raccoon River at Redfield, Iowa

\begin{tabular}{|c|c|c|c|c|c|c|c|c|c|c|c|}
\hline Day & March & April & May & Day & March & April & May & Day & March & April & May \\
\hline & 9,520 & 7,250 & 470 & $11 \ldots$ & 507 & 1,610 & 284 & $21 \ldots$ & 840 & 394 & 216 \\
\hline$\sigma_{0}$ & 7,310 & 4,300 & 416 & $12 \ldots$ & 1,130 & 1,160 & 268 & $22 \ldots$ & 676 & 376 & 322 \\
\hline ? & 3,640 & 2,780 & 372 & $13 \ldots$ & 2,460 & 865 & 256 & 23 & 426 & 356 & 431 \\
\hline & 1,360 & 3,210 & 352 & $14 \ldots$ & 3,010 & 695 & 244 & $24 \ldots$ & 348 & 370 & 458 \\
\hline & 780 & 8,340 & 340 & $15 \ldots$ & 3,600 & 745 & 240 & $25 \ldots$ & 332 & 831 & 546 \\
\hline 6. & 640 & 5,130 & 320 & $16 \ldots$ & 3,770 & 695 & 290 & $26 \ldots$ & 322 & 1,140 & 1,950 \\
\hline 7. & 508 & 2,520 & 300 & $17 \ldots$ & 12,500 & 605 & 304 & $27 \ldots$ & 440 & 951 & 2,660 \\
\hline & 500 & 2,300 & 304 & $18 \ldots$ & 2,800 & 538 & 256 & $28 \ldots$ & 891 & 760 & 1,960 \\
\hline 9. & 493 & 2,010 & 392 & $19 \ldots$ & 1,120 & 474 & 236 & 29 & 1,470 & 632 & 1,060 \\
\hline 10 & 441 & 1,820 & 333 & $20 \ldots$ & 955 & 434 & 213 & $30 \ldots$ & 2,390 & 542 & 935 \\
\hline & & & & & & & & 31 & 5,030 & $\ldots$ & 860 \\
\hline \multirow{3}{*}{\multicolumn{9}{|c|}{$\begin{array}{l}\text { Monthly mean discharge, in cubic feet per second } \\
\text { Runoff, in inches } \\
\text { Runoff, in acre feet }\end{array}$}} & 2,265 & 1,794 & 567 \\
\hline & & & & & & & & & 2.64 & 2.03 & 0.66 \\
\hline & & & & & & & & & 139,300 & $1 c 6,800$ & 34,890 \\
\hline
\end{tabular}

Gage height, in fect, and drscharge, in cubic fect per second, at indacated time, 1965

\begin{tabular}{|c|c|c|c|c|c|c|c|c|c|c|c|c|}
\hline Date & Hour & $\begin{array}{l}\text { Gage } \\
\text { height }\end{array}$ & $\begin{array}{c}\text { Dis- } \\
\text { charge }\end{array}$ & Date & Ilour & $\begin{array}{c}\text { Gagc } \\
\text { height }\end{array}$ & $\begin{array}{c}\text { Dis- } \\
\text { charge }\end{array}$ & \multicolumn{2}{|c|}{ Date } & Ilour & $\begin{array}{c}\text { Gage } \\
\text { height }\end{array}$ & $\begin{array}{c}\text { Dis- } \\
\text { charge }\end{array}$ \\
\hline Mar. 14 & 0000 & 8.04 & 2,860 & Mar. 18 & 0600 & 9.93 & 4,260 & Apr. & 8 & 0400 & 6.61 & 1,880 \\
\hline & 1000 & 8.01 & 2,840 & & 0700 & 8.50 & 3,180 & & & 1400 & 7.57 & 2,530 \\
\hline & 1800 & 8.37 & 3,090 & & 0800 & 7.63 & 2,570 & & & 2000 & 7.73 & 2,640 \\
\hline & 2400 & 9.00 & 3,560 & & 1000 & 6.50 & 1,810 & & & 2400 & 7.40 & 2,410 \\
\hline & & & & & 1200 & 5.98 & 1,490 & & & & & \\
\hline 15 & 0400 & 9.33 & 3,810 & & 2400 & 5.59 & 1,270 & May & 25 & 2400 & 4.09 & 510 \\
\hline & 1400 & 8.68 & 3,320 & & & & & & & & & \\
\hline & 2400 & 9.46 & 3,900 & Apr. 4 & 2400 & 8.24 & 3,000 & & 26 & 0200 & 4.48 & 690 \\
\hline & & & & & & & & & & 0300 & 4.93 & 915 \\
\hline 16 & 0400 & 9.77 & 4,140 & 5 & 0200 & 8.21 & 2,980 & & & 0400 & 5.34 & 1,130 \\
\hline & 1200 & 8.99 & 3,550 & & 0400 & 8.84 & 3,440 & & & 0800 & 6.08 & 1,550 \\
\hline & 1600 & 8.45 & 3,150 & & 0500 & 9.87 & 4,220 & & & 1000 & 6.28 & 1,670 \\
\hline & 1800 & 10.19 & 4,470 & & 0600 & 11.23 & 5,300 & & & $1100^{1}$ & 7.23 & 2,290 \\
\hline & 2000 & 9.02 & 3,580 & & 0800 & 13.19 & 7,130 & & & 1300 & 8.33 & 3,060 \\
\hline & 2200 & 8.43 & 3,130 & & 1000 & 14.76 & 8,890 & & & 1400 & 8.37 & 3,090 \\
\hline & 2300 & 9.12 & 3,650 & & 1600 & 16.98 & 11,800 & & & 1900 & 7.07 & 2,180 \\
\hline & 2400 & 11.00 & 5,120 & & 1700 & 16.99 & 11,800 & & & 2400 & 7.45 & 2,440 \\
\hline & & & & & 2200 & 16.38 & 11,000 & & & & & \\
\hline 17 & 0100 & 13.13 & 7,070 & & 2400 & 14.80 & 8,940 & & 27 & 1200 & 7.63 & 2,570 \\
\hline & 0300 & 14.94 & 9,110 & & & & & & & 2200 & 8.24 & 3,000 \\
\hline & 0500 & 17.16 & 12,000 & 6 & 0200 & 13.07 & 7,010 & & & 2400 & 8.28 & 3,030 \\
\hline & 1300 & 19.60 & 15,800 & & 0600 & 11.74 & 5,750 & & & & & \\
\hline & 1600 & 19.02 & 14,900 & & 1200 & 10.84 & 4,990 & & 28 & 0200 & 8.29 & 3,030 \\
\hline & 2000 & 17.45 & 12,500 & & 1800 & 9.69 & 4,080 & & & 0400 & 8.24 & 3,000 \\
\hline & 2200 & 16.75 & 11,500 & & 2400 & 8.74 & 3,360 & & & 0600 & 7.54 & 2,510 \\
\hline & 2400 & 14.35 & 8,400 & & & & & & & 1000 & 6.64 & 1,900 \\
\hline & & & & 7 & 0800 & 7.80 & 2,690 & & & 1600 & 5.90 & 1,440 \\
\hline 18 & 0200 & 12.50 & 6,440 & & 1600 & 7.12 & 2,210 & & & 2400 & 5.45 & 1,190 \\
\hline & 0400 & 11.32 & 5,380 & & 2400 & 6.72 & 1,950 & & & & & \\
\hline
\end{tabular}


(304) 5-4845. Raccoon River at Van Meter, Iowa

Location.--Lat $41^{\circ} 32^{\prime} 00^{\prime \prime}$, long $93^{\circ} 57^{\prime} 10^{\prime \prime}$, in SW $\frac{1}{4} \mathrm{SW} \frac{1}{4}$ sec. 22, T. $78 \mathrm{~N}$, R. 27 W., on right bank $100 \mathrm{ft}$ downstream from highway bridge, 0.3 mile northeast of Van Meter, 1.2 miles downstream from confluence of North and South Raccoon River, and 30 miles upstream from mouth.

Drainage area.- $-3,441 \mathrm{sq} \mathrm{mi}$.

Gage-height record.-Water-stage recorder graph, except Mar. 6-9, 19-27, May 16 when wire-weight gage readings were available. Datum of gage is $841.16 \mathrm{ft}$, above mean sea level, datum of 1929 .

Discharge record.- Stage-discharge relation defined by current-meter measurements below $40,000 \mathrm{cfs}$. Backwater from ice Mar. 1-31.

Maxima.-March-May 1965: Discharge, 22,300 cfs 0300 hours Apr. 6 (gage height, $18.35 \mathrm{ft}$ )

1915 to February 1965: Discharge, 41,200 cfs June 13, 1947; gage height, $21.77 \mathrm{ft}$ July $3,1958$.

Mean discharge, in cubic feet per second, 1965

\begin{tabular}{|c|c|c|c|c|c|c|c|c|c|c|c|}
\hline Day & March & April & May & Day & March & April & May & Day & March & April & May \\
\hline 1 & 8,000 & 13,100 & 2,190 & $11 \ldots$ & 1,000 & 14,200 & 1,420 & $21 \ldots$ & 3,300 & 2,090 & 1,350 \\
\hline & 10,000 & 11,700 & 1,930 & $12 \ldots$ & 1,300 & 12,300 & 1,490 & 22 & 2,300 & 1,870 & 1,310 \\
\hline & 8,300 & 11,400 & 1,720 & $13 \ldots$ & 2,800 & 9,940 & 1,320 & 23. & 1,600 & 1,710 & 1,440 \\
\hline & 5,800 & 11,700 & 1,570 & $14 \ldots$ & 5,000 & 7,660 & 1,200 & 24. & 1,300 & 1,700 & 1,680 \\
\hline & 3,800 & 16,700 & 1,450 & $15 \ldots$ & 6,400 & 5,720 & 1,110 & 25 & 1,100 & 2,350 & 2,170 \\
\hline & 2,500 & 19,600 & 1,360 & $16 \ldots$ & 7,600 & 4,660 & 1,050 & $26 \ldots$ & 980 & 3,580 & 3,830 \\
\hline & 1,700 & 16,700 & 1,270 & $17 \ldots$ & 16,000 & 3,850 & 1,300 & $27 \ldots$ & 900 & 3,830 & 6,520 \\
\hline & 1,400 & 16,100 & 1,240 & $18 \ldots$ & 11,000 & 3,240 & 1,500 & $28 \ldots$ & 960 & 3,410 & 8,600 \\
\hline & 1,150 & 14,600 & 1,250 & 19 & 7,000 & 2,780 & 1,750 & 29. & 2,000 & 3,000 & 9,460 \\
\hline 10 & 1,000 & 14,000 & 1,270 & $20 \ldots$ & 4,800 & 2,400 & 1,560 & 30. & 3,000 & 2,580 & 9,160 \\
\hline & & & & & & & & & 8,000 & $-\cdots$ & 5,210 \\
\hline \multirow{3}{*}{\multicolumn{9}{|c|}{$\begin{array}{l}\text { Monthly mean discharge, in cubic feet per second } \\
\text { Runoff, in inches } \\
\text { funoff, in acre-feet }\end{array}$}} & 4,258 & 7,949 & 2,570 \\
\hline & & & & & & & & & 1.43 & 2.58 & 0.86 \\
\hline & & & & & & & & & 261,800 & 473,000 & 158,000 \\
\hline
\end{tabular}


Gage height. in feet, and discharge, in cubic feet per second, at indicated time. 1965, of Raccoon Riler at Van Meter, Iowa

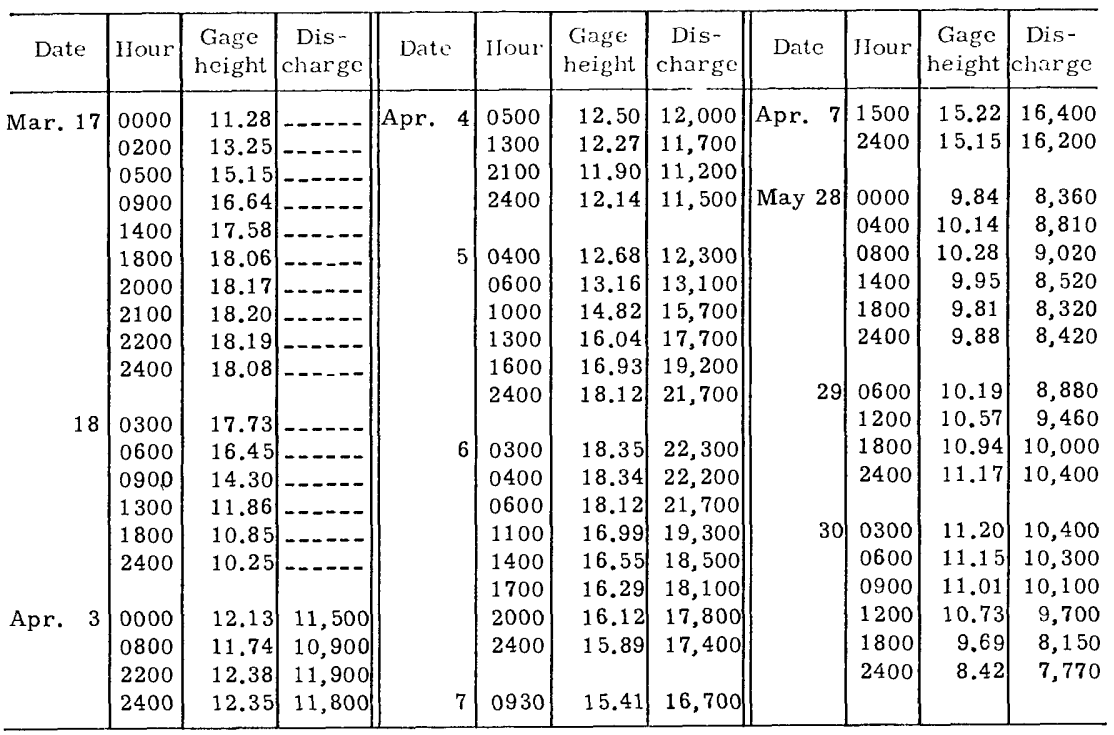

(305) 5-4855. Des Moines River below Raccoon River, at Des Moires, Iowa

Location.-Lat $41^{\circ} 34^{\prime} 30^{\prime \prime}$, long $93^{\circ} 35^{\prime} 40^{\prime \prime}$, in NE $\frac{1}{4} \mathrm{SE} \frac{1}{4}$ sec.10, T.78 N., R.24 W., on right bank $10 \mathrm{ft}$ downstream from South East 14th Street bridge, 0.8 mile downstream from Raccoon River and Scott Street Dam, and at mile 200.7.

Drainage area. $-9,879 \mathrm{sq} \mathrm{mi}$

Gage-height record.-Water-stage recorder graph. Datum of gage is $762.52 \mathrm{ft}$ above sea level, datum of 1929 .

Discharge record.-Stage-discharge relation defined by current-meter neasurements below 65,000 cfs. Backwater from ice Mar. 14-18, 24-27.

Maxima.-March-May 1965: Discharge, 65,500 cfs 0400 hours Apr. 11 (gage height, $29.78 \mathrm{ft}$ ).

1940 to February 1965: Discharge, 77,000 cfs June 26, 1947 (gage height, $20.8 \mathrm{ft}$ in gage well, $21.6 \mathrm{ft}$ from outside flood mark, at site above Scott Street dam, $0.8 \mathrm{mile}$ upstream at datum $11.16 \mathrm{ft}$ higher).

Maximum stage known since at least 1893, that of June 26, 1947 .

Cooperation.-Three discharge measurements furnished by Corps of Engineers. 
Mean discharge, in cubic feet per second, 1965, of Des Moines River below Raccoon River at Des Moines

\begin{tabular}{|c|c|c|c|c|c|c|c|c|c|c|c|}
\hline Day & March & April & May & Day & March & April & May & Day & March & April & May \\
\hline & 7,740 & 17,600 & 12,000 & $11_{-}$ & 3,210 & 65,000 & 7,270 & $21_{-}$ & 7,800 & 17,90 า & 7,760 \\
\hline & 13,700 & 22,200 & 11,100 & 12 & 3,170 & 61,600 & 7,720 & 22 & 5,740 & 16,300 & 7,460 \\
\hline 3 & 16,000 & 22,800 & 10,300 & $13 \ldots$ & 3,860 & 52,800 & 7,700 & 23. & 4,370 & 15,000 & 7,370 \\
\hline & 15,700 & 24,600 & 9,430 & $14 \ldots$ & 5,000 & 43,400 & 7,350 & $24 \ldots$ & 3,300 & 14,500 & 7,460 \\
\hline & 12,900 & 29,000 & 8,700 & $15 \ldots$ & 7,000 & 37,000 & 6,930 & 25 & 2,900 & 14,500 & 7,930 \\
\hline & 8,970 & 43,800 & 8,040 & $16 \ldots$ & 9,000 & 33,000 & 6,690 & 26. & 2,600 & 15,200 & 10,300 \\
\hline 7. & 6,100 & 52,400 & 7,700 & $17 \ldots$ & 18,000 & 29,500 & 6,900 & 27. & 2,500 & 15,200 & 14,600 \\
\hline & 4,840 & 57,600 & 7,880 & $18 \ldots$ & 21,000 & 26,100 & 7,850 & 28. & 2,870 & 14,900 & 19,300 \\
\hline & 4,070 & 62,800 & 7,800 & $19 \ldots$ & 14,500 & 22,800 & 8,660 & $29_{-}$ & 3,650 & 14,000 & 21,600 \\
\hline & 3,600 & 65,000 & 7,410 & $20 \ldots$ & 10,200 & 20,000 & 8,360 & 30. & 4,940 & 13,000 & 21,300 \\
\hline & & & & & & & & & 8,760 & $\ldots$ & 16,200 \\
\hline \multirow{3}{*}{\multicolumn{9}{|c|}{$\begin{array}{l}\text { Monthly mean discharge, in cubic feet per second } \\
\text { Runof, in inches } \\
\text { Runoff, in acre-feet }\end{array}$}} & 7,675 & 31,320 & 9,905 \\
\hline & & & & & & & & & 0.90 & 3.54 & 1.16 \\
\hline & & & & & & & & & 471,900 & $1,863,000$ & 609,100 \\
\hline
\end{tabular}

Gage herght, in fect, and discharge, in cubic feet per second, at indicated time, 1965

\begin{tabular}{|c|c|c|c|c|c|c|c|c|c|c|c|}
\hline Date & Hour & $\begin{array}{l}\text { Gage } \\
\text { height }\end{array}$ & $\begin{array}{c}\text { Dis- } \\
\text { charge }\end{array}$ & Date & llour & $\begin{array}{l}\text { Ciagc } \\
\text { height }\end{array}$ & $\begin{array}{c}\text { Dis- } \\
\text { charge }\end{array}$ & Date & Ilour & $\begin{array}{c}\text { Gage } \\
\text { height }\end{array}$ & $\begin{array}{c}\text { Dis- } \\
\text { chitrge }\end{array}$ \\
\hline or. 5 & 0000 & 22.22 & 25,300 & Apr. 7 & 2400 & 28.20 & 54,400 & Apr. 11 & 1800 & 29.65 & 64,600 \\
\hline & 0600 & 22.50 & 26,200 & & & & & & 2400 & 29.58 & 64,100 \\
\hline & 1200 & 23.09 & 28,300 & 8 & 0600 & 28.46 & 56,200 & & & & \\
\hline & 1800 & 23.98 & 31,400 & & 1800 & 28.85 & 59,000 & 12 & 0600 & 29.46 & 63,200 \\
\hline & 2400 & 24.89 & 35,100 & & 2400 & 29.08 & 60,600 & & 1200 & 29.28 & 62,000 \\
\hline & & & & & & & & & 1800 & 29.02 & 60,100 \\
\hline 6 & 0600 & 25.83 & 39,200 & 9 & 1200 & 29.43 & 63,000 & & 2400 & 28.70 & 57,900 \\
\hline & 0900 & 26.30 & 41,600 & & 2400 & 29.65 & 64,600 & & & & \\
\hline & 1200 & 26.78 & 44,500 & & & & & 13 & 1200 & 27.06 & 52,700 \\
\hline & 1800 & 27.37 & 48,600 & 10 & 1200 & 29.73 & 65 , & & 2400 & 27.2 .4 & 47,700 \\
\hline & 2400 & 27.67 & 50,700 & & 2400 & 29.77 & 65,400 & & & & \\
\hline & & & & & & & & 14 & 1200 & 26.58 & 43,300 \\
\hline 7 & 0600 & 27.80 & 51,600 & 11 & 0400 & 29.78 & 65,500 & & 2400 & 25.88 & 39,500 \\
\hline & 1800 & 28.02 & 53,100 & & 1200 & 29.73 & 65,100 & & & & \\
\hline
\end{tabular}

(306) 5-4860. North River near Norwalk, Iowa

Location.-Lat $41^{\circ} 27^{\prime} 25^{\prime \prime}$, long $93^{\circ} 39^{\prime} 10^{\prime \prime}$, in NW $\frac{1}{4} \mathrm{SW} \frac{1}{4}$ sec. $20, T .77 \mathrm{~N} ., \mathrm{R} .24 \mathrm{~W}$. , on left bank $10 \mathrm{ft}$ downstream from highway bridge, $1-3 / 4$ miles southeast of Norwalk, 8 miles northwest of Indianola, 8.7 miles upstream from Middle Creek, and 9 miles south of Des Moines.

Drainage area. -349 sq mi.

Gage-height record.- Water-stage recorder graph except Mar. 1-10, 22-25. Daily wire-weight readings available for period. Datum of gage is 788.45 above rean sea level, datum of 1929 (levels by Corps of Engineers).

Discharge record.- Stage-discharge relation defined by current-meter measurements below $9,100 \mathrm{cfs}$ and extended to $32,000 \mathrm{cfs}$ on basis of area-velocity studies. Backwater from ice Mar. 1-30.

Maxima.-March-May 1965: Discharge, about 8,000 cfs Mar. 18 (gage height, $22.86 \mathrm{ft}$, backwater from icel.

1940 to February 1965: Discharge, 32,000 cfs June 13, 1947 (gage height, $25.3 \mathrm{ft}$, from floodmark).

Cooperation.-One discharge measurement furnished by Corps of Engineers. 
Mean discharge, in cubic feet per second, 1965. of North River near Norwalk, Iowa

\begin{tabular}{|c|c|c|c|c|c|c|c|c|c|c|c|}
\hline Day & March & April & May & Day & March & April & May & Day & March & April & May \\
\hline & 600 & 1,870 & 134 & $11_{\text {_ }}$ & 82 & 456 & 78 & 21 & 1,500 & 130 & 44 \\
\hline 2. & 1,200 & 1,990 & 117 & 12. & 80 & 445 & 68 & 22 & 800 & 120 & 49 \\
\hline 3. & 1,500 & 1,400 & 105 & 13. & 78 & 305 & 62 & 23 & 450 & 110 & 62 \\
\hline 4 & 800 & 1,080 & 97 & 14 & 90 & 252 & 59 & 24 & 250 & 104 & 85 \\
\hline 5. & 350 & 1,260 & 95 & 15 & 600 & 282 & 57 & 25 & 130 & 192 & 90 \\
\hline 6. & 200 & 2,730 & 94 & $16 \ldots$ & 1,000 & 254 & 58 & 26 & 120 & 341 & 223 \\
\hline 7. & 150 & 3,140 & 92 & 17. & 2,500 & 216 & 59 & 27. & 120 & 305 & 641 \\
\hline 8. & 110 & 1,140 & 101 & 18. & 6,400 & 188 & 54 & 28. & 250 & 232 & 279 \\
\hline 9. & 90 & 836 & 136 & $19 \ldots$ & 6,600 & 162 & 49 & 29 & 600 & 187 & 140 \\
\hline 10 & 86 & 552 & 94 & $20 .-$ & 3,000 & 144 & 46 & & 1,000 & 156 & 138 \\
\hline & & & & & & & & & 1,540 & $--\cdots$ & 222 \\
\hline \multirow{3}{*}{\multicolumn{9}{|c|}{$\begin{array}{l}\text { Monthly mean discharge, in cubic feet per second } \\
\text { Runoff, in inches } \\
\text { Runoff, in acre -feet }\end{array}$}} & 1,041 & 686 & 117 \\
\hline & & & & & & & & & 3.44 & 2.19 & 0.39 \\
\hline & & & & & & & & & 64,020 & 40,600 & 7,200 \\
\hline
\end{tabular}

Gage height, in feet, and discharge, in cubic feet per second, at indicated time, 1965

\begin{tabular}{|c|c|c|c|c|c|c|c|c|c|c|c|}
\hline Date & IIour & $\begin{array}{c}\text { Gage } \\
\text { height }\end{array}$ & $\begin{array}{c}\text { Dis - } \\
\text { charge }\end{array}$ & Date & llour & $\begin{array}{c}\text { Gage } \\
\text { height }\end{array}$ & $\begin{array}{c}\text { Dis- } \\
\text { churge }\end{array}$ & Date & IIour & $\begin{array}{c}\text { Gage } \\
\text { height }\end{array}$ & $\begin{array}{c}\text { Dis- } \\
\text { charge }\end{array}$ \\
\hline 17 & $\begin{array}{l}0000 \\
0600 \\
1200 \\
1800 \\
2400 \\
0600 \\
1200 \\
1800 \\
2400 \\
0600 \\
0900 \\
1200 \\
2200 \\
2400 \\
0600 \\
1200 \\
1800 \\
2400 \\
1600 \\
1800\end{array}$ & $\begin{array}{l}10.34 \\
10.64 \\
11.12 \\
11.95 \\
13.00 \\
14.10 \\
15.82 \\
17.60 \\
18.60 \\
19.05 \\
19.17 \\
19.19 \\
19.55 \\
19.73 \\
20.57 \\
20.92 \\
21.21 \\
21.65 \\
22.85 \\
22.86\end{array}$ & $\begin{array}{l}-- \\
--- \\
--- \\
--- \\
--- \\
--\end{array}$ & Mar. 18 & $\begin{array}{l}2400 \\
0600 \\
1200 \\
1800 \\
2400 \\
0600 \\
1200 \\
1800 \\
2400 \\
\\
2400 \\
0630 \\
0900 \\
1100 \\
1200 \\
1300 \\
1400 \\
1600 \\
2000 \\
2400\end{array}$ & $\begin{array}{l}22.79 \\
22.69 \\
22.55 \\
22.33 \\
21.99 \\
21.67 \\
21.48 \\
21.21 \\
20.84 \\
\\
17.23 \\
15.80 \\
16.29 \\
16.52 \\
17.12 \\
17.30 \\
18.60 \\
19.25 \\
19.48 \\
19.63\end{array}$ & $\begin{array}{r} \\
-1,080 \\
\\
850 \\
926 \\
963 \\
1,060 \\
1,090 \\
1,370 \\
1,600 \\
1,700 \\
1,820\end{array}$ & pr. 6 & $\begin{array}{l}0800 \\
1400 \\
1800 \\
2200 \\
2400 \\
0300 \\
0600 \\
1200 \\
2000 \\
2400 \\
0200 \\
0400 \\
0600 \\
0900 \\
1200 \\
1400 \\
1600 \\
1800 \\
2000 \\
2400\end{array}$ & $\begin{array}{l}19.94 \\
20.39 \\
20.87 \\
21.17 \\
21.20 \\
21.13 \\
20.99 \\
20.68 \\
20.22 \\
19.91 \\
19.60 \\
19.02 \\
18.37 \\
17.45 \\
16.54 \\
16.12 \\
15.85 \\
15.72 \\
15.70 \\
15.77\end{array}$ & $\begin{array}{r}2,050 \\
2,540 \\
3,510 \\
4,250 \\
4,330 \\
4,150 \\
3,800 \\
3,080 \\
2,300 \\
2,030 \\
1,820 \\
1,550 \\
1,330 \\
1,120 \\
966 \\
899 \\
858 \\
838 \\
835 \\
846\end{array}$ \\
\hline
\end{tabular}


(307) 5-4864.9 Middle River near Indianola, Iowa

Location.-Lat $41^{\circ} 25^{\prime} 25^{\prime \prime}$, long $93^{\circ} 35^{\prime} 05^{\prime \prime}$, in SW $\frac{1}{4} \mathrm{SE} \frac{1}{4} \mathrm{sec} .35, \mathrm{~T} .77 \mathrm{~N}$., R.24 W., on right bank $10 \mathrm{ft}$ downstream from county highway bridge, 0.5 mile upstream from Cavitt

Creek, and 4.5 miles northwest of Indianola.

Drainage area. -503 sq $\mathrm{mi}$.

Gage-height record.-Water-stage recorder graph. Datum of gage is $776.15 \mathrm{ft}$ above mean sea level, datum of 1929 (Corps of Engineers bench mark).

Discharge record.-Stage-discharge relation defined by current-meter measurements. Backwater from ice Mar. 1-16, 21-30.

Maxima.-March-May 1965: Discharge, 9,700 cfs 1930 hours Mar. 17; gage reight, $21.00 \mathrm{ft} 0630$ hours Apr. 6.

1940 to February 1965: Discharge, $34,000 \mathrm{cfs}$ J une 13, 1947 (gage height, $28.27 \mathrm{ft}$, from floodmark).

Cooperation.-One discharge measurement furnished by Corps of Engineers.

Mean discharge, in cubic feet per second, 1965

\begin{tabular}{|c|c|c|c|c|c|c|c|c|c|c|c|}
\hline Day & March & April & May & Day & March & April & May & Day & March & April & May \\
\hline $1-$ & 930 & 2,340 & 239 & $11 \ldots$ & 145 & 953 & 161 & 21. & 350 & 234 & 101 \\
\hline 2. & 1,180 & 1,390 & 215 & $12 \ldots$ & 150 & 696 & 144 & 22. & 250 & 217 & 104 \\
\hline 3. & 800 & 822 & 203 & $13 \ldots$ & 160 & 493 & 136 & 23. & 200 & 203 & 137 \\
\hline$\therefore$ & 300 & 1,110 & 185 & $14 \ldots$ & 310 & 414 & 130 & 24 & 190 & 206 & 171 \\
\hline 5 & 200 & 4,360 & 189 & $15 \ldots$ & 820 & 427 & 124 & $25_{-}$ & 170 & 509 & 176 \\
\hline 6. & 180 & 7,640 & 192 & $16 \ldots$ & 2,400 & 395 & 122 & $26_{-}$ & 160 & 660 & 195 \\
\hline 7. & 170 & 1,690 & 181 & $17 \ldots$ & 8,500 & 367 & 121 & $27_{-}$ & 210 & 463 & 586 \\
\hline 8. & 165 & 1,840 & 215 & $18 \ldots$ & 6,620 & 310 & 114 & 28 & 350 & 412 & 326 \\
\hline 9. & 160 & 2,710 & 318 & $19 \ldots$ & 3,350 & 276 & 108 & $29_{-}$ & 600 & 319 & 198 \\
\hline 10 & 150 & 1,070 & 190 & $20 \ldots$ & 791 & 251 & 102 & $30_{-}$ & 1,100 & 290 & 339 \\
\hline & & & & & & & & 3 & 1,770 & 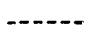 & 234 \\
\hline \multicolumn{9}{|c|}{ Monthly mean discharge, in cubic feet per second.............. } & 1,059 & 1,102 & 192 \\
\hline \multicolumn{9}{|c|}{$\begin{array}{l}\text { Runoff, in inches } \\
\text {. }\end{array}$} & 2.43 & 2.44 & 0.44 \\
\hline \multicolumn{9}{|c|}{ Runoff, in acre - feet } & 65,120 & 65,590 & 11,810 \\
\hline
\end{tabular}

Gage height, in fect, and descharge, in cubic feet per sccond, at indicated time, 1965

\begin{tabular}{|c|c|c|c|c|c|c|c|c|c|c|c|}
\hline Date & Hour & $\begin{array}{c}\text { Gage } \\
\text { height }\end{array}$ & $\begin{array}{c}\text { Dis - } \\
\text { charge }\end{array}$ & Dite & Hour & $\begin{array}{c}\text { Gage } \\
\text { height }\end{array}$ & $\begin{array}{c}\text { Dis- } \\
\text { charge }\end{array}$ & Date & Hour & $\begin{array}{c}\text { Gage } \\
\text { height }\end{array}$ & $\begin{array}{c}\text { Dis- } \\
\text { charge }\end{array}$ \\
\hline \multirow[t]{12}{*}{ Mar. 17} & 0000 & 13.96 & 3,890 & \multirow[t]{11}{*}{ Mar. 18 } & 1200 & 17.10 & 6,160 & \multirow[t]{14}{*}{ Apr. } & 0900 & 9.65 & 1,230 \\
\hline & 0100 & 15.22 & 4,770 & & 1800 & 16.00 & 5,280 & & 0930 & 10.73 & 1,710 \\
\hline & 0200 & 16.78 & 5,940 & & 2400 & 15.70 & 5,080 & & 1000 & 11.50 & 2,060 \\
\hline & 0300 & 18.19 & 7,210 & & & & & & 1030 & 12.45 & 2,530 \\
\hline & 0600 & 19.23 & 8,270 & & 0600 & 15.31 & 4,760 & & 1100 & 13.77 & 3,260 \\
\hline & 0700 & 19.19 & 8,230 & & 0900 & 14.87 & 4,450 & & 1130 & 14.83 & 3,870 \\
\hline & 0800 & 19.84 & 8,940 & & 1200 & 13.68 & 3,660 & & 1200 & 15.47 & 4,280 \\
\hline & 0900 & 19.57 & 8,640 & & 1500 & 11.57 & 2,400 & & 1300 & 16.73 & 5,130 \\
\hline & 1400 & 20.07 & 9,210 & & 1800 & 10.62 & 1,880 & & 1400 & 17.89 & 6,030 \\
\hline & 1600 & 20.16 & 9,330 & & 2400 & 9.36 & 1,240 & & 1600 & 19.18 & 7,200 \\
\hline & 1930 & 20.45 & 9,700 & & & & & & 1800 & 19.80 & 7,820 \\
\hline & 2400 & 20.03 & 9,160 & Apr. 4 & 2400 & 8.93 & 935 & & 2400 & 20.47 & 8,540 \\
\hline \multirow[t]{2}{*}{18} & 0600 & 19.12 & 8,100 & 5 & 0500 & 8.95 & 942 & & 0300 & 20.72 & 8,810 \\
\hline & 0900 & $17.81\}$ & 6,800 & & 07001 & 9.07 & 990 & & 0630 & 21.001 & 9,120 \\
\hline
\end{tabular}


Gage height, in feet, and discharge, in cubic feet per second, at indicated time, 1965, of Middle River near Indianola, Iowa-Continued

\begin{tabular}{|c|c|c|c|c|c|c|c|c|c|c|c|}
\hline Date & Hour & $\begin{array}{l}\text { Gage } \\
\text { height }\end{array}$ & $\begin{array}{c}\text { Dis- } \\
\text { chargc }\end{array}$ & Date & Hour & $\begin{array}{c}\text { Gage } \\
\text { height }\end{array}$ & $\begin{array}{c}\text { Dis- } \\
\text { charge }\end{array}$ & Date & Hour & $\begin{array}{l}\text { Gage } \\
\text { height }\end{array}$ & $\begin{array}{c}\text { Dis- } \\
\text { charge }\end{array}$ \\
\hline \multirow[t]{10}{*}{ Apr. } & 0900 & 20.90 & 9,010 & \multirow[t]{10}{*}{ Apr. ? } & \multirow[t]{2}{*}{2400} & \multirow[t]{2}{*}{9.05} & \multirow[t]{2}{*}{980} & \multirow[t]{10}{*}{ Apr. 9} & 0300 & 15.55 & 4,320 \\
\hline & 1200 & 20.33 & 8,380 & & & & & & 0500 & 15.93 & 4,570 \\
\hline & 1600 & 19.01 & 7,030 & & 0430 & 8.88 & 913 & & 0700 & 15.54 & 4,320 \\
\hline & 2000 & 17.78 & 5,940 & & 0600 & 8.92 & 931 & & 0800 & 14.92 & 3,930 \\
\hline & 2200 & 16.80 & 5,180 & & 1000 & 9.97 & 1,370 & & 0900 & 13.75 & 3,240 \\
\hline & 2300 & 16.07 & 4,670 & & 1400 & 10.97 & 1,820 & & 1100 & 12.23 & 2,420 \\
\hline & 2400 & 14.83 & 3,870 & & 1700 & 11.85 & 2,230 & & 1400 & 10.98 & 1,830 \\
\hline & 0200 & 12.92 & 2,790 & & 1900 & 13.00 & 2,830 & & 1700 & 10.49 & 1,610 \\
\hline & 0500 & 11.43 & 2,030 & & 2200 & 14.02 & 3,390 & & 2400 & 9.83 & 1,310 \\
\hline & 1200 & 10.12 & 1,440 & & 2400 & 14.67 & 3,770 & & & & \\
\hline
\end{tabular}

(308) 5-4874.70 South River near Ackworth, Iowa

Location.-Lat $41^{\circ} 20^{\prime} 15^{\prime \prime}$, long $93^{\circ} 29^{\prime} 05^{\prime \prime}$, in $\operatorname{SE} \frac{1}{4} \mathrm{SE} \frac{1}{4}$ sec.34, T.76 N., R.23 W., on right bank $15 \mathrm{ft}$ downstream from county highway bridge, 2 miles southwest of Ackworth and 0.6 mile downstream from Otter Creek. Prior to Oct. 1, 1961, at site 3.8 miles downstream.

Drainage area.- $460 \mathrm{sq} \mathrm{mi}$. Prior to October 1, 1961, $474 \mathrm{sq} \mathrm{mi}$.

Gage-height record.-Water-stage recorder graph, except May 11-28, 30, when daily wire weight gage readings were available. Datum of gage is $769.97 \mathrm{ft}$ above mean sea level, datum of 1929 (levels by Corps of Engineers).

Discharge record.-Stage-discharge relation defined by current-meter measurements below 30,000 cfs. Backwater from ice Mar. 1-29.

Maxima.-March-May 1965: Discharge, 12,100 cfs 1030 hours Mar. 17 (gage height, $25.47 \mathrm{ft}$ ).

1940 to February 1965: Discharge, 34,000 cfs June 5, 1947 (gage height, $24.60 \mathrm{ft}$, at site 3.8 miles down stream at datum $8.01 \mathrm{ft}$ lower).

Flood in June 1930 reached a stage of $24.5 \mathrm{ft}$, from information by local residents (discharge, about $30,000 \mathrm{cfs}$ ), at site 3.8 miles down stream.

Cooperation.--One discharge measurement furnished by Corps of Engineers.

Mean discharge, in cubic feet per second, 1965

\begin{tabular}{|c|c|c|c|c|c|c|c|c|c|c|c|}
\hline Day & March & April & May & Day & March & April & May & Day & March & April & May \\
\hline & 250 & 1,640 & 195 & $11 \ldots$ & 45 & 2,030 & 199 & 21. & 150 & 96 & 63 \\
\hline 20 & 100 & 542 & 156 & $12_{\ldots}$ & 35 & 668 & 156 & 22. & 100 & 84 & 73 \\
\hline 3. & 150 & 460 & 120 & $13 \ldots$ & 40 & 350 & 125 & 23. & 70 & 79 & 96 \\
\hline 4. & 120 & 671 & 250 & $14 \ldots$ & 150 & 288 & 106 & 24 & 64 & 321 & 88 \\
\hline 5. & 90 & 2,810 & 262 & $15 \ldots$ & 700 & 312 & 153 & 25 & 58 & 3,350 & 82 \\
\hline 6. & 80 & 3,680 & 203 & $16 \ldots$ & 1,700 & 249 & 300 & 26. & 90 & 1,320 & 239 \\
\hline 7. & 70 & 863 & 135 & 17. & 10,000 & 189 & 143 & 27. & 170 & 624 & 195 \\
\hline 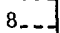 & 62 & 3,400 & 1,560 & 18. & 3,000 & 149 & 96 & 28. & 900 & 719 & 99 \\
\hline 9. & 56 & 2,700 & 1,550 & $19 \ldots$ & 400 & 127 & 75 & 29 & 1,400 & 372 & 72 \\
\hline 10. & 50 & 1,000 & 365 & $20 \ldots$ & 200 & 106 & 66 & 30. & 1,580 & 251 & 237 \\
\hline & & & & & & & & & 2,010 & $-\ldots-n$ & 181 \\
\hline \multicolumn{9}{|c|}{ Monthly mean discharge, in cubic feet per second } & 771 & 982 & 246 \\
\hline \multicolumn{9}{|c|}{ Runoff, in inches } & 1.93 & 2.38 & 0.62 \\
\hline \multicolumn{9}{|c|}{ Runoff, in acre-feet } & 47,390 & 58,410 & 15,150 \\
\hline
\end{tabular}


Gage height, in feet, and discharge, in cubic feet per second, at indicated time, 1965 , of South River near Ackworth, Iowa

\begin{tabular}{|c|c|c|c|c|c|c|c|c|c|c|c|}
\hline Date & llour & $\begin{array}{l}\text { Gage } \\
\text { height }\end{array}$ & $\begin{array}{c}\text { Dis- } \\
\text { charge }\end{array}$ & Date & IIour & $\begin{array}{c}\text { Gage } \\
\text { height }\end{array}$ & $\begin{array}{c}\text { Dis- } \\
\text { charge }\end{array}$ & Date & Hour & $\begin{array}{c}\text { Gage } \\
\text { height }\end{array}$ & $\begin{array}{c}\text { Dis- } \\
\text { charge }\end{array}$ \\
\hline Mar. 16 & $\begin{array}{l}0000 \\
0100 \\
0230 \\
1000 \\
1400 \\
1500 \\
1800 \\
2100 \\
2400 \\
0200 \\
0400 \\
0600 \\
0700 \\
0900 \\
1030 \\
1300 \\
1400 \\
1500 \\
1600 \\
1900 \\
2100 \\
2400\end{array}$ & $\begin{array}{l}11.10 \\
11.25 \\
11.76 \\
10.94 \\
10.85 \\
10.87 \\
12.00 \\
13.30 \\
15.05 \\
18.80 \\
21.90 \\
22.65 \\
24.70 \\
25.33 \\
25.47 \\
25.30 \\
25.06 \\
25.10 \\
24.98 \\
24.77 \\
24.38 \\
22.40\end{array}$ & 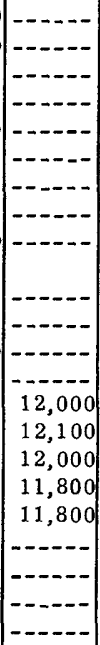 & Apr. 4 & $\begin{array}{l}0200 \\
0400 \\
0600 \\
0800 \\
1000 \\
1400 \\
1800 \\
2100 \\
2400 \\
2400 \\
0300 \\
0800 \\
0900 \\
1000 \\
1300 \\
1900 \\
2400 \\
0600 \\
1000 \\
1400\end{array}$ & $\begin{array}{r}20.35 \\
17.55 \\
15.35 \\
13.70 \\
12.63 \\
11.37 \\
10.98 \\
10.65 \\
9.83 \\
7.22 \\
\\
7.19 \\
7.37 \\
10.65 \\
13.00 \\
14.52 \\
16.01 \\
16.52 \\
16.62 \\
15.55 \\
13.87\end{array}$ & $\begin{array}{r}536 \\
528 \\
581 \\
1,780 \\
2,950 \\
3,760 \\
4,660 \\
5,010 \\
5,080 \\
4,380 \\
3,380\end{array}$ & Apr. 6 & $\begin{array}{l}0600 \\
1200 \\
1800 \\
2400 \\
0500 \\
0800 \\
1000 \\
1200 \\
1400 \\
1600 \\
1700 \\
1930 \\
2200 \\
2400 \\
0600 \\
1000 \\
1400 \\
1800 \\
2400\end{array}$ & $\begin{array}{r}8.73 \\
8.12 \\
7.57 \\
7.26 \\
7.13 \\
9.00 \\
12.00 \\
14.15 \\
16.10 \\
17.65 \\
18.30 \\
18.57 \\
17.65 \\
16.80 \\
14.38 \\
13.22 \\
11.42 \\
9.68 \\
8.57\end{array}$ & $\begin{array}{r}1,010 \\
806 \\
641 \\
548 \\
512 \\
1,120 \\
2,450 \\
3,540 \\
4,720 \\
5,800 \\
6,260 \\
6,450 \\
5,800 \\
5,210 \\
3,680 \\
3,060 \\
2,160 \\
1,390 \\
954\end{array}$ \\
\hline
\end{tabular}

(309) 5-4879.8 White Breast Creek near Dallas, Iowa

Location.-Lat $41^{\circ} 14^{\prime} 45^{\prime \prime}$, long $93^{\circ} 15^{\prime} 50^{\prime \prime}$, in NE $\frac{1}{4} N W^{\frac{1}{4}}$ sec.3, T.74 N., R.21 W., on left bank $15 \mathrm{ft}$ downstream from county highway bridge, $\frac{1}{2}$ mile downstream from Kirk Branch, and $2 \frac{1}{4}$ miles northwest of Dallas.

\section{Drainage area. $-342 \mathrm{sq} \mathrm{mi}$.}

Gage-height record.-Water-stage recorder graph, except April 29 to May 1. Oncedaily tape gage readings available for this period. Datum of gage is $759.12 \mathrm{ft}$ above mean sea level, datum of 1929 (Corps of Engineers bench mark).

Discharge record.-Stage-discharge relation defined by current-meter measurements below 6,400 cfs. Backwater from ice Mar. 1-16, 19-30.

Maxima.-March-May 1965: Discharge, 6,640 cfs 1100 hours Mar. 17 (gage height, $22.49)$.

1962 to February 1965: Discharge, 12,000 cfs (estimated) June 11, 1962 (gage height, $28.87 \mathrm{ft}$, from flood mark).

Flood of June 6, 1947, may have been slightly higher than the flood of June 11, 1962.

Cooperation.- One discharge measurement furnished by Corps of Engineers. 
Mean discharge, in cubic feet per second, 1965, of White Breast Creek near Dalias, Iowa

\begin{tabular}{|c|c|c|c|c|c|c|c|c|c|c|c|}
\hline Day & March & April & May & Day & March & April & May & Day & March & April & May \\
\hline & 450 & 985 & 142 & $11 \ldots$ & 66 & 2,070 & 163 & 21. & 200 & 112 & 38 \\
\hline & 350 & 492 & 127 & $12 \ldots$ & 60 & 866 & 112 & 22. & 150 & 98 & 38 \\
\hline & 270 & 439 & 107 & $13 \ldots$ & 70 & 316 & 86 & 23 & 130 & 91 & 40 \\
\hline & 220 & 638 & 370 & 14 & 150 & 270 & 71 & 24 & 110 & 177 & 39 \\
\hline & 180 & 2,680 & 156 & $15 \ldots$ & 500 & 548 & 100 & $25 \ldots$ & 90 & 2,390 & 41 \\
\hline & 150 & 3,130 & 119 & $16 \ldots$ & 1,100 & 383 & 116 & $26 \ldots$ & 80 & 1,590 & 114 \\
\hline $1-$ & 120 & 1,780 & 94 & $17--$ & 5,540 & 244 & 119 & 27 & 140 & 521 & 130 \\
\hline & 100 & 2,150 & 131 & $18 \ldots$ & 2,380 & 184 & 69 & 28 & 300 & 481 & 78 \\
\hline 9 & 85 & 2,010 & 899 & $19 \ldots$ & 700 & 148 & 47 & 29 & 700 & 280 & 45 \\
\hline \multirow[t]{2}{*}{$10_{-}-$} & 75 & 1,130 & 738 & $20 \ldots$ & 350 & 126 & 41 & $30 \ldots$ & 850 & 200 & 40 \\
\hline & & & & & & & & & 1,040 & $-\cdots$ & 120 \\
\hline \multicolumn{9}{|c|}{ Monthly mean discharge, in cubic feet per second } & 539 & 884 & 146 \\
\hline \multicolumn{9}{|c|}{ Runoff, in inches } & 1.82 & 2.88 & 0.49 \\
\hline \multicolumn{9}{|c|}{ Runoff, in acre-feet } & 33,140 & 52,620 & 8,990 \\
\hline
\end{tabular}

Gage height, in fret, and discharge, in cubic feet per second, al indacaled time, 1965

\begin{tabular}{|c|c|c|c|c|c|c|c|c|c|c|c|}
\hline Date & Ilour & $\begin{array}{l}\text { Gage } \\
\text { height }\end{array}$ & $\begin{array}{c}\text { Dis- } \\
\text { charge }\end{array}$ & Date & Hour & $\begin{array}{c}\text { Gage } \\
\text { height }\end{array}$ & $\begin{array}{c}\text { Dis- } \\
\text { charge }\end{array}$ & Datc & Hour & $\begin{array}{l}\text { Gage } \\
\text { height }\end{array}$ & $\begin{array}{c}\text { Dis- } \\
\text { charge }\end{array}$ \\
\hline \multirow[t]{12}{*}{ Mar. 15} & 0000 & 7.91 & - & \multirow[t]{19}{*}{ Mar. 18} & 2100 & 13.46 & 2,080 & \multirow[t]{37}{*}{ Apr. 6} & 0800 & 16.42 & 3,360 \\
\hline & 0400 & 8.00 & $\ldots-\ldots$ & & 2400 & 13.52 & 2,110 & & 1200 & 15.82 & 3,060 \\
\hline & 1000 & 8.23 & $-\ldots$ & & & & & & 1700 & 14.67 & 2,570 \\
\hline & 1100 & 8.47 & -...- & & 0300 & 13.57 & 2,130 & & 2100 & 14.19 & 2,380 \\
\hline & 1300 & 8.39 & ----- & & 1000 & 13.42 & 2,070 & & 2400 & 14.09 & 2,340 \\
\hline & 1500 & 8.49 & - & & 1600 & 13.32 & $-\cdots---$ & & & & \\
\hline & 1700 & 9.30 &..--- & & 2000 & 13.37 & $---\cdots$ & & 0800 & 13.96 & 2,280 \\
\hline & 1900 & 10.23 & -.-.-. & & 2400 & 12.80 & $\cdots-\cdots$ & & $1 C 00$ & 13.89 & 2,260 \\
\hline & 2000 & 10.56 & - & & & & & & 1200 & 13.64 & 2,160 \\
\hline & 2200 & 10.78 & & & 0500 & 10.55 & $--m-n$ & & 1400 & 12.82 & 1,830 \\
\hline & 2400 & 10.84 & & & 0800 & 8.95 & $-\ldots$ & & 1700 & 11.09 & 1,300 \\
\hline & & & & & 1000 & 8.37 & $1--0-0$ & & 2100 & 9.72 & 886 \\
\hline \multirow[t]{10}{*}{16} & 0300 & 10.97 & $-\infty-\infty$ & & 1200 & 8.00 & $--n-n$ & & 2400 & 8.88 & 670 \\
\hline & 0600 & 10.94 &.---- & & 1400 & 7.67 & $--m--\infty$ & & & & \\
\hline & 1030 & 10.83 & $\ldots-.-$ & & 1700 & 7.47 & $-\cdots-n$ & & $0 乞 00$ & 8.55 & 588 \\
\hline & 1300 & 11.07 & - . - - & & 2000 & 7.42 & $-\cdots-n$ & & 0400 & 8.49 & 572 \\
\hline & 1400 & 10.86 &.---1 & & 2200 & 7.41 & $-\cdots-n$ & & 0500 & 8.48 & 570 \\
\hline & 1700 & 12.84 & $-\cdots$ & & 2400 & 7.31 & $-\cdots---$ & & 0600 & 8.62 & 605 \\
\hline & 2000 & 13.79 & 2,220 & & & & & & 0700 & 9.01 & 702 \\
\hline & 2200 & 14.14 & 2,360 & \multirow[t]{19}{*}{ Apr. } & 2400 & 8.60 & 600 & & 0730 & 10.00 & 970 \\
\hline & 2400 & 15.60 & 2,950 & & & & & & 0800 & 11.30 & 1,360 \\
\hline & & & & & 0400 & 8.39 & 548 & & .0900 & 12.60 & 1,750 \\
\hline \multirow[t]{11}{*}{17} & 0130 & 18.00 & 4,150 & & 0500 & 8.37 & 542 & & 1000 & 13.70 & 2,180 \\
\hline & 0300 & 20.65 & 5,540 & & 0600 & 8.42 & 555 & & 1100 & 14.68 & 2,570 \\
\hline & 0400 & 21.39 & 5,980 & & 0700 & 9.74 & 892 & & 1300 & 15.45 & 2,880 \\
\hline & 0600 & 21.84 & 6,250 & & 0800 & 11.24 & 1,340 & & 1700 & 16.38 & 3,340 \\
\hline & 1000 & 22.44 & 6,610 & & 1000 & 13.62 & 2,150 & & 1800 & 16.43 & 3,360 \\
\hline & 1100 & 22.49 & 6,640 & & 1200 & 15.72 & 3,010 & & 2000 & 16.42 & 3,360 \\
\hline & 1200 & 22,41 & 6,600 & & 1400 & 17.22 & 3,760 & & 2030 & 16.16 & 3,230 \\
\hline & 1400 & 21.91 & 6,300 & & 1530 & 17.41 & 3,860 & & 2400 & 15.48 & 2,890 \\
\hline & 1700 & 20.65 & 5,540 & & 1730 & 17.37 & 3,840 & & & & \\
\hline & 2400 & 17.38 & 3,840 & & 1900 & 19.12 & 4,710 & & 0300 & 15.03 & 2,710 \\
\hline & & & & & 2000 & 19.43 & 4,860 & & 0800 & 13.71 & 2,180 \\
\hline \multirow[t]{5}{*}{18} & 0500 & 15.12 & 2,750 & & 2100 & 19.41 & 4,860 & & 1300 & 13.00 & 1,900 \\
\hline & 0900 & 13.78 & 2,210 & & 2300 & 19.21 & 4,760 & & 1800 & 12.35 & 1,680 \\
\hline & 1100 & 13.37 & 2,050 & & 2400 & 18.92 & 4,610 & & 2400 & 10.65 & 1,160 \\
\hline & 1300 & 13.22 & 1,990 & & & & & & & & \\
\hline & $1630 \mid$ & 13.20 & 1,980 & & 0500 & 17.05 & 3,680 & 10 & 0400 & 9.53 & 832 \\
\hline
\end{tabular}


Gage height, in feet, and discharge, in cubic feet per second, at indicated time, 1965, of Whitebreast Creek near Dallas, lowa-Continued

\begin{tabular}{|c|c|c|c|c|c|c|c|c|c|c|c|}
\hline Dite & IIour & $\begin{array}{l}\text { Gage } \\
\text { height }\end{array}$ & $\left|\begin{array}{c}\text { Dis - } \\
\text { charge }\end{array}\right|$ & Date & Ilour & $\begin{array}{l}\text { Gage } \\
\text { height }\end{array}$ & $\begin{array}{c}\text { Dis- } \\
\text { charge }\end{array}$ & Date & IIour & $\begin{array}{c}\text { Gage } \\
\text { height }\end{array}$ & $\begin{array}{c}\text { Dis- } \\
\text { charge }\end{array}$ \\
\hline pr. 10 & $\begin{array}{l}0600 \\
1000 \\
1730 \\
1900 \\
2000 \\
2100 \\
2300 \\
2400\end{array}$ & $\begin{array}{r}9.16 \\
9.37 \\
9.00 \\
10.10 \\
12.50 \\
13.90 \\
15.65 \\
16.06\end{array}$ & $\begin{array}{r}740 \\
792 \\
700 \\
1,000 \\
1,720 \\
2,260 \\
2,980 \\
3,180\end{array}$ & pr. 11 & $\begin{array}{l}0130 \\
0200 \\
0300 \\
0600 \\
0800 \\
1000 \\
1800 \\
2400\end{array}$ & $\begin{array}{l}16.18 \\
16.13 \\
15.25 \\
13.65 \\
13.20 \\
13.00 \\
12.64 \\
12.19\end{array}$ & $\begin{array}{l}3,240 \\
3,220 \\
2,800 \\
2,160 \\
1,980 \\
1,900 \\
1,760 \\
1,630\end{array}$ & pr. 12 & $\begin{array}{l}0300 \\
0600 \\
0900 \\
1200 \\
1500 \\
1800 \\
2100 \\
2400\end{array}$ & $\begin{array}{r}11.60 \\
10.76 \\
9.83 \\
9.13 \\
8.66 \\
8.29 \\
8.00 \\
7.77\end{array}$ & $\begin{array}{r}1,450 \\
1,200 \\
919 \\
732 \\
615 \\
523 \\
465 \\
419\end{array}$ \\
\hline
\end{tabular}

(310) 5-4885. Des Moines River near Tracy, Iowa

Location.-Lat $41^{\circ} 16^{\prime} 55^{\prime \prime}$, long $92^{\circ} 51^{\prime} 30^{\prime \prime}$, in NW $\frac{1}{4} \operatorname{SE} \frac{1}{4}$ sec.19, T.75 N., R.17 W., on right bank $250 \mathrm{ft}$ upstream from abandoned Bellefountaine Bridge, 0.5 mile downstram from bridge on State Highway $92,0.8$ mile east of Tracy, 3.1 miles upstream from Cedar Creek, and 6.4 miles downstream from English Creek, and at mile 130.3.

Drainage area. $-12,479 \mathrm{sq} \mathrm{mi}$.

Gage-height record.-Water-stage recorder graph except Mar. 1-5, 17-23, 24. Daily outside readings available for these periods. Datum of gage is $670.91 \mathrm{ft}$ above mean sea level, datum of 1929 .

Discharge record.-Stage-discharge relation defined by current-meter measurements below 155,000 cfs. Backwater from ice Mar. 1-15.

Maxima.-March-May 1965: Discharge, 77,300 cfs 1930 hours Apr. 11 (gage height, $23.17 \mathrm{ft}$ ).

1920 to February 1965: Discharge 155,000 cfs June 14, 1947 (gage height, $23.5 \mathrm{ft}$ ).

Maximum stage known since 1881, that of June 14, 1947. Flood of May 31, 1903, reached a stage of about $25 \mathrm{ft}$ (discharge, about $130,000 \mathrm{cfs}$ ).

Cooperation.-Three discharge measurements furnished by Corps of Engineers.

Mean discharge, in cubic feet per second, 1965

\begin{tabular}{|c|c|c|c|c|c|c|c|c|c|c|c|}
\hline Day & March & April & May & Day & March & April & May & Day & March & April & May \\
\hline & 000 & 7,800 & 15,000 & 120 & 5,000 & 76 , & 20 & $10=$ & 100 & 24,600 &, 800 \\
\hline 2 & 6,000 & 22,900 & 13,700 & 12 & 4,500 & 75 , & 40 & 22. & 13,100 & 20,700 & 320 \\
\hline & 8,400 & 25,100 & 12,600 & $13 \ldots$ & 4,300 & 69,700 & 8,620 & 23. & 9,420 & 18,200 & 8,180 \\
\hline & 11,000 & 26,600 & 11,500 & $14 \ldots$ & 5,000 & 62,400 & 8,560 & 24. & 6,920 & 17,200 & 8,060 \\
\hline & 15,000 & 29,500 & 11,400 & $15 \ldots$ & 7,600 & 54,200 & 8,200 & 25 & 5,570 & 21,600 & 8,120 \\
\hline & 16,000 & 39,400 & 10,200 & $16 \ldots$ & 16,700 & 47,000 & 8,000 & 26 & 4,840 & 26,500 & 8,800 \\
\hline & 13,000 & 48,600 & 9,440 & $17 \ldots$ & 28,300 & 41,400 & 7,760 & $27-$ & 4,470 & 20,800 & 11,300 \\
\hline & 11,000 & 55,900 & 9,000 & $18 \ldots$ & 38,400 & 37,300 & 7,660 & 28 & 5,660 & 00 & 15,000 \\
\hline & 8,000 & 66,800 & 10,700 & $19 \ldots$ & 38,200 & 33,300 & 8,380 & 25 & 8,340 & 18, & 00 \\
\hline & 5,400 & 75,300 & 10,900 & 20 & 33,900 & 28,900 & 9,020 & 30 & 9,400 & 16,300 & 19,900 \\
\hline & & & & & & & & & 12,300 & & 20,800 \\
\hline \multicolumn{9}{|c|}{ Monthly mean discharge, in cubic feet per second } & 12,250 & 37,890 & 10,760 \\
\hline \multicolumn{9}{|c|}{ Runoff, in inches } & 1.13 & 3.39 & 0.99 \\
\hline \multicolumn{9}{|c|}{ Runoff, in acre-feet } & 753,200 & $2,255,000$ & 661,400 \\
\hline
\end{tabular}


Gage height, in feet, and discharge, in cubic feet per second, at indicated time, 1965, of Des Moines River at Tracy. Iowa

\begin{tabular}{|c|c|c|c|c|c|c|c|c|c|c|c|}
\hline Date & Ilour & $\begin{array}{c}\text { Gage } \\
\text { height }\end{array}$ & $\begin{array}{c}\text { Dis- } \\
\text { charge }\end{array}$ & Date & Hour & $\begin{array}{c}\text { Gage } \\
\text { height }\end{array}$ & $\begin{array}{c}\text { Dis- } \\
\text { charge }\end{array}$ & Date & rr & $\begin{array}{c}\text { Gage } \\
\text { height }\end{array}$ & $\begin{array}{c}\text { Dis- } \\
\text { charge }\end{array}$ \\
\hline pr. 1 & 0000 & 11.17 & 14,400 & Apr. 5 & 1100 & 15.74 & 28,400 & Apr. 9 & 1000 & 22.28 & 67,200 \\
\hline & 0300 & 11.22 & 14,500 & & 1300 & 15.88 & 28,900 & & 1800 & 22.69 & 71,700 \\
\hline & 1300 & 12.70 & 18,400 & & 1800 & 16.41 & 30,900 & & 2100 & 22.65 & 71,200 \\
\hline & 2000 & 13.35 & 20,300 & & 2400 & 17.09 & 33,700 & & 2400 & 22.87 & 73,700 \\
\hline & 2400 & .65 & 21,300 & & & & & & & & \\
\hline & & & & 6 & 0300 & 17.65 & 36,200 & 10 & 0400 & 23.02 & 75,500 \\
\hline 2 & 0400 & 13.86 & 22,000 & & 2200 & 19.06 & 43,200 & & 0800 & 23.07 & 76,100 \\
\hline & 1200 & 14.18 & 23,000 & & 2400 & 19.22 & 44,100 & & 1800 & 22.98 & 75,100 \\
\hline & 2400 & 14.54 & 24,200 & & & & & & 2400 & 22.98 & 75,100 \\
\hline & & & & 7 & 1200 & 20.02 & 48,900 & & & & \\
\hline 3 & 0400 & 14.65 & 24,600 & & 2400 & 20.54 & 52,600 & 11 & 0600 & 23.01 & 75,400 \\
\hline & 1200 & 14.83 & 25,200 & & & & & & 1600 & 23.16 & 200 \\
\hline & 1800 & 14.91 & 25,500 & 8 & 0500 & 20.88 & 55,000 & & 1930 & 23.17 & 77,300 \\
\hline & 2400 & 14.98 & 25,700 & & 0800 & 21.00 & 55,800 & & 2400 & 23.15 & 77,100 \\
\hline & & & & & 1200 & 21.01 & 55,900 & & & & \\
\hline 4 & 1200 & 15.22 & 26,600 & & 1900 & 21.11 & 56,700 & 12 & 1300 & 23.05 & 75,900 \\
\hline & 1800 & 15.35 & 27,000 & & 2100 & 21.20 & 57,400 & & 2400 & 22,81 & 73,000 \\
\hline & 2400 & 15.45 & 27,400 & & 2400 & 21.39 & 58,900 & & & & \\
\hline 5 & 0600 & 15,59 & 27.900 & 9 & 0800 & 21831 & 62800 & 13 & $\begin{array}{l}1200 \\
2400\end{array}$ & $\begin{array}{l}22.51 \\
22.20\end{array}$ & $\begin{array}{l}69,700 \\
66,400\end{array}$ \\
\hline & & & & & & & & & & & \\
\hline
\end{tabular}

(311) 5-4890. Cedar Creek near Bussey, Iowa

Location.-Lat $41^{\circ} 13^{\prime} 10^{\prime \prime}$, long $92^{\circ} 54^{\prime} 25^{\prime \prime}$, on south line in SW $\frac{1}{4} \mathrm{SW} \frac{1}{4}$ sec.11, T.74 N., R.18 W., on left bank at downstream side of bridge on State Highway $156,1.6$ miles northwest of Bussey, and 8.9 miles upstream from mouth.

Drainage area.- $-374 \mathrm{sq} \mathrm{mi}$.

Gage-height record.-Water-stage recorder graph, except Mar. 20-23. Datum of gage is $682.15 \mathrm{ft}$ above mean sea level, datum of 1929 (levels by Corps of Engineers).

Discharge record.-Stage-discharge relation defined by current-meter measurements below 28,000 cfs. Backwater from ice Mar. 1-16, 19-28.

Maxima.-March-May 1965: Discharge, 6,500 cfs 0100 hours Mar. 18 (gage height, $20.51 \mathrm{ft}$ ).

1947 to February 1965: Discharge, 29,300 cfs May 9, 1950; gage height, $28.06 \mathrm{ft}$ July 2, 1958.

Mean discharge, in cuble feet per second, 1965

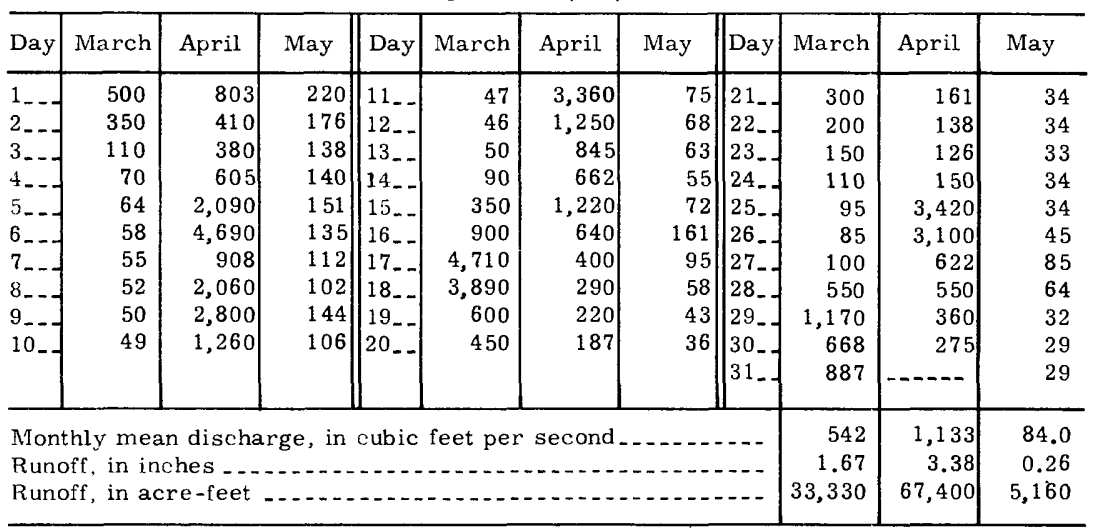


Gage height, in feet, and discharge, in cubic feet per second, at indicated time, 1965, of Cedar Creek near Bussev, Iowa

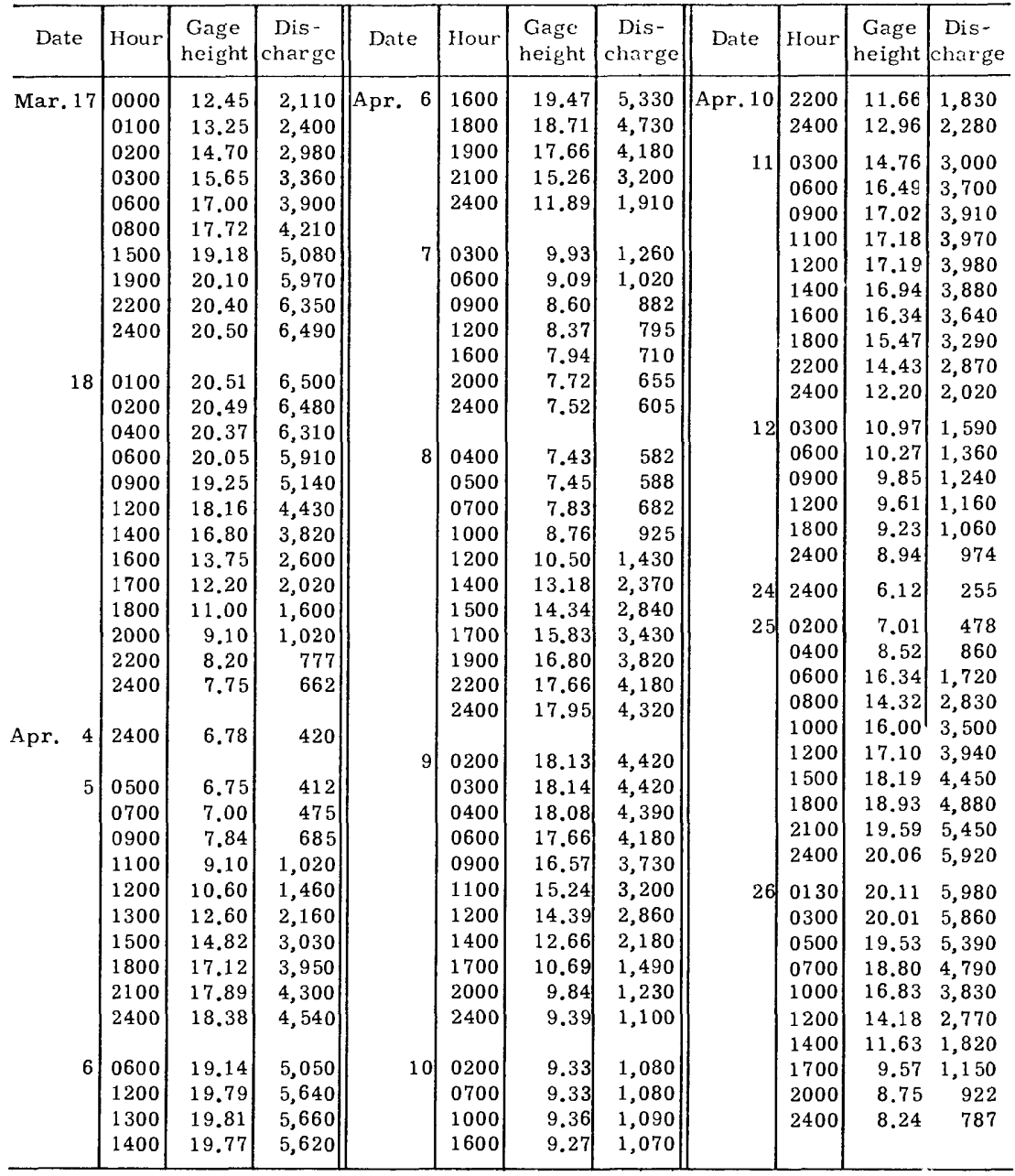


(312) 5-4895. Des Moines River at Ottumwa, Iowa

Location.-Lat $41^{\circ} 00^{\prime} 40^{\prime \prime}$, long $92^{\circ} 24^{\prime} 40^{\prime \prime}$, in $\mathrm{SE} \frac{1}{4} \mathrm{NE} \frac{1}{4}$ sec.25, T.72 N., R.14 W., on right bank $15 \mathrm{ft}$ downstream from Wabash Railroad Bridge at Ottumwa, 6.5 miles upstream from Village Creek, 9.5 miles downstream from South Avery Creek, and at mile 94.1.

Drainage area. $-13,374 \mathrm{sq} \mathrm{mi}$

Gage-height record.-Water-stage recorder graph. Datum of gage is $622.00 \mathrm{ft}$ above mean sea level, datum of 1929.

Discharge record.-Stage-discharge relation defined by current-meter measurements below 130,000 cfs. Backwater from ice. Mar. 3, 10-13.

Maxima.-March-May 1965: Discharge, 78,600 cfs 0800 hours Apr. 11 (gage height, $18.33 \mathrm{ft})$.

1917 to February 1965: Discharge, 135,000 cfs June 7, 1947 (gage height, $20.2 \mathrm{ft}$, at site 1,100 ft downstream at Vine Street Bridge at datum $0.77 \mathrm{ft}$ higher).

Flood of June 7, 1947 is the highest stage known since 1850. Flood of May 31, 1903, reached a stage of $19.4 \mathrm{ft}$, former site and datum at Vine Street Bridge or about $22 \mathrm{ft}$ at Market Street Bridge, from information by Corps of Engineers and U.S. Weather Bureau (discharge estimated as 140,000).

Cooperation.-Three discharge measurements furnished by Corps of Engineers.

Mean discharge, in cublc feet per second, 1965

\begin{tabular}{|c|c|c|c|c|c|c|c|c|c|c|c|}
\hline Day & March & April & May & Day & March & April & May & Day & March & April & May \\
\hline & 620 & 00 & & $1-$ & 00 & 77,600 & 9,670 & & 0 & 00 & 9,10 \\
\hline & 5,820 & 22,000 & 14,500 & 12 & 5,000 & 77,300 & 8,650 & $22=$ & 00 & 100 & 8,710 \\
\hline 3. & 7,200 & 25,500 & 13,300 & 13 & 4,650 & 74,000 & 8,200 & $23 \ldots$ & 12,400 & 19,300 & 8,350 \\
\hline & 9,790 & 26,000 & 12,200 & $14 \ldots$ & 5,350 & 67,400 & 7,570 & 24. & 8,530 & 18,500 & 8,290 \\
\hline & 12,400 & 28,400 & 12,300 & $15 \ldots$ & 6,950 & 60,200 & 7,990 & 25 & 6,490 & 24,500 & 8,260 \\
\hline & 14,900 & 36,100 & 11,200 & $16 \ldots$ & 12,200 & 51,600 & 7,930 & 26 & 5,680 & 29,200 & 8,890 \\
\hline & 16,200 & 41,000 & 9,910 & $17 \ldots$ & 34,600 & 44,200 & 8,050 & 27 & 4,700 & 00 & 10,600 \\
\hline 8. & 13,500 & 47,900 & 9,250 & $18 \ldots$ & 31,800 & 38,600 & 7,600 & 28 & 6,100 & 00 & 14,300 \\
\hline & 9,350 & 53,900 & 9,790 & 19 & 35,100 & 33,800 & 7,960 & 29. & 10,200 & 19,300 & 18,100 \\
\hline & 6,000 & 67,500 & 11,600 & $20 \ldots$ & 34,400 & 30,000 & 8,950 & $30 \ldots$ & 11,200 & 17,600 & 20,400 \\
\hline & & & & & & & & & 12,100 & $-\ldots$ & 21,400 \\
\hline \multicolumn{9}{|c|}{ 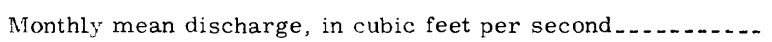 } & & & 0,940 \\
\hline \multicolumn{9}{|c|}{ Runoff, in inches } & 1.10 & 3.17 & 0.94 \\
\hline \multicolumn{9}{|c|}{ Ruinoff, in acre -feet } & 784,300 & $\approx, 262,000$ & 672,400 \\
\hline
\end{tabular}

Gage height, in feet, and disiharge, in cubic feet per second, at indicated time, 1965

\begin{tabular}{|c|c|c|c|c|c|c|c|c|c|c|c|}
\hline Date & Hour & $\begin{array}{c}\text { Gage } \\
\text { height }\end{array}$ & $\begin{array}{c}\text { Dis- } \\
\text { charge }\end{array}$ & Date & Hour & $\begin{array}{l}\text { Gage } \\
\text { height }\end{array}$ & $\begin{array}{c}\text { Dis- } \\
\text { charge }\end{array}$ & Date & HIour & $\begin{array}{l}\text { Gage } \\
\text { height }\end{array}$ & $\begin{array}{c}\text { Dis- } \\
\text { charge }\end{array}$ \\
\hline Apr. 10 & 0000 & 16.81 & 59,900 & Apr. 11 & 1200 & 18.27 & 77,800 & Apr.13 & 1230 & 17.97 & 74,500 \\
\hline & 0800 & 17.31 & 65,200 & & 2400 & 18.21 & 77,100 & & 2400 & 17.70 & 70,500 \\
\hline & 1800 & 17.72 & 70,700 & & & & & & & & \\
\hline & 2400 & 18.14 & 76,300 & 12 & 1200 & 18.27 & 77,800 & 14 & $24 ! 0$ & 17.20 & 64,200 \\
\hline 11 & 0800 & 18.33 & 78,600 & & 2400 & 18.14 & 76,300 & & & & \\
\hline
\end{tabular}


(313) 5-4905. Des Moines River at Keosauqua, Iowa

Location.-Lat $40^{\circ} 43^{\prime} 45^{\prime \prime}$, long $91^{\circ} 57^{\prime} 45^{\prime \prime}$, in $\mathrm{SE} \frac{1}{4} \mathrm{SW} \frac{1}{4}$ sec. 36 , T.69 N., R.10 W., on right bank $10 \mathrm{ft}$ upstream from bridge on State Highway 1 at Keosauqua, 4.0 miles downstream from Chequest Creek, and at mile 51.3 (revised).

Drainage area. $-14,038 \mathrm{sq} \mathrm{mi}$.

Gage-height record.-Water-stage recorder graph. Datum of gage is $557.36 \mathrm{ft}$ above mean sea level, datum of 1929.

Discharge record. - Stage-discharge relation defined by current-meter measurements below $125,000 \mathrm{cfs}$. Backwater from ice Mar, 1-18.

Maxima.-March-May: Discharge, 79,800 cfs 0400 hours Apr. 12 (gage height, $19.36 \mathrm{ft}$ ). 1903-06, 1910 to February 1965: Discharge, 146,000 cfs June 1, 1903 (gage height, $27.85 \mathrm{ft}$, from floodmark).

Flood of June 1, 1851, reached a stage of $24 \mathrm{ft}$ (discharge not determined).

Cooperation.-Two discharge measurements furnished by Corps of Engineers.

Mean discharge, in cubic feet per second, 1965

\begin{tabular}{|c|c|c|c|c|c|c|c|c|c|c|c|}
\hline Day & March & April & May & Day & March & April & May & Day & March & April & May \\
\hline & 700 & 15,000 &, 100 & $1-$ & 7,000 & 74,600 & 10,700 & $1^{21}$ & 33,300 & 27,900 & 8,880 \\
\hline & 5,000 & 20,700 & 14,500 & 122 & 5,500 & 79,200 & 8,740 & 22. & 19,100 & 23,400 & 8,990 \\
\hline & 7,500 & 24,700 & 13,300 & 13 & 5,000 & 77,400 & 8,180 & 23. & 14,100 & 19,900 & 8,260 \\
\hline & 9,500 & 26,700 & 12,200 & 14 & 6,000 & 73,800 & 8,350 & 24. & 9,950 & 18,200 & 8,120 \\
\hline & 12,000 & 30,200 & 11,600 & 15. & 8,000 & 72,500 & 8,380 & 25. & 7,120 & 24,000 & 8,070 \\
\hline & 15,000 & 43,400 & 11,500 & $16 \ldots$ & 12,000 & 63,000 & 8,100 & 26. & 6,050 & 31,100 & 8,290 \\
\hline & 17,000 & 43,400 & 10,100 & $17 \ldots$ & 35,000 & 53,400 & 8,010 & 27 & 5,320 & 28 & 9,720 \\
\hline & 15,000 & 53,300 & 9,270 & 18. & 36,000 & 44,800 & 7,790 & 28. & 5,070 & 20,800 & 12,300 \\
\hline & 11,000 & 59,900 & 9,080 & 19. & 38,200 & 38,100 & 7,560 & 29. & 9,240 & 19,400 & 16,400 \\
\hline & 9,000 & 61,800 & 10,800 & 20 & 38,200 & 32,800 & 8,240 & 30 & 11,200 & 17,900 & 19,100 \\
\hline & & & & & & & & & 11,400 & $\ldots$ & 20,500 \\
\hline \multirow{3}{*}{\multicolumn{9}{|c|}{$\begin{array}{l}\text { Jonthly mean discharge, in cubic feet per second } \\
\text { Rut:off, in inches } \\
\text { Ru, }\end{array}$}} & 13,760 & 40,650 & 10,680 \\
\hline & & & & & & & & & 1.13 & 3.23 & 0.88 \\
\hline & & & & & & & & & 845,900 & $2,419,000$ & 656,800 \\
\hline
\end{tabular}

Gage height, in fect, and discharge, in cubic fect per second, at indicated time, 1965

\begin{tabular}{|c|c|c|c|c|c|c|c|c|c|c|c|}
\hline Date & Hour & $\begin{array}{l}\text { Gage } \\
\text { height }\end{array}$ & $\begin{array}{c}\text { Dis- } \\
\text { charge }\end{array}$ & Date & Hour & $\begin{array}{l}\text { Gage } \\
\text { height }\end{array}$ & $\begin{array}{c}\text { Dis- } \\
\text { charge }\end{array}$ & Date & Hlour & $\begin{array}{l}\text { Gage } \\
\text { height }\end{array}$ & $\begin{array}{l}\text { Dis- } \\
\text { charge }\end{array}$ \\
\hline \multirow[t]{5}{*}{ Apr. 10} & 0000 & 15.39 & 58,100 & Apr. 11 & 2400 & 19.30 & 79,400 & \multirow[t]{4}{*}{ Apr. 14} & 1200 & 18.27 & 73,600 \\
\hline & 0600 & 15.62 & 59,300 & \multirow{4}{*}{12} & \multirow{4}{*}{$\begin{array}{l}0400 \\
1200 \\
2400\end{array}$} & \multirow{4}{*}{$\begin{array}{l}19.36 \\
19.30 \\
19.13\end{array}$} & \multirow{4}{*}{$\begin{array}{l}79,800 \\
79,400 \\
78,400\end{array}$} & & \multirow{3}{*}{$\begin{array}{l}2000 \\
2400\end{array}$} & 18.01 & 72,200 \\
\hline & 1200 & 15.99 & 61,200 & & & & & & & 18.22 & 73,300 \\
\hline & 1800 & 16.43 & 63,700 & & & & & & & & \\
\hline & 2400 & 17.20 & 67,800 & & & & & 15 & 0400 & 18.49 & 74,800 \\
\hline 11 & 0600 & 17.99 & 72,000 & 13 & 1200 & 19.00 & 77,700 & & 1200 & 18.21 & 73,300 \\
\hline & 1200 & 18.52 & 75,000 & & 2400 & 18.70 & 76,000 & & 2400 & 17.35 & 68,600 \\
\hline & 1800 & 19.00 & 77,700 & & & & & & & & \\
\hline
\end{tabular}


(314) 5-4910. Sugar Creek near Keokuk, Iowa

Location. -Lat $40^{\circ} 26^{\prime} 45^{\prime \prime}$, long $91^{\circ} 28^{\prime} 55^{\prime \prime}$, in $\mathrm{SE} \frac{1}{4} \mathrm{NW} \frac{1}{4}$ sec.7, T.65 N., R.5 W., on left bank $10 \mathrm{ft}$ downstream from highway bridge, 4.1 miles upstream from mouth, and 6 miles northwest of Keokuk.

Drainage area. - $105 \mathrm{sq} \mathrm{mi}$.

Gage-height record.-Water-stage recorder graph, except Mar. 18, 19, 21 to Apr. 4, Apr. 22, 23, 26-28, when no wire weight gage readings were available. Mar. 20, Apr. 5, 6, 24, 25 when wire weight readings were used. Datum of gage is $510.20 \mathrm{ft}$ above mean sea level, datum of 1929 .

Discharge record.-Stage-discharge relation defined by current-meter measurements below $3,800 \mathrm{cfs}$ and extended on basis of logarithmic plotting. Backwater from ice Mar. 1-6, 9-14, 20.

Maxima.-March-May 1965: Discharge, 4,580 cfs 0130 hours Apr. 6 (gage height, $12.65 \mathrm{ft}$, from floodmark).

1905 to February 1965: Discharge, 33,000 cfs June 9, 1905 (gage height, $20.6 \mathrm{ft}$, from floodmark), estimated on basis of area-velocity study.

Mean discharge, in cubic feet per second, 1965

\begin{tabular}{|c|c|c|c|c|c|c|c|c|c|c|c|}
\hline Day & March & April & May & Day & March & April & May & Day & March & April & May \\
\hline $\begin{array}{l}1 \ldots- \\
2 \ldots- \\
3 \ldots- \\
4 \ldots- \\
5 \ldots- \\
6 \ldots- \\
7-- \\
8 \ldots- \\
9 \ldots- \\
10--\end{array}$ & $\begin{array}{l}65 \\
50 \\
45 \\
42 \\
50 \\
40 \\
19 \\
16 \\
15 \\
14\end{array}$ & $\begin{array}{r}60 \\
50 \\
60 \\
160 \\
1,850 \\
2,080 \\
355 \\
531 \\
717 \\
411\end{array}$ & $\begin{array}{r}28 \\
23 \\
20 \\
18 \\
18 \\
18 \\
17 \\
278 \\
217 \\
45\end{array}$ & 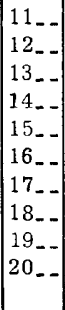 & $\begin{array}{r}13 \\
12 \\
17 \\
60 \\
173 \\
364 \\
2,080 \\
450 \\
95 \\
55\end{array}$ & $\begin{array}{r}816 \\
309 \\
96 \\
125 \\
611 \\
219 \\
86 \\
56 \\
44 \\
34\end{array}$ & $\begin{array}{c}26 \\
22 \\
16 \\
12 \\
21 \\
45 \\
17 \\
10 \\
7.6 \\
6.1\end{array}$ & $\begin{array}{l}21_{-}- \\
22-- \\
23- \\
24_{-}- \\
25_{-} \\
26_{-} \\
27- \\
28_{-} \\
29_{-} \\
30_{-} \\
31_{-}\end{array}$ & $\begin{array}{r}45 \\
38 \\
32 \\
28 \\
25 \\
23 \\
100 \\
400 \\
250 \\
120 \\
80\end{array}$ & $\begin{array}{r}28 \\
20 \\
16 \\
41 \\
680 \\
300 \\
80 \\
50 \\
41 \\
34 \\
\end{array}$ & $\begin{array}{c}5.0 \\
4.7 \\
4.3 \\
4.0 \\
4.3 \\
13 \\
16 \\
6.1 \\
4.3 \\
3.4 \\
2.8\end{array}$ \\
\hline $\begin{array}{l}\text { Mont } \\
\text { Rund } \\
\text { Runc }\end{array}$ & $\begin{array}{l}\text { hly mea } \\
\text { ff, in in } \\
\text { ff, in ac }\end{array}$ & $\begin{array}{l}\text { discha } \\
\text { ches } \\
\text { re-feet }\end{array}$ & in & ubic & . & $\cdots$ & $\because$ & - & $\begin{array}{r}155 \\
1.71 \\
9,550\end{array}$ & $\begin{array}{r}332 \\
3.53 \\
19,760\end{array}$ & $\begin{array}{r}30.1 \\
0.33 \\
1,850\end{array}$ \\
\hline
\end{tabular}

Gage height, in feet, and discharge, in cubic feet per second, at indicated time, 1965

\begin{tabular}{r|r|r|r||r|r|r|r||r|r|r|r}
\hline Date & Hour & $\begin{array}{c}\text { Gage } \\
\text { height }\end{array}$ & $\begin{array}{c}\text { Dis - } \\
\text { charge }\end{array}$ & Date & Hour & $\begin{array}{c}\text { Gage } \\
\text { height }\end{array}$ & $\begin{array}{c}\text { Dis- } \\
\text { charge }\end{array}$ & Date & Hcur & $\begin{array}{c}\text { Gage } \\
\text { height }\end{array}$ & $\begin{array}{c}\text { Dis - } \\
\text { charge }\end{array}$ \\
\hline Apr. 5 5 & 0000 & 6.00 & 546 & Apr. 6 & 0300 & 12.50 & 4,450 & Apr. 7 & 0620 & 5.57 & 498 \\
& 0600 & 7.70 & 970 & & 0800 & 11.07 & 2,510 & & 1220 & 4.42 & 279 \\
& 1200 & 9.35 & 1,560 & & 1200 & 8.97 & 1,400 & & 1870 & 3.85 & 182 \\
& 1800 & 11.00 & 2,380 & & 1500 & 7.49 & 967 & & 2490 & 3.58 & 143 \\
& 2400 & 12.58 & 4,400 & & 2400 & 6.69 & 746 & & & & \\
\hline
\end{tabular}




\section{FOX RIVER BASIN}

(315) 5-4943. Fox River at Bloomfield, Iowa

Location.-Lat $40^{\circ} 46^{\prime} 10^{\prime \prime}$, long $92^{\circ} 25^{\prime} 10^{\prime \prime}$, in SW $\frac{1}{4} \mathrm{SE} \frac{1}{4} \sec .13, \mathrm{~T} .69 \mathrm{~N}$., R.14 W., on left bank $15 \mathrm{ft}$ downstream from highway bridge, $1 \frac{1}{4}$ miles north of Bloomfield, and 8.6 miles downstream from North Fox Creek.

Drainage area. $-87.7 \mathrm{sq} \mathrm{mi}$.

Gage-height record.-Water-stage recorder graph, except May 8, 9. Datum of gage is $755.57 \mathrm{ft}$ above mean sea level, datum of 1929 .

Discharge record.- Stage-discharge relation defined by current-meter measurements below $5,400 \mathrm{cfs}$ and by slope-area measurement at $8,600 \mathrm{cfs}$. Backwater from ice Mar. 1-15, 17-27.

Maxima.-March-May 1965: Discharge, 5,060 cfs 1200 hours Apr. 8 (gage height, $19.67 \mathrm{ft}$ ).

1957 to February 1965: Discharge, 8,600 cfs May 6, 1960 (gage height, $24.02 \mathrm{ft}$ ).

Floods of June 9,1905, and June 18,1946, were highest known (stage and discharge unknown).

Mean discharge, in cubic feet per second, 1965

\begin{tabular}{|c|c|c|c|c|c|c|c|c|c|c|c|}
\hline Day & March & April & May & Day & March & April & May & Day & March & April & May \\
\hline $\begin{array}{l}1 \\
2 \ldots \\
3 \ldots \\
4 \ldots \\
5 \ldots \\
6 \ldots \\
7 \ldots \\
8 \ldots \\
9 \ldots \\
10\end{array}$ & $\begin{array}{c}90 \\
50 \\
28 \\
15 \\
11 \\
10 \\
9.6 \\
9.2 \\
8.8 \\
8.4\end{array}$ & $\begin{array}{r}83 \\
60 \\
96 \\
130 \\
543 \\
490 \\
142 \\
2,360 \\
251 \\
739\end{array}$ & $\begin{array}{r}22 \\
17 \\
14 \\
14 \\
15 \\
15 \\
12 \\
46 \\
112 \\
27\end{array}$ & $\begin{array}{l}11 \ldots \\
12 \ldots \\
13 \ldots \\
14 \ldots \\
15 \ldots \\
16 \ldots \\
17 \ldots \\
18 \ldots \\
19 \ldots \\
20 \ldots\end{array}$ & \begin{tabular}{|c}
8.2 \\
8.0 \\
12 \\
150 \\
350 \\
761 \\
2,570 \\
150 \\
90 \\
45
\end{tabular} & $\begin{array}{r}1,480 \\
129 \\
59 \\
241 \\
846 \\
126 \\
60 \\
36 \\
28 \\
24\end{array}$ & $\begin{array}{r}17 \\
15 \\
10 \\
7.2 \\
7.7 \\
7.7 \\
5.9 \\
5.6 \\
5.2 \\
4.2\end{array}$ & 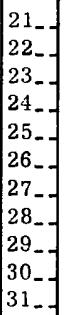 & $\begin{array}{r}34 \\
30 \\
27 \\
25 \\
24 \\
23 \\
60 \\
317 \\
236 \\
100 \\
88\end{array}$ & $\begin{array}{r}20 \\
15 \\
14 \\
12 \\
441 \\
140 \\
64 \\
58 \\
40 \\
31 \\
\end{array}$ & $\begin{array}{c}3.8 \\
8.6 \\
10 \\
7.2 \\
3.1 \\
91 \\
152 \\
32 \\
17 \\
14 \\
10\end{array}$ \\
\hline \multicolumn{9}{|c|}{$\begin{array}{l}\text { Monthly mean discharge, in cubic feet per second } \\
\text { Runoff, in inches } \\
\text { Runoff, in acre-feet }\end{array}$} & $\begin{array}{r}173 \\
2.27 \\
10,610\end{array}$ & $\begin{array}{r}292 \\
3.71 \\
17,370\end{array}$ & $\begin{array}{r}23.5 \\
0.31 \\
1,440\end{array}$ \\
\hline
\end{tabular}

Gage height, in feet, and discharge, in cubic feet per second, at indicated time, 1965

\begin{tabular}{|c|c|c|c|c|c|c|c|c|c|c|c|}
\hline Date & Hour & $\begin{array}{l}\text { Gage } \\
\text { height }\end{array}$ & $\begin{array}{c}\text { Dis - } \\
\text { charge }\end{array}$ & Date & Hour & $\begin{array}{l}\text { Gage } \\
\text { height }\end{array}$ & $\begin{array}{c}\text { Dis- } \\
\text { charge }\end{array}$ & Date & Hour & $\begin{array}{c}\text { Gage } \\
\text { height }\end{array}$ & $\begin{array}{l}\text { Dis - } \\
\text { charge }\end{array}$ \\
\hline \multirow[t]{15}{*}{ Apr. 8} & 0000 & 4.31 & 102 & \multirow[t]{5}{*}{ Apr. 8} & 2000 & 13.14 & 1,800 & \multirow[t]{15}{*}{ Apr. 10} & 0300 & 4,60 & 137 \\
\hline & 0400 & 4.27 & 97 & & 2100 & 11.24 & 1,250 & & 0600 & 4.51 & 124 \\
\hline & 0530 & 4.31 & 102 & & 2300 & 8.99 & 768 & & 0700 & 4.50 & 123 \\
\hline & 0600 & 5.89 & 290 & & 2400 & 8.09 & 615 & & 0800 & 4.53 & 127 \\
\hline & 0630 & 9.49 & 859 & & & & & & 0900 & 4.57 & 133 \\
\hline & 0700 & 13.19 & 1,820 & \multirow[t]{10}{*}{ Apr. 9} & 0100 & 7.46 & 513 & & 1100 & 6.24 & 334 \\
\hline & 0730 & 14.64 & 2,340 & & 0300 & 6.66 & 398 & & 1200 & 8.09 & 615 \\
\hline & 0800 & 15.99 & 2,920 & & 0500 & 6.15 & 328 & & 1300 & 10.11 & 988 \\
\hline & 0830 & 16.99 & 3,410 & & 0700 & 5.77 & 273 & & 1400 & 10.39 & 1,050 \\
\hline & 0900 & 18.04 & 4,040 & & 1000 & 5.37 & 274 & & 1500 & 10.34 & 1,030 \\
\hline & 1000 & 18.97 & 4,600 & & 1500 & 5.01 & 180 & & 1800 & 9.76 & 907 \\
\hline & 1200 & 19.67 & 5,060 & & 1630 & 4.96 & 180 & & 1830 & 9.54 & 870 \\
\hline & 1400 & 19.19 & 4,730 & & 1700 & 4.96 & 180 & & 1930 & 9.55 & 872 \\
\hline & 1800 & 16.81 & 3,310 & & 2000 & 4.82 & 161 & & 2000 & 9.69 & .903 \\
\hline & 1900 & 15.16 & 2,540 & & 2400 & 4.66 & 138 & & 2100 & 12.39 & 1,580 \\
\hline
\end{tabular}


Gage height, in feet, and discharge, in cubic feet per second, at indicated time 1965, of Fox River at Bloomfield. Iowa-Continued

\begin{tabular}{|c|c|c|c|c|c|c|c|c|c|c|c|}
\hline Date & Hour & $\begin{array}{c}\text { Gage } \\
\text { height }\end{array}$ & $\begin{array}{c}\text { Dis- } \\
\text { charge }\end{array}$ & Date & Hour & $\begin{array}{c}\text { Gage } \\
\text { height }\end{array}$ & $\begin{array}{c}\text { Dis- } \\
\text { charge }\end{array}$ & Date & Hour & $\begin{array}{c}\text { Gage } \\
\text { height }\end{array}$ & $\begin{array}{c}\text { Dis- } \\
\text { charge }\end{array}$ \\
\hline \multirow[t]{3}{*}{ Apr. 10} & 2200 & 14.26 & 2,170 & \multirow[t]{9}{*}{ Apr. 11} & 0630 & 16.55 & 3,180 & \multirow{9}{*}{$\begin{array}{l}\text { Apr. } 11 \\
\text { Apr. } 12\end{array}$} & \multirow{9}{*}{$\begin{array}{l}040 \\
043 \\
090 \text { ? } \\
140 ? \\
200 ? \\
240 ?\end{array}$} & \multirow[t]{2}{*}{5.37} & \multirow[t]{2}{*}{224} \\
\hline & 2400 & 15.74 & 2,800 & & 0700 & 16.41 & 3,100 & & & & \\
\hline & & & & & 0800 & 15.54 & 2,710 & & & 4.94 & 178 \\
\hline \multirow[t]{6}{*}{11} & 0130 & 16.03 & 2,930 & & 0900 & 13.90 & 2,060 & & & 4.96 & 180 \\
\hline & 0200 & 16.05 & 2,940 & & 1030 & 11.04 & 1,200 & & & 4.60 & 137 \\
\hline & 0230 & 16.13 & 2,970 & & 1200 & 9.66 & 896 & & & 4.34 & 104 \\
\hline & 0330 & 16.07 & 2,950 & & 1500 & 7.43 & 508 & & & 4.17 & 87 \\
\hline & 0500 & 16.38 & 3,090 & & 1800 & 6.39 & 356 & & & 4.07 & 74 \\
\hline & 0530 & 16.56 & 3,180 & & 2200 & 5.62 & 259 & & & & \\
\hline
\end{tabular}

(316) 5-4950. Fox River at Wayland, Mo.

Location.-Lat $40^{\circ} 23^{\prime} 45^{\prime \prime}$, long $91^{\circ} 35^{\prime} 50^{\prime \prime}$, in NW $\frac{1}{4}$ sec.31, T.65 N., R.6 W'., on left bank $90 \mathrm{ft}$ downstream from bridge on U.S. Highway 136, three-quarters of a mile west of Wayland, and 5 miles downstream from Brush Creek.

Drainage area. $-400 \mathrm{sq} \mathrm{mi}$, approximately.

Gage-height record.-Digital recorder tape punched at 15 minute intervals. Datum of gage is $501.52 \mathrm{ft}$ above mean sea level, datum of 1929 .

Discharge record.-Stage-discharge relation defined by current-meter measurements.

Maxima.-March-May 1965: Discharge, 5,370 cfs 0345 hours Apr. 6 (gage height, $15.97 \mathrm{ft}$ ).

1922 to February 1965: Discharge, 25,000 cfs June 29, 1933 (gage l eight, $21.53 \mathrm{ft}$, from floodmarks).

Mean discharge, in cubic feet per second, 1965

\begin{tabular}{|c|c|c|c|c|c|c|c|c|c|c|c|}
\hline Day & March & April & May & Day & March & April & May & Day & March & April & May \\
\hline & 139 & 310 & 136 & $111_{--}$ & 126 & 2,660 & 101 & $2 \pi$ & 266 & 145 & 29 \\
\hline 2 & 383 & 256 & 109 & $12=-$ & 109 & 2,620 & 71 & 20 & 212 & 134 & 27 \\
\hline 3. & 178 & 208 & 92 & $13-$ & 121 & 910 & 57 & 23 & 167 & 147 & 28 \\
\hline 4 & 82 & 304 & 80 & & 379 & 430 & 49 & & 118 & 122 & 25 \\
\hline 5 & 49 & 2,200 & 75 & 1 & 889 & 1,790 & 112 & & 119 & 1,080 & 26 \\
\hline 6 & 99 & 4,780 & 90 & $16 \ldots$ & 1,720 & 1,830 & 93 & 26. & 114 & 2,410 & 40 \\
\hline 7. & 98 & 1,880 & 81 & $17_{--}$ & 4,240 & 610 & 48 & 27. & 103 & 727 & 48 \\
\hline 8 & 81 & 891 & 80 & $18 \ldots$ & 4,630 & 345 & 40 & 28. & 126 & 333 & 49 \\
\hline 9. & 114 & 2,420 & 114 & $19_{-}$ & 2,260 & 224 & 35 & 29 & 781 & 231 & 116 \\
\hline 10 & 136 & 2,730 & 98 & $20--$ & 456 & 169 & 32 & 30 & 876 & 174 & 60 \\
\hline \multirow{3}{*}{\multicolumn{9}{|c|}{$\begin{array}{l}\text { Monthly mean discharge, in cubic feet per second } \\
\text { Runoff, in inches } \\
\text { Runoff, in acre-feet }\end{array}$}} & 632 & 1,102 & 67.1 \\
\hline & & & & & & & & & 1.82 & 3.07 & 0.19 \\
\hline & & & & & & & & & 38,830 & 65,590 & 4,120 \\
\hline
\end{tabular}




\section{BEAR CREEK BASIN}

(317) 5-4955. Bear Creek near Marcelline, Ill.

Location.-Lat $40^{\circ} 08^{\prime} 34^{\prime \prime}$, long $91^{\circ} 20^{\prime} 14^{\prime \prime}$, between secs.20 and 21, T.2 N., R. 8 W., on right bank at downstream side of highway bridge, 0.9 mile downstream from Grindstone Creek, $2 \frac{1}{4}$ miles northeast of Marcelline, and 12 mile upstream from mouth.

Drainage area.-348 sq mi.

Gage-height record.-Water-stage recorder graph. Datum of gage is $504.52 \mathrm{ft}$ above mean sea level, datum of 1929 .

Discharge record.--Stage-discharge relation defined by current-meter measurements below $15,200 \mathrm{cfs}$ and extended above by logarithmic plotting.

Maxima.-April-May 1965: Discharge, 11,400 cfs 1615 hours Apr. 6 (gage height, $19.46 \mathrm{ft}$ ).

1944 to March 1965: Discharge, 21,200 cfs July 22, 1951 (gage height, $26.07 \mathrm{ft}$ ).

Mean discharge, in cublc feet per second, 1965

\begin{tabular}{|c|c|c|c|c|c|c|c|c|}
\hline Day & April & May & Day & April & May & Day & April & May \\
\hline $\begin{array}{l}1 \\
2 \\
3 \\
4\end{array} \ldots \ldots$ & $\begin{array}{r}359 \\
275 \\
177 \\
218 \\
1,660 \\
8,620 \\
1,890 \\
390 \\
244 \\
497\end{array}$ & $\begin{array}{l}48 \\
42 \\
38 \\
36 \\
35 \\
34 \\
30 \\
29 \\
29 \\
28\end{array}$ & $\begin{array}{l}11 \ldots \\
12 \ldots \\
13 \ldots \\
14 \ldots \\
15_{\ldots} \\
16 \ldots \\
17_{\ldots} \\
18 \ldots \\
19_{\ldots} \\
20 \ldots \\
\ldots\end{array}$ & $\begin{array}{r}1,140 \\
560 \\
219 \\
181 \\
1,080 \\
521 \\
237 \\
166 \\
130 \\
106\end{array}$ & $\begin{array}{r}23 \\
21 \\
19 \\
18 \\
64 \\
473 \\
199 \\
52 \\
31 \\
24\end{array}$ & $\begin{array}{l}21 \ldots \ldots \\
22 \ldots \ldots \\
23 \ldots \ldots \\
24 \ldots \ldots \\
25 \ldots \ldots \\
26 \ldots \ldots \\
26 \ldots \ldots \\
28 \ldots \ldots \\
29 \\
30 \ldots \ldots \\
31 \\
31 \ldots \ldots\end{array}$ & $\begin{array}{r}92 \\
80 \\
72 \\
63 \\
223 \\
145 \\
89 \\
73 \\
63 \\
55 \\
\end{array}$ & $\begin{array}{l}20 \\
15 \\
12 \\
11 \\
9.3 \\
14 \\
23 \\
23 \\
16 \\
12 \\
9.3\end{array}$ \\
\hline \multicolumn{7}{|c|}{$\begin{array}{l}\text { Monthly mean discharge, in cubic feet per second } \\
\text { Runoff, in inches }\end{array}$} & $\begin{array}{r}654 \\
2.10\end{array}$ & $\begin{array}{l}46.4 \\
0.15\end{array}$ \\
\hline
\end{tabular}

Gage height, in feet, and discharge, in cubic feet per second, at indicated time, 1965

\begin{tabular}{c|c|r|r||r|r|r|r||r|r|r|r}
\hline Date & Hour & $\begin{array}{c}\text { Gage } \\
\text { height }\end{array}$ & $\begin{array}{c}\text { Dis - } \\
\text { charge }\end{array}$ & Date & Hour & $\begin{array}{c}\text { Gage } \\
\text { height }\end{array}$ & $\begin{array}{c}\text { Dis- } \\
\text { charge }\end{array}$ & Date & Hour & $\begin{array}{c}\text { Gage } \\
\text { height }\end{array}$ & $\begin{array}{c}\text { Dis - } \\
\text { charge }\end{array}$ \\
\hline Apr. 5 & 0000 & 5.16 & 271 & Apr. 5 & 2200 & 14.10 & 5,500 & Apr. 7 & 0200 & 12.00 & 3,820 \\
& 0300 & 5.14 & 267 & & 2400 & 14.55 & 5,900 & & 0600 & 10.00 & 2,420 \\
& 0600 & 5.10 & 258 & & & & & & 1200 & 8.35 & 1,460 \\
& 0800 & 5.10 & 258 & & 0200 & 15.10 & 6,400 & & 1800 & 7.30 & 967 \\
& 1000 & 5.35 & 312 & & 1000 & 17.55 & 8,880 & & 2400 & 6.33 & 586 \\
& 1100 & 5.80 & 424 & & 1615 & 19.46 & 11,400 & & & & \\
& 1200 & 6.60 & 687 & & 2100 & 18.00 & 9,420 & & 0400 & 5.95 & 466 \\
& 1300 & 7.50 & 1,050 & & 2200 & 16.15 & 7,390 & & 1500 & 5.46 & 337 \\
& 1600 & 8.80 & 1,710 & & 2300 & 15.00 & 6,310 & & 2400 & 5.26 & 293 \\
& 1800 & 9.40 & 2,060 & & 2400 & 14.10 & 5,500 & & & & \\
\hline
\end{tabular}




\section{WYACONDA RIVER BASIN}

(318) 5-4960. Wyaconda River above Canton, Mo.

Location.-Lat $40^{\circ} 08^{\prime} 30^{\prime \prime}$, long $91^{\circ} 33^{\prime} 55^{\prime \prime}$, in SE⿺ sec. 28 , T.62 N., R.6 W., on left bank on downstream side of bridge on State Highway 16, 1 mile upstream from Sugar Creek and 2 miles west of Canton.

Drainage area.- $-393 \mathrm{sq} \mathrm{mi}$,

Gage-height record.-Water-stage recorder graph except Mar. 19-22, Apr. 18-24 for which graph was constructed on basis of once-daily readings of wire-v'eight gage.

Datum of gage is $515.41 \mathrm{ft}$ above mean sea level, datum of 1929 .

Discharge record.- Stage-discharge relation defined by current-meter measurements.

Maxima.-March-May 1965: Discharge, 4,850 cfs 1200 hours Apr. 7 (gage height, $20.5 \mathrm{ft}$ ). 1932 to February 1965: Discharge $17,700 \mathrm{cfs}$ June 30,1933 (gage height, $30.00 \mathrm{ft}$, from floodmarks).

Mean discharge, in cubic feet per second, 1965

\begin{tabular}{|c|c|c|c|c|c|c|c|c|c|c|c|}
\hline Day & March & April & May & Day & March & April & May & Day & March & April & May \\
\hline & 370 & 355 & 121 & $11 \ldots$ & 179 & 2,340 & 67 & 21 & 288 & 123 & 22 \\
\hline & 400 & 280 & 91 & $12_{-}$ & 186 & 2,430 & 43 & 22. & 206 & 106 & 20 \\
\hline ב- & 206 & 213 & 74 & $13 \ldots$ & 220 & .850 & 36 & 23. & 172 & 143 & 20 \\
\hline 4. & 161 & 400 & 64 & $14 \ldots$ & 628 & 340 & 31 & 24 & 111 & 110 & 18 \\
\hline 10 & 179 & 1,580 & 58 & 15 & 1,250 & 1,400 & 37 & 25. & 110 & 901 & 18 \\
\hline 6. & 128 & 3,830 & 62 & $16 \ldots$ & 1,800 & 1,700 & 99 & $26 \ldots$ & 118 & 2,180 & 22 \\
\hline 7 & 146 & 4,550 & 63 & $17 \ldots$ & 3,000 & 630 & 66 & 27. & 100 & 1,100 & 29 \\
\hline 8 & 133 & 1,890 & 53 & $18 \ldots$ & 3,630 & 310 & 38 & 28. & 123 & 362 & 31 \\
\hline 9. & 186 & 1,800 & 51 & 19. & 3,930 & 206 & 28 & $29_{-}$ & 868 & 213 & 42 \\
\hline 10 & 206 & 1,320 & 65 & 20 & 941 & 156 & 23 & 30. & 1,170 & 154 & 31 \\
\hline & & & & & & & & 31. & 490 & $\ldots \ldots$ & 25 \\
\hline \multirow{2}{*}{\multicolumn{9}{|c|}{$\begin{array}{l}\text { Monthly mean discharge, in cubic feet per second } \\
\text { Runoff, in inches }\end{array}$}} & 698 & 1,066 & 46.7 \\
\hline & & & & & & & & & 2.05 & 3.03 & 0.14 \\
\hline & \multicolumn{8}{|c|}{ Runoff, in acre-feet. } & 42,910 & 63,420 & 2,870 \\
\hline
\end{tabular}

\section{FABIUS RIVER BASIN}

(319) 5-4970. North Fabius River at Monticello, Mo.

Location.-Lat $40^{\circ} 06^{\prime} 30^{\prime \prime}$, long $91^{\circ} 42^{\prime} 55^{\prime \prime}$, in SW $\frac{1}{4} \mathrm{SE} \frac{1}{4}$ sec.6, T.61 N., R.7 W., near center of span on downstream side of bridge on State Highway 16, 1 mile south of Monticello, and 19 miles upstream from Middle Fabius River.

Drainage area.- $-452 \mathrm{sq} \mathrm{mi}$.

Gage-height record.-Graph based on once or twice-daily wire-weight, gage readings. Datum of gage is $540.73 \mathrm{ft}$ above mean sea level, datum of 1929 .

Discharge record.-Stage-discharge relation defined by current-meter measurements.

Maxima.-March-May 1965: Discharge, 6,640 cfs 0910 hours Mar. 18 (gage height, $21.30 \mathrm{ft}$ ).

1874 to February 1965: Discharge, 17,400 cfs June 30, 1933 (gage height, $30.8 \mathrm{ft}$, from floodmarks). 
Mean discharge, in cubic feet per second, 1965, of North Fabius River at Monticello, Mo.

\begin{tabular}{|c|c|c|c|c|c|c|c|c|c|c|c|}
\hline Day & March & April & May & Day & March & April & May & Day & March & April & May \\
\hline $\begin{array}{l}1 . \\
2 \ldots \\
3 \ldots \\
4 \ldots \\
5 \ldots \\
6 \ldots \\
7 \ldots \\
8 \ldots \\
9- \\
10 .\end{array}$ & $\begin{array}{r}356 \\
315 \\
102 \\
71 \\
71 \\
94 \\
114 \\
137 \\
170 \\
248\end{array}$ & $\begin{array}{r}376 \\
345 \\
670 \\
620 \\
2,280 \\
5,070 \\
3,510 \\
1,230 \\
1,920 \\
1,110\end{array}$ & $\begin{array}{r}156 \\
122 \\
110 \\
94 \\
81 \\
79 \\
79 \\
67 \\
62 \\
86\end{array}$ & 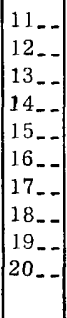 & $\begin{array}{r}228 \\
216 \\
239 \\
720 \\
1,440 \\
2,640 \\
4,820 \\
5,770 \\
1,640 \\
745\end{array}$ & $\begin{array}{r}4,160 \\
2,420 \\
570 \\
510 \\
2,480 \\
1,620 \\
370 \\
325 \\
248 \\
186\end{array}$ & $\begin{array}{l}81 \\
74 \\
54 \\
39 \\
38 \\
64 \\
53 \\
35 \\
34 \\
32\end{array}$ & $\begin{array}{l}21- \\
22- \\
23-- \\
24-- \\
25_{-} \\
26-- \\
27- \\
28- \\
29- \\
30_{-} \\
31\end{array}-$ & $\begin{array}{r}431 \\
335 \\
325 \\
266 \\
221 \\
212 \\
212 \\
292 \\
1,050 \\
960 \\
475\end{array}$ & $\begin{array}{r}131 \\
521 \\
521 \\
521 \\
82 \mathrm{C} \\
1,93 \mathrm{C} \\
67 \mathrm{C} \\
431 \\
257 \\
22 \varepsilon \\
-----\end{array}$ & $\begin{array}{l}32 \\
27 \\
23 \\
26 \\
27 \\
30 \\
31 \\
28 \\
28 \\
27 \\
26\end{array}$ \\
\hline \multicolumn{9}{|c|}{$\begin{array}{l}\text { Monthly mean discharge, in cubic feet per second. } \\
\text { Runoff, in inches } \\
\text { Runoff, in acre-feet }\end{array}$} & $\begin{array}{r}804 \\
2.05 \\
49,420\end{array}$ & $\begin{array}{r}1,202 \\
2.97 \\
71,520\end{array}$ & $\begin{array}{r}56.3 \\
0.14 \\
3,460\end{array}$ \\
\hline
\end{tabular}

(320) 5-4980. Middle Fabius River near Monticello, Mo.

Location.-Lat $40^{\circ} 05^{\prime} 40^{\prime \prime}$, long $91^{\circ} 44^{\prime} 10^{\prime \prime}$, in $\mathrm{SE} \frac{1}{4}$ sec.12, T.61 N., R.8 W., near center of span on upstream side of bridge on State Highway 16, $2 \frac{1}{2}$ miles southwest of Monticello, 8 miles downstream from Radish Branch, and 17 miles upstream from mouth.

Drainage area.-393 sq mi.

Gage-height record.-Graph based on once or twice-daily wire-weight gage readings and crest-stage gage. Datum of gage is $540.46 \mathrm{ft}$ above mean sea level, datum of 1929 .

Discharge record.- Stage-discharge relation defined by current-meter measurements.

Maxima.-March-May 1965: Discharge, 4,300 cfs 1400 hours Mar. 17 (gage reight, $16.02 \mathrm{ft}$ ).

1945 to February 1965: Discharge, 16,200 cfs June 7, 1947 (gage height, $26.28 \mathrm{ft}$ ).

A fiood of unknown date reached a stage of $26.6 \mathrm{ft}$, from floodmark (discharge, $16,800 \mathrm{cfs})$.

Mean discharge, in cubic feet per second, 1965

\begin{tabular}{|c|c|c|c|c|c|c|c|c|c|c|c|}
\hline Day & March & April & May & Day & March & April & May & Day & March & April & May \\
\hline 1 & 363 & 315 & 116 & $11 \ldots$ & 295 & 2,070 & 41 & $21 \ldots$ & 950 & 108 & 23 \\
\hline 2 & 418 & 241 & 93 & $12 \ldots$ & 265 & 2,070 & 39 & $22 \ldots$ & 237 & 94 & 20 \\
\hline 3 & 245 & 368 & 83 & $13 \ldots$ & 295 & 1,680 & 36 & $23 \ldots$ & 195 & 116 & 20 \\
\hline 4. & 118 & 442 & 72 & $14 \ldots$ & 620 & 368 & 34 & $24 \ldots$ & 129 & 364 & 18 \\
\hline 5. & 69 & 1,200 & 63 & $15 \ldots$ & 1,250 & 770 & 34 & $25 \ldots$ & 102 & 920 & 18 \\
\hline 6. & 108 & 3,900 & 60 & $16 \ldots$ & 2,110 & 1,220 & 46 & $26 \ldots$ & 131 & 1,430 & 22 \\
\hline 7. & 87 & 3,200 & 58 & $17 .-$ & 4,000 & 800 & 68 & $27 \ldots$ & 125 & 1,910 & 22 \\
\hline 8 & 135 & 3,350 & 55 & $18 \ldots$ & 3,300 & 285 & 55 & 28 & 131 & 432 & 24 \\
\hline 9. & 201 & 755 & 55 & $19 \ldots$ & 3,000 & 190 & 46 & 29 & 554 & 172 & 26 \\
\hline 10. & 305 & 950 & 48 & $20 \ldots$ & 2,310 & 147 & 29 & $30_{-}$ & 980 & 120 & 28 \\
\hline & & & & & & & & $31 \ldots$ & 455 & $\ldots$ & 27 \\
\hline \multirow{3}{*}{\multicolumn{9}{|c|}{$\begin{array}{l}\text { Monthly mean discharge, in cubic feet per second } \\
\text { Runoff, in inches } \\
\text { Runoff, in acre-feet }\end{array}$}} & 758 & 1,000 & 44.5 \\
\hline & & & & & & & & & 2.22 & 2.84 & 0.13 \\
\hline & & & & & & & & & 46,580 & 59,480 & 2,740 \\
\hline
\end{tabular}


(321) 5-5000. South Fabius River near Taylor, Mo.

Location.--Lat $39^{\circ} 53^{\prime} 50^{\prime \prime}$, long $91^{\circ} 34^{\prime} 50^{\prime \prime}$, in SW $\frac{1}{4} \mathrm{NW} \frac{1}{4}$ sec. 21, T.59 N., R.6 W., on right bank at downstream side of highway bridge, $4 \frac{1}{2}$ miles southwest of Tay'or, 5 miles downstream from Grassy Creek, and 5.3 miles upstream from confluence with North Fabius River.

Drainage area. $-620 \mathrm{sq} \mathrm{mi} 630 \mathrm{sq} \mathrm{mi}$ at site used prior to May $14,1936$.

Gage-height record.-Water-stage recorder graph except Mar. 19-21, May 14-16, May 22-30 for which graph was constructed from once-daily wire-weight gage readings. Datum of gage is $482.91 \mathrm{ft}$ above mean sea level, datum of 1929 (levels by Corps of Engineers).

Discharge record.--Stage-discharge relation defined by current-meter measurements.

Maxima.-March-May 1965: Discharge, 7,940 cfs 0300 hours Apr. 6 (gage height, $12.49 \mathrm{ft})$.

1934 to February 1965: Discharge, 19,700 cfs June 8, 1947 (gage height, $19.5 \mathrm{ft}$ ), from rating curve extended above $11,000 \mathrm{cfs}$.

Flood in 1928 reached a stage of $18.49 \mathrm{ft}$, from floodmark.

Mean discharge, in cubic feet per second, 1965

\begin{tabular}{|c|c|c|c|c|c|c|c|c|c|c|c|}
\hline Day & March & April & May & Day & March & April & May & Day & March & April & May \\
\hline & 1,230 & 655 & 130 & $11 \ldots$ & 458 & 2,230 & 49 & 21. & 1,060 & 210 & 48 \\
\hline 2. & 1,050 & 478 & 116 & $12 \ldots$ & 530 & 2,230 & 44 & 22 & 402 & 170 & 36 \\
\hline 3. & 780 & 613 & 98 & $13 \ldots$ & 705 & 1,200 & 40 & 23. & 357 & 154 & 26 \\
\hline 4 & 374 & 1,460 & 88 & 14 & 1,350 & 610 & 35 & 24 & 273 & 140 & 21 \\
\hline 5. & 248 & 3,520 & 81 & 15. & 2,230 & 1,200 & 31 & $25 \ldots$ & 205 & 142 & 20 \\
\hline 6. & 238 & 6,440 & 72 & $16 \ldots$ & 2,790 & 1,380 & 31 & $26 \ldots$ & 188 & 136 & 20 \\
\hline & 232 & 5,140 & 67 & $17 .-$ & 5,740 & 780 & 51 & 27. & 205 & 471 & 20 \\
\hline & 297 & 4,150 & 62 & 18 & 5,440 & 430 & 58 & $28 \ldots$ & 366 & 466 & 20 \\
\hline & 510 & 1,150 & 58 & 19 & 5,140 & 300 & 83 & $29_{-}$ & 1,110 & 208 & 21 \\
\hline & 442 & 927 & 54 & $20 \ldots$ & 3,430 & 235 & 60 & $30 \ldots$ & 1,600 & 160 & 64 \\
\hline & & & & & & & & & 1,140 & & 55 \\
\hline \multicolumn{9}{|c|}{ Monthly mean discharge, in cubic feet per second } & 1,294 & 1,246 & 53.5 \\
\hline \multicolumn{9}{|c|}{ Runoff, in inches } & 2.41 & 2.24 & 0.10 \\
\hline \multicolumn{9}{|c|}{ Runoff, in acre-feet } & 79,580 & 74,150 & 3,290 \\
\hline
\end{tabular}




\section{NORTH RIVER BASIN}

(322) 5-5010. North River at Palmyra, Mo.

Location.-Lat $39^{\circ} 49^{\prime} 05^{\prime \prime}$, long $91^{\circ} 31^{\prime} 15^{\prime \prime}$, in SE $\frac{1}{4} \mathrm{SW} \frac{1}{4} \mathrm{sec} .13$, T.58 N., R.6 W., on right bank $100 \mathrm{ft}$ upstream from city waterworks dam, 1,000 ft upstream from bridge on U.S. Highways 24 and 61 , half a mile north of Palmyra, and 7 miles upstream from mouth.

Drainage area. -373 sq $\mathrm{mi}$.

Gage-height record.-Digital-recorder tape punched at 15 minute intervals. Datum of gage is $464.81 \mathrm{ft}$ above mean sea level, datum of 1929 (levels by Corps of Engineers).

Discharge record.-Stage-discharge relation defined by current-meter measurements. Backwater from Mississippi River Apr. 10 to May 13.

Maxima.-March-May 1965: Discharge, 9,620 cfs 0915 hours Apr. 6 (gage height, $20.89 \mathrm{ft}$ ).

1934 to February 1965: Discharge, 27,400 cfs Apr. 11, 1944; gage height, 24.42 ft May 28, 1955.

A flood of unknown date reached a stage of about $28.0 \mathrm{ft}$, from floodmarks, at site $1,000 \mathrm{ft}$ downstream, present datum.

Mean discharge, in cubic feet per second, 1965

\begin{tabular}{|c|c|c|c|c|c|c|c|c|c|c|c|}
\hline Day & March & April & May & Day & March & April & May & Day & March & April & May \\
\hline 1 & 1,220 & 270 & 72 & $11 \ldots$ & 298 & 1,200 & 29 & 21. & 214 & 140 & 19 \\
\hline 2 & 698 & 209 & 62 & $12 \ldots$ & 387 & 550 & 27 & 22 & 182 & 130 & 17 \\
\hline 3 & 329 & 657 & 54 & $13 \ldots$ & 601 & 380 & 25 & $23_{-}$ & 174 & 120 & 15 \\
\hline 4 & 217 & 984 & 50 & $14 \ldots$ & 1,010 & 370 & 23 & 24 & 140 & 115 & 15 \\
\hline 5 & 170 & 2,070 & 46 & $15 \ldots$ & 1,060 & 270 & 21 & 25 & 118 & 110 & 14 \\
\hline 6. & 150 & 6,770 & 42 & $16 \ldots$ & 1,090 & 240 & 25 & 26 & 108 & 115 & 16 \\
\hline 7. & 137 & 2,090 & 39 & $17 \ldots$ & 5,180 & 560 & 26 & 27. & 108 & 300 & 16 \\
\hline 8. & 208 & 592 & 36 & $18 \ldots$ & 2,490 & 250 & 21 & 28 & 312 & 170 & 14 \\
\hline 9. & 428 & 332 & 33 & $19=$ & 727 & 180 & 18 & $29_{-}$ & 785 & 110 & 13 \\
\hline 10 & 307 & 380 & 31 & $20_{-}$ & 290 & 160 & 21 & $30 \ldots$ & 619 & 92 & 12 \\
\hline & & & & & & & & 31. & 387 & - - - & 11 \\
\hline \multicolumn{9}{|c|}{ Monthly mean discharge, in cubic feet per second } & 650 & 664 & 27.8 \\
\hline \multicolumn{9}{|c|}{ Runoff, in inches... } & 2.01 & 1.99 & 0.09 \\
\hline \multicolumn{9}{|c|}{ Punoff, in acre-feet } & 39,960 & 39,500 & 1,710 \\
\hline
\end{tabular}




\section{BEAR CREEK BASIN}

(323) 5-5020. Bear Creek at Hannibal, Mo.

Location.-Lat $39^{\circ} 40^{\prime} 43^{\prime \prime}$, long $91^{\circ} 24^{\prime} 33^{\prime \prime}$, in $\mathrm{SE} \frac{1}{4} \mathrm{NW} \frac{1}{4}$ sec.1, T.56 N., R.5 W., on right bank $400 \mathrm{ft}$ downstream from upstream bridge on dual U.S. Highway 61 at Hannibal, 4-3/4 miles upstream from mouth.

Drainage area. $-31.0 \mathrm{sq} \mathrm{mi}$.

Gage-height record.-Digital-recorder tape punched at 15 minute intervals. Datum of gage is $508.91 \mathrm{ft}$ above mean sea level, datum of 1929 .

Discharge record.--Stage-discharge relation defined by current-meter measurements.

Maxima.--March-May 1965: Discharge, 1,020 cfs 1900 hours Apr. 5 (gage height, $5.79 \mathrm{ft})$.

1938-42, 1947 to February 1965: Discharge, 6,500 cfs Aug. 3, 1957 (gage height, $14.05 \mathrm{ft})$.

Remarks.-High flow regulated by Bear Creek Reservoir since Aug. 7, 1961.

Mean discharge, in cubic feet per second, 1965

\begin{tabular}{|c|c|c|c|c|c|c|c|c|c|c|c|}
\hline Day & March & April & May & Day & March & April & May & Day & March & April & May \\
\hline $\begin{array}{l}1 \\
1 \\
2 \ldots- \\
3 \ldots- \\
4 \ldots- \\
5 \ldots-1 \\
6 \ldots- \\
7 \ldots- \\
8 \ldots- \\
9 \ldots- \\
10 \ldots-1\end{array}$ & $\begin{array}{c}242 \\
37 \\
14 \\
11 \\
10 \\
9.4 \\
13 \\
44 \\
61 \\
26\end{array}$ & $\begin{array}{c}13 \\
11 \\
60 \\
37 \\
119 \\
541 \\
298 \\
5.2 \\
92 \\
15\end{array}$ & $\begin{array}{r}0.6 \\
.6 \\
.5 \\
.5 \\
.5 \\
.4 \\
.4 \\
.4 \\
.4 \\
.4\end{array}$ & 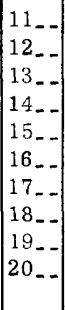 & $\begin{array}{r}31 \\
55 \\
64 \\
90 \\
54 \\
22 \\
528 \\
333 \\
18 \\
13\end{array}$ & $\begin{array}{c}5.8 \\
2.4 \\
2.0 \\
12 \\
3.8 \\
2.1 \\
1.7 \\
1.5 \\
1.2 \\
1.2\end{array}$ & $\begin{array}{l}0.4 \\
.4 \\
.4 \\
.4 \\
.4 \\
.4 \\
.4 \\
.4 \\
.4 \\
.4\end{array}$ & $\begin{array}{l}21- \\
22-- \\
23- \\
24-- \\
25- \\
26-- \\
27- \\
28- \\
29_{-}- \\
30-- \\
31_{-}\end{array}$ & $\begin{array}{l}12 \\
11 \\
11 \\
9.0 \\
8.8 \\
8.3 \\
14 \\
52 \\
57 \\
24 \\
16\end{array}$ & $\begin{array}{r}1.1 \\
1.0 \\
1.0 \\
1.5 \\
3.8 \\
1.1 \\
1.0 \\
1.0 \\
.8 \\
.8 \\
\end{array}$ & $\begin{array}{r}38 \\
113 \\
174 \\
166 \\
149 \\
48 \\
3.6 \\
2.6 \\
2.4 \\
2.4 \\
2.2\end{array}$ \\
\hline \multicolumn{9}{|c|}{$\begin{array}{l}\text { Monthly mean discharge, in cubic feet per second } \\
\text { Runoff, in inches } \\
\text { Runoff, in acre-feet }\end{array}$} & $\begin{array}{r}61.2 \\
2.28 \\
3,770\end{array}$ & $\begin{array}{r}41.3 \\
1.49 \\
2,460\end{array}$ & $\begin{array}{r}22.9 \\
0.85 \\
1,410\end{array}$ \\
\hline
\end{tabular}




\section{SALT RIVER BASIN}

(324) 5-5080. Salt River near New London, Mo..

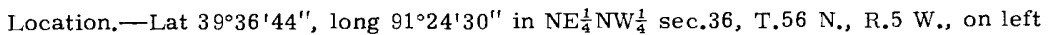
bank $180 \mathrm{ft}$ upstream from upstream bridge on dual U.S. Highway 61,2 miles north of New London, and 8 miles upstream from Spencer Creek.

Drainage area. $-2,480 \mathrm{sq} \mathrm{mi}$, approximately.

Gage-height record.-Water-stage recorder graph. Datum of gage $477.03 \mathrm{ft}$ above mean sea level, datum of 1929.

Discharge record.-Stage-discharge relation defined by current-meter measurements.

Maxima.-March-May 1965: Discharge, 26,000 cfs 1500 hours Apr. 7 (gage height, $21.53 \mathrm{ft}$ ).

1922 to February 1965: Discharge, 64,700 cfs Aug. 2, 1958 (gage height, $29.92 \mathrm{ft}$ ).

Flood of July 14, 1858 reached a stage of about $27.6 \mathrm{ft}$, present site and datum, based on comparison of June 1928 flood crest of stone marker 1 mile downstream from gage.

Mean discharge, in cubic feet per second, 1965

\begin{tabular}{|c|c|c|c|c|c|c|c|c|c|c|c|}
\hline Day & March & April & May & Day & March & April & May & Day & March & April & May \\
\hline & 4,810 & 2,110 & 416 & $11 \ldots$ & 2,510 & 5,110 & 180 & $21 \ldots$ & 5,300 & 660 & 205 \\
\hline & 7,210 & 1,480 & 371 & $12 \ldots$ & 2,920 & 11,600 & 172 & 22. & 1,480 & 554 & 169 \\
\hline U- & 4,640 & 2,220 & 332 & $13 \ldots$ & 4,330 & 6,620 & 162 & 23 & 1,060 & 490 & 144 \\
\hline & 2,430 & 12,200 & 302 & $14_{\ldots}$ & 6,510 & 2,350 & 148 & 24. & 865 & 470 & 131 \\
\hline & 1,410 & 11,700 & 277 & $15 \ldots$ & 8,560 & 2,040 & 138 & 25 & 716 & 617 & 108 \\
\hline & 995 & 20,500 & 261 & $16 \ldots$ & 8,300 & 2,750 & 127 & 26. & 571 & 1,030 & 121 \\
\hline & 898 & 25,400 & 244 & 17. & 15,900 & 3,280 & 121 & 27 & 516 & 1,240 & 118 \\
\hline & 898 & 18,700 & 221 & 18 & 23,400 & 1,970 & 138 & 28 & 716 & 1,130 & 105 \\
\hline & 1,240 & 9,880 & 209 & 19. & 21,100 & 1,160 & 144 & 29 & 2,270 & 648 & 90 \\
\hline & 1,970 & 2,590 & 190 & $20 .-$ & 11,600 & 832 & 144 & 30. & 4,130 & 485 & 88 \\
\hline & & & & & & & & 31 & 3,460 & & 85 \\
\hline \multicolumn{9}{|c|}{ Monthly mean discharge, in cubic feet per second } & 4,926 & 5,061 & 183 \\
\hline \multicolumn{9}{|c|}{ Runoff, in inches } & 2.29 & 2.28 & 0.08 \\
\hline \multicolumn{9}{|c|}{ Runoff, in acre-feet. } & 302,900 & $301,10 \mathrm{C}$ & 11,230 \\
\hline
\end{tabular}


(325) 5-5130. Bay Creek at Nebo, Ill.

Location.--Lat $39^{\circ} 26^{\prime} 35^{\prime \prime}$, long $90^{\circ} 47^{\prime} 45^{\prime \prime}$, in NW $\frac{1}{4}$ sec.19, T.7 S., R. 3 W., on left bank $400 \mathrm{ft}$ downstream from highway bridge, $500 \mathrm{ft}$ upstream from Spring Creek, a quarter of a mile west of Nebo, and 1.6 miles upstream from Chicago and Alton Railroad bridge. Records include flow of Spring Creek.

Drainage area.-162 sq $\mathrm{mi}$, including that of Spring Creek.

Gage-height record.-Digital recorder tape punched at 15-minute intervals. Prior to April 28, 1965, water-stage recorder graph. Datum of gage is $462.56 \mathrm{ft}$ above mean sea level, datum of 1929 (levels by Corps of Engineers).

Discharge record.- Stage-discharge relation defined by current-meter measurements below $9,000 \mathrm{cfs}$ and extended above by logarithmic plotting.

Maxima.-April-May 1965: Discharge, 2,140 cfs 0900 hours Apr. 11 (gø ge height, $9.49 \mathrm{ft}$ ).

1939 to March 1965: Discharge, 23,500 cfs Aug. 16, 1946 (gage height, $19.31 \mathrm{ft}$ ).

Mcan discharge, in cublc feet per second, 1965

\begin{tabular}{|c|c|c|c|c|c|c|c|c|}
\hline Day & April & May & Day & April & May & Day & April & May \\
\hline & 53 & 43 & $11 \ldots$ & 1,110 & 25 & 21. & 61 & 18 \\
\hline $2 \ldots$ & 46 & 39 & $12_{\ldots}$ & 202 & 22 & $22 \ldots$ & 53 & 17 \\
\hline $3 \ldots$ & 46 & 36 & $13 \ldots$ & 112 & 20 & $23 \ldots$ & 49 & 17 \\
\hline 4 & 51 & 33 & $14 \ldots$ & 119 & 22 & $24 \ldots$ & 86 & 16 \\
\hline ì. & 291 & 32 & $15 \ldots$ & 381 & 21 & $25 \ldots$ & 175 & 16 \\
\hline $6 \ldots$ & 562 & 32 & $16 \ldots$ & 145 & 20 & $26 \ldots$ & 94 & 22 \\
\hline $7 \ldots$ & 134 & 31 & $17 \ldots$ & 102 & 19 & $27 \ldots$ & 62 & 32 \\
\hline $8 \ldots$ & 94 & 46 & $18 \ldots$ & 84 & 20 & $28 \ldots$ & 56 & 26 \\
\hline $9 \ldots$ & 75 & 39 & $19 \ldots$ & 72 & 20 & $29 \ldots$ & 51 & 18 \\
\hline $10 \ldots$ & 289 & 29 & $20 \ldots$ & 64 & 19 & $30 \ldots$ & 47 & 15 \\
\hline & & & & & & $31 \ldots$ & . & 14 \\
\hline \multirow{2}{*}{\multicolumn{7}{|c|}{$\begin{array}{l}\text { Monthly mean discharge, in cubic feet per second } \\
\text { Runoff, in inches }\end{array}$}} & 159 & 25.1 \\
\hline & & & & & & & 1.09 & 0.18 \\
\hline
\end{tabular}

Gage helght, in fect, and deschonge, in cubie feot per second, at indicated time, 1965

\begin{tabular}{|c|c|c|c|c|c|c|c|c|c|c|c|}
\hline Date & Hour $\mathrm{r}$ & $\begin{array}{l}\text { Gage } \\
\text { height }\end{array}$ & $\begin{array}{c}\text { Dis - } \\
\text { charge }\end{array}$ & 1)ate & Hlour & $\begin{array}{l}\text { Gage } \\
\text { hoight }\end{array}$ & $\begin{array}{c}\text { Dis- } \\
\text { charge }\end{array}$ & Date & Hour & $\begin{array}{c}\text { Gage } \\
\text { height }\end{array}$ & $\begin{array}{c}\text { Dis- } \\
\text { charge }\end{array}$ \\
\hline \multirow[t]{8}{*}{ Apr. 10} & 0000 & 1.67 & 67 & \multirow[t]{8}{*}{ Apr. 11} & 0300 & 4.35 & 539 & \multirow[t]{7}{*}{ Apr. 11} & \multirow{3}{*}{$\begin{array}{l}2200 \\
2400\end{array}$} & \multirow{3}{*}{$\begin{array}{l}4.29 \\
3.68\end{array}$} & 526 \\
\hline & 1200 & 1.67 & 67 & & 0500 & 5.89 & 947 & & & & \multirow{2}{*}{392} \\
\hline & 1400 & 4.28 & 524 & & 0600 & 7.87 & 1,540 & & & & \\
\hline & 1500 & 5.90 & 950 & & 0700 & 9.35 & 2,080 & & \multirow{5}{*}{$\begin{array}{l}0600 \\
1200 \\
2400\end{array}$} & \multirow{5}{*}{$\begin{array}{l}2.85 \\
2.48 \\
2.17\end{array}$} & \multirow{5}{*}{$\begin{array}{l}230 \\
171 \\
127\end{array}$} \\
\hline & 1800 & 3.62 & 378 & & 0900 & 9.49 & 2,140 & & & & \\
\hline & 2000 & 3.08 & 270 & & 1200 & 7.60 & $1 ; 460$ & & & & \\
\hline & 2300 & 5.07 & 718 & & 2000 & 5.07 & 718 & & & & \\
\hline & 2400 & 4.96 & 690 & & & & & & & & \\
\hline
\end{tabular}




\section{CUIVRE RIVER BASIN}

(326) 5-5145. Cuivre River near Troy, Mo.

Location.-Lat $39^{\circ} 00^{\prime} 59^{\prime \prime}$, long $90^{\circ} 59^{\prime} 00^{\prime \prime}$, in $\mathrm{SE} \frac{1}{4}$ sec. $14, \mathrm{~T} .49$ N., R.1 W., on downstream side of center pier of bridge on U.S. Highway $61,1 \frac{1}{2}$ miles downstream from confluence of North and West Forks and 2 miles north of Troy.

Drainage area.- $-903 \mathrm{sq} \mathrm{mi}$.

Gage-height record.-Water-stage recorder graph except Mar. 25-26, Apr. 22-24, Apr. 29 to May 31 for which graph was constructed from once-daily wire-weight gage readings. Datum of gage is $450.27 \mathrm{ft}$ above mean sea level, datum of 1929 .

Discharge record.- Stage-discharge relation defined by current-meter measurements.

Maxima.-March-May 1965: Discharge, 11,200 cfs 1400 hours Apr. 6 (gage height, $20.54 \mathrm{ft}$ ).

1888 to February 1965: Discharge, 120,000 cfs Oct. 5, 1941 (gage height, $33.4 \mathrm{ft}$, from floodmarks).

Mean discharge, in cubic feet per second, 1965

\begin{tabular}{|c|c|c|c|c|c|c|c|c|c|c|c|}
\hline Day & March & April & May & Day & March & April & May & Day & March & April & May \\
\hline & 2,200 & 282 & 164 & $11 \ldots$ & 635 & 3,080 & 75 & 21 & 210 & 232 & 23 \\
\hline $2-$ & 2,350 & 200 & 143 & 12 & 575 & 4,270 & 68 & 22. & 160 & 182 & 22 \\
\hline 3. & 1,250 & 271 & 125 & 13 & 695 & 1,250 & 64 & 23. & 148 & 150 & 24 \\
\hline & 470 & 2,850 & 113 & 14 & 575 & 515 & 56 & 24 & 135 & 132 & 22 \\
\hline & 298 & 1,490 & 100 & $15 \ldots$ & 398 & 4,670 & 42 & 25 & 125 & 1,370 & 22 \\
\hline o. & 222 & 8,440 & 177 & $16 \ldots$ & 282 & 2,250 & 38 & 26. & 118 & 970 & 30 \\
\hline & 200 & 3,550 & 105 & $17--$ & 1,320 & 930 & 33 & 27. & 118 & 470 & 28 \\
\hline & 272 & 1,370 & 90 & 18 & 3,260 & 590 & 31 & 28 & 470 & 320 & 34 \\
\hline & 1,290 & 575 & 87 & 19 & 970 & 398 & 29 & 29. & 1,290 & 240 & 32 \\
\hline & 1,010 & 385 & 82 & 20 & 360 & 300 & 26 & $30^{-}$ & 890 & 182 & 26 \\
\hline & & & & & & & & 31. & 470 & $\ldots$ & 16 \\
\hline \multirow{3}{*}{\multicolumn{9}{|c|}{$\begin{array}{l}\text { Monthly mean discharge, in cubic feet per second } \\
\text { Runoff, in nches } \\
\text { Runoff, m acre-feet }\end{array}$}} & 734 & 1,397 & 62.2 \\
\hline & & & & & & & & & 0.94 & 1.73 & 0.08 \\
\hline & & & & & & & & & 45,160 & 83,140 & 3,820 \\
\hline
\end{tabular}

\section{ILLINOIS RIVER BASIN}

(327) 5-5855. Illinois River at Meredosia, Ill.

Location.-Lat $39^{\circ} 49^{\prime} 36^{\prime \prime}$, long $90^{\circ} 33^{\prime} 53^{\prime \prime}$, in SE $\frac{1}{4} \mathrm{NE} \frac{1}{4}$ sec. 21, T.16 N., R.13 W., on left bank 0.3 mile downstream from bridge on State Highway 104 in Meredosia, $4 \frac{1}{2}$ miles upstream from McKee Creek and at mile 71.1 .

Drainage area. $-25,300 \mathrm{sq} \mathrm{mi}$, approximately.

Gage-height record.-Water-stage recorder graph. Datum of gage is $418.00 \mathrm{ft}$ above mean sea level, datum of 1929 (levels by Corps of Engineers).

Discharge record.--Stage-fall-discharge relation defined by current-meter measurements.

Maxima.-April-May 1965: Discharge, 55,000 cfs 0500 hours April 18; gage height, $17.65 \mathrm{ft} 1000$ hours to 1700 hours Apr. 19.

1938 to March 1965: Discharge, 123,000 cfs May 26-28, 1943; gage height, 28.61 ft May 26, 1943. 
Mean discharge, in cubic feet per second. 1965, of Illinois River at Meredosia, Ill.

\begin{tabular}{|c|c|c|c|c|c|c|c|c|}
\hline Day & April & May & Day & April & May & Day & April & May \\
\hline $\begin{array}{l}1 \ldots \ldots \\
2 \ldots \ldots \\
3 \ldots \ldots \\
4 \ldots \ldots \\
5 \ldots \ldots \\
6 \ldots \ldots \\
7 \ldots \ldots \\
8 \ldots \ldots \\
9 \ldots \ldots \\
10 \ldots \ldots\end{array}$ & $\begin{array}{l}31,900 \\
32,100 \\
31,800 \\
31,100 \\
31,400 \\
34,000 \\
38,600 \\
41,300 \\
43,000 \\
44,400\end{array}$ & $\begin{array}{l}49,600 \\
49,800 \\
49,700 \\
49,600 \\
49,200 \\
47,700 \\
46,500 \\
46,000 \\
45,600 \\
45,800\end{array}$ & $\begin{array}{l}11 \ldots \\
12 \ldots \\
13 \ldots \\
14 \ldots \\
15 \\
16 \ldots \\
17 \ldots \\
18 \ldots \\
18_{\ldots} \ldots \\
20 \ldots\end{array}$ & $\begin{array}{l}46,500 \\
49,500 \\
50,500 \\
51,000 \\
52,000 \\
53,200 \\
53,200 \\
54,400 \\
54,000 \\
53,200\end{array}$ & $\begin{array}{l}45,600 \\
45,000 \\
44,300 \\
43,100 \\
40,900 \\
39,900 \\
38,800 \\
38,100 \\
36,200 \\
34,900\end{array}$ & $\begin{array}{l}21 \ldots \\
22 \ldots \\
23 \ldots \\
24 \ldots \\
25 \ldots \\
26 \ldots \\
27 \ldots \\
28 \ldots \\
26_{-} \\
30 \ldots \\
31 \ldots \\
31 \ldots\end{array}$ & $\begin{array}{l}52,400 \\
51,800 \\
51,200 \\
49,500 \\
47,700 \\
46,600 \\
46,700 \\
48,000 \\
48,800 \\
48,800\end{array}$ & $\begin{array}{l}34,100 \\
32,800 \\
31,200 \\
29,800 \\
28,300 \\
27,900 \\
27,200 \\
26,300 \\
24,600 \\
21,900 \\
17,900\end{array}$ \\
\hline \multicolumn{7}{|c|}{$\begin{array}{l}\text { Monthly mean discharge, in cubic feet per second } \\
\text { Runoff, in inches. }\end{array}$} & $\begin{array}{r}45,600 \\
2.01\end{array}$ & $\begin{array}{r}38,300 \\
1.75\end{array}$ \\
\hline
\end{tabular}

(328) 5-5870. Macoupin Creek near Kane, Ill.

Location.-Lat $39^{\circ} 14^{\prime} 00^{\prime \prime}$, long $90^{\circ} 23^{\prime} 45^{\prime \prime}$, in SE $\frac{1}{4}$ sec. 11, T.9 N., R.12 W., near center of span on downstream side of bridge on U.S. Highway 67 (alternate), 1.4 miles downstream from Link Branch, and $3 \frac{1}{2}$ miles northwest of Kane.

Drainage area. $-875 \mathrm{sq} \mathrm{mi}$.

Gage-height record.-Graph based on twice-daily readings of wire-weight gage, except Apr. 24, May 6, 10, 26. Datum of gage is $426.77 \mathrm{ft}$ above mean sea level, datum of 1929.

Discharge record.-Stage-discharge relation defined by current-meter measurements, Discharge for periods of no gage-height record estimated on basis of records for nearby stations.

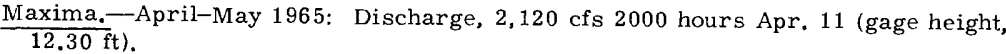

1921-33, 1940 to March 1965: Discharge, 40,000 cfs May 18, 1943 (Eage height, $28.5 \mathrm{ft}$ ).

Mean discharac, in cubic feet per second, 1965

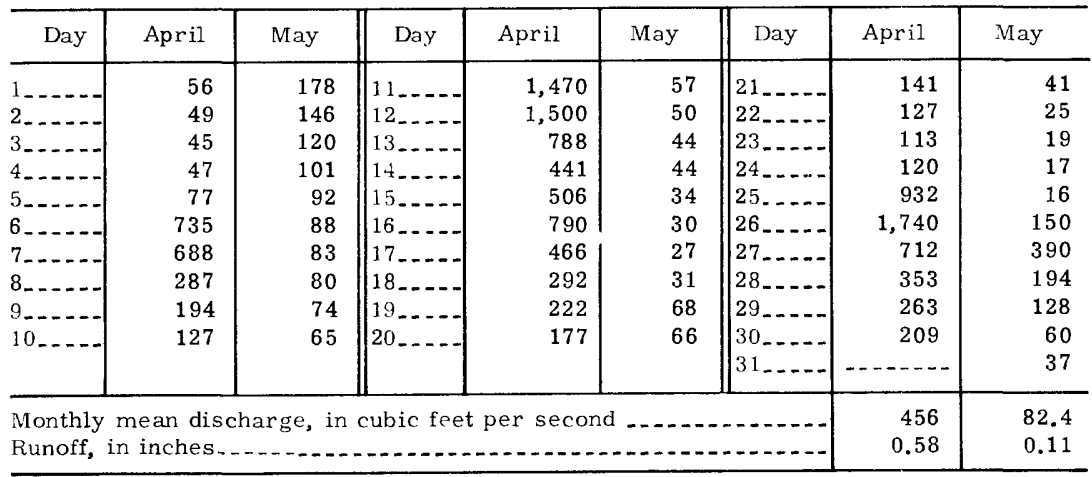




\section{MISSISSIPPI RIVER MAIN STEM}

(329) 5-5875. Mississippi River at Alton, Ill.

Location.-Lat $38^{\circ} 53^{\prime} 06^{\prime \prime}$, long $90^{\circ} 10^{\prime} 51^{\prime \prime}$, in sec.14, T.5 N., R.10 W., near left bank in downstream end of intermediate lock wall of lock and dam 26 at Alton, $300 \mathrm{f}$. downstream from Missouri and Illinois Bridge and Belt Railroad bridge, 7.7 miles upstream from Missouri River, and at mile 202.7 above Ohio River.

Drainage area. $-171,500 \mathrm{sq} \mathrm{mi}$, approximately.

Gage-height record.-Water-stage recorder graph. Datum of gage is at mean sea level, datum of 1929 (levels by Corps of Engineers).

Discharge record.-Stage-discharge relation defined by current-meter measurements. Fall used as a factor.

Maxima,-March-May 1965: Discharge, 380,000 cfs 0700 hours to 2300 hours May 3; gage height, $420.75 \mathrm{ft} 1500$ hours to 1800 hours Apr. 16.

1927 to February 1965: Discharge, 437,000 cfs May 24, 1943 (gage height, 429.91 ft), includes 90,000 cfs floodwater overflow from Missouri River.

Flood in June 1844 reached a stage of $432.10 \mathrm{ft}$ (discharge unknown).

Remarks.- Natural flow of stream affected by many reservoirs and navigation dams in upper Mississippi River basin.

Cooperation.--Alton gage-height record furnished by Corps of Engineers.

Mean discharge, in cubic feet per second, 1965

\begin{tabular}{|c|c|c|c|c|c|c|c|c|c|c|c|}
\hline Day & $\operatorname{arch}$ & April & May & Day & March & April & May & Day & March & April & May \\
\hline $\begin{array}{l}2 \\
3 \\
1 \\
5 \\
5 \ldots \\
6 \\
7 \ldots \\
8 \ldots \\
9 \\
10 \\
10\end{array}$ & $\begin{array}{r}62,400 \\
98,800 \\
118,000 \\
115,000 \\
119,000 \\
122,000 \\
133,000 \\
164,000 \\
164,000 \\
163,000\end{array}$ & $\begin{array}{l}155,000 \\
150,000 \\
154,000 \\
172,000 \\
188,000 \\
208,000 \\
219,000 \\
240,000 \\
261,000 \\
278,000\end{array}$ & $\begin{array}{l}369,000 \\
377,000 \\
380,000 \\
378,000 \\
374,000 \\
374,000 \\
373,000 \\
373,000 \\
368,000 \\
363,000\end{array}$ & \begin{tabular}{|l}
$11 \ldots$ \\
$12_{-}$ \\
$13 \ldots$ \\
$14 \ldots$ \\
$15_{\ldots}$ \\
$16_{-}$ \\
$17 \ldots$ \\
$18 \ldots$ \\
$19_{-}$ \\
$20_{-}$
\end{tabular} & \begin{tabular}{|l|}
165,000 \\
167,000 \\
176,000 \\
180,000 \\
176,000 \\
170,000 \\
181,000 \\
199,000 \\
212,000 \\
223,000
\end{tabular} & $\begin{array}{l}287,000 \\
297,000 \\
313,000 \\
330,000 \\
343,000 \\
351,000 \\
363,000 \\
369,000 \\
377,000 \\
375,000\end{array}$ & \begin{tabular}{|}
356,000 \\
344,000 \\
337,000 \\
328,000 \\
318,000 \\
307,000 \\
297,000 \\
281,000 \\
265,000 \\
243,000
\end{tabular} & $\begin{array}{l}21 \ldots \\
22 \ldots \\
23 \ldots \\
24 \ldots \\
25 \ldots \\
26 \ldots \\
27 \ldots \\
28 \ldots \\
29 \\
30_{-}\end{array}$ & $\begin{array}{r}233,000 \\
207,000 \\
149,000 \\
126,000 \\
111,000 \\
93,200 \\
81,700 \\
91,700 \\
102,000 \\
113,000 \\
146,000\end{array}$ & $\begin{array}{l}367,000 \\
358,000 \\
350,000 \\
345,000 \\
345,000 \\
342,000 \\
339,000 \\
339,000 \\
348,000 \\
357,000 \\
\ldots \ldots\end{array}$ & $\left\{\begin{array}{l}225,000 \\
209,000 \\
197,000 \\
188,000 \\
184,000 \\
179,000 \\
77,000 \\
166,000 \\
71,000 \\
172,000 \\
172,000\end{array}\right.$ \\
\hline \multicolumn{9}{|c|}{$\begin{array}{l}\text { Monthly mean discharge, in cubic feet per second } \\
\text { Ruroff, in inches } \\
\text { Runoff, in acre-feet }\end{array}$} & $\begin{array}{r}147,200 \\
0.99 \\
* 9,048\end{array}$ & $\begin{array}{r}297,300 \\
1,93 \\
* 17,690\end{array}$ & $\begin{array}{r}285,300 \\
1.92 \\
* 17,540\end{array}$ \\
\hline
\end{tabular}

*In thousands 


\section{MISSOURI RIVER MAIN STEM}

(330) 6-9345. Missouri River at Hermann, Mo.

Location.-Lat $38^{\circ} 42^{\prime} 36^{\prime \prime}$, long $91^{\circ} 26^{\prime} 21^{\prime \prime}$, in SW $\frac{1}{4}$ sec.25, T.46 N., R.5 W., on downstream side of third pier from right abutment of bridge on State Highway 19 at Hermann.

River mile, 97.9.

Drainage area. $-528,200 \mathrm{sq} \mathrm{mi}$, approximately.

Gage-height record.-Water-stage recorder graph. Datum of gage is $481.56 \mathrm{ft}$ above mean sea level, datum of 1929 .

Discharge record.--Stage-discharge relation defined by current-meter measurements.

Maxima.-March-May 1965: Discharge, 258,000 cfs 1400 hours Apr. 8 (gage height, $23 . \overline{50} \mathrm{ft}$ ).

1897 to February 1965: Discharge, 676,000 cfs June 6, 7, 1903; gag : height, $33.33 \mathrm{ft}$ July $19,1951$.

Flood of June 1844 reached a gage height of $35.5 \mathrm{ft}$ (discharge about $892,000 \mathrm{cfs}$, computed by Corps of Engineers).

Remarks.-Flow partly regulated by many reservoirs above station.

Mean discharge, in cubic feet per second, 1965

\begin{tabular}{|c|c|c|c|c|c|c|c|c|c|c|c|}
\hline Day & March & April & May & Day & March & April & May & Day & March & April & May \\
\hline & 42,800 & 68,200 & 65,200 & $11 \ldots$ & 80,500 & 226,000 & 57,900 & 21. & 186,000 & 85,500 & 51,300 \\
\hline & 48,500 & 69,600 & 59,000 & 12. & 92,300 & 224,000 & 64,800 & 22. & 148,000 & 77,500 & 54,900 \\
\hline 3 & 114,000 & 74,100 & 53,800 & 13. & 88,500 & 202,000 & 57,500 & 23. & 124,000 & 72,800 & 51,600 \\
\hline & 160,000 & 140,000 & 51,600 & 14. & 84,500 & 176,000 & 56,400 & 24. & 101,000 & 66,500 & 51,000 \\
\hline 5. & 123,000 & 178,000 & 51,600 & 15 & 82,000 & 180,000 & 63,100 & 25. & 80,000 & 63,100 & 62,300 \\
\hline 6 & 96,700 & 223,000 & 53,400 & 16. & 95,000 & 154,000 & 59,400 & 26. & 66,100 & 58,300 & 92,300 \\
\hline 7. & 79,000 & 246,000 & 54,900 & 17 _ & 117,000 & 141,000 & 51,600 & $\mid 27 \ldots$ & 59,000 & 62,300 & 77,500 \\
\hline & 65,200 & 256,000 & 52,400 & $\mid 18$ _ & 170,000 & 128,000 & 49,600 & $\mid 28 \ldots$ & 53,400 & 84,000 & 76,500 \\
\hline & 59,000 & 254,000 & 49,200 & $19 \ldots$ & 208,000 & 110,000 & 47,200 & $29 \ldots$ & 52,000 & 87,000 & 99,400 \\
\hline & 67,400 & 237,000 & 47,500 & $20 \ldots$ & 217,000 & 98,400 & 46,500 & 30 & 57,400 & 75,500 & 114,000 \\
\hline & & & & & & & & & 64,800 & & 94,500 \\
\hline \multicolumn{9}{|c|}{ Monthly mean discharge, in cubic feet per second....... } & 99,440 & 137,300 & 61,870 \\
\hline \multicolumn{9}{|c|}{ Runnf, in inches } & 0.22 & 0.29 & 0.14 \\
\hline \multicolumn{9}{|c|}{ Rutotf, in acre-feet } & *6,115 & $* 8,168$ & *3,804 \\
\hline
\end{tabular}

*In thousands 


\section{LOUTRE RIVER BASIN}

(331) 6-9355. Loutre River at Mineola, Mo.

Location.-Lat $38^{\circ} 53^{\prime} 20^{\prime \prime}$, long $91^{\circ} 34^{\prime} 30^{\prime \prime}$, in $\mathrm{SE} \frac{1}{4} \mathrm{NW} \frac{1}{4}$ sec.34, T.48 N., R.6 W., at downstream side of left pier of bridge in Mineola, 0.2 mile upstream from Sallee Branch, $1 \frac{1}{4}$ mile downstream from Interstate Highway 70 .

Drainage area. $-202 \mathrm{sq} \mathrm{mi}$.

Gage-height record.-Water-stage recorder graph except for Mar. 3, 6-9, days of no gage-height record and Mar. 1, 2, 10-12, from graph based on gage readings recorded range of stage and partial recorder record.

Discharge record.-Stage-discharge relation defined by current-meter measurements. Rate of change in stage used as a factor for stages above $8 \mathrm{ft}$.

Maxima.-March-May 1965: Discharge, 5,580 cfs 0400 hours Apr. 6 (gage height, $15.27 \mathrm{ft}$ ).

1947 to February 1965: Discharge, 12,900 cfs June 30, 1957 (gage height, $20.88 \mathrm{ft}$ ). Flood of June 1928 reached a stage of about, $28.9 \mathrm{ft}$, from information by local resident.

Mean discharge, in cubic feet per second, 1965

\begin{tabular}{|c|c|c|c|c|c|c|c|c|c|c|c|}
\hline Day & March & April & May & Day & March & April & May & Day & March & April & May \\
\hline 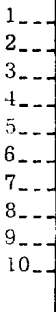 & $\begin{array}{r}498 \\
253 \\
120 \\
59 \\
50 \\
45 \\
40 \\
50 \\
100 \\
280\end{array}$ & $\begin{array}{r}72 \\
55 \\
1,090 \\
682 \\
685 \\
2,210 \\
372 \\
202 \\
130 \\
107\end{array}$ & $\begin{array}{l}38 \\
33 \\
28 \\
26 \\
23 \\
22 \\
20 \\
17 \\
16 \\
14\end{array}$ & $\begin{array}{l}11 \ldots- \\
12_{-}- \\
13_{-} \\
14_{-} \\
15_{-} \\
16_{-} \\
17 \ldots \\
18_{-} \\
19_{-} \\
20_{-}\end{array}$ & $\begin{array}{r}151 \\
212 \\
182 \\
114 \\
78 \\
51 \\
1,480 \\
386 \\
132 \\
81\end{array}$ & $\begin{array}{r}997 \\
280 \\
125 \\
334 \\
1,720 \\
386 \\
199 \\
130 \\
97 \\
76\end{array}$ & $\begin{array}{r}12 \\
11 \\
9.6 \\
8.2 \\
7.4 \\
6.4 \\
5.4 \\
8.7 \\
5.2 \\
4.2\end{array}$ & $\begin{array}{l}21 \\
22_{-} \\
23_{-} \\
24_{-} \\
25_{-} \\
26_{-} \\
27- \\
28_{-} \\
29_{-} \\
30_{-} \\
31_{-}\end{array}-$ & $\begin{array}{r}62 \\
54 \\
70 \\
71 \\
53 \\
46 \\
96 \\
470 \\
456 \\
163 \\
97\end{array}$ & $\begin{array}{r}65 \\
55 \\
50 \\
45 \\
197 \\
167 \\
87 \\
66 \\
54 \\
46 \\
-\end{array}$ & $\begin{array}{l}3.7 \\
3.2 \\
2.9 \\
2.7 \\
2.5 \\
5.6 \\
5.4 \\
4.2 \\
5.0 \\
4.0 \\
3.2\end{array}$ \\
\hline \multicolumn{9}{|c|}{$\begin{array}{l}\text { Monthly mean discharge, in cubic feet per second } \\
\text { Rmoff, in inches } \\
\text { Rumoff, in acre-feet }\end{array}$} & $\begin{array}{r}194 \\
1.10 \\
11,900\end{array}$ & $\begin{array}{r}359 \\
1.98 \\
21,380\end{array}$ & $\begin{array}{l}11.5 \\
0.07 \\
709\end{array}$ \\
\hline
\end{tabular}

\section{COLDWATER CREEK BASIN}

(332) 6--9365. Coldwater Creek near St. Louis, Mo.

Location.-Lat $38^{\circ} 48^{\prime} 50^{\prime \prime}$, long $90^{\circ} 13^{\prime} 50^{\prime \prime}$, in sec.16, T.47 N., R.7 E., on right wingwall on downstream side of bridge on U.S. Highway $67,1.7$ miles upstream from mouth, 3.5 miles south of West Alton, and 6.0 miles north of St. Louis city limits.

Drainage area. $-43.6 \mathrm{sq} \mathrm{mi}$.

Gage-height record.-Digital recorder tape punched at 15 minute intervals. Ditum of gage is $442.63 \mathrm{ft}$ above mean sea level, datum of 1929 (Missouri Highway Dejartment bench mark).

Discharge record.--Stage-discharge relation defined by current-meter measurements. Discharge on Apr. 12,13, obtained from adjoining good record and weather records.

Maxima.-March-May 1965: Discharge, 1,210 cfs 0015 hours Apr. 6 (gage height, $6.94 \mathrm{ft})$.

1959-61, 1962 to February 1965: Discharge, 6,170 cfs June 29, 1960 (gage height, $17.13 \mathrm{ft})$. 
Mean discharge, in cubic feet per second, 1965, of Coldwater Creek near St. Louis. Mo.

\begin{tabular}{|c|c|c|c|c|c|c|c|c|c|c|c|}
\hline Day & March & April & May & Day & March & April & May & Day & March & A pril & May \\
\hline 1. & 73 & 32 & 33 & 11. & 34 & 44 & 30 & 21 & 25 & 34 & 24 \\
\hline 2. & 156 & 31 & 26 & $12 \ldots$ & 33 & 39 & 29 & 22. & 28 & 33 & 24 \\
\hline 3. & 44 & 96 & 23 & $13_{-}$ & 31 & 35 & 28 & 23. & 51 & 33 & 22 \\
\hline 4. & 49 & 39 & 39 & $14 \ldots$ & 30 & 54 & 28 & 24 & 31 & 32 & 33 \\
\hline 5. & 53 & 288 & 37 & $15 \ldots$ & 29 & 102 & 27 & 25 & 29 & 160 & 24 \\
\hline 6. & 46 & 258 & 50 & $16 \ldots$ & 29 & 46 & 24 & 26. & 37 & 38 & 82 \\
\hline 7. & 48 & 65 & 34 & $17 \ldots$ & 57 & 39 & 27 & 27. & 34 & 35 & 40 \\
\hline 8. & 50 & 54 & 31 & $18 \ldots$ & 30 & 33 & 61 & 28. & 30 & 39 & 23 \\
\hline 9. & 42 & 47 & 32 & $19 \ldots$ & 28 & 34 & 24 & 29. & 31 & 32 & 21 \\
\hline 10 & 36 & 56 & 30 & $20 \ldots$ & 28 & 35 & 24 & $30_{-}$ & 32 & 31 & 17 \\
\hline & & & & & & & & 31 & 32 & 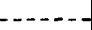 & 18 \\
\hline \multirow{3}{*}{\multicolumn{9}{|c|}{$\begin{array}{l}\text { Monthly mean discharge, in cubic feet per second } \\
\text { Runoff, in inches } \\
\text { Runoff, in acre-feet }\end{array}$}} & 41.5 & 63.1 & 31.1 \\
\hline & & & & & & & & & 1.10 & 1.62 & 0.82 \\
\hline & & & & & & & & & 2,550 & 3,760 & 1,910 \\
\hline
\end{tabular}

\section{MISSISSIPPI RIVER MAIN STEM}

(333) 7-0100. Mississippi River at St. Louis, Mo.

Location.--Lat $38^{\circ} 37^{\prime} 44^{\prime \prime}$, long $90^{\circ} 10^{\prime} 47^{\prime \prime}$, on downstream side of west pier of Eads Bridge at St. Louis, 15 miles downstream from Missouri River, 19.2 miles upstream from Meramec River, and at mile 180.0 above Ohio River.

Drainage area.-701,000 sq $\mathrm{mi}$, approximately.

Gage-height record.-Water-stage recorder graph. Datum of gage is $379.94 \mathrm{ft}$ above mean sea level, datum of 1929 .

Discharge record.- Stage-discharge relation defined by current-meter measurements.

Maxima.-March-May 1965: Discharge, 525,000 cfs 1430 hours Apr. 16 (gage height, $28.83 \mathrm{ft}$ ).

1861 to February 1965: Daily discharge, 1,019,000 cfs June 10, 11, 1903; gage height, $40.28 \mathrm{ft}$ July 22, 1951.

Flood in April 1785 may have reached a stage of $42.0 \mathrm{ft}$.

Flood in June 1844 reached a stage of $41.32 \mathrm{ft}$, from floodmarks (discharge, 1,300,000 cfs), computed by Corps of Engineers.

Remarks.- Natural flow of stream affected by many reservoirs and navigation dams in upper Mississippi River basin and by many reservoirs and diversions for irrigation in Missouri River basin.

Mean discharge, in cubic feet per second, 1965

\begin{tabular}{|c|c|c|c|c|c|c|c|c|c|c|c|}
\hline Day & March & April & May & Day & March & April & May & Day & March & April & May \\
\hline 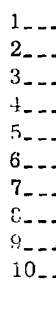 & $\begin{array}{l}102,000 \\
131,000 \\
164,000 \\
225,000 \\
275,000 \\
254,000 \\
231,000 \\
243,000 \\
240,000 \\
230,000\end{array}$ & $\begin{array}{l}218,000 \\
221,000 \\
218,000 \\
248,000 \\
324,000 \\
394,000 \\
444,000 \\
479,000 \\
500,000 \\
509,000\end{array}$ & $\begin{array}{l}431,000 \\
429,000 \\
427,000 \\
423,000 \\
416,000 \\
414,000 \\
414,000 \\
416,000 \\
414,000 \\
408,000\end{array}$ & $\mid \begin{array}{l}11 \ldots \\
12 \ldots- \\
13 \ldots \\
14 \ldots \\
15 \ldots \\
16 \ldots \\
17 \ldots \\
18 \ldots \\
19 \ldots \\
20 \ldots\end{array}$ & $\begin{array}{l}234,000 \\
246,000 \\
265,000 \\
270,000 \\
263,000 \\
260,000 \\
279,000 \\
324,000 \\
388,000 \\
425,000\end{array}$ & $\begin{array}{l}513,000 \\
516,000 \\
520,000 \\
516,000 \\
516,000 \\
523,000 \\
511,000 \\
500,000 \\
488,000 \\
474,000\end{array}$ & $\begin{array}{l}400,000 \\
396,000 \\
394,000 \\
384,000 \\
370,000 \\
362,000 \\
351,000 \\
335,000 \\
317,000 \\
299,000\end{array}$ & $\begin{array}{l}21-- \\
22-- \\
23-- \\
24-- \\
25-- \\
26-- \\
27-- \\
28-- \\
29- \\
30- \\
31-\end{array}$ & $\begin{array}{l}440,000 \\
408,000 \\
324,000 \\
263,000 \\
216,000 \\
178,000 \\
152,000 \\
147,000 \\
154,000 \\
159,000 \\
192,000\end{array}$ & $\begin{array}{l}459,000 \\
440,000 \\
425,000 \\
414,000 \\
412,000 \\
404,000 \\
398,000 \\
398,000 \\
414,000 \\
429,000\end{array}$ & $\begin{array}{l}279,000 \\
265,000 \\
255,000 \\
245,000 \\
237,000 \\
237,000 \\
263,000 \\
254,000 \\
243,000 \\
265,000 \\
286,000\end{array}$ \\
\hline $\begin{array}{l}\text { Ru } \\
\text { Ru }\end{array}$ & $\begin{array}{l}\text { If, in } \\
\text { if, in }\end{array}$ & $\begin{array}{l}\text { hes } \\
\text { e-fee }\end{array}$ & - - & & & & & & $\begin{array}{r}247,800 \\
0.41 \\
* 15,240\end{array}$ & $\begin{array}{r}427,500 \\
0.68 \\
* 25,440\end{array}$ & $\begin{array}{r}342,900 \\
0.56 \\
* 21,080\end{array}$ \\
\hline
\end{tabular}

\footnotetext{
*In thousands
} 


\section{REFERENCES CITED}

Benson, M. A., 1962a, Evolution of methods for evaluating the occurrence of floods: U.S. Geol. Survey Water-Supply Paper $1580-\mathrm{A}, 30 \mathrm{p}$.

-1962b, Factors influencing the occurrence of floods in a humid region of diverse terrain: U.S. Geol. Survey WaterSupply Paper 1580-B, 62 p.

-1964, Factors affecting the occurrence of floods in the southwest: U.S. Geol. Survey Water-Supply Paper 1580-D,70 p.

Dalrymple, Tate, 1960, Flood-frequency analyses: U.S. Geol. Survey Water-Supply Paper 1543-A, $77 \mathrm{p}$.

Ericson, D. W., 1961, Floods in Wisconsin, magnitude cnd frequency: U.S. Geol. Survey open-file report, $109 \mathrm{p}$.

Mitchell, W. D., 1954, Floods in Illinois, magnitude and frequency: Div. of Waterways, Ill. Dept. Public Waters and Building $\varsigma 386 \mathrm{p}$.

Patterson, J. L., and Gamble, C. R., 1968, Magnitude and froquency of floods in the United States; Part 5, Hudson Bay and Mississippi River basins: U.S. Geol. Survey Water-Supply Papor 1678.

Prior, C. H., 1949, Magnitude and frequency of floods in Minnesota: Div. of Waters, Minn. Dept. Conservation Bull. 1, 128 p.

Prior, C. H., and Hess, J. H., 1961, Floods in Minnesota, magnitude and frequency: Div. of Waters, Minn. Dept. Conservation Bull. 12, $142 \mathrm{p}$.

Searcy, J. K., 1955, Floods in Missouri, magnitude and frequency: U.S. Geol. Survey Circ. 370, 126 p.

Schwob, H. H., 1953, Iowa floods, magnitude and frequency: Iowa Highway Research Board Bull. 1, 171 p.

-1966, Magnitude and frequency of Iowa floods: Iowa Highway Research Board Bull. 28, pt. 1, 47 p., pt. 2, 376 p.

U.S. Congress, Special House Subcommittee to Inspect Flooded Areas in the Upper Mississippi River Basin, 1965, Upper Mississippi River basin floods of April-May 1965: U.S. 89th Cong., 1st sess., House Comm. Print No. 13, 66 p. 


\section{INDEX}

A

Apple River near Hanover, Ill. near Somerset, $W$ is.

Apple River basin

Arkansaw Creek tributary near Arkansaw, Wis.

B

Bad Axe River, North Fork, near Genoa, Wis...............................

Bad Axe River basin.

Baraboo River near Baraboo, Wis.

Bashaw Brook near Shell Lake, Wis.

Bassett Creek at County Highway 66, Golden Valley, Minn................... at Fruen Mill Co., Minneapolis, Minn .... Bassett Creek basin . ........................ Bay Creek at Nebo, Ill.

Bear Creek at Hannibal, Mo.

near Marcelline, Ill

near Monmouth, Iowa.

Bear Creek basin

429,4

Bear Lake at Haugen, Wis. . . ..............

Beaver Creek at New Hartford, Iowa . ........

Big Bear Creek at Ladora, Iowa...........

Big Cedar Creek near Varina, Iowa . ..........

Big Creek near Mount Pleasant, Iowa ........

Big Eau Pleine Reservoir near Knowlton, Wis...............................

Big Eau Pleine River near Stratford, Wis....

Big St. Germain Lake near Lake Tomahawk, Wis....................................

Big Stone Lake at Ortonville, Minn . .........

Birch Lake at Birchwood, Wis.............

Black River at Neillsville, Wis................ near Galesville, $W$ is.

Black River basin . ... . . . . . . . . . . .

Blackhawk Creek at Hudson, Iowa..........

Blue Earth River, East Branch, near Bricelyn, Minn

East Branch, tributary near Blue Earth,

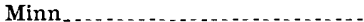
near Rapidan, Minn Boone River near Webster City, Iowa........ Buckatabon Lake near Conover, Wis.......... Buffalo Creek tributary near Brownton, Minn. Burnt Rollways Reservoir near Eagle River, Wis.

C

Cannon River at Welch, Minn Cannon River basin.
Page

A329
Castle Rock flowage near Mauston, Cedar Creek near Bussey, Iowa . . . . . . . . . $\quad 422$ Cedar River at Cedar Rapids, Iowa......... $\quad 375$ at Charles City, Iowa................ 362 at Janesville, Iowa. . . . . . . . . . . . . . . ..... 364 at Waterloo, Iowa............ 372 near Austin, Minn ...................... 360 near Conesville, Iowa................... 377 near Rochester, Iowa .................. 376

West Fork, at Finchford, Iowa........... 365 Chippewa River at Bishops Bridge, near Winter, Wis at Chippewa Falls, Wis................. 236 at Durand, Wis.......................... 245 below diversion dam, near Watson, Arinn .. 184 diversion near Watson, Minn............... 184 near Bruce, Wis...... 226 near Milan, Minn . . . . . . . . . . . . 182 Chippewa River Basin..................... 224 Clear Creek near Coralville, Iowa ............ $\quad 354$ Cobb River tributary near Mapleton, Minn.. 197 Coldwater Creek near St. Louis, Mo.......... . 441 Coldwater Creek basin...................... 441 Coralville Reservoir near Coralville, Iorra... $\quad 352$ Cottonwood River near New Ulm, Minn .... 192 Crane Creek near Lourdes, Iowa. ........... 316 tributary near Saratoga, Iowa............ 316

Crow River at Rockford, Minn ........... 163 North Fork, near Regal, Minn ............ 158 South Fork, at Cosmos, Minn ........ 160 at Hutchinson, Minn. .................. 160 near Mayer, Minn ... tributary near Mayer, Minn. ........... 163 Crow River Basin........................ 158 Crow Wing River at Nimrod, Minn . . ....... 149 Crow Wing River basin.................. 149 Cuivre River near Troy, Mo ............... 437 Cuivre River basin . .

\section{D}

Deerskin Lake near Eagle River, Wis....... 286 Des Moines River at Fort Dodge, Iowa ...... 402 at Keosauqua, Iowa................ 425 at Ottumwa, Iowa..................... 424 below Raccoon River, at Des Moines, Iowa 414

East Fork, at Algona, Iowa ........... 400 at Dakota City, Iowa near Burt, Iowa near Boone, Iowa......... 404 near Saylorville, Iowa. .................. 405 near Tracy, Iowa 
Des Moines River at Fort Dodge, Iowa-Con. Page

West Fork, at Emmetsburg, Iowa . . . . . . . A397 at Estherville, Iowa at Humboldt, Iowa $\ldots \ldots \ldots \ldots . . . . . . . . . . . .398$ at Jackson, Minn ...................... 395 below Talcott Dam near Dundee, Minn. 394 near Ottosen, Iowa . . . . . . . . . . . . . .... 397 tributary near Jackson, Minn ............ 394 tributary near Lakefield, Minn......... 394

Des Moines River basin. . . . . . . . . . . . . . . . 394 Dry Creek near Jeffers, Minn .................

E

Eagle Creek near Fountain City, Wis.

Eau Claire River at Kelly, Wis near Fall Creek, Wis

Eau Galle River at Spring Valley, Wis

Edwards River near New Boston, Ill........

Edwards River basin.

Elk River near Big Lake, Minn

Elk River basin

English River at Kalona, Iowa.

Fabius River basin

Flambeau flowage near Mercer, Wis........... Flambeau River at Babbs Island near Winter, Wis.

near Bruce, Wis .........................

South Fork, near Phillips, Wis.........

Four Mile Creek near Traer, Iowa.

Fox River at Bloomfield, Iowa.

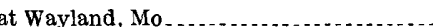

Fox River basin

G

Galena River at Buncombe, Wis

Galena River basin.

Gilmore Creek at Winona, Minn

ore Creek basin ..........................

Glaisby Brook near Kettle River, Minn......

Grant River at Burton, Wis. . . . . . . . . . . . . .

Grant River basin. ..........................

Green River near Geneseo, Ill.

Gull Lake near Brainerd, Minn. .............

Gull River at Gull Lake Dam, near Brainerd, Minn

\section{$\mathrm{H}$}

Half Mile Creek near Gladbrook, Iowa . . .... Hardin Creek, East Fork, near Churdan, Iowa .

Hay River at Wheeler, Wis................ Henderson Creek near Oquawka, Ill. . ....... Henderson Creek basin.

Hillman Creek near Pierz, Minn

\section{I}

Illinois River at Meredosia, Ill

Illinois River basin

Indian Creek near Mingo, Iowa
Iowa River at Iowa City, Iowa ... ......... A355

at Marengo, Iowa

at Marshalltown, Iowa . . . . . . . . . . 345

at Wapello, Iowa. ....................... 378

East Branch, above Hayfiel 1, Iowa...... . . 342

near Garner, Iowa

near Hayfield, Iowa...................... 342

near Klemme, Iowa ..................... 343

tributary near Garner, Iowa .............. 343

near Lone Tree, Iowa

near Rowan, Iowa................... 344

Iowa River basin $\ldots . . . . . . . . . .342$

J

Johnson Creek near St. Augusta Minn...... 155

tributary near St. Augusta, Minn _..... 154

Johnson Creek basin. ...................... 154

Judicial ditch 1-A near New Sw 3den, Minn . 200

Jump River at Sheldon, Wis............... 234

$\mathbf{K}$

Kickapoo River at La Farge, Wis........... 312 at Steuben, Wis........................ 313

Kinnickinnic River tributary at River Falls, Wis

\section{L}

Lac qui Parle River near Lac qui Parle, Minn.- $\quad 180$ Lac Vieux Desert near Land O'Lakes, Wis... 282 La Crosse River near West Salem, Wis........ 263 La Crosse River basin $\ldots . . . . . . . . . . . . . . . .263$ Lake Chippewa near Winter, Wis........... 224 Lake Dubay near Stevens Point. Wis........ 302

Lake Nokomis at Bradley, Wis............... 294

Lake Wissota near Chippewa Fa'ls, Wis. . . . . $\quad 235$

Lemonweir River at New Lisbon, Wis....... . $\quad 307$

Le Suerrr River near Rapidan, N'inn........ 198

Lightning Creek at Almena, Wis ............. 242

Little Cedar River near Ionia, Icwa _. . . . . . . $\quad 363$

Little Frog Creek near Minong, Wis . . ........ 209

Little Maquoketa River near Durango, Iowa.. 321

Little Maquoketa River basin................. 321

Little Minnesota River near Peerer, S. Dak _. 171

Little Paint Creek tributary neer Waterville, Iowa

Little St. Germain Lake near Eagle River, Wis............................. 288

Little Trimbelle Creek near Bay City, Wis... 221

Little Wapsipinicon River at Elma, Iowa..... $\quad 335$ near Acme, Iowa. ................. 335 tributary near Riceville, Iowa ........... 334

Lizard Creek near Clare, Iowa ............... 401

Long Lake near Brill, Wis . . . . . . . . . . . . 240 near Phelps, Wis...................... 286

Loutre River at Mineola, Mo ............... 441

Loutre River basin ........................ 441

Lower Ninemile Lake near Eagle River, Wis.. $\quad 284$

M

437 Macoupin Creek near Kane, Ill...............

Maple River tributary near Mapleton, Minn . . 197 
Maquoketa River near Manchester, Iowa_ ... $\begin{array}{r}\text { Page } \\ \text { A326 }\end{array}$ near Maquoketa, Iowa

Maquoketa River basin

Middle Fabius River near Monticello, $\mathrm{M}_{0}$

Middle Fork Crow River near Spicer, Minn. -

Middle Raccoon River at Panora, Iowa.......

Middle River near Indianola, Iowa..........

Mill Creek at Milan, Ill. tributary near Chatfield, Minn...........

Minnehaha Creek at 50th Street, Edina, Minn. at Minnehaha Ave., Minneapolis, Minn. . at Minnetonka Mills, Minn. ..........

Minnehaha Creek basin.

Minnesota River at Interstate Highway $35 \mathrm{~W}$ at Bloomington, Minn........... at Mankato, Minn.

at Montevideo, Minn. . . at Ortonville, Minn.

near Carver, Minn.

near Lac qui Parle, Minn.

Minnesota River basin. . . . . . . . . . . . . . . .

Minocqua Lake near Minocqua, Wis . . . . . ....

Mississippi River at Aitkin, Minn.

at Alton, Ill.

at Burlington Iowa

at Clinton, Iowa.

at Davenport, Iowa

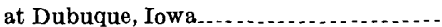

at Elk River, Minn.

at Keokuk, Iowa.

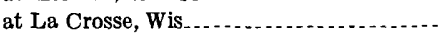

at Lansing, Iowa.

at lock and dam 3 near Red Wing, Minn

at lock and dam 4 near Alma, Wis. ...... at lock and dam 5 near Minneiska, Minn. at McGregor, Iowa

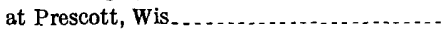

at St. Louis, Mo

at St. Paul, Minn.

below Sandy River near Libby, Minn

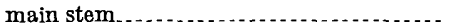
$152,157,168,219,221,248,253,256,264$, $277,280,323,332,337,381,391,439,442$ near Anoka, Minn.

near Royalton, Minn

Missouri River at Hermann, Mo........... main stem

Moose Lake near Winter, Wis . . . . . . . . . .

Mormon Creek near La Crosse, Wis...........

Mormon Creek basin

Morris Creek tributary near Norwalk, Wis... -

\section{$\mathbf{N}$}

Namekagon River near Trego, Wis . . . . . . . . . Nine Mile Creek at Bloomington, Minn....... North Fabius River at Monticello, Mo........ North Pelican Lakes near Rhinelander, Wis.. North Raccoon River near Jefferson, Iowa. .. near Sac City, Iowa.

North River at Palmyra, Mo. near Norwalk, Iowa.

North River basin.

North Skunk River near Sigourney Iowa
168
0

Page

Otsego Creek near Otsego, Minn $\ldots \ldots \ldots$
Otsego Creek basin $\ldots \ldots \ldots \ldots$

Otter Creek near Lester Prairie, Minn........ $\quad 160$

tributary near Lester Prairie, Minn....... 161$$
P
$$

Paint Creek at Waterville, Iowa . . . . . . . . 278 near Waterville, Iowa (crest-stage stc tion). $\quad 279$

Paint Creek basin . . . . . . . . . . . . . . . . . . 278

Pelican Lake near Pelican Lake, Wis... ....... 290

Petenwell fiowage near Necedah, Wis ......... 304

Pickerel Lake near Lake Tomahawk, Wis..._ 289

Pine Creek near Cannon Falls, Minn........ 222 East Branch, tributary near Dallas, Wis .- 241

Pine River at Cross Lake Dam at Cross Lake,

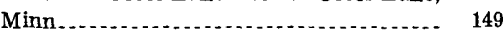

Pine River basin.......................... 148

Pine River Reservoir at Cross Lake, Minn _.. 148

Platte River near Rockville, Wis ........ . . . . $\quad 320$

Platte River basin $\ldots \ldots \ldots \ldots \ldots$............... 152, 320

Plum River below Carroll Creek, near Savanna, Ill........................... 331

Plum River basin..... 331

Pomme de Terre River at Appleton, Minn... $\quad 179$

Pope Creek near Keithsburg, Ill ............. 380

Pope Creek basin...................... $\quad 380$

Poplar River near Owen, Wis. . ............. 260

Prairie River near Merrill, Wis............... 295

\section{R}

Raccoon River at Van Meter, Iowa.......... 413 Rainbow Lake near Lake Tomahawk, Wis... 290

Ralston Creek at Iowa City, Iowa........... 356 South Branch; at Iowa City, Iowa........ $\quad 357$

Rapid Creek near Iowa City, Iowa. .......... 353

Raven Stream tributary near New Prague, Minn - 203

Red Cedar Lake at Mikana, Wis ............ $\quad 239$

Red Cedar River at Menomonie, Wis........ 243 near Colfax, Wis. ..................... 242

Redwood River at Marshall, Minn .......... 188 near Redwood Falls, Minn ............... 190

Rest Lake near Manitowish, Wis .............. 228 Rice Lake tributary near Montgomery, Minn. 203 Richland Creek near Haven, Iowa . .......... 347 Robinson Brook near Onamia, Minn ........ 165

Rock River at Moline, Ill . . . . . . . . . . . . . . $\quad 340$

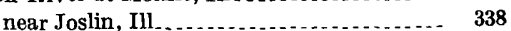

Rock River basin .......................... 338

Root River below South Fork near Houston, Minn...................... 275 near Houston, Minn near Lanesboro, Minn. ................... 267 near Rushford, Minn $\ldots . . . . . . . .269$ South Branch, at Lanesboro, Minn...... 266 at Preston, Minn ................. 266 South Fork, near Houston, Minn ......... 273

Root River basin. ...................... 265

Rum River at Isanti, Minn. . . . . . . . . . . . . . 166 at Spencer Brook, Minn 165 at West Point, Minn $\ldots 166$ near St. Francis, Minn . ................. 167 tributary near Onamia, Minn . . ........ 165 
Rum River basin $\ldots \ldots$ Page

Rush Creek near Rushford, Minn............ 269

$\mathrm{S}$

St. Croix River at Prescott, Wis

at St. Croix Falls, Wis

near Grantsburg, Wis . ......................

near Rush City, Minn....................

St. Croix River basin.

St. Francis River at Santiago, Minn.......... near Big Lake, Minn.

Salt Creek near Elberon, Iowa. ............

Salt River near New London, Mo ............. Salt River basin

Sand Creek at Jordan, Minn

near New Prague, Minn

tributary near Montgomery, Minn

Sauk River near St. Cloud, Minn............

Sauk River basin.

Sawyer Creek near Shell Lake, Wis........... School Lake Creek tributary near St. Michael, Minn

Scotch Creek tributary near Edgar, Wis..... Sevenmile Lake near Eagle River, Wis...... . . Shell Rock River at Shell Rock, Iowa.

near Northwood, Iowa.

Skunk River at Augusta, Iowa.....................

Skunk River basin..........................

Snake River near Pine City, Minn.

South Fabius River near Taylor, Mo.........

South Raccoon River at Redfield, Iowa......

South River near Ackworth, Iowa ............

South Skunk River below Squaw Creek near Ames, Iowa

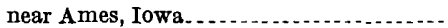

near Oskaloosa, Iowa.

Spirit River flowage near Tomahawk, Wis

Spring Creek near Durand, Wis...............

near Sleepy Eye, Minn

Squirrel Lake near Mmocqua, Wis ............

Stanchfield Creek tributary near Day, Minn. .

Stony Brook tributary near Foley, Minn.....

Story Brook near Petersburg, Minn...........

Sugar Camp Reservoir near Eagle River, Wis.

Sugar Creek near Keokuk, Iowa.

Sunrise River near Stacy, Minn

$\mathrm{T}$

The Sny basin

Timber Cres near Marshallon

Trempealeau River at Arcadia, Wis

at Dodge, Wis.

Trempealeau River basin

Trimbelle Creek basin.

Turkey River at Garber, Iowa.

at Spillville, Iowa

Turkey River basin.

Twin Lakes near Phelps, Wis
U

Page

Upper Iowa River at Decorah, Iowa......... A276

Upper Iowa River basin

\section{V}

Vermillion River basin. .................... 221

Vermillion River tributary rear Hastings, Minn

W

Walnut Creek near Hartwick, I wa......... 349

Wapsipinicon River at Indepen lence, Iowa-.- $\quad 335$ near De Witt, Iowa.......... 336

near Elma, Iowa....................... 333

Wapsipinicon River basin .................. 333

Watonwan River near Garden City, Minn... 195

North Fork, near Delft, Minn.......... 195

Waumandee Creek basin. . . . . . . . . . . . . 255

Wexford Creek basin ...................... 277

Wexford Creek near Harpers Ferry, Iowa..... 277

Whetstone River near Big Stone City, S. Dak - 173

White Breast Creek near Dallas Iowa........ 419

Whitewater River, South Fork. near Altura, Minn.

Whitewater River basin .................... 253

Willow Creek near Eau Claire, Wis.......... 238

Willow Reservoir near Hazelhurst, Wis . . . . . . 293

Winnebago River at Mason City, Iowa . ..... 367

Wisconsin River at Bridgeport, Wis........... $\quad 314$

at Merrill, Wis ...................... 296

at Muscoda, Wis........ 311

at Rothschild, Wis........................ 299

near Wisconsin Dells, Wis................. 309

at Wisconsin Rapids, Wis.............. 303

Wisconsin River basin . . . . . . . . . . . . . . . . $\quad 282$

Wolf Creek tributary near Sandstone, Minn . - 213

Wyaconda River above Canton, Mo......... 430

Wyaconda River basin. . . . . . . . . . . .

\section{$\mathbf{Y}$}

Yellow Bank River near Odesse, Minn...... 177

Yellow Medicine River near Granite Falls, Minn. ........................... 187

South Branch, at Minneota, Minn........ 186

Yellow River at Babcock, Wis.............. 305

at Cadott, Wis..................... 235

\section{Z}

Zumbro River at Zumbro Falls, Minn........ 251

North Fork, tributary near Wanamingo,

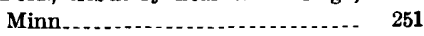

South Fork, near Rochester, Minn........ 249

Zumbro River basin........................ 249 